Marine Biological Laboratory Library Woods Hole, Massachusetts

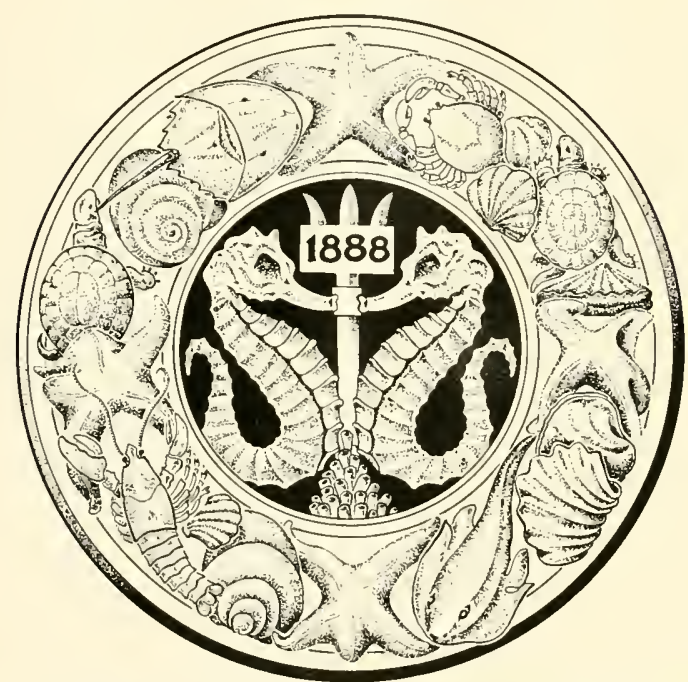

Gift of Douglas Marsland - 1978 
Pinckares

Ouggar

Packard

$\downarrow$

Marsland 



BIOLOGICAL EFFEC'TS OF RADIA'TION

VOLI ME I 


\section{COLLABORATING EDITORS}

Janet Howell Clark, Associate Professor of Physiology, School of Hygiene and Public Health, The Johns Hopkins University, Baltimore

Kenneth S. Cole, Assistant Professor of Physiology, College of Physicians and Surgeons, Columbia University, New York City

Farrington Daniels, Professor of Chemistry, University of Wisconsin, Madison

Gioacchino Failla, Physicist, Memorial Hospital, New York City

Charles Packard, Assistant Professor of Koology, Institute of Cancer Research, Columbia University, New York City

Henry W. Popp, Associate Professor of Botany, Pennsylvania State College, State College 


\section{BIOLOGICAL \\ EFFECTS OF RADIATION}

Mechanism and Measurement of Radiation, Applications in Biology, Photochemical Reactions, Effects of Radiant Energy on Organisms and Organic Products

\section{VOLUME I}

EDITED BY

\section{BENJAMIN M. DUGGAR}

Professor of Plant Physiology and Applied Botany,

Cniversity of Wisconsin

WITH THE COOPERATION OF

JANET HOWELL CLARK KENNETH S. COLE

FARRINGTON DANIELS
GIOACCHINO FAILLA CHARLES PACKARD

HENRY W. POPP

First Edition

Prepared under the Auspices of the Committee on Radiation

Division of Biology and Agriculture, National Research Council, Washington

MCGRAW-HILL BOOK COMPANY, INC.

NEW YORK AND LONDON 
Copyright, 1936, by the

McGraw-Hill Book Company, Inc.

PRINTED IN THE UNITED STATES OF AMERICA

All rights reserved. This book, or parts thereof, may not be reproduced in any form without permission of the publishers. 


\section{PREFACE}

The "Biological Effects of Radiation" constitutes a collective contribution that has been developed as a by-product from one of the activities of a committee now known as the Committee on Radiation, in the Division of Biology and Agriculture, National Research Council. In April, 1928, a group of investigators presented to the Division a suggestive program for the encouragement and promotion of investigations on the "Effects of Radiation on Organisms" and a request for assistance in securing the necessary funds with which to maintain grants stimulating research in this field. Approving action was taken, and a committee was promptly named. The activities of this committee, under the chairmanship of Dr. W. C. Curtis, 1928-1934, have been fully developed in his "Cumulative Report," as well as in annual reports, to the Division.

For the purpose of clarifying the scope of interest and for the integration of pertinent information relating to the irradiation of organisms and other biological materials, there was organized a Subcommittee on Survey. This subcommittee, and others invited to participate in a discussion of radiation problems, met in Washington in March, 1930. Initially this suhcommittee was constituted of four groups as follows: Representing genetics: A. F. Blakeslee, L. J. Stadler; general physiology: Janet Howell Clark, Max Ellis, S. O. Mast; animal development: E. E. Just; plant physiology: B. M. Duggar (Chairman), C. Stuart Gager, W. W. Garner, H. W. Popp. Each group proceeded to arrange for a conference-discussion of interested individuals, each group bringing together a score or more of persons (investigators and representatives of certain apparatus manufacturers) for participation in its colloquium. The expense of these meetings was met through the generosity of the General Electric X-ray Corporation, of the General Electric, CooperHewitt, Westinghouse, and Burdick companies, and of the National Research Council. Aside from the stimulative and informative value of the conferences held, the chief result, eventually, was the determination to bring together in the form of a Survey a collation of the available material-theoretical, factual, and methodological. Such material should adequately represent the present status of scientific knowledge in as many as practicable of the aspects of radiation in relation to biological problems.

Accordingly the subcommittee was reorganized to include those persons who were selected and who were willing to assist the editor by assuming certain responsibilities in relation to the contributions to be requested in the five major aspects of the Survey as planned; that is, 
physics, photochemistry, certain biological products, zoology, and botany. In short, the representatives of these fields served both as an editorial board and as a publication committee (see list of editorial collaborators, page ii). It is obvious that those aspects of physical and biological sciences embracing the relations of biological materials, physiological processes, and the responses of organisms to radiation are far too vast for detailed review in any single work. It is, however, indispensable to the effective work of the biologist that a careful selection of this material should be so organized and presented that an adequately comprehensive view of the whole may be obtainable with a minimum of time and labor. A complete bibliography alone would be an extensive undertaking, and this could have none of the qualities of factual presentation, critical analysis, and systematization of method that are required for better orientation of the research or teaching biologist, the interested physicist or photochemist, and the qualified general reader.

This Survey purposely excludes to a large extent practical considerations relating to plant production. Likewise it excludes those phases of applied radiology which are generally regarded as purely clinical or therapeutic; but at the same time it is recognized that clinical effects are necessarily more clearly analyzed and interpreted through the avenue of sound experimental findings, while the reasonableness of therapeutic practice is logically based upon facts well established in the laboratory. On the other hand, the applications in plant science, in medicine, and in technology have, of course, notably stimulated research and enriched the opportunities for fundamental work. Without extending too far the limits of this work it was not possible to include chapters on applied phases of absorption spectroscopy, emission spectral analyses of tissues, and radiation problems relating to the phenomenon of vision in higher animals and man.

The publication of this book has been made possible by financial support from the Committee on Radiation through contributions received for the radiation program by the National Research Council from the General Education Board, the Rockefeller Foundation, and the Commonwealth Fund. 'The editor wishes particularly to express his thanks to the collaborating editors-Drs. Cole and Failla, Daniels, Clark, Packard, and Popp, representing the five primary fields of interest referred to previously - for their indispensable and cooperative assistance. The two-score of contributors-authors of the papers included-have earned our gratitude not alone for their contributions but also for their sympathetic consideration of details requisite for a certain harmonious treatment of the whole.

MADISON, Wis.,

March, 1936.

B. M. DugGar, Editor 


\section{CON'TEN'TS}

I. Photons and Electrons.

Karl K. Darrow, Research Physicist, Bell Telephone Laboratories, New York City

II. Measurement of X-rays and Radium

Lauriston S. Taylor, Associate Physicist, National Bureau of Standards. Washington, D.C.

III. Ionization and Its Bearing on the Biological Effects of RADIATION

G. Failla. Physicist, Memorial Hospital, New York City

IV. Measurement and Application of Visible and Near-visible RADIATION .

F. S. Brackett, Consulting Physicist, Smithsonian Institution, Washington, D.C.

V. The Intensity of Solar Radiation as Recenved at the Surface of the Earth and Its Variations with Latitude, Altitude, the Season of the Year and the Time of Day . . . . . . . Herbert H. Kimball, Research Associate, Blue Hill Meteorological Observatory of Harvard University, Washington, D.C.

Irving $F$. Hand, Meteorologist, U.S. Weather Bureau, Washington, D.C.

VI. Statistical Treatment of Biological Problems in Irradiation Lowell J. Reed, Professor of Biostatistics, The Johns Hopkins University, Baltimore

VII. Photochemistry . . . . . . . . . . . . . . . Farrington Daniels, Professor of Chemistry, University of Wisconsin, Madison

VIII. The Effect of Radiation on Proteins

Janet Howell Clark, Associate Professor of Physiology, School of Hygiene and Public Health, The Johns Hopkins University, Baltimore

IX. Radiation and the Vitamins

Charles E. Bills, Research Director, Mead Johnson and Company, Evansville, Indiana 
PAPER

X. The Effects of Irradiation on Venoms, Toxins, Antibodies, and Related Substances

S. C. Brooks, Professor of Zoology, University of California, Berkeley

XI. The Effects of Radium and X-rays on Embryonic DevelopMENT . . . . . . . . . . . . . . . . Elmer G. Butler, Associate Professor of Biology, Princeton University, Princeton

XII. Effects of X-rays and Radium upon Regeneration

W. C. Curtis, Professor of Zoology, University of Missouri, Columbia

XiII. The Biological Effectiveness of X-Ray Wave-lengths. Charles Packard, Assistant Professor of Zoology, Institute of Cancer Research, Columbia University, New York City

XIV. The Physiological Effects of Radiation upon Organ and Body Srstems . M.D. Associate Professor of Medicine in charge of Division of Radiology, University of Rochester, School of Medicine and Dentistry, Strong Memorial Hospital, Rochester

XV. Short Electric Wave Radiation in Biology.

G. Murray McKinley, Instructor in Zoology, University of Pittsburgh, Pittsburgh

XVI. Biological Effects of Alpha Particles . . . . . . . .

R. E. Zirkle, Fellow in Medical Physies, Johnson Foundation, University of Pennsylvania, Philadelphia

XVII. Motor Responses to Light in the Invertebrate Animals

S. O. Mast, Professor of Zoology in charge of General Physiology, The Johns Hopkins University, Baltimore

XVIII. The Action of Radiations on Living Protoplasm.

L. V. Heilbrunn, Associate Professor of Zoology, University of Pennsylvania, Philadelphia

Daniel Mazia, Department of Zoology, University of Pennsylvania, Philadelphia

XIX. PHOTOPERIODISM

W. W. Garner, Principal Physiologist in charge of Tobacco and Plant Nutrition, Bureau of Plaut Industry, U.S. Department of Agriculture, Washiugton, D.C.

XX. Plant Growth in Continuous Illumination

John M. Arthur, Biochemist, Boyce Thompson Institute for Plant Research, Ine, Yonkers, N.Y.

XXI. The Effects of Light Intensity upon Seed Plants.

Hardy L. Shirley, Associate Silviculturist, Lake States Forest Experiment Station, University Farm, St. Paul, Minnesota 
XXII. Effects of Different Regions of the Visible Spectrum dpon SeEd Plants. . . . . . . . . . . . . . .

H. W. Popp, Associate Professor of Botany, Pennsylvania State

College, State College

F. Brown, Instructor in Botany, Pennsylvania State College, State College

XXIII. Effect of the Visible Spectrum upon the Germination of Seeds and Fruits

William Crocker, Managing Director, Boyce Thompson Institute for Plant Research, Inc., Yonkers, N.Y.

XXiV. The Effects of Visible and Ultra-violet Radiation on the Histology of Plant Tissues.

J. T. Buchholz, Professor of Botany, University of Illinois, Urbana

XXV. Some Infra-red Effects on Green Plants.

John M. Arthur (see Paper XX)

XXVi. The Effect of Ultra-violet Radiation upon Seed Plants . H. W. Popp and F. Brown (see Paper XXII)

XXVII. The Effects of Radiation on Fungl

Elizabeth C. Smith, Research Assistant, University of Wisconsin, Madison

XXViII. The Problem of Mitogenetic Rays .

Alexander Hollaender, Research Associate (under auspices of National Research Council), University of Wisconsin, Madison

XXIX. Effects of X-rays upon Green Plants

Edna L. Johnson, Associate Professor of Biology, University of Colorado, Boulder

XXX. The Effects of Radium Rays on Plants

C. Stuart Gager, Director, Brooklyn Botanic Garden, Brooklyn

XXXI. The Light Factor in Photosynthesis

H. A. Spoehr, Chairman, Division of Plant Biology, Carnegie Institution of Washington, Stanford University, California

J. H. C. Smith, Staff Member, Division of Plant Biology, Carnegie Institution of Washington, Stanford University, California

XXXII. The Influence of Radiation on Plant Resplikation and FerMENTATION.

Charles J. Lyon, Professor of Botany, Dartmouth College, Hanover, N.H.

XXXiII. Growth Movements in Relation to Radiation

Earl S. Johnston, Assistant Director, Division of Radiation and Organisms, Smithsonian Institution, Washington, D.C. 
XXXIV. Chlorophyll and Chlorophyll Development in Relation to RAdition . . . . . . . . . . . . . . 1093

O. L. Inman, Director, Kettering Foundation for the Study of Chlorophyll and Photosynthesis, Antioch College, Yellow Springs, Ohio

Paul Rothemund, Research Chemist, Kettering Foundation for the Study of Chlorophyll and Photosynthesis, Antioch College, Yellow Springs, Ohio

C. F. Kettering, Director of Research, General Motors Corporation

XXXV. Radiation and Anthocyanin Pigments. . . . . . . . . . . 1109

John M. Arthur (see Paper XX)

XXYVI. Effects of Radiation on Bacteria . . . . . . . . . . . . . . 1119 B. M. Duggar, Professor of Plant Physiology and Applied Botany, University of Wisconsin, Madison

XXXVII. The Effects of Radiation on Enzymes.

Harold A. Schomer, Fellow in Botany, University of Wisconsin, Madison

XXXVIII. Induced Chromosomal Aberratrons in Animals.

Theodosius Dobzhansky, Assistant Professor of Genetics, California Institute of Technology, Pasadena

XXXiX. Radiation and the Study of Mutation in Animals . . . . 1209 Jack Schultz, Research Assistant, Carnegie Institution of Washington, California Institute of Technology, Pasadena

Xl. Induced Mutations in Plants. . . . . . . . . . . . 1263

L. J. Stadler, Senior Geneticist, Bureau of Plant Industry, U.S. Department of Agriculture, and Professor of Field Crops, University of Missouri and Agricultural Experiment Station, Columbia

XLI. Induced Chromosomal Alterations

T. H. Goodspeed, Professor of Botany and Director Botanical Garden, University of California, Berkeley

XLII. Induced Chromosomal Alterations in Majze.

E. G. Anderson, Department of Biology, California Institute of Technology, Pasadena

XliII. Brological Aspects of The Quantum Theory of Radiation Absorption in Tissues

John W. Gowen, Associate Member, Department of Animal and Plant Pathology, The Rockefeller Institute for Medical Research, Princeton 


\title{
PHOTONS AND ELECTRONS
}

\author{
Karl K. Darrow
}

\section{Research Physicist, Bell Telephone Laboratories, New York}

Waves and Corpuscles. Monochromatic light and measurement of wave-length. External photoelectric effect and measurement of photon energy. Units of wave-length, wave number, frequency, and photon energy. Regions of the spectrum. Absorption of light by atoms. Continua in absorption spectra, and ionization by light. Theory of absorption lines. Terms. Absorption in X-ray region. Emission of light. X-ray emission spectra. Production of X-rays. Production of light of the optical spectrum. Scattering of light without change of frequency. Scattering of light with change of frequency. Scattering of X-rays. Transmutation of electron-pairs and photons.

The title of this essay may appear unduly obscure to some of my readers and unduly restrictive to others. Though it is now more than a quarter of a century since the corpuscular picture of light made its definitive entry into physics (in a somewhat apologetic but quite irresistible paper by Einstein), the undulatory picture is still the more widespread, and the name "photon" for the corpuscle of light seems not thus far to be universally familiar. With electricity, the situation is reversed; the name and the conception of the elementary particle of negative electricity have existed since before this century began, whereas the corresponding wave conception has yet to complete its first decade as a part of the structure of science. Physicists themselves have not greatly modified their usage; even the most modern of them will speak of the wave-length of a beam of monochromatic light far oftener than of the energy or momentum of its photons, and of the velocity of an electron stream far oftener than of its wave-length. Thus in speaking of "photons and electrons" I am employing a relatively familiar conception of negative electricity, a relatively unfamiliar conception of light; and I am implying that the properties of light which are suitable for mention in this essay-because they are connected with the general topic of this treatise-involve the interactions of light with negative electricity and that the corpuscular picture of both entities is the more fitted for interpreting them.

No reader of this article is unaware that light is observed only by virtue of its interactions with matter. It is probably not so widely known that all of these interactions, with but a few exceptions (very 
little known as yet), are between light and that subtlest variety of matter to which I have already referred under the name of "negative electricity" or "electrons." To the best of our knowledge and belief, all of what we normally call matter consists of atoms, each of which consists of a certain number of "orbital" electrons (varying from one element to another) and a relatively massive "nucleus" bearing a positive charge equal in magnitude and opposite in sign to the sum of the negative charges of the electrons. With the nuclei, light has no transactions, save in the rare exceptional cases to which I have just alluded. Absorption of lightrefraction, dispersion, seattering of light-excitation of one kind of light by another kind-the electrical, the thermal, the beneficent, and the injurious effects of light, on inorganic and on organic matter-are almost exclusively due to transactions between light and orbital electrons. It would, of course, be grossly extravagant to pretend that all of these phenomena are fully understood; but so much of each of them is understood, that the foregoing statement may be made as confidently as any statement in physics which is not merely a description of an actual observation.

It would doubtless be suitable at this point to define the word "light," but to do this properly would be a formidable task. I might say "light is electromagnetic radiation," but this is rather a description than a definition-a description of a theory of light which is verified by the phenomena of which I have to speak, in addition to a number of others. To say that "light is that which is perceived by the eye" would be to commit a very bad anachronism, since this definition has been out of date for physicists for 134 years, and even for the general public since the term "ultra-violet light" became of common usage. I shall evade the issue by listing the various special terms for various kinds of light which the general name includes: visible light, ultra-violet light, infrared light, thermal radiation (now a rare term, superfluous in view of the present use of "infra-red"), X-rays, gamma rays. To these should be added, for completeness, Hertzian or "radio" waves and (probably) some cosmic rays; but these last fall outside of the scope of this treatise. These diverse forms of light are best delimited from one another (though the delimitation has little importance) by citing the ranges of wave-length which they have; but inasmuch as the corpuscular picture of light is to be by far the more prominent in this essay, it is desirable to introduce the brief allusion to wave-lengths by saying something of the relations between waves and corpuscles.

Wave theory and corpuscular theory, as they are nowadays imagined in dealing with light (and, incidentally, with electricity and matter), are not antagonistic. It must be confessed that the union of the two conceptions is still far from perfected, but at least much progress has 
been made toward achieving it. Probably the best way to employ the two pictures in tandem is to imagine that the energy and momentum of a beam of light-its substance, insofar as light has any substanceare concentrated in diminutive particles, which are guided in their motion by impalpable waves. Whenever we wish to eompute how a beam of light is going-how it is to be refracted in passing across, e.g., a boundary between air and glass, how it is to be partially reflected and partially transmitted in encountering a thin film of water, how it is to be polarized in traversing a transparent crystal, how it is to be focused by a lens, how it is to be diffracted by a slit or by a grating-whenever we wish to calculate how a beam of light is going to be affected by any of these adventures, we should conceive it as a train of electromagnetic waves, and apply the electromagnetie wave theory as it was developed in the last half of the nineteenth century by Maxwell and his followers. This theory will predict that, if the beam of light falls on a plate of glass, the wave motion will be partially refracted and partially reflected; the angle of refraction will conform to Snell's famous law of sines, affeeted only by a single constant characteristic of the glass; the ratio of the amplitudes of the transmitted and the reflected wave trains will be determined by that constant and by the thickness and the backing of the glass plate and by the angle of incidence. Similarly the theory will prediet that if the beam of light falls on a diffraction grating, the amplitude of the waves proceeding from the grating will vary from point to point in space in a remarkable and characteristic way. Having utilized the wave theory to make these predictions of the amplitude of the waves, we now reintroduce the corpuseular pieture by assuming that the concentration or number per unit volume of the corpuseles at any point in space is proportional to the square of the amplitude of the waves which we have just computed for that point.

The perfect adaptation of wave picture and corpuscle picture to one another is by no means easy to attain, and in fact it has never been attained. It forms a large part of the difficult and incomplete field of theoretical physies known as "quantum mechanies." There is, however, no great difficulty about showing that the adaptation cannot be made without assuming certain relations between wave-length $\lambda$ of the guiding waves on the one hand, energy $E$ and momentum $p$ of the guided corpuscles on the other hand. These are two of the fundamental relations of Nature, valid for matter and electricity as well as light. They are:

$$
\begin{aligned}
p & =\frac{h}{\lambda} \\
E & =\frac{h c}{\lambda}=h \nu
\end{aligned}
$$


Herein $h$ stands for a constant which must have the same value in both equations; $c$ for the speed of the waves; and $\nu$ for the quotient of wave speed by wave-length, which is by definition the frequency of the waves. Nothing in the theory prescribes the values of $h$ and $c$. The value of the former is found, by experiments of many kinds (some of which I shall later describe), to be this:

$$
h=6.55 \cdot 10^{-27} \mathrm{erg} / \mathrm{sec} .
$$

with an uncertainty of less than two units in the third significant figure. It is known as Planck's constant. The value of $c$ is found, by experiments on the velocity of light signals ${ }^{1}$ in air or vacuum, to be so close to $3 \cdot 10^{10}$

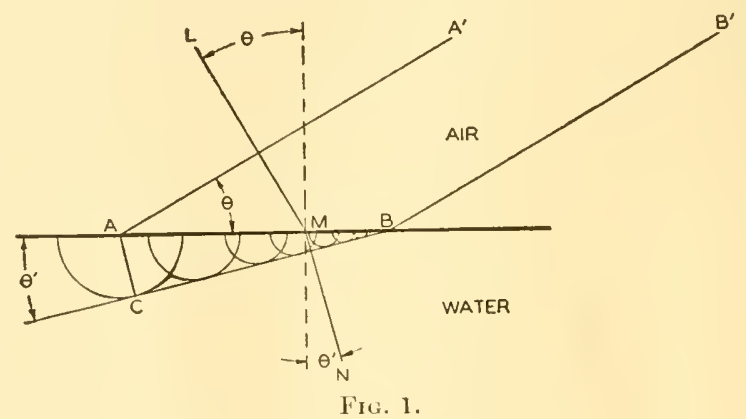

that this simple figure is quite accurate enough for most purposes. Actually the value in vacuum (to which alone the symbol $c$ should in strictness be applied) is given as $2.99796 \pm 0.00004 \mathrm{~cm}$. $/ \mathrm{sec}$. (times $10^{10}$ ).

The derivation of equation (1) is so interesting in itself, and in its relation to a famous controversy of a century ago, that it is worthy of retracing here. We shall interpret one of the simplest of optical phenomena-the refraction of a ray of light at a surface of discontinuity between two contrasting media, air and water, for example-by the wave picture and the corpuscle picture in succession.

The reasoning from the wave theory is usually made in graphic fashion by showing "Huygens' construction" (Fig. 1) which should remind many a reader of his high-school days! This is a very crude form of wave

${ }^{1}$ There is a peculiar and difficult point in this connection. What is measured in such experiments is not the wave speed but a thing entirely different in principle, the so-called "group speed." The two are very different in transparent solid or liquid media such as carbon bisulphide, but in a medium in which wave speed does not vary with wave-length they should be the same, and such appears to be the case in the medium which we know as empty space. I have developed this point at length in pp. 28-30 of an article "Elementary Notions of Quantum Mechanies" in Reviews of Moderu Physics, 6, January, 1934. 
theory, but for the present purpose it will do. Here $A A^{\prime}$ is the trace, on the plane of the paper, of a wave front moving through air (say) in the direction $L M$ toward the boundary between air and water. It is the trace of the wave front at a particular moment, say $t$; at a later moment, say $t^{\prime}$, the front has moved on to another position, $B B^{\prime}$. Denote by $v$ the speed of the wave front in air; then the perpendicular distance between $B B^{\prime}$ and $A A^{\prime}$ is equal to $v\left(t^{\prime}-t\right)$. While the wave is advancing through this distance, its intersection with the boundary of the water sweeps over the distance $A B$, which we shall denote by $D$. Designate by $\theta$ the angle between wave front and boundary, the "angle of incidence." From the diagram one sees immediately:

$$
\sin \theta=\frac{v\left(t^{\prime}-t\right)}{D}
$$

Now in Huygens' view, whenever the oncoming wave front passed over an atom in the boundary surface, it incited that atom to enit a "wavelet." The cireles drawn around various points on the line $A B$ are the traces on the plane of the paper, of halves of those spherical wavelets - the halves expanding downward into the water. According to Huygens' prineiple the ongoing wave front in the water is the envelope of these spheres. In Fig. 1 they and the ongoing wave front are represented for the moment $t^{\prime}$ when the wave in the air reaches $B$. The radius $A C$ of the wavelet expanding from $A$ is then the distance which light traverses in water during time $\left(t^{\prime}-t\right)$, for that wavelet started when the wave in the air reached $A$. Denote by $v^{\prime}$ the speed of light in water and by $\theta^{\prime}$ the angle between the new wave front and the boundary; then from the diagram:

$$
\sin \theta^{\prime}=\frac{v^{\prime}\left(t^{\prime}-t\right)}{D}
$$

rombining which with the previous equation, we get:

$$
\frac{\sin \theta}{\sin \theta^{\prime}}=\frac{v}{v^{\prime}}
$$

which conforms with the empirical law of refraction (Snell's law) in that the ratio of the sines of $\theta$ and $\theta^{\prime}$, the angle of incidence, and angle of refraction are found not to depend on either angle.

We now apply the eorpuscle picture. The line $L M N$ of Fig. 1 is to be redrawn as a heavy line, and those at right angles to it are to be left out; for $L M N$, one of the "rays" of light, is now to be interpreted as the path of a corpuscle, and there are no wave fronts. 
So long as the corpusele is too far from the boundary surface to feel any force from the water, it moves in a straight line with unchanging momentum; for the forces exerted on it by the air, being equally applied in all directions, balance one another out. In the region near the boundary this remains the truth for the components of force parallel

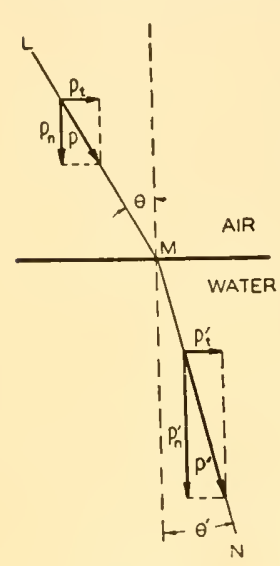

FIg. 2. to the surface; but the components along the normal, applied respectively from the direction toward the air and the direction toward the water, need not be perfectly equal. After the corpuscle has gone through the transition region and reached the depths of the water, it continues in a straight line with a momentum of which the component parallel to the boundary is still the same as it was in the air, while the normal component is changed. Denote by $p_{t}$ and $p_{n}$ these two components of the original momentum of the particle through the air; by $p$ the magnitude of their resultant which is the magnitude of the original momentum; by $p_{t}^{\prime}, p_{n}^{\prime}$, and $p^{\prime}$ the corresponding quantities for the final flight of the corpuscle through the water. From Fig. 2 we see:

$$
\sin \theta=\frac{p_{\iota}}{\sqrt{p_{t}^{2}+p_{\imath}}}=\frac{p_{\ell}}{p} ; \quad \sin \theta^{\prime}=\frac{p^{\prime}}{p^{\prime}}
$$

and since $p_{t}^{\prime}$ and $p_{t}$ are equal,

$$
\frac{\sin \theta}{\sin \theta^{\prime}}=\frac{p^{\prime}}{p}
$$

Putting this side by side with equation (3), we notice that the two equations may be rendered compatible with one another, that the two pictures may be interchanged at will, provided that we assume that the momentum of the corpuscles varies inversely as the speed of the wave fronts.

Nothing has yet been said about wave-length in the course of this argument, and for a very good reason: in Huygens' construction from which equation (3) was derived, there is no allusion whatever to periodic vibration or undulatory motion or any sort of rhythm. Indeed when Huygens drew the diagram of which Fig. 1 is essentially a remote copy, he meant the line corresponding to $A A^{\prime}$ to represent what we should now call a single "pulse," and while he said that one should suppose many such pulses emerging one after another from a candle flame, he emphasized that one should not (!) suppose them following one another at equal intervals. In using the word "wave front" I have been implying a train of regular waves, of which the line $A A^{\prime}$-or rather, the plane of which it is the trace-is a locus of constant phase. This implication was foreign 
to Huygens' mind, but it is essential to our present conception of monochromatic light, and to the explanation of the many and variegated phenomena of diffraction.

When the undulatory theory is formulated in mathematical language, ${ }^{2}$ one finds that the progress of a wave front-in the sense of the foregoing implication-across a refracting surface may be predicted by Huygens' construction. The wavelets of Fig. 1 acquire new attributes, first imposed on them by Fresnel. Huygens in effect assumed that the amplitude of each wavelet is zero except at the point where it touches the envelope common to all. In the undulatory theory, the amplitude of a wavelet varies continuously from a maximum value at the point just defined to zero at the point diametrically opposite (the wavelet being a full sphere, not a hemisphere as indicated in Fig. 1). This modification is necessary to account for diffraction, but does not affect the case of refraction of an extended wave front. We may continue to accept equation (3), and the equation obtained by comparing (3) and (4):

$$
\frac{p^{\prime}}{p}=\frac{v}{v^{\prime}}
$$

where now $v$ and $v^{\prime}$ stand for the wave speed of the train of monochromatic waves.

The final step is taken by remembering that in the two equations connecting wave speed, frequency, and wave-length, one for each side of the bounding surface,

$$
v=\nu \lambda \quad v^{\prime}=\nu^{\prime} \lambda^{\prime}
$$

the frequencies $\nu$ and $\nu^{\prime}$ must be equal; for it is of the nature of vibration, that if two or more continuous media are in continuous oscillation, the frequency (or frequencies) of that oscillation must be the same throughout the entire system, as otherwise the media could not remain in contact. Accordingly we write in place of $(5)$ :

$$
\frac{p^{\prime}}{p}=\frac{\lambda}{\lambda^{\prime}}
$$

which amounts to writing

$$
p=\frac{\text { const. }}{\lambda}
$$

which is the same as equation (1) except for lacking any intimation of the value of the multiplying constant.

${ }^{2}$ Cf., for instance, an article of mine in the Bell System Technical Journal, 7 : 281-320, 1928. 
The relation (1) between wave-length of waves and momentum of corpuscles is therefore one which we are obliged to accept if we wish to use both the wave picture and the corpuscle picture of light.

The relation (2) may be derived from (1) by means of a classical theorem concerning the ratio between the energy-density of a beam of light and the pressure which the beam exerts upon an object on which it falls. Conceive a stream of radiation in the form of an extremely long train of plane waves, flowing against a blackened plate faced normally against the direction in which they advance, which totally absorbs them. Say that the beam has intensity $I$, which means that an amount of energy $I$ appears, in the form of heat, in unit area of the blackened plate in unit time. The light exerts a pressure, say $P$, against the plate; this means that unit area of the plate acquires in unit time an amount of momentum $P$. The classical undulatory theory predicts that $P$ should be equal to the quotient of $I$ by $c$, and this is confirmed by experience. Now $I$ is equal to the product of $E$, the energy of a photon, by $N$, the number of photons which strike the plate in unit time; while $P$ is equal to the product of $p$, the momentum of a photon, by the same number $N$. Hence $E$ must be equal to $p c$, and this statement is none other than equation (2).

Equations (1) and (2) may also be proved jointly valid by invoking the Compton effect, and equation (2) by itself may be proved valid by invoking the external photoelectric effect of metals, both of which will be treated in later sections of this article. Before going further, however, we should consider more closely how the undulatory theory is tested and how wave-length is determined.

\section{MONOCHROMATIC LIGHT AND MEASUREMENT OF WAVE-LENGTH}

As Newton found in his classical experiments in optics, a narrow beam of white light is converted by a prism into a diverging beam of which the color varies from one side to the other of the beam, but if a sufficiently narrow portion of this divergent beam is isolated (by means of a slit in an opaque screen) and sent through a second prism, it is neither broadened nor changed in color. Light of the latter character is called monochromatic and has the power of forming diffraction patterns indicating a single wave-length. Every "measurement" of a wave-length is an observation on a diffaction pattern. Owing to the importance of this matter I will develop the simplest case.

Imagine two beams of identical monochromatic light moving parallel to the $x y$-plane, one making an angle $\varphi$ and the other an angle $-\varphi$ with the $x$-axis, so that they make an angle $2 \varphi$ with each other. Were they purely corpuscular, they would cross each other without interference. (The reader may object that corpuscles of the two beams would collide 
and bounce out of their paths, but actually no such sidewise scattering of one beam by another is observed, and this can be interpreted by saying that the corpuscles are so excessively small that collisions are too rare to be appreciable.) What is observed is different: In the region conmon to the two beams there is a peeuliar distribution of light intensity known as a "system of fringes," the intensity varying from point to point according to the formula

$$
\text { Intensity } \propto 1+\cos (2 m y \sin \phi)
$$

where $m$ stands for a constant to be determined by measurements of the pattern (e.g., by measuring the distance between two consecutive "dark fringes" or lines of vanishing intensity, which are obviously parallel to the $x$-axis and are separated ${ }^{3}$ by distances $\frac{\pi}{m \sin \varphi}$ ). Now this is exactly the law of variation of the squared amplitude of the wave pattern occurring when two identical sine-wave trains intersect one another at angle 20. Suppose the amplitude, frequency, and wave-length of each to be denoted by $A, n / 2 \pi$, and $\lambda$, respectively, and denote $2 \pi / \lambda$ by $m$. Then the individual wave trains following the directions of the aforesaid beams are described by the expressions

$A \cos (n t-m x \cos \phi-m y \sin \phi), A \cos (n t-m x \cos \varphi+m y \sin \varphi)$ respectively; being added, these give the resultant expression

$$
A \sqrt{2(1+\cos 2 m y \sin \varphi)} \cos (n t-m x \cos )
$$

which describes a wave motion of which the squared amplitude is given thus:

$$
(\text { Amplitude })^{2}=2 A^{2}(1+\cos 2 m y \sin \varphi)
$$

and since in all wave theories square of amplitude is taken to be proportional to intensity, there is agreement with equation (7), as I remarked. Determining the ware-length thus eonsists in measuring $m$ and equating it to $2 \pi / \lambda$.

All measurements of wave-length are made in essentially this fashion, though the type of diffraction pattern employed raries widely from ease to case and is never (except in demonstration experiments) so simple as this example. If anyone speaks of "measuring ware-length" in any

${ }^{3}$ The experiment must be performed by separating a single beam of light into two (e.g., by means of a half-silvered and hence semitransparent mirror, or a pair of Fresnel mirrors) and causing the two to recross one another's paths. The fringes are commonly said to constitute an interference pattern, but this is not essentially different from other types of diffraction pattern. 
other way, he means either that his apparatus has been calibrated against some diffraction apparatus, or that he has measured the energy of the photons and has computed $\lambda$ from them by equation (2).

Measurements of wave-length in the visible and ultra-violet ranges of the spectrum are generally made upon the diffraction patterns produced by diffraction gratings which are metal plates ruled with parallel grooves numbering several thousands to the centimeter. When monochromatic light falls through a slit against such a grating, the pattern is of such a nature that a photographic plate set at an adequate distance receives a sequence of linear imprints (images of the slit) of which the position of any one suffices to show the wave-length of the radiation; hence the term "line" for monochromatic light. Gratings for use in the infra-red are sometimes made of parallel wires. Various other instruments producing diffraction patterns, such as the echelon and diverse types of interferometer, are sometimes used in these regions of the spectrum; their patterns often bear no resemblance to the sharp lines formed by a grating. For $\mathrm{X}$-rays the use of ruled gratings is becoming extensive, though it is not likely to supersede the original method, i.e., that of using crystals of which the regular alignments of atoms serve as the rulings of a natural threedimensional grating. In the gamma-ray region it is necessary to measure the energy of the photons and deduce $\lambda$ from it by equation (2).

\section{EXTERNAL PHOTOELECTRIC EFFECT OF METALS, AND MEASUREMENT OF PHOTON ENERGY}

Having spoken of the method of measuring wave-length of light, I shall now speak of the principal method for measuring energy of photons, though this procedure requires me to describe out of its due order one of the most important phenomena of the interaction of light and electricity: the external photoclectric effect of metals, often designated simply as "the photoelectric effect."

Let a beam of monochromatic light of wave-length $\lambda$ be sent against a metal target enclosed in an evacuated tube, the target being connected to a wire passing through the tube wall so that it becomes an electrode, and the tube being fitted with other electrodes and metallic grids or gauzes connected to wires and so arranged that electric fields can be applied to the target. If $\lambda$ is greater than a certain "threshold wavelength" depending on the target metal, nothing happens. If $\lambda$ is less than this threshold wave-length, electrons emerge from the metal. If the target and the other electrodes are charged to suitably chosen relative potentials, the electric field will draw these emerging electrons to some other electrode, and so long as the light continues to shine steadily, there will be a steady photoelectric current from the target. It will be sufficient for our purpose to visualize the simplest possible arrangement: 
two plane parallel electrodes enclosed in a tube, one of them being the illuminated target, the other the so-called "collector." If the potential difference $V$ between collector and target is positive-collector more positive than target-we observe this photoelectric current $i_{m}$. If $V$ is negative and its absolute value is large, there is no current at all; for all of the electrons which emerge from the target are driven back into it by the adverse field, and the net effect is the same as though they had never come out. If $V$ is negative and its absolute value is small, there is some current, $i$, though not so great as $i_{m}$. To speak more precisely: if $V$ is varied continuously from large positive to large negative values, $i$ remains at first constant and equal to $i_{m}$, then drops gradually to zero; it becomes zero at a certain negative value of $V$, which we will call $V_{0}$.

These facts are interpreted by supposing that the light ejects electrons from the metal, and the electrons escape in various directions with various amounts of kinetic energy, these amounts ranging from zero up to a maximum value $E_{\mathrm{max}}$. When $V>0$, or even (in an ideal arrangement with infinitely extensive plates and no space charge) when $V=0$, all of these particles attain the collector. When, however, $V$ is made progressively smaller than zero, the adverse field prevents first some and finally all of them from reaching the collector-first the slowest and the most oblique, then the faster and the less oblique, and finally the fastest which shoot out normally from the target, are held up and driven back whence they came. The particular value $V_{0}$ of adverse voltage just defined is the one which just suffices to hold up these particular electrons of kinetic energy $E_{\max }$ and velocity directed normally to the plates; and it is related to $E_{\max }$ by the equation, ${ }^{4}$

$$
E_{\max }=\frac{e V_{0}}{300}
$$

Let us now suppose that $(a)$ the emerging electrons were originally among the "free" electrons which circulate within the metal and are responsible for its high electrical conductivity; $(b)$ the kinetic energy of these free electrons, so long as they do not absorb any light, never exceeds a certain maximum value $\mathrm{eW}$ ( $\mathrm{I}$ choose this odd notation because it is used in the theory); (c) each of them which escapes does so by virtue of having absorbed the entire energy $h c / \lambda$ or $h \nu$ of a photon; $(d)$ in escaping each must sacrifice not less than a certain minimum amount $e W_{a}$ of its kinetic energy in separating itself from the metal.

${ }^{4}$ The kinetic energy of a particle of charge $e$ which is just able to overcome an adverse potential difference $V$ is equal to $e V$, provided that all three quantities $E$, $e$, and $V$ are expressed in c.g.s. units. The factor 300 in equation $(9)$ is introduced when $E$ and $e$ are expressed in c.g.s. units, but $V$ is expressed in volts. Note that $V$ always includes contact potential difference. 
If all of these assumptions are valid, it follows that $E_{\max }$ must conform to the equation,

$$
E_{\max }=\frac{h c}{\lambda}-\left(e W_{a}-e W_{i}\right)
$$

and consequently (a) $V_{0}$ must be a linear function of $\lambda^{-1}$, its slope the same for all metals; (b) $V_{0}$ must be independent of the intensity of the light (which does not appear anywhere in the equation); and (c) the total number of electrons emerging per unit time from the metal (the quotient of $i_{m}$ by $e$ ) must be proportional to the number of photons falling per unit time on the metal and therefore to the intensity of the light.

All of these expectations are fulfilled. It was the second of these laws--the law that the intensity of the light is without influence on the energy of the escaping electrons-which served as the earliest incontrovertible evidence that the behavior of light cannot wholly be explained by conceiving it as a pure wave motion. For in trying to interpret the photoelectric effect in the classical manner, one must imagine the electromagnetic waves entering into the metal and setting the indwelling electrons into forced oscillations, by virtue of the continuously varying field strength and energy which accompany them. The oscillations of such an electron would grow steadily wider; the speed with which it dashed through its middle position would grow larger and larger; at last it would be torn from its moorings. One would predict that the greater the intensity of the light, the greater the energy acquired by the electron in each cycle of its forced oscillation would be, the greater the energy with which it would finally break away. But $E_{\max }$ (as indeed the whole distribution in energy of the photoelectrons) is independent of the intensity; it is as though the waves beating upon a beach were doubled in their height and the powerful new waves disturbed four times as many pebbles as before, but did not displace a single one of them any farther nor agitate it any more violently than the former gentler waves did the pebbles that they washed about.

The second and the third of the foregoing laws thus speak powerfully for the doctrine that, at least in respect of absorption, a beam of light is to be regarded as a hail of corpuscles; and they justify us in using the linear relation between $V_{0}$ and $\lambda^{-1}$ which now $\mathrm{I}$ write in the form

$$
V_{0}=\frac{A_{1}}{\lambda}+A_{2}
$$

as a basis for determining the constant $h$; for $h$ is equated simply to $e A_{1} / 300 c$. Incidentally, the theory as a whole requires that $\left(e W_{a}-e W_{i}\right)$ should vary from one metal to another just as does the Volta potential or contact potential, and is further strengthened by the fact that $A_{2}$ 
is found to vary thus from metal to metal. There are also many additional verifications of the assumptions $(a),(b)$, and $(d)$; but to analyze them all would lead us much too far afield. ${ }^{5}$

It is obvious that if $A_{1}$ has been determined once for all and $A_{2}$ has been determined for a given metal by experiments with light of known wave-lengths, the maximum kinetic energy of the electrons ejected from that metal by light of unknown constitution will give us the photon energy and hence the wave-length of the light if this is monochromatic (or that of the shortest-wave constituent of the light, if it is a mixture of various wave-lengths). A very similar method is used to ascertain the photon energies of X-rays and gamma rays, especially when this is too small to produce a measurable diffraction pattern with any available grating or other diffraction apparatus. We shall consider it later.

\section{UNITS OF WAVE-LENGTH, WAVE NUMBER, FREQUENCY, AND PHOTON ENERGY}

a. The ultimate unit of wave-length is, of course, the centimeter, but various submultiples of this universal unit are of customary use in various parts of the spectrum. These comprise:

The "micron" (symbol $\mu$ ) equal to $10^{-4} \mathrm{~cm}$., sometimes employed in the extreme infra-red.

The "millimieron" (symbol $m \mu$, formerly $\mu \mu$ ) equal to $10^{-7} \mathrm{~cm}$., occasionally employed in the infra-red, the visible, and the ultra-violet, but not so common as the unit next mentioned.

The "Ångström" (symbol A or $\AA$ ) equal to $10^{-8} \mathrm{~cm}$., usually employed in the near infra-red, the visible, and the ultra-violet.

The "X-unit" (symbol $\mathrm{X}$ ) equal to $10^{-11} \mathrm{~cm}$., employed in the $\mathrm{X}$-ray and gamma-ray regions.

b. "Wave number" is by definition the reciprocal of wave-length. When a wave number is to be calculated from a wave-length, the latter is generally expressed in centimeters, so that the customary unit of wave number is the reciprocal centimeter (symbol $\mathrm{em}^{-1}$ ). This is also the case when the values of "terms" (page 22) are expressed in wave numbers.

$c$. "Frequeney" is by definition the product of wave number by $c$, the speed of light in vacuo. The first factor is commonly expressed in

${ }^{5}$ It was formerly thought that $\mathrm{W}_{i}$ of equation $(10)$ is negligibly small, but quantum mechanics has taught us differently. Actually, the distribution in energy of the free electrons inside of a metal does not have a perfectly sharp upper limit $W_{i}$ except at the absolute zero of temperature. At temperatures higher than $0^{\circ} \mathbf{K}$., there are some free electrons having energies greater than $W_{i}$, and consequently some photoelectrons escaping with energies greater than the value of $E_{\max }$ which would be observerl at zero; the distribution in energy of these electrons agrees so well with that predicted from the quantum-mechanical theory, as to afford extra proof of the fundamental ideas. 
$\mathrm{cm}^{-1}$, the second in centimeters per second or $\mathrm{cm} . / \mathrm{sec}$. , the frequency, therefore, in reciprocal seconds (symbol sec. ${ }^{-1}$ ). The reader will seldom see numerical values of frequency cited.

$d$. The c.g.s. unit of energy is the "erg." The energy of a photon of light of frequency $\nu$ is computed in ergs by multiplying $\nu$ expressed in sec. ${ }^{-1}$ by the value of $h$ expressed in c.g.s. units (gm. cm. ${ }^{2}$ sec. ${ }^{-1}$ ), which is $6.55 \cdot 10^{-27}$. The erg is far too large a unit for convenience in dealing with any portion of the spectrum, and the customary unit of energy is the "electron volt" (symbol Ev) equal to $1.59 \cdot 10^{-12} \mathrm{erg}$. Sometimes this is called the "equivalent volt," and of ten simply the "volt of energy" or "volt," a usage much to be reprehended. As the name implies, it is the amount of kinetic energy acquired by an electron in passing unimpeded between two points of which the latter is at a potential one volt higher than the former. In the gamma-ray region, photon energies are sometimes expressed in millions of electron volts (symbol MEv).

To get photon energy in electron volts from wave-length in Ångströms, it is often convenient to remember that photons of one electron volt correspond to waves of $12,337 \AA$. If the reader finds it easier to hold in mind the number 12,345 than the other, he commits no serious error in doing so.

\section{THE REGIONS OF THE SPECTRUM}

While the several regions of the spectrum derive their names from physiological and historical accidents rather than from fundamental reasons, it is important to have some notion of their locations and extents.

The visible spectrum extends from about $3600 \AA$ at the end of the violet to about $8500 \AA$ at the end of the red, the exact limits varying from one eye to another. The corresponding photon energies are about 3.4 and $1.4 \mathrm{Ev}$, respectively.

From the red end of the visible spectrum, in the direction of increasing wave-lengths, extends the infra-red, beyond which lies the spectrum of Hertzian waves generated by oscillating electrical circuits. Until about 1924 there was a gap between these ranges -i.e., an intermediate range of wave-lengths corresponding to no radiations which had ever been observed-and the infra-red was defined as extending to the shortwave end of this gap. Subsequently the gap was closed, and this convenient definition lost; the infra-red is now commonly considered as extending to about $1000 \mu$ or one millimeter, corresponding to a photon energy of only about 0.001 Ev. People sometimes speak of the "near" or the "far" infra-red, but the distinction is extremely loose, unless perchance the near infra-red be defined as that range over which photographic plates are sensitive. This range is constantly being extended 
by research in photochemistry but still remains relatively restricted. Beyond it, infra-red light must be detected by its heating effect or by its action on a radiometer.

From the violet end of the visible spectrum, in the direction of decreasing wave-lengths, extends the ultra-violet. Like the infra-red, it formerly was terminated by a gap, beyond which lay the X-rays. This gap was closed at about the same time as that other, and now the limit of the so-called ultra-violet has to be fixed by arbitrary choice somewhere in the zone which is alternatively called that of far ultra-violet or that of soft X-rays. As acceptable a frontier as any is the wave-length $12.3 \AA$ corresponding to a photon energy of $100 \mathrm{Ev}$; but no frontier is really good. Subdivisions of the ultra-violet are the "zone of transmission by the earth's atmosphere" extending from the edge of the violet out to about $2900 \AA$ ( $4.3 \mathrm{EV})$, at which the atmosphere ceases to transmit and beyond which the spectra of the sun and stars cannot be followed; the Schumann region between about 2000 and $1250 \AA$ ( 6.4 and $10 \mathrm{Ev})$, in which fluorite must be used for lenses and prisms and special photographic films are necessary; and the Lyman region, extending thence to about $500 \AA$ (about $25 \mathrm{Ev}$ ), in which still more exceptional films are requisite and only the thimnest strata of solid matter (or better, none at all) may be put across the light beam. ${ }^{6}$

Beyond the ultra-violet stretches the $X$-ray region, and beyond that the gamma-ray spectrum, any boundary imposed between the two being of necessity even more arbitrary than any imposed between the X-rays and the ultra-violet. If we could limit the $\mathrm{X}$-ray spectrum to the characteristic X-ray "lines" (see page 25), we could put a frontier at about $0.12 \AA$ (about $10^{5} \mathrm{Ev}$ ), but this would be doing too great a violence to our classical definitions of X-rays, while on the other hand no other sharp limit can be set except the photon energy corresponding to the highest voltage thus far applied to an $\mathrm{X}$-ray tube, and this leaps upward year by year. If the reader insists on a boundary, he may take $25 \mathrm{X}\left(5 \cdot 10^{5} \mathrm{EV}\right.$ or $\left.0.5 \mathrm{MEV}\right)$.

People who work with X-rays frequently refer to the infra-red, the visible, and the ultra-violet regions collectively as the "optical spectrum."

\section{ABSORPTION OF LIGHT BY ATOMS}

The absorption spectrum of a not too dense gas consists chiefly, if not entirely, of discrete lines and is known either as a line spectrum or as a band spectrum, the distinction depending on the arrangement of the lines. Band spectra are exhibited by gases in which the atoms are grouped into molecules (notable examples being hydrogen, oxygen,

${ }^{6}$ Schumann and Lyman were the first to devise methods for observing spectra in these respective regions. 
nitrogen, as well as all gaseous chemical compounds) and will be treated farther on. Line spectra are exhibited by monatomic gases, a class which at ordinary temperatures comprises only the noble or inert gases (helium, argon, neon, and the very rare gases krypton and xenon) and the vapors of certain metals, among which mercury vapor is by far the easiest to study, while those of the alkali metals are the next easiest. Many, if not all, of these monatomic gases show spectra of both types, but in such cases the band spectra are attributed to occasional molecules mixed with the uncombined atoms. Some of the molecular gases show line spectra when heated or exposed to electrical discharges, but these are attributed to atoms which have been set free by the dissociation of molecules. It happens that for the commonest gases, both monatomic and molecular, most or all of the lines of the absorption spectrum are in the ultra-violet, so that these gases seem to be perfectly transparent so long as no account is taken of any but visible light.

The line spectra, then, are the characteristic spectra of gases consisting of single atoms. When such a gas is made more rarefied and cooler, the lines of its absorption spectrum become sharper and narrower; and it appears that we can approach quite closely to the ideal spectrum of absolutely isolated and stationary atoms not colliding or interfering with one another in any way. This limiting spectrum is the subject of what next follows.

Suppose that we have measured as many as possible of the absorption lines of a given gas. Let $\lambda_{i}$ be the general symbol for the wave-length of any one among them. I have said that in respect of absorption, monochromatic light of wave-length $\lambda$ behaves like corpuscles of energy $h c / \lambda$ or $h \nu$. The existence of the absorption-line-spectrum thus signifies, that atoms of the gas in question are able to absorb quantities of energy of the amounts $h c / \lambda_{i}$ or $h \nu_{i}$. The atoms are not able to absorb intermediate amounts of energy, for there are gaps in the spectrum between the lines. Atoms are capable of absorbing only certain specific diserete quantities of energy.

Another way of saying the same thing is: Atoms are capable of existing only in certain specific discrete states, each distinguished by a characteristic energy value; absorption of light by an atom entails the transfer or "transition" of the atom from one of these states to another, and consequently the product of $h$ by the frequency $c / \lambda_{i}$ of any absorption line is the difference between the energy values of two of the stationary states of the atom in question. This is one of the fundamental principles discerned by Niels Bohr.

In cool, rarefied, and unexcited monatomic gases the vast majority of the atoms at any moment are in one particular state, the "normal state" or "ground state." All of the absorption lines of such a gas correspond to transitions out of this special state into various others, 
known collectively as "excited states." The values of $h c / \lambda_{i}$ for such a gas are then the energy values of these excited states reckoned from a common zero of energy, viz., the energy of the normal state. If we measure the lines of the absorption spectrum of such a gas, we are at the same time determining the energy values of various states of the atoms all reckoned from this eommon zero.

What happens if a photon meets an atom which has already been transferred into an excited state by absorbing a previous photon? Usually this is not a likely occurrence, as an excited atom usually reverts to the normal state within an exceedingly short time, of the order of $10^{-8}$ see. It ean, however, be made to oecur sufficiently often to be observed, by using a very intense beam of light and a not too rarefied gas. Moreover, atoms in exeited states_-"exeited atoms" - can be made relatively abundant in a gas by instigating an electrical discharge in it (a glow or an are), or even in some cases merely by heating it. It turns out (as can be easily foreseen) that for every exeited state there is a new absorption spectrum, with new values of $\lambda_{i}$ such that the products $h c / \lambda_{i}$ give the energy values of other states reckoned from the energy of that state as zero. The absorption spectrum of a gas full of exeited atoms can thus be extremely intricate (as the emission spectrum actually is), and it was on this account that in the previous paragraph I specified a "cool, rarefied, and unexcited gas."

Reverting to such a gas: in the simplest cases the absorption lines form a converging sequence or "series" in which every line is closer to its neighbor of higher frequency than to its neighbor of lower frequeney, and the gap between consecutive lines approaches zero as the frequency rises, so that there is a "convergence frequency" or "limit frequency." The most famous series is a certain series of monatomic hydrogen, of which the frequencies of the various lines are obtained by assigning the values of the integers (from 3 upward) to the symbol $n$ in the equation

$$
\nu=R\left(\frac{1}{4}-\frac{1}{n^{2}}\right) \quad n=3,4,5 \cdots
$$

where $R$ stands for a certain constant. This series is named after Balmer who discovered the formula (not the series). His formula implies an infinite number of lines, converging upon the limit frequency $\nu_{\lim }=R / 4$. Actually, we observe, of course, a finite number of lines (about 50), each of them fainter than the next lower, and the highest of them merging into a broad haze or "continuum." Only a few other series conform to algebraic expressions as simple as Balmer's, but there are many of which the lines can be fitted by formulas of the same general type, implying an infinitc sequence of converging lines of which we observe only the first few dozen (or sometimes only the first few) as diserete lines, while 
the remainder fade away into a continuum. We infer that in such a case, the excited states of the atom may be considered to form a theoretically infinite sequence, such that the difference between the energy values of any two consecutive states approaches zero as one traverses the sequence in the direction of increasing energies. Actually there may be many such sequences displayed by a single kind of atom, and the complexities of even the simplest spectra are rather formidable.

Since a spectrum line corresponds to a transition between two states of an atom, it may be denoted by the symbols designating these two states. Thus, the most prominent line (and the one most frequently employed in research with ultra-violet light) in the ultra-violet spectrum of mercury - to wit, the line of wave-length $2537 \AA$-is sometimes denoted in this fashion:

$$
1^{1} S-2^{3} P_{0}
$$

the symbols $1^{1} S$ and $22^{3} P_{0}$ being applied by spectroscopists respectively to the normal state and to a particular excited state of the mercury atom, and the photons of wave-length $2537 \AA$ causing a transition from the former state to the latter when they are absorbed. This is a most unwelcome usage for anyone but a practiced spectroscopist, not only because the symbols are intricate but because they are frequently changed, generally in the direction of greater complexity: I recall at least five alternatives for $2^{3} P_{0}$. The biologist is not very likely to meet with this usage, but if he does he had better appeal to some professional spectroscopist for a translation of these symbols into wave-lengths.

Another difficulty which a biologist may, but is not likely to, encounter: a betterment of spectroscopic apparatus frequently leads to the discovery that what has hitherto been regarded as a single line is actually a cluster of lines very close together, i.e., differing very little in wavelength. After such a discovery the cluster is still frequently described as a single line, except in researches on the individual members of the group. The aforesaid "line" of mercury is known to be a cluster of at least five lines.

\section{CONTINUA IN ABSORPTION SPECTRA, AND IONIZATION BY LIGHT}

In the previous section I spoke of the continum which lies beyond the highest distinctly separable lines of a series. This consists in part of the uppermost lines of the series which are too close together to be distinguished or "resolved"; but there is also a part of it which extends beyond the limit of the series. The existence of this latter part is a point of little practical but great theoretical importance, for when the gas is irradiated by light of any wave-length comprised within this part, the light expels electrons from the atoms: in this particular portion of the 
spectrum, absorption of light by an atom entails detachment of an electron from the atom, a process known as "ionization." The convergence frequency $\nu_{\text {lim }}$ of a series in an absorption spectrum is the least frequency for which ionization takes place, ${ }^{7}$ and consequently the energy $h \nu_{\text {lim }}$ of the corresponding photons is the energy which just suffices to detach an electron from the atom. This is known as the "ionizing energy" or "ionization potential" of the atom. When the atom has thus been ionized by light of frequency $\nu_{\text {lim }}$, we may consider the ionized atom and its divorced electron as a system in a state, the "state of the ionized atom," having an energy equal to $h v_{\text {lim }}$ when reckoned from our regular zero.

\section{THEORY OF ABSORPTION LINES}

Consider an absorption spectrum consisting of a line series having limit frequency $\nu_{\text {lim }}$, and of the adjoining continuum. Photons of frequencies greater than $\nu_{\lim }$ are absorbed by atoms and expel electrons. Photons of certain discrete frequencies $\nu_{i}$ smaller than $\nu_{\lim }$ are absorbed by atoms but do not expel electrons. It is natural to infer that while a photon of an absorption line does not have energy enough to detach an electron completely from the atom, it does succeed in shifting an electron partway outward from its equilibrium position in the atom. The various stationary states of energy $h \nu_{i}$ (reckoned from that of the normal state as zero) would then involve different positions of an electron in the atom. The normal state would involve the position of permanent equilibrium; the excited states, positions of merely temporary equilibrium; the transition of an atom from one state to another would involve the transfer of an electron from one position to another.

This also is one of the fundamental ideas of Bohr. In his earliest papers Bohr (and subsequently many other theorists) went much further in defining the pictures or models of these stationary states. They considered that each stationary state involves a definite and characteristic orbit of the electron in the atom. Take, for example, the simplest case of all, that of hydrogen, the atom of which consists of a single electron of charge $-e$ and a nucleus of charge $+e$. Let it be assumed that the electron may revolve around the nucleus only in one or another of certain circular $^{8}$ orbits, to wit, those circular orbits in which the angular momentum of the atom is some integer multiple of $h / 2 \pi$-is equal to $n h / 2 \pi$, where $n$ stands for any integer greater than zero. (This is not a purely ad hoc assumption, but one suggested by general quantum theory!)

${ }^{7}$ Except when ionization occurs as the result of two or more processes acting simultaneously.

${ }^{8}$ Certain elliptical orbits are also permissible, but it would complicate this brief account too much to take due note of them. 
On computing the energy possessed by the atom when the electron is in one or another of these various orbits, it is found that the energy values agree precisely with those of the stationary states deduced from the Balmer series and other series in the spectrum of hydrogen! The computed energy value corresponding to the $n$th orbit (that for which the angular momentum is $n h / 2 \pi$ ) when reckoned from that of the first orbit taken as zero of energy, is given by the formula, ${ }^{9}$

$$
h \frac{2 \pi^{2} \mu m e^{4}}{h^{3}}\left(1-\frac{1}{n^{2}}\right)
$$

Let us subtract the energy value for the second orbit from that for the $n$th orbit, and divide the difference by $h$ to get the frequency of that photon, the absorption of which should transfer the electron from the second orbit to the $n$ th. We get:

$$
\nu=\frac{2 \pi^{2} \mu m e^{4}}{h^{3}}\left[\left(1-\frac{1}{n^{2}}\right)-\left(1-\frac{1}{4}\right)\right]=\frac{2 \pi^{2} \mu m e^{4}}{h^{3}}\left(\frac{1}{4}-\frac{1}{n^{2}}\right)
$$

and now comparing this with Balmer's formula we see that they are identical if the combination $2 \pi^{2} \mu m e^{4} / h^{3}$ is equal to the observational constant $R$-and this turns out to be so!

One immediately inquires: should there not also be a series corresponding to transfers of the electron from the first orbit into the outer orbits, given therefore by the formula:

$$
\nu=\frac{2 \pi^{2} \mu e^{4}}{h^{3}}\left(1-\frac{1}{n^{2}}\right)
$$

So there is! It is the Lyman series, discovered much later than the Balmer series for the sole reason that it lies far out in the ultra-violet, while that described by Balmer's formula is very conveniently placed in the visible spectrum. The Lyman series is the better of the two to keep in mind, for its lines correspond to absorption by normal atoms- the case to which I have hitherto restricted my exposition-whereas the Balmer lines correspond to absorption by excited atoms.

Were I to attempt to give even an inkling of the ways in which Bohr's ideas were developed in order to interpret more complicated spectradevelopments in the course of which many other striking numerical agreements between the theory and the data were discovered-the character of this essay would be entirely distorted. It must suffice to say that the successes of the theory were such, that the word "orbit" has become practically a synonym for "stationary state" in the common language of physicists. Even theorists who in their own thinking make

${ }_{9}$ The symbol $m$ stands for the mass of the electron, the symbol $\mu$ for the ratio $M /(M+m)$, where $M$ represents the mass of the nucleus. 
little or no use of the picture of a revolving electron, are likely enough to speak or write of the " 1 1 $S$ orbit of mercury" instead of the "stationary state of the mercury atom denoted by $1^{1} S . "$ There is, in fact, no compelling reason why the practicing spectroscopist and the user of light as a tool should not continue to employ both the word and the picture. The important point is that the stationary states and their energy values are now so fully verified as to have risen practically to the rank of facts of experience, unaffected by alterations, in the models by which theoretical physicists are accustomed to represent them. When the reader realizes this, he may safely designate a stationary state by such words as "orbit," or "position of an electron," or "arrangement of the electrons," without concerning himself unduly with the fact that some physicists use different descriptive words or none at all.

\section{TERMS}

Reverting to Balmer's formula: the frequency of any line of the Balmer series is given as the difference $\frac{R}{4}-\frac{R}{n^{2}}$, which we may write thus instead:

$$
\nu=\frac{-R}{n^{2}}-\left(\frac{-R}{4}\right)
$$

The reason for this algebraically correct but oddly appearing revision is seen by inspecting the theoretical equivalent of Balmer's formula, equation (13). The energy values of the $n$th and the second orbits, respectively, are $h R\left(1-\frac{1}{n^{2}}\right)$ and $h R\left(1-\frac{1}{4}\right)$ when reckoned from our former zero, viz., the energy of the normal state. The quantities $-h R / n^{2}$ and $-h R / 4$ are the energy values of these orbits, reckoned from a new zero greater by $h R$ than the old one. From equation (13) we can divine the meaning of this new zero. The quantity $h R$ is $h \nu_{\text {lim }}$, the energy corresponding to the limit of the Lyman series, therefore the energy just sufficient to extract an electron from a hydrogen atom initially in the normal state. In passing from the old zero of energy to a new zero greater by $h R$, we have passed from the "normal state of the neutral atom" to the "state of the ionized atom" as the new standard from which energy values are to be reckoned. The quantity $-h R / n^{2}$ is the energy of the hydrogen atom with the electron in its $n$th orbit, reckoned from the energy of the ionized atom with its electron at infinity.

The practical importance of this point is as follows: In spectroscopic tables and elsewhere, the frequency of a spectrum line belonging to a series is often written as the difference between two "terms,"

$$
\nu=A-B=-B-(-A)
$$


These are to be multiplied by $h$; then the negative quantities $-h B$ and $-h A$ are the energy values of two stationary states of the atom, reckoned from the energy of the ionized atoms as zero. To obtain the energy values of these states reckoned from the normal state of the neutral atom, one must add the ionizing energy of the atom to $-h A$ and $-h B$. Quantities such as $A$ and $B$ are technically known as "terms"; they are sometimes tabulated instead of lines, and are usually expressed in wave numbers.

\section{ABSORPTION IN THE X-RAY REGION}

In the X-ray absorption spectra there is a striking difference from those which we have hitherto been considering; instead of absorption lines being prominent and continua being inconspicuous, the continua of an $X$-ray absorption spectrum are its principal features.

Like the others, these X-ray continua signify absorption of photons attended by expulsion of electrons. Generally, any one of them presents a relatively sharp edge toward the low-frequency side, and a gradual fading out toward the high-frequency side: as the frequency is increased from an initial low value, the absorption coefficient suddenly rises from a very low to a high value, thereafter to decrease gradually and smoothly as the frequency is further raised. (At frequencies slightly below that of the absorption edge, discrete absorption lines may be observed, recalling those of the line series adjoining a continuum in an optical spectrum.) Denote by $\nu_{0}$ the frequency of an absorption edge: then $h \nu_{0}$ represents the energy just sufficient to detach an electron from the atom. Let the atoms (we will imagine a stratum of some chemical element, lead or argon, for example) be irradiated by a beam of $\mathrm{X}$-rays of frequency $\nu$ greater than $\nu_{0}$. The expelled electrons will then possess kinetic energy $K$ given by the formula

$$
K=h \nu-h \nu_{0}
$$

The similarity with equation $(8)$ is manifest: we have a linear relation between kinetic energy of expelled electrons and frequency of incident light-its slope is $h$-there is an additive constant of negative value. There are, however, striking differences: The electrons all have about the same kinetic energy, instead of a distribution of energies from zero to a maximum value to which alone the equation is applied; and the additive constant is not equal to $\left(e W_{i}-e W_{a}\right)$, but as a rule it is very much greater. All this signifies that the electrons in question were not originally free electrons circulating in the metal (if the irradiated element was a metal) but "bound" electrons definitely attached to atoms in a fixed and constant way, the energy $h \nu_{0}$ being that required to overcome the binding and make 
that definite change in the structure of the atom which is entailed by the extraction of one of its constituent parts. ${ }^{10}$

In the absorption spectrum of a massive element, such as gold, lead, or uranium, there are several of these continua and absorption edges, and therefore several different values of $h \nu_{0}$. Each corresponds to the extraction of an electron belonging to a certain definite class, which we may picture as being normally located in a certain orbit or at a certain distance from the nucleus of the atom. The absorption edge of highest frequency corresponds to the class of electrons of which it takes the greatest amount of energy to dislodge a member. Electrons of this class are therefore conceived as occupying the innermost orbit or the nearest of all available locations to the nucleus itself. They are called $K$ electrons; the corresponding absorption edge and its frequency are known as the $K$ absorption edge and the $K$ absorption frequency, and the latter is denoted by $\nu_{K}$. The succeeding absorption edges in the sense of decreasing frequency are marked by the symbols $L_{\mathrm{I}}, L_{\mathrm{II}}, L_{\mathrm{III}} ; M_{\mathrm{I}}, M_{\mathrm{II}}, M_{\mathrm{II}}, M_{\mathrm{rv}}, M_{\mathrm{v}} ; N_{\mathrm{I}}$, and others which the reader will seldom encounter. To each there corresponds a class of electrons in the atom, and (in the model) an orbit or a location for the electrons of this class; and the symbols are employed for these classes and for the frequencies of their absorption edges. A list of the absorption frequencies of an atom is thus the first step toward a map of the atom itself.

As we pass along the table of the elements from the heaviest (uranium) down toward the lightest (hydrogen), each absorption edge is shifted step by step toward lower frequencies, and each in turn fades out. The shifting is due to the decrease in nuclear charge; the positive charge at the nucleus of the silver atom, for instance, is only a little more than onehalf that on the nucleus of the uranium atom, and it attracts its $K$ electrons correspondingly less strongly, so that much less energy (actually about a quarter as much) is required to tear one of them away. The vanishing of the edge is due to the fact that each atom has one electron fewer than the one next beyond it in the table of the elements, so that one class after another is emptied as we proceed down the list.

The facts that absorption of X-rays entails extraction of electrons and that these electrons are often endowed with a large kinetic energy, are of the first importance in respect to all the actions and powers of $\mathrm{X}$-rays. Practically all of the electrical effects produced by a beam of X-rays traversing a sheet of matter are due primarily to these electrons-often called "secondary electrons"-which themselves plough through the matter ionizing the atoms very abundantly until they use up all their

${ }^{10}$ The reader will see that the quantity $\left(e W_{i}-e W_{a}\right)$ should be included in $h v_{0}$, but it is usually so very small a fraction of $h \nu_{0}$ that it is commonly ignored. 
energy and are brought to a stop; and the biological effects must be due either to these electrical effects or to something as yet unknown.

It must be mentioned at this point that the so-called "absorption coefficient" of a given substance for a given beam of X-rays is frequently so defined as to take account not only of absorption in the foregoing sense, but also of scattering - a phenomenon to be discussed in a later section.

\section{X-RAY EMISSION SPECTRA}

Emission of light is the converse phenomenon to absorption. While I began the discussion of absorption by speaking of the optical spectrum, there are reasons for reversing the procedure in dealing with emission, and beginning with that of X-rays.

$\mathrm{X}$-ray emission spectra are line spectra; and each line (with the exception of some extremely faint ones) has a frequency which is the difference between the frequencies of two absorption edges.

This principle has an important meaning. For definiteness let us take the line, in the spectrum of some heavy element, of which the frequency is the difference between that of the $K$ absorption edge and that of the $L_{\mathrm{II}}$ absorption edge. The photons of the line have each an energy which is the difference between the energy required to extract a $K$ electron and the energy required to extract an $L_{11}$ electron. We now imitate the usage introduced in dealing with absorption series in optical spectra. The energy required to extract a $K$ electron is the difference between the energy of the atom in its normal state with all of its electrons present, and the energy of the atom in a particular abnormal state with one $K$ electron missing - one vacancy in the $K$ class, let me say. The energy required to extract an $L_{\mathrm{II}}$ electron is the difference between the energy of the atom in its normal state aforesaid and the energy of the atom in another particular abnormal state with a vacancy in the $L_{\mathrm{II}}$ class. Subtract the latter from the former: the result is the difference between the energy of the atom with a vacancy in the $K$ class, and the energy of the atom with a vacancy in the $L_{\mathrm{II}}$ class. This becomes much more concrete if I introduce a metaphor. A photon of the line in question will be emitted, when an electron falls from its place in the $L_{\mathrm{II}}$ class to a vacancy existing in the $K$ class, and the atom releases the energy thus made free.

Each of the principal lines of an X-ray emission spectrum is correlated in this manner with a transition, in which an electron passes from one class -or, to use a commoner word, from one "shell" - to another, leaving a vacancy in the shell whence it comes and filling a vacancy in the shell to which it goes. We may also say that the atom makes a transition between a state distinguished by a vacancy in one shell, and a state distinguished by a vacancy in another shell. Like other pictures to which 
I have already alluded in this essay, that of the electron literally falling from one place to another in the atom is probably too definite, but there is no reasonable objection to making use of its convenience while remembering that a contemporary theorist might prefer a different image or none at all.

The line which I used as an illustration is called the $K \alpha_{2}$ line. It belongs to the $K$ series, which is composed of all the lines due to electrons which fall from various outer shells into a vacancy in the $K$ series. The corresponding transitions thus have in common their initial state, the state of an atom with a vacancy in the $K$ shell; they have different final states, one being the state of an atom with a vacancy in the $L_{\mathrm{II}}$ shell, another the state of an atom with a vacancy in the $L_{\text {III }}$ shell, and so forth. The lines of the $K$ series of a heavy element lie relatively close together and well apart from all the other lines, having considerably greater frequencies (five or ten times as great) than the rest. There is also an $L_{\mathrm{I}}$ series, composed of lines due to electrons which fall from various outer shells into a vacancy in the $L_{\mathrm{I}}$ shell; an $L_{\mathrm{II}}$ series, and series for various other shells. All of these series lie closer together (on the frequency scale) than the $K$ series to any of them, and some overlap, making a very intricate spectrum. The notation for $\mathrm{X}$-ray emission lines is complicated and obscure.

\section{PRODUCTION OF X-RAYS}

$\mathrm{X}$-rays are produced chiefly by projecting streams of electrons against blocks or "targets" of metal, occasionally by projecting X-rays from another source against the metal from which it is desired to evoke the characteristic rays. The theory outlined in foregoing sections enables us to understand some striking peculiarities of these modes of production.

Suppose first a stream of electrons, each having kinetic energy $U$, impinging upon a block of an element having $K$ absorption frequency $\nu_{K}$. According to the theory, no line of the $K$ series can be emitted unless there is a vacancy in the $K$ shell, into which an electron may fall from some outer shell. The lines of the $K$ series cannot therefore be produced, unless $K$ electrons are constantly being knocked out of the atoms. To achieve this the impinging electrons must have at least that energy $h \nu_{K}$ which we have just identified as the energy needful to extract a $K$ electron from an atom. We must therefore expect that as $U$ is increased, none of the lines of the $K$ series will appear before, and all of them will appear when $U$ attains the value $h \nu_{K}$.

This is verified by experiment. It is a remarkable experience to compare a spectrum obtained with $U$ just below $h \nu_{K}$ with a spectrum obtained when $U$ is only a little above $h \nu_{K}$; the whole $K$ series, entirely absent from the former, is conspicuous in the latter. The corresponding 
rule holds for each of the other series. When the impinging beam consists of photons instead of electrons, the like is observed. Photons are not capable of producing any line of an X-ray series, unless their frequency is superior to that of the absorption edge corresponding to the series.

However, the spectrum of the X-rays produced by fast electrons impinging against a metal does not consist exclusively of the lines of which I have just been speaking. Indeed it consists chiefly of a continuum; there are $\mathrm{X}$-rays of every frequency from the least which can be detected up to a sharply defined maximum $\nu_{\max }$ which is related to the kinetic energy $U$ of the electrons by the familiar-looking equation

$$
h \nu_{\max }=U^{\top}
$$

This is interpreted as follows: The impinging electrons, as they penetrate into the metal, swiftly lose their energy; some by spending it in dislodging electrons from the atoms and thus bringing about the eventual emission of X-ray spectrum lines, but others-and the majority-simply by making collisions (presumably with atoms or electrons) in which they are abruptly slowed down or "braked," and the kinetic energy which they lose is transformed immediately into photons. Different electrons may lose different fractions of their energy in different collisions, but no electron can lose more than the total of its initial energy $U$ in a single collision, and therefore no photon can have an energy greater than $U$. The X-rays of the frequency $v_{\max }$, which constitutes the upper limit of the continuum, are due to the relatively few cases in which impinging electrons lose the whole of their energy in one single operation the very moment they strike the metal; while all the other X-rays of the continuum are due to cases in which impinging electrons lose their energy in steps.

The X-ray spectrum of an ordinary X-ray tube thus consists of a continuum, on which various spectrum lines are superposed. It is quite possible to choose a particular energy $U$ for the electrons bombarding a target of a particular metal, such that $U / h$ is greater than the frequencies of one or more of the $K$ lines, but less than the frequency $\nu_{K}$ of the $K$ absorption edge. In such a case the continuum runs smoothly through the frequencies of the $K$ series without exhibiting a single peak at any of them; the peaks spring up above it when, and only when, $U$ is increased past $h v_{K}$ and the upper limit of the continuum has moved on past $v_{K}$. If we select a very narrow range of frequencies around some particular frequency $\nu$, measure the energy $E$ of the $\mathrm{X}$-rays lying within this range, and plot it as a function of $U$, we find that as $U$ is increased, $E$ remains zero until $U$ reaches $h \nu$, after which it rises very sharply and rapidly. If $\nu$ coincides with the frequency of some line of the $K$ series, the rate of 
increase of $E$ with increasing $U$ becomes sharply greater when $U$ reaches $h \nu_{K}$. If we hold $U$ constant, divide the X-ray spectrum into consecutive small equal ranges of wave-length, measure $E$ for each of these, and plot $E$ as function of wave-length, we obtain the distribution-in-energy curve of the continuum: this has a maximum at a wave-length about half again as great as that of the upper frequency limit, and after passing the maximum, it drops sharply to the axis of abscissae at $\lambda=c h / U$. The general shape of the curve varies little from one element to another, and its upper end is the same for all elements, as we have just seen; but its height and the area under it - the total energy radiated in the form of $\mathrm{X}$-rays - increase rapidly in passing in the direction of increasing atomic weight along the table of the elements. At best, however, the total energy radiated in X-rays is less than 1 per cent of what the impinging electrons possess; the rest appears as optical radiation or as heat.

$\mathrm{X}$-rays may be generated by projecting another beam of X-rays against a piece of matter; when so produced, they are known as "fluorescent X-rays." They comprise only the spectrum lines of the elements present in the irradiated mass, without a continuum (except for such a feeble one as may be produced by electrons released within the matter and stopped before they emerge from it). This is desirable in many ways; but unfortunately fluorescent $\mathrm{X}$-rays are always far weaker than the beams which may be generated by strong electron bombardment. The law connecting the lines which are produced with the frequency of the irradiating rays may be deduced by the reader from what has already been said.

An effect alternative to the production of X-ray spectrum lines is of much theoretical interest. Imagine an atom from which a $K$ electron has been expelled, leaving behind a vacancy which is presently filled by an electron dropping into it from the $L_{\mathrm{II}}$ shell. As I have already said, it is common for this second process to be accompanied by the emission of a photon, bearing off with it the energy $\left(h \nu_{K}-h \nu_{L_{I I}}\right)$. On the other hand, it is possible for extra electrons to be emitted in place of the photon, precisely as if this corpuscle of light had been reabsorbed in the very atom whence it was about to emerge, and had employed its energy in ejecting, say, an $M_{I}$ electron; we find electrons coming out of the substance possessing the kinetic energy $\left(h \nu_{K}-h \nu_{L 11}-h \nu_{M I}\right)$, which when added to the energy $h \nu_{M I}$ required to extract the $M_{\mathrm{I}}$ electron is none other than the energy of the vanished photon. It is possible for two or three electrons to be ejected simultaneously in this fashion. The effect is known as the Auger effect or as "internal conversion." Whether one should imagine the photon as enjoying a temporary though very brief existence between the inward falling of the electron which starts the process, and the outward springing of the observed emerging electrons, or 
whether one should eschew such a picture and simply imagine the entire process of infalling and emergence taking place en bloc, may be left to the individual's taste.

\section{PRODUCTION OF LIGHT OF THE OPTICAL SPECTRUM: ELECTRICAL MEANS}

The theoretically most intelligible way of producing light of the optieal spectrum is to bombard a gas with electrons of definite and controllable speed. Suppose such a gas as mereury vapor or sodium vapor, which when cool, rarefied, and unexeited (page 16) displays an absorption speetrum consisting of a line series. Denote by $\nu_{1}, \nu_{2}, \nu_{3}, \ldots$ the frequencies of the suceessive lines of this series, starting from that of lowest frequency; by $\nu_{\text {lim }}$ the limit frequeney. As I have said already, the quantities $h \nu_{1}, h \nu_{2}, h \nu_{3}$ are the energies of various stationary states of the atom of the vapor in question, referred to that of the normal state as zero of energy.

If the law of production of spectrum lines were the same in this region as in the $\mathrm{X}$-ray region, an emission spectrum composed of all of these lines should suddenly appear in its completeness when, and not before, the kinetic energy of the impinging electrons attains the value $h \nu_{\text {lim }}$. This, however, is not the case. Under ideal experimental conditions these lines appear successively in emission, the first when the energy $U$ of the electrons attains $h \nu_{1}$, the seeond when $U$ attains $h \nu_{2}$, and so forth. This is just what is to be expected, in view of the fact that photons having these energies are absorbed by the atoms. The electrons, of which the transitions are responsible for these lines, are able at any time to go from the normal location or "orbit" to any one of the outer orbits. We say that this is beeause the outer orbits concerned in optieal spectra are normally empty, while those concerned in X-ray spectra are normally full. A $K$ electron in a gold atom cannot be transposed into the $L_{\mathrm{II}}$ orbit or shell (these two words are used almost interchangeably) because there is already the preseribed number of electrons in this shell and no more can be added. It must either be left altogether alone or thrown elear out of the atom. ${ }^{11}$ But the electron in the normal state of the hydrogen

11 If a photon or an electron of sufficient energy should impinge on an atom just when there happened to be a vacancy in, say, the $L_{\mathrm{II}}$ shell, there is certainly no obvious reason why it should not push a $K$ electron up into that vacancy; but the chance of an impact at just such a moment is so slight that, so far as I know, the effect has not been observed.-It also seems to be possible for $K$ electrons to be transferred into vacant orbits in the periphery of the atom, but the effect is probably of no practical importance (it discloses itself in narrow spectrum lines near an absorption edge). 
atom can be transposed at any time into any one of those outer orbits for which the energy values are given by the formula $R h\left(1-\frac{1}{n^{2}}\right)$, with any integer substituted for $n$; for these orbits are always vacant, except when that electron itself has just been shifted to one of them.

Production of spectrum lines in this manner-by a stream of electrons of definite and controllable energy, just sufficient to excite one or a few lines - is unusual, except for definite purposes of research. It is sometimes fairly easy to get the first line of a scries by itself ("single-line spectrum"), but the following lines are so close together that it is often difficult and before long impossible to form an electron beam of sufficiently sharply defined speed to bring one line out clearly without bringing out the next one appreciably. Then too, the elcetron beam must not be very strong-i.e., there must not be too many electrons per centimeter squared per second-or atoms will suffer impacts from two electrons in quick succession and lines will be emitted which no single impact could produce. Usually the gas is made the theater of an electrical discharge.

There is a great variety of electrical discharges, and a great part of the art of spectroscopy consists in knowing which to use and for which spectrum lines. In all of them, the gas is traversed by electrons having kinetic energy superior to the ionizing energy of the atoms or molecules, so that at any moment a certain proportion of these are ions (rarely, however, more than a few per cent) while neutral particles are constantly being converted into ions to keep the balance while ions are resuming the neutral state by capturing electrons. Nearly always the voltage between cathode and anode, when multiplied by the charge of an electron, gives a product $e V$ greater than the ionizing energy of the gas-we say that the voltage is greater than the ionization potential; but there are some curious exceptions to this rule, for there may be places in a discharge where the potential is lower than at the cathode or higher than at the anode, or there may be electrical oscillations capable of communicating more energy to an electron than it could get by falling unimpeded from cathode to anode. The cathode may be cold (i.e., not incandescent), in which case the potential difference between it and the anode must be of the orders of hundreds or thousands of volts; or it may be intensely hot, in which case the potential difference may amount to only a few volts. The latter discharge or "are" is generally far hotter and more luminous than the former discharge or "glow." The latter is sometimes maintained in a gas of atmospheric density, the former nearly always in a tube where the gas pressure is only a few thousandths of that characteristic of the atmosphere. Intermediate cases may be realized by making the cathode in the form of a filament to which heat may be supplied from an independent battery. 
Owing to the variety of the electron speeds represented in a discharge, one must not expect to get emission spectra composed of a single line or even of a single series. When an atom has been "excited" to one of its upper stationary states, it need not (sometimes indeed it cannot) return direct by a single transition to its normal state. There is a tremendous network of possible transitions between the many stationary states of which an atom is capable, and in a discharge a great many of these transitions are perceptibly represented, giving rise to spectra immensely more rich and complicated than the absorption spectrum of the rarefied, cool, and unexcited gas. With certain kinds of atoms spectroscopists have found it necessary to produce the absorption spectrum (sometimes a difficult matter, in the cases of metals of high boiling point) before they could make much headway with the analysis of the emission spectrum.

While the brilliance of the arc makes it often the most convenient source of spectrum lines, this advantage is often bought by the sacrifice of other desirable qualities, such as sharpness and narrowness of the lines. Hotness tends to broaden a line, because the speed at which an atom is moving affects the wave-length of its radiation; density of gas tends to broaden it, because when two atoms come close together, the electric field of each perturbs the other and alters the energy values of its stationary states. The gas in an arc is hotter and (usually) denser than the gas in a glow, and this produces the undesired effects. Indeed, a striking and paradoxical effect is well known to occur, by virtue of which an arc may fail to send out precisely those wave-lengths which one expects to be the most prominent in its spectrum. Suppose a vapor having a strong spectrum line corresponding to a transition between the normal state and some excited state, the wave-length of this line being given in the tables as $\lambda_{0}$. Form a glow discharge in a cool and rarefied sample of this vapor; the line will be very narrow, extending, say, between two wavelengths $\lambda_{0}-x$ and $\lambda_{0}+x$, where $x$ is only a few millionths of $\lambda_{0}$. Form an arc discharge in a dense sample of this vapor; the line will be very broad, extending, say, between wave-lengths $\lambda_{0}-y$ and $\lambda_{0}+y$, where $y$ may be hundreds of times as great as $x$. The light, however, will be emitted from the "core" or central portion of the gas containing the are, and this core will be surrounded by cooler and relatively less excited gas. Atoms in this region will absorb photons of the same wave-lengths as they could emit, which is to say, photons of wave-lengths between $\lambda_{0}-x$ and $\lambda_{0}+x$. The photons of the middle of the broadened line, those having the wave-length assigned to the line in the tables, will be absorbed before they escape from the region of the arc, while those of the "wings" of the broadened line will make their exit without impediment. The result is the same as if the are, instead of emitting the line $\lambda_{0}$, emitted two lines one on each side of it! This is very serious in cases where the light 
is to be used to produce a resonance-effeet or some other which only the wave-length $\lambda_{0}$ ean have. The effeet is known as "self-reversal."

The eleetric spark is a souree of light distinguished by high voltage and high current density, qualities bought at the sacrifice of duration. Its spectrum displays lines of the gas through which it passes and lines of the elements to be found in the electrodes between which it springs. Some of these are not found in the ordinary are spectra of these elements and are known as "spark lines." Shortly after the advent of Bohr's atom model, it was realized that these are speetrum lines of atoms which have lost one or more of their normal quota of electrons, the transitions responsible for these lines taking place among the electrons remaining. Conditions in the spark are evidently more favorable than those in the are for maintaining a pereeptible pereentage of such ionized atoms in excited states. Of late years the study of these lines has grown enormously, partly because of the development of the technique of spectroseopic researeh in the far ultra-violet (Sehumann and Lyman regions) where most of them-though not, of eourse, the early-discovered spark lines-are to be found; partly beeause they ean now be produced in steady glow diseharges of moderately high voltages with independently heated cathodes. It is even possible to map the speetra of eertain atoms (tellurium for instance) which lack no fewer than eight of their normal quota of electrons! There are, for instance, no fewer than seven known spectra of tellurium, designated in their order by the symbols Te I (i.e., tellurium I), Te II, . . Te VII, in which the Roman numeral stands for one more than the number of missing electrons. There are consequently also no fewer than seven speetra-all of them known, and designated as palladium I, silver II, eadmium III, indium IV, tin V, antimony VI, tellurium VII--corresponding to various atoms all with the same number of electrons, i.e., 46, and nuclear eharges rumning from 46 to 52 , inclusive. The detailed comparisons between all of these spectra are of great importance for atomic theory.

\section{PRODUCTION OF LIGHT OF THE OPTICAL SPECTRUM: OPTICAL MEANS}

When a beam of light of frequency $\nu$ falls upon a eool, rarefied, and unexeited gas, the results depend upon whether or not $\nu$ coineides with the frequeney of one of the absorption lines. If there is no eoincidence, we observe the phenomenon of seattering-with or without change of frequeney-which will be the topie of subsequent sections. If there is coincidence, some of the photons will be absorbed by atoms, putting these into exeited states. From these excited states the atoms may spontaneously return to normal, emitting photons exactly like those which they absorbed. This is the simplest of all cases of the produetion of light by light, the new kind being identical with the old. The atoms may, 
however, pass spontaneously into other states, intermediate between the normal state and the one into which they were transferred by the absorption; they then emit photons of lesser energy and lesser frequency than those which they absorbed. This is a more complex case of production of light, the new kind being of different quality from the old.

The former phenomenon is most striking in such a case as that of sodium vapor irradiated by the yellow line $\lambda 5896 \AA$. The atoms are transferred into an excited state which is "next" in energy to the normal state, in the sense that there is no state of intermediate energy. It is therefore impossible for them to quit their excited state in any other way than by radiating the same kind of photon which they received (unless they have interactions with other atoms or other photons, as I shall later mention); and the emission of $\lambda 5896$ in all directions from a tubeful of sodium vapor, into which a beam of such light is projected from a single direction, can be spectacular. Another case in which this effect is very strong is that of mercury vapor irradiated by the ultra-violet line $\lambda 2536$, although here the excited state is not next (in the foregoing sense) to the normal state, and the invisibility of the light deprives the phenomenon of its spectacular quality. In general, one expects a conspicuous effect of this kind when a vapor is irradiated by the first line (i.e., the line of lowest frequency) of its absorption series; $5896 \AA$ of sodium and $2536 \AA$ of mercury are of this class. These are known as cases of "resonance radiation," the first line of an absorption series being called the "resonance line."

The more complex phenomenon-the production, by light eapable of transferring atoms from the normal state $N$ to an upper excited state $A$, of photons due to transitions from $A$ to other excited states $B, C, D, \ldots$ intermediate (in energy) between $N$ and $A$-can easily be demonstrated. It is most striking with gases in which the atoms are joined into diatomic molecules, on account of the extraordinary profusion of stationary states having encrgies very close together and the consequent extraordinary profusion of lines. Thus, if sodium vapor or iodine vapor (both of which contain a large proportion of diatomic molecules $\mathrm{Na}_{2}$ or $\mathrm{I}_{2}$, as well as atoms $\mathrm{Na}$ or I) be irradiated by a beam of monochromatic light of almost any frequency whatever (in the visible or ultra-violet) the chances are very good that this frequency will be capable of transferring the molecules into some stationary state from which they will then be capable of making transitions into dozens, nay even hundreds, of intermediate states. The single irradiating frequency will then evoke a "resonance spectrum" of dozens or hundreds of lines; and another irradiating frequency differing from the first one by only a few parts in a hundred thousand will evoke another resonance spectrum of dozens or hundreds of totally different lines. 
From my description of the mechanism of the production of light by light, the reader will infer that the produced light is always of lesser frequency and greater wave-length, or else of the same frequency and wave-length, as the producing light. This is a nearly but not quite universal rule of experience; it was discovered long before the advent of the current notions of light and atomic structure and bears the name "Stokes's law."

The exceptions to Stokes's law do not invalidate these current notions but are brought about by interesting special causes. With molecular gases there is no single state sufficiently distinguished from its neighbors to be called "the normal state." Instead, the state of lowest energy may have dozens of neighboring states, whereof the energy values exceed its own by so little that in a gas at room temperature there are great numbers of molecules in each of them. Say that in order of increasing energy these states are denoted by $N_{0}, N_{1}, N_{2}, \ldots$. It is then possible for the following pair of events to occur: a molecule in, say, state $N_{2}$ absorbs a photon of energy $h \nu$ and is thereby transferred into an excited state $A$, from which it subsequently makes a spontaneous transition into the state $N_{0}$. The photon emitted in this second event has an energy which is greater than that of the original photon by the amount of the energy difference between the states $N_{0}$ and $N_{2}$; the produced light is of higher frequency than the producing light, and there is an exception to Stokes's law, sometimes called an "anti-Stokesian" line. Exceptions of another type will be mentioned presently.

Another interesting phenomenon occurs when the irradiating light is very intense. Imagine an atomic gas, and consider four of the stationary states of its atoms: the normal state $N$ and excited states $A, B, C$. Suppose the gas illuminated by light of two frequencies simultaneously: the frequency $N A$-by which I mean that of which the photon energy is just equal to the energy differenee between $N$ and $A-$ and the frequency $A C$. The photons of the former will transfer the atoms which they strike into the state $A$. The photons of the latter will have no effect on normal atoms; but if one of them happens to strike an atom which is in the state $A$ by virtue of having just been struck (within a very short time interval-see below) by a photon of the former frequency, it may be absorbed and transfer the atom into state $C$. From this state the atom may proceed spontaneously to state $B$, so that emission of the line $B C$ will be produced by simultaneous irradiation with the two frequencies $N A$ and $A C$, though not by irradiation with either frequency separately. Now suppose that the intensity of both of the irradiating lines is doubled. The intensity of the line $B C$ emitted from the gas will then be quadrupled; for the chance of a given atom being struck by a photon of the frequency $A C$ within a short time interval after it is struck by a photon of the 
frequency $N A$ will be proportional to the product of the numbers of both kinds of photons. However, the intensity of such a line as would be designated by the symbol $A N$, emitted by virtue of a spontaneous transition occurring after a normal atom has been struck by a photon of the frequency $N A$ and not requiring the collaboration of a second photon, will be doubled. This difference in the behavior of the two kinds of lines has been observed.

The "short time interval" of which I have just spoken is the "life" or duration of the excited state-the state $A$, in this illustration. If we could put a large number of atoms into the same excited state and measure in each individual case the length of time elapsing before it leaves this state by a spontaneous transition into some other state of lower energy, we should almost certainly find that these time lengths are not all the same but are distributed according to the exponential law or "law of chance." The mean time length or "mean life" can be measured, or at least estimated, in a variety of ways, and for excited states such as we have been considering it is of the order of $10^{-8}$ sec.- - a very small value indeed! The reader must not, however, infer that if he were to project a beam of, say, the resonance line of sodium into sodium vapor, and were to interrupt it with infinite suddenness at a certain instant, the vapor would absolutely cease to emit photons of the resonance frequency within a few times $10^{-8}$ sec. after that instant. The photons emitted by some of the atoms are captured by others, absorbed, reemitted, sometimes absorbed and reemitted yet again and again; and this process of handing around the energy from atom to atom may go on for so long that the vapor in a tube of ordinary size is still emitting photons for several hundred times $10^{-8}$ sec. after the irradiation is discontinued. For the same reason, it may be observed that when a sharply defined narrow beam of light is sent into the vapor of which it is the resonance line, the vapor emits similar photons not merely from the path of the beam but also from some distance outside of the beam.

Peculiar and important phenomena occur when a mixture of gases is irradiated with light which one of them is capable of absorbing and another is not. For definiteness consider the first of them to be discovered, which happens when mereury and thallium vapors are mixed and the mixture is illuminated with the resonance line of mercury, $2536 \AA$. As the term "resonance line" signifies, the photons of this light are capable of transferring mercury atoms from the normal state $N$ to a certain excited state $A$, because their energy is equal to the energy difference $(4.88 \mathrm{Ev}$ ) between the states $N$ and $A$. Falling on mercury vapor, they cause the emission of identical photons; but falling on pure thallinm vapor they cause no emission at all, for thallium atoms have no stationary state $4.88 \mathrm{Ev}$ above the normal, and therefore cannot absorb 
these photons nor be excited by them. Yet it is observed that when exposed to the irradiation, the mixture of vapors emits not only the line $2536 \AA$ of mercury, but several lines of the spectrum of thallium! and the like effect occurs when the vapor mixed with the mercury, instead of being thallium, is any one of a number of different elements (for instance, cadmium, silver, lead, bismuth, indium, zinc).

It appears therefore that there is a mechanism of direct transfer of energy between atom and atom, not involving the emission of a photon from one and its absorption by the other. This process occurs at collisions, and those at which it occurs are called "collisions of the second kind," a name very much open to criticism for its total lack of descriptive quality! In the cases which I have just mentioned, some of the energy absorbed from the incident light is reradiated in the form of light of different (usually lower) frequency, so that these may be considered as additional cases of the production of light by light. Another case of this type is displayed by sodium: here we have a normal state $N$ and two excited states $A_{1}, A_{2}$ very close together, the transitions between $N$ and $A_{1}$ and those between $N$ and $A_{2}$ corresponding to the famous yellow $D$ lines of sodium; when rarefied sodium vapor is irradiated by either $D$ line, photons of the same line only are emitted, but when the same vapor is mixed either with hydrogen or with a much larger quantity of sodium, the other line appears, proving that collisions between two sodium atoms or between one sodium atom and a hydrogen molecule are capable of transferring one of the atoms from the state $A_{1}$ to the state $A_{2}$, or vice versa!

Even in these cases, not all of the energy given up by the primarily excited atom reappears as light. Sometimes none of it reappears as light, and we have, not a special case of the production of light by light, but a special case of the absorption of light with its total conversion into some other form of energy. Thus when a mixture of mercury vapor and hydrogen is irradiated with $2536 \AA$, the excited mercury atoms are able to dissociate the hydrogen molecules; this is especially interesting, since light of this wave-length is not able to dissociate hydrogen molecules without the aid of such an intermediary. (There are, however, numerous cases of molecules which can be dissociated by direct absorption of light ${ }^{12}$ ). Again, the excited atoms may ionize atoms of the other kind present in the mixture. There are also many chemical changes which may be brought about by excited atoms, though the light which excites them cannot by itself bring on the changes which after excitation they are capable of producing; but this idea is no novelty to anyone acquainted with photochemistry.

${ }^{12}$ See, for instance, an article of mine in Chemical Reviews 5: 451-466. 1928; the subject has, however, been much developed since then. 
The general term for the production of light by light is, of course, the familiar word "fluorescence," which is often applied to most if not to all of the phenomena described in this section. "Phosphorescence" is a word applied by tradition to cases in which the emission of light endures for a readily perceptible time after the irradiating light is cut off. It is not exhibited by gases and therefore is still comparatively obscure. It appears to be most strikingly (if not exclusively) exhibited by mixtures in which one component is present in very small proportion compared with another.

\section{SCATTERING WITHOUT CHANGE OF FREQUENCY}

As has just been said, a beam of monochromatic light traversing a gas provokes the emission, from the gas, of photons of identical frequency, provided that the common frequency of incident and of emitted light is coincident with that of one of the absorption lines of the gas. We interpret this as meaning that photons of the incident beam are absorbed by the atoms and put these into excited states, from which they return to the normal state by emitting photons identical with the absorbed ones.

An apparently similar phenomenon occurs when there is no coincidence between the incident frequency and that of any of the absorption lines: it is known as "scattering without change of frequency." It is very much feebler than the other; in order to perceive it with the eye under laboratory conditions, one must send a very strong beam of light through dust-free gas in a chamber with blackened walls, and look at the path of the beam from the side with a well-rested and dark-adapted eye. Dust in the gas will conceal the effect entirely by superposing on it a very much stronger scattering from the dust particles.

Scattering without change of frequency is one of the phenomena of light which are most readily interpreted by the classical undulatory theory. The electrons in the atoms are supposed to be set into forced vibrations by the cyclically varying electric field in the light waves, and thereby to become radiators of light themselves. If the intensity of the light is held constant and its frequency is varied, the amplitude of the forced vibrations of the electrons should increase rapidly with frequency (as the square thereof). This accounts for the blue color of the clear, fog-free, and dust-free sky; for the color is chiefly dominated by that part of the visible spectrum for which the scattering is strongest, and that is the blue part. In the $\mathrm{X}$-ray region of the spectrum, the agreement of the classical theory with observation is so good that estimates of the total number of electrons in atoms of various metals were made over a quarter of a century ago from observations on the intensity of the scattering of X-rays, these two being proportional to one another and the factor of proportionality being given by the classical theory. (This last state- 
ment is, however, valid only if the energy of the photons is considerably greater than the binding energies of the various classes of electrons in the atoms.)

With the corpuscular image there is no such elegant interpretation of scattering without change of frequency. One cannot say that the incident photon of frequency $\nu$ is absorbed, raises the atom to a stationary state, and is then reemitted, because we are dealing with cases in which there is no stationary state of energy superior by $h \nu$ to that of the normal state. It is not altogether satisfactory to say that the incident photon simply bounces off the atom, for this picture is inadequate for some of the finer details of the process. One may say that the incident photon is absorbed, raises the atom to a "virtual" state, and is instantaneously reemitted; while this sounds like a mere trick of words, it has certain advantages in respect of the next process to be considered.

\section{SCATTERING OF LIGHT WITH CHANGE OF FREQUENCY : RAMAN EFFECT; SELECTION PRINCIPLES}

There are two types of scattering with change of frequency, one of which is observed in the optical spectrum, the other in the X-ray and gamma-ray regions.

So far as the change of frequency is concerned, the former of these processes - the Raman effect-may be described by a simple picture of the corpuscular type. A photon of energy $h \nu$, entering a molecular gas (the effect is almost confined to molecular gases, and to liquids and solids) of which the molecules have an excited state $A$ of which the energy reckoned from the normal state has a value $E_{a}$ less than $h \nu$, impinges on one of these molecules in its normal state; it spends the portion $E_{a}$ of its energy in exciting this molecule, and flies off with the remainder of its energy $\left(h \nu-E_{a}\right)$; owing to this reduction in its energy, its frequency is reduced from $\nu$ to $\left(\nu-\frac{E_{a}}{h}\right)$. Thus when a molecular gas is illuminated by a beam of monochromatic light, the light emitted from the gas contains various spectrum lines, each of which is shifted downward in frequency from the incident light by an amount equal to the energy value of some excited state of the molecule divided by $h$. (Cases also occur of lines shifted upward in frequency, for a reason which the reader can easily derive from the explanation given of "anti-Stokesian lines" in a previous section.)

Objection may be made to this image of a photon striking a molecule and bouncing off with reduced energy, on the ground that we usually think of photons with different energy as being essentially different photons. However, this is probably not a sound objection; when a material object, a tennis ball, for instance, rebounds from the ground to a 
slightly smaller height than that from which it was dropped, we do not think of it as a different ball-we think of it as the same ball with a different kinetic energy and a different momentum; and it may be expedient to train ourselves to think in the same way of photons. A more serious objection is that this image is necessarily inadequate, because it does not account for the finer details of the process. I will speak of one of these, which gives me opportunity to mention a feature of stationary states and spectra not as yet introduced into this essay.

To take the simplest case: it is easy to pick out, from among the many stationary states of any atom, a group of three states which have peculiar interrelations. I will denote the members of any such triad by $S, P, D$, and take the normal state as $S$. The peculiar interrelations are these: in the emission spectrum of the gas constituted of these atoms appear the lines $P S$ and $D P$ due to transitions from $P$ to $S$ and transitions from $D$ to $P$, and in the absorption spectrum of the cool and rarefied gas appears the line $S P$, while in the absorption spectrum of the gas when excited by an electric discharge appears the line $P D$; but the line $D S$ never appears (or perhaps appears only under very abnormal conditions) in the emission spectrum, and the line $S D$ never appears in the absorption spectrum. Direct transitions in both senses occur between $S$ and $P$, and between $P$ and $D$; but direct transitions in either sense between $S$ and $D$ do not occur-they are said to be "forbidden." The $P$ state is a sort of necessary stepping-stone between the $S$ state and the $D$ state; the atom cannot pass between these two without making use of it (or some other like it); it alone is accessible from both $S$ and $D$.

Now it is a remarkable feature of the Raman effect, that when the incident photons cause the atoms-or rather, the molecules-to make transitions from the normal state to various excited states, the transitions which occur are generally of the class $S D$ ! This suggests that the sentence in the section about scattering without change of frequency, "One may say that the incident photon is absorbed, raises the atom to a 'virtual' state, and is instantaneously reemitted," may not be a mere trick of words after all; for perhaps when scattering with change of frequency occurs, it is necessary for the photon to put the atom momentarily into a state which shares the character of the $P$ state aforesaid, in that it is a necessary stepping-stone from the initial normal state to the final excited state. If a photon is totally absorbed, the atom goes from the $S$ state to the $P$ state and stays there for the $10^{-8}$ sec. or thereabouts mentioned previously; but if only part of the energy of the photon is to be absorbed, the atom goes from the $S$ state to a sort of "virtual $P$ state," and then without pause goes on to the $D$ state. This picture, like all pictures, is still inadequate; but at least it is more nearly adequate than that of the photon merely bouncing off the molecule. (Note that 
the "virtual $P$ state" must be supposed to have energy superior to that of the $D$ state, though in the previous paragraph the real $P$ state is supposed intermediate between $S$ and $D$, as is usually the case.)

I must remove the impression possibly left by my phrasing, that an atom or molecule has only three states with these peculiar interrelations. As a matter of fact, there is a great number of $S$ states, a great number of $P$ states, a great number of $D$ states, and various other categories; transitions can occur between any $P$ state and any $S$ or $D$ state, but they cannot occur between any $S$ state and any $D$ state, or between any two states distinguished by the same letter. The art of analyzing spectra consists largely in classifying states according to their relations of permitted or forbidden transitions with other states. The rules of the classification are called "selection principles" and in many cases are admirably explained by the contemporary atom models.

\section{SCATTERING OF X-RAYS WITH CHANGE OF FREQUENCY: COMPTON EFFECT}

We have already become acquainted with two of the interactions between $\mathrm{X}$-rays and matter: true absorption in atoms, and scattering without change of frequency. A third and important interaction has features in common with the former, and also with the Raman effect which we have just been considering. Like true absorption, it involves the ejection of high-speed electrons from the matter; but these are originally the most loosely bound of the electrons in the atoms, or perhaps some of them are actually the "free" electrons roaming in the substance (there is no sharp distinction between these two classes), while true absorption often involves the ejection of tightly bound electrons such as the $K$ electrons. As in the Raman effect, the photons themselves are not totally absorbed but reappear with lessened energy and lessened frequency.

This Compton effect may be interpreted as an elastic impact between corpuscles of matter and corpuscles of light, and as such it is the most beautiful example of a phenomenon amenable to an extreme corpuscular explanation and the most beautiful demonstration of equations (1) and (2) as applied to light. I now develop the equations of elastic impact between electrons and photons, employing the expressions for energy and momentum of material particles (such as electrons) which are used in relativistic mechanics, ${ }^{13}$ and assuming the electrons to be initially stationary and free. The symbols $\nu, \lambda$ refer to the incident $\mathrm{X}$-rays (photons before impact); $\nu^{\prime}, \lambda^{\prime}$ to the scattered $\mathrm{X}$-rays (photons after

${ }^{13}$ As many a physicist must have found out for himself, the attempt to make the equations look simpler and be more easily solvable by using ordinary mechanics has precisely the opposite effect from that desired. The theory of the Compton effect affords one of the cases where the wisest policy is also the easiest. 
impact); $u$ stands for the speed of an electron after impact; $\phi$ and $\theta$ for the angles made with the direction of the incident photon by those of the recoiling electron and the scattered photon, respectively.

Conservation of energy requires that the energy of the incident photon, $h \nu$, be equal to the sum of the energy of the scattered photon, $h \nu^{\prime}$, and that of the recoiling electron, which latter in relativistic mechanics is given by the expression

$$
E=m c^{2}=\frac{m_{0}}{\left(1-\overline{\left.\beta^{2}\right)^{1 / 2}}\right.} c^{2}
$$

where $m_{0}$ stands for the rest mass of the electron and $\beta$ for $u / c$. Thus,

$$
h \nu=h \nu^{\prime}+\frac{m_{0}}{\left(1-\beta^{2}\right)^{1 / 2}} c^{2}
$$

Conservation of momentum requires the following two equations, referring respectively to the momentum components parallel and perpendicular to the direction of the incident photon, in the plane common to the three directions of incident photon, scattered photon, recoiling electron:

$$
\begin{aligned}
\frac{h \nu}{c} & =\frac{h \nu^{\prime}}{c} \cos \theta+\frac{m_{0} u}{\left(1-\beta^{2}\right)^{1 / 2}} \cos \phi \\
0 & =\frac{h \nu^{\prime}}{c} \sin \theta+\frac{m_{0} u}{\left(1-\beta^{2}\right)^{1 / 2}} \sin \phi
\end{aligned}
$$

Eliminating $\phi$ and $u$ between these three equations, we arrive at a relation between $\nu^{\prime}, \nu$, and $\theta$, which may immediately be translated into the following relation of simpler aspect:

$$
\lambda^{\prime}-\lambda=\frac{h}{m c}(1-\cos \theta)
$$

This is a prediction of the wave-length which should belong to the $\mathrm{X}$-rays scattered at angle $\theta$; that is to say, an observer placing his detecting apparatus somewhere along the line leading outward from the scattering substance at an angle $\theta$ to the incident beam should detect $\mathrm{X}$-rays of the wave-length given by formula (23). This prediction is verified by experiment. Eliminating $\theta$ and $\nu^{\prime}$ between equations (20), (21), and (22), one gets a relation between $u, \nu$, and $\phi$. This may be translated into a prediction of the energy which should belong to electrons recoiling in the direction $\phi$; that is to say, an observer placing a device for detecting electrons, somewhere along the line leading outward from the scattering substance at an angle $\phi$ to the incident beam, should observe electrons 
of the energy given by the relation in question. This prediction also is verified (sometimes by using the Wilson chamber, observing the electron tracks which start off in various directions from atoms of the gas in the chamber, estimating the corresponding electron energies and correlating them with the corresponding values of $\phi$ ).

In general, when a beam of X-rays or gamma rays falls upon a piece of matter, some of the electrons which fly out are "Compton electrons" which acquire their energy in the foregoing matter, and some are photoelectrons which have been ejected from atoms by total absorption of $\mathrm{X}$-ray quanta such as I described in a previous section. Compton electrons are relatively more abundant the higher the frequency of the $\mathrm{X}$-rays and the lower the atomic number or atomic weight of the scattering element, i.e., the higher the ratio of the energy of the photons to the energy required to extract electrons from the atoms. Also, when a beam of $\mathrm{X}$-rays or gamma rays falls upon a piece of matter, some of the photons which emerge are the photons of reduced frequency recoiling from impacts with electrons, of which we have just been thinking; some are photons scattered without change of frequency; some are $\mathrm{X}$-rays emitted from atoms as an aftereffect of the extractions of electrons by incident photons. The reduction in intensity which a beam of X-rays or gamma rays suffers in traversing a stratum of matter is largely due to these three processes jointly; the two former, as compared with the last-named, are relatively more prominent the higher the frequency of the incident rays and the lower the atomic weight of the scattering substance. With gamma rays of great energy, a novel process enters upon the scene.

\section{TRANSMUTATION BETWEEN ELECTRON-PAIRS AND PHOTONS}

It is fitting to end this essay by a brief allusion to one of the most striking phenomena of Nature, unguessed as lately as three years ago. In addition to the negative electrons of which so much has just been said, there are positive electrons, first observed in 1932 among the cosmic rays. These are apparently identical with those of the negative variety in every respect except the sign of their charge. They are observed immediately as they come into being, for they may be created in the laboratory by the transmutation of light into electricity. When a beam of gamma rays (the photons must have energy superior to about one MEV) is projected against a stratum of matter, pairs of electrons of opposite sign spring forth. Each pair is formed out of a photon. The mass and the energy of the photon are converted almost totally into mass and energy of the two electrons, a trifling fraction being transferred to an atom in order to satisfy the law of the conservation of momentum. The reverse process occurs when positive electrons are fired into a stratum 
of matter: they merge with negative electrons, and the two oppositely charged electrical particles which combine in such a merger are converted into one or two particles of light. A portion of the reduction in intensity which a beam of high-energy gamma rays suffers in traversing a stratum of matter is due to the first of these processes, and a portion of the "scattered" radiation arises from the second.

Manuscript received by the editor September, 1934. 


\title{
MEASUREMENT OF X-RAYS AND RADIUM
}

\author{
LAURISTON S. TAYLOR
}

Department of Commerce, National Bureau of Standards, Washington, D. C.

Production of X-rays. The measurement of X-ray quality. Secondary methods of expressing X-ray quality. Standard measurement of X-ray quantity or intensity. Recommendations concerning $X^{X}$-ray standards adopted by the National Laboratories: Measurement of quality-Measurement of the roentgen-Calibration of dosemeters. Types of standard chambers. Standard measurement of ionization currents. Secondary ionization chambers: Wethod of calibration. Types of thimble chamber roentgenometers. Measurement of X-ray output. Comparison of X-ray generators. Measurement of very low and very high voltage $X$-rays: Grenz rays (3 to $15 \mathrm{kv}$.). Ultra-high voltage $X$-rays. Radium measurement.

\section{THE PRODUCTION OF X-RAYS}

$X$-ray Tubes.-As pointed out in other chapters, X-rays are produced by the sudden stoppage or deceleration of fast moving electrons. In practice, this effect is brought about in an evacuated tube having two electrodes between which a high potential difference, $E$ rolts, may be applied. Any electrons in the space between the electrodes are thus moved toward the positively charged eleetrode or target, with a velocity $v$, which is given approximately by the relation $v=5.95 \sqrt{E} \times 10^{7}$ $\mathrm{cm}$./sec. Upon striking the target, the electron is brought to rest in a relatively short time and an $\mathrm{X}$-ray produced which may have a minimum wave-length $\lambda_{\min }=12354 . \div E$, where $\lambda$ is expressed in Angströms $\left(10^{-8} \mathrm{~cm}.\right)$.

In the ordinary high-vacuum tube, the number of free electrons in the interelectrode space is negligibly small. In the older type of gas $\mathrm{X}$-ray tube, electrons in sufficient number were released from the eathode by the bombardment of positive ions produced in the eleetrical discharge. However, it was difficult to maintain steady gas pressures within the tube, and hence the positive ion and electron eurrents were unsteady and uncertain. In the present-day Coolidge tube (9), eleetrons are produced by means of a hot cathode in a tube so highly evacuated that the small amount of residual gas plays no important part in its operation. The electrons released from the filament by thermionic emission are then accelerated in the electric field and a steady space current ensues. 
High-voltage Generators.- The Coolidge tube also has the properties of a rectifier-permitting current to pass through it in only one direction - and hence, for accelerating the electrons, it may be used directly on a high-voltage transformer. However, larger electron currents and higher voltages may be used with less stress on a given tube when the voltage is rectified before being applied to the tube. A number of such rectifying circuits with the corresponding resultant voltage wave forms are shown in Fig. 1. The voltage relationships indicated on the figures are approximate. If, in the full-wave circuit $A$ two diametrically opposite rectifying tubes, $a$ and $c$, are removed, a half-wave generator results, and although

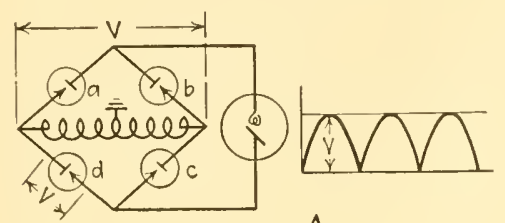

A

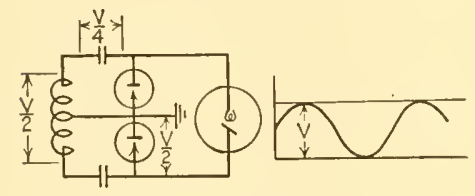

C
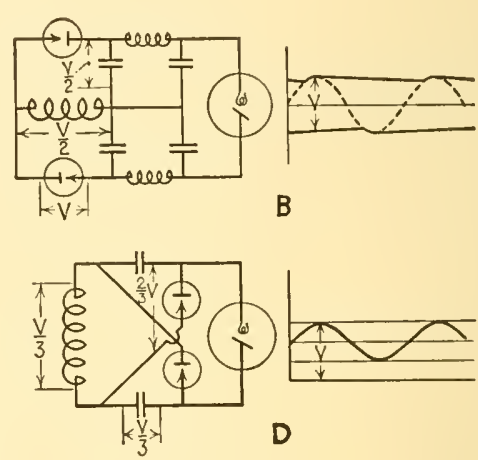

F1G. 1.-Typical X-ray generator circuits. $a$, Full wave; $b$, constant potential (Hull); c, voltage doubling (Villard); $d$, voltage tripling (Witka). (See Table 7 for comparative outputs.)

the voltage stress per valve tube is reduced to half, the circuit is not so well adapted for large currents (over $100 \mathrm{ma}$.). In circuit $B$ the ripplage is approximately proportional to the tube current and inversely proportional to the square of the capacity and the cube of the frequency. In circuits $C$ and $D$, the effect of decreasing the capacity or increasing the current is to effectively raise the time axis, cutting off the bottoms of the waves. Circuits of types $C$ and $D$ are, in general, therefore, only economical for relatively small currents-less than 10 to 20 ma. - the cost of condensers becoming prohibitive beyond that point.

Owing to space charge in the $\mathrm{X}$-ray tube, the electron current is not, in general, proportional to the voltage but approaches a saturation value depending principally upon the electron emission of the filament and the geometrical structure of the tube. Without going into detail, it is therefore evident that the $\mathrm{X}$-ray emission varies with the wave shape of the applied voltage- a factor which at once complicates the control of $\mathrm{X}$-ray output as shown below. 


\section{THE MEASUREMENT OF X-RAY QUALITY}

Early X-ray Measurements.-Since the discovery of X-rays it was known in a general way that the strength or intensity was proportional to the electron current passing through the tube, and the penetration or hardness was at least remotely related to the potential between the tube electrodes. As experimental technique and tube construction developed, it was found that current and voltage alone were insufficient for describing an X-ray beam, and more elaborate means were required. Later, as knowledge of the subject increased, the ideal method given below was established - only to be left in abeyance because of the practical difficulties involved.

It was early realized that voltage alone did not accurately specify the penetration of X-rays because of two principal reasons: $(a)$ the uncertainty in measuring the voltage, and $(b)$ the wide variations in voltage wave form. Consequently, the penetration of X-rays soon came to be measured in terms of their relative absorption in different metals. Best known of such devices was Benoist's penetrometer (4) of 1902, which consisted of a thin silver disk surrounded by 12 numbered aluminum sectors of which the thickness varied in equal steps up to $12 \mathrm{~mm}$. Laid on a photographic plate and placed in the X-ray beam, that sector was noted through which the transmitted radiation was the same as for the silver center (as shown by the darkening of the emulsion). The thickness of the matching sector obviously increases with the hardness or penetration of the radiation. It is interesting to note (see below) that present-day methods of measuring quality differ from early mainly in the matter of refinement.

Various effects known to be related to the intensity of an X-ray beam will be briefly noted. Attempts have been made to utilize all of them for practical measurements, although most of them have proved to be unfeasible. The ideal method for measuring the intensity or energy of an X-ray beam is with a thermal or heat-measuring device such as a calorimeter which completely converts all radiation into heat (35). This is the only method known to give results independent of the radiation quality. Unfortunately, the energy available in an ordinary $\mathrm{X}$-ray beam of $1 \mathrm{~cm}^{2}$ cross section is of the order of $10^{-3}$ to $10^{-5} \mathrm{cal} . / \mathrm{sec}$, and to measure so small an amount of energy is exceedingly difficult even in the bestequipped physical laboratory.

Photographic methods are likewise extremely difficult because of the complicated laws of photographic blackening, and the involved technique necessary to control adequately the enulsion exposure and the conditions for development $(6,5)$. The action of $\mathrm{X}$-rays on selenium is similar to that of light, but the utilization of selenium cells in accurate intensity 
measurements is rendered difficult because of time lag and hysteresis in the cells. The many chemical effects produced by X-rays are all complicated and their relationship to the radiation intensity is seldom known except by calibration. In 1902, Holzknecht (22) introduced pastiles which, when exposed to X-rays, changed color to a degree depending upon the quantity of radiation absorbed. Attendant color-matching charts were calibrated in arbitrary units. Fluorescence in certain minerals has also been used in X-ray-intensity measurements but complicated fluorescence-excitation laws have prevented its general use.

Lastly, and most important, is the ability of X-rays to ionize gases. This property was recognized soon after their discovery, and in 1908 Villard (54) proposed a quantitative unit of X-ray intensity based on the ionization produced in air. Essentially, this unit is the one generally recognized today, and it will be discussed in detail below.

Ionization Produced by X-rays.-Of the various ways of measuring $\mathrm{X}$-ray intensity outlined in the foregoing, all but the calorimetric method depend in their indication upon the quality or spectral-energy distribution of the radiation. Therefore, for intensity measurement, it is necessary that the dependence on quality be known to a degree which is commensurate with the desired overall accuracy of the determination. Consequently, in measuring X-ray intensity, the method must be employed in which the dependence upon quality is best understood, or at least most readily reproduced and controlled. The power of X-rays to ionize gases renders the air-ionization method most suitable for practical purposes.

As already shown (cf. Darrow, Paper I), the amount, or degree, of ionization produced in air is directly proportional to the quantity of radiation absorbed by a given mass of air. As will be discussed in detail, the unit of X-ray quantity (the roentgen) is defined in terms of the degree of ionization produced in $0.001293 \mathrm{gm}$. of air. Therefore, since the absorption of X-rays by matter depends upon the wave-length of the incident radiation, it is evident that the unit of $\mathrm{X}$-ray quantity depends upon those wave-lengths composing the beam in question.

$X$-ray Spectral Distribution.-Corresponding to any value of a steady or instantaneous voltage applied to an X-ray tube is a particular distribution of energy among the wave-lengths of the resultant $X$-ray spectrum. Likewise, this distribution depends upon the material of the targetusually tungsten, in medical or biological application. Below a certain potential for a given target material (70 kv. for tungsten) the spectrum is continuous (Fig. 2) whereas above this critical value of the potential, certain series of lines appear superposed on the continuous background, which, in their spectral position, are characteristic of the target material. As the exciting potential is increased above the critical value, the propor- 
tion of the total energy contained in the lines increases, although it never becomes a major portion of the total. In addition, in passing through various filters, the wave-lengths composing the incident radiation are absorbed unequally according to known laws of absorption, so that the resultant spectral energy-distribution curve may be very different in general form from the initial.

It is, therefore, evident that to measure properly the quantity or intensity of an X-ray beam, the actual or effective spectral distribution of the measured radiation must first be known. For simplicity, the spectral distribution of an X-ray beam is referred to as X-ray quality.

Effect of Voltage Wave Form.-The direct determination of X-ray quality is complicated by a number of factors, most important of which is the fact that only rarely is the exciting potential of a truly steady value. Actually, the applied tube voltage is usually fluctuating over all ranges from zero-tomaximum to values steady within a few per cent of maximum (ripple voltage). Since, as noted, the spectral distribution or quality changes with voltage, if a varying voltage be applied to the

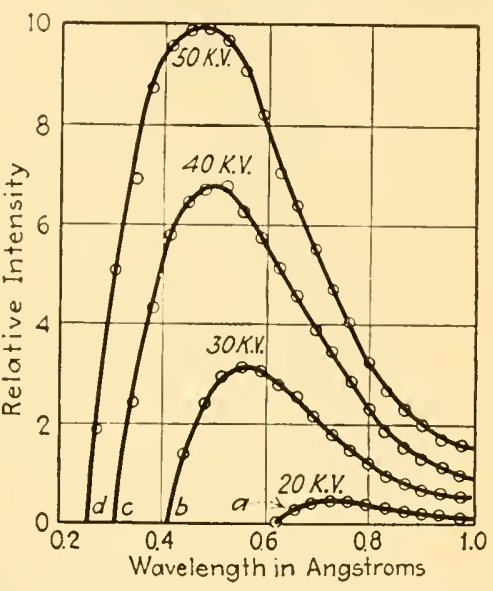

FIG. 2.-X-ray continuous spectra. (Ulrey.) tube, a composite spectrum results which is made up of all the component simple spectra corresponding to all instantaneous values of the impressed voltage.

For example, suppose a varying voltage as represented by $A$ in Fig. 3 be applied to an X-ray tube and that at time $t_{a}$ the voltage is $a=20 \mathrm{kv}$.; the spectral distribution is represented by curve $a$ in Fig. 2 (53). Similarly, at time $t_{b}$ the voltage is $b=30 \mathrm{kv}$., and the spectral distribution is given by curve $b$ in Fig. 2, and so on. If we are chiefly interested in $40-$ to $50-\mathrm{kv}$. X-rays, we note that they are present only between times $t_{c}$ and $t_{e}$ or less than one-third of the cycle. If, on the other hand, the voltage wave form is that shown in Fig. $3, B$, it is seen that the 40 - to $50-\mathrm{kv}$. X-rays are present from $t_{c}^{\prime}$ to $t_{e}^{\prime}$, or about four-fifths of the cycle. For the wave form shown in Fig. $3, C$, the spectral distribution lies always between curves $c$ and $d$ (Fig. 2). Although the peak voltage is the same in all three cases, a wave form such as $3, C$ gives a preponderance of the more penetrating radiation over wave forms $3, A$ and $3, B$.

Relationship of Voltage to Quality.-It is clear that, since the resultant or composite spectral distribution is the summation of the simple distribu- 
tions corresponding to the particular voltage wave form used, there can be no direct or simple relationship between the qualities of X-ray beams of very different voltage origin. On the other hand, when constant voltage is applied to a tube, the spectral distribution remains the same and is characteristic of that particular voltage. In such cases, it would be sufficient for fixing the quality to state only the target material, voltage, and filter. It is obvious from the foregoing discussions that peak voltages in particular may be very misleading. Under most practical conditions $(52,50)$ the effective (root mean square) voltage will give a much more nearly consistent measure of the quality, insofar as voltage measurements alone are concerned.

Direct determination of the spectral-energy distribution is possible only by means of the ionization spectrometer $(23,24)$. This process is,

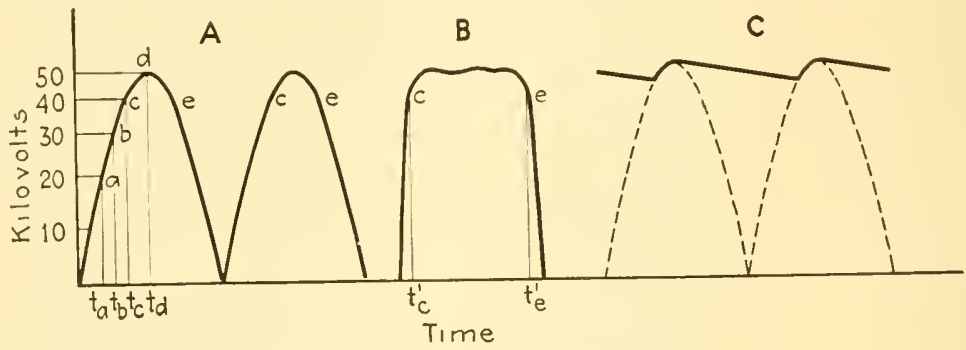

FIG. 3.-Typical X-ray voltage wave forms.

however, so complicated and laborious as to be precluded in practice. Consequently, indirect methods of quality determination are usually resorted to, most of them based upon the laws of X-ray absorption in copper and aluminum.

Complete Absorption Curve.-It has been shown theoretically by Silberstein (39) that a given X-ray spectral distribution will yield a discrete absorption curve (in, say, copper); and conversely, that from a given absorption curve can be derived the spectral distribution with sufficient accuracy for most practical purposes. Therefore, a statement of the complete absorption curve in copper or aluminum is taken as the quality of the radiation $(55,51)$.

To obtain the complete absorption curve, filters of the absorbing material (copper or aluminum) are successively placed in the X-ray beam and the transmitted intensities read with an ionization chamber (discussed in detail later on). If $I_{0}$ is the intensity of radiation, incident on a filter $f_{1}$, and $I$ the intensity transmitted through $f_{1}$, then $\log _{10} 100 \frac{I}{I_{0}}$ is the logarithm of the percentage transmission for filter $f_{1}$. Plotting the values of $\log _{10} 100 \frac{I}{I_{0}}$ against a number of values of $f$ then gives a complete 
absorption curve in its most useful form. Such a curve is shown by $A C B$ in Fig. 4, plotted on the $X-, Y$-axes for $\mathrm{X}$-rays excited by $150 \mathrm{kv}$. (constant) in a thin-walled cerium glass tube.

Until recently, the complete copper-absorption-curve method has had the disadvantage of not being expressible by a single numerical magnitude. This, however, has been removed $(50,51)$ by finding that, within reasonably satisfactory limits, the complete composite absorption curve of radiation excited by the different potential wave forms in use may be matched by the instantaneous absorption curve of the same

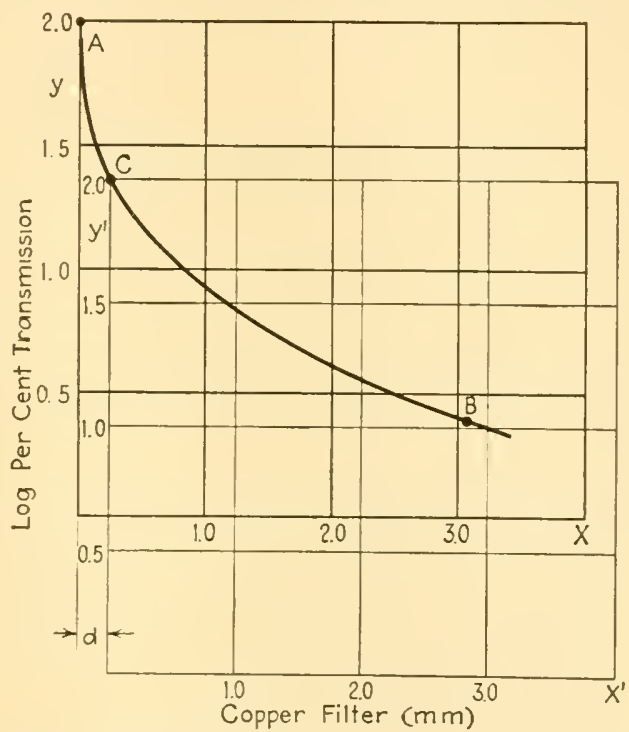

FIG. 4.- Hypothetical logarithmic absorption curves to show the effect of filtration.

material for radiation excited by some definite constant potential. It is further found that such beams having equivalent absorption curves are, over a considerable range of initial filtration, closely alike as regards their intensity clistribution in a large body of low-atomic-number material such as a water phantom. Since the spectral distribution of radiation excited by constant potential is perfectly reproducible, a family of complete absorption curves of constant voltage radiation would constitute a very convenient and adequate standard of reference for inferring the constant-potential equivalent ${ }^{1}$ of any given radiation.

Equivalent X-ray Beams.- It is, of course, obvious that two equivalent X-ray beams might be produced by voltages of very different peak

${ }^{1} \mathrm{By}$ "constant-potential equivalent" is meant the constant potential necessary to apply to an X-ray tube to yield an absorption curve of the same form as the composite absorption curve in the same material for the unknown radiation in question (51). 
values; hence one beam might have shorter wave-lengths, not present at all in the other. However, the proved equivalence, where the peak voltages are very different, signifies that while there are some shorter wave-lengths in one spectrum than in the other, they are present to a negligible extent. As the peak value of an exciting voltage approaches the equivalent constant voltage, it signifies that the energy in the shorter wave-lengths becomes relatively greater. This, however, is taken care of in the specification of quality by the fact that for a given peak voltage, if the shorter wave-lengths gain in importance, the equivalent voltage is correspondingly increased. For example, with $\mathrm{X}$-rays produced by mechanical, full-wave, or Villard rectifiers (Fig. 1) operated at the same

Table 1.-Absorption of General X-radiation in Copper

(Tube wall $1.29 \mathrm{~mm}$. cerium glass)

$I_{0} \equiv$ incident radiation; $I \equiv$ transmitted radiation; $100 I / I_{0}=$ percentage transmission

\begin{tabular}{|c|c|c|c|c|c|c|c|c|c|c|c|c|}
\hline \multirow[b]{2}{*}{$\begin{array}{l}\text { Copper filter, } \\
\text { (mm.) }\end{array}$} & \multicolumn{2}{|c|}{$100 \mathrm{kv}$} & \multicolumn{2}{|c|}{$110 \mathrm{kv}$} & \multicolumn{2}{|c|}{$120 \mathrm{kv}$. } & \multicolumn{2}{|c|}{$130 \mathrm{kv}$} & \multicolumn{2}{|c|}{$140 \mathrm{kv}$. } & \multicolumn{2}{|c|}{$150 \mathrm{kv}$. } \\
\hline & $\begin{array}{l}100 \\
I / I_{0}\end{array}$ & $\begin{array}{l}\log \\
100 \\
I / I_{0}\end{array}$ & $\begin{array}{l}100 \\
I / I_{0}\end{array}$ & $\begin{array}{l}\log \\
100 \\
I / I_{0}\end{array}$ & $\begin{array}{l}100 \\
I / I_{0}\end{array}$ & $\begin{array}{c}\log \\
100 \\
I / I_{0}\end{array}$ & $\begin{array}{l}100 \\
I / I_{0}\end{array}$ & $\begin{array}{l}\log \\
100 \\
I / I_{0}\end{array}$ & $\begin{array}{l}100 \\
I / I_{0}\end{array}$ & $\begin{array}{l}\log \\
100 \\
I / I_{0}\end{array}$ & $\begin{array}{l}100 \\
I / I_{0}\end{array}$ & $\begin{array}{l}\log \\
100 \\
I / I_{0}\end{array}$ \\
\hline 0 & 100 & 2.00 & 100 & 2.00 & 100 & 2.00 & 100 & 2.00 & 100 & 2.00 & 100 & 2.00 \\
\hline 0.14 & 20.40 & 1.310 & 22.08 & 1.344 & 23.80 & 1.377 & 25.82 & 1.412 & 27.65 & 1.442 & 30.35 & 1.482 \\
\hline 0.20 & 15.39 & 1.187 & 17.84 & 1.251 & 19.92 & 1.299 & 21.81 & 1.339 & 23.42 & 1.370 & 25.68 & 1.410 \\
\hline 0.25 & 12.89 & 1.110 & 15.18 & 1.181 & 17.37 & 1.240 & 19.19 & 1.283 & 20.85 & 1.319 & 22.85 & 1.359 \\
\hline 0.30 & 11.15 & 1.047 & 13.17 & 1.120 & 15.23 & 1.183 & 17.00 & 1.230 & 18.71 & 1.267 & 20.55 & 1.313 \\
\hline 0.40 & 8.78 & 0.943 & 10.28 & 1.012 & 12.12 & 1.084 & 13.75 & 1.138 & 15.37 & 1.187 & 16.98 & 1.230 \\
\hline 0.50 & 7.15 & 0.854 & 8.50 & 0.924 & 10.05 & 1.002 & 11.57 & 1.063 & 13.06 & 1.116 & 14.42 & 1.159 \\
\hline 0.75 & 4.53 & 0.656 & 5.77 & 0.761 & 7.04 & 0.848 & 8.32 & 0.920 & 9.55 & 0.980 & 10.68 & 1.029 \\
\hline 1.00 & 3.08 & 0.489 & 4.13 & 0.616 & 5.18 & 0.714 & 6.35 & 0.803 & 7.41 & 0.870 & 8.43 & 0.926 \\
\hline 1.50 & 1.77 & 0.248 & 2.42 & 0.384 & 3.13 & 0.496 & 4.00 & 0.602 & 4.86 & 0.687 & 5.75 & 0.760 \\
\hline 2.00 & 1.03 & 0.013 & 1.60 & 0.210 & 2.06 & 0.314 & 2.73 & 0.436 & 3.38 & 0.529 & 4.13 & 0.616 \\
\hline 2.50 & $\ldots \ldots$ & $\ldots \ldots$ & 1.02 & 0.009 & 1.46 & 0.164 & 2.00 & 0.301 & 2.50 & 0.398 & 3.12 & 0.494 \\
\hline 3.00 & & & & $\ldots$ & 1.09 & 0.037 & 1.48 & 0.170 & 1.93 & 0.286 & 2.49 & 0.396 \\
\hline
\end{tabular}

NoтE: All second-place decimal figures given in italics are approximate values.

peak values, the equivalent voltage approaches respectively nearer the peak value, indicating an increasing relative intensity of the shorter wave-lengths. Correspondingly, it is found that the absorption curves indicate respectively harder composite radiations after the same initial filtration.

It is also to be expected that two beams which yield like absorption curves with a low or moderate initial filtration [up to $1.5 \mathrm{~mm}$. Cu at 150 kv. (constant)], may, after a higher initial filtration, furnish somewhat divergent curves-the curve for the radiation having the higher peak voltage falling above the other. By matching the curves above the point corresponding to the initial filtration, however, a new equivalent voltage 


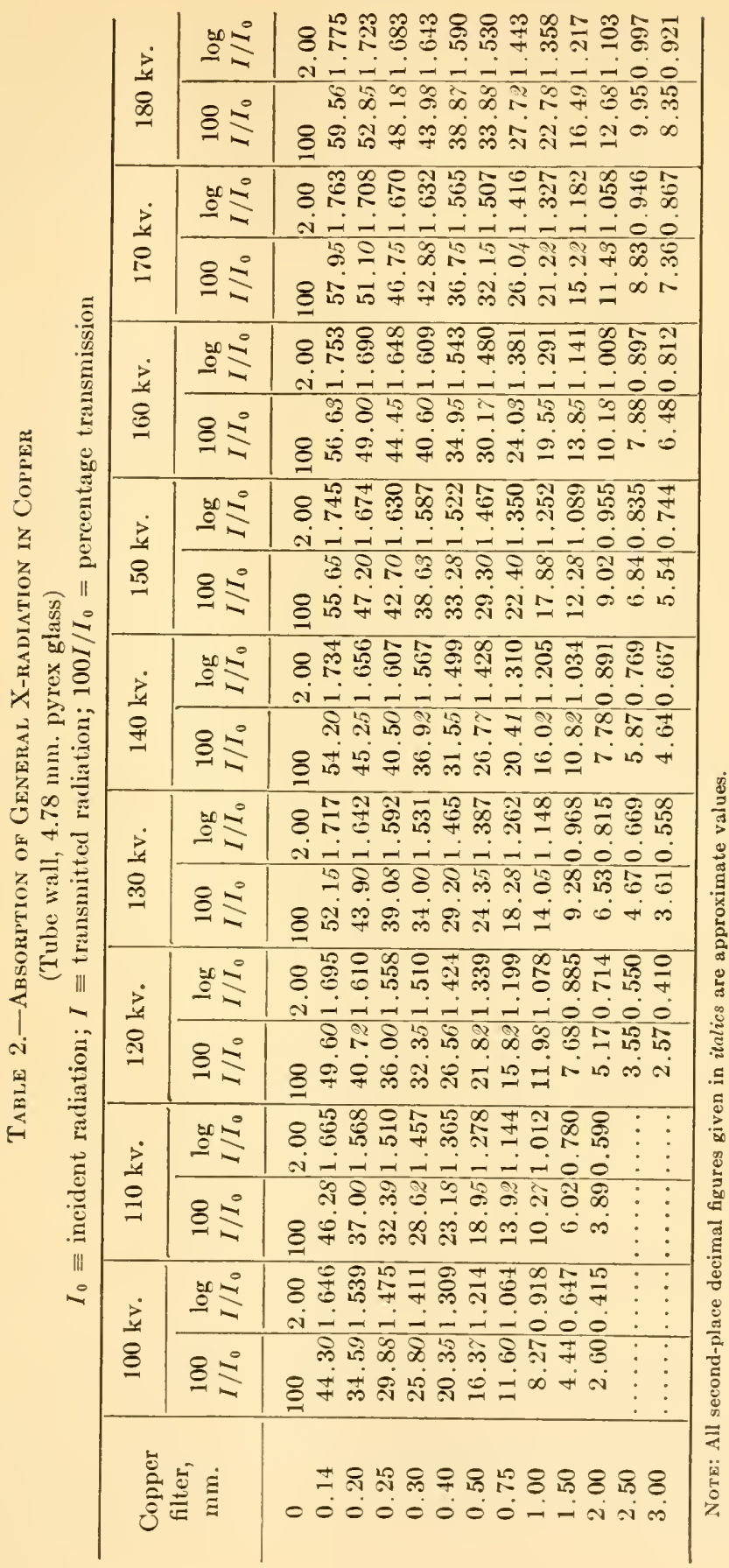


may be found which specifies the radiation more closely. This is perfectly justifiable, since the radiation not passing through the filter is lost.

Standard Absorption Curves from 60 to $180 \mathrm{Kv}$.- The specification of $\mathrm{X}$-ray quality, by using the complete absorption curve, has been recommended by the X-ray Standardization Committee of the Radiological Society of North America. ${ }^{2}$ (56).

\section{Table 3.-Absorption of General X-radiation in Aluminum}

(Tube wall, $1.29 \mathrm{~mm}$. cerium glass)

$I_{0} \equiv$ incident radiation; $I \equiv$ transmitted radiation; $100 I / I_{0}$ percentage transmission

\begin{tabular}{|c|c|c|c|c|c|c|c|c|c|c|c|c|}
\hline \multirow{2}{*}{$\begin{array}{l}\text { Aluminum } \\
\text { filter, } \\
\text { mm. }\end{array}$} & \multicolumn{2}{|c|}{$60 \mathrm{kv}$. } & \multicolumn{2}{|c|}{$70 \mathrm{kv}$. } & \multicolumn{2}{|c|}{$80 \mathrm{kv}$. } & \multicolumn{2}{|c|}{$90 \mathrm{kv}}$. & \multicolumn{2}{|c|}{$100 \mathrm{kv}$. } & \multicolumn{2}{|c|}{$110 \mathrm{kv}}$. \\
\hline & $\begin{array}{c}100 \\
I / I_{0}\end{array}$ & $\begin{array}{l}\log \\
100 \\
I / I_{0}\end{array}$ & $\begin{array}{c}100 \\
I / I_{0}\end{array}$ & $\begin{array}{l}\log \\
100 \\
I / I_{0}\end{array}$ & $\begin{array}{l}100 \\
I / I_{0}\end{array}$ & $\begin{array}{l}\log \\
100 \\
I / I_{0}\end{array}$ & $\begin{array}{c}100 \\
I / I_{0}\end{array}$ & $\begin{array}{l}\log \\
100 \\
I / I_{0}\end{array}$ & $\begin{array}{c}100 \\
I / I_{0}\end{array}$ & $\begin{array}{l}\log \\
100 \\
I / I_{0}\end{array}$ & $\begin{array}{l}100 \\
I / I_{0}\end{array}$ & $\begin{array}{l}\log \\
100 \\
I / I_{0}\end{array}$ \\
\hline 0 & 100 & 2.00 & 100 & 2.00 & 100 & 2.00 & 100 & 2.00 & 100 & 2.00 & 100 & 2.00 \\
\hline 1 & 32.1 & 1.507 & 33.88 & 1.530 & 36.04 & 1.557 & 38.90 & 1.590 & 40.39 & 1.606 & 42.42 & 1.628 \\
\hline 2 & 19.0 & 1.279 & 20.81 & 1.318 & 32.92 & 1.360 & 25.28 & 1.403 & 27.20 & 1.435 & 29.40 & 1.468 \\
\hline 3 & 12.8 .5 & 1.109 & 14.40 & 1.158 & 16.52 & 1.218 & 18.42 & 1.265 & 20.38 & 1.309 & 22.35 & 1.349 \\
\hline 4 & 9.43 & 0.970 & 10.78 & 1.033 & 12.64 & 1.102 & $\mid 14.41$ & 1.159 & $|16.20|$ & 1.210 & 18.02 & 1.156 \\
\hline 5 & 7.00 & 0.845 & 8.44 & 0.926 & 10.08 & 1.003 & 11.60 & 1.064 & 13.40 & 1.127 & 15.02 & 1.177 \\
\hline 6 & 5.41 & 0.733 & 6.79 & 0.832 & 8.25 & 0.916 & 9.69 & 0.986 & 11.32 & 1.054 & 12.88 & 1.110 \\
\hline 7 & 4.35 & 0.639 & 5.53 & 0.743 & 6.88 & 0.838 & 8.33 & 0.921 & 9.80 & 0.991 & 11.22 & 1.050 \\
\hline 8 & 3.55 & 0.550 & 4.61 & 0.664 & 5.84 & 0.766 & 7.21 & 0.858 & 8.58 & 0.934 & 9.92 & 0.997 \\
\hline 10 & 2.44 & 0.387 & 3.42 & 0.534 & 4.38 & 0.642 & 5.44 & 0.736 & 6.63 & 0.822 & 7.85 & 0.895 \\
\hline 15 & 1.16 & 0.065 & 1.79 & 0.253 & 2.40 & 0.380 & 3.17 & 0.501 & 4.06 & 0.609 & 4.80 & 0.681 \\
\hline 20 & 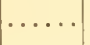 & & 1.00 & 0.000 & 1.40 & 0.147 & 2.01 & 0.303 & 2.60 & 0.415 & 3.17 & 0.501 \\
\hline
\end{tabular}

Note: All second-place decimal figures given in italics are approximate values.

Table 1 gives the copper absorption values for radiation from a thin tube in which the wall absorption is about the same as for $0.02 \mathrm{~mm}$. Cu. In the first column are given filter thicknesses at practical intervals. In the other double columns headed by the applied tube voltage are given respectively the percentage transmission of the filter and its corresponding logarithm to the base 10 .

Similar data for a thick pyrex tube having a wall absorption about the same as for $0.1 \mathrm{~mm}$. Cu are given in Table 2 .

2 Quotation from Par. 7 of the 1933 Committee report: "For most practical purposes, the quality of the X-radiation may be satisfactorily specified in terms of the copper or aluminum absorption curve combined with a statement of the initial filtration. In lieu of an absorption curve, the equivalent constant potential applied to the tube terminals to yield the same curve may be stated as a single numerical magnitude. Up to $100 \mathrm{kv}$. (constant) aluminum absorption curves and above $100 \mathrm{kv}$. (constant) copper absorption curves shall be used to establish the equivalent potential." 
Below $100 \mathrm{kv}$. (constant), absorption in aluminum has been recommended as a standard, hence Table 3 gives aluminum absorption data. In making these measurements the same thin glass tube as for Table 1 was used.

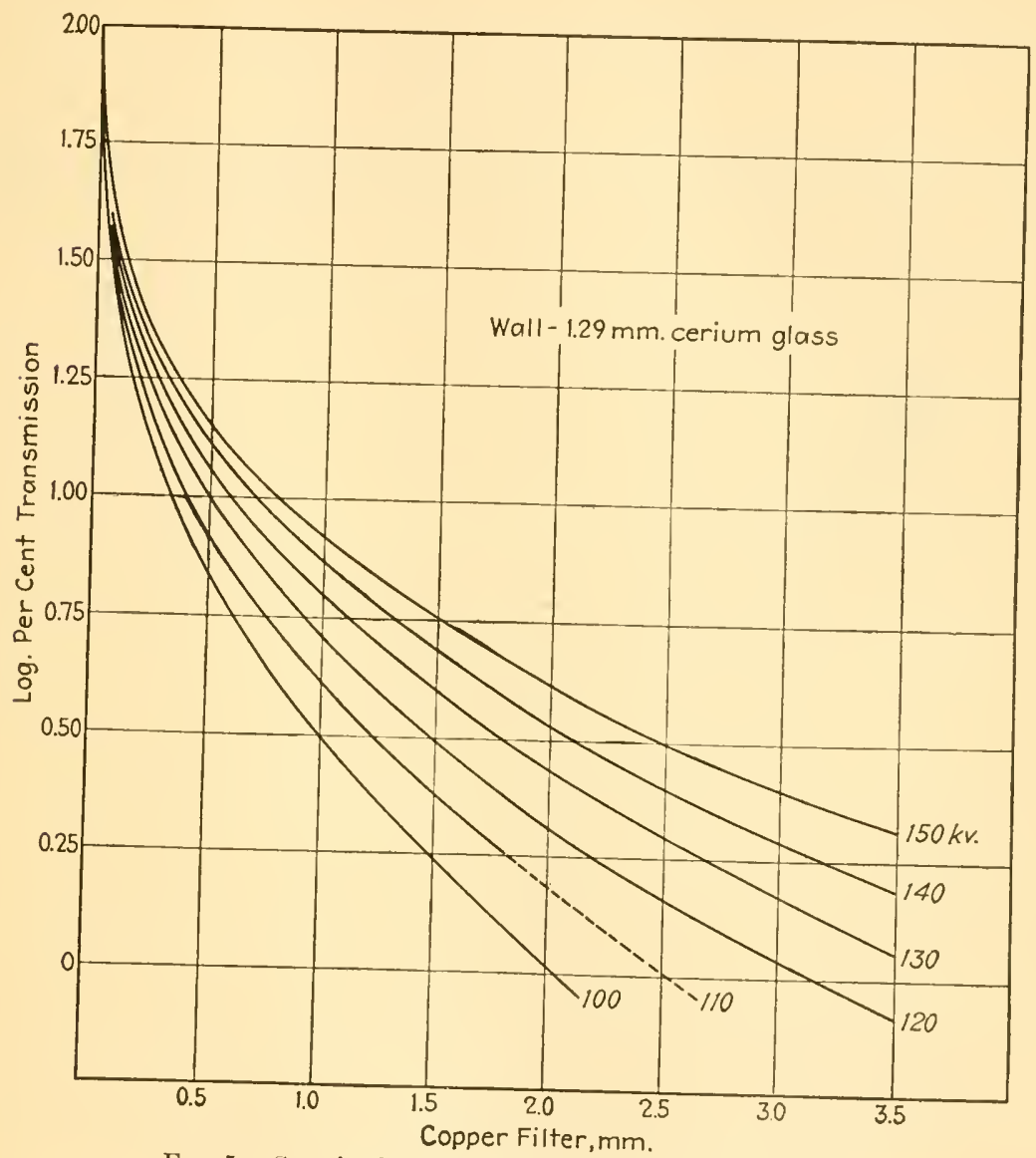

Fia. 5.-Standard copper absorption curves-thin tube.

Figures 5, 6, and 7 give, respectively, for various constant-potential excitations, the logarithmic absorption curves for copper and aluminum as plotted from Tables 1, 2, and 3, respectively (51). If it is assumed that, for the same excitation potential, the spectral distribution of the radiation emitted by the anticathodes of the two tubes is the same, then the difference between the copper absorption curves corresponding to the same potential in Figs. 5 and 6 arises entirely from the difference in the absorption of the two tube walls $-1.3 \mathrm{~mm}$. cerium glass, and 4.8 mm. pyrex glass. 
Interpretation of Absorption Curves.-To bring out the general information to be inferred from the absorption curves of Figs. 5, 6, and 7, and from this to correlate the curves of Fig. 6 with those of Fig. 5, we shall take a hypothetical absorption curve $A B$ (Fig. 4), in which $x$ gives the thickness of copper filter and

$$
y=\log \left(100 \frac{I}{I_{0}}\right)
$$

gives the corresponding values of log percentage transmission. Here it will be assumed that the incident radiation $I_{0}$ is obtained from an ideal

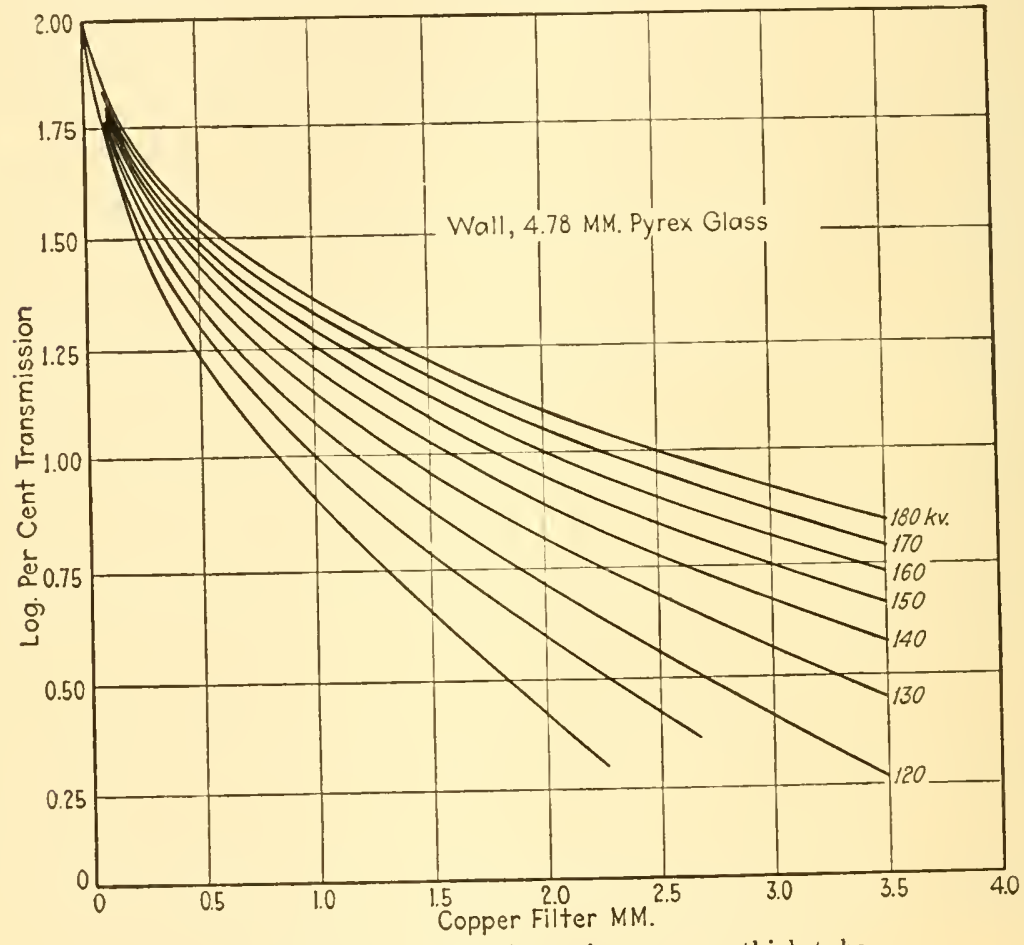

Fig. 6.-Standard copper absorption curves-thick tube.

X-ray tube of zero wall thickness so that $A B$ is the basic absorption curve corresponding to the given excitation potential.

As a first case, we wish to show how, from this basic curve, the absorption curve is obtained for the same radiation after it has been modified in quality by traversing a given thickness $d$ of the filter. In this case, $I_{d}$, the intensity emerging from the thickness $d$, becomes the incident radiation for the new curve. Against new thickness values,

$$
x^{\prime}=x-d
$$


of the filter, are plotted new log percentage transmissions,

$$
\begin{aligned}
y^{\prime} & =\log \left(100 \frac{I}{I_{d}}\right) \\
& =y+2-\log \left(100 \frac{I_{d}}{I_{0}}\right) \\
& =y+\text { const. }
\end{aligned}
$$

Thus all points on the new eurve differ in position from corresponding ones on the basic curve by the same amount; that is, the new curve is

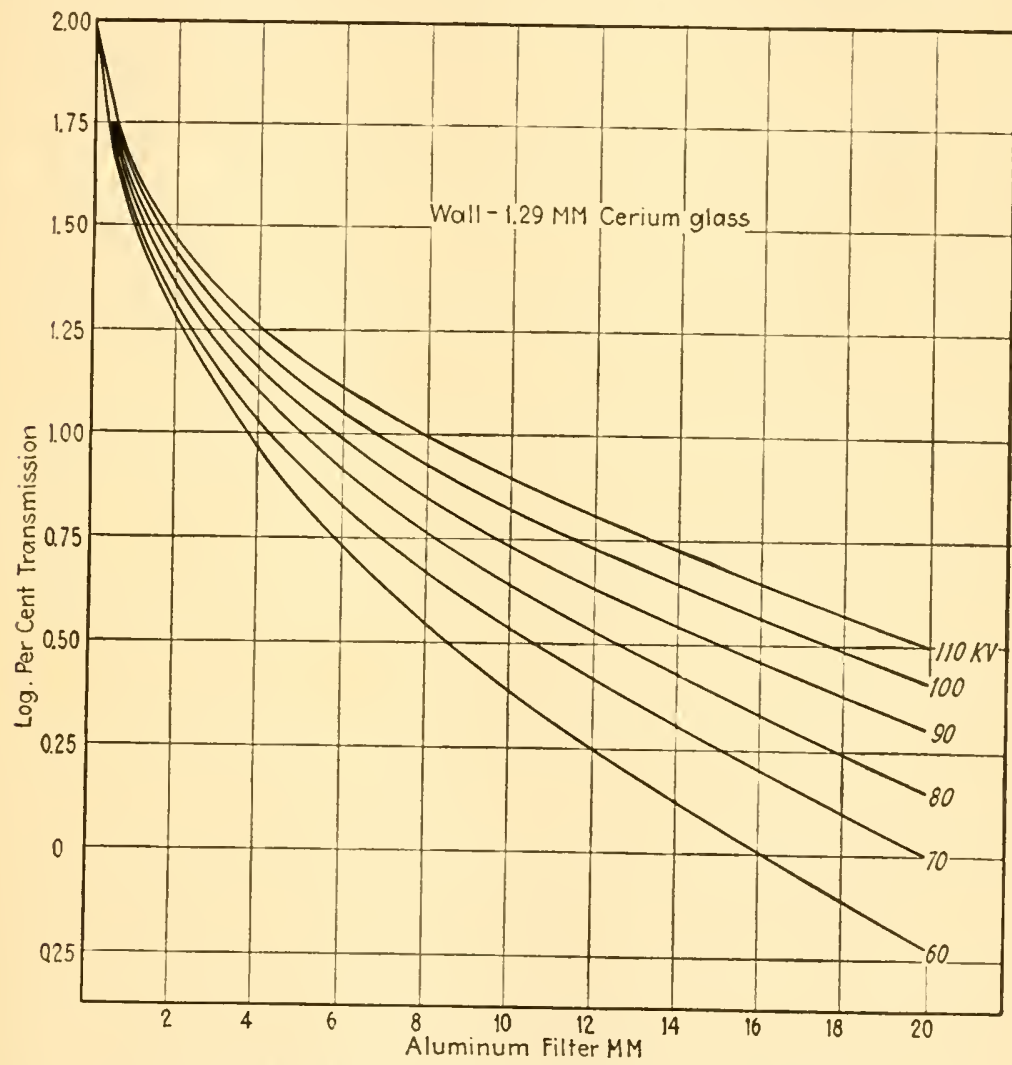

Frg. 7.-Standard aluminum absorption curves-thin tube.

obtained from the basic eurve by merely shifting the origin of coordinates to the point $x=d$ and $y=-\left[2-\log 100\left(\begin{array}{c}I_{d} \\ I_{0}\end{array}\right)\right]$.

The second case deals with the converse problem: given two absorption curves of the same radiation, but subjected to different amounts of filtration; to find the difference in thickness of the initial filters. Here we are supposed to have two separate absorption curves of Fig. $4-A B$ on 
the $X Y$-axes; and $C B$ plotted (on transparent paper) on the $x^{\prime} y^{\prime}$-axes. The second is laid orer the first and shifted to that position where it is found to fit the first. The difference $d$ in the two filter thicknesses, for corresponding points on the two curves, is given by the difference $\left(x-x^{\prime}\right)$ for the point $C$ and is the magnitude sought.

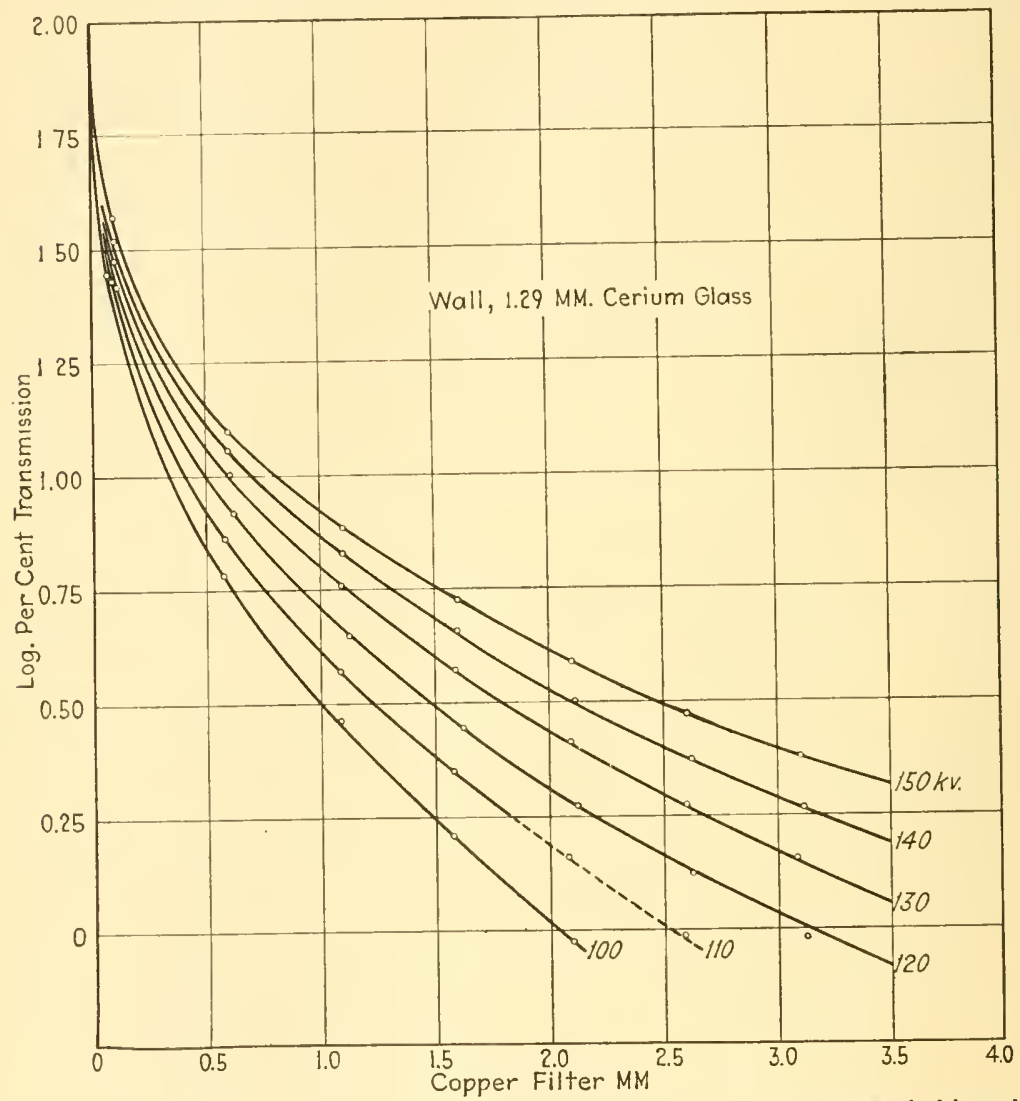

FIG. 8.- Superposition of copper absorption curves obtained with thick and thin tubes.

Having outlined the general procedure, it will next be inquired if like excitation potentials in the two tubes of Figs. 5 and 6 produce absorption curves which are subject to the same correlation as curves $C B$ and $A B$ (Fig. 4) just treated. In other words, is the radiation from the thick-wall pyrex tube (Fig. 6) of the same quality as that from the thin-wall tube (Fig. 5) after passing through some mknown thickness of copper?

By taking the curves of Fig. 6 and fitting them as just described to the curves of like excitation potential in Fig. 5, a very satisfactory correlation is obtained. The curves of Fig. 8 are those of Fig. 5 reproduced; the 


\begin{tabular}{|c|c|c|c|}
\hline 胥 & $\stackrel{\overbrace \vec { \dot { m } }}{\Xi}$ & 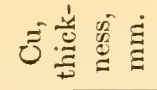 & 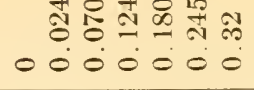 \\
\hline 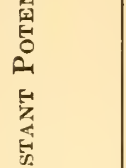 & 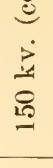 & 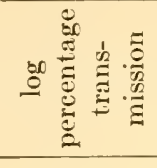 & 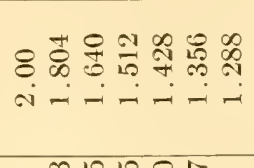 \\
\hline$\underbrace{2}_{\infty}$ & İ & 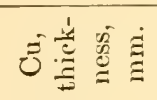 & 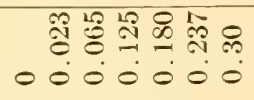 \\
\hline$\sum_{\infty}^{\infty}$ & 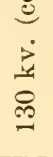 & 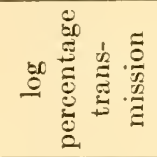 & 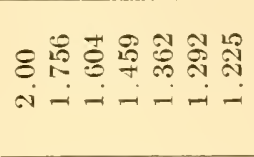 \\
\hline 星 & & 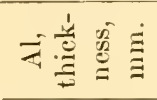 & 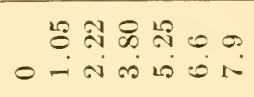 \\
\hline 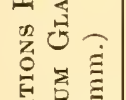 & $\stackrel{0}{0}$ & 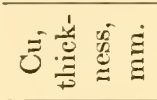 & 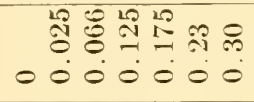 \\
\hline 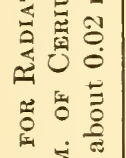 & 章 & 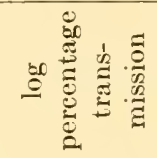 & 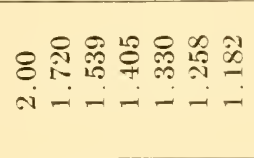 \\
\hline 先 & I & 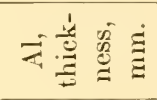 & 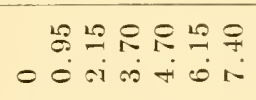 \\
\hline 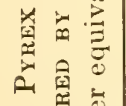 & $\underset{0}{\stackrel{0}{0}}$ & 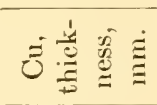 & 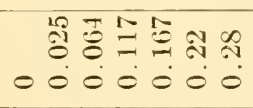 \\
\hline 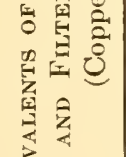 & 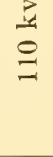 & 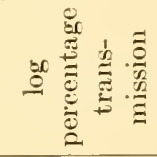 & 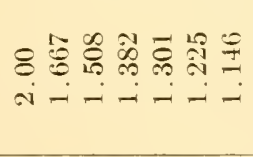 \\
\hline (9) & & 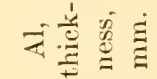 & 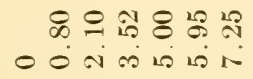 \\
\hline 苔 & 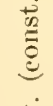 & $\tilde{U}$ & 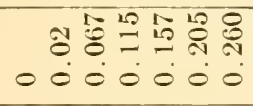 \\
\hline $\begin{array}{l}\text { दे } \\
\text { दे } \\
\text { 롱 }\end{array}$ & $\underline{8}$ & 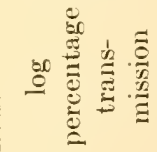 & 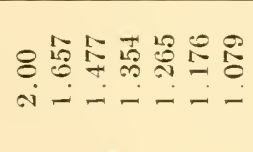 \\
\hline Ti & & $\sum_{-1}^{0}$ & $O N+O \infty O N$ \\
\hline
\end{tabular}


plotted points on these curves are from the corresponding curves of Fig. 6, each point on the extreme left indicating the thickness of copper (about $0.1 \mathrm{~mm}$.), which would make up the difference in effect of the walls of the two tubes. As may be expected, this thickness varies somewhat with the excitation potential, because the spectral absorption in glass is not the same as in copper.

From the consistency of this correlation, in which it is obvious that absorption curves corresponding to different excitation potentials do not fit, it is to be concluded that the shape of the absorption curve is characteristic of the excitation potential. On the other hand, the coordinate

Table 5.-Aldminum Equivalents of Lime Glass for Radiations Excited by Various Constant Potentials and Filtered by 0.45 mm. of Lime Glass

\begin{tabular}{|c|c|c|c|c|c|c|}
\hline \multirow{2}{*}{$\begin{array}{l}\text { Thick- } \\
\text { ness of } \\
\text { lime } \\
\text { glass, } \\
\text { mm. }\end{array}$} & \multicolumn{2}{|c|}{$60 \mathrm{kv}$. (const.) } & \multicolumn{2}{|c|}{$80 \mathrm{kv}$. (const.) } & \multicolumn{2}{|c|}{$100 \mathrm{kv}$. (const.) } \\
\hline & $\begin{array}{c}\log \\
\text { percentage } \\
\text { transmission }\end{array}$ & $\begin{array}{c}\mathrm{Al}, \\
\text { thickness, } \\
\mathrm{mm} .\end{array}$ & $\begin{array}{l}\log \\
\text { percentage } \\
\text { transmission }\end{array}$ & $\begin{array}{c}\mathrm{Al}, \\
\text { thickness, } \\
\text { mm. }\end{array}$ & $\begin{array}{c}\log \\
\text { percentage } \\
\text { transmission }\end{array}$ & $\begin{array}{c}\text { Al, } \\
\text { thickness, } \\
\text { mm. }\end{array}$ \\
\hline 0 & 2.00 & 0 & 2.00 & 0 & 2.00 & 0 \\
\hline 0.5 & 1.607 & 0.42 & 1.690 & 0.47 & 1.751 & 0.48 \\
\hline 1.0 & 1.516 & 0.84 & 1.558 & 0.86 & 1.605 & 0.88 \\
\hline 1.5 & 1.403 & 1. 26 & 1.461 & 1.38 & 1.526 & 1.33 \\
\hline 2.0 & 1. 295 & 1.72 & 1. 373 & 1.76 & 1.459 & 1.78 \\
\hline 2.5 & 1. 185 & 2.16 & 1. 286 & 2.23 & 1.395 & 2.24 \\
\hline 3.0 & 1.072 & 2.56 & 1.199 & 2.74 & 1.322 & 2.77 \\
\hline
\end{tabular}

displacement of the given curve from that of the basic curve is characteristic of the filtration between the target and the point of measurement. Having a family of such curves (as in Fig. 5), in which the constant excitation potential varies by steps of practicable magnitude, it is clear that instead of using the complete absorption curve to specify the quality of the radiation, we may use the more convenient magnitudes: excitation potential (constant) and initial filtration. Since the radiation from various wave forms of excitation potential may be simply and adequately equated to that of some equivalent constant-potential excitation, the quality of any given radiation may be specified by its equivalent constant excitation potential and the initial filtration. This initial filtration is desirably expressed in terms of the equivalent copper or aluminum.

Absorption in Tube Walls.-For the purpose of evaluating the absorption of radiation by the glass tube walls, copper and aluminum equivalents of lime and pyrex glass are given in Tables 4 and 5 . At least in the case of the thick pyrex glass tubes now commonly used, the tube-wall filtration should be taken into consideration when describing the radiation quality. 
Standard Absorption Curves from 225 to $550 \mathrm{Kv}$.-To permit extension of the complete absorption-curve method of specifying radiation quality to tube potentials above $250 \mathrm{kv}$., the copper absorption data for voltages $u p$ to $550 \mathrm{kv}$. (constant) are given in Table 6 . The initial filtration in this case is $6.4 \mathrm{~mm}$. of steel plus $13 \mathrm{~mm}$. of water, which together are equivalent to about $6 \mathrm{~mm}$. Cu. Absorption curves obtained with greater initial filtration can be readily correlated with curves plotted from Table 6, in the same manner as those of Fig. 6 with Fig. 5; but not so with curves of lower initial filtration, because of the uncertainty with which the magnitudes can be extrapolated toward the higher transmissions. In

Table 6.-Absorption of General X-radiation in Copper

(Tube wall, $6.4 \mathrm{~mm}$. steel $+13.0 \mathrm{~mm}$. water)

\begin{tabular}{|c|c|c|c|c|c|c|c|c|c|c|}
\hline \multirow{2}{*}{$\begin{array}{c}\text { Filter, } \\
\text { mm. }\end{array}$} & \multicolumn{10}{|c|}{$\log _{10}$ percentage transmission } \\
\hline & $\begin{array}{l}225 \\
\mathrm{kv} .\end{array}$ & $\begin{array}{l}250 \\
\mathrm{kv} .\end{array}$ & $\begin{array}{l}275 \\
\text { kv. }\end{array}$ & $\begin{array}{l}300 \\
\mathrm{kv} .\end{array}$ & $\begin{array}{l}325 \\
\mathrm{kv} .\end{array}$ & $\begin{array}{l}350 \\
\text { kv. }\end{array}$ & $\begin{array}{l}400 \\
\mathrm{kv} .\end{array}$ & $\begin{array}{l}450 \\
\mathrm{kv} .\end{array}$ & $\begin{array}{l}500 \\
\text { kv. }\end{array}$ & $\begin{array}{l}550 \\
\mathrm{kv} .\end{array}$ \\
\hline 0 & 2.00 & 2.00 & 2.00 & 2.00 & 2.00 & 2.00 & 2.00 & 2.00 & 2.00 & 2.00 \\
\hline 2 & 1.685 & 1.745 & 1.781 & 1.798 & 1.812 & 1.825 & 1.845 & 1.861 & 1.873 & 1.883 \\
\hline 4 & 1.450 & 1.550 & 1.605 & 1.630 & 1.653 & 1.672 & 1.705 & 1.733 & 1.757 & 1.777 \\
\hline 6 & 1. 240 & 1.384 & 1.447 & 1.478 & 1.507 & 1.532 & 1.575 & 1.612 & 1.645 & 1.674 \\
\hline 8 & 1.075 & 1.220 & 1.300 & 1.340 & 1.375 & 1.406 & 1.458 & 1.502 & 1.540 & 1.574 \\
\hline 10 & 0.920 & 1.060 & 1.158 & 1.208 & 1.248 & 1.283 & 1.343 & 1.393 & 1.438 & 1.476 \\
\hline 12 & $\ldots \ldots$ & & 1.021 & 1.072 & 1.118 & 1.161 & 1.233 & 1.290 & 1.340 & 1. 384 \\
\hline 14 & & & 0.890 & 0.948 & 1.000 & 1.046 & 1.125 & 1.190 & 1.245 & 1. 296 \\
\hline 16 & & & & 0.825 & 0.887 & 0.940 & 1.026 & 1.095 & 1.152 & 1.203 \\
\hline
\end{tabular}

other words, although these curves can be of ralue as standards, they do not have the range of application reserved to basic curves, i.e., of radiation subjected to zero filtration.

\section{SECONDARY METHODS OF EXPRESSING X-RAY QUALITY}

Effective Wave-length.-From the same absorption data necessary for the determination of the equivalent voltage, and plotted as in Figs. 5 to 7 , there may be determined an effective absorption coefficient and a corresponding effective wave-length, both of which have found some use in practice. The slope of a semilogarithmic absorption curve at any point gives directly the absorption coefficient $\mu_{e}$ in copper of the heterogeneous radiation corresponding to the particular thickness for which the point is chosen.

This follows from the absorption law

$$
I=I_{0} \epsilon^{-\mu_{e} x}
$$

where $I_{0}$ is the incident intensity, $I$ the transmitted intensity through a filter of thickness $x$, and $\mu_{e}$ is the effective absorption coefficient. 
Rewriting (4), we have

$$
\log _{\epsilon} \frac{I}{I_{0}}=2.3 \log _{10} \frac{I}{I_{0}}=-\mu_{e} x
$$

which is the equation of a straight line of slope $-\mu_{e}$ when $\log _{\epsilon} I / I_{0}$ is plotted against $x$. (Note that logarithms to the base 10 are used in

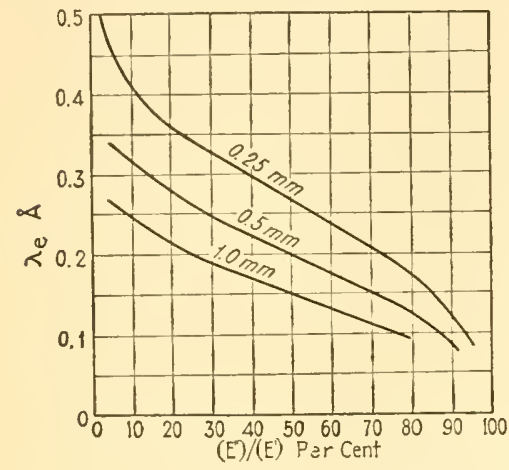

Fig. 9.-Effective wave-length (Duane) as a function of the percentage transmission of given filters.
Figs. 5 to 7 ; hence the slopes must be multiplied by 2.3 in determining $\mu_{e}$.)

Corresponding to $\mu_{e}$ may be assigned a so-called effective wave-length $\lambda_{e}$. From monochromatic X-ray absorption data in copper or aluminum may be found a wave-length $\lambda$, for which the monochromatic absorption coefficient $\mu$ is numerically equal to $\mu_{e}$. Then $\lambda=\lambda_{e}$, the true effective wave-length (44). Thus we have the definition that the effective wave-length of heterogeneous radiation is numerically equal to the wave-length of that monochromatic radiation which has the same absorption coefficient as the heterogeneous radiation in question.

Where tables giving absorption data are not available, $\mu$ may be calculated from Richtmyer's equations (34)

$$
\begin{aligned}
& \text { (Copper) } \frac{\mu}{\rho}=153 \lambda^{3}+0.2 \\
& \text { (Aluminum) } \frac{\mu}{\rho}=14.45 \lambda^{3}+0.15
\end{aligned}
$$

where $\mu$ is the absorption coefficient for wave-length $\lambda$ and $\rho$ is the filter density $\left(\rho_{\mathrm{Cu}}=8.94 \mathrm{gm} . / \mathrm{cc}\right.$. and $\rho_{\mathrm{Al}}=2.70 \mathrm{gm} . / \mathrm{cc}$.). These equations apply closely for effective wave-lengths between 0.12 and $0.4 \AA$.

An approximation to the effective wave-length $\lambda_{e}$ described above is obtained by a less laborious (and less accurate) procedure originally proposed by Duane (19). From equations 5 and 6 he calculates $(a)$ the percentage by which the $\mathrm{X}$-ray beam is reduced in passing through a given fixed filter, and $(b)$ the wave-length of the monochromatic beam which is reduced by the same amount, the latter being defined by him as the effective wave-length, $\lambda_{\text {eff }}$. Figure 9 gives a plot of the Duane effective wave-length $\lambda_{\text {eff }}$ against the percentage transmission of the given filter. The final result by this method is given through the slope of the cord to a point on the complete absorption curve as against, more properly, the 


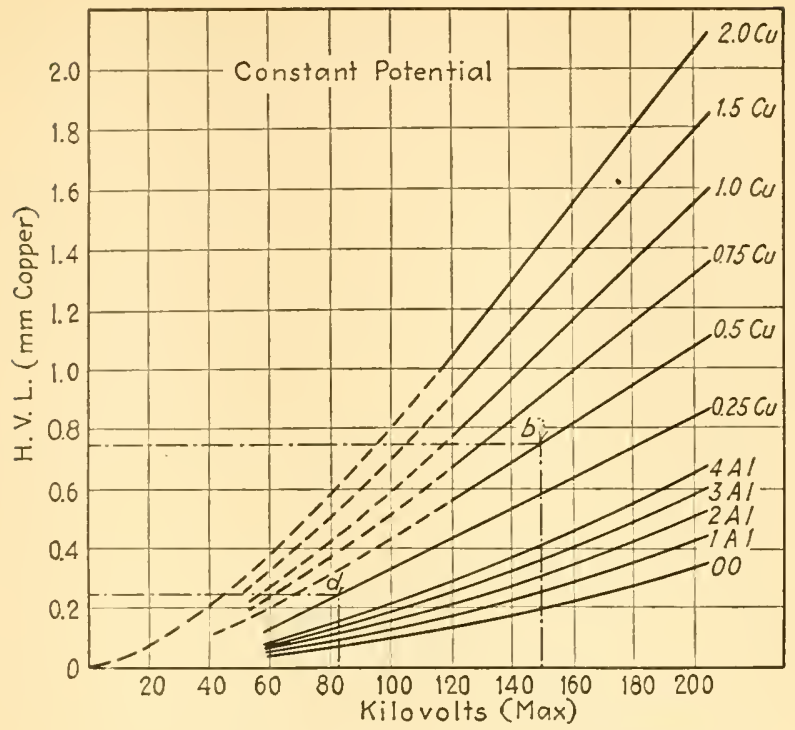

Fig. 10.-Half-value layers of copper. (Holthusen.)

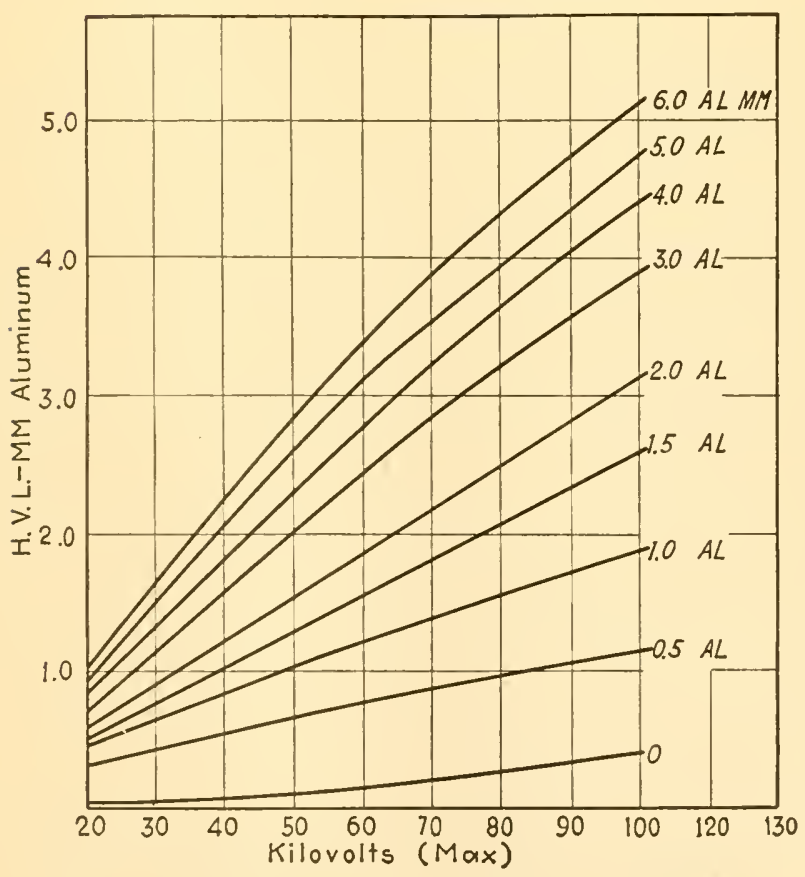

Fig. 11.-Half-value layers of aluminum. (Holthusen.) 
slope of the tangent. Of course, where the eurvature is very small, the tangent and cord nearly coineide and the two effective wave-lengths are nearly alike. The main drawback to Duane's method is that it gives a different result for every filter thickness employed (33).

Half-value Layer.-Another means of expressing quality, known as the half-value method, is used extensively in Europe (8). By this, a given radiation quality is described as the thickness of copper (or aluminum) which placed in an X-ray beam reduees the intensity to half its incident value. The result is expressed in millimeters of copper (or aluminum). For the higher voltages, the H.V.L. of copper is used. On dropping to lower voltages the H.V.L. of copper deereases rapidly and when it becomes less than $0.1 \mathrm{~mm}$., H.V.L. values of aluminum are used instead.

To obtain the H.V.L. for a given beam, filters of gradually increasing thickness are placed in the beam and the transmitted intensity read by a suitable ionization chamber. The transmitted intensities are then plotted against the corresponding filters and that filtration corresponding to the desired half-intensity is read from the graph. It is important to note that the H.V.L. measurements should be made on the beam actually used for treatment, not on the original unfiltered beam (unless the unfiltered is being used). Figures 10 and 11 give half-value layers for copper and aluminum for various constant voltages and various filtrations (21).

\section{STANDARD MEASUREMENT OF X-RAY QUANTITY OR INTENSITY}

International Definition of X-ray Quantity.-As noted above, the property of X-rays which permits them to ionize a gas forms the presentday basis of X-ray-intensity measurements. The ionization produced by a given beam is proportional to the energy absorbed from the beam by the gas between $\lambda 0.1$ and $1.5 \AA$. This in turn is a function of the density of the gas, or of the number of atoms encountered by the $\mathrm{X}$-ray in a given distance along its path. The absorption is likewise approximately proportional to the fourth power of the atomic number of the gas and to the third power of the wave-length of the X-rays. As already pointed out, in detail, the wave-length dependence of the absorption is the factor which necessitates the quality speeifieation in intensity measurements. The dependence upon the atomic number of the gas would be of utmost importance if various gases were commonly employed in practice. This undesirable eomplication is avoided by the arbitrary choice of a single gas for all comparative purposes.

For the ionized medium, air has been chosen for two particular reasons: (a) because its effective atomic number is very nearly the same as for body tissue (the X-ray absorption per gram is nearly the same for air, 
water, and body tissue over a wide range of wave-lengths), and (b) for convenience and ready reproducibility. Therefore, the internationally accepted unit of X-ray quantity is determined by the ionization produced in $0.001293 \mathrm{gm}$. of air. The definition accepted by the United States (56) differs from the international definition only in being less ambiguous for voltages above $200 \mathrm{kv}$., and reads as follows: "The roentgen is the quantity of X-radiation which, when the secondary electrons are fully utilized and the effects of all scattered radiation avoided, produces in 1 cubic centimeter of atmospheric air at $0^{\circ} \mathrm{C}$. and $76 \mathrm{~cm}$. mercury pressure such a degree of conductivity that 1 E.s.U. of charge is measured under saturation conditions."

The problem of realizing the roentgen is then resolved, essentially, into two parts: $(a)$ segregating a known volume of air which is ionized

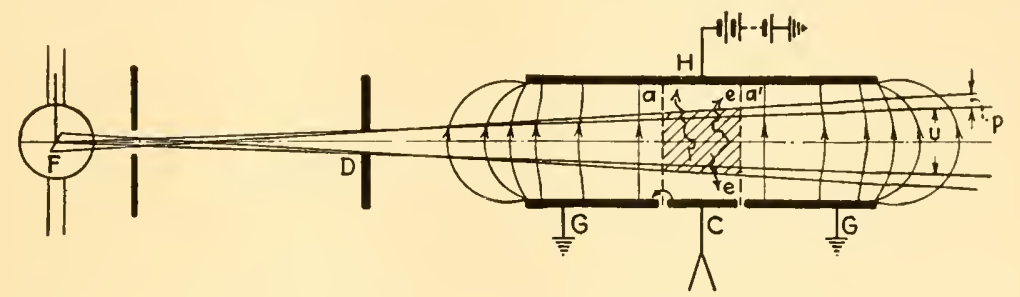

Fig. 12.-Parallel-plate standard ionization chamber.

by the beam in question, and $(b)$ measuring the number of ions produced in that volume. Actually neither of these quantities is obtainable directly, although the indirect methods to be described below do not introduce any uncertainties, at least up to $200 \mathrm{kv}$.

Standard Free-air Ionization Chamber.-The roentgen may be realized by means of the conventional ionization chamber shown diagrammatically in Fig. 12-(the figure is drawn out of proportion in order to emphasize certain essential features). The chamber proper consists of a flat plate $H$ which is maintained at a suitable potential by a battery. The ion collector $C$ is connected to an electrometer of such type that when in operation, $C$ is maintained at ground potential (cf. page 72). $G, G$ are guard plates on each side of $C$ along the direction of the beam.

The electric field (lines of force) between $H$ and the three plates $G, C, G$ are roughly as shown, bulging outward at the ends and gradually becoming parallel at the center of the chamber. It has been found experimentally that the electric field between a pair of parallel plates becomes uniform at a distance from the ends about $1 \frac{1}{2}$ times the plate separation $(11,17,42)$. Hence, in the figure, if the guard-plate widths are $1 \frac{1}{2}$ times the spacing $G H$, then the region between the lines $a a^{\prime}$ will have a uniform electric field (or parallel lines of force). X-rays in passing between the plates produce ions which are drawn to the plates along 
the paths of the force lines, and it is, therefore, necessary to know exactly which lines terminate on the grounded guard plates and on the insulated plate $C$ which is connected to the electrometer.

Since there must be a finite spacing between the edges of plates $C$ and $G$, and the lines of force are supposed to be parallel in these regions, the plate $C$ has an effective width greater than its actual width by an amount equal to half the total gap between the collector $C$ and the two guards $G$. The effective width of $C$ is also affected by its potential with respect to the guard plates, being that given above only when the potential of $C$ is the same as $G(42)$. On the other hand, according as $C$ is above or below the potential of $G$, its effective width may be less or greater than the correct value. This is caused by field distortion, where, owing to the small spacing between $C$ and $G$, the field strength may be comparatively large. In accurate measurements a potential difference of 0.5 volt between guard and collector spaced $0.5 \mathrm{~mm}$. apart may introduce a noticeable error in the results even though the field strength between $C$ and $H$ be as much as 200 volts $/ \mathrm{cm}$.

In order to maintain the collector plate at the same potential as the guards (earth) during measurements, it is necessary to employ a null or other equivalent current-measuring device $(2,3,12,13,25,45)$. A sensitive galvanometer is suitable for such measurements, although for very weak X-ray beams the ionization current is usually too small to permit the use of this method. Various electrostatic and vacuum-tube measuring devices have been successfully used for the whole practical range of X-ray intensities, but space does not permit their discussion here (see pages 75 and 77 ).

Effective Volume of Air Ionized.-The volume of air ionized by a limited beam of X-rays cannot be determined accurately with the requisite accuracy, the reason therefor being evident from Fig. 12. The focal spot $F$ is usually at least $8 \mathrm{~mm}$. in diameter; hence a beam after limitation by a diaphragm $D$ consists of an umbral region $u$ of uniform flux density and a penumbral region $p$ of nonuniform flux density. Moreover, ions are formed along the secondary electron tracks $e$, and form scattered X-rays both of which may be outside of the defined beam. It is obvious from these considerations alone that the direct volume determinations are rendered impracticable.

Its indirect determination is rendered simple and exact by the fact that all of the X-ray flux incident on the area of the aperture $D$ traverses the chamber, a fraction of which is absorbed in producing ions between $a$ and $a^{\prime}(3,11,41)$. The only assumption involved is that absorption of the X-rays by the air between $D$ and $a$ is negligible in comparison with total flux. This is valid except for the very soft X-rays - below $60 \mathrm{kv}$. - where a correction must be made. The effective rolume 
of air ionized is then taken as that of a cylinder of cross sections equal to the area of $D$ and length equal to the effective length of the collector electrode $C$ (the distance $a$ to $a^{\prime}$ ).

Secondary Effects.-To insure full utilization of the X-ray flux through the chambers two other factors must be taken into consideration: $(a)$ The spacing between $C$ and $H$ must be suffieient to permit the recoil electrons $e$ to expend their total energy in producing ions before striking the plates. For a narrow $(1 \mathrm{~cm}$.) beam of $200-\mathrm{kv}$. (peak) X-rays a spacing of $10 \mathrm{~cm}$. between $C$ and $H$ is sufficient. This spacing varies with the exciting X-ray voltage, although for practical purposes it does not appear to increase indefinitely with increasing voltage. (b) Precaution must also be taken that suffieient electric field be provided between $C$ and $H$ to produce saturation, that is, pull all the ions to $C$ or $H$ before an appreciable number are lost by recombination. For most X-ray flux densities encountered in practice, field strength of 100 to 150 volts $/ \mathrm{cm}$. is sufficient. For very high ion eoneentrations, greater fields may be required.

Since the ionization current is a function of the mass absorption of $\mathrm{X}$-rays by air, correction must be made for the air temperature and pressure as stipulated in the definition. Aceordingly, the air volume is redueed to that under normal conditions, $0^{\circ} \mathrm{C}$. and $76 \mathrm{~cm}$. $\mathrm{Hg}$ pressure, by Charles's and Boyle's law.

In further satisfaction of the definition of the roentgen, secondary effects from the walls and plates of the chamber are redueed to a minimum by making them of low-atomic-number material, carbon or aluminum. Thus any tertiary X-rays exeited by secondary X-rays from the main beam in striking the plates are of such long wave-length as to be quickly absorbed before having an opportunity to produce an appreeiable number of ions in the measuring volume. Following out the procedures given above, the intensity of an X-ray beam as measured in terms of roentgens is given by the following formula:

$$
r / \text { sec. }=\frac{i}{l \times a} \times \frac{T}{273} \cdot \frac{760}{P}
$$

where $i$ is the current to the collector electrode measured in electrostatic units, $l$ the effective length of the collector electrode, $a$ the area of the limiting diaphragm in square centimeters, $T$ the absolute temperature, and $P$ the pressure in millimeters. Where the eurrent is measured in amperes, the result must be multiplied by $1 / 3 \times 10^{-9}$.

\section{RECOMMENDATIONS CONCERNING X-RAY STANDARDS ADOPTED BY THE NATIONAL LABORATORIES}

As a result of an intercomparison of their standards $(1,46)$, the National Laboratories of the United States, England, Germany, and 
France have drawn up certain recommendations (57) on the technique of measuring the roentgen.

\section{THE MEASUREMENT OF QUALITY}

$X$-ray Quality (Hardness, Spectral Distribution).-The term X-ray quality relates to the composition of an $\mathrm{X}$-ray beam as determined by the spectral range and energy distribution. In the case of homogeneous $X$-rays, the quality may be expressed in terms of the wave-length of the radiation, measured in $\AA$ ngström units $\left(1 \AA=10^{-8} \mathrm{~cm} .=1000\right.$ X.U. $)$. In the case of the heterogeneous $X$-rays which occur in practice, a convenient practical classification quality is given below: Following the designation of the rays, there are given in order, first the approximate range of applied potential (kilovolt peak), and then the equivalent end-radiation limits in Ångströms, thus,

Ultra-soft Grenz radiation, 5 to $10 \mathrm{kv}$., 2.5 to $0.6 \AA$.

Soft radiation for diagnostic work and superficial therapy, 20 to $120 \mathrm{kv}$., 0.6 to $0.1 \AA$.

Hard radiation for deep therapy, 120 to $250 \mathrm{kv}$., 0.1 to $0.05 \AA$.

Extra-hard or super-hard radiation for highest potential therapy, over $250 \mathrm{kv}$., less than $0.05 \AA$.

Quality Specification.-In medical practice it is not convenient to express the quality of heterogeneous radiation in terms of its spectral distribution. The quality may, however, be expressed with sufficient accuracy for such purposes by a statement of generating conditions and absorption data, e.g.:

$A$. Generating conditions: (a) The target material. (b) Either the peak or r.m.s. value of the potential applied to the X-ray tube. (c) The potential wave form (e.g., constant or pulsating). (d) The type of filtration.

B. Absorption data: (a) Form of the total absorption curve in celluloid for ultra-soft radiation (5 to $29 \mathrm{kv}$.); aluminum for soft radiation (20 to $120 \mathrm{kv}$.); copper for hard radiation (100 to $250 \mathrm{kv}$.); copper or lead for ultra-hard radiation (above $250 \mathrm{kv}$.). (b) Frequently, it is sufficient for practical purposes to state the thickness in millimeters of absorbing material (celluloid, aluminum, copper or, lead) which, by insertion in the beam of radiation reduces the irradiation-ionometric intensityto one-half (half-value layer).

\section{MEASUREMENT OF THE ROENTGEN}

Recent international comparison has shown that an agreement to within at least \pm 1 per cent is attainable between the standard X-ray dosage measurements of the participating laboratories. The following 
experimental precautions should be adopted in standard measurements:

$X$-ray Tube.-The X-ray tube should be of the hot-cathode type with tungsten target. The focal spot should be as small and as uniform as possible and the target should not be appreciably pitted. Tube current and applied potential shall be kept constant with the requisite degree of accuracy during a test.

$X$-ray Beam.-A diaphragm of suitable aperture shall be mounted as near as possible to the $\mathrm{X}$-ray tube so that the $\mathrm{X}$-ray beam utilized shall include a maximum of radiation from the focal spot, and a minimum of off-focus radiation. The uniformity of the radiation field of the test beam shall be verified by ionization or photographic methods. For measurements made at different focal distances, deviation from the inverse square law should not exceed the specified measurement accuracy. The certainty of this shall be verified by special test. Where, for practical reasons, the inverse square law does not hold, the accuracy of the results is likely to be increased by conducting the measurements as far as possible from the X-ray tube.

Standard Ionization Chamber.-A. Chambers for either soft or hard radiation: Chambers used for measurement (in roentgens) of either soft or hard X-rays shall be of the open-air type (free-air, Fasskammer) wherein the measured ions are produced in a column of air which is not bounded by walls. Different forms of such chambers have been found to be satisfactory by the participating laboratories. For full constructional details the several publications of the laboratories may be referred to. The following general conditions shall be satisfied by standard chambers:

a. The ionization chamber shall be of such dimensions and so constructed that:

1. The spacing between the measuring and the high-potential electrodes is sufficient to insure full utilization of the secondary electrons.

2. The electric field between electrodes is sufficient to insure saturation. Chamber forms which make the attainment of saturation difficult are unsuitable.

3. Distortion of the electric field between the measuring and the highpotential electrodes is aroided as far as possible, for example, by the use of suitable guard electrodes or a guarded field.

b. A chamber diaphragm (entrant diaphragm, limiting diaphragm) shall be mounted to provide an entrance for the X-ray beam to the chamber and shall conform to the following general conditions:

1. The material out of which the diaphragm is made shall be of the minimum thickness which will permit not more than one part in a thousand $(1000)$ of the measured radiation to pass through the material itself. 
2. The diameter of its aperture should be approximately equal to the diameter of the focal spot of the X-ray tube, but always such as to limit the beam entering the chamber to one of uniform intensity of cross section.

3. The aperture should preferably be cylindrical on account of the ease of construction and accuracy of measurement. A covering material such as cellophane shall not be used.

4. The distance of the chamber diaphragm from the measuring electrode shall not be unnecessarily large, in order that the correction for air absorption of the rays along the path of the beam between the diaphragm and the electrode shall be a minimum.

5. Precautions shall be taken to avoid a measurable amount of scattered electrons or secondary radiation originating at the rim of the chamber diaphragm from reaching the measuring volume. For example, a second larger diaphragm whose rim is not struck by the direct beam of radiation may be placed between the chamber diaphragm and measuring electrode.

c. The ionization current is preferably measured by a null method so that a distorting electric field does not exist between the guard and measuring electrodes. Radioactive compensators should be of the constant capacity type.

$B$. Chambers for soft radiation: For the field of diagnostics and superficial therapy (soft radiation), a chamber smaller than for the hard radiation may be used to advantage. This should, of course, agree with the larger chamber over the greatest possible quality range.

$C$. Chambers for ultra-soft and super-hard radiation: While preliminary studies have indicated the probability that the standard ionization chamber employed for soft and hard radiation may be satisfactorily modified for use with ultra-soft and super-hard radiation, sufficient experience has not yet been gained to justify international agreement thereon.

\section{CALIBRATION OF X-RAY DOSEMETERS}

(Roentgenometers, Dosemeters, Irradiometers, Intensimeters, Etc.)

Small Ionization Chambers.--(a) Small ionization chambers such as are frequently used in practice should be so designed and constructed as to be independent of quality within the spectral range for which they are intended. (b) Such chambers shall be equipped with suitable arrangements for checking the constancy of their calibration. (c) There shall be no ionization in the instrument extraneous to that in the snall chamber itself.

Calibration of Small Ionization Chambers.-(a) Calibrations of small chambers may be carried out by either a direct-replacement method; or a 
method depending upon the inverse square law when its validity has been proved. (b) Approximately the same portion of the test beam should be used for both standard chamber and small chamber. This should be chosen from a portion of the main beam which is uniform. (c) Walled chambers of the thimble type shall be completely enveloped by the test beam. (d) Filters necessary in the calibration of small chambers shall be so placed in the X-ray beam that the effect of secondary radiation therefrom is not included in the measurements.

Reports on the Calibration of Small Chambers.-In reports on the above types of instruments, the participating laboratories will normally include the following: $(a)$ The equivalent in roentgens of the overall (or other desired) reading of the scale for each quality of test beam used. (b) Data on the quality of each test beam used (in accordance with the specifications given in the last paragraph. (c) The temperature and pressure at which the calibration was conducted, together with correction factors for other temperatures and pressures. (d) Data on the irradiation (Dosisleistung, ionometric intensity) which was used for the calibration, hence for which the calibration is valid. (e) Data on the means provided to check the secular constancy of the sensitivity of the instrument.

\section{TYPES OF STANDARD CHAMBERS}

Standard ionization chambers of the open-air type may have various forms, all of which should be equally reliable when properly constructed.
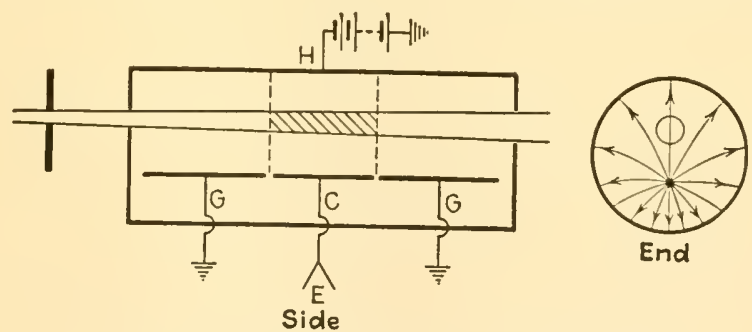

FIG. 13.-Barrel or cylindrical ionization chamber.

The simple parallel-plate type has already been discussed in sufficient detail so that the others may be but briefly described-further details being obtainable in the original publications.

The barrel or cylindrical chamber (Fig. 13) is practically the same in principle as the parallel-plate type (3). Here the high-potential electrode is a closed cylinder $H$ which surrounds the collector electrode $C$ and the guards $G$, all of which are rods of small diameter which may be placed either coaxially or eccentrically (parallel to the axis). The electric field is not uniform as for the parallel-plate chamber, but is radial. To insure a field strength sufficient for saturation at a given distance from the 
collector requires greater total plate potentials than the parallel-plate chamber, and while this offers no serious objections, care must always be taken that the beam passes through the same portion of the chamber (36). A field strength just sufficient to produce saturation at a given point would be insufficient at a point farther from the collector. Field distortion along the length of the chamber is essentially the same as in the parallel-plate type.

A third type known as the drumhead ionization chamber (Fig. 14) has some desirable features (12), although it has not yet been extensively used as a standard. In this the high-potential and collector electrodes are parallel plates arranged as shown, the radiation now passing through, and

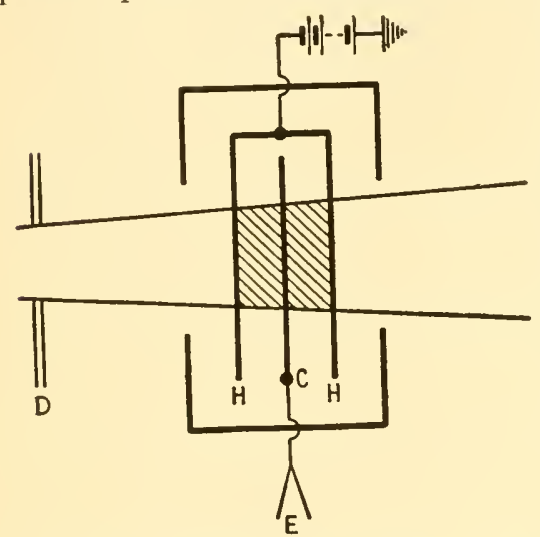

FIG. 14.- "Drumhead" ionization chamber. not just between the plates. Where the plates are very thin and made of low-atomic-number material their effect in producing secondary X-rays and electrons is negligible. Suitable plates at least for higher voltage radiations have been made of silk cloth, goldbeater skin, or a silk thread mesh, each rendered suitably conducting by light coatings of India ink or "aquadag."

The ionized volume is given by the product of length of the path between the two high-potential electrodes $H$ and the area of the dia-

phragm $D$. Except in extreme cases, field distortion is of no importance. Such a chamber has the advantage of offering a relatively large volume of ionized air, with consequent increase in ionization current.

In the three types of chambers discussed in the foregoing it is necessary, in general, that the electrode system be surrounded by a metal case which serves the double purpose of providing electrostatic shielding and shutting off any stray X-ray radiation. Such a shield may, if placed too close to the electrodes, introduce undesirable field distortion. In the case of the parallel-plate chamber a separation between the shield and high-potential electrode (11) approximately equal to the plate spacing, is sufficient to avoid field distortion. This means that for a $10-\mathrm{cm}$. collector electrode the complete ionization chamber will have a minimum total length of $65 \mathrm{~cm}$., and about $40 \mathrm{~cm}$. between the collector electrode $C$ and the limiting diaphragm $D$.

The primary standard X-ray ionization chamber employed by the National Bureau of Standards is a modification of the parallel-plate type (47). The principal feature of this is the avoidance of ficld dis- 
tortion by the use of guard wires $a, b, c, \ldots$, which are spaced uniformly between the guards and high-potential plates (Fig. 15, A). The wires are maintained at definite, equally graded, potentials by means of a highresistance potential divider as shown. Essentially, this system prevents the field from "bulging out" at the ends of the chamber. This in turn permits a shortening of the guard plates and allows the surrounding shield to be brought to within 3 or $4 \mathrm{~cm}$. of the electrodes.

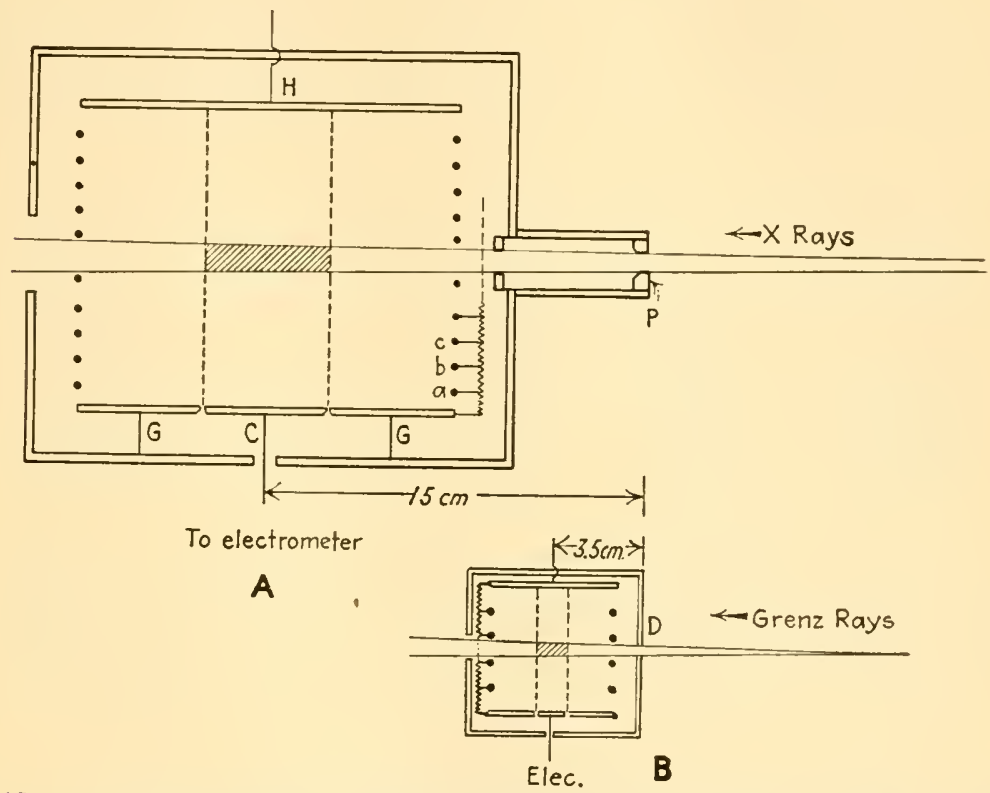

Fis. 15. - a, National Bureau of Standards guarded field standard ionization chamber $(50-200 \mathrm{kv}$.) ; $b$, guarded field standard ionization chamber for Grenz rays $(3-90 \mathrm{kv})$.

By thus shortening these distances, it is possible to bring the limiting diaphragm $D$ to within 12 to $15 \mathrm{~cm}$. of the measuring electrode. This extends the range of usefulness to considerably lower-voltage X-rays without necessitating large corrections for air absorption between diaphragm $P$ and collector $C$.

Standard Measurement of Ionization Currents.-Measurement of the ion current produced in primary standard chambers necessitates apparatus for which the calibration is direct as well as permanent. The ordinary galvanometer would be very adaptable, except that sufficiently large ion currents to produce a deflection camnot usually be obtained without introducing other and more serious errors. As a result, most standardization laboratories employ some form of electrostatic measuring device embodying the principle of current compensation (Fig. 16). One circuit, $A$, consists of an electrometer $E$, variable condenser $C$, and a 
battery of known potential $V(11)$. As the charge $+Q$ from the ionization chamber $I$ flows onto the insulated system for a time $t$, the condenser is changed through a capacity $\left(C_{2}-C_{1}\right)$ inducing an opposite charge $-Q$ on the system, so as to always maintain the electrometer at zero deflection. The current is then given by $Q / t=v\left(C_{2}-C_{1}\right) / t$. An alternative method (45) is to keep $C$ constant and vary $v$ (Fig. 15, B) whence the current $i=Q / t=C\left(V_{2}-V_{1}\right) / t$. Since the accuracy of these methods depends only on measurements of capacitance, voltage, and time, it is obvious that a high precision is obtainable. Circuits $A$ and $B$ are well adapted to a simple calibration when $E$ is such an instrument as a string or quadrant electrometer. Where $E$ is a vacuum-tube electrometer, the
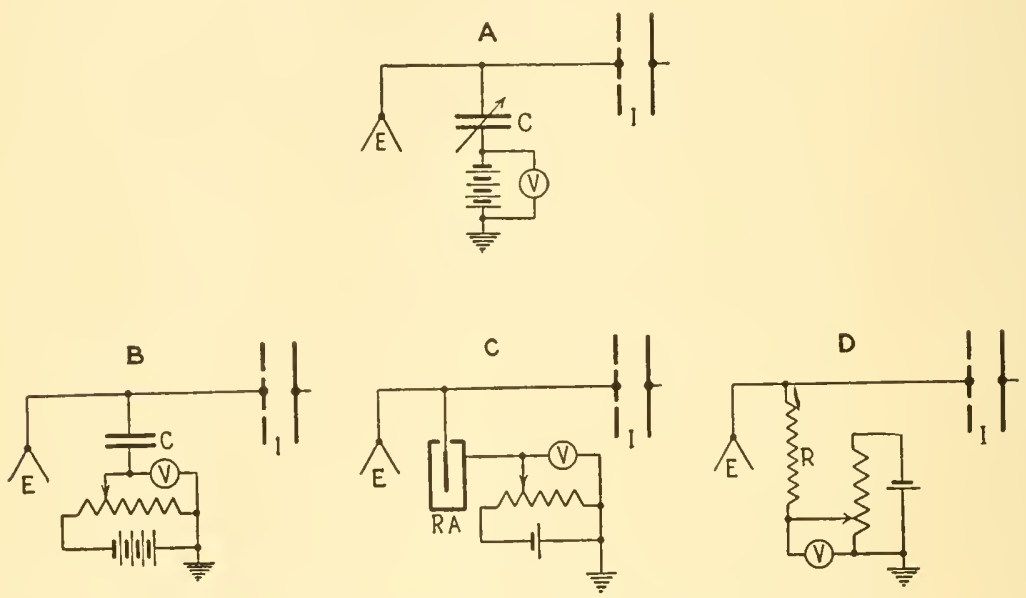

FIf. 16. - Standard measuring systems for very small currents.

calibration becomes less direct and more uncertain, depending upon the constancy of the vacuum-tube characteristics.

A third type of measuring circuit (Fig. 16, C) employs a radioactive compensator $R A$ for neutralizing the ion current (13). A small amount of radium is sealed in a tube and placed in the chamber $R A$, to which may be applied a known variable potential. The relationship of the radioactive ionization current to $V$ is determined by separate calibration. A convenient modification consists in applying a fixed potential to a variable radioactive source of ionization (25) so designed that the capacity of the system remains constant during any change in the compensator.

In a fourth method (Fig. 16, D) a known potential $V$ is applied across a high resistance $R$ of the order of $10^{10} \mathrm{ohms}$, the current balance being noted by the electrometer $E$. Knowing $R$, the current is given directly by Ohm's law $I=V / R$. For accurate measurements it is necessary that $R$ be calibrated frequently or until its constancy is assured. 


\section{SECONDARY IONIZATION CHAMBERS}

For the measurement of X-rays for biological purposes it is of course obvious that the large chambers discussed in the foregoing are impractical, principally because they lack mobility. This lack has led to the development of the small so-called, thimble chamber. Such chambers (Fig. 17) usually consist of an outer shell of some conducting material which is earthed, and a central electrode which is connected to a suitable current-measuring device and charged to a potential sufficient to insure saturation between it and the surrounding shell. When placed in an
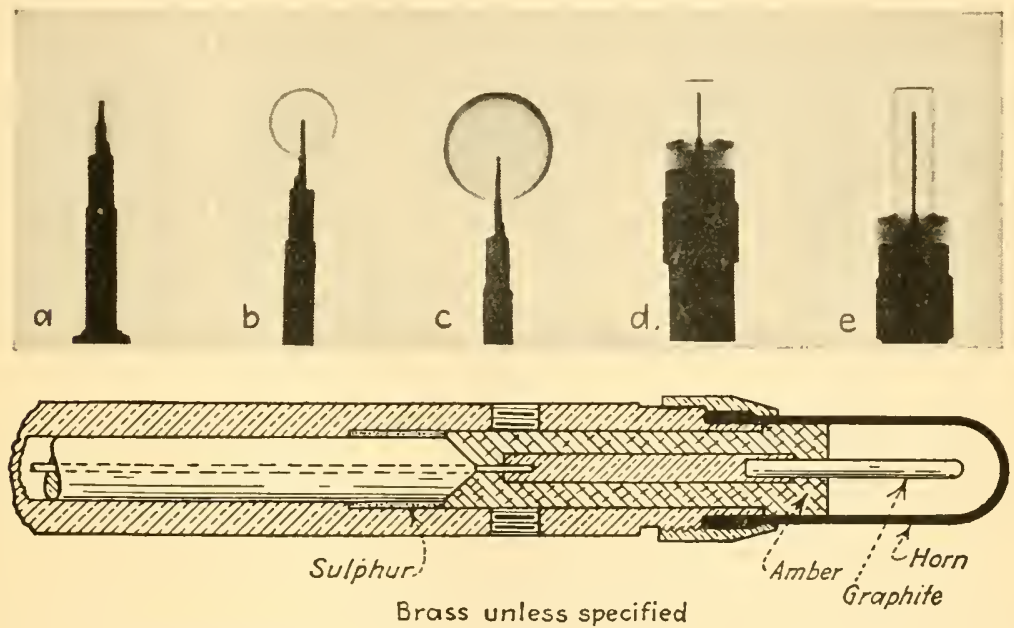

FIG. 17.-Thimble ionization chambers. Upper radiographs by Glasser; lower, Friedrich chamber.

$\mathrm{X}$-ray beam, the air within the shell is ionized, causing the charge to leak off the central electrode. The loss of charge $Q$ being proportioned to the X-ray quantity is given by the fall in potential of the charged system as indicated on the electroscope by the relation $Q=C\left(V_{1}-V_{2}\right)$, where $C$ is the electrostatic capacity of the complete insulated system and $V_{1}$ and $V_{2}$ are the potentials at the beginning and the end of the exposure of the chamber to the X-rays.

Wall Effects.-In general, the ionization per unit volume of air in a thimble chamber will not be the same as that in an open-air standard, nor even proportional to it, as the radiation quality changes. This is due to the so-called "wall effect" of the thimble chamber. X-rays striking the walls and collector electrode of the chamber produce both soft secondary X-rays and photoelectrons, both of which contribute to the ionization within the chamber in a manner different from that in the 
open-air chamber. The degree of the ionization from this source depends principally upon the atomic number of the wall material.

With a wall made of some material having the same atomic number as air, the ionization in the enclosed volume due to secondary $\mathrm{X}$-rays and electrons should theoretically be the same as the free air in a large chamber. It has likewise been demonstrated experimentally $(14,15)$ that a wall material having effectively the same atomic number $N_{\text {eff }}$ as air will, within a fairly wide range of wave-lengths $(0.1$ to $1.5 \AA)$, have the same effect on the ionization current as air. The effective atomic number $N_{\text {eff }}$ is given by the relation

$$
N_{\text {eff }}=\sqrt[3]{\frac{a_{1} N_{1}^{4}+a_{2} N_{2}^{4}+\cdots}{a_{1} N_{1}+a_{2} N_{2}+\cdots}}
$$

where $a$ is the fractioual quantity of material of atomic number $N$. By suitably choosing $a$ and $N$ in the materials composing a thimble chamber, its effective atomic number can be adjusted to 7.69 , that of air.

By a cut-and-try method, the material, shape, and position of the walls and collector electrode may be adjusted so that the photoelectrons from them produce the same net effect as air. It is thus possible to make a chamber having a graphite shell with aluminum collector which will give readings nearly proportional to the open-air chamber over a wide wave-length range of the harder radiations.

Method of Calibration. - Without going into further detail, it is evident that a thimble chamber cannot be relied upon to indicate true roentgens unless calibrated against a standard open-air ionization chamber. Even in production manufacture, it has not been found feasible to mechanically construct thimble chambers having identical characteristics without resorting to individual calibrations. To calibrate a thimble chamber, the intensity of an $\mathrm{X}$-ray beam, after suitable limitation by diaphragms, is measured in roentgens per minute at a given point with a standard free-air ionization chamber. Holding the $\mathrm{X}$-ray output steady, the standard chamber is removed from the beam, the center of the thimble chamber placed at the exact position of the front edge of the limiting diaphragm ( $D$, Fig. 12) of the standard chamber, and the time-rate of deflection noted for the thimble-chamber indicator. This must be done for each quality of radiation for which the instrument is to be used. Where the dependence of the deflection upon quality is small, only a few calibration qualities are necessary, it then being permissible to interpolate for intermediate qualities. Experience has shown that it is seldom safe to extrapolate such a calibration.

Several other sources of error must be guarded against $(7,43,57)$; for example, there may be some uncertainty as to the effective center of a thimble chamber when placing it in a test beam. To avoid undue errors 
( 1 to 2 per cent) from this source a thimble chamber should not be calibrated nearer than $50 \mathrm{~cm}$. from the tube target and preferably as much as $100 \mathrm{~cm}$. It must likewise be assured by test that the $\mathrm{X}$-ray beam is of uniform intensity over an area of diameter somewhat larger than the length of the thimble chamber.

When it is desired to read the thimble chamber and standard chamber ionization simultaneously, the two may be set at different distances from the tube and in such an alignment as not to interfere with each other. In such case, the ralidity of the inverse square law must be separately tested for each chamber, in addition to insuring that the test beam is uniform at position of each. The replacement method is usually preferable.
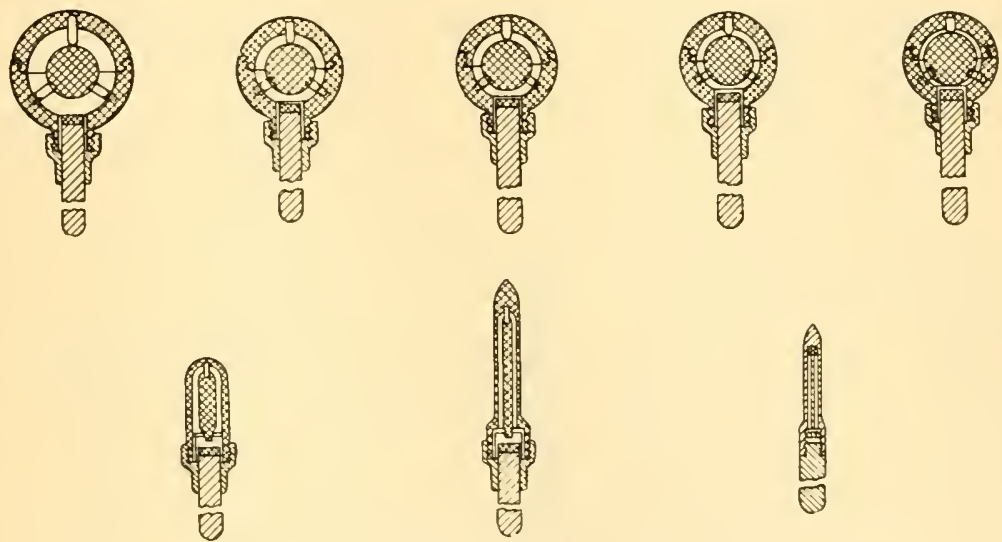

FIG. 18.-Condenser, thimble ionization chambers full size. (Sievert.)

Correction must likewise be made for the air density in the thimble chamber at the time of ealibration. This eorrection is obtained from the atmospheric temperature and pressure in the same manner as for the standard chamber.

Types of Thimble-chamber Roentgenometers.-Thimble-chamber roentgenometers may be divided into three groups, $(a)$ electroscope type, $(b)$ condenser type, and $(c)$ racuum-tube type. Instruments of any type, ordinarily read the total number of roentgens per given deflection or indieation, and by noting the time of deflection the $\mathrm{X}$-ray intensity is given in roentgens per minute. In the first two types the voltage sensitivity of the electrometer may vary over different parts of the seale; hence, for example, the number of roentgens may be different for the first and last half of the scale. Since this does not change with quality, a single correction curve may be determined by noting the time rate of change of the indicator between successive scale positions. Nost Ameri- 
can-made instruments of this type require little or no scale correction, although this should be assured by test for each instrument.

The condenser type $(19,37,38)$ of thimble chamber has definite fields of application in that the thimble chamber can be disconnected from the electrometer while being exposed to X-rays. To use, the chamber is attached to the electroscope and charged in the usual manner as indicated on the scale; it is then removed from the electroscope, the exposed lead covered with a metal cap for shielding, and exposed to X-rays, thereby discharging it; finally, it is replaced on the electroscope which now indicates its loss of charge and hence the strength of the X-ray beam.

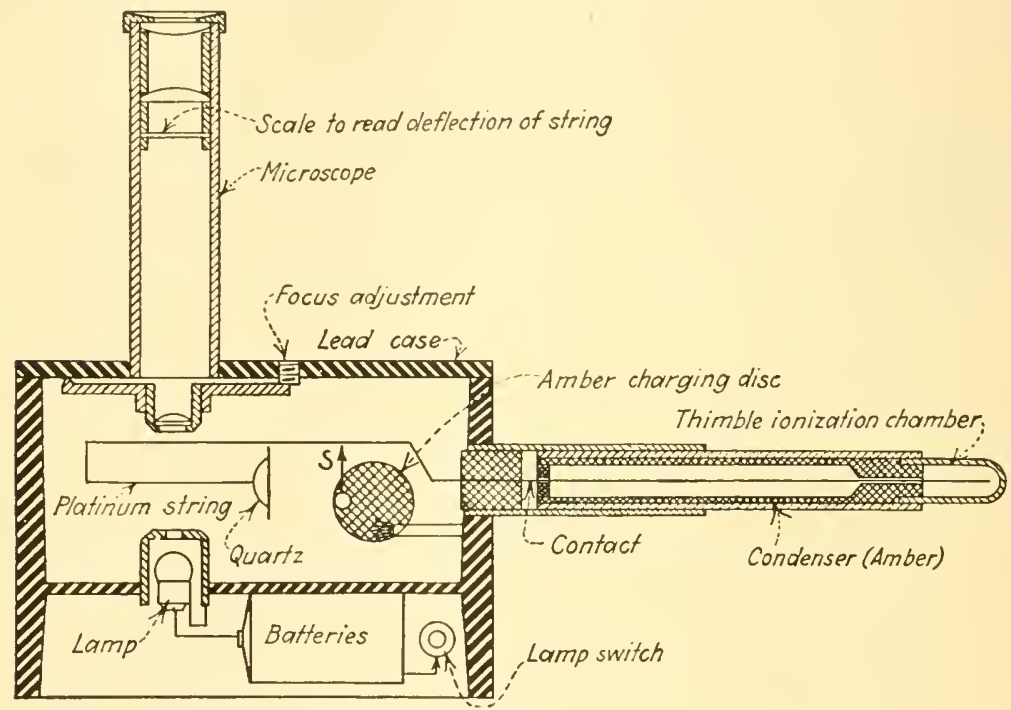

Fig. 19.-Condenser roentgenometer. (Glasser-Seitz.)

These chambers have also been made of the shape and size of radium needles (Fig. 18) thus permitting their direct insertion into tissue for the measurement of radium and X-ray depth dosage.

Figure 19 shows a condenser type of roentgenometer extensively used in this country. The ionization chamber and short section of the connecting lead, as a single unit, are inserted into the tube connected to the electroscope. The entire insulated system is then charged by means of the small bakelite electrostatic charging disk which is connected to the insulated system by the switch, $S$, during the charging operation. The potential of the system is indicated by the deflection of the conducting quartz fiber, illuminated by a 3-volt lamp, and viewed with a low-power microscope having a suitable ocular scale. When fully charged, the chamber is removed and placed in the X-ray beam, after which it is again inserted into the fully charged electroscope. The loss of charge 
is then indicated by the decrease in the deflection of the fiber. The scale is calibrated directly in roentgens. The constancy of the electrical calibration of the instrument is readily checked by inserting the ionization chamber completely discharged into the fully charged electroscope and noting the deflection after the charge has been divided between the two parts of the system.

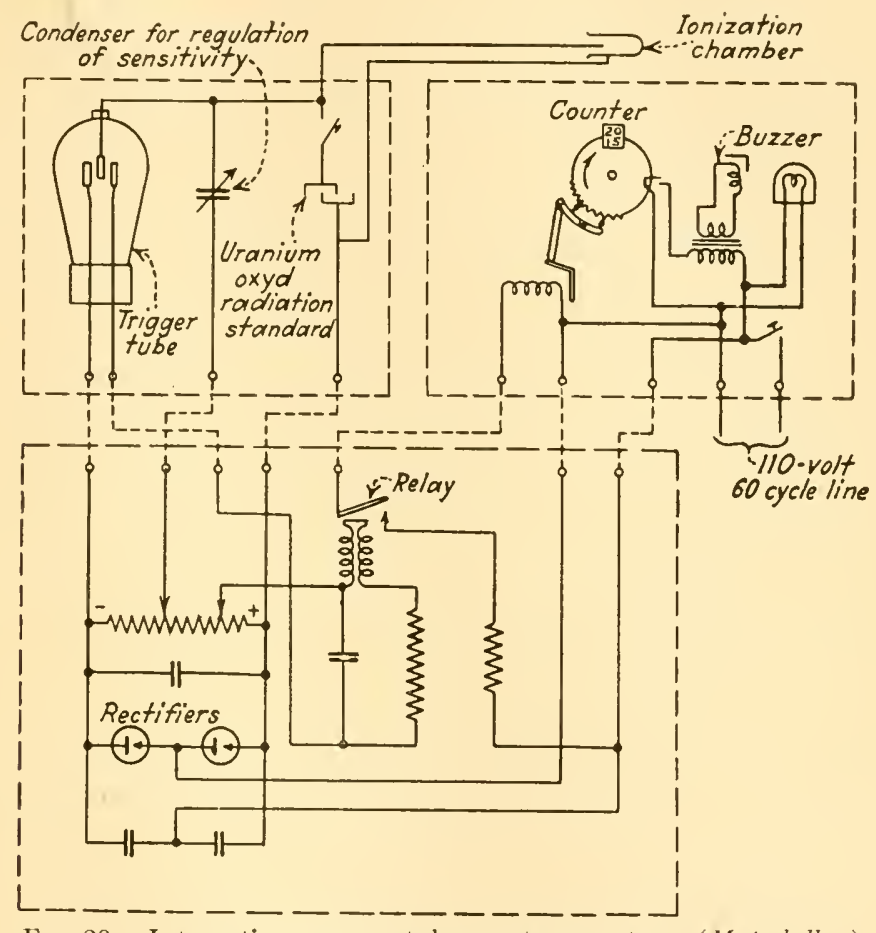

Thimble chambers having vacuum indicators are usually of the "relay type," that is, a certain small quantity of radiation causes a relay to operate and move an indicator one step, these steps being successively added up to give the total indication on a dial $(31,40)$. Such instruments require no scale correction and are frequently referred to as integrating meters.

Figure 20 shows one form of such instrument manufactured in this country. Several other similar types are made abroad (21). The ionization current flowing through the chamber is caused to accumulate upon one electrode of a gricl-glow tube until a definite potential is developed upon which an ionization discharge takes place in the tube. This discharge initiates the flow of a separate current through the tube which in turn operates the relay and a counting or recording mechanism. 
Thus every time a certain definite quantity of radiation has fallen on the ionization chamber, the glow tube functions, and the counting mechanism indicates directly the number of roentgens. The constancy of the electrical calibration is tested by connecting into the system a constant current which is produced by a small uranium oxide ionization chamber. The circuit is then adjusted by means of a variable condenser to produce a redetermined time rate of indication.

Measurement of X-ray Output.-In the measurement of the output of an $\mathrm{X}$-ray tube at a given point with a thimble chamber there are few precautions which are not self-erident from the preceding discussion. Scattering from nearby objects should be avoided, for example, by keeping the thimble chamber always at least $15 \mathrm{~cm}$. from any filters. There has been some question in biological practice whether or not to include in the measurement the back scattering from the irradiated object. Although a knowledge of back scattering is of interest, its exact measurement is very difficult in that it depends, among other factors, upon: (a) size of field, $(b)$ size and shape of irradiated object, $(c)$ size and shape of thimble chamber, $(d)$ material of thimble chamber, and $(e)$ depth to which the chamber is immersed in the surface of the irradiated material. To avoid the complication of these factors, the preferred method of $\mathrm{X}$-ray dosage measurement is to use the thimble chamber placed at the position of the surface of the irradiated object but surrounded by at least $15 \mathrm{~cm}$. of air. This gives the intensity of the X-ray beam in roentgens per minute without modification by scattering.

In view of the foregoing discussions it may be convenient to summarize the factors which it is necessary to state in describing a biological application of X-radiation. Such factors include at least the following: $a$.

Table 7.-Comparative X-ray Outputs for Different Types of Generator Factors: (a) Pyrex tube ahout $5 \mathrm{~mm}$. thick; (b) 50-cm. target to ionization chamber; (c) filter $0.557 \mathrm{~mm} . \mathrm{Cu}+1.0 \mathrm{~mm} . \mathrm{Al}$

\begin{tabular}{c|c|c|c|c}
\hline \multirow{2}{*}{$\begin{array}{c}\text { lilovolts } \\
\text { (peak) }\end{array}$} & \multicolumn{3}{|c}{ Output (roentgen) per milliampere of tube current } \\
\cline { 2 - 3 } & Coust.pd. & Full wave* & Villard $\dagger$ & Witka \\
\hline 120 & 1.45 & 0.8 & 0.79 & \\
140 & 2.30 & 1.2 & 1.39 & \\
160 & 3.30 & 1.8 & 2.00 & 1.61 \\
180 & 4.45 & 2.3 & 2.70 & 2.08 \\
200 & 5.65 & 2.9 & 3.42 & 2.50 \\
\hline
\end{tabular}

* About the same for half-wave and mechanical rectifiers.

$\dagger$ Voltage doubling (Fig. 1, C).

$\ddagger$ Voltage tripling (Fig, $1, D$ ).

Note: Outputs are about 9 per cent greater for thin glass tubes. Ratios between outputs are nearly the same as given, for filtrations up to $2.0 \mathrm{~mm}$. Cu. 
voltage (constant-potential equivalent); $b$. filter (including tube wall); $c$. target-skin distance; $d$. area, diameter, and number of fields; $e$. roentgens per minute in air at position of field; $f$. total number of roentgens delivered; $g$. duration of exposure; $h$. interval between exposures; $i$. size, shape, and position of irradiated volume.

Comparison of X-ray Generators.-For general guidance, Table 7 gives approximate outputs of several standard $\mathrm{X}$-ray machines illustrated diagrammatically in Fig. 1, as measured at $50 \mathrm{~cm}$. from the tube target $(20,32,49,50,52)$. With the exception of constant-potential excitation, large variations may occur between different generators of any one group and the mean values given in the table and subject to variations which may become as large as \pm 10 per cent.

\section{MEASUREMENT OF VERY LOW- AND VERY HIGH-VOLTAGE X-RAYS}

GRENZ RAYS (3 TO $15 \mathrm{KV}$.)

It has been adequately demonstrated that the general principles involved in the measurement of 70 - to $200-\mathrm{kv}$. X-rays may, with certain precautions, be extended to the accurate measurement of 3 - to $15-\mathrm{kv}$. X-rays. The principal correction to be applied in the use of a standard for $10-\mathrm{kv}$. radiation is that for absorption of the radiation between diaphragm and collector. This may amount to 40 to 140 per cent in using 10- to 6 -kv. (peak) unfiltered radiation. Figure 16, $B$ shows one form of such chamber (48) which by the use of the guarded field reduces the diaphragm-to-collector distance to a minimum (about $5 \mathrm{~cm}$.) while still fulfilling the requirements of a free-air chamber. This particular form of chamber is adequate for radiations up to $90 \mathrm{kv}$. and by thus overlapping the range of the larger chamber, permits a direct comparison between the two, using radiations of such high voltage $(90 \mathrm{kv}$.) as to require very small air-absorption corrections (but less than 2 per cent).

Secondary chambers of the thimble type have been adapted to the measurement of 3 - to 15 -kv. X-rays, although their accuracy is questionable in many cases $(18,21,26)$. Wall effects become relatively large and even the thimnest of wall materials absorbs a large percentage of the radiation, thus preventing its contribution to the ionization within the chamber. These two difficulties make it necessary to calibrate the secondary chamber for the exact radiation for which it will be used, and it has been shown that it is unsafe to interpolate between the radiation qualities used in the calibration. The accurate use of such thimble chambers is so beset with uncertainties that it is usually more satisfactory to use a small open-air standard for biological X-ray measurements at such low voltages (48).

Ultra-high-voltage $X$-rays. - At the time of writing, it may be said that the measurement of $\mathrm{X}$-rays excited by potentials above $250 \mathrm{kv}$. (peak) 
is not yet in a very satisfactory state, and it is still an open question whether or not the range of the large open-air standard can be extended sufficiently to measure such radiations. The particular difficulty is introduced by the increased relative importance of scattered radiation at the higher voltage.

The international definition of the roentgen (Paris, 1931) does not mention scattered radiation except that from the "wall effect," and it is only by fortunate circumstances that this omission has not caused difficulty in that below $150 \mathrm{kv}$. (r.m.s.) its effect is almost negligible.

One of the most important uses of the roentgen at higher voltages, is to permit a proper evaluation of X-ray dosage in comparison with that at ordinary voltages. It is, therefore, necessary that over the whole voltage range the roentgen be the measure of a quantity which can be translated into actual energy absorbed by tissue. Since the roentgen is proportional to the energy absorbed from the beam by air, it is necessary to restriet conditions so that the absorption will be known. At tube voltages below $150 \mathrm{kv}$. (r.m.s.) the absorption is largely photoelectric, whereas at the higher voltages, absorption is due principally to the energy removed from the beam by the recoil electrons in the Compton scattering process (27) (28).

The question then arises as to whether the definition should or should not include the effect of scattered radiation (56). If it should, we are confronted with the problem of how to devise an apparatus which will definitely include all of it. Technical diffieulties at the higher voltagesabove $150 \mathrm{kv}$. (r.m.s.)-seem at present to render this impossible. Should we then include only a definite fraction of the scattered radiation in the measurement, and if so, what shall that fraction be, and how shall we define and determine it? As this does not appear feasible with our present knowledge, the only alternative in making measurements is to avoid, as far as possible, all effective secondary radiation, and to reword the definition so as to embody the necessary conditions.

The revised definition given above appears capable of realization at high voltages without going to excessively large ionization chambers. Photographs made in a Wilson eloud expansion chamber $30 \mathrm{~cm}$. in diameter show that for a narrow 700-kv. X-ray beam practically all of the ion paths are confined within $10 \mathrm{em}$. of the beam, and the bulk of them within the geometrical beam. Furthermore, it is seen that practically no ion tracks originate in the space outside the geometrical beam. This shows clearly that $(a)$ the effect of radiation seattered from the chamber walls is negligible, and $(b)$ there is no appreciable ionization due to photo- or recoil electrons, produced by secondary (degraded) radiation scattered out of the main beam. The presence of tracks originating outside the beam indieates the entrance of a scattered quantum into that 
region with the subsequent collision with an air atom and the production of measurable ions.

The revised definition can probably be realized by the use of (a) suitable diaphragms to stop secondary X-rays and secondary electrons from the filters and diaphragm edges from reaching the measuring volume, $(b)$ a narrow and well-defined X-ray beam, and $(c)$ materials of low atomic number for the walls and electrodes of the chamber. If the diameter of the beam is sufficiently small in comparison with the distance in which the beam loses, say, half its energy, then the fraction of the degraded radiation which is absorbed in the measuring volume directly, or scattered back from the surroundings, may be neglected. Since the energy absorbed from the beam is transferred largely to recoil electrons and thence to ions, it may be determined directly from the measured ion current, which gives the number of ion pairs. It is obvious that the inclusion of an appreciable amount of scattered radiation would render such a determination very uncertain at best.

There is no definite information at present to indicate that a suitable thimble chamber can be constructed to measure accurately $\mathrm{X}$-rays above $250 \mathrm{kv}$., although certain work indicates that gamma radiation can only be measured in roentgens with a thimble chamber. There is also some evidence that ultra-high-voltage radiations may be measured with the drumhead type of chamber. These questions are still too controversial to warrant a discussion here.

\section{RADIUM MEASUREMENT}

The measurement of gamma rays from radium has been largely of a comparative and not absolute nature. Since radium dosage has been expressed in terms of geometrical factors and the amount of Ra element employed, it has been impossible to correlate dosages and biological effects of gamma rays as compared with X-rays. In many biological experiments this has naturally retarded the proper choice as to which radiation should be used.

The difficulties in the measurement of gamma rays in roentgens are somewhat analogous to those for ultra-high voltages, only accentuated $(13,27,29,30)$. For gamma rays, the avoidance of scattering becomes exceedingly difficult and moreover there is no certainty as to how much, if any, of the secondary scattering effects should be included in the measurement. It is not easy to segregate a narrow well-defined beam of gamma rays without any scattered radiation whatever, for passage through an ionization chamber. Use of a broad beam again introduces uncertainties due to scattering, and would require an excessively large chamber for its measurement. 
As already noted, it seems possible that gamma rays may be measured in roentgens with some form of thimble chamber. While many such chambers have been devised, there is not yet uniformity of opinion regarding their use. A number of observers using thimble chambers $(16,30)$ have experimentally determined the number of roentgens per milligram-hour at $1 \mathrm{~cm}$. from a point source of radium filtered with $0.5 \mathrm{~mm}$. Pt, finding a mean value of 8.6 roentgens.

Primary standardization of radium element is carried out almost exclusively by the national laboratories of several countries, each of which possesses radium standards that have been compared with the standard retained by the International Bureau of Weights and Measures at Sèvres, France. The unknown samples are directly compared with the standards by a replacement method, the ionization being measured with a heavily lead-shielded electrometer which serves also as the ionization chamber.

Since, as noted, the present-day expression of gamma-ray dosage is largely empirical, each measurement is so intimately related to its biological application that matters of filtration and intensity distribution will be discussed in connection with the biological application of radiation.

\section{REFERENCES}

1. Behnken, H. Zur Frage der Roentgen Dosiseinheit. Strahlentherapie 29: 192-198. 1928.

2. Beнnken, H. Die Roentgendosimetrie. Handbuch der gesamten Strahlenheilkunde, Biologie, Pathologie und 'Therapie. Bergmann; München, 1929.

3. Behnken, H. Die Absolute Bestimmung der Dosiseinheit "1 Roentgen" in der Physikalisch-Technischen Reichsanstalt. Strahlentherapie 26: 79-100. 1927.

4. Benoist, L. Definition expérimentale des diverses sortes de rayons-x par le radiochromomètre. Compt. Rend. 134: 225-227. 1902.

5. Bouwers, A. Measurement of the intensity of X-rays. Acta Radiol. 4: 368-377. 1925.

6. Bouwers, A. Over het meten der intensiteit van Roentgenstralen. Dissertation. Utrecht, 1924.

7. Braun, R., and H. Küstner. Zur Physik der Fingerhutkammer. Strahlentherapie $32: 550-581.1929 ; 32: 739-758.1929$.

8. Christen, Th. Messung und Dosierung der Roentgenstrablen, aus "Archiv und Atlas der normalen und pathologischen Anatomie." Fortsch. Geb. Roent. Erg.-Bd. 28: 1913.

9. Coolidge, W. D. A powerful roentgen ray tube with a pure electron discharge. Phys. Rev. (N.S.) 2 : 409-430. 1913.

10. Duane, W., and J. C. Hubson. The measurement of effective wave lengths. Amer. Jour. Roent. 20: 241-245. 1928.

11. Fallla, G. Criteria for the design of a standard ionization chamber. Amer. Jour. Roeut. 21: 47-63. 1929.

12. Fallla, G. A new instrument for measuring X-radiation. Radiology 15: 437-449. 1930.

13. Fallla, G., and P. Henshaw. The relative biological effectiveness of X-rays and gamma rays. Radiology $17: 1-43.1931$. 
14. Fricke, H., and O. Glasser. The secondary electrons produced by hard X-rays in light elements. Proc. Nat. Acad. Sci. 10:441-447. 1924.

15. Fricke, H., and O. Glasser. Eine theoretische und experimentelle Untersuchung der kleinen Ionisationskammer. Fortsch. Geb. Roent. 33: 239-250. 1925.

16. Glasser, O., and F. R. Mautz. The measurement of the intensity of gamma rays in r-11nits. Radiology $15: 93-100.1930$.

17. Glasser, O., and U. V. Portmann. The standardization of the roentgen ray dose. Amer. Jour. Roent. 19: 47-61. 1928.

18. Glasser, O., and U. V. Pormann. The physical and clinical foundations of oversoft Grenz ray therapy. Amer. Jour. Roent. 19: 442-452. 1928.

19. Glasser, O., U. V. Portmann, and V. B. Seitz. The condenser dosimeter and its use in measuring radiation over a wide range of wave lengths. Amer. Jour. Roent. 20: 505-513. 1928.

20. Gross, M. J., and Z. J. Atree. Roentgen-ray output of therapy eireuits and tubes. Amer. Jour. Roent. 30: 229-233. 1933.

21. Holthusen, H., and R. Braun. Grundlagen und Praxis der Roentgenstrahlendosierung. Georg Thieme; Leipzig, 1933. (Very complete bibliography to date.)

22. Holzknecht, G. Das Chromoradiometer. Cong. Intern. Electrologie et Rad. Méd. 2 : 377.1902.

23. Hull, A. W. Roentgen ray spectra. Amer. Jour. Roent. 2: 893-899. 1915.

24. Hull, A. W. The X-ray spectrum of tungsten. Gen. Elect. Rev. 19: 603-610. 1916.

25. Jakger, R. Ein neues Dosismesserprinzip. Strahlentherapie 33: 542-550. 1929.

26. Küstner, H. Die Dosierung der Buckyschen Grenzstrahlen nach R-Einheiten mit dem Eichstandgerät. Strahlentherapie 27: 124-145. 1928; 30: 334-342. 1928.

27. Lauritsen, C. C. Energy considerations in high voltage therapy. Amer. Jour. Roent. 30:380-387. 1933.

28. Ladritsen, C. C. Energy considerations in medium and high voltage therapy. Amer. Jour. Roent. 30: 529-532. 1933.

29. Mayneord, W. V., and J. E. Roberts. Measurements of high voltage X-rays. British Jour. Radiol. 6 : 321-340. 1933.

30. MAYNeORD, W. V., and J. E. Roberts. The ionization produced in air by X-rays and gamma rays. British Jour. Radiol. 7: 158-175. 1934.

31. Mutscheller, A. A simple integrating dosis measuring instrument. Radiology $20: 207-210.1933$.

32. Müller, W. Über den Betrieb von Therapieröhren an versehiedenen Apparaltetypen. Strahlentherapie 49: 132-140. 1934.

33. Quimby, Edith. A comparison of practical methods of measuring X-ray quality for therapy. Amer. Jour. Roent. 21: 64-72. 1929.

34. Richtmyer, F. K. The laws of absorption of X-rays. Phys. Rev. 18: 1-30. 1921.

35. Rump, W. Energiemessungen an Roentgenstrahlen. Zeitsch. Physik 43: 254-295. $1927 ; 44: 396 . \quad 1927$.

36. Schlechtmans, J. Über die Intensitätsmessung der Roentgenstrahlen nach der Ionisationsmethode. Annalen Physik 5, 5: 153-195. 1930.

37. Sievert, R. M. Eine einfache, zuverlässige Vorrichtung zum Messen von Tiefendosen. Acta Radiol. 5: 468-484. 1926. 
38. Sinvert, R. M. Eine Methode zur Messung von Rocntgen-, Radium- und Ultrastrahlen nebst einigen Untersuchungen über die Anwendbarkeit derselben in der Physik und der Medizin. Acta Radiol. Supplement XIV, 1932. (171 references on the particular subject up to 1932.)

39. Silberstein, L. Spectral composition of an X-radiation determined from its filtration curve. Phil. Mag. 15: 375-394. 1933.

40. Strauss, S. Das "Mekapion," der integrierende Roentgendosiszähler mit Selbstkontrolle. Strahlentherapie 24: 348-364. 1927.

41. TAYLor, L. S. Analysis of diaphragm system for the X-ray standard ionization chamber. Bur. Stand. Jour. Res. 3: 807-827. 1929; Radiology 15: 49-68. 1930.

42. TAYlor, L. S. Precise measurement of X-ray dosage. Bur. Stand. Jour. Res. 2 : 771-785. 1929; Radiology 14: 372-387. 1930.

43. TAYlor, L. S. The calibration of the "Fingerhut" ionization chamber. Bur. Stand. Jour. Res. 4: 631-644. 1930; Radiology 15:227-243. 1930.

44. TAYlor, L. S. Absorption measurements of the X-ray general radiation. Bur. Stand. Jour. Res. 5: 517-536. 1930; Radiology 16:302-322. 1931.

45. TAYLOR, L. S. Accurate measurement of small electric charges by a null method. Bur. Stand. Jour. Res. 6: 807-818. 1931; Radiology 17: 294-306. 1931.

46. TAYlor, L. S. International comparison of X-ray standards. Bur. Stand. Jour. Res. 8: 9-24. 1932; Radiology 18: 99-114. 1932.

47. TAYlor, L. S., and G. Singer. An improved form of standard ionization chamber. Bur. Stand. Jour. Res. 5: 507-516. 1930; Radiology 15: 637-649. 1930.

48. TAylor, L. S., and C. F. Stoneburner. The measurement of low voltage X-ray intensities. Bur. Stand. Jour. Res. 9: 769-780. 1932.

49. TAylor, L. S., and C. F. Stoneburner. Operation of thick-walled X-ray tubes on rectified potentials. Bur. Stand. Jour. Res. 10:233-247. 1933.

50. TAylor, L. S., G. Singer, and C. F. Stoneburner. A basis for the comparison of X-rays generated by voltages of different wave form. Bur. Stand. Jour. Res. 11 : 293-308. 1933; Amer. Jour. Roent. 30:368-379. 1933.

51. TAYLOR, L. S., and G. Singer. Standard absorption curves for specifying the quality of X-radiation. Bur. Stand. Jour. Res. 12: 401-420. 1934; Radiology 22 : 445-460. 1934.

52. Thylor, L. S., G. Singer, and C. F. Stoneburner. Effective applied voltage as an indicator of the radiation emitted by an X-ray tube. Bur. Stand. Jour. Res. 9: 561-569. 1932; Amer. Jour. Roent. 30: 221-228. 1933.

53. Ulrey, C. T. An experimental investigation of the energy in the continuous X-ray spectrum of certain elements. Phys. Rev. 11:401-410. 1918.

54. Villard, P. Instruments de mésure à lecture direct pour les rayons-X. Arch. Ëlec. Méd. 16: 692.1908.

55. Wilsey, R. B. The specification of X-ray quality. Radiology 17: 700-713. 1931.

56. Report of committee on the standardization of X-ray measurements. Radiology 22 : 289-294. 1934.

57. X-ray standards and units. Standardizing procedure of the National Laboratories. Amer. Jour. Roent. 31: 815-818. 1934.

\section{GENERAL REFERENCES}

58. Brenzinger, M., A. Janitzky, and E. Wilhelmy. Allgemeine Grundlagen der Physik und der Technik des Roentgenverfahrens. leipzig, 1930. 
59. Compton, A. X-rays and electrons. D. Van Nostrand Company, Inc.; New York, 1925.

60. Holthusen, H., and R. Braun. Grundlagen und Praxis der RoentgenstrahlenDosierung. Georg Thieme; Leipzig, 1933.

61. KAYE, G. W. C. Roentgenology. Paul B. Hoeber; London, 1928.

62. KaYe, G. W. C. X-rays. Longmans, Green \& Company; New York, 1926.

63. Siegbahn, M. The spectroseopy of X-rays. Oxford University Press; New York, 1925.

64. Terrill, H. M., and C. T. Ulrey. X-ray technology. D. Van Nostrand Company, Inc.; New York, 1930.

65. Weatherwax, J. L. Physics of Radiology. Paul B. Hoeber; New York, 1931.

66. X-ray Studies. Research Laboratory, General Electric Co., 1919.

Manuscript received by the editor September, 1934. 



\title{
IONIZATION AND ITS BEARING ON THE BIOLOGICAL EFFECTS OF RADIATION
}

\author{
G. Failla \\ Memorial Hospital, New York
}

Fundamental character of ionization. Distribution of ions in an ionizcd medium. Interaction of radiation and matter. Influence of the quality of radiation on the distribution of ions in living matter. Intensity of radiation and inverse square law. Influence of matter on the intensity of radiation. Secondary phenomena. Filtration. Essential factors for the correlation of ionization and biological effects. Effective intensity of radiation. Significance of ionization measurements. Knowledge of ionization produced in irradiated organism all important. Effective quantity of radiation. References.

The law of conservation of energy tells us that if a change has been brought about in a system by external means, then some energy must have been supplied to the system from the exterior. Thus, if a living cell is altered in any way by exposure to radiation, it must have received some energy which at least initiated the change. In this sense the cell may be eompared to a radio receiver and the source of radiation to a broadcasting station. Under certain conditions the radio "picks up" a signal which initiates a series of changes in the reeeiver, and the listener hears it. The energy required to produce the audible signal is very much greater than that in the incoming wave which actuated the detector, and this energy is supplied by the local source at the receiver. But energy, however small in amount, must be supplied to the detector in order to initiate the change. Furthermore, the energy must be delivered in a certain form-in this ease electromagnetic waves of a certain frequeney range. Similarly, the living cell exposed to radiation receives a small amount of energy which initiates eertain changes requiring much more energy to bring to completion, this energy being supplied by chemical changes in the cells. The initial actuating energy must also be in a certain form-visible light, X-rays, ete. Energy ean be transferred to the cell by the radiation only through the interaction of matter and radiation. Hence the understanding of this interaction is essential to the proper interpretation of the biological effects of radiation.

\section{FUNDAMENTAL CHARACTER OF IONIZATION}

The conditions necessary and sufficient for the production of ions by radiation in a given material have been set forth by $K, K$. Darrow. 
Paper I. In the case of X-rays and gamma rays, with which we are chiefly concerned here, these conditions are always fulfilled. The interaction of matter and radiation in this case is an atomic phenomenon whereby the radiation transmits some of its energy to an electron in the atom and causes it to leave the atom with a high velocity and therefore a certain amount of kinetic energy. This high-speed electron, then, spends most of its energy in removing electrons from those atoms in its path which tend to impede its motion. The wandering electrons thus created attach themselves to other atoms, forming negative ions, whereas the atoms which have lost electrons remain positively charged and form the positive ions. In a gas where the atoms are not closely packed, the ions are relatively far apart; on the other hand, they can move with considerable freedom. Through thermal agitation and the force of attraction between positive and negative charges, ions of different polarity soon come together, an interchange of electrons takes place, and neutral atoms result. This process is called recombination. Ionization and recombination take place constantly in matter through which radiation is passing, and at any one time but a very small fraction of the total number of atoms present is in the ionized state. Recombination in a gas may be reduced greatly, or actually prevented under certain conditions, by the application of an electric field, which makes the ions of different polarity move in opposite directions. In a liquid or solid the atoms are closely packed, ions are nearer to one another, and motion is more difficult. Recombination takes place also, and, in fact, it is much more difficult to prevent it by the application of even a strong electric field. That thermal agitation plays an important part in the recombination of ions may be demonstrated very vividly by the following experiment: A phosphorescent material exposed to cathode rays (high-speed electrons) glows brilliantly at room temperature. If it is then immersed in liquid air, the phosphorence stops, but it reappears after removing the material from the liquid air, when it assumes a higher temperature. (It will be recalled from Darrow's discussion-Paper I- that emission of light in a phosphorescent material involves a rearrangement of electrons in the atoms.)

We have seen how radiation transfers some of its energy to matter and how this energy is used up in the formation of ions. So far as we know at present this is the only way in which matter abstracts energy from ionizing radiations. Therefore all other effects are subsequent to ionization and must be closely related thereto. Since thermal agitation plays an important part in the recombination of ions, it follows that a good many ions of opposite polarity are brought together primarily by atomic or molecular collisions. The force of attraction between positive and negative ions is not sufficiently large to bring them together unless the distance is very short and there are no obstacles (other molecules) in 
the way. Furthermore, when a positive ion is formed, the electron which leaves the atom or molecule attaches itself to any atom or molecule which happens to be in its path. There is some eridence also that ions are apt to form clusters with neutral atoms or molecules. The final result of all these chance encounters is that some atoms or molecules are brought together under conditions which faror a regrouping of the atoms, and consequently chemical changes take place. A great many chemical changes produced by ionizing radiations have been observed (2). The change may be a breaking up of complex molecules into simpler ones, or vice versa, depending on the conditions of the experiment. But, in general, the tendency is to transform complex molecules into simpler compounds or elements. Thus volatile hydrocarbons and carbon dioxide are liberated from paraffin under the action of very intense radiation. Water is decomposed into hydrogen and oxygen, but at the same time some hydrogen peroxide is formed. Organic compounds with complex molecules are affected more readily.

It should be emphasized at this point that the chemical changes brought about by ionizing radiations are always very slight when the commonly arailable sources are employed. It has already been mentioned that only a small proportion of the atoms in an irradiated material are ionized. Of the ions thus formed, only some make subsequent encounters which fulfil the conditions conducive to chemical changes. On the other hand, with powerful beams of three-million-rolt cathode rays, ${ }^{1}$ very marked alterations have been produced in substances which are influenced only slightly by even long exposure to ordinary sources of radiation.

The biological action of radiation may be accounted for by the chemical effects which radiation produces. The sequence of events may be assumed to be: $(a)$ ionization, $(b)$ chemical changes, and (c) biological changes. There may then be further chemical changes as a consequence of the first biological changes, and so forth. This view of the process accounts, at least to some extent, for the delay (latent period) in the appearance of the effects of radiation on living organisms. The foregoing "picture" of the biological action of radiation is very rudimentary, and it is no more than a statement of certain steps in the process which we either know or surmise. It does not provide the modus operandi necessary for the proper interpretation and correlation of the known biological effects of ionizing radiations.

Whatever the mechanism of the biological action of radiation may be, we know definitely that ions are produced in the material and that the

${ }^{1}$ The cathode-ray effects referred to in this sentence are due in part to the heat liberated when the electrons are stopped. (Personal communication from A. Brasch and Fritz Lange.) 
presence of these ions is apt to produce chemical changes or electrical disturbances which must affect biological processes. Therefore, a knowledge of the ionization in the living organism which one is irradiating, is the sine qua non of any quantitative experimental study. Accordingly, it is desirable to examine this question in detail.

It has already been mentioned that the secondary beta particle 'fast moving electron) which is set in motion by the photon, removes electrons from some of the atoms in its path. The beta particle is deflected somewhat at each encounter and follows a zigzag course. In an organic medium it is apt to encounter the different complex molecules in any orientation and therefore may remove an electron from any one of the component atoms-hydrogen, oxygen, nitrogen, ete. Similarly, the electron which has thus been removed from one atom, in attaching itself to a molecule may adhere to any one of the component atoms. On this basis there would be in the material molecules with unbalanced positive or negative charges at different points in their configurations. The resultant chemical changes would then depend, to some extent at least, on the orientation of the two molecules at the time they collided. Accordingly, recombination of ions, which, in effect, means neutralization of charges, need not always result in a rearrangement of the atoms in the molecules involved. When readjustments do oceur, the resultant molecules need not necessarily be the same, and therefore more than one chemical ehange is apt to take place. Aceording to this view, only part of the total ionization produced in the material would be ehemically, and ultimately biologically, effective, first, beeause not all the ion recombinations would result in chemical ehanges, and further because not all the possible chemical readjustments would necessarily influence life processes.

\section{DISTRIBUTION OF IONS IN AN IONIZED MEDIUM}

It is obvious that the time rate of recombination of ions depends on their space concentration. The more ions of opposite polarities there are in a given space under given conditions, the more rapidly positives and negatives will come together. Since ions are produced in pairs by radiation, the concentration of positives and negatives is the same, and the rate of recombination is proportional to the square of the number of ion pairs present at any time. Other conditions being equal, the number of ion pairs per cubic eentimeter produced per second is proportional to the intensity of the radiation. Therefore, recombination should take place more rapidly the higher the intensity of the radiation. This would certainly be true if the ions were distributed perfeetly uniformly throughout the material. In a homogeneous medium which is uniformly irradiated the ions may be considered to be uniformly distributed, but only in 
the macroscopic sense of the same number per unit volume. If the ion distribution could be viewed with a microscope, it would be found that the ions are really concentrated along certain tortuous lines which mark the paths of the secondary beta particles. Accordingly, there are islets in the material in which at any one time no ions are produced. However, since the point at which a secondary beta particle is liberated is merely a matter of chance, the probability of ionization of every molecule in a homogeneous medium is the same. Therefore, all the molecules may be ionized at one time or another in the course of an indefinitely long exposure, or during a finite exposure to an extremely high radiation intensity.

In addition to the special concentration of the ions along certain lines in the material, we must consider also the distribution along these lines. It is well known that the number of ions produced per centimeter of path of particles traveling at high speed depends on the speed of the particles. In fact, it has been determined experimentally that the greatest concentration of ions occurs near the end of the path, when the speed of the particle is relatively low. It follows from the foregoing considerations that the ions in a homogeneous medium, uniformly irradiated, are concentrated largely within very small regions interspersed throughout the material.

Living tissue, considered on the submicroscopic scale, with which we are dealing, is not homogeneous. Its atomic components are largely hydrogen, oxygen, nitrogen, and carbon, but there are also present in small or minute quantities elements of higher atomic numbers, such as iron or calcium. The transformation of electromagnetic radiation (photons, cf. K. K. Darrow, Paper I) into high-speed electrons by which ionization is produced, depends very largely on the atomic number of the element in the path of the radiation. The higher the atomic number, the larger by far the amount of energy abstracted from the radiation and the larger the resultant ionization. It follows, therefore, that in a living tissue, the ionization will be most intense in the neighborhood of the molecules containing elements of higher atomic numbers. This results in further localization of the ionization.

Recombination, which is influenced markedly by the number of ion pairs per unit volume, is much more rapid than it would be if all the ions were evenly distributed throughout the material. When the intensity of radiation is increased, the total number of ions produced per second in a given material is increased in the same proportion. This increase is brought about by a proportional augmentation of the number of the betaparticle tracks, which we may call the ionization loci. The distribution of ions at each locus remains the same as before, unless perchance two of the loci coincide wholly or in part. In liquids or solids, where the mean free 
path of the molecules is very short, thermal agitation does not disturb appreciably the localized character of the ionization and therefore the rate of recombination of the ions must depend almost entirely on the linear distribution of the ions in the loci and not appreciably on the number of loci present in a given volume. Accordingly, the rate of recombination should be sensibly independent of the intensity of radiation within rather wide limits. ${ }^{2}$

It is possible that the rate of recombination of ions influences the chemical, and hence the biological, effectiveness of a given ionization. Conceivably the more rapidly recombination takes place, the larger will be the proportion of the total number of ions produced per unit time, which would return to the initial electrically neutral state without undergoing a rearrangement of the atoms in the molecule. The settlement of this question must be left to future experimentation. At first one might think that the established validity of the Bunsen-Roscoe law (biologically stated, equal effects are produced by equal doses in which intensity $\times$ time of irradiation $=$ constant, within limits of time and intensity) in certain chemical reactions, brought about by radiation, precluded any appreciable influence of the rate of ion recombination on the chemical action of radiation. However, as already pointed out, the rate of recombination of ions produced in a liquid or solid must be governed largely by the linear distribution of the ions in the ionization loci, and not appreciably by the number of loci per unit volume (which is the factor that depends on the intensity of radiation). It might be well to remember also that the validity of the Bunsen-Roscoe law in the $\mathrm{X}$-ray region has been established only for a few chemical reactions within rather narrow limits of radiation intensity (3). Exceptions have also been noted (5). From the foregoing viewpoint, one would not expect a breakdown of the Bunsen-Roscoe law until the intensity of radiation became so high that the ion density throughout the material approached that obtaining in the ionization loci themselves. This point has not been tested experimentally.

The localized character of the ionization produced in a biological material by X-rays has certain important theoretical implications. For this reason it may be well to pursue it a little further. In particular, it is desirable to study the influence of the wave-length of radiation on the ionization loci.

${ }^{2}$ In support of this idea may be mentioned the well-known difficulty of obtaining saturation current in a gas ionized by alpha rays, even when the average ionization is very low, on account of the great concentration of ions along the alpha-ray paths. It is also known that saturation current camnot be obtained in the case of liquids, irrespective of the type of radiation which produces the ionization. 


\section{INTERACTION OF RADIATION AND MATTER}

The transfer of energy from photons to matter takes place through the intermediary of electrons which are set in motion, and then, as we have seen, ionize the material. But there are two processes by which the first step may be brought about (ef. Darrow, Paper I) : By the (a) photoclectric effect, in which case all the energy of the photon is transformed at once and the secondary electron leaves the atom with a kinetic energy less than that of the photon, by the amount necessary just to separate the electron from the atom. To bring about this drastic transformation of a highenergy photon, the latter must "collide" with an electron which figuratively is capable of "withstanding the full shock before running away." That is, this reaction takes place with tightly bound electrons in the atom, such as those of the $K$ region. If the photon has an amount of energy considerably higher than that required to remove the electron from the atom (varying with the atomic number of the element), the emergent electron travels at a high speed and ionizes a large number of atoms. The energy of the photon which is utilized in just removing the electron from the atom is not lost but reappears as another photon as soon as a new electron takes the place of the one previously dislodged from the atom (cf. Darrow, page 24). Since this photon does not ionize until it also sets an electron in motion, we need not consider it at this time.

The elements of which living matter is largely made up have low atomic numbers, and even the electrons in their $K$ regions are not very rigidly bound. Accordingly, when the energy of the photon is very high (as in the case of high-voltage $\mathrm{X}$-rays), the conditions for the immediate and complete transformation of a photon are not favorable and few photoelectric encounters of this type occur. Most of the energy which is spent in ionizing living matter irradiated by high-roltage $\mathrm{X}$-rays is abstracted from the radiation through a different process, that is, (b) the Compton effect (see Darrow, page 39). In this case the transfer of energy from the photon to the electron takes place according to the laws of elastic impact and depends on the angle which the path of the emergent electron makes with the path of the impinging photon. It should be noted that a photon can transmit practically all its energy to an electron in the event of a head-on collision which sends the electron hurtling through space substantially in the direction which the photon would have followed had it not been stopped. If the electron is projected in any other direction, the energy imparted to it is always less than this amount; its speed is lower and the number of ions which it ean produce is smaller. No electrons can be emitted backward (i.e., toward the source of radiation) by this process. At most, an electron ean be projected at right angles to the path of the photon, but in this case it will abstract 
practically no energy from the photon. On the other hand, it should be remembered that the electron which is emitted initially in the general direction of the photon path will deviate from its course by impacts with atoms and may then travel in the opposite direction. The energy of the photon which is not transmitted to the electron by the Compton-effect process appears as a photon of lower energy (longer wave-length) emitted in a direction which is compatible with the laws of elastic impact. As in the case of the photoelectric effect, the energy of the secondary photon is not immediately available to produce ions. But these photons, of course, are identical with primary photons, although their energy is less, and therefore are capable of undergoing exactly the same transformations that we have just diseussed (photoelectric effect and Compton effect). When these oecur, they will produce beta particles which further contribute to the ionization of the medium. The transformation of photons into high-speed electrons and other photons, goes on indefinitely until practically all the energy of the original photons has been transferred to electrons through repeated steps of this process. Mention might be made here of the converse process, whereby secondary electrons traveling at high speeds may be stopped suddenly, in which ease their kinetic energy appears in the resultant photon. (This is how $\mathrm{X}$-rays are produced in the first place, except that the electrons are accelerated artificially in the $\mathrm{X}$-ray tube.) This is not a common oceurrence in organic materials, but in any case it simply retards the inevitable disappearance of all photons impinging on matter through the transformation of their energy into ionization.

\section{INFLUENCE OF THE QUALITY OF RADIATION ON THE DISTRIBUTION OF IONS IN LIVING MATTER}

We may now consider the influence of the quality of radiation on the distribution of ions in living matter, on the basis of the processes just described. The quality (wave-length range and energy distribution in this range) varies with the voltage applied to the X-ray tube. ${ }^{3}$ But in a beam of $\mathrm{X}$-rays produced at a given peak voltage there cannot be wave-lengths present which are shorter than a theoretical minimum, given by the expression

$$
\lambda_{\min }=\frac{12,337}{\text { peak volts }}
$$

The upper limit is rather indefinite, but may be taken as that wave-length which is capable of traversing the walls of the X-ray tube. Radiation of the minimum wave-length is actually present in negligible proportion.

${ }^{3}$ The influence of filtration on the quality of radiation will be discussed later. See also paper by L. S. Taylor for other factors which affect quality. 
In general, most of the energy is carried by waves in the neighborhood of double this wave-length.

In the case of soft X-rays, produced, for instance, at 50,000 volts, the minimum wave-length is $0.247 \AA$, and reciprocally the energy of the secondary electrons liberated by such radiation, by either the photoelectric or Compton effect, cannot exceed 50,000 Ev. Indeed, a negligible number would even approach this energy limit. Fifty thousand volt electrons will travel a distance of about $5 \mathrm{~cm}$. in atmospheric air, which corresponds to approximately $0.06 \mathrm{~mm}$. in living tissues. This is the theoretical maximum range, but actually most of the ionization loci are much shorter, perhaps less than 0.01 or $0.02 \mathrm{~mm}$. In this short distance several hundred ion pairs are liberated. As the voltage at which X-rays are produced increases, the path of the fastest secondary electrons increases also. Hence, some relatively long ionization loci are now present which were entirely absent in the case of softer X-rays. Furthermore, the average locus is also longer.

If the number of ion pairs produced per centimeter were the same throughout the path, the total distance traveled by the secondary electron would be proportional to the voltage required to give it its initial speed, because it takes the same amount of energy to produce a pair of ions at every point in the path, in a given material. This is approximately true in the first part of the trajectory of high voltage-high-speed - electrons. But as the speed reaches a certain relatively low value, the rate of ionization increases rapidly and a large number of ions are produced in a short distance. The number of ions per centimeter set free by high-speed beta particles, therefore, is approximately constant (actually it increases somewhat) along most of its trajectory, and then becomes several times larger near the end of its path. A two million volt electron, such as some of the beta particles of radium, can liberate in living tissue a total of 60,000 ion pairs, at the rate of about 3000 to 4000 per millimeter throughout most of its trajectory. In the last part of its path, amounting to a small fraction of a millimeter, the ionization rate is several times higher. The total distance traveled through tissue by such a beta particle is considerably longer than $1 \mathrm{~cm}$., but on account of the tortuous path which the electron follows the actual range is generally shorter than $1 \mathrm{~cm}$. For lower-voltage beta particles the range is correspondingly shorter (approximately).

Remembering that in an organic material the abstraction of energy from hard radiation takes place largely by the Compton effect and that through this process electrons of low as well as high speed are produced, it follows that even in the case of gamma rays a large proportion of the secondary electrons do not travel very far in living tissue. The ionization

${ }^{4}$ It is nevertheless a very high speed. 
loci, therefore, consist of some long tracks (of the order of $1 \mathrm{~cm}$.) and many shorter ones ranging down to a very small fraction of $1 \mathrm{~mm}$. The difference in the distribution of ions produced by soft and hard X-rays may be visualized most readily as follows: A living tissue irradiated with soft X-rays is interspersed with ionization loci which are very short and have the appearance of dots. When the material is irradiated by hard radiation, some of the ionization loci have the appearance of dots and some are more like commas, with short or long tails, depending on the speed of the electrons which produced them. The ion concentration in the dot loci and at the head of the comma loci is substantially the same in all cases. The concentration along the tails decreases rapidly at first and then slowly toward the end of the tail. When the tail is very long, the ion concentration is substantially constant along most of its length. The fraction of the total ionization which is found in the tails of all the comma loci depends on the quality of the radiation and increases with its penetrating power. In the case of ordinary X-rays produced at voltages under $200 \mathrm{kv}$. peak, this fraction is always small. Recombination, depending markedly on the ion concentration, is much more rapid in the dot loci than in the tails of the comma loci. Accordingly, if the rate of recombination of the ions influences their chemical and biological effectiveness, the same total number of ion pairs liberated in a given material will not produce the same chemical and biological changes when, in one case, the ionization loci are dots and, in the other, they are dots and commas. Such a difference, if it existed, would not be apparent in the usual range of X-ray wave-lengths, but it should be noticeable when the comparison is extended to the gamma-ray region. It should also be particularly marked when the ionization is produced by the hard beta rays of radium.

\section{INTENSITY OF RADIATION AND INVERSE SQUARE LAW}

The distribution of ionization in a biological medium has been discussed so far on the assumption that the radiation is uniformly distributed throughout the material. In practice this is seldom (and strictly speaking, never) the case. As already pointed out, the intensity of radiation determines the number of ionization loci in a given volume, and not the shape of the loci. Hence, the variation of the intensity of radiation from point to point in the naterial results simply in the variation of the concentration of ionization loci. We shall discuss now the factors which influence the intensity of radiation. In this connection it is important to have a clear idea of the meaning of this term as used here. (The same expression is also used to express an entirely different quantity, as we shall see later.) The fundamental concept may be presented most simply on the quantum theory of radiation. It will be recalled that in 
this theory a source of radiation emits "particles" of energy, known as photons, which in a vacuum travel in straight lines with the velocity of light. The factor which, on the undulatory theory, is called wave-length, is represented in this case by the amount of energy in the photon. The shorter the wave-length, the larger is the amount of energy in the photon. Monochromatic radiation consists of photons all of which carry exactly the same amount of energy. A very small source-"point source"-of monochromatic radiation emits, let us say, $N$ photons per second. Since the emission takes place at random, during a certain time the photons are projected uniformly in all directions. If the source is at the center of a sphere, all the photons pierce the surface of this sphere, and furthermore, in a given time the same number pass through equal areas of the surface. The area of a sphere of radius $x \mathrm{~cm}$. is $4 \pi x^{2} \mathrm{~cm} .{ }^{2}$. Therefore the number of photons traversing each square centimeter per second is $N / 4 \pi x^{2}$. If each photon carries an amount of energy $h \nu$ (cf. Darrow, page 3), the energy passing through each square centimeter per second is $N h \nu / 4 \pi x^{2}$. This is the intensity of radiation at the distance $x$ from the point source in question. If the radiation is polychromatic, it can be divided into its monochromatic components and the intensity of radiation at a given point will then be the sum of the intensities of the individual components. The mathematical relation for the intensity of radiation

$$
I=\frac{N h \nu}{4 \pi x^{2}}
$$

can be written in the form

$$
I=\frac{N h \nu}{4 \pi} \cdot \frac{1}{x^{2}}
$$

or, since for a given source and in a vacuum, $N h \nu / 4 \pi$ is constant,

$$
I=\frac{K}{x^{2}}
$$

This is the well-known inverse square law, which states that in a vacuum the intensity of radiation at any distance from a point source is inversely proportional to the square of the distance.

In most X-ray tubes employed in practice, the radiation is emitted from a small area, the "focal spot" of the target. The distance at which the material to be irradiated is placed is relatively long, and therefore the intensity of radiation follows the inverse square law very closely, in vacuo. The discrepancy introduced by the presence of air can be neglected unless the radiation is very soft. ${ }^{5}$ But when radium is used

${ }^{5}$ Certain complications which cannot be discussed here arise in the case of gamma rays, on account of the long range of the secondary beta particles. 
as the source of radiation, one is dealing generally with distributed sources of considerable dimensions compared with the distance at which the material is placed. The inverse square law is not applicable to such a source. For practical purposes one may subdivide it into very small areas, each of which can then be considered to be a point source, and the simple mathematical relation may be applied to each without introducing a large error. The resultant intensity at the point is then the sum of the intensities due to the assumed point sources. It should be noted in this connection that the intensity of radiation from each point source represents the flow of energy through a surface of unit area perpendicular to the direction of travel of the photons, at the point in question. Since photons will reach this point from different directions, the surfaces through which the flow of energy is reckoned have different orientations. The summation of the individual intensities is justified in the case under consideration, because we are really interested in the number of photons (and their respective energies) reaching a very small volume surrounding the point. While the intensity of radiation is expressed in the c.g.s. system of units in terms of the energy flow per square centimeter, it does not follow that the area of the surface must be $1 \mathrm{~cm} .{ }^{2}$. In fact, it should always be at least small enough to insure a practically uniform distribution of radiation throughout the area.

The inverse square law holds strictly in a vacuum. If matter is in the neighborhood of the point at which the intensity of radiation is desired, or near the source, certain complications arise which will be discussed presently. At this time, it is important to note that with a given constant source of radiation the same amount of energy in the form of photons is distributed over a larger and larger area as the radius of the sphere (distance from the source) increases. Therefore, the energy flow per unit area (i.e., the intensity) must decrease correspondingly. The influence of matter on the intensity of radiation at any point is superimposed on this geometrical effect. On the basis of the inverse square law alone, therefore, the intensity of radiation is different at points in a biological object which are located at different distances from the source. The variation of intensity with distance is particularly important in the practical use of radium on account of the short distances commonly employed. This will be readily appreciated from the following numerical examples: Suppose that the object to be irradiated is $1 \mathrm{~cm}$. thick. If a radium container of very small dimensions (approximately a point source) is placed at a distance of $1 \mathrm{~cm}$. from the object, the intensity on the far side will be only one quarter of that on the near side of the object, on the basis of the inverse square law alone. If the radium is placed at $0.5 \mathrm{~cm}$. distance, the intensity on the far side is only 19 of that on the near side. On the other hand, in the case of X-rays, 
where much longer distances can be used, the decrease of intensity from one side of the object to the other is much less. Thus, for a targetto-object distance of $30 \mathrm{~cm}$., it amounts to less than 7 per cent. The main thing to remember in this connection is that the controlling factor is the ratio of tissue depth to distance of source. The smaller this ratio, the less variation of intensity there is between the surface and any chosen depth. In the first example the depth, or thickness, of the object, $1 \mathrm{~cm}$., is the same as the distance of the source, and the intensity at the far side is 25 per cent of that on the surface. In the last example the depth is only $1 / 30$ th of the distance and the variation of intensity is small. If in this case the depth were increased to $30 \mathrm{~cm}$., the intensity at this point would also be 25 per cent of that at the surface. ${ }^{6}$ Similarly, if in the case where the radium is placed at a distance of $1 \mathrm{~cm}$. the thickness of the object were $1 / 3 \mathrm{~mm}$., the difference between the intensities on the two sides would be less than 7 per cent.

\section{INFLUENCE OF MATTER ON THE INTENSITY OF RADIATION}

We may now proceed to the discussion of the influence of matter on the intensity of radiation. Let us examine first what happens to monochromatic radiation when it reacts with matter. For the present we shall neglect the reaction between the radiation and the air which surrounds the source, and we shall concern ourselves solely with liquids or solids of organic constituents. In the first very thin layer of the material some of the photons which make up the beam undergo photoelectric transformations whereby most of their energy is transferred to the secondary beta particles. The remainder of the energy reappears subsequently as photons of much lower energy. ${ }^{7}$ These photons, it will be recalled, are emitted when electrons fall into the spaces in the atoms left vacant by the electrons which were expelled through photoelectric encounters. They are, therefore, the characteristic radiations of the elements in the material and, in the case under discussion, are low-energy photons. (The maximum photon energies of the $K$ regions for the principal elements of organic matter are: $\mathrm{H} 13.6, \mathrm{C} 25.1, \mathrm{~N} 33.8, \mathrm{O} 50.2 \mathrm{Ev}$.) Their emission, depending only on the resumption of the normal state of the atoms, is a random phenomenon, and in the aggregate these photons are emitted equally in all directions. Since for each photon of the original beam which undergoes a photoelectric change one low-energy photon and a high-speed electron are liberated, the total number of photons remains

${ }^{6}$ Neglecting all influence of matter on the radiation.

7 It is tacitly assumed in this discussion that the beam of X-rays under consideration is of medium hardness such as is ordinarily used at present. As already mentioned, the energy of the initial photon is subdivided between secondary electron and photon in different proportions, depending on the hardness of the radiation. 
the same. ${ }^{8}$ But $(a)$ the total photonic energy is less than it was before the beam entered the thin layer of material, and $(b)$ most of the resultant low-energy photons do not travel in the direction of the primary photons. In fact, some travel in the opposite direction. Furthermore, these photons transfer their energy to matter within a very short distance and most of them cannot leave the thin layer of material in which they were formed.

In the first thin layer of the material there occur also transformations of the Compton type. Here again the total number of photons at the end is the same, but their energy is less since some was transmitted to the secondary electrons ejected in the process. As in the case of the secondary photoelectric photons, those produced in Compton encounters may be emitted in any direction but with one very important limitation. The direction of emission and the proportion of the primary-photon energy carried by the secondary photon are governed by the laws of elastic collisions and must therefore bear certain relations to the direction of the primary photon and its energy content. The aggregate distribution of the secondary photons in this case is such that those of highest energy travel in substantially the same direction as the primary photons, those of lowest energy travel in the opposite direction, while those of intermediate energy travel in appropriate directions between these two extremes. In this case, most of the secondary photons are able to leave the thin layer of material. ${ }^{9}$ However, only those emitted substantially in the direction of the primary photons reach the next layer. Thus, while the total number of photons produced in the first layer by photoelectric or Compton encounters is practically the same as the number of photons which have been transformed, the number ${ }^{9}$ which enters the next layer is appreciably lower than the number of primary photons. Since in addition some of the photons incident on the second layer have less energy than the original ones, the intensity of radiation at this level is still less.

Similar transformations take place in the next thin layer. More of the primary photons lose their identity by transferring their energy partly to electrons and creating new photons of lower energy. Continuing in this way it is evident that at each step more and more of the primary photons disappear and a larger proportion of the transmitted radiation consists of lower-energy photons. At the same time the number of photons traveling in directions differing from that of the primary beam increases. These photons consist of those which have been produced through photoelectric and Compton energy interchanges (largely

${ }^{8}$ This is not strictly true on account of the "Auger effect" (cf. K. K. Darrow).

${ }^{9}$ The actual proportion depends, of course, on the wave-length of the original beam and the thickness of the layer under consideration. 
the latter) and those primary photons which have been scattered without loss of energy. ${ }^{10}$ This process has not been mentioned so far because no ionization results from it. The main effect is a deviation of some photons from their straight-line paths with a resultant widening of the radiation beam, and consequently a decrease of the intensity of radiation along the axis.

\section{SECONDARY PHENOMENA}

The interactions of radiation and (organic) matter just described lead to some very important results.

$A$. It is evident that monochromatic radiation becomes polychromatic (heterogeneous) after traversing even a small thickness of matter, on account of the presence in the transmitted beam of photons of lower energy.

$B$. The proportion of lower-energy photons in the transmitted beam increases with the thickness of the material, that is, the radiation becomes gradually softer. But, since the lower the photon energy the more easily it is transferred to electrons and thence utilized in producing ions, the softer components of the radiation do not travel so far in the material as the primary radiation. Accordingly, beyond a certain thickness the energy distribution of the photons in the transmitted beam (i.e., the quality) remains substantially constant, since the photons of lower energy are eliminated by the very layer of matter in which new ones of the same energy distribution are produced. When this condition is reached, it is said that the radiation is in "equilibrium with its secondaries." In the end, however, provided the thickness of material is large enough, the "degeneration" of the radiation is complete and all its energy is finally transferred to ions.

$C$. Since secondary photons are emitted in all directions, a material body traversed by a beam of X-rays acts in some respects as a new source of radiation. The most important difference is in the distribution of the rays around the new source. The part of the secondary radiation which is due to the photoelectric effect, being extremely soft, is utilized in situ almost entirely. What comes out of the material body as secondary radiation, therefore, is made up very largely of photons liberated by the Compton effect. However, as already stated, these photons are pro-

${ }^{10} \mathrm{It}$ might be well to mention at this point that most authors think of the Compton effect as a scattering process, whereby the photon collides with an electron and bounces off with diminished energy (longer wave-length). To distinguish this type of scattering from the one mentioned above in which no change of wave-length occurs, they refer to it as "Compton scattering," or scattering with change of wavelength. The writer feels that for the purpose of this presentation it is preferable to call the photon of lower energy resulting from the Compton collision a secondary photon. Of course, the final result is identical irrespective of the terminology. 
jected most copiously in the forward direction and their energy content is also greatest in this direction. Hence, the radiation which leaves the material body ${ }^{11}$ is hardest and most intense in the forward direction and gradually becomes softer and less intense as the angle between the axis of the primary beam and the direction of emission of the secondary radiation increases to $180 \mathrm{deg}$.

$D$. Since the energy carried by all the secondary radiation is derived from the primary beam, and since by the very process which produces it, some energy is transferred to electrons, the energy of the secondary rays is always much less than that of the primary. A fortiori, in any one direction the intensity of the secondary radiation is much lower than that of the primary beam at the level of the material object. This is generally true whether the radiation is hard or soft. For, if the radiation is hard, it has a high penetrating power and it undergoes few photoelectric and Compton transformations by which alone it can transmit energy to secondary rays. If it is soft, it undergoes many transformations in its passage through matter, but the secondary radiation is still softer, and a large proportion is unable to leave the material body. ${ }^{12}$

$E$. Mention has been made of the fact that some secondary radiation leaves the body in a direction opposite (or nearly so) to that of the primary beam. This constitutes what is usually called the "backscattered radiation," or simply (and improperly) the "back scatter." It plays an important part in many practical cases, and should always be taken into account in biological work. Considering the process of its formation described in the preceding paragraphs, the following qualitative conclusions may be reached: The contribution of the (characteristic) photons liberated by the photoelectric effect to the back-scattered radiation outside the body is generally negligible. This radiation is extremely soft and can traverse only a small thickness of air. The photons derived from Compton encounters, which constitute the main part of the back-scattered radiation, have all less energy than the primary photons. In addition, they differ in energy content among themselves because they do not all leave the surface (they are not all emitted) in the same direction. Hence, this radiation is very heterogeneous, even when the primary beam is monochromatic. The relative intensity of the backscattered radiation varies with the wave-length (photon energy) of the primary rays. For soft radiation it is small, since in this case the photoelectric effect predominates. For hard radiation it is an appreciable

${ }^{11}$ Obviously the size of the body and the quality of the primary radiation must also be taken into account in any actual case.

${ }_{12}$ Also, in the case of soft radiation the photoelectric effect predominates and therefore most of the energy is transferred directly to electrons. 
proportion ${ }^{13}$ of the intensity of the incident radiation. For very hard $\mathrm{X}$-rays or gamma rays it is again small because Compton "scattering" in this case takes place largely in the forward direction. It is obvious that the proportion of back-scattered radiation must increase with the thickness of the irradiated body, up to a certain point. When the thickness is such that photons liberated at the far side lose all their energy before reaching the near side, there can be no further increase. This critical thickness depends, of course, on the wave-length of the incident radiation. It is evident also that within certain limits, the cross sections of the irradiated object and the beam must influence the proportion of the back-scattered radiation.

No mention has been made of the secondary electrons liberated by the hypothetical monochromatic beam in its passage through organic matter. From the discussion of this subject already given, and the preceding considerations, it follows that: (a) The maximum length of the ionization loci is set by the wave-length (photon energy) of the primary radiation, and is the same throughout the material. (b) But the relative number of loci of this length decreases with the depth in the material at which they are produced. This is a direct consequence of the softening of the radiation by its passage through organic matter. (c) Near the surface there is a sharper separation in the length of the ionization loci, because those produced by the back-scattered radiation are much shorter than those produced by the primary photons. In the deeper layers, loci of intermediate length increase in number. These three conclusions refer to the relative distribution of loci of different lengths at different levels of the material. In addition, $(d)$ the total number per cubic centimeter decreases with the depth on account of the effect of the inverse square law and the abstraction of energy from the primary beam by the intervening matter. The loci are, therefore, more sparsely distributed in the deeper layers than at the surface. Also, it should be noted that $(c)$ a beam of radiation which has a well-defined cross section before entering the material, produces ionization outside of its geometrical contour on account of the secondary rays which are emitted in all directions. The ionization loci in this peripheral region are always shorter than within the confines of the geometrical bean proper.

What takes place when a polychromatic or heterogeneous beam of $\mathrm{X}$-rays passes through organic matter may be surmised readily from the preceding discussion. The interaction with matter is essentially the same for the different components, but it varies in degree. It may

13 The back-scattered radiation as ordinarily measured appears to have a high "intensity," even up to 50 per cent of that of the primary beam, in the case of $200-\mathrm{kv}$. filtered X-rays. This is due to the use of the term "intensity" in a different sense from the one given so far (see later). 
happen, therefore (and in practice this is usually the case), that the hardness of the radiation increases with the depth, up to a certain point, because the soft components are unable to penetrate beyond the superficial layers of the material. Obviously, under these conditions the concentration of ionization loci drops very rapidly with increasing depth.

\section{FILTRATION}

The hardening effect just mentioned, which can be enhanced by suitable choice of material, is made use of in practice to obtain beams of a more homogeneous character (i.e., less difference in the component wave-lengths) than that of the radiation emitted by an X-ray tube or radium. The process by which this is accomplished is called filtration. The choice of the material to be used as a filter is governed by the following considerations: The longer wave-length components lose energy more readily through both photoelectric and Compton transformations. But in the case of the photoelectric effect the wave-length (energy) of the secondary photon depends only on the atomic number of the material, whereas in the case of the Compton effect it depends on the wave-length of the incident radiation and the angle at which the secondary photon is emitted. The photoelectric effect is small, or even negligible, when the radiation is hard and the material has a low atomic number. The Compton effect is predominant under these conditions, and vice versa. One may conclude, therefore, that the proper material to use in any given case is one of such atomic number that the photoelectric effect takes a predominant part in removing the softer components of the beam. At the same time, the atomic number must not be so high that the photoelectric effect is also large for the harder components of the beam, since this would diminish unduly the intensity of the desired radiation. Also, it should be noted that, while the characteristic radiation of materials of low atomic number resulting from photoelectric interchanges is negligible, it assumes greater importance with the higher atomic-number elements. In practice it has been found that aluminum is a satisfactory filter for soft X-rays, produced at voltages not exceeding $100 \mathrm{kv}$. peak. Copper is ordinarily used for voltages in the neighborhood of $200 \mathrm{kv}$. peak. In this range, when high filtration is desired, say, $2 \mathrm{~mm}$. Cu, Thoraeus (4) has shown that it is more economical to use tin. However, this introduces certain complications because its characteristic radiation ( $K$ radiation $0.424 \AA$, corresponding to photon energy of $29 \mathrm{kv}$.) is in the range of wave-lengths which one wishes to eliminate. ${ }^{14}$ As a result, some fairly long radiation in the primary beam is transmitted readily (anomalously) through the filter, and some soft characteristic radiation is also emitted. To overcome this difficulty a sheet of copper is added to the tin, of

${ }^{14}$ An element is particularly transparent to its own characteristic radiation. 
sufficient thickness to absorb the soft radiation passing through the tin. Of course, copper has its own characteristic radiation, which is still softer than that of tin, and to remove it a sheet of aluminum is added to the filter. The characteristic radiation of this metal is so soft that a few centimeters of air suffice to eliminate it. It is important to note that a composite filter of this sort must always be used in such a way that the radiation traverses it from the material of highest to that of lowest atomic number.

A filter removes from a heterogeneous beam of radiation its softest components, reduces considerably the intensity of the components of medium hardness, and reduces somewhat the intensity of the hardest components. Thus the transmitted radiation, while much harder on the average, is not monochromatic. The minimum wave-length is the same in the filtered as in the unfiltered beam. What degree of filtration one should use depends on the purpose for which the radiation is to be employed. In radiotherapy with $200-\mathrm{kv}$. X-rays, a filter of $0.5 \mathrm{~mm}$. Cu and $0.5 \mathrm{~mm}$. Al is satisfactory. Filters of 2 or $2.5 \mathrm{~mm}$. Cu or equivalent composite filters are also used. With $200-\mathrm{kv}$. X-rays the increase in hardness with increase in filter beyond $0.5 \mathrm{~mm}$. Cu is slight, while the loss of radiation is considerable. The only way to obtain monochromatic $\mathrm{X}$-rays is by means of a spectrometer. Unfortunately, however, the intensity of a monochromatic beam thus produced is usually too low for most biological investigations. Beams of considerable homogeneity may be produced in certain wave-length ranges by taking advantage of the characteristic radiation and anomalous transmission of suitable materials. For this purpose the metal used as a target in the $\mathrm{X}$-ray tube, the roltage applied to the latter, and the filter material, must be properly chosen.

In the case of radium, filtration is essential if one wishes to use only the gamma radiation. It may be recalled that radium in equilibrium with its disintegration products emits three types of ionizing radiations: (a) The alpha particles, or rays, which are helium nuclei projected at high speed. They are completely absorbed by a sheet of paper of ordinary thickness. (b) The beta particles, or rays, which are electrons traveling at very high speed. It takes approximately $2 \mathrm{~mm}$. of brass, or $1 \mathrm{~mm}$. $\mathrm{Pb}$, or $0.5 \mathrm{~mm}$. Au or $\mathrm{Pt}$ to stop all the primary beta rays. (c) The gamma rays, which are photons having on the average much higher energies than are found in X-ray photons. Those of highest energy can traverse many centimeters of lead. The spectrum of gamma rays is very complex and is not very well known on account of experimental difficulties arising from the short wave-lengths involved and lack of small sources of very high power. However, it may be mentioned that the same filtration which is required to eliminate the primary beta rays also reduces effectively the soft gamma-ray components. For most 
practical purposes, it is not adrisable to push the filtration much beyond this point. The secondary radiation emitted by the filter in the case of gamma rays is very troublesome. It includes electrons which may travel at a speed approaching that of the primary beta rays. They are produced, of course, by photoelectric or Compton collisions as in the case of $\mathrm{X}$-rays, but they travel much faster because the energy of the gamma photons is much higher. Since the ionization produced in a given length of path is very much greater in the case of a beta particle than in that of a photon of equal energy, the secondary electrons emitted by the filter may produce marked spurious effects. In biological experiments it is very desirable to have an organic material several millimeters thick as a secondary filter.

\section{ESSENTIAL FACTORS FOR THE CORRELATION OF IONIZATION AND BIOLOGICAL EFFECTS}

The subject matter presented so far enables the biologist to form a mental picture of the primary reactions which occur in a biological material while it is being irradiated. Particular emphasis has been laid on ionization-its origin, spacial and linear distributions, and the influence of the quality of radiation thereon. Ionization is the only thing we know of to which, rightly or wrongly, we may attribute all other effects of radiation. Hence, any attempt at a correlation of the biological effects of radiation requires of necessity a quantitative knowledge of the ionization in the living materials studied. But this essential information is very difficult to obtain. In the first place, there is no way of measuring directly the ionization produced by radiation in a living, or even a dead, organism. The usual method employed in determining the degree of ionization in gases by measuring the largest electric current-saturation current-which the ions are capable of conveying, cannot be used in the case of tissues. For, any electric field applied to the material to obtain the saturation current (were this possible) sets up simultaneously a much larger current which has nothing to do with the ionization produced by the radiation. In other words, the material is a fairly good conductor to start with, and any increase in conductivity due to the radiation is utterly imperceptible. Therefore the best one can do is to attempt to determine the ionization in the living material by indirect means. Whenever indirect means are employed to get quantitative data, assumptions are likely to be made, explicitly or implicitly. It is then extremely important in making use of the data, to bear in mind the assumptions made and the restrictions which they impose. While the necessity of such caution is obvious, radiological literature nevertheless shows that it is rarely exercised. In the remaining paragraphs an attempt will be made to point out the principal limitations of the indirect method 
generally employed for determining the ionization in biological materials.

The term ionization as used in the preceding paragraph is quite indefinite. We must state, therefore, just what it is that we wish to know about the ionization produced in a living organism. Ions are liberated in the material during the entire time that it is exposed to radiation. In this time a definite number of ion pairs will have been produced, which represents the total amount of energy transferred to the material by the radiation. Remembering that it takes energy to produce any change, we may readily conclude that one of the factors we wish to know is the total number of ion pairs set free. The same number of ions may be distributed through a small or large volume, and obviously the effect on the individual living cells will be different. Hence it is important to know the number of ion pairs per unit volume liberated during the exposure to radiation. We must know this for every point in the material since, as already stated, the distribution of radiation is seldom strictly uniform. Having this information it is possible to correlate the observed biological change or changes with the amount of energy, in the form of ionization, supplied to the living cells. If (direct or indirect) means are available for the measurement of the biological changes, the correlation can be quantitative. Investigations of this type provide the fundamental data essential to the development of the science. The next step is the formulation of generalizations. If pursued far enough this will lead to the study of the mechanism by which the ionization produces the biological changes. Here the fact that the ions are concentrated along what we have called ionization loci cannot be ignored. This is so whether one undertakes to develop a theory on the basis of "direct hits" of a hypothetical sensitive center of the cell (1) or otherwise. For, even on the simple assumption that the chemical constitution of the medium is altered somewhat, the degree of change may depend on the concentration of ions in the loci (due to recombination or other factors). Then almost certainly the rate of diffusion of the chemically altered substances from the loci outward, must play some part in the biological effectiveness of the ionization. Therefore one should know also the distribution of the ionization loci and the ion concentration therein.

The discussion of the phase of the subject given in this chapter, provides the necessary qualitative data. In order to get the quantitative data applicable to any particular practical case, the biologist is referred to some of the textbooks listed at the end of the chapter. ${ }^{15}$ The desired information is not readily available, since little attention has been paid to it in the past, from the biologist's viewpoint. However, enough is

${ }^{15}$ Especially "Radiations from Radioactive Substances," by Rutherford, Chadwick, and Ellis. Many references to original papers will be found there. 
known to permit one to make a fairly close estimate of the distribution of ions in the loci under the conditions met with in practice.

The spacial distribution of the ionization in a given material is independent of the duration of the irradiation, in the sense that on the average each element of volume receives the same amount of energy, compatible, of course, with the variation of the intensity of radiation from point to point in the material. This is analogous to saying that every square foot of a plane exposed to rain receives the same number of drops, provided the exposure is not too short. It follows, therefore, that the number of ions per unit volume produced at a given point by a constant source of radiation, is directly proportional to the duration of the exposure. Accordingly, the main problem is to determine the number of ions liberated in unit volume of the material per unit time under the action of radiation of a given intensity and a known quality. From the definition of intensity given so far it is evident that, all other conditions remaining the same, this number is directly proportional to the intensity, since the latter determines the rate at which energy is supplied to a given volume of the material. However, when the quality of the radiation is not the same, the relation between the number of ions per cubic centimeter per second and intensity is much more complex. Remembering that even monochromatic radiation becomes heterogeneous in its passage through a biological material, it is evident that the quality of radiation is always involved. This question requires very careful consideration.

Let us examine the ionization produced by two hypothetical beams of monochromatic radiation, the wave-length of which is very different. For the sake of definiteness, imagine two point sources of equal ("candle") power, one emitting (soft) X-rays and the other gamma rays. Per unit time each source, therefore, emits the same amount of energy in all directions. Remembering the derivation of intensity of radiation previously given, the two sources will provide the same intensity of radiation at a given distance from either. A biological material placed at this distance (from either source) will receive the same amount of energy per unit time. The complete utilization of this amount of energy through ionization of the material produces the same number of ions in both cases. But, owing to the greater penetrating power of gamma rays, the same number of ions is distributed through a much larger depth and volume of the material than in the case of $\mathrm{X}$-rays. Hence the number of ions produced per second per eubic centimeter of tissue at a given depth is much smaller in the case of the gamma-ray source, under the conclitions stipulated above. It is evident, therefore, that the rate at which ions are liberated at a given point in the material depends on something more than the intensity of radiation, and that this additional factor is the quality of the radiation at the point in question. The 
quality determines what fraction of the total energy traversing a certain layer of the material is immediately available to produee ions in the same layer. ${ }^{16}$ Theoretieally, then, knowing the intensity and the quality of the radiation at a given point in the material, one would know also the rate at which ions are being produced per unit volume. The quantitative relationships involved, however, are such as to preelude their utilization in the great majority of practieal eases. It is almost impossible at the present time to measure with reasonable aceuracy the intensity of a beam of hard X-rays in terms of the energy flow per unit area. It is equally diffieult to determine the exaet quality of radiation at every point of the material (the reasons are of no particular interest to the biologist). However, many of the practieal difficulties may be overcome by a tour de force.

The argument involved is as follows: As already explained, ionization cannot be measured directly in living tissues. Fortunately, however, the interaetion of radiation and matter depends (almost) entirely on the atomie numbers of the elements and the respeetive proportions in which they are present in the material, and not on their state of aggregation. Sinee the atomic number of hydrogen is 1 , its contribution to the ionization in a living organism is very small. Praetically all the ionization is due therefore to the oxygen, nitrogen, and carbon components. Atmospheric air, consisting largely of nitrogen and oxygen, is a close approximation to living tissue, insofar as its quantitative reaction with radiation is eoncerned. Being a gas, the ionization which is produced therein can be measured readily. By virtue of this atomie relation, the number of ions produced in $1 \mathrm{gm}$. of living matter is substantially the same as the number of ions liberated in $1 \mathrm{gm}$. of air, under the same conditions of irradiation. Furthermore, the one-to-one eorrespondence is (approximately) independent of the quality of radiation. Hence, by measuring ionization in air under suitable conditions, one can determine the ionization produeed in a living organism, without a quantitative knowledge of the true (vide infra) intensity of radiation, the quality, ${ }^{17}$ and the laws of "absorption" of radiation by organic matter. It should not be assumed, however, that all diffieulties have been eliminated. For, the determination of the "suitable eonditions" alluded to above, presents a problem of the first magnitude, whieh has not yet been solved completely.

16 The usual presentation of this subject is based on the concept of "absorption" of energy by the material. It is then said that the part of the radiation which is available to produce ions, chemical and biological changes, is that which is absorbed. The fundamental concept is the same in the foregoing discussion, but the writer has purposely avoided the use of the term absorption (in fact, throughout the paper), to circumvent certain difficulties which would complicate the presentation materially.

${ }^{17}$ A knowledge of the quality of radiation is necessary, of course, in order to interpret some biological effects in terms of the ionization loci. 


\section{EFFECTIVE INTENSITY OF RADIATION}

We have seen how two sources of monochromatic radiation of widely different quality (ordinary $\mathrm{X}$-rays and gamma rays), which deliver the same intensity of radiation at the point where the biological material is placed, do not produce the same ionization in the material. It has been explained that this results from the fact that the more penetrating radiation passes through the material much more easily and leaves behind very little energy in the form of ionization. Exactly the same thing happens in the ionization chamber (ef. L. S. Taylor, Paper II) of a measuring instrument. The ionization produced therein represents a certain quantity of energy which was derived from the beam of radiation, but it tells us nothing about the total amount of radiant energy which was flowing through the chamber at the time of the measurement. In other words, the reading of the instrument per se gives us no indication of the intensity of the radiation. Nevertheless, in the art this is what is meant by the intensity of a beam of X-rays. In order to aroid confusion we shall refer to it here as the effective intensity. The distinction between the two must be kept clearly in mind. To reiterate, the true intensity of radiation at a given point in the beam represents the amount of radiant energy passing through a surface of unit area perpendicular to the direction of travel of the photons at the point in question per unit time. On the other hand, the effective intensity of radiation at a given point in the beam represents that part of the radiant energy passing through $1 \mathrm{~cm} .^{3}$ of air in the ionization chamber per unit time which is required to produce the observed number of ion pairs per cubic centimeter of air per unit time at the point in question. We have called this the "effective" intensity because it is the attribute of a beam of radiation which determines the degree of ionization (that is, the number of ion pairs per cubic centimeter per second) produced in air. In view of the similarity between the atomic numbers of the principal components of air and living tissues, this is also the attribute of a beam of radiation which is of chief interest to us. Accordingly in practice we need not concern ourselves with the true intensity of radiation. For this reason and the fact that ionization measurements always determine something more or less closely related to the effective intensity, it is customary to refer to it simply as the "intensity." This would cause no trouble if its significance were always borne in mind. Unfortunately, however, the two meanings of the term are often confused. Perhaps a simple analogy in the realm of visible light, with which we are more familiar, will serve to clarify our ideas. We may think of the intensity of light as the factor which determines how easily we can read a printed page. Projecting on the paper, successively, lights of different colors ranging from red to violet, we may find 
that we can read most easily with the yellow light. We would then conclude that this light has the greatest intensity. Let us now photograph the printed page when illuminated by the different colored lights under the same conditions as before, using the same exposure time. Upon development the plate exposed to the red light would show hardly any effect, whereas that exposed to the violet light might be eompletely fogged. According to this criterion the light of highest intensity would be the violet one. Measuring the true intensity of radiation of each light by substituting a suitable thermopile for the printed page, we may find that it is the same for all the colored lights. In the ease of the visual observation we determined the effective intensity of the light by its physiological action on the retina. With the photographic test the effective intensity was determined according to the ability of the radiation to produce a change in the emulsion. The two values of the effective intensity thus determined for each of the colored lights, differ between themselves and are both different from the true intensity of the light. Furthermore, it is obvious that the results depend also on the eye (i.e., whether color blind or not) and on the kind of photographic plate used.

\section{SIGNIFICANCE OF IONIZATION MEASUREMENTS}

The fact that in the foregoing example the effective intensity is found to depend on the effect and "apparatus" by which it is measured serves to bring out another important point in ionization measurements. The effect by which we have chosen to measure X-rays is ionization. As ionizable medium we have adopted atmospheric air primarily because its constituent elements do not differ materially in atomic number from those of living matter which are chiefly responsible for the ionization. But we have said nothing about the apparatus to be used. Since we are interested in the determination of the number of ion pairs produced per second in a definite mass of air by its interaction with the radiation at a given point in a beam, it is evident that we must know the volume (also the temperature and pressure) of the air in which the measured ionization is produced. This may be done by means of a closed ionization chamber, in which case the volume is that of the chamber, or by means of an open ionization chamber of the standard type (cf. L. S. Taylor) in which the ionized volume is determined indirectly. In the case of a closed chamber it is obvious that the radiation cannot reach the air therein without first traversing the enclosing structure. In so doing, it reacts with the material of the enclosure and produces secondary electrons and photons. Some of these reach the air in the chamber and produce ions. Consequently, the ionization which is measured in the enclosed air is made up of two parts: one which is due to the interaction of the radiation with the air in the chamber, and the other which is derived from the interaction 
of the radiation with the matter in the enclosure. Remembering that a secondary electron spends its energy in a relatively short distance (the ionization locus) by ionizing the atoms in its path, it is evident that those secondary electrons which are produced in the walls of the chamber and then reach the enclosed air will contribute materially to the ionization of the air. The same thing applies to the secondary electrons produced by the interaction of photons and the air itself. On the other hand, most of the secondary photons produced in the walls-or in the aircontribute relatively little to the ionization which is being measured. It is clear, therefore, that such a chamber does not measure, in general, the ionization which would be produced by the radiation in the same volume of air if the material walls of the chamber were not present. From what has been said so far, it might be inferred that (on account of the contribution of secondary electrons by the walls) the ionization in the chamber is greater than it would be in free air. This, however, is not necessarily so for reasons which will be apparent presently.

The relative proportions of secondary electrons ionizing the air which originate in the walls, and those which originate in the air itself, depend on the geometrical factors of the chamber and the quality of the radiation. (That the atomic composition of the materials of which the chamber is made must play an important part is obvious. In our discussion we are considering only ionization chambers which are made of organic materials or such combinations of organic materials as will approximate the atomic composition of air or living tissues.) When the radiation is soft, the secondary electrons carry little energy and the ionization loci are very short. Hence only those electrons which are liberated at or very near the inner surface of the chamber walls reach the air within it. In traversing this thickness of matter, the (soft) radiation loses considerable energy and its intensity within the chamber is therefore much less than it would be at the same point in the beam if the chamber walls were not present. The secondary electrons liberated in the air by this weaker radiation will be fewer than otherwise and hence the total ionization produced in the air of the chamber may be too small in spite of the contribution made by the wall electrons. When the radiation is hard, the situation may be reversed and the measured ionization may be too high. However, this is not so likely to happen, for a reason which deserves special attention.

$\mathrm{X}$-rays of the hardness commonly employed in practice (i.e., X-rays produced at $200 \mathrm{kv}$. peak and filtered by $0.5 \mathrm{~mm}$. Cu) in their passage through atmospheric air, liberate secondary electrons which may travel several or even many centimeters before their energy is all utilized in prorlucing ions. Whether liberated by photoelectric or Compton encounters, the electrons which travel farthest are emitted initially in the same 
general direction followed by the primary photons. In their subsequent motion through the air some of these fast electrons are deviated and may travel eventually in the opposite direction. There are also Compton electrons (of lower speed) which are emitted more or less sideways with respect to the line of motion of the primary photons. Accordingly, one may picture a beam of hard X-rays passing through air as being accompanied by a swarm of electrons darting in all directions, in a region which extends beyond the confines of the beam itself. It should be remembered, of course, that at any one time there are more electrons traveling forward than backward and that the former carry most of the energy which subsequently appears as ionization. By analogy with the case of secondary photons previously discussed, it is evident that after the radiation has traversed a certain thickness of air, a sort of equilibrium has been established between the primary photons and the secondary electrons. This thickness depends on the quality of the radiation and is greater the harder the radiation. The actual value is rather difficult to determine in the case of very hard X-rays and gamma rays. Obviously, it must be closely related to the path of the secondary electrons, but this is so long for some of them that in the same thickness of air the secondary photons will also liberate an appreciable proportion of electrons. One must then take into account also the equilibrium between primary and secondary photons and between the latter and their secondary electrons. To make the picture more concrete let us suppose that we can take an instantaneous photograph of the photons and high-speed electrons in a beam of radiation passing through air. In $1 \mathrm{~cm}^{3}$ of air we would then find a certain number of: $(a)$ primary photons, $(b)$ secondary photons, and $(c)$ beta particles. ${ }^{18}$ The number of primary photons per cubic centimeter (in the picture) decreases with the distance from the source, due to the inverse square law, as already explained. Therefore it is necessary to consider the relative numbers of the three energy carriers in $1 \mathrm{~cm} .{ }^{3}$ at different distances from the source. Let us assume that (by means of a strong magnetic field) all electrons have been eliminated at a certain cross section of the beam. At this point, then, our photograph will show only photons in the cubic centimeter of air, and for simplicity we shall assume that they are primary photons. A short distance beyond this cross section, there will be in the cubic centimeter of air in addition to photons, some beta particles, but their number is small in comparison with the number of photons. From this point on the number of beta particles per primary photon increases with the distance, rapidly at first and then slowly. The number of secondary photons per primary photon

${ }^{18}$ This term is used here instead of "secondary electrons" because some of the high-speed electrons are produced by secondary photons and might therefore be considered to be "tertiary electrons." 
increases also, but more gradually. Since in the cubic centimeter of air under scrutiny there are also ions which have been produced by the beta particles, we may extend the comparison to them. The number present per primary photon increases also with the distance in much the same way as that of the beta particles, except that at the greater distances the increase is still less marked. This is because on the average the beta particles there have less energy, since some of them have been produced by secondary photons. In the case of the gamma rays of radium this problem has been investigated experimentally in the writer's laboratory with the following results: Starting with radium filtered by $2 \mathrm{~mm}$. of brass and $4 \mathrm{~mm}$. of bakelite, the number of ions produced per second per centimeter of atmospheric air increases rapidly with the distance from the source up to about $100 \mathrm{~cm}$. At this point it is approximately 60 per cent higher than very near the source. Beyond the $100-\mathrm{cm}$. distance the increase is less rapid. Nevertheless, at a distance of $900 \mathrm{~cm}$. (the maximum used in the experiments) the value is more than twice that obtained very near the source, and the curve indicates a further increase beyond this distance. Since there is no very sharp break in the curve, it is impossible to say just what thickness of air is necessary to bring about equilibrium between the primary photons and the secondary beta rays. For practical purposes $300 \mathrm{~cm}$. probably suffice. The above results indicate that most of the secondary electrons produced by gamma rays are unable to emerge beyond a 100-cm. layer of atmospheric air around the source. However, the actual distance covered by the beta particle in its zigzag path may be much greater then this. An appreciable proportion of the secondary electrons have enough energy to carry them beyond the 2-, and even 3-meter layers of air.

In view of the foregoing considerations it is evident that the ionization produced in a small volume of air at a given point in a beam of (hard) $\mathrm{X}$-rays is due partly to the secondary electrons which are liberated there, and partly to electrons which have been liberated elsewhere and subsequently reach the volume. If this volume with imaginary boundaries is now surrounded by walls of dense matter, such as the solid material of which ionization chambers are made, it is to be expected that in general the ionization in it will not be the same as before. Most, if not all, of the secondary electrons liberated in the surrounding air, which before reached the volume under consideration, are now stopped by the chamber walls. On the other hand, other secondary electrons set free in the material of the chamber, contribute to the ionization of the air within. Under certain conditions the loss and gain through the presence of the walls may balance. In this case, the chamber measures the actual ionization which would be produced in the same volume of free air. Such a chamber may be said to have no "wall effeet." But, it is very important to note 
that this can be strictly true only for one definite set of conditions. In particular when the quality of radiation is varied, the balance of loss and gain of ionization by the presence of the wall may be upset considerably. By a suitable adjustment of materials, wall thickness, and volume, closed ionization chambers have been constructed which are remarkably free from wall effect in the range of wave-lengths commonly employed in practice. The error introduced by the wall effect on the long-wave side may be ascertained by comparison with a standard ionization chamber (cf. L. S. Taylor, Paper II). On the short-wave side, approaching the gamma-ray region, this comparison cannot be made, for reasons which cannot be given in detail here. ${ }^{19}$

\section{KNOWLEDGE OF IONIZATION PRODUCED IN IRRADIATED ORGANISM ALL IMPORTANT}

The point which is of chief interest to the biologist is this: Placing a living organism at a certain point in a beam of radiation (X-rays or gamma rays), he wants to know the number of ion $\mathrm{s}^{20}$ liberated per second per cubic centimeter at every point of the material. Knowing the duration of the irradiation (exposure time), he can calculate the total number of ions produced per cubic centimeter at any point of the material during the treatment. Then he has something which he can reasonably call the "cause" and can relate it to the observed "effect." Now granting that this is the desideratum, it follows that the biologist is not particularly interested in the ionization produced in the atmosphere at a point where he expects to place his material, unless he can deduce therefrom the ionization which is subsequently produced in the biological object. It has been shown how placing an ionization chamber with solid walls in a beam of radiation in general disturbs the ionization in the air at that point. Since it is assumed that the chamber is made of organic materials, whose atoms react individually with the radiation in the same way as the atoms of air (or living tissue), the disturbance is due to the greater concentration of atoms in the chamber materials. Or, to be more specific, it is due to the introduction of solid materials. Obriously the living

${ }_{19}$ The results of the author's work on this subject were presented recently at the Fourth International Congress of Radiology, held in Zurich. They will be published in the near future in "Acta Radiologica" (Stockholm).

${ }^{20}$ The biologist should note that the number of ions of both polarities is the controlling factor in his case. Physicists are primarily interested in ion pairs, and in general when they speak of a "certain number of ions" produced in an ionization chamber, for instance, they mean actually the number of ion pairs. This is rather confusing at times, and the biologist who makes use of such data given in texthooks on physies should ascertain whether the numbers refer to single ions or ion pairs. In this paper both terms have been used, but-it is hoped-without introducing any uncertainty. 
organism, having a density about 800 times greater than that of atmospheric air, will also disturb the ionization at the point where it is placed. However, the disturbance in this case must be considered from the point of view of the ionization produced in the solid material itself. As in the case of the closed ionization chamber, it will be seen by analogous reasoning that, under certain conditions, compensation may occur, whereby the number of ions liberated per second per cubic centimeter of the biological object is higher than the number of ions liberated per second per cubic centimeter of atmospheric air at the same point, in the ratio of the densities of the two materials. That is, the ionization per cubic centimeter of tissue will be roughly 800 times greater than that per cubic centimeter of atmospheric air at the same point. As one might expect, the compensation or balance just spoken of depends on the quality of the radiation and on the size of the biological object (including container, holder, etc.) placed in the beam. For very small objects it obtains reasonably well in the usual range of wave-lengths employed in practice. In such cases it is sufficient to measure the ionization in air in order to deduce that in the tissues. This can be done for the usual qualities of X-rays by means of either a standard chamber or a properly calibrated "air-wall" chamber. When this procedure is not justified, the problem of determining, or even estimating, tissue ionization becomes more involved. Realizing that the principal complications arise from the great difference in density between living tissue and atmospheric air, one might attempt to solve the problem by measuring the ionization in an organic material of density comparable with that of living matter. All efforts made in this direction so far have not been successful, but the work should be continued. For the present, however, being limited to measurements made in (gaseous) air, great care should be exercised in planning biological experiments, to make sure that the conditions under which the living organism is irradiated, permit a reasonably close estimate of the ionization produced therein.

The selection of the proper conditions of irradiation depends on many factors and no general rules can be given here. The following suggestions will be found helpful: Experiments may be undertaken for the main purpose of describing hitherto unknown effects of radiation. These are exploratory experiments, in which normally no attempt is made to establish quantitative relationships. In such cases the choice of experimental conditions is not important. However, it is necessary to give sufficient information about the experimental set-up and technique to enable someone else to duplicate the results. The primary factors, such as voltage, milliamperes, filter, distance, etc., stated in the preceding paper (L. S. Taylor) suffice for this purpose. If all these factors are carefully determined and described, they are sufficient, also, to 
enable the investigator or others to make a rough estimate of the tissue ionization (amount and distribution) at a subsequent time, when more information may be available. Strietly quantitative experiments are more exacting. Since it is simpler to insure a substantially uniform distribution of radiation within the material than to determine its effective intensity from point to point, the experiment should be planned with this idea in mind. A given object is irradiated more uniformly: (a) the longer the distance from the source at which it is placed (this follows from the inverse square law, as already explained); (b) the more penetrating and homogeneous the radiation used; $(c)$ the smaller the dimensions of the object to be irradiated, particularly the thickness traversed by the rays. When radium is employed as the souree of radiation, the amount available is usually too small to permit its use at long distances. It is then especially important to use a thin layer of the material to be treated. Better distribution of radiation may be obtained by placing the test object between two equal sources suitably placed. In the case of gamma rays one may obtain a fairly elose estimate of the distribution of radiation by means of the inverse square law alone, and from that decide on the distance at which the material should be placed.

The determination of the ionization produced in a biological material is, in general, a difficult matter, because all the necessary information is not readily available. The biologist should exercise great care in so doing, and should preferably consult a physicist who is familiar with the subject. In addition, he should describe the process by which the tissue ionization was arrived at and give all the primary factors of the irradiation (voltage, milliamperes, distance, etc.). The desirability-indeed the necessity - of stating the effective intensity of radiation at the beam cross section where the material was placed is obvious. However, it should be remembered that the ionization chamber by which this is measured, may influence the result markedly. In this connection it is advisable to state the type of instrument used, also how, by whom, and when it was calibrated.

\section{EFFECTIVE QUANTITY OF RADIATION}

This brings us to the next topic to be discussed here, but a brief digression is necessary to introduce the subject properly. The international unit of X-radiation-the roentgen-is a unit of "quantity" of radiation. Since nothing has been said about this so far, some explanatory remarks are in order. The distinction between true and effective intensity of radiation, which has already been brought out, is also involved in this case. A source which emits radiation at a constant rate delivers energy to a certain point in space at a constant rate, and therefore 
the true intensity of radiation at the point remains constant with respect to time. The energy which passes through $1 \mathrm{~cm} .{ }^{2}$ of a surface perpendicular to the line of motion of the photons at a certain point in a given time is the product of the true intensity of radiation and the time. This is known as the quantity of radiation. It should be noted particularly that by this definition "quantity" refers only to $1 \mathrm{~cm} .^{2}$ of area (unit area) and not to the entire cross section of the beam. Analogously, the product of effective intensity and time may be called the effective quantity of radiation. It represents the amount of energy spent by the radiation in producing ions in $1 \mathrm{~cm} .{ }^{3}$ of atmospheric air at a given point in the beam in a given time, under the conditions stipulated by the definition of the roentgen (page 62 ). Since in practice one is dealing almost entirely with effective intensity and quantity, the adjective is omitted. It should always be borne in mind, however, that the two terms (intensity and quantity) have this special meaning in radiology and that certain limitations are imposed thereby. The factor which is expressed in roentgens is the (effective) quantity of radiation. By the mathematical relation between intensity and quantity it follows that (effective) intensity may be expressed in roentgens per second or roentgens per minute.

Now, if the scale of a measuring instrument is graduated in roentgens, or roentgens per minute, its calibration must be made under the conditions imposed by the definition of the unit. In instruments with elosed ionization chambers this is done usually by comparison with a standard chamber and the ealibration holds for a more or less restricted range of wave-lengths. If the instrument is used to measure radiation outside of this quality range, the readings are incorrect. The error may be small or large, depending on circumstances. Furthermore, another experimenter using a different ionization chamber is apt to get a different reading for the same beam of radiation. This makes it difficult for others to duplicate the results of the first experimenter.

In radiology it is of ten the practice to place a small ionization chamber on the skin of the patient, with the idea of including in the measurement the back-scattered radiation. If the biologist must irradiate a test object of large dimensions, it is preferable to make the ionization measurement in air at the cross section of the beam where the surface of the material will be, and to state this factor in the report. On account of the marked heterogeneity of the back-scattered radiation, the wall effect of the chamber is apt to be larger in this case than for the primary beam of radiation. Also the geometrical relation of the chamber on the surface of the material will determine to a considerable extent what proportion of the back-scattered radiation is included in the measurement. Accordingly, the uncertainty in such measurements is apt to be greater than in those made without the back-scattered radiation. 
The fact that the roentgen is a unit of quantity of radiation-albeit in a restricted sense- -has tended to emphasize unduly this factor. One of ten finds the statement that the quantity of radiation (usually referred to as the dose) administered to a certain patient or organism was so many roentgens, omitting any reference to the duration of the treatment. The same quantity of radiation may be administered, of course, in a short or long time, and the biological effect in general is not independent of the duration of the irradiation. For this reason it is necessary to state also the time during which a certain quantity of radiation was delivered to the test object. From this point of view it is preferable to think of (effective) intensity of radiation, expressed in roentgens per minute, as the prime factor. Then a treatment may be said to have been given by using an intensity of radiation of so many roentgens per min. for a certain number of minutes. By this notation less confusion is apt to occur also in another sense. Quantity of radiation as defined here refers only to $1 \mathrm{~cm} .^{2}$ of the beam cross section (or $1 \mathrm{~cm}^{3}$ of air according to the definition of the roentgen $\left.{ }^{21}\right)$. Therefore, the quantity of radiation expressed in roentgens in the specification of a radiation treatment does not represent the total quantity of radiation falling on the entire object, but the quantity delivered per square centimeter. This restriction is visualized more readily when one speaks of the exposure of a living organism to a certain intensity of radiation for a certain length of time.

In view of the numerous restrictions and qualifications which must be borne in mind when the foregoing terminology is employed, it is not surprising that considerable confusion has resulted therefrom. In this paper the factor which has been emphasized most strongly is the ionization in the biological material itself, since, so far as we know at present, this is the primary factor which controls, directly or indirectly,

21 The effective intensity of radiation at a certain point in a beam need not be, and in general is not, determined by measuring the rate of ion production in exactly $1 \mathrm{~cm} .{ }^{3}$ of air. The question then arises as to whether the same result is obtained irrespective of the shape and size of the volume in which the ionization is measured. In the case of the standard chamber (cf. L. S. Taylor) this is very nearly so, provided the radiation is so penetrating that the decrease of intensity due to the thickness of air traversed in the volume is negligible, and the intensity throughout the cross section of the beam which is utilized is practically constant. In the case of the closed chamber, the thickness, at least, of the air volume should be small in comparison to the distance from the source at which the measurement is made, in order to avoid too great a difference between the intensity of radiation at the point of incidence and that at the point of emergence. To minimize further errors due to the variation of intensity with distance, the small ionization chamber should be placed with its center at the point for which the measurement is desired. (It might be mentioned that the effective center of an ionization chamber is not its geometrical center. However, the factors which determine the effective center are so complex that the only practical thing to do is to use its geometrical center for this purpose.) 
all subsequent effects. A complete specification of the ionization in the biological material is essential for the proper interpretation of the biological effects produced by radiation. One must know, therefore, for every point of the material, $(a)$ the number of ions produced per second per gram, or, if preferred, per cubic centimeter; $(b)$ the characteristics of the ionization loci, especially as regards the relative proportions of loci of the different lengths present, and the linear distribution of ions therein. Since these data cannot be determined by direct measurements, they must be obtained by indirect means. The similarity of the interaction between radiation and organic matter on the one hand, and air on the other, furnishes the basis for the indirect method of attack which has been found most practical so far. By virtue of this similarity the ionization (factors $a$ and $b$, above) in living tissue and air produced by radiation under comparable conditions is substantially the same. Strictly speaking, the conditions are not comparable unless the mass concentration, that is, the density, of the air is at least approximately the same as that of living matter. ${ }^{22}$ Since it has not been found practical to measure ionization in such a dense medium, one is forced to the use of atmospheric air. The density of this medium being about 1/800th of that of living tissues, many difficulties arise. Bearing in mind that the ultimate aim of ionization measurements in air is to make possible the determination or estimation of the ionization in the living organism, the experimental conditions must be such as will introduce the least uncertainty in the final result (i.e., tissue ionization). The conditions stipulated in the definition of the international roentgen fulfil this requirement in a limited range of wave-lengths and for small irradiated objects, with satisfactory accuracy. It can be demonstrated, however, that in the gamma-ray region, the conditions imposed by this definition not only cannot be fulfilled in practice, but in addition are not in general those which will permit the evaluation of the ionization in the living material in the simplest way. ${ }^{23}$ In this case still greater care must be exercised.

In view of the inherent difficulties of the subject, and the restricted sense in which some technical terms are used, the biologist is urged to concentrate his attention on the ionization produced in the biological material under the conditions of his experiments. He will do well, for instance, to think of a "dose" or quantity of radiation in terms of the number of ions per unit volume which it produces in the material under

22 This condition would be met by liquid air, if the ionization could be measured in it.

${ }^{23}$ A detailed discussion of this problem will be found in the author's article already referred to, which will be published in Acta Radiologica. It may be stated here, however, that the chief difficulty is due to the long range and tortuous path of the secondary beta particles. 
consideration, ${ }^{24}$ and of the quality of radiation as the factor which determines the number and relative lengths of the ionization loci. In many instances one needs only the relative values of these data, while in others absolute values are required. Unfortunately, this field of investigation has received very little attention heretofore, from the point of view presented here. The biologist, therefore, will have considerable difficulty in finding the necessary data in the desired form. However, the effort will be amply repaid not only by the result but by the grasp of the fundamental principles of the subject which will be acquired through such a search.

\section{REFERENCES}

1. Holweck, F., and A. Lacassagne. Action sur les levures des rayons- $\mathrm{X}$ mous (K du fer). Compt. Rend. Soc. Biol. [Paris] 103: 60-62. 1930.

2. Lind, S. C. The chemical effects of alpha particles and electrons. 2nd Ed. 252 pp. Chemical Catalog Company, Inc.; New York, 1928.

3. Quimby, Еdith H., and Helen R. Downes. A chemical method for the measurement of quantity of radiation. Radiology $14: 468-481.1930$.

4. Thoraeus, Robert. A study of the ionization method for measuring the intensity and absorption of roentgen rays and of the efficiency of different filters used in therapy. Acta Radiol. Suppl. 15: 88. 1932.

5. Woodard, Helen Q. Factors influencing the decomposition of iodides by roentgen and gamma rays. Amer. Jour. Roent. and Radium Ther. 33: 227. 1935.

\section{OTHER REFERENCE HANDBOOKS}

1. Baсhem, A. Principles of X-ray and radium dosage. 274 pp. A. Bachem; Chicago, 1923.

2. Broglie, M. de. X-rays. (Transl. by J. R. Clarke.) E. P. Dutton \& Company, Inc.; New York, 1926.

${ }^{24}$ It has been tacitly assumed that the number of ions produced in one gram of living tissue is the same as the number which would be produced in one gram of air under the same conditions. The similarity in the atomic composition of air and liviug tissues really allows us to conclude that substantially the same amount of energy is abstracted from a beam of ordinary X-rays by the same mass of air or living tissue irradiated under identical conditions. This energy makes its appearance as highspced electrons in both cases, and is then available to produce ions. In the case of air it is known that the average amount of energy required to produce an ion pair in this way is approximately 32 electron volts. Hence knowing the total amount of energy abstracted from the radiation, one can calculate the number of ions which will be produced in the air. On the other hand, the average energy required to produce an ion pair in living tissue is not known and, therefore, the number of ions produced in the tissue by a certain known amount of energy, carried by the secondary electrons, cannot be determined. Fortunately, however, for the same amount of radiant energy absorbed by one gram of air or tissue, the relation between the respective numbers of ions produced in the two materials is probably the same for all wave-lengths, and the two are therefore related by a simple multiplying factor. In the absence of definite information on this point, and as a first approximation, one may assume this multiplying factor to be one. 
3. Bruzau, M. Sur la distribution spatiale du rayonnement gamma du radium dans les milieux dispersifs légèrs. (Thesis.) Masson et Cie.; Paris, 1928.

4. Clark, G. L. Applied X-rays. McGraw-Hill Book Company, Inc.; New York, 1932.

5. Clark, J. G., and C. C. Norris. Radium in gynecology. (Chapter on physics by G. Failla.) J. B. Lippineott Company; Philadelphia, 1927.

6. Colwell, H. A., and C. P. WAKeley. An introduction to the study of x-rays and radium. Oxford University Press; New York, 1926.

7. Compton, A. H. X-rays and electrons. D. Van Nostrand Company, Inc.; New York, 1928.

8. Den Howd, D. Over de Werking van Harde Roentgenstrahlen en Gammastrahlen van Radium. (Thesis.) J. H. deBussy; Amsterdam, 1924.

9. Holthusen, H. Grundlagen und Praxis der Roentgenstrahlendosierung. Georg Thieme; Leipzig, 1933.

10. Janeway, H. H., B. S. Barringer, and G. Failla. Radium therapy in cancer. Paul B. Hoeber; New York, 1917.

11. Millikan, R. A. The electron. 2nd Ed. Chicago University Press; Chicago, 1924.

12. Perussia, F., and E. Pugno-Vanoni. Trattato di Roentgen-e di Curie-Terapia. Fratelli Treves; Milan, 1934.

13. RUtherford, E. Radioactive substances and their radiations. University Press; Cambridge, England, 1913.

14. Rutherford, E., J. Chadwick, and C. D. Ellis. Radiations from radioactive substances. The Macmillan Company; New York, 1930.

15. Science of radiology. American Congress of Radiology. Charles C. Thomas; Springfield, 1933.

16. Sievert, R. M. Eine Methode zur Messung von Roentgen-Radium- und UltraStrahlung nebst einigen Untersuchungen über die Anwendbarkeit derselben in der Physik und der Medizin. Acta Radiol. Suppl. (14) 179 pp. 1932.

17. Weatherwax, J. L. Physics of radiology. $240 \mathrm{pp}$. Paul B. Hoeber; New York, 1931. 


\title{
MEASUREMENT AND APPLICATION OF VISIBLE AND NEAR-VISIBLE RADIATION
}

\author{
F. S. Brackett
}

Smithsonian Institution, Washington, D.C.

Introduction: Electromagnetic spectrum-Systems of measurement-Units and definitions-General concepts-Black body. Mcasurement of total radiation: Absolute detector-Comparison of black-body emission-Thermocouple technique-Radiometers. Comparison of detectors. Monochromatic measurements. Filters and reflectors: Filters-Reflectors. Sources of continuous emission: Sun-Tungsten filament-Carbon arc. Selective detectors: Black body plus filter-Photocell-Photochemical. Application of selective detectors. References.

\section{INTRODUCTION}

In the first paper, the duality of our conception of radiation and matter has been indicated. The fact that both matter and radiation behave on the one hand as though made up of particles and on the other hand as though conditioned by wave motion seems ineseapable. In dealing with the problems of measurement, we are chiefly concerned with the propagation of radiation, i.e., a type of transfer of energy from one point to another. Here the wave-motion aspeet of radiation dominates our picture. In considering the interaction of radiation and matter, we must resort to the particle coneeption. This conception has particular applieability to the problems of emission and absorption of radiation. Regarding radiation as a type of transfer of energy from one point to another, we recognize two essential characteristics to be measuredquantity and quality. In the absolute measurement of quantity, we shall base our observations upon the fundamental unit of the c.g.s. system, the erg, or some convenient multiple of that unit. In the evaluation of quality, we shall appeal to our wave picture and base our measurements upon wave-lengths, the units being a convenient submultiple of the centimeter, either the Angström $\left(10^{-8} \mathrm{~cm}\right.$.), the millimicron $\left(10^{-7} \mathrm{~cm}\right.$.), or the micron $\left(10^{-4} \mathrm{~cm}\right.$.), depending upon the magnitude of the wavelength to be dealt with.

Since the velocity of wave propagation is constant for free space, the relation of wave-length to frequency is given by the simple relation $\lambda \nu=C$, where $C=2.998 \times 10 .{ }^{10}$ In propagation through matter, 
the velocity is reduced, the wave-length modified, but the frequency remains unchanged. Furthermore, in absorption and emissionphenomena of interaction between radiation and matter-the relations are most readily expressed in terms of frequency. These observations constitute a strong argument in favor of expressing our data in terms of frequency. Nevertheless, because of established usage, the preponderant amount of existing data is to be found in terms of wave-length, the wavelengths being evaluated for either free space or air. For our purpose the difference is not significant. While, for convenience, we shall follow the established usage of speaking in terms of wave-length, immediate conversion to frequency may always be made and will sometimes be desirable.

\section{ELECTROMAGNETIC SPECTRUM}

While most of our conceptions are based upon those observations of which we are cognizant through our physiological senses, experimentation in recent years has shown that this visible range of wave-lengths constitutes an extremely small portion of the great electromagnetic spectrum for which we have data through physical measurements. Different ranges of wave-lengths give rise to such markedly different observable effects that they have been given names associated with the effects produced. Only recently have the fundamental characteristics common to all radiation been recognized and enabled us to arrange a fairly complete assignment according to wave-length, extending from 0.00002 or $0.00003 \AA$ to 20,000 meters, a spread of 19 orders of magnitude. Thus, on a common logarithmic scale, the spectrum covers a range of almost 19 units. This is shown, together with a number of related physical quantities, in Fig. 1 (27). Of this tremendous range, we shall restrict ourselves in this section to a single order of magnitude, namely, from 0.2 to $2.0 \mu$ (2000 to $20,000 \AA)$, or one unit on the logarithmic scale.

In biological material, water plays such a dominant role that its characteristic absorption of radiation is a matter of great importance. Its rapid increase of absorption to wave-lengths shorter than $0.2 \mu$ and to wave-lengths longer than $2.0 \mu$ sets a natural limit to the range of wavelengths to be considered. Thus, the range of wave-lengths under consideration represents an interval of relative transparency for water. The outstanding characteristic of matter with respect to radiation in this region is that of high selectivity, that is, rapid changes of absorption with varying wave-length. It is from this selectivity that the great variety of effects, both biological and physical, arise. At the same time, it is this very selectivity which makes the problems of measurement difficult and which has led to a considerable degree of confusion. 


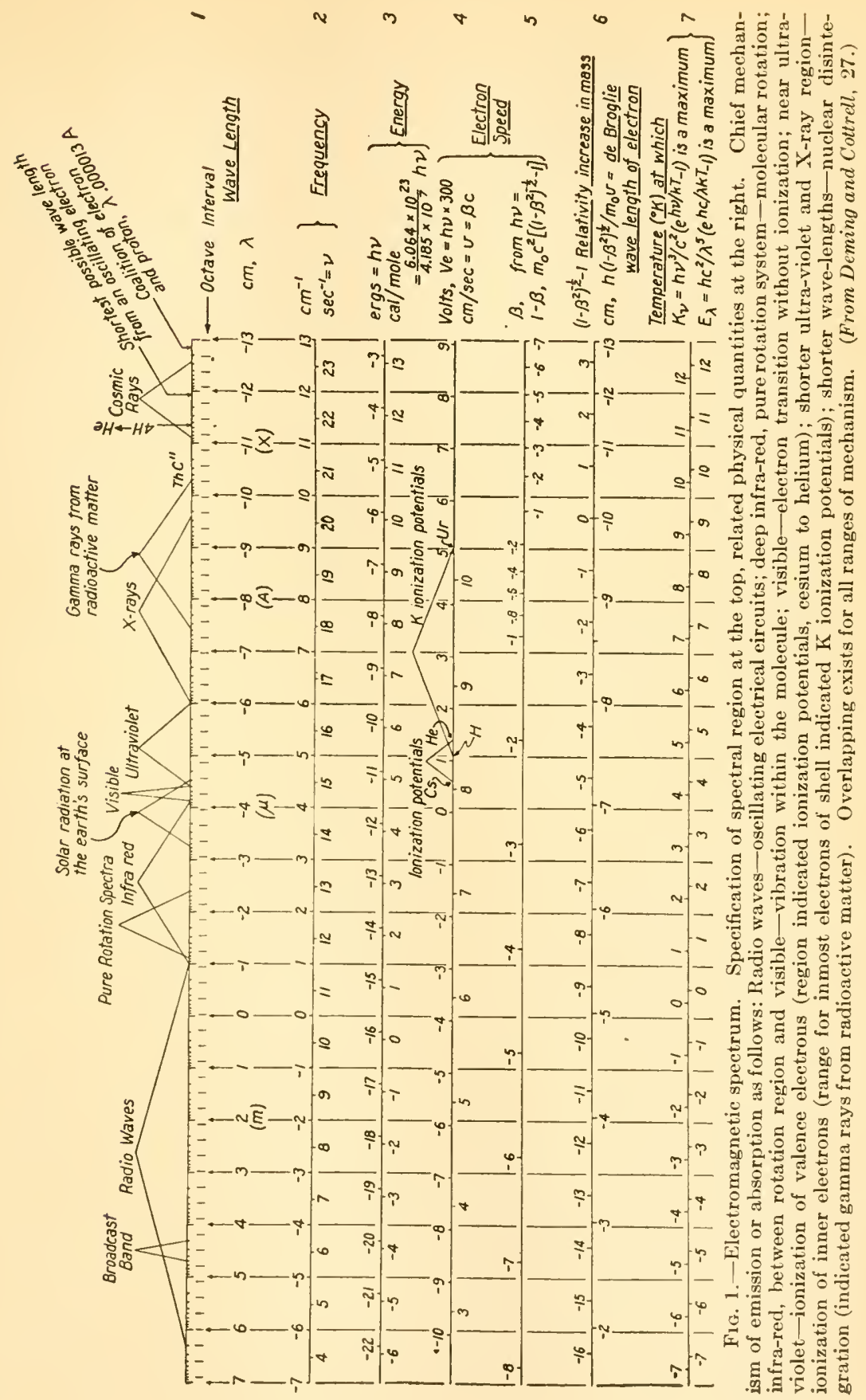




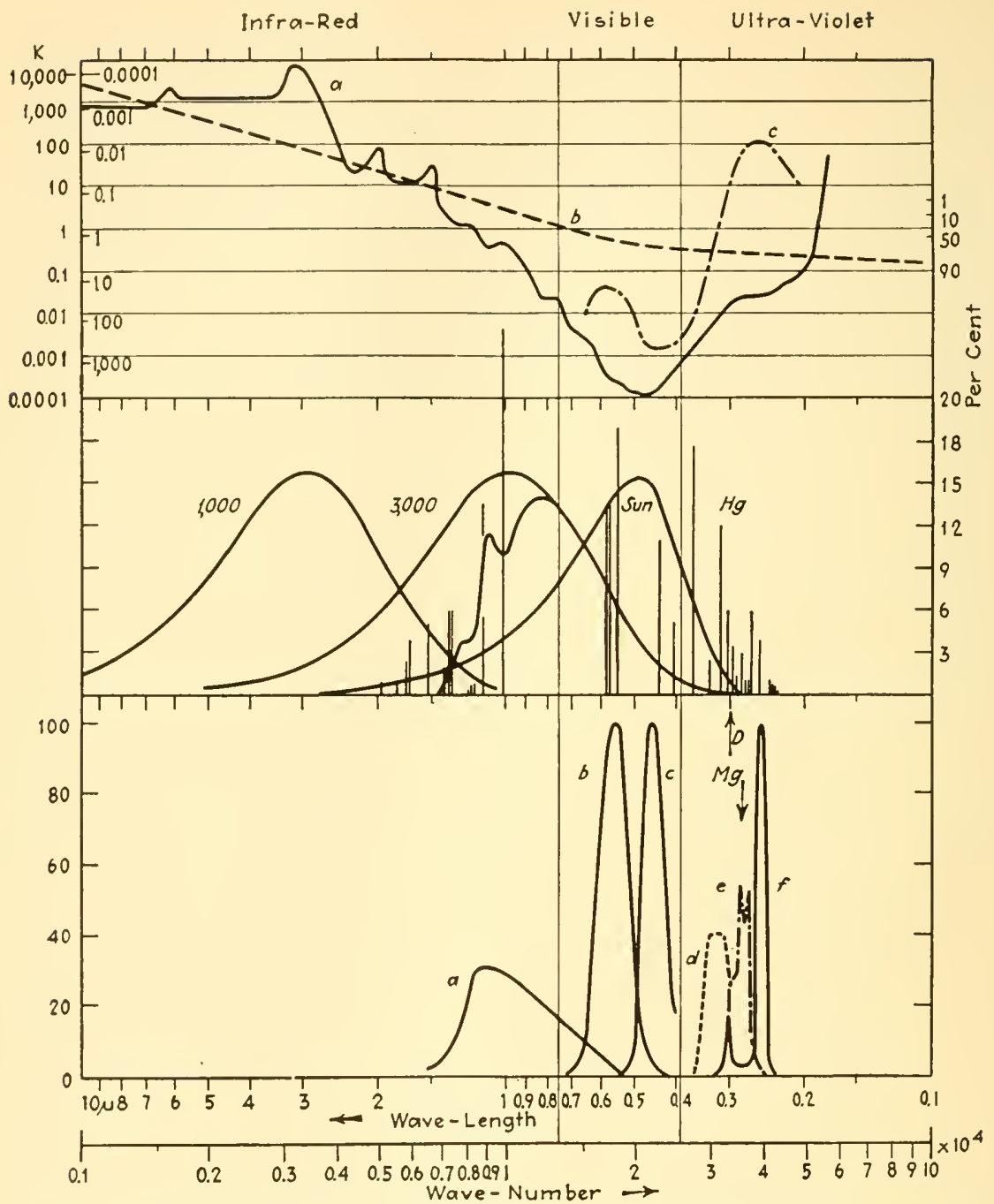

Fig. 2,-Graphs of physical and biological phenomena in the visible and near visible.

Upper section: Absorption. a, Water, ultra-violet, visible, and infra-red. Ordinates: Transmissive exponents $\kappa$ in $\quad I=I_{0} e^{-\kappa x} \quad$ (outside left). Thickness transmitting half intensity in centimeters (inside left). Transmission of $1 \mathrm{~cm}$. thickness (right). Abscissas: Wave-lengths in microns, $\mu$ (bottom). Wave numbers, waves per centimeter. $b$, Water, X-ray (same ordinates). Wave-lengths in Angströms (instead of microns as indicated at bottom). $c$, Ozone (same coordinates as in $a$; gas at standard conditions). Atmospheric transmission is equivalent to about $3 \mathrm{~mm}$. and can be found by shifting scale (right) up by approximately half a division.

Middle section: Radiation. Relative emission from body at $1,000^{\circ} \mathrm{K}$. (dull-red therapeutic lamp). Relative emission from body at $3,000^{\circ} \mathrm{K}$. (high-temperature tungsten lamp). Relative emission from sun. Relative emission from mercury arc in quartz.

Lower section: Biological phenomena. a, Transmission of flesh $(1 / 2 \mathrm{~cm}$. thick) in per cent. $b$, Relative visibility. $c$, Relative phototropism. $d$, Vitamin A. Absorption and vitamin value disappears when radiated. $e$, Ergosterol. Absorption disappears under 
In forming a general picture of the wave-length range to be dealt with, we may turn to Fig. 2 (11). In the upper section, the transmissive exponent $\kappa$ (cf. $i$, Table 5), as a function of wave-length, has been indicated by the full-line curve $a$. Remembering that the ordinates are on a logarithmic scale, one realizes the significance of the rapid and violent changes in absorption characteristics in proceeding through the range of wave-lengths indieated. Water is so transparent in the blue-green that radiation can penetrate through several hundred meters. On the other hand, at a little less than $0.2 \mu$, even a layer of $0.25 \mathrm{~mm}$. of water becomes practically opaque. The same is true in the relatively near infra-red in the region of $2.0 \mu$. Contrast these abrupt changes with the more gradual change indicated by the dotted curve $b$ for the region from 0.1 to $10 \AA$ (reading the abscissas as Angströms instead of microns).

Selectivity is also to be found in the radiation emitted by many materials. The mercury are, for instance, as shown in the middle section, emits radiation covering only a small fraction of an Angström in spread, at each of a multitude of isolated wave-lengths. A solid body, on the other hand, emits energy over a continuous range of wave-lengths from the deep infra-red rising to a maximum and then falling rapidly as one proeeeds to shorter wave-lengths. The wave-length at which this maximum occurs depends upon the temperature of the source.

Selectivity is again to be found in the interaction of biological material with radiation. For illustration, a number of such effects have been graphically indicated in the lower section. Curve $a$ shows the transmission of $0.5 \mathrm{~cm}$. of flesh; curve $b$, the response of the eye, in arbitrary units, to equal energy; curve $c$, the relative phototropic sensitivity of an oat seedling: curve $d$, the relative absorption of vitamin A to wave-lengths destructive to its biological effectiveness; curve $e$, the absorption of ergosterol, at least a portion of which may contribute during irradiation to its activation; curve $f$, the relative erythemal effectiveness. Thus, we see that for each biologieal phenomenon the contribution of equal energy but different wave-length will depend in characteristic manner upon wave-length.

Building upon the concept of radiation as the transfer of energy from one point to another by means of an electromagnetic wave motion, our problem is to determine the amount of energy transferred and the wave-lengths associated with this transfer. Our measurements will be based entirely upon the erg, centimeter, and second, all other units

radiation, which produces activation yielding therapeutic value of vitamin D. $f$, Relative erythemal effectiveness, zero degree (very light). For extreme erythema, fourth or fifth degree, the relative intensities of the two maxima are reversed.

Long wave limit of lethal effect indicated by $\uparrow \mathrm{D}$. Position of magnesium line in region of great absorption by ergosterol indicated by $\mathbf{M g}_{\downarrow} \downarrow$. (For bibliography of this figure see Brackett, 11.) 
being derived from these, simply as a matter of convenience in expressing the spatial distribution of energy transfer and in dealing with different orders of magnitude. Such a system is entirely independent, therefore, of the characteristics of vision or any other biological response. Other systems are, however, in common use, so that it will be desirable to discuss them briefly. Since our concepts are largely based on visual impressions, and since for many purposes observers have been chiefly concerned with those visual impressions, a complete system of measurement has sprung up, which is dependent upon the characteristics of the visual mechanism. In an effort to gain a universal basis for such measurement, a standard observer has been defined. Unfortunately, however, a large proportion of the published measurements depend upon the characteristics of some particular observer, and even the so-called standard observer has been redefined a number of times.

\section{SYSTEMS OF MEASUREMENT}

Three parallel types of measurement have sprung up: (a) radiometry, (b) spectrophotometry (illumination), (c) colorimetry. Each has developed its system of terminology, its units, and its methods. In description of apparatus and specification of characteristics, we shall find sometimes one system and sometimes another. Often the specifications are inadequate and indeterminate. It is important, therefore, to recognize the place and scope of each system, and as far as possible, the interrelation between them, wherever a definite relation exists.

Radiometry is the system based entirely upon the objective physical methods and specified in fundamental c.g.s. units. It will be the basis of our discussion throughout.

Spectrophotometry is an extension of photometry. In photometry, the quantitative aspect of radiation is evaluated in terms of its effectiveness as the stimulus of vision. A standard source is made the basis of comparison. The starting point is thus wholly arbitrary. The magnitude of effectiveness of other sources is determined by visual comparison. Obviously, the relative effectiveness of different sources will depend upon the relative wave-length distribution. If sources differ too greatly as to color, difficulties are encountered in evaluating the comparative effectiveness. Spectrophotometry, by a combination of visual and physical means, compares two radiations at the same wave-length, the eye being used for the most part as a null instrument.

In the definition of a standard observer, the relative effectiveness of different wave-lengths is arbitrarily specified. From the standpoint of practicability, it is desirable that this standard observer approximate the average characteristics of a large number of observers, but from the standpoint of definiteness and reproducibility, this is of no consequence. 
If the relative effectiveness for the standard source is fixed for each wavelength, the only necessary demand is that the standard source be constant and reproducible. Then other sources can be evaluated for each wave-length in terms of the standard source, and the total contribution obtained by addition. As a matter of practical procedure, however, appeal is made to the absolute system of radiation measurements and the standard observer defined as to his relative response to equal energy at each wave-length. At once, when the response of the standard observer is defined for each particular wave-length for a given energy, the photometric system presents a known relationship to absolute radiation measurements. Thus, so long as radiation occurs in the range of visibility, conversion may be made from one system to another, though such conversion may be tedious and inconvenient. Unfortunately, however, common procedure is made dependent upon the characteristics of the particular observer. Consequently, discrepancies may arise through his departure from the standard observer.

In pure research, one might prefer to avoid discussion of spectrophotometry. Practically, this is not possible for two reasons: (a) Radiometric apparatus is most commonly specified in spectrophotometric units. (b) Spectrophotometric methods may be the most readily available.

Colorimetry, the third system, deals not only with intensity as it affects the eye, but also with wave-lengths and combinations of wavelengths as they affect the eye. Thus, a multiplicity of combinations of wave-lengths and intensities may produce an identical effect, so far as the observer is concerned. Their color characteristics are therefore identical. Thus, the system of colorimetry deals with radiation wholly from the standpoint of the visual observer. It is concerned with the objective characteristics of radiation only so far as it is necessary in order to understand the subjective response. No detailed discussion of this system will be undertaken.

In the first chapter, terms which have arisen in connection with these three systems have been used more or less indiscriminately, the assumption being that at all times our attention has been focused on the purely objective phenomena and that measurements would be made in a purely physical system. If we could avoid a discussion of photometry and colorimetry, no further distinctions would be necessary. Since, however, in practical procedure, it is not possible to follow this simple expedient, it will be necessary to distinguish sharply between the concepts of the different systems. In making such a distinction, it is further necessary that ambiguity in the use of the terms be avoided.

Thus we shall draw a sharp distinction between the purely physical phenomenon of radiation, the objective stimulus of vision, i.e., light, 
and the subjective response to light, termed color. While a wide range of terminology has been developed to describe color, three essential attributes are commonly recognized: brilliance, hue, and saturation. The following table shows the relation between the terms describing subjective response (color), objective stimulus (light), and physical phenomenon (radiation).

TABLE 1

(56, ef. page 178)

\begin{tabular}{|c|c|c|}
\hline Physical phenomenon & Objective stimulus & $\begin{array}{l}\text { Subjective } \\
\text { response }\end{array}$ \\
\hline $\begin{array}{l}\text { Radiation } \\
\text { Intensity (beam) } \\
\text { Wave-length distribution }\{\end{array}$ & $\begin{array}{l}\text { Light } \\
\text { Brightness } \\
\text { Dominant wave-length(s) } \\
\text { Purity }\end{array}$ & $\begin{array}{l}\text { Color } \\
\text { Brilliance } \\
\text { Hue } \\
\text { Saturation }\end{array}$ \\
\hline
\end{tabular}

Table 2 indicates the hue which we commonly associate with the different representative wave-lengths of the pure spectrum.

TABLE 2

\begin{tabular}{c|l||c|c}
\hline Wave-length, $\lambda$ in $\mu$ & Hue & Wave-length, $\lambda$ in $\mu$ & Hue \\
\hline 0.410 & Violet & 0.580 & Yellow \\
0.470 & Blue & 0.600 & Orange \\
0.520 & Green & 0.650 & Red \\
\hline
\end{tabular}

Other hues are combination effects of different wave-lengths. For instance, purple is due to the combination red and blue.

\section{UNITS AND DEFINITIONS}

The complete physical measurement of radiation requires a determination of the energy for each wave-length and its relation to space and time. Table 3 defines the units commonly used.

In many problems it is assumed that one might have a finite amount of energy transferred by a parallel beam, i.e., a wave motion in which maxima and minima lie in parallel planes. For such an idealized ease, the flux density in a direction normal to the wave plane is often spoken of as the "intensity" or "beam intensity" and measured in watts $/ \mathrm{cm} .{ }^{2}$.

Practically, such a condition cannot be realized. It may be approached where the radiation is restricted to a small solid angle. In such cases, the term intensity or beam intensity is commonly used.

Quantities expressed in units of the absolute system cannot be converted into quantities expressed in terms of the illumination system, 


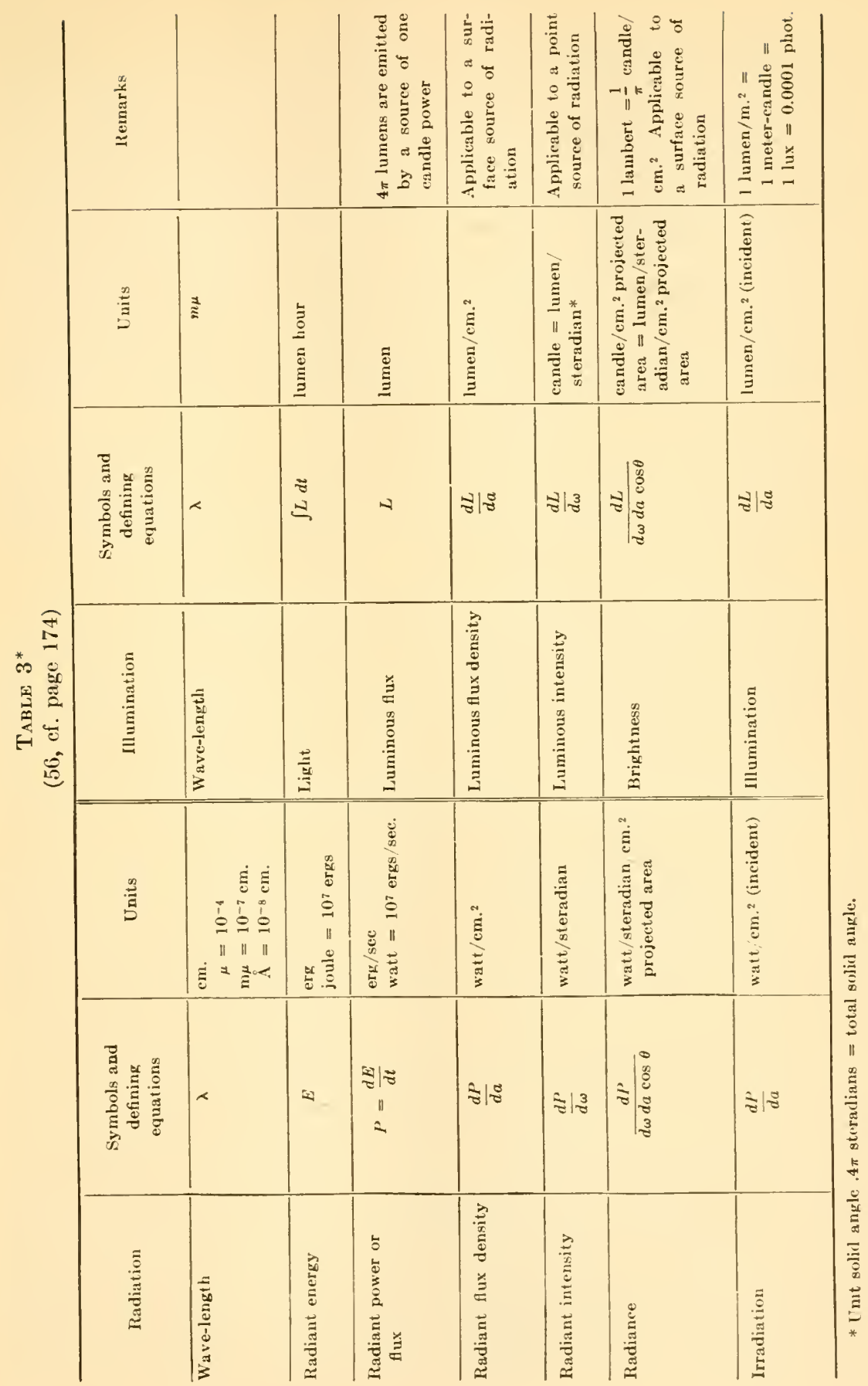


unless both source and observer are specified. A watt of radiant power of a wave-length giving rise to a blue sensation when observed will not appear so bright to the eye as a watt expended at a wave-length which appears yellow. Thus, equal luminous flux does not imply equal radiant power for different wave-lengths. Since different sources of light emit different amounts of power in different wave-lengths, the luminous equivalents will depend upon the wave-length distribution of power. As a first step toward relating these two systems of units, the relative luminous efficiency of different spectral regions has been determined for a large number of observers. A standard relative-visibility curve has been defined which closely approximates the observed average. Table 4 gives the standard relative visibility agreed upon at the last International Commission on Illumination.

TABLE 4 (41)

\begin{tabular}{|c|c|c|c|}
\hline Wave-length $\lambda, \mathrm{m} \mu$ & Relative visibility & Wave-length $\lambda, m \mu$ & Relative visibility \\
\hline 380 & 0.00004 & 580 & 0.870 \\
\hline 390 & 0.00012 & 590 & 0.757 \\
\hline 400 & 0.0004 & 600 & 0.631 \\
\hline 410 & 0.0012 & 610 & 0.503 \\
\hline 420 & 0.0040 & 620 & 0.381 \\
\hline 430 & 0.0116 & 630 & 0.265 \\
\hline 440 & 0.023 & 640 & 0.175 \\
\hline 450 & 0.038 & 650 & 0.107 \\
\hline 460 & 0.060 & 660 & 0.061 \\
\hline 470 & 0.091 & 670 & 0.032 \\
\hline 480 & 0.139 & 680 & 0.017 \\
\hline 490 & 0.208 & 690 & 0.0082 \\
\hline 500 & 0.323 & 700 & $0.00+1$ \\
\hline 510 & 0.503 & 710 & 0.0021 \\
\hline 520 & 0.710 & 720 & 0.00105 \\
\hline 530 & 0.862 & 730 & 0.00052 \\
\hline 540 & 0.954 & 740 & 0.00025 \\
\hline 550 & 0.995 & 750 & 0.00012 \\
\hline 560 & 0.995 & 760 & 0.00006 \\
\hline 570 & 0.952 & 770 & 0.00003 \\
\hline
\end{tabular}

Note: The original article contains interpolations to $1 \mathrm{~m} \mu$.

In specifying relative visibility, the maximum is taken as unity. This maximum occurs at 555 millimicrons or $0.555 \mu$, as will be seen from the curve $V_{\lambda}$ in Fig. 33 . In order to obtain the absolute visibility, or the ratio of luminous flux to radiant power or flux, $V_{\lambda}^{\prime}=L_{\lambda} / P_{\lambda}$, it is only 
necessary to establish the value of the maximum absolute visibility, $V_{0.555 \mu}^{\prime}$. The most recent determination of this value is $V_{0.555 \mu}^{\prime}=624$. The reciprocal of this value, known as the least mechanical equivalent of light, is 0.001602 watt per lumen $(67,40)$. (This is, of course, the ratio of the unit lumen to the unit watt for wave-length $0.555 \mu$.)

Thus, if one knows for a given source, the power $P_{\lambda}$ emitted at each wave-length, one obtains the luminous flux by multiplying the radiant power by the absolute visibility.

$$
L_{\lambda}=V_{\lambda}^{\prime} P_{\lambda}\left(=V_{\lambda} \times V_{0.555_{\mu}}^{\prime} P_{\lambda}\right)
$$

Integrating over the wave-length range, one obtains the total luminous power of the source.

$$
L=\int L_{\lambda} d \lambda=\int V_{\lambda}^{\prime} P_{\lambda} d \lambda
$$

Often it is most convenient to perform the integration graphically, plotting $L_{\lambda}$ as a function of $\lambda$, and measuring the area under the curve by means of a planimeter.

\section{GENERAL CONCEPTS}

Having so agreed upon our methods of specification of radiation and light, we may now attack the simple problem of the passage of a parallel beam of radiation through a portion of matter bounded by two parallel flat surfaces. At the first surface, a portion of the radiation is turned back, a portion continues through. Of this, the portion which is turned back is spoken of as reflected; the portion continuing, as transmitted. As the radiation passes through the body of the material, part of the energy is lost by absorption. At the second surface, a portion is again reflected and a residue transmitted. In the case where the incident beam falls vertically upon the surface, the terms in Table 5 may be used to specify the phenomenon.

Since $t=\sqrt{\top}=\sqrt[b]{E_{2} / E_{1}}=e^{-i}$, then $E_{2}=E_{1} e^{-i b}$, a common expression for Lambert's law.

For a wide range of solutions, the absorption of radiant energy is proportional to the number of molecules of dissolved material in the path. For such cases, the terminology in Table 6 has been established. Here it will be seen that $\mathrm{t}=\sqrt[b c]{\mathrm{T}}$ may be written $\mathrm{T}=e^{-\mathrm{ibc}}$, the exponent being proportional to the concentration and the length of path. This form is perhaps most frequently given to Beer's law. The specific transmissive exponent $\mathrm{i}$ depends upon the type of dissolved material. While the specific transmissive index $\mathrm{k}$ is commonly spoken of as the absorption coefficient, the specific transmissive exponent $i$ is of ten referred to by the same term, so that the logarithmic base must be indicated whenever the term is used. 
Table 5.-Terms Relating to the Rectilinear Transmission of Homogeneous

Radiant Energy throdgh a Homogeneous, Isotropic, Nonmetallic Medium

in the Fori of a Plate with Plane, Polished, Parallel Surfaces

Perpendicular to the Direction of Propagation* (56, ef. page 177 )

Let

$b=$ distance between the bounding surfaces.

$E_{1}=$ radiant energy incident on the first surface.

$E^{\prime}=$ radiant energy reflected by the first surface.

$E_{\mathrm{I}}=$ radiant energy transmitted by the first surface.

$E_{2}=$ radiant energy incident on the second surface.

$E^{\prime \prime}=$ radiant energy reflected by the second surface.

Then

$E_{\text {II }}=$ radiant energy transmitted by the second surface.

$$
\begin{array}{rlrl}
\rho & =E^{\prime} / E_{1}=E^{\prime \prime} / E_{2} & =\text { reflectance. } \\
\pi & =E_{\mathrm{I}} / E_{1}=E_{\mathrm{Il}} / E_{2} & =\text { admittance. } \\
T & =E_{11} / E_{1} & & \text { transmission. } \dagger \\
\mathrm{T} & =E_{2} / E_{\mathrm{I}} & & =\text { transmittance. } \\
t & =\sqrt[b]{\mathrm{T}} & & \text { transmissivity. } t \\
O & =1-T & & \text { obstruction. } \\
A & =1-\mathrm{T} & & \text { absorptance. } \\
D & =-\log _{10} T & & \text { density. } \\
i & =-\log _{e} t & & \text { transmissive exponent. } \\
k & =\log _{10} t & & \text { transmissive index. }
\end{array}
$$

* In deriving these relations, multiple reflections between the bounding surfaces have been neglected. This is permissible for a single plate of the ordinary refractive index for nonmetallic substances. It is not permissible in case $\rho$ has a high value.

$\dagger$ In addition to this restricted meaning of transmission, the term is also used, as are absorption and reflection, to denote a general subject or phenomenon.

$\ddagger$ This relation is known as Lambert's law.

It is obviously a matter of great importance to determine the characteristics of materials used experimentally with regard to their reflectance and transmissivity. Certain ideal conceptions are a convenience. A perfect reflector would have a reflectance 1 . A perfectly transparent body would have a transmissivity 1 . While such ideal reflectors and transmitters are closely approached for certain wave-lengths, one is never completely free from surface reflection. For a polished surface, the reflection $R$ of highly transparent materials depends upon the index of refraction $n$ of the material (i.e., the ratio of the velocity of radiation in free space to that in the body). In case of normal incidence

$$
R=\left(\frac{n-1}{n+1}\right)^{2}
$$

For glass, the reflection varies between 4 and 8 per cent in the visible. A black body may be described as one of zero reflectance and zero transmission. Practically, it may be approached by a distribution of lampblack over a surface. The small opening into an inclosure proves equivalent to a black surface. 
Table 6.-Terms Relating to a Substance in Homogeneous Solution in a Solvent Contained in a Cell with Plane, Parallel Sides Perpendicular to the Direction of Propagation, the Propagation through the Cell and Soldtion Being Rectilinear (56, ef. page 177)

Let

$T_{\mathrm{sol}}=$ transmission of a given cell containing the solution.

$T_{\mathrm{sov}}=$ transmission of the same (or a duplicate) cell containing pure solvent. Then*

$\mathrm{T}=T_{\mathrm{sol}} / T_{\mathrm{sov}}=\mathrm{T}_{\mathrm{sol}} / \mathrm{T}_{\mathrm{sov}}=$ transmittancy

$\mathbf{t}=\sqrt[b c]{\mathbf{T}}=$ specific transmissivity $\dagger$

where $c=$ concentration of the solution

and $b=$ thickness of the solution

$\mathbf{i}=-\log _{e} \mathrm{t}=$ specific transmissive exponent.

$\mathbf{k}=-\log _{10} \mathbf{t}=$ specific transmissive index. $\ddagger$

* The symbols $\mathrm{T}, \mathrm{t}, \mathbf{i}$, and $\mathbf{k}$ are distinguished from the terms $\mathrm{T}, t, i$, and $k$, of Table 5 , in handwriting and typewriting by using the underscore, and in printing by the use of bold face type.

$\dagger$ This relation is known as Beer's law. In many cases it is only approximate.

$\ddagger$ This term has been commonly designated as extinction coefficient.

\section{THE BLACK BODY}

Throughout the discussion so far, we have tacitly assumed a basis for determining the energy associated with a given radiation. The actual method by which we arrive at such a speeification is directly dependent upon our concept of a black body. The black body has been in fact the key to the development of our understanding of radiation. Since we measure temperature on an absolute thermodynamic basis, we may relate our observations of the energy absorbed by a body to its rise in temperature. Since the black body absorbs totally all the radiation falling upon it, it becomes our logical starting point. Knowing the heat capacity of a black body, it may be placed in a beam of radiation and the rise of temperature observed for a given length of time. Thus the rate of rise in temperature must be related to the rate of supply of energy. Investigation of the radiation enitted by black bodies of different temperatures in this way showed that the amount of energy emitted per unit time was greater for greater temperatures. In faet, the radiation was found to increase as a high power of the temperature. Sinee, however, this diselosure indieates that bodies all radiate and that the radiation depends upon the temperature, the black body which we have ehosen as a detector must itself be regarded as a radiator. Thus, our measurement becomes one dependent upon net gain or loss, this exchange depending upon the temperature of the souree, the temperature of the detector, and the temperature of the surroundings. Thus, Stefan observed that the total radiation emitted by a black body is given by the expression

$$
E=\sigma T^{4}
$$


where $\sigma=5.735 \times 10^{-5} \mathrm{erg} / \mathrm{em}^{2} / \mathrm{deg} .{ }^{4} / \mathrm{sec}$., the present accepted value for the Stefan-Boltzmann constant. The radiation exchange between two bodies would then be

$$
E=\sigma\left(T^{4}-T_{1}^{4}\right)
$$

or for small differences in temperature, we may write

$$
E=4 \sigma T^{3}\left(T-T_{1}\right)
$$

We have therefore an absolute method of determining the radiation exchange between blaek bodies. A number of instruments have been developed on this basis, known as pyroheliometers and pyranometers. These will be described below.

Since an ideal black body would absorb totally all wave-lengths, such a type of measurement may be used regardless of the wave-length distribution of radiation of the souree. Assuming for the moment that a given wave-length may be separated from other portions of radiation by some means, we may then explore the energy for each wave-length from a given souree. On the basis of elassical physies, it was predicted that a black body would emit radiation according to the following law:

$$
J_{\lambda}=c_{1} \lambda^{-5} e^{-\frac{c 2}{\lambda T}}
$$

While this exhibited a satisfactory relation to the observed facts for short wave-lengths, a marked failure was evident in the long-wave-length range. In an effort to overcome this difficulty, Planck postulated that radiation existed in small bundles $h \nu$, where $\nu$ is the frequeney and $h$ a universal constant, $h=6.547 \times 10^{-27} \mathrm{erg}$. sec., and on this assumption worked out the following law:

$$
J_{\lambda}=\frac{c_{1}}{\lambda^{5}}\left(e^{\frac{c_{2}}{\lambda T}}-1\right)^{-1}
$$

where

$J_{\lambda}=$ the energy radiated in $\mathrm{erg} / \mathrm{sec} . / \mathrm{em} .{ }^{2} /$ unit solid angle in a direction normal to the area of the black-body surface per centimeter wave-length.

$$
\begin{aligned}
& c_{1}=2 h c^{2}=1.178 \times 10^{-5} \mathrm{erg} / \mathrm{sec} . / \mathrm{cm} .^{2} \\
& c_{2}=\frac{h c}{k}=1.433 \mathrm{~cm} .-\mathrm{deg} .
\end{aligned}
$$

Figure 3 shows a family of curves for representative temperatures of $300^{\circ}$ to $6000^{\circ} \mathrm{K}$. The logarithm of the radiance $J_{\lambda} \mathrm{in} \mathrm{erg} / \mathrm{steradian} / \mathrm{cm} .{ }^{2}$ per eentimeter wave-length in a direction normal to the surface is plotted against wave-length $\lambda$ on a logarithmic scale in microns, increasing to 
the right, and wave numbers in reciprocal centimeters on a logarithmic scale increasing to the left. Values of radiance may be converted into

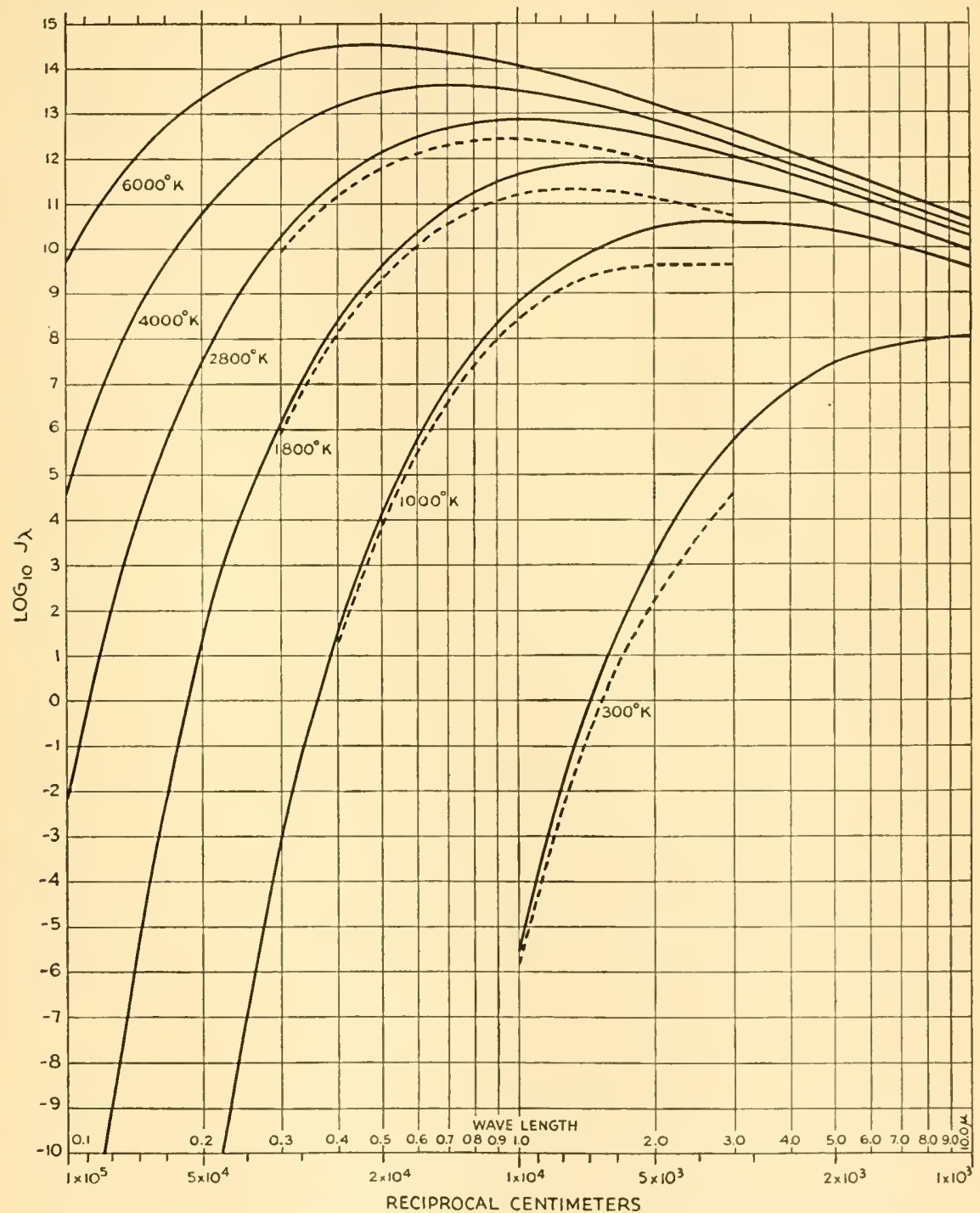

Fig. 3.-Spectral distribution of radiation from black body and tungsten. Ordinates give $\log _{10}$ radiance in ergs $/$ steradian $/ \mathrm{cm} .2 / \mathrm{cm}$. wave-length in a direction normal to the surface. Abscissas give wave-lengths in $\mu$ increasing to the right, and wave numbers in reciprocal centimeters increasing to the left. Full-line curves are for black body. Dotted curves are for tungsten.

watts/steradian/cm. ${ }^{2}$ by subtracting 7.0 from the logarithm. In order to obtain the radiation in the entire hemisphere, these values must be 
multiplied by $\pi$. The corresponding values of radiance in a direction normal to the surface for a tungsten filament are shown by dotted lines. ${ }^{1}$

Table 7 (31) gives the numerical values for black-body radiation from which the curves have been prepared, together with those for a tungsten filament. The values of emissivity upon which the data for tungsten depend are incomplete and approximate, those for $2800^{\circ} \mathrm{K} .^{2}$ being based entirely upon extrapolation. A more adequate basis for these values is urgently needed.

It may be shown that Stefan's law, mentioned above, follows as a consequence of Planck's equation (by adding up the effects for all wavelengths). For the condition that $J_{\lambda}$ be maximum, we obtain the simple relation

$$
\lambda_{m} T=\text { const. }(0.2884 \text { cm.-deg.) }
$$

Thus, the higher the temperature of the body, the shorter the wavelength of maximum radiation. For $6000^{\circ} \mathrm{K}$., the approximate temperature of the sun, the maximum intensity occurs in the visible at about $0.48 \mu$; for $3000^{\circ} \mathrm{K}$., a common temperature for a tungsten filament lamp, it occurs in the near infra-red, at $0.96 \mu$; for $1000^{\circ} \mathrm{K}$, at $2.88 \mu$; for $300^{\circ} \mathrm{K}$., approximate room temperature, at $9.6 \mu$.

For convenience in visualizing the shift of maximum and the relative distribution, one may refer to the center section of Fig. 2.

Thus, since the radiation from a black body is predictable from its temperature, such a black body may be made the standard of radiation for all wave-lengths. To determine the intensity of any other source of radiation, it is only necessary to make a comparison of the radiation for that wave-length with that of a black body. If the source of radiation is compared, wave-length by wave-length, with the black body, one need not use a black-body detector. The difficulty which this method has presented arose from the fact that black bodies were not available of such a high temperature as to radiate with sufficient intensity in the short-wave-length ranges.

A black-body source consisting of a hollow enclosure immersed in freezing platinum has been developed for use as an absolute standard of candle power by the National Bureau of Standards (66). This provides a black-body source at $2046^{\circ} \mathrm{K}$., the fixed point at which pure platinum freezes. By using freezing iridium $(38,67)$ instead of platinum, a source

1 Note: The conventional use of the centimeter for wave-length in $J_{\lambda}$ should be particularly noted. When the smaller customary units are used, values indicated must be multiplied by the corresponding factor: for Ångtröms by 10-8; for millimicrons by $10^{-7}$; for $\mu$ by $10^{-4}$.

${ }^{2} \mathrm{~K}$ refers to absolute temperature. When not otherwise specified, temperature refers to centigrade scale, $\mathbf{C}$. 


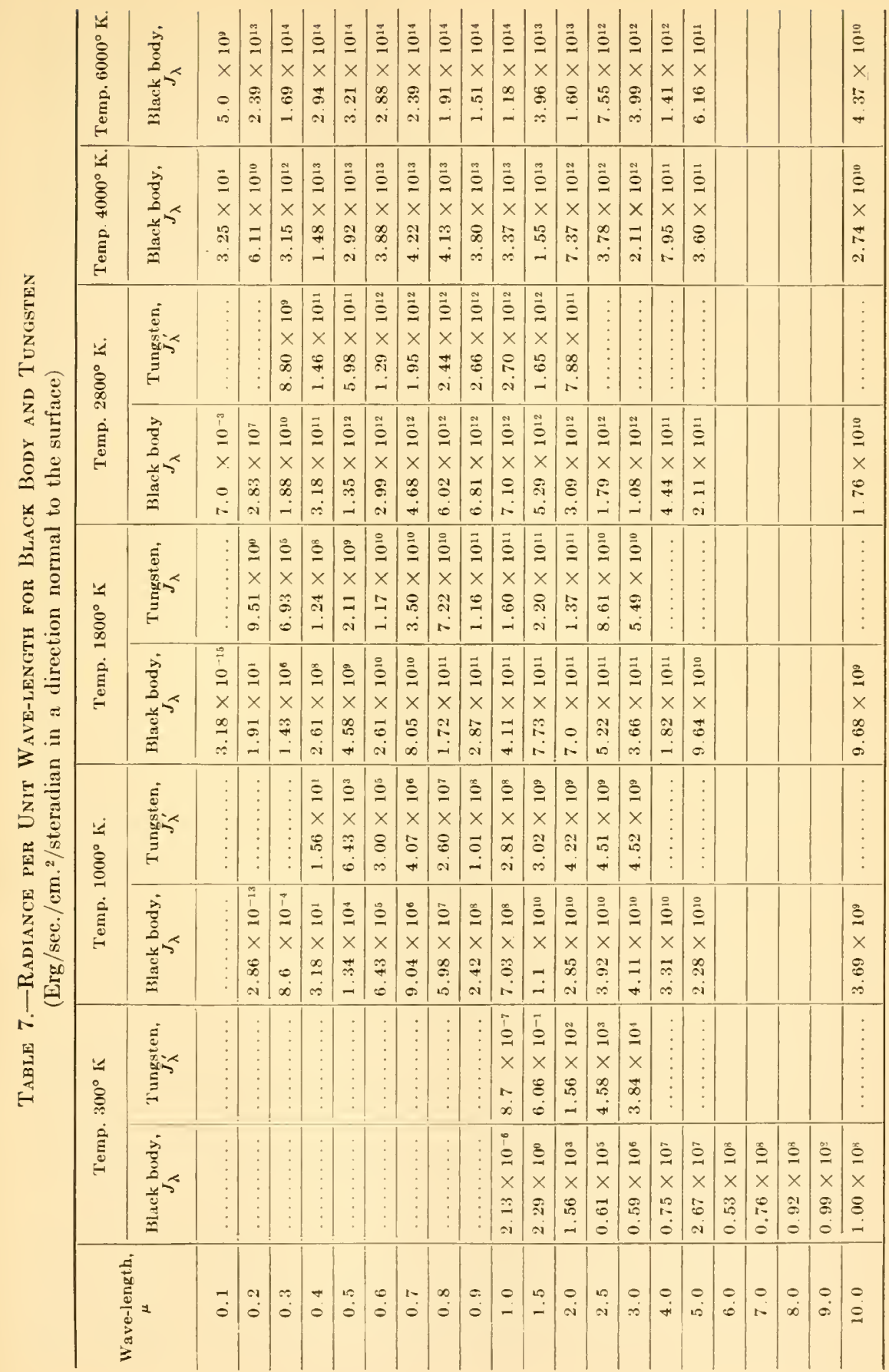


of black-body radiation at $2727^{\circ} \mathrm{K}$. has been made available. This higher temperature promises to make practieable standardization in the region of shorter wave-lengths by direct comparison with a black body. A brief description of this type of source therefore seems desirable.

The arrangement consists of a crucible containing the platinum (or iridium) in whieh is immersed a small tube which serves as the actual source (see Fig. 4). The erucible and immersed tube are made of fused thorium oxide (thoria), ground to a fine powder and then molded to
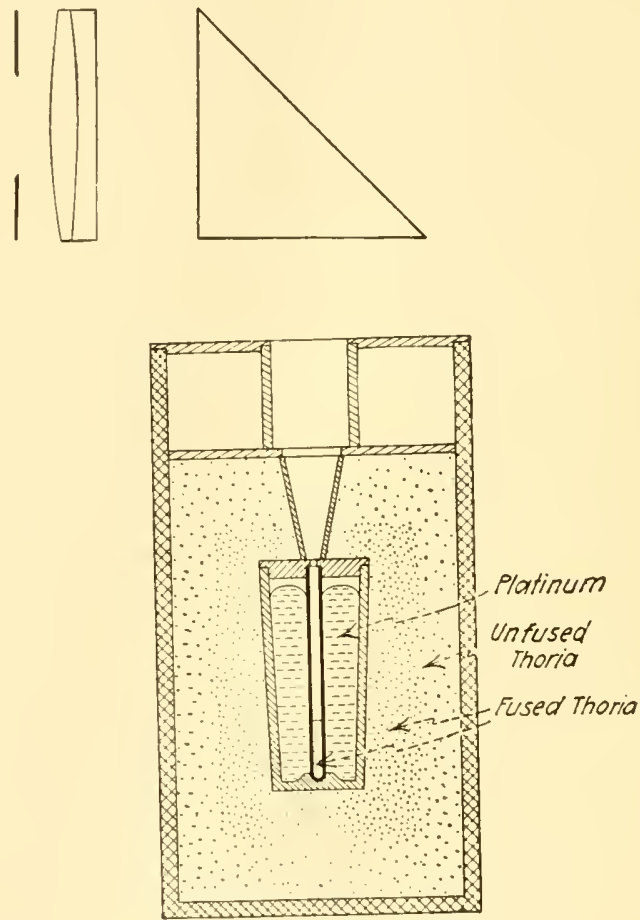

F1G. 4.-Diagram of standard black body and collimating system. (National Bureau of Standards.)

shape, using thorium chloride as a binder. It has been found that sueh erucibles, even after extensive use, do not contaminate metals of the platinum group. Powdered thoria is used for insulation. A small quantity of powdered thoria is placed in the lower portion of the tube to conceal the colder bottom of the tube.

The metal is melted by means of a high-frequency induetion furnace. After the metal has been heated a few degrees above its melting point, the power is eut off. Successive observations of the radiation show decreasing intensity until the metal begins to freeze, a constant intensity during the period of freezing, and again a falling intensity as the solid metal 
cools. The intensity of radiation during the period of freezing, usually lasting from 5 to $15 \mathrm{~min}$., has been found to be reproducible within the experimental error of observation, i.e., less than 0.25 per cent. For further details, the reader should consult the original papers (62).

This type of source has been made the standard of both brightness and color temperatures. For a discussion of its application to color temperature problems, the reader is referred to the publication of the National Bureau of Standards (65). A brief discussion of its application to brightness is not only of value in itself, but will serve to illustrate the interrelation between the photometric or illuminating system and the absolute system.

Since $J_{\lambda}$ is the power emitted per square centimeter per unit solid angle in a direction normal to the surface, if one multiplies by the absolute visibility, one obtains the brightness per unit wave-length. If, now, this value is integrated over the entire wave-length range, one obtains the total brightness of the source. As has been noted before, the absolute risibility is obtained from the relative visibility by multiplying by the maximum absolute visibility, or the reciprocal of the least mechanical equivalent of light. The expression for brightness becomes, therefore

$$
B=\frac{1}{0.001602} \int_{0}^{\infty} J_{\theta \lambda} V_{\lambda} d \lambda
$$

the constant factor having been taken outside the integral sign. In the application of this formula it should be noted that usage differs as to the quantity $J$. Our values apply to the power emitted in unit solid angle, whereas the values given in the original article refer to the total power emitted in the hemisphere. Where the latter is the case, $\pi$ appears in the denominator of the constant factor.

TABLE 8

\begin{tabular}{c|c||c|c||c|c}
\hline $\begin{array}{c}\text { Tempera- } \\
\text { ture, }{ }^{\circ} \mathrm{K} .\end{array}$ & $\begin{array}{c}\text { Brightness, } \\
\text { candles/cm. }\end{array}$ & $\begin{array}{c}\text { Tempera- } \\
\text { ture, }{ }^{\circ} \mathrm{K} .\end{array}$ & $\begin{array}{c}\text { Brightness, } \\
\text { candles/cm. }\end{array}$ & $\begin{array}{c}\text { Tempera- } \\
\text { ture, }{ }^{\circ} \mathrm{K} .\end{array}$ & $\begin{array}{c}\text { Brightness, } \\
\text { candles/cm. }\end{array}$ \\
\cline { 1 - 3 } 1550 & 1.273 & 2000 & 44.46 & 2400 & 351.7 \\
1600 & 2.077 & $2046.6 \dagger$ & 58.91 & 2450 & 434.9 \\
1650 & 3.295 & 2050 & 60.09 & 2500 & 533.3 \\
1700 & 5.091 & 2100 & 80.09 & 2550 & 648.8 \\
1750 & 7.679 & 2150 & 105.4 & 2600 & 783.7 \\
1800 & 11.33 & 2200 & 137.0 & 2650 & 940.1 \\
$1828^{*}$ & 14.02 & $2239 \ddagger$ & 167.1 & 2700 & 1121 \\
1850 & 16.38 & 2250 & 176.1 & $2727 \S$ & 1227 \\
1900 & 23.24 & 2300 & 223.9 & 2728 & 1231 \\
1950 & 32.41 & 2350 & 282.0 & & \\
\hline
\end{tabular}

* Palladium point. † Platinum point. $\$$ Rhodium point. $\quad$ Iridium point. 
For convenience in calculating the brightness of a black body of specified temperature $\theta$, an empirical formula has been evolved (67) which is in excellent agreement with the calculated and observed values over the range from $1800^{\circ}$ to $2728^{\circ} \mathrm{K}$.

$$
B=2495\left(\frac{\theta}{2955}\right)^{\frac{461.4}{\sqrt{\theta}}}
$$

Table 8 gives the brightness of the black body for various temperatures.

\section{MEASUREMENT OF TOTAL RADIATION}

\section{ABSOLUTE DETECTOR}

Instruments which yield an absolute measurement of radiation apply a calorimetric method to the black body. The pyroheliometers used for the standardization of solar-radiation measurements represent this type of instrument. The water-flow pyroheliometer used by Abbot (7) will serve as an illustration. It consists of two identical blackened chambers through which water can be circulated, one stream entering each chamber at the same temperature. Radiation is allowed to fall upon one chamber, producing a rise in temperature in the water stream. An equivalent rise in temperature is produced by a known electrical current through a known resistance in the other chamber. An electrical thermometer is used to secure equality in the temperature of the two identical streams. The heat supplied electrically to the second chamber is thus equal to the heat supplied by radiation to the first and can be evaluated either in calories per second or in watts. Knowing the area of the receiver, the irradiation can thus be determined. In practice, the influence of the surroundings is minimized by surrounding the chambers with an evacuated space, thereby eliminating convection. Since radiation loss from the two chambers is identical, it does not enter into the calculation.

A second type of absolute detector is the melikeron pyranometer (8). In this form, a metal strip blackened and so shaped as to constitute effectively a hollow inclosure is heated by radiation. The rise in temperature is measured by a thermocouple-galvanometer system. An equivalent rise in temperature is then produced electrically and the heat supplied determined, so that in this respect it is equivalent to the pyroheliometer, in that energy rate of supply is measured under presumably identical conditions.

A compensation pyroheliometer (9) has been constructed by Ångström, with two identical blackened strips, one of which is heated by radiation and the other by electrical energy, identical rises in temperature 
being produced, as indicated by thermocouples. In general, this type of instrument has not been constructed of sufficient sensitivity to be used for sources furnishing markedly less radiant energy than the sum.

\section{COMPARISON WITH BLACK-BODY EMISSION}

A second method of absolute determination involves the use of a black-body receiver whose temperature can be measured by a suitable device, wherein the deflection is simply proportional to the energy falling upon the receiver. With such a secondary type of instrument, yielding only a deflection proportional to total energy, it is necessary that its deflection be calibrated by some other method, either by means of a known source, such as a black body at known temperature, or by comparison with an absolute instrument. In practice, a standard incandescent lamp, generally carbon filament, is commonly used as a known source. Such a lamp, properly seasoned, may be secured from the Bureau of Standards, or a suitable lamp may be calibrated by them (23). It is only necessary for this purpose that the total radiation in a given direction be known for a specified condition of operation. One of the simplest instruments of the secondary type is the silver-disk pyroheliometer (2). It consists of a high-grade mercury-in-glass thermometer, bedded in a silver disk whose surface is blackened and the area accurately determined.

Another widely used instrument is the bolometer (3). Here two identical blackened strips of metal are placed in an evacuated space. The two strips become the two arms of a Wheatstone's bridge, whose balance is determined by a galvanometer. When radiation is allowed to fall on one of these strips, a rise in temperature is produced which unbalances the bridge by change of resistance. The chief difficulty with this type of instrument is that it requires a constant current to be passed through the two strips, thus necessitating a constant source of potential.

A third form, by far the most commonly used, is simply a thermocouple with a blackened receiver. Where two dissimilar metals join, an electromotive force is developed which depends upon the temperature. Thus, if a circuit is made up of two parts, one copper and the other constantan, electromotive forces will be developed in opposite directions at the two junctions. If these are at identical temperatures, no current will flow. If, however, a metallic receiver is attached to one of the junctions and its surface blackened, its temperature may be raised by radiation. For small temperature changes, the unbalanced electromotive force is proportional to the temperature rise and hence to the radiant power impinging upon the receiver. If a galvanometer is so constructed as to produce a deflection proportional to the current, the combination of thermocouple and galvanometer yields a deflection strictly proportional to irradiation. 


\section{THERMOCOUPLE TECHNIQUE}

The highest sensitivity available in present practice is obtained by using bismuth for one lead, and bismuth with 4.5 to 5 per cent of tin alloy for the other. In the most sensitive forms, the thermocouple is placed in an evacuated space. It is then possible to measure rises in temperature of the order of $10^{-5}$ degree. With such small rises in temperature, the influence of surrounding bodies is of paramount impor-
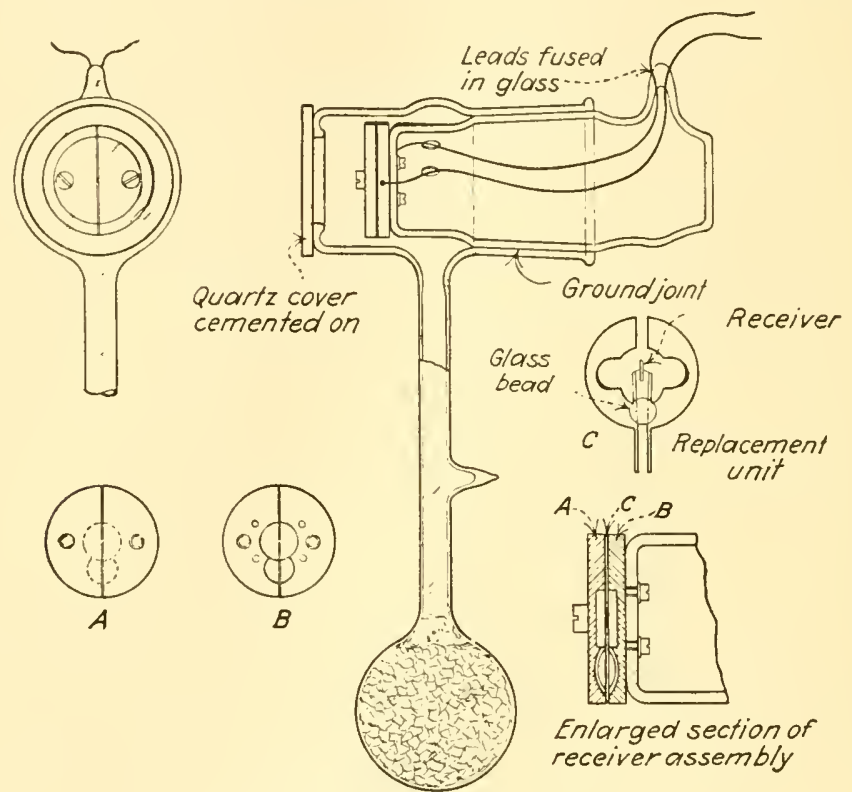

Enlarged section of receiverassembly

FIG. 5.-Housing for vacuum thermocouple.

tance. It is necessary, therefore, that the thermocouple be protected from radiation disturbances, as well as from convection. This may be accomplished in the following way: The blackened receiver is placed at the center of a surrounding shield whose temperature is that of the cold junction. An example of such a thermocouple housing is illustrated in Fig. 5. The thermocouple is constructed upon a convenient replacement unit $C$. Two thin pieces of copper are joined by a glass bead, extensions projecting toward the center of each unit. Upon these projections are welded the fine thermocouple leads. The wires are so selected as to yield a resistance of from 10 to $30 \mathrm{ohms}$. For spectroscopic purposes, the receiver is about $0.1 \mathrm{~mm}$. wide and $4 \mathrm{~mm}$. long. One side is blackened to receive the radiation and the other left bright to reduce the loss by 
radiation to the surroundings. This receiver unit $C$ is clamped at the center of a split housing $A B$. Each half of the housing is made of two parts separated by a sufficient distance to secure electrical insulation. Thus the assembled housing occupies practically the entire solid angle surrounding the receiver. Radiation can be introduced only through the narrow slit opening in $A$. The whole assembly is then placed in an evacuated space, radiation being introduced through a suitable window, quartz for radiation lying between $2000 \AA$ and $2.5 \mu$, salt if the instrument is to be used for the infra-red between $2.5 \mu$ and $15 \mu$. Where such extreme precautions have to be taken to avoid the influence of surrounding bodies upon the detector, it is necessary that the galvanometer and leads also be maintained at constant temperature. Although the entire electrical circuit may be made up of copper (except for the junction), electromotive forces may arise from impurities (which result in inhomogeneities in the circuit) and from other similar causes.

Wherever windows are used, their transmission characteristics must be taken into account. For total radiation measurements, where some of the radiation lies in the infra-red, bare thermocouples should be used. Such thermocouples do not have the high sensitivity of the vacuum thermocouple, owing to the greater heat loss by convection. The method of shielding described is of even greater importance in the use of such bare thermocouples. The sensitivity of such an instrument depends upon:

(a) The choice of alloys having the quantity

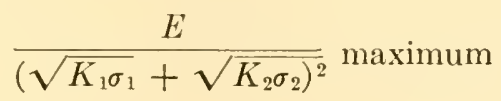

where $E=$ thermoelectric power.

$K_{1}$ and $K_{2}=$ heat conductivities.

$\sigma_{1}$ and $\sigma_{2}=$ specific resistances of the lead wires, respectively.

(b) The proper relation of size of receiver, length of wire, and resistance of the couple.

(c) The sensitivity of the current-measuring instrument. For most purposes, a high-sensitivity d'Arsonval galvanometer is preferred. For even greater sensitivity, amplification may be applied to the galvanometer. For a more complete discussion, the reader is referred to the literature (12).

\section{RADIONETERS}

Another type of instrument which has been used to some extent for spectroseopic purposes is the radiometer (5). It consists of a suspension with two symmetrical receivers, mounted on a light quartz fiber and 
placed in a gas-filled space at somewhat reduced pressure. When the surface of one receiver is heated by radiation, convection currents are set up which produce a deflection. It is important in this type of instrument that the front and back surfaces of the receiver be at as great a temperature difference as possible. For this reason, the receivers are generally laminated in character. Thus, mica is sometimes employed. The mica is subjected to a pearling process, producing a multiplicity of cleavages between the separate layers of the crystal.

A very sensitive radiometer has been constructed by Abbot wherein the receiver was built up of several layers of fly's wings (5). With such an instrument, it has been possible for him to measure the spectral distribution of radiation from stars, using the 100-in. telescope at Mt. Wilson.

In order to secure a maximum sensitivity in radiometer measurements it is necessary that optimum gas pressure be maintained. For details regarding the construction of such instruments, the reader is referred to a more complete discussion by Coblentz (19). A recent discussion of the theory has been given by Marsh, Condon, and Loeb (48).

\section{COMPARISON OF DETECTORS}

As a matter of convenience in comparing the sensitivity of the different types of instruments available for radiation measurements, and as a guide in the selection of suitable current-measuring devices, Table 9 has been prepared. For spectroscopic purposes, a small narrow receiver is desirable. If continuously recording methods are required, a small time of response is at a premium. Thus, in comparing the different instruments available, it is important to take these characteristies into account. Thermocouples referred to in Table 9 have been selected because of their small areas of receiver. If radiation is to be measured over an extended area, thermocouples can be constructed having much larger areas of receiver, the sensitivity to irradiation increasing roughly in proportion to the area. For a small-area instrument, the single couple is superior to any multiple-junction thermopile so far constructed.

Table 9 is divided into three parts, the first dealing with the characteristics of the detector unit, the second with the characteristics of the measuring device, galvanometer, amplifier, etc., and the third with the sensitivity, $(a)$ of the active element, and $(b)$ of the overall combination. For instance, the sensitive thermocouple has a resistance of $17 \mathrm{ohms}$, an area of $0.003 \mathrm{~cm} .{ }^{2}$, an inherent time of practical equilibrium of 0.4 sec. A suitable galvanometer to be used with this couple should have practically the same resistance. If it has a 5 -sec. time of response, it will have a voltage sensitivity of $2 \times 10^{-9}$ and a current sensitivity of $4 \times 10^{-11}$ amp. for the least measurable deflection under favorable conditions. 
VISIBLE AND NEAR-VISIBLE RADIATION

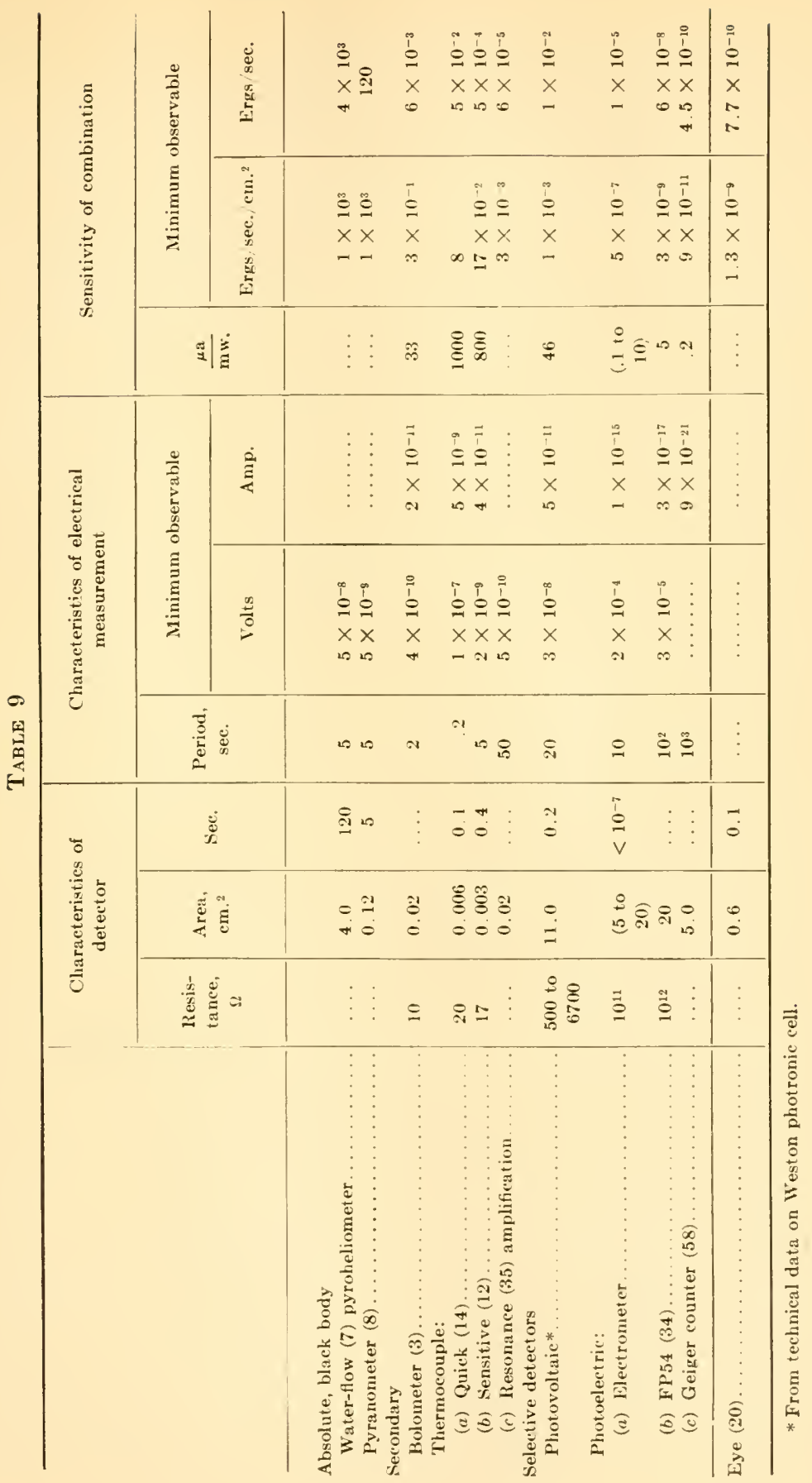


Such a couple will yield $800 \mu$ a. current for $1 \mathrm{mw}$. absorbed radiant power. It will measure as a minimum $0.17 \mathrm{erg} / \mathrm{sec} . / \mathrm{cm}^{2}$ irradiation, which for this size of receiver amounts to a least detectable radiant power $5 \times 10^{-4}$ $\mathrm{erg} / \mathrm{sec}$. If a sensitive thermocouple is combined with a resonance amplifier, the least detectable radiant power is $6 \times 10^{-5} \mathrm{erg} / \mathrm{sec}$. With a photovoltaic cell, such as the photronic cell, having a large receiver, $11 \mathrm{~cm} .{ }^{2}$ in area, an irradiation of $10^{-3} \mathrm{erg} / \mathrm{sec} . / \mathrm{cm} .{ }^{2}$ can be detected. Owing to its large receiver, however, it is absorbing a far greater amount of energy, the radiant power incident upon the surface being $10^{-2} \mathrm{erg} / \mathrm{sec}$.

In case of a photoelectric cell with electrometer measurement, we may be dealing with a detector whose internal resistance is $10^{11} \mathrm{ohms}$, its area may be anywhere from 5 to $20 \mathrm{~cm} .{ }^{2}$, its inherent time of response is extremely small, probably of the order of $10^{-7}$ sec. An electrometer which might be used for this purpose might have a period of 10 sec., a voltage sensitivity of $2 \times 10^{-4}$, a current sensitivity of $10^{-15}$. Such a photocell, depending upon the type selected, may yield a current anywhere from 0.1 to $10.0 \mu \mathrm{a}$. for $1 \mathrm{mw}$. incident radiant power. With such an arrangement, irradiation may be detected as small as $5 \times 10^{-7}$ $\mathrm{erg} / \mathrm{sec} . / \mathrm{cm} .{ }^{2}$ radiant power for a $5-\mathrm{cm} .{ }^{2}$ area. If one does not require a small time of response, the photoelectric cell may be used with an FP54 amplifier, requiring 100 sec. for time of observation. Such an arrangement may have a least measurable voltage characteristic of $3 \times 10^{-5}$, corresponding to $3 \times 10^{-17} \mathrm{amp}$. Such a device would respond to a least observable irradiation of $3 \times 10^{-9} \mathrm{erg} / \mathrm{sec} . / \mathrm{cm} .{ }^{2}$. This corresponds for a $20-\mathrm{cm} .^{2}$ area to $6 \times 10^{-8} \mathrm{erg} / \mathrm{sec}$. of incident radiant power. It will be noticed that so far only with this extremely sensitive device is the sensitivity of the eye approached, which is able to detect an irradiation of $1.3 \times 10^{-9} \mathrm{erg} / \mathrm{sec} . / \mathrm{cm} .{ }^{2}$, but owing to its very small effective area (pupil), the corresponding radiant power is only $7.7 \times 10^{-10} \mathrm{erg} / \mathrm{sec}$.

The most sensitive of all radiation detectors is the photocell Geiger counter. With this arrangement, irradiation of $9 \times 10^{-11} \mathrm{erg} / \mathrm{sec} . / \mathrm{cm} .^{2}$ has been detected. This corresponds to $4.5 \times 10^{-10} \mathrm{erg} / \mathrm{sec}$. of incident radiant power. This is the only instrument which exceeds the sensitivity of the eye to radiant power. However, for the same period of response, none even approach it.

In this table, we have presented first the absolute black-body detectors, having only a very low sensitivity; second, the secondary black-body instruments, whose deflection when properly constructed is proportional to the incident radiation; third, the selective detectors of the photoelectric type whose sensitivity varies markedly with wave-length. The sensitivity as a function of wave-length of these detectors will be taken up in detail in a later section. For most practical radiation measurements, a secondary black-body instrument is used in conjunction with a standard 
lamp. Such a standard lamp must be calibrated in terms of a black body.

\section{MONOCHROMATIC MEASUREMENTS}

Three general types of problems present themselves for monochromatic analysis:

$A$. The determination of the intensity distribution from sources as a function of wave-length.

$B$. The determination of the absorption characteristics of transparent or partially transparent materials.

$C$. The provision for monochromatic irradiation of materials.

Unquestionably the preferred method of attacking these problems involves the use of a monochromator, together with suitable detectors, either black-body type or selective. In selecting a monochromator for a given undertaking, one is concerned with: (a) Its dispersion and resolution, determining the wave-length range isolated. (b) Its numerical aperture, which determines the solid angle of radiation which the instrument is able to transmit. (c) The percentage transmission of the optical parts. $(d)$ The extraneous or scattered light transmitted by the instrument. (e) The slit characteristics.

For our purpose, we may regard a monochromator (see Fig. 6(a)) simply as an optical system which takes the radiation in a given solid angle through a narrow opening (slit) $S_{1}$ and forms an image of that narrow opening or slit upon a second slit $S_{2}$ which permits the escape from the instrument of a narrow wave-length range of known wave-length maximum and extent. Such instruments are commercially available in numerical apertures varying from $f 12$ to $f 4$ and rarely to $f 2$, the numerical aperture signifying the ratio of the focal length of the collimator (first lens) $L_{1}$ and camera (second lens) $L_{2}$, assuming as is customary that the two have the same focal length, to the diameter of the limiting aperture (prism or lens size) $P$. Thus a spectrograph of numerical aperture $f 4$ utilizes radiation from a given point on the slit having a solid angle of $1 / 16$ of a steradian. Since most sources emit radiation uniformly over a fairly large solid angle, the light-gathering power of the instrument is directly proportional to the solid angle subtended by the limiting aperture, or inversely proportional to the square of the numerical aperture. Thus, an instrument of $f 4$ would have a light gathering power nine times as great as one of $f 12$.

Two characteristics determine the overall transmission of the optical system: (a) The number of surfaces (since each normal surface reflects approximately 4 to 8 per cent), and the angle of incidence on the surfaces (since the reflceting loss increases greatly with a greater angle of incidence). (b) The absorption characteristics of the refracting materials 
used for the particular region of the spectrum. (Grating instruments are seldom used for photochemical or biological purposes, where high intensity is required.) For example, a single quartz monochromator yielded a transmission of 40 per cent at $2250 \AA$ and rose to 50 per cent at $6000 \AA$.

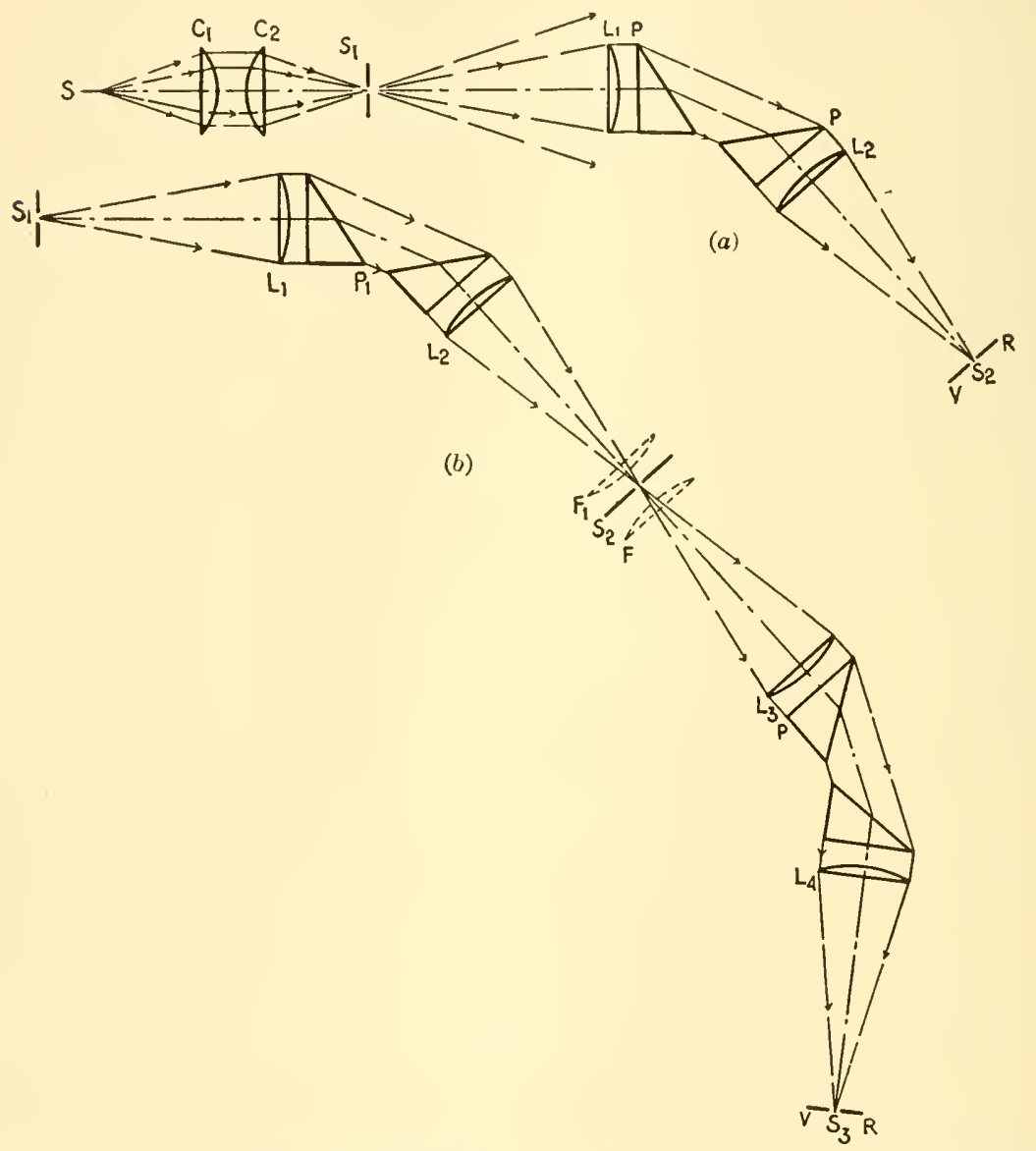

Fig. 6.-Monochromatic illuminators. (a) Single monochromator, showing condensing system. (b) Double monochromator. Two single monochromators may be combined in tandem. Where a special instrument is built, field lenses $F F_{1}$ are used, wavelength change being accomplished by a motion of slit $S_{2}$.

In addition to the radiation which forms a part of the true image falling on the second slit, radiation of other wave-lengths also falls upon the slit, which arises from surface imperfections, bubbles, and inhomogeneitics of the refractive material within the optical parts, together with light returned to the beam by surfaces not intended to contribute to the 
illumination. In a well-constructed instrument, this usually amounts to about 1 per cent in the vicinity of a strong region of emission, i.e., spectral line. That is, a background of intensity where there should be no radiation will exist to about 1 per cent of the intensity of the strong line. In instruments constructed of fused material, containing striations, this impurity may be many times greater. For most critical work, a double monochromator should be used (see Fig. $6(b)$ ), the radiation from one

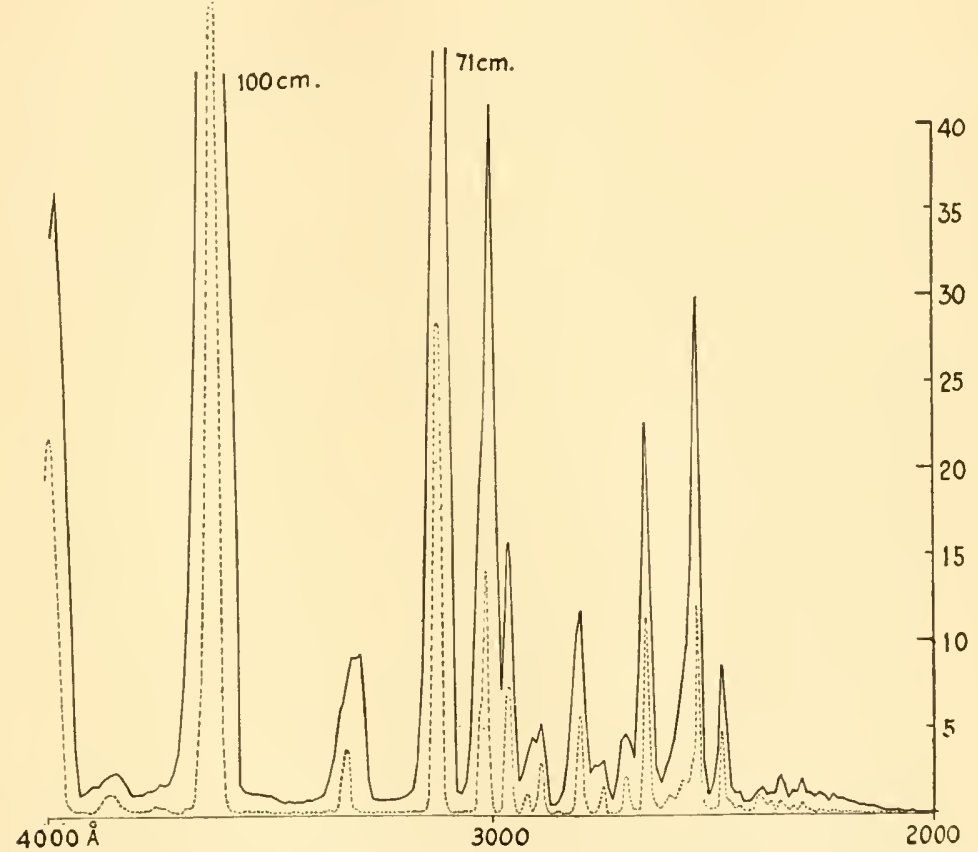

Fig. 7.-Comparison of spectra, using single and double monochromators at the same slit width. Full line shows single monochromator. Dotted line shows double monochromator.

monochromator passing through a second monochromator, the second slit of the first monochromator serving as the first slit of the second. Where no change in slit width is made, the scattered light will then be reduced to one-fourth of its original value. The total transmission of the instrument now becomes 16 to 25 per cent, but in a properly constructed double monochromator, the resolution (purity) will be doubled without change in slit width. Figure 7 (57) shows the observations of mercury-are spectrum with one monochromator, and the dotted line shows the spectral distribution as determined with two identical instruments combined into a double monochromator, the slit widths being unchanged. 
The slit characteristics of an instrument are extremely important in all forms of monochromator. The second slit must coincide in geometrical form with the image formed by the optical system of the first slit, or else the normal advantages will not be secured. Since the range of wave-lengths yielded by the instrument is critically dependent upon the slit width, it must be accurately determined and readily reproducible. In prism instruments, the off-axis deviation for a given wave-length is considerably greater than that of the central point. Consequently, the image formed of a straight first slit is curved thus demanding a curved second slit. For many purposes, it is more convenient to work with a straight second slit. It may therefore be desirable for the curvature to be imposed upon the first slit. In order to secure satisfactory results, the slits must be so adjusted that the image for a given wave-length falls precisely upon the second slit. This requires adjustment for both focal length and orientation. Since the amount of energy is dependent upon the slit length, provided it can be illuminated uniformly, and upon the width of the slits, the selection of suitable slit width and length is a matter of great importance. The selection of suitable slit width is, of course, dictated by the spectral purity required, while the slit length is a matter of instrument design.

With a suitably chosen monochromator placed in correct adjustment, the proper illumination from a source must be secured. With some sources it is feasible to bring the source so close to the slit, that the slit is directly illuminated uniformly over its extent and width, and that the solid angle subtended by the collimating lens is more than completely filled from all portions of the slit. Such an arrangement is ideal, since no added losses are incurred by the intervention of a condensing lens. This method is, however, often precluded by the character of the source, either because of its temperature, in that it cannot be brought sufficiently close to the slit, or because it does not present an extended area of uniform brightness. In such a case, it is customary to use a condensing lens, or lenses, as shown by $C_{1}$ and $C_{2}$ in Fig. $6(a)$, between the source and the first slit, which forms an image of the source upon the slit. In general, it is then desirable that a uniform portion of the source entirely cover the slit. Any convenient magnification or reduction of the image formed, as compared to the source, may be used, provided the condensing lens is of sufficient diameter and subtends a uniformly illuminated area. It is necessary that the condensing lens be of sufficient diameter that the solid angle of convergent radiation falling upon the slit be greater than the solid angle subtended by the collimating lens. A safe rule is that the diameter of illumination falling upon the plane of the collimating lens be twice the diameter of the lens. This avoids any dangers in the case of using narrow slits, from inhomogeneity of illumination due to 
the diffraction characteristies arising from the slit. In absolute intensity measurements, it is necessary to take into consideration the transmission of the condenser lens as well as that of the monochromator. A third method of slit illumination will be taken up more specifically in connection with absolute measurements of intensity of source, the above providing methods of procedure more widely applicable to various problems.

ABSOLUTE MEASUREMENTS OF THE INTENSITY DISTRIBUTION OF RADIATION FROM SOURCES AS A FUNCTION OF WAVE-LENGTH

Our problem is to determine the amount of energy of a given wavelength range supplied by a source of defined extent through a specified solid angle. We are thus able to arrive at the radiance of the source for a given wave-length, this corresponding in radiometric measurements to the quantity termed brightness in photometric measurements. A knowledge of this quantity will enable us to determine the irradiation provided by the source under various eonditions.

One method is to use a source whose area can be determined, at a sufficient distance from the first slit that the solid angle of illumination thus provided less than fills the lens and prism system. The dimensions of the source thus determine the aperture utilized. The slit area determines the solid angle of radiation measured from any point on the source. One obtains, therefore, an average value of the radiance of the exposed source. If a black-body detector, such as a thermocouple, is used, the energy escaping from the second slit may be completely absorbed by the thermocouple. The thermocouple-galvanometer sensitivity may then be determined by comparison with the total radiation from a standard lamp. This comparison provides the information-energy per second for a given galvanometer deflection. Multiplying the deflection observed by this factor, one obtains the radiant power from the second slit of the instrument under the conditions specified. Dividing this by the transmission of the instrument, one obtains a value for the radiant power $P_{s}$ passing through the first slit, provided the second slit has been so adjusted that it does not obstruct the energy of the given wave-length. Dividing this value by the solid angle subtended by the slit, one obtains the radiant intensity (watts per steradian). Or dividing by the area $A_{s}$ of the slit, one obtains the irradiation (watts per square centimeter) provided by the source at that distance. If one wishes to determine the radiance $R$, one must further divide the radiant intensity (watts per steradian) by the projected area $A$ of the source. In the case where the source is a selective emitter, the instrument has been adjusted for a given wave-length, and all other wave-lengths excluded by the second slit. One now has all the necessary data on the source for the wavelength indicated. 
600

$\mu w \cdot / \mathrm{cm}^{2}$

300

24

$24 \quad 26 \quad 28 \quad 30$

$30 \quad 35$

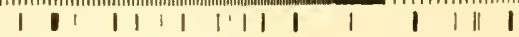

$50 \quad 70$

1 1

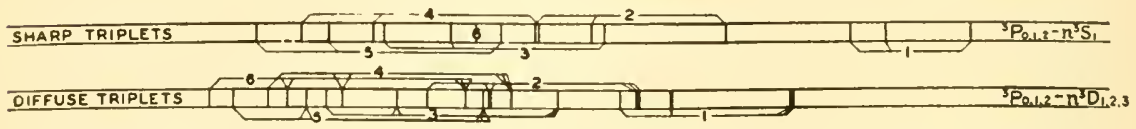

DIFFUSE SINGLETS

$1 \mathrm{LI}+\mathrm{P}_{1}-\mathrm{n}_{1}$

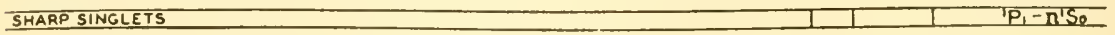

COMBINATIONS

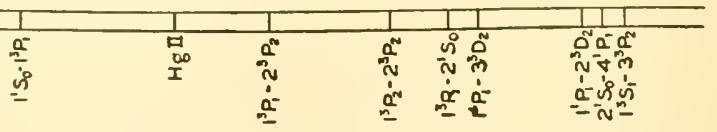

FIG. 8.-Intensity distribution from high-intensity mercury arc. Ordinates give $\mu \mathrm{W} . / \mathrm{cm} .{ }^{2}$ irradiation at $25 \mathrm{~cm}$. from a $20-\mathrm{mm}$. exposed midsection of a 250 -volt arc, operated on 143.5 volts and $4.5 \mathrm{amp}$. Abscissas give wave-lengths on prismatic scale, increasing to the right, with photographic spectrum below. Spectral identification shown diagrammatically. (From McAlister, 50.) 
If, however, one is working with a continuous source, the value obtained is representative of a wave-length band, the extent being determined by the slit width and dispersion of the instrument. Further experiments are necessary in order to evaluate the effective wave-length range. This may be done either analytically or by the use of an auxiliary spectrograph or monochromator.

An example of absolute measurement of the spectral characteristics of a source is furnished by the measurements on the quartz mercury arc by MeAlister, and illustrated in Fig. $8(50)$. These data are given in terms of the irradiation at $25 \mathrm{~cm}$., furnished by a $20-\mathrm{mm}$. midsection of a 250 -volt uviare, operated on 143.5 volts, $4.5 \mathrm{amp}$, the temperature having been maintained to provide these charaeteristics.

If a condensing lens is used, the limiting aperture must be determined. If the prism of the monochromator provides the limiting aperture, its projected area at the condenser can be computed. This area, together with the distance of the condensing lens from the source, determine the solid angle. The region of the source imaged upon the slit determines the projected area of the source eontributing. If this image is magnified or reduced, the magnification factor must be taken into aceount in computing the effective area of the souree, i.e., the slit area must be divided by the magnification due to the condensing lens.

In determining the radiance $R$ one proceeds as follows: The radiant power $P_{s}$, passing through the first slit is divided by the solid angle (determined by the projected area of the limiting aperture on the condensing lens and the distance of the condensing lens from the source). One thus obtains the radiant intensity. This in turn is divided by the projected area of the effective source (slit area $A_{s} /$ magnification $M_{c}$ of the condenser). Thus

$$
R=\frac{P_{s} M_{c}}{\omega_{s} A_{s}}
$$

The first method outlined may be preferable provided sufficient energy is available for measurement. The second method must, however, be resorted to for weak sources. In practice, often a combination of the two is used, the absolute values for strong lines being obtained by the first method and the values for the weaker lines interpolated by the seeond method. This procedure was followed by Dr. McAlister in the work illustrated.

The type of undertaking deseribed above is one of the most difficult in optical practice. Such determinations cannot be made unless extensive preparation and a great deal of time are devoted to them. This accounts for the fact that so few absolute-energy data are available coneerning even the most frequently used sources. 
Qualitative data, however, are available for most sources, in most cases photographic spectrograms, where on reduction the intensity is indicated by an arbitrary specification from 1 to 100 . In general, the plate sensitivity is either not indicated or is only roughly determined. For certain restricted types of spectroscopic investigation, photographic methods have been highly developed. These methods present even greater complexities than do the radiometric methods. For the procedure of this method, the reader is referred to the work of Harrison $(36,37)$.

In the methods outlined above, it has been assumed that a black-body detector has been used in conjunction with a monochromator. Since such black-body detectors of adequate sensitivity require calibration by means of a known source of total radiation, we must be provided with a standard lamp. If a source is available, known as to wave-length distribution of intensity, a somewhat simpler procedure is available, that of differential comparison. The unknown source may be interchanged with the known source, either mechanically or by a suitable optical system which provides an identical method of illumination. Thus, a black body of known temperature may be interchanged with any continuous source of radiation. Then one obtains simply a factor (aperture, transmission, and resolution all canceling out). Or again, an accurately calibrated mercury arc may be interchanged with a mercury are of unknown intensity distribution. If, however, a selective emitter, such as a mercury are, be compared with a continuous source, such as a black body, care must be taken to take duly into account the fact that the radiation from the continuous source occupies a wave-length range greater than the resolution of the instrument, while the selective emission of a given line occupies a wave-length range less than the resolution of the instrument. Thus, in the case of the selective source, a change of slit-width only changes the area over which radiation is subtended, the second slit merely obstructing extraneous wave-lengths. In the case of the continuous emitter, the second slit restricts the wave-length band which escapes the monochromator. Furthermore, in calculating the extent of this band, one must take into account the intensity distribution according to wave-length of such transmitted radiation, integration being performed over the wave-length range. For the substitution method, provided that one is using similar types of sources and that the slit width is such that no appreciable change in sensitivity takes place over the range of wave-lengths involved, selective detectors may be used instead of a black body. It is necessary, however, either to assure oneself that the response is linearly proportional to the power received, or if nonlinear, that the detector be calibrated for power as a function of deflection. 
DETERMINATION OF ABSORPTION CHARACTERISTICS OF TRANSPARENT OR PARTIALLY TRANSPARENT MATERIALS

This second general type of problem presents far less difficulty. The material the charaeteristies of which are to be determined may be introduced either before or after the monochromator, depending upon the characteristics of the sample. If the material presents optical parallel surfaces, and produces no appreciable seattering of the radiation, it may be introduced either in a parallel beam or in a somewhat convergent beam, provided compensating focal adjustment is made. The former method is preferred. In this ease, one may use a compound condensing lens, such as $C_{1}$ and $C_{2}$ in Fig. 6, made up of two parts separated by a sufficient distance that the material may be placed between the two components, being illuminated in this way by parallel light and so producing no appreciable change in the foeal length of the condensing lens. (A slight change is actually produced because of the effective ehange in separation of the components. However, in general this may be neglected.) If the material whose transmission characteristies are to be determined cannot be secured in sufficient size as not to restriet the aperture of the condensing lens, it may be necessary to place the sample elose to the slit, making compensating foeal adjustment for focal condition of the condenser system. In any method which places the sample before the slit, care must be taken that the image of the source upon the slit is not displaced, as such displacement may place upon the slit an element of area of different radiance. If one is dealing with material which not only absorbs but seatters, the figure obtained for absorption will represent loss both by absorption and by seattering, as the speetrograph system will effectively exclude most of this scattered radiation. In ease of scattering, if one wishes to obtain a figure for absorption alone, it is necessary to use a detector of very much larger receiver area than the beam incident upon the sample. Ideally, such a detector should be either a hollow inclosure with a sufficiently large opening, or a portion of a spherical surface of sensitive material whose radius of curvature lies approximately at the eenter of the sample. The thickness of the sample should be small compared with the diameter of the beam. The portion of the spherical surface must be large enough to include practically all seattered radiation. Even under these favorable conditions, the apparent transmission will be lowered owing to the greater path traveled by the deviated radiation. The rigorous analysis of such cases is beyond the scope of this discussion. A comprehensive treatment of such eases has been prepared by Mestre (53). Calculations of eharaeteristics for nonscattering solid or liquid material are made according to Tables 5 and 6 , respectively. 
PROVISION FOR MONOCHROMATIC IRRADIATION OF MATERIALS

In this case, the combination, source plus monochromator, becomes the source of monochromatic radiation. The second slit of the monochromator may be treated as a new source. Where selective emitters are used and the resolution of the instrument is such that only a single line passes through the second slit, the wave-length range of major intensity is limited by the inherent line width, which for all practical photochemical or biological work may be neglected, the only impurity being the scattered radiation discussed above. If, however, a continuous source is used, the wave-length range will depend upon the slit adjustment and dispersion of the instrument. With a single monochromator of $10 \mathrm{~cm}$. focal length, a quartz prism of $60-\mathrm{deg}$. angle, and $0.1-\mathrm{mm}$. slit width, the wave-length band will range from roughly $5 \AA$ at $2250 \AA$ to $200 \AA$ at $6500 \AA$. With the double monochromator, wave-lengths will be restricted to one-half this range. This is offered only to give a rough idea of the practical values available. For twice the slit width, there would be four times the wave-length range and correspondingly greater intensity.

The outstanding difficulties presented by such a source are: (a) The size of the source is limited to the size of the slit, namely, from 0.1 to $2.0 \mathrm{~mm}$. in width, and from 1.0 to $5.0 \mathrm{~cm}$. in length, depending, of course, on the size of the instrument and the purity required. (b) The solid angle of available radiation from such a source is again limited by the numerical aperture of the instrument. (c) Practical considerations of expense.

In using such a souree, a secondary black-body detector, such as a thermocouple, may be used to evaluate the power output by direct comparison with the total radiation from a standard lamp. In practice, however, it is desirable to use a secondary control either upon a portion of the beam from or through the spectrograph, or directly upon the radiation from the source. Any type of dependable detector may be used for this purpose. A photocell is often placed near the slit or condensing lens and read at intervals during the exposure in order to evaluate the changes and fluctuations in the source. This enables one to make correction on intensities determined before and after exposure. In such irradiation experiments, it may be desirable to know not only the power output which, of course, enables one to compute radiance and irradiation at the sample, but also the amount of absorbed energy.

Two general methods for evaluating the amount of absorbed energy present themselves: (a) To evaluate by computation from a knowledge of the absorption characteristics of the sample and the irradiation provided. (b) To make black-body determinations of radiation incident 
upon and emergent from the sample. A further precaution is necessary in that if appreciable diminution of intensity takes place throughout the sample, different portions of the sample are then subjected to different intensities of radiation and consequently to different resulting effects, which in most cases depend upon the (time $\times$ intensity) dosage for a given wave-length.

The determination of the absolute sensitivity of a selective detector as a function of wave-length is important in view of the great sensitivity available in such commercial detectors, photocells, for example. Provided with a source of monochromatic radiation of any desired wavelength, the procedure is simple. The intensity of the radiation for each wave-length is determined as has been indicated in this section, and the response of the detector measured. If such a detector, however, presents an extended area, the sensitivity should be determined for each portion of the area, since marked differences may exist for different parts.

\section{FILTERS AND REFLECTORS}

\section{FILTERS}

In a large range of biological experiments, the objections indicated above, due to either the size of the organism or the intensity require-

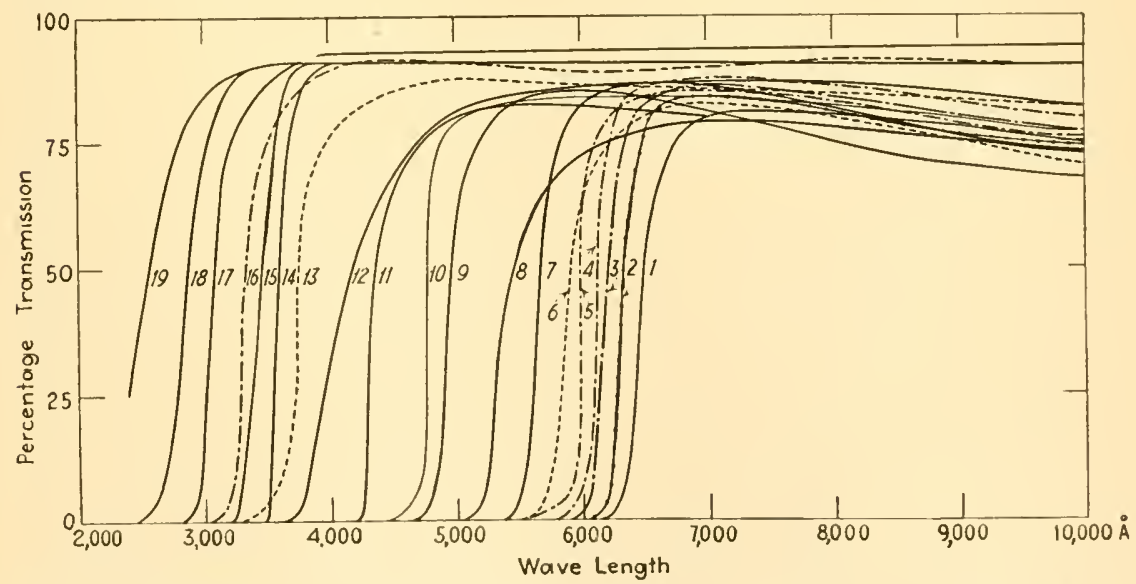

FIg. 9.-Short-wave cut-off filters-glass. (Corning.) See Table 10 for identification.

ments, may be such as to prohibit the use of the monochromator technique. One is then driven to the use of extended sources together with filters. Long discharge tubes or a multiplicity of incandescent lamps may serve to provide such a source. For this purpose, it will be desirable to examine the types of filters available. Very few satisfactory filters can be secured which transmit only a restricted region of spectrum. 


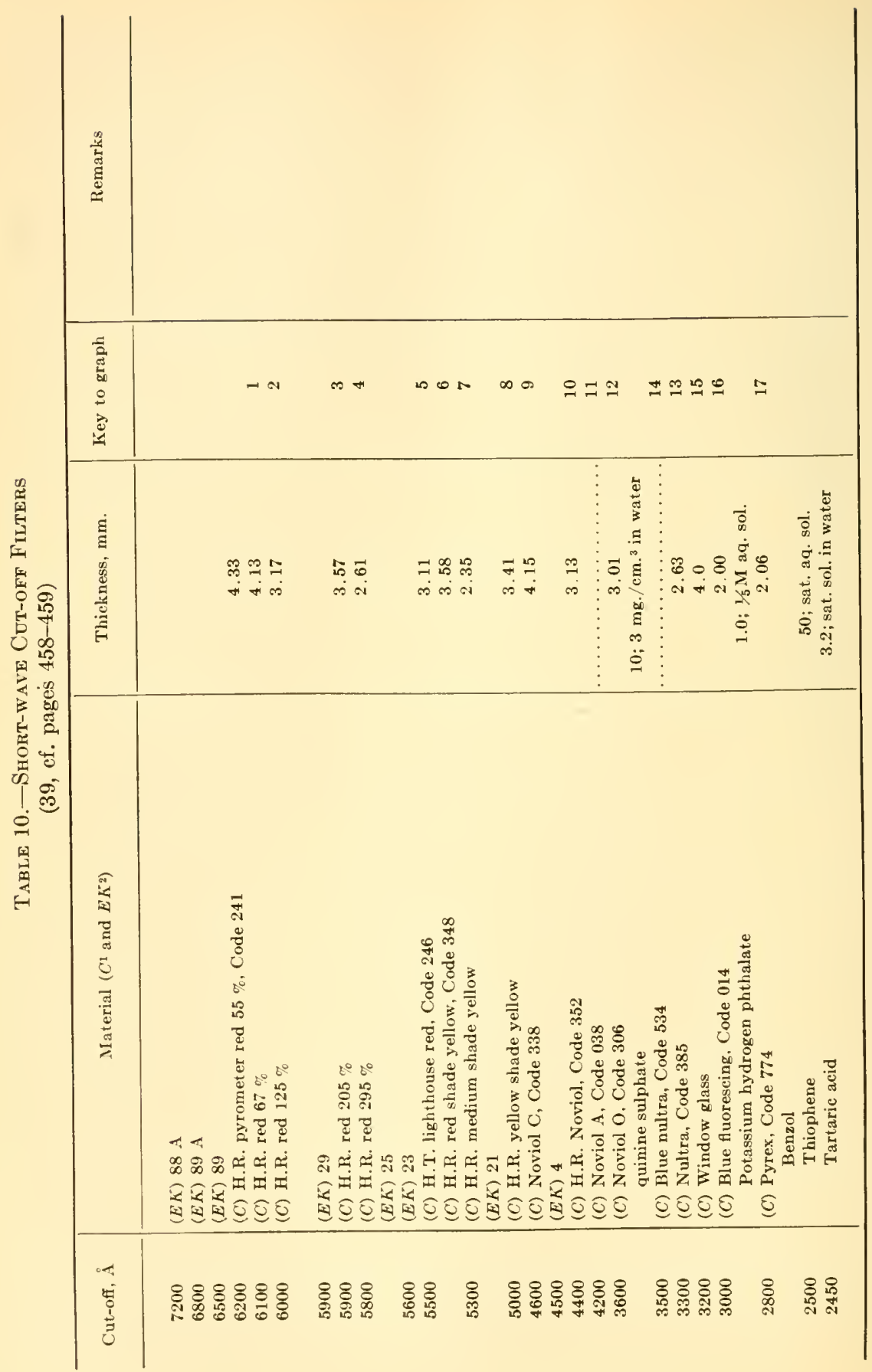




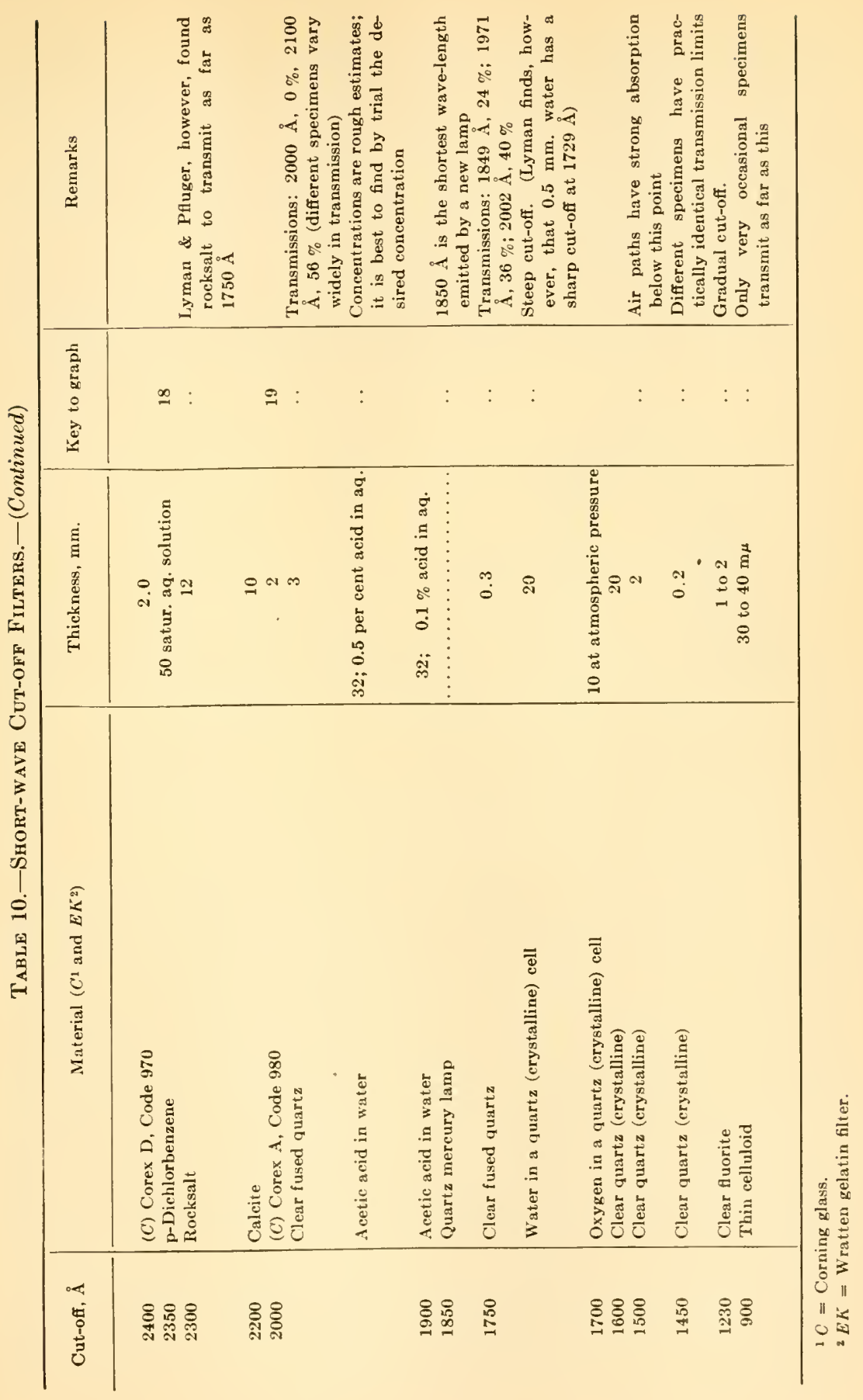


A wide range of filters is available, however, which cut off all shorter wave-lengths and transmit all longer wave-lengths. For convenience, these will be termed short-wave cut-off filters. The transmission curves typical of such filters, varying in cut-off from 6500 to $2200 \AA$ are indicated in Fig. 9. Table 10 (39, cf. pages 458-459) gives specifications of filters, chiefly glass (Corning) and gelatin (Eastman Kodak Company) and approximate short-wave cut-offs from 7200 to $900 \AA$. The cut-off indicated is for practically no transmission.

A quantitative criterion would be valueless because of the unavoidable variability of the material. Liquid filters of similar characteristics are available. For shorter wave-lengths than $2000 \AA$, the absorption due to

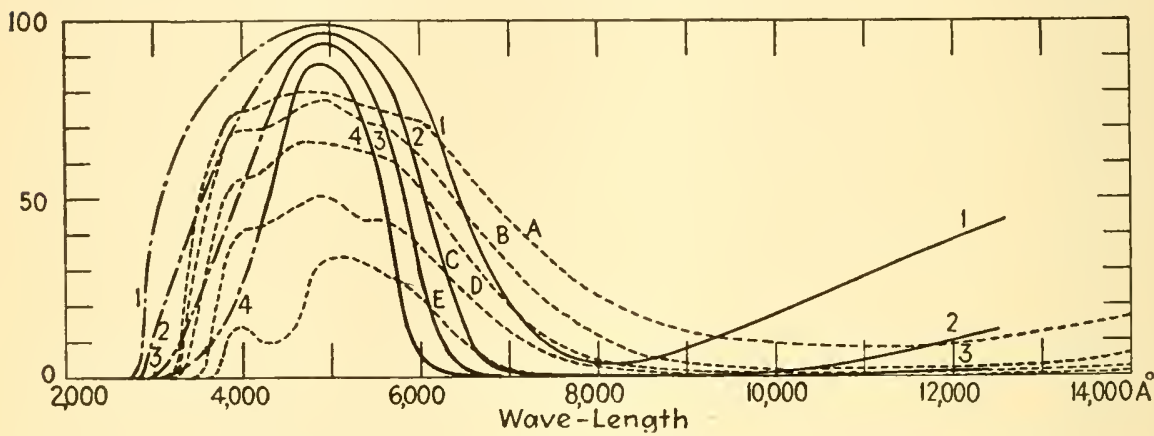

Fig. 10.-Heat-absorbing filters. Full-line curves show copper chloride in $1.0 \mathrm{~cm}$. cell: 1. One-tenth normal. 2. One-fourth normal. 3. One-half normal. 4. Normal. Dotted curves are glass filters. (Corning.)

$A$, Heat resisting, heat absorbing, extra light shade, $2.75 \mathrm{~mm}$. in thickness. $B, I b i d$., light shade, $2.66 \mathrm{~mm}$. C, Ibid., light medium shade, $2.62 \mathrm{~mm}$. $D$, Ibid., dark medium shade, $2.97 \mathrm{~mm}$. $E$, Ibid., dark shade, $2.82 \mathrm{~mm}$.

air must be taken into consideration. Since many photochemical reactions present a long-wave-length threshold, i.e., do not proceed for any longer wave-lengths and do proceed for shorter wave-lengths, short-wave cut-off filters have a wide range of applicability.

It is of ten desirable to exclude infra-red radiation, and for this purpose heat-absorbing filters must be used. Glass filters are available whose transmission characteristics are shown in Figure 10 by dotted curves, $A$ to $E$. For many types of experiments, a liquid filter made of copper chloride or copper sulphate may even better serve the purpose. Characteristics of copper chloride (15) are indicated by curves 1 to 4 . Difficulties are encountered in using copper sulphate because of its low solubility.

For many types of experiments, a water filter may suffice. In order to enable one to make a selection of suitable thickness, Fig. 11 has been prepared, indicating the percentage transmission and also the energy transmitted per second per centimeter wave-length from a square centi- 
meter area of a tungsten filament lamp, at temperature $2755^{\circ} \mathrm{K}$. This is roughly equal to the total energy emitted by a 100 -watt lamp if transmitted through the indicated thicknesses of water, since the area of the filament is about $1 \mathrm{~cm} .^{2}$

Typical band-pass filters, which transmit a more or less limited wavelength range are shown in Fig. 12. Since in practice, one often wishes to use a combination of seleetive emitter with "monochromatic filter," Table 11, A, B, and $\mathrm{c}$, has been prepared, showing some of the possi-

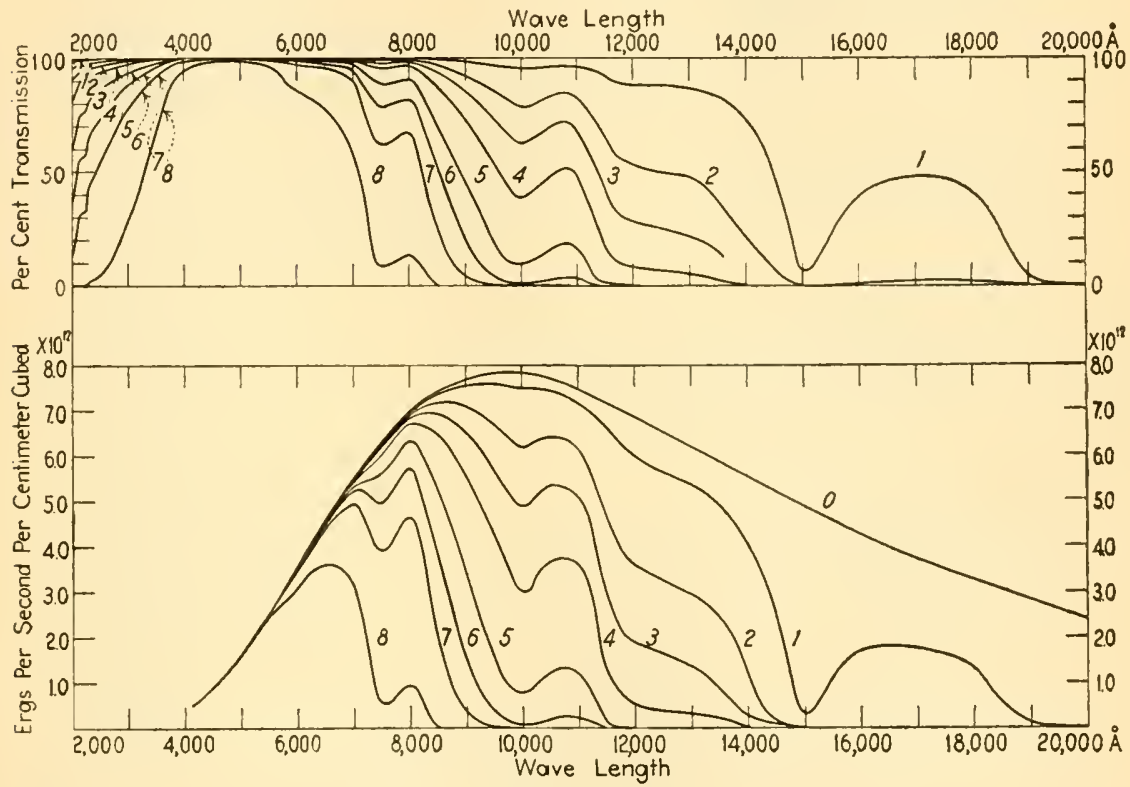

FIG. 11.-Upper-transmission of water of cell thicknesses: $1.0 .1 \mathrm{~cm}, 2.0 .5 \mathrm{~cm}$. 3. $1.0 \mathrm{~cm}$. 4. $2.0 \mathrm{~cm}$. 5. $5.0 \mathrm{~cm}$. 6. $10.0 \mathrm{~cm}$. 7. $20.0 \mathrm{~cm}$. $8.100 .0 \mathrm{~cm}$. Lowertransmitted radiation in ergs sec. $/ \mathrm{cm}^{3}$ from tungsten lamp at approximately $2755^{\circ} \mathrm{K}$., for corresponding cell thicknesses. (0. direct.)

bilities of such combinations. With a 250 -volt uviare, operated on 143.5 volts and $4.5 \mathrm{amp}$. at $25 \mathrm{~cm}$., one obtains from a 2 -cm. midsection the irradiation values indieated (50). By means of this table, it is possible to compute for a given distance and length of arc, intensities available for sueh a combination. Since the radiance is fairly uniform over the length of the tube, one may simply multiply by the relative length exposed. Other wave-lengths of monoehromatie radiation may conveniently be obtained from helium and hydrogen. Since, however, such sources are not subjeet to any degree of standardization, it is possible to give only order-of-magnitude ideas as to intensities. At different operating conditions of voltage and current, line intensities vary in both absolute and relative magnitude. Intensities indieated must not be used as the 
basis for quantitative experimentation. They are intended only as a convenience to aid in the selection of suitable equipment. A convenient group of liquid and glass filters is offered by Wood (68). Note especially the long-wave cut-off filter No. 2, Fig. 13.

A third type of filter, the Christiansen filter, has been subject to a limited use. It consists of a finely divided transparent solid, immersed in

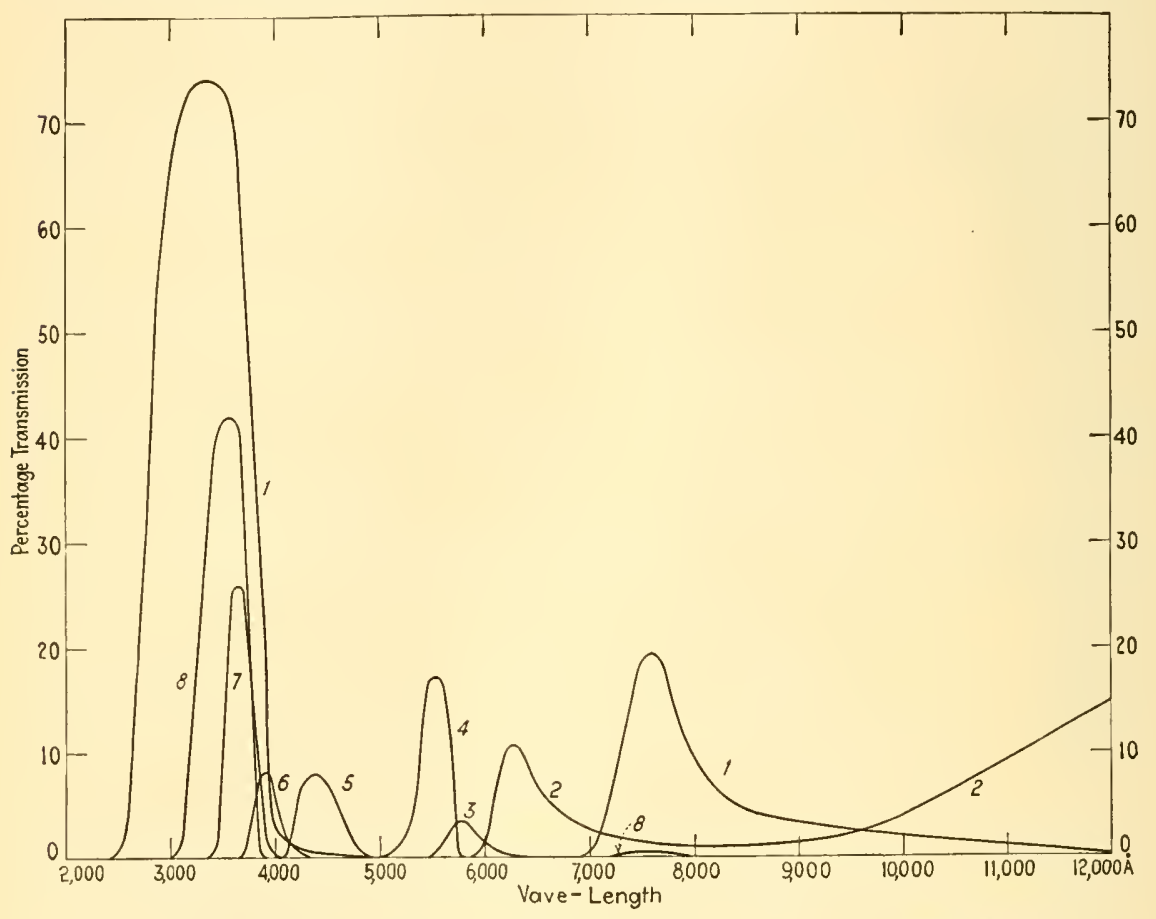

FIG. 12.-Monochromatic filters: 1. Red purple ultra-violet transmitting, $5.89 \mathrm{~mm}$. (thickness). 2. Heat resisting red $247 \%, 3.49 \mathrm{~mm}$. Light shade blue green, $3.11 \mathrm{~mm}$., Code 428. 3. Heat resisting red shade yellow, $3.38 \mathrm{~mm}$., Code 348 . Dark shade blue green, $3.70 \mathrm{~mm}$., Code 430 . 4. Heat resisting yellow shade yellow, $2.66 \mathrm{~mm}$., Code 351 . Didymium, $5.05 \mathrm{~mm}$., Code 512 . Dark shade blue green, $3.59 \mathrm{~mm}$., Code 430 . 5. Noviol A, $3.06 \mathrm{~mm}$., Code 038. Violet, $3.25 \mathrm{~mm}$., Code 511 . 6. Noviol O, $2.15 \mathrm{~mm}$., Code 306 . Red purple ultra, $6.00 \mathrm{~mm}$., Code 597. Light shade blue green, $3.98 \mathrm{~mm}$., Code 428. 7. Light blue nultra, $1.50 \mathrm{~mm}$., Code 534 . Red ultra, $4.04 \mathrm{~mm}$., Code 584 . Light shade blue green, $3.91 \mathrm{~mm}$., Code 428. 8. Red ultra, $8.87 \mathrm{~mm}$., Code 584.

a liquid whose dispersion is much greater, but whose index of refraction coincides with that of the solid at some wave-length. That particular wave-length will be transmitted without deviation if the cell is made with parallel windows. All other wave-lengths will be scattered. If such a filter is placed in an optical system, with a limited source, preferably circular, and a restricting diaphragm placed at the image of the source, most of the scattered light will be lost and a fairly monochromatic beam 


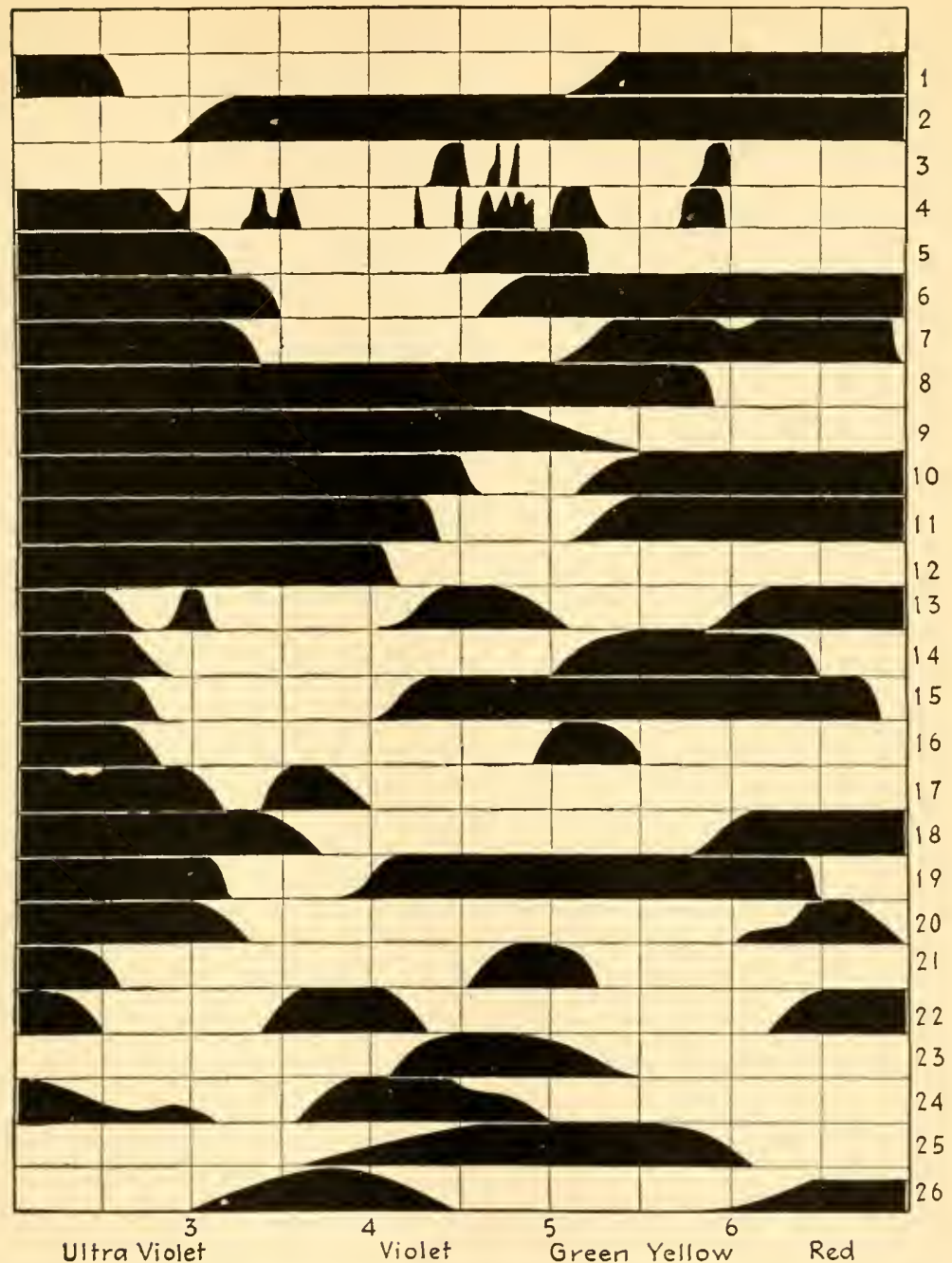

FIG. 13.-Filters recommended by R. W. Wood. (Wood, 68.) 1. Cobalt chloride in alcohol. 2. Potassium film (see his paper on Optical Properties of Metals). 3. Praseodymium chloride. 4. Neodymium chloride. 5. Uranine (Na salt of fluorescein). 6. Cuprammonium. 7. Cobalt glass. 8. Corning signal red glass. 9. Corning G34 glass. 10. Corning sextant green. 11. Signal green glass. 12. Sodium nitrate (saturated solution $2 \mathrm{~cm}$.). 13. Corning glass 984B. 14. 985B. 15. G986A. 16. Cyanosine. 17. Potassium chromate. 18. Copper nitrate. 19. Corning glass G586J. 20. Cobalt chloride in acetone. 21. Cobalt sulphate in water. 22. Nickel chloride. 23. Iodine in $\mathrm{CCl}_{4}$. 24. Nitrosodimethylaniline in water. 25. Bromine vapor. 26. Chlorine. 


\begin{tabular}{|c|c|c|c|c|c|c|c|c|c|c|c|c|c|c|c|}
\hline 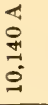 & 00 & $\ddot{-i n}$ & 00 & $\begin{array}{l}00 \\
0.0 \\
\infty ழ \\
\infty\end{array}$ & 00 & 00 & $\ddot{\infty}$ & 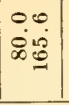 & $\ddot{0}$ & 00 & $\begin{array}{l}\infty \infty \\
\dot{8}: \vec{J}\end{array}$ & 00 & 00 & 00 & 00 \\
\hline \begin{tabular}{l}
$\infty$ \\
$\stackrel{0}{\circ}$ \\
\hdashline \\
0 \\
\end{tabular} & $\dot{8}$ & $\dot{\infty} 0$ & 00 & $\begin{array}{l}00 \\
\infty 0^{\circ}\end{array}$ & 00 & 00 & 00 & $\left|\begin{array}{ll}0 & 0 \\
\dot{\infty} & 0 \\
\infty & 0\end{array}\right|$ & 90 & 00 & 00 & 00 & 00 & 00 & $\infty$ \\
\hline $\begin{array}{l}\text { तै } \\
\text { ஸे } \\
0 \\
0\end{array}$ & $0^{\infty}$ & $\begin{array}{l}0 \infty \\
8 \times\end{array}$ & $\mid \begin{array}{l}\infty \\
00 \\
00\end{array}$ & $\begin{array}{l}0-1 \\
\dot{\infty} \\
\dot{\infty}\end{array}$ & $\begin{array}{l}0 \overrightarrow{0} \\
00\end{array}$ & $\begin{array}{l}n \mathrm{~N} \\
\mathrm{nO}\end{array}$ & $\infty$ & $\left|\begin{array}{l}0 r \\
0-1 \\
0\end{array}\right|$ & 00 & 00 & 00 & $8^{\circ}$ & 00 & 00 & 00 \\
\hline $\begin{array}{l}8 \\
\infty \\
15 \\
0\end{array}$ & 00 & 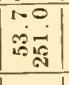 & $\mid \begin{array}{l}\infty \pi \\
\therefore 0\end{array}$ & $\left|\begin{array}{ll}0 & 0 \\
0 & 0 \\
0 & 5\end{array}\right|$ & 00 & oे & $\infty$ & 00 & 00 & 00 & 00 & 00 & 00 & 00 & 00 \\
\hline $\begin{array}{l}-1 \\
00 \\
+11 \\
\text { is }\end{array}$ & 00 & 00 & 00 & $\infty$ & $\begin{array}{l}0 \infty \\
\therefore: 0 \\
=0\end{array}$ & $\begin{array}{l}0 \infty \\
\infty \neq i \\
\infty \\
+\infty \\
-\infty\end{array}$ & $\left|\begin{array}{c}0 \\
150 \\
0\end{array}\right|$ & $\left|\begin{array}{l}0 \\
0 \\
0 \infty \\
0 \\
10 \\
0\end{array}\right|$ & 00 & 00 & 00 & 00 & 00 & 00 & 00 \\
\hline $\begin{array}{l}\stackrel{0}{\sigma} \\
\stackrel{+}{*}\end{array}$ & 00 & 100 & 100 & 00 & 00 & 00 & 00 & ${ }^{\circ}$ & 00. & 00 & 100 & 00 & 00 & 100 & $\infty 0$ \\
\hline 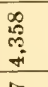 & 00 & 00 & 00 & 00 & 00 & 00 & 00 & 00 & $\begin{array}{l}+\infty \\
\text { जis } \\
\text { Nis }\end{array}$ & 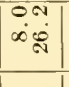 & {$\left[\begin{array}{ll}0 & 0 \\
0 & 0\end{array}\right]$} & 00 & 00 & 00 & 00 \\
\hline 范 & 00 & 00 & 00 & $\infty$ & $\infty 0$ & 00 & 00 & ${ }^{\circ}$ & 00 & 00 & $\begin{array}{l}+\infty \\
\infty \\
\infty\end{array}$ & $\begin{array}{l}+\infty \\
+\infty \\
+\infty\end{array}$ & $\begin{array}{ll}0 \\
100\end{array}$ & 00 & 100 \\
\hline 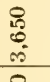 & 00 & 00 & 00 & 00 & 00 & 00 & 00 & 00 & 00 & 00 & 00 & 00 & $\left|\begin{array}{cc}0 & 0 \\
-1 & 0 \\
7 & N\end{array}\right|$ & 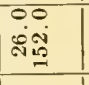 & 00 \\
\hline 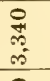 & $\infty$ & 00 & 00 & $\infty$ & 00 & 00 & 00 & 00 & 00 & 00 & 00 & 00 & $\begin{array}{ll}0 & 0 \\
0 & 0 \\
8 & -\end{array}$ & 00 & \begin{tabular}{|l}
$\frac{y}{a}$ \\
0 \\
$\vdots$ \\
100
\end{tabular} \\
\hline 品 & 00 & 00 & 00 & 00 & 00 & 00 & 00 & 00 & 00 & 00 & 00 & 00 & $\infty$ & 00 & $\dot{0}$ \\
\hline & 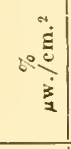 & ¿ُ & $\underbrace{\stackrel{8}{8}}$ & 暍 & $\frac{\stackrel{0}{\rightleftarrows}}{3}$ & $e^{\circ}$ & 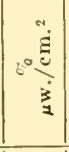 & 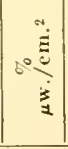 & 谙 & 䜦 & 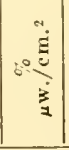 & $\frac{3}{3}$ & 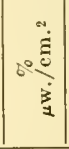 & ชำ & 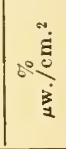 \\
\hline 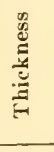 & 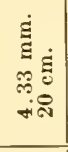 & 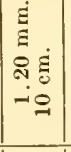 & 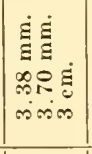 & & 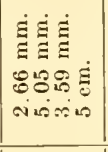 & 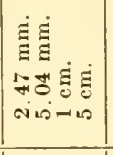 & & & 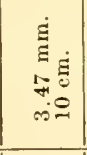 & 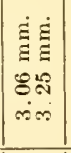 & & 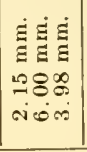 & $\begin{array}{l}\dot{\Xi} \\
\Xi \\
\infty \\
\infty \\
\infty\end{array}$ & 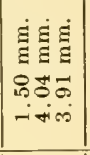 & \\
\hline 政赵 & & & $\infty$ & & $\pi$ & & & & & م & & 0 & $\infty$ & $r$ & 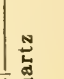 \\
\hline 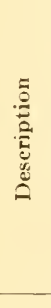 & 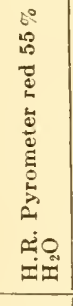 & 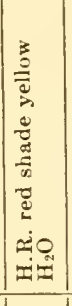 & 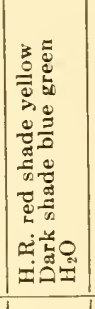 & 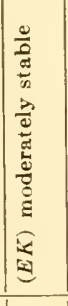 & 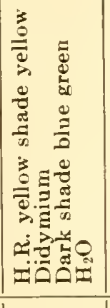 & 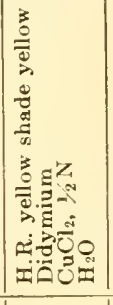 & 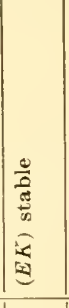 & 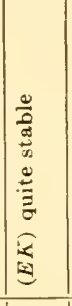 & 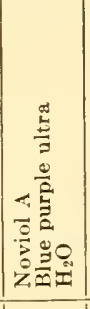 & 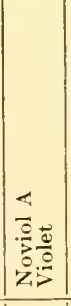 & 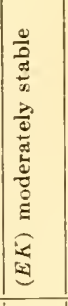 & 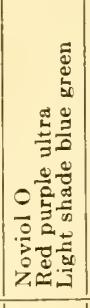 & | & 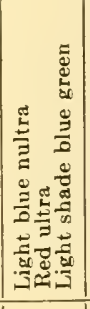 & 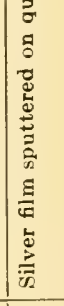 \\
\hline $\begin{array}{l}\dot{0} \\
z \\
\dot{0} \\
\dot{0}\end{array}$ & & 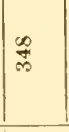 & 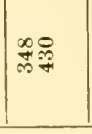 & ลี & 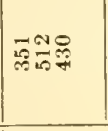 & 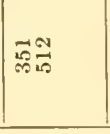 & ชิ & $\leqq$ & 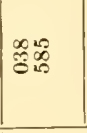 & $\frac{\infty}{8 \pi}$ & is & 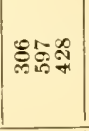 & $\vec{\infty}$ & 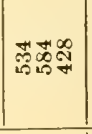 & \\
\hline or. & $\begin{array}{l}0 \\
\delta \\
\dot{0}\end{array}$ & $\begin{array}{l}0 \\
0 \\
1 \\
i\end{array}$ & & & $\begin{array}{l}\overrightarrow{0} \\
\text { is }\end{array}$ & & & & 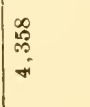 & & & $\begin{array}{l}\text { ta } \\
\dot{j} \\
\dot{j}\end{array}$ & $\begin{array}{l}8 \\
0 \\
0 \\
\infty\end{array}$ & & $\frac{\stackrel{9}{-}}{\infty}$ \\
\hline
\end{tabular}


TABLE $11 \mathrm{~B}$

\begin{tabular}{|c|c|c|c|c|c|c|c|c|}
\hline$\AA$ & $\begin{array}{c}\text { Code } \\
\text { No. }\end{array}$ & Description & Thickness & & 3,889 & 5,875 & 6,678 & $10,829 \AA$ \\
\hline 10,829 & 254 & $\begin{array}{l}\text { Heat transmitting } \\
\mathrm{H}_{2} \mathrm{O}\end{array}$ & $\begin{array}{l}3.5 \mathrm{~mm} . \\
2 \mathrm{~cm} .\end{array}$ & $\begin{array}{c}\% \\
\mu \mathrm{w} \cdot / \mathrm{cm} .{ }^{2}\end{array}$ & $\begin{array}{l}0 \\
0\end{array}$ & $\begin{array}{l}0 \\
0\end{array}$ & $\begin{array}{l}0 \\
0\end{array}$ & $\begin{array}{l}26.0 \\
26.7\end{array}$ \\
\hline 6,678 & & $\begin{array}{l}\text { H. R. red, } 67 \% \\
\mathrm{H}_{2} \mathrm{O}\end{array}$ & $\begin{array}{l}4.13 \mathrm{~mm} . \\
20 \mathrm{~cm} .\end{array}$ & $\begin{array}{c}\% \\
\mu w \cdot / c m .2\end{array}$ & $\begin{array}{l}0 \\
0\end{array}$ & $\begin{array}{l}0 \\
0\end{array}$ & $\begin{array}{r}68.0 \\
7.2\end{array}$ & $\begin{array}{l}0.1 \\
0.1\end{array}$ \\
\hline 5,875 & 348 & $\begin{array}{l}\mathrm{H} \text {. R. red shade } \\
\text { yellow } \\
\mathrm{H}_{2} \mathrm{O} \\
\mathrm{CuCl}_{2}, 1 / 4 \mathrm{~N}\end{array}$ & $\begin{array}{l}1.35 \mathrm{~mm} \\
10 \mathrm{~cm} \\
1 \mathrm{~cm}\end{array}$ & $\begin{array}{c}\% \\
\mu w \cdot / \mathrm{cm}^{2}\end{array}$ & $\begin{array}{l}0 \\
0\end{array}$ & $\begin{array}{l}42.0 \\
11.5\end{array}$ & $\begin{array}{l}5.4 \\
0.5\end{array}$ & $\begin{array}{l}0.1 \\
0.1\end{array}$ \\
\hline 3,889 & $\begin{array}{l}597 \\
512\end{array}$ & $\begin{array}{l}\text { Red purple ultra } \\
\text { Didymium } \\
\mathrm{H}_{2} \mathrm{O}\end{array}$ & $\mid \begin{array}{l}3.76 \mathrm{~mm} . \\
4.88 \mathrm{~mm} . \\
20 \mathrm{~cm}\end{array}$ & $\begin{array}{c}\% \\
\mu \mathrm{w} \cdot / \mathrm{cm} .{ }^{2}\end{array}$ & $\begin{array}{r}45.0 \\
2.8\end{array}$ & $\begin{array}{l}0 \\
0\end{array}$ & $\begin{array}{l}0.4 \\
0.04\end{array}$ & $\begin{array}{l}0 \\
0\end{array}$ \\
\hline
\end{tabular}

TABLE $11 \mathrm{C}$

\begin{tabular}{|c|c|c|c|c|c|c|c|c|}
\hline$\AA$ & $\begin{array}{l}\text { Code } \\
\text { No. }\end{array}$ & Description & Thickness & & 4,101 & 4,341 & 4,862 & $6,563 \AA$ \\
\hline 6,563 & & $\begin{array}{l}\text { H. R. red, } 93 \% \\
\mathrm{H}_{2} \mathrm{O}\end{array}$ & $\begin{array}{l}4.89 \mathrm{~mm} . \\
10 \mathrm{~cm} .\end{array}$ & $\begin{array}{c}\% \\
\mu \mathrm{w} . / \mathrm{cm} .\end{array}$ & $\begin{array}{l}0 \\
0\end{array}$ & $\begin{array}{l}0 \\
0\end{array}$ & $\begin{array}{l}0 \\
0\end{array}$ & $\begin{array}{l}70.0 \\
70.0\end{array}$ \\
\hline \multirow[t]{2}{*}{4,862} & $\begin{array}{l}338 \\
440\end{array}$ & $\begin{array}{l}\text { Noyiol C } \\
\text { Signal green } \\
\mathrm{H}_{2} \mathrm{O}\end{array}$ & $10 \mathrm{~cm}$. & $\begin{array}{c}\% \\
\mu \mathrm{w} . / \mathrm{cm} .^{2}\end{array}$ & $\begin{array}{l}0 \\
0\end{array}$ & $\begin{array}{l}0 \\
0\end{array}$ & $\begin{array}{r}32.0 \\
8.0\end{array}$ & $\begin{array}{l}0 \\
0\end{array}$ \\
\hline & $75 n$ & $\begin{array}{l}(E K) \text { moderately } \\
\text { stable }\end{array}$ & & $\begin{array}{c}\% \\
\mu \mathrm{w} . / \mathrm{cm} .{ }^{2}\end{array}$ & $\begin{array}{l}0 \\
0\end{array}$ & $\begin{array}{l}0 \\
0\end{array}$ & $\begin{array}{r}17.0 \\
4.3\end{array}$ & $\begin{array}{l}0 \\
0\end{array}$ \\
\hline 4,341 & $\begin{array}{l}038 \\
585\end{array}$ & $\begin{array}{l}\text { Noviol A } \\
\text { Blue purple ultra } \\
\mathrm{H}_{2} \mathrm{O}\end{array}$ & $\begin{array}{l}3.47 \mathrm{~mm} . \\
10 \mathrm{~cm} .\end{array}$ & $\begin{array}{c}\% \\
\mu \mathrm{w} \cdot / \mathrm{cm} .{ }^{2}\end{array}$ & $\begin{array}{l}0 \\
0\end{array}$ & $\begin{array}{r}23.0 \\
1.8\end{array}$ & $\begin{array}{l}1.3 \\
0.33\end{array}$ & $\begin{array}{l}0.2 \\
0.2\end{array}$ \\
\hline
\end{tabular}

obtained through the diaphragm aperture. If great purity is to be secured, source and final aperture must be small. Such an optical system must be just as precise as that of a spectrograph, and of the same general characteristics, the Christiansen filter taking the place of the prism. It will be seen, however, that source and emergent aperture must be limited in both directions, while in a spectrograph the slit need only be narrow. Consequently, the Christiansen filter set-up is in no sense superior to a monochromator. It has the following objections: (a) It is critically dependent upon the temperature-sensitive dispersion curve of the liquid. In most cases, the wave-length of coincidence of indices varies some 
$100 \AA$ per degree, and consequently the cell must be subjected to accurate thermostatic control. (b) It has the same objections as the monochromator in that it produces only a limited area and solid angle of effective source.

If such a Christiansen filter is used with extended source and without a small restricting aperture, one secures a wave-length distribution fairly

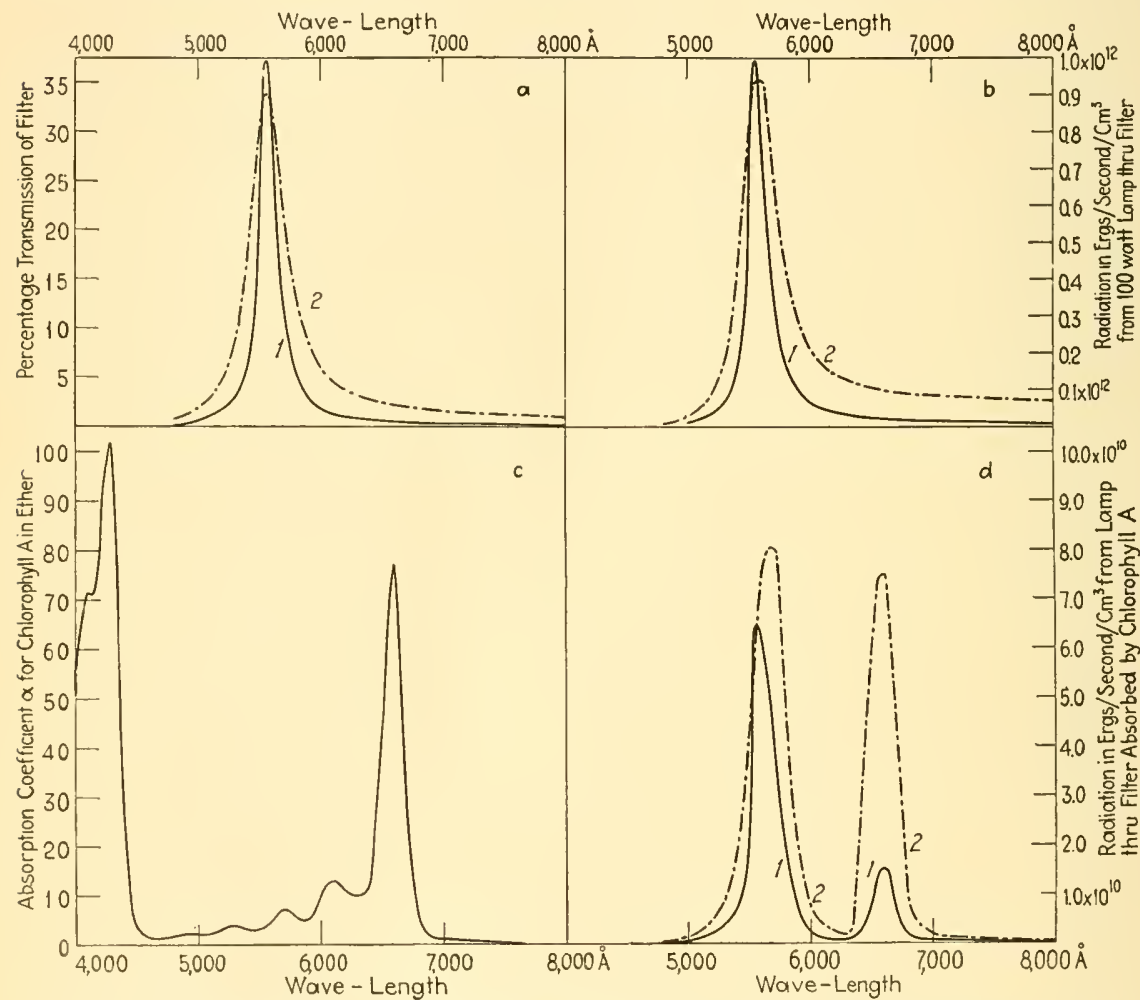

F1G. 14.-Effectiveness of Christiansen filter: Upper left-transmission of filter: 1. small area illumination; 2. large area illumination. Upper right-radiation transmitted by filters. Lower left-absorption coefficient $\alpha$ base 10 of chlorophyll $A$ in ether. Lower right-radiation absorbed by chlorophyll A solution from transmitted beam, showing maxima both in region of maximum transmission and also in region of maximum absorption. Note wave-length shift with increased area of illumination.

narrow at one-half intensity, but spreading out rapidly at low intensities. This is more aggravated on the long wave-length side. In favor of the Christiansen filter, wave-length bands may be secured anywhere in the visible or near ultra-violet. Wave-length change can be made over a limited range by change in temperature, and over a wider range by varying the liquid. For work in the visible, ordinary optical crown glass, index of refraction 1.52 to 1.54 , serves satisfactorily as the transparent 
solid, and mixtures in various proportions of ethyl alcohol, index 1.36 to 1.37 , benzene, 1.49 to 1.51 , and carbon disulfide, 1.62 to 1.635 , for the liquid, which enables one to obtain any desired wave-length of coincidence. In the ultra-violet, quartz is used instead of glass. Particles may be selected of fairly uniform size anywhere from 1 to $3 \mathrm{~mm}$. in diameter. The coarser the particles the thicker the cell which is required. For 2-mm. particles, the thickness would be from 3 to $4 \mathrm{~cm}$., depending upon the spectral purity required. For further details, the reader is referred to a recent paper (45).

Wherever filter-source combinations are used, producing a spread of intensities over a wave-length range, it must be borne in mind that three things must be taken into consideration in arriving at an interpretation of observed effects: (a) The intensity distribution of wavelength from the source. (b) The transmission characteristics of the filter. (c) The absorption characteristics of the material irradiated. The range of wave-lengths actually effective may appear surprisingly different from those which one would off-hand expect from the filter characteristics.

Consider an example where one is dealing with a photochemical reaction arising from chlorophyll in ether. Thus, in Fig. 14a (64) we have the transmission characteristics of two Christiansen filters, $(a)$ where a cell of $4 \mathrm{~cm}$. thickness is placed in a critical optical system with small restricting apertures (full line), and (b) (dash-dot) where a cell of the same thickness is placed in an optical system composed of a motion-picture projection-lamp filament and a mirror producing a magnification of the source to an area of $4 \mathrm{~cm}$. wide by $8 \mathrm{~cm}$. high. Figure $14 \mathrm{~b}$ shows the radiation transmitted by these filters from a 100 -watt lamp, assuming all the radiation utilized. (To compute the radiant power per centimeter wave-length actually available one would have to multiply by the fraction of the entire solid angle subtended by the filter. If a lamp of larger filament area, but the same temperature, $2755^{\circ} \mathrm{K}$, were used, this figure should be multiplied by the filament area. To compute total power, one must integrate over the wave-length range, taking wavelength in centimeters.) Figure $14 c(70)$ shows the absorption coefficient (base 10) or specific transmissive index of chlorophyll $A$ in ether. Figure $14 d$ shows the energy absorbed by chlorophyll $A$ under these conditions (if such a thickness of cell and concentration is used as to absorb 75 per cent at the red maximum). Curve $1(d)$ indicating the radiation absorbed where a precise optical system is used, presents two maxima, one in the region of greatest illumination, and the other occurring in the region of greatest absorption. Curve $2(d)$ shows the radiation absorbed where the filter has been used to produce an extended area of illumination. (As before, the figures are for the whole solid angle.) Here the absorption occurring near the region of principal illumination has been shifted 
markedly to longer wave-lengths, and a second maximum appears of almost coordinate magnitude in the region of maximum absorption. Of course, a similar difficulty will be encountered wherever filters produce a relatively impure spectrum. In a case where the absorption coefficient of the material irradiated is known, an interpretation may be arrived at, but in cases where one is irradiating a biological material of unknown absorption coefficient, the difficulties of interpretation become aggravated.

\section{REFLECTORS}

In the course of the construction of equipment, it is often desirable to select suitable materials for reflecting radiation. As a convenience,

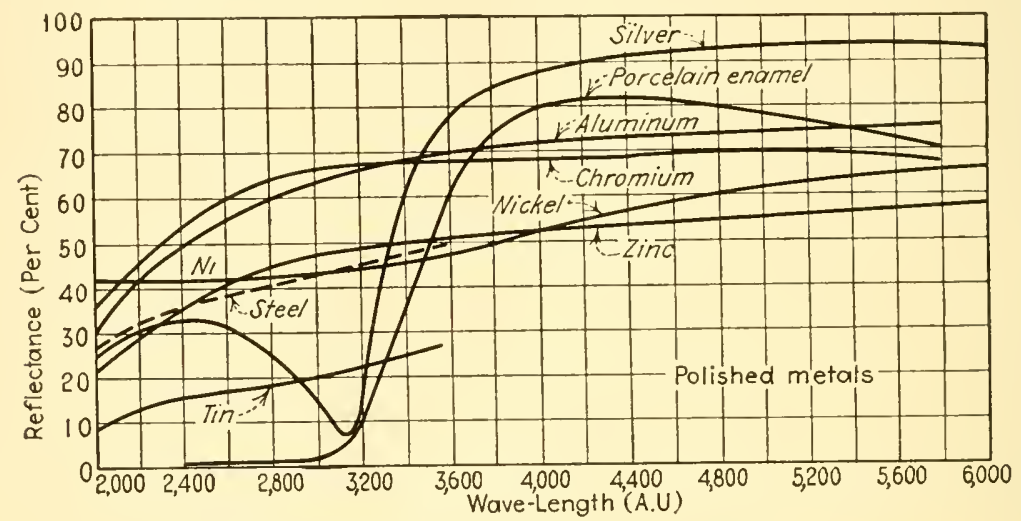

FiG. 15.-Reflectance of polished metals. (Luckiesch, 47.)

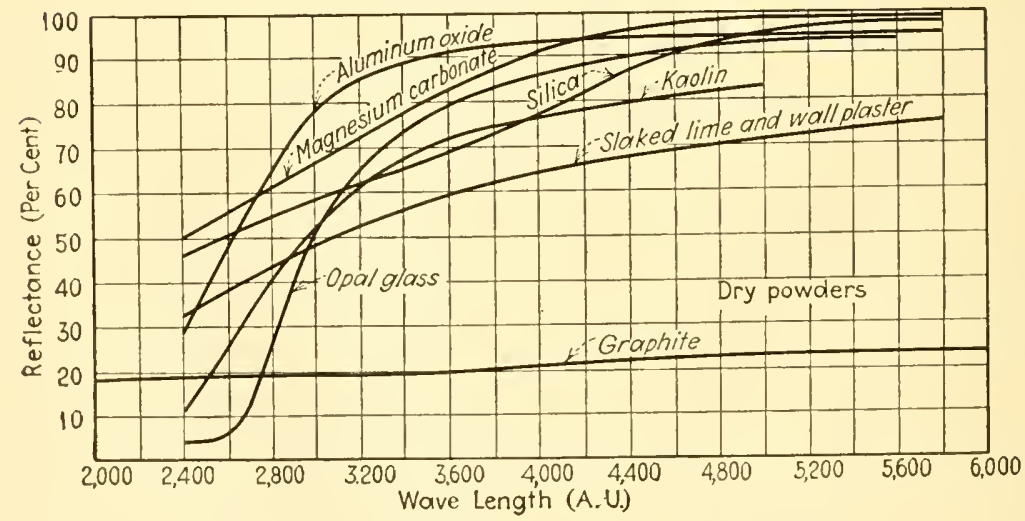

FIg. 16.-Reflectance of dry powders. (Luckiesch, 47.)

therefore, we are including curves of reflectance, the monochromatic determination of reflectance for wave-lengths varying from 2000 to $6000 \AA$. Where one wishes to obtain specular reflection, Fig. 15 (47) shows the types of metals available for construction of reflectors. More 
recently, aluminum mirrors have been developed commercially which exhibit a much higher reflectance over the entire range (better than 80 per cent in the ultra-violet, and exceeding slightly tarnished silver throughout the visible). Quantitative data, however, are not yet available.

If a diffusing surface is desired, powders may be applied to a tacky varnish surface (Fig. 16, shows the refleetance of the various materials).

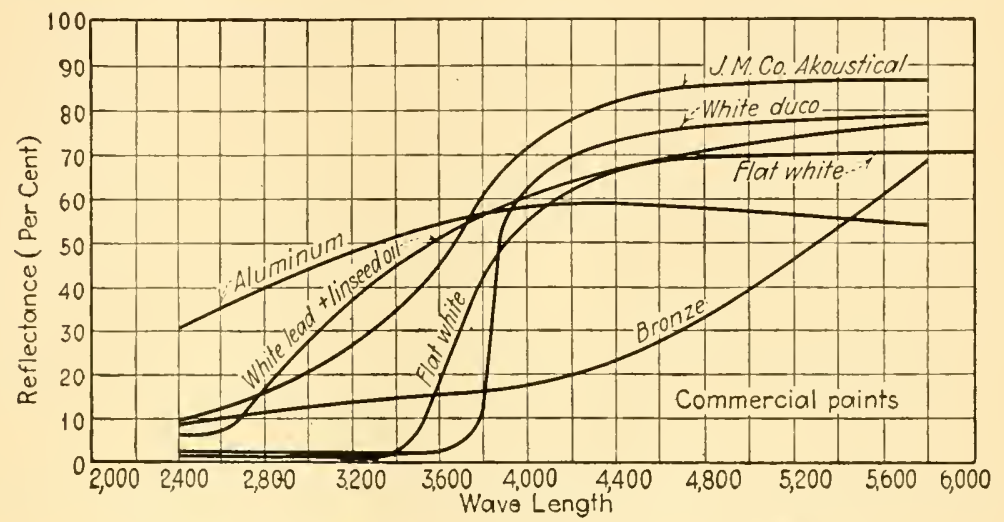

FIG, 17.-Reflectance of commercial paints. (Luckirsch, 47.)

In ease commercial paints are used, seleetion may be made in aecordance with Fig. 17 (47).

\section{SOURCES OF CONTINUOUS EMISSION}

SUN

Despite the extensive development of artificial sources of radiation, even for experimental purposes the sun must be considered a source of primary importanee, beeause of its high intensity and desirable wavelength distribution. The chief difficulties which one eneounters in using this souree arise from the great variability of the radiation as it reaches the surface of the earth. The causes of this variability are as follows: variation in distance from sun to earth; influence of the earth's atmosphere, affected by altitude or air mass; variability of water vapor and ozone; and the presence of materials due to artificial conditions such as dust and smoke.

During a clear summer day at sea level (Washington, D. C.), the total solar radiation received on a horizontal surface varies according to the dotted curve in Fig. 18, a record from the Oetober, 1924, Monthly Weather Review by Kimball (43, cf. page 478).

A portion of this total radiation is due to scattering from the sky. While the sky radiation attains a maximum at noon, its relative con- 
tribution is greater for lower sun, when the direct radiation travels a greater air path, i.e., through a greater air mass. The contribution of sky radiation is shown by the dash-dot curve. At noon, it amounts to 12.5 per cent of the total, i.e., the direct is seven times the sky radiation. At midmorning or midafternoon it amounts to 20 per cent of the total, or one quarter of the direct, and exceeds the direct near sunrise and

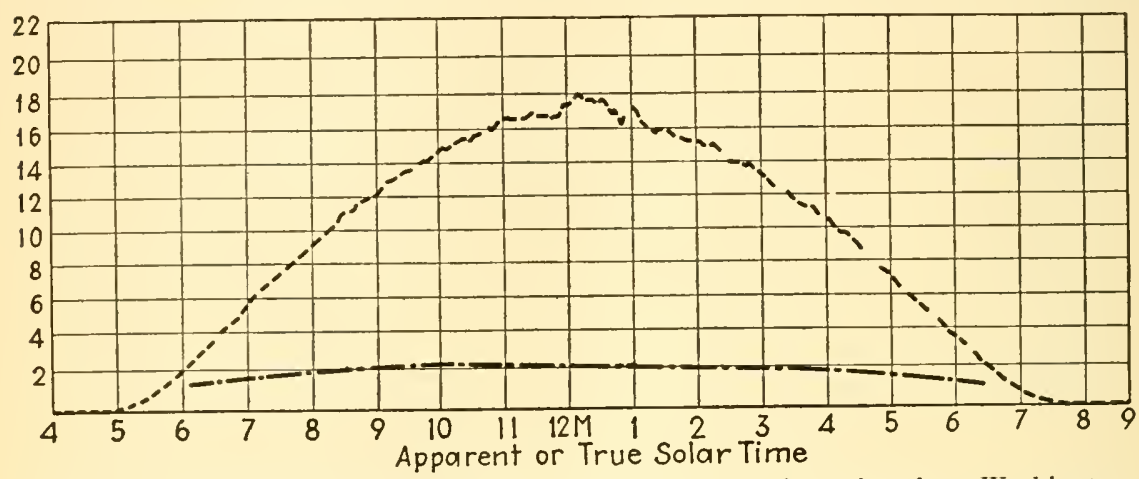

FIG. 18.- Record of total solar radiation received on a horizontal surface, Washington, D. C., on July 11, 1924. (Kimball, 43.) Dotted curve shows total radiation. Dash-dot curve shows sky radiation.

sunset. Representative values from the same source are given in Table 12.

Table 12.-Ratio of Direct Solar Radiation Received on a Horizontal Surface to Diffuse Sky Radiation

\begin{tabular}{|c|c|c|c|c|c|c|c|c|c|c|c|c|c|}
\hline & \multicolumn{13}{|c|}{ Solar zenith distance } \\
\hline & 7.5 & 25 & 30 & 48.3 & 55 & 60 & 66.5 & 70.7 & 73.6 & 75.7 & 77.4 & 78.7 & 85.0 \\
\hline Washington D.C. & & (2) & & 700 & & & ? & & 97 & 248 & & & \\
\hline Winter..... & & $\cdots$ & $\cdots \cdots$ & 1.02 & $\cdots \cdots$ & 5.18 & 4.02 & 3.30 & 2.98 & 2.48 & 2.11 & 1.70 & \\
\hline Spring.... . & & $\ddot{8} 72$ & $\cdots \cdots$ & 7.02 & $\cdots \cdot$ & 5.18 & 4.02 & 3.36 & 2.97 & 2.48 & 2.11 & 1.70 & \\
\hline Summer... & & $\begin{array}{l}8.72 \\
4.25\end{array}$ & $\cdots$ & 6.53 & $\cdots$ & 4.87 & 3.91 & 3.10 & 2.56 & 2.14 & 1.83 & 1.53 & \\
\hline Madison, Wis.... & $\cdots$ & $\begin{array}{l}4.25 \\
5.25\end{array}$ & $\cdots$ & 3.76 & $\cdots$ & 3.18 & 2.72 & 2.25 & 1.96 & 1.71 & 1. 64 & 1.50 & \\
\hline Hump Mountain... & $\ldots$ & $\begin{array}{c}5.25 \\
\ldots .\end{array}$ & $\ldots$ & 5.25 & $\cdots$ & 3.17 & 3.00 & 2.85 & 1.94 & 1.78 & & & \\
\hline Mount Wilson.... & 6.27 & $\cdots$ & $\cdots$ & 11.00 & $\cdots$ & 8.97 & 7.19 & 6.78 & 5.52 & 5.33 & 4.69 & 4.13 & \\
\hline & & 6.10 & 6.00 & 5.08 & 4.13 & 3.90 & 3.23 & 2.70 & $\ldots$ & 2.12 & $\cdots$ & 1.62 & 0.82 \\
\hline
\end{tabular}

The maximum intensity commonly attained at sea level for the latitude of Washington is $1.5 \mathrm{gm}$. cal. $/ \mathrm{min} . / \mathrm{cm} .{ }^{2}$ or $105 \mathrm{mw} . / \mathrm{cm} .{ }^{2}$. For high altitudes, it may be as high as $1.64 \mathrm{gm}$. cal. $/ \mathrm{min} . / \mathrm{cm}^{2}{ }^{2}$ (Mt. Whitney, 4420 meters). Even a light cloud over the sun on an otherwise clear day may, however, reduce the total radiation to one-third of its clear value. 
In order to gain an idea of the influence of dust and smoke, we may turn to the Weather Bureau's records for Chicago (Fig. 19). October 23 was a clear day with the strong wind purging the atmosphere of smoke. On October 22, dense city smoke prevailed until 10:51, when it was swept away by a shift of the wind. October 26 shows the effect of combined smoke and clouds.

From the high intensities attained on clear days in June, the midday maximum falls to a lowest value in Deeember. With the corresponding shortening of the days, the total energy received per day may fall to

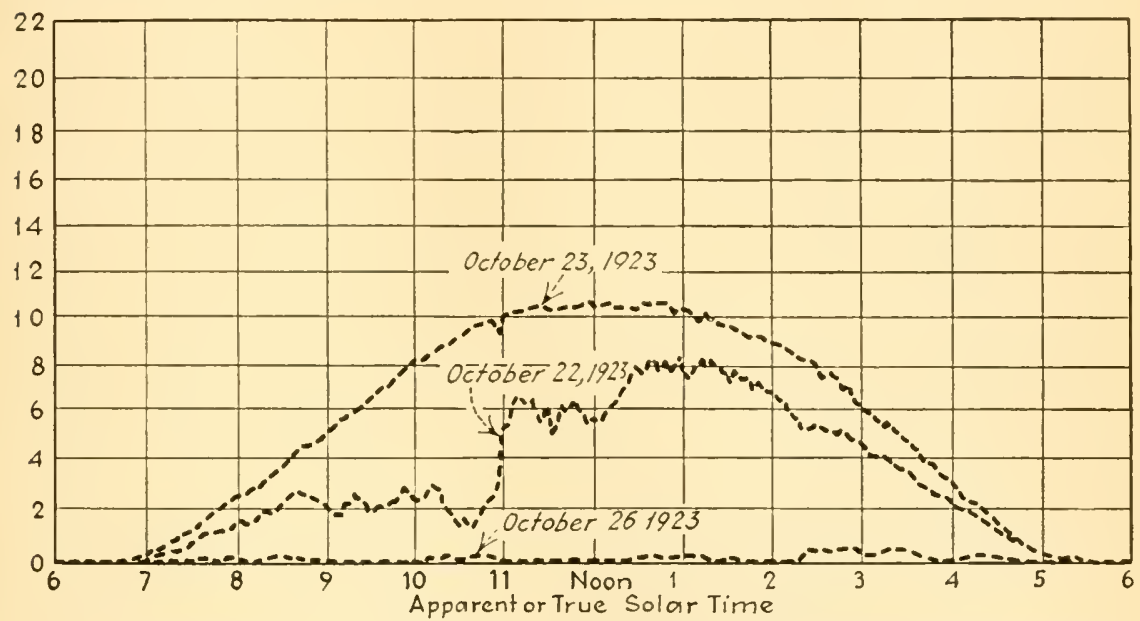

Fig. 19.--Records of total solar radiation received on a horizontal surface at the University Observatory, Chicago, Ill.

one-tenth of its summer value for average sky in the city. [See Kimball (44), also page 224, for a group of typical curves of seasonal fluctuation in total daily energy.] For an average sky in summer, noon intensity commonly reaches $1 \mathrm{gm}$. cal. $/ \mathrm{min} . / \mathrm{cm}^{2}$ at sea level.

In order to form an idea of the spectral distribution of direct solar radiation, Fig. 20 has been plotted, showing the extrapolated value for radiation outside the atmosphere and the effect of successively greater integral air masses. Air mass is defined as unity for the sun at zenith (and approximately equals the secant of the zenith distance for the other positions). Only the outstanding bands of atmospheric absorption are shown. Those in the infra-red are chiefly due to water vapor, the a mount being moderately great, $1.37 \mathrm{~cm}$. preeipitable water. These curves have been prepared from Abbot's data (4) and are intended to be representative of an average day in Washington. Note the marked decrease between 4000 and $5000 \AA$ with increasing air mass, as well as the deepening of the infra-red absorption bands. 
Contrast with this the high altitude records for Montezuma, plotted for the small precipitable water $0.05 \mathrm{~cm}$. in Fig. 21 (4).

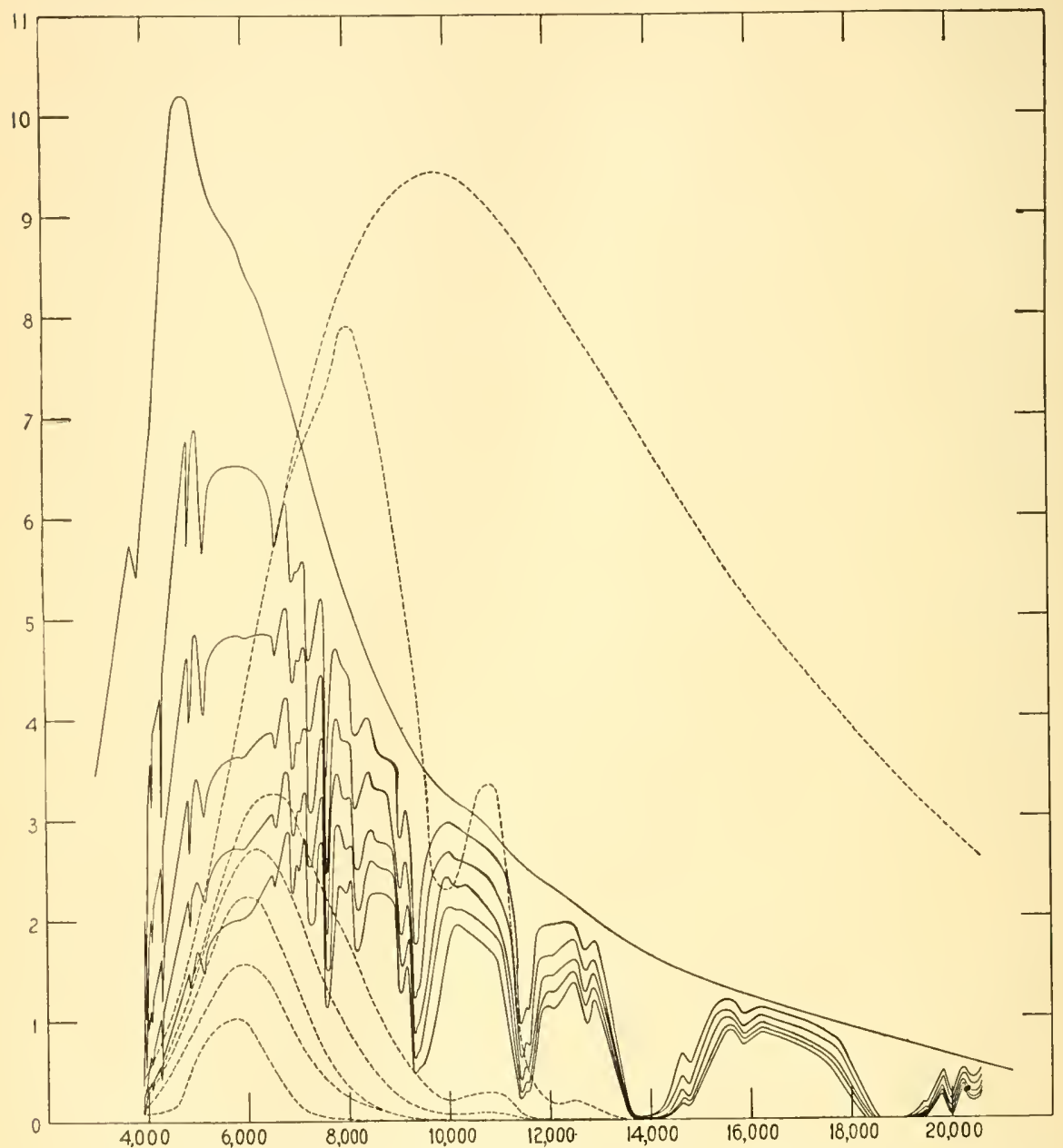

FrG. 20.-Spectral distribution of radiation from the sun, outside the atmosphere, and at air masses $1,2,3,4$, and 5, on an average day in Washington, precipitable water $1.37 \mathrm{~cm}$. Dotted curves indicate distribution from artificial sources, i.e., radiation from 100 -watt tungsten lamp, at approximately $2755^{\circ} \mathrm{K}$., same through $3-\mathrm{cm}$. water cell, through 3-cm. water cell and the following filters (all heat-resisting, heat-absorbing): extra light shade, $2.75 \mathrm{~mm}$. (thickness); light shade, $2.66 \mathrm{~mm}$.; light medium shade, $2.62 \mathrm{~mm}$.; dark medium shade, $2.97 \mathrm{~mm}$; dark shade, $2.82 \mathrm{~mm}$. (From data of Abbot, 4.)

These curves do not give any idea of the wave-length distribution below $4000 \AA$, i.e., in the ultra-violet. From 1 to 5 per cent of the energy occurs in this region. A typical distribution curve of relative intensity for wave-lengths between 2900 and $3600 \AA$ is plotted from data of 
Forsythe and Christison (28), Fig. 30. The shortest detectable wavelength is near $2900 \AA$, varying markedly with locality air mass, and season. Thus, Dorno reports for Davos $(46$, ef. pages 36,53$)$ that the limit varies from $2973 \AA$ at midday in July to $3093 \AA$ in December.

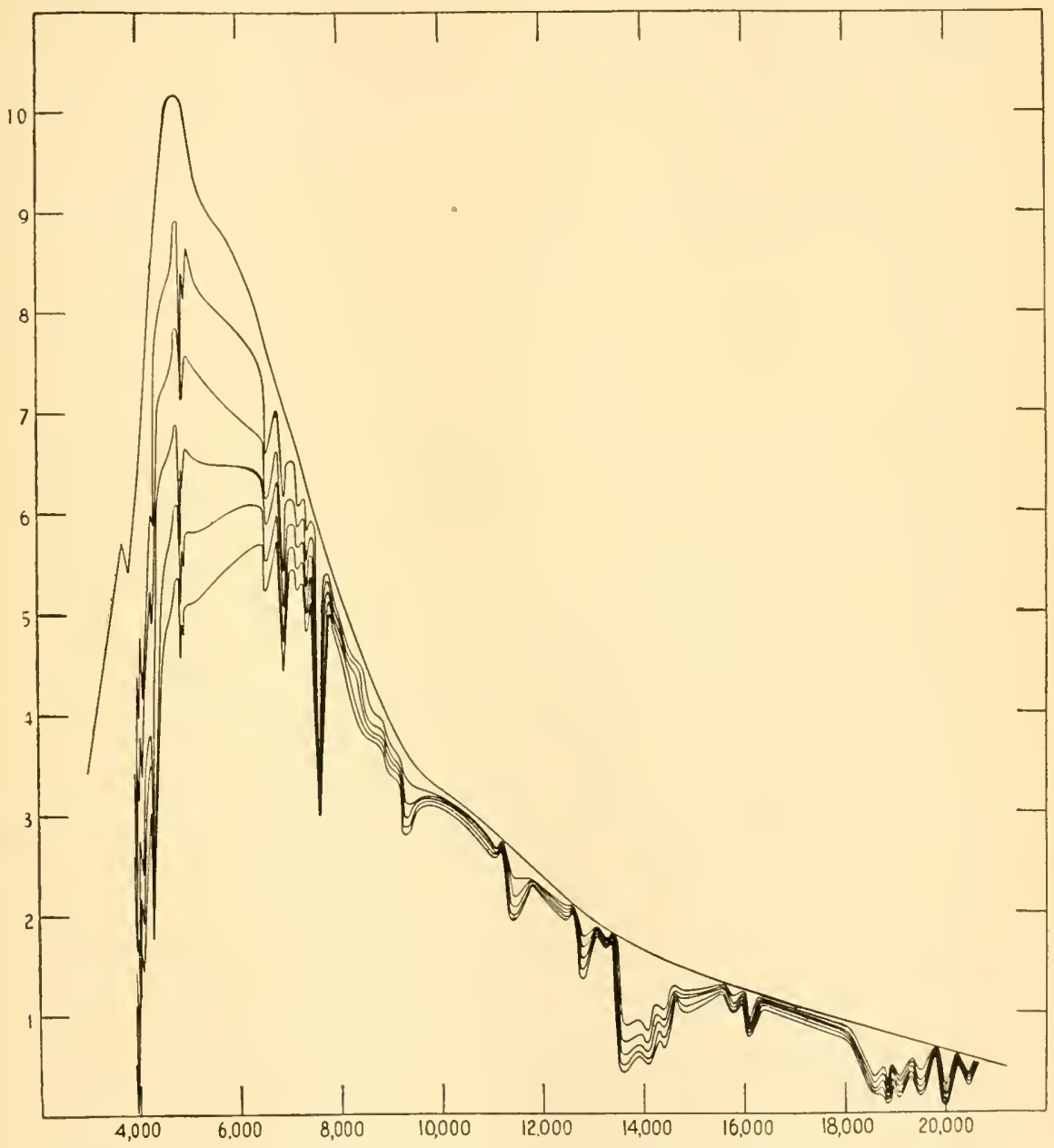

FIG. 21. - Spectral distribution of radiation from the sun, outside the atmosphere, and at air masses $1,2,3,4$, and 5 , on a clear dry day at Montezuma, precipitable water $0.05 \mathrm{~cm}$. (From data of Abbot, 4.)

On August 29, 1909, the value at noon was $2963 \AA$, while from 5 A.M. to 6 A.м., it was $3202 \AA$. Even with sun at zenith, the fall in intensity in proceeding to shorter wave-lengths is extremely great. Laurens (46, cf. page 38 ) gives the value at $2929 \AA$ as only $1 / 10,000$ of that for $3140 \AA$. Consequently, if one undertakes to evaluate intensities 
in this region, the type of long-wave cut-off employed will drastically influence the values obtained.

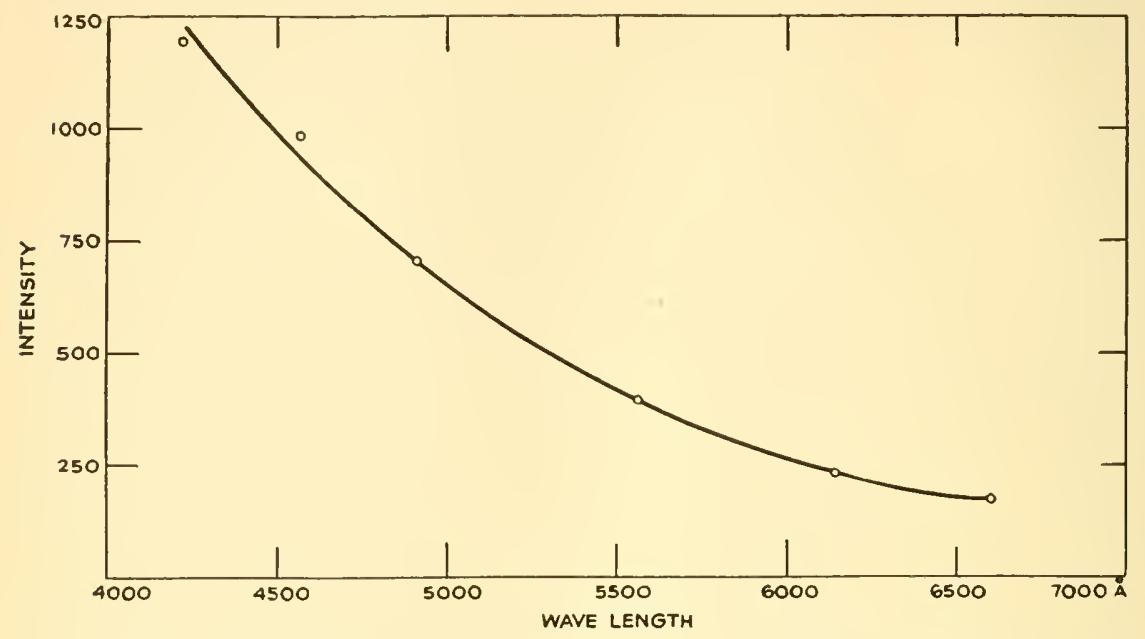

Frg. 22.-Sky radiation, intensity in arbitrary units. (From data of Abbot, 1.)

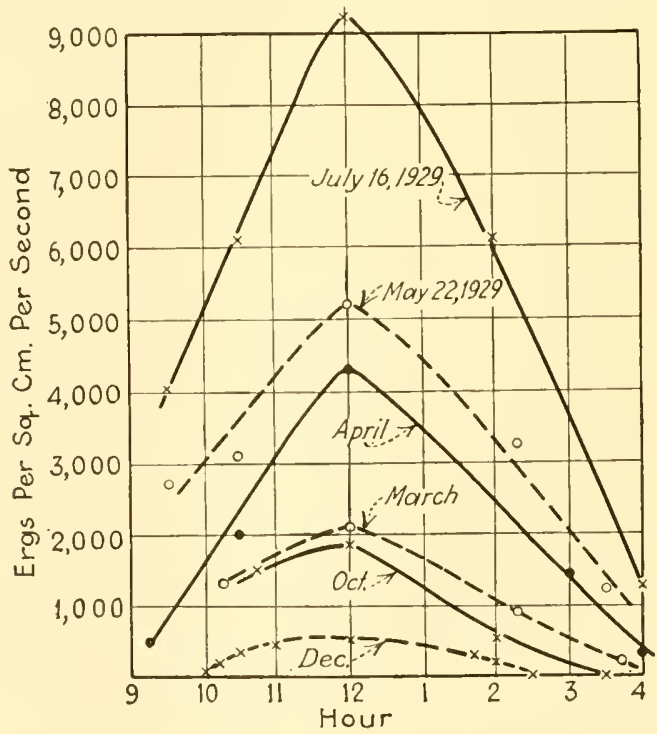

FIG. 23.-Ultra-violet radiation on clear days. (Zinc sulfide measurements by Clark, 18.)

The contribution of sky radiation is much greater for short wavelengths, as will be seen from Fig. 22, plotted from Abbot's data (1). The sky radiation in the region 2900 to $3200 \AA$ often exceeds the direct radiation even for high sun. 
Using the darkening of ZnS as a detector, Clark (18, cf. page 246) has observed the ultra-violet, cut off by a microscope slide (see curve 15 , Fig. 9) for both daily and seasonal variation at Baltimore, Md.

Figure 23 (18) shows the daily fluctuation for clear days at different representative times of the year. It will be noticed on comparison with Fig. 18 that the ultra-violet rises more sharply to a maximum and then decreases more rapidly than the total radiation, owing to the greater influence of atmospheric absorption.

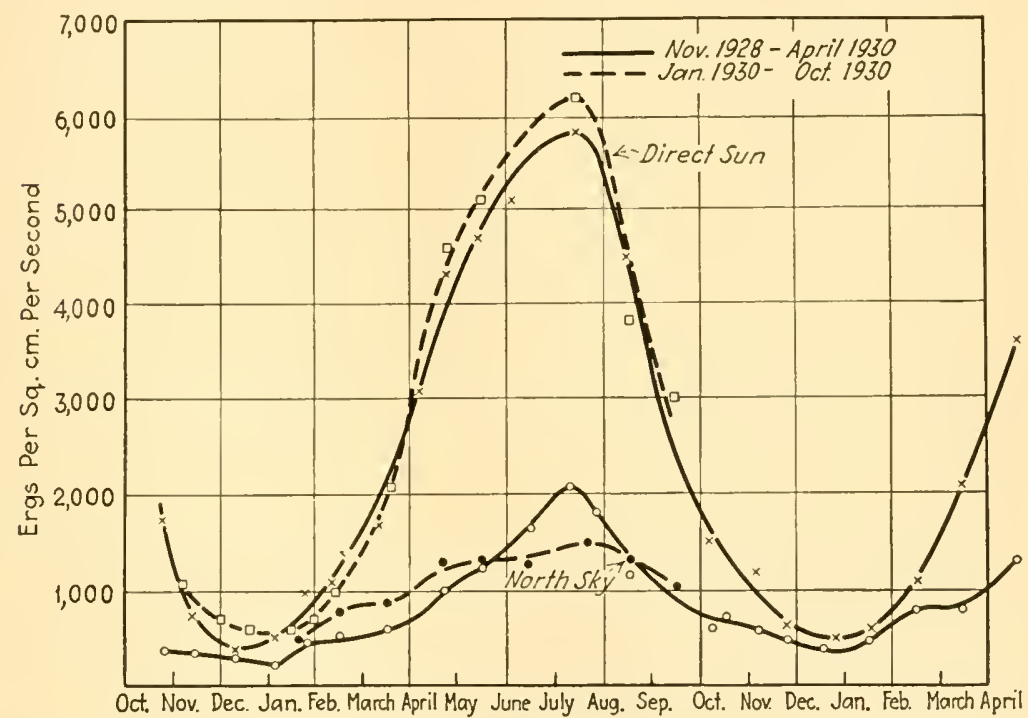

FIG. 24. -Seasonal variation in ultra-violet radiation. (Zinc sulfide measurements by Clark, 18.)

The annual variation in ultra-violet radiation lags somewhat behind that of the total radiation, as will be seen by comparing Clark's values, Fig. 24, with those of Kimball (page 224).

The variations in ultra-violet intensity and short-wave-length limit have been attributed by many observers to the absorption of atmospheric ozone. According to Dobson (18), the effective depth of ozone in the atmosphere amounts to 0.3 to $0.5 \mathrm{~cm}$. at normal temperature and pressure. Figure 25 shows the seasonal fluctuation in effective depth observed by Dobson at Oxford averaged over the years 1925 to 1928, inclusive. The absorption coefficients for ozone under standard conditions are given by curve $C$, upper section of Fig. 2. Corresponding percentage transmissions are given for $1.0 \mathrm{~cm}$. depth at the right. $\mathrm{By}$ shifting this scale up one-half division, one obtains the value for $0.3 \mathrm{~cm}$. depth. This indicates an effective cut-off in the region of $2900 \AA$, rising 
to 1 per cent transmission at $3000 \AA$ and 50 per cent or over at $3100 \AA$. From this crude estimation, we see that such a theory finds support in the known characteristics of ozone absorption. While Dobson's observations have been obtained by ozone determinations in the ultra-violet, Wulf (69) has shown that determinations by the visible ozone-absorption band are in substantial agreement. The fact that ozone attains its least effective depth well into July has been offered as the explanation for the fact that the ultra-violet reaches a maximum intensity in July, whereas the naximum total radiation occurs in June. The ozone theory of ultra-

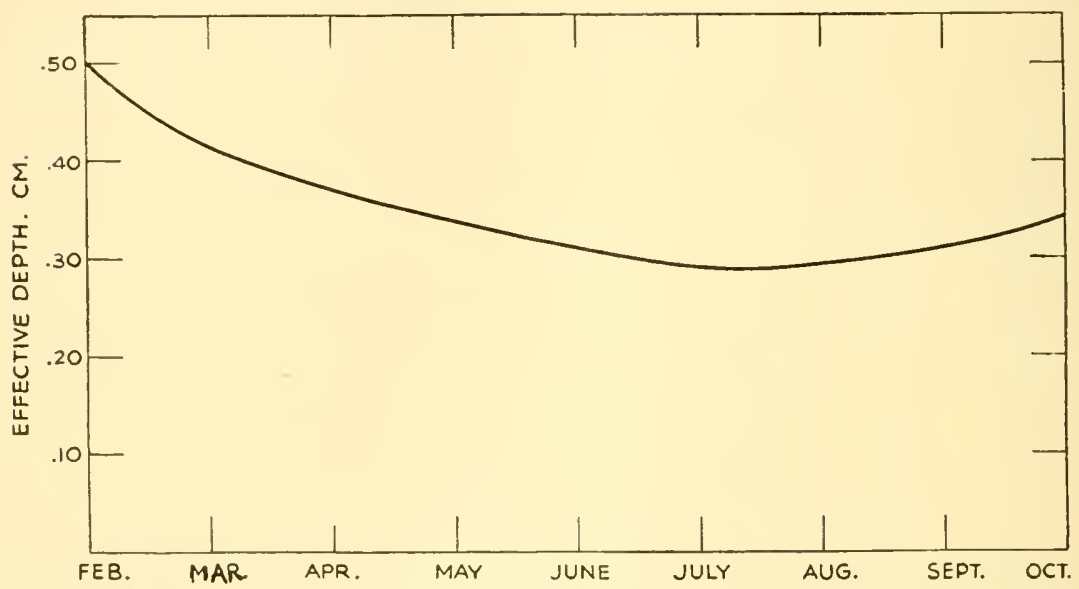

FIG. 25. - Seasonal variation in ozone. Effective depth in centimeters at standard conditions.

violet fluctuation has been severely attacked, however, by several observers (61).

The north-sky radiation, shown in Fig. 24, represents only half the solid angle and less than half the sky radiation, as the scattering is greater near the sum, which for this latitude generally occurs in the southern hemisphere.

For the region between 2900 and $3130 \AA$, Cohlentz and Stair have determined the values of ultra-violet radiation given in the curves plotted by Laurens and shown in Fig. 26 (46, ef. page 68). (Values given in the original publication (22) were increased 50 per cent on later calculations.) Here it will be seen that the maximum amounts to 86 mierowatts, as compared with between 105 and 110 milliwatts maximum total radiation for Washington, i.e., about 0.1 per cent. Clark's value for a somewhat greater wave-length range shows a maximum of about 1 per cent.

In summary, we may say that less than 0.1 per cent of the total radiation lies short of $3130 \AA, 1$ to 5 per cent lies in the ultra-violet, from 
41 to 45 per cent lies in the visible, and from 50 to 58 per cent lies in the infra-red. With high sun, the wave-length of maximum intensity per unit wave-length occurs at about $5000 \AA$, corresponding roughly to a black-body temperature of $6000^{\circ} \mathrm{K}$. Abbot's (6) estimates of total radiation outside the atmosphere of 1.90 to $1.97 \mathrm{gm}$. cal. $/ \mathrm{min} . / \mathrm{cm}^{2}$ correspond, however, to the total radiation of a black body with temperature nearer $5000^{\circ} \mathrm{K}$. The energy reaching the earth for high sun commonly varies from 0.5 to $1.5 \mathrm{gm}$. cal. $/ \mathrm{min} . / \mathrm{cm} .{ }^{2}$. With decreasing altitude of sun, the increasing air mass reduces the relative intensity of ultra-violet and blue, as compared with the red and infra-red, so that the

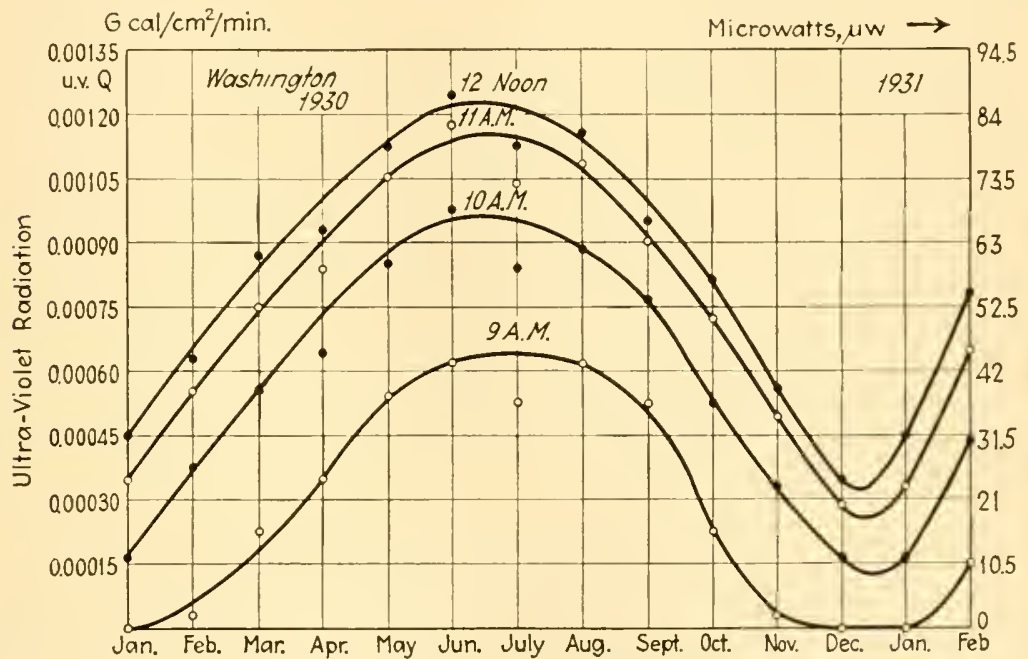

Fig. 26.-Average ultra-violet solar radiation intensities of wave-lengths less than $0.313 \mu$. (Coblentz and Stair, 46.)

distribution corresponds to a lower black-body temperature. Thus Abbot computes the distribution of direct solar radiation, equiralent to a black-body (or color) temperature varying from $6300^{\circ} \mathrm{K}$. for air mass zero to $2760^{\circ} \mathrm{K}$. for air mass 10 . The sky radiation, however, is much stronger in the blue and ultra-violet, so that its color temperature is much higher. Priest found for Washington that this varied from $6000^{\circ}$ to $24,000^{\circ} \mathrm{K}$. On overcast days, it is near the lower limit, $6300^{\circ}$ to $7000^{\circ} \mathrm{K}$. On clear days, the usual range is from $8000^{\circ}$ to $15,000^{\circ} \mathrm{K}$. Thus, the sky contribution raises the color temperature of total radiation over the direct.

Since the color temperature varies markedly with air mass, or height of the sun, the luminous equivalent varies correspondingly. Kimball gives the values in foot-candles for $1 \mathrm{gm}$. cal. $/ \mathrm{min} . / \mathrm{cm} .{ }^{2}$. 
Table 13.-Illumination Equivalent of a Gram Calorie per Minute per Square Centimeter of Radiation with the Sun at Different Zenith Distances (43, ef. page 479)

\begin{tabular}{|c|c|c|c|c|c|c|c|c|c|}
\hline Air mass....... & 1.1 & 1.5 & 2.0 & 2.5 & 3.0 & 3.5 & 4.0 & 4.5 & 5.0 \\
\hline Solar zenith distance... & 25 & 47.3 & 60.0 & 67.6 & 70.7 & 73.6 & 75.7 & 77.4 & 78.7 \\
\hline $\begin{array}{r}\text { Direct solar illumination, } \\
\text { F.C. } \ldots \ldots \ldots \ldots \ldots \ldots\end{array}$ & 7020 & 6880 & 6740 & 6650 & 6580 & 6520 & 6460 & 6410 & 6370 \\
\hline $\begin{array}{l}\text { Total illumination on a hori- } \\
\text { zontal surface, F.C...... }\end{array}$ & 7000 & 6740 & 6740 & 6320 & 6260 & 6220 & 6200 & 6200 & 6200 \\
\hline
\end{tabular}

Thus we obtain for $1.5 \mathrm{gm}$. cal./min. $/ \mathrm{cm}^{2} 10,500$ foot-candles as the highest value commonly attained at sea level. He arrives at an average value 6700 foot-candles for $1 \mathrm{gm}$. cal. $/ \mathrm{min} . / \mathrm{cm}^{2}$, which is somewhat low for high sun, and high for low sun. From this value for $1 \mathrm{gm}$. cal./min./cm. ${ }^{2}$, we obtain 103.0 lumens/watt for the average luminous efficiency of solar radiation (the range being 98 to 107 lumens/watt).

Obviously, no black-body or tungsten-filament source could simulate the solar distribution. Daylight glasses and also solution filters have been developed which give requisite color temperature in the visible, but they generally fail in both ultra-violet and infra-red. For the sake of comparison, the relative distribution has been plotted, shown by dotted curves, Fig. 20, on an arbitrary scale for a tungsten filament, temperature $2755^{\circ} \mathrm{K}$., together with curves for combination with water filter and heat-absorbing filters.

\section{TUNGSTEN FILAMENT}

Undoubtedly, the most convenient artificial source is the tungsten lamp filament. It differs from the black body as a result of the reflection of the metallic surface of the filament which of course contributes to a correspondingly lower emission of radiation. For convenient comparison with the radiation from a black body, the radiation from tungsten at the same temperature has been plotted in dotted curves in Fig. 3. It will be seen that while the radiation is less for all wave-lengths, thus corresponding in magnitude to a lower temperature black body, the relative distribution of intensity according to wave-length corresponds to a higher temperature, i.e. the maximum occurs at a shorter wave-length, and the intensity at short wave-lengths is greater in comparison with the long wave-lengths. This arises from the increase in reflectivity with increasing wave-length. Table 7 gives the values for the radiance in $\mathrm{erg} / \mathrm{sec} . / \mathrm{cm} .{ }^{2} / \mathrm{steradian}_{\mathrm{cm}}$. wave-length in a direction normal to the surface, for both black body and tungsten.

Other radiation properties of tungsten have been gathered together in Table $14(29,30)$. Most of these data deal with the convenient conversion factors by which one obtains the values for tungsten radiation from 


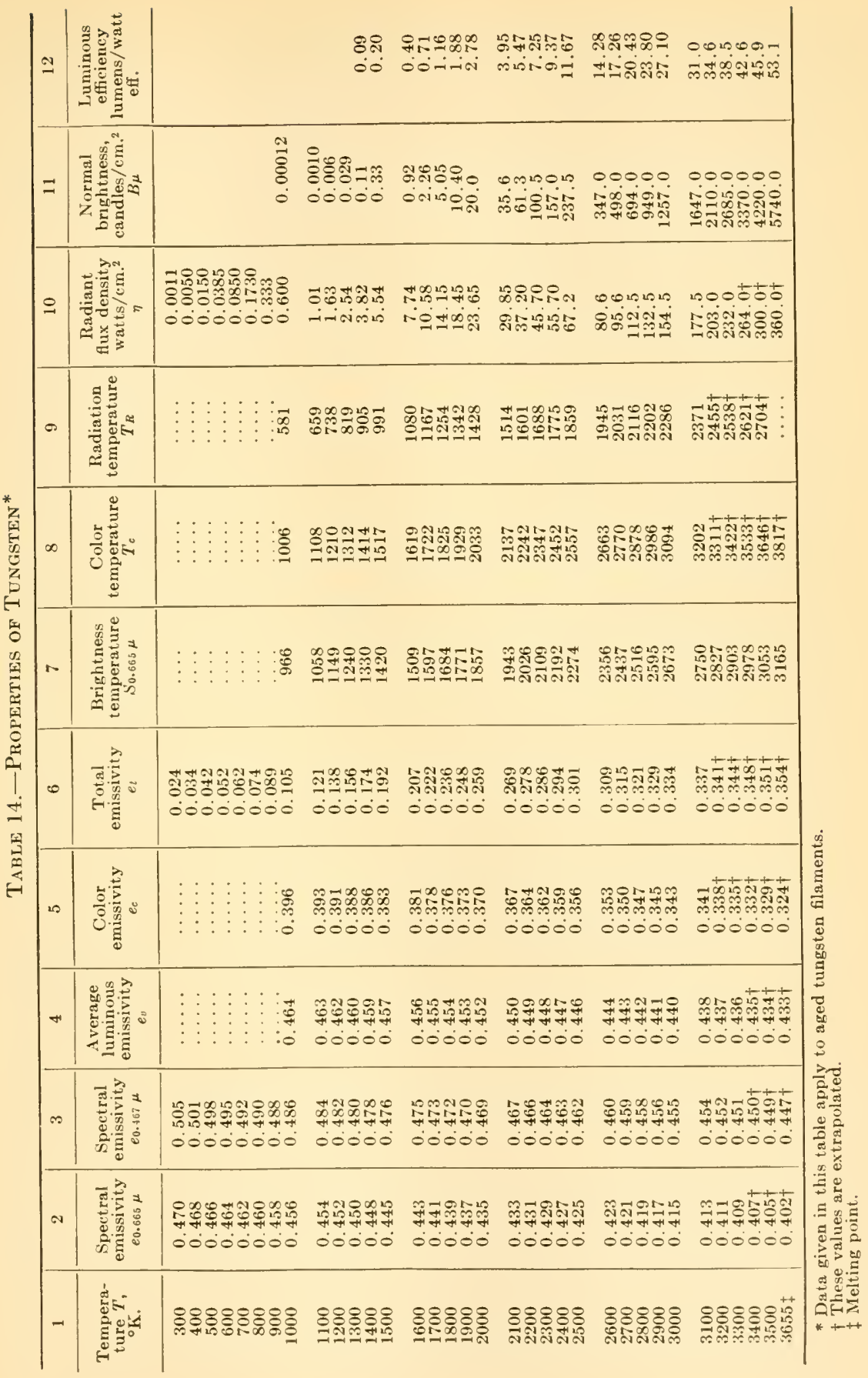


those of the black bocty, which is the underlying basis of all radiation measurements. The first column gives the actual temperature in degrees Kelvin. The second, spectral emissivity, is the ratio of the radiance of tungsten to that of the black body at the same temperature for $0.665 \mu$ or $6650 \AA$. Thus a tungsten filament at $1000^{\circ} \mathrm{K}$. emits 0.456 as much radiation at $6650 \AA$ in the red as would a black body of that temperature. From the third column, we find the corresponding value of 0.486 for $0.467 \mu$ or $4670 \AA$ in the blue, i.e., tungsten emits 0.486 as much in the blue as the black body at the same temperature. The fourth column gives the average relative emissivity $e_{v}$ throughout the visible. Thus tungsten at $3000^{\circ} \mathrm{K}$. emits 44 per cent of the number of lumens radiated by a black body at that temperature. As we have seen from Fig. 3, the wave-length distribution of tungsten corresponds to a higher temperature of black body. The term color temperature (eighth column) gives the temperature of a black body which would most nearly match that of tungsten at the given temperature, in relative distribution or color, but not in magnitude (or brightness). Since tungsten emits less for all wave-lengths than the black body at the same temperature, it emits still less in comparison with the black body at the color temperature, which is higher. Thus, we find the color emissivity $e_{c}$ (Column 5) which is relative to the black-body emission at the color temperature less than the luminous emissivity $e_{v}$. As has been noticed, tungsten emits much less relatively in the infra-red than in the visible. Hence comparing the total emission with that of a black body at the same temperature, one finds a much lower value for the total emissivity $e_{t}$ than for the luminous emissivity $e_{v}$. These are given in Column 6. The brightness temperature (Column 7) for $6650 \AA$ is the temperature for which the black body would emit the same luminous intensity as tungsten for that wave-length. These values are, of course, always lower.

The radiation temperature is that for which the black body would yield the same total radiation. From Column 9 we see that this is much lower than the given temperature. The radiant flux density, sometimes termed total radiant intensity (Column 10) enables us to compute the total power emitted from any tungsten surface of known temperature and area. In a similar way, from Column 11, (normal brightness in candles per square centimeter) we can compute the candlepower for known temperature and area. It is also convenient to remember that this normal brightness is equal to the number of lumens $/ \mathrm{steradian} / \mathrm{cm} .^{2}$ in a direction normal to the surface. The last column gives the lumens output for 1 watt input. Since most of the electrical energy is dissipated by radiation, this is close to the value for the relation of lumens to watts in the radiation.

It is interesting to compare these values with those of solar radiation, which we found to be about 103 lumens/watt. A high-temperature 
tungsten-filament lamp, $3200^{\circ} \mathrm{K}$., yields only one-third of this value. However, even in the case of the sun, less than 50 per cent of the energy lies in the visible. Furthermore, since the blue and red are much less effective than the yellow, it is not surprising to find the total (103

Table 15.-Temperature, Color Temperature, Brightness, and Efficiency of VARIOUS INCANDESCENT LAMPS*

\begin{tabular}{|c|c|c|c|c|}
\hline Lanıp & $\begin{array}{c}\text { Lumens/ } \\
\text { watt }\end{array}$ & $\begin{array}{l}\text { Maximum } \\
\text { tempera- } \\
\text { ture, }{ }^{\circ} \mathrm{K} .\end{array}$ & $\begin{array}{l}\text { Average } \\
\text { color } \\
\text { tempera- } \\
\text { ture, }{ }^{\circ} \mathrm{K} \text {. }\end{array}$ & $\begin{array}{l}\text { Maximum } \\
\text { brightness, } \\
\text { candles/ } \\
\mathrm{cm}^{2}\end{array}$ \\
\hline \multicolumn{5}{|l|}{ Vacuum lamps: } \\
\hline 50 -watt untreated carbon.......... & 2.5 & 2095 & 2080 & 55 \\
\hline 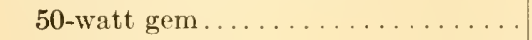 & 4.0 & 2130 & 2195 & 78 \\
\hline 50 -watt tantalum .............. & 4.9 & 2180 & 2260 & 53 \\
\hline 10 -watt tungsten............. & 7.7 & 2355 & 2390 & 128 \\
\hline 25 -watt tungsten. . . . . . . . . . & 9.8 & 2450 & 2493 & 193 \\
\hline 40 -watt tmgsten . . . . . . . . . & 10.0 & 2460 & 2504 & 206 \\
\hline 60 -watt tungsten.............. & 10.1 & 2465 & 2509 & 211 \\
\hline \multicolumn{5}{|l|}{ Regular gas-filled lamps: } \\
\hline $50-$ watt. . . . . . . . . . . . & 10.0 & 2685 & 2670 & 469 \\
\hline 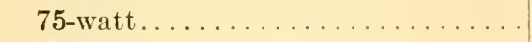 & 11.8 & 2735 & 2705 & 563 \\
\hline $100-$ watt. . . . . . . . . . . . & 12.9 & 2760 & 2740 & 605 \\
\hline $200-$ watt. . . . . . . . . . . . . . . & 15.2 & 2840 & 2810 & 781 \\
\hline \multicolumn{5}{|l|}{ 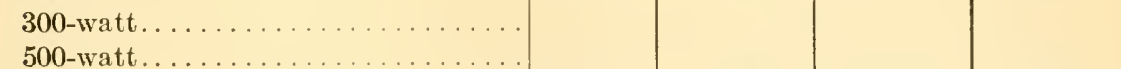 } \\
\hline 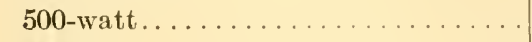 & & & & \\
\hline 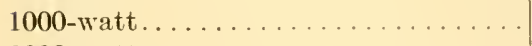 & 20.0 & 2990 & 2980 & 1225 \\
\hline 2000 -watt. . . . . . . . . . . . . . . & 21.2 & 3020 & 3000 & 1350 \\
\hline \multicolumn{5}{|l|}{ Special lamps: } \\
\hline 1000 -watt stereopticon . . . . . . . . . . & 24.2 & 3185 & 3175 & 2065 \\
\hline 900 -watt movie. . . . . . . . . . & 27.3 & 3290 & 3220 & 2660 \\
\hline $10-k w \ldots \ldots \ldots \ldots \ldots \ldots$ & 31.0 & 3350 & 3300 & 3050 \\
\hline $30-\mathrm{kw} \ldots \ldots \ldots \ldots \ldots \ldots \ldots$ & 31.0 & 3350 & 3300 & 3050 \\
\hline \multicolumn{5}{|l|}{ Daylight lamps: } \\
\hline 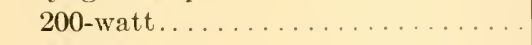 & 10.0 & 2860 & & \\
\hline 500 -watt. . . . . . . . . . . & 11.2 & 2960 & & \\
\hline \multicolumn{5}{|l|}{ Photographic: } \\
\hline 750 -watt...... & $\ldots$ & 3065 & & \\
\hline 1500 -watt . . . . . . . . . . . & $\ldots$ & 3105 & & \\
\hline
\end{tabular}

* From Forsythe and Watson (29).

lumens/watt) much less than the maximum luminous efficiency of 624 lumens/watt at $5560 \AA$.

It may be well now to consider some of the difficulties encountered in using tungsten lamps. Tungsten-filament sourees are subject to variation resulting not only from the difficulty of maintaining a constant current through the lamp, but also resulting from deterioration. Deteri- 
oration produces: (a) An increase in resistance of the filament due to loss of metal by evaporation, thus changing the operating characteristics of the lamp. (b) An accumulation of metallic deposit upon the walls, thus introducing effectively a filter of high absorbing power which varies markedly in its characteristics with wave-length. Furthermore, it is not satisfactory to use the specified temperature of a lamp for certain operating conditions as a basis for assignment of corresponding curves representing radiation as a function of wave-length. It is necessary that the aetual operating conditions be determined at the time of the exposure, unless a special mode of procedure has been carefully followed. A lamp for standardization purposes should be seasoned for a considerable length of time; second, its characteristies for different operating conditions should be accurately determined by a competent laboratory; third, it should be operated at a relatively low temperature; and fourth, it should be very little used. It is necessary, therefore, for a laboratory undertaking careful radiation work to maintain a number of lamps to serve as secondary and tertiary standards used only during brief intervals of cxposure. Refer to Bureau of Standards publication on radiation standards (23). Table 15 gives data on a number of commercial lamps. For further information, the reader is referred to the original articles (30).

CARBON ARC

Carbon arcs offer the highest available artificial temperatures, $4000^{\circ} \mathrm{K}$. being easily attainable. The positive crater is used as a source of continuous radiation. The objections to these sources are: (a) They are difficult to maintain under steady operating conditions, owing to the gradual deterioration of the crater. (b) Absorption and emission inevitably occur, owing to the hot gases surrounding the crater. By introducing salts into the core of the carbon, selective emission in the hot gases may be taken advantage of to enrich different parts of the spectrum. Extensive literature is furnished by the manufacturers, on wave-length distribution of available impregnated carbons.

\section{SOURCES OF SELECTIVE EMISSION}

Electrieal diseharge through gases furnishes a class of sources of great value in radiation work. Such sources produce relatively pure monoehromatic radiation at a number of particular wave-lengths. Thus, spread out in a spectrum, lines appear corresponding to radiation generally extending over only a few Ångströms of wave-length range, and often over only a fraction of an Angström. For photochemical purposes, these may be considered as ideally monochromatic, their width being in most cases far below the resolution of a monochromator for irradiation purposes. 
Table 16.-Monochromator Meastrements of High- and Low-1ntensity Arcs

\begin{tabular}{|c|c|c|c|c|c|c|c|}
\hline \multirow{2}{*}{$\begin{array}{l}\text { Wave- } \\
\text { length, } \\
\AA\end{array}$} & \multicolumn{2}{|c|}{$\begin{array}{l}\text { Galvanometer } \\
\text { reading, mm. }\end{array}$} & \multicolumn{3}{|c|}{$\begin{array}{c}\text { Absolute intensity } 250 \mathrm{~mm} \text {. from } \\
\text { a } 20 \text {-mm. midsection of the arc, } \\
\mu \mathrm{w} . / \mathrm{cm} .^{2}\end{array}$} & \multicolumn{2}{|c|}{$\begin{array}{l}\text { Amount excluded by } \\
\text { barium flint and } \\
\text { transmitted by quartz } \\
\text { water cell, } \mu w . / \mathrm{cm} .^{2}\end{array}$} \\
\hline & High int. & Low int. & High int. & $\begin{array}{l}\text { High int. } \\
\text { new arc }\end{array}$ & Low int. & High int. & Low int. \\
\hline 1 & 2 & 3 & 4 & 5 & 6 & 7 & 8 \\
\hline 6908 & 2.7 & 0.06 & 7.5 & $\ldots \ldots$ & 0.17 & 0.0 & 0.0 \\
\hline 6234 & 0.9 & 0.03 & 2.6 & $\ldots \ldots$ & 0.09 & 0.0 & 0.0 \\
\hline 5780 & 161 & 3.73 & 468 & 473 & 10.85 & 0.0 & 0.0 \\
\hline 5461 & 130 & 9.05 & 384 & 384 & 26.70 & 0.0 & 0.0 \\
\hline 4916 & 4.5 & 0.03 & 13.5 & $\ldots$ & 0.09 & 0.0 & 0.0 \\
\hline 4358 & 106 & 7.90 & 327 & 353 & 24.40 & 0.0 & 0.0 \\
\hline 4047 & 62 & 480 & 195 & 204 & 15.10 & 1.5 & 0.12 \\
\hline 3906 & 2.6 & 0.04 & 8. 2 & $\ldots \ldots$ & 0.13 & 0.1 & 0.00 \\
\hline 3654 & 183 & 5.50 & 585 & 643 & 17.60 & 17.6 & 0.53 \\
\hline 3341 & 16 & 0.36 & 52 & 54 & 1.17 & 3.4 & 0.47 \\
\hline 3130 & 110 & 5.10 & 359 & 377 & 16.70 & 321 & 14.92 \\
\hline 3022 & 57 & 1. 16 & 187 & 194 & 3.82 & 168 & 3.44 \\
\hline 2967 & 29.5 & 1.05 & 97.1 & 95.1 & 3.46 & 86.5 & 3.08 \\
\hline 2925 & 4.0 & 0.11 & 13.2 & $\ldots \ldots$ & 0.36 & 11.6 & 0.32 \\
\hline 2894 & 10.7 & 0,33 & 35.4 & 34.7 & 1.09 & 31.2 & 0.96 \\
\hline 2804 & 22.4 & 0.44 & 74.7 & 71.7 & 1.47 & 65.9 & 1.29 \\
\hline 2752 & 7.0 & 0.18 & 23.4 & $\ldots \ldots$ & 0.60 & 20.6 & 0.53 \\
\hline 2699 & 9.5 & 0.16 & 31.9 & $\ldots \ldots$ & 0.54 & 28.1 & 0.48 \\
\hline 2652 & 40.5 & 0.86 & 137 & 130 & 2.91 & 121 & 2.56 \\
\hline 2602 & 2.7 & 0.04 & 9.2 & $\ldots \ldots$ & 0.14 & 78 & 0.12 \\
\hline 2576 & 5.4 & 0.07 & 18.4 & $\ldots \ldots$ & 0.24 & 14.7 & $\begin{array}{ll}0 & 19\end{array}$ \\
\hline 2536 & 52 & 4.73 & 178 & 154 & 16.20 & 136 & 12.30 \\
\hline 248.3 & 15.8 & 0.44 & 54.6 & 48.6 & 1.52 & 37.8 & 1.05 \\
\hline 2463 & 2.1 & 0.06 & 7.3 & $\ldots \ldots$ & 0.21 & 5.0 & 0.15 \\
\hline 2447 & 1.8 & 0.03 & 6.3 & $\ldots \ldots$ & 0.10 & 4.3 & 0.07 \\
\hline 2399 & 5.8 & 0.14 & 20.3 & 23.3 & 0.49 & 13.6 & 0.33 \\
\hline 2378 & 4.9 & 0.11 & 17.2 & 226 & 0.39 & 11.0 & 0.25 \\
\hline 2352 & 4.5 & 0.07 & 15.9 & 16.7 & 0.25 & 9.6 & 0.15 \\
\hline 2323 & 1.8 & 0.03 & 6.4 & $\ldots$. & 0.11 & 3.7 & 0.06 \\
\hline 2300 & 1.4 & 0.06 & 5.0 & $\ldots \ldots$ & 0.21 & 2.9 & 0.12 \\
\hline 2283 & 0.9 & 0.04 & 3.2 & $\ldots \ldots$ & 0.14 & 1.8 & 0.08 \\
\hline 2250 & 0.7 & $\ldots$ & 2.6 & $\ldots \ldots$ & $\ldots \ldots$ & 1.3 & \\
\hline \multirow{3}{*}{\multicolumn{3}{|c|}{$\begin{array}{l}\text { Total for } 143.5 \text { volts. } \ldots \ldots \ldots \ldots \\
\text { Total corrected to } 150 \text { volts } \ldots \ldots \ldots \\
\text { Total less than and including } \lambda 3.130 \\
\text { for } 143.5 \text { volts . . . . }\end{array}$}} & $3,302,3$ & $\ldots \ldots$ & 147.3 & 1,126 & 43.6 \\
\hline & & & 3,450 & $\ldots \ldots$ & $\ldots$ & 1,180 & \\
\hline & & & 1,303 & & 51.0 & & \\
\hline \multirow{2}{*}{\multicolumn{3}{|c|}{$\begin{array}{l}\text { Total less than and including } \lambda 3,130 \\
\text { (corrected to } 150 \text { volts) } \ldots \ldots \ldots\end{array}$}} & \multicolumn{5}{|c|}{ For "total are" multiply all values by 7.1} \\
\hline & & & 1,362 & & & & \\
\hline
\end{tabular}



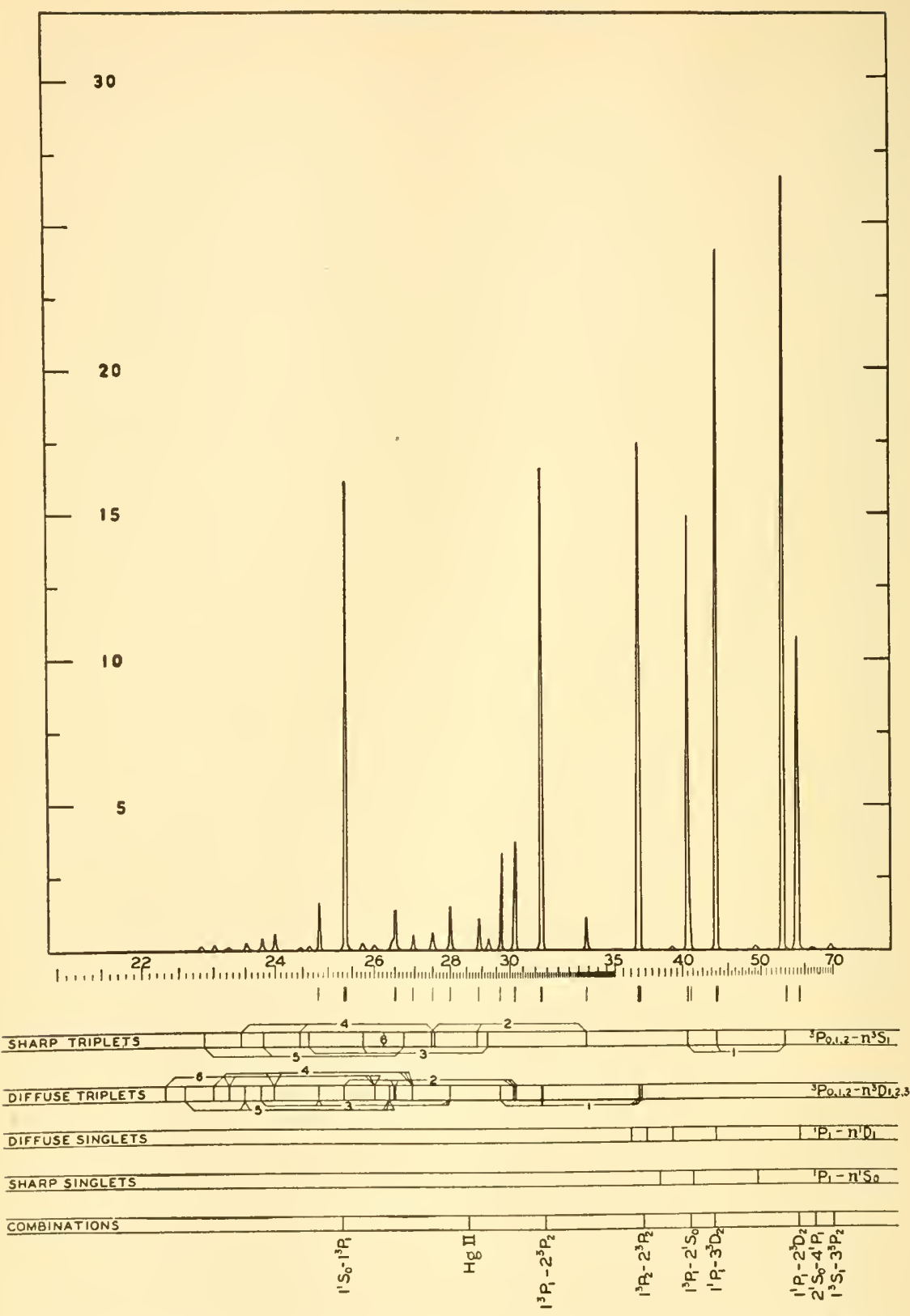

FIG. 27.- Intensity distribution from a low-intensity mercury arc. Ordinates give $\mu \mathrm{w} . / \mathrm{cm} .^{2}$ irradiation at $25 \mathrm{~cm}$. from a $20-\mathrm{mm}$. exposed midsection of a 250 -volt arc, operated on 44 volts and 3.0 amp. (From data of McAlister, 50.) Abscissas wave-lengths on prismatic scale, $\AA / 100$. Spectral designation indicated below. 
The most used source of this type is the mereury are. Efforts have been made reeently by the Illuminating Engineers' Society to produce mercury ares which will serve as rough standards of monochromatic radiation. The intensities of the lines for these arcs have been measured and agreement secured between a number of laboratories to about 5 per cent. Such sources are, however, subject to rariability, not only
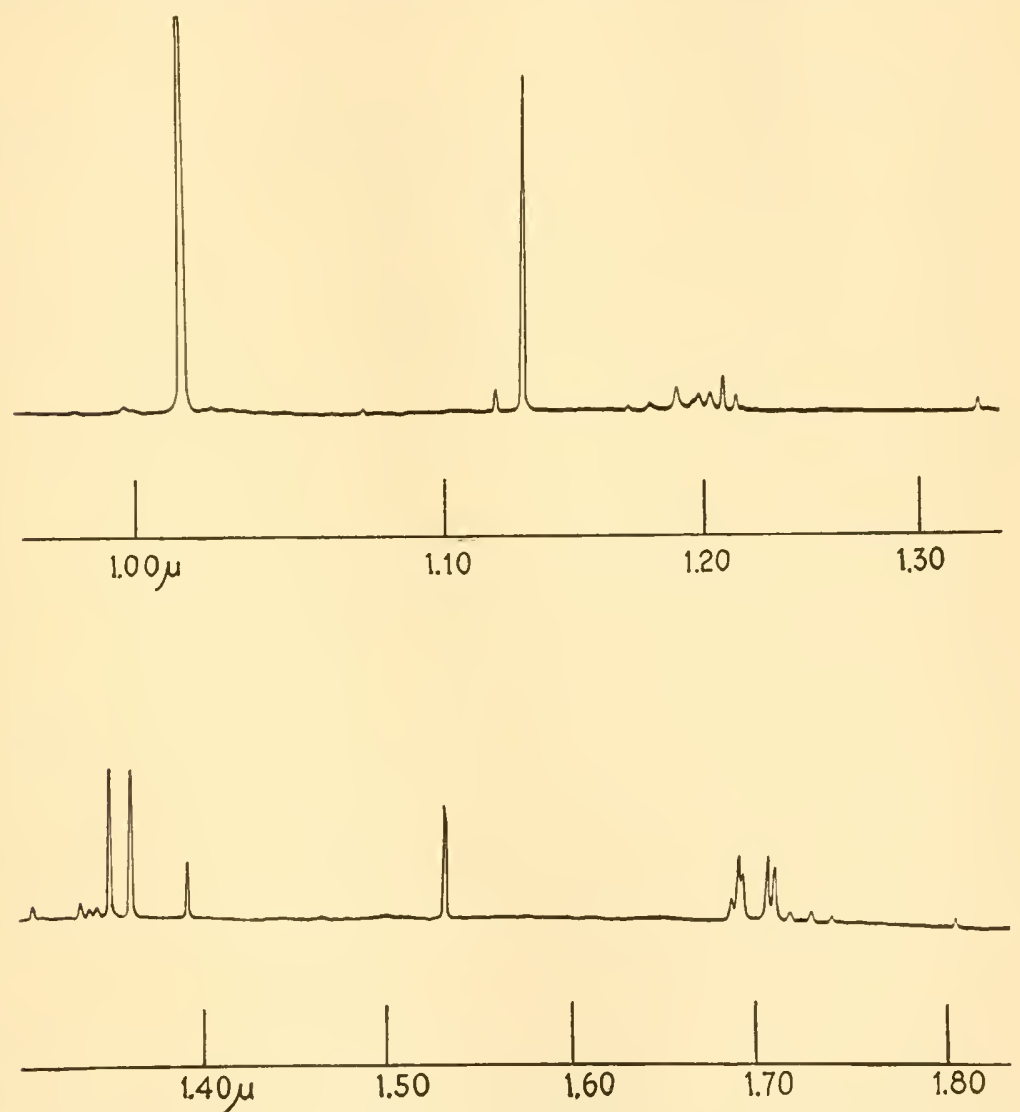

FIG. 28.- Near infra-red spectrum from a horizontal mercury arc, viewed end-on, operated at 86 volts and $3.8 \mathrm{amp}$. (From data of .1/ $\mathrm{cAlister}, 49$.

because of the difficulty of maintaining constant operating conditions of voltage and temperature, but also owing to the variability resulting from the changing transmissivity of quartz with time of operation, and the gradual deposition of metal on the surfaces because of the gradual destruction of the electrodes.

Absolute-intensity data, obtained by McAlister (50), on such an are at two different conditions, (a) at "high intensity," 143.5 volts potential 
drop across the arc, $4.5 \mathrm{amp}$. current, and (b), at "low intensity," 44 volts across the are and $3 \mathrm{amp}$. current, are given in Table 16, together with data on a new are (Column 5) for comparison. The line intensities have also been shown graphically for the high-intensity condition in Fig. 8, those for the low intensity in Fig. 27. Comparison of the data for highand low-intensity operation indieates the drastic change in both absolute and relative distribution resulting when the current is dropped from 4.5 to 3 amp. This brings out the absolute necessity of maintaining accurately controlled operating conditions.

Figure 28 shows the distribution in the infra-red for another type of mercury arc, viewed end-on. For complete wave-length and intensity data, the reader is referred to the original paper (49).

Since lines are available only at certain definite wave-lengths, if a wide range of representative points is to be secured in any photochemical study, it is necessary to use other sources which emit radiation at different wave-lengths. Another source which is available commercially is the helium arc. It produces a brilliant yellow line and a moderately strong red line. Recently, sodium ares have been placed on the market. These are chiefly valuable because of the intense radiation of the yellow $D$ lines. Another valuable source is the hydrogen discharge tube, producing an intense red line, and other weaker lines in the blue and violet. The chief objection here is that such sources are not available for highintensity operation in a commercial lamp. If the line speetrum referred to above is to be obtained, the lamp must be operated with the hydrogen in atomic condition. In order to obtain hydrogen in atomic condition, it is necessary to operate the tube with a contimuous flow of wet hydrogen, usually supplied by electrolysis. Only that part of the positive column remote from the electrodes can be used. If hydrogen is present in molecular form, a band spectrum presenting a multiplicity of lines is obtained. These lines cannot be readily isolated, so that such a source is not of any value for monochromatic irradiation.

Only in case of the mercury arc have sources been constructed sufficiently standardized to make intensity determinations of any great value. A wide range of sources furnishing other lines can be used. For ultra-violet work, some of the most valuable are given in Table 17 (63, ef. pages $507-508)$.

The are lines are obtained when operated in a low-voltage are and are due to the nonionized metal. The spark lines are obtained at high voltage or spark discharge and are due to the once-ionized element.

Figure 29 (56, ef. page 185) shows the sources commonly used for purposes of spectroseopic calibration. For a more complete list of the stronger lines, see Smithsonian Physical Tables 616, 617, and 618 (63, cf. pages 509-510). 


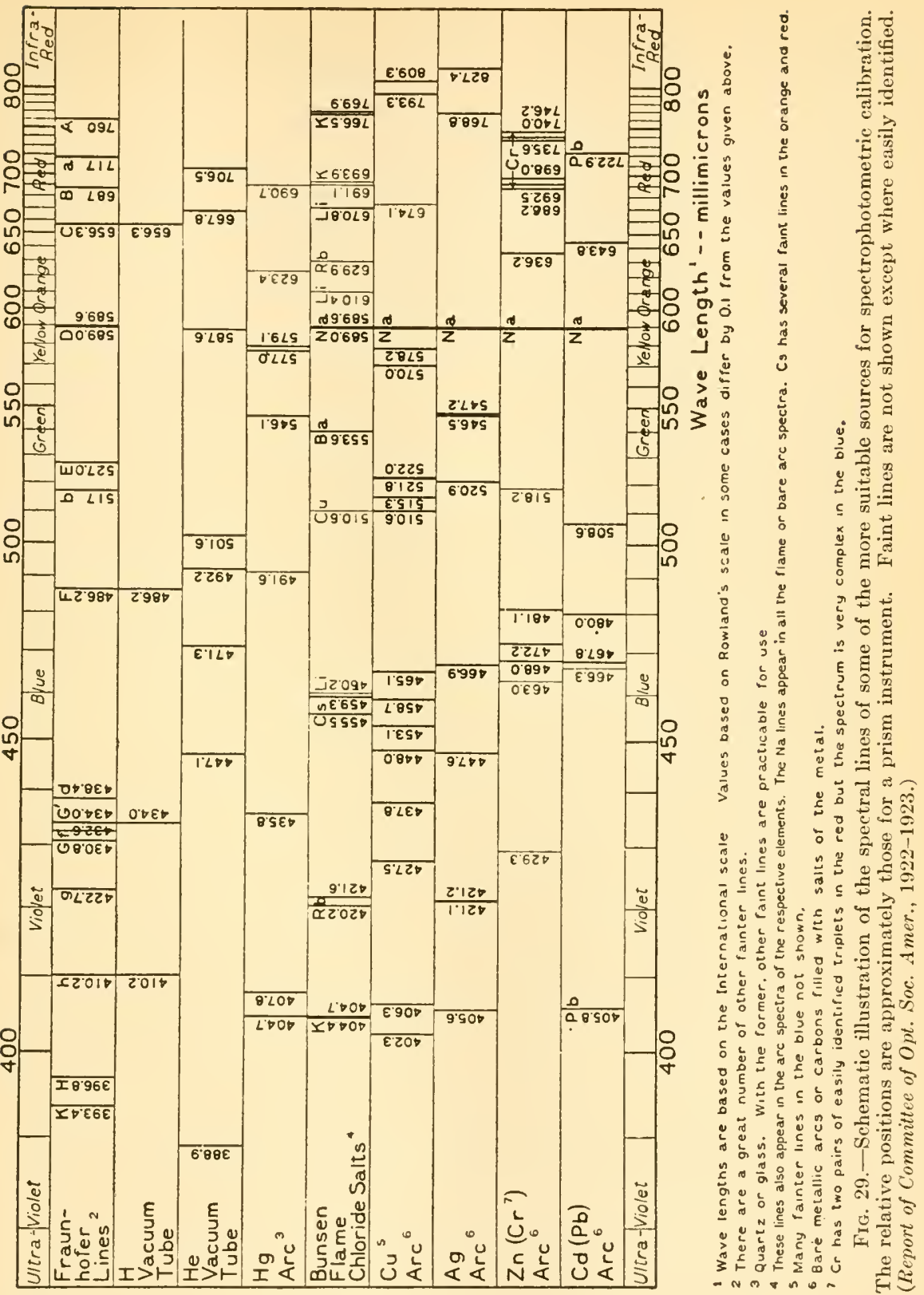


TABLE 17

\begin{tabular}{c|c|c}
\hline Metal & Arc $\lambda$ & Spark $\lambda$ \\
\cline { 1 - 3 } $\mathrm{Al}$ & 3961.55 & \\
& 3944.03 & \\
$\mathrm{Cn}$ & 3247.55 & 2247.00 \\
$\mathrm{Ag}$ & 3280.66 & 2437.81 \\
$\mathrm{Zn}$ & 2138.61 & 2061.96 \\
& 3075.88 & \\
$\mathrm{Crl}$ & 2288.02 & 2265.04 \\
& 3261.04 & \\
\hline
\end{tabular}

Two other types of sourees deserve mention. First, where gas discharge is maintained under a high pressure, atmospheric or greater, an intense practically continuous emission is obtained in the blue and ultraviolet. This is often the only available source of continuous radiation in that region. The other type of source is the ring discharge, where the excitation is produced by high-frequency induction. These sources are free from difficulties due to electrode deterioration, but since the excitation generally corresponds to very high potentials, an entirely different spectrum from the ordinary arc spectrum is obtained. Some of these sources are now manufactured for aviation-field illumination. They are the most efficient illuminants known.

By a combination of these sources of selective emission with suitable monochromatic filters, lines can of ten be isolated, thus yielding a very satisfactory monochromatic source of irradiation. Table $11 \mathrm{~A}$ shows various combinations which are possible with the mercury arc. Table $11 \mathrm{~B}$ shows corresponding possibilities with helium. Table $11 \mathrm{c}$ has been worked out for hydrogen. Assuming an arbitrary intensity 100 for the red line, the intensities given would roughly correspond for other lines available. It must be particularly emphasized that the relative intensities of lines from such sources vary over a tremendous range with differing conditions of excitation. The characteristics are influenced by temperature, pressure, voltage, and current. Furthermore, the shape and size of the tube are of great importance, as lines are reabsorbed within the source in varying degree. Figures presented are intended to serve only as a basis for selection of equipment, not as dependable operating characteristics.

\section{SELECTIVE DETECTORS}

In a wide range of problems, one wishes to evaluate energy available in restricted ranges of wave-lengths without resorting to complete monochromatic analysis. This is the case where it is not feasible to employ a monochromator, either on account of the mechanical arrange- 
ment or owing to the expense. The possibilities are then as indicated in the three sections following.

\section{BLACK BODY PLUS FILTER}

One may use a black-body detector, such as a thermocouple, in combination with filters. For this purpose, it is necessary to refer only to the filter-transmission characteristics as the black body is nonselective. The sensitivity is obtained by multiplying the sensitivity of the blackbody detector by the filter transmission for the specified wave-length. Such an arrangement is particularly convenient where one wishes to evaluate the energy for all wave-lengths longer than some specified wavelength, the procedure being to use a short-wave cut-off filter with the black-body detector. If one wishes to obtain an evaluation of energy for all wave-lengths shorter than some specified value, it is possible to use a differential thermocouple where the two components are opposed and produce no deflection if equally illuminated. If one junction is then covered by a short-wave cut-off filter and the other by a transparent material having the same transmission characteristics for all warelengths longer than the region of cut-off, the response will then correspond to the difference in energy transmitted by the two filters. A similar method may be employed for securing sensitivity to a band of wavelengths. Two short-wave cut-off filters are employed having the same transmission characteristics for the long-wave-length region. The sensitivity of the combination will then correspond to the difference between the transmissions of the two filters. A detector of this type has been developed by Coblentz, which has been adopted internationally as the standard method of measurement of radiation lying chiefly in the range from 2400 to $3200 \AA$.

Figure 30, upper section, shows the transmission characteristics of the following materials: quartz, corex A, corex D, and a special barium flint glass of the type chosen by Coblentz (26). In the middle section, the corresponding difference curves have been plotted (full line), which when used with a differential thermocouple, constitute relative-sensitivity curves. Thus, $Q-B F$ is the quartz-barium-flint combination chosen by Coblentz and recently adopted as the international standard of ultraviolet measurement. It will be noted that this combination is practically nonselective from 2500 to $3100 \AA$, a region of great biological importance. The cut-off, however, is not so sharp as might be desired. Dropping fairly abruptly to $3600 \AA$, it extends with appreciable sensitivity to $3900 \AA$ or even as far as $4400 \AA$, depending on the particular filter. The barium-flint filter indicated here and referred to in Table 16 differs slightly from that used by Coblentz. When used with a mercury arc, no great correction is necessary if one assumes that the measurement is for $3130 \AA$ 
and shorter wave-lengths, $2536 \AA$ being the last line of any great intensity emitted by the mercury arc. Referring to Table 16, we find from

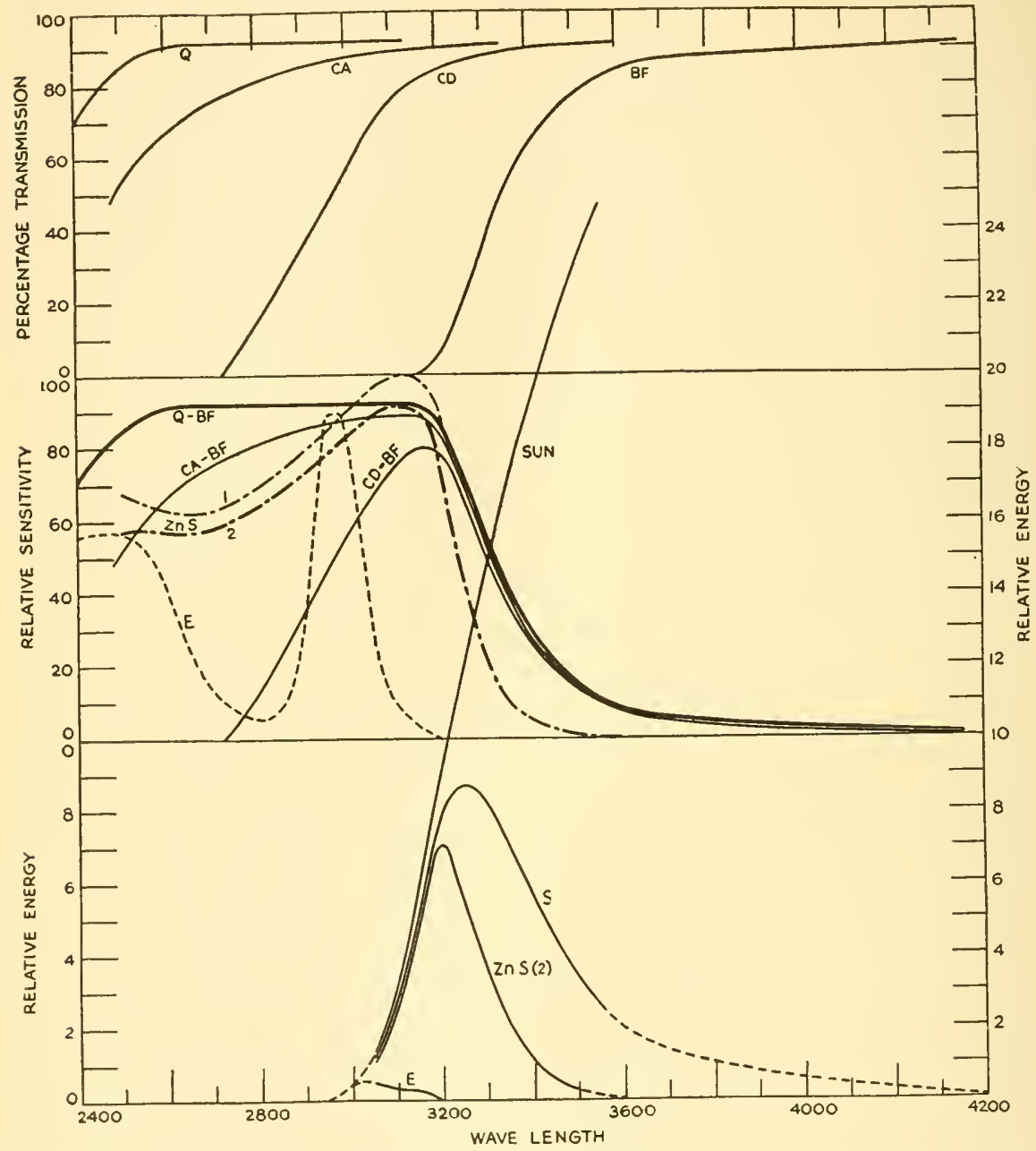

F1G. 30.-Upper section-transmission of filters: $B F$-barium flint, $3.18 \mathrm{~mm}$. (thickness) (50); $C D$ - corex D, $2.3 \mathrm{~mm}$. (21); $C A$-corex A, $2.86 \mathrm{~mm}$. (21); $Q$ - fused quartz, $4.7 \mathrm{~mm}$. (21). Center section-sensitivity of detectors and erythemal response curve: $Q-B F$, balanced thermocouple with quartz and barium-flint filters; $C A-B F$, balanced thermocouple with corex A and barium-flint filters; $C D-B F$, balanced thermocouple with corex $\mathrm{D}$ and barium-flint filters; $E$, erythema (24); ZnS-1, lithopone A (13) (Brickwedde); ZnS-2, balanced zine sulphide method with quartz and barium-flint filters. Lower section-response to solar energy: Sun, solar energy (28) (Forsythe and Christison); S, response of standard balanced thermocouple to solar energy; ZnS-2, response of balanced zinc sulphide detector to solar energy; $E$, erythemal response to solar energy.

Column 7 that the amount detected by this method in microwatts/ square centimeter at $25 \mathrm{~cm}$. from a $2.0-\mathrm{cm}$. midsection of arc shows a 
calculated difference from the value 1362 , obtained by absolute measurement of the lines in the region of $3130 \AA$ and shorter wave-lengths, of 18 per cent, and an observed difference of 15 per cent. Where correction factors computed from the transmission curves are applied, our observations on standard-type mereury ares have indieated an error of the order of 2.5 to 3 per cent. If, however, a great intensity exists in the region of cut-off, much more serious errors will be introduced.

For instance, if one is concerned with the measurement of solar energy in the region specified, much greater difficulties present themselves. Apparent solar distribution is shown in arbitrary units indicated on the right; the relative distribution of response, as measured by the international ultra-violet standard, is indicated by Curve $S$ in the lower section. Obviously, much the greater part of the energy lies in the region of wave-lengths longer than $3200 \AA$. Consequently, corrections of a rather large order must be applied in order to seeure an evaluation of the extreme region.

In the curve $C D-B F$, we have a typical case of a band selectivity secured by the differential thermocouple method, combining corex $D$ with barium flint. In using such a method, however, precautions must be taken to avoid a change in seleetivity resulting from an increased opacity of the glass due to ultra-violet exposure.

\section{PHOTOCELL}

By far the commonest selective detector in use is the photoclectric eell. Commercially, a wide wave-length range of sensitivity has been desirable. The majority of such cells employ a sensitive surface composed of cesium on cesium oxide on silver. Such a sensitive surface is placed in an evacuated tube and a potential applied between an auxiliary electrode and the sensitive surface. When illumination falls upon the sensitive surface, electrons escape and produce a current between the electrodes. This is measured either directly by a galvanometer or indirectly by means of an amplification circuit. Such a detector is capable of the greatest sensitivity of any known method. If the intensities are extremely small, the individual electrons may be counted by the method developed by Geiger and Müller (58).

Figure 31 shows the sensitivity curves of a group of commereially available photocells. The full-line curves 1 to 4 are representative of the various types of cells of cesium on cesium oxide on silver, where the tube is completely evacuated and the surface sensitized by a special process. These present two regions of maximum sensitivity, one lying near $3600 \AA$, and the other varying from 7000 to $8700 \AA$. They all present a minimum sensitivity between the two maxima in the region of $5000 \AA$. A large amount of technical investigation has been expended in order to 


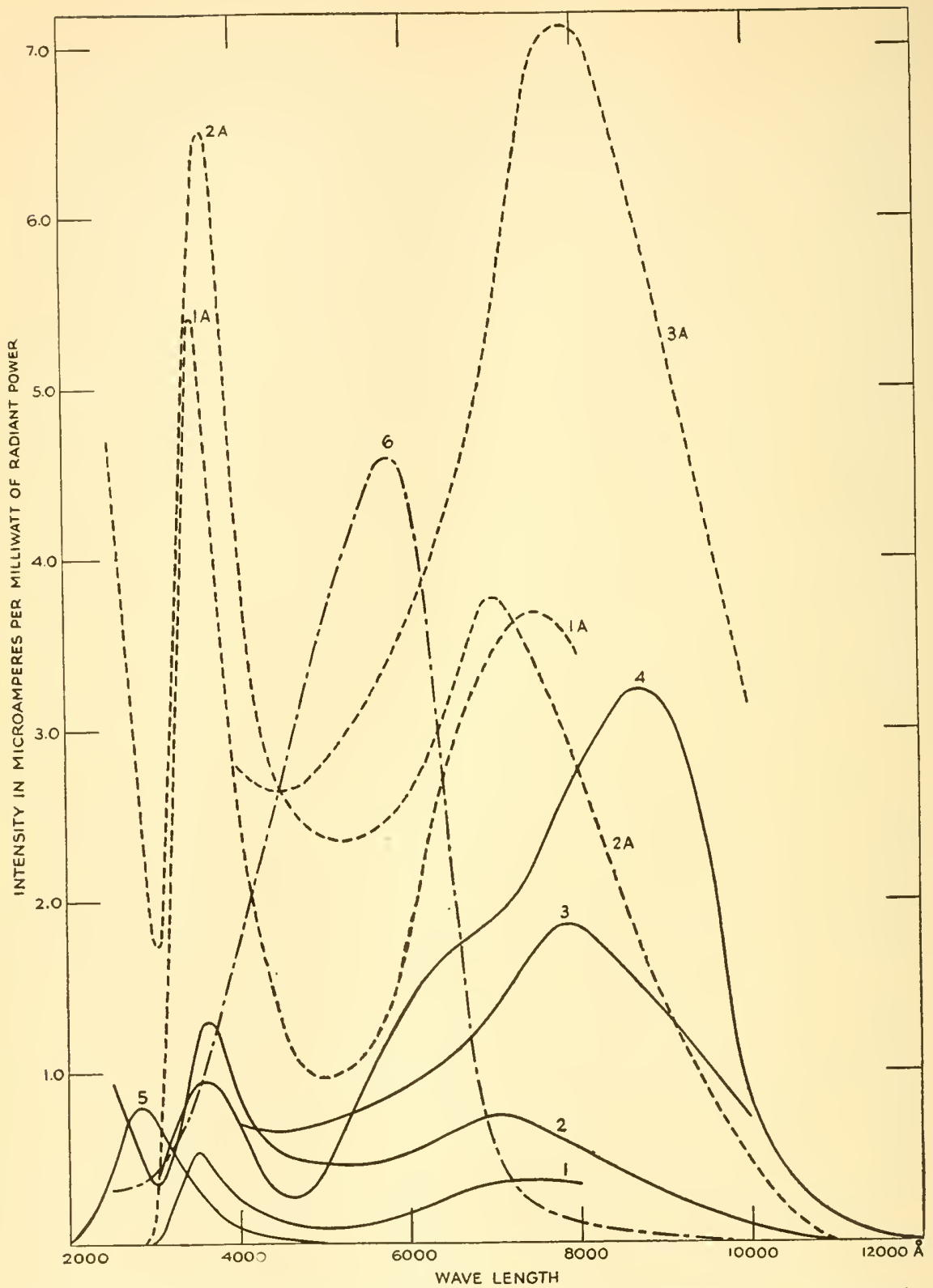

FIG. 31.- Relative-sensitivity curves of commercial photocells (in each case the area in sq. in. and the name of the manufacturer are given within parentheses): 1. PJ-22 vacuum $(0.9$, Gen. Elec.); 1A. PJ-23 gas-filled $(0.9$, Gen. Elec. $) ; 2$. Visitron type AV, quartz envelope, vacuum $(1.1, G . M.) ; 2 A$. Visitron type $\mathrm{A}$, quartz envelope, gas-filled $(1.1, G . M$.$) ;$ 3. Western Electric, vacuum (Western El.); $3 \mathrm{~A}$. Western Electric, gas-filled (Wcstern El.); 4. FJ-114 (0.9, Gen. El.); 5. FJ-76 (2.1, Gen. El.); 6. Weston Photronic cell (1.7 Weston El.). 
minimize this weakness. The short-wave-length limit of sensitivity arises from the absorption characteristics of the windows. If a quartz window is used, the sensitivity rises again in going from 3000 to $2400 \AA$. The long-wave-length limit occurs somewhere between 11,000 and 12,000 $\AA$. Number 5 shows the typical sensitivity of an alkali metal cell, employing a sodium surface. In all such cells, the sensitivity varies widely, depending upon the methods of manufacture. Individual cells of the same type also vary over a considerable range. If such a photocell is filled with gas instead of evacuated, the primary electrons produce secondary electrons in the gas and a correspondingly higher sensitivity results, the increase generally varying from four to ten times. The dotted eurves indicate the sensitivity of corresponding gas-filled cells. Another type of photoelectric cell deserves particular mention, namely, the photovoltaic cell. That a cuprous oxide rectifier cell $\left(\mathrm{Cu}_{2} \mathrm{O}\right.$ on $\left.\mathrm{Cu}\right)$ shows a response to light was observed by Grondahl in 1927 (33). Since then, such cells have been widely developed experimentally as light detectors $(10)$. They consist of two or three thin layers in close contact, and in common practice they are not evacuated. Another example is iron on selenium on lead, the iron being so thin as to be practically transparent.

The sensitivity curve of a commercial photovoltaic cell (Weston photronic) is indicated on a reduced scale by the dash-dot curve, No. 6, Fig. 31. The sensitivity values for this cell must be multiplied by 10 . The cell of copper oxide on copper shows a longer wave-length maximum and extends deeper into the infra-red.

The type of cell indicated by curve 6 has received wide application for three reasons: (a) As it generates electromotive force in the presence of light, it need only be connected with a galvanometer in order to secure a deffection. (b) Such cells produce a current higher by an order of magnitude than other types of photoelectric cells, and consequently require a less sensitive current-measuring device. (c) It exhibits a sensitivity curve very similar to the visibility curve of the eye, and hence has a wide applicability for illumination purposes.

If it is possible to prepare special cells or to secure them, a wide range of sensitivity characteristics is available. Figure 32 is representative of the general possibilities. Section (1) of this figure (39) shows the relativesensitivity characteristics of the different alkali metals, exhibiting maximum sensitivity varying from 2800 to $5500 \AA$, with long-wave-length thresholds varying from $5000 \AA$ into the infra-red. For photocells whose long-wave-length thresholds occur in the blue and ultra-violet, a number of possibilities are indicated in (2) and (3) of this figure (data through courtesy of H. C. Rentschler, Westinghouse Electric Co.). The shortwave-length limits of these cells, as indicated, arise chiefly from the win- 
dows employed. Thus, in the case of cesium, thalium, and uranium, Fig. 32 , (3), the sensitivity continues to rise from 3000 to $2400 \AA$, as shown by the dotted lines where a quartz window replaces the customary thin

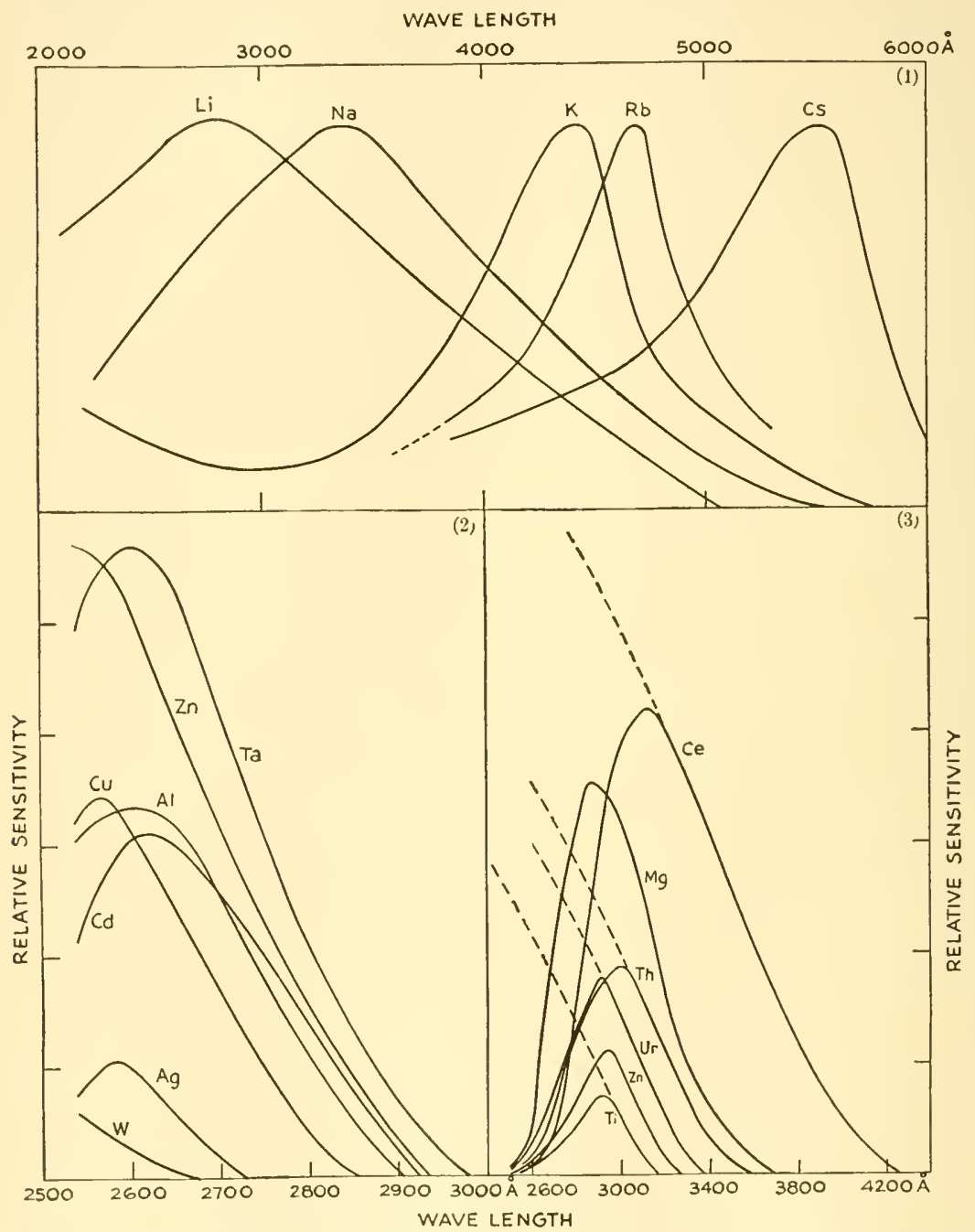

Fig. 32.- Relative sensitivity of special photocells: Sec. (1) alkali metals (Hughes and DuBridge); Li, Na, K, Rb (Pohl and Pringsheim); Cs (Campbell and Ritehie). Sec. (2) and Sec. (3) Westinghouse ultra-violet photoelectric cells. (Rentschler.) Full-line curves in thin glass; dotted curves in quartz.

glass window. From this group, a photocell can be selected having its long-wave-length threshold anywhere from 2675 to $4200 \AA$. By combination with short-wave cut-off filters indicated in Table 10 and Fig. 9, any required short-wave-length limit can be secured. Hence, by a 
combination of photocell and short-wave cut-off filter, a band of sensitivity can be secured anywhere in the visible or ultra-violet. It should be pointed out, however, that the materials indicated in Fig. 32, (2) are markedly superior in reproducibility of sensitivity to any of the other materials.

Through refinement of manufacture, the commercial cells, however, have been brought to a high degree of perfection. Even so, the experimenter must be cautioned in the use of photocells. No two photocells are alike, nor is any photocell permanent. The sensitivity curve of the particular cell must be determined at the time of use by means of a standard source. For an exhaustive discussion of photocell technique, the reader is referred to Hughes and DuBridge (39).

\section{PHOTOCHEMICAL METHODS}

Another general type of selective detector is the photographic or photochemical method. Such a method derives its wave-length sensitivity from the photochemical sensitivity of the reaction. Thus, the darkening of the photographic plate may serve as an indicator for the range of sensitivity of the plate. Photographic plates are of course readily prepared with a wide range of sensitivity distribution. (See Eastman Kodak Co. literature.) These in turn may be combined with various filters in order to secure modified ranges of selective response. Such methods, however, are nonlinear as to intensity and depend upon the time of exposure. They are, therefore, nonlinear indicators of time $\times$ intensity dosages. It is difficult to bring them in line with other quantitative methods. They require the difficult operation of evaluating darkening. However, because of their convenience and simplicity, they may be recommended for restricted types of observation. These methods have been highly developed for spectroscopic purposes and are, when properly used, capable of high precision. For a comprehensive discussion of this field, the reader is referred to the works of Harrison (37) and of O'Brien (55).

Another photochemical method deserves particular mention. When lithopone or better, pure zinc sulphide, moistened with lead acetate, is exposed to the ultra-violet, a marked darkening takes place. In order to make the results of this method reproducible, only the purest chemicals must be used. The darkening must be brought to a certain specified standard value and the time required accurately determined. This method has been extensively applied to solar observations by Clark. In order to give an idea of the sensitivity distribution, values obtained by Brickwedde (13) are plotted in the middle section of Fig. 30, marked $\mathrm{ZnS}-1$. In order to further restrict the long-wave-length cut-off, Clark (18) developed a differential method in which the time of darkening is 
obtained both under glass $T_{g}$ and under quartz $T_{q}$. Since the effective intensity is inversely proportional to the time of darkening,

$$
\text { Intensity }=\left(\frac{1}{T_{q}}-\frac{1}{T_{g}}\right) \times C
$$

where $C$ is a calibration factor. By the use of a mereury arc, Clark arrived at a calibration factor,

$$
C=72 \times 10^{4} \mathrm{erg} / \mathrm{cm}^{2}
$$

The relative wave-length sensitivity of this differential zinc sulfide method is given by curve ZnS-2 in the center seetion. While this method is more restricted as to wave-length range than Coblentz's differential thermocouple method, it is nonlinear over the whole region, showing a minimum in the region of $2650 \AA$ and a maximum at $3130 \AA$, falling rapidly between 3200 and $3300 \AA$, and is practically zero at $3500 \AA$. This selectivity might be a definite advantage in case the biological effectiveness of dosage were to closely follow the sensitivity distribution. Otherwise, it is rather difficult to evaluate its significance. Measurements by this method have been specified in terms of zinc sulfide units. This procedure is good, inasmuch as both wave-length distribution and magnitude are implied. But without a standardization as to glass filter and an accurate determination of the sensitivity distribution of a standard method of procedure, it is difficult to convert these observations into other units. Thus, the curves previously shown for daily and seasonal fluctuation in solar ultra-violet give an excellent idea of the fluctuations, but the wave-length range represented is in a large measure indeterminate, following roughly the curve shown in the lower section of Fig. 30, marked ZnS-2. The maximum response for the particular solar distribution assumed [taken from Forsythe and Christison's observations (28)] occurs at $3200 \AA$ and drops to zero roughly at $3600 \AA$. When, however, the short-wave cut-off of solar distribution varies, the wave-length of maximum response will also vary.

\section{APPLICATION OF SELECTIVE DETECTORS}

We may now ask the general question: What are the value and range of applicability of selective detectors, granting that we have quantitative reprodueible methods? They are valuable as control instruments for determining irradiation and dosage. The moment the sensitivity as a function of wave-length of a biological phenomenon becomes known, it is of ten possible to eonstruct a selective deteetor whose sensitivity curve matches in wave-length extent and distribution the sensitivity curve of the phenomenon. With such an instrument, we may evaluate the effectiveness of a given source in producing such a biological effect and 
we may evaluate the dosage, i.e., the total amount of energy over a specified time, which has contributed to the biological effect. Measurement then becomes theoretically proportional to effectiveness. Such observations are not, however, true energy measurements, but weighted energy measurements. If the selectivity curve of the detector departs from the sensitivity curve of the phenomenon, the result will be erroneous. It is impossible to emphasize too greatly the need of caution in the use of such methods. Where such a method becomes thoroughly standardized, names are generally given to weighted energy measurements, the units being proportional to effectiveness. The most completely worked out system of this character is the illuminating system referred to above. The visibility curve of the standard observer (full-line curve $V_{\lambda}$, Fig. 33, upper section) is the standard weighting coefficient for the phenomenon vision. Wherever the sensitivity curve or weighting is known, absolute energy measurements may be converted to the special units of the system. Too often, however, the fact that a weighting curve must be employed is overlooked and the observations for a particular phenomenon used for purposes to which they are not applicable. This has been responsible for much of the chaotic condition of radiation measurements.

Where one wishes to know the absolute sensitivity of a photocell, one is generally given values in lumens. Yet, with few exceptions, the sensitivity curves do not in the least correspond to the visibility curve. Consequently, the response of such an instrument will vary widely with the type of source used. Unless the source is specified, observations indicated in illumination units are absolutely meaningless. In our efforts to present quantitative data on photocells, we find satisfactory data arailable in only two or three cases. Relative-sensitivity curves are generally given, but the absolute magnitude of the response can be arrived at in only a very approximate fashion. Consequently, the curves presented must not be used for quantitative measurements but only for guidance in selection of cells. It is therefore with some hesitation that the application of selective detectors to a number of biological problems will be illustrated. Thus,

Vision.-The sensitivity curve of vision may be approximated as indicated in Fig. 33 by combination of photronic cell with filter. Such a detector will produce deflections proportional to illumination, provided, (a) the sensitivity curve of the eye has been accurately duplicated by the sensitivity curve of the combination; $(b)$ the response is linearly proportional to the illumination. Neither of these requirements is generally satisfied. Thus, the dotted curve in the upper section of Fig. 33 is an exceptionally good approximation to the standard observer. Nevertheless, applied to sources of selective emission, it might yield results greatly in error if lines occurred in the region from 4000 to $5200 \AA$. 


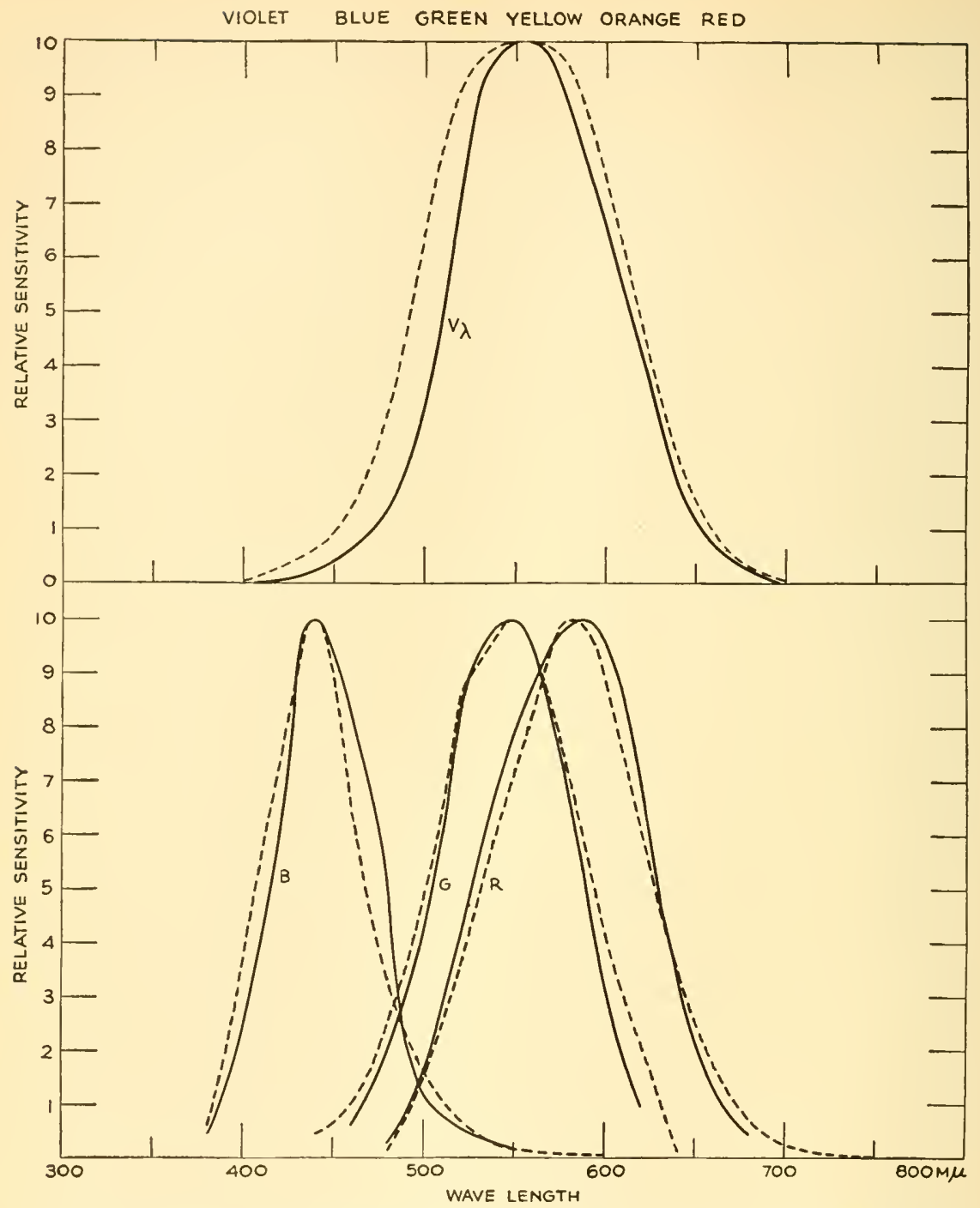

FIG. 33.--Visibility and special detectors: V $\lambda$-visibility; dotted curve - sensitivity of Weston photronic cell + heat resisting heat absorbing dark, $2.82 \mathrm{~mm}$. thickness + Noviol O, $16 \mathrm{~mm}$. thickness (Corning filters). Color evaluation: Full-line curves-1922 standard observer: $B$-blue; $G$-green; $R$-red; sensitivity reduced to same maximum. Dotted curves - corresponding special detectors: Blue - heat resisting lantern blue, $3.0-\mathrm{mm}$. thickness + Noviol O, 3.0-mm. thickness + copper chloride $1 / 2 N$ in 1.0-cm. cell; Green-signal green* + signal yellow, thin* + copper chloride $1 / 10 N$ in $1.0-\mathrm{cm}$. cell; Red-signal yellow, thick* + heat resisting heat absorbing dark, 2.82-mm. thickness + copper chloride $1 / 10 N$ in $1.0-\mathrm{cm}$. cell.

* Manufacturer does not specify thickness, due to variability of color with melt. 
Color Vision.-Figure 33, lower section, shows combinations which approximate the trichromatic sensitivity curves of the 1922 standard observer. Such arrangements offer a possibility for the quantitative determination of the color characteristics of materials. In order to arrive at such quantitative results, the following procedure is necessary: (a) The combined sensitivity, filter plus detector, of the particular arrangement to be used must be determined; $(b)$ on the basis of such actual sensitivity curves, conversion factors must be computed by which the corresponding color data can be found; $(c)$ the sensitivity characteristics of the apparatus must be verified from time to time.

For calibration and computation of conversion factors, the reader is referred to the Bureau of Standards (42).

Ultra-violet Phenomena.-The various biological effects of radiation in the ultra-violet have been lumped together and an attempt made to arrive at a universal system of measurements intencled to be applicable to all of these various effects. Probably, a group of such effects arises from ionization within the cell. Furthermore, since cells possess certain common characteristics, there is some measure of similarity in the region of effectiveness of these different phenomena. The essential differences may, however, be just the points of paramount importance. In order to bring out these characteristic differences, the relative wavelength distribution curves for a number of these phenomena have been brought together in Fig. 34. The full-line curves in the upper section indicate the percentage of bacteria killed for equal energy dosage: (a) $50 \mathrm{erg} / \mathrm{mm} .^{2}$, (b) $100 \mathrm{erg} / \mathrm{mm} .^{2}$, (c) $250 \mathrm{erg} / \mathrm{mm} .{ }^{2}$, (d) $500 \mathrm{erg} / \mathrm{mm} .{ }^{2}$ for staphylococcus, observed by Gates (32). Dotted curve, e, Fig. 34 shows the relative erythemal effectiveness as compiled from a group of observers by Coblentz and assoeiates $(24,25)$. Dash-dot curves 1, 2, and 3 are equal-energy lethal-effectiveness curves for the alga Chlorella vulgaris, obtained by Meier (52). While these follow fairly closely the bactericidal curves, the dosages corresponding are greater by an order of magnitude or more. Exact comparison is impossible beeause the percentage killing could not be determined in the densitometer methods applied to the algae. In the center section, curve $A$ gives the absorption coefficient for nonirradiated ergosterol, coneentration $80 \mathrm{mg}$. per liter, and for comparison, Curve $B$, the first product of irradiation (2800 to $3100 \AA$ ) by Reerink and van Wijk (60). While there is some rough similarity between the absorption curve $A$, which is probably related to the activation of sterols in the production of vitamin D and the lethaleffectiveness curves, one finds little similarity with the erythemal curve. Though we might be well justified in developing a method for evaluating the lethal effectiveness of different sources, there seems to be little basis for an evaluation of therapeutic effectiveness. It does not follow that 


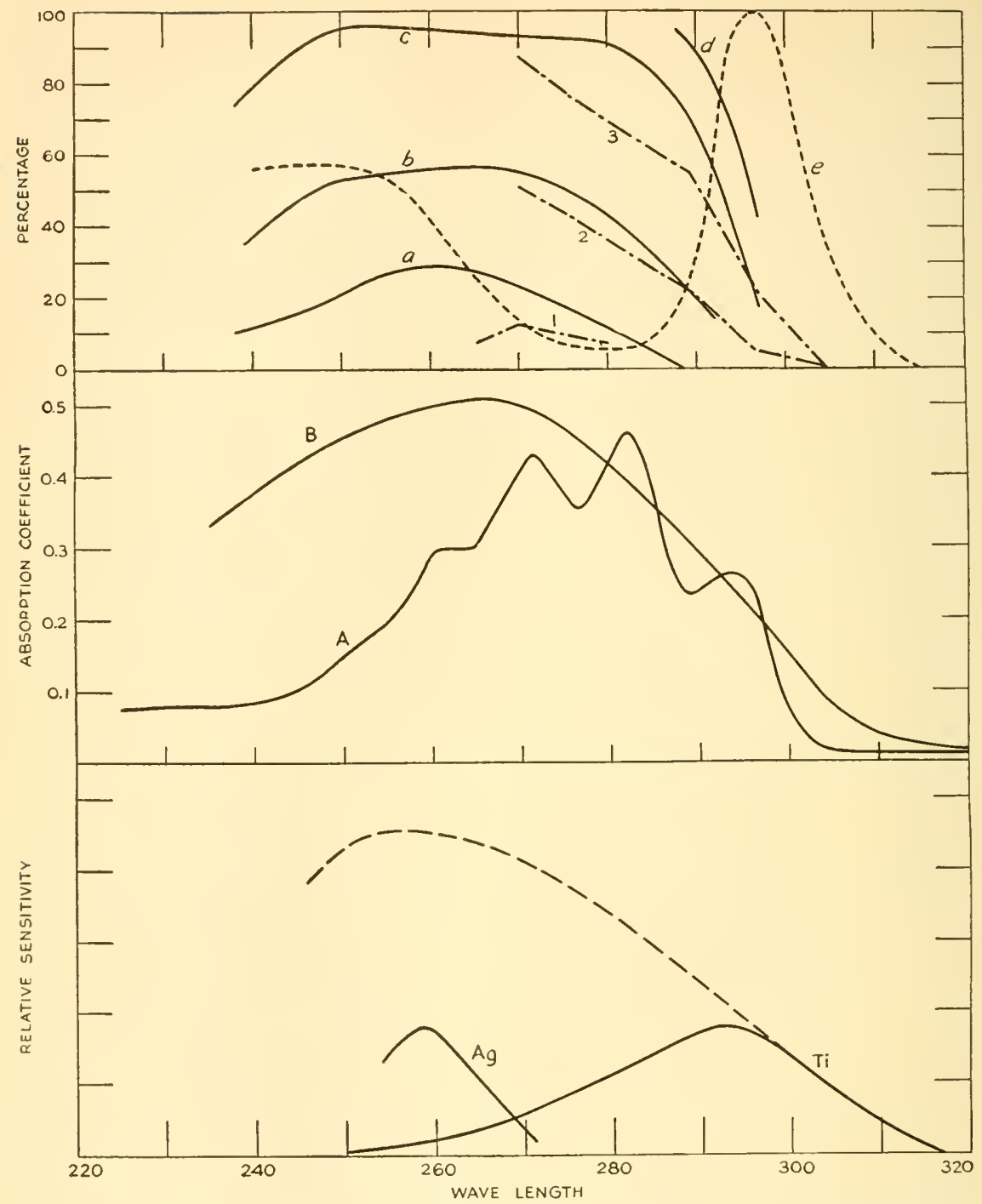

Fig. 34.-Upper section: Full-line curves-lethal effectiveness for staphylococcus. (Gates.) Dosage: $a, 50 \mathrm{erg} / \mathrm{mm}^{2} ; b, 100 \mathrm{erg} / \mathrm{mm}^{2} ; c, 250 \mathrm{erg} / \mathrm{mm}^{2}{ }^{2} ; d, 500 \mathrm{erg} / \mathrm{mm} .{ }^{2}$ Dash-dot curves - relative lethal effect for the alga Chlorella vulgaris for different dosages. (Meier.) Dotted curve $e$, erythemal effectiveness. (Coblentz.) Middle section: $a$, Absorption coefficient of unirradiated ergosterol, concentration $80 \mathrm{mg}$. per liter. (Reerink and van Wijk.) b. First product of irradiation. (Reerink and van Wijk.) Lower section: Relative sensitivity of silver and titanium photocells. Full-line curves in corex $D$. Dotted curves in thin corex $A$. 
the photochemical effectiveness in producing vitamin $\mathrm{D}$ should coincide with the absorption curves. In fact, there is considerable evidence that the long-wave-length portion of the absorption curves contributes to the development of antirachitic material, while the shorter wave-length region may be destructive to the biologically active component, so that it may be desirable in evaluating the dosage from a particular ultra-violet source to determine the relative distribution in at least two portions of the region independently. Furthermore, there seems to be little basis for concluding that the therapeutic effectiveness in the application of ultraviolet to a patient necessarily coincides with the production of the biologically active material in vitro. It is still more difficult to see why the erythemal effectiveness should be used for determining the therapeutic value. In fact, one might be definitely detrimental to the other. In such a case, the region of $0.280 \mu$ might be preferable.

Turning again to Fig. 30, the erythemal curve has been reproduced, dotted curve $E$, for direct comparison with the relative-sensitivity curves of the two methods of ultra-violet measurement in most common use, shown by the heary curves, $Q-B F$ and $\mathrm{ZnS}-2$. It is immediately evident that these weighting curves have little in common with the biological effectiveness of any of the phenomena as they are known. The value of such methods lies simply in that they enable an operator to reproduce a given dosage for a given type of source. They will tell us nothing, however, as to the relative effectiveness of two different types of sources. In the ease of the sun, the relative energy effective in erythema has been plotted in the lower section, curve $E$, Fig. 30, for comparison with the relative-response curves of the standard and zine sulfide methods. These points are brought out not as a criticism of the methods which have been developed, which undoubtedly serve a useful purpose in dosage control, but rather to indicate the definite limitations of such methods and the dangers of too wide generalization.

In the lower section of Fig. 34 the relative-sensitivity curves of two photocells have been plotted which may be of especial value in working with phenomena occurring in this region. The wave-length threshold of the titanium cell, occurring at about $3160 \AA$, is very convenient as to both the erythemal threshold and the probable absorption of ergosterol. If the titanium cell is used in thin corex $A$ (dotted curve), its sensitivity continues well beyond the region where any effective radiation is likely to be available from a quartz-housed source. When combined with a suitable glass filter, the short-wave-length limit may be adjusted as desired. Silver, on the other hand, has a threshold in the vicinity of $2750 \AA$, the short-wave-length limit depending again upon the type of window. By a combination of these two cells, the relative intensities in different portions of the region from 2400 to $3200 \AA$ can be fairly well evaluated. 
It has uniformly been the policy of the National Bureau of Standards to evaluate radiation from ultra-violet sources in absolute units, and the effort has been to secure as far as is experimentally possible a method of observation which is nonselective over the region of importance, such as, for example, the balanced-thermocouple method, with the barium-flint filter.

The practical necessity of establishing some criterion for the comparative evaluation of different types of ultra-violet sources for medical purposes cannot be escaped. This has led to the adoption of certain special units. Homogeneous radiation of wave-length $2967 \AA$ and radiant flux density $10 \mu \mathrm{w} . / \mathrm{cm} .{ }^{2}$ has been adopted tentatively by the Council on Physical Therapy (16) as a Finsen unit (F.U.). It has been observed that $2 \mathrm{~F}$.U. for $15 \mathrm{~min}$. is a representative requirement for minimum perceptible erythema. Thus it has been proposed that one erythemal unit be clefined as $2 \mathrm{~F}$.U. or $20 \mu \mathrm{w} . / \mathrm{cm}^{2}{ }^{2}$ for $15 \mathrm{~min}$. of homogeneous radiation of wave-length $2967 \AA$. On this basis, the equivalence of the erythemal unit for various ultra-violet sources has been determined. It should be pointed out that on such a basis the units are both definite and reproducible, but that the equivalence is dependent upon a number of vital considerations. Where a source produces heterogeneous radiation in the region of erythemal effectiveness, the evaluation of equivalence can be no more definite than the evaluation of erythemal effectiveness. Only by the arbitrary adoption of a standard curve of erythemal effectiveness can the erythemal unit be definitely evaluated for other sources. If such a standard weighting curve is adopted, the danger arises that subsequent observations will show that the adopted standard curve does not coincide sufficiently well with the physiological basis to make the determinations of great biological significance. Thus, we are led to the probability of frequent modifications in the standard weighting curve, as has been the experience in the case of the illuminating system.

Furthermore, the difficulty of establishing a satisfactory erythemal weighting curve is far greater than that presented by the determination of visibility. Granting, however, that such a standard weighting curve of erythemal effectiveness is adopted, it must be borne in mind that even small changes in operating conditions of a source will change the radiation erythemal equivalents. These points have been frequently emphasized by Coblentz and must be taken into consideration in using such a table of equivalents as may be found in the publication of the Council on Physical Therapy on The Acceptance of Sun Lamps (16).

More important still, let us reiterate our previous contention that there is no necessary relation between erythemal effectiveness and 
therapeutic value. Consequently, the establishment of a system based on erythemal effectiveness must not be viewed as a solution to the problem of measurement in the ultra-violet for general biological application.

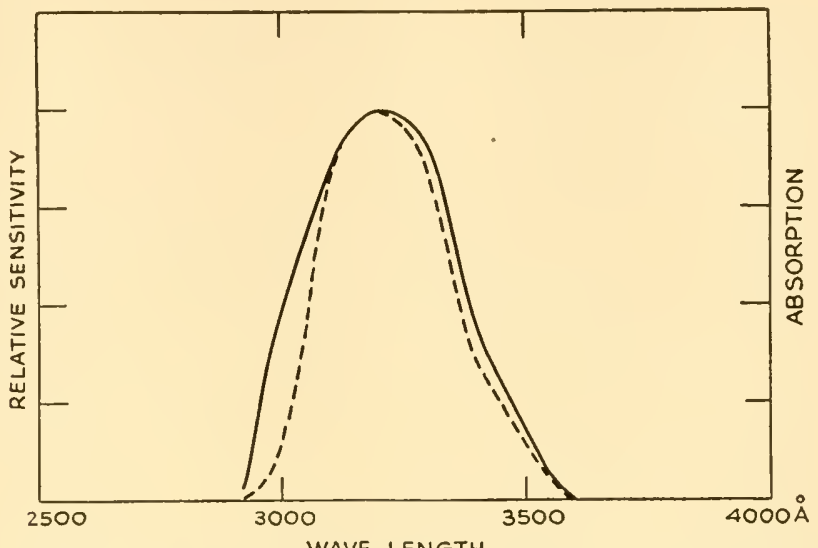

FIG. 35.-Vitamin A absorption and special detector. Full-line curve-absorption curve of vitamin A. (Morton and Heilbron.) Dotted curve-relative sensitivity of thorium photocell + pyrex, 4-mm. thickness

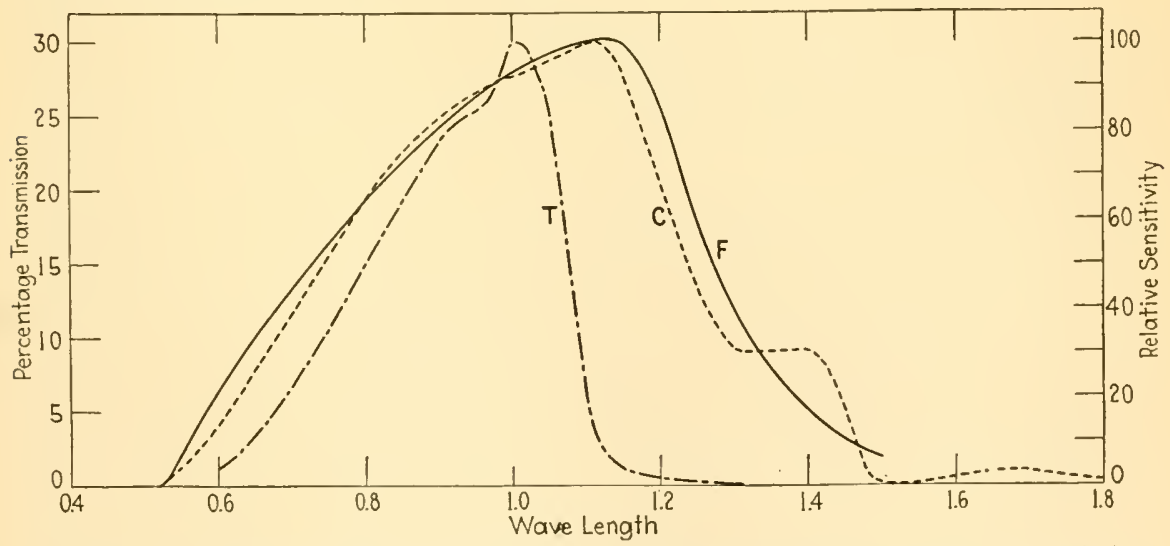

Fig. 36.-Flesh transmission and special detectors. Full-line curve $F$-transmission of $5.0-\mathrm{mm}$. thickness of flesh. (Cartwright, Forsythe et al.) Dotted curve C-thermocouple with filters, amethyst + heat resisting yellow shade yellow. Dash-dot curve $T$ thalofide cell.

Vitamin A.-The absorption of vitamin A is shown in Fig. 35 (54) Energy absorbed in this band eontributes to the destruction of the biologically active material. The absorption eurve can be closely approximated as indicated by the dotted eurve. Such a selective detector would be valuable in evaluating the destructive component of sources used in irradiating materials such as foodstuffs. 
Flesh Transmission.-The transmission curve of a $5.0-\mathrm{mm}$. thickness of flesh determined by Cartwright (17), and corrected for reflection by Forsythe and others, is shown by the full-line curve $F$ in Fig. 36. A thermocouple-filter combination, yielding a very similar curve, is offered, shown by the dotted Curve $C$. Such a detector should be useful in determining the effectiveness of sources in raising the temperature of underlying tissue. It should not be assumed, however, that this is a measure of therapeutic value.

The reliability of measurements of this general type can be no greater than the reliability of the observations of the biological effect. If the response curve of the detector departs markedly from the relative effectiveness of equal energy in producing the specified biological phenomenon, the results may be wholly misleading. We therefore urge great care in the application of selective detectors, such as has been illustrated in this chapter.

In the course of this discussion, we have attempted to outline sound procedure, to offer a convenient assembly of pertinent data, and to emphasize the many sources of difficulty encountered in radiation measurements.

\section{REFERENCES}

1. Аввот, C. G. The quality of diffused skylight. Ann. Astrophys. Observ. Sinithsonian Inst. 2 : 153-157. 1908.

2. Аввот, C. G. The silver dise pyroheliometer. Smithsonian Mise. Collect. 56: 1-9. 1911.

3. Аввот, C. G. New instruments for solar eonstant work. Ann. Astrophys. Observ. Sinithsonian Inst. 4: 45-87. 1922.

4. Аввот, C. G. The radiation of the planet carth to space. Smithsonian Mise. Collect. 82: 1-12. 1929.

5. Аввот, C. G. Radiometer. Ann. Astrophys. Observ. Smithsonian Inst. 5: 97-102. 1932 .

6. Аввот, C. G. Periodicities in solar radiation. Ann. Astrophys. Observ. Smithsonian Inst. 5: (ef. pp. 258, 278, 286). 1932.

7. Аввот, C. G., and L. B. AlDrich. An improved water-flow pyroheliometer and the standard seale of solar radiation. Smithsonian Mise. Colleet. 87 : 1-8. 1932.

8. AldRich, L. B. The melikeron-an approximately black-body pyranometer. Smithsonian Misc. Collect. 72:1-11. 1922.

9. Angströn, КNvт. Ueber absolute Bestimmungen der Wärmestrahlung mit dem elektrisehen Compensations-Pyrheliometer (u.s.w.). Ann. Phys. Chem. N.F. $67: 633-648.1899$.

10. Bergmann, L. von. Ueber eine Selen-Sperrschicht-Photozelle. Physikal. Zeitsch. $32: 286-288.1931$.

11. Brackett, F. S. Graphic correlation of radiation and biological data. Smithsonian Mise. Collect. 87 : 1-14. 1932.

12. BracketT, F. S., and E. D. MCAlister. Automatic recording of the infra-red at high resolution. Rev. Sci. Instruments 1: 181-193. 1930. 
13. Brickwedde, F. G. The light-sensitiveness of zine and silver salts. Jour. Opt. Soc. Amer. $14: 312-322.1927$.

14. Burt, R. C. Scientific Laboratory Bulletin. p. 301.

15. Carr, Emma P., and Mary L. Sherrill. Bibliography of absorption spectra of solutions. Internat. Crit. Tables 5 : 326-35S. 1929.

16. Carter, H. A. (Sec. Council Phys. Ther.). Acceptance of sun lamps. Jour. Amer. Med. Assoc. 102 : 42-44. 1934.

17. Cartwright, C. H. Infra-red transmission of the flesh. (Abst.) Jour. Opt. Soc. Amer. 20: 81-84. 1930.

18. Clark, Janet H. The zine sulfide method of measuring ultra-violet racliation and the results of three years' observations on Baltimore sunshine. Jour. Opt. Soc. Amer. 21 : 240-247. 1931.

19. Совlextz, W. W. Instruments and methods used in radiometry. Bull. Bur. Stand. 4: 391-460. 1908; 9: 7-63. 1913; 14: 507-536. 1919.

20. Coblentz, W. W., and W. B. Emerson. Relative sensibility of the average eye to light of different colors and some practical applications to radiation problems. Bull. Bur. Stand. 14: 167-236. 1918.

21. Coblentz, W. W., and R. Stair. Data on ultra-violet solar radiation and the solarization of window materials. Bur. Stand. Jour. Res. 3: 629-689. 1929.

22. Coblentz, W. W. and R. Stair. Measurements of extreme ultraviolet solar radiation using a filter method. Bur. Stand. Jour. Res. 6: 951-976. 1931.

23. Coblentz, W. W., and R. Stair. The present status of the standards of thermal radiation maintained by the Bureau of Standards. Bur. Stand. Jour. Res. 11: 79-87. 1933.

24. Coblentz, W. W., and R. Stair. Data on the spectral erythemic reaction of untanned human skin to ultra-violet radiation. Bur. Stand. Jour. Res. 12: 13-14. 1934.

25. Coblentz, W. W., R. Stair, and J. M. Hogue. The spectral erythemic reaction of the untanned human skin to ultra-violet radiation. Bur. Stand. Jour. Res. 8: 541-547. 1932.

26. Coblentz, W. W., R. Stair, and J. M. Hogue. Tests of a halanced thermocouple and filter radiometer as a standard ultra-violet dosage intensity meter. Bur. Stand. Jour. Res. 8: 759-778. 1932.

27. Dening, W. E., and F. G. Cottrell. Chart of some electromagnetic relations. Rev. Sci. Instruments 3 : 296-297. 1932.

28. Forsythe, W. E., and Frances L. Christison. Ultra-violet radiation from the sun and from heated tungsten. Gen. Elect. Rev. 32:662-667. 1929.

28a. Forsythe, W. E., and Frances L. Christison. The absorption of radiation from different sources by water and by body tissue. Jour. Opt. Soc. Amer. 20: 693-700. 1930.

29. Forsythe, W. E., and E. M. Watson. Resistance and radiation of tungsten as a function of temperature. Jour. Opt. Soc. Amer, 24: 114-118. 1934.

30. Forsythe, W. E., and A. G. Worthing. The properties of tungsten and the eharacteristies of tungsten lamps. (Abst.). Bull. Lamp Dev. Lab. Gen. Elect. Co. 2: 6-12. 1930.

31. Fowle, F. E. Black body radiation. Internat. Crit. Tables 5 : 239-240. 1929.

32. Gates, F. L. A study of the bactericidal aetion of ultra-violet light. Jour. Gen. Physiol. 13 : 231-260. 1929.

33. Grondahl, L. O., and P. H. Geiger. A new electronic rectifier. Jour. Amer. Inst. Elect. Eng. 46: 215-222. 1927.

34. Hafstad, L. R. The applieation of the FP-54 pliotron to atomic disintegrationstudies. Phys. Rev, 44: 201-213. 1933. 
35. HARDY, J. D. A theoretical and experimental study of the resonance radiometer. Rev. Sci. Instruments 1: 429-449. 1930. (Cf. also 5: 120-124. 1934.)

36. Harrison, G. R. Precision densitometers for photographic photometry. Jour. Opt. Soc. Amer. 10: 157-168. 1925.

37. Harrison, G. R. Current advances in photographic photometry. Jour. Opt. Soc. Amer. 24 : 59-72. 1934.

38. Henning, F., and H. T. Wensel. The freezing point of iridium. Bur. Stand. Jour. Res. 10: 809-821. 1933.

39. Hughes, A. L., and L. A. DuBridge. Photoelectric phenomena. MeGraw-Hill Book Company, Inc.; New York, 1932.

39a. Illuminating engineering nomenclature and photometric standards. Trans. Illum. Eng. Soc. 28 : 265-279. 1933.

40. Ives, H. E. Mechanical equivalent of light. Internat. Crit. Tables 5 : 436-438. 1929.

41. JUDD, D. B. Extension of the standard visibility function to intervals of one millimicron by third-difference osculatory interpolation. Bur. Stand. Jour. Res. 6: 465-471. 1931 .

42. JUDD, D. B. The 1931 I. C. I. standard observer and coordinate system for colorimetry. Jour. Opt. Soc. Amer. 23 : 359-374. 1933.

43. Kimball, H. H. Records of total solar radiation intensity and their relation to daylight intensity. Monthly Weather Rev. 52(10): 473-479. 1924. (See pp. $478,479$.

44. Kimball, H. H. Solar radiation as a meteorological factor. Rev. Mod. Phys. 4: 259-277. 1932.

45. Knudsen, E. Chromatische disperse Zweiphasensysteme unter besonderer Berücksichtigung ihrer Verwendung als Lichtfilter. Kolloid Zeitsch. 66:257-266. 1934.

46. Laurens, H. Physiological effects of radiant energy. Chemical Catalog Company; New York, 1933.

47. Luckiesh, M. Spectral reflectances of common materials in the ultra-violet region. Jour. Opt. Soc. Amer. 19: 1-6. 1929.

48. Marsh, H. E., E. Condon, and L. B. Lozb. The theory of the radiometer. Jour. Opt. Soc. Amer. 11: 257-262. 1925.

49. McAlister, E. D. The spectrum of the neutral mercury atom from $1 \mu$ to $2 \mu$. Phys. Rev. 34: 1142-1147. 1929.

50. McAlister, E. D. Absolute intensities in the visible and ultra-violet spectrum of a quartz mercury are. Smithsonian Misc. Collect. 87: 1-18. 1933.

51. McAlister, E. D. (Unpublished data from Division of Radiation and Organisms. Smithsonian Inst. 1934.)

52. Meier, Florence. Lethal action of ultra-violet light on a unicellular green alga. Smithsonian Misc. Collect. 87: 1-11. 1932.

53. Mestre, Harold. Absorption of energy by leaves and algae. Cold Spring Harbor Symposia on Quantitative Biology 3 (in press). 1935.

54. Morton, R. A., and I. M. Heilbron. The absorption spectrum of vitamin A. Biochem. Jour. 22 : 987-996. 1928.

55. O'Brien, Brian, and V. L. Parks. Photographic reciprocity and intermittency defects near the long wave-length limit of plate sensitivity. (Abstr.) Phys. Rev. 41: 387-388. 1932.

56. Report Opt. Soc. Amer. Progress Committee for 1922-1923. Spectrophotometry. Jour. Opt. Soc. Amer. 10: 169-242. 1925.

57. Report Division of Radiation and Organisms. Rept. Smithsonian Inst. (Appendix 8) 1931, pp. 125-137. 1932. 
58. Rajewsky, B. von. Ueber einen empfindlichen Lichtzähler. Physik. Zeitsch. $32: 121-124.1931$.

59. ReErink, E. H., and A. van WiJk. Isolation of a crystalline antirachitic reaction product from irradiated ergosterol. In Chem. at Centen. Mecting Brit. Assoc. Adv. Sei. 1931, pp. 156-158. Heffer and Sons; Cambridge, 1932.

60. Reerink, E. H., and A. van Wijk. The vitamin D problem. Biochem. Jour. 23: 1294-1307. 1929; 25: 1001-1010. 1931 .

61. Rockwood, R. S., and R. A. SAwYER. The ultra-violet transmission coefficient of the earth's atmosphere. Jour. Opt. Soc. Amer. 22: 513-524. 1932.

62. Roeser, Wm. F., F. R. Caldwell, and H. T. Wensel. The freezing point of platinum. Bur. Stand. Jour. Res. 6: 1119-1129. 1931.

63. Smithsonian Phys. Tables. Smithsonian Misc. Collect. 88: (Cf. pp. 507-510.) 1933. (Prepared by F. E. Fowle.)

64. Smithsonian lnst., Unpublished data, Div. Radiation and Organisms. 1934.

65. Wensel, H. T., D. B. Judd, and W. F. Roeser. Establishment of a scale of color temperature. Bur. Stand. Jour. Res. 12: 527-536. 1934.

66. Wensel, H. T., W. F. Roeser, L. E. Barbrow, and F. R. Caldwell. The Waidner-Burgess standard of light. Bur. Stand. Jour. Res. 6: 1103-1117. 1931 .

67. Wensel, H. T., W. F. Roeser, L. F. Barbrow, and F. R. Caldwell. Derivation of photometric standards for tungsten filament lamps. Bur. Stand. Jour. Res. 13 : 161-168. 1934.

68. Wood, R. W. Physical Optics. 846 pp. (Cf. p. 16.) Macmillan \& Company; London, 1934.

69. Wulf, Oliver. The determination of ozone by spectrobolometric measurements. Smithsonian Misc. Collect. 85 : 1-12. 1931.

70. Zscheile, F. P. An improved method for the purification of chlorophylls A and B; quantitative measurement of their absorption spectra; evidence for the existence of a third component of chlorophyll. Botan. Gaz. 95: 529-562. 1934. Manuscript received by the editor August, 1934. 



\title{
THE INTENSITY OF SOLAR RADIATION AS RECEIVED AT THE SURFACE OF THE EARTH AND ITS VARIATIONS WITH LATITUDE, ALTITUDE, THE SEASON OF THE YEAR AND THE TIME OF DAY
}

\author{
Herbert H. Kimbali \\ Blue Hill Meteorological Observatory of Harvard University \\ and Irving F. Hand \\ U. S. Weather Bureau
}

Introduction. Measurements of solar-radiation intensity at normal incidence. The vertical component of the total solar radiation (dircet + diffuse) receivcd at the surface. of the earth: Solar-radiation intensities in tropical, subtropical, and Arctic rcgions-The effects of cloudiness on the total solar radiation reccived on a horizontal surface. Summary. References.

\section{INTRODUCTION}

This paper treats of the intensity of solar radiation as received at the surface of the earth. The subject is divided naturally into two main topics, as follows: (a) The intensity of direct solar radiation as received on a surface normal to the incident solar rays; $(b)$ the intensity of the total solar radiation (direct + diffuse) as received upon a horizontal surface.

The Standard of Pyrheliometry.-In the United States, the pyrheliometric standard has been the Smithsonian Standard of Pyrheliometry of 1913 (1). In Europe the Angström compensation pyrheliometer (5) is extensively used as a standard. The ratio, Smithsonian standard/ Ångström standard $=1.035$, has been employed at some European observatories, notably at the observatory at Davos, Switzerland (6), to reduce measurements by the Angstrom pyrheliometer to the Smithsonian scale. It is necessary, therefore, in comparing pyrheliometric measurements made in different countries, to have in mind this difference in standards. Recently the Smithsonian Institution revised its standardization tests, and announces that its standard pyrheliometry of 1913 is about 2.3 per cent high (2). On account of the long series of measurements and deductions therefrom already published, it was considered less confusing to adhere to the 1913 scale of pyrheliometry, but to make clear the amount by which it is in error. The U. S. Weather Bureau has 
followed the Smithsonian Institution in adhering to the 1913 standard. In this paper radiation intensities are expressed in units of this scale unless some other scale is specified.

\section{MEASUREMENTS OF SOLAR-RADIATION INTENSITY AT NORMAL INCIDENCE}

Figure 1 illustrates the marked increase in solar-radiation intensity with altitude above sea level. The horizontal lines indicate the proportion of the radiation reaching the outer limit of the atmosphere that is transmitted to a place of observation, with the sun at different zenith distances; or, conversely, the depletion of radiation intensity in passing through the atmosphere is shown. With the sun in the zenith, the solar rays reach the earth by the shortest possible path, and the air mass passed through is called 1.0. With the sun at zenith distance $60^{\circ}$, the path is twice as long and the air mass is 2.0 . With the sun at zenith distance $70.7^{\circ}$, the air mass is 3.0 , and for greater zenith distances the air mass increases much more rapidly, reaching a value of approximately 30 when the sun is just above the horizon. ${ }^{1}$

The path is through much less atmosphere on a high mountain than at sea level, and in some investigations unit air mass is considered to be with the sun in the zenith with air pressure $760 \mathrm{~mm}$. If, therefore, the air pressure is only $684 \mathrm{~mm}$., with the sun in the zenith, and at zenith distances $60.0^{\circ}$ and $70.7^{\circ}$, respectively, the corresponding air masses will be $0.9,1.8$, and 2.7 , and with atmospheric pressure $456 \mathrm{~mm}$., the air masses will be $0.6,1.2$, and 1.8 , etc.

The curved lines in Fig. 1 clearly show the decrease in atmospheric depletion with increase in altitude at the stations named, and its increase with solar-zenith distance. The effect of increase in altitude is much more marked near sea level than on high mountains, partly because at elevated stations the pressure decrease for a given increase in elevation is less, and partly because the moisture and dust content of the atmosphere is much less. Dust has a marked diffusing effect, and water vapor absorbs strongly in the infra-red, or long-wave radiation (see Brackett, pages 171-175).

Curves 25 and 27, Fig. 1, for Washington in February and June, respectively, show much greater depletion in summer than in winter, as also do curves 21 and 26 for Lincoln, Neb., for February and August. For Davos, Switzerland, at an elevation of 1600 meters, lines 19 and 20, show for February and June, respectively, much less difference in radiation intensities than has been indicated for stations at lower elevations.

${ }^{1}$ For a more detailed statement of the relation between solar-zenith distanee and air mass, see Smithsonian Meteorological Tables, 5th revised Ed., 1931, p. lxxix, and Table 100, p. 226. 
In eomputing the atmospheric depletion of solar radiation as shown in Fig. 1, the effect of the varying distance of the earth from the sun has been eliminated. In considering the average intensities actually measured at different seasons of the year, however, the effect of this rariable distance must not be overlooked. Thus, in early July the distance of the earth from the sun is so much greater than in early January that with the same air masses, other conditions being equal, early January solarradiation intensities should exceed July intensities by 7 per cent, while in early April and early October the earth is at its mean distance from the

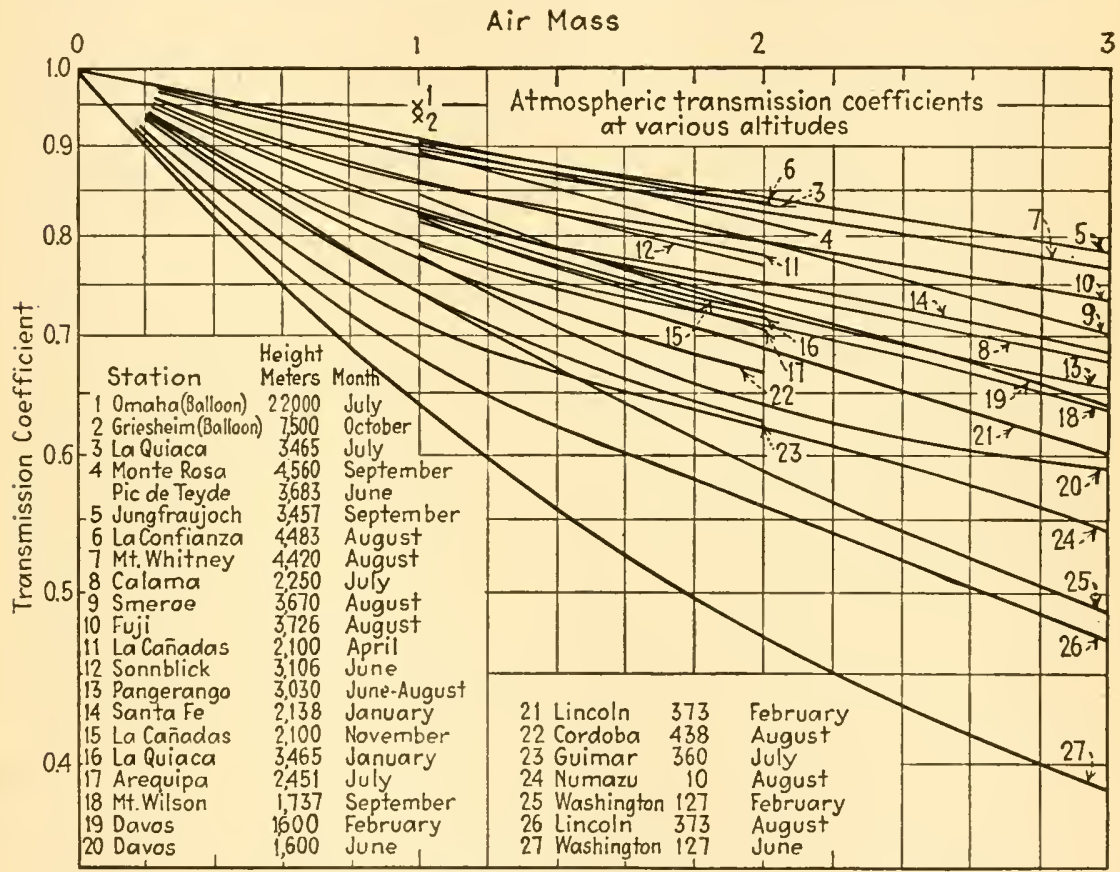

FIG. 1.-Atmospheric transmission coefficients at various altitudes.

sun. As an illustration of the effect of this varying solar distance of the earth, in Table 1 it is shown that at Washington, on August 22 and on December 23, the measured noon solar-radiation intensity averages the same, or $1.19 \mathrm{gm}$. cal. $/ \mathrm{min} . / \mathrm{em}^{2}$. When reduced to mean solar distance, however, the August intensity becomes 1.217, and the December intensity 1.151 , or 62.7 and 59.3 per cent, respectively, of the mean value of the solar constant 1.94 .

In Table 1 are given the average measured hourly intensities of solar radiation at normal incidence at five stations in the United States, on dates when the sun is at its maximum south declination, $-23^{\circ} 27^{\prime}$ on December 23; on the equator, March 21 and September 23, farthest north, $+23^{\circ} 27^{\prime}$, on June 21 , and halfway between the equinoxes and the 


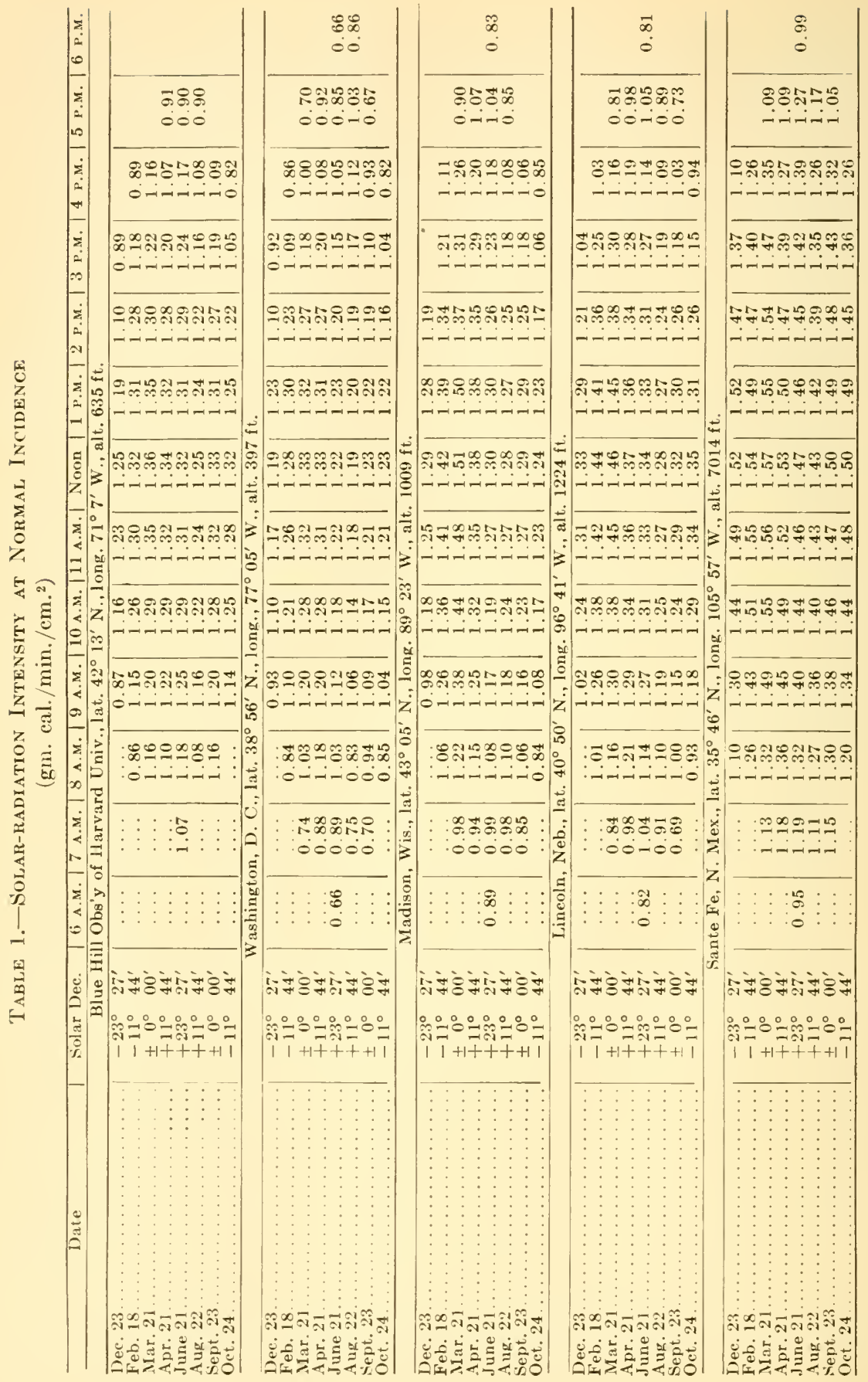


winter and summer solstices, or $-11^{\circ} 44^{\prime}$ on February 18 and Oetober 24, and $+11^{\circ} 44^{\prime}$ on April 21 and August 22. Note first of all the marked minimum noon intensity at Sante Fe in August, whieh is also seen to a lesser degree at the other stations, and is only exceeded in some other month of the year at Madison, Wis., the most northern station, in December. While this August depression is partly due to the greater solar distance of the earth at that season, it is principally due to the increased water vapor and dust content of the atmosphere in late summer. At Davos, Switzerland [(6), Table 1], farther north than any station in the United States, and with a low humidity eontent of the atmosphere, there is a noon minimum in July, and a deeided maximum in April, with a secondary maximum in August. The absolute minimum here also occurs in December, as we would expect.

Just before sunset or immediately after sunrise, if the sky is clear, the radiation received on a surface normal to the rays is still strong. Measurements made at Washington, D. C. (7), on November 9, 1909, give the following intensities, on the Angström scale:

Table 2.-Radiation Intensity with Sun Approaching Horizon

\begin{tabular}{|c|c|c|c|c|c|c|c|c|c|c|c|}
\hline & $\circ$ & $\circ$ & 0 & $\circ$ & $\circ$ & 0 & $\circ$ & 0 & 0 & $\circ$ & $\circ$ \\
\hline $\begin{array}{l}\text { Solar-zenith } \\
\text { tance.......... }\end{array}$ & 60.0 & 75.7 & 80.7 & 83.2 & 84.7 & 85.8 & 86.9 & 87.6 & 88.2 & 88.7 & 89.2 \\
\hline Air mass. . . . & 2.0 & 4.0 & 6.0 & 8.0 & 10.0 & 12.0 & 15.0 & 18.0 & 21.0 & 25.0 & 29.0 \\
\hline $\min . \mathrm{cm}^{2} \ldots \ldots$ & 1.230 & 0.999 & 0.804 & 0.653 & 0.531 & 0.454 & 0.365 & 0.292 & 0.236 & 0.176 & 0.131 \\
\hline
\end{tabular}

Table 3.-Coordinates of Stations Recording the Total (Direct + Diffuse) Radiation Received on a Horizontal Surface, and Trpe of Pyrhelioneter

EMPLOTED

\begin{tabular}{|c|c|c|c|c|c|}
\hline Stations & $\begin{array}{l}\text { Lati- } \\
\text { tude }\end{array}$ & $\begin{array}{l}\text { Longi- } \\
\text { tude }\end{array}$ & $\begin{array}{c}\text { Alti- } \\
\text { tude, } \\
\mathrm{ft} .\end{array}$ & Period of time & Instrument \\
\hline & , & , & & & \\
\hline Miami, Fla...... & $2541 \mathrm{~N}$. & $8012 \mathrm{~W}$. & $\ldots$ & Nov. '29-Aug. ' 34 . & Callendar \\
\hline New Orleans, La. & $2956 \mathrm{~N}$. & $9007 \mathrm{~W}$. & 100 & Mar. '31-Aug. '3t. & Eppley \\
\hline Riverside, Calif. . & 3358 N. & $11720 \mathrm{~W}$. & 1070 & June '33-Aug. '34. & Eppley \\
\hline Fresno, Calif ...... & $36+3 \mathrm{~N}$. & $11949 \mathrm{~W}$. & 330 & Oct. '28-Aug. '34. & Eppley \\
\hline Washington, D. C... & $3856 \mathrm{~N}$. & $7705 \mathrm{~W}$. & 397 & Oct. '14-Aug. '3t. & Eppley \\
\hline Pittsburgh, Pa.... & $4032 \mathrm{~N}$. & $8020 \mathrm{~W}$. & 1114 & Dec. '29-Aug. '34. & Eppley \\
\hline New York, N. I... & $4046 \mathrm{~N}$. & $7358 \mathrm{~W}$ & 156 & Apr. '24-Aug. '34. & Eppley \\
\hline Lincoln, Neb..... & $4050 \mathrm{~N}$. & $9641 \mathrm{~W}$. & 1225 & July '15-Aug. '34. & Callendar \\
\hline Chicago, Ill.... & $4137 \mathrm{~N}$. & $8735 \mathrm{~W}$. & 688 & Sept. '23-Aug. ' 34. & Eppley \\
\hline Blue Hill, Mass... & $4213 \mathrm{~N}$ & $7107 \mathrm{~W}$. & 635 & Dec. '32-Aug. '34. & Eppley \\
\hline Twin Falls, Itlaho. & $4229 \mathrm{~N}$. & $11425 \mathrm{~W}$. & 4300 & June '26-Aug. ' 34 . & Eppley \\
\hline Madison, Wis..... & $4305 \mathrm{~N}$. & $8923 \mathrm{~W}$. & 1009 & Apr. '11-Aug. '34. & Callendar \\
\hline Fairbanks, Alaska... & $6452 \mathrm{~N}$ & $14739 \mathrm{~W}$. & 500 & Aug. '31-July '34. & Eppley \\
\hline
\end{tabular}




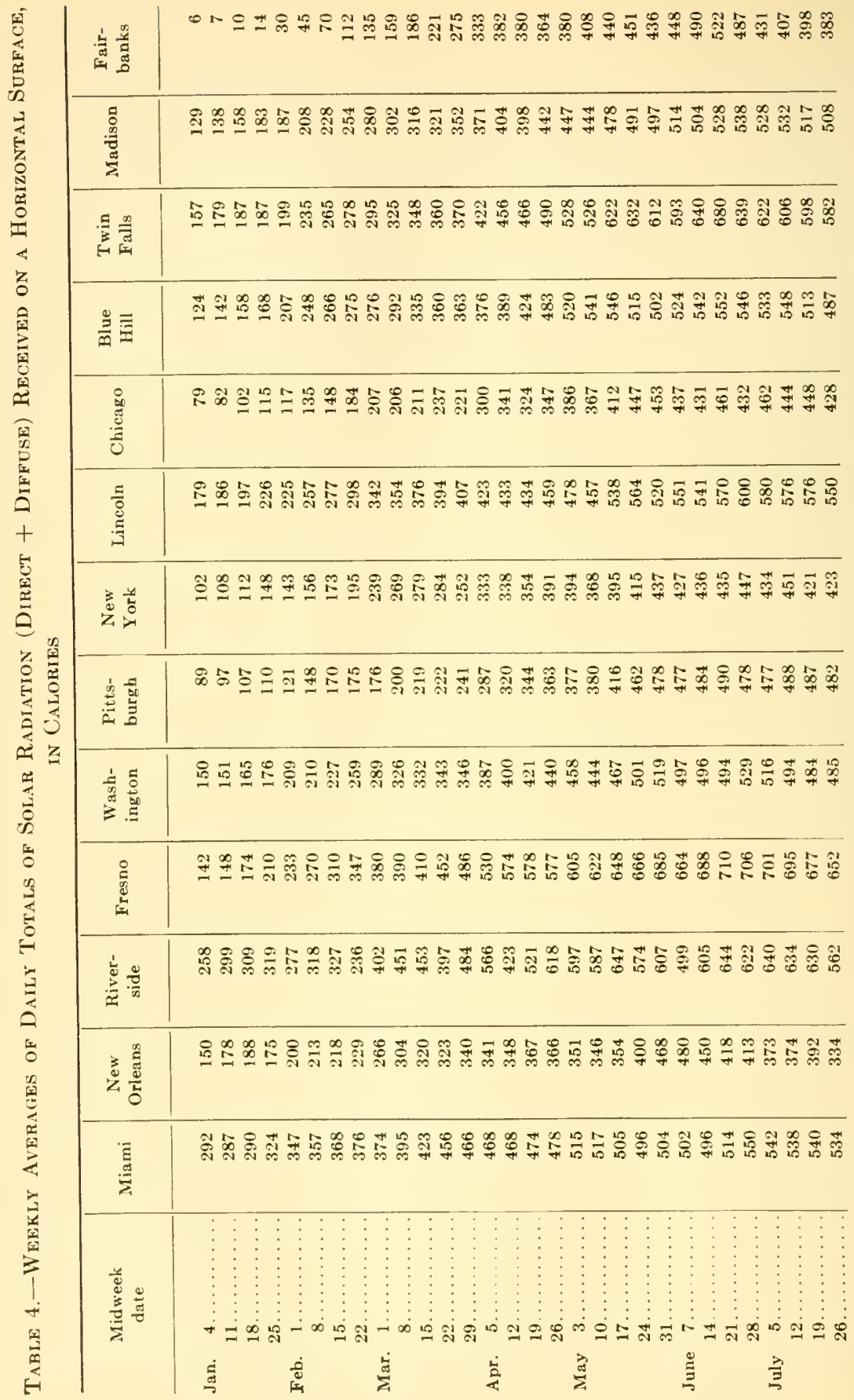




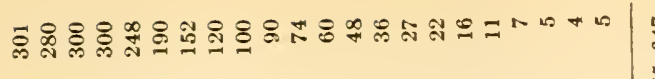

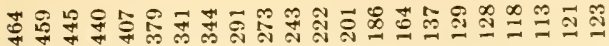

\begin{tabular}{|c|}
\hline 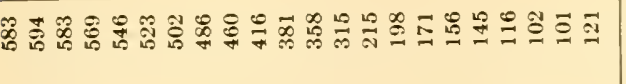 \\
\hline
\end{tabular}

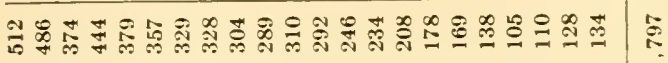

ב

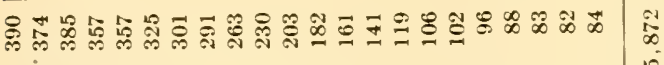

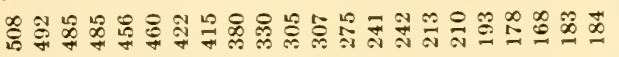

$\ddot{\infty}$

贻

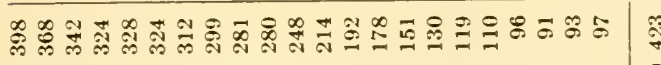

\begin{tabular}{l|l} 
& 8 \\
\hline
\end{tabular}

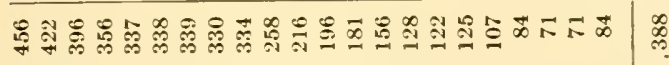

\begin{tabular}{|c|}
\hline 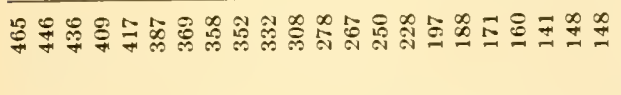 \\
\hline
\end{tabular}

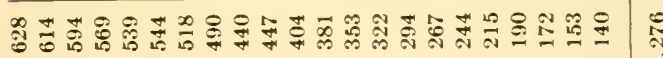

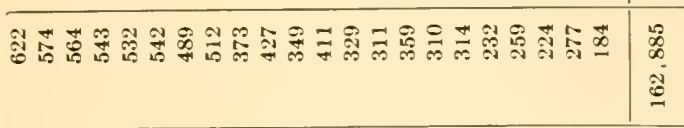

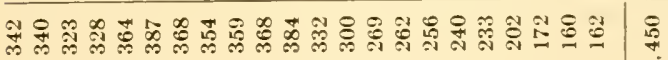

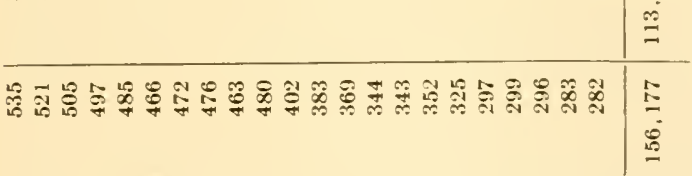

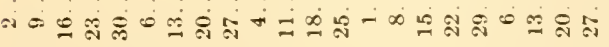

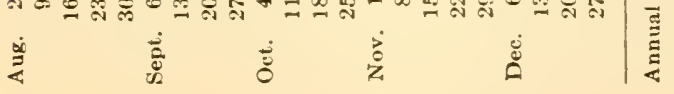




\section{THE VERTICAL COMPONENT OF THE TOTAL SOLAR RADIATION (DIRECT + DIFFUSE) RECEIVED AT THE SURFACE OF THE EARTH ${ }^{2}$}

As already noted under the previous section, a considerable percentage of the radiation received from the sun is scattered, or diffused, by atmospheric gas molecules, and by dust and other foreign particles that are always present in the atmosphere. The measurement of the vertical component of the total radiation (direct + diffuse) is easily accomplished, since the sensitive element exposed to radiation is fixed in a horizontal position, and the mechanism, or the labor necessary to keep instruments for measuring the intensity of the direct solar rays pointed accurately toward the sun, is avoided. The measurements of the intensity of the vertical component of solar radiation are, therefore, usually continuously recorded by automatic instruments, thus giving very complete records of this, the most important component of solar radiation, at least to vegetation, and thus indirectly, if not directly, to animal life, including man.

A considerable collection of data of this character has already been summarized by one of us, and published in the Monthly Weather Review $(8,9)$. Table 3 gives the coordinates of stations in the United States at which this component of the intensity of solar radiation is continuously recorded, and in Table 4 are given the average weekly means of daily totals. In the footings of this table are given the average annual totals of radiation for each station. Table 4, therefore, for stations in the United States, shows variations with latitude, altitude, and the season of the year in the daily amount of radiation received on a horizontal surface. It also shows that smoky cities like Pittsburgh, New York, and Chicago, receive about one-third less than stations like Riverside, Fresno, and Twin Falls, and one-fourth less than Blue Hill and Washington, which latter are on about the same latitude as the first-named cities.

In Table 5 are given the hourly averages of the vertical component of solar radiation for weeks that include the dates for which the radiation at normal incidence is given in Table 1. It therefore resembles Table 1 in that it shows variations in the intensity of solar radiation with latitude, altitude, the season of the year, and the time of day, but for the vertical component, including that received diffusely from the sky, instead of the intensity at normal incidence.

\section{SOLAR-RADIATION INTENSITY IN TROPICAL, SUBTROPICAL, AND ARCTIC REGIONS}

Table 6 gives the coordinates of stations in the above-named regions for which solar-radiation data are available for considerable periods of

${ }^{2}$ For a reproduction of a record of the two components of the total radiation received on a horizontal surface, see Fig. 18 of the paper by Brackett, p. 172. 
Table 5.-Hourly Averages of the Total Solar Radiation Received on a Horizontal Surface for Weeks That Include the Summer and Winter Solstices, the Equinoxes, and the Times When the Solar Declination Is Halfway between These Extremes

1. Miami, Florida

\begin{tabular}{|c|c|c|c|c|c|c|c|c|c|}
\hline \multicolumn{2}{|c|}{ Hours from noon*...... } & \multirow{2}{*}{$\begin{array}{c}0 \\
43.8\end{array}$} & \multirow{3}{*}{$\begin{array}{c}1 \\
43.4 \\
38.7\end{array}$} & \multirow{3}{*}{$\begin{array}{c}2 \\
39.7 \\
35.5\end{array}$} & \multirow{3}{*}{$\begin{array}{c}3 \\
26.7 \\
25.2\end{array}$} & \multirow{3}{*}{$\begin{array}{c}4 \\
15.3 \\
12.1\end{array}$} & \multirow{3}{*}{$\begin{array}{c}5 \\
5.8 \\
3.2\end{array}$} & \multirow[t]{3}{*}{6} & \multirow[t]{3}{*}{7} \\
\hline Dec. 23, & A.M... & & & & & & & & \\
\hline & P.M... & $\ldots$ & & & & & & & \\
\hline \multirow[t]{2}{*}{ Feb. 18, } & A.M... & 61.1 & 54.1 & 41.3 & 34.8 & 21.0 & 5.0 & & \\
\hline & P.м... & $\ldots$ & 54.0 & 42.7 & 30.9 & 18.9 & 6.8 & 0.7 & \\
\hline \multirow[t]{2}{*}{ March 21, } & A.M... & 66.5 & 64.5 & 57.2 & 41.7 & 24.3 & 11.0 & 2.3 & \\
\hline & P.M... & $\cdots$ & 60.3 & 53.1 & 40.8 & 24.5 & 11.0 & 2.6 & \\
\hline \multirow[t]{2}{*}{ April 21, } & A.M... & 67.3 & 63.2 & 60.4 & 43.6 & 31.1 & 17.9 & 7.0 & \\
\hline & P.м... & $\ldots$ & 57.9 & 47.5 & 36.9 & 24.4 & 12.8 & 4.9 & \\
\hline \multirow[t]{2}{*}{ June 21, } & A.M. . & 66.8 & 63.7 & 60.6 & 49.4 & 38.3 & 23.0 & 9.2 & 2.5 \\
\hline & P.M.. . & $\ldots$ & 62.1 & 57.9 & 41.1 & 29.9 & 17.5 & 9.3 & 1.6 \\
\hline \multirow[t]{2}{*}{ Aug. 22, } & A.M... & 65.5 & 63.2 & 58.6 & 49.0 & 32.9 & 16.7 & 5.8 & \\
\hline & P.M.. & $\ldots$ & 61.6 & 50.2 & 41.9 & 29.3 & 14.0 & 5.0 & \\
\hline \multirow[t]{2}{*}{ Sept. 23, } & A.M.... & 61.4 & 64.5 & 55.2 & 45.5 & 31.0 & 15.3 & 4.6 & \\
\hline & P.M. . & $\cdots$ & 55.2 & 46.2 & 31.2 & 20.5 & 9.5 & 1. 2 & \\
\hline \multirow[t]{2}{*}{ Oct. 24, } & A.M... & 50.4 & 51.9 & 49.3 & 41.4 & 31.5 & 17.2 & 6.3 & \\
\hline & P.M.... & $\ldots$ & 42.6 & 33.1 & 22.0 & 13.1 & 4.1 & & \\
\hline
\end{tabular}

2. Washington, D. C.

\begin{tabular}{|c|c|c|c|c|c|c|c|c|c|}
\hline \multicolumn{2}{|c|}{ Hours from noon....... } & \multirow{2}{*}{$\begin{array}{c}0-1 \\
26.4 \\
25.4\end{array}$} & \multirow{2}{*}{$\begin{array}{c}1-2 \\
22.6 \\
20.0\end{array}$} & \multirow{2}{*}{$\begin{array}{l}2-3 \\
16.8 \\
14.4\end{array}$} & \multirow{2}{*}{$\begin{array}{l}3-4 \\
8.1 \\
7.4\end{array}$} & \multirow{2}{*}{$\begin{array}{l}4-5 \\
1.8 \\
2.0\end{array}$} & \multirow[t]{2}{*}{$5-6$} & \multirow[t]{2}{*}{$6-7$} & \multirow[t]{2}{*}{$7-8$} \\
\hline Dec. 23, & $\begin{array}{l}\text { А.м. } \\
\text { Р.м. } \ldots \ldots\end{array}$ & & & & & & & & \\
\hline \multirow[t]{2}{*}{ Feb. 18} & A.M... & 40.0 & 36.2 & 27.8 & 16.0 & 4.6 & 0.6 & & \\
\hline & P.M... & 40.2 & 34.0 & 25.4 & & 7.8 & 0.7 & & \\
\hline \multirow{2}{*}{ Mlarch 21} & А.м... & 44.6 & 43.4 & 35.7 & 20.2 & 11.6 & 2.8 & 0.1 & \\
\hline & P.м... & 47.6 & 45.9 & 35.4 & 26.2 & 14.9 & 4.8 & 0.2 & \\
\hline \multirow{2}{*}{ A pril 21, } & A.M... & 53.2 & 54.6 & 51.0 & 34.2 & 20.4 & 9.2 & 1.6 & \\
\hline & P.M... & 53.6 & 47.1 & 38.6 & 31.8 & 22.8 & 11.6 & 2.9 & \\
\hline \multirow[t]{2}{*}{ June 21, } & A.M. & 71.6 & 69.6 & 58.7 & 42.6 & 26.6 & 14.1 & 5.3 & 0.5 \\
\hline & P.M.. & 61.9 & 45.5 & 40.4 & 33.1 & 24.9 & 17.6 & 5.1 & 0.6 \\
\hline \multirow[t]{2}{*}{ Aug. 22} & А.м... & 52.7 & 45.0 & 35.3 & 28.5 & 15.5 & 6.9 & 1.9 & \\
\hline & P.M... & 56.9 & 55.3 & 46.6 & 33.0 & 20.8 & 9.1 & 2.3 & \\
\hline \multirow[t]{2}{*}{ Sept. 23, } & A.M. . W & 49.7 & 45.5 & 40.4 & 29.0 & 16.2 & 4.7 & 0.2 & \\
\hline & P.M. . . & 48.5 & 47.4 & 37.9 & 27.8 & 14.8 & 4.8 & 0.2 & \\
\hline \multirow[t]{2}{*}{ Oct. 24, } & A.M. $\ldots \ldots$ & 43.2 & 40.0 & 30.0 & 17.6 & 6.4 & 1.4 & & \\
\hline & P.м.... & 45.7 & 42.8 & 32.0 & 20.6 & 10.7 & 1.6 & & \\
\hline
\end{tabular}

* Probably noon, 75 th meridian time, which is about 20 minutes faster than local solar time at $M$ iami in April and June, and 36 minutes faster in late October and early February. 
Table 5.-Hourly Averages of the Total Solar Radiation Received on a Horizontal Surface for Weeks That Include the Summer and Winter Solstices, the Equinoxes, and the Times When the Solar Declination Is Halfway between These Extremes.-(Continued)

3. Lincoln, Neb.

\begin{tabular}{|c|c|c|c|c|c|c|c|c|c|}
\hline \multicolumn{2}{|c|}{ Hours from noon....... } & \multirow{2}{*}{$\begin{array}{c}0-1 \\
31.6 \\
33.2\end{array}$} & \multirow{2}{*}{$\begin{array}{c}1-2 \\
\\
26.4 \\
29.4\end{array}$} & \multirow{2}{*}{$\begin{array}{c}2-3 \\
18.5 \\
19.8\end{array}$} & \multirow{2}{*}{$\begin{array}{l}3-4 \\
\\
9.2 \\
9.0\end{array}$} & \multirow{2}{*}{$\begin{array}{l}4-5 \\
1.2 \\
1.6\end{array}$} & \multirow[t]{2}{*}{$5-6$} & \multirow[t]{2}{*}{$6-7$} & \multirow[t]{2}{*}{$7-8$} \\
\hline Dec. 23 , & $\begin{array}{l}\text { A.M. } \ldots \ldots \\
\text { P.M. } \ldots \ldots\end{array}$ & & & & & & & & \\
\hline Feb. 18, & $\begin{array}{l}\text { А.M. } \ldots \ldots \ldots \\
\text { P.M. }\end{array}$ & $\begin{array}{l}47.4 \\
47.4\end{array}$ & $\begin{array}{l}40.6 \\
41.9\end{array}$ & $\begin{array}{l}31.6 \\
30.8\end{array}$ & $\begin{array}{l}19.0 \\
16.2\end{array}$ & $\begin{array}{l}6.6 \\
6.0\end{array}$ & $\begin{array}{l}0.4 \\
0.7\end{array}$ & & \\
\hline March 21 , & $\begin{array}{l}\text { А.м. } \\
\text { Р.м. } \ldots \ldots \ldots \ldots\end{array}$ & $\begin{array}{l}57.3 \\
53.6\end{array}$ & $\begin{array}{l}51.7 \\
41.3\end{array}$ & $\begin{array}{l}41.8 \\
39.8\end{array}$ & $\begin{array}{l}28.6 \\
28.0\end{array}$ & $\begin{array}{l}15.0 \\
15.3\end{array}$ & $\begin{array}{l}3.4 \\
5.7\end{array}$ & 0.1 & \\
\hline April 21, & $\begin{array}{l}\text { A.M. . . . } \\
\text { P.M.... }\end{array}$ & $\begin{array}{l}50.4 \\
53.4\end{array}$ & $\begin{array}{l}51.4 \\
52.0\end{array}$ & $\begin{array}{l}44.3 \\
47.1\end{array}$ & $\begin{array}{l}38.2 \\
32.3\end{array}$ & $\begin{array}{l}22.8 \\
21.5\end{array}$ & $\begin{array}{r}9.3 \\
10.8\end{array}$ & $\begin{array}{l}1.3 \\
2.3\end{array}$ & \\
\hline June 21, & $\begin{array}{l}\text { А.м....... } \\
\text { Р.м...... }\end{array}$ & $\begin{array}{l}65.0 \\
63.9\end{array}$ & $\begin{array}{l}71.4 \\
54.6\end{array}$ & $\begin{array}{l}66.0 \\
43.7\end{array}$ & $\begin{array}{l}53.2 \\
39.9\end{array}$ & $\begin{array}{l}35.2 \\
33.3\end{array}$ & $\begin{array}{l}19.3 \\
19.9\end{array}$ & $\begin{array}{l}5.2 \\
8.5\end{array}$ & $\begin{array}{l}0.1 \\
1.9\end{array}$ \\
\hline Aug. 22, & $\begin{array}{l}\text { А.M. } \ldots \ldots \ldots \\
\text { Р.M. } \ldots \ldots \ldots\end{array}$ & $\begin{array}{l}62.9 \\
64.7\end{array}$ & $\begin{array}{l}58.5 \\
58.4\end{array}$ & $\begin{array}{l}52.5 \\
46.2\end{array}$ & $\begin{array}{l}42.9 \\
35.3\end{array}$ & $\begin{array}{l}25.9 \\
25.2\end{array}$ & $\begin{array}{l}11.1 \\
10.2\end{array}$ & $\begin{array}{l}1.2 \\
2.2\end{array}$ & \\
\hline Sept. 23, & $\begin{array}{l}\text { А.м. } \ldots \ldots \ldots \\
\text { Р.м. } \ldots \ldots \ldots\end{array}$ & $\begin{array}{l}56.1 \\
57.6\end{array}$ & $\begin{array}{l}52.8 \\
50.7\end{array}$ & $\begin{array}{l}43.7 \\
39.4\end{array}$ & $\begin{array}{l}30.6 \\
27.3\end{array}$ & $\begin{array}{l}17.8 \\
13.7\end{array}$ & $\begin{array}{l}4.7 \\
3.9\end{array}$ & $\begin{array}{l}0.1 \\
0.1\end{array}$ & \\
\hline Oct. 24 , & $\begin{array}{l}\text { A.M... } \\
\text { P.M. . }\end{array}$ & $\begin{array}{l}46.5 \\
45.1\end{array}$ & $\begin{array}{l}42.1 \\
41.0\end{array}$ & $\begin{array}{l}31.0 \\
30.6\end{array}$ & $\begin{array}{l}18.2 \\
20.1\end{array}$ & $\begin{array}{l}7.9 \\
7.9\end{array}$ & $\begin{array}{l}0.6 \\
1.0\end{array}$ & & \\
\hline
\end{tabular}

4. Twin Falls, Idaho

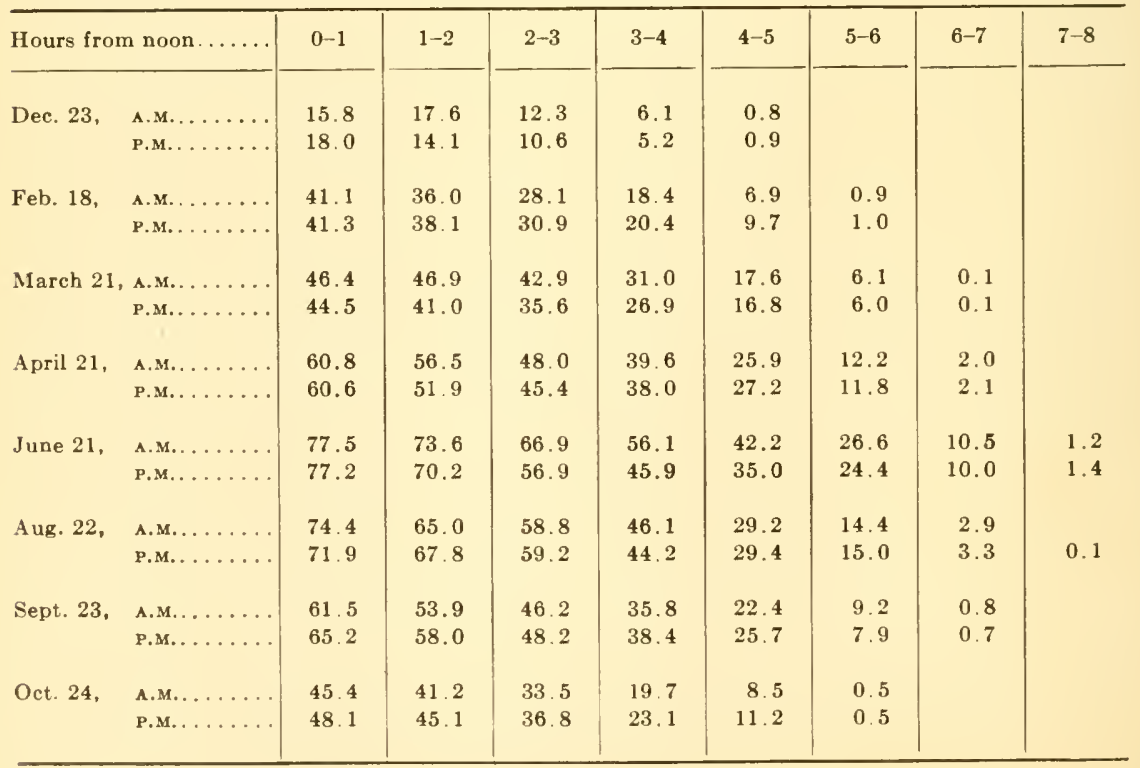


Table 5.-Hourly Averages of the Total Solar Radiation Received on a Horizontal Surface for Weeks That Include the Summer and Winter Solstices, the Equinoxes, and the Times When the Solar Declination Is Halfway between These Extremes.-(Continued)

5. Fresno, Calif.

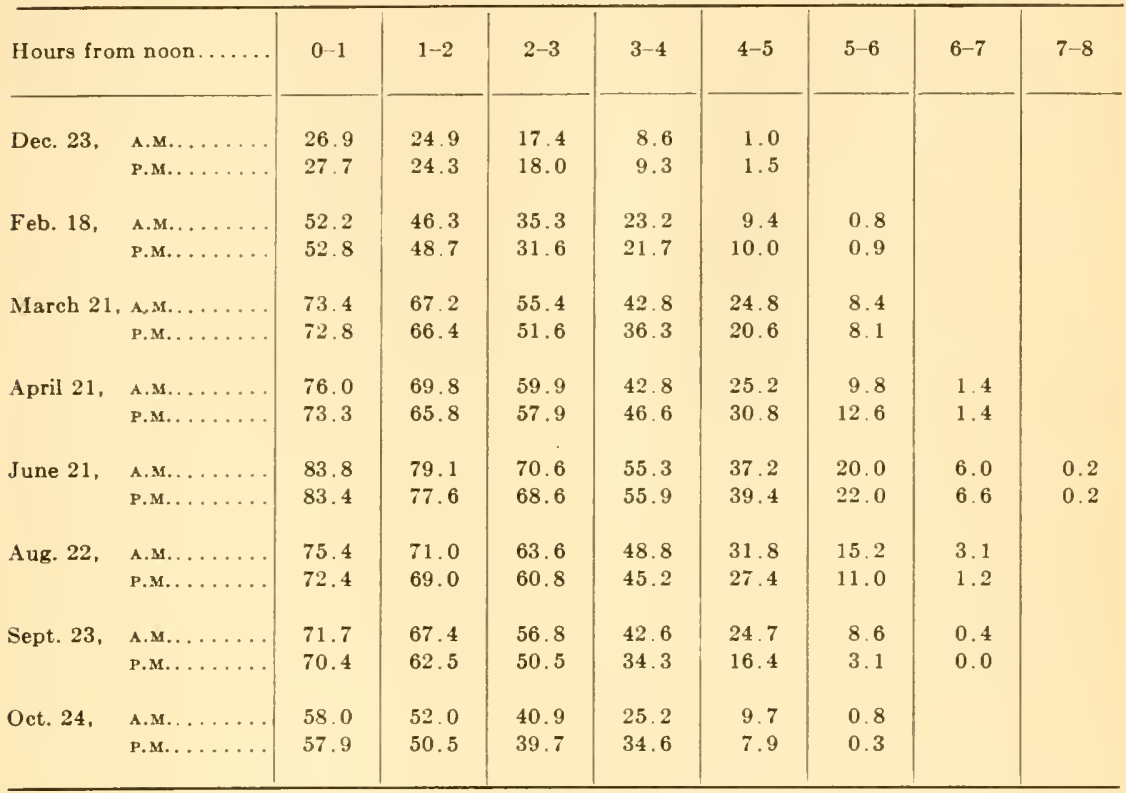

6. Fairbanks, Alaska

\begin{tabular}{|c|c|c|c|c|c|c|c|c|c|c|c|c|}
\hline \multicolumn{2}{|c|}{ Hours from noon. } & $0-1$ & 1-2 & $2-3$ & $3-4$ & $4-5$ & $5-6$ & $6-7$ & $7-8$ & 8-9 & $9-10$ & $10-11$ \\
\hline Dec. 23, & $\begin{array}{l}\text { A.M. } \ldots \\
\text { P.M. }\end{array}$ & $\begin{array}{l}2.2 \\
1.7\end{array}$ & $\begin{array}{r}0.8 \\
0.8\end{array}$ & $\begin{array}{l}0.0 \\
0.2\end{array}$ & & & & & & & & \\
\hline Feb. 18 , & $\begin{array}{l}\text { A.M.... } \\
\text { P.M. }\end{array}$ & $\begin{array}{l}12.3 \\
14.4\end{array}$ & $\begin{array}{r}9.7 \\
12.2\end{array}$ & $\begin{array}{l}5.8 \\
7.5\end{array}$ & $\begin{array}{l}2.2 \\
3.5\end{array}$ & $\begin{array}{l}0.2 \\
0.4\end{array}$ & & & & & & \\
\hline March 21 & A.M... & 31.8 & 28.6 & 24.2 & 16.8 & 9.2 & 3.3 & 0.3 & & & & \\
\hline & P.s..... & 32.2 & 27.6 & 22.8 & 14.8 & 8.0 & 2.8 & 0.2 & & & & \\
\hline A pril 21 & $\begin{array}{l}\text { А.M. } \ldots \\
\text { P.M. }\end{array}$ & $\begin{array}{l}45.6 \\
43.6\end{array}$ & $\begin{array}{l}42.6 \\
40.4\end{array}$ & $\begin{array}{l}37.0 \\
35.1\end{array}$ & $\begin{array}{l}29.9 \\
27.5\end{array}$ & $\begin{array}{l}20.4 \\
20.6\end{array}$ & $\begin{array}{l}12.2 \\
12.2\end{array}$ & $\begin{array}{l}5.4 \\
5.8\end{array}$ & $\begin{array}{l}1.2 \\
1.3\end{array}$ & & & \\
\hline June 21 & $\begin{array}{l}\text { А.М. } \ldots \\
\text { Р.М. }\end{array}$ & $\begin{array}{l}58.4 \\
60.7\end{array}$ & $\begin{array}{l}55.5 \\
56.9\end{array}$ & $\begin{array}{l}44.8 \\
47.0\end{array}$ & $\begin{array}{l}37.2 \\
39.7\end{array}$ & $\begin{array}{l}28.7 \\
34.1\end{array}$ & $\begin{array}{l}22.6 \\
30.9\end{array}$ & $\begin{array}{l}15.1 \\
22.6\end{array}$ & $\begin{array}{r}7.4 \\
11.0\end{array}$ & $\begin{array}{l}2.6 \\
4.6\end{array}$ & $\begin{array}{l}1.0 \\
1.4\end{array}$ & $\begin{array}{l}0.1 \\
0.3\end{array}$ \\
\hline Aug. 22, & $\begin{array}{l}\text { A.M... } \\
\text { Р.M... }\end{array}$ & $\begin{array}{l}38.6 \\
40.1\end{array}$ & $\begin{array}{l}36.0 \\
36.3\end{array}$ & $\begin{array}{l}30.4 \\
32.4\end{array}$ & $\begin{array}{l}20.6 \\
23.8\end{array}$ & $\begin{array}{l}11.9 \\
16.7\end{array}$ & $\begin{array}{r}7.3 \\
10.1\end{array}$ & $\begin{array}{l}3.2 \\
3.8\end{array}$ & $\begin{array}{l}0.6 \\
0.8\end{array}$ & & & \\
\hline Sept. 23, & $\begin{array}{l}\text { A.M. } \ldots \\
\text { P.M. }\end{array}$ & $\begin{array}{l}22.6 \\
18.6\end{array}$ & $\begin{array}{l}21.2 \\
16.0\end{array}$ & $\begin{array}{l}164 \\
15.3\end{array}$ & $\begin{array}{l}11.2 \\
10.2\end{array}$ & $\begin{array}{l}6.1 \\
5.5\end{array}$ & $\begin{array}{l}2.5 \\
1.8\end{array}$ & $\begin{array}{l}0.1 \\
0.1\end{array}$ & & & & \\
\hline Oct. 24. & $\begin{array}{l}\text { A.M. } \\
\text { P.M. }\end{array}$ & $\begin{array}{l}10.9 \\
10.9\end{array}$ & $\begin{array}{l}8.8 \\
9.6\end{array}$ & $\begin{array}{l}5.6 \\
6.0\end{array}$ & $\begin{array}{l}2.0 \\
2.4\end{array}$ & $\begin{array}{l}0.1 \\
0.2\end{array}$ & & & & & & \\
\hline
\end{tabular}


Table 6.-Coordinates of Stations in Tropical, Subtropical, and Arctic Regions

1. Radiation intensity at normal incidence

\begin{tabular}{|c|c|c|c|c|c|}
\hline Station & Latitude & Longitude & $\begin{array}{l}\text { Alti- } \\
\text { tude, } \\
\text { ft. }\end{array}$ & Period & Instrument \\
\hline Apia, Samoa (13)...... & $13^{\circ} 48^{\prime} \mathrm{S}$. & $171^{\circ} 46^{\prime} \mathrm{W}$. & 7 & $1925-1927$ & Gorezyriski \\
\hline Abisko, Sweden (12) ......... & $68^{\circ} 21^{\prime} \mathrm{N}$ & $18^{\circ} 49^{\prime} \mathrm{E}$ & 1280 & $\begin{array}{l}\text { July } 2- \\
\text { Sept. } 13,1913\end{array}$ & Ångström \\
\hline Mount Evans, Greenland (10).... & $66^{\circ} 51^{\prime} \mathrm{N}$ & $50^{\circ} 50^{\prime} \mathrm{W}$. & 1291 & $\begin{array}{l}\text { Sept. } 1927 \text {, } \\
\text { April, } 1928\end{array}$ & Moll \\
\hline Treurenberg, Spitzbergen (14)... & $79^{\circ} 55^{\prime} \mathrm{N}$ & $16^{\circ} 52^{\prime} \mathbf{E}$ & 30 & $\begin{array}{l}\text { Sept. } 1899 \text {, } \\
\text { April-July, } \\
1900\end{array}$ & Ângström \\
\hline
\end{tabular}

2. Radiation intensity on a horizontal surface

\begin{tabular}{|c|c|c|c|c|c|}
\hline Habana, Cuba (8)........... & $23^{\circ} 09^{\prime} \mathrm{N}$ & $82^{\circ} 21^{\prime} \mathrm{W}$. & 131 & $\begin{array}{l}\text { March, 1925- } \\
\text { May, } 1926\end{array}$ & $\begin{array}{c}\text { Weather Bureau } \\
\text { thermoelectric }\end{array}$ \\
\hline Johannesburg, S. Afr. (8)....... & $26^{\circ} 11^{\prime} \mathrm{S}$. & $28^{\circ} 4^{\prime} \mathrm{E}$. & 6102 & $\begin{array}{l}1908- \\
\text { June, } 1913\end{array}$ & Callendar \\
\hline Abisko, Sweden (4).......... & $68^{\circ} 21^{\prime} \mathrm{N}$. & $18^{\circ} 49^{\prime} \mathrm{E}$. & 1280 & $\begin{array}{l}\text { July } 2- \\
\text { Sept. } 13,1914\end{array}$ & Ångström \\
\hline Green Harbor, Spitzbergen (11). . & $78^{\circ} 00^{\prime} \mathrm{N}$. & $14^{\circ} 05^{\prime} \mathrm{E}$. & & $\begin{array}{l}\text { Sept.- } \\
\text { Oct. } 18,1927\end{array}$ & Moll-Gorczyński \\
\hline Sveanor (3)...... & $79^{\circ} 56^{\prime} \mathrm{N}$. & $18^{\circ} 18^{\prime} \mathrm{E}$. & & $\begin{array}{l}\text { July } 2- \\
\text { Aug. } 10,1931\end{array}$ & Robitzsch \\
\hline
\end{tabular}

Table 7.-Radiation Intensities at Normal Incidence (gm. eal./min./cm. ${ }^{2}$ )

Apia, Samoa

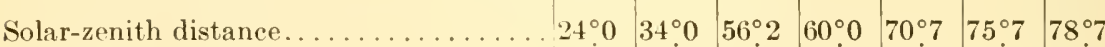

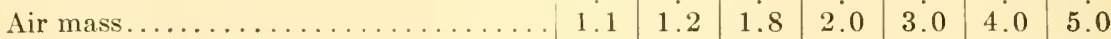

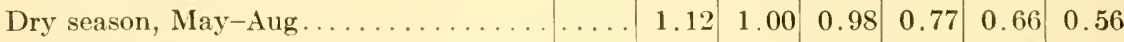

\begin{tabular}{ll|l|l|l|l|l|l|l} 
Equatorial season, Mar.-Apr., Sept.-Oet. & 1.17 & 1.15 & 1.04 & 0.98 & 0.83 & 0.74 & 0.62
\end{tabular}

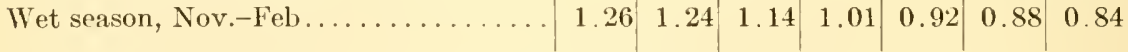

Abisko, Sweden

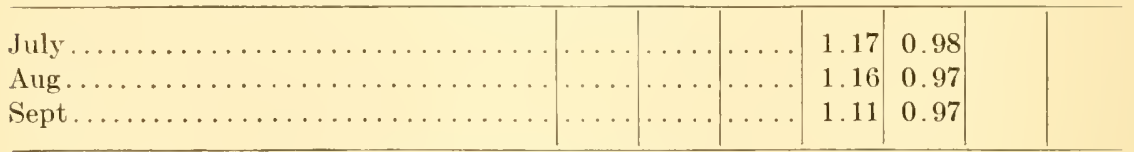

Mount Evans, Greenland

Feb

March

April

Aug

Sept

Oct.

\begin{tabular}{r|r|r|r}
$\ldots \ldots$ & $\ldots \ldots$ & $\ldots$ & 1.25 \\
$\ldots \ldots$ & 1.39 & 1.31 & 1.18 \\
1.45 & 1.28 & 1.12 & 1.03 \\
1.22 & 1.07 & 0.96 & 0.85 \\
1.32 & 1.25 & 1.15 & 1.08 \\
$\ldots \ldots$ & $\ldots$ & 1.25 & 1.18 \\
\hline
\end{tabular}


Table 7.-Radiation Intensities at Normal Incidence,-(Continued)

Treurenberg, Spitzbergen*

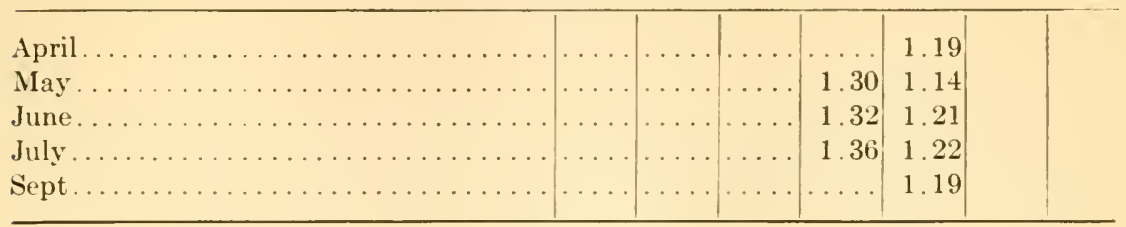

* Intensities are given in units of the Ångström pyrheliometric scale.

Table 8.-Solar-Radiation Intensities on a Horizontal Surface

(Daily averages, gm. cal./day/cm. ${ }^{2}$ )

\begin{tabular}{|c|c|c|c|c|c|}
\hline Mouth & $\begin{array}{c}\text { Johannesburg, } \\
\text { South Africa }\end{array}$ & $\begin{array}{l}\text { Habana, } \\
\text { Cuba }\end{array}$ & $\begin{array}{l}\text { Abisko, }{ }^{*} \\
\text { Sweden }\end{array}$ & $\begin{array}{c}\text { Green* } \\
\text { Harbor, } \\
\text { Spitzbergen } \\
\end{array}$ & Sveanor* \\
\hline $\operatorname{Jan} \ldots \ldots \ldots$ & 556 & 367 & & & \\
\hline Feb......... & 490 & 448 & 23 & 2 & \\
\hline March........ & 455 & 598 & 135 & 59 & \\
\hline April........ & 405 & 620 & 379 & 273 & \\
\hline May......... & 380 & 575 & 463 & 457 & \\
\hline June. . . . . . . & 365 & 610 & 468 & 546 & \\
\hline July . . . . . . . & 368 & 656 & 366 & 363 & 407 \\
\hline Aug.......... & 440 & 568 & 228 & & \\
\hline Sept......... & 531 & 519 & 143 & 86 & \\
\hline Oet... & 567 & 439 & 51 & 5 & \\
\hline Nov........ & 606 & 332 & 7 & & \\
\hline Dec........ & 579 & 330 & & & \\
\hline Year..... & 125,696 & 183.797 & & & \\
\hline
\end{tabular}

* Radiation intensities are believed to be recorded in units of the Angström pyrheliometric scale.

time. Intensities at normal incidence are summarized in Table 7, and daily averages of the total received on a horizontal surface are summarized in Table 8.

THE EFFECT OF CLOUDINESS ON THE TOTAL SOLAR RADIATION RECEIVED ON A HORIZONTAL SURFACE

The relation between $Q_{0}$, the radiation received with a clear sky, and $Q_{s}$, the amount received with an overcast, or partly overcast sky, has been given by Angstrom in the form of the following equation:

$$
Q_{s}=Q_{0}[a+(1.00-a) S]
$$

where $S$ is the percentage of possible hours of sunshine prevailing. Angström found $a$ to vary between 0.22 and 0.25 . Our own researches 
from measurements made at Washington, D. C., Madison, Wis., and Lincoln, Neb., have led us to adopt $a=0.22$, giving for the equation

$$
Q_{s}=Q_{0}[0.22+0.78 S]
$$

The value of $a$ must vary with the character of the cloud layer and perhaps also the surface conditions, as for instance, with a snow cover, a surface covered with vegetation, or a sandy surface.

Jan. Feb. Mar. Apr. May June July Aug. Sept. Oct. Nov. Dec.

I II 21 31 1020212221112111121311020301020309192981828818287172771727

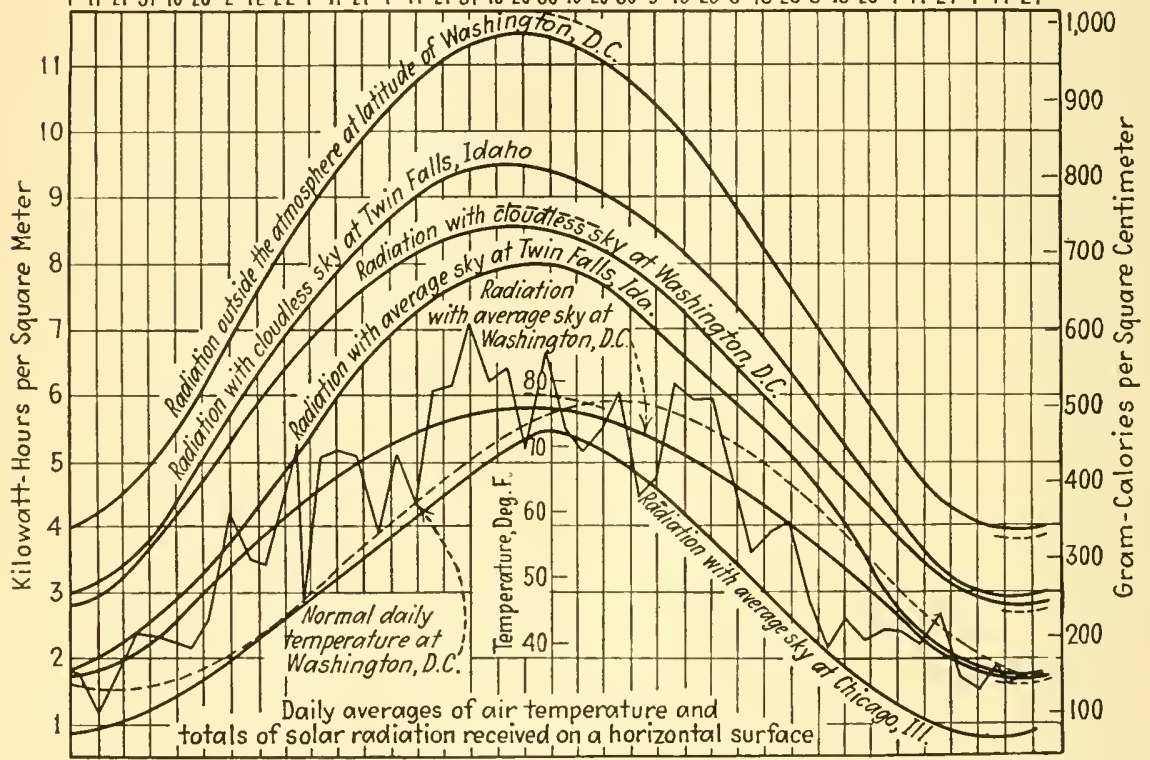

FIG. 2.-Curves for Twin Falls, Washington, and Chicago, showing the annual variation in the total radiation received on a horizontal surface with a clear sky and with average cloud conditions.

Figure 2 gives curves for Twin Falls, Washington, and Chicago, showing the annual variations in the total radiation received on a horizontal surface with a clear sky and with average cloud conditions. Superposed upon the latter for Washington is an irregular trace containing weekly averages for the year 1925 . These weekly values show variations as great as 50 per cent of the normal values and from 30 to 40 per cent of the value at the outer limit of the atmosphere. The importance of cloudiness in its effect upon the radiation received at the surface of the earth is therefore apparent.

\section{SUMMARY}

The following may be mentioned as of special interest: At the three most northerly stations, Sveanor, Treurenberg and Green Harbor, the 
zenith distance of the sun is less than $60^{\circ}$ only from about May 15 to the end of July. At Mount Evans, Greenland, and at Abisko, Sweden, the solar-zenith distance is at $60^{\circ}$ or less from about April 15 to the end of August. Table 6 shows that at Treurenberg with zenith distance $60^{\circ}, m=2$, the solar-radiation intensities average 1.30, 1.32, and $1.36 \mathrm{gm}$. cal./min./cm. ${ }^{2}$ for May, June, and July, respectively; for Mount Evans for April, August, and September, the intensities are $1.45,1.22$, and 1.32, respectively, and for Abisko for July and August, 1.17 and 1.16 , respectively.

For stations in the United States for which radiation intensities are summarized in Table 1 , the Weather Bureau file gives for average summer intensities for $m=2$, the following values: at Blue Hill, 1.08; for Washington, 0.94; Madison, 1.02; Lincoln, 1.10; Santa Fe, 1.27. For Samoa the corresponding intensity is 0.98 .

If we reduce the intensities measured at Arctic stations with the sun $60^{\circ}$ from the zenith to mean distance of the earth from the sun, we obtained the following transmission coefficients: Treurenberg, May to July, 0.718, which Fig. 1 shows to be about the same as for Mount Wilson in September; Abisko, July to September, 0.541, which is close to that for Lincoln in August; Mount Evans in April, and in August and September, respectively, 0.753 and 0.665 , the first of which is about the same as that for Sante Fe in January, and the second close to that for Lincoln in February. With the exception of that for Abisko, these are high coefficients for the summer season.

\section{REFERENCES}

1. Аввот, C. G., and L. B. Aldrich. Smithsonian pyrheliometry revised. Smithsonian Misc. Coll. 60 (No. 18): 1-7. 1913.

2. Аввот, C. G., and L. B. Aldrich. The standard seale of solar radiation. Smithsonian Mise. Coll. 92 (No. 13). 1934.

3. Angström, Anders. Scientific results of the Swedish Norwegian aretic expedition in the summer of 1931 . VII. On the total radiation from sun and sky at Sveanor. Geografiska Annaler $15(2 / 3)$ : 151-160. 1933.

4. Ångström, Anders, and Olaf Tryselius. Total radiation from sun and sky at Abisko. Geografiska Annaler 16 (1): 53-69. 1934.

5. Angström, KNut. The absolute determination of the radiation of heat with the electric compensation pyrheliometer, and examples of the application of this instrument. Astrophys. Jour. 9: 332-346. 1889.

6. Dorno, C. Tägliche, jährliche und säkulare Schwankungen der Sonnenstrahlung in Davos. (Rapport fait à la $\mathrm{I}^{\mathrm{re}}$ Conférence internationale de la Lumière, Lausanne-Leysin, 10-13 septembre, 1928.)

7. Kimball, Herbert H. Solar radiation, atmospheric absorption, and skylight polarization, at Washington, D. C. Bull. Mt. Weather Observ. 3: 69-126. 1910. (See p. 92, Table 2.)

8. Kimball, Herbert H. Measurements of solar radiation intensity, and determinations of its depletion by the atmosphere, with bibliography of pyrheliometer 
measurements. Monthly Weather Rev. 55: 155-169. 1927. (See Table 2, pp. $155-156$.

9. Kimball, Herbert H. Measurements of solar radiation intensity, and determinations of its depletion by the atmosphere. Monthly Weather Rev. 58: 43-52. 1930.

10. Kimball, Herbert H. Solar radiation intensities in Aretic regions. Gerland's Beitr. Geophys. 32: 101-105. 1931. [Summarizes data submitted by C. R. Kallquist. See also (11) below.]

11. Kimball, Herbert H. Solar radiation intensities within the Aretic Circle. Monthly Weather Rev. 59: 154-157. 1931.

12. Lindholm, F. Sur l'insolation dans la Suède septentrionale. Kungl. Svens. Vetenksapsakad. HandI. Stockholm 60 (No. 2): 1-24. 1919.

13. Thomson, Andrew. Solar radiation observation at Apia, Samoa. Monthly Weather Rev. 55: 266-267. 1927.

14. Westman, J. Mesures de l'intensité de la radiation solaire faites en 1899 et en 1900 à la station d'hivernagé suédoise à la baie de Treurenberg, Spitzberg. Mission suédois. Stockholm. Tôme 2. Section VIII. B. 1904.

Manuscript received by the editor November, 1934. 


\title{
STATISTICAL TREATMENT OF BIOLOGICAL PROBLEMS IN IRRADIATION
}

\author{
LowelL J. REed
}

\section{Department of Biostatistics, Johns Hopkins University, Baltimore}

Percentage comparisons. Treatment of measurements. Treatment of lethal dose. Curve fitting. References.

Biological problems have a degree of variability such that when treated quantitatively they become automatically statistical in character, and when we add to this degree of variability that inherent in the field of radiation, it becomes doubly necessary to treat the observations obtained with appropriate statistical technique. The problems encountered in this field involve no processes of quantitative logic not discussed in some of the various textbooks on statistical method and it would be inappropriate in this place to attempt to cover the ground usually elaborated by such books. It may, however, be of value to consider certain types of problems that occur reasonably often in the study of the biological effects of radiation and to present methods for analyzing them statistically. Since space prevents a detailed discussion of all the points involved, the reader will be referred for certain routine treatments to such standard textbooks as those of Yule (10), Pearl (5), and Tippett (9). The emphasis of the present discussion is placed rather on the interpretation of the statistical constants than on their derivation, and although it cannot hope to train workers in this field in statistical method, it aims to give direction to their studies.

\section{PERCENTAGE COMPARISONS}

In the study of the effect of irradiation on biological forms, the simplest statistical problem arises when one group of animals has been subjected to radiation of some type, a similar group of animals has not been subjected to radiation, and the observed end result is of the yes or no type, that is, the animals died or survived, the eggs hatched or did not hatch, etc. Such problems lead to a comparison of the percentages of animals that reacted in the experimental and control groups. Comparison of these percentages is so simple and direct that the only statistical question involved is that of the degree of reliability of the observed 
percentages. This question is treated under what is called the sampling error of a percentage, by which we mean the degree of variability in percentages, as determined by repeated samples of a fixed size, when as far as we can determine these samples have been drawn from the same universe or the same experience.

A discussion of sampling error can be approached most simply in terms of an actual example and we shall consider cases taken from a paper by Sievert and Forssberg (8), in which they discuss the lethal effect on Drosophila eggs of varying dosages of $\mathrm{X}$-rays at different intensities. We shall make use of their results for the dose $165 \mathrm{r}$, which are presented in Table 1.

Table 1.-Mortality Rates of Drosophila Eggs Irradiated with an X-ray Dose of 165 r at VARYiNg INTENSITIES

\begin{tabular}{c|c|c|c}
\hline Intensity, r per min. & $\begin{array}{c}\text { Number of eggs } \\
\text { irradiated }\end{array}$ & Number of deaths & Death rate, per cent \\
\cline { 1 - 2 } 2.8 & 608 & 244 & 40.1 \\
5.5 & 1186 & 648 & 54.6 \\
22.0 & 2340 & 1247 & 53.3 \\
49.5 & 2141 & 1098 & 51.3 \\
88.0 & 1497 & 828 & 55.3 \\
198.0 & 1644 & 889 & 54.1 \\
297.0 & 1022 & 499 & 48.8 \\
792.0 & 363 & 185 & 50.9 \\
1240.0 & 1267 & 679 & 53.6 \\
1860.0 & 1182 & 625 & 52.9 \\
2810.0 & 1181 & 601 & 50.9 \\
3750.0 & 854 & 463 & 54.2 \\
4690.0 & 864 & 461 & 53.3 \\
\hline
\end{tabular}

The question involved is the comparison of the percentages dying at different intensities. These percentages could be compared just as they stand, but since we know from experience that a repetition of the experiment would not give an exact duplication of these results, the comparison becomes fruitful only after we have some estimate of the stability of these results under repetition. If we consider the first two exposures, the evidence can be presented completely in what is known as a fourfold table (see Table 2).

Table 2.-Fourfold Table

\begin{tabular}{c|c|c|c|c}
\hline $\begin{array}{c}\text { Intensity, r per } \\
\text { min. }\end{array}$ & $\begin{array}{c}\text { Number of } \\
\text { deaths }\end{array}$ & $\begin{array}{c}\text { Number of } \\
\text { survivors }\end{array}$ & Total & $\begin{array}{c}\text { Death rate, } \\
\text { per cent }\end{array}$ \\
\hline 2.8 & 244 & 364 & 608 & 40.1 \\
5.5 & 648 & 538 & 1186 & 54.6 \\
\hline Total............ & 892 & 902 & 1794 & 49.7 \\
\hline
\end{tabular}


Before concluding that the mortality rate at intensity $5.5 \mathrm{r}$ per min. is greater than that at $2.8 \mathrm{r}$ per min. we must test the statistical significance of the difference between these two percentages. This question ean be framed as follows: How probable is it that if two random samples of sizes 608 and 1186 were drawn out of a universe or experienee having a basie mortality of 49.7 per cent, they would differ as mueh in their mortality as they do in the present illustration? We ask this question because if we find it quite likely that two random samples would exhibit a difference at least as great as in the present ease when these samples were drawn out of the same experience, we shall hesitate to conclude that this observed difference is due to anything other than chance.

To answer this question we need to know something of the variability of pereentages determined by a number of samples of the same size, all drawn from the same experience. For a complete discussion of this subject the reader is referred to the treatment of the point binomial in any standard textbook on statistical method. It will be sufficient to say here that theoretical considerations and experimental tests have shown that such percentages are distributed in a normal probability eurve, having a mean equal to $p$ and a standard deviation equal to the square root of $p q / n$, where

$$
\begin{aligned}
& p=\text { the pereentage in the universe sampled. } \\
& q=100-p \\
& n=\text { size of sample drawn. }
\end{aligned}
$$

Since the normal curve is completely determined by its mean and standard deviation, it is possible, having determined these eonstants, to state the distribution of deviations of varying size. Table 3 gives the probability, $P$, that a sample will deviate from the eentral position by an amount equal to, or greater than, any multiple of the standard deviation, $\sigma$.

Table 3.-Probability of Occurrence of Deviations of Varying Size, as Given BY tile Normal Probability Curve

\begin{tabular}{c|c|c||c}
\hline $\begin{array}{c}\text { Dev. from mean } \\
\text { standard dev. } \\
x / \sigma\end{array}$ & $\begin{array}{c}\text { Probability of } \\
\text { dev. } \bar{\Sigma} x / \sigma\end{array}$ & $\begin{array}{c}\text { Dev. from mean } \\
\text { standard dev. } \\
x / \sigma\end{array}$ & $\begin{array}{c}\text { Probability of } \\
\text { dev. } \bar{\Sigma} x / \sigma \\
P\end{array}$ \\
\hline 0.00 & 1.0000 & 3.25 & 0.0012 \\
0.25 & 0.8026 & 3.50 & 0.00047 \\
0.50 & 0.6171 & 3.75 & 0.00018 \\
0.75 & 0.4533 & 4.00 & 0.000063 \\
1.00 & 0.3173 & 4.25 & 0.000021 \\
1.25 & 0.2113 & 4.50 & 0.0000068 \\
1.50 & 0.1336 & 4.75 & 0.0000020 \\
1.75 & 0.0801 & 5.00 & 0.00000057 \\
2.00 & 0.0455 & 5.25 & 0.00000015 \\
2.25 & 0.0244 & 5.50 & 0.000000038 \\
2.50 & 0.0124 & 5.75 & 0.0000000090 \\
2.75 & 0.0060 & 6.00 & 0.0000000020 \\
3.00 & 0.0027 & & \\
\hline
\end{tabular}


The theoretical distribution of deviations as obtained from the normal probability curve and given in Table 3 is visualized in Fig. 1. The abscissal scale shows the size of the deviations from the mean in units of standard deviation. The total area under the curve is unity, and probabilities, or relative frequencies, are measured in terms of the area between the curve and the horizontal axis, appropriate to the probability question asked. For example, the probability of the occurrence of a deviation greater than the mean, of a size between one and two standard deviations, is given by the area $A B C D$. We may compare Fig. 1 with Table 3 by noting that the shaded area which represents deviations from the mean in both directions, of a size greater than one standard deviation, constitutes about one-third of the total area, the value given in Table 3 being 0.3173 .

With sampling variability expressed as in Table 3 , it is possible for us to determine an answer to the question as to the significance of the differ-

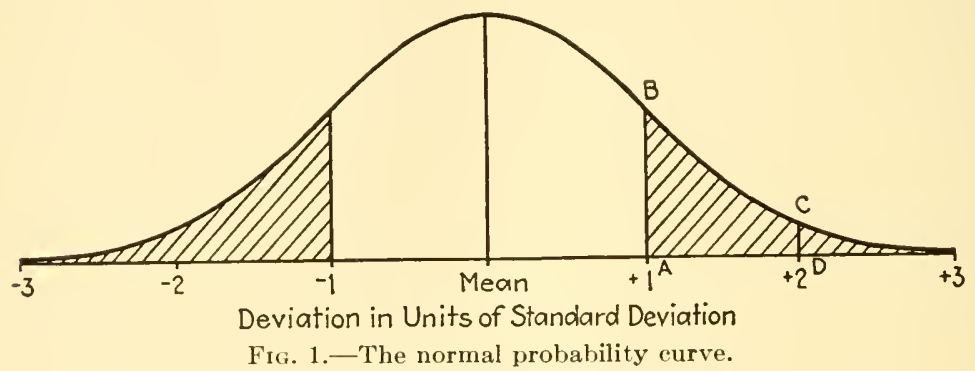

ence between the observed percentages. The first sample of size 608, if drawn from a universe having a basic percentage of 49.7 , would have a standard deviation,

$$
\sigma_{1}=\sqrt{\frac{(49.7)(50.3)}{608}}=2.0 \text { per cent }
$$

For the second sample the standard deviation would be

$$
\sigma_{2}=\sqrt{\frac{(49.7)(50.3)}{1186}}=1.5 \text { per cent }
$$

We are interested, however, in the difference between these two samples and we shall therefore need to consider not merely the sampling variability of a percentage as determined on a sample of a given size, but the sampling variability of the difference between two quantities, the degree of variability of each quantity being known. It can be shown that when two quantities are independent, the standard deviation of the difference between these quantities is given by

$$
\sigma_{\text {diff }}=\sqrt{\sigma_{1}^{2}+\sigma_{2}^{2}}
$$


Applying this equation to our ease, we have

$$
\sigma_{\text {diff }}=\sqrt{(2.0)^{2}+(1.5)^{2}}=2.5 \text { per cent }
$$

Thus we have two samples, one giving a death rate of 40.1 , the other a death rate of 54.6 per cent. The difference between these two death rates is 14.5 per cent and the standard deviation of this difference as derived above is 2.5 per cent. The difference measured in units of standard deviation is therefore

$$
\frac{\text { diff. }}{\sigma_{\text {diff }}}=\frac{14.5}{2.5}=5.8
$$

Taking this value into Table 3 , we see that the probability of these two samples coming out of the same experience is less than 0.00000001 . Thus it is extremely improbable that these two death rates are samples out of the same universe and we feel with a high degree of certainty that the mortality rate at intensity $2.8 \mathrm{r}$ per min. is definitely less than the mortality rate at intensity 5.5 .

A similar comparison of the death rates at intensity $5.5 \mathrm{r}$ per min. and $22.0 \mathrm{r}$ per min. gives a probability of 0.47 that a difference at least as great as the observed would occur when two samples of sizes 1186 and 2340 are drawn out of the same experience (53.7 per cent). Thus we hesitate to say that there is a real difference in the effect of these two intensities sinee there is such a large probability that the observed difference might arise from sampling alone.

An examination of all the succeeding intensities in Table 1 shows that the death rates fluetuate about a value in the neighborhood of 50 per cent. We might test these in successive pairs by the method just outlined, but since they have so little variation, we should like to test them as a group. We may do this by asking whether the death rates for the intensities 5.5 to $4690 \mathrm{r}$ per min., inclusive, differ from each other by amounts that are greater than would be expected under sampling. To answer this question we need the death rate for the entire experience covered by these intensities. The total number of eggs observed being 15,541 , and the number of deaths, 8223 , this death rate is 52.9 per cent. Using this general death rate, 52.9 per cent, as the value of $p$ and taking the number of eggs at each intensity as $n$, we can calculate the standard deviation for each death rate and then determine how far each death rate deviates, in multiples of its standard deviation, from the general death rate. These 12 results are given in Table 4, together with the expected number in each class, determined by applying the probabilities obtained from Table 3.

The agreement between the observed results and those expected under simple sampling would lead one to say that there is a high degree of 
probability that the variation in the results over this range of intensities is nothing more than would be expected by chance in sampling from the same universe, and it would therefore be dangerous to elaborate a scientific theory to explain this variation.

It should be emphasized that significance tests are but preliminary steps and do not constitute an analysis of the problem. If we find that Table 4.-Distribution of Observed Mortality Rates Compared with a Chance Distribution

\begin{tabular}{|c|c|c|}
\hline \multirow{2}{*}{$\begin{array}{l}\text { Distance from general death } \\
\text { rate in units of stand. dev. }\end{array}$} & \multicolumn{2}{|c|}{ Number of rates } \\
\hline & Observed & Expected \\
\hline Less than $1 \ldots$ & 7 & 8.2 \\
\hline $1-2 \ldots \ldots \ldots$ & 4 & 3.3 \\
\hline $2-3 \ldots \ldots$ & 1 & 0.5 \\
\hline 3 and over....... & 0 & 0.0 \\
\hline Total. & 12 & 12 \\
\hline
\end{tabular}

an observed difference is not significant, we shall attempt no explanation of this difference; but if the observed difference is significant, we proceed to the examination of the possible hypotheses which might account for the observed facts.

\section{TREATMENT OF MEASUREMENTS}

The preceding section dealt with problems in which the character under observation was one that had a two-way classification, such as died or did not die, but in many cases we are interested in a particular factor of the biological form that is subject to measurement on some scale. In such cases we have a measurement for each individual organism of an experimental and control group and the statistical problem is that of summarizing the measurements in such a way that the two groups may be readily compared. We may, if we choose, select some particular point on the scale and state for the experimental and the control group the percentage of animals that fall above and below this point, and then compare these percentages by the method of the preceding section. In so doing, however, we lose most of the advantage of the measurement, for the treatment proceeds as though the measurements had been taken on a scale having but one division point. A better treatment of such measurements gives us some notion of the point on the scale at which the measurements center and some idea of the extent to which the individuals vary from this centering position.

While there are an infinite number of centering points that may be defined, there are but three that are in common use, the arithmetic mean, 
the median, and the mode. The arithmetic mean is the sum of all the measurements divided by the number of measurements, and next to a simple percentage is probably the most commonly used statistical constant. The median is the point on the scale such that half the measurements are above this point and half the measurements are below. It is obvious that this definition is determinate if the number of observations is odd and not too great, but if the number of observations is even or if the number of measurements is great, the definition becomes indeterminate and some method of interpolation must be used. Such methods will be illustrated later in connection with the use of probability paper for representing a frequency distribution. The mode is defined as the point on the scale where the maximum number of individuals occur, and although this point may be determined directly from the observations, it is subject to such a degree of sampling rariation that it is probably useless to treat the mode without some form of frequency curve discussion. The difficulty of determination of the mode makes it the least used of these three centering constants.

As with the centering constant, the statistical indices of scatter are numerous, the extent of the variation of the individuals from a centering point being measured in terms of average deviation, standard deriation, quartile limits, ete. Average deviation is just what its name indicates, the average amount that the individuals deviate from the center, and may be stated separately for positive deviations (above the center) and negative deviations (below the center) or may be stated without regard for the sign of the deviation. Standard deviation is the most commonly used measure of scatter when the arithmetic mean is used as the centering point. It is defined as the square root of the average of the squares of the deviations of the individuals from the mean. Although not so simple an index to grasp as the average deviation, it is used because of its connection with the theory of errors and the normal probability curve. Standard deviation is measured in the same unit as the mean, that is, the yardstick of measurement, and it becomes a unit on this scale in terms of which the variability of the individuals is measured. In Table 3 has been presented the probability that an individual would deviate from the mean by an amount equal to or greater than any multiple of this unit of variation. These probabilities have been determined on the assumption that the material is distributed according to the normal probability curve, and Table 3 should be used only when this assumption is reasonably well justified. The quartile limits are two points on the scale of measurement of the same character as the median, and are appropriately used in connection with the median as a measurement of variation. The lower quartile is the point below which one-quarter of the observations occur, and the upper quartile is the point below which 
three-quarters of the observations occur. These limits have, however, the same difficulty of determination as the median.

Since the mean and standard deviation are the most commonly used constants in summarizing a series of measurements, the discussion in this section will be limited to the treatment of these two constants. Before proceeding to the determination of these constants for a set of observations, it is usually convenient to order the observations into what is called a frequency distribution. A frequency distribution is nothing more than a table giving values on the scale of measurement and opposite each the number of individuals found to have that particular seale value. If the unit of measurement is very small compared with the degree of variation between the smallest and largest individuals under discussion, such a frequency table becomes very long and it is customary to group the observations into classes, giving in the frequency distribution the numbers of individuals falling within continuous evenly spaced classes on the scale. Grouping of this type amounts to nothing more than a decision that the original measurements were taken with too fine a scale division, and that they might just as well have been made on a scale whose unit was equal to the unit of grouping.

Some of the various treatments that can be given a frequency distribution and the determination of the mean and standard deviation may perhaps best be presented by analyzing some actual data and we shall take for this purpose measurements from a paper by Muriel Robertson (6) in which she compared the length of the protozoon Bodo caudatus in a sample subjected to gamma-ray radiation with that of a nonirradiated group. Five hundred bodos were included in the irradiated and the same number in the control group, and their lengths were measured in units of $0.5 \mu$. These measurements arranged in frequency distributions are shown in Table 5, the first column giving the scale of the measurements in $0.5 \mu$ and the second column the number of individuals having the length indicated. The distributions are presented graphically in Fig. 2.

The mean and standard deviation of these 500 items might be computed by following the definitions directly, but the amount of labor can be very much reduced by using the frequency distribution and following the process given below. An arbitrary scale labeled $x$ is selected, the origin of this scale being placed at any point, but for convenience near the center of the distribution, and with this new scale the columns $x f$ and $x^{2} f$ are computed as indicated. The mean and standard deviation can then be obtained by the following equations:

$$
\text { Mean }=m=\text { origin }+\frac{\Sigma x f}{\Sigma f}
$$


where by origin we mean the scale reading corresponding to the zero position for $x$, and $\Sigma$ stands for summation,

$$
\text { Standard deviation }=\sigma=\sqrt{\frac{\Sigma x^{2} f}{\Sigma f}-\left(\frac{\Sigma x f}{\Sigma f}\right)^{2}}
$$

Since these measurements are expressed in terms of $0.5 \mu$, it is necessary to multiply the mean and standard deviation by 0.5 in order to get the values in terms of microns. This process was followed in determining the means and standard deviations given in Table 5. If, as is not

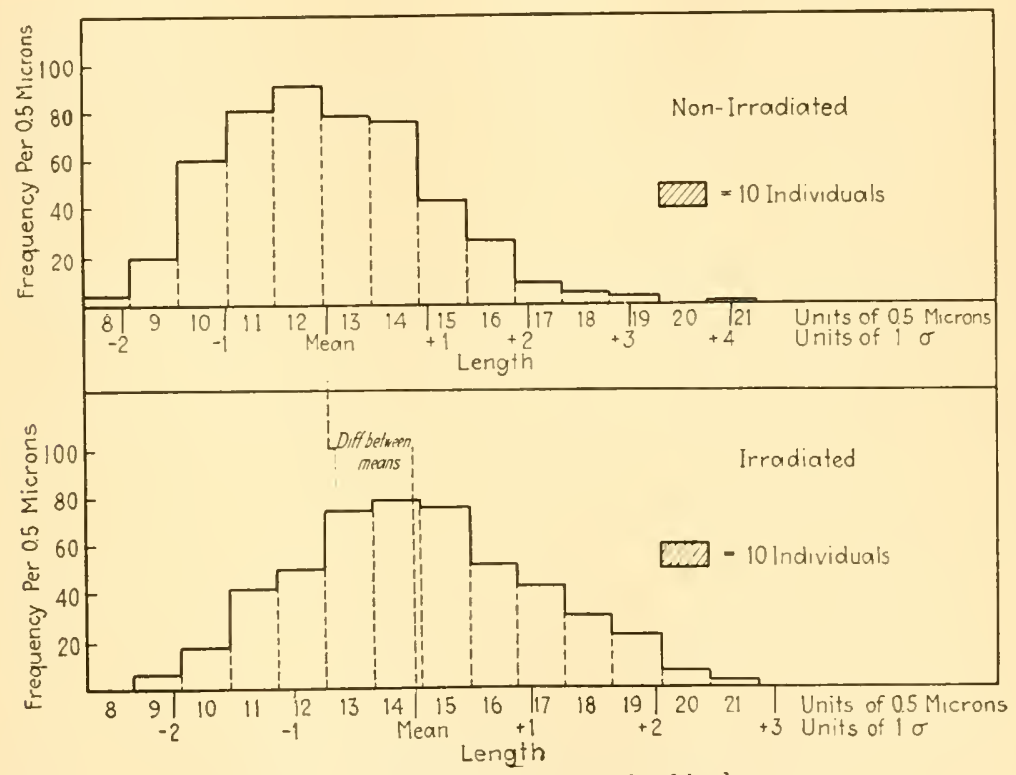

FIG. 2.-Distribution of length of bodos.

infrequently the case, a change of one in the $x$ scale corresponds to a change of something other than one in the scale of measurement, adjustment for this scale change must be made in the mean and standard deviation.

Before proceeding to the interpretation of these constants it will be necessary to consider their sampling variation, that is, the extent to which they vary under repeated tests. Study of the theory of errors shows that determination of the arithmetic mean from repeated samples out of the same experience leads to a set of means that are distributed according to a normal probability curve. The standard deviation of this curve is called the standard error of the mean and measures the variation in the 
Table 5.-Distribution of Length of Bodos in Nonirradiated and Irradiated Cultures

\begin{tabular}{|c|c|c|c|c|c|c|c|}
\hline \multirow{2}{*}{\multicolumn{2}{|c|}{$\begin{array}{l}\text { Length in units of } \\
0.5 \mu \text { (midpoint of } \\
\text { class) }\end{array}$}} & \multirow{2}{*}{$\begin{array}{c}\text { Frequency } \\
f\end{array}$} & \multirow{2}{*}{$x$} & \multirow{2}{*}{$x f$} & \multirow{2}{*}{$x^{2} f$} & \multicolumn{2}{|c|}{ Accumulated frequency } \\
\hline & & & & & & Number & Percentage \\
\hline \multicolumn{8}{|c|}{ Nonirradiated ${ }^{*}$} \\
\hline & 8 & 5 & -4 & -20 & 80 & 5 & 1.0 \\
\hline & 9 & 20 & -3 & -60 & 180 & 25 & 5.0 \\
\hline & 10 & 60 & -2 & -120 & 240 & 85 & 17.0 \\
\hline & 11 & 81 & -1 & -81 & 81 & 166 & 33.2 \\
\hline & 12 & 91 & 0 & 0 & 0 & 257 & 51.4 \\
\hline & 13 & 79 & 1 & 79 & 79 & 336 & 67.2 \\
\hline & 14 & 77 & 2 & 154 & 308 & 413 & 82.6 \\
\hline & 15 & 43 & 3 & 129 & 387 & 456 & 91.2 \\
\hline & 16 & 26 & 4 & 104 & 416 & 482 & 96.4 \\
\hline & 17 & 9 & 5 & 45 & 225 & 491 & 98.2 \\
\hline & 18 & 5 & 6 & 30 & 180 & 496 & 99.2 \\
\hline & 19 & 3 & 7 & 21 & 147 & 499 & 99.8 \\
\hline & 20 & 0 & 8 & 0 & 0 & 499 & 99.8 \\
\hline & 21 & 1 & 9 & 9 & 81 & 500 & 100.0 \\
\hline Total & . & 500 & $\ldots$ & 290 & 2404 & & \\
\hline \multicolumn{8}{|c|}{ Irradiated $\dagger$} \\
\hline & 8 & 0 & -4 & 0 & 0 & 0 & 0.0 \\
\hline & 9 & 7 & -3 & -21 & 63 & 7 & 1.4 \\
\hline & 10 & 18 & -2 & -36 & 72 & 25 & 5.0 \\
\hline & 11 & 42 & -1 & -42 & 42 & 67 & 13.4 \\
\hline & 12 & 50 & 0 & 0 & 0 & 117 & 23.4 \\
\hline & 13 & 74 & 1 & 74 & 74 & 191 & 38.2 \\
\hline & 14 & 79 & 2 & 158 & 316 & 270 & 54.0 \\
\hline & 15 & 75 & 3 & 225 & 675 & 345 & 69.0 \\
\hline & 16 & 51 & 4 & 204 & 816 & 396 & 79.2 \\
\hline & 17 & 42 & 5 & 210 & 1050 & 438 & 87.6 \\
\hline & 18 & 30 & 6 & 180 & 1080 & 468 & 93.6 \\
\hline & 19 & 22 & 7 & 154 & 1078 & 490 & 98.0 \\
\hline & 20 & 7 & 8 & 56 & 448 & 497 & 99.4 \\
\hline \multirow{2}{*}{\multicolumn{2}{|c|}{ Total. }} & 3 & 9 & 27 & 243 & 500 & 100.0 \\
\hline & & 500 & & 1189 & 5957 & & \\
\hline$*$ & $\begin{array}{r}\text { Standard d } \\
\text { Standard error } \\
\text { tandard error of }\end{array}$ & $\begin{aligned} \text { Mean } & =6.2 ! \\
\text { eviation } & =1.0 \\
\text { of mean } & =0.0 \\
\text { st. dev. } & =0.0\end{aligned}$ & & $\dagger$ & $\begin{array}{l}\text { Stand } \\
\text { tandard } \\
\text { ndard er }\end{array}$ & $\begin{array}{r}\text { Mean } \\
\text { rd deviation } \\
\text { ror of mean } \\
\text { of st. dev. }\end{array}$ & $\begin{array}{l}=7.189 \mu \\
=1.251 \mu \\
=0.056 \mu \\
=0.040 \mu\end{array}$ \\
\hline
\end{tabular}


mean to be expected from sampling alone. This standard error is given by the following equation:

$$
\text { Standard error of mean }=\sigma_{m}=\frac{\sigma}{\sqrt{n}}
$$

where $\sigma$ indicates the standard deviation of the individuals and $n$ the number of individuals. A similar treatment of the standard deviation determines its standard error as follows:

$$
\text { Standard error of st. dev. }=\sigma_{\sigma}=\frac{\sigma}{\sqrt{2 n}}
$$

These standard errors together with Table 3 may be used to indicate the likelihood that the mean and standard deviation would vary by simple sampling to any assigned degree. The numerical values of the standard errors for the illustration under consideration are given at the bottom of Table 5 .

A comparison of the average length of the irradiated and the nonirradiated animals shows a small difference in favor of the irradiated group, this group being on the average $0.899 \mu$ longer than the nonirradiated. To test the significance of this difference we follow the procedure used for percentages and state that the standard error of this difference is given by

$$
\sigma_{\text {diff. }}=\sqrt{\sigma_{m_{1}}^{2}+\sigma_{m_{2}}^{2}}=\sqrt{(.047)^{2}+(.056)^{2}}=0.073
$$

Turning to Table 3 with the value

$$
\frac{\text { Diff. }}{\sigma_{\text {diff. }}}=\frac{0.899}{0.073}=12.3
$$

we find the probability to be less than two in a billion that the observed difference is due to simple sampling, and we are therefore willing to proceed to a discussion of the reasons for this difference. Similar treatment of the standard deviation shows that the irradiated group has a standard deviation $0.194 \mu$ greater than the control, and the standard error of this difference being 0.052 , the difference is 3.7 times its standard error. From Table 3 we find that the probability of such a difference occurring by simple sampling is less than 2 in 10,000 and the difference would therefore be considered significant. Thus the individuals in the irradiated group are not only longer on the average than the nonirradiated individuals, but they show a greater degree of absolute variation in length.

In addition to discussing the absolute variability of these forms it is of interest to consider their relative variability and this is indicated by 
a statistical constant called coefficient of variation and defined as

$$
\text { C. of V. }=\frac{100 \sigma}{m}
$$

this index number stating the variability as a percentage of the mean. The standard error of the coefficient of variation is given approximately by

$$
\sigma_{\mathrm{C} . \text { of } \mathrm{v} .}=\frac{\mathrm{C} . \text { of } \mathrm{V}}{\sqrt{2 n}}
$$

In the illustration under consideration the difference between the coefficient of variation in the irradiated and that in the nonirradiated group is 0.60 with a standard error of 0.76 . The difference is thus only 0.8 of its standard error and there are approximately 40 chances in 100 that such a difference would arise from simple sampling. We shall therefore call this difference insignificant and conclude that, relative to their means, the irradiated and nonirradiated animals show the same degree of variation.

The statistical constants that have been derived may be seen in graphical relationship to each other and to their respective frequency distributions in Fig. 2.

Since the distribution of frequency is so often compared with that of the normal probability curve, a paper has been designed for the graphical treatment of frequency problems. This paper is called arithmetic probability paper and has for one axis a uniform scale representing the scale of measurement and for the other axis a scale of accumulated percentages, this scale being so adjusted that for normally distributed material the accumulated percentages of the frequency plot as a straight line. To apply this paper to the present illustration we have given in Table 5 the accumulated frequency stated as percentage of the total and these accumulated frequencies are shown on arithmetic probability paper in Fig. 3. On the vertical scale of this figure we have the length in $0.5 \mu$ and on the horizontal scale we have a statement of the percentage of individuals occurring below the indicated length. The points for the irradiated and nonirradiated forms fall approximately on straight lines, indicating that the distributions of the measurements are nearly normal. Straight lines have been drawn through these points, more attention being paid to the points in the center, since points at the extremes are always subject to a higher degree of variation on this paper.

Not only can this paper be used to test whether a distribution approximates the normal curve, but it can also be used as a computing device. Since the distributions are approximately normal the means will be at the 50 per cent position, and reading the length corresponding to this point 
for the control animals, we have 12.5 units, indicating a length of $6.25 \mu$ to be compared with the value 6.290 obtained by the computation. For the irradiated animals the length as given by the graph is $7.15 \mu$ to be eompared with the eomputed value of 7.189. To find the standard deviation, we note from Table 3 that deviations in both directions from the mean, of a size greater than one standard deviation, should form 31.73 per cent of the observations. Since the distribution is symmetrical, deviations on one side of the mean greater than one standard deviation would constitute one-half this value, or 15.87 per cent. From the graph

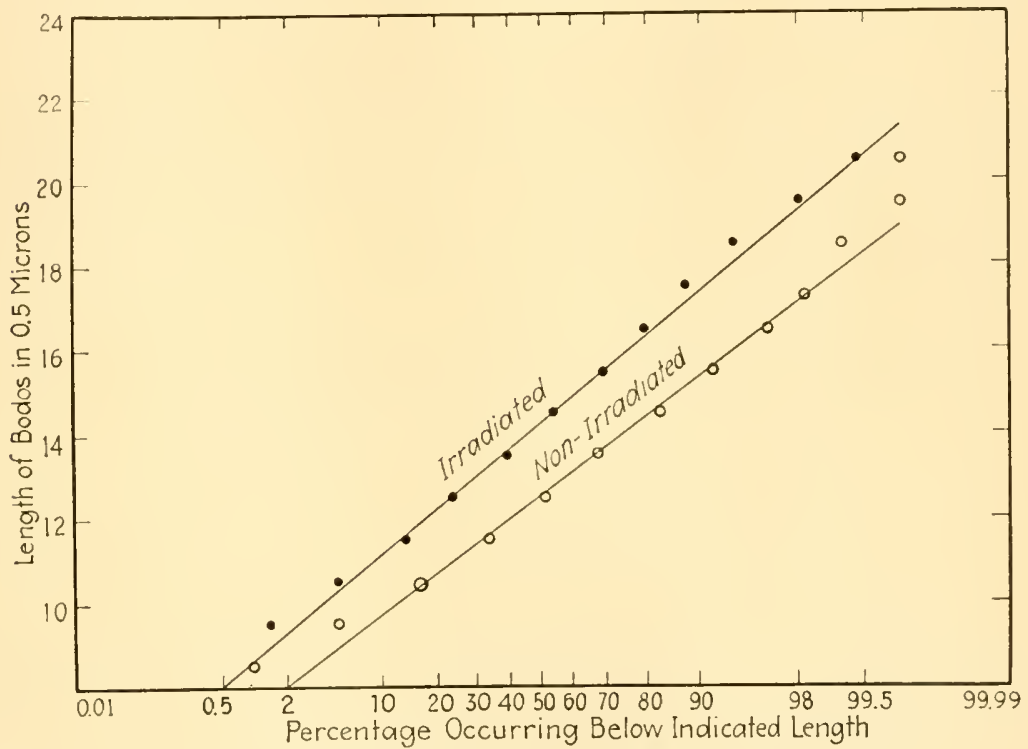

FIg. 3.-Accumulated frequency distribution of lengths of Bodo caudatus plotted on arithmetic probability paper.

for the control group, the pereentage 15.87 eorresponds to a measurement reading of 10.25 , which differs from the reading at the mean (12.5) by 2.25 units. Thus the standard deviation obtained graphically is $1.125 \mu$, while that given by the computation was 1.057. For the irradiated animals the difference between the reading at 15.87 and that at 50 per eent is 2.50 units, indieating a standard deviation of $1.25 \mu$ as compared with the eomputed value of 1.251 .

At times when we are comparing the difference between means for an experimental and control group we have a series of means such that taking them in eorresponding pairs, the experimental group does not differ signifieantly from the eontrol for any single pair and yet the difference is always in the same direetion, whieh supports the view that a real difference exists. We may aceumulate this evidence and test the signifi- 
cance of the difference between the two series as a whole by deriving the difference divided by the standard error of the difference for each pair of means and averaging these ratios. If there is no difference between experimental and control groups, these ratios for a series of differences should average to zero, with a standard deviation of unity. The standard error of the average of these ratios will therefore be $1 / \sqrt{ } n$, where $n$ is the number of pairs of values to be compared. If this mean is significantly different from zero we may conchude that the entire series of observations indicates a significant difference between experimental and control.

The process may be illustrated by a set of observations from the paper by Muriel Robertson (6), in which she compares at various times during a growth period of $23 \mathrm{hr}$., the mean length of a sample of irradiated bodos with a similar sample not irradiated. Table 6 presents the mean values and their standard errors for the irradiated and nonirradiated groups at five different times within the 23 -hr. interval.

Table 6.-Mean Lengths of Irradiated and Nonirradiated Bodos for Different Tines in a Twenty-three-hour Growth Period

\begin{tabular}{|c|c|c|c|c|c|c|c|c|c|c|}
\hline & \multicolumn{10}{|c|}{ Time of growth, hr. } \\
\hline & \multicolumn{2}{|c|}{7.5} & \multicolumn{2}{|c|}{10.5} & \multicolumn{2}{|c|}{13} & \multicolumn{2}{|c|}{19} & \multicolumn{2}{|c|}{23} \\
\hline & $m$ & $\sigma_{m}$ & $m$ & $\sigma_{m}$ & $m$ & $\sigma_{m}$ & $m$ & $\sigma_{m}$ & $m$ & $\sigma_{m}$ \\
\hline Nonirradiated..... & 14.10 & 0.21 & 12.90 & 0.21 & 13.26 & 0.20 & 13.22 & 0.21 & 12.17 & 0.14 \\
\hline Irradiated........ & 14.12 & 0.19 & 13.36 & 0.20 & 13.45 & 0.17 & 13.69 & 0.17 & 12.98 & 0.20 \\
\hline Diff. of means......... & 0.0 & 02 & 0.4 & & 0.1 & 19 & 0.4 & & & 81 \\
\hline Stand. error of diff..... & 0.2 & 28 & 0.2 & & 0.2 & 26 & 0.2 & 27 & 0.2 & 24 \\
\hline Diff. $/ \sigma_{\text {diff }} \ldots \ldots \ldots \ldots$ & 0.0 & & 1.5 & & 0.7 & & 1.7 & & 3. & 38 \\
\hline
\end{tabular}

The ratio of the difference between these means to the standard error of the difference is small enough so that at no point except possibly the last one are we assured that the difference may not quite likely be due to sampling. The average ratio of the difference to its standard error is 1.502 and the standard error of this average is $1 / \sqrt{5}$ or 0.447 . Thus the average is 3.36 times its standard error and interpolating in Table 3 , we find that the probability is less than 1 in 1000 that such a series of observations would arise by chance if no real difference between the irradiated and nonirradiated group existed. Thus we may conclude that the differences observed at the various times are really significant differences in spite of the fact that they did not show up to be significant on the basis of the individual tests. 
The preceding illustrations have presented methods for treating a series of measurements in a few of the simplest statistical constants, but since we are in general interested in as direct and simple a summary of the observations as possible, these methods will usually be found to be sufficient. In certain cases the measurements may have some more elaborate statistical characteristics, such as skewness or bimodality, that are capable of biological interpretation and are therefore worthy of investigation. These factors will obviously demand more complex treatment, but their development will follow natural extensions of the logic so far employed.

\section{TREATMENT OF LETHAL DOSE}

Since many forms of radiation destroy living matter if sufficient dosage is used, one of the very common problems encountered in the study of the effect of radiation on organisms is that of the size of dose necessary to kill the organism. It is usually found that if a group of organisms is subjected to a sufficiently small dosage, none of the forms dies, but that as the dosage is increased, an increasing percentage dies until ultimately a dosage is reached at which all of them are killed. If we plot the percentage of survivors against dosage an S-shaped curve results running from 100 per cent for small doses down through to zero per cent for large doses. This curve describes the lethal power of the form of radiation used on the particular organism subjected to experimentation. As an abbreviated statement of the lethal power of the radiation on the organism, the term lethal dose has been used. The words lethal dose have not been consistently used by different writers; some have used the words to indicate the dosage below which all animals would live but at which one or two would begin to die, others have used the words to indicate the minimal dosage at which all the animals would be killed, and still others have called the lethal dose that at which 50 per cent of the animals would die. All three of these definitions are statistical in character and are based on the above described S-shaped curve. The first two definitions are dependent on the end points of the range of the curve, while the third definition involves a centering point. Since range is a statistical constant having a high degree of variability, the end points are statistically less stable than the centering point and therefore the third definition is to be preferred and is the one which will be indicated whenever the words lethal dose are used in this chapter.

The accurate determination of lethal dose is obviously often a tedious and expensive procedure, for it calls for the determination at a varicty of dosages of the percentage of animals killed, and therefore at each dosage enough animals must be used to determine a percentage with a reasonable degree of precision. If the biological form used for experi- 
mental purposes is a bacterium, some protozoon form, or the well-known fruit fly Drosophila melanogaster, it is easily possible to determine the percentage of deaths at a variety of dosages with a high degree of accuracy, but if some of the larger forms are used, such as the rat or the rabbit, the expense of the accurate determination of these percentages is usually prohibitive. For that reason our best illustrations of lethal-dose curves are found when the smaller forms are used, as for instance those given by Packard $(3,4)$ for Drosophila eggs, two of which are given in Fig. 4.

Since the ehange in percentage mortality is an S-shaped curve, similar to that of accumulated frequencies of the normal probability

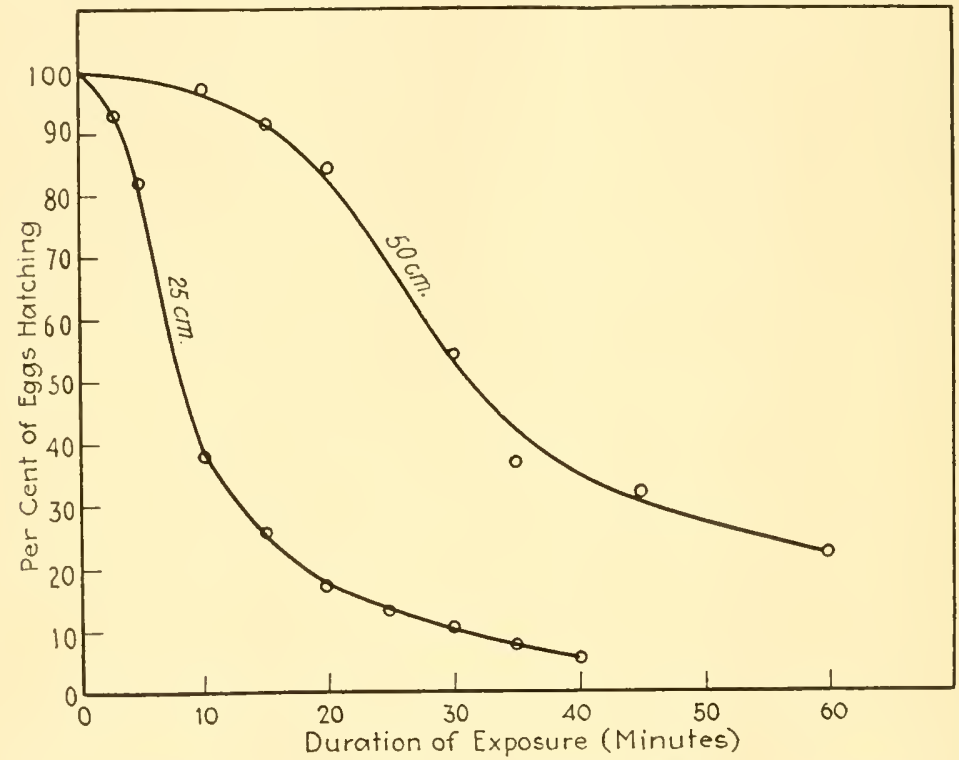

Frg. 4.-Survivorship rate of Drosophila eggs exposed to X-rays of different intensities.

curve, the probability paper previously described will be found useful for its graphic representation. In some cases the observed points will plot on such paper in essentially linear fashion and when a straight line is drawn through the points the 50 per cent position representing the lethal dose may be read directly from the graph. We may also read from such graphs the standard deviation of the curve, which would be a measure of the variation in the response of the organism to the radiation. In other cases, such as those of Packard (3), referred to above, the percentage mortality does not plot in linear fashion against the dosage on arithmetic probability paper, due to the skewness of the S-shaped curve. This skewness can sometimes be taken account of and a linear plot obtained by using the logarithm of the dosage and making a plot on arithmetic 
probability paper, or by using specially prepared logarithmic probability paper. In either of these cases we have a quick and reasonably accurate method of arriving at the value of the lethal dose.

The method just outlined is satisfactory when it is possible to use a large number of animals for the determination of each percentage. But when we are limited to a few animals, an approximate determination of lethal dose will have to be made and the procedure given in the following illustration will usually be found satisfactory for summing up the evidence contained in the observations. In Table 7 is presented a series of observations made by Russ et al. (7) for the determination of the dosage of penetrating X-rays necessary to kill the eat. The total number of animals used was 27 , and these animals in groups, varying in size from 2 to 10 were subjected to dosages over a range from $0.22 T_{1}$ to $1.1 T_{1}$.

Table 7.-Mortality of Cats Subjected to X-ray Radiation

\begin{tabular}{|c|c|c|c|c|c|c|c|}
\hline \multirow{3}{*}{$\begin{array}{l}\text { Duration of } \\
\text { radiation in } \\
\text { terms of } T_{1}\end{array}$} & \multicolumn{3}{|c|}{$\begin{array}{l}\text { Observed number of } \\
\text { animals }\end{array}$} & \multicolumn{3}{|c|}{$\begin{array}{l}\text { Implicit number of } \\
\text { animals }\end{array}$} & \multirow{2}{*}{$\begin{array}{c}\text { Probability } \\
\text { of dying } \\
n+1 \\
\end{array}$} \\
\hline & & & & Toutod & & & \\
\hline & Tested & viving & Dying & $m+n$ & $\begin{array}{c}\text { viving } \\
m\end{array}$ & $\begin{array}{l}\text { Dying } \\
n\end{array}$ & $m+n+2$ \\
\hline 0.22 & 2 & 2 & 0 & 8 & 8 & 0 & 0.10 \\
\hline 0.28 & 2 & 2 & 0 & 6 & 6 & 0 & 0.13 \\
\hline 0.36 & 2 & 1 & 1 & 5 & 4 & 1 & 0.29 \\
\hline 0.45 & 10 & 3 & 7 & 11 & 3 & 8 & 0.69 \\
\hline 0.55 & 7 & 0 & 7 & 15 & 0 & 15 & 0.94 \\
\hline 0.74 & 2 & 0 & 2 & 17 & 0 & 17 & 0.95 \\
\hline 1.1 & 2 & 0 & 2 & 19 & 0 & 19 & 0.95 \\
\hline
\end{tabular}

Inspection of Table 7 shows that it is obviously impossible to determine the percentage dying at each of the various dosages, but we can make an approximation to the lethal curve by making use of the two following assumptions: (a) All animals tested at any given dosage and found to survive would have survived if subjected to a smaller dosage. (b) All animals tested at a given dosage and found to die would have died if subjected to a larger dosage. Applying these two assumptions to the observations in the table, we can set up the columns headed $m$, implicit survivals, and $n$, implicit deaths. From the probability theorem that if an event has happened $n$ times and has failed to happen $m$ times, then the probability that on the next trial the event will happen is given by $(n+1) /(m+n+2)$, we may arrive at a figure indieating the likelihood that a eat will die at each of the dosages tried. Plotting these probabilities against the dosages on arithmetic probability paper, drawing a straight line through the points, and reading the dosage corresponding 
to the 50 per cent position, we may determine the lethal dose. For this particular illustration the lethal dose, as read from Fig. 5, is approximately $0.40 T_{1}$.

While the procedure just outlined is obviously an approximate method, it will determine the lethal dose as accurately as figures of this sort warrant. If we also read from the graph the number of units of dosage corresponding to the standard deviation, we have a measure of

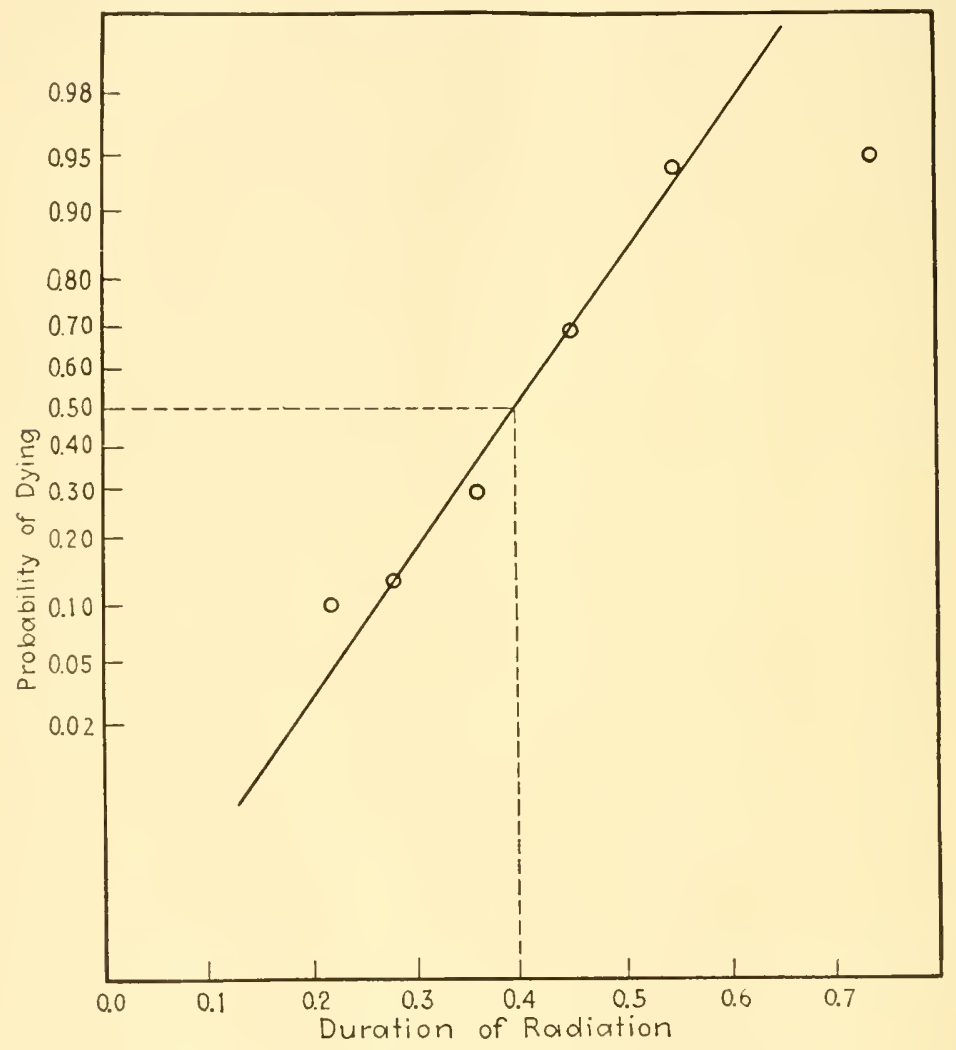

Fig. 5.-Graph for the determination of lethal dose.

the individual variability of animals in their reaction to the radiation. The value of the standard deviation so read is slightly higher than it should be, for although the two assumptions given above do not disturb the centering point, they do tend to increase the spread of the S-shaped curve describing the lethal action.

\section{CURVE FITTING}

The effects of radiation will often be measured in terms of more than one variable and a summary of the results of such cases will necessitate 
putting the variables concerned into relationship to each other. Where there are but two variables we may at times take the ratio of one variable to the other and give our solution in terms of this ratio, but in most problems the solution will involve the formulation of some other equational relationship. Such problems will involve curve fitting by either an analytical or graphical method, and the equational relationships may be based on some form of rationalization or may be entirely empirical. Since in seience we aim at as simple a rationalization as possible, the results of many problems are stated either in terms of a linear equation or in terms of an exponential equation. These two equations allow the treatment respectively of the case where one variable changes at a uniform absolute rate with regard to the other, and the case where one variable changes at a constant relative rate with regard to the other. Since these two equations are so widely applied, the methods of treating them will be developed in the two following illustrations:

As a possible application of a linear equation, let us consider the observations reported by Laurens (2) on the effeet of carbon-arc radiation on essential hypertension in man. We may limit our discussion to two of his measurements, that of systolic blood pressure before and after

Table 8.-Systolic Blood Pressure of Subject F before and after Treatment with Carbon-arc Radiation

\begin{tabular}{|c|c|c|c|c|c|c|c|c|}
\hline \multirow{2}{*}{$\begin{array}{l}\text { Visit } \\
\text { No. }\end{array}$} & \multicolumn{2}{|c|}{$\begin{array}{c}\text { Blood pressure, } \\
\mathrm{mm} . \mathrm{Hg}\end{array}$} & \multirow{2}{*}{$\begin{array}{l}\text { Visit } \\
\text { No. }\end{array}$} & \multicolumn{2}{|c|}{$\begin{array}{c}\text { Blood pressure, } \\
\text { mm. } \mathrm{Hg}\end{array}$} & \multirow{2}{*}{$\begin{array}{l}\text { Visit } \\
\text { No. }\end{array}$} & \multicolumn{2}{|c|}{$\begin{array}{c}\text { Blood pressure, } \\
\text { mm. } \mathrm{Hg}\end{array}$} \\
\hline & Before & After & & Before & After & & Before & After \\
\hline 1 & 230 & 220 & 20 & 168 & 162 & 42 & 188 & 178 \\
\hline 2 & 190 & 180 & 21 & 190 & 182 & 43 & 198 & 190 \\
\hline 3 & 165 & 168 & 22 & 178 & 168 & 44 & 191 & 178 \\
\hline 4 & 176 & 178 & 25 & 200 & 192 & 45 & 188 & 162 \\
\hline 5 & 166 & 175 & 27 & 186 & 180 & 46 & 188 & 176 \\
\hline 6 & 182 & 170 & 28 & 186 & 182 & 47 & 208 & 188 \\
\hline 7 & 178 & 145 & 29 & 196 & 180 & 48 & 190 & 178 \\
\hline 8 & 165 & 153 & 30 & 200 & 188 & 49 & 196 & 172 \\
\hline 9 & 170 & 150 & 31 & 198 & 176 & 50 & 172 & 180 \\
\hline 10 & 160 & 140 & 32 & 178 & 168 & 51 & 210 & 186 \\
\hline 11 & 180 & 178 & 33 & 210 & 198 & 52 & 170 & 168 \\
\hline 12 & 180 & 170 & 34 & 178 & 176 & 53 & 168 & 168 \\
\hline 13 & 188 & 178 & 35 & 182 & 148 & 54 & 174 & 178 \\
\hline 14 & 190 & 192 & 36 & 178 & 150 & 55 & 215 & 195 \\
\hline 15 & 180 & 162 & 37 & 170 & 188 & 56 & 186 & 195 \\
\hline 16 & 182 & 168 & 38 & 170 & 166 & 57 & 185 & 190 \\
\hline 17 & 180 & 176 & 39 & 175 & 176 & 58 & 188 & 198 \\
\hline 18 & 186 & 170 & 40 & 190 & 178 & & & \\
\hline 19 & 188 & 184 & 41 & 210 & 196 & & & \\
\hline
\end{tabular}


radiation. For the purposes of this illustration we shall treat the measurements on subject $F$ which are presented in Table 8 .

Although the effects of radiation are in this case given in terms of two measurements, blood pressure before and after radiation, the fact that we are interested in the possible rise or fall in blood pressure would lead us immediately to a consideration of the difference between the two pressures. If we form this difference for the $\mathbf{5 5}$ visits for which treatment was given, we find that there was an average fall in pressure of $9.1 \mathrm{~mm}$. $\mathrm{Hg}$. If this fall in pressure had occurred uniformly throughout the series of observations, it would only be necessary to determine its degree of variation and the problem would be resolved by the treatment of one variable alone, that is, the fall in pressure. Examination of the measurements, however, shows that there is a decided tendency for the larger falls to occur in the cases where the initial pressure was high, and we must therefore consider the difference in relation to the initial pressure. By plotting the fall in pressure against the initial pressure for each of the observations, we see that they are not in direct ratio to each other, and therefore a treatment of the drop in pressure as a percentage of the initial reading would be inappropriate. We may, however, derive an equational relationship that will give us the average drop in pressure for each initial pressure by treating this as a problem in simple linear correlation.

Simple linear correlation analysis merely means the determination of a straight line to represent the average values of one variable for given values of the other, and of some measure of the degree of variation of the points about the line. This solution may be outlined as follows: Let $y$ represent the fall in pressure and $x$ the initial pressure. Then a linear relation between these variables may be represented by the equation

$$
y=a+b x
$$

in which $a$ is dependent upon the choice of origin and is therefore relatively unimportant, and $b$ represents the change in the fall of blood pressure for each unit change in the blood pressure itself. The values of $a$ and $b$ derived by the method of least squares will be given by the following equations:

$$
\begin{aligned}
& a=m_{y}-b m_{x} \\
& b=r \frac{\sigma_{y}}{\sigma_{x}}
\end{aligned}
$$

where $m_{x}, m_{y}, \sigma_{x}, \sigma_{y}$, represent the means and standard deviations of $x$ and $y$ as defined in the section treating measurements, and $r$ is called the coefficient of correlation and is given by the formula

$$
r=\frac{(\Sigma x y / n)-m_{x} m_{y}}{\sigma_{x} \sigma_{y}}
$$


The only additional computation necessary for the determination of $r$ after the means and standard deviations have been derived is the multiplication of each $x$ value by the corresponding $y$ value, the summation of these products, and the division of this sum by the number of pairs of values involved. When there are a relatively small number of pairs of observations as is the case in this illustration, this calculation may be done directly, but if the number of observations is very much greater, the arithmetic procedure may be simplified by making use of a correlation table and by its treatment as outlined in standard textbooks on statistical method.

Applying the direct method to the observations in Table 8, we have the following results. The mean systolic pressure before radiation is $185 \mathrm{~mm}$.; the variation in these initial readings is indicated by a standard deviation of $14 \mathrm{~mm}$. ; the mean drop in pressure is $9.1 \mathrm{~mm}$.; and the standard deviation of this drop is $10.9 \mathrm{~mm}$., indicating a high degree of variation in the effect of the radiation on different days. The correlation between the drop in pressure and the initial pressure is indicated by the coefficient of correlation, $r$, of 0.32 . The values of $a$ and $b$ as determined by the above equations are -37.4 and 0.25 , respectively, so that the equation relating the drop in pressure to the initial pressure is

$$
y=0.25 x-37.4
$$

This equation written in the form

$$
y=0.25(x-150)
$$

shows that when the systolie pressure before radiation was $150 \mathrm{~mm}$., the average drop was zero, and that for initial pressures below $150 \mathrm{~mm}$., the average drop was negative, indicating a rise in pressure on the average, after radiation. The value of the $b$ constant shows that for each unit that the initial pressure stood above $150 \mathrm{~mm}$. the drop increased on the average $0.25 \mathrm{~mm}$. Thus we have in two statistical constants $a$ and $b$ a statement of the way in which, for this individual, the average drop in systolic blood pressure following radiation ehanged according to the initial pressure.

In a similar way we might study the variation of drop in systolic pressure according to duration of irradiation, and the results of the two solutions might be thrown together by the method of partial correlation, as outlined in any standard text. The satisfactory statistical treatment of this problem in a general sense would involve the determination and analysis of a series of $b$ constants for a variety of patients.

Other than the straight line, the most commonly used equational relationship is the exponential form, or the equation for geometric 
increase or decrease. This equation may be written in a variety of forms, among them being

$$
y=e^{\alpha+\beta x}=10^{a+b x}=A B^{x}
$$

where $e^{\alpha}=10^{a}=A=$ the value of $y$ when $x=0$

and $e^{\beta}=10^{b}=B=$ the ratio of the $y$ value at any $x$ position to the $y$ value at the position, $x-1$. Since the equation may also be written in the form

$$
\log y=a+b x
$$

we see that the logarithm of $y$ is related to $x$ in linear fashion and the constants $a$ and $b$ may be derived by using the logarithms of the $y$ values with the corresponding $x$ values and following the procedure just outlined for the straight line. To facilitate the treatment of such relationships, arithlog plotting paper has been developed having an arithmetic scale on one axis and a logarithmic scale on the other. On such paper we may plot our $x$ values arithmetically and our $y$ values logarithmically, and if the observed material is following an exponential relationship, the points obtained will fall on a straight line except for experimental variations.

This exponential equation might be appropriately applied to the observations taken by Henshaw (1) in his study of the effect of roentgen rays on the time of the first cleavage of marine invertebrate eggs. Different lots of the eggs were irradiated for different lengths of time and inseminated at varying times after the beginning of irradiation, the delay Table 9.-Effect of Roentgen Rays on the Retardation in Onset of First Cleavage in Arbacia Eggs According to Duration of Exposure and Time OF INSEMINATION

\begin{tabular}{|c|c|c|c|c|c|c|c|c|c|}
\hline \multicolumn{2}{|c|}{5} & \multicolumn{2}{|c|}{10} & \multicolumn{2}{|c|}{20} & \multicolumn{2}{|c|}{40} & \multicolumn{2}{|c|}{60} \\
\hline $\begin{array}{l}\text { Interval, } \\
\text { min. }\end{array}$ & $\begin{array}{l}\text { Retar- } \\
\text { dation, } \\
\text { min. }\end{array}$ & $\begin{array}{c}\text { Inter- } \\
\text { val, } \\
\text { min. }\end{array}$ & $\begin{array}{l}\text { Retar- } \\
\text { dation, } \\
\text { min. }\end{array}$ & $\begin{array}{c}\text { Inter- } \\
\text { val, } \\
\text { min. }\end{array}$ & $\begin{array}{l}\text { Retar- } \\
\text { dation, } \\
\text { min. }\end{array}$ & $\begin{array}{c}\text { Inter- } \\
\text { val, } \\
\text { min. }\end{array}$ & $\begin{array}{c}\text { Retar- } \\
\text { dation, } \\
\text { min. }\end{array}$ & $\begin{array}{c}\text { Inter- } \\
\text { val, } \\
\text { min. }\end{array}$ & $\begin{array}{l}\text { Retar- } \\
\text { dation. } \\
\text { min. }\end{array}$ \\
\hline 5 & 15.5 & 10 & 24.8 & 20 & 30.2 & 40 & 46.8 & 80 & 55.8 \\
\hline 15 & 14.0 & 20 & 21.5 & 30 & 26.8 & 50 & 42.0 & 100 & 44.5 \\
\hline 25 & 11.8 & 30 & 198 & 40 & 23.2 & 60 & 38.5 & 120 & 38.2 \\
\hline 45 & 10.5 & 50 & 17.8 & 60 & 21.8 & 80 & 32.8 & 140 & 35.5 \\
\hline 65 & 9.5 & 70 & 160 & 80 & 18.2 & 100 & 28.0 & 180 & 28.8 \\
\hline 85 & 8.0 & 90 & 13.8 & 100 & 16.8 & 120 & 24.8 & 220 & 20.2 \\
\hline 125 & 5.8 & 130 & 10.5 & 140 & 13.0 & 160 & 18.5 & & \\
\hline 165 & 5.5 & 170 & 8.5 & 180 & 9.0 & 200 & 14.2 & & \\
\hline
\end{tabular}

Exposure, $\min$.

Note: Interval, $\min =$ Interval from start of irradiation to insemination, minutes. Retardation, min, = Retardation in cleavage, minutes. 
in cleavage time being recorded for each of these conditions. Some of his results are shown in Table 9 , and if these results are plotted on arithlog paper, the points for each of the different degrees of exposure fall essentially on a straight line. If we take the logarithm of the delay in cleavage and determine a linear relationship between these logarithms and the insemination time, we have the following values of the constants for each degree of exposure:

\begin{tabular}{c|c|c}
\hline $\begin{array}{c}\text { Length of } \\
\text { exposure, min. }\end{array}$ & $a$ & $b$ \\
\hline 5 & 1.168 & -0.00289 \\
10 & 1.396 & -0.00281 \\
20 & 1.519 & -0.00306 \\
40 & 1.780 & -0.00319 \\
60 & 1.956 & -0.00291 \\
\hline
\end{tabular}

While the values of $a$ inerease with additional exposure, the $b$ values are astonishingly constant. The standard error of these $b$ constants is of the order of 0.00010 and it is obvious that the values do not differ from each other more than would be expeeted by simple sampling. If we treat the entire series of observations simultaneously, we obtain as our value of $b,-0.00298$, and using the previously derived values of $a$ with this value of $b$, we have for our equations representing the relationship between the delay in cleavage and the time after irradiation of insemination,

Exposure, $\min$.

5

10

20

40

60
Equation

$$
\begin{aligned}
& y=10^{1.168-.00298 x}=14.7(0.99316)^{x} \\
& y=10^{1.396-.00298 x}=24.9(0.99316)^{x} \\
& y=10^{1.519-.00298 x}=33.0(0.99316)^{x} \\
& y=10^{1.780-.00298 x}=60.3(0.99316)^{x} \\
& y=10^{1.956-.00298 x}=90.4(0.99316)^{x}
\end{aligned}
$$

These equations are plotted on an arithlog scale in Fig. 6 and we see that the agreement between theory and observation is excellent except for the case of the 40-min. exposure. In this case the rate of decline seems to be slightly greater than that given by the theoretical expression. This divergence is not sufficient, however, to make us seriously question our assumption that the rate of decline is constant for different degrees of exposure.

Turning to the interpretation of these equations, we see that the general order of the delay in cleavage, as represented by the constant $A$, increases with increasing exposure to radiation. But for each duration of exposure the amount of retardation of cleavage, $y$, diminishes with delay in insemination, $x$. This approach of the cleavage time toward the normal 
time proceeds at a rate represented by $B(0.993)$. Thus the eggs recover as to their cleavage time at a rate of 0.7 per cent per min. of delay in insemination. This rate of recovery in cleavage time with increasing delay of insemination is constant for various degrees of exposure.

Having derived any equational relationship between two variables, such as the ones just discussed, we naturally want some measure of the accuracy with which the equations represent the observed facts. The variability of the observed points about the curve is usually measured in

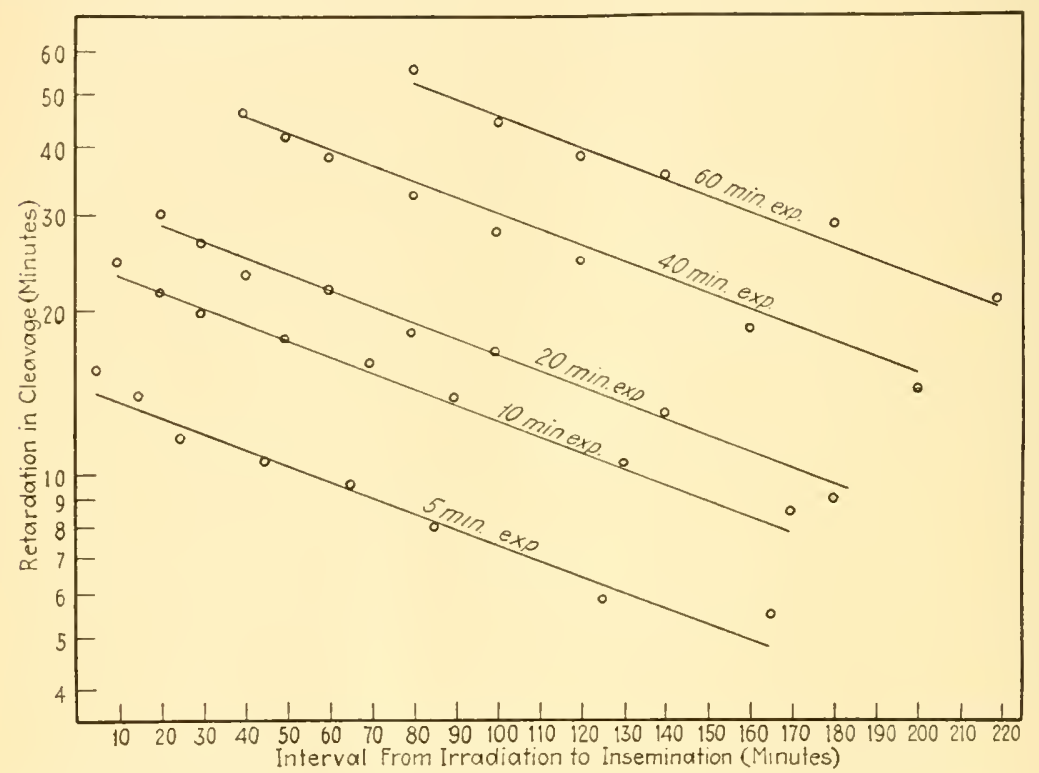

FIG. 6.-Recovery of Arbacia eggs from the effect of roentgen rays on their cleavage time.

terms of a standard deviation which we shall represent by the symbol $\sigma_{y \cdot x}$. This standard deviation is obtained by calculating from the equation a $y$ value for each point on the $x$ scale where an observation has been made, and then following the equation

$$
\sigma_{y \cdot x}=\sqrt{\frac{\Sigma\left(y_{\text {obs }}-y_{\text {cale }}\right)^{2}}{n-\alpha}}
$$

where $n$ is the number of points to which the equation has been fitted and $\alpha$ represents the number of derived constants. This standard deviation may be used in connection with Table 3 to determine the extent of the variation between the observed and calculated values. It is also the basis for the computation of the degree of sampling variation of the derived constants of the equation.

The methods outlined in this paper are characteristic of statistical treatment as a whole, and although they may seem varied in type, they 
all have the same general aim. They attempt, broadly speaking, to take a number of observations in quantitative form and sum them up in a few meaningful constants, the sampling variation of these constants always being taken into consideration. The value of the statistical method does not lie, however, in the mere determination of these constants, but rather in the fact that by expressing our scientific ideas in quantitative form we tend toward a more logical treatment of the particular problem in which we are interested. One not infrequently sees papers in which the observations have been given statistical treatment, but the constants seem to have been derived because they are fashionable, since at no time have they been used for the development of the scientific argument. Statistical constants should not be derived hit or miss, but the treatment given any particular problem should be in line with the scientific question asked, in which case the results will lend themselves directly to the development of our line of thought.

\section{REFERENCES}

1. Henshaw, P. S. Studies of the effect of Roentgen rays on the time of the first cleavage in some marine invertebrate eggs. Amer. Jour. Roentg. and Rad. Ther. 27 : 890-898. 1932.

2. Laurexs, H. The physiological effects of radiant energy. (p. 175.) The Chemical Catalog Company; New York, 1933.

3. PACKARD, C. The biological effects of short radiations. Quart. Rev. Biol. 6: 253-280. 1931.

4. Packard, C. A biological measure of X-ray dosage. Jour. Cancer Res. 11: 282-292. 1927.

5. Pearl, R. Introduction to medical biometry and statistics. 2nd ed. W. B. Saunders Company; Philadelphia, 1932.

6. Robertson, Muriel. The effect of gamma-ray irradiation upon the growth of a protozoon, Bodo caudatus. Quart. Jour. Microse. Sci. N. S. 299: 511-541. 1932.

7. Russ, S., S. Wright, H. A. Bulmax, and L. H. Clark. The physiological and cytological effects of penetrating $\mathrm{X}$-rays upon the cat and the rabbit. Proc. Roy. Soc. Med. 23: 1671-1688. 1930.

8. Sievert, R., and A. Forssberg. The time factor in the biological action of X-rays. Acta Radiol. 12: 535-551. 1931.

9. Tippett, L. H. C. The methods of statistics. Williams and Norgate, Ltd.; London, 1931.

10. Yule, G. U. An introduction to the theory of statisties. 10th ed. C. Griffin \& Company; London, 1932. 



\title{
VII
}

\section{PHOTOCHEMISTRY}

\author{
Farrington Daniels
}

Department of Chemistry, University of Wisconsin, Madison

\section{PART 1. THEORY}

Theory of chemical reaction. Activation of molecules. Types of radiation. Types of spectra. Laws involved in photochemical reactions. Quantum theory. Photochemical phenomena. Chemical kinetics. Photochemical kinetics. Influence of various factors on photochemical reactions. Experimental technique. General procedure. Refcrences. Part 1.

Photochemical reactions are chemical reactions which are produced directly, or indirectly, by the absorption of radiation. Qualitative photochemistry has been studied for a very long time, but only within the past decade or so has the quantitative study of photochemical reactions been reduced to an exact basis. The theory of photochemistry also has received important impetus within the last few years through advances in the interpretation of spectra and in the new developments of chemical kinetics. In order to understand photochemical reactions a brief survey must be given first of our present theories of chemical reactivity.

\section{THEORY OF CHEMICAL REACTION}

It is now generally accepted that chemical reaction must be preceded by an activation process. The mere existence of slow chemical reactions supports this view. If activation were not necessary, it would be expected that all molecules would be in similar states of reactivity and that the reaction would be nearly instantaneous. It is true that many instantaneous reactions are known, but these occur at very high temperatures where there is a high activation or they occur between ions, particularly in aqueous solutions, and these ions may be regarded as atoms in a state of activation.

The heat of a chemical reaction is not a measure of the energy required to activate the molecules. The heat of a chemical reaction is equal to the difference between the energy required to activate the forward reaction and the energy evolved when the products are formed from the activated reactants. This relationship is shown in Fig. 1. Activation in ordinary chemical reactions is assumed to be caused by collisions between molecules, and is thus due to heat. In a given number of molecules at a definite temperature some of the molecules will be moving rapidly and some slowly and the distribution of the velocities among the 
different molecules follows a probability curve as shown at $A$ in Fig. 2. This relation is known as the Maxwell-Boltzmann distribution law. In ordinary reactions which proceed slowly, only those molecules which are moving with very high velocities can produce activation sufficient to carry on the chemical reaction. For example, only those molecules which have a velocity greater than $V$ can take part in a chemical reaction. The

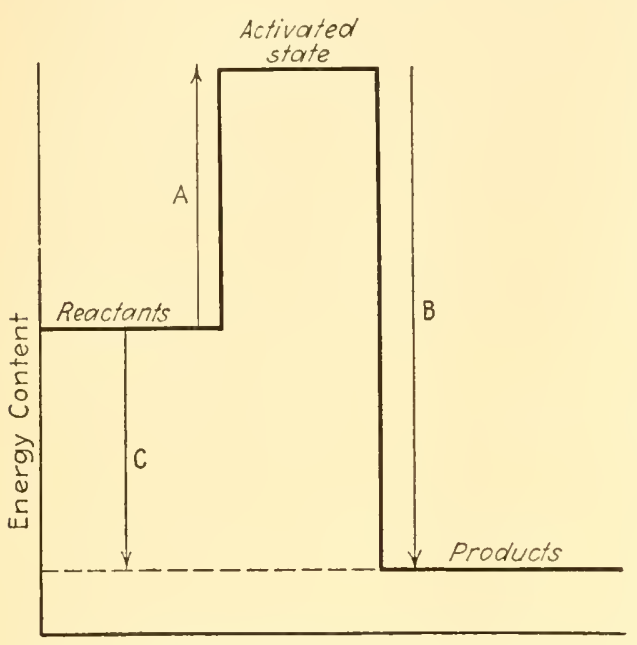

Fig. 1.-Relation between energy of activation and heat of reaction. A. Energy absorbed in activating reactants. $B$. Energy evolved when activated reactants react to give products. $C$. Net heat evolved in the reaction. value of $V$ will, of course, be different for different chemical reactions. When the temperature is raised, the distribution of velocities among the different molecules is changed in a manner suggested by the curve $B$. It will be noted that the forms of these curves are such that at the higher temperatures there is a very great increase in the number of molecules having the high velocities. For example, the ordinate $V D$ is much greater than the ordinate $V C$, but there is not so much difference between the ordinates of the two curves at the slower velocities at the left. This large increase in the number of molecules having high velocities at the higher temperature explains the fact that temperature has such a large effect in accelerating a chemical reaction. The number of molecules having sufficiently high velocities to become activated increases rapidly with the temperature. (The broken lines in Fig. 2 will be discussed later.)

Another concept which is found useful in interpreting chemical reactions is that of the variation with distance of the attractive force between the atoms. This relationship shown in Fig. 3 was due originally to Franck. In the case of some atoms (helium, for example) there is no attraction and the atoms repel each other at all distances shown in curve $A$. In other systems, shown at $B$, the atoms attract each other and the attraction becomes greater the nearer the atoms approach to each other, as in the case of electrically charged bodies. However, when the atoms come too close to each other, they are repelled. There is then a stable position at a definite distance, below which the atoms will repel each other and beyond which they will attract each other. This position is represented by the point $C$. When the energy is sufficiently great so that the atoms can no longer attract each other, dissociation occurs as 
indicated by the horizontal line $B$ at the right. The difference between this energy at dissociation and the energy in the stable position of the

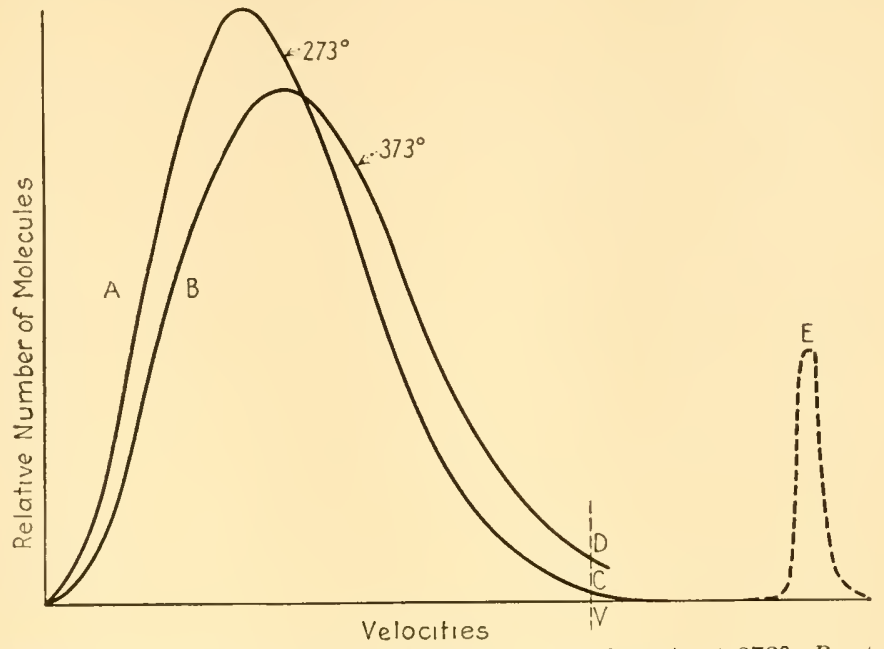

FIG. 2.-Distribution of energy among a group of molecules: $A$, at $273^{\circ} ; B$, at $373^{\circ}$. $E$ represents extra energy supplied by some external agent.

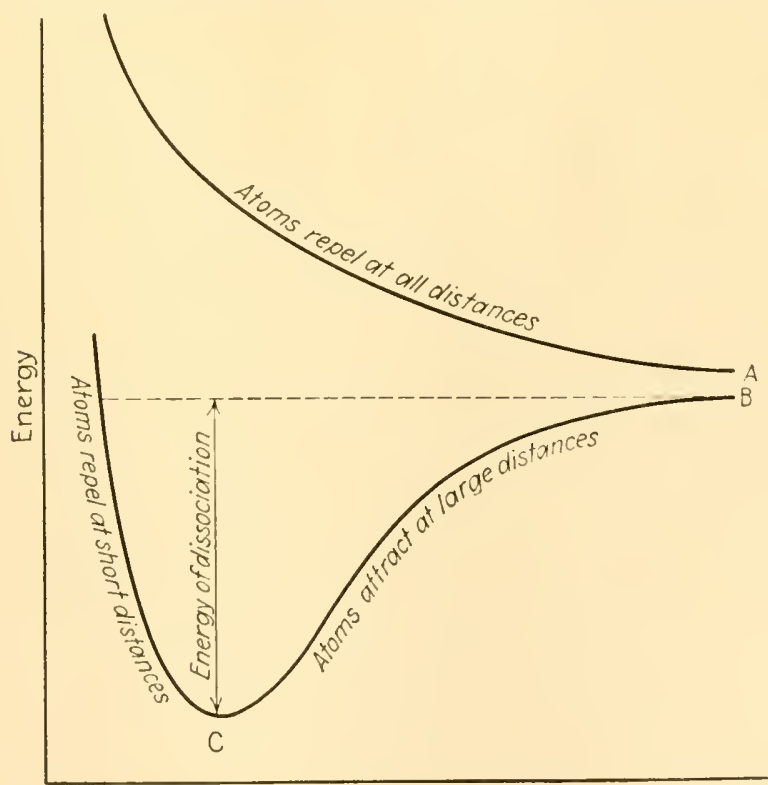

Distance Between Atoms

FIG. 3.-Energy relations existing between two atoms as a function of the clistance separating their centers.

molecule represented by $C$, represents the energy required to dissociate the molecule. It is possible to gain some insight regarding the nature 
of the dissociation process from infra-red absorption and from band spectra because they are intimately connected with the displacement of atoms within the molecule.

The energy required to break chemical bonds and thus make possible chemical reaction amounts to many thousand calories per gram molecule of material. Reactions which proceed with measurably slow velocities at room temperature usually require in the neighborhood of 25,000 cal. and the most difficult reactions, those which go only at very high temperatures, require up to $100,000 \mathrm{cal}$. and more. When a new product is formed and new chemical bonds result, energy is given out corresponding to the energy which is absorbed in the breaking of these bonds. It is clear that the energy of activation is partially offset by the energy of combination. The heat of reaction, then, is the difference. This heat of reaction must be less than the energy of activation and it usually happens that the energy released by the products is so much greater than the energy required to activate the reactants that heat is evolved in the chemical reaction. It is a common observation that exothermic reactions are much more common than endothermic reactions at room temperatures.

Activation of Molecules.-The thermal activation of molecules has just been discussed. There are various other ways in which molecules can be activated. Collisions with electrons, for example, will provide sufficient energy to produce the necessary activation of the molecule. The energy of the collision depends on the velocity of the electron, and this can be controlled by the applied voltage. Important calculations can be made from the ionization potential and the resonance potential which are determined by finding the applied voltage at which the electrons are absorbed by the molecules. Activation of molecules may be produced also by collision with the alpha particles emitted by radioactive substances. An alpha particle with a mass four times that of the hydrogen atom traveling with one-tenth the velocity of light contains sufficient energy to ionize and activate 100,000 molecules. Chemical activation may be effected also by collisions of molecules with units of radiation, called photons. These photons are presumably distributed in a random manner in a beam of radiation and each photon contains a quantum of energy, the value (in ergs) depending on the wave-length. This collision process between molecules and photons forms the foundation of photochemistry.

Again, molecules can be activated by collisions with other molecules which have already become activated or excited. For example, a mercury atom which has absorbed ultra-violet light contains an abnormal amount of energy, and when this excited mercury atom collides with a molecule of ammonia, for example, the ammonia molecule is decomposed. 
In all of these cases where molecules can receive an abnormal amount of energy from an external source, the reaction may proceed in ways which would not be expected from ordinary thermal activation. The situation is illustrated by the dotted lines at $E$ in Fig. 2, where the second hump represents the number of molecules containing a large amount of energy. It is evident that the large number of molecules having this high energy cannot come from ordinary thermal collisions and the Maxwell distribution of velocities, but must come from some external source as just described.

Extra energy for this activation may be introduced not only by collisions with photons, electrons, or other materials of high energy but also by intermediate chemical changes. Under these conditions the reaction is said to be catalytic and the extra material which accelerates the reaction is called the catalyst. The catalyst is often effective in mere traces and it is not consumed in the course of the reaction. Catalysis is quite specific and the prediction of catalytic properties is, in our present state of knowledge, more of an art than a science. One important branch of catalysis has to do with reactions at the surface of a solid; but catalysis may be effected also by gases, liquids, or dissolved material. The catalyst effects the loosening of the bonds and alters the reaction so that it is not necessary to supply the complete energy of activation as shown in Fig. 1.

\section{TYPES OF RADIATION}

There are a great many different kinds of radiation depending on the wave-length. These have been discussed on page 14 . The shorter the wave-length the more energy is contained in a given unit of radiation. The radio waves and the infra-red waves do not contain enough energy to effect ordinary chemical reactions. X-rays contain sufficient energy to completely ionize the molecules and to produce chemical reactions, but they are so penetrating that only a small portion is absorbed in a reaction system of ordinary dimensions. Ultra-violet and visible light, particularly in the blue end of the spectrum, may be very effective in bringing about chemical reactions.

Radiation in the ultra-violet and visible is due to a displacement of outer electrons in atoms or molecules and the return of these displaced electrons to their normal states. X-rays are due to a similar cause, except that the displacements of inner electrons close to the nucleus of the atom are involved. Infra-red radiation involves the displacements of atoms in the molecule. These displacements may be caused by heat, and all solids give about the same distribution of radiation among the different wave-lengths at a given temperature. Figure 4 shows a typical curve of so-called black-body radiation. The amount of radiation is plotted against the wave-length.

A black body is defined as a solid material 
which absorbs all the incident radiation and reflects none. It gives, accordingly, the maximum radiation when heated. A heated enclosure such as a furnace with a small opening furnishes a practical means of obtaining this black-body radiation. Further details concerning black-

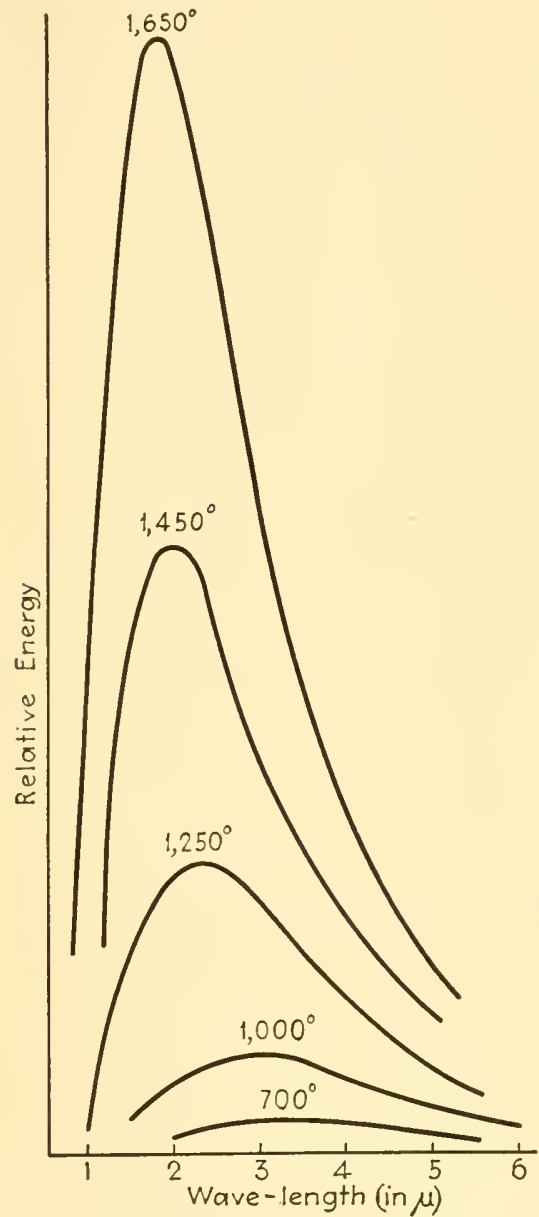

Fig. 4.-Distribution of radiant energy emitted by a heated solid. body radiation are given on page 135 . It is evident from Fig. 4 that a heated solid, a tungsten filament, for example, offers a good source of light in the visible region of the spectrum but not in the ultra-violet.

When atoms or molecules in the gas phase are subjected to extra energy, the radiation may be emitted at specific wave-lengths, as distinguished from the continuous radiation of heated solids. The passage of an electrical current between metal electrodes causes the emission of a discontinuous spectrum as in the case of the well-known mercury arc or iron arc.

\section{TYPES OF SPECTRA}

Spectra are classed as emission spectra and absorption spectra. Absorption spectra are obtained when white light of continuously varying wave-lengths is passed through absorbing material. Light of certain wave-lengths passes through umaffected, while light of other wavelengths is able to displace electrons (or atoms) in the molecules of the absorbing material. When the light rays are spread out in a spectrum with a prism or grating, those wave-lengths which have been absorbed are missing and a dark region results. The darkest regions in the absorption spectrum correspond to the greatest absorption and the most probable displacements within the molecule. As a rule, the absorption spectra, particularly in the case of solutions, are not so definite and the lines are not so sharp as in the case of emission spectra.

Band Spectra.-In the case of simple atoms, electron displacement usually leads to fine lines and discontinuous spectra. When molecules 
are involved, there is a chance to utilize some of the energy in displacements of the atoms within the molecule and in rotations of the molecules around a common center of gravity. These displacements and rotations involve comparatively small amounts of energy of varying size so that by combining them with the electron displacements a series of bands is obtained. Such bands are very common. Whereas the bands will appear under low resolution to be completely absorbing, high dispersion reveals the fact that they are made up of a large number of fine lines. The fine structure of these bands can be detected in emission spectra and in absorption spectra of gases, particularly at low pressures. Only in special cases, however, can the fine structure of absorption bands be detected in solution. The intimate contact of the solvent tends to blur out these lines. Sometimes they can be detected, however, by cooling the system with liquid air. Electronic displacements are not involved in the infra-red bands.

In the far infra-red the radiant energy is absorbed and converted into rotation of the molecule. Again large dispersion reveals the fact that these bands are made up of a number of discontinuous lines. In the near infra-red, (8000 to $200,000 \AA$ ) the absorption bands are due to a combination of molecular rotation and internal displacements, but they, too, can be resolved into discontinuous lines provided the spectrometer has sufficient resolving power. The centers of the band correspond to the displacements of atoms within the molecule, and additions or subtractions of small amounts of energy absorbed in rotation of the molecule lead to a broadening of the region of absorption and the production of an absorption band.

\section{LAWS INVOLVED IN PHOTOCHEMICAL REACTIONS}

The most important and the most obvious law in photochemistry is that of Grotthus according to which only that radiation, which is absorbed, is capable of producing chemical reaction. It is clear that those radiations which pass through a system without absorption are incapable of affecting the system. Radiation which is absorbed may, possibly, produce chemical action, but in most cases the radiation is converted into increased kinetic energy of the molecules and the temperature is raised without effecting any chemical change. Photochemical reactions result only when the conditions are such that the activated molecule can produce a chemical reaction.

The absorption of light in the simplest case follows the differential equation

$$
\frac{-d I}{d l}=k l
$$

which is so frequently met with in physical and chemical phenomena. Integration of this equation gives 


$$
I=I_{0} e^{-k l}
$$

or in logarithmic form

$$
k=\frac{-1}{l} \ln \frac{I}{I_{0}}
$$

In these formulas $I_{0}$ represents the incident light, $I$ the transmitted light, $l$ the thickness of the absorbing medium and $k$, a constant. This formula expresses Lambert's law comnecting the transmission of light with the thickness of the absorbing material. The absorption coefficient $k$ depends on the nature of the absorbing material but it is constant for any given material. This relationship may be expressed as follows in logarithms to the base 10 instead of in natural logarithms:

$$
E=\frac{1}{l} \log \frac{I_{0}}{I}
$$

where $E$ is defined as the extinction coefficient.

A similar formula applies to the influence of concentration $c$ of absorbing material on the intensity of transmitted light. This formula is known as Beer's law.

$$
I=I_{0} e^{-k^{\prime} c} \quad \text { or } \quad k^{\prime}=\frac{-2.303}{c} \log \frac{I}{I_{0}}=\frac{2.303}{c} \log \frac{I_{0}}{I}
$$

It applies to gases and to solutions when there is no other complicating change involved in the dilution process. In solution it applies only when the solvent is transparent. One of the most convenient ways of determining whether this law applies to a given system is to plot the logarithm of the transmitted light intensity against concentration, with a given thickness of material. If Beer's law is applicable, a straight line results. If the line is not straight it may be suspected that chemical changes such as dissociation or combination with the solvent are involved. In Fig. 5 a typical plot of this kind is shown for gaseous acetone.

Reflection of light $I_{r}$ at right angles to a boundary between two different media is related to the incident light $I_{0}$ by Fresnel's Law as given in equation $(6)$.

$$
I_{r}=\frac{(a-1)^{2}}{(a+1)^{2}} I_{0}
$$

The term $a$ occurring in this formula represents the ratio of the refractive indices in the two media. The refractive indices of glass, quartz, water, and most liquids are sufficiently close together so that the amount reflected at interfaces between these materials is negligible in most photochemical investigations. When a beam of light strikes a glass-air interface at right angles, it can be calculated from this formula that about 4 per cent of the light will be reflected backward. This reflection occurs at every interface in which a gas is in contact with a solid or liquid surface.

Scattering of light occurs in all materials, but it is negligible in most photochemical reactions unless there are a large number of surfaces such 
as are present in colloid systems. In such cases a considerable quantity of light may be scattered so that the amount absorbed by the system and the amount transmitted will not necessarily be equal to the total incident light.

The photochemical law of greatest theoretical importance is that of Einstein, according to which one molecule is activated for each quantum absorbed. It does not necessarily follow that one molecule will react for each quantum absorbed because there may be many complicating second-

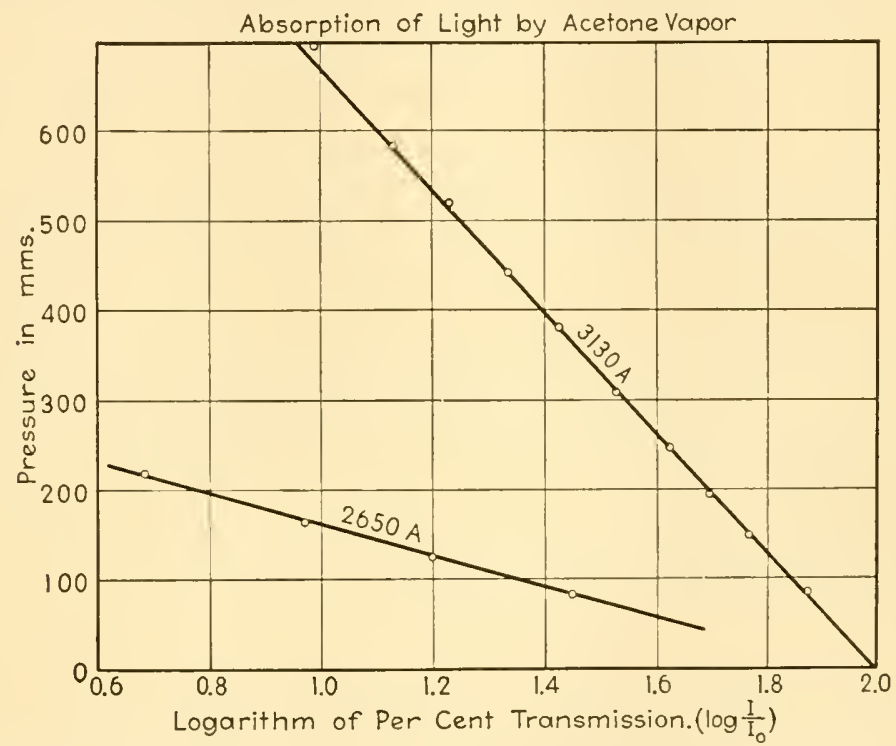

FIG. 5.-Graph illustrating Beer's law. The absorption of light by $10 \mathrm{~cm}$. of acetone vapor.

ary reactions. In fact, these secondary effects so frequently mask the primary process that the Einstein law was not accepted at first. The ratio $\Phi$ of molecules reacting to quanta absorbed is known as the quantum yield and the experimental determination of this quantum yield under different conditions constitutes one of the important approaches to the theoretical study of a photochemical reaction. The number of cases in which the quantum yield is unity is very small and the more refined experimental measurements of the last few years have not increased the number. The quantum yield varies all the way from a few hundredths or less to a million or more. These cnormous variations serve simply to emphasize the fact that the primary process is almost always followed by other complicating phenomena. Most photochemists accept the view that the Einstein relationship applies to the primary process.

\section{QUANTUM THEORY}

The quantum theory has been responsible for extraordinary developments in the fields of physics and physical chemistry. It was first pro- 
posed by Planck, in 1906. Previous to this time formulas had been developed on the basis of classical theories of light which expressed the relationship between intensity of radiation emitted by a heated solid and wave-length, both in the infra-red region of the spectrum and in the ultra-violet region. There was no satisfactory way of expressing the distribution of energies throughout the whole spectrum as given in Fig. 5. In attempting to find a satisfactory equation for the distribution of energy from a black body, Planck was led to the assumption that the radiation is emitted not continuously but discontinuously in small units of radiation which he called quanta. On this assumption he obtained a comparatively simple formula which reproduced the experimental facts (shown in Fig. 5) with extraordinary exactness throughout the whole spectrum. The assumption of the discontinuity of radiation was so radical at that time that Planck's quantum theory probably would not have been accepted, except for the fact that the concept of quanta immediately became of great importance in widely different fields. In the study of photoelectric phenomena and specific heat, in the interpretation of the data of spectroscopy, and in various other fields it became at once evident that the quantum theory provided the necessary means for correlating and explaining an enormous number of experimental facts. At the present time the quantum theory seems to be fully established and exceedingly useful.

The fundamental equation of the quantum theory is

$$
\epsilon=h \nu
$$

according to which the energy of one photon, one quantum $\epsilon$, is directly proportional to the frequency of the light $\nu$. The proportionality factor $h$ has the value $6.554 \times 10^{-27}$ erg-sec. This value has been checked by careful experiments in many different fields and is a constant of universal importance. It has been fully established that there are $6.06 \times 10^{23}$ molecules in a gram molecule (or atoms in a gram atom) and this number is known as the Arogadro number $N$. If one takes $6.06 \times 10^{23}$ photons one has the number of photons equal to the number of molecules in a gram molecule and this quantity is useful in photochemistry. It has been given the name "Einstein." One Einstein of radiation, then, absorbed by a given system produces the activation of a gram molecule of the material. Only in case secondary effects are absent will the Einstein of radiation produce a gram equivalent of the chemical product. According to equation $(\tilde{\tau})$ the energy required to produce a mole of activated molecules is then equal to $N h \nu$.

It is clear that the greater the frequency of radiation the greater is the energy contained in the radiation, and this fact is illustrated in Table 1. It is frequently necessary to convert wave-lengths or frequencies into energies. In so doing, it should be remembered that 1 Angström unit $(1 \AA)$ is one 100 millionth of a centimeter, $\left(10^{-8} \mathrm{~cm}\right.$.), and that $1 \mathrm{m \mu}$ 
Table 1.-Energy in Variods Types of Radiation

\begin{tabular}{|c|c|c|c|c|c|c|c|}
\hline Description & $\begin{array}{c}\text { Wave- } \\
\text { length, } \\
\AA\end{array}$ & $\begin{array}{c}\text { Wave- } \\
\text { length, } \\
\mathrm{m} \mu\end{array}$ & $\begin{array}{l}\text { Wave } \\
\text { number }\end{array}$ & Frequency & $\begin{array}{c}\text { Erg per } \\
\text { quantum }\end{array}$ & $\begin{array}{l}\text { Cal. per } \\
\text { Einstein }\end{array}$ & $\begin{array}{l}\text { Elec- } \\
\text { tron } \\
\text { volts }\end{array}$ \\
\hline $\mathrm{X}$-rays..... & 1 & 0.1 & $1 \times 10^{8}$ & $3 \times 10^{18}$ & $1.96 \times 10^{-8}$ & $2.84 \times 10^{8}$ & 12,340 \\
\hline Ultra-violet..... & 1,000 & 100 & 100,000 & $3 \times 10^{15}$ & $1.96 \times 10^{-11}$ & 284,500 & 12.3 \\
\hline Ultra-violet..... & 2,000 & 200 & 50,000 & $1.5 \times 10^{15}$ & $9.82 \times 10^{-12}$ & 142,300 & 6.17 \\
\hline Ultra-violet...... & 3,000 & 300 & 33,333 & $1 \times 10^{15}$ & $6.55 \times 10^{-12}$ & 94,840 & 4. 11 \\
\hline Visible (violet) ....... & 4,000 & 400 & 25,000 & $7.5 \times 10^{14}$ & $4.12 \times 10^{-12}$ & 71,120 & 3.09 \\
\hline Visible (blue-green).... & 5,000 & 500 & 20,000 & $6 \times 10^{14}$ & $3.93 \times 10^{-12}$ & 57,000 & 2.47 \\
\hline Visible (orange)....... & 6,000 & 600 & 16,666 & $5 \times 10^{34}$ & $3.27 \times 10^{-12}$ & 47,400 & 2.06 \\
\hline Visible (red)..... & 7,000 & 700 & 14,286 & $4.3 \times 10^{14}$ & $2.81 \times 10^{-12}$ & 40,600 & 1.76 \\
\hline Visible (red)... & 8,000 & 800 & 12,500 & $3.7 \times 10^{14}$ & $2.42 \times 10^{-12}$ & 35,500 & 1.54 \\
\hline Near infra-red. & 10,000 & $1 \mu$ & 10,000 & $3 \times 10^{14}$ & $1.96 \times 10^{-12}$ & 28,450 & 1.23 \\
\hline Infra-red...... & 100,000 & $10 \mu$ & 1,000 & $3 \times 10^{13}$ & $1.96 \times 10^{-13}$ & 2,845 & 0.12 \\
\hline Far infra-red.... & $1,000,000$ & $100 \mu$ & 100 & $3 \times 10^{12}$ & $1.96 \times 10^{-14}$ & 284 & 0.01 \\
\hline
\end{tabular}

is equal to $10 \AA$. The wave number is a unit, frequently used in spectroscopy. It is obtained by dividing the wave-length, expressed in centimeters, into unity. The frequency of light is obtained by dividing the wave-length of light expressed in centimeters into the velocity of light, $3 \times 10^{10} \mathrm{~cm}$. It is obvious that wave numbers are converted into frequencies by multiplying by the velocity of light, $3 \times 10^{10}$. In the last column the energy of the radiation is expressed in electron volts by dividing the wave-length in Angströms into $12336 \AA$. This relation follows from equating $h \nu$ to the product of the voltage and the charge on the electron, expressed in the proper units.

These relationships may be illustrated with a specific example using light of $5000 \AA$ (blue-green light). The wave-length of this light in $\mathrm{cm}$. is $5 \times 10^{-5} \mathrm{~cm}$. The wave number is 20,000 per $\mathrm{cm}$. The frequency is $6 \times 10^{14}$ per sec. The energy per photon, $h \nu$, is

$$
\left(6.55 \times 10^{-27}\right) \times\left(6 \times 10^{14}\right)=3.93 \times 10^{-12} \mathrm{erg} .
$$

Multiplying the energy of one photon by the number of photons required to activate a gram mol and converting ergs to calories, it is evident that one Einstein of this radiation contains

$$
\left(3.93 \times 10^{-12}\right) \times\left(6.06 \times 10^{23}\right) \div\left(4.18 \times 10^{7}\right)=57,000 \mathrm{cal} .
$$

The energy in electron volts is 2.47 .

\section{PHOTOCHEMICAL PHENOMENA}

Absorption of Radiation.-In photochemical reactions the energy necessary to start the process is supplied by the absorption of radiation. This absorption of radiation in the visible or ultra-riolet region of the spectrum results in a displacement of the electrons. If this energy of electronic excitation is transferred to give displacement of the atoms within the molecule, chemical reaction may result. If this energy of atomic displacement becomes sufficiently great, the atoms are driven apart and the molecule dissociates. If the atoms are displaced to a 
considerable extent but are not completely expelled, these molecules with the displaced atoms may become activated so that they will react with other molecules. The mechanism by which this electronic activation is transferred to energy of atomic displacement is not fully understood. In some cases at least collisions with other molecules are necessary. In still other cases, collisions of these electronically excited molecules with inert molecules are able to remove the energy of electronic excitation in the form of increased kinetic energy and thus tend to prevent chemical reaction. The energy of radiation must be at least as great as the energy required for activation. It does not necessarily follow that if the energy of the exciting light is as great as or greater than the energy of activation that activation will automatically occur; for the energy of activation may be immediately dissipated in other ways.

It might be expected that infra-red radiation which is immediately connected with the displacement of atoms in the molecule would lead directly to chemical reaction. In this case it would not be necessary to effect a transfer of electronic excitation into energy of atomic displacement. However, as seen from Table 1, the energy contained in the infrared radiation is less than that required for most ordinary chemical reactions (perhaps 25,000 cal. for those occurring at room temperature).

It is an observational fact that photochemical reactions are much more often produced by ultra-violet light than by blue light and that those produced by blue light are more common than those produced by green or red light. Such a situation is to be expected from the fact that the shorter wave-lengths of light contain greater amounts of energy as shown in Table 1. It must be realized that the intensity of energy in these photons is the significant thing rather than the total amount of energy contained in a beam of light. The greater the intensity of the beam of light the more quanta are available and the faster is a given photochemical reaction, provided that the quanta are large enough. However, if the individual photons in this particular beam of light contain an insufficient amount of energy (i.e., wave-length is too long) no photochemical reaction can occur at all, no matter how many quanta are introduced.

Chemiluminescence.-A few chemical reactions are known in which light is emitted. A common example is the glow of yellow phosphorus when exposed to air. The luminescence of the firefly, of decaying wood and certain marine bacteria are all attributed to the emission of light which accompanies the oxidation of luciferin, a substance which is found to occur in these bacteria and in insects. Rapid and violent reactions also are known to emit light. For example, when chlorine reacts with metals, a flame is produced. This is not due to incandescence of small particles, as in an ordinary flame (temperature radiation). Occasionally, among the less violent reactions examples of chemiluminescence are 
found. The Grignard reagent (phenyl magnesium bromide) when exposed to air gives a faint purplish glow which can readily be seen in the dark. Substituted compounds of this type are known to give more brilliant reactions and a considerable amount of research has been done in this field (7). One of the most striking chemiluminescent reactions has been described recently. When 3 -aminophthalhydrazide is dissolved in ordinary hydrogen peroxide, the whole solution becomes brightly luminescent so that it ean be seen even in daylight (10). A few other chemiluminescent reactions are known, but they are comparatively rare. It may be assumed that the phenomena of chemiluminescence would become very much more common if sufficiently sensitive means for the detection of light were available. In most chemical reactions the new products are formed with the evolution of heat and accompanying it there may occur frequently the production of some radiation. In many cases light may be produced in a reaction, but this light in turn may be absorbed by surrounding molecules so that little, if any, finally emerges from the reacting system. The light emitted from molecules close to the surface is more likely to emerge from the reacting system. General rules for chemiluminescence have not yet been developed. The phenomenon seems to be rather specific. It may be that violent reactions of a particular type are necessary, together with an absorption coefficient for the emitted radiation such that only slight absorption occurs within the system.

Chemiluminescence in the infra-red has not been investigated, except in flames and explosions, but it may be quite common. Chemiluminescence in the visible is rather infrequent. Chemiluminescence in the ultraviolet is very rare. It is natural to expect that a small fraction of the chemical energy may be converted into radiation and that under suitable conditions this may be given out from a system in the form of chemiluminescence. The smaller the amount of energy in a quantum, the more readily can such a quantum be produced. For these reasons one would expect to find that chemilumineseence is more frequently found with long wave-lengths than with short. As pointed out previously, it is not the heat of reaction but the energy of activation that determines the course of a chemical reaction. Aceordingly, since the wave-length of the chemiluminescent radiation is governed by the quantum theory, the minimum wave-length of the emitted light can be calculated from the energy of activation of the reverse reaction, by expressing this in ergs, setting equal to $N h \nu$ and solving for $\nu$.

Fluorescence.-When a system is excited by absorbing radiation, it is possible that some of the exeited molecules may return to a normal state with the emission of radiation having a different wave-length from that of the exciting radiation. This emission of light, different in wavelength from that of the exciting light, is known as fluorescence. As a 
rule, there is no time lag between the excitation and the emission of the fluorescent light. The wave-length of the emitted light is usually longer than that of the exciting light because there is a natural degradation of energy from quanta of large energy to the quanta of smaller energy. The generalization that the fluorescent light is of longer wave-length than the exciting light is known as Stokes' law. There are a few exceptions to this relation which can be explained as special cases in which additional energy from some other source is involved. Fluorescence caused by the energy-rich ultra-violet light is quite common and it is well known that many substances give off visible light when exeited by ultra-violet light. A few examples may be cited-fluorescein, eosin, many minerals (particularly if mixed crystals are involved), teeth and various parts of animal tissue. Solutions of quinine when excited by blue light emit red light.

In all these cases it is assumed that the exciting light displaces an electron in the absorbing molecule and that this electron then returns to a state of lesser energy. If the electron returned to exactly the same state from which it started by a single step, the wave-length of the emitted light would of course be exactly the same as that of the exciting light and the fact of the emission would not ordinarily be detected.

\section{CHEMICAL KINETICS}

The extent to which a chemical reaction proceeds can be predicted on the basis of thermodynamies when sufficient data are available. The calculation of equilibrium constants is comparatively simple in principle. It is a much more difficult problem, however, to determine the rate at which this equilibrium is established. Chemical kinetics endeavors to answer the question as to how fast a reaction will go and also strives to learn something regarding the nature of the reaction and the mechanism by which it occurs. In very rapid reactions, such as occur in electrolytic systems (ions) or at very high temperatures, the simple rules of thermodynamics and equilibrium constants can be readily applied and they give a completely satisfactory prediction of the results. In slow reactions, including the majority of reactions in organic chemistry and biological chemistry, the speed of the reaction becomes a matter of great importance. Among the reactions of organic chemistry a great many different products may result, all of which are possible according to thermodynamic calculation. The product which is formed in actual practice is the one which is produced by the fastest reaction. It is a matter of experience and technique to accelerate or retard these simultaneously occurring reactions in such a way as to obtain the desired product. For example, catalysts are used which will accelerate one reaction to a much greater extent than other competing reactions. 
The various competing reactions may be quite complicated. There may be a reverse reaction or a side reaction involving the same reactants, or one reaction may follow closely on the heels of another and a whole series of successive reactions may occur. In actual practice the slowest reaction in the series is the one measured and there may be many steps in the complete reaction which proceed at an immeasurably rapid rate. In case two reactions are proceeding at approximately the same rate the situation becomes quite confused. When one reaction is considerably slower than any of the others, it can be made the object of experimental investigation.

Order of Reaction.- - In studying reaction rates it is desirable to express the concentration as a function of the time. A graph in which concentration $c$ is plotted against time $t$ may be extrapolated within reasonable limits. Often the relationship between concentration and time can be expressed with mathematical formulas. The rate of a first-order reaction is directly proportional to the concentration as given by the following formula:

$$
-\frac{d c}{d t}=k c
$$

In the integrated form this equation becomes

$$
-\ln c=k t+C
$$

and integrating between limits, the form $(10)$ is obtained:

$$
k=\frac{2.303}{t_{2}-t_{1}} \log \frac{c_{1}}{c_{2}}
$$

It will be noted that formula $(9)$ is of such a nature that when the logarithm of the concentration is plotted against time, a straight line is produced. In fact, one of the best ways to determine whether a reaction is of the first order is to plot the logarithm of the concentration against the time. If a straight line is produced, the reaction is first order, and the slope of the line multiplied by 2.303 gives at once the specifie reaction rate or velocity constant. When this constant is known, the concentration at any time may be calculated.

In a second-order reaction the rate of the reaction depends on the product of the concentration of two reacting substances. If the initial concentrations are designated respectively by $a$ and $b$ and the amount reacting by $x$, the following mathematical formula applies:

$$
-\frac{d x}{d t}=k(a-x)(b-x)
$$

On integration this equation becomes

$$
k=\frac{2.303}{t(a-b)} \log \frac{b}{a} \frac{(a-x)}{(b-x)}
$$

This equation is of such a nature that when $\log b(a-x) / a(b-x)$ is plotter against time, a straight line is produced and the slope of the line multi- 
plied by $2.303 /(a-b)$ gives the specific reaction rate $k$. Third-order reactions are known in which the rate is proportional to the product of three different substances, but these third-order reactions are uncommon. Zero-order reactions are reactions which are entirely independent of the concentration, as given by equation (13).

$$
-\frac{d c}{d t}=k
$$

They are frequently found among photochemical reactions.

Chain Reactions.- When the products of reaction are formed with the liberation of sufficient energy to activate additional reactants, a chain reaction results. An energy chain is defined as one in which the series of reactions are continued simply by the transfer of energy. Stoichiometrical chain reactions are, however, much more common. In the case of a stoichiometrical chain it is possible to write a series of chemical reactions in which a product, such as an atom, combines with reactants and again produces the product which can react with more material and continue in a manner such as is illustrated for the hydrogen-chlorine chain given below.

$$
\begin{aligned}
\mathrm{Cl}_{2}+\text { light } & =2 \mathrm{Cl} \\
\mathrm{Cl}+\mathrm{H}_{2} & =\mathrm{HCl}+\mathrm{H} \\
\mathrm{H}+\mathrm{Cl}_{2} & =\mathrm{HCl}+\mathrm{Cl} \\
\mathrm{Cl}+\mathrm{H}_{2} & =\mathrm{HCl}+\mathrm{H} \\
\text { etc. } &
\end{aligned}
$$

These chain reactions can involve few or many molecules, depending on conditions. The hydrogen and chlorine chain reaction may involve up to about a million molecules for each molecule of chlorine which is dissociated. Other chain reactions are of much shorter length.

Temperature Effect.-Aside from finding the order of the reaction and, if possible, a mathematical relationship connecting concentration with time, one of the most important studies in chemical kinetics involves the finding of the temperature coefficient. The fundamental formula which relates the velocity constant with the temperature is given by equation (14).

$$
\frac{d \ln k}{d T}=\frac{E}{R T^{2}}
$$

On integration equation (15) is obtained.

$$
\log \frac{k_{2}}{k_{1}}=\frac{E\left(T_{2}-T_{1}\right)}{2.303 R\left(T_{2} \times T_{1}\right)}
$$

The nature of this equation is such that when the logarithm of the velocity constant $k$ is plotted against the reciprocal of the absolute temperature a straight line is obtained and the slope of this line multiplied by 2.303 and by the gas constant $R$ ( 2 cal.) gives the energy of activation for the reaction. Obviously, if two or more reactions are involved, the 
results are not easily interpretable. This energy of activation is of great importance in the theory of ehemical kinetics. It represents the energy which must be introduced into a gram molecule of the reactants in order to give them sufficient activation so that the reaction may take place. Combining the effect of temperature and concentration, the general formula (16) is obtained:

$$
k=s e^{-E / R T}
$$

This is a very useful equation which applies to most reactions and expresses the specific reaction rate $k$ as a function of the temperature and the energy of activation. The constant $s$ has the physical significance of collision frequency when the reaction is bimolecular. When the reaction is unimolecular, the significance is not so clear, but it may be connected with the oseillation of atoms within the molecule and this in turn is related to the infra-red absorption spectrum. In most unimolecular reactions $s$ has a value in the neighborhood of $10^{-12}$, and in many reactions $E / R T$ has a value in the neighborhood of 40 . In gas-phase bimolecular reactions the frequency of collision $s$ ean be calculated from standard formulas of the kinetic theory of gases involving the molecular diameter. The value of the energy of activation $E$ is usually about 25,000 cal. for unimolecular reactions which proceed with a measurable velocity at room temperature. Reactions having an energy of activation in the neighborhood of 50,000 eal. usually require a temperature in the neighborhood of $400^{\circ} \mathrm{C}$. in order to give a measurable reaction rate. It must be emphasized that the range of measurable reactions is somewhat limited. These statements are, of course, only rough approximations.

\section{PHOTOCHEMICAL KINETICS}

Photochemical kineties constitute a complicated and difficult field. Not only are photochemieal reactions subject to all the rariations which affect thermal reactions, but they involve also complications from the absorption of radiation. In the study of photochemical reactions it is desired to determine the primary photochemical process caused by the absorption of the radiation. As explained before, this primary process is very frequently obscured by secondary reactions. The photochemical reaction may institute a series of successive reactions constituting a chain. The primary reaction may be of the same nature as a reaction which is proceeding in the dark but at a slower rate. Again, the photochemical reaction may be reversed by a thermal process when the light is removed or the velocity of the primary process may vary with the intensity of the light absorbed. This in turn may change with the absorption and the extent of the chemical reaction. When the absorption of light is complete, the study of the photochemical kinetics is somewhat simplified. At least the change in the amount of absorption is not a variable in the 
rate of the reaction. There is still, however, a complication in the fact that the reactants in the front of the cell are exposed to a much greater intensity of light than those deeper in the cell. In some cases this difference has no effect, but in others it leads to complications. When the light is partially absorbed, it becomes necessary to measure the intensity of the transmitted light as well as that of the incident light, and frequent measurements are necessary if the absorption changes during the photochemical reaction. Usually it is necessary to stir a liquid system to maintain uniform conditions and to distribute throughout the whole cell the chemical products produced by the beam of light. In the case of gases the diffusion is so rapid that stirring is unnecessary.

The order of a photochemical reaction may be determined in the same manner as that described in the preceding section. The rate of the reaction at constant intensity of light is determined experimentally. If this rate is the same at all concentrations, the reaction is of zero order. If it is directly proportional to the concentration, it is first order, and if it is proportional to the square of the concentration, it is second order. In the case of chain reactions the situation is complicated and in many cases the determination of the order of the photochemical reaction is without significance.

Photochemical measurements enable one to determine conveniently the length of chains. The number of quanta absorbed by the reacting system is measured and the number of molecules reacting is determined experimentally. The ratio of the molecules reacting to the quanta absorbed gives a measure of the quantum yield. In case many molecules react per quantum absorbed the reaction is a chain reaction.

Photocatalyst.-In many cases a reacting system is unaffected by radiation until an additional material is introduced. Such a material is sometimes called a photocatalyst. It is unaffected by the reaction and may be recovered unchanged. Examples are common. The radiation may pass through the reacting system without absorption but when stopped by the photocatalyst, photochemical reaction may result. The photocatalyst may give rise to an entirely different reaction from that which oceurs in its absence, and the way in which it functions may vary considerably. In some cases it forms a loose compound with the reactants and this compound is excited by the absorbed light. In other cases the photocatalyst is excited by the absorbed radiation and transmits its energy to a reacting molecule by ordinary collision.

Induction Period.- Sometimes the catalyst of the photochemical reaction is produced by the reaction itself. Under these conditions a time lag or induction period is observed and the photochemical reaction does not proceed until a considerable period has elapsed. The situation is similar to that of autocatalysis in ordinary chemical kinetics where the catalytic material is produced by the chemical reaction itself. Under 
such conditions the reaction rate increases for a time whereas in other chemical reactions the rate decreases. No generalizations can be made concerning this induction period. It is comparatively rare and in each ease the eatalyst is specific. Examples will be given later. Sometimes the eatalyst is produced by the photochemical reaction, sometimes it is produced by a slow thermal reaction, and in still other cases an inhibitor is present and this inhibitor is destroyed by the photochemical reaetion. After destruction of an inhibitor the rate of reaction inereases.

After-effects.-Not only is a time lag observed at the beginning of a reaction in some cases, but reactions are also known in which there is a time lag when the light is turned off. When the reaction proceeds in the dark after illumination, the phenomenon is known as an after-effect.

\section{INFLUENCE OF VARIOUS FACTORS ON PHOTOCHEMICAL REACTIONS}

Temperature.-Temperature has a very marked effect on ordinary chemical reactions as explained in a preceding section. It is of such a nature that most chemical reactions which proceed with measurable velocity at room temperature double or triple their rate for a rise of $10^{\circ} \mathrm{C}$. in temperature. Photochemical reactions, on the other hand, are usually rather insensitive to temperature changes. It is to be expeeted that a purely primary photo process would be largely independent of the temperature. The reason that ordinary chemical reactions have large temperature coefficients lies in the fact that activation is produced by molecules of very high energy content and the number of these highly activated molecules increases greatly with the temperature. In photochemical reactions the excitation eomes from absorption of light from an outside source and the intensity of radiation in the system is quite independent of the temperature of the absorbing system. However, the primary photo process is very frequently followed by other processes which do depend on the temperature of the reacting system. For example, in a chain reaction the chemical reaction which follows the primary photochemical reaction is subject to the same factors which influence any ordinary chemical reaction. Thus it is found that many photochemical chain reactions do have large temperature coefficients approaching those of ordinary thermal reactions. Again, the photochemical reaction may be influenced by collisions; and collisions, in turn, are influenced by a change in temperature. Sometimes the absorption of light by the reaeting system is changed by a change in temperature and this absorption may affect the photochemical reaction. Such eases are not very common. Experimentally it is found that a large number of photochemical reactions change in velocity about 10 per cent for a $10^{\circ}$ rise in temperature. At one time it was thought that most photochemical reactions could be grouped into three classes with temperature coefficients of $1.00,1.2$, and 1.4 , but such a classification is now believed to be without significance (13). In general, one may say that if the temperature coeffi- 
cient of a photochemical reaction is large, approaching that of an ordinary thermal reaction, then the reaction contains in it steps which are pureiy thermal reactions. If the reaction rate does not change at all with temperature, then the photochemical reaction is probably free from accompanying thermal reactions. The majority of photochemical reactions have slight temperature coefficients.

Wave-length.-The wave-length of light is an important factor in photochemical reactions. Obviously, the light must be of such a wavelength (or frequency) that the radiation is absorbed. If there is no absorption there can be no reaction. If there is absorption, however, it is not necessarily true that chemical reaction follows. As pointed out before, radiation of short wave-length is more likely to produce chemical reaction than radiation of long wave-length simply because the quantum of energy is greater in the light of short wave-length. When the photon of radiation is absorbed many changes may take place. The absorbing molecule may become excited and lose its energy by collision to surrounding molecules thereby increasing their kinetic energy and raising the temperature and dissipating the radiant energy into ordinary heat. The excited molecule may react with another molecule, but a dark thermal reaction may reverse the process in such a way that no result is noticed. The excited molecule may collide with other molecules and transfer its energy in a rather specific manner so that the second molecule will undergo a chemical reaction which ean be observed.

The type of absorption spectrum encountered is often very useful in understanding and predicting photochemical reactions. A discontinuous spectrum indicates that the molecule becomes excited by the absorption of light, but a continuous spectrum indicates that the molecule becomes ionized or dissociated, and that fragments are thrown out with kinetic energy. The kinetic energy is not subject to the restrictions of the quantum theory and accordingly there is a random distribution of energies over considerable ranges giving the effect of continuous absorption. The discontinuous spectrum contains fine lines in a spectrogram (images of the slit of the spectrograph) because the displacement of electrons in the atoms or molecules is subject to quantum restrictions, but in case chemical dissociation or ionization takes place, the wavelengths between these discontinuous lines can also be utilized by a combination of displacement and dissociation so that a continuous spectrum is observed. It is obvious that a discontinuous spectrum with many discrete lines will appear as a continuous spectrum when viewed with a spectrograph of low resolving power. Oftentimes spectral gratings of large size are necessary to resolve the spectrum into its fine lines, but in a truly continuous spectrum, involving this disruption of the molecule into fragments, no amount of resolution will reveal fine structure in the spectrum. 
Frequently a spectrum shows a region of discontinuous lines coming closer and closer together and merging into a region of continuous absorption, as shown diagrammatically for iodine in Fig. 6. Under these conditions the fine structure in part of the spectrum shows that the resolving power of the spectrograph is adequate and dissociation is indicated by the region of continuous absorption. The long-wave-length edge of the band of contimuous absorption marks the least amount of energy which is necessary to dissociate the molecule. Important work has been done in correlating this long-wave-length edge of the absorption band with

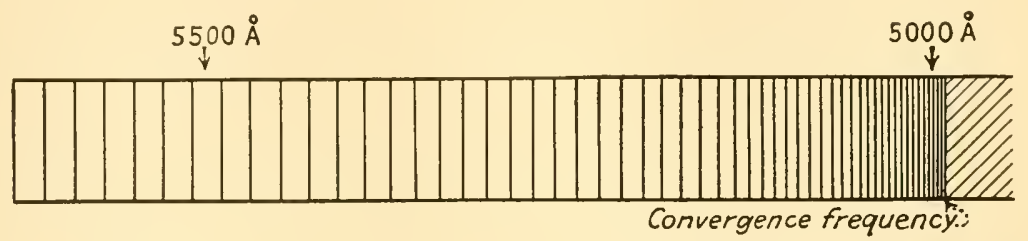

FIG. 6.--Absorption spectrum of iodine vapor, showing continuous absorption below 4995 A caused by dissociation.

the heat of dissociation as determined experimentally. In some cases there is complete agreement. In other cases the energy of dissociation, determined ehemically or thermally, is less than the energy of dissociation as calculated from the edge of the region of continuous absorption. In such cases the discrepancy is usually traced to the fact that the fragments into which the molecules are dissociated are excited. Several examples of this relationship between calculated and observed dissociation are given in Table 2.

Table 2.-Heats of Dissociation Calculated from Spectroscopy

\begin{tabular}{|c|c|c|c|c|c|}
\hline $\begin{array}{l}\text { Absorbing } \\
\text { molecule }\end{array}$ & $\begin{array}{l}\text { Maximum } \\
\text { wave- } \\
\text { length of } \\
\text { continuous } \\
\text { absorption, } \\
\AA\end{array}$ & $\begin{array}{l}\text { Heat of } \\
\text { dissoci- } \\
\text { ation, cal. } \\
\text { per mole }\end{array}$ & $\begin{array}{c}\text { Probable } \\
\text { energy of } \\
\text { excitation } \\
\text { of products }\end{array}$ & $\begin{array}{l}\text { Estimated } \\
\text { heat dis- } \\
\text { sociation } \\
\text { into normal } \\
\text { atoms }\end{array}$ & $\begin{array}{l}\text { Heat of } \\
\text { dissoci- } \\
\text { ation de- } \\
\text { termined } \\
\text { calori- } \\
\text { metrically }\end{array}$ \\
\hline $\mathrm{H}_{2} .$. & 850 & 334,000 & 234,000 & 100,000 & 101,000 \\
\hline $\mathrm{I}_{2} \ldots \ldots$ & 4995 & 56,800 & 21,600 & 35,200 & 34,500 \\
\hline $\mathrm{Br}_{2} \ldots \ldots \ldots$ & 5107 & 55,600 & 10,400 & 45,200 & 46,200 \\
\hline $\mathrm{Cl}_{2} \ldots \ldots \ldots$ & 4785 & 59,400 & 2,500 & 56,900 & 57,000 \\
\hline
\end{tabular}

In a similar manner it is possible sometimes to calculate the heat of dissociation from the wave-length at which sharp lines in an absorption spectrum change into hazy or indistinct lines. Such a spectrum of indistinct lines, which cannot be further resolved, is known as a predissociation spectrum. When such a spectrum is produced, it is supposed that the electronic and vibrational energies are subjected to the regular quantum number restrictions, but that the rotational energy is not quantized, 
i.e., restricted to definite integer multiples of a quantum, because the molecule dissociates in less time than is required for rotation. Since the kinetic energy of the fragments is not quantized, the energy absorbed from the spectrum extends over a slightly larger range of frequencies. Examples are found in the spectra of sulfur, ammonia, nitrogen dioxide, and other molecules.

Intensity.-In a simple primary photoprocess the amount of photochemical reaction should be directly proportional to the amount of light absorbed. If Einstein's relationship holds and one molecule reacts for each quantum absorbed, the extent of the reaction will depend only on the number of quanta absorbed and this in turn will depend directly upon the amount of light absorbed. This relationship is found to hold in a great many photochemical reactions. In many other photochemical reactions, however, the amount of chemical reaction is not directly proportional to the amount of light absorbed, and this deviation from direct proportionality often provides a clue as to the mechanism of the photochemical reaction. Several factors may be responsible for deviation from this simple proportionality. In some cases the molecules of absorbing material are broken up into atoms by the absorption of light and the atoms take part in the reaction. Then two atoms may be formed for each quantum absorbed and the concentration of reacting materials is twice as great as predicted on the basis of the Einstein relationship. Under these conditions the application of the mass law shows that the amount of reaction varies as the square root of the light intensity rather than as the intensity itself. Sometimes a secondary thermal reaction follows the primary photoprocess, but when the light intensity becomes very great, the primary process may become so rapid that the secondary thermal reaction cannot keep pace. Again, at high intensities the reacting materials for the primary reaction may be so greatly depleted as to slow down the reaction. A classic example of this situation is found in photosynthesis, with chlorophyll, in the so-called Blackman region where at high intensity the photosynthesis is proportionately less than at low intensities because the carbon dioxide material cannot cliffuse rapidly enough into the cells; e.g., the supply of carbon dioxide rather than the supply of photons becomes the limiting factor. The high intensity of light may also produce a congestion of reaction products in such a way that the reaction is slowed down-or perhaps accelerated. Efficient stirring becomes specially important under these conditions.

Length of Exposure.-In a simple, primary photochemical process the amount of chemical change should be directly proportional to the time of exposure, provided that the quantity of light absorbed is the same throughout the exposure. If such is not the case, the reaction is shown to be complex. Several complications may account for the failure to obtain proportionality, such as, autocatalytic effects, the destruction of 
inhibitors, changing light absorption, and various types of secondary reactions. In the same way intermittent exposure is sometimes of help in determining whether or not the primary photoprocess is followed by dark thermal reactions. A sector wheel may be revolved in the path of the light in such a way as to reduce the intensity, for example, by half. Under these conditions half of the wheel is open and half of it closed and the time of exposure is reduced to one-half by this revolving wheel irrespective of the speed at which the wheel rotates. The time between illuminations, however, is changed by changing the rate of rotation and if each period of illumination is followed by a chemical reaction which takes considerable time, the total amount of reaction may vary with the speed of rotation. In many cases, however, the chain reaction following the primary photochemical process is completed in much less time than the time between illumination. Under these conditions, of course, the intermittent illumination is unable to contribute any information concerning the reaction.

Impurities.-Many photochemical reactions are quite sensitive to small amounts of impurities such as oxygen, for example. This is particularly true with chain reactions in which a foreign substance may act to carry a chain or may act in the opposite way to inhibit the reaction by breaking a chain. As in all exact chemical work, the materials should be subjected to extensive purification. Only rarely is it possible to use commercial chemical substances without further purification. Inf many cases impurities have no particular influence on the reaction, but one must either ascertain this fact in advance or prove it experimentally.

\section{EXPERIMENTAL TECHNIQUE}

In quantitative photochemical investigations it is necessary to use light which is nearly monochromatic in character and to measure the amount of radiation absorbed. In the early researches on photochemistry these requirements were not often realized and there was serious disagreement among different workers. Frequently when the whole spectrum is used, radiation of one wave-length may produce one reaction while light of another wave-length may produce an entirely different one, and in fact may even reverse the reaction produced by the first radiation. Valuable information concerning the nature of the reaction is obtained from a knowledge of the quantum yield-the number of molecules reacting per quantum of radiation absorbed. As explained before, this quantity often enables one to determine the nature and extent of secondary chemical reactions which may accompany the primary photochemical process. For this reason considerable effort is justified in measuring accurately the amount of radiation absorbed. The energy in ergs which is transmitted from the rear of the cell is subtracted from the energy striking the front of the cell with suitable corrections 
for reflection, in order to determine the amount of energy absorbed. In case the reacting system absorbs all the radiation which enters the cell, the measurement of transmitted light may be omitted, but usually it is preferable to arrange the conditions so that some light is transmitted.

Light Sources.-The purest monochromatic light is usually obtained with a monochromator (or preferably a double monochromator) employing a prism which refracts the light into its different wave-lengths. Light sources which give discontinuous spectra lead to a greater purity of monochromatic light and the greater the distance separating the spectral lines, the easier is the separation. The mercury are is very convenient and particularly suitable for photochemical investigation. Other metal arcs are also available although they are less convenient (9).

Monochromators are more effective in giving monochromatic light than most filters, but they are subjected to the handicap of low intensity. Only a small portion of the light from the source enters the monochromator slit and a large portion of this light is scattered and lost in the monochromator. Accordingly, the intensity of the exit beam is very low. A considerable amount of effort has been expended in improving monochromators to give greater intensities. The prisms and lenses have been increased in size and the focal length of the lenses has been shortened. Also the intensity of the light has been greatly increased by using capillary lamps (5) which concentrate most of the light of the lamp on the monochromator slit.

The principle of focal isolation has been used in rendering light monochromatic. Light of longer (or shorter) wave-length is stopped by focusing the polychromatic light onto a screen provided with a suitable small hole. In the short ultra-violet this method may be superior to the monochromator method. The relative efficiencies of the two methods in both intensity and purity have been studied by Heidt and Forbes (8).

Satisfactory filters for different wave-lengths have been worked out in the visible region and various solutions and several convenient glass filters are now available $(4,11)$. The situation in the ultra-violet is less satisfactory and new developments in this field would be welcome. The subject of filters and monochromators has been discussed in detail by Brackett on pages 149 to 170 .

Measurement of Intensities. ${ }^{1}$ - The most satisfactory device for measuring the intensity of light is the thermopile, which consists of junctions of unlike metal on the back of thin metal receivers which are blackened. For photochemical investigations the utmost sensitivity is not required because a fairly high intensity of radiation is necessary to effect sufficient photochemical change to measure by chemical analyses.

${ }^{1}$ Only techniques of special application to photochemistry are suggested here. 
The receivers are usually made of thin silver. Details of construction may be found in the literature $(2 ; 6$, page 435$)$. Copper and constantan wires are easy to work with as they may be readily soldered, using a flux of rosin. Large-area thermopiles ( 1 by $4 \mathrm{~cm}$.) for photochemical purposes may be conveniently made with a single receiver of thin silver $(0.01 \mathrm{~mm}$. in thickness), to the back of which the soldered and lacquered thermocouple junctions are attached with de Khotinsky cement. The thermopile is connected directly to a galvanometer having a sensitivity of 5 to $10 \mathrm{~mm}$. per microvolt and a critical damping resistance of 10 to 25 ohms, and calibrated with a carbon-filament lamp from the U. S. Bureau of Standards. For crude work, in the absence of such a standard lamp, it may be assumed that a new 60-watt tungsten-filament lamp gives radiation such that at a distance of two meters approximately $1 \mathrm{erg}$ falls on $1 \mathrm{~mm} .^{2}$ of the receiving surface per sec. Details for exact calibration $(3 ; 6$, page 437$)$ are supplied with the lamps which may be purchased from the Bureau of Standards at a small cost. It is necessary to cover the thermopile with a window in order to prevent stray currents of air. A heavy metal box surrounding the receiver helps to maintain a constant temperature and prevent drift of the zero point. It is necessary to make the window of quartz in order to standardize the thermopile because glass windows absorb a considerable quantity of the infra-red heat radiation from the standardizing lamp. Glass windows may be used if it is not necessary to calibrate the thermopile with a standard lamp. A quartz window makes the thermopile suitable for work in the ultra-violet as well as in the visible.

The quantity of light may be determined also by an actinometer which contains a photochemically sensitive system which can be followed by chemical analyses. One of the best materials for use as an actinometer is a solution of uranyl sulfate and oxalic acid. In this solution, 0.6 of a molecule of oxalic acid is decomposed for each quantum of light absorbed throughout the whole range of wave-lengths between 2500 and $4200 \AA$. Variations at the different wave-lengths amount to 0.1 . Details may be found in the paper of Leighton and Forbes (12). The decomposition of oxalic acid is readily followed by titrations with a standard solution of potassium permanganate, in sulfuric acid solution.

Chemical Change.-The course of a photochemical process may be followed in a number of different ways, either by chemical analysis or by physical measurements. Whenever possible it is desirable to use a physical measurement which does not affect the reacting system such, for example, as pressure change of a gas, or electrical conductance, or evolution of gas. A very convenient method consists in using the absorption of light as a means of determining the amount of unknown material. In fact, the thermopile readings may be used for measuring both the intensity of light absorbed and the quantity of material in solution, 
assuming the validity of Beer's law (page 260). Samples of material can be removed for chemical analysis by titration or other means. Usually very sensitive methods of measurement are necessary because the amount of change is small. Especially when making use of monochromators, micro- or semimicrotechnique is necessary. A convenient microtechnique for analyzing gases has been developed by Blacet and Leighton (1).

\section{GENERAL PROCEDURE}

In the following paragraphs the general procedure for conducting photochemical investigations will be outlined. Specific illustrations will be given later. At the outset it is safe to assume that the photochemical reaction may be quite complex and it becomes necessary to exclude as far as possible all secondary reactions and impurities before drawing conclusions regarding the main reaction. For example, the photochlorination of organic compounds is seriously affected by the presence of small amounts of oxygen. Under ordinary conditions in the air, the photochemical reaction studied will be one involving oxygen and the production of phosgene as an intermediate step. The direct addition of chlorine to an organic compound, either saturated or unsaturated, can be interpreted only in case oxygen is absent. Many other cases of similar nature are known where traces of acid or of inorganic salts are involved in the observed reaction.

It is not sufficient in quantitative work to place a vessel containing the reacting system in front of a source of light and follow the photochemical change in the ressel. As the reaction proceeds, the original matcrial is destroyed and the absorption of light may become considerably lessened. The rate of the reaction then slows down not because of any fundamental change but simply because less of the light is absorbed. Qualitatively, one can determine the nature of the product which is formed by the photochemical reaction, but no quantitative conclusions can be drawn from the rate of the reaction. In many cases it is important to know the rate and to know just how much energy is absorbed by the photoactive material. The most fundamental quantity to be measured is the quantum yield $\Phi$, the ratio of the number of molecules reacting to the number of quanta actually absorbed. When this value is known, conclusions regarding the fundamental process can be drawn and the extent of the photochemical reaction can be predicted from known values of the light intensity and the absorption and the time of exposure. In case the absorbing material is so concentrated that all the light is absorbed throughout the whole reaction, the absorption does not change during the course of the reaction and the calculations are somewhat simplified. There are certain objections to this procedıre, particularly when a large amount of material is used. 
It is necessary to measure the energy absorbed in the whole reaction ressel. The ressel is set up in the optical train back of the monochromator slit or back of a filter and the intensity of the light passing through the slit is measured with a thermopile (or actinometer). In the case of gaseous reactions the readings are taken when the cell is empty and again when the cell is filled. In the case of solutions the intensity of transmitted light is measured with a thermopile when the cell is filled with a pure solvent and again when filled with a solution. The difference in intensity in these two cases represents the energy absorbed by the solute. It is convenient to have two reaction vessels of identical dimensions placed side by side. One is empty or contains a solvent; the other contains the reacting system. A slight correction is necessary for reflections at the surface of a cell as given by Fresnel's well-known law of reflection. With flat, plane surfaces and light striking at right angles, the reflection loss at a gas-glass (or -quartz) interface is about 4 per cent of the total intensity. The reflection loss at a liquid-glass interface may be neglected. In precision work when the thermopile is placed back of the reaction cell, some of this reflected light passes back into the reaction chamber and requires a small correction. With three gas-glass surfaces, one at the rear of the cell, one at the front of the thermopile window, and a third at the back of the thermopile window, approximately 10 to 12 per cent of the light transmitted through the reaction cell will be reflected back into the reacting mixture.

The light which passes through the reaction chamber is measured by a thermopile. Usually the surface of the thermopile receivers is about $1 \mathrm{~mm}$. wide and about $1 \mathrm{~cm}$. high and the area of the reaction ressel is much larger. It is necessary then to converge, by means of a lens, all the light which passes through the reaction cell so that it all falls on the receivers of the thermopile. In case such a procedure is not convenient, the thermopile must be moved back and forth and up and down across the rear of the reaction chamber. In this way an integrated value for the total light passing through the cell is obtained. It is comparatively easy, as described on page 277 , to make thermopiles haring an area $1 \mathrm{~cm}$. wide and $4 \mathrm{~cm}$. high and a parallel beam of light issuing from a monochromator or from a filter system can be of such dimensions as to fall completely within the area of such a thermopile. When still larger cells are used, it becomes necessary to integrate even with this large-area thermopile. In accurate work it is usually necessary to thermostat the reaction vessel and it is convenient to immerse the reaction chamber and the thermopile in a ressel containing thermostated water. Copper and brass are preferred for such a thermostat because any trace of iron rust in the water produces a considerable change in the absorption of blue and ultra-violet light. The thermopile is, of course, affected by differences in temperature and it is advisable, although more troublesome, 
to immerse the thermopile in a water-tight box provided with a (quartz) window and submerged in the thermostat directly behind the reaction ressel.

Calculations.-The total number of moles reacting is determined by chemical or physical measurements. The number of moles reacting is then multiplied by the Avogadro number $6.06 \times 10^{23}$ in order to determine the number of molecules reacting. The number of ergs absorbed by the reacting mixture is determined with the calibrated thermopile and the number of seconds is recorded during which the radiation is absorbed. Next, the number of ergs in one quantum is calculated and this quantity is divided into the total number of ergs absorbed. The quotient gives the number of quanta absorbed. In calculating the number of ergs in one quantum the wave-length of light is expressed in centimeters and divided into the velocity of light $\left(3 \times 10^{10} \mathrm{~cm}\right.$.) in order to obtain the frequency of light. This frequency of light is then multiplied by Planck's constant $h\left(6.55 \times 10^{-27}\right.$ erg-sec. $)$. Having determined the number of quanta absorbed, and the number of molecules reacting, the quantum yield $\Phi$ is calculated. It is the number of molecules reacting divided by the number of quanta absorbed. A typical example illustrating the calculation of the quantum yield is given below.

Wave-length $=4360 \AA$.

Bromine reacting $=0.0054 \times 10^{-3}$ mole

Absorbed-light intensity $=12.92 \times 10^{3} \mathrm{ergs} / \mathrm{sec}$.

Time of exposure $=895$ sec.

Total quanta absorbed $=\frac{12.92 \times 10^{3} \times 895 \times 4.36 \times 10^{-5}}{6.55 \times 10^{-27} \times 3 \times 10^{10}}=$

$2.57 \times 10^{18}$

Molecules reacting $=0.0054 \times 10^{-3} \times 6.06 \times 10^{23}=3.27 \times 10^{18}$

$$
\Phi=\frac{\text { molecules reacting }}{\text { quanta absorbed }}=\frac{3.27 \times 10^{18}}{2.57 \times 10^{18}}=1.3
$$

With the best technique now arailable quantum yields in the neighborhood of unity can be determined with a precision of about 1 per cent. The absolute values of the radiation standard are probably known with an accuracy no greater than two or three per cent and in general an accuracy within 0.05 is about all that can be expected at present in the determination of $\Phi$. Usually the accuracy of the chemical analysis constitutes the limiting factor. Sometimes the determination of the quantum yield is a very difficult matter and although it is always desirable, it is oftentimes unnecessary for a particular purpose and the time is not worth the expenditure of effort which it entails. The importance of determining the value of the quantum yield lies in the fact that deductions may often be made concerning the primary and secondary processes. If the quantum yield is unity, the chances are that the primary process 
is being measured, uncomplicated by secondary thermal reactions. If the quantum yield is nearly unity, one can look for minor complications. If the quantum yield is considerably greater than unity, a chain reaction is indicated. If the quantum yield is less than unity, it may be concluded either, (a) that some other absorbing material is present and is appropriating some of the absorbed energy, so that it cannot take part in the photochemical reaction; $(b)$ that there is no photochemical reaction, i.e., the energy is being dissipated as heat because there is no other suitable chemical outlet for the energy of excitation; $(c)$ that radiant energy is going partly into chemical energy and partly into heat or $(d)$ that a reverse reaction or side reaction, not measured in the experiment, is consuming the radiant energy.

\section{REFERENCES. PART 1}

1. Blacet, F. E., and P. A. Leightox. A dry method of microanalysis. Ind. Eng. Chem., Analyt. Ed. 3: 266-269. 1931.

2. Coblentz, W. W. Various modifications of thermopiles having a continuous absorbing surface. Bur. Stand. Bull. 11: 131-187. 1914.

3. Coblentz, W. W. Measurements on standards of radiation in absolute value. Bur. Stand. Bull. 11: 87-100. 1914.

4. Corning Glass Works, Glass Color Filters. Corning, N. Y.

5. Daniels, F., and L. J. Heidt. A simple capillary mercury vapor lamp. Jour. Amer. Chem. Soc. 54 : 2381-2384. 1932.

6. Daniels, F., J. H. Mathews, and J. W. Williams. Experimental Physical Chemistry. McGraw-Hill Book Company, Inc.; New York, 1934.

7. Dufford, Nightingale, and Calitert. Luminescence of Grignard compounds; spectra and brightuess. Jour. Amer. Chem. Soc. 47: 95-102. 1925.

8. Herdt, L. J., and G. S. Forbes. Focal isolation versus the monochromator for photochemical work in the ultraviolet. Rev. Sci. Inst. 5: 253-255. 1934.

9. Hoffman, R. M., and F. Daniels. Quartz capillary are lamps of bismuth, cadmium, lead, mercury, thallium and zinc. Jour. Amer. Chem. Soc. 54: 4226-4235. 1932.

10. Huntress, E. H., L. N. Stanley, and A. S. Parker. The oxidation of 3-amino phthal hydrazide as a lecture demonstration of chemiluminescence. Jour. Chem. Educ. 11: 142-145. 1934.

11. International Critical Tables 5: (p. 271). McGraw-Hill Book Company, Inc.; New York, 1929.

12. Leighton, W. G., and G. S. Forbes. Precision actinometry with uranyl oxalate. Jour. Amer. Chem. Soc. $52: 3139-3512.1930$.

13. Tolmax, R. C. The temperature coefficient of photochemical reaction rate. Jour. Amer. Chem. Soc. 45: 2285-2296. 1923. 


\section{PART 2. PHOTOCHEMICAL INVESTIGATIONS}

The principles and experimental technique of photochemistry may be learned best from the many excellent researches that are now appearing in the scientific journals. A brief survey of a few of these is given in the following pages. The reactions are chosen to illustrate specific theories and quantitative experimentation, and no attempt is made to cover the whole field of photochemistry. Complete references to earlier work may be found in the references cited in the bibliography.

Acetylene.-Acetylene gas polymerizes to a yellow solid at wavelengths below $2534 \AA$. Light of longer wave-length is inactive. The solid, called "cuprene," is similar to a resin and it is so insoluble that its molecular weight has not yet been determined. Oxidation and analysis show that the material has the formula $(\mathrm{CH})_{x}$. Lind and Livingston (36) found a quantum yield of about seven molecules polymerized for each quantum absorbed in the region between 2334 and $2054 \AA$. A slight temperature coefficient was reported. A focal isolation method was used in which the shorter wave-lengths were converged most by a quartz lens and passed through a small opening.

Acetone.-The photolysis of gaseous acetone has been carefully investigated in several different laboratories during the last few years. The chief reaction is:

$$
\left(\mathrm{CH}_{3}\right)_{2} \mathrm{CO}+h \nu=\mathrm{C}_{2} \mathrm{H}_{6}+\mathrm{CO}
$$

According to this reaction, the decomposition produces a doubling of the pressure. Methane and sometimes hydrogen are found with the ethane, but the carbon monoxide always comprises about 50 per cent of the products.

Damon and Daniels (12) sealed off acetone vapor in a rectangular quartz cell and measured the pressure through a sensitive quartz diaphragm, provided with an extemal platinum contact which closed an electrical circuit. A measured air pressure was balanced against the pressure of the acetone and its decomposition products. The cell was placed in a water thermostat at $56.5^{\circ}$, making possible the use of acetone vapor at a pressure of one atmosphere. Monochromatic light from a capillary lamp and large quartz monochromator was passed through the cell onto a thermopile of large area, placed at the rear of the cell and contained in a submarine jacket completely immersed in the thermostat water.

For zero readings of the thermopile and galvanometer a second quartz cell, identical in dimensions with the reaction cell, was placed at the side and shifted into the beam of the light. 
The acetone vapor was partially removed by evacuation and a series of transmissions was measured at different partial pressures. The logarithm of the percentage transmission was then plotted against the partial pressure of the acetone vapor, as shown in Fig. 5 on page 261 . It is clear from the diagram that the absorption is greatest at the $2650 \AA$ line, and the straightness of the lines indicates that the absorption follows Beer's law-as it should in the case of a simple absorbing system.

In one typical experiment the radiation was absorbed at the rate of $85,200 \mathrm{ergs} / \mathrm{sec}$. over a period of $23,000 \mathrm{sec}$. This absorption of light resulted in an increase of pressure amounting to $30.4 \mathrm{~mm}$. From the known volume of the cell (about $60 \mathrm{cc}$.) it can be calculated that $5.28 \times 10^{19}$ molecules of acetone were decomposed; calculating the radiation in terms of number of quanta absorbed during the whole exposure, it was found that on the average 0.17 molecule of acetone was decomposed for each quantum absorbed. With a total of eight separate determinations, the average deviation from this averaged value was 0.02 . The quantum yield increased at low intensities and decreased at low pressures.

The photolysis of acetone has been investigated recently by Norrish, Crone, and Saltmarsh (38). The quantum yields obtained were practically the same as those already described. These investigators found that the spectrum shows discontinuity at the longer wave-lengths from 3325 to $2950 \AA$. Below $2950 \AA$ it is continuous. Ultra-violet light produces in acetone vapor a brilliant green fluorescence. At low intensities the fluorescence appears to be produced only by exciting light that falls in this discontinuous region.

The mechanism by which acetone decomposes under the influence of ultra-violet light is a matter of considerable interest. The fluorescence of acetone suggests an excitation of the molecule, whereas the continuous spectrum suggests decomposition directly into fragments. Several different mechanisms can be suggested, but there seems to be an increasing amount of evidence, in this and similar reactions, which favors photodissociation into free radicals (methyl groups) as one of the principal primary reactions.

The spectrum of acetone vapor throughout the ultra-violet has been carefully measured and interpreted by Noyes, Duncan, and Manning (41). The theory of the decomposition of ketones and aldehydes has been discussed by Norrish and his coworkers.

Aldehydes.-Formaldehyde is decomposed by ultra-violet light between 3650 and $2540 \AA$, according to the reaction:

$$
\mathrm{H}_{2} \mathrm{CO}+h \nu=\mathrm{H}_{2}+\mathrm{CO}
$$

In the longer wave-lengths of this region the quantum efficiency is 0.7 molecule per quantum of absorbed light. At the shorter wave-lengths it is 0.9 , and in the neighborhood of $3100 \AA$ it is 1.1 (39). 
The photolysis in the gas phase of propionaldehyde (32) and acetaldehyde (33) has been carefully investigated by Leighton and Blacet, using a mercury are with a quartz monochromator. Very small quantities of the decomposition products were obtained on account of the weak intensities of the monochromatic light, and the authors developed an excellent technique for the microanalysis of 50 to $100 \mathrm{~mm}^{3}$ of these gases, using fused beads of material as solid absorbents.

Photochemical decomposition and polymerization occur as two independent and simultaneous reactions. Decomposition is independent of pressure, while the apparent polymerization is directly proportional to pressure. The quantum efficiency is independent of the intensity of the light, i.e., the amount of change at a given wave-length is directly proportional to the amount of light absorbed.

The acetaldehyde decomposes chiefly into carbon monoxide, methane, and ethane, together with a little hydrogen. At $3130 \AA$ the quantum yield for decomposition is 0.51 . At $2654 \AA$ it is about 0.77 , and at $2537 \AA$ about 1.02 . The absorption spectrum of acetaldehyde shows distinct structure in the region from 3400 to $3250 \AA$. From 3250 to $3050 \AA$ the absorption lines are diffuse and from 3050 to $2700 \AA$ the lines are without structure. Throughout the whole region from 3400 to $2400 \AA$ there is continuous absorption, reaching a maximum at about $2700 \AA$.

Recent work by Leermakers (30) shows that the quantum yield increases very rapidly at high temperatures, amounting to 310 at $300^{\circ} \mathrm{C}$. This large yield appears to be due to a long chain reaction involving free radicals such as ethyl and methyl groups.

Ammonia.-Ammonia is decomposed by short ultra-violet light, which it absorbs at $2000 \AA$ and below. A mercury arc does not give sufficient intensity in this region for conducting photochemical measurements. Accordingly a spark of zinc or magnesium is used. Large disks, rotating at right angles, form convenient electrodes between which the spark passes. Either a focal isolation method or a monochromator method can be used. The quantum yield is approximately 0.14 molecule decomposed per quantum absorbed at $25^{\circ}(28)$. The products are nitrogen and hydrogen in a 1:3 ratio. The quantum yield is independent of light intensity and varies but slightly with temperature, giving a value practically twice as large at $200^{\circ} \mathrm{C}$. The low quantum yield may be due to recombination of the fragments which are thrown out in the decomposition process; or some of the absorbed energy may be dissipated as heat in vibrations of the atoms within the molecule under conditions where dissociation does not immediately take place.

Anthracene Polymerization.- When anthracene is dissolved in xylene or other solvent and exposed to ultra-violet light, the anthracene polymerizes to give double molecules, according to the reaction: 


$$
2 \mathrm{C}_{14} \mathrm{H}_{10} \rightleftarrows\left(\mathrm{C}_{14} \mathrm{H}_{10}\right)_{2}
$$

The de-polymerization of the double compound proceeds as a thermal reaction. Anthracene shows a brilliant fluorescence when excited by ultra-violet light. The di-anthracene is not affected by the ultra-violet light, giving neither fluorescence nor dissociation.

These facts lead to an understanding of the photochemical reaction. When an electron is displaced in the anthracene molecule by the absorption of a photon of ultra-violet light, the molecule becomes excited and, on collision with a second anthracene molecule, produces the di-anthracene. If the excited molecule cannot collide with the second molecule immediately, the electron returns to a lower energy level and emits visible light. The fluorescence decreases in intensity as the concentration of anthracene molecules increases. At ordinary light intensities there is a sufficient number of molecules to give reaction, and the rate of the reaction is independent of the concentration, i.e., it is a zero-order reaction. The photochemical polymerization, as in the case of other photochemical reactions, has a small temperature coefficient; the reverse reaction, the de-polymerization, is an ordinary thermal reaction and as such has a high temperature coefficient. The rate of polymerization to di-anthracene is directly proportional to the intensity of the light, and the rate of decomposition is independent of the light intensity and directly proportional to the concentration of di-anthracene. These facts can be expressed by the following equation, in which $x$ represents the concentration of di-anthracene, $I$ the intensity of the light absorbed, $k$ a velocity constant for the light reaction, and $k^{\prime}$ a velocity constant for the dark reaction:

$$
d x / d t=k I-k^{\prime} x
$$

When the rate of de-polymerization exactly offsets the rate of polymerization, $d x / d t=0$ and an equilibrium or stationary state exists, given by the following equations:

$$
k I=k^{\prime} x \quad \text { and } \quad x=k I / k^{\prime}
$$

It is evident that the concentration of di-anthracene in this stationary state will depend on the intensity of light and on temperature. The temperature change for the photochemical reaction $(k)$ for a $10^{\circ}$ rise is 1.1 ; and the temperature change for the thermal de-polymerization $\left(k^{\prime}\right)$ is 2.8 for a $10^{\circ}$ rise. The ratio of these two values, 1.1 divided by 2.8 , gives directly the influence of a $10^{\circ}$ rise in temperature on this stationary state and permits a simple experimental check of these conclusions. The experimental facts agree well with these calculations $(55,56)$.

This reaction is an interesting one in showing how the quantum yield changes with concentration and with other variables. At the very beginning of the illumination, very little di-anthracene has accumulated and the reverse reaction is negligible. The quantum yield should then 
give one excited molecule for each photon absorbed and accordingly one molecule of di-anthracene. The quantum yield should be unity, except for the slight loss of activation through fluorescence. At the stationary state, when the di-anthracene has accumulated to such an extent that the decomposition rate is equal to the rate of production, there will be no apparent change while the beam of light is being absorbed. In other words, the quantum yield will appear to be zero. Between these limits of no di-anthracene and equilibrium quantities of di-anthracene, one may obtain any apparent quantum yield ranging from 1 to 0 . In this case the significant photochemical reaction is that taking place at the beginning of the reaction. Quite frequently, when complicating reactions follow the primary reactions, one can obtain a knowledge of the primary process by working with short exposures.

Bromination of Cinnamic Acid.- When cinnamic acid and bromine are dissolved in carbon tetrachloride, the bromine very slowly adds to the cinnamic acid, forming di-bromocinnamic acid and producing a decrease in the concentration of the remaining bromine. The concentration of the bromine is easily determined by pouring the solution into an aqueous solution of potassium iodide in water and shaking thoroughly. Bromine from the carbon tetrachloride enters the aqueous solution and liberates iodine from the potassium iodide, which is accurately titrated with sodium thiosulphate.

This reaction has been studied by many investigators. It is convenient to carry out with visible light, and is easily measured by standard titrations. In a recent investigation (5), it was found that the quantum yield decreased as the bromine in solution decreased. Extrapolation to infinite dilution of bromine gave a quantum yield of one molecule of bromine reacting per quantum absorbed. These results were obtained at $30^{\circ} \mathrm{C}$. At $20^{\circ}$ the quantum yields were about one-third as much, and at $10^{\circ}$ roughly one-ninth as much. The interesting point is that all of these quantum yields at the different temperatures gave extrapolated values of unity at infinite dilution, as would be expected from the Einstein law, which applies to the primary photochemical process. Apparently, then, in this case it is possible to separate the primary photoprocess from the secondary thermal reaction. The secondary thermal reactions are subject to the large temperature effect which ordinarily applies to thermal reactions.

It has been shown recently (6) that all these photochemical measurements on the photobromination of cinnamic acid really involve an oxygeninhibited reaction. It has been found that, when the oxygen is boiled out of the carbon tetrachloride solution by evaporating part of the carbon tetrachloride with a water aspirator, the reaction goes so rapidly in weak light that it cannot be readily followed. This inhibition by oxygen of the addition of halogen to a double bond is probably quite general. 
In this case the oxygen prevents the easy addition of bromine to cinnamic acid; only when the bromine molecules are excited by the absorption of light or possibly when they are dissociated into atoms by the light, is it possible for bromine to add easily to the double bond of cinnamic acid.

Bromine-hydrogen Reaction.-Bromine vapor shows discontinuous absorption above $5107 \AA$, and at wave-lengths shorter than this it shows continuous absorption. In this region bromine molecules are dissociated into bromine atoms, one of which exists in the excited state. When mixed with hydrogen at room temperature, no reaction takes place, but at temperatures above $150^{\circ}$ there is direct union of the bromine with hydrogen when illuminated, giving hydrobromic acid. The following reaction probably represents the course of the reaction:

1. $\mathrm{Br}_{2}+h \nu=\mathrm{Br}+\mathrm{Br}$

2. $\mathrm{Br}+\mathrm{H}_{2}=\mathrm{HBr}+\mathrm{H}$

3. $\mathrm{H}+\mathrm{Br}_{2}=\mathrm{HBr}+\mathrm{Br}$, etc.

4. $\mathrm{Br}+\mathrm{Br}=\mathrm{Br}_{2}$

The kinetics of this reaction was worked out by Bodenstein and Lütkemeyer (8).

At room temperature the bromine atoms recombine (probably through collisions with a third body), regenerating the original bromine molecules, as in equation 4. At higher temperatures the bromine atoms obtain sufficient energy to react with hydrogen molecules, giving hydrobromic acid and hydrogen atoms, as shown in equation 2. When this reaction starts in, it is possible for the hydrogen atoms to continue a chain, as given by equation 3 , followed again by a bromine atom combining with another hydrogen molecule, etc. When hydrobromic acid becomes quite concentrated, the reverse of equation 2 may take place to a considerable extent. These facts, and several others, are nicely summarized by the following equation:

$$
\frac{d[\mathrm{HBr}]}{d t}=k \sqrt{I} \frac{\left[\mathrm{H}_{2}\right]}{1+k^{\prime} \frac{\left[\mathrm{HBr}^{\prime}\right]}{\left[\mathrm{Br}_{2}\right]}}
$$

where $k$ and $k^{\prime}$ are constants and $I$ is the intensity of light absorbed.

The dependence of the photochemical reaction on the square root of the light intensity is frequently found when dissociation of a molecule into two atoms is the primary process. It follows directly from the mass law. This photochemical reaction and the thermal reaction are discussed in detail by Kistiakowsky (26).

Chlorination of Benzene.-When a mixture of chlorine and benzene is illuminated with ultra-violet light or blue light, a variety of chlorinated benzene products is produced. The reaction has been studied by several different investigators, in solution and in the gas phase.

A careful study 
of this reaction has been carried out by Noyes and his associates (48). The reaction is quite complex and involves both addition of chlorine to benzene and substitution of hydrogen by chlorine. A short chain is involved in the reaction. The rate of the reaction is proportional to the square root of the light intensity and directly proportional to the pressure of the chlorine and the pressure of the benzene. The fact that the rate of reaction depends upon the square root of the light intensity indicates that chlorine atoms are involved, and the primary process is doubtless the dissociation of the chlorine molecule into chlorine atoms. The large quantum yield can be satisfactorily accounted for by a series of intermediate reaction steps involving hydrogen atoms or chlorine atoms.

The expcriments were carried out in all-glass apparatus, and the total pressure of the gases was measured with a glass diaphragm. The chlorine remaining after illumination was determined by treatment with potassium iodide, and the hydrochloric acid formed was obtained by direct analysis. Experiments were carried out at 3660 and $3130 \AA$. The interpretation of the reaction is seriously complicated by the fact that solid and liquid products condense out on the walls of the reaction chamber. These products are then subject to further chlorination. Substitution and addition take place simultaneously, particularly after the benzene has been partially chlorinated, but at low benzene pressures almost the entire initial photochemical reaction is that of addition. The final reaction products, after long periods of illumination, always approach the composition of the dodecachlorocyclohexane $\left(\mathrm{C}_{6} \mathrm{Cl}_{12}\right)$.

The chlorination of chlorobenzene was studied in a similar manner (22) in an attempt to decide the correct mechanism for the photochlorination of the benzene. The ultimate product is the same as for the benzene. The relative amounts of substitution and addition products were carefully determined, and several mechanisms were suggested and critically discussed.

Chlorination of Tetrachloroethylene.-This is an interesting reaction because there is no opportunity for chlorine to substitute for hydrogen, and the only product would appear to be a simple one,--hexachloro ethane $\left(\mathrm{C}_{2} \mathrm{Cl}_{6}\right)$. However, as in many other photochemical reactions, and thermal reactions as well, the reaction is seriously influenced by the presence of oxygen, even in small amounts. The reactions have been studied in detail by Dickinson and his associates, both in the gas phase (14) and in a solution of carbon tetrachloride $(15,31)$.

The blue line, $4358 \AA$ of the mercury are was isolated by means of filters, and the light was passed through a pyrex cylindrical cell with polished ends. The light passing through the cell was measured by a thermopile and galvanometer.

In the absence of oxygen, the rate of the chlorination was directly proportional to the concentration of chlorine and proportional to the 
square root of the number of photons absorbed by the system. The quantum yield ranged from 300 to 500 molecules reacting for each quantum absorbed. The reaction in carbon tetrachloride solution has very nearly the same characteristics, and the quantum yields are about the same, slightly higher in certain cases. According to one proposed mechanism, the large quantum yield can be explained on the basis of a chain reaction involving the radical $\mathrm{C}_{2} \mathrm{Cl}_{5}$.

The photochlorination of tetrachloroethylene is strongly inhibited by oxygen, even in small amounts, but in place of the chlorination reaction an oxidation reaction takes place. This chlorine-sensitized photooxidation of tetrachloroethylene gives chiefly trichloroacetylchloride, together with small amounts of phosgene. The rate of this reaction is proportional to the number of photons absorbed-not to the square root of the number, as in the chlorination reaction. It is proportional also to the first power of the chlorine concentration, is independent of the amount of oxygen, and is only slightly dependent upon the concentration of the tetrachloroethylene.

The quantum yield is about 300 molecules per photon in the gas phase. In carbon tetrachloride solution the quantum yield for this reaction is much less, approximately 2 molecules per photon. It is independent of concentration or light intensity in the gas reaction, but in carbon tetrachloride solution it depends on the concentration of the chlorine. An increase of $10^{\circ} \mathrm{C}$. causes the reaction to go 1.2 times as fast. In solution, after the oxygen is used up, the reaction goes much faster, as might be expected from the fact that the quantum yield is 300 in the absence of oxygen and 2 in the presence of oxygen. In comparing the experiments in gas phase and in solution, it may be pointed out that the solvent seems to stop the oxidation chain but not the chlorination chain.

Chlorine-hydrogen Combination.-This reaction has been studied many years, and more has been published on it than on any other photochemical reaction. It has been a most baffling reaction, and many apparently contradictory results have been reported.

The quantum yield is enormous, running up as high as a million molecules per photon absorbed. This fact led to the first proposal for a chain-reaction mechanism, and historically the reaction is responsible for a number of interesting developments in chemical kineties and in photochemistry. The reaction is complicated by this long chain mechanism, by an induction period, and by the fact that small amounts of air inhibit the reaction. The reaction is inhibited not only by oxygen but by traces of various impurities.

The most recent and perhaps the most exhaustive research on this reaction is due to Ritchie and Norrish $(44,40)$. The gases, chlorine, hydrogen, and, in some of the experiments, oxygen and hydrogen chloride 
as well, were contained in large reservoirs and mixed in a cylindrical quartz cell, the pressure being determined with a glass gauge. Light from a mercury-vapor lamp was passed through filters to make it approximately monochromatic, then passed through the reaction chamber and on to a thermopile.

The concentration of chlorine was determined by application of Beer's law. Previous calibration and determination of the absorption coefficient permitted a direct reading of the concentration of chlorine from the galvanometer deflection. The same galvanometer deflection was used also in determining the encrgy absorbed, and thus it was possible to determine quantum yields directly from the galvanometer deflections alone. This method for determining quantum yields is quite superior to some of the methods used previously. There is no pressure change during the reaction, and manometer measurements cannot be used for following the course of this reaction. In one method water is added, and the hydrogen chloride and the chlorine are absorbed. Both from pressure changes and from the chemical titrations the course of the reaction can then be followed. In other methods the gas mixture is frozen out in an attached U-tube, and the pressure of the remaining hydrogen is determined. These methods are less accurate and slower than the direct determination in the cell by means of Beer's law.

The primary process is the photodissociation of chlorine molecules into chlorine atoms. At the shorter wave-lengths one of the atoms is in an excited state. The chlorine atoms combine with hydrogen molecules, forming hydrochloric acid and releasing hydrogen. A series of reactions leading to a long chain is set up, as indicated by the following equations:

$$
\begin{aligned}
& \text { 1. } \mathrm{Cl}_{2}+h \nu=\mathrm{Cl}+\mathrm{Cl} \\
& \text { 2. } \mathrm{Cl}+\mathrm{H}_{2}=\mathrm{HCl}+\mathrm{H} \\
& \text { 3. } \mathrm{H}+\mathrm{Cl}_{2}=\mathrm{HCl}+\mathrm{Cl} \text {, etc. } \\
& \text { 4. } \mathrm{H}+\mathrm{HCl}=\mathrm{H}_{2}+\mathrm{Cl} \\
& \text { 5. } \quad \mathrm{Cl}+\mathrm{Cl}=\mathrm{Cl}_{2}
\end{aligned}
$$

The retarding effect of hydrochloric acid and the influence of the concentration of the hydrogen and chlorine, together with the fact that the reaction rate depends upon the intensity of the light absorbed, can all be expressed quantitatively by an empirical formula (44).

The maximum quantum yield obtained was about 100,000 molecules per quantum absorbed. The chains are continued until stopped by some chemical reaction, as for example when a chlorine atom combines with another chlorine atom to form a chlorine molecule.

It has long been known that oxygen exerts a strong inhibiting effect on this reaction. It apparently stops the reaction chains. It was found that in oxygen-rich mixtures the quantum efficiency is independent of the intensity of the absorbed light and varies inversely as the partial pressure of the oxygen (40). 
It is seen that this reaction is extremely complicated, and it is no wonder that different investigators, working under different conditions of oxygen pressure, light intensity, and size of reaction chamber, and without special apparatus to remove impurities, have failed to agree.

The Chlorination of Trichlorobromomethane.-This photochemical reaction is a fairly simple one to interpret. The overall reaction goes according to the following equation:

$$
\mathrm{Cl}_{2}+h \nu+2 \mathrm{CCl}_{3} \mathrm{Br}^{r}=2 \mathrm{CCl}_{4}+\mathrm{Br}_{2}
$$

The chlorine atom drives out the bromine atom from the compound, and there is very little chance for any other side reaction. There seems to be no chance for the complication of a chain reaction. A quantum yield of 0.9 , slightly less than unity, was obtained in the pure liquid and in solutions, even when highly diluted with carbon tetrachloride or silicon tetrachloride (19). If these solvents are not pure, but contain dissolved oxygen, the reaction is inhibited.

Eder's Reaction.-The photochemical reaction between mercuric chloride and ammonium oxalate produced by visible light in solution has been the subject of many investigations. Mercurous chloride is precipitated and carbon dioxide is evolved, as given by the following reaction:

$$
2 \mathrm{HgCl}_{2}+\left(\mathrm{NH}_{4}\right)_{2} \mathrm{C}_{2} \mathrm{O}_{4}=\mathrm{Hg}_{2} \mathrm{Cl}_{2}+2 \mathrm{NH}_{4} \mathrm{Cl}+2 \mathrm{CO}_{2}
$$

The reaction can be followed either by the evolution of the gas or by weighing the amount of the precipitate formed. The reaction involves a long chain, so that large effects are produced for small amounts of light. The reaction, however, is very complex. It involves an induction period and is very sensitive to numerous inhibitors and catalysts. It has been used sometimes as an actinometer, but it is too erratic and too subject to catalytic effects to be recommended for this purpose.

The kinetics of a similar reaction between potassium oxalate and mercuric chloride has been thoroughly investigated by Roseveare and Olson (45). The reactions can be sensitized to the longer wave-lengths of light by the addition of dyes, such as eosin and various ions.

Fulgides.-Certain substances possess the property of changing temporarily their color when exposed to light. This phenomenon is called phototropy. Prominent in this group of substances are the fulgides, which are derivatives of the compound:

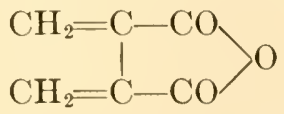

When three phenyl groups are substituted for hydrogen, a ycllow powder is produced, which turns brown on exposure to blue light and reverts to yellow when placed in the dark (49). The changes are reversible, but 
after many alternate exposures to light the sensitivity to this phototropy becomes less.

Fumaric and Maleic Acid Isomerization.-The well-known isomerization reactions of fumaric and maleic acids may be represented by the equation:

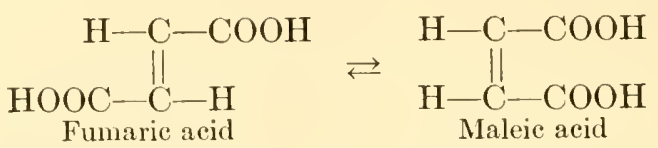

With ultra-violet light between 2000 and $2800 \AA$, fumaric acid is converted into maleic acid with a quantum efficiency of about 0.1. Maleic acid is converted into fumaric with still less efficiency-about 0.03 molecule per quantum on the average (53).

The low quantum yield in both reactions requires further explanation. The energy available in these short wave-lengths is considerably greater than that necessary to bring about the simple isomeric change. The reaction should be reinvestigated, particularly with reference to the possibility of a mechanism involving the formation of free radicals.

On the basis of these quantum yields, it can be calculated that the ratio of fumaric to maleic acid should be about $1: 3$ when the reactions have reached a steady state after long continued illumination at a constant light intensity. This value agrees closely with the value obtained experimentally.

This isomerization can be effected also by visible light in the presence of bromine. Very likely the mechanism is different. In carbon tetrachloride solution the di-ethyl ester of maleic acid is converted into the fumarate ester, with a quantum yield of about 8 at $4360 \AA$ and 4 at $5460 \AA(16)$.

Hydrogen Peroxide.-Hydrogen peroxide is readily decomposed by ultra-violet light at $3130 \AA$ and below, producing water and free oxygen. The course of the reaction may be followed readily either by the volume of oxygen liberated or by titration with potassium permanganate. The photochemical reaction is a chain reaction and quantum yiclds from 7 to 80 were obtained by Kornfeld (29) and from 4 to 100 by Allmand and Style (1). These investigators reported a dependence of the chain length on concentration and wave-length and particularly on the light intensity. They reported that the rate of decomposition varies inversely as the square root of the light intensity. The photolysis probably involves dissociation into ions according to the reaction:

$$
\begin{gathered}
\mathrm{H}_{2} \mathrm{O}_{2} \rightarrow \mathrm{H}^{+}+\mathrm{OOH}^{-} \\
\mathrm{OOH}^{-}=\mathrm{OH}^{-}+\mathrm{O}
\end{gathered}
$$

The marked influence of $\mathrm{pH}$ on this photolysis supports the view that the $\mathrm{OOH}^{-}$ion plays an important part in the photolysis. F. O. Rice 
(42) suggested that the reaction is strongly eatalyzed by dust partieles, and there is good evidence that supports this view.

Heidt (23) found that the quantum yield decreases as the concentration of hydrogen peroxide decreases and approaelies a limiting value somewhere in the neighborhood of unity. In these experiments the solutions were nearly free from dust particles and the radiation was of high intensity, eonditions that apparently lead to the suppression of the chain reaction.

Hydriodic Acid.-One of the simplest photochemical reactions is the decomposition of hydriodic acid. Gaseous hydrogen iodide is transparent through the visible region of the spectrum and to $3000 \AA$. At wavelengths shorter than this it absorbs radiation continuously without fine strueture. The energy of radiation at $3000 \AA$ corresponds to 95,000 calories per mole, and this is sufficient to break the hydrogen-iodide bond and liberate atoms. The atoms are thrown out with kinetic energy, depending on the frequency of the light. Inasmuch as this kinetic energy is not subject to the limitations of the quantum theory, it is possible for all wave-lengths to be utilized, thus giving a continuous absorption. Hydrogen molecules and iodine moleeules are formed by the combination of atoms. There is little chanee that hydrogen atoms will eombine with iodine atoms to give hydriodic acid, and there is no chance of a chain reaction. Hence the overall quantum yield is 2 molecules per quantum, as given by the following equations:

$$
\begin{aligned}
\mathrm{HI}+h \nu & =\mathrm{H}+\mathrm{I} \\
\mathrm{H}+\mathrm{HI} & =\mathrm{H}_{2}+\mathrm{I} \\
\mathrm{I}+\mathrm{I} & =\mathrm{I}_{2}
\end{aligned}
$$

The reaction is one of the simplest in photochemistry, and the quantum yield is not affected by light intensity or by concentration. It is practically the same in gases at low pressures (35) and at high pressures, and even in liquid hydriodic acid (9) or when dissolved in liquid hexane (54). This reaction has been studied by several investigators. The hydrogen iodide is placed in a quartz vessel and is submitted to monochromatic radiation. Radiation of $2536 \AA$ from a mereury-vapor lamp, filtered through a mixture of chlorine and bromine, is satisfactory. The reaction cannot be followed by pressure change because there is no change in the number of gaseous molecules. It has been followed by titrating the amount of iodine liberated after various intervals of time. It could be followed eolorimetrically by the production of iodine.

Warburg (51) found that in this reaction the number of molecules of hydriodic decomposed per quantum was the same at different wavelengths, 2070, 2536, and $2820 \AA$. The number of molecules decomposed per erg of radiation varied considerably. This is taken as one of the experimental supports for the Einstein relation and the quantum theory. 
Iodine and Ferrous Ion.-In an aqueous solution of iron salt and iodine, an equilibrium is set up in the dark according to the equation:

$$
2 \mathrm{Fe}^{++}+\mathrm{I}_{2} \rightleftarrows 2 \mathrm{Fe}^{+++}+2 \mathrm{I}^{-}
$$

When such a solution is exposed to visible light, a considerably different equilibrium point is obtained. In the presence of light the equilibrium is shifted $(43,27)$ so as to give a higher concentration of the ferric ion. The photochemical reaction probably involves the following equation:

$$
2 \mathrm{Fe}^{++}+\mathrm{I}_{3}^{-}+h \nu=2 \mathrm{Fe}^{+++}+3 \mathrm{I}^{-}
$$

The tri-iodide ion is formed in solution when iodine is mixed with an excess of potassium iodide. Kistiakowsky found a quantum yield of unity at all wave-lengths from 3660 to $5790 \AA$ under conditions where the reverse thermal reaction was negligible.

Mercury Vapor.-Mercury in the vapor state is monatomic, and when the atoms of mercury absorb radiation at $2537 \AA$ from a mercury-arc lamp they become activated to a high degree. The energy of this activation corresponds to more than 110,000 calories per mole. A great variety of chemical reactions can be produced by collision of gaseous molecules with these excited atoms of mercury. Hydrogen atoms are produced when hydrogen molecules are mixed with a small amount of mercury vapor and the mercury vapor is subjected to this light of $2536 \AA$. The atomic hydrogen, in turn, can produce a number of different hydrogenation reactions. When ethylene, for example, is added to this system, hydrogenation, decomposition, and polymerization all occur. A number of different decompositions produced by excited mercury atoms have been described by Bates and Taylor (3).

Cadmium vapor has a resonance line at $3262 \AA$, and its atoms can be excited in a similar manner by this radiation. The energy available at this wave-length is considerably smaller, and atoms excited by this radiation are not able to dissociate hydrogen, as is the case with mercury (4).

Monochloro-acetic Acid Hydrolysis.-When an aqueous solution of monochloro-acetic acid is subjected to ultra-violet light of $2536 \AA$, the following reaction takes place:

$$
\mathrm{CH}_{2} \mathrm{ClCOOH}+\mathrm{H}_{2} \mathrm{O}=\mathrm{HOCH}_{2} \mathrm{COOH}+\mathrm{Cl}^{-}+\mathrm{H}^{+}
$$

The reaction goes on in the dark also to a slight extent, and it is accelerated by hydroxyl ions. A quantum yield of practically one molecule per photon is reported for the reaction (46). As the reaction proceeds, the reaction diminishes in velocity at constant input of light, possibly because of the increased acidity produced by the reaction itself.

Nitrogen Dioxide.-Nitrogen dioxide is a brown gas. At ordinary pressures it is partially polymerized to nitrogen tetroxide. Its absorption 
spectrum is very complex, with many discrete lines and some continuous spectrum. In ultra-violet light and in the violet of the visible spectrum nitrogen dioxide is decomposed by the light that it absorbs, giving nitric oxide and oxygen. As the decomposition proceeds, nitric oxide and oxygen accumulate and the reverse reaction takes place thermally. This reverse reaction involves a triple collision between two nitric oxide molecules and one oxygen molecule; accordingly the reaction is quite slow. For short exposures this reverse reaction can be neglected, particularly at the lower pressures. When nitrogen pentoxide gas is mixed with nitrogen dioxide, the nitrogen pentoxide reacts immediately with the nitric oxide formed by the photodecomposition of the nitrogen dioxide, producing more nitrogen dioxide. In this way the reverse reaction of nitric oxide is prevented and more accurate measurements of the photodecomposition of nitrogen dioxide can be obtained by determining the amount of accumulated oxygen.

This reaction has been investigated carefully in three different laboratories $(13,25,37)$ and all are in substantial agreement.

A quantum yield of 2 , obtained with ultra-violet light by extrapolating to pure nitrogen dioxide, is explained on the basis of a collision between an activated molecule of nitrogen dioxide and a second molecule to produce two molecules of nitric oxide and one of oxygen as given by the following equations:

$$
\begin{gathered}
\mathrm{NO}_{2}+h \nu=\mathrm{NO}_{2}^{*} \\
\mathrm{NO}_{2}^{*}+\mathrm{NO}_{2}=2 \mathrm{NO}+\mathrm{O}_{2}
\end{gathered}
$$

The asterisk $\left(^{*}\right)$ indicates an excited molecule.

The lower quantum yield of about 0.5 at $4050 \AA$ is explained on the assumption that the energy of a quantum at this wave-length is not quite sufficient to bring about the chemical reaction. Part of the energy of activation must come from thermal collisions, and not every molecule that absorbs a photon contains sufficient thermal energy to effect reaction. At $4360 \AA$ there is no decomposition, i.e., the quantum yield is zero.

Norrish (37) has studied the relation of fluorescence of nitrogen dioxide to this reaction. At the longer wave-lengths he obtained a fluorescence, but at the shorter wave-lengths, where dissociation occurs, no fluorescence is detectable.

Nitrogen Pentoxide.-Nitrogen pentoxide absorbs radiation below $3000 \AA$ and it is decomposed with a quantum efficiency of 0.6 of a molecule decomposed per quantum absorbed (25). The decomposition of nitrogen pentoxide at longer wave-lengths when sensitized by nitrogen dioxide has been explained in the preceding section. It is clear that this sensitized reaction is really a chemical reaction of the nitrogen pentoxide with one of the chemical products resulting from the photodecomposition of the nitrogen dioxide. 
Nitrate ion.-When potassium nitrate is dissolved in water and subjected to radiation in the short ultra-violet region, nitrite and free oxygen are produced according to the reaction:

$$
2 \mathrm{NO}_{3}^{-} \rightarrow 2 \mathrm{NO}_{2}^{-}+\mathrm{O}_{2}
$$

The quantum yield depends on the wave-lengths of the light and on the $\mathrm{pH}$ of the solution. At a $\mathrm{pH}$ of 9.9 Villars (50) found the following values:

\begin{tabular}{|c|c|c|c|c|c|}
\hline Wave-length, $\AA \ldots . . .$. & 2540 & 2700 & 2800 & 3130 & 3660 \\
\hline Quantum vield. & 0.30 & 0.07 & 0.02 & 0.01 & 0.00 \\
\hline
\end{tabular}

At wave-length $2536 \mathrm{~A}$ the quantum yield varied from 0.05 at a $\mathrm{pH}$ of 6 to 0.3 at a $\mathrm{pH}$ of 10 to 14 . At the shortest wave-length there is probably enough energy available to cause direct rupture of the nitrate ion into the nitrite ion and atomic oxygen. At the longest wave-length the energy is insufficient for this process. The strong dependence on the $\mathrm{pH}$ of the solution indicates that the reaction is more complicated than given by the simple decomposition of the nitrite ion. The low quantum yield, less than unity, can be attributed to loss of energy by collision to the surrounding solvent or to loss of energy in vibration of the atoms in the ion, but it may be due to the existence of more complex reactions.

Oxidation of Ions by Dissolved Oxygen.-The oxidation of an aqueous solution of sodium sulfite by dissolved oxygen is typical of a large group of reactions. It is strongly inhibited by small traces of organic material, such as hydroquinone, benzaldehyde, and similar substances. This marked inhibition is characteristic of chain reactions. That the oxidation of sodium sulfite by dissolved oxygen is a chain reaction was demonstrated by Bäckström (2), who found that about 50,000 molecules react per quantum of radiation at $2536 \AA$.

The auto-oxidation of stannous chloride is a similar reaction; both the large quantum yield and the marked inhibition by various substances show the reaction to be a chain reaction. The reaction was studied in aqueous and in nonaqueous solutions by Haring and Walton $(20,21)$. The complex ion produced by the union of stannous chloride and hydrochloric acid absorbs ultra-violet light and in the presence of dissolved oxygen is changed over into stannic chloride. The reactions in water are somewhat complicated by hydrolysis, but similar results are obtained in nonaqueous solvents in which no hydrolysis can take place. Rough measurements of the quantum yield showed that many molecules react for each photon absorbed.

Picric acid was found to be one of the most powerful inhibitors. In acetic acid solvent, for example, one millimole of stannous chloride was oxidized in $2 \mathrm{~min}$, whereas, with 0.02 gram of picric acid present, less 
than 0.01 of a millimole was oxidized in $20 \mathrm{~min}$. Metadinitrobenzene, paranitrotoluene, and various other substances were found to be inhibitors. Phenol urea was found to be an accelerator. These various substances act as inhibitors not only for the photochemical reaction, but also for the thermal reaetions. The close parallelism shows that the thermal reaction as well as the photochemical reaction is a chain reaction.

Oxalic Acid Sensitized by Uranyl Ion.-The investigation of this reaction by Leighton and Forbes (34) gives perhaps the most precise photochemical measurements described in the literature. Earlier work on this reaction was unsatisfactory, and wide discrepancies in the results of different investigators were apparent. In this investigation monochromatic light from a mereury-vapor lamp and a monoehromator was passed through a quartz eell and the absolute energies were measured with a thermopile and galvanometer standardized against a standard lamp of the Bureau of Standards. Several corrections are described in detail.

Oxalic acid is decomposed by very short ultra-violet light, but it is not decomposed by the longer ultra-violet light or by visible light. However, in the presenee of the uranyl ion, this light does eause oxalic acid to decompose into carbon dioxide, carbon monoxide, and water. The oxalic acid is titrated with dilute potassium permanganate. The uranyl salts are strongly fluorescent in ultra-violet light. It is likely that a complex is formed between the oxalie acid and the uranyl ion, which absorbs the light and causes disruption of the oxalic acid molecule. The uranyl ions are not affected by the reaction and can be used over again in the decomposition of an indefinite amount of oxalie acid.

Using a solution 0.01 molal in uranyl sulfate and 0.05 molal in oxalie aeid at $25^{\circ} \mathrm{C}$., the following quantum yields were obtained at different wave-lengths:

\begin{tabular}{|c|c|c|c|c|c|c|c|}
\hline Wave-length, $\AA$. & 2550 & 2650 & 3000 & 3130 & 3660 & 4060 & 4350 \\
\hline Quantum yield. & 0.60 & 0.60 & 0.57 & 0.56 & 0.49 & 0.56 & 0.58 \\
\hline
\end{tabular}

The temperature effect for a $10^{\circ}$ rise is 1.03 .

For the shorter ultra-violet light, it has been found (17) that a tenfold dilution of this solution gives better results, and the quantum yield is not ehanged by the dilution. For practical use it is found that the uranyl oxalate is easier to purify than the uranyl salt and the oxalic acid.

This reaetion is probably the best for general use in actinometry. The quantum yield is accurately known to a very few hundredths of a unit and it is independent of concentration over wide range; it is only slightly dependent on wave-length and on temperature. It is reproducible and is free from the erratie variations that frequently accompany chain reactions. Investigators in different laboratories have obtained excellent 
checks. Furthermore, it has already been used in many researches as an actinometer, and it is generally accepted as perhaps the best standard. Although accurate only for monochromatic light, this reacting system can be used with certain definite limitations as an actinometer for polychromatic light (34).

Ozone.-Oxygen is partially converted into ozone by ultra-violet light of short wave-length-below $2000 \AA$. The absorption spectrum of oxygen in this region is discontinuous, and it is likely that the absorption of a photon produces an activated molecule, which then collides with a second molecule, producing two atoms of oxygen and one molecule of oxygen. Each of these oxygen atoms can then combine with an oxygen molecule, resulting in the formation of two molecules of ozone. If this is the process, the corresponding equations would be:

$$
\begin{aligned}
\mathrm{O}_{2}+h \nu & =\mathrm{O}_{2}^{*} \\
\mathrm{O}_{2}^{*}+\mathrm{O}_{2} & =\mathrm{O}_{2}+\mathrm{O}+\mathrm{O} \\
\mathrm{O}+\mathrm{O}_{2} & =\mathrm{O}_{3}
\end{aligned}
$$

and two molecules of ozone should be produced for each quantum absorbed. Working at high pressures, 50 to 300 atmospheres, Warburg (52) found a quantum yield approaching 2 but ranging from 2.06 to 1.3 , depending on the pressure. Although the oxygen is much less absorbing at the longer wave-lengths, there is still some production of ozone by ultra-violet light at $2536 \AA$. Short ultra-violet light from the sun's rays is absorbed by the air long before it reaches the surface of the earth and practically no ozone is produced directly from sunlight at sea level. In the stratosphere, however, considerable amounts of ozone are found.

Ozone itself absorbs light between 2300 and $2800 \AA$ and also in the red end of the visible spectrum. It is decomposed by the ultra-violet light which it absorbs with a quantum yield of 3.5 in pure ozone at $15^{\circ} \mathrm{C}$. (47).

When oxygen is radiated with polychromatic light containing both the short ultra-violet and the longer ultra-violet, an equilibrium is set up, ozone being formed and decomposed. A steady state is soon reached at which the ozone is decomposed as fast as it is formed. The concentration of ozone at this steady state depends on the relative intensities of the longer and shorter ultra-violet. Quite frequently the smell of ozone is detected around a freshly started mercury-vapor lamp but after the lamp becomes heated the ozone is largely destroyed.

Ozone can be decomposed also by light that it does not absorb, provided a suitable photosensitizer is added. Light of 4360 and $4060 \AA$ passed into a mixture of chlorine and ozone decomposes two molecules of ozone for each quantum absorbed. This photochemical yield is practically independent of the ozone concentration over a tenfold range from 0.5 to 5 per cent and it is only slightly affected by temperature, but the 
quantum yield is considerably reduced by collisions with foreign gases. The effect of these foreign gases is rather specific, helium producing a considerable effect.

The photolysis of ozone is exceedingly complex, and in spite of a considerable amount of work in various laboratories (24) the reaction is not yet satisfactorily understood. In the presence of water vapor (18) the quantum yield ranges from 1.6 to 130 , indicating a chain mechanism. The quantum yield varies inversely as a fixed power of the light intensity.

Persulfate Ion.- When potassium persulfate is dissolved in water and subjected to ultra-violet light between 2536 and $3020 \AA$, oxygen is produced and the persulfate ion is changed to sulfate ion according to the following reaction:

$$
\mathrm{S}_{2} \mathrm{O}_{8}^{--}+\mathrm{H}_{2} \mathrm{O}+h \nu=2 \mathrm{SO}_{4}^{--}+2 \mathrm{H}^{+}+1 / 2 \mathrm{O}_{2}
$$

A quantum yield of unity was found for this reaction (11). In the presence of potassium chloride or mercuric chloride it dropped to 0.7 of a molecule per quantum. The work was carried out with a thermopile and monochromator, using a quartz capillary lamp provided with a magnetic field designed to extend the life of the lamp.

\section{REFERENCES. PART 2}

1. Allmand, A. J., and D. W. Style. The photolysis of aqueous hydrogen peroxide solutions. Jour. Chem. Soc. pp. 596-623. 1930.

2. Вӥскsтröм, H. L. J. The chain-reaction theory of negative catalysis. Jour. Amer. Chem. Soc. $49:$ 1460-1472. 1927.

3. Bates, J. R., and H. S. TaYlor. Studies in photosensitization. I. Jour. Amer. Chem. Soc. 49: 2438-2456. 1927.

4. Bates, J. R., and H. S. TAYlor. Studies in photosensitization. II. A source of cadmium resonance radiation. Jour. Amer. Chem. Soc. 50: 771-773. 1928.

5. Bauer, W. H., and F. Daniels. Separation of photochemical and thermal action in the photobromination of cinnamic acid. Jour. Amer. Chem. Soc. 56: 378-385. 1934.

6. BaUer, W. H., and F. Daniels. Oxygen a factor in the photobromination of cinnamic acid. Jour. Amer. Chem. Soc. $66: 2014.1934$.

7. Berthoud, A., and J. Beranek. Photochimie des halogènes. Addition du brome a l'acide cinnamique et au stilbène. Jour. Chim. Phys. 24: 213-237. 1927.

8. Bodenstein, M., and H. Lütremeyer. Die photochemische Bildung von Bromwasserstoff und die Bildungsgeschwindigkeit der Brommolekül aus den Atomen. Zeitsch. Physik. Chem. 114: 208-236. 1925.

9. Bodenstein, M., and F. Lieneweg. Unabhängigkeit der Zersetzung des Jodwasserstoffs im Licht von Aggregatzustand und Temperatur und Versuche zur Deutung des Mechanismus des Vorgangs. Zeitsch. Physik. Chem. 119: 123-138. 1926.

10. Bonнoeffer, K. F. Anwendung der Quantentheoric auf photochemische Sensibilisierungen. Zeitsch. Physik 13: 94-105. 1923.

11. Crist, R. H. The quantum efficiency of the photochemical decomposition of potassium persulfate. Jour. Amer. Chem. Soc. 54:3939-3942. 1932.

12. Damon, G. II., and F. Daniels. The photolysis of gaseous acetone and the influence of water. Jour. Amer. Chem. Soc. $55: 2363-2375.1933$. 
13. Dickinson, R. G., and W. P. Baxter. The quantum yield in the photochemical decomposition of nitrogen dioxide. Jour. Amer. Chem. Soc. 50: 774-782. 1928.

14. Dickinson, R. G., and J. L. Carrico. The photochlorination and the chlorinesensitized photo-oxidation of gaseous tetrachloroethylene. Jour. Amer. Chem. Soc. $56: 1473-1480.1934$.

15. Dickinson, R. G., and J. A. Leermakers. The chlorine-sensitized photooxidation of tetrachloroethylene in carbon tetrachloride solution. Jour. Amer. Chem. Soc. 54 : 3852-3862. 1932.

16. Eggert, J., F. WAChholtz, and R. Schmidt. Über einige Reactionen des durch Licht angeregten Brons. Physik. Zeitsch. 26: 865-868. 1925.

17. Forbes, G. S., and L. J. Herdt. Optimum composition of uranyl oxalate solutions for actinometry. Jour. Amer. Chem. Soc. 56:2363-2365. 1934.

18. Fories, G. S., and L. J. Heidt. The influence of water upon the photolysis of ozone at $\lambda \lambda 280,254$ and $210 \mathrm{~m} \mu$. Jour. Amer. Chem. Soc. 56:1671-1675. 1934.

19. Grüsz, H. Über die Anwendung des photochemischen Äquivalentgesetzes auf verdünnte Lösungen. Zeitsch. Elektrochem. 29 : 144-150. 1923.

20. Haring, R. C., and J. H. WAlton. The autoxidation of stannous chloride. III. The photochemical reaction. Jour. Phys. Chem. 37: 375-380. 1933.

21. Haring, R. C., and J. H. Walton. The autoxidation of stannous chloride. IV. The effect of some non-aqueous solvents. Jour. Phys. Chem. 38: 153-160. 1934.

22. Hart, E. J., and W. A. Noyes, Jr. The chlorination of chlorohenzene: a comparison with benzene. Jour. Amer. Chem. Soc. 56: 1305-1310. 1934.

23. HeIDT, L. J. The photolysis of hydrogen peroxide in aqueous solution. Jour. Amer. Chem. Soc. 54 : 2840-2843. 1932.

24. Heidt, L. J., and G. S. Forbes. The photolysis of dry ozone at $\lambda 208$, at $\lambda 254$ and at $\lambda 280 \mathrm{~m} \mu$. I. Quantum yields. Jour. Amer. Chem. Soc. 56: 2365-2368. 1934.

25. Holmes, H. H., and F. Daniels. The photolysis of the nitrogen oxides; $\mathrm{N}_{2} \mathrm{O}_{5}$; $\mathrm{N}_{2} \mathrm{O}_{4}$ and $\mathrm{NO}_{2}$. Jour. Amer. Chem. Soc. 56:630-637. 1934.

26. Kistiakowsky, G. B. Photochemical processes. (Cf.pp. 104-110.) Chemical Catalog Co.; New York, 1928.

27. Kistiakowsky, G. B. The action of light on the ferrous-ferric: iodine-iodine equilibrium. Jour. Amer. Chem. Soc. 49: 976-979. 1927.

28. Kistiakowsky, G. B., and E. O. Wiig. The photochemical decomposition of ammonia. Jour. Amer. Chem. Soc. 54 : 1806-1820. 1932.

29. Konnfeld, G. Die Zersetzung des Wasserstoffsuperoxyds in ultra-violetten Licht. Zeitsch. Wiss. Photographie 21: 66-92. 1921.

30. Leermakers, J. A. The chain photolysis of gaseous acetaldehyde. Jour. Amer. Chem. Soc. 56: 1537-1541. 1934.

31. Leermakers, J. A., and R. G. Dickinson. The photochlorination of tetrachloroethylene in carbon tetrachloride solution. Jour. Amer. Chem. Soc. 54: 4648-4657, 1932.

32. Leighton, P. A., and F. E. Blacet. The photolysis of the aliphatic aldehydes. I. Propionaldehyde. Jour. Amer. Chem. Soc. 54 : 3165-3178. 1932.

33. Leigiton, P. A., and F. E. Bracet. The photolysis of the aliphatic aldehydes. II. Acetaldehyde. Jour. Amer. Chem. Soc. 55: 1766-1774. 1933.

34. Leighton, W. G., and G. S. Forbes. Precision actinometry with uranyl oxalate. Jour. Amer. Chem. Soc. $52: 3139-3152.1930$.

35. Lewis, B. The photochemical decomposition of hydrogen iodide: The mode of optical dissociation. Jour. Phys. Chem. 32: 270-284. 1928.

36. Lind, S. C., and R. Livingston. The photochemical polymerization of acetylene. Jour. Amer. Chem. Soc. 54: 94-106. 1932. 
37. Norrish, R. G. W. Photochemical equilibrium in nitrogen peroxide. III. A comparison of the thermal, photochemical and electrical decompositions, and a general theory of the change. Jour. Chem. Soc. pp. 1604-1621. 1929.

38. Norrish, R. G. W., H. G. Crone, and O. D. Saltmarsh. Primary photochemieal reactions. $\mathrm{V}$. The spectroscopy and photochemieal decomposition of acetone. Jour. Chem. Soe. pp. 1456-1464. 1934.

39. Norrish, R. G. W., and F. W. Kirkbride. The deeomposition of formaldehyde. Jour. Chem. Soc. pp. 1518-1530. 1930.

40. Norrish, R. G. W., and M. Ritchie. The photosynthesis of hydrogen ehloride. III. Mixtures containing oxygen. Proc. Roy. Soc. London. 140A : 713-740. 1933.

41. Noyes, W. A. Jr., A. B. F. Duncan, and W. M. Manning. The ultra-violet ahsorption spectrum of acetone vapor. Jour. Chem. Phys. 2: 717-725. 1934.

42. Rice, F. O. The eatalytic activity of dust particles. Jour. Amer. Chem. Soe. 48: 2099-2113. 1926.

43. Rideal, E. K., and E. G. Williams. The action of light on the ferrous-ferrie, iodine-iodide equilibrium. Jour. Chem. Soe. pp. 258-269. 1925.

44. Ritchie, M., and R. G. W. Norrish. The photosynthesis of hydrogen chloride. I. A new experimental method: The inhibiting effect of hydrogen ehloride. II. Oxygen-free systems. Proc. Roy. Soc. London. 140A : 99-125. 1933.

45. Rosevfine, W. E., and A. R. Olson. The thermal reaetion between potassium oxalate and mercuric ehloride. Jour. Amer. Chem. Soc. 51: 1716-1724. 1929.

46. RudBerg, E. Die photochemische Spaltung von Monochlor- und Monobromessigsäure und das Einsteinsche Gesetz. Zeitsch. Physik 24: 247-263. 1924.

47. Schumacher, H. J., and U. Beretta. Die Photokinetik des Ozones. II. Der Zerfall des Ozons im ultravioletten Licht. Zeitsch. Physik. Chem. 17B : 417-428. 1932.

48. Smith, H. P., W. A. Noyes, Jr., and E. J. Hart. A further study of the chlorination of benzene. Jour. Amer. Chem. Soe. 55 : 4444-4459. 1933.

49. Sтовве, H. Phototropieerseheinungen bei Fulgiden und anderen Stoffen. Ann. Chem. Liebig's. 359: 1-48. 1907.

50. Villars, D. S. The photolysis of potassium nitrate, Jour. Amer. Chem. Soc. $49: 326-337.1927$.

51. WARBURG, E. Üher den Energieumsatz bei photochemischen Vorgängen in Gasen. VII. Photolyse des Jodwasserstoffs. Sitzber. Preuss. Akad. pp. 300317. 1918.

52. Warburg, E. Zum Energieumsatz bei photochemischen Vorgängen im Gasen. Zeitsch. Elektroehem. 27: 133-142. 1921.

53. WARBURG, E. Ueber den Energieumsatz bei photoehemischen Vorgängen. IX. Photochemische Umwandlung isomerer Körper ineinander. Sitzber. Preuss. Akad. pp. 960-974. 1919.

54. WARBURG, E., and W. RUMP. Über die Photolyse von Jodwasserstoffösungen in Hexan und in Wasser. Zeitsch. Physik 47:305-322. 1928.

55. Weigert, F. Weitere Beiträge zur thermodynamischen Theorie photochemischen Prozesse. Ber. Deut. Chem. Ges. 42: 850-862. 1909.

56. Weigert, F. Über den Mechanismus der photochemischen Polymerisation des Anthracens. Naturwiss. 15: 124-126. 1927.

\section{GENERAL REFERENCES}

Bonhoeffer, K. F., and P. Harteck. Grundlagen der Photochemie. 295 pp. Theodor Steinkopff; Dresden and Leipzig, 1933.

Dhar, N. R. The Chemical Action of Light. 512 pp. Blackie and Sons, Ltd.; London, 1931. 
Faraday Society Srmposium. Trans. Faraday Soc. $27: 359-573.1931$.

Griffith, R. O., and A. McKeown. Photoprocesses in Gaseous and Liquid Systems. 691 pp. Longmans Green \& Co.; London, 1929.

Kistiakowsky, G. B. Photochemical Processes. $270 \mathrm{pp}$. The Chemical Catalog Co.; New York, 1928.

National Research Council. First Report of the Committee on Photochemistry. Jour. Phys. Chem. 32 : 481-575. 1928.

Noyes, W. A., JR. The Correlation of Spectroscopy and Photochemistry. Rev. Modern Phys. 5 : 280-287. 1933.

Style, D. W. G. Photochemistry. 96 pp. E. P. Dutton \& Company; New York, 1931.

TAYlor, H. S. Photochemistry. A Treatise on Physical Chemistry (Cf. pp. 14591518). D. Van Nostrand Co., Inc.; New York, 1931.

Manuscript received by the editor June, 1935. 


\title{
VIII
}

\section{THE EFFECT OF RADIATION ON PROTEINS}

\author{
Janet Howell Clark
}

School of Hygiene and Public Health of the Johns Hopkins University, Baltimore, $M d$.

Introduction. Absorption of radiant energy by proteins. Effect of radiation on suspensoids. Effect of radiation on emulsoids: Ultra-violet radiation-Sensitization to visible light-Effect of $\mathrm{X}$-rays and radium rays on proteins-General conclusions. Primary and secondary light reactions. Relation of protein denaturation to general physiological light reactions. References.

\section{INTRODUCTION}

A substance is said to exist in the colloidal state when, because of the large size of the molecules, or aggregates of molecules, it is unable to diffuse through certain membranes. When the internal or dispersed phase is solid and the external or continuous phase is liquid, the colloid is called a suspensoid. When the dispersed phase is liquid and the continuous phase liquid, it is an emulsoid. Generally speaking, most natural colloids are emulsoids and most artificial colloids are suspensoids.

The two great classes of colloids, emulsoids and suspensoids, differ in the state of the internal or dispersed phase, liquid in the former and solid in the latter. They differ also in the affinity of the internal phase for water, the external phase. Emulsoids are lyophile or hydrated colloids, and suspensoids are, in general, lyophobe or nonhydrated. Metallic hydroxides can combine more or less with water and, though classed as suspensoids, are more like emulsoids in many respects. This difference in hydration brings about differences in stability. Suspensoids are precipitated by the addition of small amounts of electrolytes carrying the opposite eharge, whereas emulsoids are precipitated only by large amounts of salts. Suspensoids are stable in solution only when charged and fall out of solution when they lose their charge. Some emulsoids (globulins) precipitate when uncharged and others (albumins) stay in solution even when their charge is lost. Although it is possible to prepare some suspensoids with either a positive or negative charge, suspensoids in general earry a charge of definite sign depending on the material of the dispersed phase. Emulsoids earry either a positive or negative charge depending on the hydrogen ion concentration of the medium.

Proteins are in many respects the most important members of the emulsoid class. The ehemical behavior of proteins in solution does not 
differ from that of erystalloids, for it has been shown that proteins combine stoichiometrically (i.e., by the purely chemical forces of primary valency) with acids and alkalies, forming protein salts which dissociate electrolytically. The colloidal nature of the proteins is attributed to the large size of their ions which enables them to fulfill the conditions necessary for the establishment of a Donnan equilibrium, namely, that the protein ion is prevented from diffusing through membranes which are permeable to the smaller crystalloidal ions. Proteins are formed by the condensation of a number of molecules of various amino acids, and amino acids, by virtue of their amino and carboxyl groups, react with either acids or bases to form salts (i.e., they are amphoteric electrolytes). The salts formed with a base (for instance $\mathrm{NaOH}$ ) dissociate to give a positively charged sodium ion and a negatively charged protein ion. The salts formed with an acid, such as $\mathrm{HCl}$, dissociate to form a negatively charged chlorine ion and a positively charged protein ion. Therefore at a certain hydrogen ion concentration, called the isoelectric point, protein molecules exist uncharged, but on the alkaline side of the isoelectric point they carry a negative charge and on the acid side a positive charge.

The isoelectric points of some proteins are given in Table 1.

\section{TABLE 1}

\section{Protein}

$\mathrm{pH}$, Isoelectric

Glutenin... . point

Serum albumin

Casein

Gelatin

4.7

Egg albumin

4. 7

Fibrinogen

4. 8

Serum globulin

5. 0

Oxyhemoglobin

5. 4

Gliadin. .

6.74

9.0

The osmotic pressure, viscosity, and swelling of proteins are lowest at the isoelectric point and their stability in water solution is least at this hydrogen ion concentration.

Some proteins (globulins) lose their water of hydration and precipitate spontancously at the isoclectric point. Other proteins (albumins) retain their water of hydration and stay in solution even at the isoelectric point where they are uncharged. The difference between an albumin and a globulin is therefore fundamentally a difference in affinity for water. Globulins are hydrated only when charged, and even when charged, they have a lower affinity for water than albumins, for they are more easily precipitated by salts.

The following definitions for the various changes produced in the state of a protein are given by Lewis (36). Crystallization is the precipitation in solid crystalline form of undenatured protein. Denaturation is a 
change which the natural protein is capable of undergoing and is a definite step in the transition of the natural, crystallizable protein molecules to the amorphous state represented by flocculation. This change may be brought about by various ageneies, one of which is heat. Heat denaturation is a change in the protein molecule brought about only in the presence of water. It is unimolecular in nature and appears to be associated with some internal physical or chemical change within the structure of each protein unit and is generally regarded as being of an irreversible nature, although recent work $(1,2,37,38)$ states that it may be reversible under certain conditions. The denaturation produced by radiation is apparently different from heat denaturation in some respects, but all types of denaturation produce similar changes in the solubility of proteins. A denatured albumin has the solubility characteristic of a globulin, as it no longer stays in solution at the isoelectric point and precipitates more readily with salts. Flocculation is the precipitation of protein in the neighborhood of the isoelectric point in an amorphous form. A distinction should be made between true floceulation (chain formation produced by addition of successive protein units) at or near the isoelectric point and the precipitation of a protein salt in amorphous form at a $\mathrm{pH}$ removed from the isoelectric point. In an albumin, flocculation is possible only provided the original protein has suffered the change known as denaturation. The two consecutive changes, denaturation plus flocculation, are referred to as coagulation.

\section{ABSORPTION OF RADIANT ENERGY BY PROTEINS}

In considering the effect of radiation on proteins it must be remembered that radiant energy produces changes only when energy is absorbed.

Ultra-violet Radiation.-The absorption of ultra-violet radiation by protein solutions has been studied by many investigators. Lewis $(34,35)$ found the absorption of blood serum in the ultra-violet region to be due to the proteins. Serum albumin, pseudoglobulin, and euglobulin from horse and human serum gave similar curves, although the absorption of globulins was greater than that of albumins. They all begin to absorb at approximately $3100 \AA$. The absorption increases to a maximum at $2800 \AA$, falls to a minimum at $2500 \AA$, and then rises sharply for shorter wave-lengths. Smith (45) gave similar results, which are shown in Fig. 1. He found the extinction coefficient of globulin to be twice as great as that of albumin at $\lambda 2800 \AA$ and the values for horse and human serum proteins to be the same. He also examined the absorption of a number of the constituent amino acids of the serum proteins and found the same absorption maximum and minimum in tyrosin and tryptophan, a point previously noted by Dhéré (13). He therefore attributed the absorption of serum proteins largely to these amino acids. The exact values given by different authors for the extinction coefficients of serum albumin and 
serum globulin differ (56), owing probably to the method of preparing the proteins and their purity. The values for the absorption maximum and minimum given by different authors are listed in Table 2 .

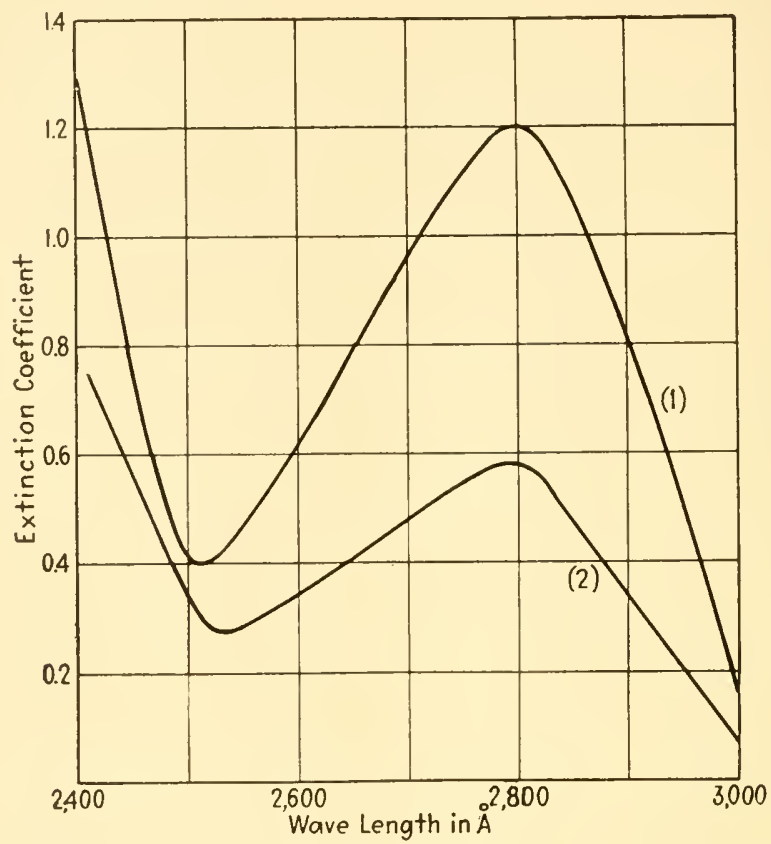

Fig. 1.-Absorption curves of: (1) serum pseudoglobulin; (2) serum albumin. (After F. C. Smith, Proc. Roy. Soc. [London] B. $104: 198$. 1929.)

Egg albumin was found to have an absorption curve similar to that of serum albumin $(3,34,35,55)$. Lewis $(34,35)$ remarks that the close similarity in form of all the curves when corrected to a common ampli-

TABLE 2

\begin{tabular}{|c|c|c|c|}
\hline Author & Protein & $\begin{array}{c}\text { Absorption } \\
\operatorname{max.,~} \AA\end{array}$ & $\begin{array}{c}\text { Absorption } \\
\text { min., } \AA\end{array}$ \\
\hline \multirow[t]{4}{*}{ Lewis. } & Human serum albumin & & \\
\hline & Human serum globulin & & \\
\hline & Horse serum albumin & 2800 & 2500 \\
\hline & Horse serum globulin & & \\
\hline \multirow[t]{3}{*}{ Smith............... } & Horse serum albumin & & \\
\hline & Horse serum pseudoglobulin & 2790 & 2520 \\
\hline & $\begin{array}{l}\text { Human serum albumin } \\
\text { Human serum pseudoglobulin }\end{array}$ & & \\
\hline Svedberg and Nichols (55). & Egg albumin & 2780 & 2500 \\
\hline \multirow[t]{2}{*}{ Svedberg and Sjorgren (56). } & Horse serum albumin & 2780 & 2500 \\
\hline & Horse serum globulin & 2850 & 2550 \\
\hline
\end{tabular}


tude, and the fact that the amplitudes are all nearly simple multiples of a common factor, point to a similarity of constitution among these proteins and to a variable "concentration" of the active group.

$X$-rays and Radium Rays. - The absorption of X-rays and gamma rays has been discussed in a previous paper and will be mentioned only briefly here. The absorption coefficient $\mu$ divided by the density $\rho$ of the absorbing screen $=\mu / \rho=$ the mass absorption coefficient. Dense substances are more absorptive, mass for mass, than light substances and $\mu / \rho$ increases rapidly with the atomic weight of the absorbing material. This increase is more noticeable with hard rays than with soft.

No experiments have been carried out on the mass absorption coefficients of the various constituents of tissue, such as proteins, but the absorption of blood and serum as a whole is not very different from that of water.

The beta rays of radium are more easily absorbed than $\mathrm{X}$-rays and gamma rays. Their penetrating power depends on the velocity of the rays and the density of the medium. The heavier alpha particles are absorbed more easily than beta rays.

Ionization Due to Absorption of Radiant Energy. - The alpha, beta, and gamma rays of radium and $\mathrm{X}$-rays all ionize matter through which they pass. Owing to their rapid absorption, alpha rays produce intense ionization in a limited depth of material. The ionization produced by the more penetrating beta rays is less dense and less concentrated. $\mathrm{X}$-rays and gamma rays are so penetrating that little energy is absorbed by matter through which they pass so that they produce relatively little ionization and the ions formed are more widely separated.

The ionization of atoms and molecules, as the result of the absorption of ultra-violet and visible radiation, is called the photoelectric effect. The majority of solids show this effect for wave-lengths shorter than about $3000 \AA$, although the threshold varies from element to element. No liquids, either organic or inorganic, have been found to show a genuine photoelectric effect with radiation longer than $1700 \AA$ except solutions of ferrocyanide salts. Water shows only a very weak photoelectric effect even at these very short wave-lengths. Photoelectric effects from organic liquids are usually due to solid films or colloidal particles on the surface, but apparently nothing definite is known in regard to the photoelectric effect from colloidal solutions such as proteins.

When atoms and molecules absorb energy from wave-lengths too long to produce ionization or decomposition, they may become "activated" and this activated state favors chemical reactions, such as oxidations and reductions, and frequently brings about isomeric changes and polymerization of molecules.

There is also evidence of increased conductivity in some liquids absorbing ultra-violet radiation (15) which may be due to the formation 
of heavy ions such as would result from the breaking up of molecules rather than from the emission of electrons.

\section{EFFECT OF RADIATION ON SUSPENSOIDS}

Before taking up the effect of radiation on proteins it is well to summarize briefly the effect on lyophobe suspensoids, and metallic hydroxides. Crowther and Fairbrother (12) radiated Bredig solutions of iron and copper which are positively charged suspensoids and a Bredig solution of silver and colloidal gold, which are negatively charged, with $\mathrm{X}$-rays from a molybdenum tube. The colloidal copper precipitated on radiation and the iron was more easily precipitated after radiation by potassium chloride. The negatively charged colloidal silver and gold, however, became more dispersed and more stable after radiation. After exposure to X-rays, colloidal gold did not precipitate on boiling. Crowther found that the effect of beta rays on a Bredig solution of copper was exactly the same as that of X-rays. They concluded from these experiments that $\mathrm{X}$-rays and radium rays coagulate positively charged suspensoids, or make them less stable, whereas they disperse negatively charged suspensoids and make them more stable.

Nordenson (41), on the other hand, found that two types of colloidal gold, prepared by different methods, one giving positively charged particles and the other negatively charged particles, showed aggregation under ultra-violet radiation and that there was a similar though less marked effect with beta rays. He concluded that radiation has a slow coagulating effect on metallic colloids like that of weak electrolytes, and that the effect is independent of the charge on the particle. The aggregation was accompanied by a decrease in the charge, whether positive or negative, which he attributed to the absorption of $\mathrm{H}^{+}$or $\mathrm{OH}^{-}$ions formed in the solvent by the radiation.

Fairbrother (16) and Crowther (11) studied the effect of X-rays and the beta rays of radium on ceric hydroxide. This solution shows changes in viscosity on radiation. When exposed in low concentrations the viscosity decreases to a minimum and then increases with final setting to a gel. In sols of higher concentration there is such a rapid gel formation that the initial decrease in viscosity is masked. If the sol is radiated to the point of minimum viscosity, it will then set without further radiation, although very slowly, whereas it sets rapidly with continued radiation. The initial decrease in viscosity is supposed to be due to discharge of the positively charged colloid, and the subsequent rise in viscosity to aggregation of the discharged colloidal particles. In comparing the effect of beta rays and $\mathrm{X}$-rays there was numerical equivalence between the effect of the two types of radiation as far as the discharge of the particles and decrease in viscosity were concerned, but the aggregation and rise in viscosity were more rapid under beta radiation. He found, 
by calculation, that the loss of charge of the colloidal particles is proportional to the energy absorbed in the sol as a whole for both beta rays and X-rays. For equal absorption of the sol as a whole the absorption of $\mathrm{X}$-rays by the colloidal particles, in the ease of both ceric hydroxide sol and colloidal copper, will be greater than for beta rays. The mass coefficient of X-ray absorption for copper and cerium is about 40 times that of water and for beta radiation about twice that of water. Since the effect is the same for the two types of radiation, this indieates that the discharge of the colloid is due to ionization of the solvent, not to ionization of the colloidal particles. The fact that only positively charged suspensoids are coagulated is attributed to the mobility of the electrons formed in the ionization of water. The positive ions formed would be too large to penetrate the skin of solvent molecules surrounding each colloidal particle, and diseharge it, as could be done by the more mobile electrons.

Similar effects are reported by Fernau and Wolfgang (22) for both cerie hydroxide sol and ferric hydroxide sol. A fall in viscosity followed by a rise in viscosity with gel formation followed radiation by beta and gamma rays, or by gamma rays only, though the effect was more marked when beta rays were present. In general, it may be stated, therefore, that $\mathrm{X}$-rays and radium rays will produce aggregation in positively charged suspensoids, probably as a result of the discharge of the charged particles by the electrons freed in the ionization of the solvent (water), and will disperse negatively eharged suspensoids making them more stable. The effect of ultra-violet radiation on suspensoids is less definite. The results reported so far indicate a slow coagulating effect independent of the charge on the colloid which is attributed to the absorption of $\mathrm{H}^{+}$ and $\mathrm{OH}^{-}$ions formed by ultra-violet radiation which is not short enough to liberate electrons.

\section{EFFECT OF RADIATION ON EMULSOIDS}

Proteins are emulsoid colloids and may be charged either positively or negatively as their eharge is due to ionization of the salts formed with acids and bases. They differ in their degree of hydration and the distinction between globulins and albumins is made on the basis of solubility. Globulins are hydrated only when they are charged so that salt-free globulin at the isoelectric point is nonhydrated and precipitates. Albumins are hydrated independently of their charge so that they stay in solution even at the isoelectric point, although at this point they are in a condition of minimum hydration. Globulins therefore are distinguished from albumins by their physical properties and are defined as proteins not soluble in water but soluble in acid, alkali, and dilute neutral salt solutions. This makes it difficult to distinguish between globulins and denatured albumins as a denatured albumin is no longer soluble at the isoelectric point. 


\section{ULTRA-VIOLET RADIATION}

Denaturation.-The effect of radiation on proteins has been reviewed by Spiegel-Adolf $(49,50)$. The most striking and important change produced in all proteins, whether globulins or albumins, whether positively or negatively charged, is a change in solubility or denaturation. Proteins are denatured by the wave-lengths of ultra-violet radiation which they absorb (i.e., $\lambda$ shorter than $3000 \AA$ ). Salt-free albumin exposed to ultra-violet radiation shorter than $3000 \AA$ shows no coagulation except near the isoelectric point, but after radiation it flocculates if brought to the isoelectric point or on dialysis (10). Pseudoglobulin becomes less soluble and precipitates at the isoelectric point like a euglobulin. Euglobulin, after radiation, has a wider zone of flocculation at the isoelectric point. All three types, after radiation, are more easily thrown out of solution by any precipitating agent, such as salts or alcohol. Denaturation, or loss of affinity for water, occurs therefore without exception when proteins are exposed to ultra-violet radiation.

Radiation denaturation has not been studied in the exact quantitative way that heat denaturation was studied by Chick and Martin $(7,8)$. Results so far indicate that radiation denaturation and heat denaturation, though resulting in the same solubility changes (10), are brought about by a different type of physicochemical change. Under certain conditions $(5,10)$ (at $\mathrm{pH}>5.4$ and $0^{\circ} \mathrm{C}$.) albumin after radiation will no longer precipitate on boiling, although it precipitates like a globulin on one-half saturation with ammonium sulphate. Spiegel-Adolf $(50,53)$ states that the increase in absorption after radiation, noted in detail below, does not occur after heat denaturation except for a slight effect at wave-lengths shorter than $2750 \AA$. Viscosity and surface-tension changes have not been compared after heat denaturation and radiation denaturation in many proteins but show differences in the case of egg albumin (10). The reversibility of heat coagulation under certain conditions has been established and it is stated by Spiegel-Adolf $(47,50)$ that light denaturation is not reversible. Heat denaturation without flocculation does not change the osmotic pressure of proteins (30), but no experiments have been carried out on the osmotic pressure of radiated proteins and it is not known if radiation denaturation is a unimolecular reaction like heat denaturation $(7,8)$ or not. Heat denaturation occurs only in the presence of water $(7,8)$, but Stedman (54) states that proteins are denatured when radiated dry, an observation confirmed by unpublished observations of the author on dried, crystalline egg albumin. Further work is necessary before definite conclusions can be drawn, but it seems, from the scattered results so far reported, that the physicochemical changes resulting in heat denaturation and radiation denaturation are different. (See footnote*, page 319.) 
Although a decrease in solubility is the most widely investigated, and probably the most important result of radiation, many other changes have been noted in radiated proteins which may be associated with the physicochemical change somewhat loosely designated as denaturation, or may be independent of it. These changes will be mentioned briefly below.

Odor.-During irradiation proteins acquire a characteristic, rather unpleasant, burnt odor similar to that produced on exposing the skin to an ultra-riolet arc. In the case of egg albumin it is independent of the purity being just as evident in material recrystallized five times as in a crude preparation. Mond $(39,40)$ found purified serum albumin developed less smell than pure egg albumin. When dried crystalline egg albumin is exposed to an ultra-violet arc, there is no odor until the radiated material is dissolved in water.

Color.-All proteins, whether dry or in solution, become more or less yellow under radiation. The color change seems to parallel the production of an odor and the two occur under the same conditions.

Optical Rotation.-Many authors have noted the fact that proteins show definite increases in levorotation after radiation. Both Chalupecky (6) and Young (63) reported this result with egg albumin. Stedman (54) found increases in levorotation in edestin in 10 per cent $\mathrm{NaCl}$ and in gliadin in 70 per cent alcohol (see also Spiegel-Adolf (49)).

$V$ iscosity. - An increase in viscosity is marked after radiation in the case of globulins (49), the change being greater for euglobulins than for pseudoglobulins and greater for pseudoglobulins than for albumins. Young (63) noted a rise in viscosity in radiated egg albumin but Clark (10) failed to find any at $\mathrm{pH}$ 6.0. This has been taken by some authors as evidence of a change in state of aggregation with radiation $(39,40)$.

Surface Tension.--After radiation there is a decrease in surface tension which, like the increase in riscosity, is greatest in the euglobulins and least in the albumins (49), although the surface-tension change is more marked in albumins than the viscosity change (10).

Temperature of Coagulation.-Chick and Martin $(7,8)$ have pointed out that the coagulation temperature is not a fixed property of proteins and depends on a variety of factors but the temperature at which, under similar conditions, a precipitate is first visible is lowered by radiation $(4,44,54)$. That is, after radiation denaturation, the proteins are in general more easily coagulated by heat. There are, however, special cases where, after radiation, proteins no longer coagulate on heating $(5,10,29)$.

Hydrogen Ion Concentration.-A number of investigators have noted that there is a change in acidity after radiation. Mond $(39,40)$ and Young (63) state that acid solutions become less acid and solutions on the alkaline side of the isoelectric point become more acid. Stedman (54) 
found a decrease in the $\mathrm{pH}$ of distilled water after radiation and concluded that the change in hydrogen ion concentration must be due to a change in the solvent and not in the protein molecule and is probably due to ionization of the water. As ultra-violet radiation does not liberate electrons from water, this assumes an increase in electrolytic dissociation.

Absorption.-The characteristic absorption bands of proteins in the ultra-violet region $(34,35,45,56)$ were found by Spiegel-Adolf and associates $(28,47,48,51-53)$ to undergo changes after radiation with ultra-violet light. When enough acid or alkali is added to prevent the visible coagulation which occurs at the isoelectric point on radiation, egg albumin, serum albumin, and serum pseudoglobulin all show increased absorption after radiation, the change being more marked in albumins than in globulins. After denaturation with ultra-violet radiation albumins and pseudoglobulins have an extinction coefficient characteristic of euglobulins in addition to the solubility of euglobulins. SpiegelAdolf notes that similar changes in absorption occur after denaturation by radium but that after heat denaturation there is no increase in absorption except a slight effect in $\lambda<2750 \AA$.

Becker and Szendrö (3) measured the extinction coefficient of egg albumin and ox serum albumin after radiation in $\mathrm{O}_{2}$ and $\mathrm{N}_{2}$. In both cases there was an increase in absorption. When radiated in nitrogen, the extinction coefficient increased with the maximum and minimum still present at $\lambda 2800$ and $\lambda 2500 \AA$ and the absorption limit was changed from $\lambda 3100$ to $\lambda 4000 \AA$. When radiated in oxygen, the increase in absorption was more marked, the maximum and minimum disappeared, and there was absorption starting at $4000 \AA$ and increasing steadily in going to shorter wave-lengths (see Fig. 2). Spiegel-Adolf (48) also noted the fact that proteins show increased absorption after radiation in either nitrogen or oxygen and concluded that light denaturation is not an oxidation. The curves given in Fig. 2, however, show some difference in the changes taking place in oxygen and nitrogen.

It has been suggested that the increased absorption in light-denatured albumin is due to a change in degree of dispersion of the protein, but as increased absorption is also evident in the ultra-filtrate from radiated proteins (especially when radiated in $\mathrm{O}_{2}$ ) it is probably not due to aggregation alone but to some more fundamental change in the protein molecule.

Fluorescence.-Wels $(59,60)$ and Becker and Szendrö (3) have shown that after radiation there is an increase in fluorescence of proteins, the fluorescent radiation having a maximum at $\lambda 3600$ to $3700 \AA$.

Oxygen a Factor.-Harris (25 to 27 ) found that plasma takes up oxygen during radiation, but the fact that changes in solubility and extinction cocfficients have been found to take place when proteins are radiated in nitrogen (48) indicates that denaturation is not due to an oxidation. It 
has been stated that radiated water contains hydrogen peroxide which may produce oxidation even when free oxygen is not present. Kernbaum (31 to 33) found $\mathrm{H}_{2} \mathrm{O}_{2}$ in water radiated with radium, probably as the result of the reaction $2 \mathrm{H}_{2} \mathrm{O}=\mathrm{H}_{2} \mathrm{O}_{2}+\mathrm{H}_{2}$. However, both Kernbaum and Tian $(57,58)$ state that this reaction does not take place in water as the result of ultra-violet radiation unless very short wave-lengths are used (probably shorter than $1700 \AA$ ) and a very long radiation is given. Oxidation as the result of the formation of hydrogen peroxide,

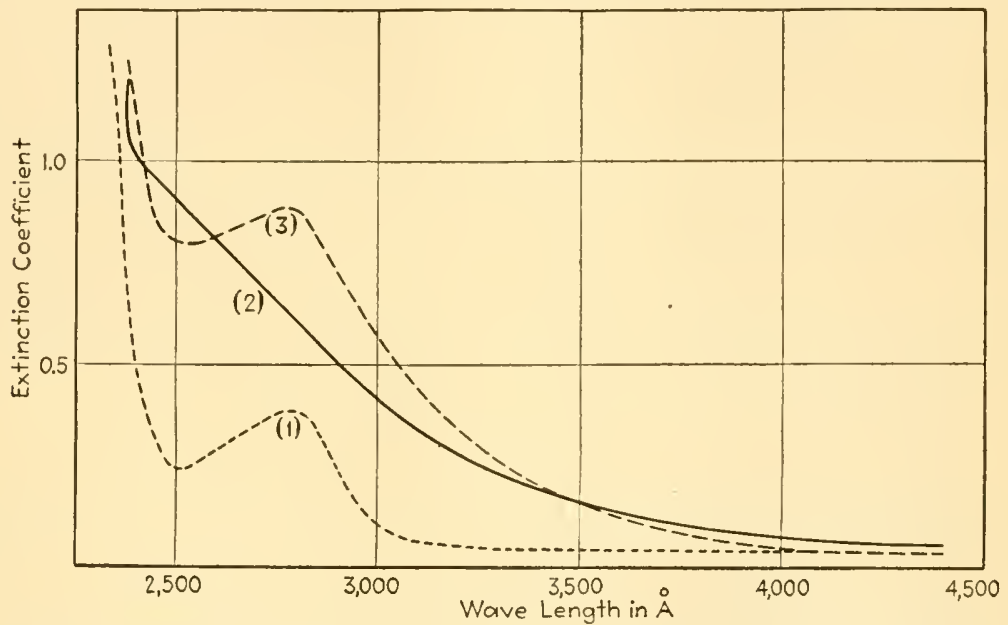

FIG. 2.- Change in absorption curve of egg albumin after ultra-violet radiation: (1) egg albumin unradiated; (2) after radiation in $\mathrm{O}_{2} ;(3)$ after radiation in $\mathrm{N}_{2}$. (After $J . P$. Becker and P. Szendrö, Pflügers Archiv. Gesam. Physiol. 228: 755. 1931.)

therefore, can hardly be a factor in the denaturation of proteins by ultra-violet radiation.

\section{SENSITIZATION TO VISIBLE LIGHT}

Ordinarily proteins are denatured only by radiation shorter than $3000 \AA$. In the presence of certain sensitizers, however, proteins are denatured in visible light in the region of the absorption band of the sensitizer (9). No investigations have been made to determine whether oxygen is necessary for this reaction or not. Eosin, mereurochrome, and a number of other dyes act as sensitizers. The most active sensitizer in vivo is a.derivative of hemin, hematoporphyrin. An animal into which it has been injected, when exposed to visible light, suffers from extensive erythema and death may result, probably from a fall in blood pressure due to extensive capillary dilatation. Howell (29) sensitized fibrinogen to visible light with hematoporphyrin with very eurious and anomalous results. Ultra-violet radiation affects unsensitized fibrinogen like other globulins, making it less soluble. Dreyer and Hansen (14) found a rise in coagulation temperature in radiated fibrinogen and Mond 
$(39,40)$ found a fall. When fibrinogen is sensitized with hematoporphyrin and radiated with visible light, it becomes more soluble and after radiation is not coagulated by thrombin, does not coagulate on heating, and does not flocculate on dialysis. This increased solubility after radiation is unique except for the fact that albumin radiated at $0^{\circ} \mathrm{C}$, at a $\mathrm{pH}>5.4$, no longer coagulates on heating.

With these two exceptions it may be stated in general terms that all proteins (albumins, pseudoglobulins, and euglobulins) are denatured by ultra-violet radiation shorter than $3000 \AA$ at any $\mathrm{pH}$, whether they are radiated in oxygen or nitrogen. After denaturation they floceulate if brought to the isoelectric point or on dialysis and are more readily precipitated by salts, alcohol or heat. This denaturation is accompanied by an increase in ultra-violet absorption, an increase in viscosity, a decrease in surface tension, an increase in levorotation, and by the development of a characteristic odor and a yellow color. The decrease in affinity for water and the increase in absorption are more marked in albumins. The increase in viscosity and decrease in surface tension are more marked in globulins.

\section{THE EFFECT OF X-RAYS AND RADIUM RAYS ON PROTEINS}

Denaturation.-In the first investigation of the effect of radium on proteins Hardy (24) found that globulin is turned to a jelly only when it is negatively charged. This is apparently a direct effect on the negative colloid produced by the positive $\alpha$-particles. The effect of beta and gamma rays is to denature proteins irrespective of their charge. Fernau (17 to 19) found that serum albumin and egg albumin remained clear after seven hours' radiation but showed evidence of denaturation as they were more easily precipitated by alcohol and the heat coagulation temperature was lower. The solutions became opalescent after several days' radiation and flocculated if radiated for still longer periods. Similar results were obtained with pseudoglobulin solutions. In subsequent experiments (21) the process of denaturation was followed by counting the number of particles in a Tyndall cone of known depth by means of an ultramicroscope. Pseudoglobulin from horse serum was exposed to beta and gamma radiation by immersing a glass capsule containing $78 \mathrm{mg}$. of radium in the solution. There was no visible change in six days, but after an exposure of one day there was a marked increase in the number of particles counted under the ultramicroscope.

Wels (59) and Wels and Thiele (62) used the ultramicroscope to follow the denaturation produced in proteins by exposure to X-rays. They radiated serum albumin and serum globulin in buffer solutions on both sides of the isoelectric point. In serum albumin the increase in the number of particles was only marked at the isoelectric point and fell off rapidly at a higher or lower $\mathrm{pH}$. Globulin solutions showed a large 
increase in the number of particles on both sides of the isoelectric point so that the formation of aggregates after radiation is independent of the charge on the particles.

As $\mathrm{X}$-rays and gamma rays are so little absorbed by protein solutions, it requires days of radiation to produce a measurable degree of denaturation, whereas short exposures to ultra-violet radiation will produce a marked effect.

Viscosity, $p H$ and Other Changes.-Fernau and Pauli (20) found that protein solutions were more acid after exposure to radium. Wels (59) and Wels and Thiele (62) state that unbuffered protein solutions, exposed to X-rays, are made more acid on the alkaline side of the isoelectric point and more alkaline on the acid side.

The viscosity of serum albumin is increased only at the isoelectric point after exposure to $\mathrm{X}$-rays. Serum globulin shows an increase on both sides of the isoelectric point, but it is necessary to radiate six hours or more to produce a perceptible change $(38,59,62)$.

The results with $\mathrm{X}$-rays and gamma rays are not so extensive as those reported with ultra-violet radiation, but as far as they go they show that the same effect is produced by all three types of radiation, i.e., they all denature proteins and decrease their solubility. The small absorption of X-rays and gamma rays makes the effects produced by them less pronounced unless a very long radiation is given.

GENERAL CONCLUSIONS

In regard to suspensoids it can be stated that positively charged lyophobe suspensoids precipitate and positively charged lyophile suspensoids set to a gel when radiated by $\mathrm{X}$-rays, or by the beta and gamma rays of radium.

Emulsoid colloids, such as proteins, are denatured by ultra-violet radiation. The denaturation occurs whether the colloid is positively or negatively charged, whether it is radiated in oxygen or nitrogen. As far as present results go, they indicate that similar effects are produced by $\mathrm{X}$-rays and by beta and gamma rays, although only after a prolonged radiation and possibly by an entirely different mechanism. After denaturation, proteins flocculate if brought to the isoelectric point and are more easily precipitated by salts, alcohol, or heat. This decrease in solubility is more marked in albumins as is also the accompanying increase in absorption. Denaturation is accompanied, or followed by, an increase in viscosity and decrease in surface tension which are more marked in globulins.

There is good evidence, in the case of suspensoids, for belicving that the effect of $\mathrm{X}$-rays, beta rays, and gamma rays is primarily due to an ionization of the solvent and that subsequent interaction of electrons with the positively charged suspensoid particles brings about precipi- 
tation or gelation. The effect of $\mathrm{X}$-rays, beta rays, and gamma rays on emulsoids may possibly be due to the same cause.

In the case of emulsoids the fact that they are denatured irrespective of their charge is evidence that an ionization of the colloid is not the cause of denaturation. As water is not ionized except by radiation shorter than $1700 \AA$, denaturation by ultra-violet radiation is probably not due to an ionization of the solvent. Unfortunately the sensitivity curve of proteins to monochromatic ultra-violet radiation has not been investigated. If it were known to be identical with the absorption curve, one could be certain that the change produced by ultra-violet radiation is a change in the protein molecule itself and not a change in the solvent. Heat denaturation has been found to be a unimolecular reaction. If radiation denaturation is also unimolecular, the change is not, primarily, one of molecular aggregation. Unfortunately no investigations have been made on the molecular weight of radiated albumins to see if, after radiation, at a $\mathrm{pH}$ where visible aggregation does not take place, the radiated albumin has the molecular weight as well as the solubility and absorption characteristic of a globulin. The aggregation seen under the ultramicroscope in globulins and the flocculation at the isoelectric point in radiated albumins is not the primary change produced by radiation but a secondary change taking place between the denatured molecules.

\section{PRIMARY AND SECONDARY LIGHT REACTIONS}

In a primary light reaction a substance $A$ is changed to form $B$ on the absorption of radiation and if $B$ is stable, the amount formed is proportional to the energy absorbed and $i \times t$ (intensity $\times$ time) is a constant. Usually $B$ is unstable. $B$ may revert to $A$ again with production of fluorescence. Or $B$ may react with other substances present (i.e., $B+C \rightarrow D)$. In that case the amount of $D$ formed is not proportional to the amount of energy absorbed and $i t^{p}=$ const. In general this is the type of reaction resulting from the absorption of radiation. The amount of $B$ formed may be proportional to the radiant energy absorbed and independent of the temperature, but the final product measured $(D)$ is not proportional to the radiant energy absorbed and is not independent of temperature, and the period of time necessary for the reaction of $B$ with $C$ is responsible for the latent period which is such a well-known characteristic of light reactions.

The precipitation of negatively charged colloids by alpha rays may possibly be a primary light reaction, though measurements to substantiate this are lacking.

The precipitation or gelation of positively charged suspensoids by beta rays, gamma rays, and X-rays is a secondary light reaction as the primary effect is apparently an ionization of the solvent. 
The denaturation of proteins by radiation may be a primary reaction per se. No experiments have been carried out as yet to settle this point. Gentner and Schwerin (23), by counting particles in a pseudoglobulin solution under the ultramicroscope, found that, as radiation time $X$ intensity increased, the rate of precipitation increased somewhat and the latent period decreased a great deal (see Fig. 3,a). They found also that if two equal values of radiation $X$ intensity are compared, the greater intensity $X$ the shorter time resulted in a greater rate of precipitation and a shorter latent period (see Fig. 3,b). Therefore $i t^{p}=$ const.
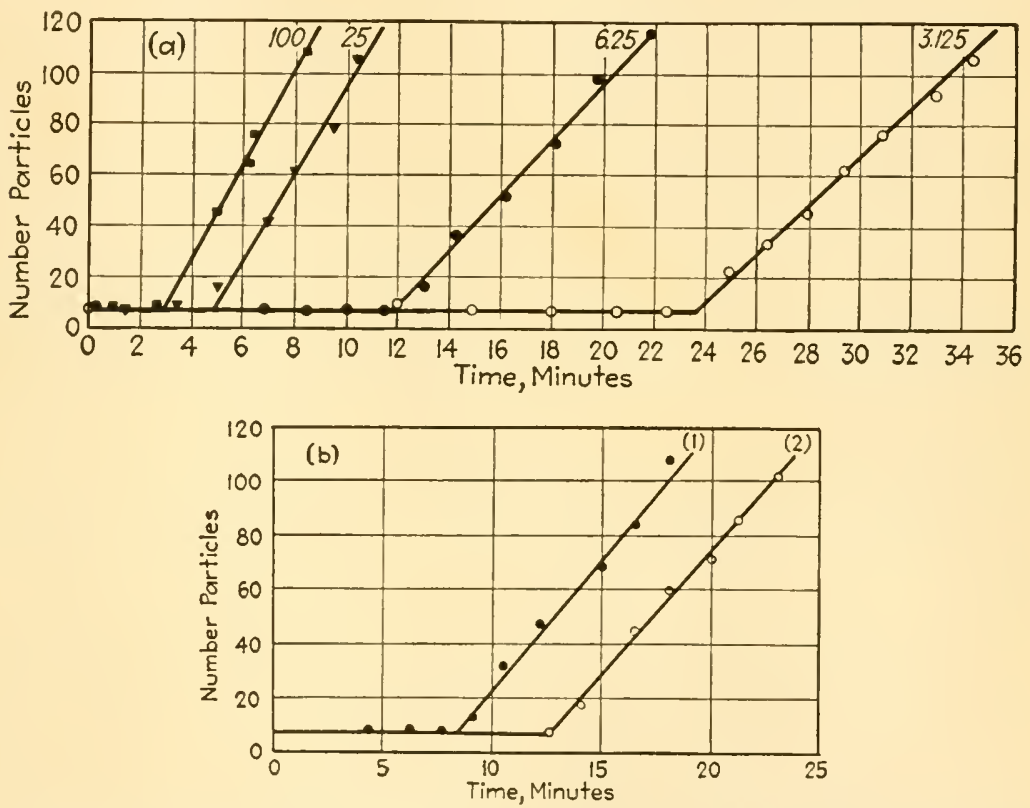

Fig. 3.-Number of particles visible in the field of the ultramicroscope a given time after radiation. $a$. (Upper diagram): Number at head of each curve $=$ product $i t$ (radiation intensity $\times$ radiation time). $b$. (Lower diagram): The product it $=$ same but in curve 1 = large intensity for a short time and in curve $2=$ small intensity for a long time. (After Gentner and Sehwerin Strahlenth. 37: 788.1930. )

( $p$ being less than 1 for the effect of ultra-violet light on protein solutions). Observations of this type, however, measure the rate of aggregation of the denatured protein particles, not the rate of denaturation. The aggregation is certainly a secondary light reaction, but the denaturation itself, which precedes aggregation, may be a primary light reaction. Experiments to investigate this point are highly desirable. (See footnote*, page 319 .)

The nature of the physicochemical change taking place in protein molecules, which results in denaturation, has not been solved. The evidence so far indicates no change in molecular size, the aggregation being probably a secondary phenomenon. It has been suggested (42) 
that the loss in solubility after radiation is due to an internal tautomeric change, possibly a rearrangement of peptide linkages into closed-ring systems, which would diminish the affinity for water, but one must confess that we are as yet ignorant of the nature of the change we speak of as "denaturation" and the mechanism by means of which it is brought about.

\section{RELATION OF PROTEIN DENATURATION TO GENERAL PHYSIOLOGICAL LIGHT REACTIONS}

The best known result of radiation is the lethal action produced on the living cell. This is probably due to a combination of several causes, one of which is the denaturation and coagulation of the cell proteins. Changes in the colloids in living cells are reversible and an irreversible change, such as that produced by radiation denaturation, would bring about the death of the cell. The sensitivity curve of microorganisms to ultra-violet radiation has been determined $(43,46)$ and the similarity of the curve to the absorption curve of proteins is good evidence that the absorption of radiation by cell proteins produces irreversible changes resulting in death. As proteins are denatured at any $\mathrm{pH}$, the primary radiation change always takes place. This may be followed by gel formation or coagulation if the protein is at its isoelectric point or if salts are present in sufficient quantity. Photographs of microorganisms killed by ultra-violet radiation show that coagulation has actually taken place.

In the eye media the cornea and lens are subject to protein denaturation in the presence of ultra-violet radiation. The lens protein is not at its isoelectric point so that it docs not coagulate after radiation unless there is an abnormal salt content, but it is very possible that denaturation of the lens protein, even without subsequent flocculation, may affect the normal nutrition of the lens.

The skin cells are killed by radiation (ultra-violet, X-rays or radium) and on disintegration liberate a substance which produces capillary dilatation and there are probably extensive denaturation and coagulation of tissue proteins leading to changes in the permeability of cells and a shift in the water balance.

The rapidly effective ultra-violet rays are so strongly absorbed by the skin that little, if any, radiation reaches the blood stream, but X-rays and gamma rays penetrate all the tissues and the changes they produce are consequently widespread. Isolated serum proteins are, rapidly denatured by ultra-violet radiation and slowly denatured by X-rays and gamma rays. When radiated as they occur in combination in the serum, however, they show no flocculation and serum is known to exercise a protective action in many radiation effects. Red cells hemolyze when radiated in isotonic salt solutions but not when radiated in serum. 
However, the serum proteins in the blood of an animal exposed to penetrating radiation may be denatured to some extent and, although no experimental evidence has been given on this point, it is probable that denaturation would result in a larger globulin-albumin ratio and this in turn would result in a change in the amount of bound water.

The fact that after radiation the water-binding capacity of proteins is much diminished would lead to physiological changes throughout the animal body and the effect of radiation may be profound in higher animals as well as in microorganisms. Fortunately the rapidly effective ultra-violet rays do not penetrate deeply enough to produce widespread injury.

\section{REFERENCES}

1. Anson, M. L., and A. E. Minsky. The preparation of completely coagulated hemoglobin. Jour. Gen. Physiol. 13: 121-132. 1929.

2. Anson, M. L., and A. E. Minsky. The preparation of insoluble globin, soluble globin and heme. Jour. Gen. Physiol. 13: 469-476. 1929.

3. Becker, J. P., and P. Szendrö. Spektralanalytische und chemische Untersuchungen über den Abbau von Eiweiss dureh ultraviolette Strahlen. Pflüger's Arch. Ges. Physiol. 228: 755-763. 1931.

4. Borie, W. T. The temperature coefficient of the coagulation caused by ultraviolet light. Science 37:373-375. 1913.

5. Bovie, W. T., and O. C. Woolpert. On the mechanism of the light action on solutions of albumen. Science 60: 70. 1924.

6. Chalupeck Ý, H. Der Einfluss der ultravioletten Strahlung auf die Augenlinse. Wien. Med. Wochensch. 63: 1986-1991. 1913.

7. Chick, Harriette, and C. J. Martis. On the "heat coagulation" of proteins. Jour. Physiol. 40: 401-430. 1910.

* Since this review was written some work carried out by the author, which is now in press, has thrown light on a number of the questions raised in this review. Salt-free isoeleetric egg albumin was given ultra-violet radiation and the amount of material subsequently preeipitated was determined quantitatively by the Tyndall beam from the opalescent solutions. Coagulation as the result of radiation was found to take place in three steps. If salt-free isoeleetric egg albumin is radiated at $4^{\circ} \mathrm{C}$. the initial change, the light-denaturation of the protein molecule, oceurs without the secondary changes which result in coagulation. This primary process was found to be unimolecular with a temperature coefficient of 1 . It must therefore be a physieal change resulting in a new configuration of the molecule with different physical and chemical properties. The second step in the coagulation process is a reaction between the light-denatured molecule and water which has the high temperature coefficient of 10 . The second step may be similar to heat denaturation of the protein molecule but occurs at a lower temperature. Heat denaturation does not proeeed at an appreeiable rate below $50^{\circ} \mathrm{C}$. whereas the heat ehange following light-denaturation takes place at an appreciable rate at $10^{\circ} \mathrm{C}$. and proceeds rapidly at $40^{\circ} \mathrm{C}$. The final step is the floceulation of the light- and heat-denatured moleeules. The change taking place in salt-free proteins as a result of radiation, therefore, which is ealled light-denaturation, is not sufficient in itself to produce coagulation. It is followed by floceulation if the radiated proteins are heated to a moderate temperature for a short time. 
8. Chick, Harriette, and C. J. Martin. On the "heat coagulation" of proteins. Pt. II. The action of hot water upon egg-albumin and the influence of acid and salts upon reaction velocity. Jour. Physiol. 43:1-27. 1911.

9. Clark, J. H. The reaction of ultraviolet light on egg albumin in relation to the isoelectric point. Amer. Jour. Physiol. 61: 72-79. 1922.

10. Clark, J. H. Studies on radiated proteins. I. Coagulation of egg albumin by ultraviolet light and heat. Amer. Jour. Physiol. 73: 649-660. 1925.

11. Crowther, J. A. The action of ionizing radiations on colloids. Philos. Mag. and Jour. Sci. Ser. 7, $7: 86,98.1929$.

12. Crowther, J. A., and J. A. V. Fairbrother. The action of X-rays on colloids. Philos. Mag. and Jour. Sci. Ser. 7, 4: 325-335. 1927.

13. Dне́ré, C. Recherches spectrographiques sur l'absorption des rayons ultraviolets par les albuminoides, les proteids et leurs dérivés. Thèse. Fribourg (Suisse), 1909.

14. Dreyer, G., and O. Hansen. Sur la coagulation des albumines par l'action de la lumière ultraviolette et du radium. Compt. Rend. Acad. Sci. [Paris] 146: 234 236. 1907.

15. Eller, W. H. Photo-conductivity in dielectric liquids. Jour. Opt. Soc. 20: 71-80. 1930.

16. Fairbrother, J. A. V. The action of X-rays on colloidal ceric hydroxide. Philos. Mag. and Jour. Sci. Ser. 7, 6: 385-401. 1928.

17. Fernau, A. Über die Wirkung der durchdringenden Radiumstrahlen auf Serumund Eialbumin. Biochem. Zeitsch. 167: 380-383. 1926.

18. Fernad, A. Über die Wirkung der durchdringenden Radiumstrahlung auf Pseudoglobulin. Biochem. Zeitsch. 189: 172-174. 1927.

19. Fernad, A., and W. Pauli. Über die Einwirkung der durchdringenden Radiumstrahlung auf anorganische und Biokolloide. I. Biochem. Zeitsch. 70: 426-441. 1915.

20. Fernau, A., and W. Pauli. Über die Einwirkung der durchdringenden Radiumstrahlung auf anorganische und Biokolloide, III. Kol. Zeitsch. 30:6-13. 1922.

21. Fernau, A., and M. Spiegel-Adolf. Ultramikroskopische Untersuchung der Wirkung durchdringender Radiumstrahlung auf Pseudoglobulin. Klin. Wochensch. $6: 1798-1800.1927$.

22. Fernau, A., and P. Wolfgang. Über die Einwirkung der durchdringenden Radiumstrahlung auf Kolloide, II. Kol. Zeitsch. 20: 20-33. 1917.

23. Gentner, W., and K. Schwerin. Über den Zeitfaktor der Strahlungsreaktion des Eiweisses. Strahlentherapie 37 : 788-794. 1930.

24. Hardy, W. B. Colloidal solution. The globulin system. Jour. Physiol. (Proc. Physiolog. Soc.) 29: 26-29. 1903.

25. Harris, D. T. The action of light on blood. Biochem. Jour. 20: 271-279. 1926.

26. Harris, D. T. Photo-oxidation of plasma. Biochem. Jour. 20: 280-287. 1926.

27. Harris, D. T. Observations on the velocity of the photo-oxidation of proteins and amino-acids. Biochem. Jour. 20: 288-292. 1926.

28. Hausmann, W., and Mona Spiegel-Adolf. Über Lichtschutz durch vorbestrahlte Eiweisslösungen. Klin. Wochensch. 6 : 2182-2184. 1927.

29. Howell, W. H. Note relative à l'action photodynamique de l'hématoporphyrine sur le fibrinogène. Arch. Internat. Physiol. 18: 269-276. 1921.

30. Huang, T. C., and H. WU. Studies on denaturation of proteins X. Osmotic pressure of denatured egg albumin and methemoglobin in concentrated urea solutions. Chinese Jour. Physiol. 4: 221-230. 1930.

31. Kernbaum, M. Action chimique sur l'eau des rayons pénétrants du radium. Compt. Rend. Acad. Sci. [Paris] 148: 705-706. 1909. 
32. Kernbaum, M. Action chimique sur l'eau des rayons pénétrants du radium. Compt. Rend. Acad. Sci. [Paris] 149: 116-117. 1909.

33. Kernbaum, M. Décomposition de l'eau par les rayons ultraviolets. Compt. Rend. Acad. Sci. [Paris] 149 : 273-275. 1909.

34. Lewis, S. J. The ultra-violet absorption spectra of blood sera. Proc. Roy. Soc. B $89: 327-335.1917$.

35. Lewis, S. J. The ultra-violet absorption spectra and the optical rotation of the proteins of blood sera. Proc. Roy. Soc. B 93: 178-194. 1922.

36. LEWIs, W. C. M. The crystallization, denaturation, and flocculation of proteins with special reference to albumin and hemoglobin, together with an appendix on the physicochemical behavior of glycine. Chem. Rev. 8:81-165. 1931.

37. Mirskr, A. E., and M.L. Ansos. The reversal of the coagulation of hemoglobin. Jour. Gen. Physiol. 13: 133-143. 1929.

38. Mirsky, A. E., and M. L. Anson. Improved methods for the reversal of the coagulation of hemoglobin. Jour. Gen. Physiol. 13 : 477-481. 1929.

39. Mond, R. Untersuchungen über die Wirkung der ultravioletten Strahlen auf Eiweisslösungen. I. Mitteilung. Pflüger's Arch. Ges. Physiol. 196: 540-559. 1922.

40. Moxd, R. Untersuchungen über die Wirkung der ultravioletten Strahlen auf Eiweisslösungen. II. Mitteilung. Pflüger's Arch. Ges. Physiol. 200: 374-378. 1923.

41. Nordenson, H. Die Bedeutung des Lichtes für die Stabilität kolloider Lösungen . Zeitsch. Physik. Chem. 90: 603-627. 1915.

42. Rimingtos, C. Protein structure and denaturation. Nature 127: 440-441. 1931.

43. Rivers, T. M., and F. L. Gates. Ultra-violet light and vaccine virus. The effect of monochromatic ultra-violet light upon vaccine virus. Jour. Exp. Med. $47: 45-49.1928$.

44. Schanz, F. Die Wirkungen des Lichtes auf die lebende Substanz. Pflüger's Arch. Ges. Physiol. 161: 384-396. 1915.

45. Sмiтh, F. C. The ultra-violet absorption spectra of certain aromatic aminoacids, and of the serum proteins. Proc. Roy. Soc. B 104: 198-205. 1929.

46. Sovve, C. Die Abhängigkeit der lichtbiologischen Reaktionen von der Wellenlänge. Strahlentherapie 23 : 45-51. 1928.

47. Spiegel-Adolf, Mona. Physikalisch-chemische Untersuchungen bestrahlter Proteine. Die Veränderungen des Seralbumins bei Ultraviolettbestrahlung und ihre Beziehungen zur Hitzegerinnung. Biochem. Zeitsch. 186: 181-193. 1927.

48. Spiegel-Adolf, Mona. Physikalisch-chemische Untersuchungen bestrahlter Proteine. Der Einfluss kurzwelliger Bestrahlung auf das Absorptionsvermögen des Serums und der Serumeiweisskörper im Ultraviolett. Biochem. Zeitsch. 197 : 197-209. 1928.

49. Spiegel-Adolf, Mona. Die Globuline. Steinkopff; Leipzig, 1930.

50. Spiegel-Adolf, MoNa. Effects of ultraviolet rays, radium and X-rays on proteins. Arch. Path. 12 : 533-542. 1931.

51. Spifgel-Adolf, Moxa. Neuere Ergebnisse über Eiweissveränderungen durch ultraviolette, Radium- und Röntgenstrahlen. Ergeb. Physiol. 27: 832-863. 1928.

52. Spiegel-Adolf, Moxa, and O. Krumpel. Physikalisch-chemische Untersuchungen bestrahlter Proteine. II. Absorption des lichtdenaturierten Seralbumins im Ultraviolett. Biochem. Zeitsch. 190: 28-41. 1927.

53. Spiegel-Adolf, Mona, and Z. Oshima. Physikalisch-chemische Untersuchungen an bestrahlten Proteinen. VI. Mitteilung. Weitere Studien zum spektroskopischen und biologischen Nachweis von Lichtveränderungen an Proteinen. Biochem. Zeitsch. 208: 32-44. 1929. 
54. Stedman, H. L., and L. B. Mendel. The effeets of radiation from a quartz mercury vapor arc upon some properties of proteins. Amer. Jour. Physiol. 77 : 199-210. 1926.

55. Svedberg, T., and J. B. Nichols. The molecular weight of egg albumin I. In electrolyte-free condition. Jour. Amer. Chem. Soc. 48: 3081-3092. 1926.

56. Svedrerg, T., and B. Sjögren. The molecular weights of serum albumin and of serum globulin. Jour. Amer. Chem. Soc. 50: 3318-3332. 1928.

57. Tian, A. Sur la nature de la décomposition de l'eau oxygénée produite par la lumière. Compt. Rend. Acad. Sci. [Paris] 151: 1040-1042. 1910.

58. Tian, A. Sur la décomposition de l'eau par la lumière ultraviolette. Compt. Rend. Acad. Sci. [Paris] 162: 1012-1014. 1911. [See p. 1196, same vol., for errata.]

59. Wels, P. Der Einfluss der Röntgenstrahlen auf Eiweisskörper. I. Mitteilung. Pflüger's Arch. Ges. Physiol. 199: 226-236. 1923.

60. Wels, P. Der Einfluss kurzwelliger Strahlen auf die Fluorescenz von Eiweisskörpern und ihren Spaltprodukten. Pflüger's Arch. Ges. Physiol. 219 : 738-752. 1928.

61. Wels, P. Der Einfluss der Quarzlampenstrahlung auf die Fluorescenz von Geweben und Zellen. Pflüger's Arch. Ges. Physiol. 223 : 369-377. 1929.

62. Wels, P., and O. Thiele. Der Einfluss der Röntgenstrahlen auf Eiweisskörper. II. Mitteilung. Pflüger's Arch. Ges. Physiol. 209: 49-64. 1925.

63. Younf, E. G. The coagulation of protein by sunlight. Proc. Roy. Soc. B 93 : 236-248. 1922. 


\title{
IX
}

\section{RADIATION AND THE VITAMINS}

\author{
Charles E. Bills
}

\section{Research Laboratory, Mead Johnson and Company, Evansville, Indiana}

The discovery of activation. Physical chemistry of activation. The individual products of irradiation. Origin of vitamin $D$ in nature. References.

It is well to preface a paper on radiant energy and the vitamins with the remark that the discussion will necessarily center on vitamin D. The formation of the other vitamins does not involve radiant energy except in the indirect relation that green plants require it for their growth, and that vitamins are elaborated in abundance by plants. There is no good evidence that any vitamin except certain forms of vitamin D is directly a photochemical product, and it is by no means certain that all forms of vitamin D require radiant energy for their formation.

Vitamin A probably never occurs in the vegetable kingdom. Moore (60) demonstrated that animals synthesize it from carotene. It is true that carotene, or provitamin $\mathrm{A}$, is a common pigment of green plants, yet it can also be elaborated by microorganisms in culture (Baumann, Steenbock, Ingraham, and Fred, 5). The B vitamins are plentiful in green plants, but even more so in yeasts which grow in the dark. Vitamin $\mathrm{C}$ is developed not only in green plants, but in germinating seeds and in the bodies of certain animals.

It seems that the effect of radiant energy on the vitamins is mainly a destructive one. The investigations on this subject are assembled in the excellent monographs by Browning (18), the Medical Research Council (58), and Laurens (52). Since all vitamins are comparatively - unstable substances, they are more or less subject to destruction by radiations, particularly by ultra-violet rays and the ozone frequently associated therewith. Inasmuch as most experimental work has been conducted with crude edible products in which the vitamins comprised only a trace of the total, very little indeed can be said regarding the stability of the vitamins per se toward radiant energy. It is, however, a point of practical significance that in the momentary exposure to ultra-violet rays which is required to endow foodstuffs such as milk with vitamin $D$, no appreciable destruction of the other vitamins is evident.

The researches of the past decade which culminated in the activation of ergosterol were, in a sense, a bringing together of old observations on the therapeutic value of fish oils and of light. In brief, they have 
shown that ultra-violet rays, acting on the sterol-bearing fats of the skin, produce a form of vitamin $\mathrm{D}$ which is similar in antirachitic action to the vitamin $\mathrm{D}$ contained in fish oils. The vitamin $\mathrm{D}$ produced by the irradiation of pure ergosterol has recently been obtained in crystalline form.

The following résumé of studies on the relation of radiant energy to vitamin $\mathrm{D}$ consists mainly of excerpts from a larger survey (9). Several thousand references to papers covering experimental rickets, and the chemistry and physiology of vitamin $\mathrm{D}$, are given in the following specialized reviews: Park (62), McCollum and Simmonds (55), Hess (30), MacLeod (56), Blunt and Cowan (16), Goldblatt (26), Browning (18), Medical Research Council (58), Laurens (52), and Weidlich (87).

\section{THE DISCOVERY OF ACTIVATION}

Sunshine, like fish oil, is an old remedy for rickets. Its importance, however, was not fully appreciated until Huldschinsky $(44,45)$, by means of radiographs, clearly demonstrated the healing action of sunlight and of the light from the quartz mercury arc. Hess, Pappenheimer, and Weinstock (31) determined that the effective wave-lengths of light are the shorter ultra-violet waves of the solar spectrum, or the still shorter waves of artificial sources. Goldblatt and Soames (27) discovered that the livers of irradiated rats, when fed to nonirradiated rats, convey some of the virtue of irradiation to the animals which eat them.

In 1924 Hess and Steenbock independently and almost simultaneously announced the discovery that exposure of edible materials to ultra-violet light endows them with antirachitic activity. Hess found that inert oils, namely, cottonseed and linseed, acquired this property of cod liver oil. Steenbock found the same to be true of muscle tissue, mixed feed, and fats. (Cf. refs. 29, 79.)

A succession of publications by Steenbock and by Hess rapidly expanded the knowledge of activation. The original brief communications were published in extenso by Steenbock and Black (81), Steenbock and Nelson (83), and Hess and Weinstock (32). These papers, together with several supplementary contributions and the Steenbock (80) patent of 1928 , revealed that edible materials in great variety are activatable, and that activation depends upon the same short wave-lengths which are effective directly in the cure of rickets. Furthermore, it appeared that activation is relatively permanent and that it is not a phenomenon of oxidation. The active moiety of irradiated linsced oil was traced to the unsaponifiable fraction, just as in the case of cod liver oil itself.

Shortly a second stage in the knowledge of activation was reached when it was demonstrated that sterols become antirachitic upon irradiation. Hess, Weinstock, and Helman (35) succeeded in activating phytosterol and cholesterol, and to a lesser extent, lanolin. Steenbock and Black (82) found that cholesterol which had been specially "purified" by 
esterification became definitely antirachitic upon irradiation, but that phytosterols from old vegetable oils aequired only slight, if any, activity. Rosenheim and Webster (67) activated cholesterol.

Thus it became evident that in foodstuffs the sterol fraction contains the acceptor of the activating rays. The course of investigation now turned to the elucidation of the chemical changes induced by irradiation in the sterols.

Hess and Weinstock $(33,34)$ introduced the use of the quartz spectrograph for investigating the chemistry of activation. They found that ordinary cholesterol was somewhat opaque to ultra-violet light, and that irradiation decreased its opacity. On the other hand, dihydrocholesterol and dihydrositosterol, which were not activatable, were practically transparent, and the transmission of ultra-violet light was not altered by irradiation. Unfortunately, a mercury are was used as the light source for the spectrograms. Since this gives a discontinuous spectrum, nothing could be learned of the spectral structure in the region of absorption.

Schlutz and Morse (75), with better technique, determined that the absorption spectrum of ordinary cholesterol is banded. Their densitometer tracings showed two maxima of absorption at approximately 2940 and $2830 \AA$. A trained observer, biased, perhaps, by later knowledge, can see in these original tracings two additional faint inflections. In retrospect it thus appears that Schlutz and Morse were the first to record what is now recognized as the provitamin absorption spectrum. They noticed that after brief irradiation the inflections gave way to general absorption, and, considering Beer's law, they postulated that either the cholesterol had been at least half metamorphosed, or else "the substance in which the absorption spectrum is changed may be a small amount of impurity in the cholesterol which is not removed by repeated crystallizations from alcohol, and which is exceedingly absorptive."

Such are the coincidences of research that, on December 10, 1926, reports from three separate laboratories confirmed the contamination hypothesis. Rosenheim and Webster (69) found that cholesterol which had been regenerated from cholesterol dibromide was so pure that it no longer showed the characteristic absorption spectrum, and was no longer activatable. They had previously noted (68) that ergosterol is activatable, but having made no quantitative determination of the degree of activatability, they did not now recognize "ergosterol" as the contaminant removed by bromination.

Heilbron, Kamm, and Morton (28) reported that fractional crystallization of cholesterol led to the accumulation, in the least soluble fraction, of the substance responsible for the characteristic absorption spectrum. They recognized a third absorption maximum, $2690 \AA$. Irradiation destroyed the three bands, leaving only general absorption. 
Pohl (63), by the technique of photoelectric photometry, also detected three absorption bands in cholesterol, which faded upon irradiation. With the knowledge that complete disappearance of the bands corresponded to the destruction of only a trivial fraction of the cholesterol, he concluded that the absorbing substance was present only in minute amount. He found that cholesterol purified via the dibromide, or via allocholesterol, and a sitosterol purified via the dibromide, did not show the usual absorption.

These simultaneously announced studies left no doubt that an impurity present in ordinary cholesterol and phytosterol is responsible for at least the greater part of the antirachitic activity conferred upon these sterols by irradiation.

After an interchange of suggestions between the investigators, the impurity, or provitamin, was presently identified as ergosterol. Pohl (64) found that the three known absorption bands of ordinary cholesterol are also exhibited by ergosterol. Moreover, ergosterol showed them in vastly greater intensity than cholesterol. Chemical studies and a biological assay were ammounced by Windaus and Hess (96). In keeping with expectations, the antirachitic potency of irradiated ergosterol was found to be far greater than that of irradiated cholesterol. At the same time, Rosenheim and Webster $(70,72)$ reported essentially the same findings, from which they concluded that the precursor of vitamin $D$ is ergosterol, or a sterol of similar constitution.

Bills, Honeywell, and MacNair (14) confirmed these remarkable discoveries in a way which seemed to leave no reasonable doubt that ergosterol is the contaminant provitamin. By the use of a hydrogen discharge tube, which emits an exceptionally continuous ultra-violet spectrum, they discovered that ordinary cholesterol exhibits a fourth absorption band, $\lambda 2600 \AA$. Exactly the same band was shown by ergosterol, making the series $\lambda \lambda 2935,2820,2700$, and $2600 \AA$, with four points of identity instead of three. An indication that the contaminant was ergosterol, and not some sterol with the same absorption bands, was obtained by comparing the rates of destruction. Acetone solutions of ordinary cholesterol, and of ergosterol-free cholesterol plus added ergosterol, were boiled with potassium permanganate. The absorption bands, identical in intensity at the outset, faded at essentially the same rates.

Despite the convincing evidence that ergosterol is the provitamin in cholesterol, Waddell (84) has recently published experiments which indicate that such is not the case. He found that irradiated cholesterol was many times more effective on chickens, rat unit for rat unit, than ergosterol which had been irradiated either by itself or in the presence of cholesterol. Waddell's experiments contain no obvious flaw, and if confirmed, will necessitate revision of the provitamin concept. 


\section{PHYSICAL CHEMISTRY OF ACTIVATION}

Morton, Heilbron, and Kamm (61) reported that the disappearance of ergosterol irradiated in dilute solution bore a linear relation to the time. Unpublished data from this laboratory show, however, that concentrated solutions do not exhibit the linear relationship. It is probably true, and certainly logieal to expeet, that under no conditions is the time-disappearance curve actually a straight line, for the products of irradiation are numerous and some of them are sufficiently absorptive to aet as filters against the remaining unchanged ergosterol.

The wave-lengths which affect ergosterol are, of eourse, those which it absorbs. Ergosterol absorbs strongly from 3050 to $2300 \AA$ in the far ultra-violet region (Cox and Bills, 21). But it exhibits a little absorptive power beyond these limits in both directions. Wave-lengths as long as $3130 \AA$ are known to activate it slightly, and there is reason to believe that still longer waves play a part in the decomposition of ergosterol and/or of its primary irradiation produets (Lahousse and Gonnard, 50). The predominance of these longer waves may explain the relatively low effieieney of sunlight in activation. In the experiments of Windaus, Borgeaud, and Brunken (92), Windaus and Borgeaud (91), and Windaus and Brunken (93), white light in the presence of optical sensitizers resulted in definite chemieal changes in ergosterol, but not in the formation of vitamin D. Cathode rays (Knudson and C. N. Moore, 48) and radium emanation (R. B. Moore and DeVries, 59) have a moderately effective activating action on ergosterol. X-rays do not activate (Goldblatt, 26). The few published reports to the contrary are not supported with acceptable bio-assays. Radio waves of high intensity and short wave-length are without action (unpublished investigation).

The early work of Pohl (63) suggested that activation may involve the addition of energy to the sterol molecule through an electron displacement. Measurement of the energy relations was attempted by Kon, Daniels, and Steenbock (49), who ventured a definite computation in ergs. In a similar undertaking, Marshall and Knudson (57) recognized that the measurement of the energy required for activation is complicated by the oceurrence of secondary reactions. Bills, MeDonald, BeMiller, Steel, and Nussmeier (15) considered that if the energy of aetivation remains resident in the activated molecule, a determination of the heat of combustion should reveal it. Actually they found no difference in this respect between plain ergosterol and a rery potent irradiation produet eontaining over 20,000 international units of vitamin D per $\mathrm{mg}$. It is interesting to observe that provitamin $\mathrm{D}$, which is as important to animals as chlorophyll is to plants in the utilization of light, does not play even a small role in the storage of energy.

The researehes of Kon, Daniels, and Steenbock (49), Webster and Bourdillon (85), and Marshall and Knudson (57) seemed to show that 
vitamin $\mathrm{D}$ is formed with equal efficiency by any wave-lengths within the region of the principal absorption of ergosterol. Strictly with reference to the formation of vitamin, and apart from side reactions and decompositions, such a conclusion may be correct. It is known, however, that the irradiation of ergosterol produces several substances in succession and/or in parallel. This fact was established by the early investigations of Rosenheim and Webster (71), Webster and Bourdillon (85), Smakula (78), Bills, Honeywell, and Cox (12), Fabre and Simonnet (25), Webster and Bourdillon (86), Delaplace and Rebière (22), Bourdillon, Fischmann, Jenkins, and Webster (17), Windaus, Westphal, v. Werder, and Rygh (102), Lahousse and Gonnard (50), Reerink and van Wijk (65), and others. It has been confirmed in many later investigations. Inasmuch as the several products of irradiation have absorption spectra different from ergosterol, they are themselves susceptible to different wavelengths. The net result is that the sequence of products obtained by irradiation varies with the wave-lengths of light employed (Reerink and van Wijk, 65; Windaus, 89; Askew, Bourdillon, Bruce, Jenkins, and Webster, 4).

The temperature coefficient of activation is small. Bills and Brickwedde (10) found that cholesterol containing 1.2 parts of "ergosterol" per 1000 parts was readily activated at $-183^{\circ} \mathrm{C}$, although the product was somewhat less potent than the product of similar irradiation at room temperature. Webster and Bourdillon (85) irradiated ergosterol at temperatures between $-195^{\circ}$ and $+78^{\circ} \mathrm{C}$. and again observed the smallness of the temperature coefficient. Since bimolecular reactions have a large temperature coefficient and are generally inhibited at very low temperatures, the conclusion from these experiments was that the change involved is monomolecular. All subsequent experience, including the analysis of crystalline preparations of vitamin $\mathrm{D}$, has borne out the view that activation consists in an isomerization of the ergosterol molecule.

The composition of the irradiation product is influenced by the purity and nature of the solvent in which the ergosterol is dissolved. Ergosterol in the dry state takes activation poorly, presumably because the first irradiation products which form on the exposed surfaces act as filters to prevent light from reaching the under layers until the surface products are destroyed. This principle may explain the observation of Windaus, Westphal, v. Werder, and Rygh (102) that agitation of the solution during exposure enhances the formation of vitamin $D$. To the extent that the solvent itself is opaque to short waves, it necessarily exerts a a filtering action on the activating rays.

The presence of dissolved oxygen in the solvent markedly affects the spectral picture of activation (Smakula, 78; Bills, Honeywell, and Cox, 12 ; Reerink and van Wijk, 65). It does so by altering the by-products of activation, rather than by affecting to any large extent the forma- 
tion or destruction of vitamin D (Bills, Honeywell, and Cox, 12). The by-products which form by oxidation make difficult the crystallization of the vitamin (Angus, Askew, Bourdillon, Bruce, Callow, Fischmann, Philpot, and Webster, 1). In the experiments of Beard, Burk, Thompson, and Goldblatt (6) extreme freedom from oxygen in a dry activation did not increase the antirachitic potency of the product. As is usual in the absence of a solvent, the potency attained was only moderate, amounting in this case to roughly one-tenth of the value expected when activation is performed in ether. The investigations of Windaus (89), Angus, Askew, Bourdillon, Bruce, Callow, Fischmann, Philpot, and Webster (1) and Bills, McDonald, BeMiller, Steel, and Nussmeier (15) show that vitamin $\mathrm{D}$, either in crystalline form or as a resin, is decidedly more stable to oxidation than some of the nonvitamin substances which are formed with it in irradiation. Its stability is about the same as that of ergosterol itself.

Apart from any light-filtering action of solvents, and from any role which they may play as carriers of dissolved oxygen, there appears to be what Bills, Honeywell, and Cox (12) termed a specific solvent effect on activation. This was revealed by parallel spectrographic and biological examinations on the course of activation in alcohol, cyclohexane, and ether. The importance of the specific solvent effect was shown by the fact that the time required for the attainment of maximum potency in ether was longer than the time required for the entire sequence of activation and destruction in alcohol. The maximum potency reached in ether was higher than in alcohol or in cyclohexane, but the spectral changes were most conspicuous in alcohol.

\section{THE INDIVIDUAL PRODUCTS OF IRRADIATION}

Attempts to characterize the individual products of irradiation and to ascertain the number of them by interpretation of absorption spectra have been numerous but not very profitable. The reason is easily understood, for with mixtures of substances having overlapping absorption bands, determinations of the position and height of the bands are unreliable. Precision methods of bio-assay and chemical methods of isolation have been the principal means of progress during the later stages of the study.

A general survey of the irradiation products, with spectral absorption curves, was given by Windaus, Lüttringhaus, and Busse (99). Setz (77) has brought the genetic relationships of the products up to date, and confirmed the view that the changes involved are not reversible. It appears from his work that when ergosterol is irradiated for not too long a period, the product produced by wave-lengths below $2800 \AA$ is chiefly tachysterol and vitamin $\mathrm{D}$ (calciferol), whereas the product of longer wave-lengths is chiefly lumisterol and calcif erol. 
The genetic series is probably best summarized in the following diagram. This is based mainly on the papers by Windaus and his colleagues, particularly Setz. There is some evidence that the scheme is not complete. Between lumisterol and tachysterol a protachysterol may occur. The reviewer has assumed that substance 248 precedes the suprasterols, because the absorption spectrum of overirradiated substance 248 (Bills, Honeywell, and Cox, 12) is essentially that of the suprasterols studied by the German workers. The name, calciferol, is retained for that form of vitamin $\mathrm{D}$ which has been isolated. It is not impossible that at least one other form of the vitamin occurs in crude irradiation product.

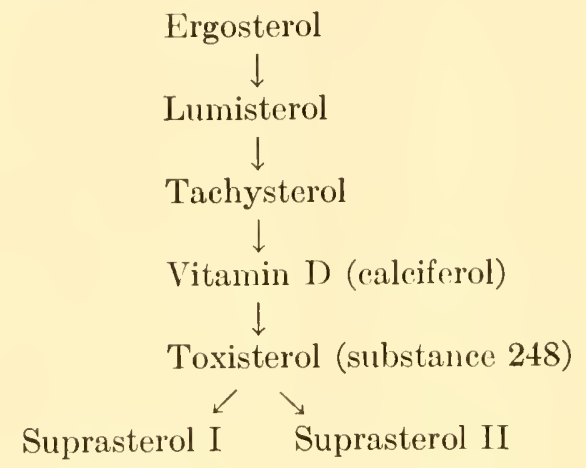

1. Ergosterol. $-\mathrm{C}_{28} \mathrm{H}_{43} \mathrm{OH} ;\left.\right|_{3} ^{=} ;$m.p. varies with hydration; $\lambda \lambda 2600$, $2700,2820,2935 \AA ;[\alpha]_{5461}^{20}=-174^{\circ} ;[\alpha]_{\mathrm{D}}^{20}=-135^{\circ}$ in chloroform.

Ergosterol, the parent substance of the series of irradiation products, has been prepared in high purity by Bills and Honeywell (11) and Callow (19). It forms colorless crystals which tenaciously retain water of erystallization. Commercial specimens of otherwise good quality contain about 5 per cent of $\alpha$-dihydroergosterol (Callow, 20).

2. Lumisterol. $-\mathrm{C}_{28} \mathrm{H}_{43} \mathrm{OH} ;\left.\right|_{3} ^{=} ;$m.p. $118^{\circ} ; \lambda \lambda 2650,2800 \AA ;[\alpha]_{546}^{19}=$ $+235.4^{\circ} ;[\alpha]_{\mathrm{D}}^{19}=+191.5^{\circ}$ in acetone.

Crude irradiation product, after removal of unchanged ergosterol by treatment with methyl alcohol or digitonin, is a nearly colorless resin. In the separation of vitamin D from this resin, a substance was recovered which was called sterol $\mathrm{X}$ by the English workers and given the name Lumisterin by the Germans. It was studied in considerable detail by Windaus, Dithmar, and Fernholz (94), who obtained it by the fractional crystallization of the acetate of the lumisterol-calciferol addition compound. The absorption spectrum of lumisterol is similar to that of ergosterol in range and intensity, but it shows only two maxima, instead of four. Lumisterol appears to be the initial product of the irradiation of ergosterol. It probably is devoid of antirachitic action, but it is converted by irradiation into vitamin D. It forms with calciferol a 
definite addition compound in the ratio of $1: 1$, this being the vitamin $\mathrm{D}_{1}$ of the German workers.

3. Tachysterol. $-\mathrm{C}_{28} \mathrm{H}_{43} \mathrm{OH} ;$ m.p. indeterminate; principal absorption band at $2800 \AA$, lesser bands at 2680 and $2940 \AA ;[\alpha]_{5461}=-86.3^{\circ}$; $[\alpha]_{\mathrm{D}}^{18}=-70^{\circ}$ in "Normalbenzin."

Windaus, Lüttringhaus, and Busse (99) removed from the crude resin another sterol which they called Tachysterin. This was the object of a special study by Windaus, v. Werder, and Lüttringhaus (101), who prepared it from the addition compound, tachysteryl acetate citraconic anhydride, via tachysteryl 3.5-dinitro-4-methyl benzoate. These products form good crystals, although tachysterol itself does not crystallize. The absorption spectrum of tachysterol is characterized by its great intensity at $2800 \AA$. Tachysterol is a source of trouble in the separation of irradiation products. Besides failing to crystallize, it exhibits an exceptional affinity for oxygen, so great that perbenzoic acid titrations to determine its degree of unsaturation have been uninformative. Tachysterol follows lumisterol and precedes calciferol in the sequence of irradiation products. It probably is devoid of antirachitic action, but it may have a slight toxic effect.

4. Vitamin D. Calciferol. $-\mathrm{C}_{28} \mathrm{H}_{43} \mathrm{OH} ;\left.\right|_{4} ^{=} ;$m.p. 115 to $117^{\circ} ; \lambda 2650 \AA$; $[\alpha]_{5461}^{20}=+122^{\circ} ;[\alpha]_{\mathrm{D}}^{20}=+102.5^{\circ}$ in alcohol.

By means of an ingenious method of fractional sublimation Askew, Bourdillon, Bruce, Jenkins, and Webster (3) were able to obtain small amounts of potent crystals from the crude resin. The method was improved, and a detailed study of the active crystals was reported by Angus, Askew, Bourdillon, Bruce, Callow, Fischmann, Philpot, and Webster (1). The name, calciferol, was given to the crystalline substance. A few months later Askew, Bourdillon, Bruce, Callow, Philpot, and Webster (2) discovered that the original calciferol was a mixture, separable via the 3.5-dinitrobenzoate into pure calciferol and two inactive sterols. One of the latter, pyrocalciferol, was merely a thermal transformation product, produced during the distillation. The other, "sterol $\mathrm{X}$," had probably come over from the original irradiation product. By the new esterification method calciferol could be obtained directly from the resin, without distillation. The antirachitic potency of pure calciferol was found to be 40,000 international units per milligram.

While the isolation of calciferol was in progress in England, Windaus and his associates in Germany were developing a different technique to the same end. Windaus (90) and Windaus, Lüttringhaus, and Deppe (100) reviewed their preliminary studies on activation, and announced that a crystalline vitamin $\mathrm{D}$ was obtained by removing the inactive components of the resin by means of maleic or eitraconic anhydride. Soon after this, Linsert (54) obtained another crystalline preparation somewhat different in its properties from the first. For a few 
weeks Windaus and Lüttringhaus (98) regarded these two preparations as distinct forms of vitamin $\mathrm{D}$ and called them vitamin $\mathrm{D}_{1}$ and vitamin $\mathrm{D}_{2}$, respectively. Presently, however, Windaus, Linsert, Lüttringhaus, and Weidlich (97) discovered that the Linsert preparation was essentially identical with Bourdillon's pure calciferol, while the first German preparation was an addition compound of calciferol with an inactive sterol.

5. Toxisterol. Substance 248.-Morton, Heilbron, and Kamm (61), extending the work of Pohl (64), showed that when ergosterol is irradiated, the three bands with maxima at 2935,2820 , and $2700 \AA$ disappear, while a single new band of great intensity develops at 2470 or $2480 \AA$, and in turn fades away to weak general absorption in the far ultra-violet region. With inadequate biological evidence, the assumption was easily made that the new band represented vitamin D. Bills, Honeywell, and Cox (12), introducing precision methods of assay, showed that the appearance of the band at $2480 \AA$ coincided not with the development, but with the destruction, of antirachitic potency. They, and also van Wijk and Reerink (88), associated substance 248 with isoergosterol. Cox and Bills (21) contributed further evidence of relationship to the isoergosterols, but noted a point of difference, namely, that substance 248 does not precipitate with digitonin.

Substance 248 has not yet been isolated. Bills, Honeywell, and Cox (12) found that irradiation products withdrawn for examination at the moment when substance 248 was at its maximum concentration, still contained some vitamin D. Further irradiation totally eliminated antirachitic activity, while only a small amount of substance 248 was destroyed. Laquer and Linsert (51) attributed a toxic quality to substance 248 , and proposed the name, Toxisterin (toxisterol). The product which they investigated still contained some vitamin $\mathrm{D}$, but not enough to account for the relatively great toxicity. It was evidently a mixture similar to that which Hoyle (42) obtained by limited overirradiation, and which he reported to have toxic-caleifying properties all out of proportion to the antirachitic potency. Such a mixture, unfortunately, seems to have been the "vitamin D" of the I.G. Farbenindustrie Aktiengesellschaft patent of 1928 (the old Vigantol of toxic repute). There is considerable evidence, both spectrographic and toxicologic, that toxisterol is formed most readily when alcohol is the solvent in which the ergosterol is irradiated (Bills, Honeywell, and Cox, 12, 13; van Wijk and Reerink, 88; Dixon and Hoyle, 23; Hoyle and Buckland, 43; Hoyle, 42; Kern, Montgomery, and Still, 47.

6. Suprasterol I. $-\mathrm{C}_{28} \mathrm{H}_{43} \mathrm{OH} ;\left.\right|_{3} ;$ m.p. $104^{\circ}$; general absorption below $2500 \AA ;[\alpha]_{\mathrm{D}}^{18}=-76^{\circ}$ in chloroform.

7. Suprasterol II. $-\mathrm{C}_{28} \mathrm{H}_{43} \mathrm{OH}$; $\left.\right|_{3} ^{=}$; m.p. $110^{\circ}$; general absorption below $2500 \AA ;[\alpha]_{\mathrm{D}}^{19}=+62.9^{\circ}$ in chloroform. 
The products of extreme overirradiation, presumably the end products of the series, were designated Suprasterine by Windaus, Gaede, Köser, and Stein (95). They recognized two isomers, suprasterol I and suprasterol II, which they separated by taking advantage of differences in the solubility of the allophanic acid esters. The suprasterols are not antirachitic and are only slightly toxic.

In the review by Bills (9) a mass of evidence was summarized which goes to show that ergosterol, lumisterol, and tachysterol are not the only sterols which become antirachitic upon irradiation. They are, however, the supremely important ones, and the only ones which develop enormous antirachitic potency. It was also brought out that several forms of vitamin $\mathrm{D}$ exist, which differ from each other in physiological action. Thus, for example, the vitamin D as ordinarily prepared from ergosterol is far more effective for rats than for chickens, while the reverse is true of the vitamin $\mathrm{D}$ contained in fish oils.

\section{ORIGIN OF VITAMIN D IN NATURE}

Vitamin D rarely, if ever, occurs in living plants, and the reason is not difficult to understand. The lower, nonpigmented, nonphotosynthesizing plants, which contain much ergosterol, thrive in dark places and perish in the light. The higher plants, containing relatively little ergosterol, possess pigments which presumably filter out the activating rays at the short end of the solar spectrum. Even if the vitamin could be formed in the superficial layers, plants could not store it, "unprovided as they are with any system for the translocation of lipoids." Dead plant tissue, by insolation, may acquire slight potency, as in the case of hay cured in the sun, coconut oil rendered by solar rays, or mushrooms struck by sunlight. Lack of color is no indication of the activatability of plant materials, for even such tissues as white iris petals are known to contain filters against ultra-violet rays.

In the animal kingdom vitamin $\mathrm{D}$ is widespread, but abundant only in the fishes. Its origin in fishes is obscure. Steenbock and Black (82) suggested that it is formed by the insolation of plankton which in turn is ingested by little fish, and these by larger fish, etc. Against this hypothesis is the observation by Leigh-Clare (53) that the marine diatom, Nitzschia closterium, which synthesizes "vitamin A," fails to effect any genesis of vitamin $\mathrm{D}$, even when grown under conditions of maximum insolation. Drummond and Gunther (24) obtained essentially negative findings with both phytoplankton and zooplankton collected from the ocean. Belloc, Fabre, and Simonnet (7) found that zooplankton collected in spring contained ergosterol but no vitamin D, while zooplankton taken in midsummer showed ergosterol with perhaps vitamin D. Their evidence, although not convincing, may have some significance in view of the July change in vertical distribution of zooplankton (Russell, 74). 
It would seem that if fish obtain vitamin D from plankton, they must salvage it with extraordinary economy.

Irradiation of the body surface appears to play no part in the formation of vitamin $\mathrm{D}$ in fish. Bills (8) observed that catfish are very sensitive to ultra-violet irradiation, yet do not elaborate more vitamin $\mathrm{D}$ as a result of it. Schmidt-Nielsen and Schmidt-Nielsen (76) found that the basking shark, Cetorhinus maximus, has comparatively little vitamin D in its liver oil, in spite of the fact that it basks for hours at the surface of the water, and feeds on plankton. Piscicultural experiments by Bills (8) made it appear probable that at least a portion of the vitamin D of fish originates by synthesis within the fish.

The higher animals lack the power to synthesize vitamin $D$, so their requirements must be met by ingesting it, or particularly by exposing the body surface to sunlight. All animal fats contain sterols, those of the skin being particularly rich in provitamin. Hess and Weinstock (33) demonstrated that irradiated skin is antirachitic, and that irradiated sterol exerts its action when administered subcutaneously. Rekling (66) found that irradiation did not protect rats from rickets when they were prevented from licking their fur. Hou and Tso (41) found that the skin of normal rabbits was slightly antirachitic, the dorsal skin more so than the ventral, but that the skin of rickety rabbits or of normal rabbits reared indoors was without protective action. Hou (39) noted that the effectiveness of ultra-violet irradiation for curing rickets in rabbits was almost lost when the skin had been previously washed with ether. Rowan (73) observed that birds of prey on a meat diet developed rickets, and that the addition of feathers to the diet supplied protection. He suggested that the preen gland is concerned with the formation of vitamin D. Hou $(36,37,38,40)$ made an elaborate study of the formation of vitamin D in birds. In brief, his findings were as follows: Birds differ from mammals in having only one gland of a sebaceous nature. This is the glandula uropygialis, or preen gland. Preen gland oil contains "ergosterol," which birds, by preening, distribute over their feathers and effectively expose to sunlight. The vitamin $\mathrm{D}$ is either ingested by swallowing the feathers, or absorbed by the skin from the feathers. The feathers and skin of normal birds were shown to be antirachitic, but in rickety birds, or birds whose preen glands had been removed, the feathers and skin had little, if any, antirachitic action. Removal of the preen gland made the birds susceptible to rickets, and rickety birds without the gland were not benefited by exposure to ultra-violet radiation or sunshine.

Thus Hou (37) was led to see that " . . . vitamin D or its precursor is principally derived from the oil secretion rather than from the diet. This may be more or less true for all birds. For although nocturnal birds, and the carnivorous animals which prey upon other forms of 
'feather and fur,' may derive their vitamin D supply mainly from their victims, yet the source of the vitamin in the prey would appear to lie in the oil secretion. This may explain the absence of oil gland in some species of birds . . . and the necessity of adding rabbits or small birds with the fur or feather intact, to the diet of young carnivora in captivity to ensure their successful development. . . Further it is commonly known that if herbivora, e.g., horses, are scrubbed thoroughly with soap and water, they do not thrive. It may thus be inferred that the sebaceous secretion, which is the homologue of the secretion of the preen gland of birds . . . , is an important source of vitamin D in the mamnial."

Hon (40) found that rickety chickens with preen glands removed could be cured by irradiating the feet, even though irradiation of the body or of the head was ineffective. In this case the substance activated was obviously neither preen gland oil nor circulating blood, but something in the tissues of the feet which was directly absorbed.

Evidence enough has been presented to show that the higher animals obtain vitamin $\mathrm{D}$ in at least three ways, the relative importance of which must vary with habits, requirements, and opportunities: (a) By eating such foods as eggs, fish, whole furred or feathered animals, and insolated dead vegetable tissues; $(b)$ by ingesting insolated sebaceous matter in the process of neatening the body-licking and preening; and (c) by directly absorbing the products of insolation formed on or in the skin. It is to be noted that in all of these sources, except possibly fish, the ultimate origin of the vitamin is traceable to sterols activated by light.

\section{REFERENCES}

1. Angus, T. C., F. A. Askew, R. B. Bourdillon, H. M. Bruce, R. K. Callow, C. Fischmann, J. St. I. Philpot, and T. A. Webster. A crystalline antirachitic substance. Proc. Roy. Soc. London B 108:340-359. 1931.

2. Askew, F. A., R. B. Bourdillon, H. M. Bruce, R. K. Callow, J. St. L. Philpot, and T. A. Webster. Crystalline vitamin D. Proc. Roy. Soc. London B 109: 488-506. 1932.

3. Askew, F. A., R. B. Bourdillon, H. M. Bruce, R. G. C. Jenkins, and T. A. Webster. The distillation of vitamin D. Proc. Roy. Soc. London B 107: 76-90. 1930.

4. Askew, F. A., R. B. Bourdillon, H. M. Bruce, R. G. C. Jenkins, and T. A. WEBster. The effects of further irradiation of the radiation products of ergosterol. Proc. Roy. Soc. London B 107: 91-100. 1930.

5. Baumanx, C. A., H. Steenbock, M. A. Ingrailam, and E. B. Fred. Fatsoluble vitamins. XXXVIII. Mieroorganisms and the synthesis of carotene and vitamin A. Jour. Biol. Chem. 103:339-351. 1933.

6. Beard, H. H., R. E. Burk, H. E. Thompson, and H. Goldblatt. The antirachitic activation of ergosterol in the absence of oxygen. Jour. Biol. Chem. 96: 307-312. 1932 .

7. Belloc, G., R. Fabre, and H. Simonnet. Contribution à l'étude de l'activité biologique des stérols. Étude des stérols de Plankton. Compt. Rend. 191: 160-162. 1930. 
8. Bulls, C. E. Antiricketic substances. VI. The distribution of vitamin D, with some notes on its possible origin. Jour. Biol. Chem. 72: 751-758. 1927.

9. Bills, C. E. Physiology of the sterols, including vitamin D. Physiol. Rev. 15: 1-97. 1935.

10. Bills, C. E., and F. G. Brickwedde. The activation of cholesterol at liquid oxygen temperature. Nature 121: 452. 1928.

11. Bills, C. E., and E. M. Honeywell. Antiricketic substances. VIII. Studies on highly purified ergosterol and its esters. Jour. Biol. Chem. 80:15-23. 1928.

12. Bills, C. E., E. M. Honeywell, and W. M. Cox, Jr. Antiricketic substances. IX. Quantitative biophysical studies on the activation of ergosterol. Jour. Biol. Chem. 80 : 557-563. 1928.

13. Bills, C. E., E. M. Honeywell, and W. M. Cox, JR. Influence of solvents on the activation of ergosterol. Jour. Biol. Chem. 92:601-604. 1931.

14. Bills, C. E., E. M. Honeywell, and W. A. MacNair. Antiricketic substances. VII. Biochemical and spectrographic studies on purified cholesterol. Jour. Biol. Chem. $76: 251-261.1928$.

15. Bills, C. E., F. G. McDonald, L. N. BeMiller, G. E. Steel, and M. NussMEIER. Heat of combustion of activated ergosterol. Jour. Biol. Chem. 93: 775-785. 1931.

16. Blunt, K., and R. Cowan. Ultraviolet light and vitamin D in nutrition. 229 pp. Univ. of Chicago Press; Chicago, 1930.

17. Bourdillon, R. B., C. Fischmann, R. G. C. Jenkins, and T. A. Webster. The absorption spectrum of vitamin D. Proc. Roy. Soc. London B 104: 561583. 1929 .

18. Browning, E. The vitamins. 575 pp. Baillière, Tindall and Cox; London, 1931.

19. Callow, R. K. The purification of ergosterol. Biochem. Jour. 25: 79-86. 1931.

20. Callow, R. K. Occurrence of $\alpha$-dihydroergosterol as an impurity in yeast ergosterol. Biochem. Jour. 25: 87-94. 1931.

21. Cox, W. M., Jr., and C. E. Bills. Antiricketic substances. X. On the relation of the isoergosterols to vitamin D. Jour. Biol. Chem. 88: 709-713. 1930.

22. Delaplace, R., and G. Rebière. Irradiation de l'ergostérol: action des rayons ultraviolets du quartz et des rayons X mous. Compt. Rend. 188: 1169-1172. 1929.

23. Dixon, W. E., and J. C. Hoyle. The effects of irradiated ergosterol in large doses. British Med. Jour. 2 : 832-835. 1928.

24. Drummond, J. C., and E. R. Gunther. Vitamin content of marine plankton. Nature 126: 398.1930.

25. Fabre, R., and H. Simonnet. Ftude comparative de la valeur de l'essai biologique et de l'essai physique de l'ergostérol irradié. Compt. Rend. 188: 424-426. 1929.

26. Goldblatt, H. Die neuere Richtung der experimentellen Rachitisforschung. Ergeb. Allgem. Path. und Path. Anat. 2. Abt. 25 : 58-491. 1931.

27. Goldblatt, H., and K. M. Soames. Studies on the fat-soluble growth-promoting factor. I. Storage. II. Synthesis. Biochem. Jour. 17: 446-453. 1923.

28. Heilbron, I. M., E. D. Kamm, and R. A. Morton. The absorption spectra of cholesterol and its possible biological significance with reference to vitamin D. I. Preliminary observations. Jour. Soc. Chem. Ind. 45: 932. 1926. (Published in extenso, 1927. The absorption spectrum of cholesterol and its biological significance with reference to vitamin D. I. Preliminary observations. Biochem. Jour. 21: 78-85. 1927.) 
29. Hess, A. F. Experiments on the action of light in relation to rickets. Trans. Amer. Ped. Soc. 36: 57-60. 1924. (Reprinted, Amer. Jour. Dis. Children 28 : 517-520. 1924.)

30. Hess, A. F. Rickets osteomalacia and tetany. $485 \mathrm{pp}$. Lea and Febiger; Philadelphia, 1929.

31. Hess, A. F., A. M. Pappenheimer, and M. Weinstock. A study of light waves in relation to their protective action in rickets. Proc. Soc. Exp. Biol. Med. 20 : 14-16. 1922.

32. Hess, A. F., and M. Weinstock. Antirachitic properties imparted to inert fluids and to green vegetables by ultra-violet irradiation. Jour. Biol. Chem. 62 : 301-313. 1924.

33. Hess, A. F., and M. Wernstock. The antirachitic value of irradiated cholesterol and phytosterol. II. Further evidence of change in biological activity. Jour. Biol. Chem. 64:181-191. 1925.

34. Hess, A. F., and M. Wernstock. The antirachitic value of irradiated cholesterol and phytosterol. III. Evidence of chemical change as shown by absorption spectra. Jour. Biol. Chem. 64: 193-201. 1925.

35. Hess, A. F., M. Wernstock, and F. D. Helman. The antirachitic value of irradiated phytosterol and cholesterol. I. Jour. Biol. Chem. 63: 305-308. 1925.

36. Hou, H. C. Studies on the glandula uropygialis of birds. Chinese Jour. Physiol. 2 : 345-380. 1928.

37. Hou, H. C. Relation of the preen gland (glandula uropygialis) of birds to rickets. Chinese Jour. Physiol. 3: 171-182. 1929.

38. $\mathrm{Hov}, \mathrm{H}$. C. Further observations on the relation of the preen gland of birds to rickets. Chinese Jour. Physiol. 4: 79-92. 1930.

39. Hov, H. C. The role of the skin in the cure of rickets by irradiation. II. The site of activation of the provitamin. Chinese Jour. Physiol. 4: 345-358. 1930.

40. Hou, H. C. Relation of preen gland of birds to rickets. III. Site of activation during irradiation. Chinese Jour. Physiol. 5 : 11-18. 1931.

41. Hov, H. C., and E. Tso. The role of the skin in the cure of rickets by irradiation. Chinese Jour. Physiol. 4: 93-116. 1930.

42. Hoyle, J. C. Further observations on the toxic effects of irradiated ergosterol. Jour. Pharmacol. 40: 351-372. 1930.

43. Hoyle, J. C., and H. Buckland. Further observations on the effects of large doses of irradiated ergosterol. Biochem. Jour. 23: 558-565. 1929.

44. Huldschissky, K. Heilung von Rachitis durch künstliche Höhensonne. Deutsche Med. Wochensch. 45: 712-713. 1919.

45. Huldschinsky, K. Die Behandlung der Rachitis durch Ultraviolettbestrahlung. Zeitsch. Orthop. Chir. 39: 426-451. 1920. (Case reports.)

46. I. G. Farbenindustrie Aktiengesellschaft. Improvements in the photochemical production of vitamin D from ergosterol. British Patent 296,093 Nov. 15, 1928. $3 \mathrm{pp}$.

47. Kern, R., M. F. Montgonery, and E. U. Still. The effect of large doses of irradiated ergosterol upon nitrogen, calcium, and phosphorus metabolism in rats. Jour. Biol. Chem. 93: 365-380. 1931.

48. Knudson, A., and C. N. Moone. Comparison of the antirachitic potency of ergosterol irradiated by uItra-violet light and by exposure to cathode rays. Jour. Biol. Chem. 81: 49-64. 1929.

49. Kon, S. K., F. Daniels, and H. Stennbock. The quantitative study of the photochemical activation of sterols in the cure of rickets. II. Jour. Amer. Chem. Soc. 50: 2573-2581. 1928.

50. Lahousse and Gonnard. Sur le spectre d'absorption de la vitamine antirachitique. Jour. Phys. et Rad. Ser. 6, 10:114S-116S. 1929. 
51. Laquer, F., and O. Linsert. Die biologische Wirkung des Toxisterins. Klin. Wochenseh. 12 : 753-754. 1933.

52. Laurens, H. The physiological effects of radiant energy. 610 pp. Chemical Catalog Company; New York, 1933.

53. Leigh-Clare, J. L. A search for vitamin D in the diatom Nitzschia closterium (W. Sm.) Biochem. Jour. 21: 368-372. 1927.

54. Linsert, O. [Cited in footnote by Windaus, Lüttringhaus, and Deppe, 1931 (cf. entry 100)].

55. McCollum, E. V., and N. Simmonds. The newer knowledge of nutrition. 4th ed., 594 pp. Macmillan; New York, 1929.

56. MaCLeOD, F. J. The chemistry of vitamin D. Jour. Nutrition 2: 517-530. 1930.

57. Marshall, A. L., and A. Knudson. The formation of vitamin D by monochromatic light. Jour. Amer. Chem. Soc. 52 : 2304-2314. 1930.

58. Merlical Research Council. Special Report Series, No. 167. Vitamins: a survey of present knowledge. 332 pp. His Majesty's Stationery Office; London, 1932.

59. Moore, R. B., and T. DeVries. The activation of ergosterol with radium emanation. Jour. Amer. Chem. Soc. 53: 2676-2681. 1931.

60. Moore, T. The relation of carotin to vitamin A. Lancet 1929, $2: 380-381$.

61. Morton, R. A., I. M. Heilbron, and E. D. Kama. The absorption spectrum of ergosterol in relation to the photosynthetic formation of vitamin D. Jour. Chem. Soc. (1927): 2000-2005. 1927.

62. PARK, E. A. The etiology of rickets. Physiol. Rev. 3: 106-163. 1923.

63. Pонl, R. Über das Absorptionsspektrum des antirachitisch wirksamen Cholesterins. Nachr. Ges. Wissensch. Göttingen, Math.-physik. Kllasse (1926), 142-145. 1927.

64. Pонц, R. Über das Absorptionsspektrum des antirachitischen Provitamins und Vitamins. Nachr. Ges. Wissensch. Göttingen, Math.-physik. Klasse (1926), 185-191. 1927.

65. Reerink, E. H., and A. van WiJk. The vitamin D problem. I. The photochemical reactions of ergosterol. Biochem. Jour. 23 : 1294-1307. 1929.

66. Rekling, E. Untersuchungen über die Wirkungsart des Lichtes bei experimenteller Rattenrachitis. Strahlentherapie 25: 568-579. 1927.

67. Rosenheim, O., and T. A. Webster. Rickets and cholesterol. Lancet 1925, 1: $1025-1026$.

68. Rosenheim, O., and T. A. Webster. The anti-rachitic properties of irradiated sterols. Biochem. Jour. 20: 537-544. 1926.

69. Rosenheim, O., and T. A. Webster. Further observations on the photochemical formation of vitamin D. Jour. Soc. Chem. Ind. 45: 932. 1926. (Published in extenso, 1927-The relation of cholesterol to vitamin D. Biochem. Jour. 21 : $127-129$.)

70. Rosenileim, O., and T. A. Webster. On the nature of the parent substance of vitamin D. Lancet 1927, 1: 306-307.

71. Rosenheim, O., and T. A. Webster. The photochemical production of vitamin D from ergosterol. Lancet $1927,2: 622-625$.

72. Rosenileim, O., and T. A. Webster. The parent substance of vitamin D. Biochem. Jour. 21: 389-397. 1927.

73. Rowan, W. Bird feathers and the antirachitic vitamin D. Nature 121: 323-324. 1928.

74. Russell, F. S. Vitamin content of marine plankton. Nature 126: 472.1930.

75. Schldtz, F. W., and M. Morse. Some spectroseopic observations on cod liver oil. Amer. Jour. Dis. Children 30: 199-209. 1925. 
76. Schmidt-Nielsen, S., and S. Schmidt-Nielsex. Der Gehalt der Knorpelfische an antirachitischem Vitamin. Zeitsch. Physiol. Chem. 189:229-238. 1930.

77. Setz, P. Photochemische Unwandlungen der Bestrahlungsprodukte des Ergosterins. Zeitsch. Physiol. Chem. 215 : 183-192. 1933.

78. Smakula, A. Zur Kenntnis des Absorptionsspektrums von Ergosterin in bestrahltem und umbestrahltem Zustande. Nachr. Ges. Wissensch. Göttingen, Math.-physik. Klasse (1928): 49-64. 1928.

79. Stensbock, H. The induction of growth promoting and calcifying properties in a ration by exposure to light. Science $60: 224-225.1924$

80. Sтеклвоск, H. Antirachitic product and process. U. S. Patent $1,680,818$. 5 pp. Aug. 14, 1928.

81. Stennbock, H., and A. Black. Fat-soluble vitamins. XVII. The induction of growth-promoting and calcifying properties in a ration by exposure to ultraviolet light. Jour. Biol. Chem. 61: 405-422. 1924.

82. Steenbock, H., and A. Black. Fat-soluble vitamins. XXIII. The induction of growth-promoting and calcifying properties in fats and their unsaponifiable constituents by exposure to light. Jour. Biol. Chem. 64 : 263-298. 1925.

83. Steenbock, H., and M. T. Nelson. Fat-soluble vitamins. XIX. The induction of calcifying properties in a rickets-producing ration by radiant energy. Jour. Biol. Chem. 62: 209-216. 1924.

84. WADDELL, J. The provitamin D of cholesterol. I. The antirachitic efficacy of irradiated cholesterol. Jour. Biol. Chem, 105: 711-739. 1934.

85. Webster, T. A., and R. B. Bourdillon. Notes on the irradiation of ergosterol. Biochem. Jour. 22 : 1223-1230. 1928.

86. Webster, T. A., and R. B. Bourdillox. The absorption spectrum of vitamin D. Nature $123: 244.1929$.

87. Weidlich, G. Die Chemie des Vitamins D. Zeitsch. Vitaminforsch. 2 : 253266. 1933.

88. van WiJk, A., and E. H. ReErink. Vitamin-D and iso-ergosterol. Nature $122: 648.1928$.

89. Windaus, A. Einige weitere Erfahrungen über das bestrahlte Ergosterin. Nachr. Ges. Wissensch. Göttingen, Math.-physik. Klasse (1930): 36-57. 1930.

90. Windaus, A. The chemistry of irradiated ergosterol. Proc. Roy. Soc. London B $108: 568-575.1931$.

91. Windaus, A., and P. Bongeaud. Über die photochemische Dehydrierung des Ergosterins. Ann. Chem. (Liebig's) 460: 235-239. 1928.

92. Windaus, A., P. Borgeadd, and J. Brdnkes. Die photochemische Oxydation und Dehydrierung des Ergosterins. Nachr. Ges. Wissensch. Göttingen, Math.physik. Klasse (1927): 313-314. 1928.

93. Windaus, A., and J. Brunken. Utber die photochemische Oxydation des Ergosterins. Ann. Chem. (Liebig's) 460: 225-235. 1928.

94. Windaus, A., K. Dithmar, and E. Fernholz. Über das Lumisterin. Ann. Chem. (Liebig's) $493: 259-271.1932$.

95. Windaus, A., J. Gaede, J. Köser, and G. Stein. Über einige krystallisierte Bestrahlungsprodukte aus Ergosterin und Dehydro-ergosterin. Ann. Chem. (Liebig's) 483:17-30. 1930.

96. Windads, A., and A. Hess. Sterine und antirachitisches Vitamin. Nachr. Ges. Wissensch. Göttingen, Math.-physik. Klasse (1926): 175-184. 1927.

97. Windaus, A., O. Linsert, A. Lüttrixghaus, and G. Weidlich. Über das krystallisierte Vitamin D2. Ann. Chem. (Liebig's) 492: 226-241. 1932.

98. Windaus, A., and A. Lüttrivghaus. Einige Bemerkungen über das antirachitische Vitamin aus bestrahltem Ergosterin. Zeitsch. Physiol. Chem, 203: 70-75. 1931. 
99. Windads, A., A. Lütrringhads, and P. Bosse. Neue Beobachtungen an Bestrahlungsprodukten des Ergosterins. Nachr. Ges. Wissensch. Göttingen, Math.-physik. Klasse (1932): 150-159. 1932.

100. Windaus, A., A. Lüttringhaus, and M. Deppe. Über das krystallisierte vitamin $\mathrm{D}_{1}$. Ann. Chem. (Liebig's) 489: 252-269. 1931.

101. Windaus, A., F. v. Werder, and A. Lüttringhaus. Über das Tachysterin. Ann. Chem. (Liebıg's) 499: 188-200. 1932.

102. Windaus, A., K. Westphal, F. v. Werder, and O. Rygh. Einige Beobachtungen über die Ultraviolettbestrahlung des Ergosterins. Nachr. Ges. Wissensch. Göttingen, Math.-physik. Klasse (1929): 45-59. 1929.

Manuscript received by the editor March, 1934. 
$\mathrm{X}$

\title{
THE EFFECTS OF IRRADIATION ON VENOMS, TOXINS, ANTIBODIES, AND RELATED SUBSTANCES
}

\author{
S. C. Brooks \\ University of California, Berkeley, Cal.
}

Irradiation in vitro: Venoms. Saponins and toxic proteins of plant origin. Bacterial toxins. Antigens (Tuberculous; Syphilitic; Bacterial; Miscellaneous). Alexin. Antibodies (Specific hemolysins; Bacteriolysins; Syphilitic antibody; Precipitins; Agglutinins; Phagocytosis and opsonins; Anaphylaxis; Antitoxins). Irradiation in vivo: Alexin. Antibodies (Specific hemolysins; Bacteriolysins, phagocytosis, and opsonins; Alexin fixation and syphilitic antibody; Agglutinins; Antitoxins). Résumé. References.

There exists a literature of about 125 papers or series of papers, excluding preliminary communications and reviews, which deal with this group of substances. About 100 of these deal with effects produced in vitro; the remainder deal with supposed effects produced in vivo by local or general irradiation of living animals. The latter are particularly difficult to evaluate and to interpret and will be treated separately at the end of this ehapter.

It should also be kept clearly in mind that very few of these so-called "substances" are really known in any other way than by their effects. Many are undoubtedly one or a few related well-defined substances, e.g., bacterial toxins; others, e.g., some snake venoms, appear to include several distinet, more or less well-defined, substances; others, such as alexin, agglutinins, and, in fact, most pathogenic antigens and antibodies are literally nothing but ill-understood properties of vaguely apprehended complex colloidal systems. Further diseussion of so involved and technical a topic is out of question here, although recent research has yielded some encouraging progress. ${ }^{1}$

Even where assuredly no real substance is now known, we shall here, for the sake of brevity, refer to "substances."

The experiments on irradiation in vitro suffer from the lack of adequate quantitative technique; workers using survival of test animals as a criterion have almost uniformly failed to use enough animals to give their results statistical validity, while experiments using hemolysis, bacteriolysis, agglutination, or the like, are usually recorded in terms of

${ }^{1}$ For further discussion of this field the reader is referred to H. G. Wells, "The Chemical Aspects of Immunity" (163) and to other works cited there. 
“-," “土," " ++," “4+," or similarly unsatisfactory terms. For this reason very few protocols are given here. Much of the work was done in the youth of immunology and could profitably be repeated with better methods, such as can now be devised.

Although, for several reasons, it is impossible to make a scientifically justifiable classification of toxins, antibodies, etc., the arbitrary classification here followed (see "Contents") has some logical basis.

\section{IRRADIATION IN VITRO}

\section{VENOMS}

Snake venoms and allied toxic materials are complex solutions usually exhibiting several properties in varying relative and absolute degree: neurotoxic, hemolytic, or hemorrhagic, anticoagulant or accelerating coagulation of the blood, and so on. Upon intravenous injection into suitable animals in sublethal doses they are also capable of giving rise to antivenins, and may, therefore, be said to act as antigens. The hemolytic and hemorrhagic effects are probably due to cytolysis of erythrocytes and capillary endothelial cells, respectively, and may be due to a single ingredient of the venom. One and the same venom may produce predominantly hemolytic effects in one species of animal and predominantly hemorrhagic effects in another. If the cytolysis is dependent upon the agent penetrating the particular cells affected, the situation would be paralleled by such hemolytic agents as glycol, glycerol, etc., which have similar specific relations with the erythrocytes of different species (89). It is entirely possible that the neurotoxic effect, which may be upon the respiratory center or the phrenic nerve which innervates the diaphragm, or upon both, may be due to the same constituent of the venom as the cytolytic effects, but there is no good evidence to support this idea. In fact, Delezenne and Fourneau (42) believe that in the case of cobra venom, a lipase in the venom produces a hemolysin in the blood stream by acting upon lecithin to produce lysocithin, an extremely hemolytic substance. The relationship between the coagulant, anticoagulant, agglutinative, and antigenic properties of venoms and the cytolytic and neurotoxic effects is similarly obscure.

Experiments purporting to show that, since heating or irradiation causes the disappearance of one effect before another, these effects must be due to independent substances, cannot be considered conclusive in the absence of exact information as to the relation between venom concentration and the different toxic effects which may vary differently with change in concentration.

For these reasons it is difficult to express on a sound quantitative basis the effects of irradiation on different venoms as studied by different workers using different criteria of toxicity. 
It is clear that most, if not all, the above-mentioned properties of venoms are weakened by appropriate irradiation. Noguchi (124) made an extensive study of the photodynamic inactivation of the venoms of the cobra (Naja naia), daboia (Vipera russellii), and rattlesnake (Crotalus adamanteus). Solutions were made up containing 0.32 or 0.08 per cent (daboia) of dried renom and 0.05 per cent of eosin or erythrosin, and these were allowed to stand in direct sunlight for $30 \mathrm{hr}$. The hemolytic action of all three venoms was substantially diminished, the effects, according to the author, being greatest on rattlesnake and least on cobra venom. Using "moderate hemolysis" as an end point, cobra venom is most stabile, and daboia and rattlesnake venoms nearly alike; using "no hemolysis" as an end point, cobra and rattlesnake venoms are almost destroyed in the presence of eosin, and daboia venom is least affected, while with erythrosin, cobra venom is unaffected, rattlesnake venom nearly destroyed, and daboia venom intermediate. Noguchi tries to relate these findings to the relative stabilities of the supposed principles neurotoxin, coagulin and hemorrhagin, but all that one can safely conclude is that all the principal toxic effects of these venoms are susceptible of photodynamic inactivation.

Noguchi also studied the globulin-precipitating and erythrocyteprotecting actions of cobra venom, which he found to be very resistant to photodynamic irradiation. Parallels between thermostability and photostability are suggested.

Most students of the photolability of venoms have used ultra-violet light. Massol (117) showed that cobra venom (1:10,000) was rapidly inactivated by the light of a 300-watt quartz-mercury-arc lamp; activity was deduced from tests of the lethal effects on white mice. The author also shows, though the data are not very numerous, an apparent protective effect of antivenin (immune horse serum) when it is mixed with venom prior to irradiation. No explanation of this is offered.

Much, Peemöller, and Haim (121) also exposed cobra venom $(1: 2000)$ to ultra-violet light from a quartz mercury arc, keeping the temperature at about 0 to $2^{\circ} \mathrm{C}$. The exposure was equivalent to 72 "skin erythema doses," and the exposed venom completely lost its power to hemolyze human erythrocytes. The authors indulge in much extravagant speculation as to the cause and significance of the effects, which they believe to be intimately connected with effects on lipoids, a not improbable assumption.

One hour of irradiation at $40 \mathrm{~cm}$. from a quartz mercury arc of unspecified characteristics was found by Arthus and Collins to "completely" destroy the in vivo toxicity of daboia and cobra venoms which are primarily coagulative and neurotoxic, respectively. The protective effects of horse serum, egg white, and Witte's peptone when present during irradiation were noted. Similar effects are commonly observed 
in other systems and will be discussed below in relation to the photolability of tetanus toxin.

Arthus and Collins studied also the influence on coagulation exerted by venoms as affected by ultra-violet radiation. In vivo cobra venom retards coagulation, while the venoms of the daboia, the fer-de-lance (Bothrops atrox) and the tropical rattlesnake (Crotalus terrificus) promote coagulation and lead to thromboses. In vitro the effects of the first two on citrated plasma appear only on addition of calcium ions, coagulation being then retarded (cobra) or accelerated (daboia), while the last two are alone able to coagulate citrated plasma. Ten minutes' irradiation, the temperature not exceeding $25^{\circ} \mathrm{C}$., was adequate to reduce all these types of effects to 5 to 10 per cent of their original strength. Data are given for various times of irradiation, and a fairly complete picture of the progressive effects on the different venoms may be obtained.

Phisalix and Pasteur (129) have made an extensive study of the effects of ultra-violet irradiation on the venom of the European asp (Vipera aspis). The 400-watt quartz-mercury-arc lamp, emitting light down to $2300 \AA$, was not sufficient to affect significantly the toxicity of this venom (1:1000 in physiological salt solution) as judged by its effect on mice. The authors suggest that there was perhaps a slight increase in toxicity due to irradiation, but this is not borne out by the data given. The irradiated venom was, however, not capable of provoking immunity, so that the antigenic power ("echidno-vaccine" of the authors) was destroyed by irradiation which left the neurotoxin and hemorrhagin essentially unaffected.

Viper venom is also capable of destroying the virus of rabies in vitro even after heating to $75^{\circ} \mathrm{C}$. for $15 \mathrm{~min}$. to destroy its toxicity: this power, as well as its immunizing properties, is destroyed by heating to $100^{\circ}$ for $10 \mathrm{~min}$. (Phisalix and Pasteur, 130). Viper serum has similar properties, and irradiation of serum destroyed the antigenic and antirabiesvirus properties while leaving the toxicity unaffected (Phisalix and Pasteur, 131).

Destruction of the toxic properties of viper venom (Vipera aspis?) and cobra venom by exposure to the radiation from a capsule of $\mathrm{RaBr}_{2}$, was observed by C. Phisalix (127). Presumably the effect was due to gamma and perhaps also beta radiation, but the description of the experiments is too meager to allow closer analysis. RaEm and its active deposit dissolved in the venom solution also inactivated it, in this case with development of turbidity and a characteristic odor, perhaps as a result of alpha and increased beta radiation (128).

C. Phisalix (128) also found the poisonous secretions of a terrestrial salamander and the common toad to be unaffected by radium radiation in similar dosage. 
Venomous secretions and venomous sera also occur in many other groups, notably fishes and amphibians. Kopaczewski (101) has made a special study of the toxic serum of the moray (Muraena helena), as well as of the venom secreted around certain of its teeth. The serum. usually referred to as "eel serum," can be destroyed by irradiation but is relatively insensitive. The venom was apparently not tested in this regard. The serum was weakened by $48 \mathrm{hr}$. exposure to direct sunlight, and lost about one-half to two-thirds of its toxicity on $30 \mathrm{~min}$. exposure to a powerful quartz-mercury-arc lamp (approximately $8000 \mathrm{cp}$.) at a distance of $5 \mathrm{~cm}$. Temperature was not a factor. Sources of ultra-violet radiation of wave-length 3000 to $4000 \AA$ were tried but were too weak to have any effect, although the effectiveness of visible light would lead one to expect some loss of toxicity at least. Exact energy measurements are needed before the destructive efficacy of different spectral regions can be compared. Kopaczewski correlates inactivation of the moray serum with an ultramicroscopically observed decrease in dispersity (102).

Schuscha, under E. Friedberger, ${ }^{2}$ also investigated the toxicity of "eel serum," which he tested by injection into guinea pigs. The inadequacy of such tests for quantitative work is probably the cause of the anomalous course of the inactivation by ultra-violet radiation as observed by him, $10 \mathrm{~min}$. irradiation producing less effect than $5 \mathrm{~min}$. His figures for the reduction of the hemolytic effect of eel serum in vitro as a result of irradiation are also quantitatively anomalous.

Friedberger himself had previously reported (66) that although the toxicity of eel serum was decreased by brief ultra-violet irradiation, its hemolytic power was not appreciably altered. Evidently this material is quite photostabile, although Kopaczewski's work seems to be adequate proof that it can be detoxicated by light.

\section{SAPONINS AND TOXIC PROTEINS OF PLANT ORIGIN}

Among the earlier investigations of the biochemical effects of light is one by Dreyer and Hanssen (46) in which it is shown that the glucosides saponin and cyclamin and the toxic proteins ricin and abrin all lose their hemolytic power under ultra-violet irradiation from a Bang Ag-electrode quartz-lamp.

Quantitative data are given illustrating the regular course of photoinactivation which the authors state was shown by the substances named, as well as by coli agglutinin and three enzymes studied at the same time. Though the details of titration and calculation are not given, it appears that a unimolecular reaction isotherm is followed, whose familiar equation is

$$
k=\frac{1}{t} \log \frac{a}{a-x}
$$

${ }^{2}$ Schuscha, A. T. Thesis, Cairo, 1915, quoted by Friedberger and Scimone (67). 
where $k$ is a constant, $t$ is time, $a$ is the original amount of photalabile substance, and $x$ the amount destroyed in time $t$. For cyclamin, $k=0.0033$ when $t$ is expressed in minutes and Briggsian logarithms are substituted for natural logarithms. The goodness of fit of the observed to the theoretical curve may be judged from Fig. 1. The agreement seems satisfactory and this research constitutes one of the pioneer demonstrations of the conformity of biological reactions to classical physicochemical laws.

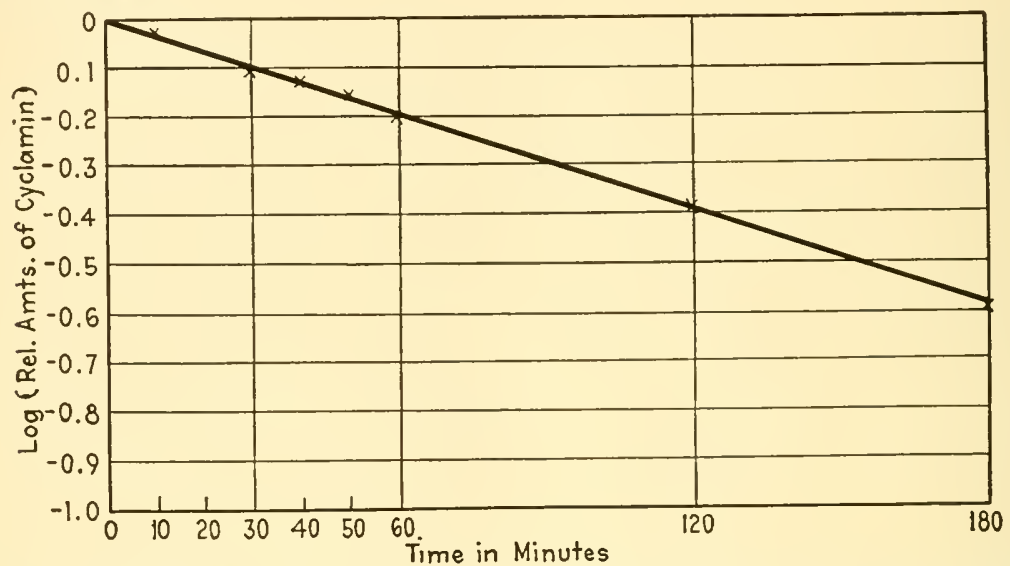

FIG. 1.-The relative hemolytic activity of cyclamin after different periods of irradiation. The straight line is the theoretical curve for $k=0.0033$, and the points represent observed values. Ordinates represent logarithms of $a-x$ (the fractional part of the original activity); abscissas, time in minutes. (After Dreyer and Hanssen, 46.)

Seyderhelm and Opitz (148) subjected purified saponin to ultraviolet radiation from a quartz-mercury-arc lamp, and found that it rapidly lost both its hemolytic power and its power to so injure leucocytes as to make them take up (become permeable to?) Congo red and trypan blue. The authors attribute the inactivation to decreased dispersion, as shown by the appearance of the Tyndall phenomenon after 5 to $6 \mathrm{hr}$., and of gelation after $7 \mathrm{hr}$. irradiation. There was also a decrease in the surface tension of the solution. Further changes in the physiological activity of saponin upon irradiation are also recorded, and Wheeler-Hill (164) in the discussion appended to the paper of Seyderhelm and Opitz (148), contributes some new data including studies on the absorption of light of $\lambda<3340 \AA$. This increases during irradiation.

Tappeiner and Jodlbauer (156) reported that ricin, the toxic protein of Ricinus, lost its agglutinating power when exposed to sunlight in the presence of oxygen and such photodynamic sensitizers as eosin, fluorescein, ete. Crotin, from Croton sp., resembled ricin.

Busck (31) investigated in detail the protective effects of normal serum or other protein colloids added to ricin-dye mixtures which were then exposed to visible light. The agglutinating power of the ricin 
in vitro was used as a measure of its activity. He attributes the protection to the formation of inactive complexes of dye and protein molecules. The paper deals also with protection of many test objects besides ricin and is primarily a contribution to the theory of the photodynamic effect.

Baroni and Jonesco-Nihaiesti (13) also found rather rapid loss of agglutinating power by ricin upon ultra-violet irradiation. Relative rates of destruction are given by these authors for various toxins, etc., but can hardly be granted quantitative significance, for reasons discussed below,

The upper wave-length limit for effective photochemical ricin inactivation, as judged by its toxicity for test animals, was shown by Carmichael to lie at about 2450 to $2540 \AA$ (32).

Schubert (142) exposed ricin, either dry or in 0.01 per cent aqueous solution, to the near ultra-violet ("Originalhöhensonne" lamp with uviol glass screen) and observed that it lost its toxicity for white mice. Welch (162) showed that its toxic and agglutinating powers were destroyed at about the same rate by ultra-violet radiation, but that its antigenic power was more resistant.

\section{BACTERIAL TOXINS}

These appear usually to be proteins or products of protein decomposition, and those produced by different organisms differ in the characteristic symptoms produced. Those whose photolability have been studied are all exotoxins, i.e., products given off to the culture medium by the growing organisms; they are usually broth filtrates, or, in one case (hemotoxin of $B$. proteus), broth cultures which have been killed by ether.

Diphtheria toxin was the first of all the substances treated in this paper to be studied from the point of view of its resistance to irradiation. Roux and Yersin (138), in 1889, published the significant observation that although quite stabile in the dark, it was almost completely inactivated by $10 \mathrm{hr}$. exposure to sunlight. When sealed up in small tubes without air, the toxin was only slightly injured by similar irradiation. The authors conclude that visible light plus oxygen is the active agency.

Emmerling (51) compared the effects of sunlight on diphtheria toxin and various enzymes, finding the toxin to be less stabile than the enzymes. Aside from this, his work serves merely to confirm that of Roux and Yersin.

Photodynamic destruction of diphtheria toxin in the presence of eosin ( 0.05 per cent) by 3 days' exposure to diffuse daylight has been observed by Tappeiner and Jodlbauer (155). Similar effects were observed with fluorescein ( 0.1 per cent), methylene blue (0.02 per cent), and dichloranthracene disulfonate. Rabbit or ox gall may also be used 
as a sensitizer (77). Huber (87) obtained similar results by 4 hr. exposure to direct sunlight, toxicity being judged by either hemolytic power in vitro or lethal effects on laboratory animals. Both papers show oxygen to be necessary for this effect. Various other data pertinent to the theory of photodynamic action are also given by Huber.

It seems much more probable that the photoprocess involves oxidation, as this work of Huber indicates, and as Roux and Yersin suggest in the case of visible light, than that it is a reduction, as suggested by Hertel (84) on the basis of experiments on this toxin and various enzymes using light of $\lambda \simeq 2800 \AA$. Hertel found 10 min. irradiation sufficient to reduce the toxicity of diphtheria toxin to much less than 25 per cent. This is much more rapid than inactivation by direct sunlight, but there are no clues as to the relative energy flux in the two cases.

Hartoch, Schürmann, and Stiner (75) observed rapid destruction of diphtheria toxin by ultra-violet light (Nogier-Triquet "Unterwasserbrenner"), toxic, antigenic, and antitoxin binding powers being about equally sensitive.

Welch (162) tested the relative stability of the original bouillon and the purified toxin prepared by Gross' method which eliminates most of the nitrogen and leaves a clear, colorless toxin. This purified toxin is much more quickly inactivated by the light of a carbon arc. The effect is presumably due to screening by components of the bouillon other than the toxin, as suggested by the author (cf. page 349 under Tetanus Toxin).

Baroni and Jonesco-Mihaiesti (13) also observed inactivation of diphtheria toxin by ultra-violet light.

X-ray effects were studied by Gerhartz (70). Various conditions of irradiation, using "soft" or "medium hard" tubes, were tried and evidence of some destruction of toxin obtained, but the small number of animals tested leaves the results far from quantitative, and Morgenroth (120) criticizes the paper severely on this basis. Gerhartz's reply in the same number of the same journal adds nothing new. Evidently an effect of X-rays on diphtheria toxin is as yet not definitely proven.

Fabre and Ostrovsky $(52,53)$, however, found detoxication when they added 1 microgram of "radium" ( $\mathrm{Ra}$ element or $\mathrm{RaSO}_{4}$ ?) to each milliliter of toxin and allowed the mixture to stand for 10 to 43 days. Many desirable details are lacking in these papers, e.g., the description seems to imply that the radium was ultimately injected into the test animals, which seems improbable and undesirable. Their conclusions, therefore, still leave some possible doubt as to any appreciable effect of radiations of large quantum value on the properties of diphtheria toxin.

Tetanus toxin was also very early found to keep less well in the light than in the dark. Tizzoni and Cattani (158), in 1891, showed that in bouillon cultures both bacteria and toxins had been destroyed by $4 \frac{1}{2}$ months in diffuse daylight, provided air (i.e., oxygen) was present. 
Controls in a hydrogen atmosphere or in the dark were practically uninjured. Less transparent media such as gelatin served to protect the toxin more or less.

Kitasato (97) obtained more rapid detoxication of bouillon filtrates by diffuse daylight than the earlier work suggested. The data, based on two mice per sample, are only roughly quantitative.

Fermi and Celli (56) found dry tetanus toxin to be completely inactivated by $48 \mathrm{hr}$. exposure to direct sunlight, as compared with the enzymes trypsin and pepsin which were not, but they believed that the relatively high temperatures $\left(40^{\circ}\right.$ to $50^{\circ} \mathrm{C}$.) incident to irradiation were responsible. If the temperature was kept at $37^{\circ}$ or below, there was no destruction of the toxin in $15 \mathrm{hr}$. At first glance this would seem to invalidate Kitasato's work in which, with temperatures of $35^{\circ}$ to $43^{\circ}$, sunlight completely inactivated tetanus toxin in 15 to $18 \mathrm{hr}$. But it is apparently the use of dried toxin rather than toxic bouillon filtrates which makes the difference. Fermi and Pernossi (57) investigated both and found that at temperatures not exceeding $37^{\circ}$ the dissolved toxin was completely inactivated by direct sunlight in $15 \mathrm{hr}$., in agreement with Kitasato, while the dried toxin was still somewhat active after $100 \mathrm{hr}$. irradiation. Similar effects of concentration or desiccation are often met with among toxins and immune bodies.

Loewenstein (107) reported inactivation of tetanus toxin by visible light from a 0.25 -amp. Nernst lamp, with and without interposition of colored-glass screens of undetermined transmission. Loewenstein also reported photodynamic inactivation by visible light acting on an undescribed toxin-eosin mixture. It had been described previously by Tappeiner and Jodlbauer (155) using 0.05 per cent eosin and diffuse daylight, and Huber (87) used eosin or erythrosin and found destruction of the hemolytic effect in vitro as well as of the general toxicity. (Cf. also above in reference to diphtheria toxin.) Oxygen is necessary.

Flexner and Noguchi (61) noted photodynamic destruction of the lytic and tetanospasmic principles of tetanus toxin both by sunlight alone and, more rapidly, by sunlight acting on toxin solutions containing 0.15 per cent eosin or related dyes. Hausmann and Pribram (78) also obtained photodynamic inactivation of tetanolysin, using ox or rabbit gall as a sensitizer.

Ultra-violet inactivation of tetanolysin, first described by Courmont and Nogier (37), in 1909, illustrated well two points: the importance of transparency to light of the effective spectral region; and the characteristic absence of the need for oxygen, which distinguishes most ultra-violet reactions from the analogous photodynamic reactions.

Influence of Dilution.- The first point is treated by Courmont and Nogier (38) in a paper in which they show that while 1 to $2 \frac{1}{2} \mathrm{hr}$. hardly inactivated undiluted toxic tetanus bouillon in their earlier experiments, 
now by diluting the bouillon 1:2000 with distilled water they could almost completely detoxicate it in "a few" minutes.

In the same issue of the Comptes Rendus there is an account of considerably more detailed experiments on tetanus-toxin dilutions by Cernovodeanu and Henri (33). The effect of diluting toxic bouillon filtrates with 0.8 per cent $\mathrm{NaCl}$ was the same as that observed by Courmont and Nogier when they used distilled water. Dilution with fresh nontoxic bouillon decreased the rate of destruction of toxin, presumably because the ratio of protective substance to toxin was increased. A screen of bouillon $5 \mathrm{~mm}$. thick interposed between the source and the toxin to be irradiated prevented inactivation, thus demonstrating the effect of the protective substances in bouillon to be principally at least a result of a purely physical shading of the toxin. Spectrograms showed that the undiluted bouillon had a pronounced absorption for light of $\lambda<2805 \AA$, while there was still considerable transmission of light of $\lambda=2399 \AA$ by a similar depth of bouillon diluted $1: 100$ with 0.8 per cent $\mathrm{NaCl}$.

These facts are of such general significance in all irradiation experiments on biological media, especially with ultra-violet light, that they have been given here in some detail. They have also been noticed in studies on alexin, on antibodies, etc., but will be mentioned hereafter only as they bear on some other point of importance.

Oxygen in Relation to Ultra-violet and Visible Irradiation.-Cernovodeanu and Henri (33) also show that irradiation of tetanus toxin in exhausted and sealed quartz vessels still produced inactivation, showing that the reactions induced by ultra-violet radiation can destroy toxin in the absence of oxygen. Inactivation by visible light, on the other hand, depends upon some component of air, presumably oxygen (Tizzoni and Cattani, 158; and Tappeiner and Jodlbauer, 155). Other substances such as ricin and tetanus toxin have also been shown by Tappeiner and Jodlbauer to be inactivated photodynamically only when free oxygen is available. In fact, photodynamic processes in general require free oxygen. Why should this difference exist? Does it mean that inactivation by visible light (with or without sensitizers) is an oxidation process, while that by ultra-violet radiation is not? Blum (20) suggests that $\lambda<2800 \AA$ may release oxygen from water so that in the presence of very short wave-lengths oxidations may procecd without free oxygen, thus leading eventually to the same end result, namely, in this case oxidation of the toxin. If inactivation is the result of oxidation by the primary splitting of water, ultra-violet radiation acts primarily on water rather than on the toxin.

Other investigators have added minor details of some interest to our knowledge of the effects of ultra-violet radiation on tetanus toxin. Schubert (142), using a Hanau "Originalhöhensonne" (quartz mercury 
arc) and keeping his samples cooled to about $0^{\circ}$ to $2^{\circ} \mathrm{C}$., found both diluted toxin and dry toxin exposed in very thin layers to be about equally labile. The lability of the dry toxin is rather surprising. He also verified a previous report, for which no citation is given, that ultra-violet irradiation increases the ultra-violet absorption of the toxin.

The fact that Schubert could inactivate tetanus toxin at $0^{\circ}$ to $2^{\circ}$ conforms to expectations based on the theoretical and observed low temperature coefficient of photochemical reactions. Cernovodeanu and Henri (33) tested this particular point and found practically no effect of temperature on the reaction rate between $0^{\circ}$ and $24^{\circ} \mathrm{C}$. The same authors claim to have shown that the light reaction proceeds according to unimolecular reaction laws, but the agreement is only approximate. Welch (162), using an air-cooled carbon are as a source of visible and ultra-violet light, distinguished between the tetanolysin and tetanospasmin, but found them to be equally affected by light.

Toxins appear to be very resistant to X-radiation. Lusztig (111), in a paper which covers a great deal of ground, but which is marred by omission of much needed details and by careless bibliography, states that 10 H.E.D. ("Haut-Erythema-Dosis") had no effect upon the toxicity (or pathogenicity) of (killed?) cultures of the organism of swine erysipelas (B. rhusiopathiae suis).

No detoxication of tetanus toxin by radiations from radium was found by Fabre and Ostrovsky $(52,53)$, although they did observe it in the case of diphtheria toxins (see page 348 ).

Toxin of B. botulinus.-Gradual loss of toxicity of meat extracts in which $B$. botulinus had been grown was observed by Van Ermengem (159) and by Thom, Edmondson, and Giltner (157) when the extracts were exposed to diffuse or direct sunlight and air.

The toxins investigated by Schoenholz and Meyer (141) required a longer time for "inactivation" than those just mentioned, either because they were originally more potent or more resistant, or because, as the authors suggest, the layer of paraffin oil with which they overlaid their toxic broth impeded the diffusion of oxygen into the broth and thus retarded detoxication.

Miscellaneous Bacterial Toxins.-Bacillus proteus yields a hemolytic toxin when cultures are killed by ether, and the ether then removed by aeration. This toxin preparation is probably susceptible to inactivation by visible light, although Ecker and Brittingham (49) found it to be unaffected by the relatively very short exposure of $3 \mathrm{hr}$. which they tried. It is about as rapidly inactivated by ultra-violet radiation as other toxins, an "Alpine lamp" at $30 \mathrm{~cm}$. reducing its titer to about 50 per cent in $5 \mathrm{~min}$. In a very rough way, ultra-violet inactivation of this toxin follows the exponential course of a unimolecular reaction, the approximate observed toxicities after various exposures being $5 \mathrm{~min}$., 50 per cent; 10 min., 25 per cent; 15 min., 5 per cent. 
Protein-free toxic filtrates produced by bacteria of the colon-typhoid group studied by Welch (162) produce two types of phenomena in vivo: a "skin reaction," and a "skin reactivity," i.e., sensitization to subsequent injections. The latter might be classed as an antigenic property. It is abolished by 60 to $70 \mathrm{~min}$. irradiation at $25 \mathrm{~cm}$. from a 55 -volt, 30-amp., direct-current carbon arc, while the skin-reaction test (toxin?) is abolished after $40 \mathrm{~min}$.

Toxins Present in Human Serum.-Macht (112) has detected by their effects on the growth of seedlings of Lupinus albus several toxic substances, among which two have been tested as to their stability on ultraviolet irradiation. Pernicious-anemia toxin, present in serum in that disease, was found to be about 20 per cent inactivated by $30 \mathrm{~min}$. irradiation. Eosin accelerated destruction: presumably a photodynamic effect accompanying visible light. Menotoxin, which Macht believes to be related to cholesterol, is apparently unaffected.

\section{ANTIGENS}

Many of the toxic materials discussed above have the power to evoke changes in mammals upon injection or introduction per os. Many materials, such as the antigens used in the Wassermann reaction, evince very little toxicity. For convenience, therefore, we shall group together here the antigenic aspects of all these substances, even though their toxicity may have been considered above.

Tuberculin.--The various tuberculins produce no toxic symptoms in normal animals, and cannot, therefore, be considered as toxins. Neither do they appear to be, strictly speaking, antigens. Their inclusion here is arbitrary. The different types all seem to be more or less sensitive to irradiation.

Jansen (93) exposed previously heat-killed and dried B. tuberculosis in layers 0.3 to $0.5 \mathrm{~mm}$. thick to concentrated carbon-arc light without effect; three types of tuberculin irradiated under similar conditions gave the characteristic odor of burned horn, but had exactly the same effects on tuberculous guinea pigs as the controls. Visible light is, therefore, by itself practically without effect.

However, the addition of eosin or erythrosin may lead to inactivation. Bouveyron (21) added 2 per cent dye to 1 per cent Institut Pasteur tuberculin (saturated with chloroform) and exposed it for several hours to clear July sunlight. Controls without dye and/or wrapped in black paper produced the typical cutaneous reactions in tuberculous guinea pigs, while the sample irradiated with dye did not. The same result was attained by $12 \mathrm{hr}$. exposure to a 1000 -cp. electric light at $20 \mathrm{~cm}$. distance.

The earlier findings of Jacobsohn (90) are in apparent conflict with this, but his exposure of the tuberculin to diffuse daylight for $24 \mathrm{hr}$. is not nearly equivalent to Bouveyron's exposure of about $12 \mathrm{hr}$. to direct 
sunlight. An exposure to diffuse daylight for several weeks or months might be expected to produce inactivation. Jacobsohn also used a different test: the "réaction précoce" (Marmorek's phenomenon); his paper should be consulted for details.

Ultra-violet radiation was used by Hausmann, Neumann, and Schuberth (77), and was found to inactivate tuberculin in dilute aqueous or saline solution ( 0.1 to 10 per cent, in $1 \frac{1}{2}$ to $2 \mathrm{hr}$.) ; only light of $\lambda<3250 \AA$, approximately, was effective.

No experiments appear to have been made with X-rays, but Fabre and Ostrovsky $(52,53)$ appear to have demonstrated a reduction in the severity of the symptoms induced in tuberculous guinea pigs when irradiated instead of unirradiated "necrotuberculin" (Ostrovsky) was used. (See page 348 for certain critical reservations needful in the case of these papers.)

Wassermann antigen usually consists of a more or less modified extract of luetic organs or of beef or other heart muscle. It is an exceptionally ill-defined material. Stiner and Abelin (154) appear to have been the first to report inactivation of syphilitic antigens by ultra-violet radiation. A few years later the effect of intense ultra-violet irradiation (quartz-mercury-arc lamp, 220 volts, $3 \mathrm{amp}$., at $7 \mathrm{~cm}$., the temperature being kept close to $0^{\circ} \mathrm{C}$.) on various antigens was reported in a preliminary way by Friedberger and Scimone (67), and in more detail by Friedberger and Shiga (68). Both luetic-liver and guinea-pig-heart extracts were photosensitive, the latter only slightly; $40 \mathrm{hr}$. irradiation-a huge dosewas required to inactivate the former. Addition of cholesterol protected both, the latter being then not perceptibly labile. Another antigen was "completely stabile."

The antigen used by Brann (24) was not found to be affected by the ultra-violet light from a 200-volt, 6-amp. Kromayer lamp at 0.5 to 1.0 $\mathrm{cm}$. in 1, $1 / 4 \mathrm{hr}$. This irradiation is short compared with that used by Friedberger et al., and Brann also used cholesterinized beef-heart extract which, according to these authors, is relatively insensitive. Under these conditions the discrepancy of results is to be anticipated.

Brann was also unable to detect any effect from X-rays (4 to 13 " $\mathrm{E} \mathrm{D}$ " - presumably erythema doses) or from suspension in a small volume of antigen of a capsule containing 20 to $24.6 \mathrm{mg}$. of $\mathrm{RaBr}$.

Bacterial Antigens.-Welch (162) and Megrail and Welch (118) have reported that the antigenic properties of tetanus toxin and of toxic protein-frce filtrates from bouillon cultures of bacteria of the colontyphoid group are somewhat more stabile under ultra-violet irradiation (from special "cored" carbons) than are the concomitant toxic principles. In the first case it was possible to prepare an atoxic antigen; in the latter case the toxic principle was inactive after $40 \mathrm{~min}$., but the antigenic principle, which sensitizes the skin to subsequent intravenous 
injection of the same filtrate (the "Schwartzmann reaction"), required 60 to $70 \mathrm{~min}$. for its inactivation.

Eberson's experiments (47) on the effect of ultra-violet light on the antigenic properties of living cultures of different strains of meningococci are principally of bacteriological interest. Brief irradiation (1 to $6 \mathrm{~min}$.) appeared to have increased, and longer exposures decreased the power of certain strains to act as antigens in the production of agglutinins for other strains; the author speaks of irradiation making the antigen "more inclusive." The experiments are difficult to interpret from a photochemical point of view. Additional controls would have been desirable.

Reference should also be made to Hasskó's (76) ultra-violet irradiation of B. typhosus, B. enteritidis (Gärtner), and B. dysenteriae Shiga with partial loss of their specific agglutinability, and to Friedberger's (66) report that ultra-violet irradiation of trypanosomes in diluted mouse blood in vitro destroyed their antigenic property in $10 \mathrm{~min}$.

Friedberger also reports (66) that sheep erythrocytes irradiated until slightly hemolyzed, still bound the corresponding amboceptor, thus retaining their antigenic properties in a certain measure.

Fiorini and Zironi (58) were unable, on the other hand, to detect any effect of X-rays, either immediate or after one to two transplants, on the agglutinability of $B$. typhosus by specific agglutinins. The dosage and type of radiation are not clearly described.

Protein Antigens. - Like the bacterial toxins studied by the same author, ricin was found by Welch (162) to maintain its antigenic power longer on exposure to ultra-violet radiation than it did its toxic and agglutinating powers.

Other proteins, such as egg white or foreign sera, do not ordinarily display great toxicity on injection into normal animals but do have considerable antigenic power leading to precipitin formation, sensitization, etc. It is, however, their capacity to act in the role of precipitable substance or "antigen" in the specific precipitin reaction which has been studied in connection with irradiation. Fleischmann (60) found that exposure of beef or horse serum or egg white to diffuse daylight led to delay or to prevention of their preeipitation, provided eosin, safranin, or methylene azure was added before irradiation.

The experiments indicate that only the precipitability, and not the power to "bind" the correspondingly specific precipitin was affected. Precipitins were also studied and found to be less stabile (cf. page 367 ).

Doerr and Moldovan (44) obtained essentially similar results with ultra-violet radiation (Kromayer lamp at 5 to $10 \mathrm{~cm}$.), using sheep, ox, and horse sera. They noted an increasingly rapid loss of precipitability with increasing dilution. The power of these sera to produce anaphylactic shock in sensitized animals was reduced at about the same rate as the precipitate-forming power. Friedberger (66) and Scott (145, 
146) have also reported loss of specific precipitability by human serum, and Hasskó reports loss of precipitability by neosalvarsan for horse serum, all as a result of ultra-violet irradiation. In the latter case, the reaction was independent of free oxygen and was accelerated by passage of bubbles of any gas. Some change of colloidal state is possibly the important factor here rather than a chemical change.

Anaphylaxis.-An animal which has received injections of proteins, such as normal or antisera, may become sensitized so that further injections of the same protein bring on characteristic further symptoms of what is called anaphylactic shock, which may lead to sudden death. The injected protein may be considered to be an antigen, both when it is used to sensitize an animal, and when it is used to induce anaphylactic shock.

The ability of proteins to sensitize animals seems not to have been the subject of irradiation experiments. The power to induce anaphylactic shock in sensitized animals is reduced by ultra-violet irradiation and finally destroyed, but it appears to be more resistant than almost any other immunological property of serum; only precipitability by specific precipitins is more resistant (Baroni and Jonesco-Mihaiesti, 14 ; and Scott, $145,146)$.

\section{ALEXIN}

Discussion of alexin or complement, as it is often called, will here be directed first to the hemolytic function which is exerted in conjunction with normal or specific hemolysins or amboceptors. There are over 20 papers dealing with this subject alone, more than for any other substance dealt with in this paper. At the end of the section brief reference will be made to analogous cytolytic properties of blood and other body fluids. Attention will be directed first to the effects of different types of radiation on alexin, with brief reference to significant accessory details, and then several papers having special theoretical bearing on the nature of alexin and the process by which light inactivates it will be discussed as a group.

Observations. - Visible light, both with and without photodynamic sensitizers, was found by Lichtwitz (105) to inactivate the alexin of both normal and specifically hemolytic sera (rabbit and goat antibeef). This is the first recorded photoinactivation of alexin. Lichtwitz found that his "controls" lacking eosin were about half inactivated by exposure of $16 \mathrm{hr}$. to direct sunlight. An ice bath prevented undue heating, which would alone have seriously affected the alexin in so long an exposure, even if the temperature had been only $40^{\circ} \mathrm{C}$. (Madsen and Watabiki, 113.)

Bovie (23), in studying the effects of tropical sunlight on alexin, maintained controls shielded from the ultra-violet of wave-length less 
than $3000 \AA$ by glass containers and kept at $10^{\circ} \mathrm{C}$.; these were about half inactivated by $1 \frac{1}{4} \mathrm{hr}$. exposure to direct sunlight.

In contrast to these workers, Jacoby and Jacoby (91) were unable to detect any effect of exposure to June sunshine at Davos, except once when a 2-hr. exposure produced slight inactivation.

No clear conclusions can be drawn from Ecker's (48) attempt to compare the effects of different parts of the visible and infra-red spectrum, because the relative intensities are not determined.

No one appears to have determined whether or not oxygen is essential for inactivation by visible light, a matter which would be of considerable theoretical interest.

Photodynamic Inactivation.-Lichtwitz's paper (105) was concerned primarily with photodynamic effects. Eosin was used as a sensitizer, and $8 \mathrm{hr}$. in direct sunlight reduced the alexin to 20 to 25 per cent of the original value; $16 \mathrm{hr}$. irradiation reduced its activity to less than 5 per cent. Appropriate tests showed that the specific hemolysin or amboceptor was much more stabile than the alexin in the same or in different sera. Pfeiffer (126) obtained similar results.

In this connection Noguchi's paper (125) is of very great interest in showing that hemolytic substances such as certain soaps become nonhemolytic except for specifically sensitized cells when dissolved in (heat inactivated) serum instead of water, and at the same time become thermolabile and sensitive to photodynamic inactivation. The soap-serum mixture is, therefore, an exceedingly good analog of alexin in at least three important respects. The analogy is worth remembering.

Ultra-violet Radiation.-Bovie's experiments (23) show that sunlight ultra-violet ( $\lambda 2800$ to $4000 \AA$ ) inactivates alexin, since about half the effect of direct sunlight was due to light of wave-length less than $3000 \AA$.

Baroni and Jonesco-Mihaiesti (13) exposed dilute guinea-pig serum ( 1 per cent in 0.9 per cent $\mathrm{NaCl}$ ) in a layer only $1.5 \mathrm{~mm}$. deep to the light of a 110-volt 4-amp. Heraeus quartz mercury are at $16.5 \mathrm{~cm}$. Its alexin was inactivated in 40 to 60 sec. Undiluted serum, on the other hand, required 17 to $20 \mathrm{~min}$. exposure for alexin inactivation. Alexin was destroyed in about one-sixth of the time required to destroy a rabbit anti-sheep amboceptor of titer 1:500.

Courmont, Nogier, and Dufourt (39) made a similar observation on human serum, and, in addition, found that there was no difference between irradiation in nitrogen, oxygen, air or in vacuo. Small differences would have escaped their attention.

Abelin and Stiner (1) first observed alexin inactivation under controlled temperature conditions $\left(10^{\circ} \mathrm{C}\right.$.) ; earlier workers had, however, satisfied themselves by appropriate controls or otherwise that heating was not a factor in their results. These authors also showed how Bacillus coli, as well as alexin, was protected by thick layers or high concentrations 
of the serum in which it was suspended, and correctly interpreted the effect as due to sereening.

The principal contribution of Delbet and Beauvy (41) was the negative one of finding no correlation between alexin destruction and the appearance of the ultramicroscopically observed particles in the serum. These particles showed active Brownian movement and negative eataphoretie charge. Normal human serum, which is hemolytic for rabbit erythrocytes, was used. This serum possesses not only the alexin property, but also another substance, a "normal hemolysin" necessary for hemolysis (ef. page 365). Only the alexin was inactivated in Delbet and Beauvy's expcriments, the hemolysin being more resistant to irradiation.

Friedberger (66), keeping his samples on ice during irradiation, was unable to inactivate the alexin of dried and powdered serum, but did inactivate alexin in solution. He also experimented with " $\mathrm{M}$ " and " $\mathrm{E}$ " fractions ("mid-piece" and "end piece") of alexin obtained by Lieffmann's method. The separation was undoubtedly very incomplete, and in view of much better work by Schubert $(142,143)$ need not be diseussed.

Neither Scaffidi (140) nor Heuer (85) added anything new in regard to ultra-violet irradiation. The same is true of Brann (24). Baker and Peacock (11) make an interesting estimate of the relative sensitivity of alexin and $B$. coli, the alexin being about 20 times the more rapidly destroyed by the light of a 70-volt 3-amp. Cooper-Hewitt quartz-mercuryare lamp.

Diacono (43) showed that the specific hemolysin in the serum of an immunized guinea pig was more stabile than the alexin of the same serum when exposed to ultra-violet light. Delbet and Beauvy had previously shown the same for a normal hemolysin in human serum.

$X$-rays and Radium Rays.- The earlier reports of attempts to inactivate alexin by $\mathrm{X}$-rays $(58,62,140)$ are alike in their failure to find any effect and in the absence of any description of the type or dosage of radiation which gives any satisfactory idea of these factors. Fiorini and Zironi come the nearest to such a description when they state that the source was a "demimous" tube at $10 \mathrm{~cm}$., and the dosage " 5 -10X."

Sehubert (142) gives a somewhat better but still meager deseription of his source, and reports slight alexin inactivation in $1 \mathrm{hr}$. He used a Coolidge tube, voltage and current not stated, and filtration through $1 \mathrm{~mm}$. Al. Merlini (119), on the other hand, did not find any alexin destruction by X-rays. His dosage amounted to 1/16 to 1/8 skin erythema dose and the conditions described suggest that he used "harder" radiation than did Schubert; this may account for the disagreement.

Lusztig (111) is able to give a much better description of his source and dosage, the latter being given in terms of H.E.D., 1 H.E.D. being 
$9 \mathrm{~min}$. irradiation at $28 \mathrm{~cm}$., through $0.5 \mathrm{~mm}$. Zn from a tube operating at $200 \mathrm{kv}$. and $4 \mathrm{ma}$. He observed partial inactivation of alexin by doses ranging from 0.5 to 4.0 H.E.D. Unfortunately, he omits mention of the concentration or depth of the irradiated serum. For some obscure reason, about the same amount of inactivation was found at all dosages. Such phenomena are, however, not uncommonly experienced by those working on alexin, as is illustrated by the work of Chambers and Russ (34) and of Brooks (28), referred to later.

Some doubt is cast on all these results with $\mathrm{X}$-rays by the experience of Baermann and Linser (10). These workers X-rayed serum containing alexin, and found it to be inactivated, but when they kept the serum cool, and grounded the container, there was no inactivation. The apparent effect of the $\mathrm{X}$-rays on the alexin was, in reality, a result of heating or perhaps of electrical-discharge phenomena. ${ }^{3}$

Chambers and Russ (34) studied the effects of radium rays on alexin. $\mathrm{RaBr}_{2}$ was used as a source and successive small volumes of serum $\left(32.5 \mathrm{~mm}^{3}\right)$ were spread in a layer about $1.5 \mathrm{~mm}$. deep on the thin mica plate overlying the thinly spread radium salt. The alpha rays could pass through the mica, and were presumably absorbed for the most part within about $20 \mu$ more or less, depending upon the thickness of the mica (unstated); the alpha particles from $\mathrm{RaC}^{\prime}$ would penetrate about 20 to $30 \mu$ further. The nature of alpha-ray absorption is such that no alpharay energy acted upon any but this relatively thin layer, at most about 3 to 4 per cent as thick as the whole layer. The beta and gamma rays with greater penetrating power were found to be without appreciable effect under the experimental conditions obtaining.

Under such conditions it is not surprising that inactivation of the alexin was slow, about 40 to $45 \mathrm{hr}$. being required for half destruction. A peculiar feature of the experiment of Chambers and Russ is the initial lag of the inactivation process. This may have a theoretical significance which will be discussed in detail below in connection with similar results obtained by Brooks.

Brann (24) also observed slight inactivation of alexin by radiations from $\mathrm{RaBr}_{2}$, but whether alpha radiation was involved is not made clear.

Theoretical Aspects.-It is manifestly impossible to discuss here the whole physical chemistry of immune bodies. The argument in favor of a simple, primarily stoichiometrical explanation has been forcefully presented by Svante Arrhenius in his "Immunochemistry" and "Quantitative Laws in Biological Chemistry." It is clear, however, from papers by Madsen and his pupils, eited by Arrhenius, that most toxins are inactivated by heat according to quite definite laws, the time curve of

${ }^{3}$ This is probably the work sometimes referred to as that of "Burmann and Tinsa." 
the process being that of an unimolecular reaction, i.e., an exponential curve of the form

$$
x=x_{o} e^{-k t}
$$

where $x_{0}$ is the initial strength of the toxin and $x$ is the strength at time $t$. Furthermore, the effects of temperature on the rate of inactivation are quite regular and predictable on a physicochemical basis. For alexin, these facts were clearly shown by Madsen and Watabiki (113). It is: therefore, of interest to examine the effects of irradiation on immune bodies from this point of view. Practically all the available data concern alexin which has been carefully studied by Brooks (28) and by Lundberg $(109,110)$, entircly independently of each other. The substantial agreement of their findings has, therefore, increased weight.

In both cases the titration of alexin was carried out with an accuracy not attainable with other immune bodies (26), determinations on a single sample being reproducible to within about 1 per cent. Triplicate irradiations of three samples of a single serum gave the following titers after irradiation: $56.8,54.4,54.7$ (28). This will serve to give an idea of the statistical ralidity of the results.

Both authors report that the inactivation of alexin by ultra-violet light is nearly independent of temperature: Brooks gives $Q_{10}$ values gradually increasing over the range from $0^{\circ}$ to $40^{\circ} \mathrm{C}$. from 1.02 to 1.18 . Lundberg gives values of $\mu$, the so-called Arrhenius temperature characteristic, varying between 0.002 and 0.003 , which corresponds to a $Q_{10}$ value of about 1.1. These values arc typical of photochemical reactions, and show that, at least in the case of ultra-violet radiation, the amount of alexin inactivated is closely proportional to the amount of photochemical reaction.

The course of the reaction was found by Brooks (28), who irradiated solutions of guinea-pig serum (5 per cent in a special physiologically balanced salt solution), to vary rather irregularly from one sample of alexin to another, but to vary around an exponential curve. Table 1

Table 1.-Values of the Rate Constant $k$ for Inactivation of Alexin by Ultra-violet light, Calculated at Various Times dering the Process, as for a Unimolecular Reaction

The values are the means of eight experiments

(After Brooks, 28)

Exposure, min.

$$
\begin{gathered}
k=\frac{1}{t} \log \frac{a}{a-x} \\
0.026 \\
0.032 \\
0.030 \\
0.028 \\
0.030 \\
0.028 \\
0.028
\end{gathered}
$$


gives the values of the term $k$ in the unimolecular reaction isotherm

$$
k=\frac{1}{t} \log \frac{a}{a-x}
$$

after different exposures, and might be interpreted as showing that the fundamental reaction proceeds at a rate proportional to the concentration of a single disappearing molecular species. Other possible interpretations are not excluded.

Lundberg obtained better agreement in single experiments than did Brooks. This was perhaps due to his irradiation of undiluted serum (hog), since he found dilution prior to irradiation to conduce to irregu-

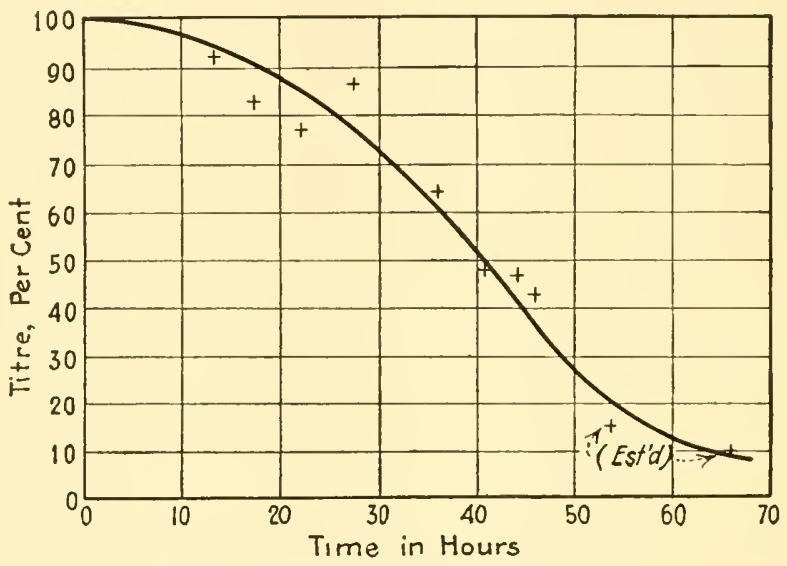

FIG. 2.-Alexin titer as affected by X-rays. Ordinates: alexin titers; abscissas: time. (After Chambers and Russ, 34.)

larities of titer and reaction rate. The reason for this is not obvious. Both authors agree in finding photoinactivation to be fundamentally a photochemical destruction of some one substance, or possibly a group of very similar substances with the same photosensitive atomic groupings.

As already mentioned, Chambers and Russ (34) found during inactivation of alexin by $\alpha$-rays an initial lag in the process (Fig. 2). Similar curves were found by Brooks for individual sera; in fact, in one case a large transitory increase of alexin titer was observed during the first 3 to 4 min. Chambers and Russ apparently did only one experiment, and their observation may well be atypical like similar observations by Brooks. On the other hand, their method of titration obviously permits errors of at least 10 per cent and possibly more in individual determinations of alexin titer, and errors of this magnitude might account for the whole deviation of their time curve from its expected exponential form. Diffusion must also be taken into account in their experiments, since all the radiation was absorbed in a small part of an unstirred sample.

However this may be, it is well to bear in mind the possibility suggested by them, and independently given in more detail by Brooks (29), 
that there exist in serum one or more precursors of alexin, or related substances, which become transformed into alexin by irradiation, or at least reenforce its action. We use the word "alexin" here to mean the principal hemolytic molecular species present in serum and acting in conjunction with amboceptors.

Another suggestive fact is that Schubert (142) found the moist globulin precipitate or "mid-piece" fraction (cf. below) to have its activity increased oftentimes by ultra-violet irradiation.

Lundberg's work also gives a clear quantitative statement of the acceleration of inactivation by dilution, the rate constant rising with dilution from 0.0364 for undiluted serum, through 0.0447 for 33 per cent, to 0.0882 for 10 per cent solution. Brooks' value for 5 per cent solution, namely, 0.030 , is somewhat snaller, probably because of the use of a weaker source of radiation (quartz-mercury arc).

That the inactivation of alexin by ultra-violet radiation does not depend upon free oxygen is apparent from Hasskó's (76) experiments on the effect of bubbling gases through various dilutions of serum ( 1 to 20 per cent) during ultra-violet irradiation. Although alexin inactivation did proceed a little faster with oxygen than with nitrogen, the difference is, in view of his obsolete and highly inaccurate method of titration, quite without significance. It is unfortunate that immunologists do not more generally grasp the possibility of discarding their obsolete methods and doing statistically valid quantitative work.

Lundberg's mention of increased opalescence upon irradiation suggests a colloidal or coagulative phenomenon. In addition to the large volume of support which this conception receives from other sources, we may mention some which are of particular interest from the point of view of irradiation.

Coagulation of proteins, which are the principal colloids of serum, is well known to involve at least two steps: denaturation and flocculation. Both of these may be reversible under appropriate conditions. ${ }^{4}$ Egg albumen is denatured by ultra-violet light and then flocculates rapidly and at first reversibly at room temperature (Bovie, 22). Is alexin similarly sensitized so that it goes on to further destruction at room temperature? As a matter of fact, alexin partially inactivated by heat may regain activity (Gramenitzki, 71). This might be thought of as a result of spontaneous reversion of the denaturation phase of protein coagulation. Brooks (27) was able to confirm Gramenitzki's claim that after heating there was a regeneration of alexin, but a considerable number of the same sera partially inactivated by ultra-violet irradiation showed no evidence whatsoever of either regeneration or of increased sensitization to heat $\left(37^{\circ}\right)$. Koopman $(100)$ has claimed to have found regeneration after partial inactivation by ultra-violet light, but his

${ }^{4}$ See, for example, Anson and Mirsky $(5,6)$. 
method of titration lacks accuracy, and his claim should not be accepted until more convincing evidence is offered to counterbalance the more extensive and more accurate determinations by Brooks, or to explain the discrepancy.

These experiments do not afford any ground for assuming that colloidal processes or coagulation play a part in alexin photoinactivation, but neither can they be regarded as evidence to the contrary. Processes of this type may well go on to irreversible change very rapidly during irradiation, not remaining in any unstabile wholly or partially denaturized stage for any appreciable time. In this connection it is interesting that Hassko (76) found the effect of irradiation upon the fluorescence of the serum to be affected by the nature of the gases present more than was the alexin activity. This fluorescence is one of the most sensitive indices of change in proteins upon irradiation.

A similar absence of any effect of irradiation was noted by Brooks (29) in testing the inactivating effects of temporary acidulation. There was no difference between control samples and irradiated samples which were, therefore, not sensitized to low $\mathrm{pH}$ conditions. Nevertheless, this experiment is suggestive in that inactivation occurs upon reaching a $\mathrm{pH}$ (4.6 to 4.4) corresponding rather closely to the isoelectric point of many globulins.

Similar pH changes are involved in the so-called "fractionation" of alexin into a mid-piece, or "M piece," which is the globulin fraction precipitated and redissolved, and an end- or "E piece," which is the residual fluid containing albumens. This fractionation has been most successfully done by Schubert (143), whose paper should be read by anyone interested. His best preparations show $\mathrm{E}$ piece to have no hemolytic power, and $M$ piece to have very slight, if any, hemolytic power in salt solutions; M piece dissolved in heat-inactivated serum becomes somewhat hemolytic, and recombined with $\mathrm{E}$ piece regains practically all its hemolytic power. Schubert regards $M$ piece as the real alexin or lysin carrier.

These $\mathrm{M}$ and $\mathrm{E}$ fractions have been the subject of endless experiment, from which it seems probable that M piece is more sensitive to light than E piece. Kagawa (95) seems to have established this fairly clearly for ultra-violet light, and Koopman (100) has made similar observations. Schubert (142) has made a still clearer demonstration because of his better fractionation of alexin. His M piece retained less than 3 per cent of its activity (upon recombination with $\mathrm{E}$ piece) when given an irradiation which left the $\mathrm{E}$ piece totally unaffected (see also Kodama and Shinomiya, 98a).

These facts acquire increased significance when one notes that according to Young (166) purified serum albumen is many times more susceptible to coagulation by ultra-violet light than the corresponding globulin fraction. The former corresponds to $\mathrm{E}$ piece, which is little 
affected by ultra-violet light in its hemolytic properties, while M piece, the globulin fraction, is extremely susceptible. Evidently there is associated with the globulin fraction of scrum a lytic principle, the true alexin, which may be inactivated by light independently of coagulation of the globulin, but which, nevertheless, cannot cause hemolysis unless the globulin is in a certain very definite state which depends upon $\mathrm{pH}$, salts, other proteins (e.g., E piece and amboceptor), etc.

'The nature of this lysin or alexin proper is still in question, but Noguchi's work (125) on emulsions of soap in protein sols, similar suggestive experiments by Schubert (142), and other evidence cited by Brooks (29) lead the writer to the view that the lysin itself probably contains fatty acids, probably unsaturated, either as soaps, lipins, or the like. Irradiation experiments have been of considerable importance in suggesting and supporting such a view. If this hypothesis is correct, the great comparative lability of alexin is probably due to the presence of a double bond which is readily oxidized photochemically.

It should be noted, however, that the prevailing opinion favors the view expressed by Wells (163) that alexin is simply serum proteins, particularly globulins, in a particular physicochemical and colloidal state.

Properties Related to Hemolytic Alexin.-Blood serum possesses cytolytic powers affecting cells other than erythrocytes. It has not been shown that such properties are in reality due to the same substance, although that would not appear improbable. Instances of destructive effects of irradiation on cytolytic properties of serum are not numerous. Busck (31) studied the toxicity of serum for paramecia, ealling this property alexin, and found it to be destroyed by midday sunlight when fluorescein dyes, methylene blue, cyanin, or anthracene dyes were mixed with the serum. The same effect could be obtained by using ultraviolet light in the absence of dyes. Condensed light from a carbon arc (e.g., 45 volts, 50 amp.) was used, and the inactivation was produced by the light which could not pass through glass, hence of ware-length $\lambda<3000 \AA$.

Eidinow (50) reported that defibrinated blood given ultra-riolet irradiation in vitro lost its bactericidal power for Staphylococcus albus. This loss of bactericidal power may represent alexin destruction. It would probably have been much more rapid if erythrocytes had not been present to absorb so much of the incident light.

The hemolytic property of the cerebrospinal fluid is apparently quite different from alexin; it is not merely thermostabile at $70^{\circ} \mathrm{C}$, , but upon ultra-violet irradiation actually increases in amount, even when irradiation is carried far enough to make the fluid deep brown in color and to smell strongly of "burnt skin" (Danielopolu, 40). In the same fluid there is, however, a substance which protects erythrocytes against 
hemolysis by sodium taurocholate, and this substance, or property, to which no name is given, is quite rapidly destroyed by the same irradiation. This work has been critically examined by Achard and Foix $(2,3)$ who find similar results with blood serum, as to both hemolytic and antihemolytic powers, when very long exposures are given. They also criticize Danielopolu's method of titration. Obviously we are here dealing with extensive photochemical changes which lie outside of the realm of immunology. More purely chemical methods of study would seem to be demanded.

\section{ANTIBODIES}

Antibodies may be defined as sera or other body fluids which have, in response to the introduction of toxin or other foreign substances, developed special properties, usually aiding to defend the body against further introduction of the same agent. Sometimes by rapidly attacking the agent they produce exaggerated reactions such as anaphylaxis. The most important types include specific hemolysins, antitoxins, agglutinins, the syphilitic antibody which, in the presence of an appropriate antigen, "binds" alexin, precipitins, etc.

Specific Hemolysins (Amboceptors). - When erythrocytes of one species of mammal are intravenously injected into another, the serum of the latter acquires, to a high degree, the power to hemolyze the erythrocytes of the former. In the presence of an adequate concentration of alexin, i.e., any normal serum, small traces of serum from the animal thus immunized will cause hemolysis in vitro of erythrocytes of the donor species. Such a serum, usually heated to inactivate its own alexin, is most often called amboceptor, from Ehrlich's concept of it as a physical intermediary between alexin and the erythrocyte. Similar amboceptors may be present in normal sera and are then distinguished as normal hemolysins.

These bodies have never been reported to be affected by visible light, either with or without photodynamic sensitization, possible exception being made of studies by Yanagihara (165). His brief note describes the effects of acid and alkali on the inactivation of a hemolysin by direct sunlight, eosin, methylene blue, or neutral red being dissolved in the serum. Lack of details, especially as to the nature of the hemolysin, make it impossible to tell whether it was alexin or a hemolysin which was inactivated, or to be sure just what the results mean. Except for the fact that when hydrochloric acid was added ( $\mathrm{pH}$ not stated) methylene blue was a relatively ineffective sensitizer, the data do not show anything very convincingly.

Definite negative evidence is given by Lichtwitz (105) who failed to find any photodynamic effect on a specific hemolysin. 
With ultra-violet light the situation is much clearer. It has already been noted that when a serum containing both alexin and a hemolysin is irradiated, the alexin is destroyed first (Delbet and Beauvy, 41; and Diacono, 43). This corresponds to the relatively high thermostability of hemolysin, and appears to be borne out by comparisons of the photosensitivity of the two principles taken separately. This was first pointed out by Baroni and Jonesco-Mihaiesti (13) and confirmed by Stiner and Abelin's two papers, one (1) on alexin and the second (154) on amboceptor (hemolysin).

They found that, as might be expected, it took longer to completely inactivate hemolysins of high titer, and that diluted sera were more quickly rendered ineffective. So long as "complete" destruction is used as an end point, so long will it be impossible to say whether such experiments do or do not indicate differences in sensitivity to irradiation: they may mean only that it takes longer to reach a given low level of titer from a high start than from some intermediate level. We cannot even be sure that alexin is more sensitive to light than are hemolysins. Reaction-rate constants (see page 359 ) or even the relative times required for a given fractional reduction in titer, say, to one-half, would have given this much needed information. We now stand on most uncertain ground.

Stiner and Abelin also found that the normal hemolysins of human blood were inactivated by ultra-violet light. Inactivation was again complete sooner than for the specific hemolysins, but whether because of greater lability or because of lower titer, it is impossible to say. Scott $(145,146)$ was the first after Baroni and Jonesco-Mihaiesti to observe inactivation of hemolysins by ultra-violet irradiation. Heuer (85) adds nothing new, and Hasskó (76) appears in this connection to have merely confirmed Stiner and Abelin's findings.

Friedberger (66), in additional experiments like Stiner and Abelin's, also tested the effect of ultra-violet radiation on hemolysin fractions analogous to those obtained from alexin-containing sera. As in the case of alexin, the albumen fraction ( $\mathrm{E}$ piece of alexin) was stabile, while the globulin fraction (M piece of alexin) was destroyed by $20 \mathrm{~min}$. irradiation. This suggestive finding has never been made the basis for further work so far as the literature shows, although some further details are given by Friedberger and Scimone (67).

As with alexin, X-ray experiments on hemolysins are inconclusive. Negative results are reported by Fiorini and Zironi $(58,59)$, Scaffidi (140), and Konrich (99). Fiorini and Zironi state only the dosage used: 5 to $10 \mathrm{X}$; Scaffidi gives almost no information as to the source used; Konrich fails to state voltage. Lusztig (111) reported about $2 / 3$ inactivation by 3 H.E.D., but in view of the findings of Baermann and Linser (10) 
in regard to alexin (page 358 ) one may be permitted to doubt the finality of Lusztig's findings.

There seem to have been no experiments on the effects of radium radiation on hemolysins. Quite probably, if tried, they were not described because of lack of positive results.

Specific Bacteriolysins.-In addition to normal bacteriolytic powers, possibly of the alexin type, referred to above (page 363), blood serum may acquire specific bacteriolytic powers analogous to specific hemolysins. Baroni and Jonesco-Mihaiesti (13) found that such a lysin, from the serum of a cholera-immune horse, was inactivated by ultra-violet irradiation in about the same time as a specific hemolysin which they also studied, i.e., in $7 \frac{1}{2}$ to $9 \mathrm{~min}$. as compared with $4 \frac{1}{2}$ to $6 \mathrm{~min}$. Heuer (85) made similar observations on a rabbit anticholera bacteriolysin.

Syphilitic Antibody and Alexin Fixation.-Sera of patients with active syphilis usually contain a principle whose protective powers are, to say the least, doubtful, but which in conjunction with an appropriate antigen in vitro inactivates any alexin present. This inactivation appears to be a variant of the complement fixation or Bordet-Gengou phenomenon, which results when approximately equivalent amounts of a specific antibody, e.g., antityphoid serum, and its corresponding antigen, in this case $B$. typhosus, but not $B$. coli, or some other species, are mixed, and form a compound or amicronic precipitate. This compound either combines with or adsorbs alexin, or in some way so affects the mixture that its alexin property is lost. (Excessive amounts of alexin are only partially inactivated.) Syphilitic antibody will react with many types of antigen, including those devoid of any relation to syphilis, being thus nonspecific in contrast to true alexin fixation. It is regarded by some authors as an entirely distinct phenomenon.

According to Friedberger and Scimone (67), the antibody of syphilis is destroyed by ultra-violet irradiation; but Brann (24) was unable to detect any such effect. Both papers include experiments on normal sera, which were not so altered by irradiation as to give any alexin fixation ("positive Wassermann reaction"). Hasskó (76), using irradiation even weaker than either of the above, reports that syphilitic antibody was inactivated. It seems probable that this is true, and that Brann was mistaken in claiming the contrary. Friedberger and Scimone (67) also report both fractions, analogous to alexin $\mathrm{M}$ and $\mathrm{E}$ pieces, to be more affected by irradiation than the controls. In another experiment they destroyed by ultra-violet irradiation the normal ability of rabbit serum to act like syphilitic antibody.

Brann (24) also tried to inactivate syphilitic antibody by $\mathrm{X}$ - and radium radiation: $\mathrm{X}$-rays up to 13 erythema doses had no effect on either positive or negative sera ("Metrorohr," voltage and amperage not given, filtration through $3 \mathrm{~mm}$. Al). Radium also had no effect, capsules 
containing 20 to $24.6 \mathrm{mg}$. $\mathrm{RaBr}_{2}$ being suspended in, or close to, the sera. No information is given as to whether alpha rays could reach the sera or not. This lack of information also vitiates the experiments of Friedberger and Shiga (68) who used $85 \mathrm{mg}$. of $\mathrm{RaBr}_{2}$ " in a metal capsule in needle form $2 \mathrm{~cm}$. long" (translation mine). These authors claim to have found some weakening of syphilitic antiserum, the process continuing after the end of irradiation. But the omission of essential details, such as the composition of the radiation incident on the serum, robs both of these papers of most of their meaning.

Other complement-fixing antibodies have been shown to be destroyed by ultra-violet irradiation in vitro, e.g., cholera antiserum [Baroni ancl Jonesco-Mihaiesti (13)].

Precipitins may be thought of as antibodies which form a visible precipitate instead of the supposed compound or amicronic precipitate responsible for alexin fixation. This property is destroyed by photodynamic irradiation, according to Fleischmann (60), and possibly also to a very slight extent by visible light alone. He also considered that oxygen may be essential, but this point was left unproven. The precipitins tested were rabbit sera precipitating beef or horse sera or egg white; sensitizers were eosin or safranin, 0.5 per cent, or methylene azure, 0.01 per cent; $8 \mathrm{hr}$. illumination resulted in complete inactivation. Fleischmann also showed that irradiated precipitin, even if incapable of producing a precipitate, still combined with or otherwise so affected the precipitable substance that it could not be precipitated by the addition of nonirradiated precipitin. When the precipitable substance was irradiated a similar situation resulted.

Doerr and Moldovan (44) also used rabbit anti-egg white serum, and found it to be inactivated by a few hours' ultra-violet irradiation from a Kromayer quartz-mercury-arc lamp. The first effect of irradiation was more in the nature of retarding precipitate formation than of reducing the amount; but in time the amount of precipitate formed was also reduced and finally no precipitate formed. The proper understanding of this retardation effect is important for titration of irradiated precipitins.

Lusztig (111) was unable to affect rabbit anti-horse serum albumen precipitins by X-radiation up to 10 H.E.D.

Agglutinins are antibodies which precipitate bacterial or other cells, and are, therefore, related to precipitins. There seems to be no record of their inactivation by visible or photodynamic irradiation, but presumably this would occur since they are easily affected by shorter wavelengths. This was first shown by Dreyer and Hanssen (46) who made a special study of the course of the inactivation process. Table 2 shows the extraordinary agreement which they found between experiment and theory, assuming the reaction to follow a unimolecular reaction isotherm. Unfortunately they do not describe their method of agglutinin titration, 
Table 2.-Observed and Calculated Titers of B. coli Agglutinin at Various Times during Inactivation by Ultra-violet Light*

\begin{tabular}{c|c|c}
\multicolumn{2}{c}{ After Dreyer and Hanssen (46) } \\
\hline Time, min. & $\begin{array}{c}\text { Observed } \\
\text { titer }\end{array}$ & $\begin{array}{c}\text { Calculated } \\
\text { titer }\end{array}$ \\
\cline { 3 - 3 } 0 & 350 & $(350)$ \\
10 & 264 & 267.4 \\
20 & 205 & 204.2 \\
30 & 156 & 156.0 \\
40 & 120 & 119.1 \\
50 & 91 & 91.0 \\
60 & 70 & 69.5 \\
90 & 32 & 31.0 \\
120 & 13 & 13.8 \\
\hline
\end{tabular}

* The calculated titers are based upon the assumption that the reaction follows the unimolecular reaction isotherm, namely, $k=1 / t \log [a /(a-x)]$, where $a$ is the initial titer, $a-x$ the titer at any time, $t$; and $k$ a constant.

and it hardly seems probable that they could have obtained values accurate to the third significant figure. Unless this was so, the perfection of the agreement must have been more or less fortuitous. It should be emphasized that this would not invalidate their conclusion that the

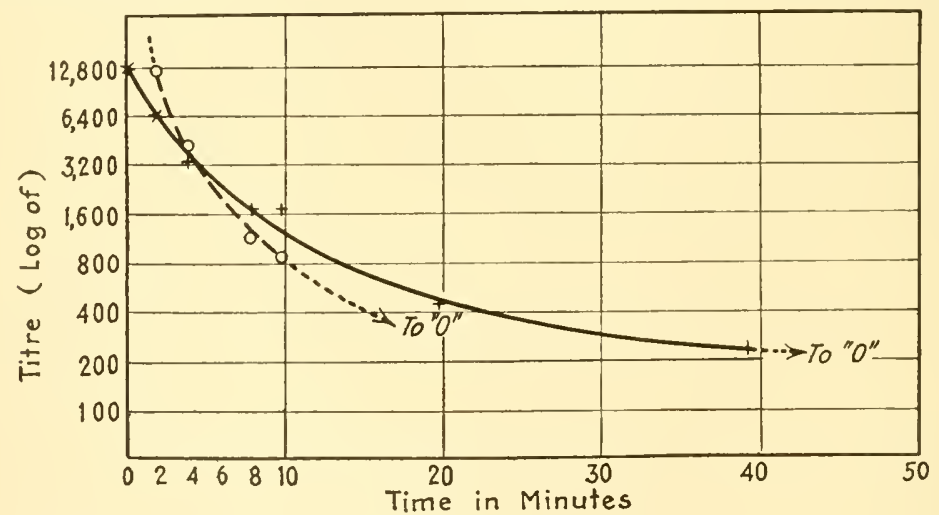

FIG. 3.-The time course of inactivation of typhoid $(x$

$x$ ) and paratyphoid $B$ $(o \ldots o)$ agglutinins. Ordinates represent titers on a logarithmic scale, and abscissas time on an arithmetic scale. If the processes followed the course of unimolecular reactions the curves should be straight lines. (After Heuer, 85.)

inactivation follows the course of a unimolecular reaction, as was also true for a large group of toxins and ferments studied by them at the same time (see page 345 ).

Heuer (85) has also given time curves of agglutinin inactivation by ultra-violet radiation; he used serum of asses immunized against typhoid, paratyphoid B, and cholera. His results do not accord very closely with what would be expected if the reaction obeyed the unimolecular reaction equation. Figure 3 shows the low degree of agreement; it is redrawn 
after Heuer's figures. It is possible that Hener's use of diluted sera ( 1 or 0.1 per cent) may have been responsible for his irregular results, just as Lundberg (110) states to be the case with alexin (see page 360 ).

Baroni and Jonesco-Mihaiesti (13) exposed horse anticholera serum to ultra-violet light and found that it lost its agglutinin property in 18 to 20 min., which was about double the exposure required to inactivate the bacteriolysin of the same serum. In their second paper (14) the same authors make a detailed comparison of the agglutinin of an antityphoid serum with its power to produce anaphylactic shock. The latter was more resistant to ultra-violet light.

Hasskó (76) gives various details as to the relation of original titer and dilution to the rate of inactivation by ultra-violet light, and by absorption on dead bacteria, separates a residual " $\mathrm{O}$ " agglutinin from an absorbed " $\mathrm{H}$ " agglutinin which is the more resistant to irradiation. The agglutinins used were active against Bacillus enteritidis Gärtner, B. typhosus, and B. dysenteriae Shiga, and had titers varying from 1600 to 50,000 .

$\mathrm{X}$-radiation was found not to affect agglutinins against either $B$. typhosus or B. pullorum. Fiorini and Zironi (59) exposed the former to doses up to $10 \mathrm{X}$, and Lusztig (111) exposed the latter to as much as 5 H.E.D.

Skrop (149) records a decrease in typhoid agglutinin titer produced by beta radiation, and an increase produced by alpha radiation (149a). He attributes these effects to decrease or increase in positive charge on agglutinin particles (micelles?) due to the absorption of negatively or positively charged beta or alpha particles respectively. The evidence and the conclusions are most suggestive.

Phagocytosis and Opsonins.-It is sometimes difficult to distinguish here between effects on leucocytes and effects on opsonins, which are defined as antibodies facilitating the phagocytosis of a particular type of cell. Thus Reiter's paper (137) records that radium radiation of blood increased its phagocytic power; the effect may have resulted from changes in either leucocytes or serum. The work of Chambers and Russ (34) makes it appear probable that the increase was due to effects on leucocytes, since all that Chambers and Russ could find upon $\alpha$-radiation of serum alone was a progressive loss of opsonin. Their technique has been described above (see page 358 ) and the effect was here again due solely to $\alpha$-radiation, the $\beta$ - and $\gamma$-rays being without effect. The course of opsonin inactivation was approximately that of a unimolecular reaction, or, as the authors say, "of a general exponential type." But the titer was taken as the ratio between the number of bacteria ingested per leucocyte in the irradiated citrated plasma and the corresponding number in the controls. This method is with justice considered by the authors theoretically vulnerable, and they accordingly caution against accepting 
the idea that the process of inactivation of opsonin by $\alpha$-radiation follows "rigorously" the course of a unimolecular reaction. Nevertheless, in view of similar findings in the case of alexin (Brooks, Lundberg) and various venoms, toxins, and immune bodies (Dreyer and Hanssen), this observation of Chambers and Russ seems significant.

Anaphylaxis.-Presumably when serum from a specifically sensitized animal is injected into a normal animal and induces the same type of anaphylactic sensitization in this animal, the effect is to be attributed to something analogous to an antibody. Doerr and Moldovan (44) irradiated the serum of a rabbit sensitized to egg white, and found that it had lost this power to confer passive sensitivity. This effect of ultraviolet radiation was rather slow, complete destruction requiring several times as long an irradiation as for the anaphylactic toxicity of the same serum.

Antitoxins.-Sera which protect animals against toxins, either from actual infections or injected, are called antitoxins. They appear to combine with toxins in vitro with resulting detoxication, and there is considerable evidence that the combination is stoichiometric.

Photodynamic inactivation of antitoxins was briefly mentioned by Tappeiner and Jodlbauer (155), and Huber reported inactivation of diphtheria and tetanus antitoxins by $4 \mathrm{hr}$. exposure to sunlight, eosin being present; free oxygen was necessary as for the corresponding toxins, already mentioned.

Ultra-violet radiation inactivates diphtheria antitoxin (Baroni and Jonesco-Mihaiesti, 13; Scott, 146). Particular interest attaches to Scott's experiment which was made in the hope that the danger of anaphylactic shock as a result of injecting antitoxic sera might be reduced by ultra-violet irradiation of the antitoxin. It transpired, however, that the antitoxic property was destroyed first, leaving the serum still able to produce anaphylactic shock. Scott (145), in an abstract of a paper read before the Pathological Society, gives several further details as to coagulability, salting out, and ionization of the serum proteins, particularly the globulin fraction. The report is so condensed as to make undesirable an extended consideration of the hypotheses advanced.

Lusztig (111) failed to find any significant effect of X-rays on tetanus antitoxin, even when 10 erythema doses were given.

Fermi (55) allowed direct sunlight to act on horse antirabies serum for 10 to $100 \mathrm{hr}$. He found that the longer the scrum was irradiated, the more quickly after infection of mice with rabies virus was it necessary to inject the antiserum in order to keep the mice alive. Insolation for $100 \mathrm{hr}$. made the antirabies serum totally inactive.

\section{IRRADIATION IN VIVO}

The irradiation of animals may have either direct or indirect effects upon the immune properties of their blood plasma. Earlier workers in 
some cases interpreted their observations in terms of direct effects of the radiation on circulating immune substances. This interpretation hardly seems justified in view of more recent work on the role of the reticulo-endothelial system in immune phenomena, and the practical certainty that at least some parts of this system will be affected by any type of irradiation. Jaffé (92) has recently reviewed the relation between the reticulo-endothelial or macrophage system and immune phenomena, and it need not, be further discussed here. The whole subject of irradiation in vivo is so involved with the theories of inmunity that it cannot be adequately considered here, but it is of such theoretical and therapeutic importance that a cursory survey and bibliography are given. The latter is certainly far from complete, but the nature of the evidence is such that no one paper is of great importance: it is only by the agreement of many papers that possibility becomes probability. It is probably not too much to say that there is no single paper whose conclusions are based upon statistically adequate data: most of them are pitiably inadequate. Under such circumstances disagreement and confusion need cause no surprise. And under such circumstances it does not seem worth while to assemble all the available references, even though doubtless there have been experiments along some lines which are here not touched upon.

The different groups of immune bodies may best be considered here about as above, except that the few references to toxins and antigens will be treated in connection with their corresponding antibodies.

\section{ALEXIN}

There seem to have been practically no experiments on the effect of visible light alone. Pincussen (132, page 415) refers briefly to unpublished tentative experiments by Lippmann and himself on guinea pigs with and without previous injection of eosin as a photodynamic sensitizer. Without dye no effect was observed, but with dye the alexin content of the serun decreased.

Mixed visible and ultra-violet radiation was studied by Huntemüller (88) who exposed healthy persons to general irradiation from Jesionek (quartz mercury) lamps long enough to cause moderate erythema. He claims to have observed a transient increase, a subsidence, and a secondary increase to 2 to 3 times the normal alexin titer. The paper is statistically very weak but derives some support from similar results obtained previously by Koopman (100) with guinea pigs. Koopman also noted an alexin decrease if irradiation was prolonged.

As to X-rays, the results are conflicting. Quadrone $(135,136)$ in two papers gives scantily supported evidence that weak daily doses lead to alexin increase in man and experimental animals. This is supported by Manoukhine's experiments $(114,115)$ upon which he based a theory of the mechanism of immunity which is now not accepted. (See Manoukhine, 116.) Manoukhine thought that X-rays caused leucocytolysis, 
and that alexin and antibodies were thus liberated into the blood stream. Bauer (15) and Heeren (79) do not confirm these results. Bauer's clinical data include no statement of dosage, but Heeren's dose, at least in the case of the one rabbit used, was bigger than Manoukhine's: 3 H.E.D. (which is roughly equivalent to $9 \mathrm{H}$.) as compared with $0.5 \mathrm{H}$., and was given but once and Manoukhine's daily.

Lusztig reports one experiment on one guinea pig which received an X-ray dose of 0.5 H.E.D. and the next day had an alexin titer 0.4 of its previous value (111). This is not very satisfying evidence.

The data neither prove nor exclude the possibility that the alexin content of the blood is increased by moderate ultra-violet or X-irradiation; apparently fatal or nearly fatal doses decrease it (Koopman, 100; Quadrone, 136).

\section{ANTIBODIES}

\section{Specific Hemolysins}

The treacherous nature of the evidence available in all experiments with irradiation in vivo is aptly illustrated by the papers of Konrich (99) and of Bessemans and coworkers $(18,19)$ on specific hemolysins and other antibodies. Both used approximately erythema doses from therapeutic quartz-mereury-arc lamps on shaven rabbits; both used as antigens sheep erythroeytes and killed $B$. typhosus; both used a considerable number of animals (5 to 12) for each experimental group, and both kept control nonirradiated groups for comparison. These are distinctly the most careful of the papers on hemolysins, and yet Konrich (99) finds hemolysins distinctly increased, and typhoid agglutinins not certainly affected or if anything retarded, while Bessemans and Nélis (18) find no effect on hemolysins, and Bessemans and Seldeslachts (19) a distinct increase in typhoid agglutinins. The contradiction is perfect. All that the reviewer can do is to assume that both sets of data are statistically inadequate, and that nothing has been proven.

Several investigators found under some conditions of X-irradiation an increased hemolysin titer at some time (Manoukhine, 114; Konrich, 99; Lusztig, 111). Lusztig found repeated irradiation to lead finally to partial or complete loss of hemolysin. Hektoen $(81,82)$ found only a loss, which was specially marked when irradiation preceded antigen injection, presumably injuring the reticulo-endothelial system so that it could no longer react effectively to the subsequent injection of antigen. Fiorini and Zironi $(58,59)$ obtained only negative results. Negative results were also obtained with alpha radiation from injected thorium $\mathrm{X}$ in various doses of the order of magnitude of $15 \mathrm{E.s.U}$. per $\mathrm{kg}$. in rabbits (Lippmann, 106).

It is evident that sufficiently heavy dosage of any of these agents will inhibit antibody production, and it is not impossible that appropriate 
irradiation after immunization is in progress may increase the titer of the free circulating hemolysin in the blood, even if only temporarily. This effect is perhaps analogous to the effects of injections of certain chemical reagents such as pilocarpin on antitoxins (Salomonsen and Madsen, 139) or $\mathrm{MnCl}_{2}$ on antitoxins (Walbum, 160) and hemolysins (Walbum and Schmidt, 161). It is impossible with the information given to draw any conclusions as to the dosages most favorable for demonstrating stimulation or inhibition.

There is no basis for concluding that hemolysins once formed are directly affected in vivo by irradiation of any kind.

\section{Bacteriolysins, Phagocytosis and Opsonıns}

Bacteria in blood may become incapable of colony production because the plasma kills them or because under the influence of opsonins they are ingested and killed by leucocytes. Although these may be independent processes there are so many experiments which do not separate the two that they are here considered as a group.

Azzi (9) interpreted his experiments on guinea pigs as showing that ultra-violet irradiation of infected wounds promoted both bacteriolysis and phagocytosis; overexposure decreased the latter.

Colebrook (35) observed an increase in the bactericidal power of blood on exposure of normal and pathological men to sunlight. Since serum did not show this change, he attributed it to an effect on phagocytosis, but in a later paper with Eidinow and Hill (36) a slight effect on the bactericidal power of serum was also claimed to occur in rabbits exposed to ultra-violet light or other irritants producing erythema. Genner (69) disagreed with these findings on serum and blood of ultra-violet-irradiated rabbits and men, but Eidinow (50) appears to have presented fairly clear evidence of the reality of the effect provided overdosage is avoided. No significant effect was produced by light of $\lambda>3100 \AA$, unless eosin or hematoporphyrin was injected to produce photodynamic effects; while increases up to 65 per cent resulted when light of $\lambda 2500$ to $3100 \AA$ was used in dosages producing mild erythema. Bamnerman (12) also obtained results supporting the conclusions of Colebrook and of Eidinow.

The promotion of the bactericidal power of blood by ultra-violet irradiation appears probable insofar as the production of substances or conditions promoting phagocytosis is concerned, but doubtful with respect to an increase in bacteriolysins. However, even the former is subject to some reservation, since Pincussen (133) was able to observe a greater proportion of phagocytes active in vitro after irradiation of guinea pigs with infra-red or long-wave-length visible light $(\lambda>7000 \AA)$ while the visible and near ultra-violet portion of the light from the "Vitalux" lamp, i.e., $\lambda 2800$ to $7000 \AA$, was inactive. Dark heating led to similar results (cf. Lüdke, 108). In Eidinow's and Colebrook's experi- 
ments heating of the blood close to the body surface as a result of infrared components of the light used may not have been avoided. It may well account for their results.

It is not certain whether similar criticism might not be directed at $\mathrm{X}$-ray experiments also, but here there is more pronounced leucocytosis or leucopenia depending on the dosage and the region irradiated. Increase in bacteriolytic power was reported by Manoukhine (114), Heidenhain and Fried (80), and Fried (65). Relatively small doses were given in all these cases, much less than an erythema dose. Both human and laboratory animal studies are included. Läwen (103) found no effect, perhaps because of the dosage he used which was apparently rather higher than those mentioned, or because he failed to make observations at the right time to detect what is at best a quickly transitory effect.

Hektoen (82) claims to have measured opsonins in sera of immunized animals, but since he nowhere makes any explicit statement and his tables refer either to hemolysin or to "antibody" otherwise unspecified, we can only guess his findings by analogy with those on hemolysin. If the analogy is valid, massive X-ray doses after immunization have no effect, as found by earlier workers; but heavy doses some days preceding immunization inpede or prevent opsonin formation.

The work of Dresel and Freund (45) on "plakin," the B. anthracis lysin of sera of horses, rabbits, etc., appears to refer to something other than a bacteriolysin. At best the bacteriolysins studied by all these workers seem to be far from specific in their action (cf. 65).

Several workers have noticed increased resistance of patients or laboratory animals to purulent infections after X-irradiation, and suggest increased bactericidal power of the blood as a probable reason. It is not clear how such cases should be classified, and the evidence is far from convincing (Manoukhine, 114, 115; Serena, 147; Lusztig, 111; also, for thorium X, Lippmann, 106).

Changes in the bactericidal power of blood as a result of opsonin production or destruction by the radiations of radioactive substances do not appear to occur, at least when tolerable dosages are administered (Reiter, 137; Albela, 4).

\section{Alexin Fixation and Syphilitic Antibody}

Attempts to produce syphilitic antibodies in the blood of syphilitic patients with negative Wassermann reactions by injection of thorium $\mathrm{X}$ were unsuccessful (Lippmann, 106). At the same time it was not found possible to abolish even by fatal doses of ultra-violet radiation the normal positive Wassermann reaction of rabbit blood serum (Friedberger and Scimone, 67).

Although Fiorini and Zironi $(58,59)$ speak of "Komplement-Ablenkung," they evidently refer not to deviation but to fixation of alexin. 
They were unable by $\mathrm{X}$-irradiation in doses of about " $16 \mathrm{X}$ " (about 3 H.E.D.) to affect this property of typhoid-immune rabbits.

\section{Precipitin}

Precipitin formation may be impeded or prevented by previous heavy $\mathrm{X}$-irradiation as shown by Benjamin and Sluka (16); this was confirmed by Hektoen (82). Benjamin and Sluka also observed that the injected antigen disappeared very slowly from the blood stream after irradiation. They used single or divided doses of 60 to $100 \mathrm{H}$., i.e., about 20 to 30 H.E.D. Leidenfrost's (104) failure to observe any similar effects of $\mathrm{X}$-irradiation appears to be referable to the much lower dosage used, and Lusztig's negative experiments (111) with exposures up to 10 H.E.D. after immunization confirm similar findings by the earlier workers. It is evident that $\mathrm{X}$-radiation sufficiently heavy to cause heavy injury to the reticulo-endothelial system interferes with subsequent antibody production, but it has not been shown to have any marked effect on antibody already produced.

\section{Agglutinins}

Visible and Ultra-violet Light.-Stäubli (153) refers briefly to some work which he did with v. Gonzenbach showing that at high altitudes animals lost their typhoid agglutinin more rapidly than at low altitudes, especially if exposed to sunlight. But this is probably misleading even if generally true, which seems doubtful. Clinical and experimental studies of the effects of exposure to a "Höhensonne" or similar source of visible and ultra-violet light on typhoid-agglutinin titer lead to no clear-cut conclusion. Hansen (72) reported irradiation to increase the titer of the serum of tuberculous patients who had previously been vaccinated against typhoid; and later $(73)$ he obtained similar results with rabbits. His experiments unfortunately are open to eriticism as to their statistical significance. Bessemans and Seldeslachts (19), confirming Hansen, and Konrich (99), failing to confirm, are, as mentioned above, both of quite reasonable statistieal validity, and their diametric disagreement is difficult to explain. A possible clue lies in Potthoff and Heuer's experiments (134) in which colored animals showed an increase, and unpigmented animals a decrease, as a result of daily irradiation during the development of immunity. Conditions affecting the amount and spectral distribution of the light reaching the capillary cireulation may be a major disturbing factor. On the whole it seems likely, though unproven, that irradiation of this general type can, under the right conditions, promote high agglutinin content of the serum.

Berntsen's finding (17) that agglutinins against B. tuberculosis were inereased in the sera of surgical-tuberculosis patients upon irradiation is based upon rather slender evidence. 
$X$-rays and Radioactivity.-Müller (122) claimed to have shown an increase in typhoid agglutinin in irradiated rabbits, but his data are totally inadequate. Läwen (103) found either no effect or an inhibition of subsequent agglutinin formation, which is more clearly evident in the work of Fränkel and Schillig (64). Fiorini and Zironi $(58,59)$ found essentially the same. Nevertheless, it was claimed by Manoukhine (114) and by Lusztig (111) that small X-ray doses (about 1/3 H.E.D.) increased the typhoid and cholera (Manoukhine) and B. pullorum (Lusztig) agglutinins of previously immunized animals. Their evidence is not very strong and needs confirmation.

Irradiation prior to immunization certainly inhibits agglutinin formation as it does that of other autibodies (Hektoen, 82; Hempel, 83), undoubtedly as a result of injury to the reticulo-endothelial system.

On the clinical side Kaznelson and Lorant (96), like Manoukhine (114) and Lusztig (111), claim to have found that small X-ray doses administered during the falling phase of typhoid agglutinin titer may lead to a temporary arrest of the fall, or even to an increase.

This is essentially what was found by Schütze (144) when he allowed rabbits to breathe radium emanation or injected radium salts in small doses, and also agrees with Lippmann's (106) observation on the effect of injecting thorium X. Fränkel and Gumpertz (63) find no effect with small doses of thorium $\mathrm{X}$, and a decrease with large doses. This may be because their thorium $\mathrm{X}$ was not administered at the right time.

On the whole there appears to be reason to suspect that small doses of $\mathrm{X}$-radiation or radioactivity given during the falling phase of agglutinin titer may give rise to a temporary increase. Irradiation before immunization leads to no change unless the dosage is large, and then only to an inhibition or prevention of agglutinin formation.

\section{Antitoxins}

Many supposed cases of protection of animals against disease by irradiation have been attributed to effects on antitoxin production. Here again as in the case of bacteriolysis (see above) the mechanism is obscure. Other explanations may be offered, such, for example, as the accelerated inactivation of toxins produced by momentary heating of the blood as it passes through the irradiated region. Sonne $(150,151,152)$ regards this as almost certainly the only cause of the protection afforded by visible light when guinea pigs are given a lethal dose of diphtheria toxin and then irradiated. Sonne used a 50-volt, 70-amp. carbon arc, condensing the light and filtering it to eliminate most of the infra-red and ultra-violet light. This, he points out, raises the blood stream to 47 to $48^{\circ} \mathrm{C}$. as it passes close to the surface of the body, and he calculates that a 2 -hr. irradiation would destroy as much toxin as a whole day of general fever. 
of $42^{\circ} \mathrm{C}$. Since toxin inactivation is accelerated enormously more than the toxic effect itself in the range between body temperature and $47^{\circ} \mathrm{C}$., this artificial fever should have inereased the length of survival of the guinea pigs used. Experiments showed that the mean length of life of the animals was: irradiated, 8.8 days; control, 3.5 days. This Sonne believed to mean that 40 per cent of the toxin had been destroyed. Other explanations he thought had only a speculative basis.

However, Lüdke (108) among others has shown that warming increases the amount of certain antibodies in the blood stream of immunized animals, and in particular notes this for diphtheria agglutinin.

Colebrook, Eidinow, and Hill (36), as well as Hartley (74), were unable to find any effect of visible or visible plus ultra-violet irradiation on the antitoxin titer of serum from animals immunized against diphtheria and then irradiated. Since their sources used only a few hundred watts compared with Sonne's 3500, and besides that were not colleeted and intensified by lens systems, the two sets of experiments are hardly comparable, and their failure to find any effect does not cast doubt on Sonne's findings, nor does it eliminate possible ehanges in antitoxin titer as possible participants in the effects observed by him. Moreover, Jodlbauer and Tappeiner $(94)^{5}$ seem to have given adequate proof that photodynamic irradiation saves animals from lethal doses of the toxin ricin by photochemieal effect, since survival is possible only when eosin has been recently injected at the site of toxin injection. Elsewhere it has relatively little effect. Accordingly we are also led to suspect the participation of direct photochemical inactivation of toxin in Sonne's experiments. Nevertheless, the probable participation of other mechanisms must not lead us to forget, here or elsewhere, that the action postulated by Sonne is, qualitatively at least, inevitable; it oeeurs regardless of the modifying effects of other simultaneous processes.

Gerhartz (70), too, adopted the explanation that toxin was destroyed in vivo by irradiation, this time with $\mathrm{X}$-rays, to explain the longer survival of rabbits given a lethal dose of diphtheria toxin and then irradiated. The conclusiveness of his data was attacked by Morgenroth (120) and the ensuing polemies add little to our knowledge but uncertainty. In any event, the considerations mentioned above for visible light apply here also.

Lusztig (111) was unable to find that $\mathrm{X}$-rays afforded any protection against diphtheria toxin alone, although small doses did seem to lengthen the life of animals given fatal injections of a toxin-antitoxin mixture, and to protect against Shiga dysentery toxin either alone or mixed with antitoxin. But the data are statistieally inconclusive, and speculation as to possible explanations therefore uncalled for.

${ }^{5}$ Photodynamic protection against toxins was also described by Tappeiner and Jodlbauer (155) and by Flexner and Noguchi (61). 
Buchner's experiment (30) on X-irradiation of mice vaccinated against Trypanosoma brucei or $T$. equiperdum showed similarly a decreased mortality from subsequent infection on the part of irradiated mice, but the author recognizes the result as statistically unconvincing. It may correspond to a real effect, although it is less marked than the protection against pneumococcus infection which Lippmann claimed to have been afforded by injections of thorium $\mathrm{X}$ into mice. Lippmann's findings (106) do not appear to have been confirmed.

Effects of visible plus ultra-violet irradiation on certain cutaneous immune reactions in tuberculosis have also been recorded (Müller, 123; Hirschmann, 86). The explanations of all these phenomena are entirely unknown.

\section{RÉSUME}

Reviewing the work of the last three or four decades on the effects of irradiation on toxins and immune bodies one might hope to be able to give answers to at least the following questions: (a) How sensitive to irradiation are these immune bodies, either absolutely or relative to better defined substances? $(b)$ What is the relative photolability of the different individual bodies or classes of them? (c) What are the relative effects of different types of radiation, both corpuscular and electromagnetic in relation to wave-length or quantum energy? $(d)$ What are the mechanisms and reactions involved in different cases? (e) What information is afforded as to the nature of toxins and immune bodies? To none of these questions can we give satisfactory answers. The prime difficulty is obvious as soon as we try to answer the first question.

a. An approach could be made by using monochromatic radiation of known energy flux, with appropriate irradiation chambers so that we could know how much and what kind of energy was being absorbed. But even then we would have no guarantee that the energy was being absorbed by any one chemical species, either the "body" being studied if that be a chemical individual, or any other which might be responsible for its inactivation. Until we do use appropriate irradiation conditions and can isolate the immune body free from other substances which absorb and dissipate energy, we shall have to content ourselves with something like the following answer:

All the substances in question, so far as they have been studied, are affected by irradiation, especially ultra-violet. The effect is usually inactivation, although in the case of alexin there is ground for suspicion that under some circumstances increased activity may result from brief irradiation. The dosage required is in general rather small, even where serum is used undiluted so that there is a great deal of light absorption by presumably inert components, i.e., components unrelated to the property measured. In general, we may hazard an estimate that toxins, alexin, 
and antibodies are more sensitive to most types of radiant energy than are most enzymes, simple protein solutions, etc. Even this estimate requires the reservation that it may only be that immunologieal methods are more sensitive indieators of ehange than are the methods in use in other fields.

$b$. The relative lability of various toxins, ete., under irradiation might again be stated with some confidence if we had pure substances having known optical characteristies, and standard conditions of (monochromatic) irradiation. A table can be made for all experiments with quartz-mercury-are lamps, arbitrary guesses being made as to corrections to be introduced for wattage, current density and temperature in the are (which affect the spectral composition of the light), distance from source, thickness of absorbing layer, and effectiveness of stirring, ete., and figures thus deduced which do not vary more than about tenfold for any one substance. For some substances such as alexin the margin of doubt is not so appalling, but nevertheless such a tabulation is not worth publishing. Perhaps the greatest obstacle to progress in this field is the obstinacy with which its workers, mostly ignorant of the basic principles of physical ehemistry, eling to worthless criteria such as "complete hemolysis" or "completely destroyed" as end points, leaving the reader to guess whether the process is, for example, 0.9 or 0.9999 complete.

We are thus left with the following slender harvest: in mammalian sera similarly diluted, under roughly equal intensity of ultra-violet irradiation, alexin is half inactivated in about $0.5 \mathrm{~min}$., those properties including lysins, agglutinins, precipitins, opsonins, ete., which are rather generally believed to be simply different measures of a single thing, are half inactivated in 2 to $20 \mathrm{~min}$, and antitoxin in perhaps $10 \mathrm{~min}$. Venoms and plant toxins have always been irradiated in rather transparent media, often in 1:10,000 dilution, and eomparisons with serum media would rest on treacherous ground. In these media they are half inactivated in perhaps 2 to 3 min. Exception must be made for moray serum, irradiated undiluted, which might on the same basis as antisera, be guessed to be half inactivated in about $4 \mathrm{~min}$. It should also be noted that Phisalix and Pasteur $(129,131)$ were unable to detoxicate the venom or serum of the asp (Vipera aspis) by ultra-violet irradiation. This result is so out of line with all other known facts that further investigation is needed. Bacterial toxins in bouillon media require perhaps a few seconds to 5 min.; purified toxins are more sensitive.

To summarize: it seems probable that alexin is the most sensitive to ultra-violet irradiation, antibodies somewhat less so, and, allowing for the greater transpareney of the media, venoms and toxins about the same as antibodies or more stabile. Data on other antigens do not justify even a guess, and it must be remembered that the above statement is only a guess. The order of stability as far as concerns the actual reactivity of 
the "bodies" concerned may just as well be utterly different, for all that we really know.

c. Experiments with other than ultra-violet radiation are for the most part so seattering that intercomparison of different substances (under $b$ above) or different types of radiation is difficult. This is especially true because of the meagerness of description of the physical nature of the radiation, the absorbing properties of the irradiated system, and, in the region of $\mathrm{X}$-rays and radioactive rays, the multiplicity of independently variable units, none of which is very satisfactory in practice. One can say with a good deal of confidence, however, that the properties under consideration are little if at all affected by visible light, except when photodynamic sensitizers are used; that from about $\lambda 3000$ to $2800 \AA$ on, lability increases with decreasing wave-length until excessive absorption makes experiment impracticable, while X-rays and gamma rays are relatively ineffective, and alpha rays effective. Much of this appears to be a question of how effectively the radiation is absorbed: there is good reason to believe that if absorbed the energy of any quantum greater than that corresponding to $\lambda \simeq 3000 \AA$ is utilized, and that the change induced is very closely proportional to the energy absorbed by the substances whose chemical ehange leads to inactivation.

$d$. If this be true, the mechanisms involved may well be quite similar except for electromagnetic radiation of $\lambda>3000 \AA$, approximately. Since at longer wave-lengths oxygen is required, while with larger quantum energy it may perhaps be made available by irradiation in the absence of free oxygen, it seems not improbable that inactivation of toxins and immune bodies by radiation may be due to oxidation following primary activation of oxygen. One must nevertheless keep in mind the process of protein denaturation and the ensuing colloidal changes, which, however, seem to be rather slower than the ehanges in toxins and immune bodies. Electrical discharge of colloidal particles by alpha and beta rays has also been suggested as a possible mechanism. Reduction processes have also been suggested. The last two hypotheses now seem relatively improbable, and oxidation the most probable. No one type of reaction may account for all or even any one of the changes described.

$e$. Irradiation tells us little about the chemical and physical nature of toxins and immune bodies. It is easy to conclude from analogy that they are single molecular species, that they possess such and such chemical nature or groupings; but analogy is not proof. The irradiation of alexin has led to suggestive hypotheses as to its dual nature: lytic substance with special dependence on the physicochemical state of serum proteins (globulins in particular) (see page 363). This amplifies the more generally accepted view that alexin is the latter only. Future work along physicochemical lines, including the better planned use of irradiation as a tool, should show whether or not this suggestion has worth. 
In any event studies of irradiation in immunochemistry have made and should in the future continue to make contributions to our knowledge of this field, which in spite of the welter of published papers still continues to be a field for pioneer research.

\section{REFERENCES}

1. Abelin, S., and O. Stiner. Die Einwirkung des ultravioletten Lichtes auf das Komplement des Meerschweinchenserums. Zeitsch. Immunitätsf. und Exp. Ther. Orig. 19: 1-8. 1913.

2. Achard, Сh., and Ch. Forx. Propriétés hémolytiques thermostabiles et propriétés antihémolytiques thermolabiles des sérums normaux pour les globules rouges de chien. Compt. Rend. Soc. Biol. [Paris] 73 : 593-595. 1912.

3. Achard, Ch., and Ch. Forx. Sur la pouvoir hémolytique thermostabile du sérum normal. Compt. Rend. Soc. Biol. 74: 658-660. 1913.

4. Albela, D. Deutsch. Med. Wochensch. 48:1347. 1922.

5. Anson, M. L., and A. E. Mirsky. Protein coagulation and its reversal. Serum albumen. Jour. Gen. Physiol. 14 : 725-732. 1931.

6. Anson, M. L., and A. E. Mirsky. The reversibility of protein coagulation. Jour. Phys. Chem. 35: 185-193. 1931.

7. Arrhenius, S. Immunochemistry. The Macmillan Company, New York; 1907.

8. Arthus, M., and H. N. W. Collins. Actions des rayons ultra-violets sur quelques venins et antivenins. Arch. Intern. Physiol. 30:250-266. 1928.

9. Azz1, A. Azione dei raggi ultravioletti sulla fagocitosi batterica. HaematoIogica 1: 435-449. 1920.

10. Baermann, G., and P. Linser. Über die lokale und allgemeine Wirkung der Röntgenstrahlen. Münch. Med. Wochensch. 51: 996-999. 1904.

11. Baker, S. L., and P. R. Peacock. The susceptibility of the infective agent of the Rous chicken-sarcoma to the action of ultraviolet rays. British Jour. Exp. Pathol. 7 : 310-316. 1926.

12. Bannerman, R. G. Über einige Wirkungen der Bestrahlung nittels Höhen- . sonne auf das Blut. Strahlentherapie 25 : 727-729. 1927.

13. Baroni, V., and C. Jonesco-Mihaiesti. Sur la destruction par les rayons ultra-violets des principes actifs des sérums normaux et préparés. Compt. Rend. Soc. Biol. [Paris] 68: 393-395. 1910.

14. Baroni, V., and C. Jonesco-Mrhaiesti. Sur la destruction par les rayons ultraviolets de la propriété antisensibilisine du sérum de cheval. (Contribution à l'étude du mécanisme de l'anaphylaxie). Compt. Rend. Soc. Biol. [Paris] 69 : $273-275 . \quad 1910$.

15. Bauer, R. Über den Komplementgehalt des menschlichen Serums. Med. Ǩln. 26: 1635-1636. 1930.

16. Benjamin, E., and E. Sluka. Antikörperbildung nach experimenteller Schädigung des hämatopoetischen Systems durch Röntgenstrahlen. Wien. Klin. Wochensch. 21: 311-313. 1908 .

17. Berntsen, A. Inquiry into the action of the carbon are light upon the blood in surgical tuberculosis. Hospitalstid. 61: 513-524. 1918.

18. Bessemans, A., and P. NÉlis. L'irradiation ultra-violette et l'élaboration des hétéro-hémolysines. Compt. Rend. Soc. Biol. [Paris] 99 : 329-331. 1928.

19. Bessemans, A., and A. Seldeslachts. Action des rayons ultra-violets sur l'élaboration des agglutinines bactériennes. Réduction et suppression de la phase de latence. Compt. Rend. Soc. Biol. [Paris] 99: 628-630. 1928. 
20. Budm, H. F. Physiological effects of light. Proc. Soc. Exp. Biol. and Med. 30: 718-721. 1933.

21. Bouverron, A. Action de la lumière sur la tuberculine en solution colorée par l'eosine ou l'erythrosine. Compt. Rend. Soc. Biol. [Paris] 87 : 1018-1020. 1922.

22. Bovie, W. T. The temperature coefficient of the coagulation caused by ultraviolet light. Science 37: 373-375. 1913.

23. Bovie, W. T. The action of the extreme ultra-violet of tropical sunlight on the complementing power of serum. Jour. Med. Res. 38: 335-344. 1918.

24. BRANN, G. Beitrag zur Wirkung der Röntgen-, Radium-, und Ultraviolettstrahlen auf die Komponenten der Wassermannschen Reaktion. Zeitsch. Immunitätsf. und Exp. Ther. Orig. 44:27-32. 1925.

25. Brooks, S. C. Complement destruction as a measure of the effects of radiation. Jour. Med. Res. 38 : 345-351. 1918.

26. Brooks, S. C. Precise titration of complement. Jour. Med. Res. 41: 399-409. 1920.

27. BRooks, S. C. The regeneration of complement after radiation or heating. Jour. Med. Res. 41: 411-424. 1920.

28. Brooks, S. C. The kinetics of inactivation of complement by light. Jour. Gen. Physiol. 3 : 169-183. 1920.

29. Brooks, S. C. The mechanism of complement action. Jour. Gen. Physiol. 3: 185-201. 1920.

30. Buchner, A. Über Kombinationen von spezifischer Immunisierung und Einwirkung von Röntgenstrahlen, von Hochfrequenzströmen und ultravioletten Strahlen. Zeitsch. Hyg. 74: 411-437. 1913.

31. Busck, GunNi. Die photobiologischen Sensibilisatoren und ihre Eiweissverbindungen. Biochem. Zeitsch. 1: 425-540. 1906.

32. Carmichael, E. B. The influence of chemical and other agents upon the toxicity and antigenic power of ricin. II. The detoxification of ricin by various agencies. Jour. Pharmacol. 35 : 193-221. 1929.

33. Cernovodeanu, P., and V. Henri. Action de la lumière ultraviolette sur la toxine tétanique. Compt. Rend. Acad. Sci. [Paris] 149: 365-368. 1909.

34. Chambers, Helen, and S. Russ. The action of radium radiations upon some of the main constituents of normal blood. Proc. Roy. Soc. [London] B 84: 124-136. 1911.

35. CoLEвrook, L. The influence of sunlight upon the bactericidal power of human blood. British Med. Jour. 1924, $2: 11$.

36. Colebrook, L., A. Eidenow, and L. Hill. The effect of radiation on the bactericidal power of the blood. British Jour. Exp. Pathol. 5: 54-64. 1924.

37. Courmont, J., and Th. Nogier. Action de la lampe en quartz à vapeurs de mercure sur la toxine tétanique. Compt. Rend. Acad. Sci. [Paris] 148: 655. 1909.

38. Courmont, J., and Th. Nogier. Sur la faible pénétration des rayons ultraviolets à travers les liquides contenant des substances colloïdes. Compt. Rend. Acad. Sci. [Paris] 149: 364-365. 1909.

39. Courmont, P., Th. Nogier, and A. Dufourt. Disparition de l'alexine des sérums par les rayons ultra-violets. Compt. Rend. Soc. Biol. [Paris] 74: 11521153. 1913.

40. Danielopold, D. Action des rayons ultra-violets sur le liquide céphalorachidien. Compt. Rend. Soc. Biol. [Paris] 73: 666-668. 1912.

41. Delbet, P., and A. Beauvy. Étude comparée de l'action des rayons ultraviolets sur la pouvoir hémolytique et sur l'état colloïdale du sérum sanguine. Compt. Rend. Acad. Sei. [Paris] 159: 278-281. 1914. 
42. Delezenne, C., and E. Fourneau. Constitution du phosphatide hémolysant (Lysocithine) provenant de l'action du venin de cobra sur la vitellus de l'oeuf de poule. Bull. Soc. Chim. 15: 421-434. 1914.

43. Diacovo, H. Nouvelles recherches expérimentales sur l'hémolyse. Arch. Inst. Pasteur, Tunis 15: 318-331. 1926.

44. Doerr, R., and J. Moldovax. Die Wirkung des ultravioletten Lichtes auf das Eiweissantigen und seinen Antikörper. Wien. Klin Wochsch. 24: 555-559. 1911 ,

45. Dresel, E. G., and H. Freund. Studien zur unspezifischen Reiztherapie. 2. Mitteilung: Über die experimentelle Steigerung der Anthrakozidie im Blute. Arch. Exp. Path. und Pharm. 91: 317-331. 1921.

46. Dreyer, G., and O. Haxssex. Recherehes sur les lois de l'action de la lumière sur les glycosides, les enzymes, les toxines, les anticorps. Compt. Rend. Acad. Sci. [Paris] 145: 564-566. 1907.

47. Eberson, F. Effect of ultraviolet rays on antigenic properties. I. Studies on meningococcus. Jour. Immunol. 5: 345-362. 1920.

48. Ecker, E. E. The action of the visible spectrum on complement. Jour. Infect. Dis. 31: 356-360. 1922.

49. Ecker, E. E., and H. H. Brittinghalr. Proteus hemotoxin. Amer. Jour. Hyg. 5: 662-668. 1925.

50. Eininow, A. Über die baktericide Wirkung der ultravioletten Strahlen. Strahlentherapie $25:$ 730-734. 1927.

51. Emmerling, O. Die Einwirkung des Sonnenlichts auf die Enzyme. Ber. Deut. Chem. Ges. 34: 3811-3814. 1901.

52. Fabre, G., and E. Ostrovsky. Action du sulfate de radium sur les toxines. Compt. Rend. Assoc. Franc. Av. Sci. 40: 122-123. 1911.

53. Fabre, G., and E. Ostroysky. Action du radium sur les toxines. Compt. Rend. Assoc. Franc. Av. Sci. 40: 1004-1006. 1912.

54. Famulener, L. W., and T. Madsen. Die Abschwächung der Antigene durch Erwärmung. (Vibriolysin, Tetanolysin, Ziegenserum-Hämolysin). Biochem. Zeitsch. 11: 186-209. 1908.

55. Fermi, C. Azione della luce solare sul siero antirabbico. Arch. Farmacol. Sper. 12 : 159-160. 1911 .

56. Ferni, C., and F. Celli. Beitrag zur Kenntniss des Tetanusgiftes. Centralbl. Bakt. und Paras. 12: 617-619. 1892.

57. Fermi, C., and L. Pernossi. Ueber das Tetanusgift. Centralbl. Bakt. und Paras. 15 : $303-310.1894$.

58. Fiorini, M., and A. Zironi. Immunicorps et rayons Röntgen. Arch. Eléctr. Méd. 24: 113-116. 1914.

59. Fiorini, M., and A. Zironi. Immunisatorische Phänomene und Milzbestrahlung. Strahlentherapie 6: 457-465. 1915.

60. Fueischuans, P. Die bei der Präzipitation beteiligten Substanzen in ihrem Verhalten gegenüber photodynamischen Stoffen. Münch. Med. Wochensch. 52: 693-694. 1905.

61. Flexner, S., and H. Noguchi. The effect of eosin upon tetanus-toxin and upon tetanus in rats and guinea pigs. Jour. Exp. Med. 8: 1-7. 1906.

62. Fränkel, E. Der Einfluss der Röntgenbestrahlung auf das hämolytische Komplement des Meerschweinchenserums. Berl. Kilin. Wochensch. 49: 20302032. 1912 .

63. Fränkel, E., and F. Gumpertz. Ueber die Einwirkung von Thorium X-Injektionen auf die Agglutinine. Berl. Klin. Wochensch. 51:209-210. 1914.

64. Fränkel, E., and K. Schillig. Ueber die Einwirkung der Röntgenstrahlen auf die Agglutinine. Berl. Klin. Wochensch. 50 • 1299-1301 1913. 
65. Fried, C. Bakterizidie nach Röntgenbestrahlung. Strahlentherapie 21: 56-72. 1926.

66. Friedberger, E. Weitere Versuche über ultraviolettes Licht. III. Mitteilung. Berl. Klin. Wochensch. $51:$ 1402-1405. 1914.

67. Friedberger, E., and V. Scimone. Zur Wirkung der ultravioletten Strahlen auf Antikörper, Antigene und auf die Komponenten der Wassermannschen Reaktion. Zeitsch. Immunitätsf. und Exp. Ther. Orig. 37: 341-362. 1923.

68. Friedberger, E., and A. Shiga. Ueber den Einfluss der Radiumstrahlen auf die Komponenten der Wassermannschen Reaktion. Zeitsch. Immunitätsf. und Exp. Ther. Orig. 39: 445-451. 1924.

69. Genner, V. Action des rayons chimiques de la lumière sur la pouvoir bactéricide du sang et du sérum. Compt. Rend. Soc. Biol. [Paris] 94: 603-606. 1926.

70. Gerhartz, H. Diphtheriagift und Röntgenstrahlen. Berl. Ḱlin. Wochensch. 46 : 1800-1802. 1909.

71. Granenitzki, M. Über die Regeneration des Komplements (nach Erwärmung). Biochen. Zeitsch. 38: 501-515. 1912.

72. HANSEN, $T$. The influence of the universal Light Bath on the amount of typhoid agglutinin in human blood, and some observations as to the effect of the light bath on rabbits injected with killed typhoid bacillus. Act. Med. Scand. 56: 629-639. 1922.

73. Hansen, T. Der Einfluss des allgemeinen Lichtbades auf die Menge des Typhusagglutinins im menschlichen Blut und einige Beobachtungen über dic Wirkung des Lichtbades auf mit abgetöteten Typhusbazillen behandelte Kaninchen. Strahlentherapie 16: 114-123. 1924.

74. HartLey, P. The effect of radiation on the production of specific antibodies. Jour. Exp. Pathol. 5 : 306-313. 1924.

75. Hartoch, O., W. Schürmans, and O. Stiner. Ueber die Einwirkung des ultravioletten Lichtes auf das Diphtherietoxin. Zeitsch. Immunitätsf. und Exp. Ther. 21: 643-653. 1914.

76. Hassкó, A. Vergleichende Untersuchungen über die Wirkung des ultravioletten Lichtes auf Komplement, Amboceptor, Agglutinin, die Wassermannsehe Reaktion, und die präcipitinogene Eigenschaft des Serums in Vitro. Biochem. Zeitsch. 226 : 462-481. 1930.

77. Hausmann, W., W. Neumann, and K. Schuberth. Der Einfluss des Lichtes auf Tuberkulin. I. Mitteilung. Zeitsch. Tuberk. 46:32-36. 1926.

78. Hausmann, W., and E. Pribram. Über die zerstörende Wirkung der Galle auf Toxine und Antitoxine bei Belichtung. Biochem. Zeitsch. 17: 13-20. 1909.

79. HeEren, J. Komplementäre Eigenschaften des Blutserums oder Alexinreaktion nach Röntgenbestrahlung. Strahlentherapie 42: 189-197. 1931.

80. Heidenhain, L., and C. Fried. Röntgenstrahlen und Entzündung. Klin. Wochensch. 3 : 1121-1122. 1924.

81. Hектоen, L. The influence of the X-ray on the production of antibodies. Jour. Infect. Dis. 17: 415-422. 1915.

82. Heкtoen, L. Further studies on the effects of the Roentgen ray on antibodyproduction. Jour. Infect. Dis. 22 : 28-33. 1918.

83. Hempel, K. Experimentelle Untersuchungen über den Einfluss von Röntgenstrahlen auf die Bildung von spezifischen Agglutininen. Strahlentherapie 26: 379-385. 1927.

84. Hertel, E. Ueber Beeinflussung des Organismus durch Licht, speziell durch die chemisch wirksamen Strahlen. Vergleichend-physiologische Untersuchungen. Zeitsch. Allgem. Physiol. 4: 1-43. 1904. 
85. Heder, G. Der Einfluss der ultravioletten Strahlen auf Antikörper in vitro. Zentralbl. Bakt. Paras. und Inf., 1 Abt., Orig. 88: 380-387. 1922.

86. Hinschiann, E. Über den Einfluss der Aureollampenbestrahlung auf die Immunität Lungentuberkulöser. Strahlentherapie 11: 420-428. 1920.

87. HUBER, HI. Weitere Versuche mit photodynamischen, sensibilisierenden Farbstoffen. Arch. Hyg. $64: 53-88.1905$.

88. Huntemüller, O. Die Wirkung der Lichtstrahlen auf die natürlichen Abwehrkräfte (Alexine) in Blutserum. I. UV.-Bestrahlung. Strahlentherapie 35: 489-500. 1930.

89. Jacoвs, M. H. Osmotic hemolysis and zoölogical classification. Proc. Amer. Phil. Soc. 70 : 363-370. 1931.

90. JАсовsohs, D. La fluorescence et la tuberculine reaction précoce. Compt. Rend. Soc. Biol. [Paris] 66 : 713-714. 1904.

91. JАсовY, M., and M. JАсовY. Über die Einwirkung der Sonnenbestrahlung auf das Komplement. Biochem. Zeitsch. 151:314-317. 1924.

92. JAFF́, R. H. The reticulo-endothelial system in immunity. Physiol. Rev. 11: 277-327. 1931.

93. Jansen, H. Ueber die Resistenz des Tuberkulins dem Licht gegenüber. Centralbl. Bakt. Paras. und Inf., 1 Abt. Orig. 41:677-680. 1906.

94. Jodlbauer, A., and H. von Tappeiner. Utber die Wirkung fluoreszierende Stoffe auf Toxine. Deut. Arch. Klin. Med. 85 : 399-415. 1905.

95. Kagawa, T. Contribution à l'étude de l'action des rayons actiniques. Sur le mécanisme de l'inactivation du complément par les rayons ultra-violets. Le Progr. Méd. III 36: 311-318. 1921.

96. Kaznelson, P., and J. St. Lorant. Allgemeine Leistungssteigerung als Fernwirkung therapeutischer Röntgenbestrahlungen. Münch. Med. Wochensch. 68: $132-134.1921$.

97. Kiтasato, S. Experimentelle Untersuchungen über das Tetanusgift. Zeitsch. Hyg. 10: 267-305. 1891.

98. Klingmüller, V., and L. Halberstaedter. Ueber die bakterizide Wirkung des Lichtes bei der Finsenbehandlung. Deut. Med. Wochensch. 31: 539-542. 1905.

98a. Kodama, T., and S. Shinomya. Taiwan Igakkai Zasshi. No. 214. 1921. [English abstract in: Jap. Med. World 1:28. 1921, with title: "A comparative experiment on hemolytic and bacteriolytic complement."]

99. KonRICH, Fr. Beitrag zur Wirkung von Röntgen- und Ultraviolettstrahlen auf Antikörper in vivo und vitro. Centralbl. Bakt. Paras. und Inf., Abt. 1, Orig. 95: 237-244. 1925.

100. Koopman, J. Über den Einfluss der ultravioletten Strahlen auf das Blut. Deut. Med. Wochensch. 50:277-278. 1924.

101. Kopaczewski, W. Influence des radiations lumineuses sur la toxicité du sérum de la murène. Compt. Rend. Soc. Biol. [Paris] 80: 884-885. 1917.

102. Kopaczewski, W. Recherches sur le sérum de la murène. Annales Inst. Pasteur 32: 584-612. 1918.

103. LÄwEN, A. Experimentelle Untersuchungen über das Verhalten röntgenisierter Tiere gegen bakterielle Infektionen unter besonderer Berücksichtigung der Bildung spezifischer Antikörper. Mitt. Grenzgeb. Med. Chir. 19: 141-186. 1909.

104. Leidenfrost, H. Experimentelle Untersuchungen über die Wirkung der Röntgenstrahlen auf tierisches Blutserum. Zeitsch. Röntgenk. 12: 186-191. 1910.

105. Lichtwitz, L. Ueber die Wirkung fluoreszierender Stoffe (des Eosins) auf normale und hämolytische Sera. Münch. Med. Wochensch. 51: 1589. 1904. 
106. Lippmann, H. Studien über die Steigerung der Resistenz und des Antikörpergehaltes durch Knochenmarksreizmittel: Thorium X, Arsenikalien usw. Zeitsch. Exp. Path. Therap. 16: 124-140. 1914.

107. Loewenstein, E. Über aktive Schutzimpfung bei Tetanus durch Toxoide. Zeitsch. Hyg. 62 : 491-508. 1909.

108. LÜDkE, H. Über die Bedeutung der Temperatursteigerung für die Antikörperproduktion. Deut. Arch. Klin. Med. 95: 425-456. 1909.

109. Lundberg, E. G. Sur la photolabilité du complément. Compt. Rend. Soc. Biol. [Paris] 85: 758.1921.

110. Lundberg, E. G. On the Photolability of Serum Complement. Jour. Immunol. 7 : 389-407. 1922.

111. Lusztig, A. Die Wirkung der Röntgenstrahlen auf die spezifischen Immunkörper. Zentralbl. Bakt. Paras. und Inf., 1 Abt., Orig. 111: 244-266. 1929.

112. МАснт, D. I. The influence of ultra-violet irradiation of menotoxin and pernicious anemia toxin. Proc. Soc. Exp. Biol. and Med. 24: 966-968. 1927.

113. Madsen, T., and T. Watabikt. Recherches sur la thermolabilité des compléments. Overs. K. Danske Vidensk. Selskabs Forh. 125-159. 1915.

114. Manókhine, I. I. Sur le rôle des globules blancs et de la rate dans la production de l'alexine, des hémolysines, des agglutinines et de les bacteriolysines. Compt. Rend. Soc. Biol. [Paris] 74 : 1221-1222. 1913.

115. Manoukhine, I. I. Sur l'influence de l'irradiation de la rate sur la tuberculose chez des singes et des cobayes. Compt. Rend. Soc. Biol. [Paris] 74 : 1263-1265. 1913.

116. Manoukhine, I. I. The treatment of infectious diseases by leucocytolysis produced by Röntgenization of the spleen. Lancet 200:685-687. 1921.

117. Massol, L. Action des radiations de la lampe en quartz a vapeurs de mercure sur le venin de cobra et sur son antitoxine. Compt. Rend. Soc. Biol. [Paris] 71: 183-185. 1911.

118. Megrail, E., and H. Welch. Antigenic power of ultra-violet irradiated tetanus Toxin. Proc. Soc. Exp. Biol. and Med. 28: 494-495. 1931.

119. Merlini, A. Raggi Roentgen e complemento. Giorn. Batt. Immunol. 4: 522-535. 1929.

120. Morgenroth, J. Diphtheriegift und Röntgenstrahlen. Berl. Kilin. Wochenseh. 46: 1962.1909.

121. Much, H., F. Peemöller, and A. Haim. Lichtwirkung und Kobragift. Münch. Med. Wochensch. 74: 1365-1366. 1927.

122. Müller, P. T. Über den Einfluss erhöhter Aussentemperatur und der Röntgenbestrahlung auf die Antikörperproduktion. Sitzungsber. K. Akad. Wiss. Wien. Math.-Nat. K1. 114(3): 479-496. 1905.

123. Müller. W. Eine Analyse der Wirkung nichtspezifiseher Mittel bei chirurgischer Tuberkulose. Münch. Med. Wochensch. 62: 1077-1078. 1915.

123a. Namiki, J. Jap. Jour. Dermatol. 32: 1023-1068. 1932. [English: pp. 117120.]

124. Noguchi, H. The photorlynamic action of eosin and erythrosin upon snake venom. Jour. Exp. Med. 8: 252-267. 1906.

125. Noguchi, H. Üher gewisse chemische Komplementsubstanzen. Biochem. Zeitsch. $6: 327-357.1907$.

126. Pfeiffer, H. Ueber die Wirkung fluoreszierender Stoffe (Eosin) auf normales Serum und rote Blutkörperehen. Wien. Klin. Wochensch. 18: 328-330. 1905.

127. Phisalix, C. "Influence des radiations du radium sur la toxicité du venin de vipère. Compt. Rend. Acad. Sci. [Paris] 138: 526. 1904.

128. Phisalix, C. Influence de l'émanation du radium sur la toxicité des venins. Compt. Rend. Acad. Sci. [Paris] 140: 600-602. 1905. 
129. Phisalix, M., and F. Pastedr. Actions des rayons ultraviolets sur le venin de vipère aspic. Compt. Rend. Acad. Sei. [Paris] 186: 538-540. 1928.

130. Phisalix, M., and F. PAsteur. Les rayons ultraviolets détruisent le pouvoir rabicide du venin de la vipère aspic. (Vipera aspis L.) Compt. Rend. Acad. Sci. [Paris] 186 : 975-977. 1928.

131. Phisalix, M., and F. Pasteur. Actions des rayons ultraviolets sur le sérum de la vipère aspic. Compt. Rend. Acad. Sci. [Paris] 186: 1756-1758. 1928.

132. Pincussen, L. Photobiologie. Georg Thieme; Leipzig, 1930.

133. Pincussen, L. Das Verhalten der Blutphagozyten voll bestrahlten Meerschweinchen. Strahlentherapie 39: 103-108. 1930.

134. Potthoff, P., and G. Heuer. Der Einfluss der ultravioletten Strahlen auf die Antikörper in vivo. Zentralbl. Bakt. Orig. 88: 299-306. 1922.

135. Quadrone, K. Klinische und experimentelle Untersuchungen über die Wirkungen der Röntgenstrahlen. Zentralbl. Inn. Med. 26: 521-526. 1905.

136. Quadrone, K. Klinische und experimentelle Untersuchungen über die Wirkung der Röntgenstrahlen. Zentralbl. Inn. Med. 26 : 593-597. 1905.

137. Reiter, H. Einfluss der Radium-Emanation auf die Phagocytose (Opsonine). Zentralbl. Röntgenstr. Rad. 1: 237-244. 1910.

138. Roux, E., and A. Yersin. Contribution a l'étude de la diphtérie. Ann. Inst. Pasteur 3: 273-288. 1889.

139. Salomonsen, C. J., and T. Madsen. Influence de quelques poisons sur la pouvoir antitoxique du sang. Compt. Rend. Acad. Sci. [Paris] 126: 1229-1233. 1898.

140. Scaffid, V. Einfluss des Schüttelns, der ultravioletten Strahlen und der Röntgenstrahlen auf das Komplement und den hämolytischen Amboceptor. Biochem. Zeitsch. 69 : 162-180. 1915.

141. Sсhoenholz, P., and K. F. Meyer. Effect of direct sunlight, diffuse daylight and heat on potency of botulinus toxin in culture mediums and vegetable products. Jour. Infect. Dis. 35 : 361-389. 1924.

142. Schubert, J. Studien über die Ultraviolettbestrahlung von Tetanustoxin, Rizin, hämolytischem Komplement und Fettsäuren. Zeitsch. Immunitätsf. und Exp. Ther. Orig. 58: 106-122. 1928.

143. Schubert, J. Komplementspaltung und Komplementwirkung. Zentralbl. Bakt. Paras. und Inf., I Abt. Orig. 122: 82-86. 1931.

144. Sснӥтze, A. Ueber den Einfluss des Radiums auf die Produktion von Antikörpern im tierischen Organismus. Med. Klin. 7 : 1729-1730. 1911.

145. Scort, W. M. Some effects of ultra-violet rays on serum. Jour. Pathol. Bacteriol. 16: 148. 1911.

146. Scotr, W. M. The effect of exposure to ultra-violet light on blood-serum. Proc. Cambridge Phil. Soc. 16: 124-125. 1911.

147. Serena, M. I concetti di Manoukhine sulla leucocitolisi provocata artificialmente coll'azione dei raggi X. Riv. Ospedal. 4: 419-422. 1914.

148. Seyderilelm, R., and G. Opitz. Über den Einfluss der ultravioletten Bestrahlung auf die Beziehung zwisehen Gift und Zellmembran. Strahlentherapie 28: 122-124. 1928.

149. Skrop, F. Aclátok az Immunsavók Physikai-Chemiai Jellegére Vonatkozólag. Magyar Orvosi Archivum 26:50-60. 1925. (See also (a) Ibid. 26: 399-400. 1925.)

150. Sonne, C. Essais expérimentaux au sujet de l'influence exercée par la bain de lumière universel sur l'action de la toxine diphthérique dans l'organisme. Compt. Rend. Soc. Biol. [Paris] 85: 759-761. 1921.

151. Sonne, C. Investigations on guinea-pigs relating to the influence of the Light Bath on the action of diphtheria toxin in the organism. Act. Med. Seand. 56: 619-628. 1922. 
152. Sonne, C. Untersuchungen an Meerschweinchen über den Einfluss des Lichtbades auf die Wirkung des Diphtherietoxins im Organismus. Strahlentherapie 16: 104-113. 1924.

153. StÄubli, C. Das Höhenklima als therapeutischer Faktor. Ergebn. Inn. Med. Kinderheilk. 11: 72-118. 1913.

154. Stiner, O., and S. Abelin. Ueber den Einfluss des ultravioletten Lichtes auf hämolytische Ambozeptoren. Zeitsch. Immunitat. 20: 598-605. 1914.

155. Tappeiner, H., and A. Jodlbauer. Ueber die Wirkung fluoreszierende Stoffe auf Diphtherietoxin und Tetanustoxin. Münch. Med. Wochensch. 51: 737-739. 1904.

156. Tappeiner, H., and A. Jodlbauer. Monograph. Leipzig, 1907.

157. Thом, C., Ruth B. Edmondson, and L. T. Giltner. Botulism from canned asparagus. Jour. Amer. Med. Assoc. 73: 907-912. 1919.

158. Tizzoni, G., and G. Cattani. Ueber die Widerstandsfähigkeit der Tetanusbacillen gegen physikalische und chemische Einwirkungen. Arch. Exp. Path. 28: 41-60. 1890.

159. Van Ermengem, E. Ueber einen neuen anaëroben Bacillus und seine Beziehungen zum Botulismus. Zeitsch. Hyg. 26: 1-56. 1897.

160. Walbum, L. E. Action exercée par le chlorure de manganèse et d'autres sels métalliques sur la formation de l'antitoxine diphtérique et l'agglutinine du B. coli. Compt. Rend. Soc. Biol. [Paris] 85: 761-764. 1921.

161. Walbum, L. E., and A. Schmidt. Die Bedeutung der Metallsalze für die Ambozeptorbildung. Zeitsch. Immunitätsf. und Exp. Ther., Orig. 42: 32-43. 1925.

162. Wexch, H. The effect of ultra-violet light on molds, toxins and filtrates. Jour. Prev. Med. 4: 295-330. 1930.

163. Wells, H. G. The Chemical Aspects of Immunity. New York, 1929.

164. Wheeler-Hill. Discussion of Seyderholm and Opitz q.cf. Strahlentherapie 28: 124.1928.

165. Yanagihara, H. Tappeiner's photodynamic phenomenon in acid and alkali solutions. Jour. Orient. Med. 1: 71-72. 1923.

166. Yoong, E. G. The coagulation of protein by sunlight. Proc. Roy. Soc. London B 93 : 235-248. 1922. 


\title{
THE EFFECTS OF RADIUM AND X-RAYS ON EMBRYONIC DEVELOPMENT
}

\author{
Elmer G. Butler
}

Department of Biology, Princeton University, Princeton, N. J.

Historical. Modifications in early stages of development. Fertilization-Cleavage -Gastrulation. Modifications in later stages of development. Differential sensitivity in embryonic development. Summary. References.

\section{HISTORICAL}

Many studies have been made during the last thirty years on the effects which radium and $\mathrm{X}$-rays exert on developing eggs and embryos. Two motives appear to have guided investigators into this type of work. First, there has been the desire to investigate, through the use of embryonic material, the fundamental nature of the alterations which are produced in living cells by exposure to radium and $\mathrm{X}$-rays. For investigations of this type the eggs of aquatic animals are particularly favorable, because of their uniformity and the ease with which they can be obtained in great abundance. Secondly, there have been studies which have been more particularly embryological in character. In these studies radium and $\mathrm{X}$-rays have been used simply as instruments of research. The method of experimental embryology is that of producing abnormal development in order more completely to understand the factors which underlie normal development. For this purpose various mechanical and chemical methods have long been employed, and to these has been added the method of radiation. In the following review no attempt will be made to separate these two aspects of the work which has been done on the radiation of eggs and embryos. Indeed, it has often been true that in a single investigation both aspects, the radiological and the embryological, have received equal attention.

In a survey of the literature dealing with the effects of radium and $\mathrm{X}$-rays on embryonic development one is struck by the scarcity of reliable quantitative data. The work which has been done in this field has been, for the most part, qualitative in nature, and in many cases, particularly in the earlier investigations, the qualitative results are not thoroughly trustworthy. In many cases the amount of radiation which reached the egg or embryo was either unknown or at least unstated, the area of the embryo which came under the influence of the radiation was undeter- 
mined, and little attention was given to the influence of external factors other than radiation, such as change of temperature or chemical changes in the medium. Only rather recently have investigators been occupied with the collection of reliable quantitative data with regard to the effect of specific dosages on specific phases of embryonic development. It is this field which at the present time offers the greatest possibilities for future research.

So far as known to the writer, the first recorded observations of the action of radium or of $\mathrm{X}$-rays on embryonic development are those of Bohn $(22,23)$, who submitted amphibian larvae and also the ova of the sea-urchin, Strongylocentrotus lividus, to the action of radium bromide. Bohn simply floated a glass tube containing a few centigrams of radium bromide on the water of a dish in which he had placed the larvae or the eggs which he used. He found that the general effect was a retardation of growth. Moreover, as a result of the radiation many of the amphibian larvae died in later stages of development and others developed into monsters which exhibited various abnormalities. With regard to the developing eggs of the sea-urchin he made the following generalizations. If the developing eggs were radiated before they began to gastrulate, development progressed only as far as gastrulation and then ceased. If gastrulation had already begun at the time of radiation, it might be completed, but the larvae which developed were abnormal. Bohn also exposed the ova and the spermatozoa of the sea-urchin beforefertilization, and reported that the ova withstood the radiation better than the spermatozoa. In a few cases he found artificial parthenogenesis induced by the radiation.

After the work of Bohn, several other investigators soon entered the field. Perthes (79) studied the ova of Ascaris, Schaper (85) studied the eggs and larvae of the frog and the newt, Gilman and Baetjer (39) worked on the developmental stages of the newt and the chick, and Tur (89) and Bordier and Galimard (25) also studied the developing chick.

The work of Schaper (85), which was completed after Schaper's death by Levy (63), represents one of the more critical of the early studies, and has served as a basis for subsequent work on amphibians. Schaper used larvae of the frog and the newt which he subjected to the action of radium bromide. He found that development was retarded, and that in later stages many developmental abnormalities appeared. These abnormalities were associated especially with the neural tube, the retina, the olfactory organs, the heart, and the blood vessels. Schaper and Levy extended their investigations to include a study of the action of radium in preventing regeneration in amphibian larvae, and also made some of the earliest observations on the effect of radium in suppressing mitosis.

Gilman and Baetjer (39) in their study of the effects of X-rays on the development of the newt and the chick reported that in each case there 
was an initial acceleration of development which occurred during a short period after radiation. They found, however, that the general effect of radiation was developmental retardation, and that many abnormalities appeared in radiated newts and chicks. Tur (89), who used radium bromide, and Bordier and Galimard (25), who used X-rays, also reported that arrested development resulted from the radiation of chick embryos.

The work of Perthes (79) is probably the most systematic of the earlier investigations. He exposed the ova of the nematode worm, Ascaris megalocephala, to various dosages of $\mathrm{X}$-rays and to $10 \mathrm{mg}$. of radium bromide for various lengths of time, and studied the changes which were brought about in the early stages of development. The general result, when the ova were exposed either to X-rays or to radium bromide was a slowing of development. The extent of the developmental retardation Perthes found to be proportional either to the X-ray dosage or to the length of exposure to radium bromide. Moreover, in addition to being much slower, cleavage in the radiated ova was in many cases very irregular and abnormal embryos often resulted. These abnormal embryos were usually irregular masses of cells which possessed low vitality.

All of the early investigations were important primarily in demonstrating that both radium and $\mathrm{X}$-rays exert a pronounced and often far-reaching effect on embryonic development in such widely separated animals as the echinoderms, the nematodes, the amphibians and the birds. The most commonly reported effect was general developmental retardation, which in some cases might be preceded by an initial acceleration of short duration. In connection with the retardation of development many structural abnormalities appeared. Later work has dealt for the most part with an analysis of the effect of radium and $\mathrm{X}$-rays on particular phases of embryonic development, and with a more critical examination of the abnormalities which are produced.

\section{MODIFICATIONS IN EARLY STAGES OF EMBRYONIC DEVELOPMENT}

FERTILIZATION

The effect of radiating one or both of the germ cells previous to fertilization has been the subject of considerable investigation. In his studies on amphibian development Bardeen $(18,19,20)$ reported that exposure of spermatozoa to X-rays reduced both their motility and their capacity to fertilize ova. Moreover, ova which were fertilized with radiated spermatozoa usually failed to develop beyond the gastrula stage, and the few gastrulac which continued development formed abnormal larvae. A few experiments by McGregor (65) gave substantially the same results. According to Bardeen, radiation of ova before fertilization had little effect on their capacity for fertilization by normal spermatozoa. Radiated ova, however, gave rise to a large percentage of abnormal larvae. 
In an extensive series of experiments by $\mathrm{O}$. Hertwig and his associates and by Packard the effect of radiating germ cells previous to fertilization has been carefully investigated. In the frog O. Hertwig (52) and G. Hertwig (48) found that radiation of either spermatozoa or ova previous to fertilization resulted in fewer abnormalities than when the fertilized egg was radiated. In addition, they made the surprising discovery that when normal ova were fertilized with spermatozoa, which had been radiated for a long time, the resulting abnormalities were fewer than when the spermatozoa had been radiated for a short time. In other words, the longer the radiation, the fewer were the abnormalities which resulted; the shorter the radiation, the greater were the number of abnormalities. This paradox was explained by the Hertwigs as follows: In the case of short exposures the chromatin content of a spermatozoon was so injured that after fertilization it was unable to funetion normally, it interfered with the normal movement of the egg chromosomes, and abnormal development of the fertilized egg ensued. On the other hand, if the radiation of a spermatozoon had been for a long period of time, for example, as long as $12 \mathrm{hr}$., then the ehromatin of the spermatozoon was so thoroughly injured that it took no part at all in fertilization. In this ease a spermatozoon simply served as an aetivating agent which incited the egg to development without the fusion of male and female chromatin. Development, therefore, was purely parthenogenetic. Likewise, the Hertwigs found that in the case of radiated ova which were fertilized with normal spermatozoa the damage which was produced was inversely proportional to the length of exposure. The longer the ova were exposed, the fewer were the abnormalities. Ova exposed for $15 \mathrm{~min}$., for example, gave more abnormalities and lived a shorter time than ova radiated for several hours. The Hertwigs eoncluded that in this ease, as in the case of the spermatozoa, the longer exposures so completely injured the chromatin of the egg nucleus that after fertilization the female chromatin failed to fuse with the male chromatin and, therefore, took no part in development. The development of the egg in such a case as this, therefore, was androgenetic.

The early conclusions of the Hertwigs, which were based on their experiments on amphibian spermatozoa and ova, were without cytological evidenee, but later G. Hertwig (49) repeated the experiments, fertilizing normal sea-urchin eggs with radiated spermatozoa. He was able to demonstrate clearly that sea-urchin ova which were fertilized with radiated spermatozoa developed parthenogenetieally. Development was of ten abnormal, however, because of a rather passive interference by the abnormal sperm head. Other experiments, in which either the spermatozoon or the ovum was radiated before fertilization, were made by P. Hertwig (56) using the eggs of Triton and the frog and by Oppermann (70) using trout eggs. Recently Simon (87) and Dalcq (35) have repeated 
the experiments of the Hertwigs on frog eggs, and in addition to radium these investigators have used X-rays and ultra-violet radiation. They report that when ultra-violet is employed the effects which the Hertwigs obtained occur more frequently than when either radium or X-rays are used.

Packard $(71,73)$ has followed in considerable detail in Nereis and in Chaetopterus the alterations in fertilization which occur after radiation. In the case of Nereis Packard found that when normal eggs were fertilized

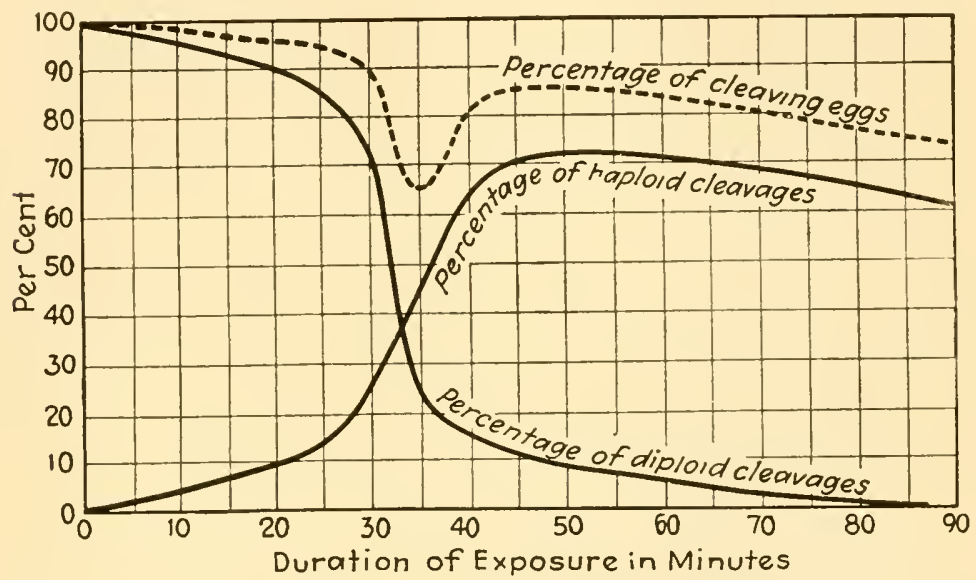

FIG. 1.-Percentages of haploid and diploid cleavages in Chaetopterus eggs, which were exposed to radium bromide and then fertilized by normal spermatozoa. The ordinate represents the percentage of eggs which cleaved, and the abscissa represents the duration of exposure in minutes. (From Packard, 73.)

by radiated spermatozoa, the extent to which the eggs were stimulated to development varied. In some cases the spermatozoon activated the egg normally and was drawn into the egg cytoplasm, but normal cleavage asters failed to develop and there was no fusion of male and female nuclear material. In other cases, however, the radiated spermatozoon activated the egg only to the extent of the extrusion of jelly and the formation of a fertilization cone, but the spermatozoon was not drawn into the egg. In Nereis, when the male and female nuclei do not fuse, the egg fails to develop. In this respect the situation is different from that in Ascaris. When Nereis ova were radiated, the maturation phenomena became irregular and the ova failed to develop properly, even though they were fertilized by normal spermatozoa. Packard found, also, that radiation of fertilized eggs resulted in more extensive developmental abnormalities than radiation of unfertilized ova.

In the case of Chaetopterus Packard was able to obtain decisive quantitative data with regard to nuclear behavior in radiated eggs which were fertilized by normal sperm. These data are shown graphically in Fig. 1, in which the ordinate represents the percentage of radiated eggs which 
cleaved and the abscissa represents the duration of exposure in minutes. It will be noted that as the length of exposure increased, the number of diploid eleavages decreased, and the number of haploid eleavages increased. With regard to the pereentage of eggs which underwent cleavage, it will be seen that brief exposures and also very long exposures did not prevent eggs from dividing. But at a point midway between these extremes was a period when only about 65 per cent of the eggs divided.

The question of relative sensitivity of spermatozoa and ova has been considered by Mavor and De Forest (67), using Arbacia, and by H. and M. Langendorff $(59,60)$, using Psammechinus. According to the quantitative results of these investigators, retardation in development is always greater when spermatozoa are radiated than when ova are radiated. The eytological basis for this result is not clear.

The only fertilization studies on higher animals are those of Asdell and Warren (8), who inseminated female rabbits with rabbit spermatozoa which had received large doses of $\mathrm{X}$-rays. Litters of apparently normal young were obtained from two rabbits so inseminated. It is difficult to correlate these results with the work of previous investigators on lower animals. Further studies on mammals would be of value.

\section{CLEAVAGE}

Probably no other single phase of development has reeeived so much attention as that of cleavage. Dividing egg cells have supplied suitable material, not only for a study of the effect of radiation on cleavage as such, but also for a study of the broader problem of the effect of radiation on eell division in general. It was indieated by the work of early investigators and has been shown conclusively by many later workers, that the application of either radium or X-rays in suitable dosages brings about a retardation in the rate at which the fertilized ovum undergoes division. Among the many investigators who have studied this problem have been Bardeen (20), O. Hertwig (52), P. Hertwig (54), G. Hertwig (49 and 51), Mottram (68), Richards (81), Packard (72), Richards and Good (83), Seide (86), Hammett, Green, and Davenport (41), and Henshaw (45).

Henshaw (45) has been able to colleet detailed quantitative data which demonstrate the effect of various doses of $\mathrm{X}$-rays on the cleavage of the Arbacia egg. In Henshaw's experiments unfertilized eggs were radiated and then fertilized with normal spermatozoa. The time required for the completion of the first cleavage in radiated eggs as compared with normal eggs was carefully recorded. The results show clearly that $\mathrm{X}$-radiation prolongs the time which elapses between insemination and the completion of the first cleavage. The degree of retardation is a function of the amount of radiation administered and also of the time intervening between the moment when radiation begins and the moment when insemination 
takes place. Such a result indicates that recovery of the egg from the effects of radiation may begin as soon as the radiation begins, with the result that the delay in cleavage dates not from the end of the radiation, but rather from the beginning of the radiation. Some of Henshaw's results are shown in Fig. 2, in which the relation of cleavage time to the length of exposure and to the minutes elapsing between radiation and insemination is demonstrated graphically. For correlation with these data it would be of value to know the nuclear and cytoplasmic changes taking place within the egg, particularly from the standpoint of the earlier fertilization

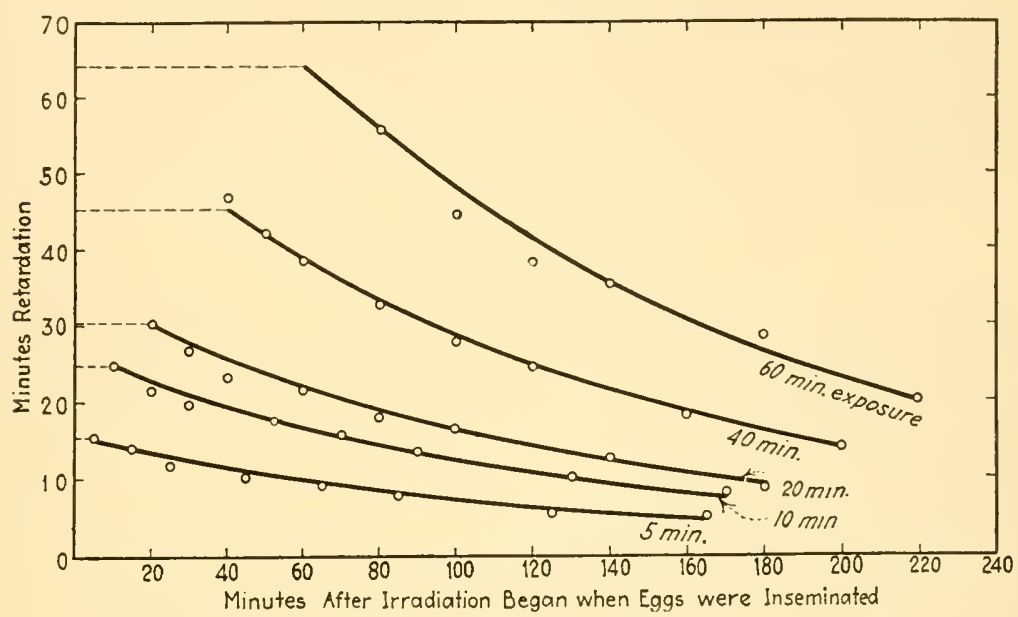

F1G. 2.- The effect of various doses of X-rays in retarding the first cleavage of Arbacia eggs. The ordinate represents the retardation of the first cleavage expressed in minutes, and the abscissa represents the minutes after irradiation began when the eggs were inseminated. (From Henshaw, 45.)

studies of G. Hertwig and of Packard which have been discussed above.

Although the data of Henshaw deal only with the retardation of the first cleavage, undoubtedly later changes are similarly affected. Differences arise, however, with regard to the exact moment at which cleaving eggs are radiated. Indeed, it has been shown that when eggs are radiated with the proper dosage at the proper time, acceleration instead of retardation of cleavage may result. Thus, Packard (72) found in the case of Arbacia that a brief but intense application of radium, during the period when the germ nuclei were approaching, accelerated the rate of division. Later exposures, which were made during the formation of the first cleavage spindle, accelerated the rate of cleavage to a greater or less degree, depending on the phase of mitosis at the time of exposure. Such results as these occur, however, only after brief radiation. Longer radiation always retards cleavage. Results similar to those of Packard have been obtained through the use of $\mathrm{X}$-rays by Richards (81) on the cleavage of Planorbis eggs and by Richards and Good (83) on Cumingia eggs. Hoffmann (57) has also reported an accelerated cleavage in frog eggs following 
light radiation. Ancel and Vintemberger (6), on the other hand, in their work on the eggs of the frog found no acceleration at all. They hold that radiation causes a retardation whatever the dosage employed.

One must come to the general conclusion, therefore, that retardation of cleavage after exposure to either radium or X-rays is the rule, and that acceleration is the exception. Light doses may accelerate cleavage; heavy doses always retard cleavage.

Extensive changes occur in the chromatin of eggs in which cleavage has been retarded by radiation. P. Hertwig (54) and Payne (78), have studied these chromatin changes in detail and have found that the chromosomes of radiated ova break up into granules and present a general disordered appearance. Packard (73) has found in Chaetopterus that the chromosomes which were apparently most seriously injured lacked spindle fibers and exhibited no signs of movement during cleavage.

\section{GASTRULATION}

As development of radiated individuals progresses, many abnormalities often become evident. The stage at which severe abnormalities first appear is, in most cases, that of gastrulation. Gastrulation is a period in development during which extensive cellular movement and cellular differentiation normally occur, resulting in the establishment of the tissues and organs of the new individual. Radiation so disturbs the orderly mechanism of development that the cells appear unable to go through the complex changes incident to gastrulation. Many investigators have observed the irregularities of gastrulation in radiated individuals. Bohn $(22,23)$ in his early study on the sea-urchin found that exposure of the blastula of ten prevented gastrulation. If gastrulation had already begun before the exposure, then the gastrula which was formed was often abnormal and short-lived. Perthes (79) found in the case of Ascaris that irregular cell masses developed instead of normal gastrulae and Tur $(90,91)$ made similar observations on the mollusc, Philine.

In the case of amphibians, imperfect gastrulation is one of the most commonly observed developmental abnormalities. Schaper (85), and later Hertwig (52), found that gastrulation in radiated amphibians was retarded and often atypical. Bardeen $(18,19,20)$, in his extensive observations on toad and frog development, found the effect on gastrulation so marked that he divided his larvae into two categories, one in which gastrulation was complete, and the other in which gastrulation was incomplete. Incomplete gastrulation sometimes ran as high as 75 per cent in eggs which were radiated during cleavage. In many cases, in which gastrulation appeared to be unaffected, no larval differentiation took place, and always there were large numbers of abnormal larvae. One of the most common results of abnormal gastrulation was the appearance of larvae with spina bifida. In one of Bardeen's experiments 
spina bifida occurred in 21.7 per cent of the individuals. Hertwig (52), and also Baldwin $(15,16,17)$, have reported the production of large numbers of animals with this defect. Recently abnormal gastrulation in radiated amphibian eggs has been studied by Simon (87), by Olivieri (69), and by Pasquini and Meldolesi $(76,77)$. Pasquini and Meldolesi have found early stages of gastrulation to be more sensitive than are later stages.

\section{MODIFICATIONS IN LATER STAGES OF DEVELOPMENT}

Except for the observations of Perthes (79), Tur (90, 91), and a few others, most of the detailed studies of developmental-abnormalities which occur after radiation have been made on amphibian larvae and on chick embryos. With regard to amphibians the most complete accounts will be found in the papers by Schaper (85), Levy (63), Hertwig (52), and Bardeen $(18,19,20)$. A survey of this work reveals that the external defects which are of most frequent occurrence are abnormally shaped heads, distended abdomens, and deformed or dorsally flexed tails. Examination of the internal organs shows that the nervous system, the eyes, and the vaseular system appear to be most susceptible to injury. Any or all of these abnormalities may occur without reference to the particular time at which radiation was made. Bardeen has shown that many of the abnormalities occur in experiments in which either the spermatozoa or the ova were radiated before fertilization. On the other hand, abnormalities are frequent also in cases where radiation was administered in relatively late stages of development.

Radiation of chick embryos in ovo results in extreme developmental disorders and often in the death of the embryo. Gaskell (38), Colwell, Gladstone, and Wakeley (30,31), Ancel and Vintemberger (2), Regaud, Lacassagne, and Jovin (80), Strangeways and Fell (88), Heim (44), and Butler (28) all describe in considerable detail the changes which occur as a result of various dosages of radiation. In ehick embryos, as in amphibian larvae, vascular disorders are common. These vascular disorders are undoubtedly associated with the fact that the vascular system is one of the first to be established and also is one of the most rapidly developing systems of the body. Moreover, the extra-embryonic circulation of the chick embryo is so extensive that small initial developmental arrests probably result in later and more extensive abnormalities in the embryonic and extra-embryonic circulatory pattern. One of the most obrious and also one of the most common occurrences is to find many areas of extravasated blood, both within the body and also in the extra-cmbryonic area of radiated chicks. Furthermore, in addition to vascular disorders, abnormal development of the nervous system, particularly the brain, is of frequent occurrence in radiated chick embryos. These nervous abnormalities conform to no particular pattern. They appear to be the result 
of early developmental arrests in the neural tube which are caused by the radiation.

The fact that the systems of the embryonic body, both in amphibians and in birds, which are most severely affected by radiation are the nervous and the vascular systems indicates a possible correlation between metabolic rate and radiosensitivity. The nervous and the vascular systems in early development are the most rapidly growing systems of the body. The regions where these are developing are undoubtedly the regions of highest metabolic rate. That these regions should be the most sensitive to radiation is very indicative of a correlation between metabolic rate and sensitivity.

Strangeways and Fell (88) have tested the possibility of a correlation between metabolic rate and sensitivity by using the method of tissue culture. Chick embryos after 6 days of incubation were exposed to $\mathrm{X}$-rays and then placed for $5 \mathrm{hr}$. in a refrigerator at $0^{\circ} \mathrm{C}$. At the end of the $5 \mathrm{hr}$. at $0^{\circ} \mathrm{C}$. tissue fragments of the heart, the sclerotic, the choroid, the intestine, and the skin were transplanted and grown in vitro. In the case of these cold-treated embryos fairly good growth was obtained from all of the tissues cultivated. On the other hand, in tissue cultures made from ehick embryos which were incubated for $5 \mathrm{hr}$. after the radiation no growth at all, or at best scanty growth, took place in any culture. From these results it appears, therefore, that the degenerative changes produced in the tissues by radiation are greatly delayed, if not completely arrested, by maintaining the embryos immediately after the radiation at a low temperature and thereby lowering the metabolic rate.

Concerning the relationship between sensitivity and metabolic rate in amphibian development, Ancel and Vintemberger $(1,6)$ have come to conclusions similar to those of Strangeways and Fell. Ancel and Vintemberger have found in their work with amphibian eggs that the extent of injury is a function of the work which normally is done after the radiation. In other words, the activity of a cell after the exposure determines to a great extent the degree to which the cell will be injured. If cellular activity after radiation is great, then the cells which are suffering from the effects of the radiation are unable normally to perform these activities and as a result abnormalities occur. On the other hand, if cellular activity after radiation is slight, then the cells may be able to recover from the effects of the radiation and no serious disturbances may result. Packard (74) has arrived at a similar conclusion in his studies on Drosophila eggs, which will be discussed later.

Another question with regard to the degenerative effects of X-rays and radium on embryonic tissues is whether these effects are due to a direct action of the radiation on the cells and tissues, or whether they are due to an indirect action resulting from the accumulation of toxic substances in the body fluids. In answer to this question, with regard to 
the chick, Strangeways and Fell have shown that fragments of normal embryonic tissues may be cultivated in vitro in a medium made from the undiluted extract of the tissue of radiated chick embryos. However, the tissue of radiated embryos was incapable of growth even in normal plasma. Such results as these indicate that death of the tissues of a radiated embryo is not due to the formation of stable toxic substances in body fluids.

Butler has come to similar conclusions with regard to amphibian development. It has been demonstrated that with proper dosage of X-rays one may prevent the development and also the regeneration of limbs in Amblystoma larvae (Butler, 27, 29). In this case the question arises as to whether the prevention of limb development and of limb regeneration is due to a direct action of the radiation on the cells which go into the formation of the limbs, or whether toxic substances are formed in the body which act upon the cells and prevent their differentiation. Recent experiments by Butler have shown that if one limb bud be shielded with lead and the remainder of the body be radiated, the shielded limb on the radiated larva will develop normally. Likewise, radiation of the posterior half of an Amblystoma larva, while the anterior half of the body is shielded, results in the growth of the anterior limbs and the complete suppression of the posterior limbs. It scems clear, therefore, from these experiments, as well as from the experiments of Strangeways and Fell, that the suppression of tissue growth and the initiation of tissue degeneration result from a direct effect of radiation on the tissues themselves and not from toxic substances contained within body fluids.

Gaskell (38) in his study of radiated chicks reported particularly on the mitotic changes which occurred within the tissues. He found that radiation brought about either a partial or a complete diminution of mitotic activity. Whether or not the embryo recovered, according to Gaskell, depended on the extent to which mitotic activity was diminished. This effect of X-rays on mitotic activity in chick embryos has also been studied by Regaud, Lacassagne, and Jovin (80) and by Butler (28).

The wall of the developing neural tube provides particularly favorable material for a study of the effects of radiation on cells in different phases of mitotic activity. Two types of cells are found in the wall of the neural tube of young chick embryos; one type, the germinal cells, with nuclei conspicuously active in mitosis, is restricted to the innermost layer of the neural wall; the other type of cell, with nuclei in the resting state and none in division, forms the main mass of the neural wall and is known as the mantle layer. Butler has shown that a study of the neural tube after radiation with a single nonlethal dose of $\mathrm{X}$-rays reveals a series of cellular changes as follows: an initial period of mitotic depression during which mitotic figures in the germinal cells disappear entirely; a period of pseudo-recovery during which mitotic activity reappears; a period of 
degeneration which is characterized by severe nuclear changes among the cells in the resting state; and finally a period of remarkable regeneration and recovery which results in a return of the tissue to its normal condition.

Except for the work on the neural tube, few detailed observations have been made on the effects of carefully controlled nonlethal doses of radiation on other tissues of the embryonic chick. However, observations by Colwell, Gladstone and Wakeley $(30,31)$ show that changes take place in probably every one of the embryonic tissues after radiation. A study of the nature of these alterations in each tissue and a determination so far as possible of the factors which govern them offer an attractive field for future investigation.

It has long been known from clinical observation and from experimental evidence that the radiation of pregnant mammals, either with radium or with X-rays, may seriously affect fetal development. The reports dealing with this subject, however, are for the most part of a very general nature and are scattered through biological and clinical literature. For more complete references than will be given here to the work in this field the reader is referred to the paper by Bailey and Bagg (14).

By far the most complete and systematic work on the effect of radiation on fetal development of mammals has been done by Bagg (9 to 12) and his collaborators. Bagg has treated breeding female mice and rats with radium and with $\mathrm{X}$-rays before and after mating, and also in late stages of pregnancy. His results show that when females after mating are injected subcutaneously with radioactive physiological salt solution, the formation of embryos is in many cases prevented. In other cases Bagg found that development began, but the embryos were either absorbed in utero or early abortion took place. In still other cases, abnormal young were born alive at term. The abnormalities which such offspring exhibited were principally serious vascular disturbances. There was a breakdown of capillary endothelium and the formation of prominent subcutaneous hemorrhagic areas. In general, these vascular disturbances appear to be of the same type as those which have been observed in radiated chick embryos by Strangeways and Fell (88) and other investigators.

Bagg found that injection of female mammals with radioactive salt solution before mating, instead of after mating, resulted in less disturbance of fetal development. However, in some cases the absorption of embryos or early abortion occurred, and in other cases the young which were born alive at term exhibited the characteristic subcutaneous hemorrhagic areas.

Bagg has also used the method of exposing the ventral abdominal wall of pregnant mammals, which were nearly at full term, to the action of the gamma rays of radium. This treatment resulted in the production of 
more extensive and more varied abnormalities than did the injection of radioactive physiological salt solution. These abnormalities became apparent sometime after the birth of the individual. Vascular disturbances, such as heretofore observed, were frequent and, in some cases, were so severe as to cause the death of the animal. Moreover, other abnormalities were often present such as gross imperfections in eye development, arrested development of certain brain regions, particularly the cerebral cortex, and in some cases arrested development of testes and ovaries. Bagg's conclusion with reference to mammals, that there is a "marked selective action of radium emanation on fast-growing embryonic structures," is in accordance with the many observations on lower animals, some of which have been described in preceding pages.

In his later studies Bagg used X-rays instead of radium. By the use of $\mathrm{X}$-rays he obtained disturbances in the development of the vascular, nervous and reproductive systems similar to those which resulted from the use of radium, and, in addition, he found serious abnormalities in the development of the kidneys (metanephroi). These kidney abnormalities were principally the absence either of one or of both kidneys, or the development of kidneys which were functionally imperfect. An embryological study of the kidney abnormalities found in the offspring of Bagg's radiated animals has been made by Brown (26).

Although the work of Bagg and his associates appears to establish the fact that radiation of pregnant mammals causes serious abnormalities in the offspring, conflicting reports are to be found in the literature. For example, Levine (62) reported that the administration of relatively large sterilizing doses of $\mathrm{X}$-rays to white mice during pregnancy brought about $n o$ defects in the offspring. An examination of the literature shows, however, that reports such as that of Levine are in the minority.

An extensive study of the possible inheritance of some of the developmental defects of radiated mice and rats, particularly defects of the eyes and the kidneys, has been made by Bagg and Little (13), Little and Bagg (64), and Bagg (12). Interesting and important as these genetical studies are, they do not properly fall within the bounds of the present discussion.

\section{DIFFERENTIAL SENSITIVITY IN EMBRYONIC DEVELOPMENT}

One of the central problems of the effects of radiation on embryonic development is that of differential sensitivity. With regard to this problem several questions arise. At what period during embryonic development is an organism most sensitive to radium and X-rays? Does sensitivity change with age? When are developmental abnormalities most likely to occur? Which organs or organ systems of the embryo are subject to the greatest alteration? The last two questions have already been dealt with in foregoing pages. Suffice it to say here, that the greatest 
amount of available evidence points to the period of gastrulation and body-axis formation as the time when abnormalities in radiated embryos most frequently oceur. On the other hand, embryos which survive this period of relative instability, or which are not radiated till gastrulation is past and the body axis is established, frequently exhibit serious abnormalities in the nervous and vascular systems and, in some cases, in the urinogenital system.

The other aspect of the problem of sensitivity, which was referred to above, deals with the relation between sensitivity and age. This problem is concerned not with the immediate or subsequent appearance of abnormalities; it is concerned rather with a determination of the amount of radiation which an egg or an embryo can withstand at a particular time and still remain viable, and with the question of a change in resistance with relation to age.

Bergonié and Tribondeau (21) have made a generalization with regard to radiosensitivity which has often been referred to as the BergoniéTribondeau law. This law holds that the sensitivity of cells varies directly with their reproductive capacity and inversely with their degree of differentiation. According to the Bergonié-Tribondeau law, therefore, sensitivity should decrease during embryonic development; older embryos should be more resistant than younger embryos. A review of the work in which any quantitative data at all are presented indicates that in most cases this generalization holds true. Bardeen (20), for example, observed that young stages in amphibian development were much more sensitive than older stages. The increase in resistance in the amphibian egg during development has been studied more recently by Woskressensky (94) who has colleeted important quantitative data. Perthes (79) in his early work on Ascaris reported that later stages in development were less sensitive than earlier stages. Holthusen (58) found in Ascaris eggs that maximum sensitivity occurred at the time of the first cleavage. After the first cleavage the sensitivity of the developing Ascaris gradually declined. In the development of the tobacco beetle, Runner (84) found that sensitivity decreased with age. Likewise, Mavor (66) has shown in Drosophila that the egg and larval stages are relatively very sensitive and that sensitivity gradually decreases during pupation. At the end of pupation an individual is approximately 35 times as resistant to X-rays as at the beginning of pupation, and nearly 100 times as resistant as at the time when the egg was laid. Figure 3, taken from Mavor's work, shows graphically the increasing resistance of the Drosophila egg during development from the egg to the imago.

In the development of a few organisms the generalization of Bergonie and Tribondeau apparently does not hold true. In the work of Strangeways and Fell (88) on the developing chick it has been shown that older chick embryos were more susceptible to destructive $\mathrm{X}$-ray effects than 
were younger embryos. Embryos which had been incubated $25 \mathrm{hr}$., for example, were more resistant to $\mathrm{X}$-rays than embryos which had been incubated 6 days. Such a result as this raises the question as to whether the tissues of the older embryos are actually less resistant than the tissues of the younger embryos, or whether, owing to the more complex organization of the older embryos, radiation produces physiological disturbances which are either absent or less pronounced in the younger embryos.

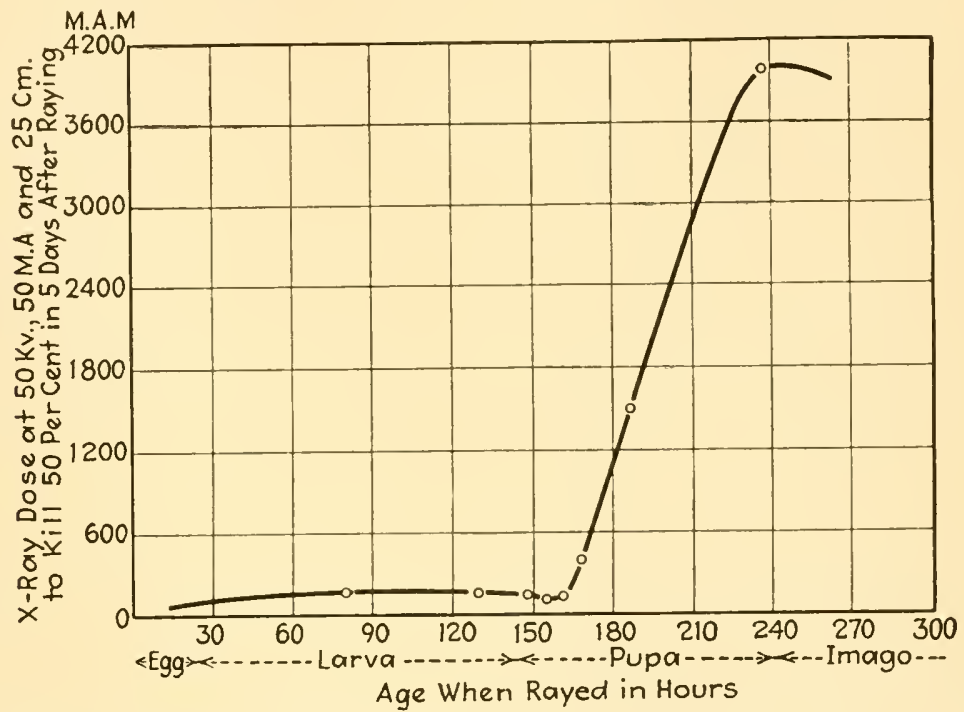

FIG. 3.-The variation in susceptibility to X-rays during the development of Drosophila. The ordinate represents the X-ray dose expressed in milliampere minutes, which was required to kill 50 per cent of the individuals within five days after the radiation. The abscissa represents the age of the individuals in hours at the time of the radiation. (From Mavor, 66.)

Tissue-culture studies of Strangeways and Fell indicate that the latter possibility is undoubtedly true, for, as they have shown, the cells, qua cells, of a 6-day chick are no more susceptible to the action of X-rays than those of a 25-hr. ehick. Strangeways and Fell believe that the death of radiated 6-day chick embryos is due, in part at least, to the interruption of gaseous exchange in the tissues of the chicks, such as might result from the clotting of blood in the vessels shortly after radiation. The developing chick embryo, therefore, becomes an exception to the generalization of Bergonié and Tribondeau, because we are dealing in the case of the chick with an indirect action of X-rays instead of with the direct action of the radiation upon the cells and tissues.

The greatest amount of evidence at hand, therefore, indicates that with a few exceptions, such as the chick, there is a gradual increase in resistance to $\mathrm{X}$-rays and radium as development of an organism progresses. Nevertheless, in some animals fluctuations occur in the upward curve of 
resistance. For example, it has been shown recently by Henshaw and Henshaw (46), using Drosophila, that during the $3 \mathrm{hr}$. after fertilization there is marked increase in resistance. This is followed during the next $2 \mathrm{hr}$. by a pronounced decrease to nearly the level at the time of fertilization. This decrease is then followed by a subsequent rise in resistance. This variation in resistance during the first $5 \mathrm{hr}$. after fertilization is not shown in Mavor's curve for Drosophila (Fig. 3). Henshaw and Henshaw have shown, furthermore, that the curve of resistance varies with the dosage of X-rays. They have demonstrated, also, that the curve of resistance to $\mathrm{X}$-rays is entirely different from the curve of resistance to alpha particles. When Drosophila eggs are most resistant to $\mathrm{X}$-rays they are most susceptible to alpha particles. This difference is apparently associated with the short penetration range of the alpha particles into protoplasm, and also with the movement of active cells during the formation of the blastula and the gastrula in the developing Drosophila egg.

Holthusen (58) has obtained interesting results in the case of Ascaris. He finds that Ascaris eggs are very resistant between fertilization and the first cleavage. At the time of the first eleavage there is a period of maximum sensitivity after which resistance gradually inereases.

The work of Woskressensky (94) on Axolotl and Drosophila larvae presents some of the most interesting and the most accurate data which are available on the relation of age to sensitivity. From the data of Woskressensky it is possible to predict accurately the time of death of a radiated embryo on the basis of the dosage of $\mathrm{X}$-rays and the age of the individual at the time of radiation. Woskressensky shows that with a given dosage the sensitivity of a developing organism deereases with age according to a hyperbolic function. He points out, furthermore, that decrease in sensitivity is synchronous with decrease in the velocity of growth. In other words, there appears to be an association between growth rate and sensitivity. Such a correlation as this must not be taken to mean, however, that sensitivity is dependent primarily on growth rate. Although an association between growth rate and sensitivity is clearly indicated, growth rate is probably only one of several factors which underlie sensitivity.

If it be true that growth rate and sensitivity are inseparably associated, then changes in external conditions which affect the growth rate should also affect the sensitivity of a developing organism. Using changes in temperature to alter the division rate of Drosophila eggs, Packard (74) has tested this hypothesis and found an indieation of a definite correlation between division rate and sensitivity. The higher the temperature at the time of exposure to $\mathrm{X}$-rays, the more sensitive are Drosophila eggs. In other words, as the temperature rises, the division rate increases and sensitivity also inereases. However, it is important to note that Packard's data show that the increase in sensi- 
tivity does not run parallel with the increase in division rate. Such a variation as this indicates the presence of other factors. Holthusen (58) and Dognon (37) have obtained results similar to those of Packard by modifying the division rate in Ascaris by temperature changes.

Still another aspect of the problem of growth rate and sensitivity must be taken into account. Strangeways and Fell, in their tissue-culture work, and Ancel and Vintemberger in their work on the ova of amphibians and birds have demonstrated the necessity in growth-rate studies of discriminating between the growth rate before or at the time of exposure, and the growth rate which would normally oceur after exposure. The growth rate and the general activity of cells, which would normally take place during the period immediately following radiation, appear to be of primary importance in determining the degree of injury which results from the radiation.

For a more complete understanding of this involved problem of growth rate and sensitivity it would be of value to possess accurate quantitative data showing the growth rate in larval stages of some animal whose growth rate eould be measured with aceuracy, and whose corresponding sensitivity to radiation could also be readily observed.

\section{SUMMARY}

Exposure to radium or to $\mathrm{X}$-rays results in pronounced and often far-reaching effects on embryonie development in all species of animals which have been investigated. Radiation either of one or of both germ cells prior to fertilization eauses abnormal fertilization, and, in some cases, leads to parthenogenetic or androgenetic development. The rate of cleavage is usually retarded by radiation, but under certain circumstances a slight but temporary acceleration of cleavage has been noted. The period of gastrulation and body-axis formation is a critical period for radiated individuals. During gastrulation and the formation of the body axis many abnormalities arise which lead in most eases to the production of monsters and frequently to death of the embryo.

Observations on the effects of radium and X-rays on avian and mammalian development have been restricted for the most part to the relatively late stages of organogenesis. In both birds and mammals radiation of an embryo affects particularly the most rapidly developing systems, such as the nervous and the vaseular systems. Abnormalities in either one or both of these systems are often so serious as to cause death of the radiated individual. Disorders in the reproductive and the nephric systems also often occur after radiation.

In general, the sensitivity to radium and $\mathrm{X}$-rays during development gradually decreases as the age of an individual increases. There is considerable evidence that decrease in sensitivity with increase in age may be associated with the decrease in growth rate. Although an 
association between sensitivity and growth rate undoubtedly exists, both in the development of the organism as a whole and in the development of individual tissues, nevertheless, it appears evident that additional factors also govern sensitivity. A determination of these factors is one of the central problems of radiation.

\section{REFERENCES ${ }^{1}$}

1. Ancel, P., and P. Vintemberger. De l'action des rayons $\mathrm{X}$ sur la segmentation et la gastrulation chez Rana fusca. Compt. Rend. Soc. Biol. [Paris] 91: 12671270. 1924 .

2. Ancel, P., and P. Vintemberger. Influence de l'activité cellulaire sur la manifestation des lésions produites dans le blastoderme de l'oeuf de Poule par les rayons X. Compt. Rend. Soc. Biol. [Paris] 91: 1425-1428. 1924.

3. Ancel, P., and P. Vintemberger. Etude de l'action des rayons $\mathrm{X}$ sur le développement embryonnaire. Les rayous $\mathrm{X}$ ont-ils une action aecélératrice? Arch. Biol. 35 : 1-43. 1925.

4. Ancel, P., and P. Vintemberger. Sur l'évolution comparée de l'embryon et du blastoderme dans les oeufs soumis à l'action des rayons X. Compt. Rend. Soc. Biol. [Paris] 92 : 172-174. 1925.

5. Ancel, P., and P. Vintemberger. Comparaison entre les effets des rayons $\mathrm{X}$ et ceux du vieillissement sur l'oeuf de Poule. Compt. Rend. Soc. Biol. [Paris] 92: 1401-1403. 1925.

6. Ancel, P., and P. Vintemberger. Influence de la rapidité d'évolution de l'oeuf irradié de Rana sur la durée de sa survie. Compt. Rend. Soc. Biol. [Paris] 93: 281-283. 1925.

7. Ancel, P., and P. Vintemberger. Influence de la température sur la radiosensibilité d'oeufs d'Oiseaux et de Batraciens. Compt. Rend. Soc. Biol. [Paris] 97: 796-799. 1927.

8. ASDELL, S. A., and S. L. WARREN. The effect of high voltage Roentgen radiation (200 kv.) upon the fertility and motility of the sperm of the rabbit. Amer. Jour. Roentgenol. and Radium Ther. 25:81-84. 1931.

9. BAGG, H. J. Disturbances in mammalian developnent produced by radium emanation. Amer. Jour. Anat. 30: 133-161. 1922.

10. BAGG, H. J. The absence of one kidney associated with hereditary abnormalities in the descendants of x-rayed miee. Proc. Soc. Exp. Biol. and Med. 21: 146-149. 1923.

11. BAGG, H. J. The absence of both kiclneys associated with hereditary abnormalities in mice. Proc. Soc. Exp. Biol. and Med. 21: 228-229. 1924.

12. BAGG, H. J. Hereditary abnormalities of the viseera. I. A morphological study with special reference to abnormalities of the kidneys in the descendants of x-rayed mice. Amer. Jour. Anat. 36: 275-311. 1925.

13. BAGG, H. J., and C. C. Litrue. Hereditary structural defects in the descendants of mice exposed to Roentgen-ray irradiation. Amer. Jour. Anat. 33: 119-145. 1924.

14. Balley, H., and H. J. BagG. Effects of irradiation on fetal development. Amer. Jour. Obstet. and Gynecol. 5: 3-15. 1923.

15. BALDWIN, W. M. The artificial production of monsters conforming to a definite type by means of x-rays. Anat. Rec. 17: 135-163. 1919.

${ }^{1}$ Mrs. R. M. Betts of the Biological Library, Princeton University, has aided in the compilation of these references. 
16. BALDWIN, W. M. The artifieial production of monsters demonstrating localized defeets as the result of injury from x-rays. Amer. Jour. Physiol. 62: 296-303. 1920 .

17. BALDWIN, W. M. The artificial production of syringomyelocele in the tadpole by means of x-rays. Anat. Ree. 22: 305-309. 1921.

18. BARDEen, C. R. Abnormal development of toad ova fertilized by spermatozoa exposed to the Roentgen rays. Jour. Exp. Zool. 4: 1-44. 1907.

19. Bardeen, C. R. Variations in susceptibility of amphibian ova to the x-rays at different stages of development. Anat. Rec. 3 : 163-165. 1909.

20. Bardeen, C. R. Further studies on the variation in suseeptibility of amphibian ova to the $x$-rays at different stages of development. Amer. Jour. Anat. 11: 419-498. 1911.

21. Bergoní, J., and L. Tribondeau. Interprétation de quelques résultats de la radiothérapie et essai de fixation d'une teehnique rationnelle. Compt. Rend. Acad. Sci. [Paris] 143: 983-985. 1906.

22. Вонs, Georges. Influenee des rayons du radium sur les animaux en voie de croissanee. Compt. Rend. Aead. Sei. [Paris] 136: 1012-1013. 1903.

23. Вонn, Georges. Influence des rayons du radium sur les oeufs vierges et féeondés, et sur les premiers stades du développement. Compt. Rend. Aead. Sei. [Paris] 136: 1085-1086. 1903 .

24. Border, H. Influence des rayons $\mathrm{X}$ sur l'évolution des vers à soie. Radium 2 : 410. 1905 .

25. Borpier, H. and J. Galimard. Action des rayons $\mathrm{X}$ sur le développement de l'embryon du poulet. Areh. d'élec. Méd. Exp. et Clin. 13: 491-494. 1905.

26. Brown, Alice L. An analysis of the developing metanephros in mouse embryos with abnormal kidneys. Amer. Jour. Anat. 47:117-171. 1931.

27. Butler, E. G. X-radiation and regeneration in Amblystoma. Science 74: 100-101. 1931.

28. Butler, E. G. On some cellular reactions to x-radiation. Jour. Exp. Biol. 9: 107-116. 1932 .

29. ButLER, E. G. The effeets of x-radiation on the regeneration of the fore limb of Amblystoma larvae. Jour. Exp. Zoöl. 65: 271-315. 1933.

30. Colwell, H. A., R. J. Gladstone, and C. P. G. Wakeley. The aetion of repeated doses of x-rays upon the developing chick embryo. Jour. Anat. 67: 1-11. 1922.

31. Colwell, H. A., R. J. Gladstone, and C. P. G. Wakeley. Action of x-rays upon the developing chick embryo. Ser. II and III. Jour. Anat. 60: 207-228. 1926.

32. Colwell, H. A., and S. Russ. Radium, x-rays and the living cell. G. Bell and Sons; London, 1924.

33. Colwell, H. A., and C. P. G. Wakeler. An introduction to the study of $x$-rays and radium. Oxford University Press, 1926.

34. DALCQ, A. À propos des effets de l'irradiation des gamètes chez les Amphibiens. Areh, d'Anat. Microscop. 25 : 336-371. 1929.

35. Dalce, A. Interprétation eytologique des effets, sur la gastrulation, de l'irradiation d'un des gamètes, ehez Rana fusca. Compt. Rend. Soc. Biol. [Paris] 104: 1055-1058. 1930.

36. Dantschakoff, V., and A. Lacassagne. Stérilisation par les rayons $\mathrm{X}$, de l'ćbauche gonadique du poulet. Ses effets sur le développement de la gonade. Compt. Rend. Soe. Biol. [Paris] 109: 1067-1069. 1932.

37. Dognon, A. Aetion de la température sur la radiosensibilité de l'ocuf d'Asearis. Compt. Rend. Soc, Biol. [Paris] 94: 466-468. 1926. 
38. Gaskell, J. F. The action of x-rays on the developing chick. Proc. Roy. Soc. [London] B 83 : 305-310. 1911.

39. Gilman, P. K., and F. H. Baetjer. Some effects of the Röntgen rays on the development of embryos. Amer. Jour. Physiol. 10: 222-224. 1904.

40. Haecker, V., and N. Lebedinsky. Über die beschleunigende Wirkung geringer Strahlendosierungen auf tierische Eier. Arch. Mikrosk. Anat. 85: 555-560. 1914.

41. Hammett, F. S., Matie Green, and Eleanor Davenport. The effect of radium on growth and development in eggs of Crepidula and Pagurus. Protoplasma 15: 415-421. 1932.

42. Hastings, S. On the action of the secondary X-rays from copper on the development of the ova of Ascaris megalocephala. Arch. Middlesex Hosp. 27: 154-156. 1912 .

43. Hastings, S., H. Beckton, and B. H. Wedd. A contribution to the study of the effects of radiation on silkworms. Arch. Middlesex Hosp. 27: 128-153. 1912 .

44. Heıм, K. Biologische Röntgenwirkungen, verfolgt beim Huhn vom Ei bis zum Organexplantat. Strahlentherapie 27:694-710. 1928.

45. Henshaw, P. S. Studies on the effect of Roentgen rays on the time of the first cleavage in some marine invertebrate eggs. I. Recovery from Roentgen-ray effects in Arbacia eggs. Amer. Jour. Roentgenol, and Rad. Ther. 27: 890-898. 1932.

46. Henshaw, P. S., and C. T. Henshaw. Changes in susceptibility of Drosophila eggs to alpha particles. Biol. Bull. 64 : 348-357. 1933.

47. Henshaw, P. S., C. T. Henshaw, and D. S. Francis. Relative effects produced by $200 \mathrm{kv}$. Roentgen rays, $700 \mathrm{kv}$. Roentgen rays, and gamma rays. III. Comparison based on effects on Drosophila eggs and on wheat seedlings. Amer. Jour. Roentgenol. and Radium Ther. 29: 326-333. 1933.

48. Hertwig, G. Radiumbestrahlung unbefruchteter Froscheicr und ihre Entwicklung nach Befruchtung mit normalem Samen. Arch. mikrosk. Anat. 77: 165-209. 1911.

49. Hertwig, G. Das Schicksal des mit Radium bestrahlten Spermachromatins im Seeigelei. Arch. mikrosk. Anat. 79: 201-241. 1912.

50. Hertwig, G. Parthenogenesis bei Wirbeltieren, hervorgerufen durch artfremden radiumbestrahlten Samen. Arch, mikrosk. Anat. 81: 87-127. 1913.

51. Hertwig, G. Strahleneinwirkung auf Wachstum und Entwickling. Handbuch der gesamten Strahlenheilkunde, Biologie, Pathologie und Therapie, Bd. 1. J. F. Bergmann; München, 1927. (Good bibliography.)

52. Hertwig, O. Die Radiumkrankheit tierischer Keimzellen. Ein Beitrag zur experimentellen Zeugungs- und Vererbungslehre. Arch. mikrosk. Anat. 77: $1-95,97-164.1911$.

53. Hertwig, O. Versuche an Tritoneiern über die Einwirkung bestrahlter Samenfäden auf die tierische Entwicklung. Arch. mikrosk. Anat, 82: 1-63. 1913.

54. Hertwig, Paula. Durch Radiumbestrahlung hervorgerufene Veränderungen in den Kernteilungsfiguren der Eier von Ascaris megalocephala. Arch. mikrosk. Anat. $77: 301-312.1911$.

55. Hertwig, Paula. Das Verhalten des mit Radium bestrahlten Spermachromatins im Froschei. Ein cytologischer Beweis für die parthenogenetische Entwicklung der Radiumlarven. Arch. mikrosk. Anat. 81: 173-182. 1913.

56. Hertwig, Paula. Durch Radiumbestrahlung verursachte Entwicklung von halbkernigen Triton- und Fischembryonen. Arch. mikrosk. Anat. 87: 63-122. 1916. 
57. Hoffmann, V. Über Erregung und Lähmung tierischer Zellen dureh Röntgenstrahlen. I. Experimentelle Untersuchungen an Froscheiern und -larven. Strahlentherapie $13: 285-298.1922$.

58. Holthusen, H. Beiträge zur Biologie der Strahlenwirkung. Pflüger's Areh. Ges. Physiol. 187: 1-24. 1921.

59. LangendorfF, H. and M. Strahlenbiologische Untersuchungen an den Keimzellen des Seeigels. Strahlentherapie 40: 97-110. 1931.

60. LangendorfF, H. and M. Strahlenbiologisehe Untersuchungen an befruchteten Seeigeleiern. Strahlentherapie 42 : 793-799. 1931.

61. Lazarus-Barlow, W. S., and H. Beckton. On radium as a stimulus of cell division. Arch. Middlesex Hosp. 30: 47-71. 1913.

62. Levine, M. The influence of Roentgen rays on white mice and their progeny. Amer. Jour. Roentgenol. and Rad. Ther. 17: 546-550. 1927.

63. Levy, O. Mikroskopische Untersuchung zu Experimenten über den Einfluss der Radiumstrahlen auf embryonale und regenerative Entwicklung. Arch. Entwicklungsmeeh. 21: 130-149. 1906.

64. Litrte, C. C., and H. J. BAGG. The occurrence of four inheritable morphologieal variations in mice and their possible relation to treatment with $\mathrm{x}$-rays. Jour. Exp. Zool. 41: 45-91. 1924.

65. McGregor, J. H. Abnormal development of frog embryos as a result of treatment of ova and sperm with Roentgen rays. Science 27: 445-446. 1908.

66. Mavor, J. W. A comparison of the susceptibility to x-rays of Drosophila melanogaster at various stages of its life-cycle. Jour. Exp. Zool. 47: 63-83. 1927.

67. Mayor, J. W., and D. M. De Forest. The relative susceptibility to x-rays of the eggs and sperm of Arbacia. Proc. Soc. Exp. Biol. and Med. 22: 19-21. 1924.

68. Motrram, J. C. On the action of beta and gamma rays of radium on the cell in different states of nuclear division. Arch. Middlesex Hosp. 30: 98-119. 1913.

69. Olvieri, F. Dell'azione dei raggi Roentgen sulle larve di Bufo vulgaris. Monit. Zool. Ital. $40\left(1 \frac{1}{12}\right): 442-444.1930$.

70. OppermanN, K. Die Entwicklung von Forelleneiem nach Befruchtung mit radiumbestrahlten Samenfäden. Arch. mikrosk. Anat. 83: 307-323. 1913.

71. Packard, Charles. The effect of radium radiations on the fertilization of Nereis. Jour. Exp. Zool. 16: 85-129. 1914.

72. Packard, Charles. The effect of radium radiations on the rate of eell division. Jour. Exp. Zool. 21: 199-212. 1916.

73. Packard, Charles. The effect of radium radiations on the development of Chactopterus. Biol. Bull. 35: 50-70. 1918.

74. Packard, Charles. The relation between division rate and the rudiosensitivity of cells. Jour. Cancer Res. 14: 359-369. 1930.

75. Packard, Charles. The biological effects of short rudiations. Quart. Rev. Biol. 6 : 253-280. 1931. (Extensive bibliography.)

76. Pasquini, P., and G. Meldolesi. Ricerehe sulla radio-sensibilità nello sviluppo delle ova d'Anfibi. I. Radiosuscettibilità differenziale dei vari stadi embrionali (Anuri). Atti. R. Accad. Naz. Lincei (Ser. 7) 10: 298-306. 1929.

77. Pasquini, P., and G. Meldolesi. Ricerehe sulla radio-sensibilità nello sviluppo delle ova d'Anfibi. II. Alterazioni specifiche e malformazioni seeondarie da radiosuscettibilità differenziale in Rana esculenta. Atti. R. Accad. Naz. Lincei (Ser. 7) 11: 705-712. 1930.

78. PAYNe, F. A study of the effect of radium upon the eggs of Asearis megalocephala univalens. Arch. Entwicklungsmech. 36:287-293. 1913. 
79. Perthes, G. Versuche über den Einfluss der Röntgenstrahlen und Radiumstrahlen auf die Zellteilung. Deut. Med. Wochensch. 30:632-634; 668-670. 1904.

80. Regaud, C., A. Lacassagne, and J. Jovin. Lésions microscopiques déterminées par les rayons X dans l'embryon de poulet. Compt. Rend. Soc. Biol. [Paris] 93 : $1587-1589.1925$.

81. Richards, A. The effect of x-rays on the rate of cell division in the early cleavage of Planorbis. Biol. Bull. 27 : 67-96. 1914.

82. Richards, A. Recent studies on the biological effects of radioactivity. Science 42 : 287-300. 1915 .

83. Richards, A., and D. J. Good. Notes on the effect of x-radiation on the development of Cumingia eggs. Biol. Bull. 37: 209-221. 1919.

84. Runner, G. A. Effect of Röntgen rays on the tobacco, or cigarette, beetle and the results of experiments with a new form of Röntgen tube. Jour. Agric. Res. $6: 383-388.1916$.

85. Schaper, A. Experimentelle Untersuchungen über den Einfluss der Radiumstrahlen und der Radiumemanation auf embryonale und regenerative Entwicklungsvorgänge. Anat. Anz. 25 : 298-314; 326-337. 1904.

86. Seide, J. Zur Kenntnis der biologischen Strahlenwirkung. Untersuchungen am Ascaris-Ei mit ultravioletten, Röntgen- und Radiumstrahlen. Zeitsch. Wiss. Zool. 124 : 252-304. 1925.

87. Simon, S. Effets de l'irradiation d'un des gamètes sur la gastrulation, chez Rana fusca. Compt. Rend. Soc. Biol. [Paris] 104: 1052-1055. 1930.

88. Strangeways, T. S. P., and Honor B. Fell. A study of the direct and indirect action of X-rays upon the tissues of the embryonic fowl. Proc. Roy. Soc. [London] B 102 : 9-29. 1927.

89. TUR, J. Sur les malformations embryomnaires obtenues pour l'action du radium sur les oeufs de la poule. Compt. Rend. Soc. Biol. [Paris] 57 : 236-238. 1904.

90. TUR, J. Sur le développement des oeufs de Philine aperta L. exposés à l'action du radium. Compt. Rend. Acad. Sci. [Paris] 149: 439-441. 1909.

91. Tur, J. Expériences sur l'action du radiun sur le développement de Pholas candida Lam. Compt. Rend. Soc. Biol. [Paris] 70: 679-681. 1911.

92. Vintemberger, P. Résultats de la suppression du blastopore, ehez Rana fusca, par irradiation localisée a l'hémisphère inférieur de la blastula, et théorie de l'origine blastoporale du dos de l'embryon. Compt. Rend. Soc. Biol. [Paris] 112 : 809-812. 1933.

93. Vintemberger, P. Sur les résults de la destruction, par irradiation localisée, de deux ou des quatre macromères du stade a huit cellules, dans l'oeuf de Rana fusca. Compt. Rend. Soc. Biol. [Paris] 112: 1083-1086. 1933.

94. Woskressensky, N. M. Über die Wirkung der Röntgenbestrahlung auf das embryonale Wachstun. Roux' Arch. Entwicklungsmech. Organ. 113: 447-461. 1928.

95. Zuppinger, A. Radiobiologische Untersuchungen an Aseariseiern. Strahlentherapie 28: 639-758. 1928. 


\title{
EFFECTS OF X-RAYS AND RADIUM UPON REGENERATION
}

\author{
IV. C. Curtis \\ University of Missouri, Columbia, Missouri
}

Porifera. Hydrozoa. Turbellaria. Nemertina. Chaetopoda. Tunicata. Amphibia. Physiological Regeneration. Diseussion. References.

The prime importance of irradiation in this field is its use as a technique. It is important that the effects of the rays upon regenerating tissues be ascertained in all their morphological, physical, and ehemical details, as in other cases where irradiation is used as a means of experimentation; but such knowledge waits upon a more extensive understanding of the effects of these radiations upon protoplasm. In the meantime, one can use the rays in a study of regeneration as one would use stains or reagents in producing results that can be utilized for experimentation, without knowing as mueh as might be desired concerning the manner in which the rays act upon the cells. In a representative series of animals it has been found that exposure to $\mathrm{X}$-rays or to radium reduces or completely destroys the power of regeneration, and in some instanees the irradiated individual remains alive for so long a period that its death seems to result from other causes, or at least to be indirectly connected with the irradiation. Healing of the wounded surface usually occurs in a normal manner, while the regenerative changes by which a complete individual is produced are inhibited in proportion to the exposure. In some of these cases the inhibition apparently results from the effects of the radiations upon specific types of eells which are neeessary for the regeneration. These cells are either destroyed or changed to such an extent that they cannot function normally; and such changes observed in cells may be regarded as the obvious, although perhaps not the only, cause for the lost power of regeneration.

An explanation that suggests itself is the well-known fact of the differential susceptibility of cells to the radiations in question. As formulated in the law of Bergonié and Tribondeau, undifferentiated germ cells, embryonic cells, and the less differentiated cells of adult animals are more susceptible to $\mathrm{X}$-rays and to radium than more differentiated cells. If regeneration involves the presence of cells with embryonic poteneies, which respond by division, movement, and differentiation and so restore the normal whole in a mutilated animal, or from a fragment, 
it may be supposed that the inhibition of regeneration by radium and $\mathrm{X}$-rays results from injury or destruction of these "embryonic" cells without which the regeneration camnot occur. To test such an hypothesis, one should inquire regarding the existence and the roles of cells with embryonic potencies in each particular case where the regeneration can be thus inhibited, and whether these cells are affected by the rays in question.

There are in theory four possible sources for new tissues in a regenerate: like cells may produce like; specialized cells may dedifferentiate and then redifferentiate into other cell types; unspecialized, "embryonic," or "formative" cells that have persisted from the early stages of the individual's development may be the source of new tissues; or the changes may include more than one of these processes. In the papers of Korschelt (32), Goetsch (19), and Hellmich (23) will be found accounts of this histological aspect of regeneration and many references. To review these histological relationships and the effects of irradiation, as described in particular cases, reference will be made to some of the recent papers which deal with the histology of regeneration in the various phyla and to studies of regeneration that have been undertaken with the technique of irradiation.

\section{PORIFERA}

The conditions in Porifera may be cited, although it can be said that true regenerative processes do not occur in sponges because the members of this phylum have no specialized organs that can be removed and then restored. Some genera, such as Spongilla, will produce new functional sponges from cuttings, but many others do not have this power, although healing and formation of some new tissue undoubtedly occur. On the other hand, the reduction bodies formed by certain species $(68,69$, and 47) have long been known to "regenerate" new sponges, and sponge gemmules develop in a manner that resembles regeneration. The formation of new sponges by the reunition masses produced from dissociated sponge cells should be mentioned, although such a process may be regarded as too extreme a reorganization to be called regeneration. In the case of reunition, recent histological studies $(68,70)$ indicate that dedifferentiation and redifferentiation into cells that may be of another type do not occur in the reassociation and subsequent changes by which the new sponge is formed, and this seems equally true in reduction and the formation of a new sponge that follows (47). The only cells that survive in numbers are the choanocytes, which lose their collars and flagella temporarily but become normal choanocytes again in the newly forming mass; and the archaeocytes, one type of which, the nucleolate cells, can form all the other cell types including choanocytes; while another type, which includes smaller and more active cells, forms only 
the new epidermal membranes upon the external surface and lining the canals. The epidermal areas and the more specialized cells of the middle region seem to have no part in the restoration. It is, however, difficult to be sure of what happens if one has recourse only to the common techniques of fixation and staining even though eheeked by study of the living material. According to Wilson (68, page 166) "it has yet to be proven that sponge cells ever dedifferentiate into a regenerative, embryonic condition, although the dissociation phenomena in hydroids lend some support to the view that this is possible"; and again, "the retention by cells of their essential nature, even after complete morphological dedifferentiation, is illustrated by the dissociated collar cells."

Conclusive evidence that archaeoeytes are essential for reunition in sponges might be obtained by means of the technique of irradiation which appears not to have been employed in the attack upon this problem. From unpublished experiments conducted by the writer upon the reunition and subsequent changes in Microciona prolifera after exposure to $\mathrm{X}$-rays, it appears that such irradiation retards the reunition proeess and formation of the new sponges; but the data are inadequate for satisfactory conclusions. The finding by Wilson and Penney (70) of three principal types of cells, which are important in the reunition of sponges, may be significant in relation to the appearance of dermal cells, gastral cells, and arehaeocytes, as the primary cell types in the differentiation of certain sponges. A comparative study, by the technique of irradiation, of differentiation in embryonic development, in gemmules, and in the reduction and reunition masses of sponges might yield important data upon the general problem of differentiation as well as data upon this problem in the Porifera.

\section{HYDROZOA}

In coelenterata the histology of regeneration has proved difficult to follow, and its study has not been undertaken in many speeies that have been used extensively for investigations upon the general physiological aspects, or what may be called the "organismal" factors in contrast with the histological factors involved. The hydras alone seem to have been extensively studied with regard to these cellular changes. In this family the trend of the recorded observations for many years favored Nussbaum's account (46) of the interstitial cells as an undifferentiated stock from which all the other cell types arose, both in reproduction and in the normal growth of the animal. The abundance of such totipotent cells explained the great powers of regeneration observed in these animals. On the other hand, Rowley (50), in a brief paper without figures, concluded that in Hydra viridis "the new cells which appear during the regeneration of the hydra are formed by division of the old cells throughout the entire piece, as in the normally growing animal." She found 
evidence for division in the large cells of both ectoderm and endoderm and raised the question whether the division of interstitial cells so commonly observed during regeneration might not be related primarily to the formation of new cnidoblasts and their nematocysts, which the regenerate produced in large numbers when tentacles and hypostome were being restored. Hadzi $(20,21)$ concluded that buds arose from interstitial cells, and Schulze noted (53) that all tissue elements of the hydra arose from such cells. Gelei (18), however, found mitoses frequently in all cell types of Hydra grisea, except cnidoblasts and the cells of the tentacles, and concluded that the whole organism was not far from an embryonic state. Kanajew $(29,30)$ regarded the regeneration in Pelmatohydra oligactis as a result of the transformation of interstitial cells into other cell types, since he found mitoses rare in the differentiated cells and abundant in the undifferentiated or interstitial cells. He described the latter as migrating through the supporting lamella and then differentiating into the new endoderm cells. In a later study (31) his earlier observations were not substantiated, and he concluded that the interstitial cells played an insignificant part in regeneration and budding, since regenerating parts and buds were formed principally at the expense of the neighboring differentiated cells, as shown by histological study and by the transplantation of parts that had been vitally stained. The observations of Strelin (61) upon Pelmatohydra oligactis further support the concept of an embryonic role for the interstitial cells; but this interpretation is not supported by the observations of McConnell (43) who described abundant mitoses in the epitheliomuscular cells of $P$. oligactis and hence concluded "there is no evidence that the indifferent interstitial cells of the ectoderm or endoderm are elaborated into the large epitheliomuscular cells," and further, in a second paper (44), that the secretory cells of the endoderm also reproduced by mitosis. There seems no reason to consider the possibility of amitosis in hydras, since only mitosis has been recorded by recent investigators.

With such contrasting views one despairs of a solution of the problem by the ordinary methods of histological study, although the presumption favors the conclusions of investigators such as Kanajew, who has changed his opinion, or McConnell, whose microphotographs demonstrate the existence of mitoses which he describes as frequent, rather than the opposite conclusions of Strelin (61) and others who may have overlooked mitoses in their material. There is also the possibility that species of hydras differ in this particular, or that the members of a species exhibit seasonal differences. A new mode of observation or experimentation might yield facts that would be more convincing than any available by older methods. If, for example, one could destroy all the interstitial cells without too great injury to the individual, it would be possible to determine the potencies of the cells that remained. It appears that such 
an elimination of interstitial cells can be virtually accomplished by the technique of irradiation.

In correlated investigations Zawarzin (71) and Strelin (62) have described the gross and also the histological effects of $\mathrm{X}$-rays upon regeneration and budding in Pelmatohydra oligactis. The exposures, given as $79 \mathrm{r}$ per min. ranged from about 100 to $395 \mathrm{r}$. The gross effects of this irradiation, as described by Zawarzin, included both a stimulation and a retardation upon budding as well as regeneration. These effects were so related to the irradiation that the stimulating effect appeared immediately after exposure, the retarding effect only after an interval or latent period. There was no latent period in the sense of a period during which no recognizable changes occurred. The total effect was the sum of these retarding and stimulating factors. As would be the case with temperature, there were optimum and maximum exposures so that the rule of Arnd-Schulze held for $\mathrm{X}$-rays in this instance. In addition to the primary stimulation by the rays, there appeared in later stages following heary exposures a sccondary stimulation as evidenced by the regeneration. The operations for regeneration resulted in an increased resistance to the rays, since operated individuals survived strong exposures better than nonoperated individuals. Spring hydras were found to be more resistant to the rays than summer hydras. The quantitative method of estimating the rapidity of regeneration by number of tentacles regenerated was regarded by Zawarzin as sufficiently accurate for estimation of the biological effects of the radiation. The failure to secure clear evidence for a stimulating effect of such radiations in the higher vertebrates Zawarzin believed due to complexities of the physiological reactions which mask any such response that may occur. $\mathrm{He}$ accepted the conclusions of others that such a stimulating effect can be demonstrated in plants, in protozoa, in various invertebrates, and even in the lower vertebrates, as shown by the more rapid growth of axolotl larvae after weak exposures to X-rays (27).

Strelin (62), in a histological study of Zawarzin's material, found a complete agreement between the occurrence and activities of interstitial rells and the power of regeneration in these $P$. oligactis. The increased rate of regeneration, which Zawarzin considered evidence for a stimulating effect by the rays, was correlated by Strelin with a more rapid differentiation of interstitial cells and hence their more rapid disappcarance from the ectoderm, with eventual retardation or loss of the power of regeneration. There were no interstitial cells to be found in the later stages of hydras receiving heavy exposures and such hydras had no power of regeneration (Fig. 1, $A$ and $B$ ). These heavily exposed individuals showed a certain degree of degeneration which Strelin attributed to the loss of functions normally performed by the interstitial cells. The most significant cases appear to be those following exposures in which some 
interstitial cells survived (Fig. 1, $C$ and $D$ ). In correlation with the destruction of interstitial cells, such an individual lost for the time being its power of regeneration; but the few surviving interstitial cells began to multiply and eventually the power of regeneration was restored. Strelin concluded that destruction of interstitial cells by the X-rays caused loss of the power of regeneration and of budding, and that the data obtained by Zawarzin and himself furnished conclusive proof that the interstitial cells functioned as a reserve of embryonic material from which the other cell types were differentiated.

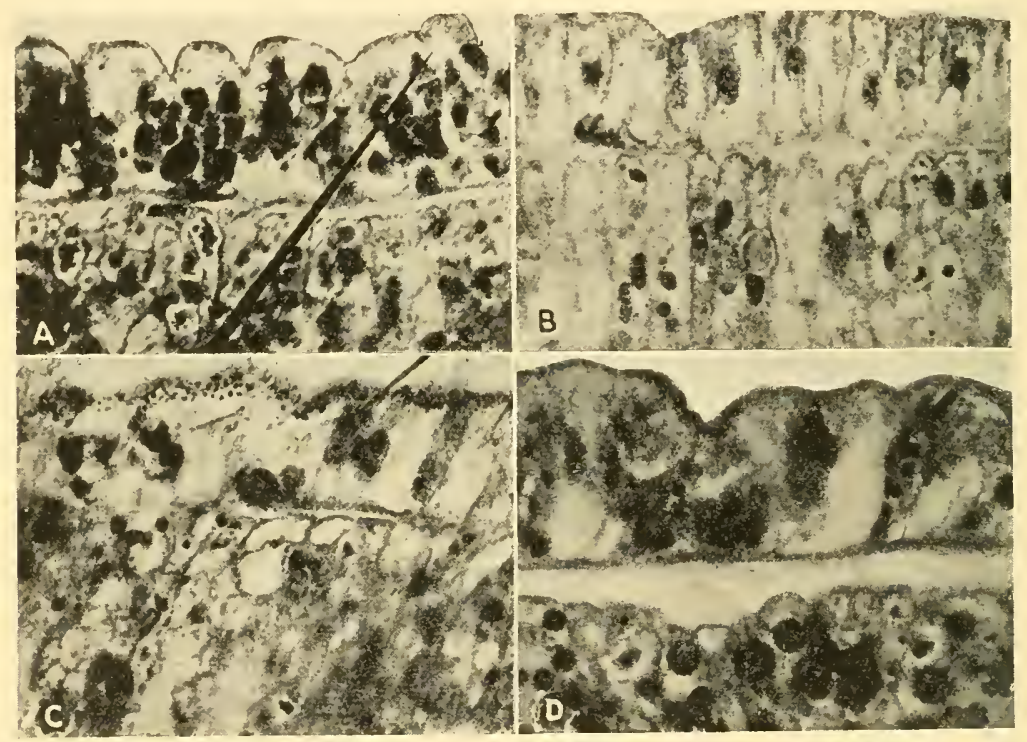

FIG. 1.-Microphotographs showing effect of X-rays upon cells of Pelmatohydra oligactis. $A$, from longitudinal section of a normal specimen showing ectoderm, supporting lamella, and part of endoderm. $B$, from similar section of an irradiated specimen showing no interstitial cells in ectoderm. C, from a 3-day irradiate showing early stage in restoration of interstitial cells from a few such cells that survived. $D$, later stage of this restoration. (From Strelin, 62.)

The results described by these two investigators seem to justify Strelin's conclusions, although it can be argued that the rays affect all the cells of the hydra and so alter the physiological state of the individual as a whole that it becomes temporarily incapable of regeneration, and with heavicr exposures its state becomes so much altered that death ensues. Moreover, one is critical of Zawarzin's interpretations where the combination of stimulating and retarding effects is intermingled in the manner described. These results should be thoroughly checked by other workers familiar with the technique of irradiation and the histology of hydras. To prove the case beyond question it would be necessary to produce hydras in which the interstitial cells had all been destroyed and in which the capacity for regeneration as well as that to perform other 
functions apparently dependent upon these cells had been lost, yet, in spite of these losses, organisms in which the power to live indefinitely persisted, just as a mammal sterilized by irradiation may live out its usual span of existence. This might be impossible with the hydra, because some necessary function of the organism, such as the formation of nematocysts, might be lost with the destruction of the interstitial cells, and further because the normal span of life could not be determined.

Many investigators have ascribed a similar embryonic role to the interstitial cells in marine hydrozoa ever since the investigations of
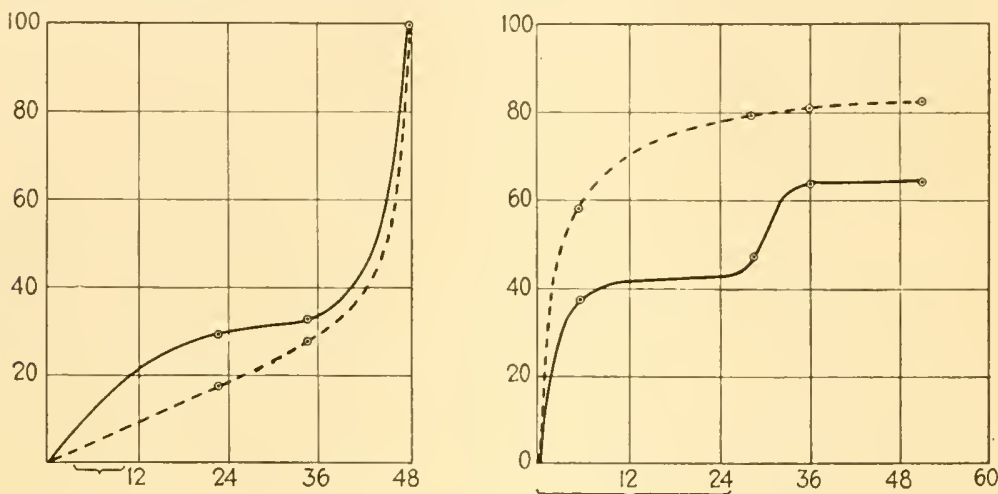

FIG. 2.-Effects of beta rays of radium upon regeneration in Tubularia crocea. Leftcurves showing acceleration of hydranth regeneration due to seven hours' exposure; ordinates indicate percentage of development; abscissas, hours from time of cutting; broken line represents controls; continuous line, irradiates; bracket indicates period of exposure. Right-curves showing retardation of hydranth regeneration from a $27-\mathrm{hr}$. exposure; details as in last. (From Congdon, 11.)

Weismann upon hydroids. Others have disputed this doctrine; but the histology of these hydrozoa has not been so exhaustively studied as that of hydra. Congdon (11) exposed pieces of the stems of Tubularia crocca, from which the hydranths had just been removed, to beta radiations from $300 \mathrm{mg}$. of impure radium for periods up to three days in length. $\mathrm{He}$ found that the shorter exposures accelerated and that the longer ones retarded the regeneration. The degree of retardation increased slowly with lengthening exposure; but the degree of retardation relative to the length of exposure decreased with lengthening exposure. In order to express the amount of regeneration numerically, the whole process of restoring a hydranth was divided arbitrarily into eight stages as observed during the three or four days involved, so that the stage of regeneration of each piece could be calculated for exposed and for control sets. The results are indicated by Fig. 2 which shows that acceleration and retardation result from different types of exposure. By means of correlation tables, appearing as his Figs. 7 and 8, Congdon showed the conditions determining whether acceleration or retardation would occur. Upon the whole it appeared that the exposures producing acceleration were the 
shorter, while the amount of retardation varied directly with the length of the exposure. He concluded further that there was "much to indicate that, if time be given for the cut pieces to partially regenerate before exposure, the effect of the beta radiation is decreased"; and that "differing amounts of regeneration before exposure played only a minor part in the various retardations and accelerations." However, "the retarding effect does not increase so rapidly as the length of exposure, though within the limits of these exposures, it does continue to increase. In other words, the sensitiveness of the hydroid decreases as the length of exposure increases." According to Congdon, this might be "simply an expression of Weber's law, that beyond a certain maximum of intensity any stimulus has a decreasing power of stimulation. Or, it may be that the early stages of regeneration are more sensitive to exposures for the same reasons that the embryo is more sensitive than the adult."

Curtis and Ritter (15), in a study of Tubularia crocea demonstrated that the power of regeneration could be completely inhibited by X-rays. The data relating to these exposures are: 65,000 v., 5 ma., thin cardboard filter, emission $31.4 \mathrm{r}$ per min. Small masses from a colony, with basal entanglement of the stems intact, were spread fanwise to form an approximately single layer in a Petri dish containing a measured amount of water that was estimated to be not more than $8 \mathrm{~mm}$. deep in any part of the dish. There was necessarily considerable difference in the depth of water that screened any one part of a stem or hydranth from the rays. With exposures in different experiments ranging from 30 to $120 \mathrm{~min}$. it was found that $90 \mathrm{~min}$. was sufficient for inhibition of hydranth regeneration in almost every instance. Thus, with a 90-min. exposure the regeneration of hydranths, which was normally accomplished within $48 \mathrm{hr}$., was completely inhibited, although the stems remained alive for upward of 20 days, which was as long as it was possible to maintain normal stems or colonies under the artificial conditions of the experiments. The irradiated stems could produce outgrowths resembling hydrorhizae, apparently by rearrangement of existing material, but never hydranths when the exposure was sufficient. Externally the stems were normal to all appearances when compared with the living controls in any experiment, and internally the normal circulation within the enteron was maintained. This latter feature indicated that the physiological state of the endoderm cells could not have been much disturbed by the radiation. Such nonregenerates could not live indefinitely without hydranths. They did live as long as normal stems and portions of the colonies remained alive under similar conditions. During such a 3-week period a control stem might have its hydranth removed 3 or 4 times with subsequent regenerations, while the $\mathrm{X}$-rayed stems after similar removals of oral portions showed only a series of healings. In this case the irradiated piece seems to meet the specifications for continued exist- 
ence during as long a period as a normal individual, but the situation is complicated by existence under laboratory conditions and by the fact that this hydroid appears in shallow waters at Woods Hole, Mass., about May 15 and dies in these habitats during July, although it may still be found in deeper water. The histological changes following the irradiation remain to be studied. It is known, however, that this species possesses many interstitial cells.

It appears, then, with coelenterates that lighter exposures to X-rays and to the beta rays of radium result in some acceleration of the rate, while heavier exposures result in a retardation and ultimately a destruction of the poyer of regeneration. In hydras both the acceleration and the retardation have been correlated with the effects of the radiations upon the interstitial cells which many investigators have thought capable of produeing all the other cell types, and therefore possessing embryonic potentialities. The destruction of embryonic cells might be expected by these radiations in accordance with the law of Bergonié and Tribondeau, and such destruction would account for the loss of regenerative power. Irradiation furnishes a technique by which the problem of differentiation in the regenerating organism may be attacked in a more advantageous manner than by any mechanical operation or by any technique of staining unless it be the use of vital dyes.

\section{TURBELLARIA}

Among platyhelminthes, the fresh-water planarians have been most extensively studied for regeneration. In recent years investigators of the histological changes have commonly agreed that the so-called "formative" or "regeneration" cells of the mesodermal region are the source from which new organs and tissues arise during regeneration. There is, however, disagreement regarding the origin of these cells (Fig. 3). Investigators such as Vandel (66) and Steinmann (55 and 56) maintain that formative cells originate by dedifferentiation, while others such as Curtis and Schulze (14) regard the formative cells as a persistent embryonic stock and maintain that the differing powers of regeneration in certain genera of planarians may be related to the relative abundance of these cells. Despite much investigation of its role in regeneration, the parenchyma of planarians, as Bartsch (4, page 199) puts it, is "ein sehr beliebtes Streitobjekt aller Planarienforscher." The technique of irradiation has been applied to this problem.

In a brief but comprehensive paper, Bardeen and Baetjer (3) deseribed the effects of $\mathrm{X}$-rays upon regeneration in Planaria maculata and $P$. lugubris. The worms were exposed to "medium soft" X-rays from 10 to $20 \mathrm{~min}$. each day for a period of from 12 to 18 days, after which they remained alive 20 to 30 days from the begimning of the various experiments. In the exposed worms, healing occurred by an initial museular 
contraction and subsequent extension of the old epithelium as in controls, but the epithelium remained thin and did not become columnar as is normally the case. There was a slight production of new tissue at the cut surfaces in some of the worms cut just before or soon after the first exposures and hence without allowance for a latent period in the action of the rays. There was no new tissue at these surfaces, or in the region where a pharynx would have been formed in a normal regenerate, when the specimens were cut well after a sufficient exposure. Microscopic examination of a few specimens, taken at varying periods after the last

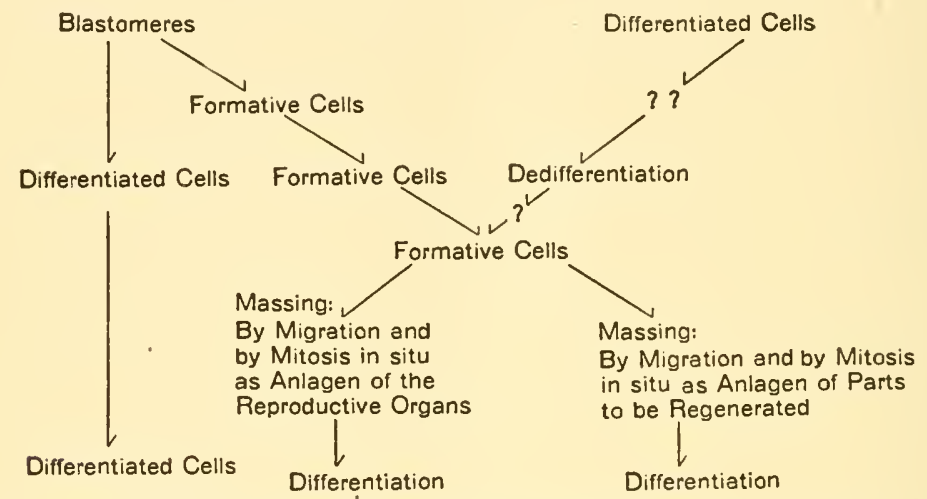

FIG. 3.-Source of formative cells in planarians according to the conflieting theories of these cells as a persistent embryonic stock and as arising by dedifferentiation. (From Curtis and Schulze, 14.)

exposure in one of the experiments, showed no marked alteration in the specialized cells of the muscular, nervous, and digestive systems; but the testes showed none of the mitotic divisions that were abundant in testes of controls. A single specimen from another experiment killed within the second $24 \mathrm{hr}$. after being cut for regeneration showed no signs of cell division, although controls showed abundant mitoses in the "tissueforming parenchymal cells" at this period. After irradiation the physiological activities of whole worms were apparently normal as shown by "normal reactions to light, to meehanieal, and to chemical (food) stimuli." The authors did not describe the external ehanges just preceding death, except to say that death resulted from a "degenerative process which began in the region of the head and extended slowly back." A few recently hatched individuals, which they observed, were "affected like the mature specimens but more quickly."

From these observations Bardeen and Baetjer concluded that "the power of regeneration may be completely destroyed by exposing planarians to the action of the roentgen rays"; that these rays "have a powerful inhibitive effect upon cell reproduction in planarians," and that this cell division "may be entirely stopped by sufficient exposure." Evidence for a latent period in the action of the rays, which was known to clinicians 
at the time of this work, was also recorded. The fact which was then being recognized that the effects of these radiations upon organisms were due "primarily to their action upon cells capable of reproductive activity" and which was soon to be formulated as the law of Bergonié and Tribondeau, was thus supported by the work of these investigators. After so comprehensive, although brief, a survey of the effects in planarians it is surprising that other studies upon the effects of such radiations upon regeneration did not follow immediately. The paper by Schaper (51) recording observations on the effeets of radium emanation upon embryonic and regenerative processes contains nothing regarding planarians, except a very brief account of a similar check upon regeneration by exposure of Planaria lugubris to radium. In none of this early work was it possible to determine exactly what exposures were being given, as can be done in current experimentation.

So far as the writer of the present article is aware, the next studies upon the effects of X-rays in the regeneration of planarians were those by himself and his students, as variously reported before the American Society of Zoologists (15) and the work of Weigand (67). These investigations were undertaken as a renewal of earlier studies upon the histological changes of regeneration of planarians (12) in which the importance of a reserve stock of "formative cells" had been indicated. It was thought that the abundance of these cells in different species might be correlated with the contrasting powers of regeneration that had long been known to occur in planarians, and this appears to be the case in Planaria maculata as compared with Procotyla fluviatilis (14). The technique of irradiation gave promise in the attack upon this problem and the results justified the expectation (Fig. 4). With exposures ranging from 2500 to $3500 \mathrm{r}$ planarians such as $P$. maculata and $P$. agilis, which have great powers of regeneration and many formative cells, were rendered incapable of regeneration, although some of the worms remained alive for 30 days or more before beginning to show abnormalities that were the forerunners of death. Somewhat lighter exposures produced individuals that regenerated slowly or imperfectly and with a minimum of new tissue at the cut surfaces. In some instances such individuals recovered from the effects of the irradiation and regenerated normally when eut 60 or 70 days after the date of exposure. When irradiated worms were sectioned, it was found that the formative cells were proportionately reduced in numbers. In the heaviest exposures these cells were so far eliminated that there seemed to be none remaining in many of the specimens (Fig. 5). The mesodermal region was thus changed from its characteristic appearance in Planaria agilis to that seen in the postpharyngeal region of Procotyla fluviatilis (14) which is incapable of regeneration. In experiments with radium, in which an exposure about equivalent to that with the $\mathrm{X}$-rays was used, regeneration was inhibited in a similar manner. 

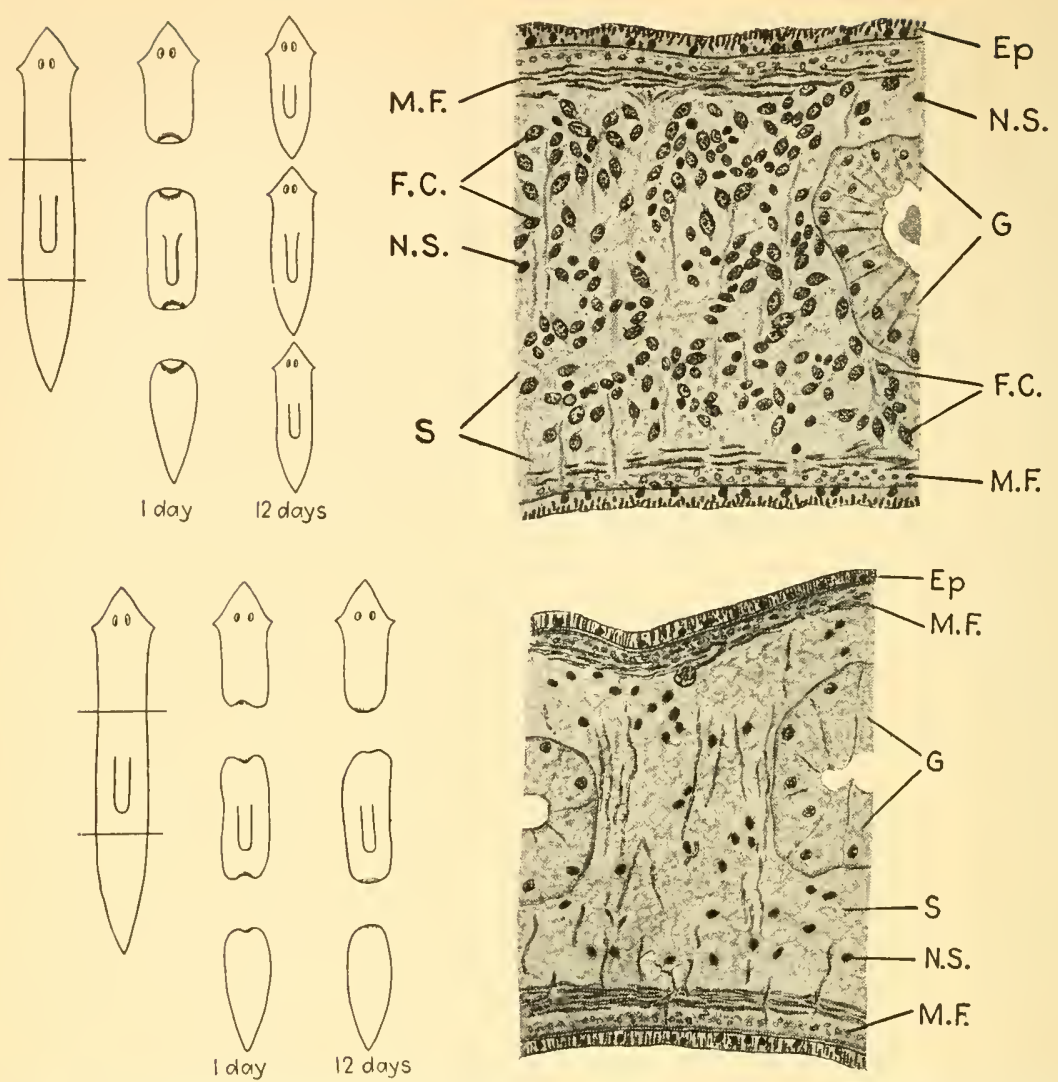

FIG. 4.-Inhibition of regeneration and destruction of formative cells in Planaria agilis by X-rays. $E p$, epithelium; F.C., formative cells; $G$, gut; $M . F$. , muscle fibers; N.S., nucleus of syncytium; $S$, syncytium. (From Curtis, 13.)

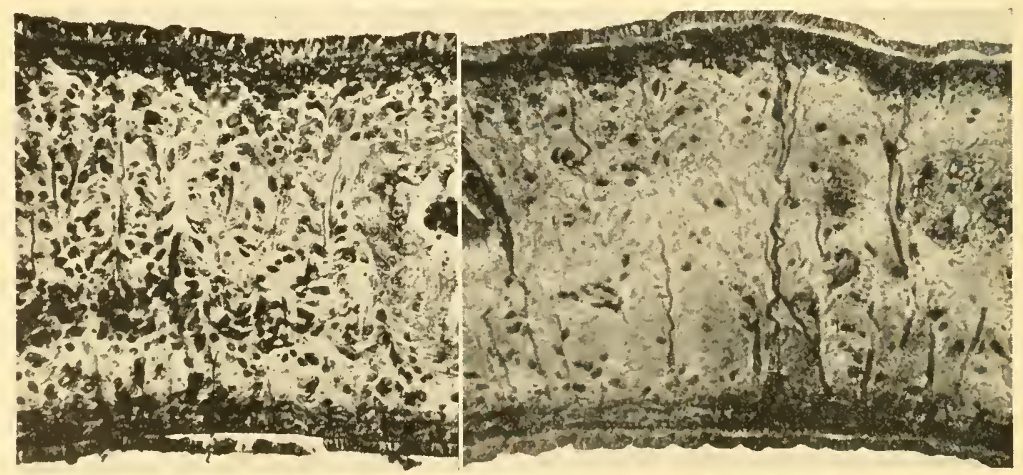

FIG. 5.-Microphotographs showing effect of X-rays (3500 r) upon formative cells of Planaria agitis. Left - part of a longitudinal section of control showing many formative cells in parenchyma. Right-similar section of an irradiate 10 days after exposure showing parenchyma without formative cells but with the syneytium, muscle fibers, etc., intact; lobes of the gut and part of nerve cord are seen. Compare with Fig. 4. 
In his extensive study of the effects of radium irradiation upon Polycelis nigra, and comparisons with Planaria torva and Planaria lugubris, Weigand (67) has considered both the gross and the histological changes involved. As a source, Weigand used a radium bromide preparation containing about $15 \mathrm{mg}$. and enclosed in a steel capsule with a mica window $10 \mathrm{~mm}$. in diameter. For longer periods the planarians were exposed three at a time in a glass container $4 \mathrm{~mm}$. deep and $10 \mathrm{~mm}$. wide and covered with thin aluminum foil. The container was filled with water and placed directly on the mica window of the capsule. For the exposures of 5, 10, and 15 min. a block of paraffin with a similar cavity was used. This procedure seemed not to result in a lack of oxygen or an undue increase of temperature, except in a few instances.

The experiments included exposures ranging from $5 \mathrm{~min}$. to $16 \mathrm{hr}$. and the period of observation ranged from 6 to 27 days between the begin-
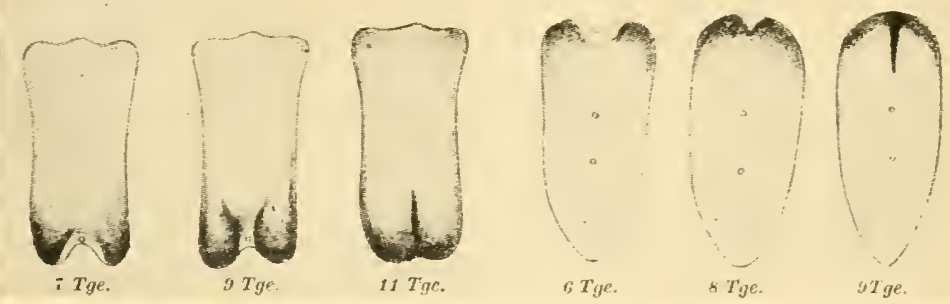

FIG. 6.-Concrescence in wound region of head and tail pieces of Polycelis nigra exposed to radium and cut immediately after. $7 \mathrm{Tge.,}$ etc., days after such exposure and operation. (From Weigand, 67.)

ning of the experiment and death or fixation of the individual specimens. In all the experiments on regeneration the worms were cut transversely, through the region of the pharynx, into head and tail pieces. When alive, the head pieces showed the typical cap of regenerative tissue at the cut surface and the new mouth, which could be compared in controls and in the irradiates; but the regenerated pharynx could be identified only as it happened to be thrust from the mouth opening. The tail pieces showed the new tissue with its subsequent development of eyes and typical head outline, the mouth, and the pharynx only when extruded. For other details sections were necessary.

In Weigand's first experiment Polycelis nigra was rariously exposed and in each case cut immediately after the exposure. It was found that the rays did not have a lethal effect, within the period of observation (13 to 21 days), if exposure did not exceed $15 \mathrm{hr}$., as in this experiment, although the specimens that were exposed more than $15 \mathrm{~min}$. showed characteristic concrescence of the margins and other deformations that eventually prove lethal (Fig. 6). All the irradiated pieces began to regenerate, at least the wound healing was completed. The amount of regenerative tissue that could be seen externally as the "cap" diminished with increased exposure and the regeneration became correspondingly 
restricted and delayed as to formation of such new tissue and mouth openings (Fig. 7). Eyes appeared only on the tail pieces exposed 5,10 , and $15 \mathrm{~min}$., respectively, and their appearance was some 4 days later than in controls. The individuals receiving these short exposures did not show the deformations found in longer exposures and the regeneration was eventually completed. Exposures of $30 \mathrm{~min}$. and longer showed progressive reduction in the amount of regeneration tissue formed and correlated failure to form the lost parts (Fig. 7), followed by increasing deformation in later stages. The head pieces seemed slightly more resistant to deformation than the tail pieces.

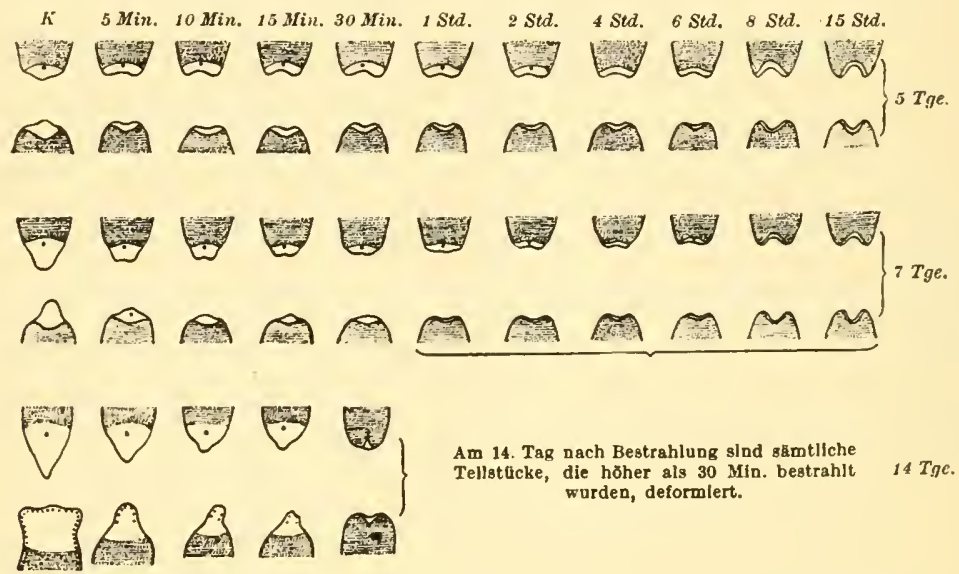

F1G. 7.- Cap of regenerative tissue on head and tail pieces of controls and irradiates of Polycelis nigra, exposed to radium from $5 \mathrm{~min}$. to $15 \mathrm{hr}$., as the cap appears at 5,7 , and 14 days, respectively. $K$, the control. The cap is white, the old tissue shaded. (From W'eigand, 67.)

In other experiments the worms were exposed and cut $1,1 \frac{1}{2}, 2$, and 3 days after exposure. In general this procedure resulted in progressively greater injury than occurred when the cutting followed immediately after exposure. In those cut at 3 days, the formation of the cap failed almost completely, the concrescence and deformation appeared earlier, and the mouth opening appeared less frequently.

From one experiment made upon Planaria torva and two upon $P$. lugubris, for comparison with the foregoing results in Polycelis nigra, it appeared that $P$. torva reacted much the same as $P$. nigra when cut immediately after exposure, while $P$. lugubris showed greater resistance to similar exposures by the formation of more regenerative tissue, by later and less inhibition of regeneration, and by less deformation.

In a series of experiments in which the worms were cut from $11 / 2$ to 9 days before exposure (Fig. 8), it was shown that the cap of regenerative tissue became more resistant to the irradiation as it grew older, that is, as its differentiation proceeded. Complete regeneration occurred when the exposure was not made until a sufficient age had been reached by the 
regenerative tissue. Feeding was shown to have no effect upon the changes following irradiation. Whole specimens, irradiated but not cut for regeneration, showed the deformations seen in later stages of head and tail pieces. The worms survived exposures up to $15 \mathrm{~min}$. without subsequent abnormalities, but longer exposures resulted in deformations which presumably would have been fatal in all cases. $P$. lugubris again showed greater resistance as whole individuals. With an aluminum filter that cut out the beta and the softer gamma rays there was much less effect than with the unfiltered irradiation. Many of the irradiates could hardly be distinguished from the controls.
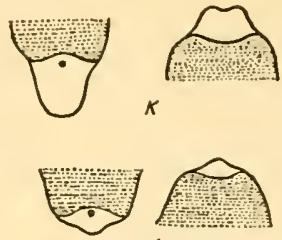

$2 \frac{1}{2} \operatorname{Tge}$
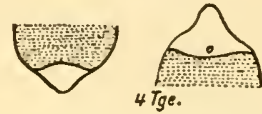

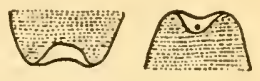

$1 \frac{1}{2}$ Tge.

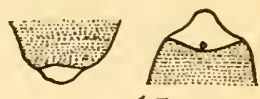

$3 \frac{1}{2}$ Tge
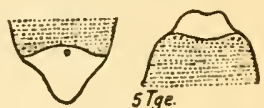

FIG. 8.-Cap of regenerative tissue on headand tailpieces of controls and irradiates of Polycel is nigra exposed to radium $2 \mathrm{hr}$., as the cap appears 7 days after the operation when time of exposure is varied. $K$, control. $11 / 2$ Tge. to 5 Tge., exposures made at $1 \frac{1}{2}$ to 5 days respectively after the operation of cutting into head and tail pieces. (From Weigand, 67.)

In the histological study, it was found that the cellular changes induced by the irradiation could be recognized as early as $2 \mathrm{hr}$., whereas external changes might not be recognized in such an individual until
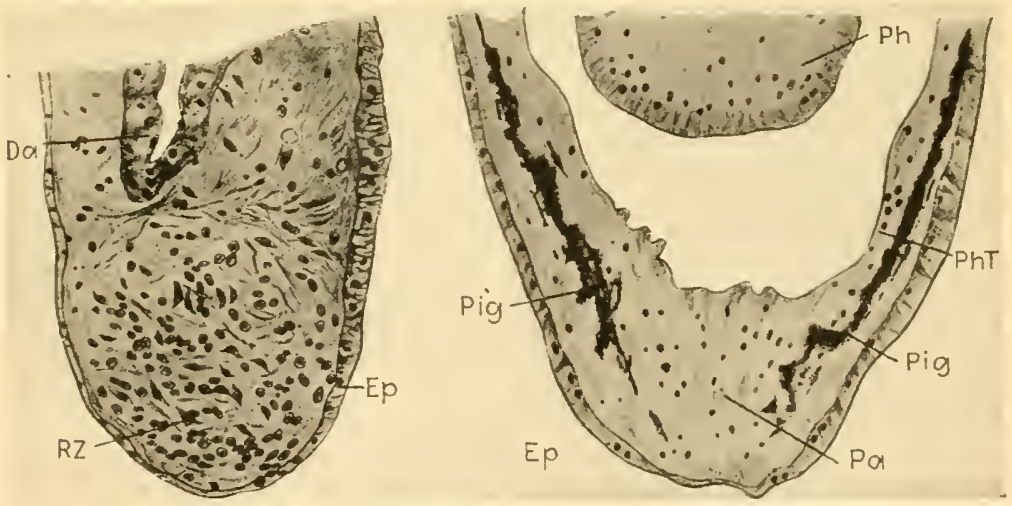

FIG. 9.-Effect of radium upon cells of Polycelis nigra. Left-a sagittal section through posterior end of a head piece, used as control and killed 4 days after operation, showing normal accumulation of cells that form the eap of regenerative tissue visible externally. Right-sagittal section through posterior end of an irradiated head piece exposed $6 \mathrm{hr}$., cut immediately thereafter, and killed 6 days after such exposure and operation. Compare the parenchyma with that shown in Fig. 5, right. There is no cap of regenerative cells. The wound has been healed by extension of the old epithelium. Da, gut lobe; Ep, epithelium; $P a$, parenchyma; $P h$, pharynx; $P h T$, pharynx sheath; $P i g$, pigment; $R Z$, regenerative cells. (From Weigand, 67.)

4 or 5 days after the exposure. In worms cut immediately after irradiation of from 3 to 15 hr. (Fig. 9) healing occurred in the irradiates in the same manner as in controls by extension of the old epithelium; but there 
was no such abundant mitosis in the cells of the parenchyma. At $24 \mathrm{hr}$. there was no beginning of a cap of regeneration cells at the cut surface, and abnormal mitoses were numerous in the parenchyma while other parts appeared normal. At $48 \mathrm{hr}$. it appeared that exposures longer than $30 \mathrm{~min}$. had hindered cell division to such an extent that no cap of regeneration cells was formed. Exposures up to 30 min. gave some abnormal cell division but did not completely check the normal mitoses and formation of the cap. In general the number of all mitoses, both normal and abnormal, declined with increasing periods of exposure. Dedifferentiation was found in both irradiates and controls. It seemed not to be influenced by the rays except as mitosis was involved. At $72 \mathrm{hr}$., with the higher exposures, there was extensive cell degeneration in the parenchyma (Fig. 10).

The worms cut 1 to 3 days after irradiation showed greater injury to the tissue than those cut immediately after similar exposures. After the shorter exposures abnormal mitoses persisted for a considerable time with subsequent cell degeneration. With the longer exposures cell division was lacking and degeneration had appeared $24 \mathrm{hr}$. after the cutting. Pieces in stages of deformation showed local degeneration of the body epithelium and a degeneration of the parenchyma as a result of the destruction of the regenerative cells (Fig. 10). The deformation as externally visible resulted from an accumulation of degenerating cell material arising either from the cells injured by the rays or by sloughing off of gut cells. Irradiated whole specimens appeared about the same as those cut for regeneration, except that distention as a result of accumulated material from the gut did not occur. In the degenerating areas of the surface there were mitoses and also cell degeneration, with the latter predominating in later stages. Brief study of the sex cells showed the oöcytes and spermatocytes in stages of degeneration 4 or 5 days after irradiation. In general the changes appeared later in the more differentiated sex cells than in the cells of the parenchyma and after longer exposures.

It was evident from the foregoing data that the histological changes involved in the first instance the formative cells or those with embryonic potencies, and that the obvious effect was upon mitosis in these cells. The law of Bergonic and Tribondeau was thus again confirmed, and Weigand thought he could distinguish as the primary effect upon mitosis (cf. 1,2) pyenosis along with the absence of mitoses that was found during the first hours after exposure, and as the secondary effect the abnormal mitoses that subsequently appeared. Since changes were thus recognizable in the cells so soon after the irradiation, Weigand questioned whether one could speak of a latent period in the morphological sense. In attempting a more complete analysis of his observations, the changes in the irradiates were correlated with the account of the normal histo- 
logical changes during the regeneration of planarians which derives the formative cells by dedifferentiation as well as by division of formative cells already in existence (Fig. 3). According to this concept, the individual has at the outset a limited number of these cells in its parenchyma. When it is cut for regeneration this initial supply becomes greatly reduced in numbers by migration to the cut surface to form the eap of regenerative tissue. As the regenerative and regulatory changes proceed, more formative cells are produced by dedifferentiation of other cells that are not too highly specialized; and this new crop multiplies and migrates as
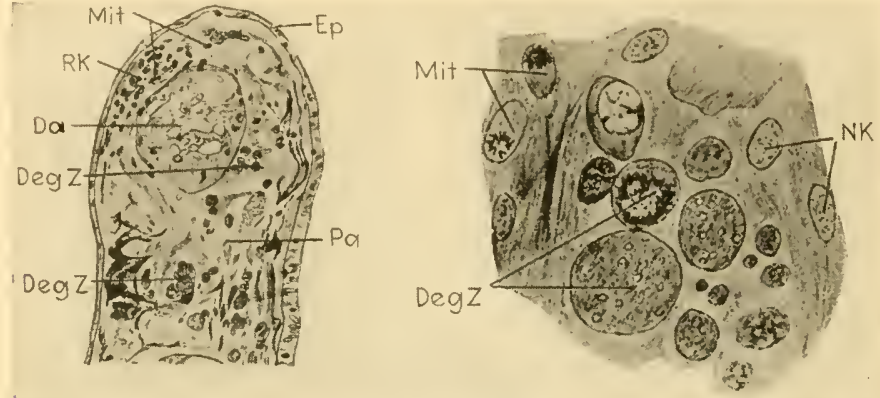

Fig. 10.- Effect of radium upon cells of Polycelis nigra. Left-a sagittal section through the cap of regenerative tissue on a tail piece exposed $30 \mathrm{~min}$. to radium, cut immediately thereafter, and killed 5 days later. In the cap region there is a very small collection of regenerative cells, some of them showing normal mitoses. Degenerating cells are abundant elsewhere in the parenchyma. Right- a detail from such a section. $D a$, gut lobe; $\operatorname{Deg} Z$, degenerating cell; $E p$, epithelium; Mit, mitoses; $N K$, normal nucleus; $P a$, parenchyma, $R K$, cap of regenerative cells. (From Weigand, 67.)

necessary $(55,56)$. With this picture in mind, Weigand concluded from his observations that the formative cells already in existence were injured by the rays, but that the dedifferentiations by which more formative cells were produced were not interrupted. Only when the dedifferentiating cell reached the state of a formative cell and mitosis began (Lang, 35) was there evidence of effects of the irradiation. The larger numbers of degenerating cells in later stages of irradiates that had been cut, as compared with irradiated whole speeimens, were interpreted as resulting from formative cells that had arisen by dedifferentiation and then degenerated. The fact that injury was greatest when the cutting was done 2 or 3 days after exposure and the regeneration thus began at that period, he explained by supposing that more of the cells capable of dedifferentiation were mobilized at that time and in an especially critical stage. The fact that a small regeneration cap could be formed after short exposures and the formative cells differentiate normally in the same was explained by supposing that some of them had escaped injury and retained their power of migration. Having once arrived at the cap they seemed more resistant to the rays, since degenerating cells were never abundant in this region; and as the eap differentiated, this resistance 
increased (Fig. 8). There was no evidence of a stimulating effect in the action of the rays. The highly differentiated cells of the gut, sense organs, nervous system, and musculature remained unchanged until affected by the final deformation and degeneration of the piece.

It appears from these studies by various investigators that the inhibition of regeneration in planarians by $\mathrm{X}$-rays and radium involves destruction, or at least injury, of the cells that exhibit embryonic potencies in normal regeneration. The changes observed in the chromatin and in the mitoses, as in other instances of irradiation, suggest that the effect is directly upon these cells and not an indirect effect resulting from a changed physiological state of the organism as a whole. In other words, cellular changes in this instance seem to precede the organismal changes appearing as the failure of regeneration and the degeneration that follows.

In a brief and presumably a preliminary statement, Meserve and Kenney (41) have reported a study of the effects of X-rays upon different sized populations of Planaria dorotocephala totaling over 800 individuals. They state that the primary effects, which appear during the first 2 weeks, are largely of a nonspecific nature, since the head forms developed resemble those induced by various depressants except for one type of head formation. After any one exposure of 4, 8, or 12 skin units, the range of distribution of types regenerated increases as crowding increases. A subsequent disappearance of tissue differentiated during the first 2 weeks seems to be an effect of $\mathrm{X}$-rays on planarian tissue that gains expression after regeneration has reached its limits and that is first apparent in the region having highest rates of metabolism.

Secondary effects were observed, in all of the X-rayed forms receiving 4,8 , and 12 skin units, as cytolytic changes, which first became prominent 34 days after exposure and resulted in death. The authors state, although there is nothing said regarding a study of the cells by means of sections or otherwise, that their results agree with those of Bardeen and Baetjer (3), "who conclude that X-rays affect cell division and cell differentiation and that the effects are probably confined to these two"; that "cell differentiation is not as much affected as cell division"; and that "the effect upon cell division is not direct." No reference is made to the work of Weigand (67) or to the very limited observations which Bardeen and Baetjer made upon the cellular changes, as noted on page 420 .

It is further stated by Meserve and Kenney, that "while both the more immediate effects and the delayed effects of X-rays may be specific upon the protoplasm, it does not necessarily follow that, because head frequency is affected by X-rays, the factors which control head frequency are specific and directly related to the activity of special formative cells. The formative cell theory of Curtis does not recognize the fact that the 
variations in head forms regenerated are the same type as those produced by other physieal and ehemical agents. It is no more necessary to assume the selective action of X-rays on formative cells than it is necessary to assume selective action of other physical and chemical agents which alter head frequeney. The first apparent effeets of $\mathrm{X}$-rays, like various other agents, seems to be not on speeial formative cells but upon nonspeeific protoplasmie factors upon which head development depends."

With regard to these statements it ean be remarked that while the work of Curtis and his students, like that of many other investigators, has shown the importance of such cells in the regeneration of planarians, the "formative cell theory" is the theory of Weismann and not of any later investigator. It seems abundantly established that the so-ealled formative or regenerative cells funetion in planarians in the formation of new parts during regeneration and that such eells give rise to the germ cells. As shown by Fig. 3, the immediate origin of these cells is not so obvious. What ean be elaimed with respect to irradiation is that radium and X-rays destroy these cells with embryonie potencies, in the same manner that these rays destroy embryonic cells in many organisms aceording to the law of Bergonié and Tribondeau. The effect appears in the eells soon after exposure, whether due to "direct hits" or to indireet effects upon the organism as a whole that quickly affect these cells without affecting others in any recognizable manner. In the absence of evidence for either possibility, the later effects of deformation as described by Weigand and the eytolysis deseribed by Meserve and Kenney may be explained as well by the toxic action of material arising from degeneration of formative cells, and aceumulating in the parenehyma, as from more direct effects of the irradiation.

It seems from these studies that the technique of irradiation provides a check upon the investigations that have shown the importance of formative eells in the regeneration of planarians. The weakness of such results with radium and $\mathrm{X}$-rays lies in the disputed origin of the formative cells, in the difficulty of recognizing formative cells as such, and in the fact that individual planarians, after becoming wholly ineapable of regeneration as a result of such irradiation, cannot be maintained alive and in an otherwise normal state for a long period. As with hydras, one should obtain individuals that possessed no formative eells and could not regenerate, but lived indefinitely without other abnormalities. If the formative cells are a persistent embryonic stock, necessary for other cell replacements and for sexual reproduction as well as for regeneration, it would perhaps be too much to expeet that the individual thus deprived of these cells could live out a normal span of life, whatever that may be for a given planarian. It might be expected, however, to live much longer than the periods indirated, and one might hope to determine the lethal conditions if it died prematurely. 


\section{NEMERTINA}

Knowledge of regeneration in nemerteans, particularly the genus Lineus, has been greatly extended by the recent studies of Coe (8). In certain species, such as Lineus socialis and L. vegetus, the power of regeneration "is so great that almost any small piece of the body, provided it contains a portion of one of the lateral nerve cords, is able to develop into a minute worm of normal proportions," and the persistence of this potency can be shown "by repeatedly cutting off portions of partially regenerated pieces until extremely minute individuals less than a hundred thousandth the size of the original are finally obtained." On the other hand, with Lineus pictifrons the ability to restore a new head from a body fragment extends only through the anterior half of the foregut region; while with $L$. ruber, which is the "broad form" of Nussbaum and Oxner (45), only that part containing the anterior ends of the nerve cords restores a missing head. In addition to his studies of the external and organismal factors, Coe has described the histological factors involved. In an attempt to analyze the organizing potencies he has concluded that the cut nerve cords liberate an agent that activates the dormant cells which are situated in the parenchyma between the organ systems in all parts of the body, and which by virtue of this activation assume the properties of regenerative cells, migrating anteriorly and posteriorly and finally collecting in large numbers beneath the new epidermis which has already covered the cut ends of the fragment. Those that migrate posteriorly are soon incorporated into the organ systems of the original body, which are restored both by the multiplication of the differentiated cells, to form cells of like nature, and by the incorporation of regenerative cells which differentiate into cells of the respective tissues, presumably under the influence of the existing parts. The cells that migrate anteriorly, on the contrary, form a true blastema consisting of an apparently undifferentiated mass of mesenchyme cells, which, like an early embryo, seems to be a self-determining system composed of cells that are multipotent and capable of differentiation into any of the new organs. The blastema is thus an essentially new individual. Coe concluded that the different regenerative capacities, or contrasting powers of regeneration in closely related species "may be dependent upon differences in the extent of distribution either of the activating agent or of the regenerative cells."

So far as the writer is aware, the technique of irradiation has not been applied to these problems of regeneration in nemerteans, except by Coldwater (10) whose work has appeared only as an abstract. Whole specimens of Lineus socialis were exposured with $100 \mathrm{kv}, 5 \mathrm{ma} ., 21 \mathrm{~cm}$. and covered by a $75-\mathrm{mm}$. bakelite filter for periods of $10,15,20$, and $30 \mathrm{~min}$., respectively, which represented approximately $400 \mathrm{r}$ per min. by 
the ionization chamber of Failla, in use at the Marine Biological Laboratory in the summer of 1930 . Immediately after irradiation the worms were divided by transverse cutting into pieces approximately $2 \mathrm{~mm}$. in length and checked against controls obtained in the same manner which regenerated normally. In each series the irradiates healed their cut surfaces. In the series exposed to 4000 and to $6000 \mathrm{r}$ there was a slight development of the brain and eyes when the pieces were killed 41 days

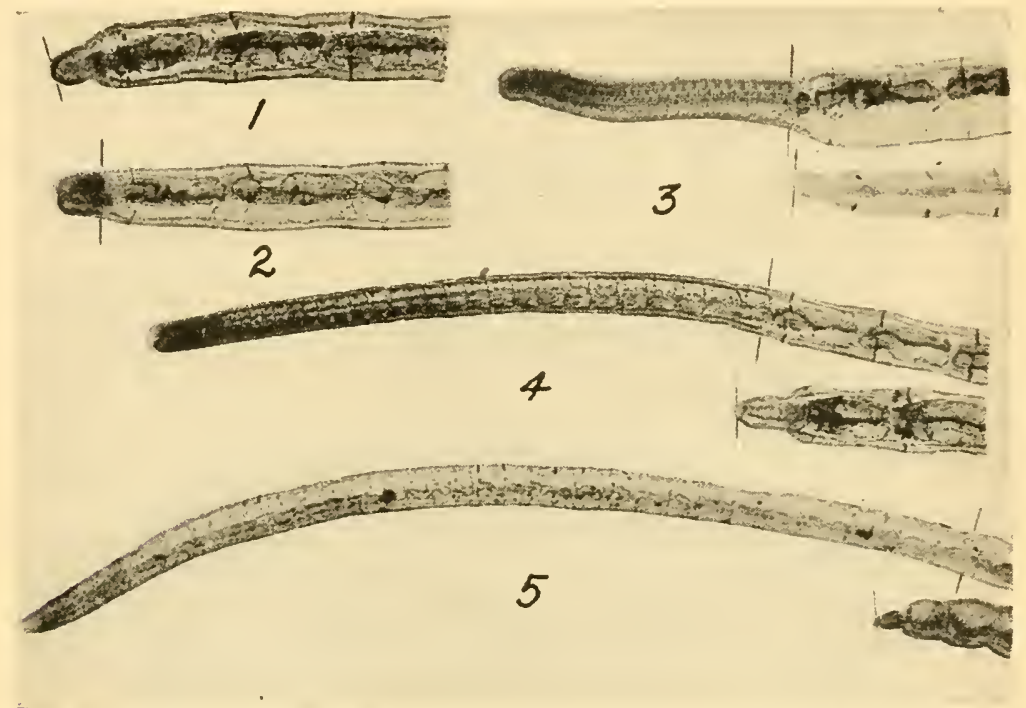

FIG. 11.-Effect of X-rays upon posterior regeneration of Tubifex tubifex as shown by mierophotographs of whole mounts. 1, X-rayed worm 7 days after removal of posterior segments; 2 , control 7 days after a similar removal; 3 , control and X-rayed specimens 14 days after such removal; 4 , control and X-rayed 21 days after removal; 5 , control and $\mathrm{X}$-rayed 30 days after removal. Plane of removal shown by line in each figure. (From Stone, 58.)

after exposure. In the $8000 \mathrm{r}$ and in the $12,000 \mathrm{r}$ series there was healing and the pieces were normal in appearance, but there was no evidence of regeneration externally when they were killed at 41 days. A preliminary histological examination showed no beginnings of regenerating organs and an absence of embryonic cells, although other cells appeared normal as seen in the sections. Since the irradiated pieces were in good condition when killed, there was as good reason to believe that death would have resulted eventually from starration as that it would have resulted from any delayed effects of the irradiation. Completion of this study by Coldwater has been delayed. In view of the general resemblances between the histological factors of regeneration in nemerteans and planarians and the fact that nemertean tissues are perhaps more favorable for such study, its publication in extended form is desirable. 


\section{CHAETOPODA}

Among the annulata regeneration has been so widely observed that some degree of this potency is presumably universal in these animals. In the oligochaetes the gross aspects of the process and its histological details have become well known in genera that are presumably representative. Thus in Limnodrilus and Tubifex $(33,34,58,59)$, posterior regeneration occurs at any level behind the tenth somite, and a full complement of somites can be eventually restored (Fig. 11). Anterior
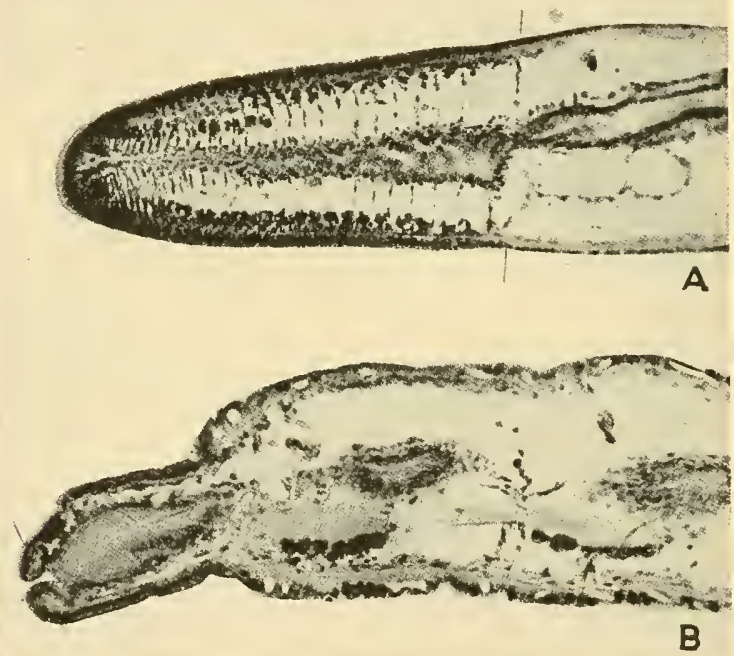

FIG. 12.-Effect of X-rays upon posterior regeneration of Tubifex tubifex as shown by microphotographs of sagittal sections. $A$, Control 9 days after removal of posterior segments; $\times 145$. $B$, Same of $\mathrm{X}$-rayed worm 12 days after a similar removal; $\times 170$. Plane of removal is shown by a line in each figure. (From Stone, 58.)

regeneration occurs in Tubifex when not more than 10 or 12 of the anterior somites are removed, but more than 3 new somites are never formed in the restoration of a normal head region (Fig. 13). In recent studies of Lumbriculus, Zhinkin $(72,73)$ and Turner $(63,64)$ have confirmed the earlier observations that in this species posterior regeneration occurs to about the same extent as in Tubifex, but that anterior regeneration occurs from any level except the extreme posterior end. The histological changes during posterior regeneration are essentially the same in the two genera. Among the peritoneal cells on the posterior faces of the septa, except in the most anterior region, there are what have been called the "reserve cells" or "quiescent neoblasts." When the posterior end of the worm is removed, the reserve cells in neighboring somites are activated to a growth or metamorphosis in the course of which they are transformed into much larger cells the cytoplasm of which is crowded 
with stored nutrient material. In their final stages these full-grown neoblasts migrate ventrally down the septa and then posteriorly along the nerve cord through the ventral openings in the septa to the cut end

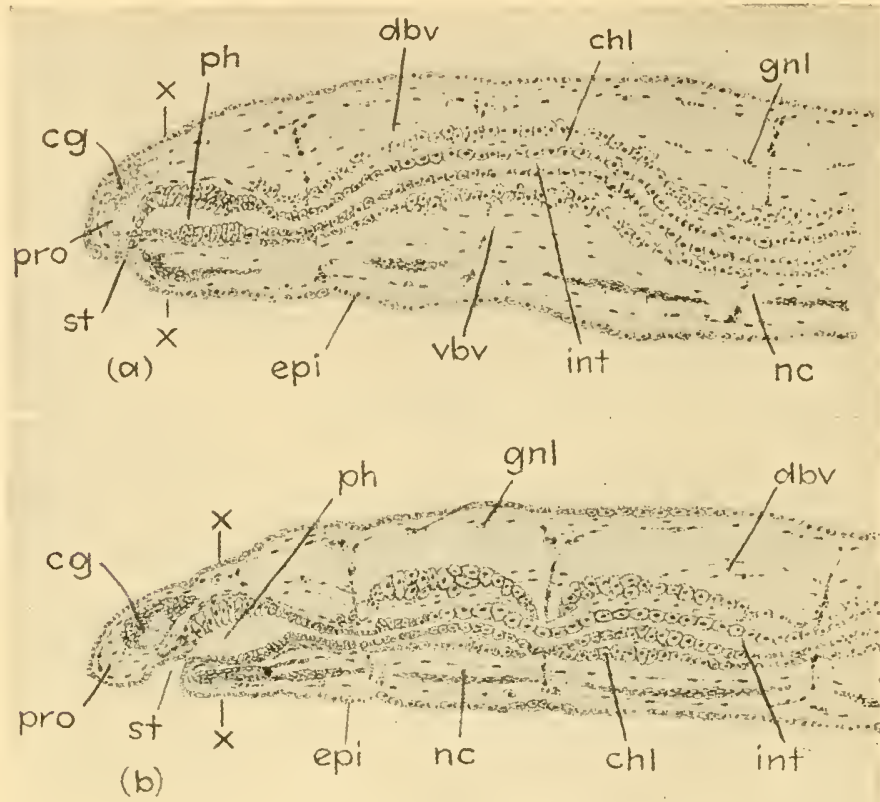

(b)

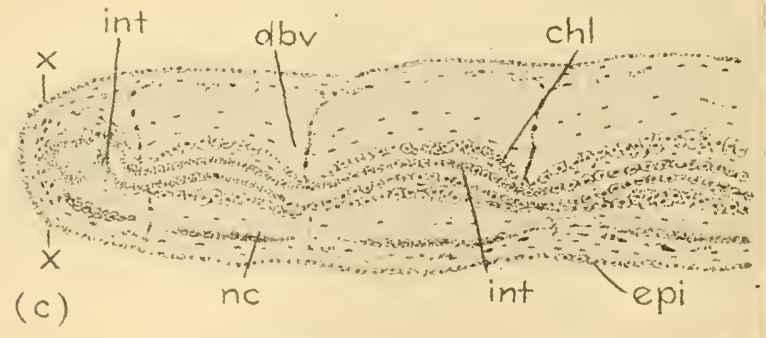

FIG. 13. - Effect of X-rays upon anterior regeneration of Tubifex tubifex. Top-sagitta section of anterior end of control 8 days after removal of three to four anterior segments. Middle-same of a control 18 days after such a removal. Bottom-sagittal section healed anterior end of an X-rayed specimen 30 days after such a removal. cg, cerebral ganglion; $c h l$, chloragogue layer; $d b v$, dorsal blood vessel; epi, epidermis; gnl, granular leucocytes; int, intestine; $n c$, ventral nerve cord; $p h$, pharynx; pro, prostomium; st, stomadaeum; vbv, ventral blood vessel; $x x$, plane of the cut. (From Stone, 59.)

of the specimen which has in the meantime become healed by rearrangement of the old tissues in advanee of any cell proliferation (Fig. 11). Only when a neoblast has reached this posterior region does it divide. From the neoblasts thus accumulating posteriorly there arises by mitosis a mass of smaller cells which differentiate into the new mesodermal 
tissues (Fig. 12). The cells of the old ectodermal epithelium divide mitotically to form the regenerating portion of the nervous system and more epithelium of this sort as the regeneration proceeds. The cells of the endodermal epithelium form more epithelium in a similar manner. In the anterior regeneration of Tubifex quiescent neoblasts may be present on somites near enough the regenerating region to become involved, as they do in Lumbriculus, but in Tubifex such neoblasts do not grow and migrate anteriorly (33, p. 438). The new parts are formed by multiplication from the old ectodermal, endodermal, and mesodermal tissues, respectively (Fig. 13). In contrast to this, the anterior regeneration of Lumbriculus involves a growth and anterior migration of neoblasts and their division and differentiation in the regenerating end similar to that which occurs posteriorly. The histological changes during the regeneration in both these genera thus proceed from cells within the regions of the respective germ layers in the general manner of embryological development. It is not clear that basal cells of the epithelia are the active units, as Stone (58 and 59) and Turner (63) have stated, since Turner (64) has concluded he was mistaken in this respect and that the "principal cells" of the two epithelia are the ones that multiply by mitosis. For the oligochaetes then, it is clear from the work of Krecker $(33,34)$ as well as these later investigators and others, such as Hämmerling (22) and Zhinkin (74), that a definite type of cell plays an important, if not an exclusive, role in the formation of the regenerating mesoderm (cf. Krecker, 34, page 42), and that the epithelia arise in the manner described.

Reserve cells are also found in many polychaetes such as Filograna implexa and Chaetopterus variopedatus (16 and 17), in which such cells are always present in normal uncut specimens and are the only cells of the body that seem to have embryonic potencies. These can be regarded as homologous with the reserve cells of oligochaetes, since they are similar in origin and since they have a similar part in growth, budding, and regeneration. However, in the sabillid, Euratella chamberlin, Stone (60), found no such reserve cells at any time. In the regeneration of this species; epidermal cells multiply at various sites and invade the middle region, where they differentiate into the new mesoderm tissues as well as the regenerating nerve cord. As with the oligochaetes, the two epithelia are self-restoring.

The problems indicated by the foregoing regenerative changes in the annulates, particularly in the oligochaetes, include: the origin and potencies of the mesodermal reserve cells; their activation to growth, migration, and differentiation; and the activation to division of the cells composing the two epithelia. The material is favorable alike for gross observation and experimentation in the living state; for study of the histology from whole mounts and sections; and for obtaining quantitative 
data by measuring the regenerated outgrowths or counting their somites. These problems have been further attacked in related material by transplantation experiments $(65,42)$; and by experiments showing that the -SH group stimulates regeneration and is apparently essential for growth during normal regeneration (9). Results have also been obtained by irradiation and this technique gives further promise.

In Tubifex tubifex following an exposure to X-rays of $7125 \mathrm{r}$, Stone (58) found that posterior regeneration never oceurred (Figs. 11 and 12). There was only the formation of a small knob by rearrangement of existing tissues without cell proliferation or differentiation. After this healing additional somites could be removed at intervals with repetition of the healing and knob formation. The inhibition of regeneration continued as long as the worms survived in the cultures, in one experiment 147 days, which was as long as the normal uncut worms were maintained. The irradiated specimens thus behaved as though "castrated" against regeneration. In contrast to this, control worms from which as many as 32 posterior somites were removed regenerated as many new ones in about 32 days.

In specimens cut for posterior regeneration Stone fortnd no neoblasts in any of the Tubifex that received the 7125-r exposure and consequently none of the histological changes dependent upon neoblast growth, migration, and differentiation. In like manner the changes in the epithelia which oceur in the normal regenerate were inhibited. A similar inhibition of anterior regeneration and of its cell multiplication in the epithelia was obtained by Stone with exposures to X-rays of $9000 \mathrm{r}$ (Fig. 13). Turner $(63,64)$ has obtained comparable results with Lumbriculus inconstans, and Zhinkin (72, 73) with L. variegatus; Rahn (49) has found that the neoblasts of naids are not present after irradiation with radium; and Stone (60) obtained inhibition of regeneration in Euratella chamberlin by exposures with radium. There scems to be no question as to the establishment of such an inhibition in the ammulata and the correlated structural and functional changes in cells as described.

By means of the irradiation it is possible to eliminate the neoblasts, as though one eut out parts of the organism by a surgical opcration. Then it is possible to follow what happens in regeneration; indeed, the embryologist has long proceeded in the same manner with analogous experimental operations that have greatly extended our knowledge of development. The results in this phylum are perhaps more satisfactory than in any of the other cases cited previously, because of the clear histological picture that is presented and the precision of the changes that can be induced by the irradiation. The problem remains of how both the reserve cells of the mesoderm and the epithelial cells are affected to render them incapable of their normal behavior following removal of a part of the individual, that is, why these cells are no longer activated. 
The effect upon early prophase stages as established in other cases cannot be postulated, unless one thinks of the early prophase as reaching farther back into the interdivision phase of the cell than is commonly supposed. Since it is a fair hypothesis that the chemicophysical changes at the cut surface may produce changes in the blood or the coelomic fluid that might affect the reserve cells, one may question whether the effect of the irradiation is upon a humoral activation of this sort or directly upon the reserve cells. It should be possible to determine these points by a combination of irradiation and other techniques. Stolte (57) has recently considered the problem of activation in Dero limosa. Coldwater (9) has made a beginning in this direction by showing that treatment with solutions containing the $-\mathrm{SH}$ group increases the rate of regeneration in Tubifex tubifex and that this group presumably functions in the normal regenerative process but does not influence the inhibition or retardation of regeneration by exposure to X-rays. Zhinkin (74) has grafted posterior somites of an irradiated Rhynchelmis upon the cut posterior end of a normal individual, with the result that neoblasts activated by cutting of the nonirradiated worm migrated back into the irradiated transplant and normal regeneration followed. Apparently the irradiated epithelial cells were stimulated, by the presence of the neoblasts, to produce new somites.

\section{TUNICATA}

In tunicata there are extensive powers of regeneration in solitary as well as colonial species. The histology of this regeneration and of the correlated processes of asexual reproduction, which are known to be essentially similar, has been studied intermittently for many years since the observations of Hjort (26) and Lefevre (36) aroused interest by contradicting the doctrines then current regarding the potencies of the germ layers. Thus in the budding of Perophora, Lefevre found amoeboid blood cells to be the most important factor in the formation of new parts and in the main his observations have been substantiated by the work of later investigators upon budding and regeneration in tunicates. His view was that the fate of any one of these totipotent cells was an outcome of the place of attachment and the stimuli received in that particular region as the bud development proceeded. The origin of these blood cells it was, of course, impossible to establish as direct descendants of the embryonic mesoderm, but there was no evidence that any of them had arisen by dedifferentiation.

In a recent study of regeneration and reduction in Clavelina, Spek (54) has described certain vacuolated cells as totipotent in regeneration and as arising in a short region of the gut just behind the stomach. Brien (5) has made a good case against these "Tropfenzellen" of Spck as the source of new material during budding and regeneration. According to Brien, such cells are nutritive in function and important during 
regeneration only to that extent. The cells that are important in the formation of new parts are certain undifferentiated cells of the mesodermal region which Brien regards as representing an embryonic reserve to be found in tunicates and in many other animals.

In a brief study of the effects of radium irradiation upon Clavelina lepadiformis, Weigand (67) has shown that the regeneration can be affected by this means in the same manner as in planarians. A retardation appeared externally in the later appearance of the caps of cells at the injured regions and the slower regenerative growth and differentiation (Fig. 14). The effect increased with longer exposures, but a complete inhibition was not obtained with the maximum ones used in Weigand's experiments. His study of the normal histology of the regeneration was brief and not conclusive, although he observed that regeneration of the branchial sac began without mitoses. The source of the regenerative cells was not clear, but the "Tropfenzellen" of Spek (54) did not participate. Mitoses were observed later in the regenerative cells as differentiation proceeded and mitoses were also observed in "Tropfenzellen." Histological study of the irradiates showed abnormal mitoses which apparently eventuated in the degeneration of the injured cells. The branchial sac regenerated more slowly because of the lesser number of normal mitoses in the early stages of the regeneration and the appearance of abnormal mitoses after the longer exposures to the rays, while the number of "Tropfenzel-

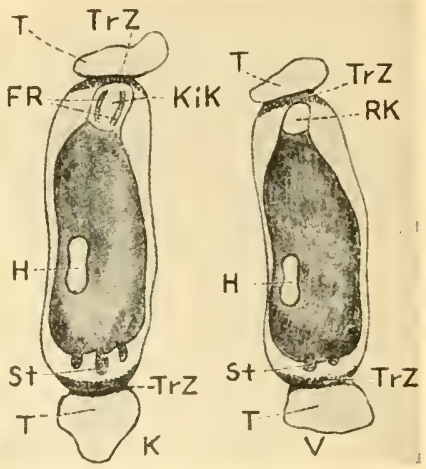

FIG. 14.-Effect of radium upon regeneration of Clavelina lepadiformis. $K$, control. $V$, irradiate exposed to radium $7 \mathrm{hr}$., cut for regeneration immediately thereafter, and killed 7 days later. Differentiation has not proceeded so far in the irradiate as in the control. $F R$, ciliated groove; $H$, heart; Kik, brachial basket; $R K$, regeneration cap; St, stolon; $T$, tunic; $\operatorname{Tr} Z$, Tropfenzellen. (From Weigand, 67.) len "was found reduced after such exposures.

In view of his limited study of the histology in the normal and the irradiated specimens and the contradiction by Brien of Spek's interpretations, which Weigand accepted, it is impossible to draw any conclusions from these experiments by Weigand, except that the irradiation exercised an inhibiting effect, as in other cases, and that abnormal mitoses were induced. If Brien's conclusions regarding undifferentiated mesenchyme cells are correct, these cells are presumably the ones most affected and the disturbance of their multiplication or their death would explain the retardation of regeneration that Weigand observed.

\section{AMPHIBIA}

In vertebrates the amphibia have presented the most favorable material for studies upon regeneration. The urodeles in particular have been 
the subject of many investigations of the superficial and organismal aspects of the process. The histological aspects, which have received less attention until recent years, have proved extremely difficult to understand. According to Hellmich $(23,24)$ who has made an exhaustive study of these changes, the cells observed in the regenerating limb of an adult salamander, such as Amblystoma mexicanum, can be grouped into two large classes, the hematogenetic and the histogenetic. "The first class includes the erythrocytes, lymphocytes, eosinophile cells, special leucocytes, and plasma cells; the second, the mesenchymal cells, fibrocytes, wandering cells, mast cells, pigment cells, giant cells, and pericytes." In the regeneration of young larvae it is impossible to make any such separation into cell types. It appears, however, that mesenchymal cells are essential for regeneration in the larval stages, since there is no regeneration following complete extirpation of a limb bud, which presumably results in extirpation of all the cells of the anlage and its immediate environment. Hellmich assumes "that in the normal growth of the limb there remains a residual number of undifferentiated mesenchymal cells," which can then function in adult as well as larval regeneration. While the histological processes of regeneration in adult salamanders are thus very complicated, proceeding from various kinds of differentiated and undifferentiated cells and connected with inflammatory conditions, the same processes in young larvae are simpler, consisting principally of the multiplication of undifferentiated mesenchymal cells and differentiation of the cells thus produced without being merely a repetition of embryonic development in a restricted part of the body.

The studies of Schaper (51) on the effects of radium irradiation upon amphibian development included a brief examination of these effects upon regeneration in Triton larvae. This investigation and the one by Levy (37), who studied Schaper's material after the latter's death, are now important only as confirmed by recent work. Like the work of Bardeen and Baetjer (3) with planarians, these papers constituted important exploratory studies that might have led to further research as an immediate outcome, since it was shown that such irradiation inhibited regeneration. No one else seems to have undertaken further studies of this sort with vertebrates during many years. Litschko $(38,39,40)$ was first in this revival of interest, although closely followed by Butler (7) and by Brunst and Scheremetjewa $(6,52)$.

In his latest paper Litschko (40) apparently includes the preliminary accounts given in the two preceding publications and summarizes numerous experiments each made with five or six animals and a similar number of controls. He considered the effects of X-rays on regeneration of limbs, tail, and dorsal fin in the axolotl, especially with regard to what caused the inhibition. The exposures, which ranged from 22 to $600 \mathrm{r}$, were either 
general, with one or both limbs sereened from the rays to the level of the thighs and the remainder of the specimen exposed; or local, with the limbs exposed and the remainder sereened. The animals were anesthetized with ether before irradiation and the exposures made in a dorsoventral direction. In most eases the limbs were amputated 1 to $2 \mathrm{hr}$. after the irradiation, except in the experiments designed to test the persistence of the effect. He found that a suffieient general exposure killed all the irradiates in any experiment, but the time of death depended upon the age of the individual as well as the strength of exposure. Thus, year-old speeimens exposed to $600 \mathrm{r}$ died 19 to 27 days later, while ninemonths-old speeimens exposed to $330 \mathrm{r}$ died at 25 to 30 days. A similar dual relationship was observed in the inhibition of regeneration. The yearlings exposed to $330 \mathrm{r}$ showed complete inhibition of regeneration when their limbs were amputated, while nine-months old individuals exposed to only $88 \mathrm{r}$ showed a similar inhibition. With lesser irradiations the period of survival varied inversely with the exposure and the regeneration was less affected until at an exposure of $44 \mathrm{r}$ there was complete survival and subnormal regeneration, and at $22 \mathrm{r}$ both survival and normal regeneration. Local exposures of limbs and fins, instead of general ones, were used exelusively after it had been found that the results in local and general exposures were similar, beeause with local exposures the experiments could be made without the complication of lethal effects due to the irradiation. The only disadvantage was that regeneration proceeded more slowly following local exposure of a limb than following a general exposure.

Despite the effects observed in animals that had received general exposures, Litsehko concluded that the inhibition was due to local action alone. The failure of regeneration of the limbs after certain loeal exposures (88 $r$ for nine months-old specimens) seemed to result from the existence in the limb of certain conditions under which the power of regeneration could not express itself and not from a complete destruction of this poteney. Histological study showed that inhibition in the outgrowing stump of a regenerating limb was correlated with the formation of a mass of eonnective tissue at the distal end into which it seemed that the muscle and other underlying tissues could not extend themselves as normally. Removal of the whole of this terminal mass of connective tissue, by a second amputation made before degeneration of the underlying tissues had begun, resulted in a complete regeneration, while lesser removals resulted in lesser degrees of regeneration. It thus appeared that the regenerative potency had not been lost but had been inhibited by the action of the rays upon the connective tissue. This aetion he regarded as a stimulation and found additional support for such an interpretation in the superregeneration observed where a portion of a locally irradiated region had been eut from the dorsal fin (Fig. 15). In 
such a case, if the underlying tissues had not been involved the regeneration exceeded the normal, because the connective tissue which composes the fin between the two epithelial layers was stimulated, as he thought, to more active growth. Again, he thought that a similar superregeneration might occur, and be similarly explained, when part of the tail was removed by a transverse cut, but he was not sure of this.

Litschko also investigated the persistence of the inhibitive effects of the irradiation by making the amputations from 1 to 8 weeks after the
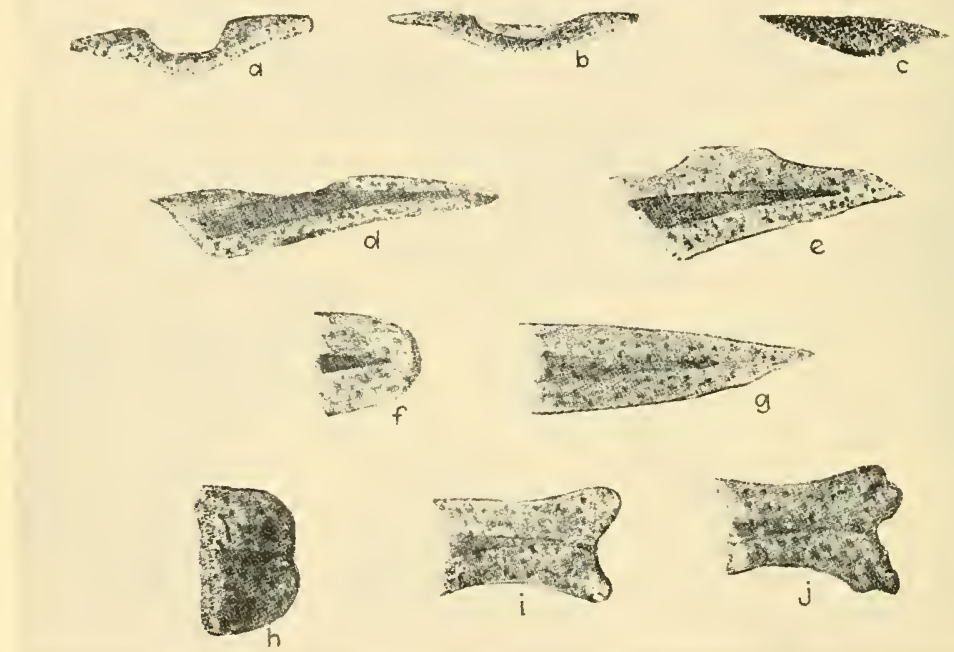

F1G. 15.-Effects of X-rays upon regeneration of tail in the axolotl. $a$, dorsal fin on second day after exposure and removal of a portion as shown; $b$, normal regeneration of the removed portion, after an exposure without injury to the underlying dorsal musculature, 1 month after exposure and operation; $c$, the same after 2 months showing the normal outline completed although there is still a slight difference between the color of new and old tissue; $d$, defective healing by scar formation 2 months after exposure and operation in a case where the underlying elements of the dorsal musculature were involved; $e$, superregeneration 10 months after the exposure and operation; $f$, tail 2 or 3 days after an amputation that removed only the distal portion of the fin which consists of connective tissue covered by epithelium; $g$, normal regeneration in such a case 2 months later; $h$, schematic representation of a more proximal amputation, involving musculature, chorda, and nerve cord, as it appears shortly after the operation; $i$, supcrregeneration in such a case 2 months later; $j$, the same after 9 months. (From Litschko, 40.)

exposures instead of 1 to $2 \mathrm{hr}$. after as in other experiments. From these experiments it appeared that the impulse to increased growth of connective tissue persisted for a long time when it was once given by the rays. A similar inhibition with stump formation was observed even when the amputation was made 8 weeks after the irradiation, but when the connective tissue tip of this stump was removed by a second amputation, complete regeneration occurred. This was his final evidence that the power of regeneration was not suppressed as a result of the irradiation, rather the raying was only a hindrance in its expression. 
Butler (7) studied the effects of X-rays upon regeneration of the fore limb in larvae of Amblystoma punctatum, using a Coolidge medium focus tube operated at $60 \mathrm{kv}$, $6 \mathrm{ma}$., $25 \mathrm{~cm}$. from target to specimen, and without a filter. The exposures are not stated in roentgens, but the foregoing factors were constant throughout. The duration of exposure was varied in different experiments, but in each case a daily exposure, of 2,3 , or 5 min., respectively, was made throughout periods of from 21 to 25 days according to the experiment. The question of the exposure necessary to prevent regeneration remained to be thoroughly investigated. It appeared that the exposures used in some of the earlier experiments were much longer than was actually necessary, since it was found in later experiments that daily exposures of $1 \mathrm{~min}$. each were as effective as the daily exposures of longer duration. The position of the larvae during exposure is not stated.

In a representative case, larvae $15 \mathrm{~mm}$. in length with fore limbs in early four-digit stage were irradiated in two lots 2 and $3 \mathrm{~min}$. daily for a period of 21 days, beginning the day following amputation of one fore limb on each irradiate. In none of these irradiates was there any sign of regeneration of the amputated fore limb. In the controls there was rapid and normal regeneration. There was no significant difference between the larvae irradiated $2 \mathrm{~min}$. and those given the 3-min. exposure. In another experiment, larvae with fore limbs in early two-digit stage were irradiated $5 \mathrm{~min}$. daily for 25 days following amputation. A similar result was obtained by amputation of the limb buds before appearance of the digits.

Comparable results were obtained by Butler in 23 experiments of a like nature. He found that the amputated limb failed to regenerate and also that there was usually a considerable resorption, so that the nonregenerating limb stump was smaller several weeks after amputation than the portion remaining attached to the body the few days after the operation. The effect of the rays upon digit formation Butler regards as significant. He found when a normal and uncut limb was about to enter the two-digit condition at the time radiation began, that the first two digits would be formed in a manner apparently normal, but no third digit would ever develop. In like manner, if the third digit was just appearing at the time radiation began, the third digit would be completely formed, but no fourth digit would ever develop. These results suggested that the X-rays acted upon differentiating tissue at a certain critical period in the process during which there was a heightened sensitivity to these radiations, and that if the differentiation had passed this period before the exposures began, it proceeded despite the irradiation. From other experiments in progress but not included in the paper under discussion, Butler thought that a similar critical period might exist in the process of regeneration. 

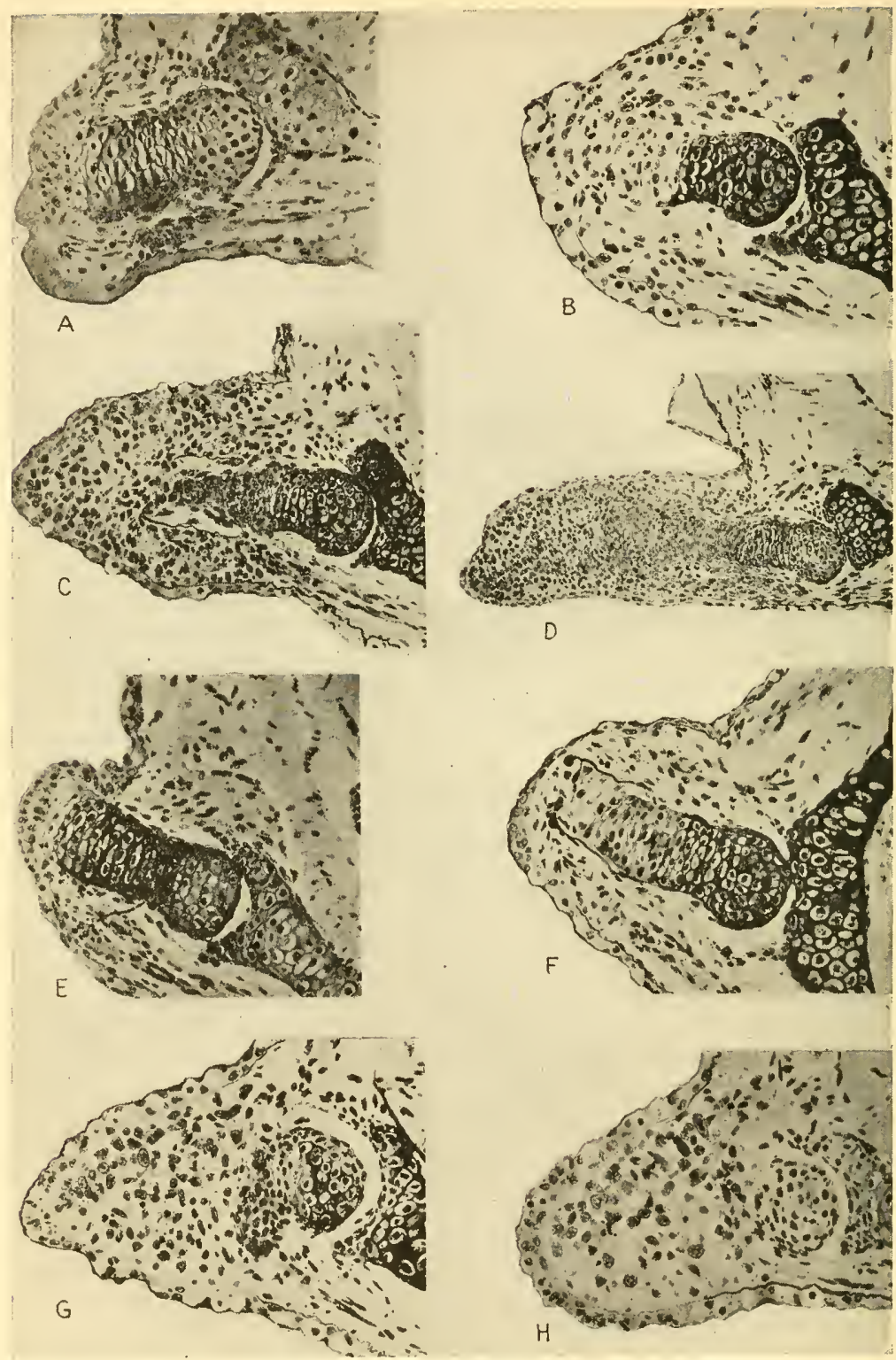

Fig. 16.-Effects of X-rays upon regeneration and cells of Amblystoma larvae. $A$, longitudinal section through a limb stump of a nonirradiated control 18 hours after amputation showing healing and beginning of normal changes in the cartilage of humerus; $B$, the same 4 days after amputation showing further normal changes in cartilage and beginning of blastema; $C$, the same 9 days after amputation showing further changes as above; $D$, the same at 12 days; $E$, longitudinal section through limb stump of an irradiated larva $11 \mathrm{hr}$. after amputation showing healing as in control; $F$, the same 4 days after amputation showing absence of the typical blastema; $G$, the same 8 days after amputation showing absence of typical blastema and degeneration of cartilage of humerus; $H$, the same 13 days after amputation ( $c f$. with $D$ above) showing further reduction of humerus. (From Butler, 7.) 
A careful study of the histological changes was included in this investigation (Fig. 16). During normal regeneration following amputation in the three-digit stage, for example, healing occurred by an extension of the epidermis without cell division. The next important change was a vacuolation of the cartilage at the cut end of the humerus, which Butler regarded as primarily a result of the mechanical injury at time of amputation. The changes in this region of the cartilage eventuated in what he regarded as a dedifferentiation of the cartilage cells into mesenchyme cells rather than a degeneration related to phagocytosis, as described by certain investigators. While these changes were taking place in the cartilage during the first 6 days after amputation, other mesenchyme cells accumulated between the epidermis and the end of the humerus; and these cells in conjunction with those arising by dedifferentiation of the cartilage apparently gave rise to the blastema from which all the new parts of the regenerating limb except epidermis and nerves were formed in the manner described by various investigators. Mitotic figures were frequently observed in the limb during normal regeneration.

In the nonregenerating limbs of X-rayed individuals the histological changes differed profoundly from these normal processes, except that the epidermal healing proceeded normally. With material from an experiment in which the larvae were exposed for $5 \mathrm{~min}$. daily, the changes during the first 2 or 3 days following amputation were not obviously different from those in the normal regeneration (Fig. 16). On about the fourth day there was observed the beginning of an extensive alteration of the cartilage of the humerus. At the outset this was not unlike the dedifferentiation described for the control specimens but was more extensive and finally resulted in complete disappearance of the humerus in 13 to 16 days, and then of the scapula some 18 days after the amputation. Butler did not regard this as a degeneration process in the ordinary sense, because there were very few evidences of degenerating cartilage cells and no accumulation of blood cells about the end of the humerus as would have been expected if phagocytosis had been in progress. As the dissolution proceeded, released cartilage cells became indistinguishably mingled with the cells of what he called the "pseudoblastema" in a manner comparable with the mingling of mesenchyme cells in this region during the normal regeneration. Thus, about 20 days after limb amputation the irradiated larva possessed a nonregenerating limb stump totally devoid of skeleton.

This dissolution of the cartilage occurred in spite of the fact that the limb of the opposite side, which was not amputated, developed from the two-digit to the three-digit stage during the same period and in the same specimens. In short, cartilage passed through stages of dissolution on one side of the body while cartilage was developing on the other. It was noted, however, that no fourth digit appeared on the unamputated 
limbs in this experiment. The effect of the irradiation seemed to be not on cartilage tissue as such but on the initiation and differentiation of new cartilage. It appeared that the critical period mentioned for the regenerating limbs might be identical for the period of cartilage initiation and differentiation, or simply the expression of the sensitivity of cartilage differentiation to $\mathrm{X}$-rays.

The "pseudoblastema" seemed fundamentally like the normal blastema, although it consisted of a much smaller number of cells and did not proceed to the normal differentiation (Fig. 16). Vacuolation appeared among the widely scattered nuclei and there was a considerable amount of intercellular material that was apparently nonliving, presumably the débris of broken-down cartilage matrix and of disintegrated cells. As with the normals, it was impossible to distinguish between cells of the pseudoblastema proper and cells released by dissolution of the cartilage. In contrast with the limb of the normal regenerate, the limb of the irradiate showed no normal mitoses. Instead there were many abnormal mitoses with tangled masses of chromosomes in no very definite orientation, in the manner that has long been known to result from X-ray exposure. Other cellular abnormalities were increase in size of nuclei as compared with those of the normal blastema; later, the appearance in these swollen nuclei of deeply stained granules; and finally, remnants of such disintegrating nuclei in the intercellular débris.

In discussing the foregoing observations and interpretations, Butler points out that the irradiation in some manner inhibits differentiation of the cells of the blastema. Whether the effect should be regarded as acting directly upon the cells of the blastema, or as an indirect effect resulting from some action of the rays upon the organism as a whole, he leaves an open question. In any case the cells of the blastema are particularly susceptible to the rays, as might be expected, since the normal blastema is a region composed of actively dividing cells. The pseudoblastema shows only a limited number of undifferentiated cells and no normal mitoses. The prevention of regeneration he regards as due "to the activity of the $\mathrm{X}$-rays in preventing cell division and more particularly in preventing differentiation of the cells of the blastema which normally give rise to the components of the regenerating limb." The second effect, namely, the dissolution and final disappearance of the cartilage, which he characterizes as like cartilage dedifferentiation "run wild," he thinks may be associated with the effect of the rays upon differentiating cells, that is, prevention of the differentiation of new cartilage within the blastema may have brought about secondarily the dedifferentiation of cartilage already formed. According to such an hypothesis, "cartilage dedifferentiation does not occur in limbs in which $\mathrm{X}$-radiation prevents digit formation, for the reason that in the process of normal digit formation dedifferentiation is not involved," and the 
irradiation "does not initiate cartilage dedifferentiation directly." The primary effect of the irradiation, therefore, he regards as an effect upon differentiation; and the extensive cartilage dissolution as a secondary effect induced by the radiation but dependent upon the effect on differentiation.

In comment upon these conclusions it may be said that instead of regarding the primary effect as upon differentiation it would be simpler to regard it as an effect upon cell division and so upon cells with differentiation potencies. As a result of this effect upon cell division the
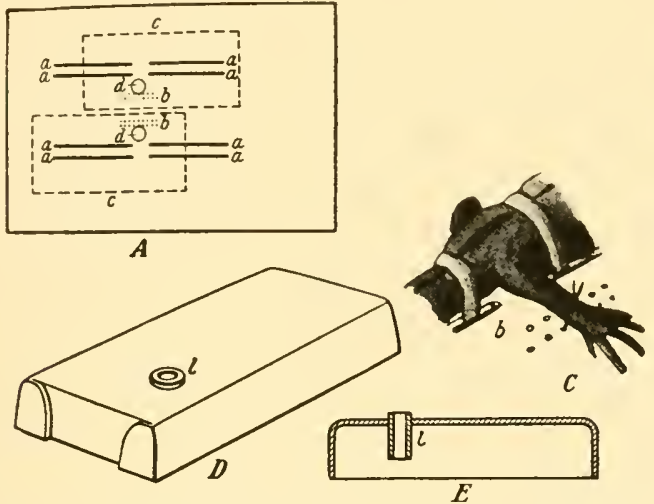

FIG. 17.-Technique for local exposure of well-grown specimens of Triton cristatus. $A$, top view of stage with slits $a a$ for fastening the animal as in $C$ and $B$, holes $b$ for fastening the limb as in $C$, and opening $d$ for the field of exposure; $B$, position for exposure of two animals at same time; $C$, detail of the fastening; $D$, lead cover with opening $l$ corresponding to $d$ in $A ; E$, the same in section. (From Brunst and Scheremetjewa,6.)

differentiation of these cells cannot proceed in the normal manner. Again, it is noted that Butler's exposures were all given after the operation for regeneration. It would be interesting to know whether the pseudoblastema would have appeared and what would have been the effect upon the cartilage, if the series of the daily exposures to the rays had begun several days before the operation, or if a single heavier exposure had been given. The check upon subsequent differentiation of digits in irradiated but uncut limbs is perhaps also referable in the first instance to a check upon cell division. As this work is being continued in Butler's laboratory (48), these questions will no doubt be given further consideration now that so excellent a beginning has been made.

The investigation by Brunst and Scheremetjewa (6) on the effects of X-rays upon regeneration of the limbs in well-grown specimens of Triton cristatus has shown again the inhibitive action of such irradiation when given locally (Figs. 17 and 19). The limit of an obvious and constant effect was 300 to $750 \mathrm{r}$, while $15,000 \mathrm{r}$ resulted in a more or less constant inhibition (Fig. 18). An exposure of $3750 \mathrm{r}$ showed on the whole a strong 
inhibitive effect, but the results were so diversified in all the experiments that differences in the reactions of the individuals to the same exposure became apparent. For this reason the authors concluded that a larger

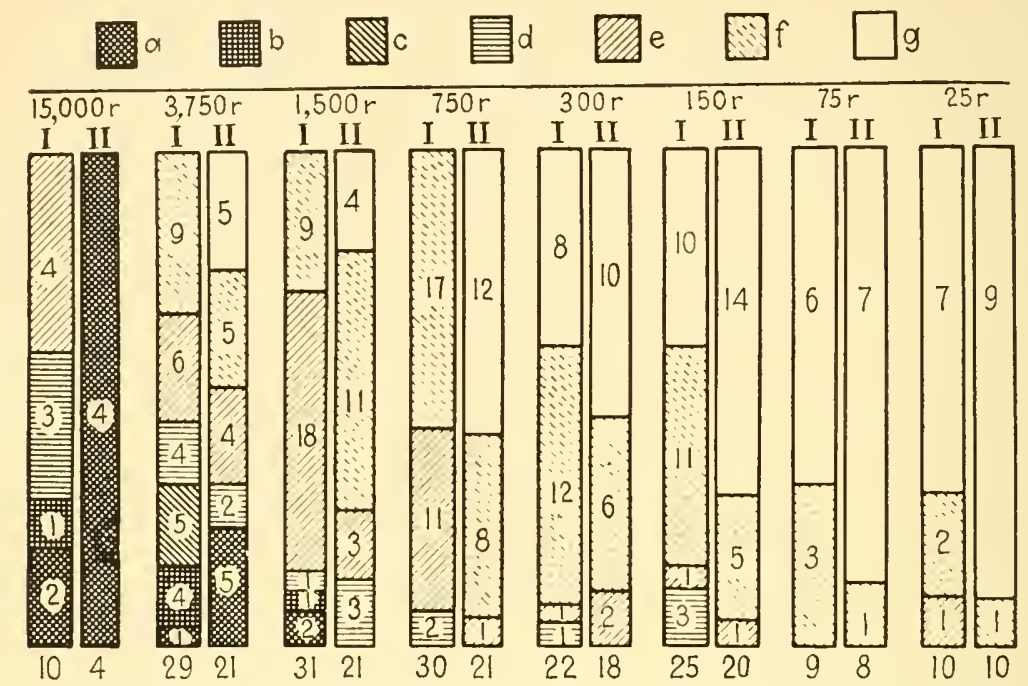

FIG. 18.-Graphic representation of the effects of various X-ray exposures upon regeneration of the limbs of the irradiated side of Triton cristatus. Each pair of columns represents an exposure, the number above the pair representing the roentgen units. Column I represents the first regeneration; Column II, the second. The numbers below the columns show the total number of individuals at conclusion of any experiment. The numbers within the columns show the number of individuals giving the reactions designated $a$ to $g$. The shading indicates these reactions as follows: $a$, no regeneration; $b$, reduction; $c$, regeneration and reduction; $d$, insignificant regenerative growth; $e$, significant delay of regeneration; $f$, insignificant delay of regeneration; $g$, normal regeneration. (From Brunst and Scheremetjewa, 6.)

number of specimens than were used in each experiment by Litschko (40) is necessary for trustworthy conclusions (Figs. 22 and 23). They explained this difference of reactions in terms of the capacity of individual organisms to react differently to the same external factor and the complex effect of irradiation involving primary and secondary factors as it
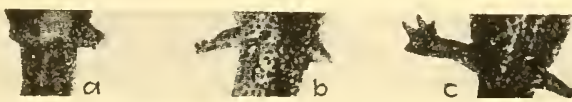

Fig. 19.-Effect of X-rays (1500 r) upon regeneration of hind limbs of Triton cristatus. a, ventral view 17 days after exposure of the right and amputation of both these limbs; $b$, dorsal view of same specimen 34 days after; $c$, dorsal view of same 51 days after. (From Brunst and Scheremetjewa,6.) does in an animal with such a degree of organization as the salamander. In studying the persistent effects of the irradiation, it was observed in cases of stronger exposure that where the first regeneration of any appendage showed a conspicuous effect its second regeneration usually showed an even stronger effect; and that where the first regeneration was not conspicuously affected its second regeneration was affected to an even smaller degree (Figs. 18, 20, and 21). 
The effect of the irradiation could not be entirely local, since it was found that with local exposures the effects became evident to some degree in the regeneration of the unexposed extremity of the same animal (Fig. 22). With weaker exposures the nonirradiated limb, or "regenerate control," developed in about the same manner as any "normal control." No evidence was found of any directly stimulating action of the rays upon the regeneration. There is nothing in this paper regarding the histological changes in controls or in irradiated individuals.

In another paper (52) these same investigators have studied the effects of $\mathrm{X}$-rays upon regeneration of the

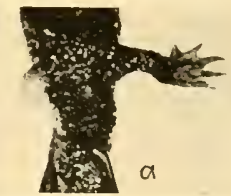

Fig. 20.-Effect of X-rays $(15,000 \mathrm{r})$ upon regeneration of hind limbs of Triton cristatus. a, ventral view 119 days after exposure of the right and amputation of both hind limbs; $b$, ventral view of a similar specimen 62 days after a second amputation of both limbs. (From Brunst and Scheremetjewa, 6.) tail in Pelobates fuscus tadpoles that were given single exposures ranging from 750 to $7500 \mathrm{r}$ (Fig. 24). Within these limits they obtained all stages in the inhibition of tail regeneration. It was found that total exposures had a greater effect than local exposures of the same order. The dorsal side, which was toward the source of the irradiation, showed greater injury than the ventral, as seen either in greater inhibition of the dorsal regeneration or in more intensive reduction on the dorsal side of the stump (Figs. 25 and 26). The tail outline beeame asymmetrical in the first case, beeause the tail of the regenerate was bent dorsally; and in the second, because the rentral part of the stump predominated. The
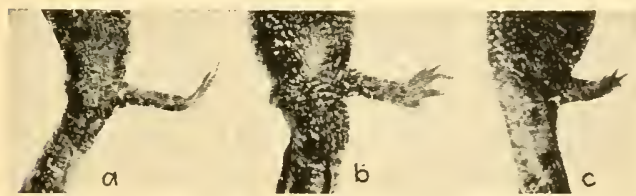

Fig. 21.- Effect of X-rays $(3750 \mathrm{r})$ upon regeneration of hind limbs of Triton cristatus. $a$, ventral view 135 days after exposure of the right and amputaspecimen 135 days after; $c$, ventral view of a similar specimen 74 days after a second amputation of both limbs. (From Brunst and Scheremetjewa, 6.) tion of both hind limbs; $b$, ventral view of a similar

optimum exposure, of those employed, for this dorsal bending was $1500 \mathrm{r}$ after which the ventral regeneration was still quite intensive. With $2250 \mathrm{r}$ the entire tail regeneration was so affected that the bending was less in evidence. The different tissues of the tail and of the stump showed differing sensitivities to the rays and morphologieal injuries that could be clearly recognized appeared in the following order: (a) pigment cells; (b) epithelium; $(c)$ chorda; $(d)$ gelatinous connective tissue; $(e)$ museulature; $(f)$ nervous tissue. The regenerative potencies of the tissues were lost in this order: (a) chorda; (b) spinal cord and musculature; (c) gelatinous connective tissue; $(d)$ blood vessels and epithelium. The differing sensitivity of chorda and spinal cord was especially noticeable. In spite of the fact that the spinal cord was nearer the source of the rays, it grew intensively after 
exposures that markedly inhibited growth of the chorda and even after exposures that brought the growth of the chorda to a complete standstill

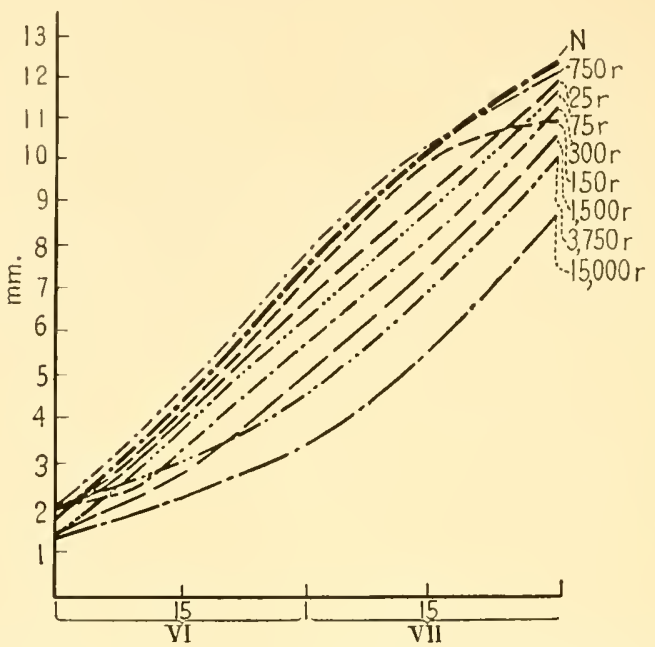

Fig. 22.- Mean growth curves for the regeneration of nonirradiated limbs in Triton cristatus, following exposure of the opposite limbs to X-rays ranging from 25 to $15,000 \mathrm{r}$. $N$, controls. (From Brunst and Scheremetjewa, 6.)

(Fig. 26). The optimum for showing this difference between chorda and spinal cord was $2250 \mathrm{r}$. None of the exposures showed a directly stimulating effect upon the regeneration. With $3750 \mathrm{r}$ there seemed to be an indirect stimulation of regeneration in the epithelium, in which it was

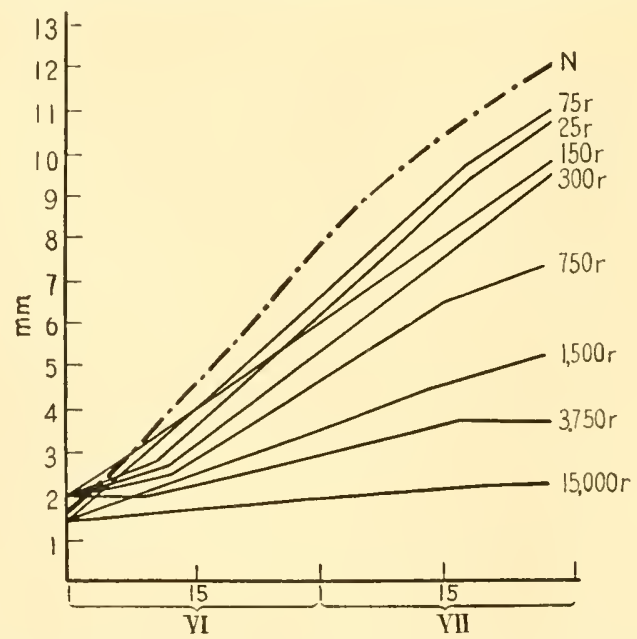

FIg. 23.-Mean growth curves for the regeneration of irradiated limbs in Triton cristatus, following exposure of same to X-rays ranging from 25 to $15,000 \mathrm{r}$. $N$, controls. (From Brunst and Scheremetjewa, 6.)

thought the degeneration products, resulting from the necrotic changes in the irradiated parts, exercised the stimulating influence. 

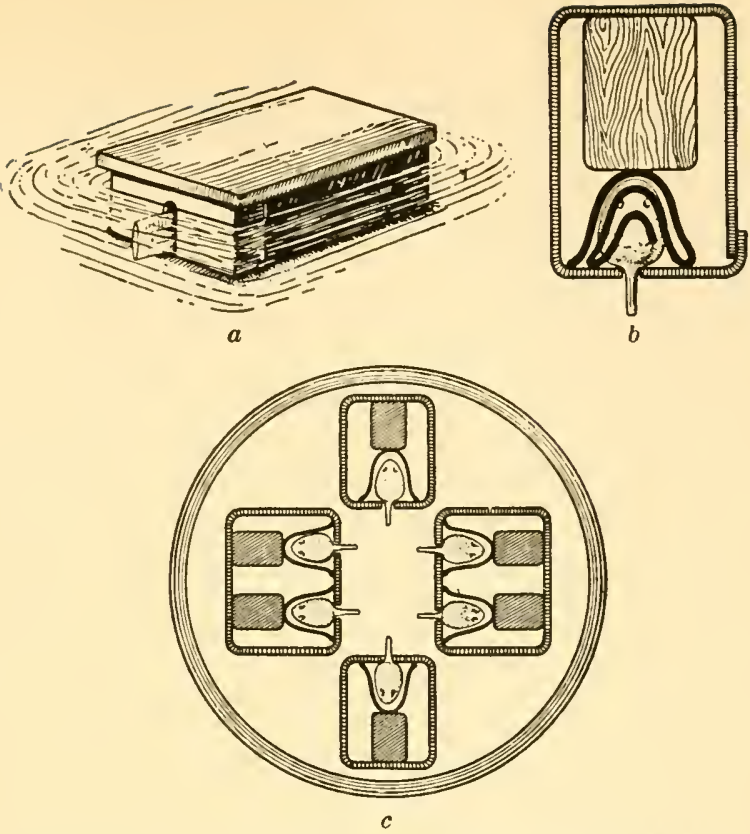

FIg. 24.- Technique for local exposure of tadpoles, Pelobates fuscus. a, external view of one of the holders shown in $c$ with lead cover in place as during exposure; $b$, method of fastening the tadpoles under the lead shield by means of a bent strip of lead and wooden block; $c$, arrangement of such holders in a crystallization dish during irradiation. (From Scheremetjewa and Brunst, 52.)
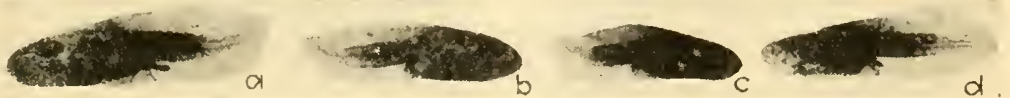

FIg. 25.-Effects of $\mathrm{X}$-rays upon regeneration of tail in tadpole of Pelobates fuscus. $a, b$, and $c$, three regenerates at 32 days after local exposures of $1500 \mathrm{r}$ and amputations immediately following; $d$, normal regeneration of control. (From Scheremetjewa and Brunst, 52.)

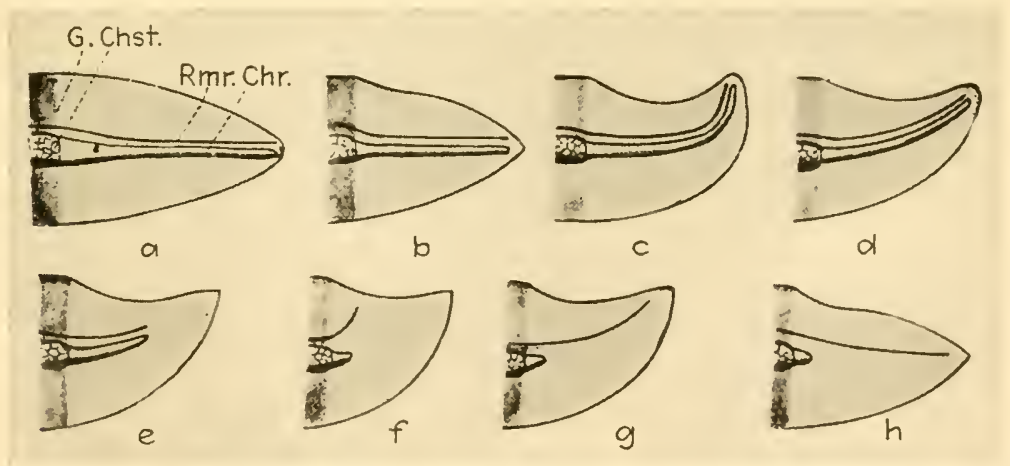

Fig. 26.-Schematic representation of different reactions in tail regeneration of tadpoles of Pelobates fuscus following X-ray exposures ranging from 1500 to $2250 \mathrm{r}$. Chr, regenerated chorda; Chst, stump of chorda; $G$, border between the new and old tissue; $R m r$, regenerated spinal cord. (From Scheremetjewa and Brunst, 52.) 
It is evident from the foregoing studies upon amphibia that members of this class among vertebrates react to radium and X-rays in a manner superficially comparable with the reactions found in other animals having conspicuous powers of regeneration. The healing again appears as a process independent of regeneration and performed by extension of the old epithelium, although the new covering may not attain normal thickness in the absence of cell additions by multiplication or otherwise. The degree of inhibition is proportional to the exposure. 'The extent to which the effects of the irradiation are local is not fully established, although the loeal effects seem to be more important than such general effects as have been described. The complexity of the histological changes in the regeneration of the adult amphibian makes it appear that any destruction of reserve cells or of cells with embryonic potencies that might oceur would be difficult to demonstrate beyond question. In the larvae, however, these changes are simpler and elues may be obtained to an understanding of adult conditions. In view of what is known of the histological changes during normal regeneration and those following irradiation in other types of animals, there is a presumption in favor of undifferentiated reserve cells as important factors, which makes this perhaps the most significant clue to be followed as the case now stands. While the vertebrate presents the disadvantage of this histological complexity and also a physiological one, it has the advantage of presenting tissues that are better known than those of any other animals and the same may be said regarding the physiological processes of vertebrates. There is also the advantage, in a case like the well-grown salamander, of an animal large enough for the easy removal of parts that can be regenerated and an animal that can be maintained for long periods under laboratory conditions. Since these advantages outweigh the disadvantages, it would seem that these amphibia and perhaps other lower vertebrates should be promising material for further investigations. If there is any animal type that might meet the specifications for "castration" against regeneration, to which reference has been made, it would seem to be the members of this group. There is also the fact that what may be accomplished with the vertebrates is more closely related to clinical knowledge and interests that may be illumined by investigations of this nature; and that existing knowledge of vertebrate physiology gives so broad a foundation for study of the organismal effects of such radiations upon these animals.

\section{PHYSIOLOGICAL REGENERATION}

What is often called "physiological" regeneration might be included in the present discussion, but it would be difficult to limit the account of such processes and there are many who question their inclusion under the head of regeneration. The effects of X-rays and radium upon the 
growth of hair has long been known and the effects upon certain glands is another indication that these radiations may inhibit normal restorative processes in a way that can be compared with their effect upon the type of regeneration under consideration. To review the literature bearing upon such a comparison would carry one far into the field of clinical medicine. As an introduction to the more general biological study of these "physiological" processes, as affected by X-rays and radium, the recent paper by Hirsch (25) regarding secretion may be cited.

\section{DISCUSSION}

The foregoing résumé of investigations in this field shows that radium and $\mathrm{X}$-rays have a similar inhibitive effect upon regeneration in animals as representative and as widely scparated as coelenterates, flatworms, segmented worms, and chordates. Externally, the same kind of changes appears in each of these cases. Healing occurs in the normal manner by an extension of the old epithelium, but the subsequent thickening and restoration of the original state of these cells that occur in normal regeneration do not follow. The regenerative processes by which the missing parts become restored are retarded or completely inhibited according to the degree of exposure to the rays. Internally, it seems that the clue to the changes observed lies in the effect of the irradiation upon whatever cells are active in producing the new tissues by division, migration, and differentiation. One pictures the normal regeneration as involving in the first instance histological changes incident to the mutilation of a given region, and also organismal changes or those which involve the individual as a whole and are not clearly recognizable in certain of its cells. These latter processes doubtless bring about other histological changes as the regeneration proceeds. While the organismal and the histological factors must be regarded as so inextricably mingled that they are merely different aspects of a unified series of events, it is convenient to consider them separately wherever definite changes of either sort become apparent. The histological aspects are more easily recognized insofar as they consist of morphological and regional changes in cells. These include: the nature and origin of the cells concerned in regencration; their growth, division, movement, and the like, as activated, perhaps, by organismal factors; and their differentiation under the influence of organizing centers. Thus by the interplay of these general and specific factors the regeneration is consummated. Whether one puts the organism or its cells first in such a case depends to some extent upon one's concept of the individual in relation to its cells as well as the data from observation and experiment.

In some instances, such as the planarian, the effect of the irradiation upon specific cells appears to be a direet effect, since it is recognizable soon after the cxposure. In a case of this sort it is easy to think in terms 
of the differential susceptibility of cells, which has been so often demonstrated in accordance with the law of Bergonié and Tribondeau, as responsible for the primary effect of the irradiation; and of later effects as an outcome of such primary effects upon these cells. For example, the degenerative changes that appear ultimately in a planarian may result from toxic products formed by destruction of the considerable amount of protoplasm composing the regenerative cells. In a case where no effect is apparent until a later period, the delay may be due to changes within certain cells not becoming recognizable soon after the irradiation or to an organismal effect that does not at first become evident as changes in any of the cells. In the absence of specific evidence it is perhaps an academic question whether the changes observed in a given cell originated as "direct hits" within that cell or have resulted, for example, from changes induced in body fluids and so affecting the cell indirectly. Even the seemingly direct action upon cells in tissue cultures is open to these two theoretical explanations. What is needed is specific evidence if it can be obtained.

Passing over this question of direct or indirect effects and beginning with recognizable cell changes, we have as the obvious explanation of what can be observed the differing susceptibility of different types of cells to these radiations. In a case, such as the planarian, it is known that the so-called regenerative or formative cells, whether they have originated by dedifferentiation or by multiplication from an embryonic stock, differentiate into the various cell types and form the missing parts during regeneration and hence may be said to have embryonic potencies. These are the cells most sensitive to the irradiation in question, and with their reduction in numbers or complete destruction regeneration is correspondingly affected. Again, in the hydra regeneration is checked and the interstitial cells, which have been described by many investigators as having embryonic potencies and as being most important in regeneration, are the cells conspicuously affected in the first instance. In oligochaetes it is the neoblasts, in Clavelina it is the cells described as active during regeneration, in the limb of Amblystoma larvae the cells of the blastema, while in the tadpoles of Pelobates the different types of cells exhibit differing susceptibilities which account for the gross differences between controls and irradiates. One could hardly expect a greater coincidence of the internal effects and of the external effects of these radiations in checking regeneration, whether in the tentacle of a hydra or the tail of a tadpole. Moreover, the histological changes indicated are in agreement with the widely demonstrated effects of radium and X-rays upon less differentiated cells, and upon cells with embryonic potencies. The consistency of all these observations is such that one feels justified in considering the potencies of specific types of cells as one of the most important factors in the regenerative process. While this 
had been indicated from earlier investigations by the familiar techniques, it is being more effectively demonstrated by the technique of irradiation.

Without expecting too much from further studies upon regeneration by means of irradiation, it may be hoped that a method of experimentation capable of destroying certain types of cells, as by an operation of far greater delicacy than could be accomplished by the finest microdissection, will yield many important results in this field. There is no other method by which more profound internal changes can be effected, in some instances without ultimate destruction of the organism. Since radium and X-rays affect primarily the cells with embryonic potentialities, one can remove these cells, as though by dissection, and hope to discover the kind of organism which then exists. That the differential in sensitivity is even finer than this appears from the way in which the cells in the cap at the anterior end of a regenerating planarian lose their extreme sensitivity as differentiation proceeds in the same manner as differentiating cells during embryonic and later development. As the converse of this one would expect an increase of sensitivity in any case of dedifferentiation that was clearly demonstrable. The finer differential is seen again in the differing effects as the time relationship between exposure and operation is shifted; and in the case where $\mathrm{X}$-rays inhibit cartilage differentiation in the amputated limb of Amblystoma during a period when differentiation of this tissue is proceeding in the opposite limb which was left intact. There is also the possibility of distinguishing critical periods in differentiation as suggested by some of the observations on planarians and on Amblystoma. The vexed questions of the potencies and of the origin of formative cells along with the problem of contrasting powers of regeneration can be attacked as by no other technique; and it should be possible to separate more effectively the histological from what we have called the organismal factors in regeneration. A study of such problems becomes a study of the general problem of cell differentiation and determinism. In all this it is highly desirable that we should learn more concerning the fundamental effects of the radium and X-rays upon protoplasm by study of material more favorable for the purpose than is the regenerating tissue of most animals.

\section{REFERENCES}

1. Alberti, W., and G. Politzer. Úber den Einfluss der Röntgenstrahlen auf die Zellteilung. Arch. Mikrosk. Anat. und Entwicklungsmech. (Roux) 100: 83-109. 1924.

2. Alberti, W., and G. Politzer. Über den Einfluss der Röntgenstrahlung auf die Zellteilung. II. Mitteilung. Arch. Mikrosk. Anat. und Entwicklungsmech. (Roux) 103: 284-307. 1924.

3. Bardeen, C. R., and F. H. Baetjer. The inhibitive action of the Roentgen rays on regeneration in planarians. Jour. Exp. Zool. 1: 191-195. 1904.

4. Bartsch, O. Die Histiogenesis der Planarienregeneräte. Arch. Mikrosk. Anat. und Entwicklungsmech. (Roux) 99: 187-221. 1923. 
5. Brien, P. Contribution à l'étude de la régénération naturelle et expérimentale chez les Clavelinidæ. Ann. Soc. Roy. Zool. Belgique 61: 19-112. 1930.

6. Brunst, V. V. and E. A. Scheremetjewa. Untersuchung des Einflusses von Röntgenstrahlen auf die Regeneration der Extremitäten beim Triton. I. Beobachtungen der Regeneration der Extremitäten beim Triton nach Bestrahlung mit verschiedenen Dosen von Röntgenstrahlen. Roux' Arch. Entwicklungsmech. Organ. 128: 181-215. 1933. [See also (52).]

7. Butler, E. G. The effects of x-radiation on the regeneration of the fore limb of Amblystoma larvae. Jour. Exp. Zool. 65 : 271-303. 1933.

8. CoE, W. R. Analysis of the regenerative processes in nemerteans. Biol. Bull. 66 : 304-315. 1934. [Literature list cites author's earlier papers in this field.]

9. Coldwater, K. B. The effect of sulphydryl compounds upon regenerative growth. Jour. Exp. Zool. 65: 43-71. 1933.

10. Coldwater, K. B. The effects of x-rays on regeneration in the nemertean, Lineus socialis. Anat. Rec. $47: 286.1930$.

11. Congdon, E. D. Effects of radium on living substance. I. The influence of radiations of radium upon the embryonic growth of the pomace-fly Drosophila ampelophila, and upon the regeneration of the hydroid Tubularia crocea. Bull. Mus. Comp. Zool. $53:$ 345-358. 1912.

12. Curtis, W. C. The life history, the normal fission and the reproductive organs of Planaria maculata. Proc. Boston Soc. Nat. Hist. 30: 515-559. 1902.

13. Curtis, W. C. Old problems and a new technique. Science 67: 141-149. 1928.

14. CurTis, W. C., and L. M. Schulze. Studies upon regeneration. I. The contrasting powers of regeneration in Planaria and Procotyla. Jour. Morph. 55: 477-513. 1934.

15. Curtis, W. C. et al. Papers before American Society of Zoologists. Abstracts in Anat. Record, as follows: 29 : 105, 1924; 31 : 304-306, 1925; 34: 145-146, 1926; $34: 150-151,1926 ; 37: 128,1927 ; 37: 134,1927$.

16. Faulkner, G. H. The anatomy and the histology of bud-formation in the serpulid Filograna implexa, together with some cytological observations on the nuclei of the neoblasts. Jour. Linn. Soc. (London) Zool. 37: 109-190. 1930.

17. Faulkner, G. H. The histology of posterior regeneration in the polychaete Chietopterus variopedatus. Jour. Morph. $63: 23-58.1932$.

18. Gelei, J. von. Über die Sprossbildung bei Hydra grisea. Angaben über die Selbstgestaltungsfähigkeit des Organismus als eines Ganzen. Roux' Arch. Entwicklungsmech. Organ. 105: 633-654. 1925.

19. Goетsсн, W. Das Regenerationsmaterial und seine experimentelle Beeinflussung. Roux' Arch. Entwicklungsmech. Organ. 117, 211-311. 1929.

20. Hadzi, J. Bemerkungen über die Knospenbildung von Hydra. Biol. Zentbl. 31: 108-111. 1911.

21. HADZI, J. Allgemeines über die Knospung bei Hydroiden. Extrait IXe Congrès Internat. Zool. 1913.1914.

22. Ḧ̈mmerling, J. Die ungeschlechtliche Fortpflanzung und Regeneration bei Aeolosoma hemprichii. Histologische und experimentelle Untersuchungen. Zool. Jahrb. Abt. Allg. Zool. Physiol. 41: 581-656. 1924.

23. Hellmich, W. Untersuchungen über Herkunft und Determination des regenerativen Materials bei Amphibien. Roux' Arch. Entwicklungsmech. Organ. 121: 135-203. 1930.

24. Helimich, W. Histology of regeneration in different species of adult and larval urodeles. Anat. Rec. 48: 303-307. 1931.

25. Hirsch, G. C. Analyse der Restitution des Sekretmaterials im Pankreas mittels Röntgenstrahlen. Beobachtungen an lebenden Zellen. Roux' Arch. Entwicklungsmech. Organ. 123 : 792-821. 1931. 
26. HзоRт, J. Beitrag zur Keimblätterlehre und Entwicklungsmechanik der Ascidienknospung. Anat. Anz. 10: 215-229. 1894.

27. Hoffman, Victor. Über Erregung und Lähmung tierischer Zellen durch Röntgenstrahlen. 1. Experimentelle Untersuchungen an Frascheiern und-larven. Strahlentherapie $13: 285-298.1922$.

28. KanAJEW, J. Über die histologischen Vorgänge bei der Regeneration von Pelmatohydra oligaetis Pall. Zool. Anz. 65:217-226. 1926.

29. Kanajew, J. Einige histologische Beobachtungen über das Entoderma der Pelmatohydra oligactis Pall. bei der Regeneration. Zool. Anz. 67: 228-234. 1926.

30. Kanajew, J. Úber den histologischen Bau des Entoderms und Mundkegels von Pelmatohydra oligactis Pall. Zool. Anz. 67:305-308. 1926.

31. KanaJew, J. Zur Frage der Bedeutung der interstitiellen Zellen bei Hydra. Roux' Arch. Entwicklungsmeeh. Organ. 122 : 736-759. 1930.

32. Korschelt, E. Regeneration und Transplantation. I. Bd. Regeneration. Borntraeger; Berlin, 1927.

33. Krecker, F. H. Some phenomena of regeneration in Limnodrilus and related forms. Zeitseh. Wiss. Zool. 95: 384-450. 1910.

34. Krecker, F. H. Origin and activities of the neoblasts in the regeneration of microdrilous annelida. Jour. Exp. Zool. 37 : 27-46. 1923.

35. Lang, P. Ueber Regeneration bei Planarien. Arch. Mikrosk. Anat. 79: 361426. 1912.

36. Lefevre, G. Budding in Perophora. Jour. Morph. 14:367-424. 1898.

37. Levy, O. Mikroskopische Untersuchungen zu Experimenten über den Einfluss der Radiumstrahlen auf embryonale und regenerative Entwicklung. Nach den hinterlassenen Präparaten von Prof. Alfred Schaper. Roux' Arch. Entwicklungsmech. Organ. 21: 130-149. 1906.

38. Liтsснко, E. J. Beobachtungen über die Regeneration der Extremitäten beim Axolotl nach Einwirkung von Röntgenstrahlen (russ.). 1930. (Cf. Brunst and Scheremetjewa, 6, pp. 182-183.)

39. Liтsснко, E. J. Further observations on the effeet of $x$-rays on regeneration in axolotl. Compt. Rend. Acad. Sci. URSS. Ser. A. 3: 65-70. 1932.

40. Liтschко, E. J. Einwirkung der Röntgenstrahlen auf die Regeneration der Extremitäten, des Schwanzes und der Dorsalflosse bein Axolotl. Acad. Sei. Un. Rép. Sov. Social., Trav. Lab. Zool. Exp. et Morph. Animaux 3: 101-140. 1934.

41. Meserve, F. G., and M. J. Kenner. The effects of X-rays on Planaria dorotocephala. Science 79: 408-409. 1934.

42. Mutscheller, F. Regenerationsversuche an Regenwurmtransplantaten. (Ein Beitrag zur Analyse der Polarität des Regenwurmkörpers.) Roux' Areh. Entwicklungsmech. Organ. 113: 640-668. 1928.

43. McConnelu, C. H. Mitosis in Hydra. Mitosis in the ectodermal epitheliomuscular cells of Hydra. Biol. Bull. 64 : 86-95. 1933.

44. McConnell, C. H. Mitosis in Hydra. Mitosis of the secretory cells of the endorlerm of Hydra. Biol. Bull. 64: 96-102. 1933.

45. Nussbaum, J. and M. Oxner. Studien über die Regeneration der Nemertinen. I. Regeneration bei Lineus ruber (Müll.). Roux' Arch. Entwicklıngsmech. Organ. 30 (erster teil): 74-132. 1910.

46. Nussbaum, M. Ueber die Theilbarkeit der lebendigen Materia. II. Beiträge zur Naturgeschichte des Genus Hydra. Arch. Mikrosk. Anat. 29: 265-366. 1887.

47. Penney, J. T. Reduction and regeneration in fresh-water sponges (Spongilla discoides). Jour. Exp. Zool. 65: 475-497. 1933. 
48. Puckets, W. O. The effects of $x$-radiation on the development and regeneration of the early fore-limb bud of Amblystoma punctatum. Anat. Rec. 54: 79. 1932.

49. RAHN, E Die Einwirkungen von Radiumstrahlen auf aie ungeschlechtliche Fortpflanzung der Naiden. Zeitsch. Wiss. Zool. 145: 113-168. 1934.

50. Rowley, H. T. Histological changes in Hydra viridis during Regeneration. Amer. Nat. 36: 579-583. 1902.

51. Schaper, A. Experimentelle Untersuchungen über den Einfluss der Radiumstrahlen und der Radiumemanation auf embryonale und regenerative Entwicklungsvorgänge. Anat. Anz. 25: 298-314, 326-337. 1904.

52. Scheremetjewa, E. A., and V. V. Brunst. Untersuchung des Einflusses von Röntgenstrahlen auf die Regeneration des Schwanzes bei den Kaulquappen von Pelobates fuscus. I. Untersuchung der Regenerate von mit verschieden Dosen einmalig bestrahlten Kaulquappen. Roux' Arch. Entwicklungsmech. Organ. 130 : 771-792. 1933. [See also (6).]

53. Schulze, P. Die Bedeutung der interstitiellen Zellen für die Lebensvorgänge bei Hydra. Sitz.-Ber. Ges. Nat. Fr. Jahrg. 1918. Berlin, 1919.

54. SPEK, J. Über die Winterknospenentwicklung, Regeneration und Reduktion bei Clavelina lepadiformis und due Bedeutung besonderer "omnipotenter" Zellelemente für diese Vorgänge. Roux' Arch. Entwicklungsmech. Organ. 111: $119-172.1927$.

55. Steinmann, P. Prospektive Analyse von Restitutionsvorgängen. I. Die Vorgänge in den Zellen, Geweben und Organen während der Restitution von Planarienfragmenten. Roux' Arch. Entwicklungsmech. Organ. 108: 646-679. 1926.

56. Steinmann, P. Prospektive Analyse von Restitutionsvorgängen. II. Über Reindividualisation, d.i. Rückkehr von Mehrfachbildungen zur einheitlichen Organisation. Roux' Arch. Entwicklungsmech. Organ. 112: 335-349. 1927. [Literature lists of (55) and (56) cite author's earlier papers in this field.]

57. Stolte, H. A. Die Herkunft des Regenerationsmaterials in der Teilungszone von Dero limosa Leidy und das Problem der Activierung dieser Zellen. Blastocystenstudien I. Zeitsch. Wiss. Zool. 143 : 156-200. 1933.

58. Stone, R. G. The effects of x-rays on regeneration in Tubifex tubifex. Jour. Morph. 53 : 389-431. 1932.

59. Stone, R. G. The effects of x-rays on anterior regeneration in Tubifex tubifex. Jour. Morph. 54: 303-320. 1933.

60. Stone, R. G. Radium radiation effects on regeneration in Euratella chamberlin. Tortugas Lab. 28: 159-166. Carnegie Inst. Pub. No. 435. 1933.

61. Strelin, G. S. von. Zur Frage über den morphologischen Bau und die Herkunft einiger Zellelemente von Pelmatohydra oligactis. Zool. Anz. 79: 273-285. 1928.

62. Strelin, G. S. von. Röntgenologische Untersuchungen an Hydren. II. Die histologischen Veränderungen im Körperbau von Pelmatohydra oligactis unter der Wirkung der Röntgenstrahlen und ihre Bedeutung für die Regeneration und Vermchrung. Roux' Arch. Entwicklungsmech. Organ. 115: 27-51. 1929.

63. Turner, C. D. The effects of x-rays on posterior regeneration in Lumbriculus inconstans. Jour. Exp. Zool. 68: 95-119. 1934.

64. Turner, C. D. The effects of $\mathrm{x}$-rays on anterior regeneration in Lumbriculus inconstans. Jour. Exp. Zool. 71:53-81. 1935.

65. Uвisch, L. V. Über die Aktivierung regenerativer Potenzen. Roux' Arch. Entwicklungsmech. Organ. 51: 33-58. 1922.

66. VANDEL, A. Recherches expérimentales sur les modes de reproduction des planaires triclades paludicoles. Bull. Biol. de la France et de la Belgique 55: 343-518. 1921. 
67. Weigand, K. Regeneration bei Planarien und Clavelina unter dem Einfluss von Radiumstrahlen. Zeitsch. Wiss. Zool. 136 : 255-318. 1930.

68. Wilson, H. V. Sponges and biology. Amer. Nat. 66:159-170. 1932.

69. Wilson, H. V. A new method by which sponges may be artificially reared. Science 25: 912-915. 1907.

70. Wilson, H. V., and J. T. Penney. The regeneration of sponges (Microciona) from dissociated cells. Jour. Exp. Zool. 66: 73-147. 1930.

71. Zawarzin, A. A. Röntgenologisehe Untersuchungen an Hydren. I. Die Wirkung der Röntgenstrahlen auf die Vermehrung und Regeneration bei Pelmatohydra oligactis. Roux' Arch. Entwieklungsmech. Organ. 115:1-26. 1929.

72. Zhinkin, L. Die Regeneration bei Lumbriculus variegatus naeh Einwirkung von Röntgenstrahlen. Zool. Anz. 100:34-43. 1932.

73. Zhinkin, L. Über die Wirkung der Röntgenstrahlen auf die Regeneration bei Lumbriculus variegatus Gr. Acad. Sci. Un. Rép. Sov. Social., Trav. Lab. Zool. Exp. et Morph. Animaux 3: 71-100. 1934.

74. Zhinkis, L. Über die Bedeutung der Mesodermanalagen bei der Regeneration von Rhynchelmis limosella. Zool. Anz. 105: 305-312. 1934.

Manuscript received by the editor October, 1934. 



\section{XIII}

\section{THE BIOLOGICAL EFFECTIVENESS OF X-RAY WAVE-LENGTHS}

\section{Charles Packard}

\section{Institute of Cancer Research, Columbia University, New York}

The radiations whose biological effects are discussed in this paper consist chiefly of roentgen rays generated at various voltages from $10 \mathrm{kv}$. to upward of $500 \mathrm{kv}$. The range of wave-lengths involved is thus very great, extending over more than five octaves, from $2.0 \AA$ to less than $0.05 \AA$. The reactions which they produce can be compared with those elicited by the alpha, beta, and gamma rays of radium, but since the intensities of the latter radiations have not been successfully measured in roentgens, such comparisons must be made with caution.

The biological effects produced by these various qualities of radiation can be considered from two points of view, the qualitative and the quantitative. Do they vary in nature depending on the wave-length of the incident beam, and do they vary in magnitude when equal amounts of radiation of different wave-lengths are given? The answers to these questions have been sought from the time that $\mathrm{X}$-rays and radium first began to be used in therapy, and yet today there is no unanimous opinion on either of them.

\section{QUALITATIVE EFFECTS}

The first radiologists believed that X-rays of different qualities were capable of producing different kinds of reactions in tissues. This was a natural inference, for it was known that in the region of visible light and the ultra-violet, specific wave-lengths produced specific effects. And experience showed that soft X-rays, filtered very lightly or not at all, produced severe but superficial burns in the skin while hard, highly filtered rays did not, because, as was supposed, the caustic rays had been removed. For a time the hard rays were believed to have healing properties. But in 1915 Rost (35) pointed out that both hard and soft rays cause the same histological changes in the skin and other tissues. Later work has confirmed his conclusions. Thus Reisner (34) remarks that "the erythema reaction of the human skin after exposure to gamma rays, that is, to the hardest radiation, does not differ fundamentally from that produced by hard, soft, or very soft X-rays." Differences in the magnitude of the reaction are due, as Rost showed, to the relative amount of 
energy absorbed per unit volume of tissue. When equal amounts are absorbed in equal volumes, the effects are the same, regardless of the different penetrating powers of the rays.

Not only do all wave-lengths of radiation, and also the beta rays of radium, produce the same histological effects, but they also elicit the same more delicate changes in the cell constituents. Fuhs and Politzer (10) find that the morphological changes in the chromosomes and the order in which they make their appearance are alike, whether $\mathrm{X}$ - or gamma rays are the exciting agents. Politzer and Pauli (31), however, reported that after exposure to cathode rays, whose intensity is very great, the cells during mitosis display abnormalities in the achromatic figure not seen after treatment with $\mathrm{X}$-rays. They therefore concluded that these rays produce a specific effect. But more recently it has been demonstrated that these same characteristics can be produced by X-rays if the latter are delivered at the rate of 40,000 $\mathrm{r}$ per min., an intensity comparable with that of the cathode rays (11). It has also been claimed that monochromatic radiations of different wave-lengths have the same effect on tissue culture cells, but mixed radiation produces a different kind of reaction which expresses itself in the changed mode of growth of the culture (39). The evidence on this point is not convincing.

Other experiments in which the criterion of effect is not a visible morphological change but rather a physiological or genetic alteration also show that the quality of radiation does not determine the nature of the effect. Hoffmann (19) finds that the reaction of the protozoan Opalina to vital dyes is the same for various X-ray wave-lengths: Feichtinger (6) has shown that the viscosity changes induced in Spirogyra by alpha and beta rays are essentially the same when equal energy per unit volume is absorbed. Finally, Hanson and Heys (15) first noted that gamma rays and $\mathrm{X}$-rays produce the same mutations in Drosophila. The results of subsequent genetic experiments with radiations are reviewed by Timoféef-Ressovsky (38).

The statement is often made that qualitative differences in the effectiveness of different wave-lengths are shown by the elective properties of the rays, a term used to describe the well-known fact that hard, highly filtered radiations may, under appropriate conditions of dosage, injure certain tissues while leaving others unharmed. When the testis is exposed to gamma rays of low intensity, the seminal epithelium alone is injured; but if X-rays of much higher intensity are used, the entire organ undergoes massive degeneration (33). Observations of this kind gave rise to the belief that gamma rays, because of their short wavelength, could produce better therapeutic results than were possible with $\mathrm{X}$-ray treatment. There was much clinical evidence to support this view. But more recent work has shown conclusively that the favorable clinical effects following the use of radium are not due to any specific 
action but rather to the fact that the intensity of the beam is very low. When $\mathrm{X}$-rays are delivered at comparable intensities, the tissue reactions are the same as to radium. The rate at which the radiation is delivered is the important factor. There is no evidence that short rays induce results which are biologically different from those brought about by longer rays, or that they possess potencies not shared by the latter.

Why, then, should beams of low intensity show elective action? The answer lies in the biological properties of the cells rather than in the physical nature of the radiations. When a beam of high intensity is absorbed in a tissue, the very extensive ionization which ensues initiates destructive changes in all of the cells whether they are naturally resistant or sensitive, and none escapes injury. But the slight damage done by a beam of low intensity can be repaired by the resistant cells as rapidly as it is inflicted, so that at the end of even a long exposure they appear to be unharmed. The reverse is true for sensitive cells and they soon succumb.

The conclusion that the nature of the reaction is not influenced by the wave-length of the incident beam is supported by physical considerations. When $\mathrm{X}$ - and gamma rays are absorbed, a primary electron is ejected from the absorbing atom. This electron, whose velocity depends on the wavelength of the incident beam, collides with atoms along its path, setting free hundreds or even thousands of secondary electrons from them, and continues so to do until it has expended all of its energy and comes to rest. The extensive ionization produced in this way is the starting point of a series of biological reactions which may lead to the injury or death of the cells. If then the same number of secondary electrons are produced by both hard and soft rays; if, in other words, the same amount of ionization occurs, we should expect the biological reaction to be the same also. And when the amount of ionization is greater in one case than another, we should expect a difference only in the magnitude of the reaction and not in its nature.

\section{QUANTITATIVE EFFECTS}

Whether equal quantitative effects are produced by equal doses of radiation having widely different wave-lengths is a question which has not yet been finally answered. Its solution involves two problems; first, the accurate measurement of the dose, and second, the accurate measurement of the biological effects which it produces. Methods for determining dosage are discussed elsewhere. At the present time the general opinion is that $\mathrm{X}$-ray dosage can be measured with reasonable accuracy when the rays are produced at voltages lying within the range commonly used in therapy. Difficulties arise when these limits are exceeded, especially on the side of very high voltages. Indeed, measurcments of $\mathrm{X}$-rays produced at $500 \mathrm{kv}$. and more are only approximate, 
while those of gamma rays, which represent voltages of the order of $1000 \mathrm{kv}$. and more, are very uncertain.

Quantitative biological effects are determined by measuring the magnitude of the reaction of various test objects to the rays. First to be used for this purpose was the human skin, a natural choice because the first radiologists were concerned almost wholly with clinical effects. Because at that time no accurate means of measuring intensities had been devised, the erythema reaction itself was used as a unit of dosage. It is still employed widely although it lacks precision. The degree of reddening of the skin is subject to considerable variation depending on the natural pigmentation of the skin, on the part of the body which is exposed, and on the age and health of the patient. Furthermore, the magnitude of the reaction varies with the area under exposure and with the intensity of the beam. For these reasons an absolute erythema dose cannot be defined in terms of physical units.

The older literature shows little uniformity of opinion regarding the effectiveness of different wave-lengths in producing a typical skin reaction. Many of the conclusions arrived at before 1928 when the international unit, or roentgen, was adopted, are questionable because of inaccurate measurement of dosage, and because no account was taken of the part played by differences in intensity. The general opinion was that soft radiations are more effective than hard. Recent observations, however, have shown that this is not true (36). When the erythema doses for beams of various degrees of softness are measured by a standard instrument they are found to be identical. So also Hess (17) whose first measurements indicated that rays generated at $60 \mathrm{kv}$. were twice as effective as those produced at either 9 or $185 \mathrm{kv}$., discovered when his dosimeter was properly calibrated, that all three doses were actually the same. Hickey and Pohle (18) and others give evidence to show that this equality of effect extends over a wide range of wave-lengths, when the area of exposure is small and the duration of exposure is constant. But Reisner who reviews the subject in detail comes to the conclusion that while within the region of moderately hard rays $(0.14$ to $0.22 \AA)$ all wave-lengths are equally effective, still softer radiation is much more active. In opposition to this view are Failla and Henshaw (7) who state that the hard gamma rays of radium are more effective than $160-\mathrm{kv}$. $\mathrm{X}$-rays. In measuring these two qualities they used both physical and biological methods. The latter consist in determining the doses of both radiations which kill the same proportion of Drosophila eggs. Equal doses thus measured are not equally effective on the skin, the gamma rays being more active.

Without doubt inaccurate measurement is in part responsible for these wide variations in opinion. But an important source of error lies in the neglect of the time factor (20). It is now known that the same total 
dose, measured in roentgens, produces very different degrees of reaction depending on the length of exposure, that is, on the intensity of the beam. Holthusen (22) has shown that an increase in the duration of exposure must be accompanied by an increase in the total dose if the same degree of erythema is to be obtained. Thus $500 \mathrm{r}$, delivered in $1 \mathrm{~min}$. produce the same results as $900 \mathrm{r}$ given in $50 \mathrm{~min}$., or $1400 \mathrm{r}$ given in about $8 \mathrm{hr}$. In these tests Holthusen used both hard and soft $\mathrm{X}$-rays and also gamma rays. The latter were calibrated by a biological method similar to that used by Failla and Henshaw, except that Ascaris eggs were used. When equal doses of gamma and $\mathrm{X}$-rays are given in equal times, the reaction is approximately the same, the gamma rays being slightly more active.

One disadvantage of the skin as a test object is that it cannot be uniformly radiated throughout. A varying amount of the incident energy is absorbed in the outermost layers, the proportion depending on the penetrating power of the rays. Furthermore, the effect produced by the primary beam is augmented by scattered radiation, especially in the case of the moderately hard rays.

Better test objects are small, growing organisms such as eggs, seeds, bacteria, and the like, all parts of which receive approximately equal amounts of radiant energy. In these also scattered radiation is negligible. There are several criteria by which the usefulness of these various organisms can be judged.

$a$. The effect of the radiation must be clear-cut and easily measured. In the case of seedlings it is the rate of growth or the proportion of specimens whose growth is completely inhibited. For eggs, it is the failure of the larva to hatch.

$b$. A high degree of sensitivity is a valuable asset, for it permits small doses and short exposures. The latter are especially desirable, for during long exposures the organisms not killed immediately continue their development and grow more and more resistant. In the accompanying Table 1 are shown the doses necessary to kill 50 per cent of the samples of various organisms, or to reduce their growth to half of that shown by the controls.

TABLE 1

$\mathrm{R}$

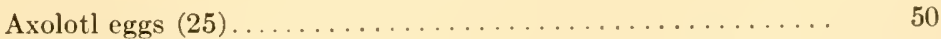

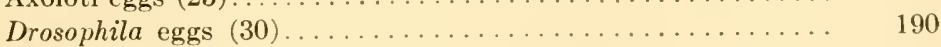

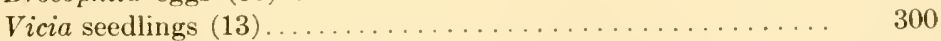

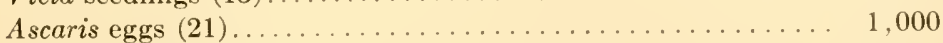

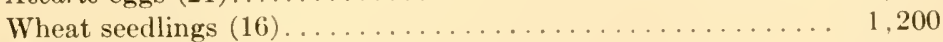

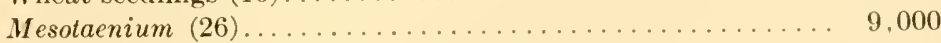

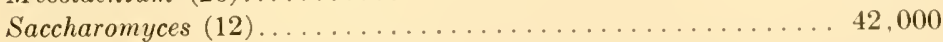

The figure given for Ascaris eggs is only approximate, for the eggs from different worms vary in sensitiveness within very wide limits. The 
very resistant alga and yeast cells must be exposed to beams whose intensity is several thousand $r$ per min. in order to reduce the length of exposure to reasonable limits.

A constant degree of sensitivity is a great advantage for it permits direct comparisons between the results of different experiments. Drosophila eggs have shown a remarkable degree of constancy; $190 \mathrm{r}$ kill half of the eggs obtained from different strains of wild flies which have been worked with for a number of years. Precisely the same dose has been found for Drosophilas tested in Germany (23).

$c$. The necessity of using small organisms in these experiments is obvious when very soft radiations are employed, for their penetrating powers are so slight that there is an appreciable difference in the amount of energy received by the upper and lower sides of the object. Even with Drosophila eggs whose greatest diameter is about $0.18 \mathrm{~mm}$., the correction for absorption of rays generated at $12 \mathrm{kv}$. amounts to 6 per cent. For still lower voltages this correction rapidly becomes much more important.

d. Genetic purity is considered important, especially in plant material. But tests with hybrid strains of wild Drosophila indicate that it is not significant.

It is evident that the choice of test objects which will fulfill these requirements is limited. Many seeds are of little value because of their high powers of resistance. This is true for the protozoa in general. Eggs are usually available for limited periods only, and often in numbers too small to furnish significant data. Drosophila eggs, however, conform to the above requirements.

The amount of normal variation which occurs in the various test objects which have been used has scarcely been mentioned in the literature, yet an evaluation of the published results of radiation experiments cannot be made without some information on this point. Bolaffio (1), who used the seedlings of Vicia Faba, remarks that "almost never do all seeds in a test act alike. Many times in one group the differences are so great that the entire test which has taken so much time and labor must be discarded. In the presentation of data there is always a strong subjective factor, so that the results represent not a measure but a preference." Lachmann and Stubbe (24), however, believe that the amount of variation can be greatly reduced by appropriate culture methods. Data given by Zuppinger (44) in his work on Ascaris eggs shows that the coefficient of variation varies in different tests between 12 and 28 per cent. But he states that differences of 5 per cent between the means of two sets of experiments are significant. An analysis of data obtained from tests with Drosophila eggs indicates a coefficient of variation ranging from 3 to 8 per cent. Table 2 gives the results of a series of 10 tests. 


\section{TABLE 2}

\section{Roentgens Delivered}

183

183

184

184

184

184

186

188

188

189

185
Per Cent Hatching

57.4

52.4

51.3

51.1

52.5

47.7

48.9

55.6

49.5

56.1

52.3

$\sigma= \pm 3.08 ; \quad C_{v}=5.90 ; \quad E_{m}= \pm 0.66$.

The data show that when equal doses, measured in roentgens are delivered, the proportion of eggs that survive is very constant. Similar series of tests in which the doses range from 50 to $300 \mathrm{r}$ furnish data for

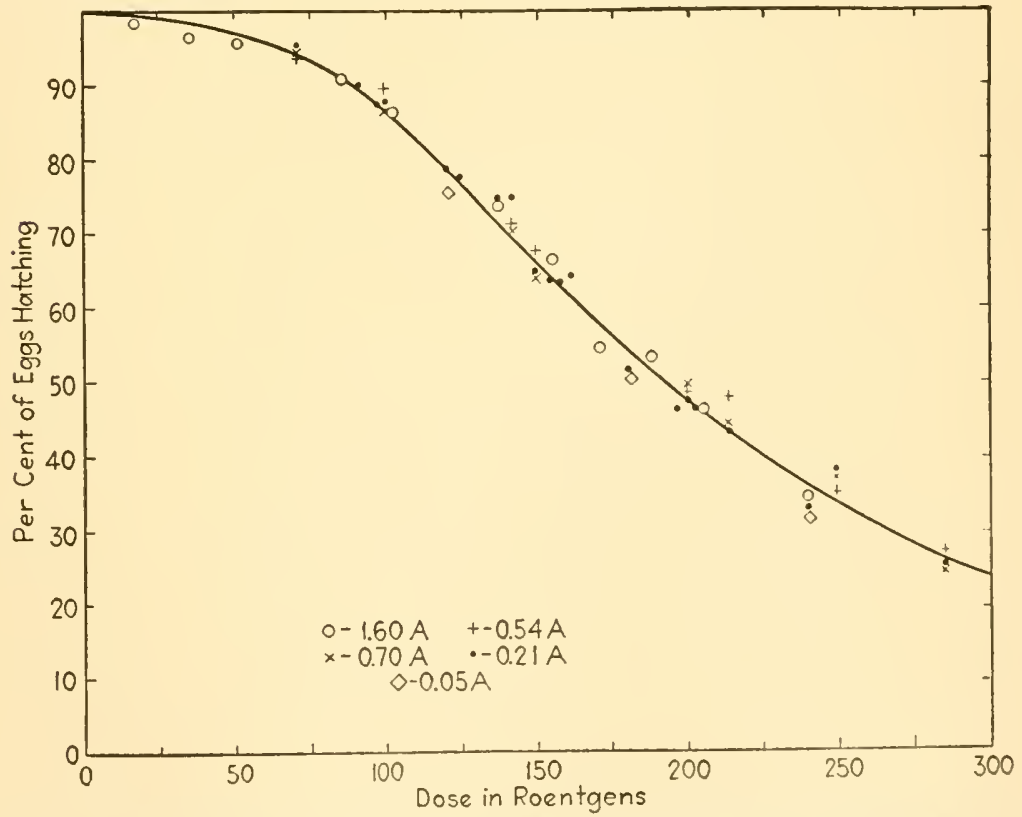

FIG. 1.-Curve showing the relation between X-ray dosage and biological effect. It is based on data derived from experiments in which different wave-lengths, as indieated by the symbols, were used.

the survival eurve shown in Fig. 1. This curve is an expression of the normal variability of the eggs to $\mathrm{X}$-radiation; it is also a means of measuring dosage (28), for the proportion of survivals is an aceurate indication of the number of roentgens which the entire sample received. When doses estimated in this way are compared with measurements made with 
an open ionization chamber, the results are found to agree within 2 per cent. By this use of the survival curve the relative effectiveness of equal doses of different wave-lengths can be determined with great precision.

The first investigators who employed small test objects in the study of the wave-length problem were handicapped by the lack of a standard unit by which to express the quantity of radiation which they used, and by the lack of accurate dosimeters. It is not surprising, therefore, that there was little agreement in their results. But as the means of measurement have improved the diversity of opinion has grown less, until at the present time nearly all are agreed that equal doses of all wave-lengths within a very wide range produce equal quantitative results.

First to demonstrate this was Wood $(40,41)$ who used mouse tumor tissue as a test object. The finely minced tumor particles were exposed to homogeneous beams having wave-lengths of 0.21 and $0.71 \AA$ and then inoculated into healthy animals. The criterion of effect was the failure of the particles to grow in the host animal. Since the tumor strain which he used always "takes," any failure to grow was due to the lethal action of the rays. The doses were measured with an open ionization chamber. Although the degree of variability in this material is fairly large, the results of numerous tests, involving thousands of animals, clearly showed that there is no constant difference in effectiveness between these two qualities of radiation.

Evidence on this question derived from the study of the reaction of various seedlings is still somewhat confused. The earlier literature which is extensive may be passed over because the experiments lacked precision both in dosage and in biological technique. The usual procedure is to radiate germinated specimens whose roots are of about the same length. The criterion of effect is the increase in the length of the irradiated roots as compared with that of the controls, or else, the proportion of seedlings which cease to grow after a definite time. The variability in this material is partly due to methods of handling. Specimens that have been soaked in water for some hours to start germination are more sensitive than those soaked for only a short time. Then also, sensitivity varies with the length of the root at the time of radiation. Injuries to the root tip are not uncommon. This leads Henshaw (16) to reject from each sample one-third of the irradiated seedlings whose root length appears to depart most widely from the average. Recently Glocker and his collaborators $(13,14)$ have made experiments on a variety of seedlings, using appropriate culture methods and carefully measured doses. They find that the dose needed to kill 50 per cent of the samples of the horse bean, Vicia Faba, and of mustard and sunflower seedlings is the same whether the radiation is hard, soft, or medium $(0.18,0.56,1.54 \AA)$. But Lachmann and Stubbe (24) who also used Vicia conclude that hard rays are much more effective than soft. However, they failed to take into account the fact 
that the latter radiation was largely absorbed in the upper layer of the root tips, the lower portion receiving only a fraction of the total dose. When a correction is made for absorption, the effeets of both radiations are found to be about equal.

A few experiments by Glocker and others have been made on certain algae (26) and on yeast (12). In each case undivided cells were irradiated, and, after incubation, the proportion of individuals which had divided only once or not at all was determined. These were reckoned as injured. The results indicate that both types of eells are much more affected by soft rays $(1.54 \AA)$ than by rays of medium hardness $(0.56 \AA)$. Very large doses were given because it is diffieult to eheck cell division completely in unicellular organisms and in fertilized eggs. For example, fertilized but uneleft frog eggs may be given a dose of 72,000 $\mathrm{r}$ and yet divide at least once. But $400 \mathrm{r}$, given at the same stage, suffice to cause death when gastrulation begins. Because of the somewhat indefinite criterion of effect, and because both yeast and algal eells are very sensitive to external conditions, these results are not wholly convineing.

On bacteria very little careful quantitative work has been done. The necessity of using only those types which ean be spread uniformly on the plates, and of preventing multiplication during the period of exposure has not been realized (43). Wyekoff (42), who used Bacillus coli, finds that the biological action of radiations having a wave-length of $1.54 \AA$ or less is proportional to their measured ionization. But very soft rays are less effective.

Braun and Holthusen (2) and others have carried on extensive studies on the wave-length problem, using Ascaris eggs as a test material. Unsegmented eggs from a single worm are irradiated and, after four days of incubation, are examined to determine the proportion of injured embryos. When eggs are exposed to soft and hard rays of the same intensity and for the same length of time the percentage of injuries in both eases is alike within the limits of experimental error. But Zuppinger (44) holds that hard rays are in general more effective than soft rays. He finds, however, that the effectiveness of the latter diminishes slightly as the wave-lengths increase to $0.53 \AA$, where it is at a minimum, and then increases more rapidly with deereasing wave-length. Dognon (3) on the contrary has stated that the soft rays are the more effective. But he remarks (4) that if due allowance is made for absorption of the softest radiations, the correeted doses indicate an equality of effeet.

In a very extensive series of experiments with Drosophila eggs, various investigators have demonstrated the complete lack of any differential action due to wave-length. From the softest rays, generated by $12 \mathrm{kv}$. to very hard rays produced at $700 \mathrm{kv}$., the quantitative effect of equal doses is always the same. The criterion of effect is the proportion of eggs which live. These eggs are almost free from disturbing environ- 
mental influences, and the influence of the time factor. Their easy availability at all times, in large numbers, makes them exceptionally good test objects.

The experiments of Packard are summarized in Fig. 1. Each point is determined from the average of several tests involving several hundred eggs. The doses were measured by open ionization chambers, the intensity of the softest radiation $(1.5 \AA)$ being obtained with special care. In this instance every source of inaccuracy was investigated and the necessary corrections made (5). The intensity determined by the biological method differed from that obtained from ionization readings by less than 2 per cent. The hardest radiations, generated at $550 \mathrm{kv}$. could not be measured with equal precision, but the biological results are in fair agreement with those obtained at lower voltages (27). Henshaw (16), who has studied the effectiveness of 700-kv. X-rays, finds that it does not differ significantly from that of much softer radiation. These results, together with those of Glocker (14), give no evidence that there is any point of maximum or minimum effectiveness throughout this very wide range of wave-lengths.

$\mathrm{A}$ comparison between the activity of gamma rays and $\mathrm{X}$-rays has been made by Simon (37). Virgin Drosophila were irradiated, then mated, and the percentage of fertile eggs determined. The intensity of radiation was measured in ergs/cc. according to the method of Stahel. The effect produced by equal doses of both radiations, when given in equal lengths of exposure, was the same. But short exposures proved to be more effective than long, the total doses being equal.

The course of the survival curve of Drosophila eggs remains constant regardless of the quality of radiation used as a lethal agent (29). This is true also for the mortality curves of Ascaris, of yeast, algae, and bacteria. But according to Glocker (14) there are a few test objects whose mortality curves are affected by changes in the wave-length of the incident beam, the soft rays being more effective in small doses while the reverse is true for large doses. This phenomenon he attributes to the size of the quanta. A discussion of the quantum theory is presented elsewhere in this volume.

One more test of a different kind may be mentioned. Fricke and Petersen (9) have investigated the action of hard and soft rays on hemoglobin. The laked blood is exposed to measured doses of three wavelengths. The hemoglobin is gradually transformed into methemoglobin, the exact amount being determined by a spectrophotometer. They conclude that radiations of 0.75 and $0.54 \AA$ produce identical results, while shorter rays $(0.25 \AA)$ are very slightly more effective. In further experiments Fricke and Morse (8) irradiated acid solutions of ferrous sulphate with homogeneous beams of approximately the same wavelengths previously used. Analysis showed that in each case the amount 
of oxidation to ferric sulphate was the same. Quimby and Downes (32) also have shown by another chemical method that within a very wide range of wave-lengths the amount of mercurous chloride precipitated from a mixture of ammonium oxalate and mercuric chloride is proportional to the length of exposure, other factors being the same. They therefore conclude that this reaction may be useful for measuring the quantity of radiation, since it is independent of wave-length effects.

The effectiveness of $\mathrm{X}$-ray wave-lengths is a problem of practical importance to the radiologist. If soft rays are more potent than hard rays and produce different kinds of changes in tissues, then each wavelength becomes a different medicament. This view, once widely held, is no longer tenable. The qualitative and quantitative effects of equal doses of both hard and soft X-rays are the same. The radiotherapist, therefore, now chooses that radiation which can penetrate to the site to be treated, knowing that the energy, in the form of short rays, actually absorbed by cells at a depth will produce the same amount of change that is produced by an equal dose of soft rays absorbed at the surface. Whether the still shorter gamma rays of radium or $\mathrm{X}$-rays of comparable wave-length, generated at potentials of a million volts, will conform to this rule cannot be finally determined until more accurate means of measuring their intensities have been devised.

\section{REFERENCES}

1. Bolaffio, M. Versuche zur luftelektrischen und biologischen Wirkung von Strahlen verschiedener Wellenlänge. Strahlentherapie 20:673-736. 1925.

2. Braun, R., and H. Holthusen. Einfluss der Quantengrösse auf die biologische Wirkung verschiedener Röntgenstrahłenqualitäten. Strahlentherapie 34: 707734. 1929.

3. Dognon, A. Action biologique des rayons $\mathrm{X}$ monochromatiques et differents longueur d'onde sur l'oeuf d'Ascaris. Compt. Rend. Acad. Sci. [Paris] 194: 2336-2338. 1932.

4. Dognon, A. Action des rayons X monochromatique de longueur d'onde differente sur l'oeuf d'Ascaris. Compt. Rend. Acad. Sei. [Paris] 196: 437-438. 1933.

5. Exner, F. M. Intensity measurements with $12 \mathrm{kv}$. X-rays. Amer. Jour. Cancer 16: 1275-1304. 1932.

6. Feichtinger, Nora. Viskositätsänderung des Protoplasmas als Folge radioaktiver Bestrahlung. Naturwissensch. 21:589-591. 1933.

7. Failla, G., and P.S. Henshaw. The relative biological effectiveness of X-rays and gamma rays. Radiology $17: 1-43.1931$.

8. Fricke, H., and S. Morse. The relation of chemical, colloidal and biological effeets of roentgen rays of different wavelengths to the ionization which they produce in air. II. Action of roentgen rays on solutions of ferrosulphate. Amer. Jour. Roentgenol. 18: 426-430. 1927.

9. Fricke, H. and B. W. Petersen. I. Action of roentgen rays on solutions of hemoglobin in water. Amer. Jour. Roentgenol. 17: 611-620. 1927.

10. Funs, H., and G. Politzer. T̈ber die Wirkung der Radiumstrahlen auf die Zelltheilung. Strahlentherapie 45 : 359-364. 1932.

11. Glocker, R., and H. and M. Langendorff. Zur Frage der "spezifischen" Wirkung der Kathodenstrahlen auf die Zelle. Naturwissenseh. 19: 251. 1931. 
12. Glocker, R., H. Langendorff, and A. Reuss. Über die Wirkung von Röntgenstrahlen verschiedener Wellenlänge auf biologische Objekte. III. Strahlentherapie $46: 517-528.1933$.

13. Glocker, R., and A. Reuss. Über die Wirkung von Röntgenstrahlen verschiedener Wellenlänge auf biologische Objekte. I. Strahlentherapie 46: 137-160. 1933.

14. Glocker, R., and A. Reuss. Über die Wirkung von Röntgenstrahlen verschiedener Wellenlänge auf biologische Objekte. V. Strahlentherapie 47: 28-34. 1933.

15. Hanson, F. B., and Florence Heys. Analysis of effects of different rays of radium in producing lethal mutations in Drosophila. Amer. Nat. 63: 201-213. 1929.

16. Henshaw, P. S., C. T. Henshaw, and D. S. Franc1s. Relative Effects produced by $200 \mathrm{kV}$ Roentgen rays, $700 \mathrm{KV}$. Roentgen rays, and gamma rays. Amer. Jour. Roentgenol. 29: 326-333. 1933.

17. Hess, P. Härteabhängigkeit der R-dosen im Vergleich zu äquivalenten Erythemen aller gebrauchlichen Strahlenqualitäten. Strahlentherapie 27: 146-160. 1928.

18. Нгскеу, P. M., and E. A. Poнle. Skin toleration doses in roentgen units and their relation to the quality of radiation. Radiology 12: 309-316. 1929.

19. Hoffmans, C. Strahlenhärte und biologische Wirkung. Strahlentherapie 43: 140-159. 1932 .

20. Holthusen, H. Der Zeitfaktor bei der Röntgenbestrahlung. Strahlentherapie 21 : 275-305. 1926.

21. Holthusen, H. Biologische Wirkungen der Röntgenstrahlen mit besonderer Berücksichtigung des Einflusses der Wellenlänge, der Intensität, und der Bestrahlungsdauer. Strahlentherapie 31: 509-517. 1929.

22. Holthusen, H., Vergleichende Untersuchungen über die Wirkung von Röntgen und Radiumstrahlen. Strahlentherapie 46:273-288. 1933.

23. JÜngling, O., and H. Langendorff. Biologische Ausdosierung von Radiumpreparäten. Strahlentherapie 48: 174-186. 1933.

24. Lachmann, E., and H. Stubbe. Über die biologische Wirkung der Grenzstrahlen verglichen mit harten Röntgenstrahlen. Strahlentherapie 43:489-516. 1932.

25. Langendorff, H. and M., and A. Reuss. Über die Wirkung von Röntgenstrahlen verschiedener Wellenlänge auf biologische Objekte. II. Strablentherapie 46: 289-292. 1933.

26. Langendorff, H. and M. and A. Reuss. Utber die Wirkung von Röntgenstrahlen verschiedener Wellenlänge auf biologische Objekte. IV. Strahlentherapie 46: 655-662. 1933.

27. PACKard, C. The quantitative biological effects of X-rays of different wavelengths. Jour. Cancer Res. 11: 1-15. 1927.

28. PACKARD, C. A biological measure of X-ray dosage. Jour. Cancer Res. 11: 282-292. 1927.

29. PACKARD, C. A comparison of the quantitative biological effeets of gamma and X-rays. Jour. Cancer Res. 12: 60-72. 1928.

30. PACkARD, C. The biological effectiveness of high voltage and low voltage $\mathrm{X}$-rays. Amer. Jour. Cancer 16: 1257-1274. 1932.

31. Politzer, G., and W. E. Pauli. Über die biologische Wirkung der Kathodenstrahlen. Strahlentherapie 33: 704-710. 1929.

32. Quimby, Edity H., and Helen R. Downes. A chenical method for the measurement of quantity of radiation. Radiology $14: 468-481.1930$.

33. Regaud, Cl., and R. Ferroux. Sur la diversité des réactions des tissus traités par les rayons X. Zeitseh. Krebsforseh. 32: 10-26. 1930. 
34. Reisner, A. Hauterythem und Röntgenstrahlung. Ergebn. Med. Strahlenforseh. Bd. 6, Geo. Thieme; Berlin, 1933.

35. Rost, G. A. Experimentelle Untersuchungen über die biologische Wirkung von Röntgenstrahlen versehiedener Qualitäten. Strahlentherapie 6: 269-329. 1915.

36. Schubert, M. Vegleichende Messungen mit Küstner Eiehstandgerät, Martius Ionometer, und Sabourand-Noire Tablette. Strahlentherapie 35: 553-560. 1930.

37. Simon, Suzanve. Action comparée des rayons $\mathrm{X}$ et des rayons gamma sur la stérilisation des femelles de Drosophila melanogaster. Le Cancer $7: 229-248$. 1930.

38. TMoféef-Ressorsky, N. W. Einige Versuche an Drosophila melanogaster über Beziehungen zwisehen Dosis und Art der Röntgenbestrahlung und dadureh ausgelöste Mutationsrate. Strahlentherapie 49: 463-478. 1934.

39. Wendrowsky, V. Die Wirkung der gemischten und monochromatischen Röntgenbestrahlung auf das Gewebswachstum der Milz in vitro. Arch. Exp. Zellforsch. 14: 412-441. 1933.

40. Wood, F. C. The effect on tumors of radiation of different wavelengths. Amer. Jour. Roentgenol. 12: 474-481. 1924.

41. Wood, F. C. Further studies in the effectiveness of different wavelengths of radiation. Radiology 5: 199-205. 1924.

42. Wrckoff, R. W. G. The killing of colon bacilli by X-rays of different wavelengths. Jour. Exp. Med. 52: 769-780. 1930.

43. Wyckoff, R. W. G., and T. M. Rivers. The effect of eathode rays on certain baeteria. Jour. Exp. Med. 51: 921-932. 1930.

44. Zuppinger, A. Radiobiologische Untersuchungen an Ascariseiern. Strahlentherapie 28: 639-758. 1928. 



\title{
XIV
}

\section{THE PHYSIOLOGICAL EFFECTS OF RADIATION UPON ORGAN AND BODY SYSTEMS}

\author{
Stafford L. Warren
}

Division of Radiology, Department of Medicine, University of Rochester School of Medicine and Dentistry and Strong Memorial Hospital, Rochester, New York

Introduction. Critical. General concept. Skin. Blood vessels and the reticuloendothelial system. Connective tissue. Dosage, wave-length, and skin. Effect of general body irradiation. Gastro-intestinal tract. Lungs and heart. Kidney. Adrenals. Eye. Ear. Nerve tissue. Bone and cartilage. Hematopoietic system. Gonadal system. Miscellaneous. Summary. References.

\section{INTRODUCTION}

The purpose of this review is to correlate the known facts concerning the physiological effects upon body and organ systems of the various radiations making up the electromagnetic spectrum.

The widespread application of various portions of the electromagnetic spectrum for therapeutic and experimental purposes has made essential a more accurate knowledge of the effects of these radiations upon body tissues. The student and investigator is rather overwhelmed by the immense volume of literature which has developed within the last ten years in the field of "radiation effects" upon tissues. For general references dealing with roentgen and radium radiation effects up to the advent of $200-\mathrm{kv}$. equipment, the reader is referred to Colwell and Russ (46), reviews of the German literature by Schwarz (331), Kuhlmann (181), Flaskamp (105), Fuhs and Konrad (108), Zwerg (403, 404), Waters and Kaplan (381), Dessauer (75), Mavor (216), critical reviews by Caspari (39), Holthusen (158), Warren (369), Packard (260), and an exhaustive summarization of the physiological effects upon certain organs by Desjardins $(70,72,73,74)$. For data concerning ultra-violet radiation, important reviews are those by Laurens (191), Ellis and Wells (93), Mayer (218), and Kuhlmann (182). Lacassagne $(184,187)$ discusses the evidence in favor of the direct and selective action of roentgen radiation upon body cells. In vitro work on embryonic tissue cells is reported in detail by Cox and Spear (52).

\section{CRITICAL}

The greatest difficulty encountered in attempting to correlate the effects of any radiations upon the body systems is due to the variations 
in dosage methods used by the different investigators. Until the international unit of measurement of roentgen radiation, the roentgen ( $\mathrm{r}$ unit), was agreed upon, these dosage factors could only be identified in a general way. Since the adoption of this $r$ unit, it is possible to compare dosages applied to the surface, at least, with one another. The roentgen postulates that ionization effects of roentgen radiation are comparable, but so far methods of measuring such effects over the other parts of the spectrum have not been available. The situation is still obseured if the unit $\mathrm{erg} / \mathrm{cm} .{ }^{2} / \mathrm{sec}$. is used; this defines the total quantity delivered upon a certain plane or surface but does not allow for that lost by transmission through the tissue. Nor does it take into consideration any specific properties of the substance irradiated, i.e., specific spectral absorption or transmission in the ultra-violet spectrum. Perhaps the matter would be simplified still further if some common energy equivalent like ergs absorbed per unit volume of tissue were used, which could be applied with greater accuracy to the whole spectrum.

Another difficulty encomtered in comparing the physiological effects of these radiations is the variation in the intensities and the time factors involved, and the lack of a clear understanding of the differential effects of the different bands of the electromagnetic spectrum from the shortest to the longest wave-lengths. Most of these differences may be based upon purely physical rather than biological phenomena. In addition, it is often difficult to interpret the results obtained, because of the failure of many of the experimenters to take into consideration the broader concepts of the effects of radiation, the authors having emphasized the clinical and empirical rather than the biological and physical viewpoints. Further, there is still a lack of agreement in the establishment of a definite biologieal unit of dosage, ehiefly because there may be certain individual differenees, as well as species differences, in the test objects, which may influence the effectiveness of a given dosage. Moreover, results obtained with experimental animals may not be direetly applied to human tissues, although in general the principles are the same, however different the doses involved may be.

The primary effect of radiation may be considered to be a destructive one. Seeondary effeets, aside from loss of function, are mainly those due to repair of this damage. The whole concept of radiation therapy is built upon the supposed greater sensitivity of the tumor cells or the abnormal cell mass treated over that of the normal near-by struetures, of necessity exposed to the radiation. Normal tissues, too, vary greatly in their suseeptibility to damage by radiation, and only rough estimates are available for a comparison of the relative doses involved in tissue damage.

The variation in the depth dosage in the tissues irradiated in experimental animals and humans must bear some important relation to the 
so-called sensitivity of the organs (376). The manner in which the dosage may be varied by organs of different density, the presence of gas and air in certain organs, and other factors was discussed by Wintz and Rump (392). Tsuzuki (354) irradiated normal rabbits with 160 per cent of human skin erythema dose (with average wave-length of $0.1 \AA$, $170 \mathrm{kv}$.) and studied the depth dose developed in various organs. This is a very rational attempt to arrive at the sensitivity of the various organs. Weatherwax and Robb (382) showed considerable difference in the depth dose when collapsed and expanded lung tissue was immersed in a water phantom. The expanded lung allows more radiation to pass through the water, although there is a reduction in the amount of scattered radiation.

A critical inspection of published data would indicate that up to the present time there are no accurate dosage tables for the total destructive dosage, or "lethal" dosage, for any of the cell types in vivo. Little consideration (except Tsuzuki, 354) has been given to the effective depth dose delivered to an organ in situ (Packard, 260), especially in experimental animals, and it is apparent that most of the effects obtained in organs following irradiation are in various degrees of totality. Most investigators have manipulated dosage to the point of obtaining an appreciable, destructive, structural change, either microscopic or gross, with some demonstrable functional abnormality. There are several important handicaps to experiment: $(a)$ the technical difficulties in handling the experimental animal or biological material and the cumbersome physical equipment in an accurate manner during the period necessary for the irradiation; $(b)$ the difficulty in localizing the radiation and measuring the amount delivered to the organ; $(c)$ the variation in the biological materials, in regard to both individual and species differences; (d) the difficulty in interpreting results where the normal variables are not well understood either in the laboratory animal or in the greatest variable, the diseased patient.

\section{GENERAL CONCEPT}

Fundamentally, there are two major types of change that radiation may bring about in a cell, and thus in an organ, namely, an acute fatal injury or an injury which is not necessarily fatal but which leads to degenerative changes of greater or less degree, such as edema and swelling, faulty mitosis, shrinkage in size, and in extreme cases, loss of nuclei and hyalinization. An infiltration of wandering cells may occur, but this is often negligible in quantity and is probably a repair reaction. With destruction of sensitive cells, the connective-tissue stroma which is sometimes also damaged, tends to collapse and become more prominent, and the repair reaction tends to bring in new connective tissue cells to fill up the spaces left by the disappearance of the cells of an organ. This does not take into consideration, of course, those cells only slightly 
injured which are able to recover completely in function and reproductive power. All variations between acute fatal injury and return to normal are relative to dosage and sensitivity.

In every organ there are probably a few cells in such a stage of "sensitivity" that a certain minimal dosage of radiation will be lethal for them. There are also, in even the more sensitive cell groups, some cells which will survive extremely large doses of radiation which will have killed all of the other cells of a given organ. These few cells, some of them abnormal now, attempt to regenerate, and the degree of success in restoring the function of the organ involved is determined, to a great extent, by the number of functioning survivors and the defeets brought about by the collapse of the destroyed cells. Lack of knowledge of many of the intimate processes going on within the eell is, of course, a handicap common to the whole field. The investigator may very well apply the methods used with the simpler biological materials (see other papers in this work) to experiments dealing with the more complex organs in animals.

A study of the time relations of some of these effects of irradiation must be divided up into two considerations: first, the immediate death of a certain number of the cells following irradiation, and the repair or failure of repair of this damaged area, and second, the prolonged degenerative processes in cells not killed and the resultant disturbances therefrom. A true "latent period," therefore, should, strictly speaking, involve only the acute injury, including death, of the cell, and occupy that time interval between the irradiation and the disturbance in function of the particular type of eell under investigation. Since the cells in a given organ are apt to vary somewhat in their sensitivity at the time of any given irradiation, one may have all stages of injury of the cells in an organ from minimal injury to death. This will be evidenced as an average effect of the total damage done and will influence the functional disturbance and histologieal and gross appearance of the organ accordingly.

In considering the effects of radiation upon the organ systems and in particular the effect of roentgen and radium radiation, it is necessary to take into consideration the methods of dosage as well as the total amount involved and the time interval between various doses. There are three main types of dosage: first, the "massive-dose" method, in which the total, usually near the erythema dose, is given at one sitting within a short or relatively prolonged period of time, usually depending upon the amount of energy available to the investigator; second, the "fractional method" in which a definite proportion of an erythema dose is given at regular short intervals, either as a dose of high intensity given over a short period of time, simple fractional method, or the same dosage of radiation given with low intensity over a relatively long period of time, protracted fractional method; third, the method of giving a large dose of 
very low intensity radiation over a very long period of time. The last usually concerns small amounts of radium or radium emanation. Most of the experimental work done upon animal tissues up to a recent date has been done with the massive-dosage method, so that the physiological effects presented concern chiefly this type of dosage, but of recent years the other methods have been employed to a considerable extent, though as a rule for therapeutic purposes only. Experimental observations made upon the skin of patients yield information of value in depicting the changes which may occur under these variations of dosage procedure.

\section{SKIN}

Although the effect of radiations on the skin has long been studied, the sequence of the changes which follow exposure and the explanation of such changes are not fully known. The reaction involves the epithelium, the blood and lymph vessels, connective tissue, and other structures which contribute to the nutrition and support of the epithelium. The amount of reaction to a definite dose seems to vary with the thickness and location of the skin (169). The epilation dose and the erythema dose for animals are almost 4 times as great as for the human skin.

The histological changes have been extensively studied $(9,183,276)$. Rost (305) describes in detail the effects of massive irradiation with roentgen rays. In the cutis there is an increase in connective tissue and endothelial cells, a decrease and atrophy of elastic fibrils, with shrinkage of the collagenous fibrils. Repair is slow and is accompanied by the formation of atypical cells. Subsequently pigmentation occurs, involving the disintegration of pigment cells. The pigment may be dispersed into the surrounding structures and into lymph channels (223). Sweat glands and the muscles of blood vessels show no change $(154,183,213)$.

Wolbach (393) has studied the changes which occur in human and guinea pig skin after repeated irradiations which extended over long periods of time. There is first a swelling of the subcutaneous collagen bundles, a reaction which results in a greatly thickened corium. Coincident with this is a gross shrinkage of the skin and a destruction of some connective tissue cells. Similar changes in the walls of blood ressels lead to the ultimate occlusion of the latter. Subsequently the epidermal cells swell and show abnormal mitoses (154). The maximum radiation effect is on the superficial layer of the corium in which necrosis occurs. Still later, the epidermis in the necrotic areas proliferates, while in the corium there is a formation of new collagen bundles. In cases of chronic roentgen ray dermatitis, normal collagen is replaced by dense, hyaline collagen rich in elastic fibers (393). There is also obliterative thrombosis in the blood vessels of the corium and subcutaneous tissues, and necrosis. Representative proliferation of the epidermis then follows (25, 57,61 ). 
Ewing (96) points out that "the enormous swelling and hyperchromatism of the epithelial nuclei after large doses of radium is a strictly specific feature of cell damage from irradiation which has never been satisfactorily explained." This damage may cause increased permeability of the cell membrane, resulting in an increased ability to absorb water. The most pronounced changes are those occurring in blood vessels, "of which the external evidence of the initial hyperemia followed by induration and relative anemia bear simple witness. The initial hyperemia seems to be merely a somewhat peculiar inflammatory process with vasodilation, exudation of serum, leucocytes, and, especially after roentgen rays, red blood cell infiltration."

Permanent epilation or loss of hair may follow massive doses of roentgen or gamma rays. Pressure anemia reduces the dosage required $(89,305)$. The hair which grows again after moderate doses is often devoid of pigment $(13,15,249,250)$. But when irradiation is given by the fractional method, very large doses ean be tolerated $(342,403)$. The hair of rabbits which had received $5600 \mathrm{r}$ by the fractional method grew again with no apparent injury. Only after doses of $9600 \mathrm{r}$ was epilation permanent. The effects are the same whether the fractional doses are given at a high or a low intensity.

Skin lesions produced by Grenz rays having a wave-length of 4 to $8 \AA$ are not unlike those which follow exposure to ultra-violet and roentgen radiations $(45,108,275,276)$. The erythema may appear within 12 to $72 \mathrm{hr}$. and increase in severity (313). With further exposure the skin becomes progressively less sensitive and may fail to respond even to large doses. Pigmentation may last as long as 10 months (307).

A comparison of the effects of very low voltage roentgen rays with those produced by cathode $(11,189,318)$ or ultra-violet rays shows that the latter are much less effective than the more penetrating radiations (237). The erythema dose for $12-\mathrm{kv}$, roentgen rays lies between 80 and $120 \mathrm{r}$, but as the voltage is decreased, the number of roentgens required to produce this reaction rises, until at $4 \mathrm{kv}$. it is $1000 \mathrm{r}(275,395)$. Expressed in terms of energy, the roentgen ray erythema is about 300 $\mathrm{erg} / \mathrm{cm} .^{2}$ For cathode rays it is about $500 \mathrm{erg} / \mathrm{cm} .^{2}$, but very much larger doses result in only a mild reaction not followed by pigmentation or epilation. In comparison, Coblentz states (43) that the erythema dose for ultra-violet waves $(2790 \AA)$ is $500,000 \mathrm{erg} / \mathrm{cm} .{ }^{2}$, and for $2800 \AA$ it is $1,500,000 \mathrm{erg} / \mathrm{cm}^{2}$ Other effects of cathode rays are discussed by Pape (264).

Carrie (35) sensitized mice with hematoporphyrin and obtained an epilation with less than the normal epilation dose of Grenz rays. Not only was the time of onset shortened, but the reaction was neither as severe nor as lasting as that produeed by high doses of Grenz rays alone. This effect resembles that produced by ultra-violet radiation rather than that of roentgen radiation. 
Ultra-violet rays have so slight a power of penetration that their effects are limited to the skin and superficial structures (191). Other effects, such as chemical changes induced in the blood, must come about through the absorption of the radiation in the capillary screen immediately beneath the skin. Keller $(173,174,175)$ reviews in detail the literature on the histological changes in the skin produced by these rays, and adds some observations of his own. The first change appears $5 \mathrm{hr}$. after exposure. The skin is sharply red, and leucocytes begin to infiltrate among the cells of the papillae and the superficial layers. Oxydase-reaction granules appear around the vessels which are full of blood. In $30 \mathrm{hr}$. the reaction is increased. The nuclei of the superficial cells, occupying a zone from 0.05 to $1.0 \mathrm{~mm}$. in thickness are dimly stained, while adjacent cells show a deeply stained protoplasm with their nuclei lying in an unstained area. Degenerating cells and leucocytes appear. The basal cells show little colloid degeneration; the pigment layer is somewhat disturbed; and here and there the cutis is separated from the epidermis. The blood vessels are still dilated and the oxydase reaction well marked.

During the next few days the general reaction subsides. But the greatest amount of swelling of connective-tissie cells occurs on the third day after exposure. The epithelium becomes much reduced in thickness, and is divided into two layers, the outer being deeply stained and the inner composed of a normal layer in which no signs of degeneration can be seen. The blood vessels and connective-tissue cells gradually assume their normal state. At no time do the sweat glands and hair papillae show any reaction. For a detailed account of these phenomena and for a discussion of the general reaction of the body to ultra-riolet rays, the original paper should be consulted.

Sensitivity to ultra-violet rays depends on the color of the skin and hair, on the age of the individual, and on the time of the year when exposure occurs. During menstruation and pregnancy it is increased $(21,22,43,91,92)$. The intensity of the reaction to ultra-violet radiation is apparently only a question of dosage, for with high dosage the deeper structures are damaged, even the endothelial cells, capillary loops, and fibroblasts. Degeneration and ulceration may be carried to extremes by increased dosage (306).

The pigmentation which develops after exposure serves as a screen by absorbing ultra-violet energy and preventing its further penetration $(9,10,132,191,232,233,358,397)$.

Many workers believe that the chemical content of the skin is not only changed by the irradiation but also is apt to influence the result (116). Adler $(3,4,5)$ thought there was a fall in the $\mathrm{Ca}$ and $K$ content with increasing doses of roentgen rays. Uhlmann (359) thinks that the acid-base shift in the blood has an important influence on epilation by 
roentgen radiation because with a low alkali reserve epilation may be produced by a dose of $300 \mathrm{r}$, while with a normal reserve, $600 \mathrm{r}$ must be given to produce the same effect. He thinks that shorter wave-lengths require higher dosages to elicit the same amount of reaction.

When radiations are combined with other injurious agents, the effect on the skin is a summation of the effects of each agent acting alone. Hawkins (141) exposed the skin of a guinea pig to $30-\mathrm{kv}$. roentgen rays, without filter, and to heat $\left(46^{\circ} \mathrm{C}\right)$. The only reaction produced by these agents acting alone was a swelling of the tissues; a combination of the two, however, induced a well-marked and persistent damage. The results were the same regardless of the sequence in which the agents were applied.

So also when roentgen rays are combined with ultra-violet radiation the result is an intensification of the dermatitis caused by the former (259). This experiment was performed in the hope that the ultra-violet might have an ameliorating effect following accidental overdosage with roentgen rays. There appears to be no antagonistic or reversal effect in the combination of ultra-violet and infra-red rays (47).

The epilation dose has been proposed by many observers as a biological unit of measurement, but the reviewer believes that it is unsatisfactory because of the variability in response among different animals in a group, and because the degree of epilation is difficult to determine. The epilation dose for rabbits has been estimated to be $1000 \mathrm{r}$ (302), $2000 \mathrm{r}$ (124), while Warren has found that the erythema stage of Miescher's classification of epilation stages $(228,231)$ is produced by 2000 to $2400 \mathrm{r}$. Perhaps a more careful study with definite quantities of radiation may serve to remove this uncertainty in dosage.

The effect of roentgen and gamma rays on the reparative processes which follow simple incision has been investigated. Takahashi (347) found that young capillaries and fibroblasts are very sensitive, their growth being stopped by a 5-min. exposure to beta and gamma rays from $15 \mathrm{mg}$. of radium $(0.2-\mathrm{mm}$. Ag filter). A $30-\mathrm{min}$. dose so injures the tissues that regeneration is induced from nearby structures. He found no evidence of stimulation. It should be emphasized that the proliferation following large doses is a regenerative process and not the result of stimulation. Pohle, Ritchie, and Wright (278) exposed the skin of rats before and after incision to doses of $1000 \mathrm{r}$, using wave-lengths of 0.34 and $0.18 \AA$. This treatment had no effect on the healing process unless it was given less than two days after incision, in which case it retarded healing. But Fukase $(109,110)$ states that doses of $400 \mathrm{r}$ on the incised skin of rabbits favor the reparative process.

Some careful work which takes into consideration the dosage-time relations between irradiation, injury (incision), the lethal dosages for the cells concerned with the repair process, etc., might give interesting 
information concerning the fundamental changes in tissues following irradiation. The effects of radiations other than roentgen and gamma rays could be studied to advantage by this procedure.

\section{BLOOD VESSELS AND THE RETICULO-ENDOTHELIAL SYSTEM}

It has already been mentioned that the erythema is a result of changes produced by radiations in the capillaries of the skin. The endothelium is damaged, as shown by a change in the permeability of the cells, and the contractile power of the capillary muscles is altered $(248,284,336)$. Very little is known about the type of injury which may be produced, aside from evidence obtained with the capillary microscope and from clinical observations. David and Gabriel $(58,60)$ first observed the reaction of the blood vessels of the skin to roentgen rays of various wavelengths by means of this microscope. They found that long waves produce a more vigorous reaction than short waves, a conclusion open to some doubt because of their uncertain dosage. The reaction appears first and is most striking in the superficial layers, and the capillary network stands out in a strongly reddened background. With the shorter wave-lengths the damage appears later and later. Dilation of the capillaries is the most characteristic change. Some observers, however, have reported an initial constriction (192). These observations should be repeated with calibrated wave-lengths and accurately measured doses. The tonus of the capillaries may be altered in other organs (111). Turano (355) points out that the damage occurs before histological changes can be seen. He believes that the capillaries in patients with vasodilation, due to disease or imbalance, are more sensitive to both ultra-violet and roentgen rays than are normal vessels. The dilation may continue for as long as two months (192). The endothelial cells show granules with vital stains after irradiation (288).

Histological studies of blood vessels in fixed tissues show only late changes $(184,282,284,309)$. The more acute changes are probably in the realm of functional response to local damage of the endothelium or to the mechanism controlling the size of the capillary itself. That is, it may be either direct or indirect. Zwerg $(403,404)$ found no change in the blood vessels or in the muscles with doses as high as 5600 to $9600 \mathrm{r}$, given by the fractional method.

The effects of both ultra-violet and roentgen radiations on the reticulohistiocytic system have been studied by Castellino (40) who observed that in guinea pigs, vitally stained with trypan blue, the number of histiocytes was increased. The cells were full of granules. Occasional Langhans cells and some fibroblasts stained blue. Similar observations have been made on mice and rats $(244,288)$. These reactions he considered to be evidence of a stimulation of this system. Phagocytosis may be increased by moderate dosages of roentgen rays $(18,286)$. 
Connective-tissue cells and the endothelial cells are very resistant to in vitro autolysis after irradiation in vivo (372). This fact might suggest that severe damage to the endothelial cells is not a primary factor in the capillary dilation which develops in the skin during the production of an erythema, but rather that some other mechanism is responsible for the fluctuation in the size of the capillaries. An investigation of this problem would be well worth undertaking because it may not only lead to further information about the function of the capillary control mechanism but would also establish more definitely the changes brought about in the capillary by radiation.

Miescher $(227,229,235,238)$ has described in detail the color changes in the human skin after roentgen radiation of various wave-lengths. His doses are uncertain but the quantities are comparable with each other, in minimal and maximal ratios of $1: 6$ and 1:8. He lists seven stages; (a) slight reddening, (b) weak reddening, (c) strong reddening, $(d)$ very strong reddening with a slight cyanotic overcast, $(e)$ reddening and swelling of the whole field, $(f)$ reddening with vesicle formation and desquamation, and $(g)$ ulceration. In addition, he determined a pigmentation scale. The pigmentation was distinguished from the redness by observing the skin under compression through a glass plate, and was divided into 4 degrees from "slight" to "very strong."

He found three waves of reaction in the skin, the "time" border of which for the first wave lay between the first and fourth day, for the second wave, between the eighth and the twenty-second day, and for the third, between the thirty-fourth and the fifty-first day. The greatest intensity occurred during the third wave. In some instances no isolated third wave was observed, while in a few cases a fourth wave appeared. The latent periods between the individual waves are not related to the dose but seem to be influenced by individual factors. In the same individual the rhythm of the curve of redness is the same for both large and small doses.

In the course of the erythema reaction the timing of the pigmentation curve showed considerable variation from the waves of reddening, but in general each wave of redness was followed by one of pigmentation. Sometimes there were more of the latter than of the former (198). It has been thought that the blond skin is more sensitive than the brunette, but probably it is not. A mild erythema is more readily visible in a pale skin. The child skin, in general, shows the same fluctuations as the adult skin $(224,303)$.

Schall (319) found a sharp reaction of redness from 4 to $8 \mathrm{hr}$. after roentgen radiation. This immediate response lasts for $24 \mathrm{hr}$. or more. The erythema is maintained at a high level for the whole period of 75 days, but there are two major waves centering around the thirty-fifth and the sixtieth days, as Miescher found. The pigment begins about 
the twelfth day and has its maximum varying from the fortieth to the seventieth day. Twenty per cent variations in the dose caused no appreciable change in the degree of reaction.

The capillaries of the skin do not completely return to normal after one roentgen ray erythema dose (273). The microscopic and macroscopic changes appear in cycles, without, however, a sharp interval between the consecutive periods. One "medium crythema dose" of ultra-violet rays produces capillary changes similar to those induced by unfiltered roentgen rays, but they last for a shorter time and are followed by complete recovery. The increased redness characteristic of the erythema is due to the increased quantity of blood in the peripheral vessels (135). According to David and Gabriel (60) not only the size but also the number of capillary loops may be increased.

Grenz rays, excited at 4 to $12 \mathrm{kv}$., produce in general the same reactions as do higher voltage roentgen rays (303). In the rabbit ear, following exposure, there is a dilation of the vessels, epilation, ulceration, and crust formation. The reaction is dependent on the intensity of the radiation and on the length of exposure $(112,113)$. In the human skin, reddening appears early and continues at its height for five days (306). It then gradually subsides during the next three months during which time pigmentation develops. The histological changes are not unlike those produced by roentgen rays. Cell destruction, swelling of the endothelium, infiltration of lymphocytes and leucocytes occur.

The erythema produced by ultra-violet irradiation may vary in intensity as much as 100 per cent in the same individual, depencling on the region which is exposed, and between different individuals (272, 273, $341,398)$. Schall and Alius $(320,321,322)$ using an "erythema meter" found that the reaction develops in a wave which has its maximum 6 to $10 \mathrm{hr}$. after exposure. The latent period before the onset of the erythema is usually from 1 to $2.5 \mathrm{hr}$. but may be more. These authors present curves illustrating the several types of reaction, detailed description of which is given.

Motojima (239) has compared the effects of ultra-violet rays, roentgen, and gamma rays on the capillaries of the frog's tongue. In each case dilation of the vessels occurred, a slowing of the blood stream with stasis and diapedesis, and edema. These responses were most severe following ultra-violet radiation, and recovery was most rapid. Beta rays produced a rapid reaction which remained longer than that following exposure to gamma rays. Roentgen rays produced the least response. The impossibility of measuring equal doses of these three types of radiation prevents strict comparisons between the degree of effect produced. The erythema following exposure to radium radiation (294) has a stronger color and is darker than that produced by roentgen rays (296). Furthermore, the height of the redness is later, pigmentation is greater, and the 
whole reaction lasts longer. The gamma-ray erythema has been studied by Reisner $(294,297)$ who reviews the literature on this subject. He finds that the doses previously given have been about 40 per cent too high. Instead of $560 \mathrm{mg}$. el hr. at $1 \mathrm{em}$. he believes that 310 to $330 \mathrm{mg}$. el hr. $(0.2 \mathrm{~mm}$. Pt and $1.7 \mathrm{~mm}$. brass filter) are adequate to produce effects comparable to those which follow roentgen radiation. Braun, however (29), finds no difference between the two when both are given by the massive-dose method.

The sequence of events following exposure of mouse tails to gamma rays is essentially that found in the human skin, that is, erythema, various degrees of epilation, desquamation, and ulceration (13). The eonstriction, dry gangrene, and amputation of the tails were due to bloodvessel thrombosis which is a late stage of severe injury.

\section{CONNECTIVE TISSUE}

The reactions of connective tissue to radiations have been mentioned in the preceding seetions $(96,369,393)$. The behavior of individual cells after exposure is best seen in tissue culture preparations $(34,52)$. The latent period which oceurs between the exposure and the first appearance of injury has been studied by Fiseher $(102,103)$ who used fibroblasts and osteoblasts from the embryo chiek. He believes that the length of this period is not dependent on the rate at which the eells are dividing, and that it cannot be increased or diminished exeept to a small degree. Contrary to the usual opinion, he holds that the absolute effect of radiation is the same whether cells are dividing rapidly or slowly (102). When very lightly irradiated cells are exposed to abnormal conditions, such as high or low temperatures or poisons, which in themselves are not injurious, the effect of the radiation is inereased. This may be simply an evidence of summation.

The connective-tissue cells and the endothelium, together with the blood-vessel-muscle cells which are relatively resistant to radiation $(163,290)$ and the nerve endings and plexus in the capillary wall (whose sensitivity is unknown) may all be involved in any changes brought about in the various organs as well as in the skin by radiation. The extent to which these tissues are involved is largely dependent upon the dosage administered to the organ. The more resistant organs may show injury only to the blood vessels while in other organs whose cells are more sensitive, the capillaries play little part in the evident damage. This indieates the great sensitivity of the cells in such organs.

\section{DOSAGE WAVE-LENGTH AND SKIN}

The relative effectiveness of different wave-lengths of radiation in producing the erythema reaction has been the subject of much research in recent years. The amount of energy required to bring about this response by means of heat is far greater than it is for ultra-violet, while 
the latter amount is greater than that needed to produce a roentgen-ray erythema. Whether equal doses of roentgen rays, gamma rays, and cathode rays are equally effective, is still debatable and must remain so until the intensity of these radiations can be measured in equivalent units. Stahel (339) compared the activity of roentgen and gamma rays on the basis of erg $/ \mathrm{cm}^{2}$ absorbed, and found that while the roentgen-ray erythema dose $(600 \mathrm{r})$ is equal to $96,000 \mathrm{erg} / \mathrm{cm}^{2}{ }^{2}$, the gamma-ray dose may rise as high as $8,000,000 \mathrm{erg} / \mathrm{cm}^{2}$ Such estimates are very questionable.

Although roentgen rays can now be measured accurately in terms of roentgens, the question is not yet settled whether all wave-lengths are equally effective $(75,76)$. Many observers $(14,15,42,77,122,151$, $153,199,252,270)$ believe that when the areas exposed are equal, as well as the intensities of the beams, and the doses, measured in roentgens, the erythema reaction is the same for all ordinary wave-lengths. Others, however, maintain that the soft rays act more vigorously $(7,97,106,118$, $121,225,226,311,327)$. A summary of some of the opinions on this point is given in the table. Glocker (123) and Pohle (281) present evidence to show that when a full erythema dose of $700 \mathrm{r}$ is given, the skin reaction is the same for all qualities of roentgen rays within the region which they explored. But softer rays appear to be more effective when given in small doses. Reisner and Neef (297) have made a careful study of this problem, using accurately measured doses. They conclude that when the area exposed is small $\left(4 \mathrm{~cm}^{2}\right)$, the reaction to equal doses of roentgen radiation having wave-lengths from 0.14 to $0.26 \AA$ are equal. Softer rays are more effective. With a larger area $\left(50 \mathrm{~cm} .{ }^{2}\right)$ the range of wave-lengths within which an equality of effect occurs is somewhat greater.

Still another view is that the effectiveness steadily increases with increasing wave-length from a minimum to about $0.45 \AA$ (311). From this point onward it decreases $(118,327)$. According to Meyer and Glasser (225) the number of roentgens required to produce an erythema by means of different roentgen ray wave-lengths, divided by the halfvalue layer in water of those beans, is practically a constant.

In recent years the method of dividing the total dose into fractions which are given at frequent intervals over a period of days or weeks has been widely used (cf. 242). Reisner (295) compared the effect of such doses with that of a single massive dose. The latter, a "tolerance dose" was $1100 \mathrm{r}$ on a field 2 by $2 \mathrm{~cm}$. Using this as a standard, he found that equal skin reactions could be obtained by giving 27 daily exposures, each of 10 per cent $(110 \mathrm{r}$ ); or 7 exposures of 30 per cent each. For a full discussion of the effects of these and other arrangements of divided doses the original paper should be consulted. In each case, when doses are small and are given over an extended period, the tolerance dose, measured in roentgens, is greatly increased. 


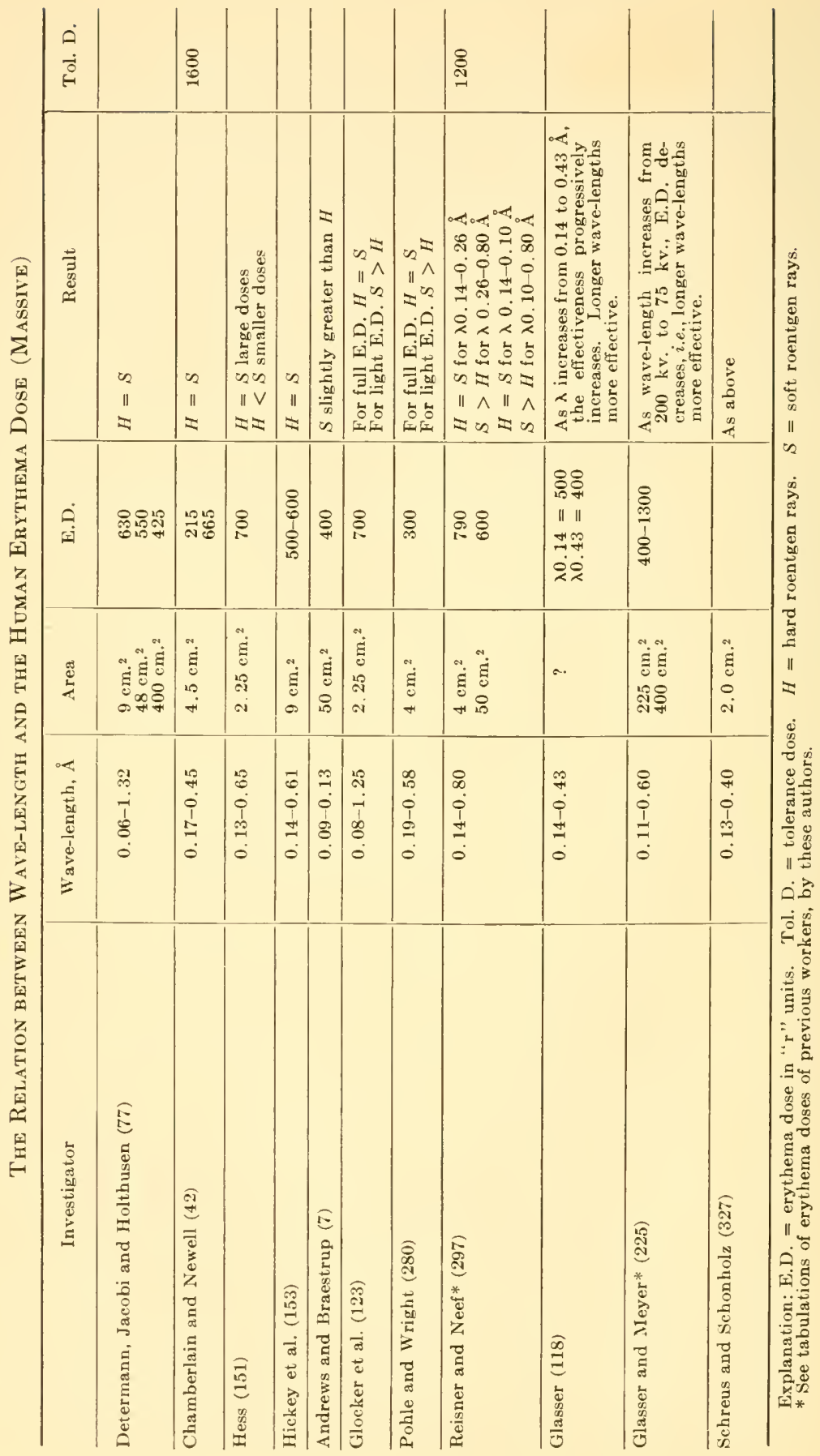


Coutard $(50,51)$ has found that either protracted or simple fractional irradiation with a total dose of 4500 to $5000 \mathrm{r}$ is followed by severe damage to the skin and mucous membranes which heal, however, and remain intact for at least 6 to 7 years. The treatment is given in two daily sessions of $1 \mathrm{hr}$. each (225 to $250 \mathrm{r}$, the intensity being 7.5 to $20.0 \mathrm{r}$ per min.) for 10 days. There is redness of the skin with edema by the end of this time, and desquamation begins, so that by the twenty-eighth day there is a total loss of the superficial epithelial cells with a denudation of the epidermis, aceompanied by pain. The lesion is usually repaired by the forty-second day "without any visible trace" of injury. With greater doses the period of reaction is longer; with smaller doses, the pain is less.

By appropriate spacing of the exposures, the time of the reaction in the mucous membranes of the mouth and throat can be separated from that of the skin. Mucositis occurs between the fourteenth and the twenty-eighth days, and is practically asymptomatic. Radio-epidermitis develops between the twenty-eighth and the forty-second day. This very interesting and puzzling sequence occurs if the radiation is given over a period of 14 days. The difference is probably due to the smaller dose received by the mucosa in contrast to that supplied to the skin. If the period is prolonged to 18 days with proportionately smaller daily doses, the damage to the skin is less and the skin heals sooner. Lesions produced by this method are considerably greater than those which follow a massive dose, but they heal with little damage, leaving the skin thin and flexible and usually free of pigment $(61,241,324,392)$. But telangectasis and permanent edema may ensue (150). For a further discussion of this topic see Zuppinger (402). Holfelder (157) by giving 50 to 60 per cent of an erythema dose every 2 to 5 days over a period of 2 to 6 weeks, was able to administer a total of 1600 to $2400 \mathrm{r}$. In his procedure, the dosage in successive treatments is gradually decreased, while the intervals are increasingly prolonged.

McNattin (221) used the Coutard method of treatment with $200-\mathrm{kv}$. radiation and compared the effects with those obtained with $700 \mathrm{kv}$. administered by the fractional method, and with the radium pack. He believes that as a first approximation the following doses may be therapeutically equal if fractionated over a period of 3 weeks or longer: $(a)$ for $200-\mathrm{kv}$. roentgen rays, 6 threshold erythema doses, (b) for $700-\mathrm{kv}$. roentgen rays, 4.7 erythema doses, (c) for gamma rays, 1.6 to 2.2 erythema doses. The threshold erythema dose is $600 \mathrm{r}$ for 200-kv. radiation (massive dose) filtered by $0.5 \mathrm{~mm}$. $\mathrm{Cu}$ and $3.0 \mathrm{~mm}$. Al and includes back-scattering. The skin reaction with the above large (tolerance) doses is very marked with the $200-\mathrm{kv}$. radiation and with gamma rays, and less severe with the 700-kv. rays. On the other hand, after the skin has healed, the mildest changes are found after the gamma-ray treatment $(66,144,149,194,311)$. 
Pfahler (266), using the theoretical concept of Kingery (176), has undertaken to use the saturation method, which consists in applying an erythema dose at high intensity within a short time in one sitting, and then maintaining this effect over a period of time by means of "additional smaller doses to correspond with the loss in effect during any given period." The rate of loss is represented by a logarithmic curve. According to Stenstrom and Mattick (342), 50 per cent of the initial dose can be added on the ninth day after a full erythema dose. But there is some evidence that the interval depends on the wave-length of the radiation employed (268). These additional doses cannot be given indefinitely, but within certain limits the method gives increased destructive effects on tumors. Reisner (293) is of the opinion that this method of dosage is not so effective as Coutard's.

The total amount of radiation which cells and tissues can tolerate is very great. Chick embryo fibroblasts, exposed in vitro, will survive doses of 6000 to $7000 \mathrm{r}$ (163), while massive doses of 1200 to $1600 \mathrm{r}$ upon small areas (4 to $25 \mathrm{~cm}^{2}$ ) of the human skin do not permanently injure the tissues $(24,231)$.

Pack and Quimby (258) believe, from their study of the skin erythema produced by different intensities, that a greater amount of roentgen radiation is required if the exposure is long. They point out, however, that there must be a differential recovery of cells after the exposure to radiation. A large number of experiments tend to support the theory that normal cells recover much more quickly than do neoplastic cells. In a review of the literature the authors find that there is a lack of agreement regarding the influence of the intensity of the beam on the effect produced. Some investigators found that the reaction to a definite dose is the same regardless of the intensity, while others believe that low intensities are more effective. The majority claim that the reverse is true, namely, that high intensities act most vigorously. Pack and Quimby emphasize the work of Regaud (291) who showed that greater differential effects could be obtained on tumor cells than on normal tissues with low intensity radiation. In their experiments on the production of the erythema on the human skin they found that "if all the times used in such an experiment are short in comparison with the duration of the life span of the cell in question, no difference will be observed with different intensities. If, on the other hand, the duration of exposure to radiation of low intensity is comparable to the life span of the cell, a different result may be expected, namely, that a greater effect is produced on the skin by a stronger source acting for a short time than by a weak source used for a correspondingly long time." (See 159, 161.)

Holthusen (160) finds that the effectiveness of roentgen radiations depends not on their wave-length but on the intensity of the beam. Five hundred roentgens delivered at the rate of $500 \mathrm{r}$ per min. produce the 
same effect as $1000 \mathrm{r}$ delivered at the rate of $15 \mathrm{r}$ per min. Equivalent amounts of radium or roentgen radiations have equivalent effects on the skin, if the intensities of both are the same. This conclusion is in agreement with that of Quimby and Pack (286), although other work $(97,287)$ seems to indicate that there may be some difference in the effectiveness of different wave-lengths. Holthusen's suggestion, that radium may produce a stronger reaction in the second wave, may be but a variation which has been pointed out by Miescher (227) of identical doses, or it may explain why it is possible to give a slightly greater total dosage of roentgen-ray and gamma radiation eombined than of either alone (286).

Experiments to test this latter point indicate that when roentgen rays, gamma rays, or ultra-violet, in various eombinations, are used to produce an erythema, about one-third more radiation must be administered than when any one of these agents is used alone. This appears to be true for combinations of gamma rays and hard beta rays, gamma rays and hard roentgen rays. With equal parts of ultra-violet and soft roentgen rays, the erythema is not produced even after a 50 -per-cent increase in the dose $(108,280,285,287)$.

The intensity of radiation, which is an important factor in determining the amount of reaction of the skin to equal doses, seems to play no part in the response of some other tissues $(14,15)$. Mouse tails exposed to equal doses of widely different intensities of beta and gamma rays respond equally. Bagg and Halter (15) have used this method to compare the relative effectiveness of roentgen and gamma rays. When very small areas are exposed, the effect of $400 \mathrm{mc} . \mathrm{hr}$. is the same as that of $2350 \mathrm{r}$. The filtration of the radon was by $2 \mathrm{~mm}$. brass and $3 \mathrm{~mm}$. bakelite. Experiments with the rabbit's ear showed that for a larger area $\left(28 \mathrm{~cm} .{ }^{2}\right)$ the emission from $1 \mathrm{gm}$. of radium at $1 \mathrm{~cm}$. distance is approximately equivalent to $100 \mathrm{r}$ per min.

Failla (97) in reviewing the effects of various wave-lengths produeed by 200 and $700-\mathrm{kv}$. roentgen rays, and gamma rays, believes that there is a true differential action of radiation for human skin as compared to other test objects, and that 700-kv. radiation is intermediate in its effects between the $200-\mathrm{kv}$. radiation and gamma rays.

Grenz rays are absorbed almost completely ( 88 per cent by $3 \mathrm{~mm}$. of human skin) in the surface, while roentgen and gamma radiation are mostly transmitted by the skin (31). While measurements of quantity are not yet established, it is thought that $250 \mathrm{r}$ produces an immediate mild erythema $(119,295)$. Reisner (295) found that doubling the dose (4-cm. ${ }^{2}$ field) gave increasingly severe reactions up to $4800 \mathrm{r}$. The reaction from $2400 \mathrm{r}$ of Grenz rays was equivalent to that produced by $1000 \mathrm{r}$ of $200-\mathrm{kv}$. roentgen rays.

It is the reviewer's opinion that clinical experience and animal experiments seem to bear out the fact that there is some difference in effect 
between the various wave-lengths of roentgen and gamma rays, and that it may be possible to bring out this differential effect by the fractionated method of dosage. One difficulty lies in the method of measuring the dosage of the various wave-lengths over the whole range of the electromagnetic spectrum so that equivalent amounts of energy may be used. Individual variation in the response of the animal or human skin is also important, often as much as 20 per cent (88).

The effects produced by the ultra-violet, visible, and infra-red radiations depend upon the character of the substances making up the skin, i.e., some of the radiation is reflected (363) and some is absorbed (44) by the stratum corneum and pigments $(142,202,343)$, the capillary bed (363), and tissue proteins $(76,391)$.

Bachem, in $1927(9,10)$, in a very extensive study of the transmissibility of the skin, has found that the stratum corneum transmits best in the middle of the ultra-violet spectrum with a secondary maximum near $2500 \AA$, for the malphigian layer has an absorption maximum at the border between the visible and the ultra-violet, so that the corneum absorbs both the shortest and the longest infra-red radiations and transmits the long ultra-violet rays and the near infra-red rays. The subcutaneous tissue receives only long wave-lengths (down to depths of 2 to $3 \mathrm{~cm}$.) centering about $6000 \AA,(9,10,53)$ while red light penetrates quite well through fingers, ears, eheeks, sinuses (frontal). In the infra-red transmission rises around $10,000 \AA$ and falls around $14,000 \AA$. The development of pigment or an erythema or ischemia retards the transmission of the ultra-violet radiation $(87,152,202,340)$, but in general the reaction is proportional to dosage and large doses of all but the visible cause destructive effects similar to that produced by roentgen rays $(173,174)$. The skin temperature may rise (even with visible radiation) owing to the reflex dilation of the skin capillaries $(53,142,175,328,330)$. The reaction is magnified, where the source is sunlight or a earbon arc, by the deeper effects of the more penetrating short infra-red and red radiation, which cause dilation of the larger vessels beneath the skin. This brings about an erythema (when mild doses are used) beyond the area irradiated (142) which is of longer duration that that due to the longer infra-red and ultra-violet radiation absorbed in the skin proper.

Dosage and the absorption spectrum of tissues are thus both important in these effects. Human skin has erythema maxima at 2500, 2800, and $3000 \AA(133,139)$, and at $3030 \AA(357,358)$, and reflects very little of the wave-lengths between 3000 and $4000 \AA$. Pigment development oceurs strongest at 2480 and $2540 \AA$, less at 2970 and $3030 \AA$ (330), but the matter of equal dosage for such experiments has not been verified, for either erythema or pigment production.

In general, the physical character of the absorbing surface or tissue (skin) determines the effectiveness of individual wave-lengths of the 
longer end of the electromagnetic spectrum but has little effect probably when the shorter (beyond $1 \AA$ ) wave-lengths are used. The dosage is the product of time and intensity. The massive tolerance dose and the total fractional dosage of roentgen rays give almost equally serere reactions from which the skin may recover though the margin of safety is probably greater with the latter. Effective differences between long and short roentgen rays of equal amounts (over equal areas) are so small as to be of little importance in practice in so far as the skin is concerned. The normal variation in the animal and human subject is probably as great as if not greater than the difference in effect between the long and short roentgen rays.

\section{THE EFFECT OF GENERAL BODY IRRADIATION}

If the whole animal organism (dog, rabbit, and most other experimental animals) is irradiated (377) by rather high massive dose of roentgen radiation, i.e., approximately $2500 \mathrm{r}$, an acute rapidly developing intoxication ensues, usually accompanied by bloody diarrhea and exitus on the fourth or fifth day. There is evidence of rather widespread damage to certain internal body structures without evidence of damage to others. This is due partly to the specific sensitivity or resistance of certain tissues to this dosage level and partly to the variation in the appearance time of evidence of damage (i.e., latent period). It is believed by some (377) that when the whole body or the abdomen is irradiated, the major cause for the fatal intoxication is the rapid destruction and disintegration of the intestinal mucosa within the 4- to 5-day period. A somewhat more complex though sharply linited syndrome is likewise produced by irradiation of the bony skeleton with the abdomen protected (334). In this type of experiment the destruction of bonemarrow cells, while just as extensive as is the case in the intestinal epithelium, causes only a slight or moderate intoxication. Exitus in this case is accompanied or precipitated by a progressive thrombocytopenia and sudden widespread capillary hemorrhage ( 8 to 10 days). Other acute syndromes may be produced by damages of the liver, etc., but these have not been thoroughly studied.

Profound chronic damage of serious and fatal type may likewise be produced by total irradiation because of the production of widespread sloughing of the skin and muscle or diffuse degeneration of intestinal epithelium, or by means of intensive localized irradiation of certain vital organs such as the heart or kidneys (\$4). In such circumstances exitus occurs usually by secondary infection of the tissues through the skin ulcers or by functional disability and failure of the organ, i.e., slow starvation from inadequate digestion and absorption of food, etc. In all areas irradiated in such a manner many other structures are damaged, such as lymphoid tissues, gonad cells, blood vessels, and comnective 
tissues, skin, etc. The amount of damage is always proportional to the dosage plus the sensitivity of the cells involved.

Tsuzuki (354) finds a great many acute changes in most of the organs when the whole body of the rabbit was irradiated, and the animal sacrificed dircetly after irradiation or at intervals of $3,13,36 \mathrm{hr}$. All of the organs, including the salivary gland, thyroid, lung, thymus, heart, liver, spleen, intestines, pancreas, kidney, adrenal, testis, and bone marrow, showed hyperemia and apparently some degeneration of cells. Much of this is gone after $36 \mathrm{hr}$. with a residual of a slight amount of atrophy.

He explained the onset of the acute intoxication which he noted following such irradiation upon the basis of this rather extensive acute cell destruction. The latter was quickly followed by repair. Some of the changes described may not be due to the irradiation, but this phase should be further studied. The dosage used and the period of observation were probably well below those necessary to enable the author to study the acute syndromes mentioned above.

Exposure of the entire body to a large quantity of radium may bring about very similar damage, i.e., destruction of lymphoid tissue in the spleen, lymph nodes, intestinal lymph follicles, and bone marrow, degeneration of the epithelium and hyperemia of the intestine, cellular degeneration in the testis or ovary, stomach and intestine, kidneys and liver, mucoid degeneration and desquamation of the columnar epithelium of the bronchi and trachea, hyperemia, alveolar exudation, and focal consolidation of the lungs $(73,74)$.

Intravenous or oral administration of sufficient doses of thorium $\mathrm{X}$ or radium salts or emanation induces a leucopenia affecting chiefly the lymphocytes, large mononuclear cells, and blood platelets, hyperemia and hemorrhage of the bone marrow, marked destruction of lymphocytes and atrophy of lymphoid follicles in the spleen and lymph nodes, inflammation and destruction of the vascular endothelium, with secondary proliferative thickening of the vessel walls, central parenchymatous necrosis of the lobules of the liver, hyperemia, exudative distention of the glomerular capsules and coagulation necrosis of the kidneys, and intense hyperemia of the lungs, with exudative distention of the alveoli. After intravenous injection, the greatest changes appear to be in the blood vessels, lymphoid structures, and liver, whereas, after oral ingestion, the large intestine, lymphoid structures, and kidneys suffer the greatest injury $(73,74,185,188,193,201)$.

These effects differ little from those brought about by intensive roentgen irradiation. The chief difference lies in the low intensity of radiation spread over a very long time interval. The fact that the intestine, kidney, and lung are sites for excretion while endothelial phagorytes, bone, liver, and spleen are sites for storage of any particular foreign material would tend to intensify the damage in such structures. 
The severe constitutional reactions from excessive exposure of large parts of the human body to ultra-violet radiation are well known. The gradual building up of the tolerance of the skin through repeated fractional dosage, similar to the fractional dosage methods for roentgen therapy, is the basis for its therapeutic use $(191,218)$. Death has followed too severe and extensive skin damage. This intoxication may have the same mechanism as that following severe burns from steam and other intense sources of radiation in the infra-red spectrum.

The body has adapted itself to live in equilibrium while receiving large quantities of infra-red radiation, particularly the longer wavelengths, and is itself a producer of these radiations. Death may be the result of upsetting this equilibrium by the intensive generalized absorption of infra-red radiations from without (artificial fever) or the production of and failure to radiate excessive amounts of infra-red radiation (spontaneous fever accompanying infections, etc.). Death may likewise follow excessive losses, i.e., exposure to cold. In frostbite a part of the body has been irradiating infra-red radiation at so great a rate that the skin and capillaries and subcutaneous tissues become damaged by the low temperature attained. This is just the opposite type of energy exchange that occurs in a "heat burn," but relatively the same damage occurs and perhaps the same type of intoxication if the damage is extensive enough.

"Radio-wave" Radiation.-In the last few years there has been considerable interest in the effect of very long electromagnetic waves, so-called "high-frequency" or radio waves. There is considerable evidence to show that the longer waves in the neighborhood of 300 meters (which are produced by the ordinary diathermy machine) produce effects in tissues which are probably related to the transference of this energy into heat. There is some evidence to show that the shorter wave-lengths may have certain specific effects in certain organs of the body, perhaps owing to differences in conductivity, or to electrolyte content, or for other reasons as yet unknown. It is thought that these high-frequency currents may build up excessive temperature gradients in certain organs of the body. Oettingen (254), has published perhaps the most comprehensive study of the lesions produced by 3 -meter waves in the liver, kidney, brain, testes, ovary, and musele of the mouse. Similar changes have been demonstrated by Schliephake (325).

Schereschewsky (323) has shown that there is evidence to prove that the shorter wave-lengths (1.0 to 4.69 meters in radio frequencies) do cause specific elevations in temperature of various organ emulsions in vitro. With the very shortest waves used (1 meter) the comparatively specific heating of these emulsions is maintained and brain, pancreas, and lung retain their property of relatively high heating. Blood serum and plasma show less specific heating than organ tissuc; red blood cells heat 
up much more than the whole blood or plasma. Certain frequencies seem to show specific heating effects. This is very difficult to prove in vivo because of the difficulty up to the present of measuring the actual temperature gradients in organs under the influence of these "highfrequency" electromagnetic fields. The changes described by Oettingen (254) and Schliephake (325) are of two types, the acute damage which is lethal, and chronic changes. Acute lethal damage is due for the most part to complete or partial coagulation of vital tissue and in part to the dehydration which may result from the elevation of the temperature of the whole animal. The elevation of the white blood count with increase in the polymorphonuclear cells and a relative decrease in lymphocytes which occurs after the exposure to this short-wave radiation is a characteristic of the elevation of the body temperature rather than a specific effect due to these electromagnetic radiations (3 to 300 meters) for the same circumstance arises in patients and animals whose temperature is elevated artificially by other methods (radiant energy, chemical poisons, etc.). In the acute lethal experiments (254) there is a generalized hyperemia of all the organs, with edema and damage to blood vessels and organ cells which in the reviewer's experience occurs at or above a body temperature of $43^{\circ} \mathrm{C}$. In the animals irradiated frequently there are some degenerative changes in all the organs with various stages of repair and disintegration. This, to the reviewer, means that the organs are damaged by local elevations of temperature within the organs. The temperature of the tissues was raised too rapidly for the blood streams and local conduction to remove the excessive encrgy which piled up to the point of damage or even to the point of coagulation. There is considerable theoretical evidence to show that it may be possible to bring about such localized heating in certain tissues without heating the other tissues if the proper wave-lengths are used.

\section{GASTRO-INTESTINAL TRACT}

Changes in the gastro-intestinal tract produced by roentgen radiation have been reviewed in some detail by Warren (369) and more recently the experimental literature has been outlined in great detail by Desjardins (74).

Upper Portions.-The mucous membrane of the mouth, pharynx, and esophagus does not differ materially in the type of reaction from that noted in the skin except that the lesions are somewhat influenced by the moist surface (and therefore the reaction depends upon the salivary secretion to some extent), by the presence of digestive juices and food (abrasion), and by the absence of the horny layer which acts as a slight protective coat in the case of the skin. The radiosensitiveness of the mucous membrane of the nose and its accessory sinuses has not been determined experimentally. Excessive irradiation of the larynx (roent- 
gen rays or radium) may cause an inflammatory reaction characterized by redness and edema of the mucosa. Several months or even years later, these disturbances may be accompanied or followed by stenotic thickening and dyspnea, and in some cases by pneumonia and death. Many examples of such reaction have been reported (Desjardins, 74).

Salivary Glands.-After exposure to roentgen radiation the submaxillary gland swells and the acini degenerate with loss of the lumen. There is some infiltration of plasma cells and lymphocytes. The gland cells become vacuolated, with granular protoplasm and abnormal nuclei. Dilation of the capillaries may occur. These changes are less severe in the parotid than in the submaxillary where regeneration first takes place $(28,316)$. The secretion of saliva in the dog is reduced after heavy radiation (37) but returns slowly to normal. The sensitivity of the buceal mucosa is temporarily diminished, a response which probably affects the sensation of taste. There is also a decrease in the ability of the dog to control the muscle movements of mastication and swallowing, an effect which may be due to the influence of the radiation of the peripheral motor nerves. This is practically the only reference to such an effect and perhaps warrants further study.

Desjardins (74) states that "there is no valid evidence that small doses of radiation increase the functional activity of the salivary glands; on the contrary, the evidence indicates that small doses have no effect, but that strong doses interfere with their function and diminish any secretion of saliva by causing metabolic inhibition and degeneration of the specific epithelium of the glands. The degree of such action, as well as its onset and duration, depend on the dosage, and the effect of irradiation may be temporary or permanent for the same reason. The salivary glands are exceptionally sensitive to irradiation. The reaction of the glands thus induced may be early, late, or combined. Early reaction oceurs in a large percentage of eases in which the region of one or more of the glands is irradiated, and consists of rapid swelling, with a sensation of heat and tension. The swelling begins a few hours after irradiation and generally subsides spontaneously within 24 to $72 \mathrm{hr}$. Females are twice as susceptible as males, and blondes more susceptible than brunettes. The later reaction consists in dryness of the mouth which appears from a few days to 2 to 3 weeks after irradiation of more or all of the salivary glands, is most pronounced 2 to 4 weeks after exposure, subsides slowly thereafter, and is due to decreased secretion of saliva. Its severity and duration vary with the dosage. Both the early and late reactions may occur in the same case."

Esophagus.-There is relatively little information regarding the effects of radiation on the esophagus because its position makes observation difficult. Probably its sensitivity is about the same as that of the skin and mucous membranes of the mouth (99). Warren (368) noted 
that $1200 \mathrm{mg}$. el $\mathrm{hr}$. of radium $(0.5 \mathrm{~Pb}$ filter $)$ produced a sharp reaction in the mucosa of the lower esophagus when the applicator was practically in contact with its walls. The effect lasted from the tenth to the twentieth day after exposure. Injuries following radiotherapy of the esophagus are not common $(74,289)$.

Stomach.-The gastric mucosa is much more sensitive than the esophagus and may be damaged by doses which leave the skin unharmed. Large quantities of radiation which cause epilation and necrosis of the skin result in the destruction of the stomach wall and perforation. Such lesions have been extensively studied $(19,64,166,374-377,394)$. The pathological changes involve chiefly the secretory epithelium. Dawson (64) observed that the chief cells are the most sensitive while the parietal cells are resistant. The latter, even when showing no histological injury, are unable to produce acid. The decrease in the acidity of the gastric juice is one of the characteristic reactions to radiation $(64,283)$. Radium from an external source produces practically the same changes; when applied locally its effect is very intense. If the dosage has not been too great, repair follows injury. There is a proliferation of the connective-tissue stroma and blood vessels, a realignment of a latent atrophic mucosa. The regenerated mucosa is thin but histologically normal. (For a review of the functional changes following irradiation cf. Desjardins, 74.)

Small Intestine.-The small intestine is considerably more sensitive than the stomach, the sensitivity being manifested mostly in the crypt cells of the villi. This commences sharply at the pyloric sphincter and extends to the ileocecal valve, the upper portion being more sensitive than the lower. The effect of the radiations is proportional to the dosage. In the dog a dose equivalent to the amount necessary to produce epilation will destroy the entire mucosa $(134,207,208,377)$.

Lethal doses of roentgen rays given over the abdomen of a normal dog are followed by a physiological reaction of remarkable uniformity (371). In the first $24 \mathrm{hr}$. the crypt epithelium shows a definite degenerative change which begins to appear as early as $2 \mathrm{hr}$. after exposure. The bone marrow, spleen, lymph nodes, and ovaries are also affected. During the next $24 \mathrm{hr}$., necrosis of this epithelium may be almost complete, while the tip epithelium of the villi remains practically intact. There is little edema and invasion of wandering cells. On the third day the small intestine from pylorus to ileocecal valve is raw and inflamed. The crypt and villous epithelium has in large part vanished, leaving a collapsed framework of mucosa in which there is some edema and invasion of wandering cells. The disintegration of the mucosa is apparently responsible for the symptoms and pathological lesions which develop. The fourth day marks the peak of intoxication, and death usually takes place at this time, preceded by vomiting, bloody diarrhea, and coma. 
The anatomical and histological picture resembles that of the third day. During this whole period there is no evidence of damage to the skin. In spite of the almost complete destruction of the mucosa of the small intestine which is almost denuded of its protective mechanism, indeed the muscularis mucosa is bare in many places, the body usually is not invaded by an overwhelming number of bacteria (374).

All of the common experimental animals respond in the same manner to doses of 2000 to $2500 \mathrm{r}$ over the abdomen. Divided doses given within a 5- to 6-day period cause practically the same effect as a single massive dose equal to the sum of the small doses. But when exposures are given at intervals of 5 days there is no evidence of a summation effect (375). The rat and guinea pig are slightly more sensitive than the dog, eat, and rabbit. By contrast, birds, frogs, and reptiles, are very resistant and may tolerate doses two or three times as great as the lethal dose for dogs $(373,375)$.

Exposure to large doses of roentgen rays will cause notable increase in the speed of autolysis (372) of the crypt or secretory epithelium of the dog's small intestine. These changes can be demonstrated readily in material obtained from dogs sacrificed $2,24,48,72$, or $96 \mathrm{hr}$. after the initial radiation. In the irradiated dogs the secretory crypt epithelium of the small intestine autolyzes first and the epithelium of the villi last, while the reverse is true in the normal control small intestine. The colon shows little change and the stomach no demonstrable changes in autolysis under like conditions. The kidney likewise is unaffected. The spleen, lymph glands, liver, and the pancreas show a moderate increase in the speed of autolysis only in tissues taken from irradiated animals within $48 \mathrm{hr}$. of the initial exposure. What the significance of this disturbance of cell ferments in the intestinal mucosa may be, one cannot pretend to say. At least these observations strengthen one's confidence in the profound functional disturbance of this important intestinal epithelium - a disturbance which it is believed is responsible for the clinical abnormalities and fatal intoxication occurring in these animals.

Martin and Rogers (208) studied the chronic lesions in the small intestines of dogs receiving various amounts of dosage over the abdomen (with approximately $100 \mathrm{kv}$.). The animals developed ulcerated areas when small doses were given, which were followed by cachexia and death in 2 or 3 weeks. Damage to a single isolated loop resulted in a more slowly progressive cachexia with exitus in 2 months with intestinal ulceration and occasionally obstruction. The fat absorption was inhibited and the mucous secretion was increased.

Further work with chronic or acute lesions in the small intestines may lead to an explanation of the intoxication which occurs from the acute injury, as pointed out by some worker's (371), for this is apparently closely related to the intoxication found in intestinal obstruction and the clinical disturbance noted after intensive irradiation of patients. 
Mottram and Kingsbury (245) suggest that following the injury of the intestine by radium radiation there is an interference in the production of mucus by the mucosa which precedes the necrosis of the epithelial cells. They found that an invasion of the tissue of the mucosa by bacteria follows the production of the desquamation already referred to. They believe that the thrombopenia which they could produce was a secondary effect, along with the presence of the bacteria in the blood, to the breaking down of the epithelial barrier of the intestine against bacterial invasion. Their experiments probably involve severe bone-marrow injury as well as intestinal injury and they were studying the combined effects of these two injuries.

Further studies on the chronie degenerative lesions might give information concerning the mechanism of absorption and secretion, provided the proper biochemical method could be set up. The same is true of similar lesions produced in the stomach and colon. The effect of radiation from radium administered from outside the body is essentially the same as that of roentgen radiation.

From clinical observations in the literature it is probable that the human small intestine is likewise sensitive to damage (16) and by the same dose (i.e., some injury may be produced by less than the human erythema dose). Sanders (317) reports stenosis of the small intestine in patients receiving radiation over the abdomen one-half year previously. The fatal intoxication following irradiation over the abdomen is dependent upon a rather critical dosage, for with a less severe reaction (i.e., from less dosage) the result is that of acute partial injury and destruction of some of the same crypt cells followed by various stages of degeneration and repair. The repair is characterized by the proliferation of connective tissue and blood-vessel capillaries into the fibrin-rich covering elinging to the denuded surface and the regeneration and rapid growth of the cell remnants, apparently from the crypt-cell group. The repaired surface is apt to contain abnormal and degenerate cells. There is frequently a fair number of mueus-secreting cells in addition. The reviewer is inclined to believe that the repaired portion, since it is covered by abnormal cells, does not permit normal digestion and absorption to occur. In extreme cases this may be almost nonexistent. This would account for the survival of the experimental animals past the acute stages, and explain the continued loss of weight and oceasionally exitus from starvation, after a considerable period of time if the lesion is extensive enough. The placing of radium within the bowel, especially within isolated loops, or the irradiation from without of isolated loops, might be a suitable method of determining how these destructive agents bring about such specific and localized disturbances of function.

On the injection of radioactive substances such as thorium $\mathrm{X}$, or radium chloride, the small intestine is damaged by the radiation given 
off as the material is excreted into the intestine (187). Usually this damage is of the chronic type because of the low dosage involved. Other parts of the body, of eourse, are subjected to irradiation from these substances, but changes in other organs will be diseussed in their proper place.

Colon.-While no great amount of work has been done directly on the colon, the experiments deseribed by many workers (Regaud, 291; Martin et al., 207; Warren et al., 371) show that it is more resistant than the small intestine, probably about equal in sensitivity to the stomach. The predominance of mucus-secreting cells in damaged areas would suggest that this type of cell is more resistant than the other types. This finding compares favorably with observations on similar cells of the stomach and small intestines. The mucosa of the rectum has essentially the same sensitivity characteristics as the mucosa of the mouth.

Bladder.-The bladder, being lined with pavement epithelium, would be expected to have about the same relative sensitivity as the skin. This similarity seems to be borne out by clinical observations following the application of radium. The acute experiments on dogs seem to support this belief, though no work has been done direetly upon the bladder. In experiments where the animal has been given generalized irradiation, the bladder shows no acute changes, though it is possible that neither the dosage nor the time intervals used were within the proper limits. The same is probably true of the rest of the genitourinary tract not including the kidney, though there is little or no experimental work to suggest this. Martin and Rogers (209) found that $53 \mathrm{mg}$. hr. of radium applied in steel needles alongside the ureter in dogs produced a partial stricture, while $75 \mathrm{mg}$. hr. produced complete stricture.

Pancreas.-In the acute experiments dealing with the effects upon the intestinal traet all the workers agree that the pancreas is not damaged by doses and time intervals within the scope of these experiments. There is no direet experimental evidence that the pancreas can be damaged by the irradiation in situ, without producing severe and eventually fatal damage to the small intestine, except for a diffuse fibrosis noted by Doub (et al., 83, 84) in 2 dogs out of a very large number exposed in the kidney region. The sugar metabolism was not disturbed. Orndorf, Farrel, and Ivy $(256,257)$ studied the effeets of $1 / 10,1 / 2$, and 1 human erythema dose on the function of a piece of pancreas transplanted under the manmary gland of the dog. They used $150 \mathrm{kv}$. (effective wavelength of $0.181 \AA$ ). One-tenth erythema dose increased the concentration of lipase and trypsin but did not change the quantity. One-half human erythema dose increased the quantity and total output of ferments. One erythema dose decreased the quantity of ferment and total 
output for 2 weeks after exposure. One animal returned to normal 47 days after exposure. They conclude that the pancreas may be temporarily injured by one erythema dose, its power to regenerate being able to compensate for this.

Liver.-There is a controversy concerning the effects of roentgen and gamma radiations on the liver. Many investigators find no evidence of histological change (134, 207-209, 291, 369-377), while others report injuries of various kinds. Pohle and Bunting (277) describe swelling and atrophy of the cells, a reaction which occurs in two cycles, somewhat similar to the rhythm noted in the skin erythema. In some instances (83) the liver may be reduced in weight and show extensive fatty infiltration, some increase of fibrous tissue, and a loss of orderly cell arrangement. Three human cases (38) showed injury to the bile ducts, with vacuolation, swelling, and necrosis of the epithelial cells, followed by a slow and atypical regeneration. Hartock and Israeleski (138) review the literature on this subject, and present evidence showing that the secretion of bile in the frog is diminished by doses of $500 \mathrm{r}$ or more. The effect lasts at least 3 weeks. Russ and Scott $(310,312)$ implanted radon seeds in the liver of a rat and found that those cells which were intensely irradiated were less injured than those which received less radiation. Furthermore, cells near the blood vessels were less injured than those at some distance. Chronic changes in the liver are rarely observed because either the subject succumbs to massive destruction from large doses, or the regeneration after smaller doses is so rapid and complete as to leave little or no residual $(59,83)$.

The nuclear damage which has been reported (204) may not be a consequence of irradiation. The livers of rats and rabbits kept under laboratory conditions are apt to show occasional pathological variations; furthermore the dosage used is comparable to that used by others who found no histological changes under similar experimental conditions. There is no doubt that transient anatomical or histological and functional damage may be produced by radiation (83). The best evidence of functional effects is presented by means of bile fistula experiments (337). There is considerable evidence of rapid recovery of the liver function. But if sufficient radiation is given repeatedly, in the proper sequence and dosage, extensive irreparable damage might be obtained. A comprehensive, well correlated study of the effects of roentgen rays on the liver is needed.

Gall Bladder.-Brahms and Dornbacher (27) in irradiating the liver region of the dogs with relatively soft irradiation $(65 \mathrm{kv}$.) found both acute and chronic changes in the gall-bladder wall consisting of hemorrhage, edema, round-cell infiltration, fibrous-tissue hyperplasia, and occasionally necrosis of the epithelium. These lesions were obtained without damage to the duodenal and pyloric mucosa, and they suggest that the 
gall bladder is more sensitive than the gastro-intestinal tract. This has not been reported by any other workers and has not been within the reviewer's experience. The illustrations published by Brahms and Dornbacker show in some cases, thickened, infiltrated gall-bladder walls suggesting chronic inflammatory changes, rather than changes typical of damage due to irradiation. This possibility should be eliminated before it is accepted that the gall bladder is very sensitive to radiation.

\section{LUNGS AND HEART}

Lungs.-It is the opinion of the reviewer that the structures within the lungs are relatively resistant to irradiation. The stroma which is composed mainly of connective tissue and blood and lymph capillaries is not very sensitive to ordinary "therapeutic" dosage, and there is very little evidence to support the proposition that the bronchial-alveolar epithelium is very sensitive, i.e., more so than the skin. The pleura must likewise be quite resistant, in fact almost as resistant as the skin. Dosage above the human erythema dose either as a single massive dose or as fractional dosage, or where the lung is struck by converging ("cross fire") beams of high dosage, may well cause damage (70, 73, 94, 131, 205). Here, too, the damage may be related to the most resistant cells of the organ. The blood-vessel endothelium is apparently less severely damaged than the bronchial epithelium, which may swell and even necrose (Ludin et al., 205). In this state secondary infection is prone to happen in experimental animals and presumably in human cases. The pleural inflammation may be noted along with that of the skin and lung parenchyma. The mediastinal structures with the exception of the thymus and lymph nodes react about the same as the pleura to equivalent dosage. Caution should be used in interpreting mild changes found in the lungs because of the common finding of some pneumonitis in ordinary animals kept under laboratory conditions $(403,404)$. Capillary damage, edema, evidence of injury to the bronchial and alveolar epithelium, localized emphysema or atelectasis may be found without and with clinical evidence of acute respiratory disease (62). Such changes may be rather uniformly distributed throughout the animal colony. Changes such as those described by the various workers $(204,205,403,404)$ following the irradiation of the animal with very large doses are probably specific changes brought about by irradiation. From the biological standpoint the lungs are fairly resistant to irradiation. From the therapeutic aspect, in the face of crossfire and extensive fractional dosage procedures, the lung may suffer extensive damage and even be severely enough injured to cause death. Human cases under treatment by radiation, however, cannot be freed from the suspicion that the amount of neoplastic involvement of the lung substance and pleura was responsible to a 
great extent for the fibrosis and other changes reported $(70,73,94,101$, $131,238,356$ ).

Blood Pressure.-There is little or no evidence that roentgen rays induce changes in blood pressure. Stephens and Flory (344) irradiated unanesthetized rabbits with large doses and failed to find any alteration either in blood pressure, in the respiratory rate, or in the size of the spleen or other abdominal viscera which they viewed through an abdominal window. This is also the opinion of Desjardins and Marquid (70) who have made an extensive study of this subject. When human patients with tumors are irradiated, a fall in blood pressure, lasting from 1-35 days may occur (263). But in all probability this reaction is not due directly to the radiations but rather to the disintegration products of the cells destroyed by them. The results of Swann (345), which appeared to indicate a fall in pressure following exposure, may have been due to currents set up in the apparatus by the roentgen equipment.

Heart Muscle.-Animal experiments indicate that the specific radiosensitiveness of the heart is relatively low, probably near the tolerance of smooth muscle and blood-vessel muscle, and much lower than that of many other structures. Doses of roentgen rays even beyond the amounts usually employed in the treatment of human patients probably have no effect on the heart. Experiments with rabbits indicate that single doses of about $600 \mathrm{r}$ produce no definite change in the size of the heart, in the histology of the myocardium, or in the nervous mechanism $(111,114,127$, $332,349,378)$. The same is true when the radiation is given by the simple fractional or protracted fractional method, with a total dose of $7000 \mathrm{r}$ (403). Even 5 months after treatment no sign of injury is found. Kawashima (172) was unable to find any changes in the contractile rate or power of isolated frog heart muscle after intense roentgen radiation or immersion in radioactive Ringer's solution. Desjardins (73), in summarizing the clinical reports, finds a few instances of apparent injury from radiation but concludes that such effects must be ascribed to indirect factors, such as injury to the pleura and lungs or to the circulation of toxic products induced by the rays in other organs.

The heart is not entirely insensitive to radiations and may be injured by doses of sufficient strength. The damage appears in the blood vessels, in the muscle cells, and in the conductive system. The early effects of large doses and the late effects of small doses on the blood vessels of the heart consist of an initial engorgement and a later necrosis followed by a rupture of the wall and consequent hemorrhage into the surrounding tissue (26). A thickening of the walls and even an obliteration of the lumen are also reported (136). Fedder and Kellner (99) find irreparable damage to the nuclei of the cells.

Changes in the muscle are described by Warthin and Pohle (379) who employed multiple exposures. The fibers present a rather "wooly" 
appearance throughout, the cross striations are less clear and distinct than in normal tissue, and the staining properties of both protoplasm and museles are poor. Fibrosis is frequent, and peculiar in that it is diffuse and in fine strands between the individual muscle fibers. In addition, there is a large amount of loose areolar tissue present and the tissues are edematous. In further experiments on rats (380) the precordium was exposed to beams of two different wave-lengths $(0.19$ and $0.34 \AA$ ). The doses varied from 500 to $1000 \mathrm{r}$. No clinical symptoms could be observed which might indicate a heart injury. The only gross pathological change consisted of a dilatation of the right ventricle. Mieroscopically, Zenker's necrosis in various degrees could be demonstrated, and an increase in stroma nuclei with small foci of lymphocytes. From these results it appears that the tolerance dose for the myocardium of adult rats is about $500 \mathrm{r}$, effective in the heart. Werthmann (385) exposed rabbits to repeated doses of $500 \mathrm{r}$ each. The total dose varied from 8500 to $21,500 \mathrm{r}$, the exposures extending over long periods. $\mathrm{He}$ reports an atrophy of the muscle fibers with fracturing, empty sarcolemma cells, simple nuclear degeneration, and changes in the connective tissue with perivascular fibrosis and sclerosis. The sarcoplasm may show some coagulation and necrosis (99). Dilation of the heart to two or three times its normal size was found by Hartmann (136). It was confined for the most part to the right auricle and right ventricle, the former being hemorrhagic and its muscle replaced by areas of hyaline degeneration. This did not occur in the ventricular walls.

The effects of radiations on the conductive system of the sheep heart have been studied by Hartmann (136). "Microscopically, the larger and smaller branches of the auriculoventricular bundle are well preserved when compared with the damage to the musele proper. The larger branches showed only thinning of the fibrils at the margin of the cells with vacuolation and distortion of the nuelei, while some of the smaller branches which lie in areas where the muscle stains poorly and is infiltrated by leucocytes, show individual cells distorted, vacuolated, poorly stained, and with shriveled nuclei. The infiltration, however, does not extend into the branches of the bundle and in no case was hemorrhage or complete destruetion of the bundle found."

When the muscle power of the heart is weakened either through changes in blood supply or direct damage of the muscle cells or conduction system, the changes in any of the hydrodynamics of the pulmonary circulation elsewhere might well be reflected in extensive cardiac dysfunction. Any disturbance of the conduction system would probably upset the rhythm, in certain cases at least. The electrocardiographic changes may be explained by changes in the conduction system or shifts in the shape of the heart, due to dilation, hemorrhage into the muscle, etc. Such changes are only produced with high dosage. 


\section{KIDNEY}

There is considerable controversy over changes which might occur in the kidney following irradiation. In general, the experimental results with animals indicate that there is no definite kidney damage to be noted histologically following doses of the order of human epilation doses in the animals (reviewed by Warren, 381; and Desjardins, 72, 74). What changes are found after such dosage are usually functional and transient $(147,224)$. Experimental animals, especially dogs, often show spontaneous changes in the kidney which might mask incipient changes due to radiation (111). Other workers (136, 137, 255, 256, 388, 389, 404) have studied changes produced in the kidney by irradiation after transplantation or some other method of delivery of the kidney directly into the field of irradiation. These experiments allow of a great increase in dosage to the kidney without the probability of damage to nearby structures, especially the intestines, as well as the skin, for if the kidney is left in situ, very large doses, depending upon the size of the animal (3000 to $5000 \mathrm{r}$ or more), are necessary to damage its substance. Large doses cause acute and chronic changes of various degrees with destruction of blood vessels, glomeruli, and tubules, with generalized sclerosis of the kidney, a condition which gradually results in secondary changes such as hypertension and cardiac hypertrophy and functional disturbances such as acidosis, nitrogen retention, polyuria, edema, and all the changes secondary to such dysfunction which might be expected to accompany the histological picture of extensive damage and resultant fibrosis.

Alterations in the vessel walls in the kidney are found resembling in all gradations those in the capillaries of the skin (111). The vessels may become sclerotic and thickened and even closed. In the glomeruli they are often hyalinized and atrophic, though occasional vessels seem to escape damage.

Swelling of the renal epithelium, particularly in the tubules, and occasionally hemorrhage into the interstitial tissues, occur immediately after the irradiation (80). Separation from the basement membrane is then noted, and later cell destruction or atrophy occurs (with casts in the urinc). There is finally complete necrosis of the tubules, followed by inflammatory cell infiltration and fibrosis and later shrinkage of the kidney. In this stage the glomeruli may be surrounded by scar tissue and very well preserved, but the tubules do not regenerate $(388,389)$ and are replaced by scar tissue $(136,137)$. Radioactive materials (thorium $\mathrm{X}$ ) placed in the kidney substance produce local damage to all nearby structures because of the high dosage (246).

In general, the kidney as a whole is probably as resistant as the blood resscls in the kidney or elsewhere in the body. Specific damage to renal epithelium is of the same dosage level as that to blood vessels. It is hardly likely that sufficient radiation may reach the kidney in situ 
under ordinary therapeutic usage in sufficient quantity to cause damage, unless very high dosage is used. Transplantation of the kidney or its delivery through an incision for the purpose of irradiation offers the simplest method of producing changes which simulate those due to chronic nephritis, without damage to other organs. The method is cumbersome, but the procedure can be used in the study of this type of nephritis.

\section{ADRENALS}

These glands have been shown to be highly resistant, and very strong doses must be given to cause the changes which oceur chiefly in the cortical portion $(71,362,369)$. The complete disappearance of the medullary portion with extensive fibrosis, after direct irradiation, has been reported (210), but Doub, Bollinger, and Hartmann (83, 84) using large doses found only chronic changes, consisting of a thickened capsule, an increase in the lipoid content of the zona glomerulosa, and moderate fibrosis of the cortex. No demonstrable adrenal insufficiency was present. They believe that marked acute damage may occur, but this is not permanent because of the great regenerative power of the gland. Frey found no change whatever except for transient hyperemia and retarded cell division (107). He used only male animals, because in females the unavoidable irradiation of the ovaries introduces complications. He remarks that "the statements in the literature as to the direct damage caused by roentgen rays and radium to suprarenals and the great sensitivity of these are not according to fact; it is a question of an indirect and not any direct, specific, or elective effect of the rays."

This subject is of considerable interest for future research because of the physiological studies which might be made if it were possible to damage certain portions specifically without causing specifie damage to other parts. Great technical handicaps must be orercome because of the well-known difficulties with which any physiological study of the adrenals is faced. Much of the older work is of doubtful value.

\section{EYE}

Roentgen, in 1897 , reported that the new rays that he had discovered had a stimulating effect on the retina. The sensation of light, when the eye is irradiated directly, has been attributed to a direct stimulative action on the retina or to the production of fluorescence $(302,346)$.

The reactions in the cornea show as plain epithelial damage and a fine stippling 5 days after the irradiation, when the lids and sclera are quite swollen and a slimy secretion is present on the sclera (168). The stipling on the surface soon becomes confluent so that great ulcerative defects invading the corneal substance appear, and a keratosis follows. The iris, the lens, and the background showed nothing pathological in this stage. Larger doses produce on the cornea a confluent ulceration, with the development later of a definite keratitis, lasting about 6 months 
with final healing ( 9 months). After a single irradiation of 250 per cent of a human erythema dose a 9 months' observation shows the corneal epithelium disturbed in regularity and order of the cells and their staining power in the different layers. There is a degeneration of the corneal cells and a beginning degeneration of the inner horny layer and the normal content of the outer layer is diminished. The retina plainly shows (from a single dose) ganglion-cell changes due to definite irradiation damage.

Cremer (54) describes a cataract with acute inflammation of the cornea in a child as a result of irradiation, following a dosage which did produce similar damage to an animal. In the rabbit, cataract is always caused by an epilation dose, but this does not occur with such constancy in man. The lens changes do not appear until a dosage up to $1340 \mathrm{r}$, or 270 per cent of human skin dose is applied (302). If the maximum safe dose for the lens of the rabbit is taken as 100 per cent (or 180 per cent human erythema dose), for the cornea and conjunctiva it is 200 per cent, for retina and ganglion cells 100 per cent, for the conjunctiva 200 per cent. The lens is more sensitive to permanent damage than the hair papillae of the lid $(168,301,302)$. The dosage necessary to produce chronic damage is not so great as that producing acute damage, provided that sufficient length of time is allowed to pass for the chronic changes to occur $(301,302)$.

Desjardins (72) has very carefully reviewed the experimental work done on the eye. He points out that the conjunctiva and the eyelids have about the same sensitivity as the skin and the resultant changes, while they are perhaps slightly more severe, pass through the same cycle of latent period, injection of vessel, loss of hair, inflammation, and ulceration. The cornea, iris, ciliary bodies, and retina are increasingly more resistant in the order named. Changes may occur in the capillaries in any portion of the eye. These are identical with changes described for the capillaries elsewhere (i.e., the skin).

The painful conjunctivitis and photophobia following exposure of the human eye to ultra-violet radiation are well known. Verhoeff and Bell (361) have done the best work on the effects of ultra-violet radiation on the eye, showing that in rabbits destructive changes can be brought about in the retina, as well as in the cornea and the lens. There is considerable discussion about the effects of various wave-lengths and whether or not the effects are primarily due to ultra-violet or infra-red after absorption $(156,191,218)$.

\section{EAR}

Desjardins (72) has pointed out that very little work has been done on the effect of radiation upon the ear. Most of the data reported concerned cases in which abnormal conditions, either surgical laceration 
or mechanical disturbances from the application of the radium, confused the issue so much that very little definite data concerning the effects upon the normal ear systems are available. Girden and Culler (117) gave various amounts of radiation at 50 to $85 \mathrm{kv}$. with various filters to dogs over the ear; the doses varied from $400 \mathrm{r}$ in 18 weeks up to $11,162 \mathrm{r}$ in 25 weeks. They obtained severe damage to the skin and an anemia. With very careful testing they seemed to show an initial rise in auditory acuity following small doses, but this lasted 2 to 3 weeks only. Deafness was not produced. The experiments may be criticized by the suggestion that the dosage was spread over too long a period of time and the conclusion may be drawn that huge amounts are necessary to bring about changes, partly because of the position of the ear within the bony structure and partly because of the difficulty in delivering a high dosage with this low voltage to the auditory mechanism. It is very likely that if this problem is investigated it will be possible to show damage to all parts of the structure of the inner ear. The musele attached to the drum, the blood vessels, and the nerves are probably as resistant to radiation effects here as elsewhere unless the proper depth dose is administered.

\section{NERVE TISSUE}

Brain.-Lyman, Kupalov, and Scholz (206), in the best review of the German and Russian literature (cf. 215, 288, 401) find general agreement that roentgen irradiation in therapeutic doses eauses no damage to the adult central nervous system or to its function. Intensive irradiation may cause changes in blood vessels and perhaps temporarily reduce the secretion of the spinal fluid (68). While the radiation in 18 to 20 human erythema doses may alter the function of the central nervous system, this does not seem to be due to primary action upon the cells of the cortex (206). The excitability of the cortical cells may be lowered, as determined by conditioned reflexes, but this seems to be secondary to changes in the blood supply. The irradiation, even when directed at a single area, causes a diffuse physiological and pathological effeet, involving the subeortex as well as the cortex and probably also the vegetative nervous system. Histological studies show diffusely seattered degenerative change in the precapillaries and capillaries of the brain within 6 weeks. One dog, allowed to live 6 months, exhibited signs of diffuse damage to the functions of the cortex and subcortex, i.e., ataxia, trembling, and weakness. Histological studies showed advanced degenerative changes with hyalinization and obliteration of the arterioles and several extensive areas of necrosis in the brain. Direct injury to the nerve parenchyma by irradiation is not probable in view of the focal destruction of fibers in the chiasm and nature of the diffuse degenerative changes which vary in different places. Furthermore, the lesions were no less 
prominent throughout the frontal region, which was not irradiated. The main injury thus was related to the blood vessels, even those of considerable size.

Bagg $(12,90)$ showed a focal destruction and occasionally cyst formation produced in the brain by the implantation of radon in glass capillaries. Cairns and Fulton (33) placed radon seed screened with $3 \mathrm{~mm}$. platinum beside the spinal cord and within the dura on the dorsal surface in the lower dorsal region. Doses varying from 9.8 to $31.3 \mathrm{mc}$. caused complete paraplegia within a few days in cats.

Pendergrass et al (265) report that after exposure of the brain and cord of dogs to $1000 \mathrm{mg}$. hr. of radium, an extensive edema of the irradiated tissues is produced with severe symptoms, suggesting the production of considerable damage and elaboration of connective tissue. This is somewhat dependent upon the region irradiated.

Peripheral Nervous System.- Swann (345) thought that the sympathetic nervous system was more sensitive after short exposure and depressed after long exposure, though his experiments are open to serious objections. Langer (190) reviews the effect of roentgen rays on the autonomic nervous system; he concludes that the irradiation has a depressing effect. Salinger and Thiel (315) irradiated in patients the superior cervical ganglion and ciliospinal center of one side. They report changes of pressure in the eyeball with protrusion of the bulb with later retraction and pupillary dilation. They posulated that roentgen rays had a definite influence on the vegetative nervous system. This has not been checked in animals and in view of the pathology present in such patients, the proof of this contention is still lacking in the reviewer's opinion (for bibliography see 41, 128, 206, 236). Regaud (292), Warren (371, 372) and others have noted the presence of apparently intact ganglion cells and plexus cells in various organs severely damaged by irradiation, especially stomach and intestine.

Where changes in growth have been obtained, this is best explained by the possibility of producing damage to the special glands connected with the central nervous system, especially the pituitary.

Del Buono (67) irradiated the pituitary region in rabbits and thought he detected slight changes in blood pressure with return to normal in 24 $\mathrm{hr}$. There were also a leucocytosis and hyperglycemia, but in general the amounts of change found are within the experimental error when rabbits are used.

The excitation of pain in the end organs in the skin when the latter are damaged does not necessarily indicate that the end organs themselves or the nerve trunks have been directly damaged by the radiation, but they are secondary indicators of the damage produced in the local structures. There are acute changes in the skin following intensive irradiation which are accompanied by pain only during the height of the skin 
reaction. The majority of the painful skin lesions reported are of the chronic, degenerative type. In areas of severely damaged skin where the production of severe pain is of such great chronicity as that described in radiologists and patients having roentgen ulcers, the mechanism is probably one of scarring and contracture of connective-tissue around nerve endings and thus related to damage of the connective-tissue cells rather than direct damage to the nerve trunk or nerve endings themselves (78).

It should be pointed out that the symptoms resulting from changes brought about in the central nervous system by irradiation will vary in accordance with the location of the lesions produced. While it is reasonable to expect that there will be certain variations in sensitivity of the various cell types which go to make up the brain and cord and ganglia, the symptomatology may be generalized rather than specific. The skull diminishes appreciably the effective dosage delivered to the brain substance. It should be possible to bring about disturbances in the spinal fluid mechanism through chronic damage of the blood vessels of the choroid plexus wherein changes might be produced, such as those brought about in capillaries elsewhere (kidney, skin, heart, etc.). It is likely that further studies, patterned after the experiments of Lyman et al (206), though complicated in execution, would yield considerable information concerning minor changes in brain cells which may be of transitory or of permanent character. Conditioned animals should be used for such experiments. Reports of changes in the vegetative nervous system are in the reviewer's opinion as yet controversial. This field is open to research but should be investigated only by those well trained in studying this system from other points of view.

\section{BONE AND CARTILAGE}

Bone and cartilage (see also section on Teeth) are resistant to roentgen radiation. It is common therapeutic knowledge, however, that cartilage may be damaged by very intensive irradiation for carcinoma, especially in the neck region where the structures of the larynx and trachea may be severely damaged and the cartilage may slough whenever intensive cross-fire dosage is used (cf. 95, 397).

Phemister (269) inserted a 12.5 -mg. needle of radium into the lateral surface of the condyle region of the femur of the dog for $18 \mathrm{hr}$. There was necrosis of the bone and cartilage with disintegration of the marrow. White fibrous connective tissue with very few cells, filled the marrow spaces; cartilage was partially replaced by fibrocartilage. Instead of the formation of a sequestrum, there was a very slow creeping replacement of the necrotic bone by new bone, unless infected. There was considerable destruction of the surrounding soft tissue which contributed to the delay in healing. Placing $37.5 \mathrm{mg}$. near the shaft of the femur for $20 \mathrm{hr}$. 
apparently killed the bone immediately around the irradiated area with slow attempt at repair at the periphery. The periosteum, cortex, and medullary contents were killed by the radium radiation. The dead bone remained attached to the living bone and survived for 250 days. Fractures occurred in some of his animals with overriding. If the bone is not used for weight bearing, the necrotic bone will be sequestrated and very slowly absorbed.

Moderate doses of roentgen radiation caused a decrease in the quantity of the callus of recently fractured bone in rabbits $(109,110)$ and seemed to increase the speed of healing. Large doses caused no callus to appear for 2 weeks and then there was an increase in the amount of callus over the normal, but the total time for healing was greatly increased. Others (247) found no change in the healing of fractured bone.

Ewing (95) has described a form of chronic osteitis following irradiation, a proliferative process which is probably condary to harm done rather than a primary stimulative reaction. Bone close to the skin when irradiated heavily may develop a productive osteitis with hard brittle bone. The periosteum being thickened and hyaline, flakes of necrotic bone appear, and the shaft is greatly increased in thickness. The bone marrow is deprived of its cells and is reduced to a mass of mucinous fat and connective tissue. It may be necessary to lay down entirely new bone, because to all intents and purposes the severely irradiated bone is dead. This is especially true of intensive irradiation of the jaw and ribs $(20,105)$. Similar sensitivity of bone to radiation has been noted by Martland (212) as the result of the deposit of radioactive substances in the bones of painters of luminous dials, in whom widespread necrosis of the jaw occurred. Here also, as is the case after roentgen irradiation, secondary bacterial invasion of bone injured by radiation is a factor. The injury to bone in dial painters is much more severe than has yet been described after roentgen irradiation, because the action of the deposited radium and mesothorium compounds is continuous over a period of years. Furthermore, alpha radiation as well as beta and gamma radiation has its source in intimate contact with the cells and irradiates them intensely. In rats, 60 per cent of the material injected was reclaimed from the mandibles (350). Such continuous action leads not only to necrosis of the shaft and increased calcification in the ends of the bones (350) but also to diffuse radiation osteitis and to injury to the hematopoietic system $(212,350,360)$. As still another possibility in the chain of evils caused by the deposition of radioactive materials in bone, Martland's (212) most recent contribution calls attention to the occurrence of osteogenic sarcoma of bone in 2 radium dial painters.

Teeth.-Leist (195) exposed the jaws of young rats to large doses of unfiltered radiation and found signs of definite injury to the odontoblasts 
which are especially sensitive. The dentine structures are destroyed, but the enamel is not much changed. Smaller doses bring about pulp atrophy. The further development of the teeth is retarded. In the dog, growth of the teeth may be stopped altogether and they may fall out. In such a case the periodontoid tissues, alveolar bone, and the bone marrow of the jaw are injured, and the development of the entire jaw retarded.

In humans even mature teeth may undergo atrophy and fall out on exposure to strong doses of roentgen rays or radium. Injury to the teeth appears to be due to the absence of saliva. Exposure of the pregnant uterus to radiation has been known to inhibit dental development in the child, but this is probably the result of interference with general growth (74).

\section{HEMATOPOIETIC SYSTEM}

A discussion of the influences of irradiation upon cellular elements of the blood must be divided up into a study of the various cells and the organs eoncerned with the production of these various elements (369).

The spleen and lymph nodes have long been known to contain large numbers of lymphocytes sensitive to radiation $(147,165,170,236,334)$. Akaiwa et al. (6) studied the changes in exeised popliteal lymph nodes of the rabbit at various intervals following one-third to 2 human skin erythema doses $(0.08 \AA)$. The destructive changes brought about were proportional to the dose. Within the first $12 \mathrm{hr}$. there is an increase in size of the lymph node with nuclear disintegration, especially of the lymphocytes, and decomposition of germinal centers with "cellular infiltration, congestion, and exudation." Mitotie figures were seen only following small doses but not after large doses. In the period between $12 \mathrm{hr}$. to 5 days after irradiation, there is phagocytosis and removal of débris. After 5 days the damaged areas are filled in with connectivetissue cells. This article and its illustrations are worth a careful perusal. The changes in the spleen resemble in every detail those noted above for the lymph node $(65,147,208,220,369,371,372)$. The spleen may be reduced to a stroma remnant showing nothing but eonnective tissue, reticulum, and blood vessels $(84,334)$ after intensive irradiation of either single massive or repeated dosage.

The extreme sensitivity of the lymphocytes has been shown by Murphy $(249,250)$ who was able with repeated very small doses of low-voltage roentgen radiation, to deplete the lymphocytes in laboratory animals without changing appreciably the numbers of other eirculating cells in the blood. The lymphocytes were depleted to their lowest level about $18 \mathrm{hr}$. after the last irradiation. The return to normal depended upon the amount of dosage in some cases requiring 1 to 2 months. The dosage used caused no appreciable change in the bone-marrow cells of these mice. 
There have been many recent studies of the circulating white blood cells of patients and workers $(81,125,143,178,180,234,243)$ showing that a leucopenia develops no matter what the method of irradiation or wave-length used, the same proportionate fall occurring after the protracted, fractional irradiation that occurs after the massive-dose irradiation or the use of Grenz rays $(112,113)$. Zwerg (404) irradiated a fold of skin on the back of rats and found that a leucopenia was produced from which the animal recovered within a week, suggesting that the effect was upon the circulating cells only. Irradiation $(600 \mathrm{r})$ of one of a parabiotic pair was followed by a similar drop and rise with a later fall in the white-blood-cell count of both animals. The changes are due to a relative fall in the lymphocytes and an apparent rise of polymorphonuclear cells in both animals. Injection of thorium $\mathrm{X}$ into mice, guinea pigs, and chickens showed similar inhibition (destruction) of lymphocytes and macrophages (364). In culture, this amount did not affect the fibroblasts but did damage the lymphocytes thus indicating even in vitro the greater sensitivity of the adult lymphocytes.

In vitro exposure of human neutrophile leucocytes (granulocytes) indicates that the chief immediate biological effect of irradiation with either roentgen or gamma rays or visible-light rays is the immobilization of the granulocytes, and structural changes followed by death do not occur until later $(186,365)$.

A few workers report a leucocytosis immediately after irradiation and apparently this is common in rabbits, but within $24 \mathrm{hr}$. there is a definite leucopenia in all animals and in the human being. The leucocytosis resulting from the injection of colloidal silver or turpentine abscesses afforded no protection against a terminal leucopenia following irradiation of the bones of dogs nor is the leucopenia affected by transfusion (334). Platelet disturbances have been reported by Lacassagne $(186,188$, see also 334$)$ in rabbits. Wright and Bullman (396) have reported a decrease in platelets in the cat after prolonged treatment. The number of circulating white blood cells in animals vary greatly from time to time. It is the reviewer's opinion that many laboratory animals are very unsatisfactory for such study, since considerable variation in the blood cells is apt to occur under laboratory conditions.

Many workers have failed to produce hemolysis of the red blood cells in vivo $(180,334)$ or in vitro $(81,180,334)$ or to change the response to hypertonic and hypotonic solution (fragility) after doses as high as 16 erythema doses $(180,334,338,348)$. Holthusen (158) was able to produce hemolysis in vivo and the production of methemoglobin in the circulating blood with 50 human erythema doses.

Goulston (129) reported in the human, rat, and fowl bloods an increase in the fragility of the red blood corpuscles after irradiation with $8400 \mathrm{mg}$. hr. of lightly filtered radium. There is an apparent difference 
between the effects upon the nucleated cells (more sensitive) and the non-nucleated cells. For cathode electron effects see Hausmann and Zakorsky (140), Langendorff et al. (189).

Kromeke (180) states that rabbits exposed over the whole body to 1 to 20 H.E.D., showed plainly a polycythemia, an increase sometimes as high as 28 per cent in the red blood cells after a latent period of 3 to 5 days. In some rabbits, the highest red-blood-cell count was reached on the 8th day, when there was an increase of about a million. This decreased later and in some rabbits anemia developed. The hemoglobin kept parallel to the red-blood-cell count, but the color index dropped somewhat in the longer experiments due to the young cells. The refractive index of the serum proteins usually decreased. There was usually a leucopenia which was more or less transitory. Nucleated red cells were not seen, nor were eosinophilic or basophilic cells ever seen in the smears of the blood. There was a normal count of polychromatophilic cells. Apparently the bone marrow had used up its reserve and was making new cells. This would account for the drop in hemoglobin and color index $(180,351)$. Some believe a stimulation of marrow cells may even be produced (Bucky and Guggenheimer, 32).

Bone Marrow.-The precursors of the cireulating blood cells in the marrow are sensitive to irradiation, perhaps even more so than the adult lymphocytes, because of the relatively smaller dose that may be given to them directly, i.e., through the bones $(291,334)$. The acute changes which occur in the hemopoietie system of dogs following total destruction of the bone marrow by $1700 \mathrm{r}$ over the skeleton with the intestines protected, produces an acute agranulocytosis, a sudden disappearance of platelets ( 7 to 8 days) and a sudden and fatal, diffuse, eapillary hemorrhage within a cireumseribed time interval ( 8 to 9 days after irradiation) (334). The elinical pieture is that of a normal active dog for 6 to 7 days, then suddenly anorexia and occasional vomiting develop but no severe intoxication. The circulating white-blood-cell counts decreased from 15,000 per $\mathrm{mm} .^{3}$ before irradiation to 25 to 200 (all polymorphonuclears and large mononuelear cells) on the sixth day and remained at the extremely low level of 25 to 100 per $\mathrm{mm}^{3}{ }^{3}$ up to exitus. Doubling the dosage or large transfusions did not change this sequence of events. Gross and mieroseopic hemorrhages were found in most tissues. The red blood corpuscles were well preserved throughout the experiment and showed no hemolysis or increased fragility. There were no nucleated forms, nor was there increase in reticulated forms. The platelets disappeared from blood smears the day before exitus. This was probably responsible for the diffuse hemorrhagie phenomena (383). Only an occasional megakaryoeyte was found in the spleen and none in the bone marrow (334). The bone marrow was depleted of all of its cellular contents except for the conneetive-tissue stroma, fat cells, blood vesscls 
and capillaries, and phagocytes. There were occasional islands of plasma cells and a few normoblasts. The substance of the spleen and lymph nodes was greatly reduced and the germinal centers were visible only as remnants. These organs contained a few round cells, many of which showed mitotic figures. Large phagocytes enclosing brown pigment and fragments of red blood cells were present in the marrow, lymph nodes, and spleen. The persistence of these phagocytes in the presence of total destruction of the lymphoid and myeloid systems suggests that they are either very resistant to radiation, which is hardly likely, or that they are formed after irradiation from local structures, probably from the capillary endothelium. The clotting power of the blood was not disturbed in these experiments. The hematocrit readings fell on the average 10 per cent during the course of the experiment, the greatest drop occurring within the last few days.

If one or more vertebrae were not included in the field of radiation, the animal recovered from the acute intoxication (334). In such cases the circulating leucocytes did not drop below 500 white blood cells per $\mathrm{mm} .{ }^{3}$ and platelets were present in the blood smears at all times. These animals slowly recovered from the leucopenia and survived. There was a total lack of tissue and white-blood-cell reaction to any intercurrent bacterial or acute respiratory infection, probably because of the leucopenia.

Localized irradiation of bone marrow or subtotal destruction of the marrow is followed by active regeneration $(36,240,335)$. Normally fatty marrow may become erythropoietic (180). The irradiated marrow cells (guinea pigs) showed changes quite early (335), consisting of degeneration and later complete destruction. These changes affected the myeloid cells as well as the erythrocytic cells. The former were more sensitive and regenerated less rapidly. Even after irradiation with small repeated doses, which caused an increase of red and white cells in the circulating blood, the histological findings in the bone marrow always showed degenerative changes. Unirradiated bone marrow at a distance from that irradiated showed changes in the morphology and distribution of the cells. The megakaryocytes showed extensive nuclear injury, the percentage of immature red cells was markedly increased, the red cells predominated over the white, and the polymorphonuclears were greatly decreased in number in the marrow, though if the doses were small both these and the myelocytes remained normal (335). Among the normal bone-marrow cells Casati (36) found that 50 days after irradiation (rabbits) round nuclei predominated, either with or without granules; there were only a few transition forms. There were also many cells which made up a part of the reticulum that contained granules similar to those of myelocytes and which might be interpreted as transition forms. They showed a tendency to develop toward the more differentiated normal forms. These changes occurred in various grada- 
tions, no matter what the dose used and what the bone irradiated. These changes were not in proportion to the changes in the circulating blood.

This procedure, of irradiating only a part of the marrow and studying the stimulus or compensatory changes brought about in unirradiated areas, should yield some valuable information regarding the response of the bone marrow to the sudden needs of blood regeneration. Such work should be done on dogs which have a stable hematopoietic system. Further work with total destruction of the bone marrow should yield very interesting information concerning the life cycle of the components of the bone marrow or the myeloid series, but of course the experiments will be complicated by the tendency of the animal to bleed, owing to the thrombocytopenia. It is impossible at present to tell whether the irradiated normoblasts in the marrow have matured into adult red blood corpuscles or have been destroyed with a few surviving in statu quo. Their precursors are evidently destroyed readily by the dosage used. Total destruction of marrow activity with essentially similar results may likewise be brought about by injection of sufficient amounts of radioactive materials $(30,49,186,188,326,350,360,383)$.

The coagulating power of the blood is not affected except in therapeutic cases irradiated for castration or hyperthyroidism, where it fluctuated in amount (200). Pagniez, Rarina, and Solomon (261) were unable to show any changes in the coagulation time of the blood in vitro, but the coagulation time was rapidly accelerated in most of their human cases of blood dyscrasia with irradiation of between 100 to $500 \mathrm{r}$. There were a few reports in which no reaction appeared $(23,308,334)$.

Pohle (274) studied the reticulo-endothelial system of rabbits exposed to doses varying from 150 to $300 \mathrm{r}$ over the spleen or orer the whole body. He points out that there is considerable variation in the effects produced and believes that there is some increase of the speed of elimination of Congo red or trypan blue following these small doses $(300 \mathrm{r})$. Doubling the dose causes the changes to approach more nearly normal values. The significance of these experiments is not clear, yet the idea behind them is of merit. The use of graded dosage and a better method of demonstrating functioning endothelial phagocytes might be of value (cf. 399, 400).

Thorsness (352) irradiated (1/4 to $1 / 2$ erythema doses) the foreign-body reaction (aleuronate abscesses) in rabbits and found that the abscesses were reduced in size and the absorption of the particulate materials increased. Apparently there is an early degeneration of fibrin before the tissues have established this function. There is destruction of lymphocytes and macrophages, and hyalinization and calcification in the areas, the true destructive changes of these cells being evident one day after irradiation exposure (see also 217). 
There has been considerable interest in the effect of radioactive materials introduced directly into the body. Perhaps the most complete experimental work is that of Thomas and Bruner (350) who injected into young rats 40 to $60 \mu \mathrm{g}$. of radium chloride over 126 to 191 days. This produced a marked secondary anemia and caused the death of some rats. The bone marrow was aplastic except for an area of hyperplasia in the central two-thirds of the shafts of the long bones. Growth was retarded and there was a decrease of the calcification in the central portion of all bones and calcification was concentrated near the joints. The lymphocytes in the spleen and lymph nodes had been destroyed and there seemed to be an increase in the number of lymphoblasts, plasma cells, and giant cells. Extramedullary myelopoiesis was marked in the spleen and lymph nodes and present to a slight extent in the liver. There was degeneration of many of the cells in the adrenals. There was some noninflammatory degeneration and fibrosis of the myocardium of a few rats with shrinkage of the liver. There was a parenchymatous nephritis with numerous areas of calcification in the kidney. Various stages of degeneration in the tubules of the testes were noted. Normal ovarian follicles were noted and the destruction of embryos was occasionally found. Frequently the periosteum at the costochondral junctions was involved by hyperplastic changes and of ten both caries and repair were present. The thyroid, parathyroid, and pancreas showed no change. There was recovered 99 per cent of the radium from the bone, though 60 per cent of this was found in the mandibles (see Martland, 212). The kidneys and intestines contained the largest amounts present in soft tissues. Embryos contained 3.6 per cent. The radioactive material was deposited with the calcium at the knee joints and vertebrae. The lungs showed a patchy pneumonia as a terminal effect but no primary damage.

Valeeff (360) studied the changes in the blood following the injection of 0.5 to $1.0 \mathrm{mg}$. of thorium $\mathrm{X}$ intravenously in rabbits. He did not think there was a direct effect of the thorium $\mathrm{X}$ upon the erythrocytes, although there was a change in their sedimentation rate on the first and second days and continuous variations in the degree of leucopenia. The sedimentation rate, he believes, is apt to depend upon damage to other organs, especially the bone marrow (271), although he believes the capillary system is damaged, too, and there may be some effect from that. The leucopenia depends upon damage of the bone marrow; the lymphocytes and monocytes were thought to be slightly more resistant than marrow cells $(30,49,186,198,326,350,360,364,383)$. Barker and Schlundt (17) point out the long duration with which radium chloride may affect the human body when introduced into the circulation or by mouth, i.e., for periods of at least 5 years (314). 


\section{GONADAL SYSTEM}

Testis.-Feroux and Regaud (100) find that the sensitive cells in the testis are the basal cells and the spermatogonads. The adult sperms are apparently insensitive since they show no change following irradiation. This is borne out by fertility and in vitro motility tests on adult sperms in rabbits (8). Wigoder (387) found that in guinea pigs and rats sterilization occurred usually at $750 \mathrm{r}$. The latent period between irradiation and complete destruction could be reduced by higher doses. The spermatocytes were most sensitive, the spermatogonia more resistant. The abnormalities included the formation of abnormal cells (giant cells) the overgrowth of basal (Sertoli) cells, and a high percentage of residual and proliferating interstitial tissue. Complete sterility with very large doses could be produced in the rat within 7 days, which is a shorter period than that reported by other observers (100). Irradiation of live guinea-pig sperm with $2000 \mathrm{r}$ at $85 \mathrm{kv}$. caused no changes, but irradiation of the testis with doses up to $7000 \mathrm{r}$ caused the sperm of the killed animal to be more active and live longer than that of the control animal. This is certainly unexpected and contrary to the usual experimental results with other types of cells. Larger doses killed the animal. A review of these experiments seemed to show that the sperm from a control testis had precisely the same activity, so that it is the reviewer's opinion that these are normal variations and not specific radiation effects (cf. 162).

Adler (2) describes a decrease of the in vitro metabolism and an increase of aerobic and anaerobic glycolysis, appearing 24 hours and reaching a maximum about 34 days after intensive irradiation. At this later time a histological examination revealed degenerative changes in the nuclei of the sperm-producing cells. Lesions develop in the seminiferous tubules in a zone of about $0.6 \mathrm{~mm}$. depth after 20 to 30 sec. exposure of the rat testis to high voltage (200 to $250-\mathrm{kv}$. cathode "rays" or electrons (167). Shorter exposure showed less penetration. The spermatogonia, spermatocytes, and spermatids showed fragmentation and karyolysis of nuclei, pycnosis, and decreased amount of mitosis. The basal (Sertoli) cells are not very much affected nor were the interstitial cells. There was considerable damage to the scrotal skin with acute necrosis of the epidermal and hair-follicle epithelium and vascular damage. The collagenous fibrils of the corium were hyalinized and fused.

Ovary.-Gricouroff and Murray (130) give probably the most complete description of the changes brought about by rocntgen irradiation in ovogenesis. In a comparison of 295 mice of a highly in-bred strain, they used 100 for control study and irradiated the remaining 195 closcly related animals with various doses of roentgen rays. Many variations are described in the control ovaries. This side-by-side comparison of the variations common to unirradiated and irradiated ovaries is essential to 
examine the changes wrought by irradiation alone. Similar age groups must be compared to show that the follicular degeneration with zona pellucida remnants is different from similar changes found among control ovaries. A dose of $54 \mathrm{r}$ produced the first detectable change, one day after irradiation, within the structure of the primary follicles, in mice between 90 and 250 days of age. Further changes are irregular in occurrence up to 139 days after irradiation when various degrees of destruction are invariably present, although structural "sterility" is not uniformly obtained. Exposure of $150 \mathrm{r}$ or more produces structural sterility, the primary follicles being absent after 2 days and all follicular elements are absent by the forty-third day after irradiation. A dosage of $54 \mathrm{r}$ is sufficient to produce replacement of normal follicular elements by a common epithelioid or luteal-like structure. The replacement is complete, after doses of $150 \mathrm{r}$ or greater, within 43 days after irradiation. A dosage of $1800 \mathrm{r}$ is lethal for adult mice of this strain in 4 days after irradiation [by diffuse acute intestinal injury?]. The ovaries from mice exposed to this massive dose show marked follicular degeneration after one day. After 4 days, all follicular structures are found to be degenerating $(369,371$, also $46,86,104,171,177,179,211,214,251,253,262,384)$.

The tertiary follicles in rabbits (170 per cent human erythema dose, 299) were most sensitive, the ovule being affected first. The interstitial cells of the corpus luteum were the most resistant. The primary follicles are not functionally damaged by this dosage. Progeny from irradiated ovaries are less numerous and show late development (85). Levine (197) demonstrated that the human erythema dose produces sterility in female mice for 5 to 6 months (329), males for 2.5 to 3 months after irradiation. The hormones controlling sexual desire may not return for 6 months after the irradiation with doses 2.5 times the castration dose (329). Unchanged primary follicles in each ovary of the rabbit were present with dosages of $1500 \mathrm{mg}$.hr. of radium placed over the ovarian site on the back (1). There were no intact primary follicles with $2000 \mathrm{mg}$.hr. or greater.

\section{MISCELLANEOUS}

Cutler (55) implanted filtered and unfiltered radon tubes in a rabbit's muscle and found three zones of the reaction regardless of the filtration: (a) zone of complete necrosis, caseation; (b) partial necrosis of coagulation necrosis; (c) zone of hyperemia. This holds true for similar dosage in other tissues. Smooth and striated muscle is quite resistant to radiation but with sufficient dosage will become atrophic or necrotic and scarred (79). In some experiments following very high massive dosage with sloughing of the skin the subcutaneous tissues and muscles may become hard and almost cartilaginous in density and hardness. Much of it may become replaced by connective tissue. Secondary infection is 
common and indolent. Blood ressels tend to thrombose in such areas $(12,393)$.

Wierig (386) reports a late injury with atrophy of the breast structurc of a young girl (17 years of age) who had received intensive short-wave irradiations over the chest for mediastinal tumor. There were skin changes and atrophy of the thorax muscles.

The capsule of the thyroid in the dog is thickened only by dosages which would produce skin ulcers (366). There is some tendency to hyperplasia of the normal thyroid and parathyroid. Within several months after irradiation there is an increase in the connective-tissue stroma of the parathyroid with a slight decrease in the blood calcium. The dosage used ( 4 to 12 doses of $375 \mathrm{r}$ ) at 7 to 14-day intervals indicated that clinical dosage did not affect the parathyroid and has relatively little effect on normal thyroid tissue.

The hyperplasia of the thyroid gland which occurs in opossums on a high-protein (meat) diet was definitely decreased, but not entirely prevented, by the irradiation with $385 \mathrm{r}$, at weekly intervals for 4 to 12 doses (367).

Lenz (196) exposed the thymus glands of young rabbits to very small doses of 200-kv. radiation, i.e., one thirty-fifth H.E.D. and less without demonstrating any stimulating effect from these small doses but on the contrary found that the normal resolution occurs more rapidly after the exposure to this intensity (cf. 398).

These changes are hardly within the scope of this review because of the wide ramifications such a discussion would entail. Too of ten the results are obscured by the fact that the irradiation is directed into a tumor mass so that the results $(115,126,145,333,395)$ are of interest from the clinical standpoint but not indicative of normal tissue changes. Much of the work in animals is likewise difficult to correlate because of the absence of suitable standards for normal findings under controlled laboratory conditions in these animals. The type of animal uscd varies a great deal. The amount of dosage and the tissues irradiated must influence the result tremendously $(48,56,63,82,83,136,148,155,164,203,276$, $279,300,353,369)$. This is very seldom considered. All too frequently the chemical changes in the blood are not correlated with a careful study of the kind or the extent of injury produced (functional and anatomical) in the tissues irradiated. Much of the animal experimental work is done with too low dosage (H.E.D. or less) usually in the expectation of postulating similar observations (usually negative) upon human cases $(63,271,279)$. When obtained, the results are not directly comparable. Adler and associates $(2,3,4,5)$, for instance, exposcd rabbits to $1000 \mathrm{r}$ over the backs and obtained a decrease in the blood-sodium level of from 6 to 15 per cent. In ill patients treated with roentgen or radium radiation in their elinic no such changes were found. 
There is need for a careful, well-correlated study of the specific and nonspecific chemical changes brought about locally and generally by both specific and nonspecific damage done to cell structure by radiation. Ideally this might be done by the collaboration of a biochemist, a physiologist, a pathologist, and a radiologist, each well versed in his field.

\section{SUMMARY}

In General.-The specific effects upon organs and tissues of the various bands of radiation in the electromagnetic spectrum depend in general upon the physical character and properties of these radiations and the manner in which they are administered. The effects upon any given organ or part of an organ may be general or specific. The extent to which damage may occur is dependent upon the penetrating and destructive power of the given band of radiation. Any given effect is primarily due to direct damage to cells, and is relative to dosage, and extends over the entire range from minimal damage to immediate or ultimate death of the cell. In producing a specified damage to a given cell type, the total quantity of the dosage, the duration of the exposure and its intensity, and the intervals between partitions of dosage, if any, the rate of growth and variations in sensitivity of the cell as well as its specific sensitivity are all interrelated. Indirect or secondary damage, through injury to blood ressels or circulation of cell-disintegration products is possible. This is especially true of blood vessels because of the occurrence of late effects from wave-lengths shorter than $8 \AA$. Repair postulates various grades of abnormal cells developed from damaged survivors. If organ cells have been extensively destroyed, capillaries and fibroblasts may invade and fill up the vacant space. Late degenerative effects may follow either relatively small dosage or primary healing of acute injury.

Since damage is related to dosage of any particular band of radiation, the physical properties and especially the penetrating power determine the effects which may be produced. Thus ultra-violet radiation may be expected to affect only the more superficial structures while roentgen radiation should affect deeper structures. Much of the apparent resistance of an organ is probably a result of the screening effect of interposed tissues. The matter of the depth dose available to tissues has not been worked out for roentgen and radium radiation in regard to such a sensitivity index. In the bodies of small animals like the mouse and rat or in vitro tissue experiments the dosage is probably close to that delivered to the surface. In larger animals and especially in the bones the dosage may be quite different.

In Particular.-A sensitivity index can be constructed roughly, for roentgen radiation. Taking all factors into consideration one is impressed by the high relative sensitivity of the bone-marrow cells and intestinal crypt epithelium. It is difficult to determine whether these 
very sensitive tissues should be placed above or below the well known sensitivity of the lymphocyte and the lymphoid structures including the spleen, in evaluating their relative position in the scale. The gonadal cells, especially the immature forms, are quite sensitive to roentgen irradiation but less so than those tissues just mentioned. In a descending scale of sensitivity, beginning with a moderate sensitivity, we may list certain tissues in the following rough order: the thymus, the stomach, colon, and bladder epithelium; salivary epithelium, probably kidney epithelium, hair papillae, blood-vessel endothelium, fibroblasts, and young connective-tissue cells including collagenous fibrils. Next in order of sensitivity are: the mucosa of the mouth, esophagus, rectum, and vagina, the lung parenchyma, pleura, skin epithelium, and the structures of the eye. The liver and adrenal (cortex more than medulla) are thought by some to be relatively sensitive but to possess tremendous regenerative power-which obseures evidence of damage. This needs elarification. Next come the smooth, striated, and cardiac musculatures, eartilage, bone including osteoblasts, teeth, normoblasts, Sertoli's eells, and stroma cells of testes and ovary. There is some doubt concerning the relative sensitivity of the panereas. The most resistant cells may be listed in the following order: adult thyroid and parathyroid (?), adult pituitary, brain and nerve cells, nerve trunks and endings, tendons and joint capsules, i.e., adult specialized eonnective-tissue structures, adult sperm cells, and red blood cells. The red blood cells and adult sperm cells have resisted the highest dosage administered to body tissues.

\section{REFERENCES}

1. АвÉ, K. Tierexperimente zur Bestimmung der Kastrationsdose des Radiums. Strahlentherapie $16: 554-559.1924$.

2. AdLer, K. Die biologisehe Wirkung der kurzwelligen Strahlen auf den Stoffwechsel der Zelle. Strahlentherapie $36: 1-31.1930$.

3. Adler, K., and Margurite Adler. Der Einfluss der Röntgenbestrahlung auf den Natriumspiegel des Blutserums. Strahlentherapie 42:584-590. 1931.

4. Adler, K., and Margurite Adler. Caleium-und Kaliumstoffweehsel unter dem Einfluss der Röntgenstrahlen bei Kaninehen. Strahlentherapie 44: 481488. 1932 .

5. Adler, K., and O. Wiederhold. Caleium und Kaliumstoffweehsel unter dem Einfluss der Röntgenstrahlen bei Kaninchen. Strahlentherapie 44: 383-392. 1932.

6. AKAiwa, H., and M. TAKEshima. The reaction of lymphoid tissue to roentgen radiation. Amer. Jour. Roentg. and Rad. Ther. 24: 42-46. 1930.

7. Andrews, G. C., and C. B. Braestrup. Skin erythema dose in terms of roentgens in superficial therapy. Amer. Jour. Roentg. and Rad. Ther. 29: 663-666. 1933.

8. ASDELL, S. A., and S. L. WARREN. The effect of high voltage roentgen radiation (200 kv.) upon the fertility and motility of the sperm of the rabbit. Aner. Jour. Roentg. and Rad. Ther. 25: 81-84. 1931.

9. BAchem, A. Physieal and biological observations on skin reaction. Radiology 9: 241. 1927 . 
10. BaChem, A. Die Lichtdurchdringung der menschlichen Haut. Strahlentherapie $39: 30-56 . \quad 1930-31$.

11. Baensch, W., and R. Finster. Über die therapeutischen Anwendungsmöglichkeiten von Kathodenstrahlen. Strahlentherapie 33: 399-434. 1929.

12. BAGG, H. J. The action of buried tubes of radium emanation upon normal and neoplastic tissues. Amer. Jour. Roentg. 7 : 536-544. 1920.

13. BAGG, H. J., and C. R. Halter. Experimental studies on tissue reaction to radiation. Amer. Jour. Roentg. and Rad. Ther. 24:640-647. 1930.

14. BAGG, H. J., and C. R. HALTER. The relation of intensity of radium radiations to tissue reactions using a segment of a growing mouse tail as a biological indicator. Amer. Jour. of Roentg. and Rad. Ther. 27: 1-13. 1932.

15. BAGG, H. J., and C. R. Halter. Relative effects produced by $200 \mathrm{kv}$. roentgen radiation, $700 \mathrm{kv}$. roentgen radiation, and gamma rays. Amer. Jour. Roentg. and Rad. Ther. 29 : 334-342. 1933

16. Ball, C. F. Abdominal deep therapy injuries. Amer. Jour. Roentg. and Rad. Ther. 13: 220-226. 1925.

17. Barker, H. H., and H. Schlundt. The detection, estimation and elimination of radium in living persons given radium chloride internally. Amer. Jour. Roentg. and Rad. Ther. 24: 418-423. 1930.

18. Benassi, E. Irradiazioni roentgen e colorazione vitale degli elementi reticoloendoteliali. Radiol. Med. 16:874-891. 1929.

19. Bensaude, R., I. Solomon, and P. Oury. Roentgen ray treatment in gastric affections of non-cancerous origin. Presse Méd. 33:841-843. 1925.

20. Blalr, V. P., J. B. Brown, and W. G. Hamm. The surgical treatment of postradiation keratosis. Radiology 19:337-344. 1932.

21. Bope, H. G. Ein weiterer Beitrag zur Histologie sensibilisierter röntgenbestrahlter Haut. Strahlentherapie 40: 515-545. 1931.

22. Bode, H. G., and E. Rı⿻cke. Zur Histologie sensibilisierter röntgenbestrahlter Haut. Strahlentherapie 35 : 265-288. 1930.

23. Bonanno, A. Produzione di agglutinine nel sangue sotto l'influenza di raggi röntgen. L'Actinoterapia 6: 65-82. 1927.

24. Borak, J. Über die epidermiolytische Bestrahlungsreaktion. Fortschr. Geb. Roentgenstr. $45: 397-406.1932$.

25. Bordier, H. Roentgen ray epithelioma cured by diathermy. Jour. Amer. Med. Assoc. 80: 283.1923.

26. Borman, M. C. Histological changes in the sino-auricular node and adjacent tissues of the dog following radium emanation. Amer. Jour. Roentg. and Rad. Ther. 18: 111-118. 1927.

27. Brahms, J., and L. Darnbacher. Effect of X-rays on the gallbladder by experimental production of cholecystitis. Radiology 13: 103-108. 1929.

28. Brancovict, M. L'hypersécrétion modifie-t-elle la radiosensibilité de la glande sous-maxillaire? Compt. Rend. Soc. Biol. [Paris] 99: 757-758. 1928.

29. Braun, R. Vergleich der biologischen Wirkung von Röntgen und Gammastrahlen. Strahlentherapie 38: 11-18. 1930.

30. Broulé et Boulin. Un cas d'anémie pernicieuse aplastique provoquée par la radium. Bull. Mem. Soc. Méd. Hospitaux Paris 12:528. 1925.

31. Buckr, G. Grenz ray therapy. Radiology 14:126-135. 1930.

32. Bucky, G., and H. GugGenheimer. Steigerung der Knochenmarksfunktion durch Röntgenreizdosen. Klin. Wochenschr. 1: 11-12. 1922.

33. Cairns, H., and J. F. Fulton. Experimental observations on the action of radon on the spinal cord. Lancet 219: 16.1930.

34. Cant1, R. G., and F. G. Spear. Effect of gamma irradiation on cell division in tissue culture in vitro, Proc. Roy. Soc. Biol. 102 : 92-101. 1927. 
35. Carrie, C. Untersuchungen über Sensibilisicrung gegen Grenzstrahlen. Strahlentherapie $46: 697-705.1933$.

36. Casati, Anvibale. Experimentelle Untersuchungen über die Röntgenwirkung auf das Knochenmark. Strahlentherapie 32: 721-738. 1929; 38: 315-321. $1930 ; 43$ : 582-58s. 1932.

37. CASE, J. T., and W. N. BoldyreFF. Influence of roentgen ray upon gastric secretion. Amer. Jour. Roentg. and Rad. Ther. 19:61-70. 1928.

38. Case, J. T., and A. S. Warthin. The occurrence of hepatic lesions in patients treated by intensive deep roentgen irradiation. Amer. Jour. Roentg. and Rad. Ther. 12: 27-46. 1924.

39. CASPARI, W. Weiteres zur biologischen Grundlage der Strahlenwirkung. Strahlentherapie 18: 17-35. 1924.

40. Castellino, P. G. L'azione dell'irradiazione roentgen ed ultraviolette sil sistema reticulo, jstiocitario cutaneo. Arch. Radiol. 6:681-689. 1930.

41. Challley-Bert, P., and A. Zimmenn. Action des radiations sur l'excitabilité vagale. Compt. Rend. Soc. Biol. [Paris] 100: 719. 1929.

42. Chamberlain, W. E., and R. R. Newell. The epilation and the erythemat dose. Radiology, 10: 280-283. 1928.

43. Coblentz, W. W. Ultra-violet radiation useful for therapeutic purposes: specification of minimum intensity or radiant flux. Jour. Amer. Med. Assoc. 98: 1082-1086. 1932; 99 : 125-127. 1932.

44. Coblentz, W. W., R. Stair, and J. M. Hague. Die spektrale Erythemreaktion der menschlichen Haut auf Ultraviolettstrahlung. Strahlentherapic 42: 373378. 1931.

45. Colaneri, L. J. Les propriétés biologiques des rayons de 4 à 8 angstroms. Bull. Mem. Soc. Radiol. Méd. France 17: 100-105. 1927.

46. Colwell, H. A., and S. Russ. Radium X-rays and the living cell. G. Bell and Sons; London, 1924.

47. Conte, E. Ulteriori ricerche intorno all'azione biologica di radiazioni a varia lunghezza d'onda. Radiol. Med. 17: 689-698. 1930.

48. Cori, C. F., and G. W. Pucher. Biological reactions of X-rays; effect of radiation on nitrogen and salt metabolism. Amer. Jour. Roentg. and Rad. Ther. 10 : 738-745. 1932.

49. Corper, H. J., and L. Heкtoen. Effect of injection of active deposit of raclium emanation on rabbits. Jour. Infect. Dis. 31: 305-312. 1922.

50. Coutard, H. Sur les délais d'apparition et d'évolution cles réactions de la peau, et de la bouche et du pharynx provoquées par les rayons $\mathbf{X}$. Compt. Rend. Soc. Biol. [Paris] 86: 1140-1142. 1922.

51. CoutARD, H. Roentgentherapy of epitheliomas of the tonsillar region, hypopharynx and larynx from 1920-1926. Amer. Jour. Roentg. and Rad. Ther. 28: 313-331. 1932.

52. Cox, S. E., and E. G. Spear. A synopsis of the radiologieal work of the Strangeways Research Laboratory, Cambridge. British Jour. Radiol., N.S. 2: 222-227. 1929.

53. Cramer, H., and G. Fechner. Hautuntersuchungen und klinische Ergebnisse bei Anwendung von sichtbarem kalten'Rotlicht. Strahlentherapic 39: 474-484. 1930-31.

54. Cremer, M. Röntgenkatarakte, deren Bedeutung und Verhütung. Strahlentherapie 36: 732-735. 1930.

55. Cutler, M. Comparison of the effects of unfiltered and filtered radon tubes buried in rabbit muscle. Amer. Jour. Roentg. and Rad. Ther. 16:535-543. 1926.

56. Czepa, A. Das Problem der wachstumsfördenden und funktionssteigernden Röntgen-Radiumwirkung. Strahlentherapie 16: 913-917. 1924. 
57. Daels, F. Contribution to the experimental provocation of tumors by means of radium. British Jour. Radiol. 30:474-476. 1925.

58. David, O. Untersuchungen über den Einfluss von Röntgenstrahlen auf Kapillaren. Strahlentherapie 23 : 366-368. 1926.

59. David, O., and G. GaBriel. Kapillarmikroskopische Untersuchungen über die Tiefenwirkung von Röntgenstrahlen. Strahlentherapie 17: 192-196. 1924.

60. David, O., and G. Gabriel. Die Kapillarmikroskopie des Röntgenerythems. Strahlentherapie $16: 372-381.1924$.

61. Davis, J. S. Clinical illustrations of deep roentgen ray and radium burns. Amer. Jour. Roent. and Rad. Ther. 29 : 43-78. 1933.

62. Davis, K. S. Intrathoracic changes following $\mathrm{X}$-ray treatment-A clinical and experimental study. Radiology $3: 301-321.1924$.

63. Davy, Leita. Studies of the systematic effect of roentgen rays. II. The acid base balance and the serum proteins of dogs before and after irradiation. Amer. Jour. Roentg. and Rad. Ther. 25 : 255-265. 1931.

64. Dawson, A. B. Histological changes in the gastric mucosa (Pawlow pouch) of the dog following irradiation. Amer. Jour. Roentg. and Rad. Ther. 13: 320-326. 1925 .

65. DAZI, A. Morphological changes of the blood following irradiation of splenic area with so-called stimulating doses. Radiol. Med. 11:552-561. 1930.

66. Dean, A. L. Results of skin tests made to determine an objective dose for radium radiations. Amer. Jour. Radiol. 10 : 654-661. 1923.

67. Dé Buono, P. Weitere Untersuchungen über die Wirkung der Röntgenstrahlen auf das vegetative System. Strahlentherapie 34:301-312. 1929.

68. Demel, R. Tierversuche mit der Röntgenbestrahlung des Cerebrums. Strahlentherapie 22 : 333-336. 1926.

69. De Renyi, G. S., M. De Renyi, and D. P. Murphy. Preconception ovarian radium irradiation of albino rat (Mus norvegicus albinus) A biological study. Amer. Jour. Roentg. and Rad. Ther. 28: 764-783. 1932.

70. Desjardins, A. U. The reaction of the pleura and lungs to roentgen rays. Amer. Jour. Roentg. and Rad. Ther. 16: 444-453. 1926.

71. Desjardins, A. U. Effect of irradiation on the suprarenal gland. Amer. Jour. Roentg. and Rad. Ther. 19: 453-460. 1928.

72. Desjardins, A. U. Action of roentgen rays and radium on the eye and ear. Amer. Jour. Roentg. and Rad. Ther. 26: 639-679, 787-819, 919-942. 1931.

73. Desjardins, A. U. Action of roentgen rays and radium on the heart and lungs. Amer. Jour. Roentg. and Rad. Ther. 28: 127-143, 271-292, 421-436, 567-578, 699-720, 843-858. 1932.

74. Desjardins, A. U. Action of roentgen rays and radium on the gastro-intestinal tract. Amer. Jour. Roentg. and Rad. Ther. 26: 145-190, 335-370, 493-510. 1931.

75. Dessauer, F. Zur Erklärung der biologischen Strahlenwirkungen. Strahlentherapie, $16: 208-221.1924$.

76. Dessauer, F. The question of the fundamental biological reaction of radiation. Radiology 14: 1-16. 1930.

77. Determann, A., H. Jасовi, and H. Holtuusen. Die Erythemwirkung verschiedener Strahlenqualitäten auf Grund von Messungen in Röntgeneinheiten mit dem Küstnerschen Eichstandgerät. Strahlentherapie 26 : 472-483. 1927.

78. Dobrovolskaia-Zavadska1A, N. Action des rayonnements du radium sur les nerfs périphériques. Compt. Rend. Soc. Biol. [Paris] 91: 1322-1324. 1924.

79. Dobrovolskaia-Zayadskaia, N. Modifications des fibres striées sous l'influence d'irradiations prolongées au moyen de foyers radifères introduits dans les muscles. Jour. Radiol. et Electrol. 8: 49-61. 1924. 
80. Domagh, G. Die Röntgenstrahlenwirkung auf das Gewebe im Besonderen betrachtet an den Nieren. Morphologische und funktionelle Veränderungen. Beitr. Path. Anat. und Allg. Path. 67: 525. 1927.

81. Doonnkaat-Koolman, M. D. Experimentelle Untersuchungen über dic Beeinflussung der Erythrozyten durch Röntgenstrahlen. Strahlentherapie 24: 668-680. 1926.

82. Doub, H. P., A. Bolliger, and F. W. Hartiran. Immediate metabolic disturbances following deep roentgen ray therapy. Prevention and treatment of untoward reactions. Jour. Amer. Med. Assoc. 85 : 1299-1303. 1925.

83. Doub, H. P., A. Bolliger, and F. W. Hartian. Immediate metabolic disturbances following deep roentgen ray therapy. Amer. Jour. Roentg. and Rad. Ther. 13 : 54-63. 1925.

84. Doub, H. P., A. Bolliger, and F. W. Hartman. The relative sensitivity of the kidney to irradiation. Radiology 8: 142-148. 1927.

85. Druessen, L. F. Keimschädigung durch Röntgenstrahlen. Strahlentherapic $16: 656-689.1924$.

86. Dryoff, R. Experimentelle Beiträge zur Frage der Nachkommenschädigung durch Röntgenstrahlen. Strahlentherapie 24: 288-312. 1927.

87. Dufestal, L. G. La pigmentation: ses consequences au point du vue du traitement actinothérapiques. Annales Inst. Action. 2: 88-89. 1928.

88. Duffy, J. J., R. F. McNattin, M. M. Copeland, and L. H. Quimby. The relative effects produced by $200 \mathrm{kv}$. roentgen rays, $700 \mathrm{kv}$. roentgen rays and gamma rays. Amer. Jour. Roentg. and Rad. Ther. 24:343-345. 1933.

89. Dres, O. Anänie, Zellalter und Radiosensibilität. Strahlentherapie 36 : 552-561. 1930.

90. Edwardes, D. J., and H. J. BAGG. Lesions of the corpus stratum by radium emanation and the accompanying structural and functional changes. Amer. Jour. Physiol. 65 : 162-173. 1923.

91. Ellinger, F. Die Atiologie des Lichterythems vom Standpunkt des Latenz stadiums aus betrachter. Strahlentherapie 40: 760-763. 1931.

92. Ellinger, F. Die Lichtempfindlichkeit der menschlichen Haut, ihre Bestimmung und Bedeutung für die lichtbiologische Konstitutionsforschung. Strahlentherapie 44: 1-82. 1932.

93. Ellis, C., and A. Wells. The chemical action of ultra violet rays. Chemical Catalog Company; New York, 1925.

94. Evans, W. A., and T. Leucutia. Intrathoracie changes induced by heavy radiation. Amer. Jour. Roentg. and Rad. Ther. 13: 203-220. 1925.

95. Ewing, J. Radiation osteitis. Acta Radiology 6:399. 1926.

96. Ewing, J. Tissue reactions to radiation. Amer. Jour. Roentg. and Rad. Ther. 15: $93-115.1926$.

97. Failla, G. Correlation of experimental results. Relative effects produced by roentgen rays $(700 \mathrm{kv}$.), $(200 \mathrm{kv}$.) roentgen radiation and gamma rays. Amer. Jour. Roentg. and Rad. Ther. 29:352-362. 1933.

98. FArkes, H. H. Radiun in malignant disease of esophagus and upper respiratory tract. Boston Med. and Surg. Jour. 186 : 705-709. 1922.

99. Fedder, L., and H. Hellner. Die Veränderungen der quergestreiften Muskulatur nach Röntgenbestrahlungen im Tierexperiment. Strahlentherapie 30:682705. 1928.

100. Feroux, R., and C. Regaud. Est-il possible de stériliser le testicule du lapin adulte par une dose massive de rayons $\mathrm{X}$ sans produire de lésion grave de la peau? Compt. Rend. Soc. 13iol. [Paris] 97 : 330-333. 1927.

101. FIKE, R. H. The oceurrence of roentgen pleuropneumonitis in treatment of breast cancer. Amer. Jour. of Roentg. and Rad. Ther. 27 : 509-512. 1932. 
102. Fischer, A. Mesothoriumbestrahlung von Gerardezellen in vitro. Strahlentherapie, 40 (Sonderband) 16:54-96. 1931.

103. Fischer, A., and M. Horowitz. Multiplikation der Wirkung kleinster Radiumdosen auf Gewebezellen in vitro. Strahlentherapie 40: 465-469. 1931.

104. Flaskamp, W. Zur Frage der Schädigung der Nachkommenschaft durch Röntgenstrahlen. Strahlentherapie 24: 282-287. 1927.

105. Flaskamp, W. Utber Röntgenschaden und Schäden durch radioaktive Substanzen. Strahlentherapie, Sonderband $12: 365$ p. Urban and Schwarzenberg; Berlin, 1930.

106. Freund, L. Neuere Arbeiten aus dem Gebiete der Röntgenstrahlen-Biologie und-Therapie. Wein. Klin. Wochensch. 39: 1491. 1926.

107. Frey, H. Experimentelle Untersuchungen über die Röntgensensibilität der Nebennieren. Acta Radiologica 9: 23-53. 1928.

108. Funs, H., and J. Konrad. Grenzstrahl-Hauttherapie. Sonderbände Strahlentherapie 16: 122 p. Urban and Schwarzenberg; Berlin, 1931. (See also Zur Angabe der Strahlenhärte bei Grenzstrahlbehandlung. Strahlentherapie 40: 196-200. 1931.)

109. Fukase, S. Über die Beeinflussung der Frakturheilung durch Röntgenstrahlen. Fortsch. Geb. Roentgenstr. 41: 581-588. 1930.

110. FUKASE, S. Über die Beeinflussung der Wundheilung durch Röntgenbestrahlung. Strahlentherapie 36: 102-115. 1930.

111. Gabries, G. Die Beeinflussung von Tierorganen durch Röntgenbestrahlung. Strahlentherapie 22: 106-124. 1926.

112. Gabriel, G. Weitere Untersuchungen über die sogennante Grenzstrahlung. Strahlentherapie 26: 189-199. 1927.

113. Gabriel, G. Physikalische und biologische Untersuchungen über die sogenannte Grenzstrahlung. Strahlentherapie 24: 534-547. 1927.

114. Gabries, G. Uther die Beeinflussung des vegetativen Systems durch die Röntgenstrahlen. Strahlentherapie 34: 813-821. 1929.

115. Gaissler, E. O. Ṫher die Auswirkung der Röntgen und Radiumbestrahlung im Stoffwechselbild von Gebärmutterkrebskranken. Strahlentherapie 37 : 361-377. 1930.

116. GaNs, O. Effect of roentgen rays on respiration of skin. (Abstract.) Jour. Amer. Med. Assoc. 80: 882. 1923.

117. Girden, E., and E. Culler. Auditory effects of roentgen radiation in dogs. Amer. Jour. Roentg. and Rad. Ther. 30: 215. 1933.

118. Glasser, O. Erythemodosen in Röntgeneinheiten. Strahlentherapie 21: 476-479. 1926.

119. Glasser, O. The physical foundation of grenz-ray therapy. Radiology 18: 713-726. 1932.

120. Glasser, O. First observations on the physiological effects of roentgen rays on the human skin. Amer. Jour. Roentg. and Rad. Ther. 28: 75-80. 1932.

121. Glasser, O., and F. R. Mautz. The measurement of the intensity of the gamma rays of radium in r-units. Radiology 15: 93-100. 1930.

122. Glasser, O., and U. V. Portmann. Erythema and tolerance doses in American clinies. Radiology 14 : 346-350. 1930.

123. Glocker, R., E. Hayer, and O. Jüngling. Ither die biologische Wirkung verschiedener Röntgenstrahlenqualitäten bei Dosierung in R-Einheiten. Strahlentherapie 32 : 1-38. 1929.

124. Glocker, R., H. Langendorff, and A. Reuss. T'ber die Wirkung von Röntgenstrahlung verschiedener Wellenlänge auf biologische Objekte. Strahlentherapie $46: 571-528.1933$. 
125. Gloor, W., and A. ZuPpinger. Blutuntersuchungen bei protrahiert-fraktionierter Bestrahlung. Strahlentherapie $40: 438-464.1931$.

126. Goldstein, M. J., and J. P. Neworoschkin. Utber die Veränderungen der Katalase und Lipase im Blut der mit Röntgenstrahlen behandeleten Kranken. Strahlentherapie $36: 736-744.1930$.

127. Gordon, B., G. F. Strong, and E. S. Emery. The effect of direet radiation over the pre-eordium on the heart size, the heart mechanism, and the myoeardiun of rabbits. Amer. Jour. Roentg. and Rad. Ther. 11:328-330. 1924.

128. Gouin, J., and A. Bienvenue. L'action des rayons $\mathrm{X}$ sur le sympathiques est-elle fait nouveau? Bull. Soc. Radiologie Méd. France. 167:127. 1930.

129. Goulston, Daphne. The inerease in the fragility of the red blood eorpuscles after exposure to radium. British Jour. Radiol., N. S. 5: 775-779. 1932.

130. Gricouroff, G. Étude histologique de l'action des rayons X sur l'ovaire à la période d'ovogenèse. Arch. Inst. Radium, Univ. Paris. 2: 1-80. 1930.

131. Grover, T. A., A. C. Christie, and E. A. Merritt. Intrathoracic changes following roentgen treatment of breast careinoma. Amer. Jour. Roentg. and Rad. Ther. 10: 471. 1923.

132. Guthmans, H., F. Anseli, and H. Papenberg. Das Verhalten des Hautelastizität während der Schwangersehaft und ihre Beeinflussung dureh Ultraviolettbestrahlung. Strahlentherapie 44: 443-474. 1932.

133. Guthmann, H., K. Schwerin, and F. Stahler. Die Ultraviolettabsorption der Eiweissbaustoffe. Strahlentherapie 39:401-441. 1930-31.

134. Halt, C. C., and G. H. Whipple. Roentgen ray intoxieation: Disturbances in metabolism produeed by deep massive doses of the hard roentgen rays. Journ. Med. Sei. 157 : 453-482. 1919.

135. Harris, M., E. T. Leddy, and C. Sineard. The speetro-photometer analysis of the eolor of skin following radiation by roentgen rays. Radiology 19: 233256. 1932.

136. Hartmann, M., A. Bolliger, and H. B. Doub. Experimental nephritis produced by irradiation. Amer. Jour. Med. Sei. 172: 487-500. 1926; Bull. Johns Hopkins Hospital. 41 (no. 1.) : 36-61. 1927.

137. Hartmann, M., A. Bolliger, and H. B. Doub. Funetional studies throughout eourses of roentgen ray nephritis in dogs. Jour. Amer. Med. Assoe. 88: 130-145. 1927.

138. Hartock, W., and M. Israeleski. Zur Wirkung der Röntgenstrahlen auf die Funktion sezernierender Organe-Lebeudbeobachtungen im Luminiszenzlicht. Strahlentherapie 44:557-574. 1932.

139. Hausser, K. W. Einfluss der Wellenlänge in der Strahlenbiologie. Strahlentherapie 28: 25-39. 1928.

140. Hausuann, W., and E. Zakovsky. Über die Wirkung von Kathodenstrahlung auf Erythroeyten. Strahlentherapie 33: 196-198. 1929.

141. HAwkins, J. A. The skin reaetion produced by alternations of heat and X-ray at various intervals. Jour. Exp. Med. 53: 405-413. 1931.

142. Haxthausen, H. Einige Untersuchungen über die Kreislaufveränderungen in der Haut nach Bestrahlung mit verschiedenen Liehtquellen. Strahlentherapie 30: 662-681. 1928.

143. HAYER, E. Blutbildbeobachtungen bei Röntgenbestrahlen auf Erythroeyten. Strahlentherapie $33: 196-198.1929$.

144. Hazen, H. H., and L. C. Milstead. Epilation in mice as a biological standard for determining roentgen-ray dosage. Amer. Jour. Roentg. and Raul. Ther. 13: 451-452. 1925.

145. Heeren, J. Komplimentüre Eigenschaften des Blutserums oder "Alexinreaktion" nach Röntgenbestrahlung. Strahlentherapie 42: 189-197. 1931. 
146. Heeren, J., and J. Pansdorf. Gewebsatmung nach Röntgenbestrahlung experimentelle Untersuchungen an Leber und Niere. Strahlentherapie 42: 307-327. 1931.

147. Herneke, H. Experimentelle Untersuchungen über die Einwirkung der Röntgenstrahlen auf innere Organe. Mitt. Grenzgeb. Med. und Chir. 14: 21-94. 1904-1905.

148. Held, A. Die Blutzuckerregulation unter Röntgenstrahlen. Strahlentherapie 38: 72-39. 1930.

149. Henshaw, P.S., C. T. Henshaw, and D. S. Francis. Relative effects produced by $200 \mathrm{kv}$. R. R., $700 \mathrm{kv}$. R. R. and gamma rays. III. Comparison based on effects on Drosophila eggs and on wheat seedlings. Amer. Jour. Roentg. and Rad. Ther. 29 : 326-333. 1933.

150. Herrnheiser, G. Vorläufige Erfahrungen mit der (protrakiert) fraktionierten Bestrahlung. Strahlentherapie 46: 435-443. 1933.

151. Hess, P. Die Erythembreite von Röntgenstrahlen verschiedener Wellenlängen. Strahlentherapie $33:$ 721-731. 1929.

152. Hickel, A. propos de l'antagonisme desr ayons infra-rouges et des rayons ultra-violets. Bull. Offieiel Soc. Française Elee. et Rad. Mai, 1928.

153. Hickey, P. M., E. A. Pohle, G. A. Lindsay, and J. M. Barnes. Skin toleration doses in roentgen units and their relation to the quality of radiation. Radiology 12 : $309-316.1929$.

154. Hilsnitz, O. Früher Nachweis histologischer Veränderungen nach Röntgenbestrahlung an der Haut. Strahlentherapie 22 : 525-537. 1926.

155. Hirsch, E. F., and A. J. Peterson. Blood with deep roentgen ray therapy: Hydrogen-ion concentration, alkali reserve, sugar and non-protein nitrogen. Jour. Amer. Med. Assoc. 80: 1505-1507. 1923.

156. Hoffmans, W. Über die Wirkung des sichtbaren Lichtes, der ultraroten und ultra-violetten Strahlen auf das Auge. Strahlentherapie 39: 93-102. 1930.

157. Holfelder, H. Die planmässige Bestimmung eines optimalen Rythmus für die Strahlentherapie bei malignen und benignen Erkrankungen. Strahlentherapie $46: 72-82 . \quad 1933$.

158. Holthusen, H. Die Wirkung der Röntgenstrahlen in biologischer Hinsicht. Strahlentherapie 18: 241-261. 1924.

159. Holtuusen, H. Der Zeitfaktor bei der Röntgenbestrahlung. Strahlentherapie 21 : $275-305.1926$.

160. Holthusen, H. Vergleichende Untersuchungen über die Wirkung von Roentgen- und Radium-Strahlen. Strahlentherapie 46: 273-228. 1933.

161. Holweck, F., and A. LaCassagne. Étude énergétique de l'action biologique de diverse radiations. Compt. Rend. Soc. Biol. [Paris] 103: 60-62. 1930.

162. HоокеR, D. R. The effect of exposure to roentgen rays on reproduction in male rats. Amer. Jour. Roentg. and Rad. Ther. 14: 327-336. 1925.

163. Hopwood, F. L., and M. Donaldson. A remarkable sequel to an attempt to determine the X-ray lethal dose for tissue cultures growing in vitro. British Jour. Radiol., N. S. 3: 69-75. 1930.

164. Hussey, R. G. Influence of x-rays on properties of blood. Jour. Gen. Physiol. 4: 511-516. 1922 .

165. IsaACs, R., and A. C. Danelian. Maintenance of leukocyte level and changes during irradiation. Amer. Jour. Med. Sei. 174: 70-87. 1927.

166. Ivy, A. C., J. E. McCarthy, and B. H. Orndorff. Studies on the effects of roentgen rays on glandular activity. Effect of exposure of abdominal and thoracic areas to roentgen rays on gastric secretions; roentgen cachexia. Jour. Amer. Med. Assoc. 83: 1977-1984. 1924. 
167. JACOBSEN, V. C. Effects of high voltage cathode rays on germinal epithelium of the rat. Arch. Path. 9: 967-983. 1930.

168. J A СовY, Dr. Experimentelle Untersuchungen über Schädigungen des Auges durch Röntgenstrahlen. Strahlentherapie 16: 492-506. 1924

169. Jolly, S. Mode d'action des rayons $\mathrm{X}$ sur les tissus peut-on-modifier expćrimentalement la radio-sensibilité? Bull. Acad. Méd. [Paris] 93: 276-279. 1925.

170. Jolly, S., and A. Lacassagne. De la résistance des leukocytes du sang vis-à-vis des rayons x. Comp. Rend. Soc. Biol. [Paris] 89:379. 1923.

171. Jost, DorA. Untersuchung über die Indikation und den Erfolg der Schwachbestrahlung und temporären Kastration mit Röntgenstrahlen bei der Fraı und ihre Bedeutung für die Nachkommenschaft, an Hand des Materials der Freiburger Universitäts-Frauenklinik. Strahlentherapie 46: 601-616. 1933.

172. KaWAshima, Dr. Über den Einfluss strahlender Energie auf die Zirkulation. Untersuchungen an Straubschen Froschherzen. Strahlentherapie 17: 381-389. 1924.

173. Keller, P. Úber die Wirkung des ultravioletten Lichtes auf die Haut unter besonderer Berïcksichtigung der Dosierung. Strahlentherapie 16: 52-74, 301-308, 537-553, 824-835. 1924.

174. Keller, P. Über die Wirkungen des ultravioletten Lichtes auf die Haut. Strahlentherapie 28: 152-162. 1928.

175. Kellwr, P. Die Strahlen-Wärmereaktion der Haut. III. Pigment- und Warmestrahlenschutz. Strahlentherapie 35: 350-360. 1930.

176. Fingery, L. B. Saturation in roentgen therapy: its estimation and maintenance. A preliminary report. Arch. Derm. and Syph. 1: 423. 1920.

177. KLEIN, H.V. Ungewöhnlicher Befund bei röntgenbestrahlten Kaninchenovarien. Strahlentherapie $25: 443-456.1927$.

178. Kolta, E., and J. Forster. Die Wirkung der Röntgen-Strahlen auf das Blut. Strahlentherapie 21: 644-667. 1926.

179. КотzarefF, A., and M. Mollow. Action of radium on the uterus. Gyn. and Obst. $6: 244-273.1922$.

180. Квомеке, F. Über die Einwirkung der Röntgenstrahlen auf die roten Blutkörperchen. Strahlentherapie 22: 608-652. 1926.

181. Kunlmann, B. Die Allgemeinwirkung der Röntgenstrahlen. Strahlentherapie 19: $817-881.1925$.

182. Kuhlmanx, B. Gesamtinhaltsverzeichnis der Bände, 1-25. Strahlentherapie 1928.

183. Küstner, H. Die Dossierung in der Hauttherapie. Verhandl. Deut. Rontgen-Ges. 23 Kongress. 3-5: 78-81. Fortsch. Geb. Röntgenstr. 46: 1932.

184. Lacassagne, A. The direct and indirect action of radiation on cancer tissues. Radiology 11 : 393-401. 1928.

185. Lacassagne, A., G. Fournier, and J. Lattes. Mise en evidence par leur différence d'action biologie, de deux groupes de rayonnements de l'uranium $\mathrm{X}$ inégalement absorbables. Comp. Rend. Soc. Biol. [Paris] 99: 1641. 1928.

186. Lacassagne, A., and H. Gricouroff. De l'action des radiations sur les leucoeytes du sang, étudie au moyen de la methode des cultures. Jour. Radiol. et Ëlect. 11 : 573-580. 1927.

187. Lacassagne, A., J. Latres, and G. Fourvier. Modifications produites dans l'epithelium seminal par les rayons de l'uranium X. Compt. Rend. Soc. Biol. [Paris] 99: 1643. 1928.

188. Lacassagne, A., J. Latres, and J. Laveden. Étude expérimentale des effets biologiques du polonium introduit dans l'organisme. Journ. Radiol. et Electrol. 9 : $67-80.1925$. 
189. LangendorfF, N., and H. Langendorfa. Die Erzielung eines specifischen Kathodenstrahleneffektes durch hohe Röntgendosen. Strahlentherapie 41: 135-141. 1931.

190. Langer, H. Roentgen rays and the autonomic nervous system. Amer. Jour. Roentg. and Rad. Ther. 18: 137-144. 1927.

191. Laurens, H. The physiological effects of radiant energy. The Chemical Catalog Company; New York, 1933.

192. Lazarew, N. W., and AnNa Lazarewa. Uber die funktionellen Veränderungen der Blutgefässe nach Röntgenbestrahlung. Strahlentherapie 23:41-77. 1926; 25 : 255-279, 458-469. 1927; 26: 347-362. 1927.

193. Lazarus, P. Radiothorium und seine klinischtherapeutische Anwendung. Deut. Med. Wochensch. 48: 451-453. 1922.

194. Leddy, E. T., and J. I. Weatherwax. Standardization of ionization measurements of intensity and measurements of scattered and secondary x-rays effective in producing an erythema. Amer. Jour. Roentg. and Rad. Ther. 10: 488-497. 1923.

195. Leist, M. Über die Einwirkung der Röntgenstrahlung und des Radiums auf Zähne und Kiefer. Strahlentherapie 24: 268-281. 1927.

196. Lenz, M. Investigation of thymus stimulation by roentgen rays. Amer. Jour. Roentg. and Rad. Ther. 13: 226-234. 1925.

197. Levine, M. The influence of roentgen rays on white mice and their progeny. Amer. Jour. Roentg. and Rad. Ther. 17: 546-550. 1927.

198. Levine, W. T. Pigmentary reaction in Rana clamitans larvae following treatments with x-rays. Proc. Soc. Exp. Biol. and Med. 28: 594-596. 1931.

199. Liеснт, A. Über den Zeitfaktor der biologisehen Strahlenwirkung. Strahlentherapie $33: 1-53.1929$.

200. Linhardt, St. V. Einfluss der Röntgenstrahlen auf die Blutgerinnungszeit und das Blutbild. Stralentherapie 16 : 754-774. 1924.

201. Loeb, L. Effects of roentgen rays and radio active substances on living cells and tissues. Amer. Jour. Roentg. 9: 497-501. 1922.

202. Lovisatti, N. Über einige strahlenbiologische Probleme der Haut. Fortsch. Geb. Röntgenstr. 44: 235-240. 1931.

203. Low-Beer, A. Experimentelle Untersuchungen zur Frage der biologischen Röntgenstrahlenwirkung. Strahlentherapie 46: 469-516. 1933.

204. Ludin, M. Leberveränderungen nach Röntgenbestrahlung. Strahlentherapie 19: 138-141. 1925.

205. Ludin, M., and A. WERThemann. Lungenveränderungen nach experimenteller Röntgenbestrahlung. Strahlentherapie 38:684-701. 1930.

206. Lyman, R. S., P. S. Kupalov, and W. Scholz. Effect of roentgen rays on the central nervous system. Arch. Neurol. and Psychi. 29: 56-87. 1933.

207. Martin, C. L., Rogers, F. T. Intestinal reaction to erythema dose. Amer. Jour. Roentg. and Rad. Ther. 10: 11-19. 1923.

208. M A rtin, C. L., and F. T. Rogers, Roentgen ray cachexia. Amer. Jour. Roentg. and Rad. Ther. 11: 280-286. 1924.

209. Martin, C. L., and F. T. Rogers, The effect of irradiation on the ureter. Amer. Jour. Roentg. and Rad. Ther. 16: 215-218. 1926.

210. Martin, C. L., F. T. Rogers, and N. F. Fisher. The effect of roentgen rays on the adrenal gland. Amer. Jour. Roentg. and Rad. Ther. 12:466-471. 1924.

211. Martios, H. Kleinschädigung durch Röntgenstrahlen. Strahlentherapie 41: 47-66. 1931.

212. Martland, H. S, P Conlon, and J. P. Knef. Some unrecognized dangers in the use and handling of radioactive substances. Jour. Amer. Med. Assoc. 85: 1769. 1925 . 
213. MAtas, R. Remarks on the delayed or remote appearance of $x$-ray burns after long periods of lateney. Amer. Jour. Roentg. and Rad. Ther. 13: 37-40. 1925.

214. Matthews, H. B. Effects of radium rays upon the ovary. Amer. Jour. Roentg. and Rad. Ther. 6: 614-618. 1923.

215. Marburg, O., and M. Sgalitzer. Die Röntgenbehandlung der Nervenkrankheiten. Strahlentherapie 37 (Sonderband 15) : 1-20. 1930.

216. Mavor, J. W. Biological effects of x-rays. Amer. Jour. Roentg. and Rad. Ther. $10: 968-974.1923$.

217. Maxmow, A. A. Studies on the ehanges produced by roentgen rays in inflamed connective tissue. Jour. Exp. Med. 37:319. 1923.

218. MAYer, E. Clinical application of sunlight and artificial radiation. The Williams and Wilkins Company; Baltimore, 1926.

219. MAYER, E. Die Wirkung von ultravioletten Strahlengemischen auf gewebekulturen. Strahlentherapie 39: 148-193. 1930-31.

220. Mayneord, W. V., and A. Piney. Some effects of $x$-radiation on blood. British Jour. Radio., N. S. 1: 257-272. 1928.

221. McNattin, R. F. The relative effects produced by $200 \mathrm{kv}$. roentgen rays, $700 \mathrm{kv}$. roentgen rays and gamma rays. Amer. Jour. Roentg. and Rad. Ther. $29: 346-351.1933$.

222. McQuarrie, I., and G. H. Whipple. Study of renal funetion of roentgen ray intoxication resistance of renal epithelium to direct radiation. Jour. Exp. Med. $35: 225-242 . \quad 1922$.

223. Memmesheimer, A. M., and R. Matthaei. Beobachtungen ïber die Pigmentierung der Haut. Strahlentherapie 35:339-352. 1930.

224. Meyer, H. Die Röntgenstrahlenempfindliehkeit der kindliehen Haut. Strahlentherapie 43 (Sonderband 17): 67-92. 1932.

225. Meyer, W. H., and O. Glasser. Erythema doses in absolute units. Radiology $6: 320-325.1926$.

226. Meyer, W. H., and O. Glasser. Further studies on the influence of raliation quality on the erythema dose measured in physical units. Radiology 8: 311316. 1927.

227. Miescher, G. Das Röntgenerythem. Strahlentherapie 16: 333-371. 1924.

228. Miescher, G. Röntgenbiologie der gesunden und kranken Haut. Strahlentherapie $27: 257-280.1928$.

229. Miescher, G. Das Problem des I,ichtsehutzes und der Lichtgewöhnung. Strahlentherapie 35: 403-443. 1930.

230. Miescher, G. Careinomtherapie mit superponierter (verzettelter) Röntgenbestrahlung. Strahlentherapie 36:434-471. 1930.

231. Miescier, G. Gegenwärtige Methoden der Krebsbestrahlung und ihre Erfolge; einmalige Höchstdosis. Strahlentherapie 37: 17-30. 1930.

232. Mrescher, G. Die Sehutzfunktionen der Haut gegenüber Lichtstrahlen. Strahlentherapie 39: 601-618. 1930-31.

233. Miescher, G. Untersuchungen über die Bedeutung des Pigments für den ult ravioletten Lichtsehutz der Haut. Strahlentherapie 45: 201-216. 1932.

234. Minot, J. R., and R. G. Spurling. Effeet on blood of irradiation, especially short wave length roentgen ray therapy. Amer. Journ. Med. Se. 168: 215-241. 1924 .

235. Mogilnitzky, B., and L. Podsjaschuk. Zur Frage über die Wirkung der Röntgenstrahlung auf das zentrale Nervensystem. Fortseh. Geh. Röntgenstr. 40: 1096-1108. 1929.

236. Mogilnitzky, B., and I. Podujaschuk. Röntgenstrahlen und sogen. "hämatonzephalische Barriere." Fortsch. Geb. Röntgenstr. 41: 66-75. 1930. 
237. Monacelli, M. Ưber Spätschädigungen nach Grenzstrahlbehandlung. Strahlentherapie $35:$ 581-591. 1930.

238. Moppetт, W. The allantoic membrane of the chick exposed to beta radiation. British Jour. Radiol., N. S. 5: 342-348. 1932.

239. Мотоліма, R. Experimentelle Untersuchungen über den Einfluss der Röntgenstrahlen auf den Ablauf von Entzündungen in Vergleich mit anderen physikalischen Methoden. Strahlentherapie 29: 30-70. 1928.

240. Motrran, J. C. Histological changes in the bone marrow of rats exposed to irradiation from radium. Arch. Radiol. and Electrother. 25: 197. 1920.

241. Motrram, J. C. Histologic changes in lymphatic glands following exposure to radium. Amer. Jour. Med. Sci. 165: 469-479. 1925.

242. Motтram, J. C. The reaction of the skin to radium exposure repeated after varying lengths of time. British Jour. Radiol. 30: 387-391. 1925.

243. Mottram, J. C. Some blood examinations of x-ray workers. British Jour. Radiol., N. S. 5: 156-158. 1932.

244. Motrram, J. C. The action of radium on blood supply. The white reaction. British Jour. Radiol., N. S. 6 : 643-646. 1932.

245. Mottram, J. C., and A. N. Kingsbury. Some researches into the action of radium and $\mathrm{x}$-rays correlating the production of intestinal changes, thrombopenia and bacterial invasion. British Jour. Radiol. 30: 18-26. 1925.

246. MüHSAM, E. Tierexperimentelle Versuche mit Thorium $\mathrm{X}$ und Radium an Nieren und Harnleitern. Strahlentherapie 44: 131-146. 1932.

247. MÜLler, W. Der Einfluss der Röntgenstrahlen auf den Knochen. Strahlentherapie 16: 1018-1019. 1924. (Abst)

248. MÜLLER, W. Über das Verhalten der Hautkapillaren im röntgenbestrahlten Gebiet. Strahlentherapie 19: 607-608. 1925.

249. Murphy, J. B. Experimental studies in roentgen ray effects. Amer. Jour. Roentg. and Rad. Ther. 11: 544-546. 1924.

250. Murphy, J. B. Monograph Rockefeller Inst. for Med. Res., 1926.

251. Murray, J. M. A study of the histological structure of mouse ovaries following exposure to roentgen irradiation. Amer. Jour. Roentg. and Rad. Ther. 25: 1-45. 1931.

252. NeEF, C. Toleranzdosen in der Röntgendiagnostik. Fortsch. Geb. Röntgenstr. $41: 414-426.1930$.

253. NÜrnberger, L. Zur Frage der Keimschädigung durch Röntgenstrahlen. Strahlentherapie 21: 577. 1926.

254. Oettingen, K. V. Pflanzen- und tierexperimentelle Untersuchungen in elektrischen Wechselfelde eines Kurzwellensenders. Strahlentherapie 41: 251-285. 1931 .

255. O'Hare, J. P., H. Altnow, J. T. D. Christian, A. W. Calhoun, and M. C. Sossman. Chronic nephritis produced by $\mathrm{x}$-ray. Boston Med. and Surg. Jour. 194 : 43-45. 1926.

256. Orndorf, B. H., J. I. Farrell, and A. C. Iry. Studies of effects of x-rays on glandular activity. Jour. Radio. 4: 189-199. 1923.

257. Orndorf, B. H., J. I. FArrell and A. C. Ivy. Studies on the effect of roentgen rays on glandular activity - external pancreatic secretion. Amer. Jour. Roentg. and Rad. Ther. 16: 349-354. 1926.

258. PАск, G. T., and E. H. Quimby. Time intensity factor in irradiation. Amer. Jour. Roentg. and Rad. Ther. 28 : 650-664. 1932.

259. PACKARD, C. The biological measurement of scattered radiation. Jour. Cancer Res. 13 : 373-382. 1929.

260. PaCkard, C. The biological effects on short radiations. Quart. Rev. Biol. 6: 253-280. 1931. 
261. Pagniez, P., A. Ravina, and Solomon. Action des rayons de roentgen sur la coagulation du sang. Jour. Radiol. et Ėlectrol. 7 : 153-157. 1923.

262. Pankow, O. Keimschädigungen dureh Röntgenstrahlen. Münchess. Med. Wochensch. $77:$ 303-306. 1930.

263. Pansdorf, H., and W. Nell. Die Blutdrueksehwankungen nach Röntgenbestrahlungen und ihre klinische Bedeutung. Strahlentherapie 38: 40-53. 1930.

264. PAPE, R. Die Hauttoleranz bei langsam bestrahlung in möglichst unfraktionierter Applikationsform. Strahlentherapie 45: 461-474. 1932.

265. Pendergrass, E. P., J. M. Haman, K. M. Houser, and V. C. Rambo. The effect of radium on the normal tissues of the brain and spinal cord of dogs and its therapeutic application. Amer. Jour. Roentg. 9: 553-569. 1922.

266. Pfanler, G. E. The saturation method in roentgentherapy, as applied to deep seated malignant disease. British Jour. Radiol. 31: 45-54. 1926.

267. Pfahler, G. E., J. V. Klauder, and J. L. Martin. Experimental studies on the combined effeets of roentgen rays and ultra-violet rays. Amer. Jour. Roentg. and Rad. Ther. 16: 150-154. 1926.

268. Pfaller, G. E., and B. P. Widman. Further observations on the use of the saturation method of radiation therapy in deep-seated malignant disease with some statisties. Radiology $11: 181-190.1928$.

269. Phemister, D. B. Radium necrosis of bone. Amer. Jour. Roentg. and Rad. Ther. 16: 340-348. 1926.

270. Piepenborn, J. Versuche über dem Wirkungsgrad von Röntgenstrahlen verseheidener Wellenlänge auf die Milz der Maus, bei gleieher Dosis, gemessen in Röntgen Einheiten. Strahlentherapie 33: 322-337. 1929.

271. Pohle, E. A. Studies on the suspension stability of the human blood. The changes of the sedimentation rate of the erythroeytes in vitro and in vivo after x-ray exposure. Radiology 6:55-58. 1926.

272. Ponle, E. A. Studies on the roentgen erythema of the human skin. Radiology 6: 236-245. 1926 .

273. Pohle, E. A. Studies of the roentgen erythema of the human skin. Radiology $8: 185-194.1927$.

274. Pohle, E. A. The effeet of roentgen rays on the retieulo-endothelial system. Amer. Jour. Roentg. and Rad. Ther. 23: 291-298. 1930.

275. Pohle, E. A., and C. H. Bunting. Skin reactions following exposure to roentgen rays. Radiology $13: 496-503.1929$.

276. Pohle, E. A., and C. H. Bunting. A study of the effect of graded doses of two different wave lengths on the skin of rats. Radiology 16:647-659. 1930.

277. Pohle, E. A., and C. H. Bunting. Studies of the effect of roentgen rays on the liver. Histological ehanges in the liver of rats following exposure to single graded doses of filtrated roentgen rays. Acta Radiologica 13: 117-124. 1932.

278. Ponle, E. A., G. Ritchie, and C. S. Wright. Studies of the effeet of roentgen rays on the healing of wounds. Radiology 16:445-460. 1930.

279. Pollet, E. A., and E. L. Sevringinaus. Studies of systemic effect of roentgen ray; blood ehemieal changes in dogs following exposure to filtered x-rays of short wave length. Amer. Jour. Roentg. and Rad. Ther. 23: 291-298. 1930.

280. Pohle, E. A., and C. S. Wright. Studies of the roentgen erythema of the human skin. Radiology 14: 351-363. 1930.

281. Polak, E. Utber die Wirkung von Radium-emanation auf die Gefässe. Aeta Radiologica 9: 169-205. 1928.

282. Pordes, F. Zum biologischen Wirkungs-mechanismus der Röntgenstrahlen. Strahlentherapie 19: 307-324. 1925. 
283. Portis, S. A., and R. Ahrens. The effects of the shorter wave length roentgen ray on the gastric secretion of dogs. Amer. Jour. Roentg. and Rad. Ther. 11: 272-280. 1924.

284. Pullinger, B. D. Causes of cell death in irradiated human tissue. Jour. Path. and Bacteriol. 35 : 527-540. 1932.

285. Quimby, Edith. The skin erythema dose with a combination of two types of radiation. Amer. Jour. Roentg. and Rad. Ther. 17: 621-625. 1927.

286. Quimby, Ерiтh, and G. T. РАск. The skin erythema for combinations of gamma and roentgen rays. Radiology $13: 306-312.1929$.

287. Qumby, Edith, and G. T. PAck. Further studies on the skin erythema with combinations of two types of radiation. Radiology 15: 30-38. 1930.

288. Rachuanow, A. Zur Frage über Wirkung der Röntgenstrahlen auf das Zentralnervensystem. Strahlentherapie 23: 318-325. 1926.

289. RАнм, H. Über Röntgenspätschädigungen. Strahlentherapie 20: 213-214. 1925.

290. RAHM, J., and W. Koose. Ein Beitrag zum Wirkungsmechanismus harter Röntgenstrahlen. Strahlentherapie 23: 195-216. 1926.

291. REgAUd, C. The sensibility of bony structures to radiations and the mechanism of radio-necrosis of bones. Jour. Radiol. et Êlectrol. 6 : 450-455. 1922.

292. Regaud, C., and T. Nogiert. Estimation différente des doses de rayon $x$ suivant les divers modes d'éclairage du chromoradiometre. Strahlentherapie 38: 19-26. 1930.

293. Reisner, A. Untersuchungen über die Veränderungen der Hauttoleranz bei verschiedener Unterteilung der Strahlendosis. Strahlentherapie 37: 779-787. 1930.

294. Reisner, A. Biologische Versuche über die Wirkung der verschiedenen Strahlenmengen bestimmter Radiumpraparate auf die Haut. Strahlentherapie 38: 19-26. 1930.

295. Reisner, A. Erythemversuche mit Grenzstrahlung. Fortsch. Geb. Röntgenstr. $45: 74-77 . \quad 1932$.

296. Reisner, A. Hauterythemverlauf bei fraktionierter Verabfolgung grosser Strahlenmengen. Fortsch. Geb. Röntgenstr. 45 : 293-307. 1932.

297. Reisner, A., and Th. C. Neef. Hauttoleranzdosis und Strahlenqualität. Strahlentherapie 34: 313-339. 1929.

298. Remer, J., and W. W. Belden. Roentgen diagnosis and therapy of the thymus in children. Amer. Jour. Roentg. and Rad. Ther. 18: 119-144. 1927.

299. Robinson, M. R. The effect of a castration dose of roentgen rays upon the rabbit ovary. Amer. Jour. Roentg. and Rad. Ther. 18: 1-24, 1927.

300. Roffo, A. H., and L. M. Correa. Die Einwirkung der Röntgenstrahlen auf den Cholesteringehalt der Karzinome. Strahlentherapie 18:871-874. 1924.

301. Rohrschneider, W. Experimentelle Erzeugung von Röntgenstrahlenkatarakt. Strahlentherapie 31: 596-600. 1928-29.

302. Rohrschneider, W. Über die Wirkung der Röntgenstrahlen auf das Auge. Strahlentherapie 38: 665-681. 1930.

303. Rominger, E., and J. JACHims. Erfährungen mit der Grenzstrahltherapie in der Kinderheilkunde. Deut. Med. Wochensch. 57: 1005-1008. 1931.

304. Rost, G. A. Die biologischen Grundlagen der Ultraviolett-Therapie. Strahlentherapie $16: 1-23.1924$.

305. Rost, G. A. Utber die biologische Wirkung der Röntgenstrahlen auf die gesunde und kranke Haut. Strahlentherapie 44: 521-540. 1932.

306. Rотнев, J. Über den Angriffspunkt der Röntgenstrahlenwirkung am biologischen Objekt. Strahlentherapie $27: 197-253.1928$.

307. Rottmann, H. G. Zur Kenntnis der Histologie des Grenzstrahlen-erythema. Strahlentherapie 27: 518-532. 1928. 
308. Rubin, E. H., and O. Glasser. The effect of roentgen irradiation on the veloeity of erythrocyte sedimentation. Amer. Jour. Roentg. and Rad. Ther. $17: 520.1927$.

309. Russ, S. Experimental studies upon the lethal doses of $x$-rays and radium for human and other tumors. British Jour. Radiol. 29: 275-292. 1924.

310. Russ, S., and G. M. Scort. The aetion of radon "seeds" on tumor and liver cells of the rat. British Jour. Radiol. 32 : 239-244. 1927.

311. Russ, S., and G. M. Scott. The differential action of x-rays and gamma rays upon some living tissues. British Jour. Radiol., N. S. 2: 301-306. 1929.

312. Russ, S., and G. M. Scott. The differential action of gamma rays. British Jour. Radiol., N. S. 5 : 814-822. 1932.

313. Saidman, J. Les propriétés biologiques et thérapeutiques des rayons de 4-9 angstroms. Bull. Mem. Soe. Radiol. Méd. France 18: 21-27. 1930.

314. Saint George, A. U., A. O. Gettler, and R. H. Muller. Radioactive substances in body five years after death. Archives of Pathol. 7: 397-405. 1929.

315. Salinger, H., and R. Thiel. Die Wirkung der Röntgenbestrahlung des Sympathikus auf das Auge. Strahlentherapie 42:96-112. 1931.

316. SAlis, H. V. Zu den Röntgenveränderungen naeh Bestrahlung der GlandSubmaxillaris. Strahlentherapie 17: 395-400. 1924.

317. Sanders, A. Zur Kenntnis der Röntgenschädigungen am Darm. Strahlentherapie 18: 457-472. 1924.

318. Schaffer, W., and E. Witte. Physikalische und biologische Versuehe an Kathodenstrahlen. Strahlentherapie, 31: 415-466. 1928-29.

319. Schall, L. Zur Hautery themfrage. Strahlentherapie $23: 354-360.1926$.

320. Schall, L. Untersuchungen über den Ablauf des Lichterythems. Strahlentherapie 28: 164-167. 1928.

321. Schall, L., and H. J. Alies. Zur Biologie des Ultraviolettlicists. II. Zur Frage der Messung der Hautreaktion. (Ein Neuer Erythem- und Pigmentmesser.) Strahlentherapie $19: 796-804.1925$.

322. Schall, L., and H. J. Alius. Zur Biologie des Ultraviolettlichts. Die Reaktion der menschliehen Haut auf die Ultraviolettbestrahlung. Strahlentherapie 23: 161-180. 1926.

323. Schereschewsky, J. W. Heating effect of very high frequency condenser fields on organic fluids and tissues. Publ. Health Repts. 48: 844-855. 1933.

324. Schinz, H. R. Gegenwärtige Methoden der Krebsbestrahlung und ihre Erfolge. Strahlentherapie $37: 31-49.1930$.

325. Schliephake, E. Über Tiefenwirkung und elektive Gewebswirkung kurzer elektriseher Wellen. Strahlentherapie 38: 655-664. 1930.

326. Schlundt, H., and G. Failla. The detection and estimation of radium in living persons. Amer. Jour. Roentg. and Rad. Ther. 26:265-271. 1931.

327. Schreus, H., and L. Schoenholz. Die Toleranzdosen der Haut in "Röntgen"Einheiten bei verschiedenen Strahlenhärten. Strahlentherapie 24: 485-500. 1927.

328. Schkötтer, H. Neue Arbeiten über Wirkung der Lieht und Wärmestrahlen auf den Organismus. Strahlentherapie 16: 96-103. 1924.

329. Schugt, P. Untersuchungen über die Wirkung abgestufter Dosen von Röntgenstrahlen versehiedener Wellenlänge auf die Struktur und Funktion der Ovarien. Strahlentherapie 27:605-662. 1928.

330. Schultze, W. Temperaturmessungen im Innern der Haut und deren Beziehung zur Abkühlungsgrösse. Strahlentherapie 39: 303-319. 1930-31.

331. Schwarz, G. Zur Kenntnis der Röntgenreaktion der Haut. Strahlentherapie 18 : 483-485, 845-848. 1924.

332. Schweizer, E. Utber spezifische Röntgenschädigung des Herzmuskels. Strahlentherapie 18: 812-828. 1924. 
333. Sharp, G. S. The $\mathrm{pH}$ of human mixed saliva during irradiation from intraoral carcinoma. Amer. Jour. Roentg. and Rad. Ther. 25:266-270. 1931.

334. Shouse, S. S., S. L. Warren, and G. H. Whipple. Aplasia of marrow and fatal intoxication in dogs produced by roentgen radiation of all bones. Jour. Exp. Med. 53 : 37-445. 1931.

335. Siciliano, L., and C. Banci-Buonamic. Studi sul Midallo osseo l'azione dei raggi x. Arch. di Radiol. 6: 1108-1136. 1930.

336. Siedemgrotzky, K. Über das Verhalten der Hautkapillarem im Röntgenbestrahltegebeit. Strahlentherapie 19: 84-123. 1925.

337. Sмүтн, F. S., and G. H. Whipple. Bile salt metabolism. II. Proteose and x-ray intoxication. Jour. Biol. Chem. 59:637-659. 1924.

338. Spurling, H., and R. Thiel. Effect on blood of irradiation, especially short wave length R. therapy. Amer. Jour. Med. Sci. 168: 215-241. 1924.

339. Stahel, E. Bestinmung der bei Gamma- und Röntgen-Strahlbehandlung vom Gewebe absorbierten Energiemengen. Strahlentherapie 33: 296-321. 1929.

340. Stahl, R., and G. Simsch. Untersuchungen über die Modifizierung der Erythem- und Pigmentbildung durch äussere Einflüsse. Strahlentherapie 27: 311-330. 1928.

341. Stahl, R., and G. Simsch. Über Beeinflussung von Erythem-und Pigmentbildung in der Lichttherapie. Strahlentherapie 28: 176-179. 1928.

342. Stenstrom, W., and W. L. Mattick. Study of skin reactions after divided roentgen ray dosage. Amer. Jour. Roentg. and Rad. Ther. 15:513-519. 1926.

343. Stenstrom, W., and M. Reinhard. Some measurements of the transparency of skin to light. Acta Radiologica 5 : 553-560. 1926.

344. Stephens, J. G., and H. Florey. An investigation of the immediate effects of $\mathrm{x}$-rays on living animal tissues. British Jour. Radiol. 32: 159-166. 1927.

345. Swann, M. B. R. A study of the immediate effects of x-rays on the functions of certain tissues and organs. British Jour. Radiol. 29 : 195-220. 1924.

346. TAFT, R. B. Concerning the visibility of roentgen rays. Amer. Jour. Roentg. and Rad. Ther. 28: 245-248. 1932.

347. Takahashi, T. The action of radium upon the formation of blood capillaries and connective tissue. British Jour. Radiol., N. S. 3:439-445. 1930.

348. Taylor, H. D., W. D. Wetherby, and J. B. Murphy. Studies on x-ray effects: I. Destructive action on blood cells. Jour. Exp. Med. 29: 53-75. 1919.

349. Thibaudeau, A. A., and W. L. Mattick. Histological findings in hearts which have been exposed to radiation in the course of treatment of adjacent organs. Jour. Cancer Res. 13 : 251-259. 1929.

350. Thomas, H. E., and F. H. Bruner. Chronic radium poisoning in rats. Amer. Jour. Roentg. and Rad. Ther. $29: 640-662.1933$.

351. Thomas, Marguerite, H. D. Taylor, and W. D. Wetherby. Studies on $x$-ray effects: II. Stimulative action on the lymphocytes. Jour. Exp. Med. $29: 75-82.1919$.

352. Thonsness, E. T. The effects of roentgen rays on cytology and absorption from experimental abscesses. Amer. Jour. Roentg. and Rad. Ther. 28: 81-86. 1932.

353. Tsukamoto, R. T'ther die Stoffwechselstörungen nach Bestrahlung der Leber mit Röntgenstrahlen. Strahlentherapie 18: 320-374. 1924.

354. Tsuzukı, M. Experimental studies on action of hard roentgen rays. Amer. Jour. Roentg. and Rad. Ther. 16: 134-148. 1926.

355. Turano, L. Sulle modificazioni dei capillari alle radiazione rontgen. Arch. Radiol. 6: 349-363. 1930.

356. Tyler, A. F., and J. R. Blackman. Effect of heavy radiation on pleura and lungs. Jour. Radiology 3 : 469-475. 1922. 
357. Uhumans, E. Über die Abhängigkeit der Pigmentbildung von der Wellenlänge der Strahlung. Strahlentherapie 35:361-368. 1930.

358. Uhlmans, E. Über histologische Untersuchungen der mit Vitalux Lampe bestrahlten Haut. Strahlentherapie 40: 765-769. 1931.

359. Uhlians, E. Experimentelle Untersuchungen über den Einfluss der Ernährung auf die Strahlenempfindlichkeit tierischen Gewebes. Strahlentherapie 46 : 550-563. 1933.

360. VALEeFF, I. Einfluss von Thorium $\mathrm{X}$ auf die Senkungsgeschwindigkeit der roten Blutkörperchen. Strahlentherapie $26: 363-378.1927$.

361. Verhoeff, F. H., L. Bell, and C. B. Walker. Pathological effects of radiant energy (U-V) upon the eye. Proc. Amer. Acad. Arts and Sci. 51: 627-818. 1916.

362. Vischia, Q. Les variations de la glycemie dans l'irradiation des surrénales. Riv. Rad. e Fisiea Medica, Jan., 1930. 71.

363. Vos Ries, J. Über die Durchlässigkeit der Haut für Ultraviolett. Schweiz. Med. Wochensch. $57: 248.1927$.

364. WAllbach, G. Über die Einwirkung von Thorium $\mathrm{X}$ auf die Leukozyten Kultur des Menschen mit besonderer Berücksichtigung der entspreehenden Benzolwirkung. Strahlentherapie 46: 675-696. 1933.

365. Wallgrex, A. Zur Kenntnis der biologischen Wirkungen der g-Strahlen. Acta Radiolog. 14: 111-121. 1933.

366. Walters, O. M., B. J. Axson, and A. C. Iry. The effect of x-rays on the thyroid and parathyroid glands. Radiology 16:52-58. 1931.

367. Walters, O. M., B. J. Axson, and A. C. Ivy. The prevention of hyperplasia of the thyroid in the opossum by x-rays. Radiology 18:583-591. 1932.

368. WARREN, S. I. A simple radium carrier and filter giving an easier approach to neoplasms of difficult access. Amer. Jour. Roentg. and Rad. Ther. 20: 563-570. 1928.

369. Warren, S. L. Physiological effects of roentgen radiation upon normal body tissues. Physiol. Review 8: 92.1928.

370. Warren, S. L., and G. H. Whipple. Intoxication. Unit dose over thorax negative over abdomen lethal epithelium of small intestine sensitive to x-rays. Jour. Exp. Med. 35: 187-202. 1922.

371. Warres, S. L., and G. H. Whipple. Intoxication-A study of sequence of clinical anatomical and histological changes following a unit dose of x-rays. Jour. Exp. Med. 35: 203-211. 1922.

372. Warren, S. L., and G. H. Whipple. Speed of autolysis of various body tissues after lethal x-ray exposures, remarkable disturbance in epithelium of small intestines. Jour. Exp. Med. 35: 213-224. 1922.

373. Warren, S. L., and G. H. Whipple. Roentgenotherapy in man in the light of experiments showing sensitivity of intestinal epithelium. Jour. Amer. Med. Assoc. 81: 1673. 1923.

374. WARREN, S. L., and G. H. Whipple. Bacterial invasion of the blood stream as influenced by x-ray destruction of the mucosal epithelium of the small intestine. Jour. Exp. Med. 38: 713-723. 1923.

375. Warren, S. L., and G. H. Whipple. The cumulative effect or summation of $\mathrm{x}$-ray exposures given at varying intervals. Jour. Exp. Med. 38: 725-730. 1923.

376. WARrex, S. L., and G. H. Whipple. The path of a beam of hard rays in the living organism. Jour. Exp. Med. 38: 731-739. 1923.

377. Warren, S. L., and G. H. Whiples. Intestinal lesions and acute intoxication produced by radiation in a varicty of animals. Jour. Exp. Med. 38: 741-752. 1923. 
378. Warthin, A. S., and E. A. Pohle. Effect of roentgen rays on the heart. Jour. Amer. Med. Assoc. 89: 1825-1829. 1927.

379. Warthin, A. S., and E. A. Pohle. Effect of roentgen rays on heart: Microscopic changes in heart muscle of rats and of rabbits following series of exposures. Arch. Int. Med. 43: 15-34. 1929.

380. Warthin, A. S., and E. A. Pohle. The effect of roentgen rays on the heart. III. The toleration dose for the myocardium of rats. Amer. Jour. Roentgen. and Rad. Ther. 25: 635-643. 1931.

381. Waters, C. A., and I. I. Kaplan. Year book of radiology, 1932 and 1933. The Year Book Publishers, Inc.; Chicago, 1932 and 1933.

382. Weatherwax, J. L., and C. RовB. Determination of radiation values in lung tissue with variable qualities of radiation. Radiology 14: 401-410. 1930.

383. Weil, E., and A. Lacassagne. Anémie pernicieuse et leucemie myéloide mortelles provoquées par la manipulation de substances radio-actives. Bull. Acad. Médecine 9 : 257. 1925.

384. We1s, H. A. Effect of radium upon rabbit ovaries. Surg. Gynéc. Obst. 36 : 373-382. 1923.

385. Werthemann, A. Experimentelle Röntgenschädigungen der Herzmuskels. Strahlentherapie 38: 702-709. 1930.

386. Wierig, A. Über Spätschädigungen durch Röntgenbestrahlung des menschlichen Körpers im Entwicklungsalter. Fortsch. Geb. Roentgenstr. 34 : 297-301. 1926.

387. Wigoder, Sylvia, B. The effect of x-rays on the testis. British Jour. Radiol., N. S. 2 : $213-222.1929$.

388. Willis, D. A., and A. Bachem. The effects of roentgen rays upon the kidney. Amer Jour. Roentg. and Rad. Ther. 18: 334-337. 1927.

389. Willis, D. A., and A. Bachem. Die Wirkung der Röntgenstrahlen auf die Niere. Strahlentherapie 27 : 121-123. 1928.

390. Wilhelmy, E. Ủber die Erythemreaktion der Haut auf Röntgenstrahlen, die 2.5-12 kv. entsprechen, und auf Kathodenstrahlen gleicher Absorbierbarkeit. Strahlentherapie 45: 388-393. 1932.

391. Winterstein, O. Lichtwirkung auf Bakterien. Strahlentherapie 39: 619-642. 1930-31.

392. Wintz, H., and W. Rump. Über die Tiefenwirkung der Röntgenstrahlen bei homogenen und inhomogenen Körpern. Fortsch. Geb. Roentgenstr. 29: 580586. 1922 .

393. Wolbach, S. B. A summary of the effects of repeated roentgen ray exposures upon the human skin, antecedent to the formation of carcinoma. Amer. Jour. Roentg. and Rad. Ther. 13: 139-143. 1925.

394. Wolfer, J. A. Chronic experimental uleer of the stomach. Jour. Amer. Med. Assoc. 87: 725. 1926.

395. Woodard, Helen, and Helen Downes. The effect of radiation on the aeidity of the blood. Amer. Jour. Roentg. and Rad. Ther. 25: 271-275. 1931.

396. Wright, S., and H. A. Bulman. Selective action of x-rays on blood cells of cat. Lancet $217: 217-219.1929$.

397. Wynen, W. Die Radiosensibilität des Knochens in ihrer Bedeutung für die Röntgenbestrahlung der Gelenkturberculose. München. Med. Wochensch. 76: 244-246. 1929.

398. Wucherpfennig, V. Biologie und praetische Verwendbarkeit der Erythemschwelle des Ultraviolette. Strahlentherapie 40 : 201-244. 1931.

399. Zacilerl, H. Beitrag zur Allgemeinwirkung der Röntgenstrahlen. Strahlentherapie $23: 272-287.1926$. 
400. ZACherL, H. Über die Bedeutung das Reticulendothelialen Apparates bei der Röntgenbestrahlung des Collumcarcinoms. Strahlentherapie 33 : 515-536. 1929.

401. Zimmern, A., and J. A. Chavany. Die Radiosensibilität des Nervengewebes. Strahlentherapie 41: 482-495. 1931.

402. Zuppinger, A. The protracted fractional method of roentgen therapy (Coutard). Amer. Jour. Roentg. and Rad. Ther. 25 : 784-792. 1931.

403. Zwerg, H. G. Die theoretischen, Experimentellen, klinischen und wirtschaftlichen Grundlagen der protrahiert-fraktionierten Röntgenbestrahlung maligner Tumoren. Strahlentherapie $43:$ 201-248. 1932.

404. ZWERG, H. G. Über die Allgemeinwirkung der Röntgenstrahlen mit besonderer Berücksichtigung ihres zeitlichen Auftretens. Strahłentherapie 45: 297-306. 1932. 



\title{
$\mathrm{XV}$
}

\section{SHORT ELECTRIC WAVE RADIATION IN BIOLOGY}

\author{
G. Murray McKinley \\ Zoological Laboratories, University of Pittsburgh
}

Introduction. Biological effects. Internal heat. Synthetic fever. An effect other than heat. Conclusions. Apparatus: General description-Push-pull oscillator-Halfwave oscillator. References.

\section{INTRODUCTION}

We are already familiar with the far-reaching effects obtaincd in biology by the use of gamma rays, of $\mathrm{X}$-radiation, of ultra-violet, of light, and of infra-red. We know that all of these form one great, continuous spectrum of electromagnetic waves, the various regions of which differ only in wave-length. Beyond the near infra-red is the radiation used by radio, which extends this spectrum into regions of very long waves, now commonly called Hertzian or electric waves.

Although this spectral band is continuous, there are still gaps not yet adequately filled by instrumental means, as, for example, the gap between $\mathrm{X}$-rays and ultra-violet. Another great gap, now being rapidly filled, lies between the near infra-red and the waves of wireless. It is only recently that short-wave radio transmission became a fact and advance in this direction has led to a continued reduction in the length of waves obtainable. This has largely been made possible by improvement of the three-electrode vacuum tube.

The modern short-wave vacuum-tube oseillator has permitted the study of the action of electric waves of a meter or less, and with such technical progress, research in the biological effects of this type of radiation has become increasingly important. The invention of the threeelectrode vacuum tube has been the door which opened to the biologist a whole new world of radiation. The region is inconceivably vast, extending from the near infra-red to a theoretical infinity, and it might seem quite difficult for the biologist to decide upon some locality as most suitable and promising for experimentation, were it not for certain practical and mechanical restrietions. The present-day vacuum tube, although one of the most marvelous of our physical instruments, is still unable to generate in adequate intensity wave-lengths of much less than 1 meter, so that the region of shortest electric waves cannot at present be utilized in biology. Again, apparatus generating wave-lengths much longer than 100 meters is not convenient in biology, and the 
experimenter has for the time being confined his researches to a range of wave-lengths of from 1 to about 100 meters.

The biologist applies this radiation by use of a tuned circuit excited by electrical oscillations of very high frequency $(3,000,000$ to $300,000,000$ cycles per sec.), the exciting oscillations being generated by a threeelectrode vacuum tube. Not being able to obtain this form of energy in sufficient amount unless the very closest possible approach is made to the radiating source, the biologist exposes his experimental material in a part of the radiating circuit itself. A part of this tuned circuit (as will be described in another part of this paper) is a high-frequency electric field which is present between the plates of a condenser. With the modern vacuum tube and associated circuits it is possible to provide in this space (in which exposures are to be made) an electric field of continuous wave current emitted sharply at the wave-length to which the circuit is tuned.

Biological material in this situation, that is, insulated between the condenser plates of the cireuit, is subjected to an electrical stress. The excitation circuit shifts the polarity of the condenser at the frequency employed, and consequently a very rapidly oscillating field is provided. No free electrons from the external circuit can enter, nor can any electrons flow out of the experimental material; but there may take place a displacement current in which electrons in the molecules of the material being exposed are stressed first in one direction and then in the other. Free electrons would tend to pass from molecule to molecule, and the molecules themselves, if bipolar, as indicated by recent researches in physics, would tend to rotate in response to the changing potential of the field.

Schereschewsky points out that this situation (an animal between the condenser plates) in equivalent electric circuit presents at least two possibilities. The first and more probable possibility is that the system (see deseription of apparatus) may be regarded as two condensers in series connected by a resistance, the first condenser being formed by one plate of the metallic condenser and one side of the body surface, the intervening glass acting as a dielectric. The second condenser is formed in similar fashion by the other side of the body, the other plate of the metallic condenser, and the intervening glass. Between these two condensers in series is the bulk of the body tissue of the animal acting as a connecting resistance. The other possibility in equivalent electric circuit is that the animal's body may act in the circuit as a dielectric of poor quality. In this case the electrical equivalent would be a condenser shunted by a resistanec.

Such an intimate "stirring" of the fundamental constituents of matter might well be expected to affect very profoundly the complex and delicately balanced structure of living material, and such the biologist finds 
to be the case. It is here, then, in the region of relatively short electric waves and with special apparatus for the concentration of the waves, that we find the biologist at work. He discovers at the very beginning of his work that in this as in other regions of the electromagnetic spectrum the waves exert a potent influence on living material.

\section{BIOLOGICAL EFFECTS}

The biological effects of high-frequency electric fields as generated by the vacuum tube appear to have been first investigated by Gosset, in 1926, and his coworkers in France. Schereschewsky (27, 28), Christie and Loomis (5), and McKinley (13) were the first American workers to use the new three-electrode vacuum tube, while Schliephake $(30,31)$ appears to have been the first in Germany.

Gosset and his coworkers, Gutman, Lakhovsky, and Magrou, studied the effects of very high frequency fields upon plant tumors caused in Geranium by Bacterium tumefaciens. They reported that exposure of the plants to this radiation brought about, eventually, a necrosis of the tumors.

Schereschewsky (27) seems to have been the first to subject animals to the action of high-frequency fields as generated by the vacuum tube. He found that exposure caused severe symptoms which might result in death if the exposure was prolonged more than a few minutes. Some, at least, of these symptoms he thought to be due to heat retention. Besides this acute lethal effect, he found that exposure caused destruction of tissue. After sublethal exposures, in many instances, small hemorrhagic areas were observed along the course of blood vessels of the ears and within a few days the ears became necrotic and dropped off. This was also true of the tail.

Schereschewsky used mice in his work and found considerable individual variation in response to the influence of the radiation and part of his early work consisted in determining the average lethal time for various frequencies. Differences in individual response to the radiation made his later work difficult and have been a source of trouble to all other workers in biology. This same individual variation was studied in 1930 by MeKinley and Charles (15) using an insect (the parasitic wasp Habrobracon) and the coefficient of variability in a series of closcly controlled experiments at a constant frequency was found to be more than 56 per cent.

Schereschewsky attempted to compare the effect of various frequencies and to establish an optimal frequency for lethal action on the mouse. He described such optimal frequencies, and came to the conclusion that the frequencies of highest lethality lay between $18,000,000$ and $66,000,000$ cycles per sec. He ascribed the phenomena at that time to the specific action of the radiation in the form of electromechanical 
vibrations in the living cell, and only in part to the generation of heat by convection currents.

However, Christie and Loomis (5) pointed out that the method of Schereschewsky for comparing the outputs of energy at different frequencies was by no means correct, because the ammeter reading in the resonating circuit, used as a standard by Schereschewsky, gives only an indication of the peak of resonance in the secondary circuit, rather than an absolute gauge of energy across the plates (see section on Apparatus). Unfortunately, however, the method used by Christie and Loomis to measure and compare the output across the plates was also incorrect. They used the rate of heating of a saline thermometer placed between the condenser plates as a gauge for comparison of the outputs developed between the plates at different frequencies. McLennan and Burton (19) pointed out that the saline thermometer could not be used in this situation because of the possibility that variation in frequency by itself leads to an altered rate of heating for the same field intensity between the condenser plates.

McLemnan and Burton proved that for several electrolytes, including solutions of sodium chloride, there exists a specific concentration for which at a constant wave-length a maximum rate of heating is developed. These two workers and others have found that the maximum heating will be shifted with the frequency independent of the field intensity developed across the plates, and therefore the rate of heating in a saline thermometer of a given concentration of sodium chloride cannot be used to compare the output for different frequencies.

Schereschewsky in his early work believed that he had demonstrated a specific frequency band in which the mouse was killed much more quickly than at other regions in this end of the spectrum, and that death was not altogether due to internal heating. Christie and Loomis concluded, albeit from procedures shown to be unsound, that there was no specific action on the part of short electric waves and that the lethal effect on mice was due solely to heat.

These two more or less contradictory experiments leading to different interpretations of the effect of the action of high-frequency electric fields started a controversy which has continued to the present time. On the one side are those workers who believe that the biological effects of this radiation are due solely to the generation of internal heat-a heat generated perhaps by the setting up in the body of the exposed animal of a displacement current. On the other side are those workers who believe their experiments demonstrate an effect other than that of simple, internal heat.

\section{INTERNAL HEAT}

Attempts to measure the degree of heat generated in the living body have given only approximate results, owing to mechanical and other 
difficulties to be pointed out later, but under strong dosage it was evident that the animal body was subjected to the equivalent of a high degree of external heat. MacCreight and McKinley (12) attempted an indirect measure of the heat generated in the body of young rats when subjected to a dosage which killed them in about 5 min. They found that although the rectal temperature at death never rose higher than $46^{\circ} \mathrm{C}$., the actual temperature to which the animal was subjected was much higher. They compared the macroscopic picture as presented by animals killed by high-frequency fields and those killed by various degrees of external heat and found that only when external temperatures reaching $160^{\circ} \mathrm{C}$. were used, did the effects of external heat compare to those of the high-frequency field.

That the amount of heating in all tissues is not the same was pointed out by Schlicphake (31). Schliephake compared the action of short electric waves (2.8 meters) with the action of ordinary diathermic currents, in the heating of particular tissues. He found that diathermy (frequencies of 100,000 cycles per sec.) selectively heated the fatty tissue, and that the short electric waves heated liver and bone to the greatest extent, these data being obtained by the use of the rate of cutaneous heating as a standard. McLennan and Burton (19) confirmed these observations, and were in accord with Schliephake regarding the depth of action of the high-frequency ficld. They were of the opinion that at these high frequencies the so-called electrical "skin effect" in tissues is of little significance, it being more probable that the radiation passes directly through the living cell rather than around it in the intercellular substance.

Further heating-effect studies were made by several other workers. Richards and Loomis (25a) found that the heating effect for electrolytes in a high-frequency field has a maximum that depends on conductivity and frequency. Kahler, Chalkley, and Voegtlin (11), working with a protozoan, Paramecium caudatum, came to the conclusion from their experiments that the lethal effect was due entirely to heat. They found that death occurred at the same temperature whether the organism was killed by an agent of external heat or by a high-frequency ficld.

\section{SYNTHETIC FEVER}

Another group of papers dealing with the temperature aspects of elevated temperatures in general and in particular with those induced by high-frequency irradiation has been adequately summarized by Carpenter and Boak (3) and these workers themselves reported that high-frequency irradiation (wave-length 30 meters) causes elevations of temperature and prevents the development of experimental scrotal chancres in rabbits.

Carpenter and Page (4), taking advantage of the very quick and certain heating effect of exposure to the high-frequency field, were able 
to obtain synthetic fevers in animals, including man, without the injection of foreign substanees. They followed the suggestion of Hosmer (9) who had studied heating effects on salt solutions of various concentrations and on small laboratory animals, and they have contributed a therapeutic agent of very great possibilities. By proceeding cautiously they were able to treat 25 human patients, obtaining fevers of $104^{\circ}$ to $105^{\circ} \mathrm{F}$. in 60 to $80 \mathrm{~min}$. The patient was suspended in the field so that the waves penetrated the body from one side to the other; the head was not exposed. They were careful to use the lowest possible current and seem to have avoided entirely the skin and muscle blistering found by MacCreight and McKinley (12) to accompany hard dosage.

Carpenter is convinced that in spite of the crudities of the present-day apparatus this method of obtaining artificial fevers is not only practical but efficient and will prove of great value to the clinician, physiologist, biochemist and bacteriologist. He also studied the relation of this synthetic fever to infectious diseases in laboratory animals and believes that two desirable effects are obtained by raising the body temperature. First, the increased heat within the body makes a less favorable environment for the multiplication of virus. Second, the heat increases the rate of those chemical processes concerned with the development of immunity and with the general defense mechanism of the body against infectious agents. The general basis for this latter belief is the unquestionable stimulation of metabolism accompanying an artificial fever. Relative data have been reported by Nasset and Warren (22) who found that in dogs subjected to this exposure there was a depletion of blood sugar and a diminution in the carbon dioxide content of the blood. These phenomena indicate that synthetic fever obtained by means of the highfrequency field leads to a markedly increased metabolism.

In this connection both Schliephake and McKinley found that attempts to prolong synthetic fevers in mice for more than $12 \mathrm{hr}$. result in a profound disturbance of the body-temperature-regulating mechanism, and after continued treatment the body temperature tends to fall below normal. Animals so treated of ten die. It is believed that only synthetic fevers of very short duration are safe.

The factor of internal heat observed in these experiments has been obvious in all work with the high-frequency field. It is, after all, the effect one would expect from an a priori examination of the nature of the electric field. Kahler and his coworkers (11) pointed out that the photochemical effect in the high-frequency field is negligible.

\section{AN EFFECT OTHER THAN HEAT}

Internal heat, then, as a major factor is admitted by all workers who are using this radiation; but there is a growing conviction on the part of several that heat, as such, is not the only factor involved-that there 
appears to be another and secondary factor which can only be demonstrated when the first is ruled out. This secondary factor is spoken of by those who are attempting to demonstrate it as "an effect other than that of simple heat."

Schereschewsky in his early work believed that the heat factor was only partly responsible for the effects observed, and he was also impressed with the possibility of a differential action on the part of various frequencies. He had observed that tumorous tissue seemed to be more susceptible to this radiation than other tissues, and to test this he chose the tissue cells of transplantable tumors. He selected for his experiments a most virulent mouse sarcoma, implantation of which yielded 95 to 96 per cent of "takes." Treatment was localized as far as was possible to the cancer cells, the tumors being placed between small insulated plates.

Soon after beginning his experiments Schereschewsky noted that in favorable cases treatment had a pronounced effect upon the tumor, the mass seeming to become much smaller and softer almost immediately after exposure. Many technical difficulties arose during the course of these experiments, but the most troublesome was the association of this tumor with a diphtheroid bacillus, which of itself is pathogenic for mice. However, despite all difficulties, of 403 mice treated 100 recovered tumorfree. No case of spontaneous recession of the tumor was observed in 230 control mice.

In these experiments Schereschewsky did not attempt to rule out the heat factor completely. Schliephake $(33,34)$ had observed differential heating effects on various tissues of the animal body and came to the conclusion that there was some indication that the biological effects of ultra-high-frequency fields may be due in part to some action other than that of simple heat, but he, like Schereschewsky, had not eliminated the heat factor.

McKinley (13), in work with the nervous tissue of the frog, attempted to rule out the heat factor by comparing the action of both external heat and the high-frequency field on this tissue. He found that in irradiations of the isolated spinal cord, exposed by dissection, definite action on the central nervous system was demonstrated. External heat over a wide range of temperatures did not duplicate the characteristic action of the high-frequency field on this tissue. Headlee and Burdette (7) found indications of differences in lethal time from high-frequency radiation in insects, the differences in time apparently corresponding to the amount of nervous tissue present.

These somewhat indefinite indications of an effect other than simple heat were given additional and very strong support by Szymanowski and Hicks (36) who were able to attenuate diphtheria toxin by means of the high-frequency field. In a series of very carefully conducted experiments these two workers completely ruled out all gross heating effects and yet 
obtained definite attenuation of the three major toxins, diphtheria, tetanus, and botulinus. The effect was obtained without the development in the toxin of temperatures that would by themselves affect the potency of the toxin.

Bennedetti (2), using diathermy, and later McKinley (13), using the modern vacuum-tube circuit, obtained a slight acceleration of growth in the early germination period of seedlings. In these experiments the heat factor was presumably ruled out.

Schliephake $(33,34)$ reported that staphylococci may be susceptible to the direct action of ultra-high-frequency currents. They were not, however, entirely convinced that the heat factor had been eliminated. The organisms were exposed to the electric field in broth cultures. Szymanowski and Hicks (35) reported on a number of attempts to obtain lethal action, or at least to stimulate dissociation, in pathogenic bacteria. They treated the Dick hemolytic streptococcus of scarlet fever in broth culture to a high-frequency field and in several experiments observed a delay in growth. They did not observe any lethality or dissociation.

Although the results of this work were essentially negative, Hicks and Szymanowski expressed the opinion that it might be possible with a smaller volume of broth, the use of a thin film of culture, and many additional technical improvements with the short-wave generator to demonstrate some action on bacteria. They also pointed out that the selective absorption of broth cultures, wherein there may be large thermal gradients between the broth, or continuous phase, and the organism, or discontinuous phase, makes it difficult to determine the anount of absorption in the bacterial cell itself. They thought that this factor might be partially eliminated by working with a suspension in which cellular elements constituted a high percentage of the total mass.

Oartel and McKinley (24) found that treatment of the organisms normally present in fragments of infected human teeth had a lethal effect in 30.5 per cent of the samples treated. The arrangement or grouping of the organisms was affected in 15 per cent, the changes noted being for the most part a change from streptococcus forms to diplococcus forms, or from long chains to short chains. Also, of the total number treated a marked reduction in size of the organisms was noted in about 15 per cent. Treatment was so adjusted in these experiments as to avoid the generation of any measurable heat in the teeth fragments.

Attempts to rule out the "heat factor" have usually been along two lines: either by use of a constant temperature system or by treatment in extremely weak fields. Szymanowski's experiment with toxins and McKinley's work with germinating corn, both already described in this paper, are cases in point. Both of these experiments, although they strongly indicate specific effects other than heat, leave something to be desired, since it is not absolutely certain that the small amount of heat 
generated in the treated material was not in some way the cause of the effects observed.

Most biological material does not lend itself readily to such a delicate test as the above, but it occurred to the writer that in the incubation of the chicken's egg there might be possibilities of a more favorable comparison of the effects of the direct application of heat and high frequencies. In this case the essential technique consisted in substituting the heating effect of the field during a part of the natural incubating period. Providing the technique was properly handled, it was thought that if high frequencies had no other effect than that of pure heating, no change would occur in the natural development of the chick.

In this experiment unpublished and incomplete data indicate that treatment of very early stages (before or during the formation of the primitive streak) in the chick has no apparent effect on development. Treatment of the chick embryo at the 48-hr. stage or later, however, has a marked effect on development. The action of the field in 76 per cent of the cases studied was that of a delayed lethal result. Macroscopic and microseopic study of the embryos failed to reveal the eause of this delayed lethal effect.

The average time of treatment in this experiment was about $10 \mathrm{hr}$.; and, since every possible precaution was taken during this time to maintain by the action of the field the correct degree of incubation temperature, this is thought to very strongly indicate that external heat and high-frequency fields are quite different in their action upon living material.

It is probable that the embryos exposed to the field while in very early stages are not affected because of the more or less indifferent nature of the tissue present before or during the formation of the primitive streak. At $48 \mathrm{hr}$. or later nervous tissue predominates and it may be, as indicated by other experiments in this paper, that this tissue was in some way injured. The injury was not, evidently, such as to kill the embryos immediately but was sufficient to stop development after a few days.

\section{CONCLUSIONS}

The experimental results of all investigators clearly demonstrate that the electric field is capable of profoundly modifying living tissue, and use of this source of energy has not only produced many interesting biological effects but has opened a number of new lines of investigation.

Synthetic fevers can be generated in the animal body, affording a new and well-controlled method for the experimental study of fever physiology and of the temperature-regulating mechanism.

Its possible utility in studies of growth is demonstrated by its power to bring about acceleration in the germination of seeds. 
Dissociation and size changes in bacteria are, apparently, produced by this agency, and such action by electric fields may be of value to the modern bacteriologist.

The possibility that this agent produces a specific effect on nervous tissue, and to a lesser degree on other tissues, opens the door to important researches in both physiology and therapeutics.

The destruction of various parts of the nervous system, especially the brain, without dissection of the tissue surrounding them is shown to be possible and this may be of some use in the study of the function of these parts.

Modification of the course of development in vertebrate embryos is a possibility and in the future the proper manipulation of this source of energy may prove of value to the experimental embryologist.

It is at least strongly indicated in this paper that high-frequency fields and external heat are in no way comparable. Many of the observed effects of electric fields could not be duplicated with external heat as the agency, and, according to modern physical theory, such duplication is not to be expected. The action of the electric field is carried to the very foundations of the matter exposed to its influence. This action is instantly distributed throughout the whole body of the specimen and does not depend, as does external heat, upon a relatively slow penetration. The specific action of high-frequency fields is not clearly understood, but in such substances as the animal and plant body, exposed as indicated in this paper, it is suggested that the following actions may occur:

Conduction currents, or the flow of ions, are set up in the tissues of the body and these currents oscillate at the frequency of the field. It is possible that the specific effects on nervous tissue are due to conduction currents.

All permanent molecular dipoles are rotated by the action of the field. In living material, with its very large protein molecules, this may be an important factor in bringing about changes in protoplasmic balance.

Under the action of high frequencies temporary dipoles would tend to form and this would introduce possible hysteresis effects, a phenomenon which accounts in part for the generation of internal heat.

Any body potentials which might be present between or within tissues might be altered by the rapidly changing potential of the field.

\section{APPARATUS}

\section{GENERAL DESCRIPTION}

The recent increase in commercial importance of the ultra-highfrequency radio spectrum has greatly advanced the apparatus and circuits available for the production of high-frequency oscillating currents in the region of 60 megacycles. 
At the present time the best method of generating oseillations of the order of 60 megaeyeles is that of using triode vacuum tubes in regenerative circuits. The physical prineiples of such eireuits are now fairly well understood and the problem of generating ultra-high frequencies resolves itself into a process of designing the equipment in such manner as to eliminate all extraneous eapacities and inductances from the main oscillatory circuit. Triode high-frequency oseillators are of two general types. One type of cireuit is composed of the usual inductance and capacitance elements, while the second type of oseillatory eircuit depends upon the rate of propagation of electrons between the elements of the triode. The latter type is capable of produeing the highest frequencies but has the disadvantage that the power obtainable is quite limited. Regenerative oseillators have been found to be the most suitable for producing the frequeneies of interest to the biologieal investigator.

Regenerative oseillators which will function in the region of 60 megacycles are not difficult to set up, provided certain factors are recognized and provided for. It is important to recognize that as the frequeney progressively increases, the values of inductance and capacitance necessary to produce resonance decrease until a point is reached where a straight piece of wire represents a considerable amount of inductance. Also, the smallest of stray eapacities at ordinary frequencies often becomes the limitation of the highest frequencies obtainable in vacuumtube cireuits. This is particularly true of the interelectrode capacitance of a vacuum tube and also the distributed capacitance of the leads connecting the base of the vacuum tube to the elements of the tube. It is therefore necessary in constructing oscillators for ultra-high frequencies to reduce these stray eapacities and inductances to the smallest possible value consistent with the mechanieal limitations involved.

From the foregoing it can be seen that the proper place to begin in the design of ultra-high-frequeney equipment is with such cireuits and tubes as will lend themselves to the reduction of all capacities and inductances which are external to the main oscillatory cireuit. The oscillatory cireuits for ultra-high frequencies are similar to those used in low-frequeney work, the only essential difference being in the values involved. At ultra-high frequencies a piece of wire a few inches long has sufficient induetance when used in connection with the interelectrode capacities of most commercial vaeuum tubes to form an oscillatory circuit, the fundamental of which is in the neighborhood of 60 megacycles.

Referring to Fig. 1, it will be noted that the circuit represented is the familiar Hartley oscillator arranged for the operation of two $71 / 2$-watt tubes in push-pull. The inductances for the grid and plate eireuit are composed of single turns of wire, the diameter of which may be varied in order to vary the fundamental frequency of the oscillator. The limiting frequency of commercial tubes is reached when these inductances 
$L 1-L 2$ have been reduced to the point of connecting directly from one tube base to the other. The circuit elements then remaining for the production of high-frequency currents consist of the grid-to-plate capacitance of the two tubes (in series) combined, and the inductance of the leads connecting these elements. Naturally, when such a point is reached, it becomes difficult to take power from the tube circuit, owing

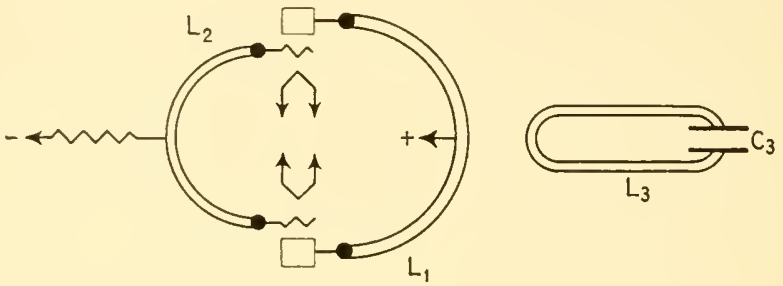

FIG. 1.

to the limited magnetic coupling possible. Therefore, the practical fundamental frequency possible when using commercial tubes is that frequency which is generated when some external inductance in the form of a loop remains for coupling to an experimental circuit where the energy will be used. Such a circuit is indicated in Fig. 1 as $L 3-C 3$. The physical

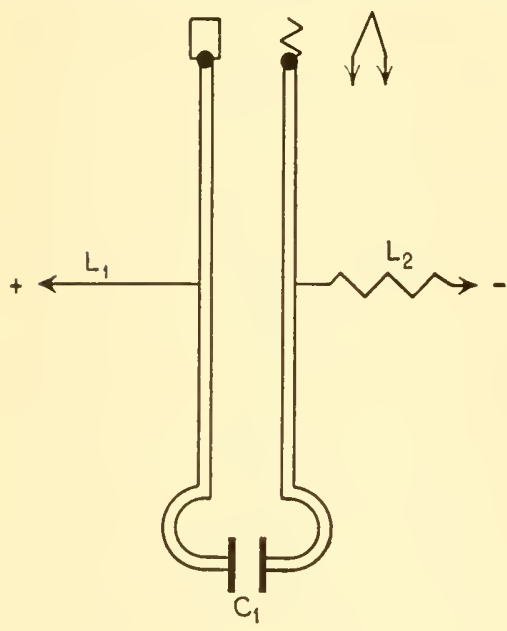

FIG. 2. details of this circuit as well as that of Fig. 2 will be discussed later.

The voltage distribution in the circuit shown in Fig. 1 is such that it is maximum at the grid and plate ends of the two inductance loops and zero at the point where the plate voltage and grid resistor are connected. It is necessary then to couple $L 3$ and $C 3$ to the oscillator circuit so as to provide a region for exposing biological material. This is provided between the plates of condenser $C 3$ in the exposure circuit. It is necessary to provide also a means of resonating the exposure circuit to the frequency of the oscillator circuit and this is usually accomplished by varying the size of loop $L 3$ after the biological material has been placed between the condenser plates in the exposure circuit. The amount of high-frequency voltage available across the plates of the condenser $C 3$ is controlled by varying the magnetic coupling factor between $L 1$ and $L 3$, or by fixing the magnetic coupling and varying the voltage applied to the oscillator tubes. All of these items have a variable effect upon the frequency of the oscillations generated, but it has been found that within 
limits such variation is not important to the biological investigator. The maintenance of constant voltages across condenser $C 3$, however, is important and the circuits used in this work were designed with this factor in mind.

The circuit in Fig. 2 is set up with the intention of simplifying the method of obtaining high frequencies for biological experimentation. As far as the mode of oscillation is concerned, this circuit is similar to that of Fig. 1. Instead of two tubes in push-pull, however, we now have one tube, and, at the other end of the inductance loops, a capacitance composed of two plates spaced apart a distance sufficient that the capacitance of the condenser so formed will roughly approximate the interelectrode capacitance of the vacuum tube at the other end of the oscillatory circuit. When this is done, the voltage distribution is a maximum at the terminals of condenser $C 1$ and at the grid and plate of the vacuum tube supplying the power to the oscillatory circuit. Plate voltages and grid-bias voltages may be fed to the circuit nidway between the tube and condenser, since the voltage at these points is zero. As will be indicated later, it is possible to place the experimental material between the condenser plates of $C 1$, and it is unnecessary to have a separate exposure circuit.

It is necessary in any regenerative type of ultra-high-frequency oscillator that it be mechanically symmetrical. This is done in order to provide the shortest possible connections and to reduce the resistance losses of the circuit to a minimum. Only the best of dielectric materials should be used in order to keep down losses and increase the power available from a given size of vacuum tube. Too much emphasis cannot be placed on the size and kind of conductors used in the construction of ultra-high-frequency oscillating circuits. Copper tubing is considered best since it provides a maximum conducting section for a given diameter of conductor. Precautions in this respect combined with a limited use of dielectric supports will provide a low-resistance circuit.

In any vacuum-tube-oscillator circuit the function of the vacuum tube is to supply the energy for oscillation. In addition to this the vacuum tubes of ordinary commercial design also become a part of the oscillating circuit they supply, and, as pointed out, increase the difficulty of obtaining high-frequency oscillations. Certain types of tubes have recently been designed in which the detrimental effects of the interelectrode capacities of the tubes have been reduced. Such tubes are, however, at the present time unavailable, and therefore the characteristics of the tube have to be considered together with the inductances and capacitance relations of the oscillator circuit.

Mention has been made of the desirability of maintaining, insofar as it is possible, a constant voltage across the plates of the condenser used to expose biological material. At first thought it would seem possible to 
accomplish this by providing an ammeter to read the current flowing to the condenser plates; and were the frequency low enough, this would be entirely practical (calculation of voltages from eapacity reactance of the condenser). However, at the extremely high frequencies used in biological work accurate current-measuring instruments are not available, and it is necessary to arrive at some approximation by other means. One method is to expose a column of neon gas to the dieleetrie field of the condenser. At the fixed frequeney used in any one series of experiments, it is found that increasing or decreasing the field of the condenser raises or lowers the height of ionization in the gas column. At a fixed frequency the indications are found to be quite sharp and it is possible by this means reasonably to standardize the dosages. This prineiple has lately been applied to volume indicators on radio-broadeast receivers where it is again used as a voltage-indicating device. It is quite true that such a device absorbs power from the oscillatory circuit and this power must be made available in addition to that required by the biological material.

\section{PUSH-PULL OSCILLATOR}

In Fig. 3 is described a high-frequency push-pull oseillator using rectified-alternating-current power supply. Two UX210 71/2-watt tubes supply power to the oscillating cireuit $L 1-L 2$. $\quad L 1-L 2$ are made of $1 / 4$-in. copper tubing formed into a half eirele connecting the grids and the plates of the tubes. The radius of this eirele is approximately 6 in. Midway along the two conductors are connected choke $X 2$ and resistor $R 1$. Choke X2 is a eoil of 100 turns of No. 38 D.C.C. wire wound on a tube $1 \mathrm{in}$. in diameter. Resistor $R 1$ is induetively wound and has a resistance of approximately $10,000 \mathrm{ohms}$. The four chokes, $X 1$, in the filament cireuit of the tubes are made of 10 turns of No. 14 wire on a core $1 \mathrm{in}$. in diameter and the turns are spaced the diameter of the wiring. By-pass condensers $C 1$ are small mica condensers having a value of 2,000 $\mu \mu \mathrm{f}$. Resistor $R 2$ provides the filament center tap and is a General Radio type 437 , having a total resistance of $60 \mathrm{ohms}$. By-pass eondenser $C 4$ has a value of $2,000 \mu \mu \mathrm{f}$, and is of sufficient voltage rating to stand the voltage of the power supply. Resistance $R 3$ is a voltage divider resistance across the D.C. output of the high-voltage vacuum-tube rectifier and is a General Radio type 446. The resistance is approximately 15,000 ohms. Condensers $C 2$ and $X 3$ comprise the 120 -eyele filter in the vaeuum-tube reetifier output. Condensers $C 2$ have a value of $8 \mu \mathrm{f}$. each and $\mathrm{X} 3$ a value of $20 \mathrm{~h}$. at $50 \mathrm{ma}$. This reetifier filter is known as a General Radio type 527-A. A UX280 full-wave rectifier tube is used for rectification. Transformer $T 1$ supplies the A. C. voltages for the rectifier and for the filament eireuit of the oseillator tubes and the reetifier tube. This transformer is a General Radio type 565-B, rated at 200 watts with a high-voltage output of 600 volts each side of the center tap. 
The power supply is mounted separately from the oscillator and the necessary leads are run to the proper points of connection in the oscillator circuit. The oscillator circuit is mounted on a baseboard in approximately the manner shown in the circuit sketch. This simplifies wiring and provides the most direct high-frequency paths for the oscillator current. The frequency range covered by this oscillator may be varied by varying the size of the loops $L 1$ and $L 2$. With the dimensions mentioned the frequency of generation will be in the neighborhood of 60 megacycles.

\section{HALF-WAVE OSCILLATOR}

Figure 4 shows the detailed connections and layout of a half-wave oscillator which supplies voltages to condenser $C 1$ for test purposes.

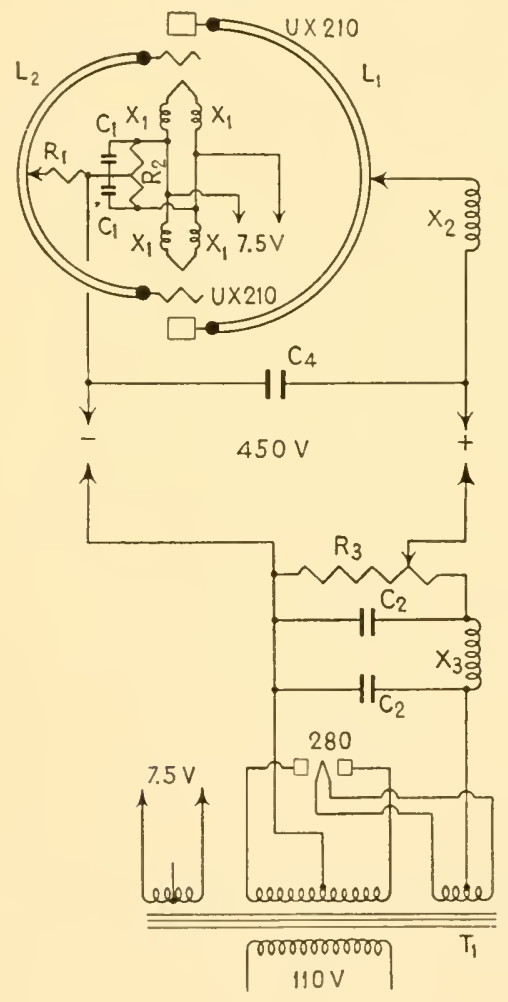

FIG. 3.

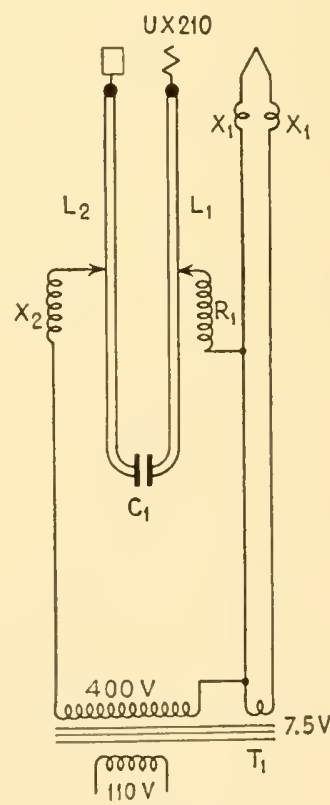

Fig. 4.

The circuit uses a UX210 tube and the values of $X 1, X 2, R 1$, and $T$ are the same as those shown in Fig. 3. The inductance rods $L 1$ and $L 2$ are made of $1 / 4-$ in. copper tubing. One end of the rods connects directly to the base of the vacuum tube, while on the other end is mounted a condenser made of two copper plates approximately 3 in. square and 
spaced at distances of from 1 to 3 in. apart. The two rods, tubes, and condenser $C 1$ are mounted on a baseboard in such a manner that the two rods, $L 1$ and $L 2$, parallel each other about 3 in. apart. The length of these rods and the capacity of condenser $C 1$ may be varied to vary the frequency of the oscillator. Shortening the rods will increase the frequency of the oscillator. The points of connection of $X 2$ and $R 1$ are important. They should be connected at the point of zero voltage along the parallel inductance rods $L 1$ and $L 2$. The proper point of connection will vary with the capacity of $C 1$ and must be found experimentally after the dielectric to be exposed between the plates of condenser $C 1$ has been inserted. Once the point is determined, the connections can be made permanent. The method used in locating the point of zero voltage is to pass a small tube containing neon gas along the conductor to a point where it ceases to glow. The voltage at this point is very near the electrical center of the system.

This circuit, when set up as shown in Fig. 4, will produce oscillations of the order of 60 megacycles and the field of $C 1$ provides an excellent exposure point for biological material.

The above circuits may be, of course, adapted to much more powerful vacuum tubes than here listed, but the standard UX210 tubes, or the like, are sufficient for most biological purposes. Circuits using tubes up to $1 / 4 \mathrm{kw}$. are fully described in the literature.

\section{REFERENCES}

1. Baldwin, W. M., and N. G. Nelson. Histological effects produced in albino rats by high frequency currents. Proc. Soc. Exp. Biol. and Med. 26:588. 1929.

2. Bennedett, E. Intorno all'azione del campo electromagnetico oscillante ad alta frequenza su alcuni germi vegetali. Atti R. Accad. Naz. Lincei Rend. Cl. Sci. Fis. Mat. e Nat. $4:(7 / 8): 324-332.1926$.

3. Carpenter, C. M., and R. A. Boak. Effect of heat produced by ultra-high frequency oscillator on experimental syphilis in rabbits. Amer. Jour. Syph. 14: 346-365. 1930.

4. Carpenter, C. M., and A. B. Page. Production of fever in man by short radio waves. Science 71: 450-454. 1930.

5. Christie, R. V., and A. L. Loomis. The relationship of frequency to physiological effects. Jour. Exp. Med. 49:302. 1929.

6. Gosset, A., A. Gutmann, G. Laknowskr, and I. Magrou. Essai de therapeutique du "cancer expérimental" des plantes. Compt. Rend. Soc. Biol. [Paris] 91: 626-628. 1924.

7. Headlee, T. J., and R. C. Burdette. Some facts relative to the effect of high frequency radio waves on insect activity. Jour. New York Ent. Soc. 37 : 59-64. 1929.

8. Horlacher, W. R. An attempt to produce mutations by the use of electricity. Science 72: 96-97. 1930.

9. Hosmer, H.R. Heating effects observed in a high frequency static field. Science 68 : 225-237. 1928.

10. Huxford, W. S. Standing waves on parallel wires. Phys. Rev. Corning, N. Y. 2nd Ser. 25: 686-695. 1925. 
11. Kahler, H., H. W. Chalkley, and C. Voegtrin. The nature of the effect of a high frequency electric field upon Paramecium. Publ. Health Repts. 44: 339-347. 1929.

12. MacCreight, J., and G. M. McKinley. Biological effect of temperature variations with high frequency oscillations. Proc. Soc. Exp. Biol. and Med. 27: 841-843. 1930.

13. McKinley, G. M. Some biological effects of high frequency electrostatic fields. Proc. Pennsylvania Acad. Sci. 4: 43-46. 1930.

14. MCKinley, G. M. The ultra-high frequency magnetic electric field in biology. Univ. of Pittsburgh Bull. 30: 2. Nov., 1933.

15. McKinley, G. M., and D. R. Charles. Certain biological effects of high frequency fields. Science 71: 490. 1930.

16. McKinley, G. M., and J. G. McKinley. The vacuum tube oscillator in biology. Quart. Rev. Biol. 6: 322-328. 1931.

17. McKinley, J. G., and G. M. MCKinley. High frequency equipment for biological experimentation. Science 71: 508-510. 1930.

18. McLennan, J. C. The heating effect of short radio waves. Jour. Maryland Acad. Sci. 2 : 44.1931.

19. Mclennan, J. C., and A. C. Burton. The heating of electrolytes in high frequency fields. Canadian Jour. Res. 3 : 224-240. 1930.

20. Mclennan, J. C., and A. C. Burton. Selective heating by short radio waves and its application to electrotherapy. Canadian Jour. Res. 5: 550-566. 1931.

21. Mellon, R. R., W. T. Szymanowski, and R. A. Hicks. An effect of short electric waves on diphtheria toxin independent of the heat factor. Science $\mathbf{7 2}$ : 174-175. 1930.

22. Nasset, E. S., F. W. Bishop, and S. L. Warren. Physiological effects of high frequency current. Amer. Jour. Physiol. 96: 439-448. 1931.

23. Nasset, E. S., and S. L. Warren. Some metabolic changes occurring in prolonged diathermy treatments. Proc. Soc. Exp. Biol. and Med. 27: 943-944. 1930 .

24. OARtel, J. S., and G. M. McKinley. Report of the effect of radiation on infected, extracted teeth. Trans. Rochester Fever Seminar, Vol. 2, Univ. Rochester Press; 1932.

25. Pierce, G. W. Piezoelectric crystal resonators and crystal oscillators applied to the precision calibration of wave-meters. Proc. Amer. Acad. Arts and Sci. 59: 81-106. 1923.

25a. Richards, A., and A. L. Loomis. Dielectric loss in clectrolyte solutions in high frequency fields. Pro. Nat. Acad. Sci. 15: 587-593. 1929.

26. SAyers, R. R. Review of literature on physiological effects of abnormal temperatures and humidities. Pub. Health Repts. 42: 933-996. 1927.

27. Schereschewsky, J. W. The physiological effects of currents of very high frequency. Pub. Health Repts. 41: 1939-1963. 1926.

28. Schereschewsky, J. W. The action of currents of very high frequency upon tissue cells-upon a transplantable mouse sarcoma. Publ. Health Repts. 43: 927-939. 1928.

29. Schereschewsky, J. W., and H. B. Andervont. The action of currents of very high frequency upon tissue cells-upon a transplantable fowl sarcoma. Publ. Health Repts. 43: 940-945. 1928.

30. Schliephake, E. Die biologische Wärmewirkung im elektrischen Hochfrequenzfeld. Verhandl. Deut. Kongr. Innere Med. 40:307. 1928.

31. Schlieprake, E. Tiefenwirkungen im Organismus durch kurze elektrische Wellen. I, II. Teil. Zeitsch. Exp. Med. 66:212-230. 1929.

32. Schliephake, E. Die Methoden der elektrischen Kurzwellenanwendung. Abderhalden's Handb. Biol. Arbeitsmeth., Abt. V, Teil 2/2, 1929. 
33. Schliephake, E. Therapeutische Versuche im elektrischen Kurzwellenfeld. Klin. Wochenschr. 9: 2333-2336. 1930.

34. Schliephake, E. Über Tiefenwirkung und elektive Gewebswirkung kurzer elektrischer Wellen. Strahlentherapie 38: 655-664. 1930.

35. Szymanowski, W. T., and R. A. Hicks. The biologic action of ultra-high frequency currents. Jour. Infect. Dis. 50: 1-25. 1932.

36. Szrmanowski, W. T., and R. A. Hicks. Further studies on the biologic action of ultra-high frequency currents. Jour. Infect. Dis. 50:466-472. 1932.

37. UNDERHILl, C. R. Electronics in surgery-the radio knife. Electronics 1: 316-319. 1930.

Manuscript received by the editor December, 1933. 


\title{
XVI
}

\section{BIOLOGICAL EFFECTS OF ALPHA PARTICLES}

\author{
R. E. ZIRKLE ${ }^{1}$
}

\begin{abstract}
Eldridge Reeves Johnson Foundation for Medical Physics, University of Pennsylvania, Philadelphia
\end{abstract}

Advantageous properties of alpha particles. Suitable sources of alpha particles. Effects upon macroscopic structures a?dd systems. Effects upon single cells, parts of cells, and small groups of cells. Summary. References.

\section{ADVANTAGEOUS PROPERTIES OF ALPHA PARTICLES 2}

Alpha particles ${ }^{3}$ are an intensely ionizing type of radiation. Hence, their most striking physical effect is the same as that of cathode, beta, gamma, and X-rays. However, their penetration in matter is very low. Those from polonium penetrate air about $3.9 \mathrm{~cm}$. and water about $32 \mu$ (Michl, 14). Since their penetration is very nearly the same in substances of equal density, $32 \mu$ is also a fair value for their penetration in living matter. This low penetration makes them useless for cancer therapy, which fact accounts for their relatively infrequent use in purely biological experiments dealing with effects of ionizing radiations.

However, alpha particles offer several advantages in such investigations if small biological objects are used. Their paths in matter are straight lines, ${ }^{4}$ and the lengths of the paths are nearly equal if only one radioactive substance is used as the source. These properties, together with the low penetration (short length of path), make it possible to attack limited portions of single cells. The number of ions produced per linear unit of an alpha-particle path varies in a characteristic fashion with the distance from the end of the path (Fig. 1). This makes it possible to place a suitably small biological test object at various distances from the end of the path and thus study directly the effect of ion concentration

1 National Researeh Fellow in the Biological Sciences.

${ }^{2}$ Unless otherwise indieated all statements of a physical nature are based upon the book by Rutherford, Chadwick, and Ellis (19).

${ }^{3}$ An alpha particle is a helium atom lacking two eleetrons and hence carrying a double positive electronic charge. It is ejeeted from an atom of a radioactive substance with an initial velocity of the order of one twentieth that of light. The exact value of the initial velocity, and hence of the initial energy, is different for particles from different radioactive substances but is the same for all particles enitted by any one substance. The same is approximately true of the total penetration in matter, for this quantity varies nearly as the cube of the initial velocity.

${ }^{4}$ Significant deviations from straight lines, due to collisions with atomic nuclei, are so rare as to be negligible in biological work. 
in the path. This is at present impracticable with any other type of radiation. Absolute values of doses of alpha particles can be determined directly with ease and accuracy, and expressed in terms of the number of particles striking in unit time a unit area at a definite distance from the source. It is to be noted that expression of dose in this manner makes possible a direct and precise calculation of the number of alpha-particle paths which traverse any cell or part of a cell during administration of any given dose. This also is at present impracticable with any other type of radiation. Duplication of dose with the same source is extremely

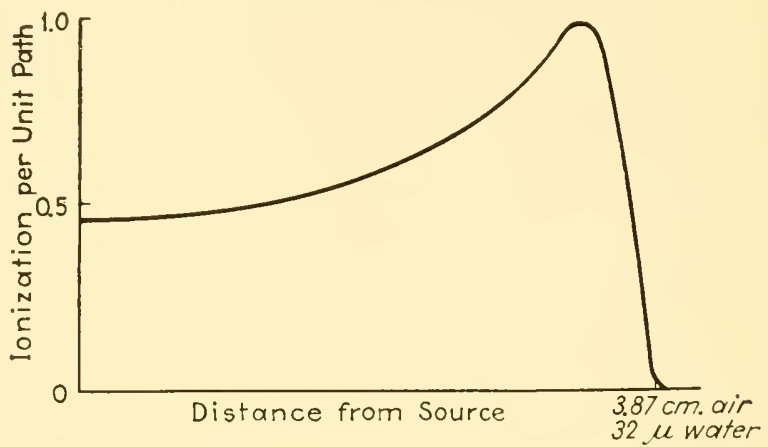

Fig. 1.- Relation between ionization per unit path of a beam of alpha particles and distance from the source (polonium). (After Curie and Behounek, 4.)

easy, because the rate of emission of alpha particles varies with time in accordance with a simple exponential law.

\section{SUITABLE SOURCES OF ALPHA PARTICLES}

Two substances-radon (radium emanation) and polonium (radium F)-have been used as sources in nearly all biological work with alpha particles.

Radon.-This substance is a chemically inert gas. Its chief advantages over polonium are: $(A)$ If necessary equipment and a suitable amount of radium are available, the preparation of sources, especially of very active ones, is much more rapid. (B) The gamma-ray activity of its decay products makes possible a rapid and accurate indirect measurement of the rate of emission of alpha particles. Its chief disadvantages are: $(a)$ It decays to half value in less than four days. This necessitates a new source every few days. (b) It has decay products which emit beta and gamma rays. These rays require special investigation, so as to make sure that the biological effects are certainly due to alpha particles. (c) Radon has two decay products-radium $\mathrm{A}$ and radium $C^{\prime}$ - which also emit alpha particles. Since the particles from these two substances have velocities different from that of the particles from radon itself, this means that the alpha-ray beam from a radon source is heterogeneous. In many types of experiments this may be of no 
importance, but in experiments such as those involving accurate control of penetration it may be very disadvantageous.

Radon is used in two ways: $(A)$ dissolved in the culture medium; $(B)$ confined in very thin-walled glass bulbs (alpha-ray bulbs). The first method is very convenient for certain types of qualitative work, but its use in quantitative work is open to several criticisms: (a) The distribution of radon between organism and culture medium is unpredictable. The radon may not be absorbed at all by the cells, it may be concentrated in the cells, or its concentration may be the same in the cells and in the medium. As with any other dissolved substance, the only way to make sure of the degree of absorption of radon is to make direct determinations upon the specific organism being used. The same is true for each of the decay products-radium $\mathrm{A}$ and radium $\mathrm{C}^{\prime}$ - which also emit alpha particles. (b) The radium $\mathrm{A}$ and radium $\mathrm{C}^{\prime}$ further complicate matters. If pure radon is introduced into the closed culture vessel, it distributes itself between the gas and liquid phases in a known way. The formation of radium $\mathrm{A}$ and radium $\mathrm{C}^{\prime}$ becomes significant almost immediately. (At the end of $3 \mathrm{~min}$., half as many, and at the end of 9 min., seveneighths as many alpha particles are being emitted by radium $\mathrm{A}$ as by radon itself.) The rates of formation are accurately known, and these substances should present no insuperable difficulties if all the radon decays in the liquid phase. However, it would be very difficult to predict what fractions of the radium $\mathrm{A}$ and radium $\mathrm{C}^{\prime}$ formed in the gas phase find their way into the liquid phase where they become effective. This uncertainty is significant whenever the volume of the gas phase is of as large an order as that of the liquid phase, for at $20^{\circ} \mathrm{C}$. the distribution coefficient of radon between water and gas phases is about $1: 4 .^{5}$ The uncertainty becomes worse if the radon is introduced along with the equilibrium amounts of radium $\mathrm{A}$ and radium $\mathrm{C}^{\prime}$, for these substances are solids deposited in a very thin layer on the walls of the original radon container and may or may not dissolve readily in the culture solution.

The use of radon in bulbs is much more satisfactory. The chicf disadvantages are: (a) The glass walls seriously reduce the penetrating ability of the emergent alpha particles. (b) Particles ejected at various points in the bulb traverse different thicknesses of glass before emerging. This introduces further heterogeneity into a beam of particles which is already heterogeneous because of different initial velocities (see above).

Polonium.-This substance has the following advantages: (a) It decays to half value in 136 days. A source of satisfactory original activity may be used upon most biological material for at least 2 or 3 months without the necessary increase in length of exposure becoming a serious factor. (b) It is the only known substance which emits alpha particles and has no radioactive decay products. (c) It can be readily

${ }^{5}$ Derived from International Critical Tables $3:$ p. 257. 
deposited upon free metallic surfaces. The radiation from such a source is homogeneous. Doses can be calculated with certainty and precision.

\section{EFFECTS UPON MACROSCOPIC STRUCTURES AND SYSTEMS}

Not many papers can be definitely placed in this category. The low penetration of the alpha particles usually insures that the observations of effects will be made upon individual cells and not upon gross morphological structures. Moreover, a number of investigations with radon cannot be included because it is uncertain whether the reported results are ascribable to alpha particles or to beta and gamma rays emitted by the decay products of radon.

Zwaardemaker (29) reports that alpha particles, as well as beta radiation, will compensate for the omission of potassium from the Ringer solution ordinarily used in maintaining the normal beat of an isolated frog's heart. He accordingly ascribes the essential role of potassium in this solution to its radioactivity. Polák (15) confirms these results. Zwaardemaker also reports that the effect of alpha particles is antagonized by an appropriate simultaneous dose of beta rays. This he ascribes to the charge of the beta rays (electrons) being opposite in sign to that of the alpha rays. As Redfield and Bright (18) point out, this seems very improbable in riew of the fact that the ionization due to both types of radiation produces electrical disturbances which are tremendous compared to those which could be produced by transfer of the charges carried by the alpha and beta particles.

This view that the biological effects of alpha particles are due to their positive charges has also been expressed by Straub and GollwitzerNeier (22) who report experiments which indicate that alpha particles facilitate the loss of negative charge and the assumption of a positive one by human red blood cells. Hardy (8) also invoked the charges of the alpha particles to explain his observation that this radiation coagulated serum globulin in alkaline solution (globulin charged negatively), while an acid solution (globulin charged positively) became clearer. However, in both these cases, the observed results could be qualitatively explained by the assumption that the fundamental effect of the alpha particles was the removal of electrons from (or ionization of) the red-cell constituents and the globulin molecules, respectively. Moreover, with this assumption, one could account for some 10,000 to 15,000 times as much removal or addition of charge as could be brought about by means of the charges carried by the particles themselves.

Chambers and Russ ${ }^{6}$ observed what may be an instance of qualitative difference in biological effects of alpha rays and of beta rays. When both alpha and beta rays were used on a suspension of bacteria, marked agglutination occurred before the lethal point was reached. If the alpha

${ }^{6}$ Original paper not available. See Colwell and Russ (2), p. 162. 
rays were screened off, no agglutination took place, although the death point was ultimately reached. Colwell and Russ (2) suggest that the charges on the particles may be responsible for the difference in action in the two eases. A more probable factor is the difference in the distribution of the ions produced by the two types of radiation.

The relatively tremendous destructive power of alpha particles is demonstrated in an early paper by Willcock (25). This author exposed Hydra viridis to $50 \mathrm{mg}$. of radium bromide in such a way that the alpha, beta, and gamma rays could all reach the organism. The animal's vitality was low at the end of $1 \mathrm{hr}$. and disintegration oceurred after $2 \mathrm{hr}$. However, when only the beta and gamma rays from the same preparation were allowed to reach the organism, the latter survived for several days after an exposure of $4.5 \mathrm{hr}$.

This great destructive ability has recently proved to be of economic and clinical importance. A number of girls, while working with radioactive luminous paint, ingested amounts of radium ranging from 10 to 180 micrograms. After several years, serious pathological conditionschiefly anemias and sareomas of the bones-developed, and death usually resulted. These effeets are almost certainly due to the alpha particles emitted by the radium and its decay products. Martland (13) has summarized these cases, particularly the clinical aspects, and Schlundt, with various collaborators, has deseribed the pertinent physical investigations (e.g., Schlundt and Failla, 20). Thomas and Bruner (24) have recently produced anemias and other pathological conditions in rats by injection of a few micrograms of radium. This work, along with the clinical observations just mentioned, emphasizes the need for investigation of the fundamental effeets of alpha particles on living matter. As Martland (13) points out, it is possible that the initiation of some malignant growths may be due to the action of radioactive substances normally present in the body in amounts minute in comparison with those concerned in the cases of radium poisoning.

Stoklasa and Pěnkava (21, page 739) describe lethal effects of radon upon guinea pigs, mice, frogs, and lizards. These effects are ascribed to alpha particles. The doses reported, however, are surprisingly small. If one calculates the maximum number of alpha partieles which could have been emitted per unit time per unit volume of animal (guinea pig or mouse), the figure obtained is less than one-twentieth of that resulting from a similar calculation of the minimum intensity used by Thomas and Bruner (24) on rats. Moreover, the time required for the rats to die under continuous exposure was about 10 times that reported by Stoklasa and his coworker for mice and guinea pigs, so that the ratio of the doses used by the two sets of workers is at least 200:1.

Favorable effects of alpha particles upon seed germination have been reported at least twice. Such an effect is very surprising, for the alpha 
particles certainly could not have penetrated through the coats of the particular seeds used. Gager (7) exposed 16 wheat grains to polonium alpha particles and found, 11 days after planting them, that the average shoot length of the resulting seedlings was $123 \mathrm{~mm}$., while the corresponding figure for the controls was $76 \mathrm{~mm}$. However, he planted the irradiated grains all in one pot and the controls in another. The observed acceleration of germination may have been due to some factor other than the radiation. Stoklasa and Pěnkava (21, pages 359-360) report a favorable effect of alpha particles upon germination of seeds of Picea excelsa and Pinus silvestris. However, their data as a whole show that the reported increases are not statistically significant. Moreover, they germinated the irradiated and control lots in separate vessels and report no use of duplicates.

Stoklasa and Pěnkava (21) report various other cases of acceleration of vital processes by minute quantities of radon and ascribe the effects to alpha particles. For example, the carbon dioxide production of fish (Cyprinus carpio) is increased (21, pages $877-879$ ) by a concentration of $2.9 \times 10^{-5} \mathrm{mc}$. of radon per liter in the gas phase of the respiration chamber. Here, as in other cases, the authors find that the alpha particles have an effect opposite to that of beta and gamma rays from a tube preparation of radon. The following figures summarize their results:

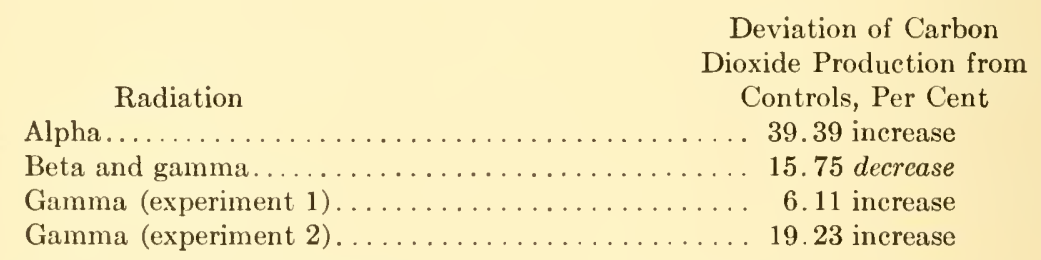

These results are very difficult to understand, since it is very doubtful that the beta rays could reach the fish. Practically all of these rays would have been stopped by $1 \mathrm{~cm}$. of water, and the authors mention no precautions to keep the fish near the top. Failures (such as this one and others mentioned above) to take properly into account the physical properties of these various radiations, are conducive to a general lack of confidence in the results reported by these authors.

\section{EFFECTS UPON SINGLE CELLS, PARTS OF CELLS, AND SMALL GROUPS OF CELLS}

Every paper reviewed under this heading describes the production of marked abnormality in the biological material. No favorable effects have been described, although in some cases these should have been observable if actually produced.

Chambers and Russ (1) found that alpha rays hemolyze red corpuscles. Two methods were used: $(a)$ solution of radon in a suspension 
of corpuscles; (b) irradiation with 2.4 or $3.3 \mathrm{mg}$. $\mathrm{RaBr}_{2}$ through miea screens. Estimates of the number of alpha particles necessary to hemolyze a corpuscle gave the figure 2000 when radon was in solution and 8000 when the rays had to penetrate through mica.

Experiments with leueocytes showed that these had a tendency to avoid or to migrate away from a region undergoing alpha irradiation. Since the serum showed a decrease in surface tension when irradiated, the behavior of the leucocytes was ascribed to this physical change in their medium.

These authors report gencral negative results when the alpha rays were screened off, only the beta and gamma rays remaining effective. However, they used under these conditions exposures of the same order of duration as those used with all three types of rays effective. If they had irradiated for longer periods, the beta and gamma rays alone would probably have produced similar effects.

Redfield and Bright (17), as a matter of fact, demonstrated this to be true. They found that 25 per cent hemolysis of sheep red corpuscles could be produeed in $5 \mathrm{hr}$. by $0.46 \mathrm{mc}$. of radon in solution or by $54.0 \mathrm{me}$. in a glass tube (alpha rays screened off). This is another illustration of the relatively enormous destructive power of the alpha particle. Hemochromolysis and stromatolysis were found to proceed at different rates, the former being complete when the latter was ouly about 50 per cent complete.

Swann and del Rosario (23) produced nonmotility in Euglena by means of radon in solution. When the percentage of survivors was plotted over time of exposure, the resulting curves were logarithmic and their slopes were proportional to the concentration of radon. (The effects of alpha particles from radium $\mathrm{A}$ and radium ' $\mathrm{C}^{\prime}$ were not considered.) From this result the authors eoncluded that the effeet upon a cell was due to a collision of a single alpha particle with some especially sensitive volume in the cell. The ealculated surface of this sensitive volume corresponded to that of a sphere $4.8 \mu$ in diameter, which is very nearly the diameter of the Euglena nucleus. However, when correction is made for a numerieal error due to a misinterpretation of the tables used, it is found that the diameter of the hypothetical sensitive volume must be of a considerably lower order than that originally calculated. ${ }^{7}$

Lethal effects of alpha particles upon yeast have been described in papers by Holweck (10), and by Lacassagne (12), and by these two authors jointly (11). Two types of effect were elassified: (a) no cell division ("immediate death"); (b) one eell division ("deferred death"). Cells accomplishing more than one division were considered unaffected. Dosage was caleulated in terms of number of ion pairs produced per

7 The value and validity of the concept of a "sensitive volume" have been discussed by Crowther (3). 
cubic micron. The doses necessary to produce deferred death and immediate death in 50 per cent of the cells (observations made $24 \mathrm{hr}$. after irradiation) are tabulated below. The results of similar calculations for soft X-rays are shown for comparison.

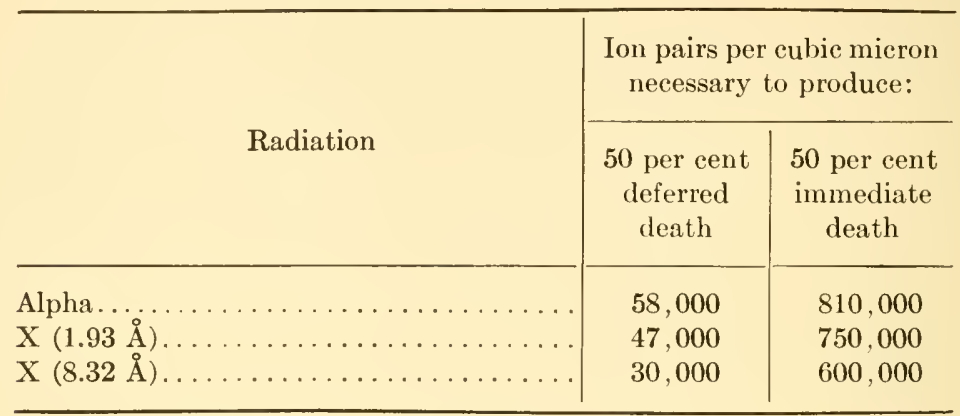

These results indicate that the amount of ionization necessary to produce a given degree of effect is of the same order for all of these radiations.

Feichtinger (5) made comparative studies of the effects of alpha rays (from polonium) and beta rays (from radium $\mathrm{C}$ and radium $\mathrm{E}$ ) upon the root-tip cells of Crepis virens. Her sources of alpha and beta rays were estimated to be of equivalent ionizing power per unit volume of protoplasm. After irradiation the roots were fixed, embedded, sectioned, and stained with iron-alum hematoxylin. Three stages of effects were recognized: (a) increased affinity of the cell contents for the stain; (b) formation of vacuoles and clumping of protoplasm, the cell as a whole appearing deeply stained; $(c)$ breaking up of the protoplasm into small, deeply staining granules which lie near the cell wall, the cell as a whole appearing rather empty. For equal estimated ionization per unit volume, the effects of the alpha and beta rays were the same. The alpha-ray effect was limited to an external layer about $30 \mu$ thick, while the beta rays were effective in all cells of the root. This, of course, was to be expected because of the difference in the penetrating powers of the two types of radiation.

In a recent paper (6) the same author reports viscosity changes in cells of Spirogyra irradiated with alpha particles. As the dosage was increased, the viscosity (as indicated by migration of chloroplasts under centrifugation) first decreased and then increased. Beta rays had a similar effect. These results appear in agreement with the observations of Williams (26) that X-rays first increase and then decrease the rate of protoplasmic streaming in various plant cells.

The low and nearly uniform penetration of polonium alpha particles makes it possible to irradiate limited portions of cells ranging in diameter 
from 20 to $100 \mu$. This advantage has been used by Zirkle (27) to compare the effects of irradiation of a nucleated fraction of the cell with those of a corresponding irradiation of an equal fraction lacking the nucleus. The spore of the fern Pteris longifolia was especially favorable material for this investigation because the nucleus $(10 \mu$ in diameter) lies at the periphery of the protoplast $(38 \mu$ in diameter) and its location can be readily ascertained from its proximity to a Y-shaped set of sutures on the spore wall. By interposition of a thin aluminum screen the penetration of the alpha particles was reduced sufficiently to insure that only the top third of each cell was irradiated. If the wall sutures of a given spore were upward, the nueleus was in the irradiated volume; if the sutures were downward, an equal total volume was irradiated but the nucleus escaped. The qualitative effects of irradiation were the same under both of these conditions. Three distinet activities of normal germination-cracking of the spore wall, chlorophyll development, and cell division-were inhibited. Division was more easily inhibited than chlorophyll development and the latter more easily than cracking. ${ }^{8}$ When the nucleus was in the irradiated volume, the dose of alpha particles needed to inhibit any one of these processes in any given percentage of individuals was far less than when the nucleus was not irradiated. An exact numerical comparison is impracticable, but a lower limit of 20:1 may be set for the ratio of effectiveness of a given dose of alpha particles under the two conditions. This result is, of course, not surprising as far as cell division is concerned. The essential role of the nueleus in mitosis is well known. It is of interest, however, to note that injury to the nucleus is also especially effective in prevention of chlorophyll formation and in prevention of absorption of enough water to crack the spore wall.

Henshaw and Henshaw (9) also deseribe results which demonstrate that alpha particles are particularly effective when they strike nuclei. These authors studied the change in susceptibility of eggs of Drosophila as development progresses. The transverse diameter of the egg of Drosophila is about $100 \mu$, and under the particular experimental eonditions the alpha particles from polonium must not have penetrated more than $15 \mu$. Hence only a relatively thin peripheral layer of each developing egg was irradiated. In the early stages of development the nuclei are located well within the egg. As they multiply, they migrate toward the periphery and form a blastoderm. Later, as gastrulation begins, many of the nuclei move inward, out of range of the alpha particles. Henshaw and Henshaw found that this migration of nuclei was definitely correlated with sensitivity, the eggs being most susceptible when the nuelei were located peripherally. This was true in spite of the fact that,

${ }^{8}$ These effects are qualitatively the same as those of soft $\mathrm{X}$-rays (unpublished data, obtained with Dr. A. L. Patterson). 
at this stage of development, the eggs were least susceptible to X-rays which, of course, penetrated to all parts of the egg.

Redfield and Bright (16) have studied a very interesting effect of alpha particles upon the egg of Nereis limbata. Immediately beneath the vitelline membrane of the unfertilized egg is a coarsely alveolar layer about $4 \mu$ thick. This layer contains a jelly which, after fertilization, is extruded through the vitelline membrane, forming a thick layer outside the egg. The emptied alveoli become filled with water, forming a thin perivitelline space between the vitelline membrane and the protoplasm. Irradiation with alpha particles caused a decrease in jelly extrusion, while the volume of the perivitelline space was correspondingly increased. This effect was also produced by beta rays, gamma rays, and ultra-violet light of wave-length less than $3000 \AA$ and hence seems to be an effect of ionizing radiations in general.

In a later paper (18) these authors demonstrate a striking relationship between ionizing power of alpha rays and degree of biological effect (thickness of perivitelline space). The source of radiation was an exceptionally thin-walled glass bulb containing radon and, of course, radium $\mathrm{A}$ and radium $\mathrm{C}^{\prime}$. The alpha particles from radon, radium $\mathrm{A}$, and radium $\mathrm{C}^{\prime}$ have different penetrating abilities. Hence the experimental curve describing the variation in ionization per unit path with the distance from such a source is not so simple as that shown in Fig. 1 for a polonium source. It has three maxima and two minima. Two of the maxima and one of the minima are quite pronounced. Redfield and his coworker exposed various lots of Nereis eggs to the same dose of alpha particles per egg but with the thin perivitelline spaces at different distances from the source. When degree of biological effect was plotted over distance from the source, the resulting curve had two maxima and one minimum. Moreover, the distances between these three critical points were the same as the corresponding distances on the ionization curve. This result shows very clearly that the biological effectiveness of the alpha particle varies along its path, and that this variation is closely related to the variable ionization per unit path.

Unfortunately, because of limited data and the heterogeneity of the alpha particles used, it is impracticable to deduce just what mathematical relationship existed between ionization per unit path and the effectiveness in prevention of jelly secretion. If the biological effectiveness could be shown to be directly proportional to the ionization per unit path, then it would be evident that the production of a given degree of effect was due only to the production of a given number of ions. It would be immaterial whether this number of ions be produced by a relatively small number of alpha particles traversing the perivitelline space at that part of the path where the ionization is relatively high, or vice versa. On the other hand, if biological effectiveness could be shown not to be directly propor- 
tional to the ionization per unit path, then it would be obvious that the distribution, as well as the number, of the ions was a factor influencing the biological effectiveness.

The possibility that the distribution as well as the number of ions may influence the biological effectiveness has been recently investigated by Zirkle (28). Polonium was used as source, and the ionization along the path of the average alpha particle varied as shown in Fig. 1. Since the total length of path was $32 \mu$, it was possible to place nuclei of various lots of fern spores ( $10 \mu$ in diameter, see above) so that some were traversed by alpha particles at the beginning of their paths where the ionization per unit path was low; some at the ends of the paths, where the ionization per unit path was high; and others in intermediate portions of the paths. The nuclei of all lots were turned upward (see above) and, by interposition of a suitable thickness of aluminum, the alpha particles could be retarded sufficiently to cause them to traverse the nuelei at any desired linear portion of the path. Seven portions of the path were investigated. The ionization produced in the nucleus by the average alpha particle under these seven conditions is shown in the second column of Table 1 (arbitrary units). The arbitrary values, it will be noted, range from 1.00 at position 1 (near the end of the path) to 0.58 at position 7 (near the beginning of the path). The biological effectiveness at the seven positions was obtained as follows: A complete survivor-dosage curve was obtained for each position. From this curve could be determined the number of alpha particles per nucleus necessary to produce a given degree of biologieal effect $(e . g$. , inhibition of cell division). The reeiprocal of this number was then a measure of biologieal effectiveness per alpha particle. The seven measures of biological effectiveness $^{9}$ thus obtained are shown in the third eolumn of Table 1 (arbitrary units). Now, if biological effectiveness of an alpha partiele were determined solely by the number of ions it produced in the nucleus, it is plain that the observed biologieal effectiveness at the seven positions should, within experimental error, be proportional to the ionization at these positions. In other words, the figures in columns 2 and 3 of Table 1 should be substantially the same. However, there is a systematie divergence in these two columns. It is hence clear that biological effectiveness is not dependent solely upon number of ions per nueleus. Distribution of ions also must be a factor. For a given amount of nuelear ionization, the maximum effect is obtained by using the fewest possible paths, with of course the highest production of ions per unit path.

9 These values were the same, regardless of the degree of effect chosen as end point; that is, the seven eurves had the same shape but different slopes. The same values, within experimental error, were obtained with all the three processes studied (eracking, chlorophyll development, division). The figures in column 3 of Table 1 are averaged from the three corresponding sets of data. 
Table 1.-Comparison of Biological Effectiveness of Alpha Particles with Their Ionizing Ability

\begin{tabular}{c|c|c|c}
\hline Nuclear position & $\begin{array}{c}\text { Ionization } \\
\text { produced in nucleus }\end{array}$ & $\begin{array}{c}\text { Observed biological } \\
\text { effectiveness }\end{array}$ & $\begin{array}{c}\text { Theoretical biologi- } \\
\text { cal effectiveness }\end{array}$ \\
\cline { 1 - 2 } 1 & 1.00 & 1.00 & 1.0 \\
2 & 1.00 & 1.00 & 0.97 \\
3 & 0.85 & 0.60 & 0.61 \\
4 & 0.77 & 0.46 & 0.47 \\
5 & 0.68 & 0.36 & 0.35 \\
6 & 0.62 & 0.28 & 0.29 \\
7 & 0.58 & 0.23 & 0.23 \\
\hline
\end{tabular}

a Based on the assumption that the biological effectiveness varies as the five halves power of the ionization per unit path.

Although biological effectiveness is clearly not a linear function of ionization per unit path, there is apparently a simple mathematical relationship between the two. This can be expressed (Zirkle, 28) by the empirical equation $B=k I^{2.5}$, where $B$ is biological effectiveness, $k$ a constant, and $I$ the ionization per unit path (compare columns 3 and 4, Table 1). Since the effective radius of the path is roughly the same throughout the length of the path, it follows that $I$ is roughly proportional to the local concentration of ions in the path. It thus appears that in different portions of the path, the biological effectiveness of the alpha particle varies with the ion concentration raised to some power of the order of the square or the cube.

These results immediately suggest the investigation of a possible concentration factor in the action of alpha particles upon other biological material. It would also be of great interest to determine whether or not the concentration effect can be modified by biological factors, such as water content.

\section{SUMMARY}

Practically all of the reported effects of alpha particles have been lethal or harmful. The few reports of favorable stimulation are all open to serious criticism.

The qualitative effects of alpha particles have usually been found similar to those of beta, gamma, and X-rays. Moreover, the doses of these various radiations necessary to produce a given degree of biological effect have been found to produce the same order of magnitude of calculated total ionization per unit cellular volume. However, the total ionization per unit cellular volume is not the only ionization factor determining the degree of biological effect, for with alpha particles it has been shown that a given number of ions may produce widely different degrees of biological effect if one uses various linear portions of the ionizing paths along which the concentration of ions varies considerably. 
Alpha particles have proved very useful in irradiating limited portions of cells. Irradiation of the nucleus has been shown, in two distinct investigations, to be particularly important in producing various harmful effects.

Alpha particles, when emitted in the mammalian body at relatively low rates for considerable periods of time, produce serious pathological conditions, including malignant growth.

\section{REFERENCES}

1. Chanbers, Helen, and S. Russ. The action of radium radiations upon some of the main constituents of normal blood. Proc. Roy. Soc. London B 84: 124-136. 1912.

2. Colwell, H. A., and S. Russ. Radium, X-rays and the living cell. $2 \mathrm{~d}$ ed. G. Bell and Sons; London, 1924.

3. Crowtifer, J. A. The action of X-rays on Colpidium colpoda. Proc. Roy. Soc. London B 100: 390-404. 1926.

4. Curie, Irène, and F. BĚHounek. Étude de la courbe de Bragg relative aux rayons du radium C. Jour. Phys. Radium. 7: 125-128. 1926.

5. Feichtinger, Nora. Über die Einwirkung der $\alpha$ - und $\beta$-Strahlen auf das Protoplasma. Protoplasma 14: 36-51. 1931.

6. Feichtinger, Nora. Viskositätsänderung des Protoplasmas als Folge radioaktiver Bestrahlung. Naturwissensch. 21:569-575, 589-591. 1933.

7. GaGer, C. S. Effeets of the rays of radium on plants. Mem. New York Botan. Gard. 4: 1-278. 1908.

8. HARDY, W. B. The action of salts of radium upon globulins. Jour. Physiol. 29 : 29-30. 1903.

9. Henshaw, P. S., and C. T. Henshaw. Changes in susceptibility of Drosophila eggs to alpha particles. Biol. Bull. 64: 348-357. 1933.

10. HoLweck, F. Etude énergétique de l'action biologique de diverses radiations. Compt. Rend. Acad. Sci. [Paris] 190: 527-529. 1930.

11. Houweck, F., and A. Lacassagne. Sur le mécanisme de l'action ey tocaustique des radiations. Compt. Rend. Soe. Biol. [Paris] 103: 766-768. 1930.

12. Lacassagne, A. Différence de l'action biologique provoquée dans les levures par diverses radiations. Compt. Rend. Acad. Sci. [Paris] 190:524-526. 1930.

13. Martland, H. S. The occurrence of malignancy in radioactive persons. Amer. Jour. Cancer 15 : 2435-2516. 1931.

14. Michl, W. Über die Reichweite der $\alpha$-Strahlen in Flüssigkeiten. Sitzungsber. Akad. Wiss., Wien, Math.-Naturw. K1. 123 (2a): 1965-1999. 1914.

15. Polák, E. Ther den Einfluss radioaktiver Strahlungen auf die automatische Tätigkeit des Froschherzens mit besonderer Rücksicht auf die Wirkung grosser Strahlendosen und auf den Antagonismus der $\alpha$ - und $\beta$-Strahlung. Pfüger's Arch. Ges. Physiol. 226 : 659-675. 1931.

16. Redfield, A. C., and Elizabetii M. Bright. The physiological changes produced by radium rays and ultraviolet light in the egg of Nereis. Jour. Physiol. 55 : 61-85. 1921.

17. Redfield, A. C., and Elizabeth M. Brigit. Hemolytie action of radium emanation. Amer. Jour. Physiol. 65: 312-317. 1923.

18. Redfield, A. C., and Elizabeth M. Bright. The physiological action of ionizing radiations. II. In the path of the $\alpha$-particle. Amer. Jour. Physiol. 68: 62-69. 1924.

19. Rutherford, E., J. Chadwick, and C. D. Ellis. Radiations from radioactive substances. Ed. 1, Macmillan; New York, 1930. 
20. Schlundt, H., and G. Failla. The detection and estimation of radium and mesothorium in living persons. III. The normal elimination of radium. Amer. Jour. Roentg. Rad. Therapy 26 : 265-271. 1931.

21. Stoklasa, J., and J. P̌̌nkava. Biologie des Radiums und der radioaktiven Elemente. I. Biologie des Radiums und Uraniums. Parey; Berlin, 1932.

22. Straub, H., and Kl. Gollwitzer-Meier. Blutgasanalysen. XIII. Mitteilung. Der Einfluss von $\alpha$-Strahlen auf Hämoglobin und Blutkörperchen. Biochem. Zeitsch. 136 : 128-139. 1923.

23. Swann, W. F. G., and C. Del Rosario. The effect of radioactive radiations upon Euglena. Jour. Franklin Inst. 211: 303-317. 1931.

24. Thomas, H. E., and F. H. Bruner. Chronic radium poisoning in rats. Amer. Jour. Roentg. Rad. Therapy 29 : 641-662. 1933.

25. Wrllcock, E. G. The action of the rays from radium upon some simple forms of animal life. Jour. Physiol. 30:449-454. 1904.

26. Williams, MAUd. Observations on the action of X-rays on plant cells. Annals Bot. 37 : 217-224. 1923.

27. Zirkle, R. E. Some effects of alpha radiation upon plant cells. Jour. Cell. and Comp. Physiol. 2 : 251-274. 1932.

28. Zirkle, R. E. Biological effectiveness of alpha particles as a function of ion concentration produced in their paths. Amer. Jour. Cancer 23 : 558-567. 1935.

29. Zwandemaker, $H$. On physiological radioactivity. Jour. Physiol. 63: 273-289. 1920. 


\title{
XVII
}

\section{MOTOR RESPONSES TO LIGHT IN THE INVERTEBRATE ANIMALS}

\author{
S. O. MAST
}

The Zoological Laboratory of the Johns Hopkins University, Baltimore

Rhizopods. Flagellates. Ciliates. Colonial forms. Coelenterates. Worms: Tubicolous annelids-Arenicola larvae-Earthworms-Turbellaria. Molluscs: Mya-Pecten -Helix-Agriolimax. Arthropods: Daphnia. Insects. References.

A motor response is often defined as a change in rate or in direction of movement of an organism or a portion of it. This definition is accepted in the following review. The space allotted is, however, so limited that it is not expedient to attempt to consider even superficially these responses in all the species of invertebrates. I shall therefore omit several groups altogether and select for discussion only a few species in the remaining groups, i.e., those which have been most thoroughly studied.

\section{RHIZOPODS}

Response to light is fairly common among the rhizopods and the results in hand indicate that it is essentially the same in all. It has, however, been thoroughly investigated in only one species, Amoeba proteus.

Amoeba proteus consists of a thin elastic outer membrane, the plasmalemma, a central relatively fluid granular mass, the plasmasol, surrounded by a relatively solid granular layer, the plasmagel, and a thin fluid hyaline layer between the plasmagel and the plasmalemma. During locomotion the plasmalemma is attached to the substratum and to the adjoining plasmagel, the plasmagel at the pasterior end is transformed into plasmasol which flows forward to the anterior end and is there transformed into plasmagel. The forward flow of the plasmasol is due to contraction of the plasmagel at the posterior end and expansion at the anterior end, owing to difference in its elastic strength in these two regions. Response in this form is due to changes in the elastic strength of localized regions in the plasmagel, or in the rate of transformation of plasmasol into plasmagel and vice versa, or in the attachment to the substratum (Mast, 148a, 151a, 153).

There are two types of responses to light in Amoeba, one of which is primarily correlated with the magnitude of change in intensity, and the other with the rate of change in intensity. The latter is closely correlated with adaptation; the former is not. 
(a) If an amoeba is kept for some time in very weak light it becomes relatively inactive; and if the light is now increased, it very gradually becomes more active again. This response is similar to the response to change in temperature. It is primarily correlated with the magnitude of the change, not with the rate of change in intensity. It is probably due to the effect of light on the rate of transformation of gel to sol and vice versa. This type of response occurs also in Difflugia (Mast, 153b), but the observations on it should be repeated and extended under carefully controlled conditions.

(b) If the intensity of light on an active amoeba is rapidly increased, movement stops suddenly (Engelmann, 57, et al.). If it is slowly increased this does not occur. This response, therefore, depends upon the rate of change in luminous intensity (Mast, 139). Pfeffer called responses of this sort "Schreckbewegung," shock-reaction.

The character and the magnitude of the response to rapid increase in light vary greatly in Amoeba. It may consist merely of momentary retardation in streaming in a localized region in a pseudopod, of total cessation throughout the entire animal with reversal in direction of streaming after recovery, or of any one of an endless number of modifications between these extremes. The character of the response is correlated with the amount of light received, as well as with the rate of reception. There is no fixed threshold and the "all-or-none law" does not apply (Mast, 153).

If an amoeba is intensely illuminated for only a very short time, movement does not cease until some time after the light has been cut off. The period between the beginning of illumination and the response is known as the "reaction time"; the time illumination must continue, the "stimulation period"; and the time it need not continue, the "latent period" (Folger, 64). There are therefore two processes involved in producing this response. The one occurs only in light, the other in light or in darkness. The action of light probably results in the formation of a substance which by its action produces, independent of light, another substance which induces the response.

After an amoeba has responded to rapid increase in illumination, some time must elapse before it will again respond to the same increase in illumination. There is therefore a refractory period, a period during which the amoeba recovers from the effect of the stimulation. During a part of this period the amoeba may remain either in light of the same intensity as that which induced the response, in light of lower intensity, or in darkness, but during the rest of the period it must be in light of lower intensity or in darkness. There are therefore two processes which occur during the refractory period, one ( 1 to 2 min.) which proceeds with or without any change in luminous intensity, and one (10 to $20 \mathrm{sec}$.) which proceeds only if the intensity is decreased. These processes result 
in the production of the physiological state which obtained before the exposure, i.e., in recovery (Folger, 64).

The latent period and the amount of light energy required to induce cessation of movement vary with the intensity of the light used; as the intensity increases, the latent period increases rapidly from about 1 sec. at $500 \pm$ meter-candles to a maximum of about 6 sec. at $1000 \pm$ meter-candles and then decreases gradually to about 0.75 sec. at $11,000 \pm$ meter-candles; and the light energy required to induce cessation of movement decreases from about $7000 \pm$ meter-candle sec. at $500 \pm$ metercandles to a maximum of about $24,000 \pm$ meter-candle sec. at $1500 \pm$ meter-candles and then increases to about $30,000 \pm$ meter-candle sec. at $11,000 \pm$ meter-candles (Folger, 64). These figures are only rather crude approximations for they were obtained by a method of calculation which yields results with a large probable error and they have not been confirmed. They are, however, sufficiently accurate to substantiate Folger's conclusion that the Bumsen-Roscoe law does not hold.

This work should be repeated and the latent period established by direct observation in all luminous intensities, instead of by calculation. This is especially desirable since recent experience makes it possible to select specimens of Amoeba proteus in which the responses are much more consistent than they were in those used by Folger.

No explanation has been offered for the variation in the latent period, with luminous intensity during the stimulation period; but it has been suggested that the variation in the amount of light energy required to induce cessation of movement is due, at least in part, to adaptation (Mast, 153). For if the light is rapidly increased and then held, streaming soon begins again, i.e., the organism recovers from the effect of the increase in light, in other words, becomes adapted (Mast, 139; Folger, 64). This shows that the effect of rapid increase in light is eliminated while the organism is continuously exposed to the light, and it indicates that there are two opposing processes involved, i.e., that increase in light induces certain changes in the organism and that internal factors tend continuously to oppose and to eliminate these changes. If this is true, the more rapidly a given amount of light is received, the less time there is for recovery, and consequently the greater will be the effect of a given quantity of light. This probably accounts for the increase in the amount of light energy required (with decrease in intensity), observed in weak light, but it does not account for the increase in the amount required (with increase in intensity), observed in strong light.

The quantity of light energy required to induce cessation of movement depends upon the chemical composition of the surrounding medium. Increase in $\mathrm{HCl}$, for example, causes increase in the quantity required, but increase in $\mathrm{CO}_{2}$ causes decrease in the quantity required. In solutions of $\mathrm{KCl}, \mathrm{CaCl}_{2}$, and $\mathrm{MgCl}_{2}$, respectively, the quantity of energy 
required increases as the salt concentration decreases, but in solutions of $\mathrm{NaCl}$ there is no consistent correlation between the quantity of energy and the concentration of the salt. In general, the quantity of energy required appears to vary directly with the viscosity of the cytoplasm (Mast and Hulpieu, 160). The observations made by these authors. extended, however, over only a very limited range of environmental variation. The conclusions reached are, therefore, not applicable to wide ranges of variations in the environment (Mast and Prosser, 162).

Increase in the illumination of any localized region of an amoeba results in increase in the thickness of the plasmagel in this region. Increase in the illumination of the entire amoeba results in increase in the thickness of the plasmagel in the tip of the advancing pseudopods and this causes cessation in movement (shock-reaction).

The shorter waves of light are more efficient in inducing this response than the longer waves (Harrington and Leaming, 76, Mast, 139), and ultra-violet is probably more efficient than visible light (Inman, Bovie, and Barr, 111), but the distribution in the spectrum of stimulating efficiency has not been precisely ascertained. Folger (64) maintains that it is not closely correlated with temperature, but he did not thoroughly investigate the problem.

In a beam of light Amoeba proteus orients fairly definitely and goes from the light (Davenport, 47). If it is illuminated from one side, pseudopods develop more freely on the shaded side than on the illuminated side. This results in gradual turning away from the light. The stronger the light the sharper the turn. Orientation is therefore brought about by retardation in the formation of pseudopods on the more highly illuminated side, owing to increase in the thickness of the plasmagel on this side (Mast, 139; Luce, 130). There is some evidence which indicates that Amoeba proteus is photopositive in very weak light (Schaeffer, 195; Mast, 153), but no carefully controlled observations have been made in reference to this.

Folger $(65,67)$ maintains that mechanical stimulation causes cessation in movement in Amoeba which appears, point for point, to involve the same processes as cessation produced by increase in illumination, and that if mechanical stimulation too weak to produce a perceptual effect is followed by increase in illumination too slight to produce by itself a perceptual effect, or vice versa, there is cessation in movement. He concludes that light and mechanical agitation produce the same kind of changes, and that since the latter cannot produce photochemical changes, response to the former is not due to photochemical processes (Folger, 65, 67).

It is well known that light produces photoelectric effects which may consist in changes in the position of the valency electrons in the molecules or in their total elimination, and that this results in the activation of the molecules. It is consequently evident that light can produce changes 
in the electric equilibrium in the molecules, which results in changes in the charge they carry, or in polarization, and it is quite conceivable that this could cause such an alignment of the molecules as to result in the formation of gel. It is also well known that mechanical agitation facilitates the alignment of molecules in the process of crystallization. This seems to indicate that it could in the same way facilitate gelation. If this is true, it supports Folger's contention that stimulation by light is due primarily to photophysical effects.

McClendon (164) maintains that localized chemical stimulation of one pseudopod of an amoeba results in cessation of movement in all the pseudopods, i.e., that such stimulation produces impulses which are transmitted to all parts of the body. Verworn (208) comes to similar conclusions. Mast (154) obtained results which are in opposition to these conclusions. He maintains that localized illumination causes, by its gelating effect, increase in thickness and in the elastic strength of the plasmagel in the region illuminated, that this effect is not transmitted to other regions and that there are no impulses produced, but that the effect of localized gelation and the consequent contraction is transmitted and that this results in coordinated action.

Response of Amoeba to electricity is due primarily to solation of the plasmagel on the anodal side (Luce, 130; Mast, 153a). The contention of Bayliss (12) that it is due to gelation has not been confirmed. Light and electricity consequently appear to be opposite in action.

A moeba does not respond to light while it is feeding. "When the food cup is being formed and the pseudopods are flowing around the prey, there is no response to light, no matter how great the intensity may be" (Folger, 64). This indicates that response to light in this organism is closely correlated with its physiological state. The effects of mechanical agitation and of chemicals in the surrounding medium support this contention.

Mast (139) and Mast and Pusch (163) maintain that the change in response correlated with change in physiological state is, under some conditions, analogous to what is called "learning" in higher animals. They found that if a pseudopod of an amoeba, going in a definite direction, comes in contact with a spot of intense light, it stops and is retracted, that then other pseudopods are formed in succession, come in contact with the light, stop, and are retracted; that after a number have thus come in contact with the light, the direction of streaming is suddenly reversed and the amoeba moves away from the spot of light; and that as the number of reversals in a given individual increases, the number of successive contacts with the spot of light required to induce a reversal decreases. This, they maintain, indicates learning.

Mast $(154,155)$ observed that if a large portion of the tip of the pseudopod is illuminated with very intense light, reversal occurs after a 
single contact and that this is associated with marked increase in the thickness of the plasmagel at the tip, and he concludes that a series of weaker contacts induces reversal, owing to cumulative gelating effect, and that we therefore have here a fairly comprehensive insight into a process which closely resembles what is usually called "learning."

\section{FLAGELLATES}

A large number of the different flagellates respond to light and many of them respond very precisely. The response has, however, been thoroughly investigated in only a few species, but it is probably fundamentally the same in all.

\section{EUGLENA}

Euglena either swims freely through the water by means of the action of its flagellum or it creeps on the substratum without the action of the flagellum, but it always rotates on the longitudinal axis as it proceeds. If the intensity of the light is rapidly changed, the organism responds by abrupt change in rate and in direction of movement. Under some conditions it responds to increase and under others to decrease in intensity. If the intensity is slowly changed it does not respond, at least not in this way. This is a typical shock-reaction.

The response to decrease in intensity results in aggregation in brilliant spots in a field of light; the response to increase results in aggregation in dark spots. These spots act like a trap; the Euglenae get into them by random movement, but when they reach the boundary on the way out, the change of intensity of the light induces the shock-reaction and prevents their exit (Engelmann, 58).

In a beam of light, Euglena usually orients very precisely and goes either toward or from the light. Verworn (207) postulates that when it is not oriented, and opposite sides are unequally illuminated, the flagellum beats more effectively in one direction than in the other, that this results in turning until opposite sides are equally illuminated, and that the flagellum then beats equally in opposite directions, and the organism continues on a straight course.

This theory is in principle essentially the same as that formulated by Ray in 1693, in reference to orientation in plants, and accepted by De Candolle (31) in 1832. According to it the effect of light on the activity of the motor mechanism is dependent upon the intensity (not upon change of intensity) of the illumination of this mechanism or the photoreceptors connected with it. The light acts continuously after orientation has been attained, as well as during the process of orientation, the only difference being that during the process of orientation the illumination on opposite sides is unequal, and consequently results in quantitatively unequal action in the motor mechanism; while after 
orientation it is equal on opposite sides and consequently results in equal action in this mechanism on opposite sides. Verworn applied this theory to ciliates as well as to flagellates. Loeb, in his earlier work, strenuously opposed it, accepting Sachs' "ray-direction" theory, ${ }^{1}$ but he adopted it later (126), applied it to higher animals, and introduced the idea that the action of the locomotor appendages is quantitatively proportional to the intensity of the light on the photoreceptors connected with them and that this is due to the effect of light on muscle tonus. This theory has been designated the "difference of intensity theory," "the continuousaction theory," "the Ray-Verworn theory," "the tropism theory," "Loeb's muscle-tonus theory," etc. (Mast 148).

Engelmann (58) maintains that only the anterior end of Euglena is sensitive to change in luminous intensity. Jennings (113) contends that owing to this, all turning from the light results in reduction in illumination and all turning toward the light in increase in illumination of the sensitive substance, that photopositive specimens consequently turn until they face the light and photonegative specimens until they face the opposite direction, and that the stimulus which induces turning then ceases and the organisms consequently continue either directly toward or from the light.

Mast (140) concludes that the photosensitive substance is confined to the concavity in the opaque portion of the eyespot, that rotation on the longitudinal axis therefore results in alternate shading and exposing of this substance, if the organisms are not directed toward or from the light, that this induces shock-reactions which result in orientation, and that the organisms remain oriented and proceed directly toward or from the light because, after they have attained either of these two axial positions, rotation no longer produces changes in the illumination of the sensitive substance in the eyespot, and they therefore continue in the direction assumed. He holds that the orienting stimulus ceases after the organism has become oriented and that the organism then continues directly toward or from the light because, owing to internal factors, it tends to take a straight course and because, if for any reason it is turned from this course, the orienting stimulus immediately acts, and induces shock-reactions which bring it back on its course. He consequently does not accept the Ray-Verworn theory, according to which the organism is held on its course by the continuous action of the light.

Bancroft (9) presented evidence against the contention that photic orientation in Euglena is due to shock-reactions and concluded that it is due to tonus effects brought about by "the continuous action of the light" (in accord with his conception of Loeb's tropism theory). Mast

${ }^{1}$ According to this theory orientation is correlated with the direction in which the light passes through the organisms, not with the relation of its intensity on opposite sides. 
(142) demonstrated, however, that if the evidence presented by Bancroft is valid, it proves that Bancroft's explanation of orientation in Euglena is not correct. Moreover, the fact that, after Euglena is oriented, the rate of locomotion is practically independent of the luminous intensity (Mast and Gover, 159) also militates against Bancroft's explanation. Bancroft's experiments should be repeated and extended under more carefully controlled conditions.

If Euglena is subjected for long periods to low iliumination or to darkness, it gradually becomes less active; and if the illumination is then increased it gradually becomes more active again. The rate of change in activity varies with the magnitude of the change in intensity, but this response is never so sudden and abrupt as the shock-reaction. There are therefore two types of responses to light in Euglena, one depending primarily upon the rate of change in luminous intensity, the other primarily upon change in the amount of light received. The one results in orientation and aggregation, the other involves the degree of activity.

In a field of light consisting of two horizontal beams crossing at right angles, Euglena orients and goes toward or from a point between the two beams. The location of this point is related to the relative intensity of the two beams in such a way that the tangent of the angle between the direction of locomotion and the rays in the stronger beam is approximately equal to the intensity of the weaker divided by that of the stronger (Buder, 30; Mast and Johnson, 161). Buder judges this to show a quantitative proportionality between the stimulus and the response. Mast and Johnson conclude that "it has no bearing on the problem concerning the quantitative relation between stimulus and response," but that it can be explained on the assumptions that the eyespot is a photoreceptor and that the stimulating efficiency of light varies with the angle of incidence.

The shorter waves in the visible spectrum are more efficient than the longer in stimulating Euglena and other flagellates. Strasburger (202) concluded that stimulation is confined to violet, indigo, and blue in the solar spectrum, with the maximum in the indigo. Engelmann (58) maintains that for Euglena the maximum is in the blue between 4700 and $4900 \AA$ and Loeb and Maxwell (128) assert that in the carbon-are spectrum it is between 4600 and $5100 \AA$. The unequal distribution of energy in the spectrum was not considered in these conclusions. Mast (144) made corrections for unequal distribution of energy and ascertained the relative stimulating efficiency of negative and positive orientation at intervals of $100 \AA$ throughout the visible spectrum, and found that as the wave-length increases the stimulating efficiency increases very rapidly from zero at about $4100 \AA$ to a maximum of 21 arbitrary units at $4850 \AA$, and then decreases equally rapidly to zero at about $5400 \AA$. He holds, however, that the limits of the stimulating region depend upon the luminous intensity. 
It is generally held that Euglena is positive in weak and negative in strong light (Famintzin, 63; Engelmann, 58; et al.). This is, however, true only in a very general sense. Increase in temperature without any change in illumination tends to make it positive, and decrease in temperature tends to make it negative (Strasburger, 202). Mast (140) observed, in strongly photopositive specimens in a beam of constant light, that as the temperature decreases, they become less strongly positive and less active until they come to rest at about $10^{\circ} \mathrm{C}$., after which they become more active, reaching a maximum at about $5^{\circ} \mathrm{C}$.; and he observed that as they become more active they become photonegative and swim as rapidly from the light as they swam toward it before the reduction in temperature.

Various other environmental factors also influence reversal. This problem has not been thoroughly investigated in reference to Euglena. The evidence in hand shows, however, that the nature of the response is not specifically correlated with the immediate environment, i.e., that internal factors-the state of adaptation, for example-play a predominant role.

\section{CILIATES}

Very few of the ciliates respond to light and only one of these, Stentor coeruleus, has been at all thoroughly investigated. If luminous intensity is rapidly increased, this organism stops, turns toward the aboral surface, and then proceeds. This is a shock-reaction, for if the intensity is slowly increased there is no response. If the intensity is decreased there is no response. If Stentor is exposed in a beam of light, it orients fairly precisely and swims from the light, i.e., it is photonegative. It rotates on the longitudinal axis as it swims, consequently when it is not oriented the oral and the aboral surfaces are alternately shaded and illuminated. The oral surface is much more sensitive than the aboral; therefore every time that this surface is carried from the shaded to the illuminated side, the result is the same as an increase in the illumination of the entire organism and it consequently responds, i.e., it turns toward the aboral surface. This continues until it is directed from the light, and rotation no longer produces changes of intensity on the two surfaces. Photic orientation in Stentor, is therefore, the result of a series of shock-reactions just as it is in Euglena. There is no evidence in support of the view that it is the result of a continuous quantitative difference in the activity of the cilia on opposite sides in proportion to the difference in the illumination of these sides. The process of orientation in this form is therefore not in accord with Verworn's theory (Jennings, 113; Mast, 137, 140).

More work should be done on responses in very low illumination and on the effect of various environmental factors on the response to light. The relations between rate of locomotion and intensity and wave-length of light have not been investigated. 


\section{COLONIAL FORMS}

\section{VOLVOX}

Volvox is a slightly elongated globular colonial organism somewhat less than $1 \mathrm{~mm}$. in diameter. It consists of numerous cells (zooids) each of which contains two flagella and an eyespot. The zooids are so arranged as to form a single layer at the surface of the colonies. The eyespot in each zooid is directed toward the posterior end of the colony, but those at the anterior end are much larger than those at the posterior end.

The colonies rotate on the longitudinal axis as they swim. This is due to the diagonal stroke of the flagella. In a beam of light they usually orient and go fairly directly either toward or from the light, i.e., they may in a beam of light be either photopositive or photonegative or neutral.

If they are swimming toward the light and the intensity is rapidly decreased without any change in the direction of the rays, rotation on the axis stops and forward movement increases greatly, but this continues for only a few seconds. If the intensity is increased, forward movement stops and rotation increases. If they are swimming from the light the reverse occurs, i.e., forward movement decreases if the intensity is increased and increases if it is decreased. If the colonies are neutral, there are no such responses to changes of intensity. These responses consist chiefly, if not entirely, of rapid changes in the direction of the stroke of the flagella. In photopositive colonies rapid decrease in illumination causes the stroke to change from diagonally backward to straight backward and increase in illumination causes it to change from diagonally backward to sidewise. In photonegative colonies precisely the reverse obtains. If the luminous intensity is slowly changed these responses do not occur. They are therefore dependent upon the rate of change of intensity, i.e., they are shock-reactions which are somewhat similar to those observed in Euglena.

If Volvox is kept in low illumination or in darkness for several hours it becomes inactive, and if the illumination is now increased it gradually becomes active again. These responses consist chiefly, if not entirely, in changes in the rate or the efficiency of the stroke, not in changes in the direction of the stroke of the flagella. They are relatively slow responses which occur even if the luminous intensity is gradually changed. They are primarily dependent upon change in luminous intensity, not upon the rate of change. There are consequently in Volvox two different types of response, typical shock-reactions and responses which are often called kinetic responses.

If a colony of Volvox in a beam of light is laterally illuminated, it turns gradually until it is oriented and then proceeds either toward or 
from the light. When it is laterally illuminated, the zooids, owing to rotation on the longitudinal axis, are continuously transferred from the dark side to the light side and vice versa. The eyespot consists of a pigment cup, a lens located at the opening of the cup, and photosensitive substance located in the cup. As the zooids pass from the light side to the dark side, the photosensitive substance in the eyespots becomes shaded by the pigment cup and as they pass from the dark side to the light side, this substance becomes fully exposed. The rapid decrease in the illumination of the sensitive substance on the dark side induces shockreactions on this side which, in photopositive colonies, consist of increase in the backward phase of the stroke of the flagella; and the rapid increase in the illumination of this substance on the light side induces shock-reactions on this side which consist of increase in the lateral phase of the stroke of the flagella. This difference in the direction of the stroke of the flagella causes the colonies to turn gradually toward the light until they are directed toward it, after which all sides are equally illuminated, rotation on the longitudinal axis no longer produces changes in the illumination of the photosensitive substance, and the shock-reactions cease. They continue to proceed directly toward the light because, in the absence of external stimulation, they tend to take a straight course and because, if they are forced out of their course, opposite sides immediately become unequally illuminated, resulting in change in the intensity of the illumination of the photosensitive substance in the eyes and consequently in reorientation.

In negative colonies the process of orientation is precisely the same as it is in positive colonies except that decrease in intensity causes increase in the lateral phase, and increase in intensity increase in the backward phase of the stroke of the flagella, resulting in more rapid movement of the illuminated than of the shaded side of the colonies and consequently in turning from the light in place of toward it.

Orientation in light is the result of qualitative difference in action of the locomotor appendages on opposite sides, due to shock-reactions, not to quantitative difference, due to continuous action of the light. It is therefore not in accord with the Ray-Verworn orientation theory (Mast, $138,143,151)$.

Mast (138), on the basis of quantitative results, concludes that the minimal difference in light intensity on opposite sides of a colony necessary to induce a response varies greatly with the physiological state of the colony, but that with colonies in a given state it varies directly with the intensity and that the ratio between it and the intensity is nearly constant, i.e., nearly in accord with the Weber-Fechner law. His observations, however, covered such a small range ( 2 to 27 meter-candles) and the probable error in the results is so large that further observations concerning this relation are highly desirable. 
The lens in the eyespot serves to bring the shorter wave of light to a focus on the photosensitive substance. This substance is much more sensitive in the central part of the cup than elsewhere. This conclusion rests on calculations based upon the relation between the location of the focal point and the direction of the incident light, and the relation between the direction of locomotion in a field of light (consisting of two horizontal beams which cross at right angles) and the relative intensity of the two beams. If photopositive colonies are exposed in such a field of light, they swim toward a point between the beams. The location of this point depends upon the relative intensity of the two beams. When the colonies are oriented, the illumination on opposite sides is unequal but the effect is equal. This is due to difference in the location of the focal point in the eyespot on opposite sides. By ascertaining, therefore, the location of these points in relation to the relative intensity of the beams, the distribution of sensitivity can be calculated (Mast, 152a; Mast and Johnson 161).

Volvox is usually positive in weak and negative in strong light but the reverse obtains under some conditions. It may be either positive or negative or neutral in every condition of illumination in which orientation occurs. If it is positive, a shadow on the photosensitive substance in the eyespots in the zooids causes change in the direction of the stroke of the flagella of the zooids from diagonal to backward, and a flash of light on this substance causes change from diagonal to sidewise; if it is negative, the reverse obtains; and if it is neutral, there is no response unless the changes in luminous intensity are great.

Reversal in the direction of orientation from positive to negative is, therefore, due to internal changes of such a nature that shock-reactions which were produced by decrease are produced by increase in the illumination of the photosensitive substance; and reversal from negative to positive is due to the reverse. The nature of the response to light in Volvox depends upon the state of adaptation and the intensity of the illumination. If it is fully adapted in a given intensity, it becomes positive if the intensity is increased and negative if it is decreased. If it is not fully adapted, it becomes negative if the intensity is increased and positive if it is decreased.

The time required for colonies of Volvox to become negative or positive after the luminous intensity has been changed (the reaction time) depends upon the degree of adaptation and the extent of the change. If colonies which have been in strong light 1 to $2 \mathrm{hr}$., followed by a variable period in darkness, are subjected to strong light, the time required to become positive (reaction time) increases with increase in the length of the period in darkness (dark adaptation) from $0.04 \mathrm{~min}$. (with $2 \mathrm{~min}$. in darkness) to a maximum of $0.52 \mathrm{~min}$. (with $16 \mathrm{~min}$. in darkness) and then decreases to $0.18 \mathrm{~min}$. (with $25 \mathrm{~min}$. in darkness). If the colonies are 
kept longer in strong light and are then subjected to darkness, the reaction time decreases to a minimum and then increases as the time in darkness increases. If they are left in darkness until they are fully dark-adapted, and are then exposed to light of different intensities, the reaction time (as the intensity increases) decreases from $29 \mathrm{~min}$. in 5.24 meter-candles to a minimum of $0.098 \mathrm{~min}$. in 7.5 meter-candles, and then increases to $0.358 \mathrm{~min}$. in 62,222 meter-candles; but the energy required to make the colonies positive varies directly with the light intensity over the whole range tested, and over most of the range the variation is nearly proportional to the variation in intensity. No satisfactory explanation of this relation is in hand.

If colonies are kept in a given intensity or in darkness they become adapted, i.e., they lose the ability to respond to light, and if the intensity is now changed they regain it. The processes associated with adaptation and those induced by change in illumination are therefore antagonistic. But the rate of these antagonistic processes varies greatly and it depends upon the magnitude of the change in intensity. For example, if darkadapted colonies are exposed to light of 22,400 meter-candles for $0.05 \mathrm{~min}$., then returned to darkness, it takes $20 \mathrm{~min}$. or more in darkness to eliminate the effect of the light. This indicates that under these conditions the processes which occur in light proceed at least 400 times as fast as the reverse processes which occur in darkness.

To account for the phenomena described it is necessary to postulate at least three interrelated processes some of which must be directly correlated with light in such a way that change in illumination of very short duration can cause complete reversal in the nature of the response. It is altogether probable that some of these processes are photochemical reactions, that others are dependent upon the results of these, and that all are closely correlated with the physiological state of the organism as a whole (Mast, 156). The evidence in hand clearly indicates that such simple processes as those postulated by Mast (138) in his first paper dealing with this problem, and those postulated by Luntz (133) in a recent paper, are very inadequate (Mast, 156).

A considerable number of other facts have been established concerning reversal in Volvox and related forms, e.g., increase in temperature and increased hydrogen ion concentration, and some anesthetics, especially chloroform, cause colonies, which are photonegative under given conditions, to become strongly photopositive under the same conditions, but they usually remain positive only a few moments and then become negative again (Mast, 145, 146). There is also a rery interesting correlation between reversal in light and response to electricity in that photopositive colonies always swim toward the cathode and photonegative colonies toward the anode (Mast, 152). These facts show that reversal in light is not due to direct action of environmental factors. They indi- 
cate that it is correlated with the rate of metabolism, but they give no information concerning the processes involved.

The distribution of stimulating efficiency in the spectrum for Volvox (Laurens and Hooker, 124) and Gonium (Mast, 144) is essentially the same as it is for Euglena; but for the closely related forms Pandorina and Spondylomorum (Mast, 144) the maximum is at $5350 \AA$ in place of $4850 \AA$, and the effective region extends from this wave-length much farther in either direction than it does for Euglena, Gonium, and Volvox.

\section{COELENTERATES}

Relatively few coelenterates respond to light and the response is not marked in those that do. They were, however, among the first animals without eyes investigated in reference to these responses. Trembley (204), in 1744, exposed to light green hydras in a jar covered with an opaque case containing an opening on one side and he found that they migrated toward this opening, but he gives no information concerning the nature of the response and the process of aggregation.

It is now known that they ordinarily move fairly directly toward the light; that they move in the opposite direction if the light is very intense; that they tend to come to rest in darkness; and that aggregation on the more highly illuminated side of a vessel is consequently not due to the relation between rate of movement and light intensity. There is no fixed orientation, but if they are photopositive, they tend to face in the direction of the light more than in other directions and they usually move only when they face in this direction. There are no definite shockreactions and nothing is known concerning the processes involved in stimulation. The anterior end is probably more sensitive than the posterior but no photoreceptors have been found. Response to light differs radically from the response to electricity (Mast, 140).

Blue probably has a higher stimulating efficiency than other regions of the spectrum. Wilson (223) maintains that daylight, the full spectrum, has a lower stimulating effect than the blue of daylight. This is important for it indicates that the action of the blue is antagonized by some of the other colors in the daylight.

The only other coelenterate which has been at all accurately studied in reference to photic response is Eudendrium. Eudendrium in the hydranth stage is a sessile animal. It bends directly toward the light in all intensities. This response is very slow, for it requires some $48 \mathrm{hr}$. to bend $90^{\circ}$. There is nothing in the nature of shock-reaction. Loeb (126) contends that the bending is due to contraction on the more highly illuminated side and that the process of orientation is identical with that in plants. The evidence in support of these contentions is, however, extremely weak. Growth appears to be involved in the process of orientation and this is doubtless related to difference in the luminous intensity 
on opposite sides, but nothing is known eoncerning the details (Mast, 140).

The planulae of Eudendrium, small ciliated organisms which move slowly on the substratum, also turn directly toward the light. The anterior end continuously swings from side to side, but there are no shock-reactions. No details concerning the process of response are known (Mast, 140).

\section{WORMS}

Some of the worms are very sensitive to light and respond very precisely, especially in the larval stages, but only a few species have been thoroughly investigated. I shall present the results obtained with these species in some detail and those obtained with some other species very briefly.

\section{TUBICOLOUS ANNELIDS}

Many of the worms give very striking shock-reactions, usually to rapid decrease in luminous intensity. Various tubicolous annelids are particularly noteworthy in this respect. If a shadow is east on them, they suddenly dart into their tubes (Dalyell, 46; Ryder, 191; Andrews, 7; et al.). This response doubtless serves to protect them against enemies. It is readily modified, and the amount of reduction in light necessary to induce it varies greatly, as does also the time the animals remain in the tubes after a response. Mrs. Yerkes (233) found, in a series of 60 successive responses of a specimen, that it remained in the tube from 10 to 710 sec. It is consequently evident that these responses are not closely correlated with the magnitude of the change in the light received. They are due to muscular contractions, but nothing is known concerning the photoreceptors, the internal processes involved in the contraction, and the stimulus and the impulse that induce it.

\section{ARENICOLA LARVAE}

The larvae of many aquatic worms are at first intensely photopositive and later photonegative. Observations made on the larvae of Arenicola lead to the following conclusions: These larvae are finger-like in form and about $0.3 \mathrm{~mm}$. long. They have two eyes and a band of cilia near either end. They swim for a time after they hatch, then settle to the bottom and crawl. They orient precisely and are strongly positive when they swim and negative when they crawl.

Swimming is the result of eiliary action, but orientation is the result of muscular contraction. The larvae rotate on the longitudinal axis when they swim, so that when they are laterally illuminated, the two eyes are alternately directed toward and away from the light, resulting in alternate increase and decrease in the intensity on them. When in the 
process of rotation one eye comes to be directed toward and the other from the light, the muscles on the illuminated side contract violently, turning the head toward the light. This occurs twice during each rotation and soon results in orientation, after which, if the light is from a single source, the two eyes are continuously equally illuminated and muscular contraction ceases.

Mast (140) contends that these muscular contractions are reflexes dependent upon the rate of change of luminous intensity in the eyes, and that the orienting stimulus consequently ceases after the organism is oriented. Garrey (75) holds that they are the result of difference of tonus in the muscles on opposite sides, due to difference in the amount of light received by the two eyes; that the tonus of the muscles on either side is continuously proportional to the amount of light received by the eye on that or the opposite side; and that the orienting stimulus consequently continues after the organism is oriented, being equal when the two eyes are equally illuminated, i.e., when the organisms swim directly toward the light. If the larvae are held so that they cannot rotate, and the light in one eye is increased or that in the other is decreased, the muscle on the more highly illuminated side contracts, but it is unfortunately not known how long this position is held. The results in hand consequently show that contraction of the muscles is correlated with difference in the intensity of the illumination of the two eyes, but they do not show whether it is due to difference in tonus in accord with Garrey's contention or change of intensity in accord with Mast's. It may, however, be said that the response occurs so rapidly that there does not appear to be time to induce what is ordinarily called tonus.

The distribution of stimulating efficiency in the spectrum for larvae of Arenicola is essentially the same as it is for Euglena viridis. The maximum is approximately at $4850 \AA$, from which it decreases rapidly in either direction (Mast, 144).

\section{EARTHWORMS}

The response to light is essentially the same in the different species of earthworms investigated. The following statements refer to Lumbricus terrestris and Eisenia foetida. These species respond very vigorously to a flash of light by violent contraction or by raising the anterior end and swinging it from side to side. If repeatedly stimulated in fairly close succession these responses become less and less definite and finally disappear (Hoffmeister, 108). If the intensity is slowly increased, they do not occur. They are, therefore, shock-reactions, i.e., responses dependent upon the rate of change in intensity.

If specimens are kept in darkness or in constant illumination they become quiet, and if the illumination is now increased, they very slowly become active. This response is not directly dependent upon the rate 
of increase in luminous intensity. There are therefore two types of response, a rapid response which is closely correlated with the rate of change in luminous intensity, and a slow response which is not closely correlated with the rate of change in this intensity. Neither type of response has been thoroughly investigated.

In the epidermis and in enlargements, near the epidermis, of some of the nerve cords, there are large cells, each of which contains a highly refractive body surrounded by a nerve net. These cells are photoreceptors. They are very abundant in the prostomium. From here posteriorly they decrease rapidly to a minimum in about the sixth segment, then remain nearly constant until near the posterior end where they increase again. Their distribution is the same as the distribution of sensitivity to light (Hesse, 107; Hess, 106).

Lumbricus and Eisenia orient fairly precisely. They are usually negative in all but very weak light in which they are positive (Adams, 1). If they are laterally illuminated without increase of intensity, usually they gradually turn directly from the light until they are oriented. If they are laterally illuminated with increase of intensity, they frequently turn directly toward the light, then swing the anterior end from side to side for a time, and finally turn from the light. If the intensity of lateral illumination is low, there is usually marked swinging of the anterior end and orientation is indefinite. The swinging of the anterior end is in the nature of exploratory movements; it serves to localize the direction of illumination more accurately. Turning from the light (orientation) may be due entirely to contraction of the shaded side or entirely to random swinging of the anterior end or to a combination of these two phenomena. Contraction of the shaded side is directly correlated with difference in the intensity of the illumination of opposite sides, but it is probably in the nature of a reflex shock-reaction. At any rate, there is no evidence indicating that it is due to difference in tonus of the muscles on opposite sides in direct proportion to differences in the amount of light received by the photoreceptors on opposite sides (Mast, 140).

Specimens with the brain removed are positive in ordinary daylight, i.e., they are positive in light of very much higher intensity than are normal specimens. In specimens with the nerve cord cut, the portion back of the cut is positive and that in front of the cut is negative. The action of the brain, therefore, increases the negativity of the worm (Hess, 105). Adaptation to light (Hess, 105), reduction in temperature, injection of depressant drugs around the brain, and injury of certain parts of the brain (Prosser, 187) have the same effect as removing the brain; rise in temperature, within certain limits, and stimulating drugs have the opposite effect. Specimens with the brain removed are less active and become more readily light-adapted than normal spccimens (Prosser, 187). These facts indicate that decrease in metabolic processes 
makes the worms more positive and that the action of the brain counteracts this. If this is true, the positiveness of the worms should vary indirectly with oxygen tension. This has not been investigated.

Prosser (187) reaches the following conclusions concerning the processes involved in these phenomena: In specimens with the brain removed, lateral illumination produces impulses in the photoreceptors on the illuminated side, which pass through the nerve cord to the muscles on this side and cause them to contract. This results in turning toward the light. In normal specimens, in addition to the impulses which pass through the cord to the muscles on the illuminated side, there are impulses which pass through the brain to the muscles on the opposite side. These come from the receptors in the highly sensitive anterior end of the worm. They are, therefore, much stronger than those which originate farther back and pass through the cord. The latter are consequently inhibited by the former; this results in contraction of the muscles on the shaded side and turning from the light.

This accounts for the observed increase in positiveness with decrease in the activation of the brain. There are, however, other possible interpretations of the results in hand. Further investigation is desirable. The observations on adaptation to darkness and light should also be extended.

The stimulating efficiency of light is closely correlated with wavelength. Beginning with zero at $4430 \AA$, it increases rapidly, with increase in wave-length, to a maximum between 4830 and $4930 \AA$, and then decreases rapidly to zero at $5240 \AA$. This indicates that the range of wave-length effective in stimulation is very small. In high intensity it is doubtless larger than indicated by the results presented (Mast, 144).

Yerkes (234) demonstrated that earthworms in a maze learn by experience to avoid regions containing salt or an electric grill. Copeland (41) found, in observations on specimens of Nereis virens in glass tubes, that if a spot of light is always flashed on the food when it is presented in front of the opening of the tubes, these worms soon learn to come out when the light is flashed on without the food; and that if now a shadow is always cast on the food when it is presented, they soon learn to come out when the shadow is thrown on without the food. He concluded that "as a result of experience, both increased and decreased illumination indicates to the worms the presence of food."

\section{TURBELLARIA}

The turbellaria creep on the substratum by means of cilia and turn by means of muscles. Many of them respond to light. If the intensity is rapidly changed, practically all of these raise the head and swing it from side to side. This response depends upon the rate of change in intensity. It is a shock-reaction. Some wander about at random until 
they get into light of a certain intensity, where they come to rest and thus aggregate in this intensity. Others orient fairly precisely; some of them are photopositive, others photonegative (Loeb, 126; Walter, 211; Mast, 140).

If the light intensity is increased after a specimen has come to rest, it remains at rest for some time, then slowly becomes active. This activity gradually increases to a maximum and then remains fairly constant (Mast, 140). The maximum depends somewhat upon the intensity (Walter, 211). This relation should be more thoroughly investigated. This response does not directly depend upon the rate of change of intensity. It may be called a kinetic response.

Taliaferro (203) made a thorough study of the process of orientation in Planaria maculata under accurately controlled conditions and came to the following conclusions: If Planaria maculata is illuminated from one side with light of moderate intensity, the opposite side contracts, resulting in gradual turning until it is oriented, after which it proceeds fairly directly from the light. If the intensity is higher, it turns more rapidly, and if it is very high, it may first swing the anterior end from side to side several times and then turn from the light. The nature of the response is therefore dependent upon the intensity of the light.

If the two eyes are removed, it does not orient. Orientation is consequently due to stimuli received through the eyes. Specimens with one eye removed orient nearly as precisely as normal specimens. If they are illuminated from the normal side, they turn directly from the light until they are oriented and then continue fairly directly from the light. If they are illuminated from the blind side, they continue without any response until, owing to random wandering or swinging or twisting of the anterior end, the light enters the intact eye. If it enters the posterior or the ventral rhabdomes in the eye, they turn toward the normal side, i.e., from the light, and soon become oriented. If it enters the anterior or the dorsal rhabdomes, they turn from the normal side, i.e., toward the light. This turning may continue until they are directed from the light, or there may be a series of successive responses which finally result in orientation. The direction of turning, i.e., the nature of the orienting response, therefore, depends upon the location of the stimulus in the eye as well as upon the intensity of the light. These responses are rapid and of short duration. They are probably dependent upon rate of change in intensity, i.e., they are probably shock-reactions. Orientation in one-eyed specimens is, therefore, due to one or more responses, consisting of rapid muscular contraction on one side. It obviously is not due to "balanced" stimulation of photoreceptors on opposite sides, resulting in "balanced" tonus of muscles on opposite sides, in accord with Loeb's tonus theory of orientation, and there appears to be 110 reason for assuming that it is essentially different in normal specimens. 
The activity of specimens without eyes depends upon the intensity of the light in which they are exposed. These responses are correlated with the amount of light energy received, rather than with the rate of change in the amount received. There are, therefore, two kinds of response in Planaria, one dependent upon the eyes, the other not. There is nothing known concerning the chemical and physical changes associated with either of these responses or with the stimuli which induce them, and practically nothing concerning the quantitative relation between the stimuli and the responses.

The distribution in the spectrum of stimulating efficiency, in these forms, has not been adequately investigated. Kühn (119) says planarians are positive in light of long wave-length and negative in light of shorter wave-length, and Beuther (18) maintains they have color vision, but Koehler (116) thinks this has not been established. Merker and Gilbert (167a) conclude that they "see" ultra-violet, but they give no information concerning discrimination between different regions in the visible spectrum.

\section{MOLLUSCS}

Response to light has been fairly intensively studied in several different molluses: Mya, Pecten, Helix, Limax, and Agriolimax.

MYA

Mya, the long-necked clam, responds very definitely to rapid increase in light by contraction of the neck, and to rapid decrease in light by closing the siphons (Nagel, 175). It contains numerous photoreceptors on the inner surface of the siphons. They are found throughout its entire length, but they are most abundant in the central region. Their distribution is closely correlated with the distribution of sensitivity, and the reaction time varies directly with the number of receptors illuminated (Light, 125).

Hecht (83 to 89) investigated in its various phases, the response to increase in light intensity in $\mathrm{Mya}$ and also in Pholas and Ciona, ${ }^{2}$ and reached the following conclusions:

The time between increase in illumination and contraction (reaction time) is composed of two periods. During the first of these periods, the exposure period, the organism must be in light; during the second, the latent period, it does not need to be in light. In a given intensity, as the exposure period increases, the reaction time and the latent period clecrease to a minimum and then remain constant. The minimum latent period does not vary with the intensity but the exposure period $t$, produc-

${ }^{2}$ Pholas is a molluse which is much like Mya in structure. Ciona is an ascidian which is not closely related to Mya, but it contains siphons which respond to increase in illumination very much like those in Mya. 
ing the minimum latent period, is inversely proportional to the intensity $i$, that is, $t i$ is constant and the response is therefore in accord with the Bunsen-Roscoe law.

With a given intensity, the exposure period is practically independent of temperature, but the latent period varies inversely with the temperature. The value of $\mu$ in the Arrhenius equation is 19,700 for Mya, 18,300 for Pholas, and 16,200 for Ciona.

During dark-adaptation the reaction time for constant intensity increases rapidly at first, and then more slowly until it reaches a minimum, after which it is constant. The rate of dark-adaptation varies directly with temperature. It is a slow process, requiring half an hour or more.

The maximum stimulating efficiency in the visible spectrum is at $5000 \AA$ for $M y a$ and at $5500 \AA$ for Pholas. From these points it decreases rapidly in either direction.

During exposure to light, a substance $S$ in the photoreceptors, is broken down into two or more substances $(P, A, C)$. One of these, $C$, is a catalyst, which during the latent period acts on another substance $L$ and produces still another substance $T$. When this substance becomes sufficiently concentrated it stimulates the nerves and causes the siphon to contract. In darkness, $P$ and $A$ unite to form $S$.

The first reaction is a reversible photochemical reaction independent of temperature. The second is dependent upon temperature. It is not a photochemical reaction. It is probably an oxidation reaction catalyzed by iron.

The processes involved are identical in the three forms investigated, but the substances involved differ.

Piéron (185) and Folger (66) repeated a portion of Hecht's observations on Mya. Both maintain that the results obtained do not support Hecht's contention that the energy $t i$, necessary to induce retraction of the siphon, is constant for different intensities. In view of these contradictory contentions it is highly desirable to have the observations on which they are based, repeated and extended.

Hecht and Wolf (95) compared the stimulating efficiency for Mya, of intermittent light with a flash frequency of 130 per sec., with that of continuous light and found it to be the same. They conclude (page 388), "the results demonstrate the validity of Talbot's law for Mya."

\section{PECTEN}

Pecten is remarkable for its numerous well developed eyes on the edge of the mantle. It responds vigorously to rapid reduction in light by closing its valves but it does not respond to increase in light (Wenrich, 219). There has been much discussion but very little experimental work concerning the function of the eyes. Wenrich maintains that if a highly illuminated small white cardboard is moved over a large vertical 
black surface in front of one of these molluses, it closes its valves even if conditions are so arranged that the illumination of the white cardboard increases as it moves. He concludes that this demonstrates "the ability of the Pecten eye to form an image."

I think that this conclusion is valid. The response observed is doubtless due to reduction in light intensity on a localized region of the retina, owing to movement of the image of the white cardboard.

\section{HELIX}

The snail, Helix, like the turbellaria, creeps by means of cilia and turns and orients by means of muscles. It is photonegative.

Buddenbrock (23) maintains that in vertical illumination specimens with one eye removed turn continuously toward the blind side, and that the rate of turning (magnitude of deflection from a straight course) varies directly with the intensity of the light. He asserts that this is due to unequal tonus of the muscles on opposite sides, owing to the difference in the illumination of the two eyes, but he contends that in lateral illumination such specimens turn directly from the light toward either side, and that this is due to reflexes. He maintains that if Helix is slowly rotated so as to change the direction of movement in relation to the source of light, it turns so as to resume its original orientation. This response he calls "Kompassbewegung." Fraenkel (68) obtained similar results with another snail, Elysia viridis. This response seems to show that the eyes of Helix and Elysia form images, and that when these move over the retina the animals turn so as to tend to retain their former position.

If the eyes form images the direction of turning is probably correlated with the location of the stimulus in the eye as it is in Planaria. If this is true, the continuous turning toward the blind side in vertical illumination is probably correlated with the location of the image in the eye, just as is the turning toward the light in lateral illumination. There consequently appears to be no necessity for assuming the former to be the result of tonus and the latter the result of reflexes.

\section{LIMAX AND AGRIOLIMAX}

The slugs, Limax and Agriolimax, like Helix, use cilia for creeping and muscles for turning. They are photonegative and geonegative. One-eyed specimens in vertical illumination tend continuously to deflect toward the blind side, i.e., they make circus movements like Helix. Normal specimens on a vertical surface in a horizontal beam of light creep upward and deflect from the source of light. The relation between the amplitude of deflection and the intensity of vertical illumination, and the relation between the angle of deflection from the vertical and the intensity of lateral illumination have been investigated by Crozier 
and Cole (43), Crozier and Federighi (44), Wolf and Crozier (230), and Crozier and Wolf (45).

They found that the amplitude of deflection from a straight course in one-eyed specimens in vertical illumination varies directly with the light intensity and inversely with the rate of locomotion, and that the angle of deflection from the vertical in normal specimens with lateral illumination varies directly with the light intensity and inversely with the time of exposure to the light.

They assume that turning is due to difference in the tonus of the muscles on opposite sides, that this difference in tonus is proportional to the difference in the amount of light received by the two eyes, that light acts continuously, and that the effects of light and gravitation on turning on a vertical surface in a horizontal beam of light are practically independent of the magnitude of the angle between the vertical and the direction of locomotion. On the basis of these assumptions and the results obtained in experimental observations, they made mathematical calculations and reached the following conclusions:

In Limax the amplitude of turning in vertical illumination (one-eyed specimens) and the angle of deflection from the vertical in horizontal illumination (normal specimens) are directly proportional to the logarithm of the intensity. In Agriolimax in horizontal illumination, the angle of deflection from the vertical and "the velocity of photic adaptation" are directly proportional to the logarithm of the intensity. "The excitation is at any moment proportional to the rate of photolysis of sensitive material $S$, undergoing a first-order decomposition by light and reconstituted by a 'dark' reaction which is second order and with positive autocatalysis." (43, page 669.)

Some of these conclusions are equivocal, for some of the assumptions upon which they rest are questionable. The effect of gravitation on turning, which is assumed to be constant, is zero when the slug faces upward, and it increases (as the angle of deflection from the vertical increases), to a maximum probably when the slug faces downward; and the stimulating efficiency of light in reference to the effect on turning, which is assumed to be constant, probably varies with the location of the image of the light in the eye, and this varies with the angle of deflection from the vertical. Moreover, Buddenbrock contends that in Helix, which is similar in structure to Limax, orientation is the result of reflexes. If this is true, the magnitude of turning is probably not proportional to the difference in the amount of light received by the two eyes, as assumed. These investigations should, therefore, be repeated and extended with the variables involved controlled or eliminated.

\section{ARTHROPODS}

The study of response to light in the arthropods is largely confined to Daphnia and a number of insects. 


\section{DAPHNIA}

Daphnia is a small crustacean with one compound median eye and two locomotor antennae, one attached to either side at the anterior end.

In diffuse constant illumination, it orients with the long axis vertical and the anterior end up, and swims about very slowly and irregularly, now sinking a little and then rising again, with but little change in location. In a horizontal beam of light, it behaves in the same way but it orients so that the back faces the light. If the light intensity is rapidly decreased, it stops, sinks a little, and then either retains its orientation and swims slowly toward the light backward or turns through $180^{\circ}$ and swims rapidly toward it forward. If the intensity is rapidly increased, it stops, sinks a little and swims from the light forward. If the intensity remains constant after it has become positive or negative, it soon becomes neutral, i.e., it rapidly becomes adapted to the changed illumination. If the intensity is slowly changed these responses do not occur. They are, therefore, dependent upon rate of change in intensity, and have the essential characteristics of shock-reactions. Ewald (60) calls them "Bewegungsreflexe."

If Daphnia is kept in darkness 12 to $24 \mathrm{hr}$., it becomes inactive and sinks to the bottom; and if it is then illuminated, it gradually becomes active and swims upward. This response occurs whether the light is slowly or rapidly increased; it is, therefore, not specifically dependent upon the rate of change in intensity (Koehler, 115).

There are at least two systems involved in response to light in Daphnia; one which is primarily dependent upon rate of change in intensity, and one which is not.

The relation between the rate of change in intensity and the magnitude of change in activity should be more thoroughly investigated.

Clarke (37) studied in some detail the Bewegungsreflexe. He was unable to make accurate measurements concerning the different phases of the response, owing to the gradual change in the activity of the organisms ("the gradual onset of the response"). This doubtless accounts in large measure for the marked variability in the results recorded. He came to the following conclusions:

The "threshold amount of reduction" in light varies inversely with the rate of reduction and the "duration of previous exposure to light" and directly with "duration of previous sojourn in dark." The latent period varies inversely with the amount of reduction and the temperature. The "speed of response" varies directly with the amount of reduction and the temperature. The "magnitude of response" varies directly with the amount of reduction and the "duration of previous exposure to light" and inversely with the "duration of previous sojourn in dark." The "duration of response" varies directly with the amount 
of reduction. The change in direction of movement from photopositive (following decrease in illumination) to photonegative (following increase in illumination) "can be accounted for by one mechanism of excitation, if it were photoreversible and properly controlled."

The photoreversible process postulated by Clarke is essentially the same as that postulated in 1907 by Mast to account for reversal in photic response in Volvox. The results obtained in more recent observations on Volvox (Mast, 152, 156) made it necessary, however, to postulate a much more complicated system to account for reversal and the factors associated with it in these organisms. This doubtless also obtains for Daphnia, for Clarke's postulated mechanism does not explain reversal in direction of locomotion from negative to positive accompanied by reversal in orientation.

Clarke and Wolf (38) observed that when Daphnia swims forward toward the light, the body is "tilted forward" and that when it swims backward from the light, it is "tilted backward" and they seem to hold that reversal is due to this change in posture, but they do not explain how this difference in the posture of the antennae would produce the difference in direction of locomotion observed. I think it is due to reversal in the direction of the effective stroke of the antennae and that the tilting of the body is due to lag in the movement of the body, owing to the horizontal pull of the antennae at the anterior end. It would probably be a simple matter to ascertain by direct observations which of these views is correct.

Increase in hydrogen ion concentration (Ewald, 60), rapid decrease in temperature (Clarke, 37) and strychnine (Clarke and Wolf, 38) have the same effect on reversal in direction of movement as rapid decrease in luminous intensity. Reversal in light is correlated with reversal in a galvanic current. When Daphnia is photopositive, it is positive to the cathode, and when it is photonegative, it is positive to the anode. It always swims backward toward the cathode and forward toward the anode (Clarke and Wolf, 38). The effect of environmental factors on reversal in Daphnia is essentially the same as it is in Volvox (Mast 145, $146,152,156)$.

Orientation.-Ewald (62) maintains that in reference to Bewegungsreflexe, the stimulating efficiency of intermittent light with a frequency of about 1 flash per sec. is 16 times as great as continuous light, that as the frequency increases, the efficiency decreases proportionally until at about 20 flashes per sec., it is equal to continuous light. He concludes that Talbot's law and the Bunsen-Roscoe law hold.

This method has been used by a considerable number of other investigators (Ewald, 61; Patten, 183; Loeb, 127; et al.) to prove that the Bunsen-Roscoe law holds. It is, however, obvious without experimental demonstration that intermittent light has for every system quantitatively 
the same effect as continuous light, provided the frequency is sufficiently high. It is therefore evident without observation that the BunsenRoscoe law holds in some respects for every system and that its establishment is of importance only when it is specifically correlated with flash frequency, i.e., the duration of the exposure period, and with the intensity of the light (Mast, 148).

Daphnia, as stated above, usually orients with its back toward the source of light. If the direction of illumination is changed, it turns directly from the light until it is again oriented. If it is held so that the body cannot turn, the eye turns so as to assume as nearly as possible its former orientation (Rádl, 189).

Clarke (37, page 184) comes to the following conclusions: "Orientation is brought about by the differential stimulation by the light of the bilaterally symmetrical organ of light reception. The consequence is that differential changes in the posture of the two swimming appendages on the two sides of the body are produced. As a result the animal is turned until equal amounts of light are received by both sides of the eye and orientation is complete." It "conforms to the general theory of phototropic orientation," meaning, I assume, Loeb's tonus theory.

He presents no evidence in support of these conclusions. It may well be that orientation is the result of change in the direction of the stroke of the antennae correlated with the location of the stimulus in the eye, rather than to "differential changes in the posture" of the two antennae correlated with the relation in the amount of light received by opposite sides of the eye. Detailed observations on the posture and the action of the antennae would doubtless yield results which would have important bearings on this problem. Moreover, according to Ewald (62), when the eye is oriented in light from two sourees, the two sides are equally illuminated only if the light received from the two sources is equal. If this is true it is obvious that when Daphnia is oriented under natural eonditions "both sides of the eye" rarely if ever" "receive equal amounts of light."

Ewald (62) maintains that intermittent light has per given quantity the same effect as continuous light on the orientation of the eye and on the position of aggregation of free specimens, and that this effect is independent of flash frequency down to 1 per sec. and consequently that the process of orientation and the Bewegungsreflexe are independent. He postulates that the former is due to stimulation of the lateral, and the latter to stimulation of the anterior ommatidia in the eye, but he presents no evidence in favor of this postulation.

Clarke (37) and Clarke and Wolf (38) observed that strychnine makes photonegative specimens photopositive without eausing any ehange in orientation and they maintain that this shows that the two processes are distinet. They eonsequently support Ewald in the contention that the 
Bewegungsreflexe and the process of orientation are independent. But they do not support him in the contention that their independence is due to different receptors involved. They maintain that the one process is correlated with rate of change in light intensity and the other with continuous aetion of light.

Color Vision.-Beginning with Bert (13) numerous investigators have attacked the problem of color vision in Daphnia. Some concluded that Daphnia is color-blind, others that it is not. Koehler (115) reviewed the literature in detail and presented the results obtained in an intensive investigation of the problem. He maintains that if Daphnia is fully adapted to light of moderate intensity, it is positive in red, orange, yellow, or green, and negative in blue or violet, no matter whether these colors are brighter or darker for Daphnia than the white light in which it is adapted, but that if they are fully dark-adapted, they are negative to all colors provided the increase in intensity is low and that if they are very strongly light-adapted, they are positive to all color's.

He concludes that if Daphnia is fully dark-adapted or very strongly adapted to intense light, it is eolor-blind, but that if it is adapted to light of moderate intensity, it can distinguish at least two eolors. "Sie unterscheiden der Wellenlänge nach, und unabhängig von der Intensität, mindestens zwei Qualitäten voneinander und ron farblosen Lichtern, nämlich langwelliges (Rot, Gelb, Grün) und kurzwelliges Licht (Blau, Violett)" (page 135). Merker (166a) maintains that he has proved that Daphnia "sees" ultra-violet, but he gives no information coneerning the rest of the speetrum.

\section{INSECTS}

More observations and more speculations have been deroted to motor responses to light in insects than in any other group of invertebrates. These observations and speculations eoncern the processes involved in orientation, the effect of light on activity, the rate of adaptation to light and to darkness, and vision.

\section{ORIENTATION}

Many insects orient very precisely in light, some are photopositive or photonegative and some tend to hold any given direction of movement that has once been assumed in relation to the direction of the most intense illumination. In light from two sources, the former usually go toward or from a point between the two sources, the latter usually directly toward one of the two sources.

If a photopositive inseet is laterally illuminated in a beam of light from a single souree, it turns toward the light until the two eyes are approximately equally illuminated, and then proceeds in a fairly straight course. If it is exposed in a field of light consisting of two beams crossing 
at right angles, it goes toward a point between the two beams. The location of this point depends upon the relative intensity of the two beams. The greater the difference, the nearer the more intense beam is this point (Mast, 140; Patten, 183; Dolley, 49).

Results obtained in observations made on Eristalis tenax by Dolley and Wierda (56) show that with the intensity of the two beams so related that when the insect is oriented the image in one eye is near the anterior end and that in the other eye is near the posterior end, the one receives 50 times as much light as the other. That is, these results show that to produce a given effect on turning, an image near the anterior end of the eye must be 50 times as intense as an image of the same size near the posterior end. This indicates that the efficiency of light to induce turning decreases rapidly as the location of the image passes forward in the eye. In an oriented insect under these conditions the two eyes are equally illuminated only when the light in the two beams is equal (Mast, 148). Parker (182) and Cole (39) demonstrated that a given amount of light in a large image has a greater effect on turning than the same amount of light in a small image. It is therefore evident that when an insect is oriented under natural conditions, the two eyes are rarely if ever equally illuminated.

If photopositive insects on a nonreflecting background are (with a concentrated source of light) illuminated on one side from behind, all the legs on the illuminated side step backward and all on the opposite side forward. If illuminated directly from the right, the front legs on both sides step toward the right. If illuminated directly from in front, all the legs on both sides step forward. This obtains also for insects with only one functional eye. The movement of the legs on both sides is controlled by photic stimulation of either eye and the direction of movement depends upon the location of the stimulus in the eye (Mast, 148, 149).

Photopositive insects with the front and the middle legs on one side removed orient nearly as precisely as normal insects. If they are laterally illuminated from either direction, the front leg is extended toward the light, attached and then flexed. Thus the anterior end is pulled either toward the operated or toward the normal side, depending upon which side is more highly illuminated. After they are oriented, the front leg is extended nearly directly forward, attached, and then flexed. Thus they proceed slightly sidewise but fairly directly toward the light, with the two eyes continuously unequally illuminated (Mast, 148).

Insects with the function of one eye destroyed tend to turn continuously toward the functional eye if they are photopositive, and toward the blind side if they are photonegative, and some of them lean in the direction in which they turn, the legs on one side being sharply flexed and those on the other side much extended (Holmes, 109; Parker, 182; Rádl, 189; Dolley, 49; Garrey, 75). 
In a beam of light they orient with the longitudinal axis at an angle with the direction of the rays and go diagonally across the beam. The angle of deflection is independent of the light intensity; but if the intensity is rapidly increased or decreased after the insects are oriented, they turn sharply from or toward the light, then gradually back until they have assumed their former direction of movement. If the intensity is slowly changed, they do not turn. These responses are therefore typical shockreactions (Dolley, 49).

The tendency to turn is much more marked in diffuse light with much reflection from the background than it is in a beam of light with little reflection from the background. It decreases with time and in some insects disappears entirely (Rádl, 189; Holmes, 109; Dolley, 49; Minnich, 170). Some contend that the decrease is correlated with experience; but Clark (33) found that it is correlated with adaptation to light and that it probably is independent of experience. Mast (149) maintains that if the ventral and the posterior surfaces of the functional eye are covered, there is no tendency to turn. He concludes that the turning is due primarily to stimulation of the rhabdomes in the posterior and the ventral portions of the eye by light reflected from the background. Dolley (49) contends that in vertical illumination the rate of deflection in turning varies directly with intensity, if the source of light is large and the background is white, but indirectly if the source of light is very small and the background is dull black.

Eristalis on the wing orients fairly accurately. If, after it is oriented in a beam of light, the source of light is raised or lowered, it turns directly upward or downward. When the position of the light is changed, the images in the two eyes are equally and simultaneously changed in location. The turning is due to this change. It cannot be due to difference in the illumination of the two eyes for they are continuously equally illuminated (Mast, 148).

If a male firefly on foot or on the wing is some distance from a female firefly when she produces a flash of light, he turns until he faces the direction from which the light came and then proceeds directly toward her. The magnitude of the angle through which he turns consequently varies from zero to practically $180^{\circ}$ depending upon the direction in which he faces in relation to the location of the female when the flash of light is produced. This obviously is correlated with the location in the eye of the image of the flash of light. If it is located in the posterior region of the eye, a chain of reflexes is initiated which results in turning through nearly $180^{\circ}$ followed by direct forward movement. If it is located in the anterior region of the eye, no turning reflexes are initiated.

If a male firefly glows in the neighborhood of a female, she raises and twists the abdomen until the ventral surface is directed toward him and then glows, no matter where he is located. Thus, after an image of a 
flash of light produced by a male has been formed on the retina of a female, she, in total darkness, directs the ventral surface of the abdomen toward any point in space, depending upon where the male chances to be when he glows. The image obviously starts a series of reflexes, the nature of which is specifically correlated with the location of the image in the eye.

The males very rarely if ever respond to the glow of other males. In some way they are able to distinguish between the flashes of light produced by the two sexes (Mast, 141).

Some insects, after they have assumed a definite axial position in relation to a source of light, tend to retain this position. This phenomenon is particularly prominent in a complex field of light, e.g., in direct sunlight in an open field and in insects on a turntable. The insects apparently tend to take such a course that the location of the images in the eye remains constant. It has been observed in the beetle Coccinella by Rádl (189), in ants by Santschi (194); in bees by Wolf (224, 225), and in a considerable number of other insects by Buddenbrock (22, 23, 26, 27), Niemczyk (177), Fraenkel (68), Willrich (222), Schulz (199), and Buddenbrock and Schulz (29).

Loeb (126), Bohn (20), Garrey (75), and many others hold that photic orientation in insects is the result of quantitative difference in the activity of the locomotor appendages on opposite sides, that this difference in activity, e.g., length of steps, is due to difference in the tonus of the muscles in the appendages on opposite sides, that the difference in tonus is directly proportional to the difference in the amount of light received by the eyes on opposite sides, in accord with the Bunsen-Roscoe law, that light acts continuously, not intermittently, that after orientation the two eyes receive the same amount of light, and that this results in equal muscle tonus and equal activity in the locomotor appendages on opposite sides.

These conceptions concerning orientation do not account for many of the facts presented above, for example: $(a)$ orientation in insects with one eye covered; $(b)$ orientation in insects with legs on one side removed; $(c)$ turning upward or downward toward the light, while the two eyes are continuously equally illuminated; $(d)$ turning toward the side on which the legs are more extended; (e) orientation in a field of light consisting of two beams unequal in intensity crossing at right angles, in which the two eyes do not receive equal quantities of light after the insect is oriented; $(f)$ shock-reactions in one-eyed insects; $(g)$ decrease in deflection in circus movement, with increase in light intensity; $(h)$ orientation in insects which tend to maintain an axial direction such that the images in the eyes remain constant in position; $(i)$ orientation of the male firefly in response to a flash of light produced by the female. 
On the basis of these facts and others, Mast $(147,148,149)$ came to the following conclusions: Orientation in insects is the result of reflexes specifically related to the location of the stimulus in the eyes. Stimulation of different regions in either eye induces different series of reflexes in which all the legs are involved. If both eyes are simultaneously stimulated, the effect produced in one is modified or neutralized by that produced in the other. If the stimuli in the two eyes are the same in location and magnitude, light has no immediate observable effect. If they are the same in location but different in magnitude, the insect turns toward the stronger. If they are the same in magnitude and different in location, the insect turns toward the one which is farther back. ${ }^{2 a}$ After the insect is oriented in a field of light, no matter how complex, orienting stimulation ceases. It is not held on this course by continuous action of the light. It tends to continue in the direction assumed, owing to internal factors. But if for any reason it deviates from the course, it is stimulated and turns back as described above. According to these conclusions the location of the stimuli in the eyes is quite as important in the process of orientation as the relative amount of light received by each of them.

Clark (35) maintains that the conclusions presented are in accord with all the facts in hand concerning photic orientation in insects except three: $(a)$ increase in stimulating efficiency with increase in the size of the source of light; $(b)$ decrease in the angle of deflection in one-eyed insects, with increase in time in light, and $(c)$ decrease in this angle with increase in the intensity of light in low illumination.

To account for these he adds to the conclusions the following assumptions: (a) The "all-or-none" principle obtains in the stimulation of the ommatidia. (b) Variation in the threshold of the ommatidia in any given region is in accord with the normal distribution curve. (c) The threshold of the ommatidia varies directly with light adaptation. The magnitude of the response varies directly with the number of ommatidia stimulated.

\section{QUANTITATIVE RELATION BETWEEN STIMULUS AND RESPONSE}

It is well known that the activity of many insects varies with the intensity of the light to which they are exposed. The butterfly Vanessa antiopa, for example, is usually much more active in diffuse sunlight than it is in direct sunlight or in darkness (Parker, 182; Dolley, 49), and blowfly larvae are under certain conditions much more active in strong than in weak light. If, for example, the light intensity is suddenly increased they usually raise the anterior end and swing it violently from

${ }^{2 a}$ These statements refer to insects which are photopositive. If they are photonegative, the opposite obtains. 
side to side and then erawl much more rapidly than they did in the lower intensity (Mast, 140).

Various attempts have been made precisely to ascertain the relation between luminous intensity and activity in insects. These attempts concern the rate of deflection toward one side with the two eyes unequally illuminated, and the rate of locomotion with the two eyes equally illuminated.

a. Rate of Deflection with the Two Eyes Unequally Illuminated.-Dolley (49) found in observations on Vanessa with one eye covered that in vertical illumination on a black background, the rate of deflection varies inversely with luminous intensity if the source of light is small, and directly if it is large and if there is much reflection from the background. The direct relation has been observed in a number of other insects. Garrey (75) says, e.g., that the robber fly on a glass cylinder surrounded by a much larger one of white mat paper illuminated from above, takes a spiral course as it proceeds toward the light and that "the brighter the light, the greater the number of spiral circuits required to make the ascent"; and Minnich (170) obtained similar results in observations on the honey bee. But they present no details concerning this relation. Clark (35), however, in observations on Dineutes, found that the rate of deflection does not increase uniformly as the intensity increases. He says that as the intensity increases, the rate of deflection increases to a maximum and then remains constant, and that on a black background the rate of increase is much more rapid than on a white background and the maximum not so high. He concludes that the rate of deflection depends upon the state of adaptation as well as upon the amount of reflection from the background and the size of the source of light.

Mast (140) maintains that if opposite sides of fly larvae are unequally illuminated, the degree of deflection from the more highly illuminated side varies directly with the differenee in the intensity of illumination of the two sides. Patten (183) confirms this.

Mast (148), in observations on Eristalis tenax in a field of light consisting of two horizontal beams which crossed at right angles, measured the angle of deflection from the median in relation to the relative intensity of the beams. The results obtained show that in reference to the effect on deflection, the rhabdomes in the posterior portion of the eye are much more sensitive than those in the anterior portion, but they have little or no bearing on the quantitative relation between stimulus and response.

Yagi (232) on the basis of results obtained in the walking stick Dixippus, concludes that "photic excitation is proportional to the logarithm of the intensity." This insect, on a vertical wall, assumes, in response to gravity, a vertical axial position. Yagi measured the angle of deflection from the vertical, in response to lateral illumination of different intensities. He maintains that this angle varies directly with the inten- 
sity and reached the conclusion presented above. This conclusion rests upon the assumptions that the magnitude of the response to gravity is independent of the axial position of the insect and that there is no difference in the sensitivity of the rhabdomes in different portions of the eye. There is, however, no evidence in support of either of these assumptions. The action of gravity on orientation doubtless varies from maximum when the insect faces downward, to zero when it faces upward, and the effect on deflection of light of a given intensity probably varies from maximum when it is received by the rhabdomes at the posterior edge of the eye, to zero when it is received by those in the center of the anterior portion of the eye. The validity of the conclusion is therefore highly questionable.

b. Rate of Locomotion with the Two Eyes Equally Illuminated.-Loeb $(126,127)$ maintains that the light received by the eyes controls the tonus of the muscles in the locomotor appendages of animals including insects, and that their activity is correlated with the muscle tonus in such a way that it varies directly with the intensity of the light in accord with the Bunsen-Roscoe law. But the evidence in support of this contention is far from convincing.

Mast (140) maintains that in fly larvae with both sides equally illuminated the rate of locomotion over a distance of $10 \mathrm{~cm}$. is a little higher in strong than it is in weak light. Herms (97) obtained similar results. These observations, as well as those cited above, indicate that the activity in fly larvae varies directly with luminous intensity. But in spite of the quantitative measurements made by all the investigators, it is (because of continuous changes in the intensity of the illumination of the photoreceptors in the larvae, brought about by alternate extension and retraction and swinging from side to side of the anterior end as they crawl) impossible to give anything definite concerning the quantitative relation between the stimulus and the response.

Dolley (50) concludes that the rate of locomotion in Vanessa with both eyes equally illuminated in a horizontal beam of constant light is practically independent of the intensity of the light, and Minnich (170) reaches the same conclusion in regard to the honey bee. Moore and Cole (173) and Cole (40) conclude, however, that in the Japanese beetle, Papillia japonica and the fruit fly Drosophila melanogaster, "the rate of response of the organism is proportional to the logarithm of the intensity of the stimulus."

Moore and Cole made their observations as follows: A considerable number of specimens were put into a tall vessel directly under a lamp. After they had aggregated at the top, the ressel was inverted and the time required for half of them to reach the top was measured. Then the ressel was again inverted, etc. The time recorded consequently includes the time required to orient and to get under way as well as the 
time required to travel from the bottom to the top of the vessel. Moreover, there were, owing to the inversion of the vessel, radical changes in the locations of the stimuli in the eyes and in the intensity of the illumination of the photosensitive elements, and the observed results only very roughly approximated the calculated values. It is therefore obvious that the conclusions reached by Moore and Cole are not well founded.

Welsh (215) maintains that as the light increases from a low intensity $\left(0.185\right.$ foot-candle) the rate of locomotion in the water mite Unionicola, ${ }^{3}$ increases rapidly to a maximum at about 70 foot-candles and then remains practically constant and that the increase in the rate is the result of increase in both the frequency and the length of the steps. He concludes: "The change in length of stride is due to tonus changes in the leg muscles; this is due to the tonic effect of light, and is directly proportional to the logarithm of the light intensity." But why he considers the change in the length of the steps due to change in tonus rather than to change in the magnitude of contraction of the muscles, is not clear. The mites were fully dark-adapted, and then light-adapted for 2 to $3 \mathrm{~min}$. before each test. They were therefore more nearly completely light-adapted in the tests made in the lower than in those made in the higher intensities. This doubtless accounts, at least in part, for the difference in rate of locomotion observed. Moreover, there was considerable change of light intensity during each test.

Clark (33) in observations on the whirligig beetle, Dineutes, avoided changes of intensity during the tests, and he ascertained the effect of adaptation as well as the effect of light intensity on rate of locomotion. He reached the following conclusions: In fully dark-adapted specimens the rate of locomotion in light of 4000 meter-candles is at first relatively high; then, as the time of exposure increases, it gradually decreases to a minimum, after which it remains constant. In light of 8000 metercandles it is at first relatively low ; then it gradually increases to a maximum (which is the same as the preceding minimum), after which it remains constant.

In specimens which are fully adapted to the light in which they are exposed, the rate of locomotion increases (with increase of intensity) to a maximum at 0.5 meter-candle, then remains constant until 8000 metercandles is reached, and then decreases. The condition of adaptation of the eyes is consequently a very important factor in the relation between the rate of locomotion in insects and luminous intensity.

As a whole, the evidence in hand does not warrant any precise conclusions, concerning the quantitative relation between the stimulus and response to light in insects, but it shows that to ascertain this relation

${ }^{3}$ Unionicola is an arachnid, not an insect. I include it because its responses to light are similar to those of many insects. 
with a degree of precision worth while it is necessary rigidly to control adaptation and the localization of the stimulus in the eye.

Hartline (78) did this in observations on the action current in the bumble bee Bombus and the grasshopper Melanoplus. He found that the initial action current (millivolts) in dark-adapted eyes is directly proportional to the time of exposure if the intensity is constant, and to the logarithm of the intensity if the time of exposure is constant, but that this obtains only over limited ranges of intensity. This method yields very consistent and valuable results, but it is obvious that these results do not directly express the quantitative relation between the stimulus and the motor response.

\section{THE NATURE OF THE STIMULUS}

It is very generally held that light causes, directly or indirectly, chemical changes in the eyes of insects and that these changes result in the production of impulses which pass to the muscles and induce the responses.

Loeb (127), Garrey (75), et al. contend that the light acts continuously, that the chemical changes in the eyes are directly proportional to the amount of light received in accord with the Bunsen-Roscoe law, and that the magnitude of the response is directly proportional to the photochemical changes, as in Hammond's mechanical dog, which is motivated and directed by the action of light in selenium cells symmetrically situated on opposite sides.

If these contentions are valid, intermittent light should have the same stimulating efficiency as continuous light. It has been demonstrated that if the frequency of intermittent light is high this obtains in blowfly larvae (Patten, 183), in the tachina fly (Archytas), the butterfly (Vanessa), and the drone fly (Eristalis) (Dolley, 50, 51, 52), in dragon-fly larvae (Sälzle, 192), and in the honey bee (IVolf, 229).

This is, however, to be expected, for just as it is impossible to distinguish between a straight line and the arc of a circle, if the circle is sufficiently large, it is impossible to distinguish between continuous and intermittent light, if the frequency is sufficiently high. The relation in question is, therefore, of importance only when it is specifically correlated with the frequency (Mast, 148).

Mast and Dolley (157), in observations on Eristalis, ascertained the critical frequency for different intensities, that is, the lowest frequency in intermittent light, which is for this insect indistinguishable from continuous light of the same intensity. It was found that this frequency varies directly with the intensity. Sälzle (192) and Wolf (227 to 229) in observations on dragon-fly larvae and the honey bee, ascertained this frequency over a much wider range of intensities and came to the same 
conclusion. They maintain, moreover, that this frequency is "a sigmoid function of the logarithm of the intensity."

Dolley (50, 51, 52), Mast (148), and Mast and Dolley (157, 158), in observations on several different insects, discovered that as the frequency decreases from the critical frequency, the stimulating efficiency increases, and that the stimulating effect of a given quantity of intermittent light at optimum frequency is, under some conditions, more than 16 times as great as that of the same quantity of continuous light. They maintain that the optimum frequency and the stimulating efficiency at this frequency vary directly with the intensity, and that stimulating efficiency at optimum frequency is practically independent of the ratio between the length of the light and the dark periods, if this ratio is $<1$, i.e., if the dark period is longer than the light period, but that it varies inversely with this ratio if it is $>1$. They conclude that "the light receptors in the eye of an insect are not continuously stimulated when they are continuously illuminated; that there are periods during which the light acts, followed by periods during which it does not act; sensitive periods followed by non-sensitive (refractory) periods; periods in which light induces, in the receptors, photochemical changes in one direction followed by periods in which the reverse (restitution) occurs; so that, when in intermittent illumination, the light and the dark periods harmonize with the sensitive and the refractory periods, respectively, all of the light received is used in stimulation, while in continuous illumination that received during the refractory period is not used." They hold that light induces, in the photoreceptors, the formation of certain substances which, as soon as a given amount has accumulated, produces an impulse, that the substances produced are then in some way converted, so as to restore the system to the original condition, after which the light acts again, etc.

If a dark-adapted eye (insect, crab, eel) is suddenly illuminated, the action current in the optic nerve increases rapidly to a maximum and then decreases rapidly, after which it decreases slowly or remains nearly constant for some time. The duration of the increase in the action current varies inversely with the intensity of the light, but it is at best only a small fraction of a second. There is no indication of regular periodicity in continuous illumination (Adrian, 2; Hartline, 78, 80; Prosser, 188).

These results indicate that the difference between the stimulating efficiency of continuous and intermittent light described above is correlated with spurts of increase in impulses after the flashes in the intermittent light rather than with periodicity in sensitiveness in the continuous light; and that the decrease in stimulating efficiency with increase in flash-frequency, is due to the decrease in the time for restitution during the dark periods. 
Hartline and Graham (80) demonstrated, however, that in singlenerve elements the impulses are periodic in continuous illumination. They demonstrated that inmediately after the receptor is illuminated the frequency of impulses increases, that this is followed by a relatively long period without impulses, after which the frequency is much lower but fairly regular, and that the frequency varies directly with the intensity of the illumination. The absence of regular periodicity in the optic nerve as a whole, is, therefore, doubtless due to fusion of the effect of the action currents in the individual fibers composing it. There probably are then, in the nerve elements, sensitive periods followed by refractory periods; periods in which light induces, in the receptors, photochemical changes in one direction followed by periods in which the reverse (restitution) occurs. But it is difficult to understand how a system which has been altered by the action of light, can be restored without any change in illumination.

\section{ADAPTATION TO LIGHT AND TO DARKNESS}

It has been observed by various investigators that the sensitivity of insects to light depends upon the degree of adaptation. Dolley (53) attempted to ascertain this relation quantitatively for Eristalis tenax by subjecting for different periods of time one eye to darkness and the other to light of a given intensity, and then noting the degree of deflection from the median diagonal in a field of light consisting of two equal beams crossing at right angles. He concludes that as the time in darkness increases, the sensitiveness increases rapidly to a maximum at about 60 min., then remains nearly constant for about $2 \mathrm{hr}$, after which it decreases rapidly again, and that after the eye has been in darkness $4 \mathrm{hr}$. it is less sensitive than the light-adapted eye.

These conclusions are, however, equirocal, owing to the fact that in the test concerning the relative sensitiveness of the two eyes, after unequal adaptation, the images in the eyes were not fixed, so that there probably were marked changes in the intensity of the light rcceived by the illuminated rhabdomes. These experiments should therefore be repeated and extended under more rigidly controlled conditions.

Hartline (79) obviated this difficulty, but his observations were made on the horseshoe crab, Limulus. He fixed the eye in relation to the source of light and measured the action current produced by a given amount of light on a given region of the retina after it had been in darkness for various periods of time. He maintains that as the time in darkness increases from zero, the action current increases rapidly at first and then more slowly and that it becomes constant after about $30 \mathrm{~min}$. in darkness. If the action current, in millivolts, is plotted against the time in darkness, the results lie with remarkable accuracy on the theoretical curve, $k t=[1 /(E \infty-E)]+C$. He observed no decrease in sensitivity of the 
eye of Limulus comparable to that observed by Dolley in Eristalis after it had been in darkness for several hours, but this may have been due to insufficient time in darkness to induce the decrease in Limulus.

\section{MODIFICATION IN RESPONSE TO LIGHT}

Bees which are normally strongly photopositive can readily be trained to go into totally dark places for food; they can be trained to go into or out of either dark or highly illuminated hives, through long tortuous dark passages, for example, an ordinary black garden hose 10 meters long, with numerous sharp curves in it; and they can be trained to select, regardless of brilliance, any one among a number of different colors or patterns associated with food or with their hives (Lubbock, 129; Frisch, 74; Bertholf, 14; Baumgärtner, 11; Opfinger, 181; Zerrahn, 235).

\section{VISION}

The behavior of many insects in the open clearly indicates that they distinguish between objects at a distance. This is especially true of robber flies and dragon flies in capturing prey; of the pupae of the 17-year locust in selecting objects to ascend for the purpose of molting, and of bees and butterflies in selecting flowers for the purpose of procuring food. These phenomena may be due to difference in the brightness, difference in the form or difference in the color of the objects distinguished.

a. Brightness Vision.-Frisch (74) and Kühn (118) in observations on bees with the training methods, obtained results which indicate that only great differences in brightness are distinguished; but Hess (103) on the basis of results obtained in observations on the positive response to light of different intensities, concluded that the ability to distinguish brightness is very nearly as acute in bees as it is in man. Bertholf (16) with a method somewhat similar to that used by Hess obtained results which indicate that bees clearly distinguish between two surfaces which differ in brightness by 30 per cent, whereas human beings clearly distinguish between the same surfaces when they differ in brightness by only 10 per cent. He concludes brightness vision is markedly less acute in bees than it is in man.

b. Form Vision.-Frisch (74), Baumgärtner (11), Hertz (100 to 102), Opfinger (181), Friedlaender (72), and Zerrahn (235) conclude on the basis of results obtained in training experiments, that bees can distinguish figures which differ only in form, but that their ability to discriminate form is rather crude. They maintain, for example, that bees cannot distinguish between circular, elliptical, triangular, and square figures of the same size. Baumgärtner concludes that bees have no true sense of form comparable to that in man. Verlaine $(205,206)$ asserts, however, that wasps are far more accomplished than bees in that they readily distinguish between circles, triangles, and squares. 
c. Color Vision.-Lubbock (129) clearly demonstrated that ants are sensitive to ultra-violet. This has by various methods been confirmed for a considerable number of different insects (Hess, 104; Kühn and Pohl, 122; Lutz and Richtmyer, 136; Lutz, 134, 135; Kühn, 119). The results obtained by these investigators are, however, qualitative. Bertholf $(16,17)$ ascertained quantitatively the distribution of stimulating efficiency in the spectrum for the honey bee and for the fruit fly, Drosophila. He presented to the insects two translucent glass plates of the same size, one illuminated with white and the other with monochromatic light produced by means of a quartz prism, and then changed the intensity of the white light until its effect on their photopositive response was equal to that of the monochromatic light in different regions of the spectrum. He came to the following conclusions:

As the wave-length decreases, the stimulating efficiency for the honey bee increases from zero at about wave-length $6450 \AA$ to a maximum of 100 arbitrary units at $5550 \AA$, then decreases to 10 at $4350 \AA$, after which it rapidly increases to a second maximum of 450 at $3650 \AA$ and then rapidly decreases to zero at about $2800 \AA$. The distribution of stimulating efficiency for the honey bee is, in the visible spectrum, nearly the same as it is for man, but in the ultra-violet the maximum is $41 / 2$ times as high as the maximum in the visible spectrum, whereas it is zero for man. The distribution of stimulating efficiency for Drosophila is essentially the same as it is for the honey bee, except that the limits of the spectrum and the maximum in the visible are shifted considerably toward the ultra-violet and the maximum in the ultra-violet is more than $5 \frac{1}{2}$, in place of $4 \frac{1}{2}$, times as high as the maximum in the visible spectrum.

Sander (193), in observations on the honey bee obtained results which differ greatly from those obtained by Bertholf. He agrees with Bertholf in the contention that there are two maxima; but he holds that the one is at $5700 \AA$ in place of at $5550 \AA$, and the other at $4700 \AA$ (blue), in place of at $3650 \AA$ (ultra-violet), and that the efficiency at the former is slightly greater than at the latter, in place of only one-fourth as great. He consequently does not agree with Bertholf in the contention that the stimulating efficiency of ultra-violet is comparatively very high. Bertholf's contention is, however, strongly supported by results obtained by Lutz $(134,135)$.

Sander's methods were similar to those used by Bertholf, except that he ascertained the frequency of selection of each of a number of lights which differed in wave-length but were equal in energy; while Bertholf ascertained the intensity of white light that resulted in the same frequency of selection as each of a series of monochromatic lights obtained from different regions of the prismatic spectrum, i.e., lights which differed both in wave-length and in energy. Sander maintains that the relation between stimulating efficiency and energy varies with wave-length, and 
that Bertholf's method is consequently inadequate. It may also be said that the difference in the stimulating efficiency of two lights is not proportional to the frequency of selection and that Sander's method is consequently also inadequate. The experiments should be repeated with Bertholf's method, but with several series of monochromatic lights equal in energy in each series but different in different series.

Hess $(103,104)$ maintains that bees and other insects distinguish different regions in the spectrum, owing to difference in brightness or brilliance, i.e., that insects do not have color vision. Lubbock (129), Frisch (74), Kühn and Pohl (122), Kühn (119), Kühn and Fraenkel (121), and Bertholf (14) conclude that bees can distinguish between colors or chromas which are for them equal in brightness or brilliance. Frisch contends that only two chromas are distinguished by bees, blue-violet and green-yellow-red. Kühn and Pohl maintain that four chromas are distinguished, yellow-green, blue-green, blue-violet and ultra-violet. Kühn and Fraenkel conclude that bees can distinguish eight chromas. Bertholf maintains that they can distinguish between the following pairs of chromas when they are equal in brilliance: blue and violet, blue and green, blue and yellow, blue and red, green and red, green and yellow.

The training method was used by all these investigators and the results obtained by all clearly show that the bees distinguished between the colors used, but in some of the experiments the possibility of selection on the basis of differences in brightness and in other characteristics was not excluded, especially brightness due to reflection and radiation of ultraviolet (Lutz 134, 135). Bertholf appears, however, to have excluded this possibility. Color vision appears consequently to be definitely established for bees. But so far recognition of only five to eight colors has been demonstrated for them; whereas man, according to Nutting (180), can distinguish 130 to 180 . Color vision is therefore probably much less acute in bees than it is in man.

d. Visual Acuity.-From results obtained with the training method Baumgärtner (11), using yellow and blue figures, concludes that bees can barely distinguish squares $5 \mathrm{~mm}$. wide at a distance of $2.5 \mathrm{~cm}$. and squares $10 \mathrm{~mm}$. wide at a distance of $10 \mathrm{~cm}$., but he thinks much smaller spots on flowers are recognized. Results obtained by him in a study of the structure of the eye support these conclusions and indicate that visual acuity is highest in the central, anterior portion of the eye and decreases rapidly dorsally and ventrally but only slightly posteriorly.

It is well known that movement of a surface containing alternate light and dark areas induces compensatory movements in insects (Rádl, 189; Buddenbrock, 22 ; et al.). These movements are doubtless types of reflex responses to rapid changes in luminous intensity on the retina of the eye. Schlieper (196) made use of this fact and tested the response of a number of different insects to movement of a background containing 
alternate stripes which varied in color and in luminous intensity, but he did not obtain very definite results.

Hecht and Wolf (94), Wolf (226 to 229), Clark (35a), Wolf and Crozier (231), and Hecht and Wald (93) perfected the method and obtained very precise results in observations on the responses of bees, the fiddler crab, and the fruit fly, Drosophila, to movement of a background containing alternate dark and light stripes which differed in width, in luminous intensity and in relative luminous intensity. They reached the following conclusions:

Under optimal conditions, the power to discriminate between surfaces which differ only in luminous intensity is about 20 times as great in man as it is in the honey bee and about 150 times as great in man as it is in Drosophila. The visual acuity is about 10 times as high in man as it is in the honey bee and about 1000 times as high as it is in Drosophila. Visual acuity increases with increase in luminous intensity in such a way that when it is plotted against the logarithm of the intensity, sigmoid curves are produced which are practically the same in form for the three species studied. It is postulated that visual aeuity varies directly with the number of ommatidia that are stimulated, that the sensitivity of the ommatidia varies, and that the number stimulated inereases as the intensity increases. This accounts for increase in acuity with increase in intensity, but sinee maximum intensity diserimination still increases with increase in intensity, after acuity has become constant, it does not account for this. It is therefore concluded that intensity discrimination is probably correlated with frequency of impulses in the optic nerves, which is in turn correlated with intensity.

\section{REFERENCES}

This bibliography is fairly complete for the years 1926 to 1933 , inclusive. For the preceding period it contains only the references cited. Extensive lists of references covering different portions of this period can be found in Mast (140), Loeb (127), and Rose (190).

1. Adams, G. P. On the negative and positive phototropism of the earthworm Allolobophora foetida (Sav.) as determined by light of different intensities. Amer. Jour. Physiol. 9 : 26-34. 1903.

2. Adrian, E. D., and R. Matruews. The action of light on the eye. Parts I-III. Jour. Physiol. 63, 64, 65 : 1927-1928.

3. Alverdes, F. Stato-, Photo- und Tangoreaktionen bei zwei Garneelenarten. Zeitsch. Vergl. Physiol. 4: 699-765. 1926.

4. Alverdes, F. Lichtsinn, Gleichgewichtsinn, Tastsinn und ihre Interferenzen bei Garneelen. Zeitsch. Wiss. Zool. 132 : 135-170. 1928.

5. Alverdes, F. Tierpsychologische Analyse der intracentralen Vorgänge, welche bei decapoden Krebsen die locomotorischen Reaktionen auf Helligkeit und Dunkelheit bestimmen. Zeitsch. Wiss. Zool. 137: 403-475. 1930.

6. Ameln, P. Der Lichtsinn von Nereis diversicolor O. F. Müller. Zool. Jahrb. $47: 685-722.1930$.

7. Andrews, E. A. Compound eyes of annelids. Jour. Morph. 5: 271-299. 1891. 
8. Baldus, K. Untersuchungen über Bau und Funktion des Gehirnes der Larve und Imago von Libellen. Zeitsch. Wiss. Zool. 21: 557-620. 1922.

9. Bancroft, F. W. Heliotropism, differential sensibility, and galvanotropism in Euglena. Jour. Exp. Zool. 16: 383-428. 1913.

10. Bartels, M. Sinnesphysiologische und psychologische Untersuchungen an der Trichterspinne Agelena labyrinthica (Cl.). Zeitsch. Vergl. Physiol. 10: 527-593. 1929.

11. Baumgärtner, H. Der Formensinn und die Sehschärfe der Bienen. Zeitsch. Vergl. Physiol. 7 : 56-143. 1928.

12. Bayliss, W. M. The Properties of colloidal systems. IV. Reversible gelation in living protoplasm. Proc. Roy. Soc. 91: 196-201. 1920.

13. Bert, P. Sur la question de savoir si tous les animaux voient les mêmes rayons que nous. Arch. Physiol. 2 : 547. 1869.

14. Bertholf, L. M. Chroma-vision in the honeybee. Maryland Agric. Soc., Md. Farm Bur. Fed. 12 : 383-389. 1928. [Extensive bibliography.]

15. Bertholf, L. M. Reactions of the honeybee to light. Jour. Agric. Res. 42 : 379-419. 1931.

16. Bertholf, L. M. The distribution of stimulative efficiency in the ultra-violet spectrum for the honeybee. Jour. Agric. Res. 43: 703-713. 1931.

17. Bertholf, L. M. The extent of the spectrum for Drosophila and the distribution of stimulative efficiency in it. Zeitsch. Vergl. Physiol. 18: 32-64. 1932.

18. Beuther, R. Über die Einwirkung verschiedenfarbigen Lichtes auf Planarien. Abhandl. Naturforsch. Ges. Rostock, III. Folge 1, 41 pp. 1926.

19. Bevelander, G. Response to light in the cercariae of Bucephalus elegans. Physiol. Zool. 6: 289-305. 1933.

20. Bohn, G. Les Tropismes. Rapport VIme Congrès Internat. Psychologie. 15 pp. Genève, 1909.

21. Brand, H. Die lokomotorischen Reaktionen von Nereis diversicolor auf Licht und Dunkelheit und der Einfluss von Eingriffen an Receptoren, Effectoren und Zentralnervensystem. Zeitsch. Wiss. Zool. 144: 363-401. 1933.

22. Bundenbrock, W. von. Die Lichtkompassbewegungen bei Insekten insbesondere den Schmetterlingsraupen. Sitzungsber. Heidelberg. Akad. Wiss., 26 pp., 1917.

23. Buddenbrock, W. von. Versuch einer Analyse der Lichtreaktionen der Heliciden. Zool. Jahrb. Abt. Allg. Zool. 37 : 315-360. 1919.

24. Buddenbrock, W. von. Einige Bemerkungen zum augenblicken Stand der Frage nach dem Farbensinn der Tiere. Zool. Anz. 84: 189-201. 1929.

25. Buddenbrock, W. von. Untersuchungen über den Schattenreflex. Zeitsch. Vergl. Physiol. 13 : 164-213. 1930.

26. Buddenbrock, W. von. Die Orientierung zu bestimmten Stellen im Raum (Wirbellose). Handb. Norm. und Pathol. Physiol. 15 : 1023-1039. 1931.

27. Buddenbrock, W. von. Beiträge zur Lichtkompassorientierung (Menotaxis) der Arthropoden. Zeitsch. Vergl. Physiol. 16: 597-612. 1931.

28. Buddenbrock, W. von, und C. Schlieper. Kritische Bemerkungen zur Arbeit A. Müller's, Über Lichtreaktionen von Landasseln. Zool. Anz. 67 : 88-102. 1926.

29. Buddenbrock, W. von, und E. Schulz. Beiträge zur Kenntnis der Lichtkompassbewegung und der Adaptation des Insektenauges. Zool. Jahrb. Physiol. 52 : 513-536. 1933.

30. Buder, J. Zur Kenntnis der phototaktischen Richtungsbewegungen. Jahrb. Wiss. Bot. 68: 105-220. 1917.

31. Candolle, A. P. DE. Physiologie végétale. 1832. [A German transl. by Röper, pub. 1835.] 
32. Chaffee, E. L., W. T. Bovie, and A. Hampsos. The electrical response of the retina under stimulation by light. Jour. Opt. Soc. Amer. $7:$ 1-45. 1923.

33. Clark, L. B. Adaptation versus experience as an explanation of modification in certain types of behavior (circus movements in Notonecta). Jour. Exp. Zool. 51: 37-50. 1928.

34. Clark, L. B. Some factors involved in the reactions of insects to changes in luminous intensity. Shock reactions in Dineutes assimilis. Jour. Exp. Zool 58 : 31-41. 1931.

35. Clark, L. B. Modification of circus movements in insects. Jour. Exp. Zool. 66:311-333. 1933.

36. Clarke, G. L. Change of phototropic and geotropic signs in Daphnia induced by changes of light intensity. Jour. Exp. Biol. 7 : 109-131. 1930

37. Clarke, G. L. Quantitative aspects of the change of phototropic sign in Daphnia. Jour. Exp. Zool. 9: 180-211. 1932.

38. Clarke, G. L., and E. Wolf. The mechanies of tropistic reactions and the strychnine effect in Daphnia. Jour. Gen. Physiol. 16: 99-105. 1932.

39. Cole, L. J. An experimental study of the image-forming power of various types of eyes. Proc. Amer. Acad. Arts and Sci. 42:335-417. 1907.

40. Cole, W. H. Note on the relation between the photic stimulus and the rate of locomotion in Drosophila. Science 55: 678-679. 1922.

41. Copeland, M. An apparent conditioned response in Nereis virens. Jour. Comp. Psych. 10: 339-354. 1930.

42. Crozier, W. J. The orientation of animals by opposed beams of light. Jour. Gen. Physiol. 8: 671-684. 1927.

43. Crozier, W. J., and W. H. Cole. The phototropic excitation of Limax. Jour. Gen. Physiol. 12 : 669-674. 1929.

44. Crozier, W. J., and H. Federighi. Phototropic circus movements of Limax as affected by temperature. Jour. Gen. Physiol. 7 : 151-169. 1924.

45. Crozier, W. J., and E. Wolf. On the place of photic adaptation. Jour. Gen. Physiol. 11 : 289-295. 1928.

46. Dalyell, Sir J. G. The powers of the Creator revealed. London, 1853.

47. Davenport, C. B. Experimental Morphology. Vol. 1, 280 pp.; New York, 1897.

48. Dietrich, W. Die lokomotorischen Reaktionen der Landasseln auf Licht und Dunkelheit. Zeitsch. Wiss. Zool. 138: 187-232. 1931.

49. Dolley, W. L., JR. Reactions to light in Vanessa antiopa, with special reference to circus movements. Jour. Exp. Zool. 20 : 357-420. 1916.

50. Dolley, W. L., JR. The rate of locomotion in Vanessa antiopa in intermittent light and in continuous light of different illuminations, and its bearing on the continuous action theory of orientation. Jour. Exp. Zool. 23: 507-518. 1917.

51. Dolley, W. L., JR. The relative stimulating efficiency of continuous and intermittent light in Vanessa antiopa. Psychobiol. 2: 137-176. 1920.

52. Doller, W. L., $J_{R}$. The relative stimulating efficiency of continuous and intermittent light in the tachina fly, Archytas aterrima. Amer. Jour. Physiol. 64: 364-370. 1923.

53. Dolley, W. L., JR. Dark adaptation in the eye of Eristalis tenax. Physiol. Zool. 2 : 483-490. 1929.

54. Dolley, W. L., JR. The relation between luminous intensity and the length of the refractory period in the eye of Eristalis tenax. Jour. Exp. Zool. 66:1S5-191. 1930.

55. Dolley, W. L., JR. Factors involved in stimulation by intermittent light. Jour. Exp. Zool. 62 : 319-326. 1932. 
56. Dolley, W. L., Jr., and J. L. Wierda. Relative sensitivity to light in different parts of the compound eye in Eristalis tenax. Jour. Exp. Zool. 53: 129-139. 1929.

57. Engelmann, T. W. Über Reizung contraktilen Protoplasmus durch plötzliche Beleuchtung. Pflüger's Arch. Ges. Physiol. 19:1-7. 1879.

58. Engeluann, T. W. Über Licht- und Farbenperception niederster Organismen. Pflüger's Arch. Ges. Physiol. 29 : 387-400. 1882.

59. Engeluann, T. W. Bacterium photometricum. Pflüger's Arch. Ges. Physiol. $30: 95-124.1883$.

60. Ewald, W. F. Über Orientierung, Lokomotion und Lichtreaktionen einiger Cladoceren und deren Bedeutung für die Theorie der Tropismen. 34 pp.; Erlangen, 1910.

61. Ewald, W. F. The applicability of the photochemical energy-law to light reactions in animals. Science $38: 236-237.1913$.

62. Ewald, W. F. Versuche zur Analyse der Licht- und Farbenreaktionen eines Wirbellosen (Daphnia Pulex). Zeitsch. Sinnesphysiol. 48: 285-324. 1914.

63. Famintzin, A. Die Wirkung des Lichtes auf Algen und einige andere nahe verwandte Organismen. Jahrb. Wiss. Bot. 6:1-44. 1867.

64. Folger, H. T. A quantitative study of reactions to light in amoeba. Jour. Exp. Zool. 41: 261-291. 1925.

65. Folger, H. T. The effects of mechanical shock on locomotion in Amoeba proteus. Jour. Morph. and Physiol. 42: 359-370. 1926.

66. Folger, H. T. Reactions to light by Mya arenaria in relation to the BunsenRoscoe law. Anat. Rec. 34: 115. 1926.

67. Folger, H. T. The relation between the responses by Amoeba to mechanical shock and to sudden illumination. Biol. Bull. 53 : 405-412. 1927.

68. Fraenkel, G. Phototropotaxis bei Meerestieren. Naturwissensch. 14: 117122.1927.

69. Fraenkel, G. Beiträge zur Geotaxis und Phototaxis von Littorina. Zeitsch. Vergl. Physiol. 5 : 585-597. 1927.

70. Fraenkel, G. Über Photomenotaxis bei Elysia viridis Mont. Zeitsch. Vergl. Physiol. 6: 385-401. 1927.

71. Fraenkel, G. Die Orientierung von Schistocerca gregaria zu strahlender Wärme. Zeitsch. Vergl. Physiol. 13: 300-313. 1930.

72. Friedlaender, Marianne. Zur Bedeutung des Fluglochs im optischen Feld der Biene bei senkrechter Dressuranordnung. Zeitsch. Vergl. Physiol. 15: 193-260. 1931.

73. Friedrich, H. Mitteilungen über vergleichende Untersuchungen über den Lichtsinn einiger mariner Copepoden. Zeitsch. Vergl. Physiol. 15: 121-138. 1931.

74. Frisch, K. von. Der Farbensinn und Formensinn der Biene. Zool. Jahrb. Abt. Allg. Zool. u. Physiol. 35 : 1-188. 1914.

75. Garrey, W. E. Light and the muscle tonus of insects. The heliotropic mechanism. Jour. Gen. Physiol. 1: 101-125. 1918.

76. Harrington, N. R., and E. Leaming. The reactions of Amoeba to light of different colors. Amer. Jour. Physiol. 3 : 9-16. 1900.

77. Hartune, H. K. The electrical response to illumination of the eye in intact animals, including the human subject; and in decerebrate preparations. Amer. Jour. Physiol. 73: 600-612. 1925.

78. Hartline, H. K. A quantitative and descriptive study of the electric response to illumination of the arthropod eye. Amer. Jour. Physiol. 83 : 466-483. 1928.

79. Hartline, H. K. The dark adaptation of the eye of Limulus, as manifested by its electric response to illumination. Jour. Gen. Physiol. 13: 379-389. 1930. 
80. Hartline, H. K., and C. H. Graham. Nerve impulses from single receptors in the eye. Jour. Cell. and Comp. Physiol. 1: 277-295. 1932.

81. HAUG, G. Die Lichtreaktionen der Hydren. (Chlorohydra viridissima und Pelmatohydra oligactis (P.) typica.) Zeitsch. Vergl. Physiol. 19: 246-303. 1933.

82. HaYASCHI, I. An experimental criticism of the theory of Lichtkompassbewegung of v. Buddenbrock. Zool. Soc. Japan, Tokyo. 1929.

83. Неснт, S. The photic sensitivity of Ciona intestinalis. Jour. Gen. Physiol. 1: 147-166. 1918.

84. Неснт, S. The nature of the latent period in the photic response of Mya arenaria. Jour. Gen. Physiol. 1:657-666. 1919.

85. Неснт, S. The effect of temperature on the latent period in the photic response of Mya arenaria. Jour. Gen. Physiol. 1:667-685. 1919.

86. Heснт, S. Time and intensity in photosensory stimulation. Jour. Gen. Physiol. 3 : 367-373. 1921.

87. Неснт, S. Intensity discrimination and the stationary state. Jour. Gen. Physiol. 6: 355-373. 1924.

88. Неснт, S. The kinetics of dark adaptation. Jour. Gen. Physiol. 10 : 781-809. 1927.

89. Неснт, S. The influence of temperature on the photosensory latent period. Jour. Gen. Physiol. 11: 649-656. 1928.

90. Hecht, S., S. Shlaer, and C. D. Verrijp. Intermittent stimulation by light. II. The measurement of critical fusion frequency for the human eye. Jour. Gen. Physiol. $17: 237-249.1933$.

91. Hecht, S., S. Shlaer, and C. D. Verrijp. Intermittent stimulation by light. III. The relation between intensity and critical fusion frequency for different retinal locations. Jour. Gen. Physiol. 17 : 251-268. 1933.

92. Hecht, S., S. Shlaer, and C. D. Verrijp. Intermittent stimulation by light. IV. A theoretical interpretation of the quantitative data of flicker. Jour. Gen. Physiol. 17 : 269-282. 1933.

93. Hechт, S., and G. WALD. The influence of intensity on the visual functions of Drosophila. Proc. Nation. Acad. Sci. 19: 964-972. 1933.

94. Hechт, S., and E. Wolf. The visual acuity of the honey-bee. Jour. Gen. Physiol. 12 : 727-760. 1929.

95. Неснт, S., and E. Wolf. Intermittent stimulation by light. I. The validity of Talbot's law for Mya. Jour. Gen. Physiol. 15 : 369-389. 1932.

96. Henke, K. Die Lichtorientierung und die Bedingungen der Lichtstimmung bei der Rollassel Armadillidium cinereum Zenker. Zeitsch. Vergl. Physiol. 13: 534-625. 1930.

97. Herus, W. B. The photic reactions of sarcophagid flies, especially Lucilia caesar Linn. and Calliphora vomitoria Linn. Jour. Exp. Zool. 10: 167-226. 1911.

98. Herter, K. Versuche über die Phototaxis von Nereis diversicolor, O. F. Müller. Zeitsch. Vergl. Physiol. 4: 103-141. 1926.

99. Herter, K. Bemerkungen zu der Arbeit P. Ameln's über den Lichtsinn vou Nereis. Zool. Jahrb. 48: 697-704. 1931.

100. Hertz, Mathilde. Die Organisation des optischen Feldes bei der Biene. Zeitsch. Vergl. Physiol. 8 : 693-748. 1929.

101. Hertz, Mathilde. Die Organisation des optischen Feldes bei der Biene. II. Zeitsch. Vergl. Physiol. 11: 107-145. 1929.

102. Hertz, Mathilde. Das optische Gestaltproblem und der Tierversuch. Verhandl. deut. Zool. Ges. 33. Vers., Marburg., Zool. Anz. Suppl. 4: 23-49. 1929. 
103. Hess, C. von. Messende Untersuchung des Lichtsinnes der Biene. Pflüger's Arch. ges. Physiol. 163 : 289-320. 1916.

104. Hess, C. von. Neues zur Frage nach einem Farbensinne bei Bienen. Naturwissensch. 8: 927-929. 1920.

105. Hess, W. N. Reactions to light in the earthworm, Lumbricus terrestris L. Jour. Morph. and Physiol. 39 : 515-542. 1924.

106. Hess, W. N. Photoreceptors of Lumbricus terrestris, with special reference to their distribution, structure, and function. Jour. Morph. and Physiol. 41: 63-93. 1925.

107. Hesse, R. Untersuchungen über die Organe der Lichtempfindungen bei niederen Thieren. I. Die Organe der Lichtempfindungen bei den Lumbriciden. Zeitsch. Wiss. Zool. 61: 393-419. 1896.

108. Hoffmeister, W. Die bis jetzt bekannten Arten aus der Familie der Regenwürmer. Braunschweig, 1845.

109. Holmes, S. J. The reactions of Ranatra to light. Jour. Comp. Neur. and Psych. 15: 305-349. 1905.

110. Holmes, S. J. Phototaxis in Volvox. Biol. Bull. 4: 319-326. 1903.

111. Inman, O. L., W. T. Bovie, and C. E. BArr. The reversal of physiological dominance in Amoeba by ultraviolet light. Jour. Exp. Zool. 43: 475-484. 1926.

112. JANDA, V. Über die Phototaxis der Larven und Imagines von Anthrenus muscoruin L. Zool. Anz, 96: 77-84. 1931.

113. Jennings, H. S. Contributions to the study of the behavior of lower organisms. Carnegie Inst. of Washington Publ. 16: 256 pp. 1904.

114. Just, G. Untersuchungen über Ortsbewegungsreaktionen. I. Das Wesen der phototaktischen Reaktionen von Asterias rubens. Zeitsch. Vergl. Physiol. 5: 247-282. 1927.

115. Koenler, O. Über das Farbensinn von Daphnia magna Straus. Zeitsch. Vergl. Physiol. 1: 84-174. 1924.

116. Koenler, O. Beiträge zur Sinnesphysiologie der Süsswasserplanarien. Zeitsch. Vergl. Physiol. 16: 606-756. 1932.

117. Kropp, B., and E. V. Enzmann. Photic stimulation and leg movements in the crayfish. Jour. Gen. Physiol. 16: 905-910. 1933.

118. KüHN, A. Zum Nachweis des Farbenunterscheidungsvermögens der Bienen. Naturwiss. 12 : 116-118. 1924.

119. KüHn, A. Über den Farbensinn der Bienen. Zeitsch. Vergl. Physiol. 5: 762-800. 1927.

120. Kühn, A. Phototropismus und Phototaxis der Tiere. Handb. Norm. und Path. Physiol. Bd. 12. 1929.

121. KüHN, A., and G. Fraenkel. Über das Unterscheidungsvermögen der Bienen für Wellenlängen im Spektrum. Nachr. Ges. Wiss. Göttingen. Math.-Phys. Kl. $4: 330-335.1927$.

122. KüнN, A., and R. Pонц. Dressurfähigkeit der Bienen auf Spektrallinien. Naturwissensch. 9: 738-740. 1921.

123. Lammert, A. Über Pigmentwanderung im Punktauge der Insekten sowie üler Licht- und Schwerkraftreaktionen der Schmetterlingsraupen. Zeitsch. Vergl. Physiol. 3 : 225-278. 1925.

124. Laurens, H., and H. D. Hooker. Studies on the relative physiological value of spectral lights. II. The sensibility of Volvox to wave-lengths of equal energy content. Jour. Exp. Zool. 30: 345-368. 1920.

125. Light, V. E. Photoreceptors in Mya arenaria, with special reference to their distribution, structure, and function. Jour. Morph. and Physiol. 49: 1-42. 1930. 
126. LokB, J. Dynamies of living matter. 233 pp. New York, 1906.

127. Loes, J. Forced movements, tropisms, and animal conduct. 209 pp. Philadelphia and London, 1918. [Extensive bibliography.]

128. Loeb, J., and S. S. Maxwell. Further proof of the identity of heliotropism in animals and plants. Univ. Cal. Pub. Physiol. 3 : 195-197. 1910.

129. Lubвоск, Sir J. On the sense of color among some of the lower animals. Part I. Jour. Linn. Soc. (Zool.), 16: 121-127. 1881.

130. Luce, R. H. Orientation to the electric current and to light in Amoeba. Anat. Rec. $32: 55.1926$.

131. Luntz, A. Untersuchungen über die Phototaxis. I. Mitteilung: Die absoluten Schwellenwerte und die relative Wirksamkeit von Spektralfarben bei grünen und farblosen Einzelligen. Zeitsch. Vergl. Physiol. 14:68-92. 1931.

132. Luntz, A. Untersuchungen über die Phototaxis. II. Mitteilung: Lichtintensität und Schwimmesgeschwindigkeit bei Eudorina elegans. Zeitsch. Vergl. Physiol. 15: 652-678. 1931.

133. Luntz, A. Untersuchungen über die Phototaxis. III. Mitteilung: Die Umkehr der Reaktionsrichtung bei starken Lichtintensitäten und ihre Bedeutung für eine allgemeine Theorie der photische Reizwirkung. Zeitsch. Vergl. Physiol. 16 : 204-217. 1932.

134. LuTz, F. E. Apparently non-selective characters and combinations of characters, including a study of ultraviolet in relation to the flower-visiting habits of insects. Ann. New York Acad. Sci. 29: 181-283. 1924.

135. Lutz, F. E. Experiments with "stingless bees" (Trigona cressoni parastigma) concerning their ability to distinguish ultraviolet patterns. Amer. Mus. Novitates No. 641. 1-26. 1933.

136. Lutz, F. E., and F. K. Richtмyer. The reaction of Drosophila to ultraviolet. Science $65: 519.1922$.

137. MAst, S. O. Light reactions in lower organisms. I. Stentor coeruleus. Jour. Exp. Zool. 3 : 359-399. 1906.

138. MAST, S. O. Light reactions in lower organisms. II. Volvox. Jour. Comp. Neur. and Psych. $17:$ 99-180. 1907.

139. Mast, S. O. Reactions in Amoeba to light. Jour. Exp. Zool. 9:265-277. 1910.

140. MAst, S. O. Light and the behavior of organisms. $410 \mathrm{pp}$. New York, 1911. [Extensive bibliography.]

141. Mast, S. O. Behavior of fire-flies (Photinus pyralis)? with special reference to the problem of orientation. Jour. Animal Behav. 2 : 256-272. 1912.

142. Mast, S. O. Orientation in Euglena with some remarks on tropisms. Biol. Centrbl. 34: 641-674. 1914.

143. MAST, S. O. The process of orientation in the colonial organism, Gonium pectorale, and a study of the structure and function of the eye-spot. Jour. Exp. Zool. $20: 1-17.1916$.

144. MAST, S. O. The relation between spectral color and stimulation in the lower organisms. Jour. Exp. Zool. $22: 471-528.1917$.

145. MAST, S. O. Effects of chemicals on reversion in orientation to light in the colonial form, Spondylomorum quaternarium. Jour. Exp. Zool. 26: 503-520. 1918.

146. MAst, S. O. Reversion in the sense of orientation to light in the colonial forms, Volvox globator and Pandorina morum. Jour. Exp. Zool. 27 : 367-390. 1919.

147. Mast, S. O. Photic orientation in insects. Proc. Nation. Acad. Sci. 8: 240-245. 1922.

148. MAst, S. O. Photic orientation in insects with special reference to the drone-fly, Eristalis tenax and the robber-fly, Erax rufibarbis. Jour. Exp. Zool. 38: 109205.1923. 
148a. Mast, S. O. Mechanies of locomotion in Amoeba. Proc. Nation. Acad. Sci. 9 : 258-261. 1923.

149. Mast, S. O. The process of photic orientation in the robber-fly, Proctacanthus Philadelphicus. Amer. Jour. Physiol. 68: 262-279. 1924.

150. Mast, S. O. Photic orientation in insects. Amer. Nat. 60:479-482. 1926.

151. MAst, S. O. Reactions to light in Volvox, with special reference to the process of orientation. Zeitsch. Vergl. Physiol. 4: 637-658. 1926.

151a. Mast, S. O. Structure, movement, locomotion, and stimulation in Amoeba. Jour. Morph. and Physiol. 41:347-425. 1926.

152. MAST, S. O. Reversal in photic orientation in Volvox and the nature of photic stimulation. Zeitsch. Vergl. Physiol. 5 : 730-738. 1927.

152a. MAst, S. O. Structure and function of the eye-spot in unicellular and colonial organisms. Arch. Protistenk. 60: 197-220. 1927.

153. MAST, S. O. The nature of response to light in Amoeba proteus (Leidy). Zeitsch. Vergl. Physiol. 15 : 139-147. 1931.

153a. MAst, S. O. The nature of the action of electricity in producing response and injury in Amoeba proteus (Leidy) and the effect of electricity on the viscosity of protoplasm. Zeitsch. Vergl. Physiol. 15 : 309-328. 1931.

153b. MASt, S. O. Movement and response in Difflugia with special reference to the nature of cytoplasmic contraction. Biol. Bull. 61:223-241. 1931.

154. MAst, S. O. Localized stimulation, transmission of impulses, and the nature of response in Amoeba. Physiol. Zool. 5: 1-15. 1932.

155. MASt, S. O. Locomotion in Amoeba proteus (Leidy). Protoplasma 14: 321-330. 1931.

156. MAST, S. O. The rate of adaptation to light and to darkness in Volvox globator. Zeitsch. Vergl. Physiol. 17: 644-658. 1932.

157. Mast, S. O., and W. L. Dolley, Jr. The relation between the stimulating efficiency of intermittent light and the length of the light and the dark periods. Amer. Jour. Physiol. 71: 163-173. 1924.

158. Mast, S. O., and W. L. Dollexy, JR. The relation between the stimulating efficiency of intermittent and continuous light. Amer. Jour. Physiol. 72: 84-88. 1925.

159. MAST, S. O., and MARY Gover. Relation between intensity of light and rate of locomotion in Phacus pleuronectes and Euglena gracilis and its bearing on orientation. Biol. Bull. 43 : 203-209. 1922.

160. MAst, S. O., and H. R. Hulpieu. Variation in the response to light in Amoeba proteus with special reference to the effects of salts and hydrogen-ion concentration. Protoplasma 11: 412-431. 1930.

161. Mast, S. O., and P. L. Johnson. Orientation in light from two sources and its bearing on the function of the eye-spot. Zeitsch. Vergl. Physiol. 16: 252-274. 1932.

162. Mast, S. O., and C. L. Prosser. Effect of temperature, salts, and hydrogen-ion concentration on rupture of the plasmagel sheet, rate of locomotion, and gel/sol ratio in Amoeba proteus. Jour. Cell. and Comp. Physiol. 1: 333-354. 1932.

163. Mast, S. O., and L. C. Pusch. Modification of response in Amoeba. Biol. Bull. 46 : 55-59. 1924.

164. McClendon, J. F. Protozoan studies. Jour. Exp. Zool. 6: 265-287. 1909.

165. Merker, E. Die Empfindlichkeit feuchthäutiger Tiere im Lichte. Zool. Jahrb. 42 : 1-174. 1925.

166. Меккек, E. Die Empfindlichkeit feuchthäutiger Tiere im Lichte. II. Warum kommen Regenwürmer in Wasserlachen um and warum verlassen sie bei Regen ihre Wohnröhren? Eine licht- und atmungsbiologische Studie. Zool. Jahrb. 42 : 487-555. 1926. 
166a. Merker, E. Sehen die Daphnien ultraviolettes Licht? Zool. Jahrb. 48: 277-348. 1930.

167. Merker, E., and G. Bräunig. Die Empfindlichkeit feuchthäutiger Tiere im Lichte. III. Die Atemnot feuchthäutiger Tiere im Lichte der Quarzquecksilberlampe. Zool. Jahrb. 43: 275-338. 1927.

167a. Merker, E., and H. Gilbert. Das Sehvermögen unserer Süsswasserplanarien im langwelligen Ultraviolett. Zool. Jahrb. 51: 441-504. 1932.

168. Meyer, ANNette E. Über Helligkeitsreaktionen von Lepisma saccharina L. Zeitsch. Wiss. Zool. 142 : 254-312. 1932.

169. Miller, H. M., Jr., and Elsie E. Mahaffy. Reactions of Cerceria hamata to light and to mechanical stimuli. Biol. Bull. 59: 95-103. 1930.

170. Minnich, D. E. The photic reactions of the honey-bee, Apis mellifera L. Jour. Exp. Zool. 29 : 343-425. 1919.

171. Mitchell, W. H. Jr., and W. J. Crozier. Photic orientation by two pointsources of light. Jour. Gen. Physiol. 11: 563-583. 1928.

172. Moore, Mary M. The reactions of Cerianthus to light. Jour. Gen. Physiol. 8: 509-518. 1927.

173. Moore, A. R., and W. H. Cole. The response of Popillia japonica to light and the Weber-Fechner law. Jour. Gen. Physiol. 3 : 331-335. 1921.

174. Müller, ANwemarie. Über Lichtreaktionen von Landasseln. Zeitsch. Vergl. Physiol. 3: 113-144. 1925.

175. NAGEL, W. A. Der Lichtsinn augenloser Thiere. 120 pp. Fischer; Jena, 1896.

176. Necheles, H. Observations on the causes of night activity in some insects. Chinese Jour. Physiol. 1 : 143-155. 1927.

177. Nı⿻мczук, E. Die Lichtkompassbewegung der niederen Tiere. Berlin, 1923. (Ungedruckte Dissertation).

178. Nomura, E. Effect of light on the movements of the earthworm, Allolobophora foetida (Sav.). Sci. Repts. Tohoku Imp. Univ. Biol. 1: 293-409. 1926.

179. Nomura, E., and Ohfuchi Shinryo. Seasonal changes of photic orientation in Allolobophora foetida. Sci. Repts. Tohoku Imp. Univ. Biol. 3 : 97-112. 1928.

180. Nutting, P. G. 1919 report of Standards Committee on visual sensitometry. Jour. Opt. Soc. Amer. and Rev. Sei. Instruments 4: 55-79. 1920.

181. Opfinger, Elisabeth. Über die Orientierung der Biene an der Futterquelle. Zeitsch. Vergl. Physiol. 15 : 431-487. 1931.

182. Parker, G. H. The phototropism of the mourning-cloak butterfly. Mark Anniversary Volume, pp. 453-469. 1903.

183. Patten, B. M. A quantitative determination of the orienting reaction of the blowfly larva (Calliphora erythrocephala Meigen). Jour. Exp. Zool. 17: 213280. 1914.

184. Peters, E. Vergleichende Untersuchungen über den Lichtsinn einheimischer Cladocerenarten. Zool. Jahrb. 43: 1-40. 1926.

185. Pí́rox, H. Les lois de l'excitabilité lumineuse des Lamellibranches. Recherches sur la loi de variation des énergies liminaires chez Mya arenaria. Bull. Sta. Biol. d'Arcachon 23 : 1-32. 1926.

186. Priebatsch, Irma. Der Einfluss des Lichtes auf Farbwechsel und Phototaxis von Dixippus (Carausius) morosus. Zeitsch. Vergl. Physiol. 19: 453-488. 1933.

187. Prosser, C. L. Effect of central nervous system on responses to light in Eisenia foetida, Sav. Jour. Neur. 69:61-86. 1934.

188. Prosser, C. L. Action potentials in the nervous system of the crayfish. I. Spontaneous impulses. 1934. [To appear in Jour. Cell. and Comp. Physiol. About 26 pp.] 
189. RÁDL, E. Untersuchungen über den Phototropismus der Tiere. 188 pp. Leipzig, 1903. [Extensive bibliography.]

190. Rose, M. La question des tropismes. 469 pp. Paris, 1929. [Extensive bibliography.]

191. Ryder, J. A. Primitive visual organs. Science 2: 739. 1883.

192. SÄLZLE, K. Untersuchungen an Libellenlarven über das Sehen bewegter Objekte. Zeitsch. Vergl. Physiol. 18: 347-368. 1932.

193. SANDER, IW. Phototaktische Reaktionen der Bienen auf Lichter verschiedener Wellenlänge. Zeitsch. Vergl. Physiol. 20:267-286. 1933.

194. Santschi. Observations et remarques critiques sur le mécanisme de l'orientation chez les fourmis. Rev. Suisse Zool. 19:117-134. 1911.

195. Schaeffer, A. A. Reactions of Amoeba to light and the effect of light on feeding. Biol. Bull. 32 : 45-74. 1917.

196. Schlieper, C. Farbensinn der Tiere und optomotorische Reaktionen. Zeitsch. Vergl. Physiol. 6 : 453-472. 1927.

197. Schlieper, C. Über die Helligkeitsverteilung im Spektrum bei verschiedenen Insekten. Zeitsch. Vergl. Physiol. 8: 281-288. 1928.

198. Schulz, W. Über die Bedeutung des Lichtes im Leben niederer Krebse. (Nach Versuchen an Daphniden.) Zeitsch. Vergl. Physiol. 7: 488-552. 1928.

199. Schulz, W. Die Orientierung des Rückenschwimmers zum Licht und zur Strömung. Zeitsch. Vergl. Physiol. 14: 392-404. 1931.

200. Segall, J. Versuche über Lichtreaktionen und Lichtempfindlichkeit beim Regenwurm. Zeitsch. Vergl. Physiol. 19: 94-109. 1933.

201. Seifert, R. Raumorientierung und Phototaxis der Anostraken Euphyllopoden. (Versuche an Chirocephalus und Artemia.) Zeitsch. Vergl. Physiol. 16: 111184. 1932.

202. Strasburger, E. Wirkung des Lichtes und der Wärme auf Schwärmsporen. Jena. Zeitsch. 12 : 551-625. 1878.

203. Taliaferro, W. H. Reactions to light in Planaria maculata, with special reference to the function and structure of the eyes. Jour. Exp. Zool. 31:59-116. 1920.

204. Trembley, A. Mémoires pour servir à l'histoire d'un genre de polypes d'eau douce à bras en forme de cornes. 324 pp. Leyden, 1744 .

205. Verlaine, L. L'instinct et l'intelligence chez les Hyménoptères. VII. l'Abstraction. Ann. Soc. Roy. Zool. Belg. 58: 59-88. 1927.

206. Verlaine, L. L'Instinct et l'intelligence chez les Hyménoptères. VIII. Note conılémentaire sur l'abstraction. Ann. Soc. Roy. Zool. Belg. 68: 240-250. 1928.

207. Verworn, M. Allgemeine Physiologie. 615 pp. Jena, 1894.

208. Verworn, M. Irritability. 264 pp. New Haven, 1913.

209. Visscher, J. P. Reactions of the cyprid larvae of barnacles at the time of attachment. Biol. Bull. 54 : 327-335. 1928.

210. Visscher, J. P., and R. H. Luce. Reactions of the cyprid larvae of barnacles to light with special reference to spectral colors. Biol. Bull. 54: 336-350. 1928.

211. Walter, H. E. The reactions of Planarians to light. Jour. Exp. Zool. 5: 35-162. 1907.

212. Wejls, G. P. Colour response in a leech. Nature [London] 129: 686-687. 1932.

213. Wersh, J. H. Reversal of phototropism in a parasitic water mite. Biol. Bull. 59: 165-169. 1930.

214. Wetsh, J. H. Specific influence of the host on the light responses of parasitic water mites. Biol. Bull. 61:497-499. 1931. 
215. Welsh, J. H. The nature and movement of the reflecting pigment in the eyes of crustaceans. Jour. Exp. Zool. 62: 173-183. 1932.

216. Welsh, J. H. Temperature and light as factors influencing the rate of swimming of larvae of the mussel crab, Pinnotheres maculata Say. Biol. Bull. 63: 310-326. 1932.

217. Welsh, J. H. Photokinesis and tonic effect of light in Unionicola. Jour. Gen. Physiol. 16: 349-355. 1932.

218. WeLsh, J. H. Light intensity and the extent of activity of locomotor muscles as opposed to cilia. Biol. Bull. 65: 168-174. 1933.

219. Wenrich, D. H. Notes on the reactions of bivalve mollusks to changes in light intensity: image formation in Pecten. Jour. Animal Behav. 6:297-318. 1916.

220. Weyradch, W. K. Untersuchungen und Gedanken zur Lichtorientierung von Arthropoden (1. Teil). Zool. Jahrb. 47: 291-328. 1930.

221. White, Gertrude M. Reactions of the larvae of the shrimp, Paleomonetes vulgaris, and the squid, Loligo pealii, to monochromatic light. Biol. Bull. 47: 265-273. 1924.

222. Willrich, Ursula. Beiträge zur Kenntnis der Lichtkompassbewegung und des Farbensinnes der Insekten. Zool. Jahrb. 49: 157-204. 1931.

223. Wilson, E. B. The heliotropism of Hydra. Amer. Nat. 25: 413-433. 1891.

224. Wolf, E. Über das Heimkehrvermögen der Bienen. Zeitsch. Vergl. Physiol. 3: 615-691. 1926.

225. Wolf, E. Über das Heimkehrvermögen der Bienen. 2. Mitt. Zeitsch. Vergl. Physiol. 6: 221-254. 1927.

226. Wolf, E. Sehschärfeprüfung an Bienen im Freilandversuch. Zeitsch. Vergl. Physiol. $14: 746-762.1931$.

227. Wolf, E. The visual intensity discrimination of the honey bee. Jour. Gen. Physiol. $16: 407-422.1933$.

228. Wolf, E. On the relation between measurements of intensity discrimination and visual acuity in the honey bee. Jour. Gen. Phýsiol. 16: 773-786. 1933.

229. Wolf, E. Critical frequency of flicker as a function of intensity of illumination for the eye of the bee. Jour. Gen. Physiol. 17: 7-20. 1933.

230. Wolf, E., and W. J. Crozier. Orientation in compound fields of excitation; Photic adaptation in phototropism. Jour. Gen. Physiol. 11: 7-24. 1927.

231. Wolf, E., and W. J. Crozier. The variability of intensity discrimination by the honey bee in relation to the visual acuity. Jour. Gen. Physiol. 16: 787-793. 1933.

232. YAGI, N. Phototropism of Dixippus morosus. Jour. Gen. Physiol. 11: 297-300. 1928.

233. Yerkes, AdA W. Modifiability of behavior in Hydroides dianthus. Jour. Comp. Neur. and Psych. 16:441-449. 1906.

234. Yerkes, R. M. The intelligence of earthworms. Jour. Animal Behar. 2 : 332-353. 1912.

235. Zerrahn, Gertrud. Formdressur und Formunterscheidung bei der Honigbiene. Zeitsch. Vergl. Physiol. $20: 117-150.1933$.

Manuscript received by the editor A pril, 1934. 



\title{
XVIII
}

\section{THE ACTION OF RADIATIONS ON LIVING PROTOPLASM}

\author{
L. V. Heilbrunn and Daniel Mazia
}

\section{Department of Zoology, University of Pennsylvania, Philadelphia}

Introduction. Permeability: Visible light-Ultra-violet rays-Radium-Roentgen rays. Surface charge. Protoplasmic colloids: Visible light-Ultra-violet rays-Roentgen rays and radium. Conclusion. References.

\section{INTRODUCTION}

It is recognized that radiation affects protoplasm and protoplasts, but whether or not this direct action may explain the effects of radiation on organisms is another problem. Generalizations by radiologists have appealed to protoplasmic effects out of all proportion to exact information concerning these. The interest in an evaluation of our information, therefore, extends beyond that of cellular physiology, which in itself must be great.

Our knowledge is admittedly slight. The number of radiation experiments which consider direct protoplasmic effects even to the extent of examining the cells microscopically is small. The observations to be discussed in this paper were necessarily culled from outside the literature of cellular physiology, from plant and animal physiology, cytology, pathology, bacteriology, and from clinical and experimental medicine. The task is to impose upon these data the point of view and the criteria of the student of protoplasm.

The change of stress from the purely radiological to the physiological implies in this case a new emphasis upon the qualitative. Quantitative experiments in the literature of radiation are characterized by the use of some conspicuous biological criterion, with reference to which the results of changing the physical variables such as intensity, frequency, and time are compared. The criteria typically used are death, change in growth rate, change in division rate, abnormal fertilization, abnormal development, etc., all complex phenomena whose components alone have physiological meaning.

It is premature to consider with great exactness the relations of the physical variables in terms of diffuse and unanalyzed "biological" effects. The first question is, what do the rays do to protoplasm-to the cell? The mathematical treatment of exact mortality curves, for example, is not a primary concern for us. The theories advanced in terms of the physics of the radiation-especially the effects of radiations 
on gases-are even less relevant. We shall be concerned with the physics of the living cells, and the effects upon these which radiations share strictly in common with other external influences-a fact of ten overlooked by the specialist in radiations.

At the present time among radiation authorities there seems to be scant appreciation of the fact that a physical study of protoplasm is at all possible. Thus Rajewsky (299), after pointing out that most of the radiation literature is concerned with end reactions and that what is needed is some research on the "Grundvorgänge," goes on to state that with present techniques no direct information concerning the primary effect of radiation on plant and animal cells is possible. He describes, therefore, experiments on proteins. A similar point of view is maintained by Dyes (85).

We shall insist emphatically that a knowledge of the effects of radiations on proteins or other inanimate materials is of secondary importance to the knowledge of the effects of radiations on living protoplasm. Over and over again, direct study of the colloid chemistry of protoplasm has shown that the living substance is unique in its behavior toward physical and chemical agents (Heilbrunn, 136). A student of blood clotting would scarcely expect to arrive at a solution of his problem by studying the colloidal properties of egg albumen and gelatin. The behavior of the blood is vastly different, we are certain, from that of a simple protein, and it is complicated by the presence of little-known substances exceedingly potent in their effects on coagulation. We can scarcely hope, therefore, for success in treating protoplasm, which is at least as complex as blood, as though it were a simple protein. We stress this analogy, for on the one hand protoplasm in its power of sudden gelation shows a colloidchemical similarity to blood (Heilbrumn, 136), and on the other hand, the various substances involved in blood clotting are all apparently present in tissue cells (Kraus and Fuchs, 200, 201, Fischer, 100). Inanimate proteins can, therefore, scarcely provide a satisfactory model for the living cell.

Some radiologists have emphasized the fact that isolated cells are less sensitive to roentgen rays than cells in mass (see Glocker and Reuss, 121), and they insist that it is unwise to consider experiments on isolated cells. As far as we can judge, however, it seems certain that the effects on single cells are essentially the same as on cells in mass, although apparently a larger dosage is necessary to affect them. One explanation for this phenomenon, or rather one factor in the explanation, is the fact that injured cells may very well have an indirect effect on cells in their neighborhood, so that when a tissue is irradiated, the more sensitive cells affect the whole mass. We shall return to this point later. But our main interest is in the primary effect of radiation on the living protoplasm and this can be best studied in isolated cells. 
Many writers stress the fact that the nucleus is more sensitive to radiation than the cytoplasm (Reiss, 311, Vintemburger, 376, Zirkle, 397). The extreme point of view, to the effect that only the nucleus is sensitive to radiation (Zirkle, 398), can certainly not be held, for irradiation of cytoplasm alone is effective (Reiss, 311, Vintemburger, 376, Tchahotine, 356, probably also Feichtinger, 97). There are indeed some who claim that the cytoplasm is more sensitive than the nucleus (Wail and Frenkel, 380, Iasswoine, 168, Jansson, 177, Rochlin-Gleichgewicht, 317). The argument need not concern us further. Certain it is that the cytoplasm alone may be profoundly affected by all types of radiation.

As yet there is little information regarding the effects of radiation-or of anything else-on the physical and physicochemical properties of the nucleus. We must, therefore, confine our discussion to the cytoplasm.

Neither the metabolic effects nor the purely cytological will be considered here, for these are discussed in other papers. The physical and physicochemical properties of the cells for which methods of study have been developed will be treated. These are $(a)$ permeability to water and dissolved substances, $(b)$ viscosity, $(c)$ electrical charges and potentials, $(d)$ reaction. In addition, the direct visible effects of radiations on living protoplasm will be discussed; the morphological aspects of degenerative changes must be accounted for before cytolysis is completely explained. The paper embraces the following types of radiations and particulate agents conveniently classified with radiations: visible light, ultra-violet rays, roentgen rays, alpha particles, beta and gamma rays, cathode rays, but not heat waves, Hertzian waves, or cosmic rays. Although infra-red radiation certainly has an effect on living material (Hertel, 143, Vogt, 377, W. Hoffmann, 156, Nelson and Brooks, 244 , etc.), the action on protoplasm has scarcely been examined. Our primary interest is in the effect of radiations on plant and animal cells, and tissues in mass are considered only when information concerning protoplasm may be derived from them. The literature on inanimate colloids is not included here. Our treatment is not exhaustive; we wish chiefly to advance a point of view that is based on what knowledge there is of the physical and colloidal properties of protoplasm, and, therefore, seems somewhat more purposeful than the prevailing "quantitative" point of view.

Many of the papers we have read have offered so little to our discussion that we do not refer to them in the text. Perhaps half of these we have included in the bibliography with the idea that perhaps the reader who cares to consult them may find more than we did.

\section{PERMEABILITY}

The cell physiologist has always been impressed with the importance of knowing the permeability of the living cell, and the changes in per- 
meability under one condition or another. No aspect of cell physiology has been more frequently studied. By many authors increase or decrease in permeability has been related to the essential phenomena in the life and death of the cell.

Numerous methods have been employed to test permeability. These include staining methods, osmotic methods, chemical analyses of cells and their surrounding fluid, measurements of electrical conductivity, etc. No one of the methods is above criticism, although some are to be preferred to others. The problem is somewhat different in plant cells which, unlike animal cells, are usually characterized by large vacuoles. In the case of enormous cells like those of Valonia or Nitella the contents of the vacuoles may be analyzed chemically. These vacuoles do not represent protoplasm, and the entrance of substances into them implies passage into the protoplasm and out of it again.

In recent years there has been an increasing tendency to examine results critically, to apply more certain methods, and to obtain quantitative results. On the whole, chemical and osmotic methods are now preferred to staining and electrical techniques. The staining of a cell depends on more than mere permeability, and when one observes increases in the electrical resistance of a mass of cells, a host of factors other than permeability are involved. Modern authors have studied the permeability of the cell membrane to water as well as to dissolved substances. (For recent reviews, see Jacobs, 172, Stiles, 349, Gellhorn, 1929, Homès, 164, Lucké and McCutcheon, 244.)

In interpreting permeability studies each experiment should be considered critically. In view of the fact that no method is entirely satisfactory, agrecment between results obtained by different methods is greatly to be desired. In the case of irradiated cells the fact that the rays cause a release of bound salts from the protoplasm (see below) is likely to be a complicating factor.

\section{VISIBLE LIGHT}

The effect of visible light upon permeability has been studied mostly by botanists. As early as 1879 Pringsheim (297), focusing a powerful beam of white light upon Spirogyra filaments, noted that when the radiation caused a breakdown of chlorophyll the cells became permeable to aniline blue, which did not penetrate normal cells. The earliest "quantitative" permeability method, the method of isotonic coefficients, was used in the study of the action of light by Lepeschkin (218) and Tröndle $(361,362)$. In such measurements, the difference between the concentration of a penetrating substance, such as $\mathrm{KNO}_{3}$, required to cause plasmolysis and the concentration of the same substance isotonic with a solution of a nonpenetrating substance (such as sucrose) which will cause the same degree of plasmolysis, is taken as the measure of the 
amount of salt that penetrates. The permeability coefficient $\mu$ is defined as $1-C / C^{\prime}$, where $C^{\prime}$ is the plasmolytic concentration of salt and $C$ the concentration physically isotonic with the plasmolytic nonelectrolyte.

Tröndle studied the palisade and parenchyme cells of the leaves of Tilia cordata and the parenchyme cells of Buxus sempervirens. Lepeschkin used the pulvinus cells of Phaseolus, Spirogyra filaments, and Rheo leaf cells. In both studies daylight of undetermined intensity was the source of radiation. Both authors found an increase in permeability to $\mathrm{KNO}_{3}$ with increased illumination. Thus, in Tilia, 28 experiments in cloudy weather gave an average $\mu$ of 0.24 ; in direct sunlight the average of 22 determinations was 0.32 . With an artificial source of light, a 32 candle-power bulb, the value of $\mu$ could become even higher. Tröndle does not report exposure time intervals, though from later experiments (362) he plotted a time curve showing that illuminated cells ultimately became less permeable than darkened cells.

Lepeschkin's results are similar. In cells of Phaseolus kept in sunlight the permeability coefficient was 1.5 to 2 times as great as that of the darkened controls.

This method of isotonic coefficients has been criticized by many reviewers $(46,101,349)$. Zycha (404) has sharply attacked the results of Lepeschkin and Tröndle, and with considerable justice. In his experiments Zycha used the same material as his predecessors and employed the same method of isotonic coefficients with much regard to exactness of technique. Examining carefully the sources of error in the method, he calculates that it can be as high as 30 per cent. His experimental results are diametrically opposed to those of Lepeschkin and Tröndle, but he discards them as well as the latter because of the intrinsic uncertainty of the method. Lepeschkin (221) criticizes Zycha's use of parallel slices of leaf for the determinations of the plasmolytic concentration of salt and of sugar, respectively; the variation among slices, he claims, does not permit this. Zycha had insisted upon this procedure because plasmolysis, occupying an hour or more, must injure the cell to such extent that the second determination is made with an abnormal cell. Both of these claims may be just and together invalidate the method entirely. Nadson and Rochline-Gleichgewicht (269) found that plasmolysis by sucrose causes a release of $\mathrm{Ca}$ in cells of Elodea and Pterygophyllum as evidenced by the formation of calcium oxalate crystals. One of us (Mazia) was able to verify this observation in Elodea cells and found, further, that plasmolysis by salt solutions could cause this $\mathrm{Ca}$ release. These facts would support Zycha's view.

Brauner (43) used a plasmolytic method, with controlled illumination. An exposure of cells from the coleoptile of Avena to a source of 50,000 meter-candle sec. for $30 \mathrm{~min}$. caused an increase of 25 per cent in the amount of $\mathrm{NaNO}_{3}$ penetrating in a fixed period of time. After 
60 min. illumination the permeability was 75 per cent below that of the controls, as in the time curves of Tröndle.

Kisselew (188) and Lvoff (246) were primarily interested in the interpretation of the opening of stomata as an action of light on the permeability of the guard cells. The former tested the guard cell permeability to dyes, diastase, saltpeter, sucrose, and by estimation of the rate of plasmolysis, to water. In all of Kisselew's measurements, the guard cells of open stomata showed a reduced permeability. Light is the agent that commonly induces stomatal opening. Lvoff measured isotonic coefficients in the leaf cells of Rumex, using controlled illumination. His results contradict those of Kisselew. He discovered that illumination caused a 60 to 100 per cent increase in the permeability coefficient of the guard cells, and a smaller but noticeable increase in $\mu$ in the neighboring cells. This author, and later Lepeschkin, criticize Kisselew's use of starch digestion as a measure of the penetration of $\mathrm{KNO}_{3}$; but Kisselew used other methods, as noted, and particularly the rate of plasmolysis, which (according to this author) is a sounder measure of permeability than the isotonic coefficient (cf. Stiles, 349, page 20), although its application is to water penetration.

A more exact osmotic method, the Höfler plasmometric method, was used by Hoffmann (154) on Spirogyra cells. The Höfler method consists of following the rate of deplasmolysis or reversal of plasmolysis when cells are immersed in a solution of a substance which penetrates slowly. Hoffmann found that Spirogyra cells in daylight showed 60 per cent higher permeability to glycerin than those in the dark. Considering possible errors, Hoffmann states that the permeability increase in the light is at least 20 to 30 per cent. Cells without nuclei behave in the same manner as normal cells.

Although plasmolytic methods such as that of Höfler are very much in favor at the present time, the results they give are not to be accepted unreservedly. The experimental studies of Weber (388) indicate clearly that in some cases, at least, the plasmolytic method does not provide a measure of the normal permeability of the cell but rather the permeability as altered by the plasmolytic agent. Here again the release of free electrolytes noted above may be a factor; Hljin (169) found that immersion of cells of Iris in isotonic salt solutions, particularly Na salt solutions, caused an increase in the osmotic pressure of the cell interior with respect to the cell membrane. Furthermore, Weber (388) has presented evidence that plasmolysis always implies a rupture of the cell membrane, which reforms very rapidly if $\mathrm{Ca}$ is present.

Various authors have attempted to determine the influence of light upon exosmosis of substances. Lepeschkin (218) placed pulvini of Phaseolus in water for a fixed period of time, some in daylight, some in darkness, and then analyzed the water for total solids. About 1.5 to 2 
times as much material (undefined) had diffused from the illuminated cells as from the controls, a figure agreeing well with the results obtained by the method of isotonic coefficients.

Blackman and Paine (28) did similar work with pulvini of Mimosa, determining by electrical conductance measurements the amount of effusing salt. Using a 50-candle Nernst lamp as a source of illumination, they observed an immediate increase followed by a decline in the rate of increase of conductivity. Dillewijn (73; cited after Zycha, 464) made similar measurements with tissue of Avena and obtained results contradicting those of Blackman and Paine. It should be pointed out that exosmosis may depend on other factors than permeability, notably the concentration of diffusible electrolytes in the cytoplasm, and, in experiments with whole tissues, the intercellular materials.

Direct measurements of tissue conductivity were used by Brauner (43) to determine permeability changes in the coleoptile of Avena; his illumination is very accurately defined. This author was able to detect a conductivity increase upon illumination superimposed upon that caused by the implantation of platinum electrodes (made fast with hot paraffin!). The inadequacies of such a method are obvious and, indeed, are well indicated by Brauner himself.

Direct chemical methods also have been applied to the study of the effects of visible light on cell permeability. Hoagland and Davis (150) and Hoagland, Hibbard, and Davis (151) measured directly the amount of anion accumulating in the sap of Nitella cells in the darkness and in white light. In the early experiments, daylight was the source of illumination, and $\mathrm{Cl}, \mathrm{NO}_{3}$, and $\mathrm{Br}$ were determined in the sap. Chloride absorption in the light against a concentration gradient sometimes left the surroundings totally free of $\mathrm{Cl}$ in a few days; in 3 days twice as much $\mathrm{NO}_{3}$ was absorbed by the illuminated cells as by the darkened ones, and in 4 to 5 days three times as much $\mathrm{Br}$. In the later work artificial sources of illumination were used, the amount of light being defined only in terms of the wattage of the lamps. Bromide, not found naturally in the sap of these cells in appreciable concentration, was determined. Light accelerated accumulation of this ion, and even cells exposed to $\mathrm{Br}$ in the dark showed increased absorption of $\mathrm{Br}$ if they had been just previously illuminated. Accumulation did not vary directly as the amount of light; intensity was the more weighty factor, contrary to the conclusion of Tröndle. These authors present their data as indicative of changes in a metabolic process which they (and Zycha) contrast with ordinary permeability phenomena, which last depend on the laws of physical diffusion. The difference of opinion depends, of course, on the chosen definition of permeability. Hoagland, Hibbard, and Davis found that the presence of $\mathrm{Ca}$ inhibited penetration, and reported a temperature coefficient of accumulation, $Q_{10}=2.0$ to 3.0. Both of these facts charac- 
terize ordinary permeability phenomena (cf. Lucké and McCutcheon, 244).

None of the work considered has taken into account the effects of light of different wave-lengths. The first investigation of this problem is that of M. M. Brooks (45), who worked with the large eells of the alga Valonia macrophysa and determined eolorimetrically the penetration of 2-, 6-dibromophenol indophenol into the sap. Her eurves show an inverse relation between permeability inerease and wave-length (Fig. 1). This permeability increase is independent of the energy transmission of

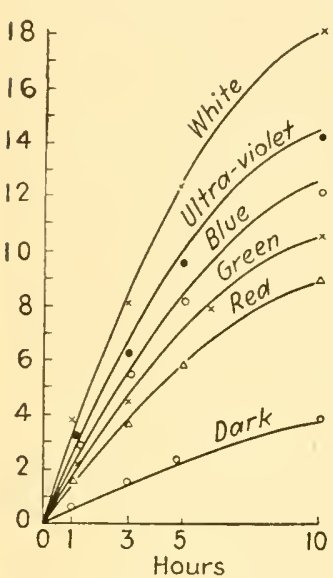

FIg. 1.-The molecular concentration of $2-, 6-$ dibromophenol indophenol in the sap of Valonia (ordinates) when the plants had been exposed to screens transmitting different wave-lengths at intervals up to $10 \mathrm{hr}$. (abscissas), at $22^{\circ} \mathrm{C}$. (After Brooks, 44.) of light on animal cell permeability is correspondingly less abundant. The most light-sensitive of animal tissues, the retina, was eonsidered by Lange and Simon (210). Frog retina was exposed to a 300-candle Osram lamp at a distance of $80 \mathrm{~cm}$. Following Embden, the authors considered the rate of phosphate production a measure of permeability. This they found to be increased by light, but as a permeability index it is highly indirect, if at all valid.

Packard (283) used Paramecium in his study of the penetration of $\mathrm{NH}_{4} \mathrm{OH}$ under illumination. The cells were first stained with neutral red, and the decoloration was taken as a measure of the penetration of the alkali. The injuries due to the alkali are not accounted for but may not be important for relative measurements. Of course, the method assumes constancy of cellular $\mathrm{pH}$ during irradiation. Cells irradiated 
with sunlight through glass were more permeable than those kept in the darkness. By the use of filters, Packard also obtained results agreeing with those of Brooks and Lepeschkin; the shorter wave-lengths had relatively more effect upon the permeability.

Increased intensity of stain in both nucleus and cytoplasm of frog leucocytes in Nile blue sulfate when irradiated with a 32-candle Osram lamp was found by Haberlandt (126). Disappearance of the color with protracted illumination, however, renders difficult any reading of a permeability factor into these results.

Recently Lepeschkin (224) has presented data showing an increase of the permeability of human and cow erythrocytes to electrolytes and nonelectrolytes under the influence of sunlight. He used three methods: (a) hematocrit, (b) osmotic hemolysis, and (c) isotonic coefficients based on the use of a penetrating and a nonpenetrating hypotonic solution to cause hemolysis. By the first method he determined the relative permeability of the blood corpuscles to glucose; by the second, permeability to glycerin; and by the third, the permeability of cow erythrocytes to $\mathrm{NaCl}$ and the permeability of human erythrocytes to glucose. In all cases light caused an increase in the rate of penetration, whether of dissolved substance or water, although the results are not translated into absolute terms.

In all, then, the evidence favors the view that visible light increases the permeability of plant and animal cells. The chief objections arise from the experiments of Kisselew and of Zycha. The former used only the very specialized guard cells; the latter's results are open to the criticism of Lepeschkin, already mentioned. The newer and semiquantitative data render a conclusion fairly safe, though the methods of control of radiation quality and intensity allow no quantitative comparisons. Even for plant cells there is yet no single set of experiments that combines a reliable permeability method with accurate determination of the illumination.

\section{ULTRA-VIOLET RAYS}

The literature on changes in permeability produced by ultra-violet rays is much smaller than that on the risible spectrum. The work is largely on animal cells, for which, unfortunately, the methods of exact permeability measurement are limited to a few types of material.

The work of Brooks and of Lepeschkin, which contained evidence for a permeability increase in plant cells by the ultra-violet portion of ordinary sunlight has already been cited. These authors made no experiments using artificial sources.

The "radiopuncture" method of Tschachotin (364 for method proper; 357 ), by which a beam of ultra-violet rays is focused on a single cell or part of a cell, has given this author results interpreted as evidencing a 
permeability increase in the membrane of Strongylocentrotus (sea urchin) eggs. After irradiation at a point on the surface of an egg, he placed the egg in hypotonic or hypertonic sea water. In hypotonic sea water he observed a protuberance, in hypertonic an invagination of the surface at the point irradiated, indicating more rapid movement of water across the membrane at this point. Tschachotin further reported that decoloration of neutral red-stained eggs exposed to alkali occurred most rapidly at the point irradiated.

Besides these experiments, most of the information concerning the action of ultra-violet on permeability concerns specialized tissues. Eckstein and von Mollendorf (87) studied the accumulation of trypan blue in the kidney epithelium of irradiated and subsequently injected rats. The dye accumulated more readily in the cells of the irradiated animals than in those of the controls. This type of result has been quoted (e.g., by Tsebrikow, 367) as evidence for a permeability change. Certainly it is too indirect to be of much value; the authors themselves do not consider permeability.

Gans and Schlossmann (110) applied the indicator method to the investigation of the penetration of $\mathrm{NH}_{4} \mathrm{OH}$ into sections of human skin. The skin, stained with neutral red, was obtained from patients irradiated with direct sunlight or a mercury-vapor lamp. Four eases are given, and in each the decoloration of the rayed section proceeded faster than that in sections of nonirradiated skin from the same patients.

They quote as further evidence for their view the data of Regelsberger (309) who measured the polarization resistance of normal and irradiated skin. His source of ultra-violet was of sufficient intensity to cause erythema 2 hr. after a 1-min. exposure. At the onset of erythema Regelsberger observed a 10 - to 200 -fold increase in the polarization resistance, which Gans and Schlossmann consider a measure of permeability. Keller (184) obtained similar results. It is obvious that in all of these experiments a tissue of many layers is being considered, so that cytolytic changes, rather than reversible membrane effects, may be responsible for the electrical fluctuations. This criticism is vigorously advanced by Lewis and Zotterman (227).

\section{RADIUM}

The investigation by Maud Williams (392) of the effects of combined beta and gamma rays on epithelial cells from the petiole of Saxifraga umbrosa includes estimations of permeability changes (a) by measurements of the stomatal apertures, following a theory of stomatal closure similar to that of Kisselew, according to which closure is caused by permeability increase; and $(b)$ by determinations of the rate of plasmolyis by sugar solutions. Her sources were $0.1 \mathrm{gm}$. of radium element and 
$0.51 \mathrm{mg}$. of bromide with a gamma-ray activity equivalent to $0.27 \mathrm{mg}$. of element. The capsules of radioactive material were placed directly on the coverslip over the preparation. The rays caused a considerable decrease in the average stomatal gap (ratio of width to length). Irradiation also caused a 2- to 20 -fold increase in the percentage of cells plasmolyzed by a given hypertonic sucrose solution in a given time, which is a fairly direct indication of an increased permeability to water.

Packard $(281,282)$ presents evidence, largely indirect and wholly qualitative, for an increase in the permeability of protozoa by radium. He used $13.4 \mathrm{mg}$. of element very near the culture. He compared the permeability (determined by the neutral red- $\mathrm{NH}_{4} \mathrm{OH}$ method) of conjugating and normal Paramecium with their relative radiosensibility. Similarly, the relative permeabilities of Paramecium and Stylonychia were compared with their sensibility to the rays. The more permeable animals always proved the more susceptible to the lethal action of radium rays. From this he concluded that permeability increase was a factor in radium cytolysis. Since $0.12 \mathrm{~mm}$. of lead obviated all these results, he attributed them to the soft beta rays. The later paper gives more direct evidence; the irradiated and nonirradiated cells were tested by the indicator method. Here Packard was able to demonstrate that permeability of Paramecium to $\mathrm{NH}_{4} \mathrm{OH}$ increased as the dose of beta rays increased.

In 1925 Van Herwerden (146) used the "reversible gelation" which rendered the nucleus and nucleolus of epithelial cells in tadpole tails visible, as a criterion of the penetration of dilute acetic acid. She exposed a preparation of $3.1 \mathrm{mg}$. of $\mathrm{RaBr}_{2}$ (activity unspecified) for an hour or two, then surrounded the cells by 0.05 to 0.07 per cent acetic acid. The gelation appeared immediately. In nonirradiated controls 20 to $30 \mathrm{~min}$. were required to bring about the same result. Van Herwerden found her results were not significantly affected by the interposition of $2 \mathrm{~mm}$. of lead, contradicting the results just mentioned of Packard who, however, probably used larger doses. The reasoning of Van Herwerden is somewhat unsatisfying; if, as is more than possible, the rays themselves cause a partial gelation, this might be summated with the acid effect, and the end point would be reached sooner without any membrane change.

The earliest evidence that erythrocyte permeability might be increased by the rays from radium is that of Henri and Meyer (141). They exposed a suspension of erythrocytes to $0.1 \mathrm{gm}$. of radium bromide for 8 to $9 \mathrm{hr}$., then determined colorimetrically the degree of hemolysis by a graded series of hypotonic solutions. Their data show a decreased osmotic resistance in the irradiated cells, indicating augmented permeability to water. This experiment has been carried out in a quantitative way by Mascherpa (254) who, however, used only radium emanation, which he dissolved in the experimental solutions and therefore permitted to act during hemolysis. Table 1 gives his results. 


\section{Table 1.-Effect of Radium Emanation on Osmotic Resistance of Dog Erythrocytes \\ (Mascherpa, 254)}

\begin{tabular}{c|c|c|c|c}
\hline & \multicolumn{3}{|c}{ Concentration NaCl acting for $12 \mathrm{hr}}$. \\
\cline { 2 - 4 } & \multicolumn{2}{|c|}{ To initiate hemolysis } & \multicolumn{2}{|c}{ To complete hemolysis } \\
\cline { 2 - 4 } $\begin{array}{c}\text { Emanation in } \\
\text { Maché units }\end{array}$ & Control & Radiated & Control & Radiated \\
\hline & & & & \\
\hline 1529.550 & 0.69 & 0.75 & 0.54 & 0.57 \\
2799.540 & 0.72 & 0.78 & 0.57 & 0.60 \\
3865.590 & 0.75 & 0.81 & 0.48 & 0.66 \\
4755.510 & 0.69 & 0.87 & 0.51 & 0.69 \\
\hline
\end{tabular}

Alpha particles, therefore, do decrease osmotic resistance in blood cells. Mascherpa questions the significance of such results as a measure of permeability, pointing out that the relations of pigment, stroma, and membrane are not understood, and that the pigment itself is changed by the radiation, the hemoglobin forming methemoglobin. (For discussion of this problem see Höber, 152, page 421.) Jacobs (173) may also be consulted for an analysis of the relation between hemolysis and permeability.

Mascherpa (254) has also presented direct evidence for an increase in the water permeability of frog skin by radium emanation. The skins, forming the membranes of simple osmometers filled with salt solutions or salt and glucose, were immersed in distilled water containing radium emanation in solution. The activity of the emanation solutions was 4755.1 Maché units. The level in the manometer rose more rapidly when the skin was immersed in the radioactive water than in the controls, as the figures in Table 2 show.

Table 2.-Effect of Radium Emanation on Water Permeability of Frog Skin (Mascherpa, 254)

\begin{tabular}{c|c|c}
\hline \multirow{2}{*}{ Minutes } & \multicolumn{2}{|l}{ Level of osmometer } \\
\cline { 2 - 3 } & Control & Radiated \\
\hline 0 & 0.105 & 0.090 \\
10 & 0.112 & 0.100 \\
20 & 0.119 & 0.115 \\
30 & 0.127 & 0.116 \\
40 & 0.134 & 0.121 \\
50 & 0.139 & 0.125 \\
60 & 0.154 & 0.140 \\
\hline
\end{tabular}


The relative permeability effects of the different products of radioactive disintegration cannot be determined from the sparse data available. It seems true, at least, that alpha rays and mixed beta and gamma rays may cause an increase in cell permeability; as for gamma rays, we have only the contradiction between the experiments of Packard and of Van Herwerden.

\section{ROENTGEN RAYS}

It is consonant with the therapeutic importance of the roentgen rays that their general cellular effect should long have been the object of thought and some research. As the physiological importance of membrane changes has become recognized, attention has been turned to the effects of roentgen rays on cell and tissue permeability. The literature is not large, but the several workers are in good agreement. There is as yet no single investigation that can be considered quantitatively valid both physiologically and radiologically.

The first direct consideration of the problem is that of Richards (314) who used four of the criteria of permeability increase then current: $(a)$ pigment release from Arenicola larvae, $(b)$ penetration of alkali into cells stained with neutral red, $(c)$ penetration of neutral red into cells made alkaline, and $(d)$ artificial parthenogenesis. Richards does not describe his source of radiation, but all of his results are negative. Irradiation caused no release of pigment from Arenicola larvae, nor did it change the "permeability" of the eggs of Asterias or Arbacia, nor did it activate them.

Brummer (48) treated human skin with a dose of $10 \mathrm{X}$-units (at 2 ma.) and determined permeability to $\mathrm{NH}_{4} \mathrm{OH}$ after the method of Gans and Schlossmann already described. The stained skin was decolorized in $8 \mathrm{~min}$., while the control required $6 \mathrm{hr}$. Softer rays $(5.3 \mathrm{ma}$.) proved more effective, although the extent of the difference is not clear. The same author (49) attempted to extend his conclusions to erythrocytes. Cells exposed to $3,6,10$, and $15 \mathrm{X}$-units were suspended in methylene blue, centrifuged after a period of time, and the intensity of the supernatant dye determined. As the dose increased, the amount of dye taken up by the cells diminished, indicating a permeability decrease, in contradiction to Brummer's results with skin.

Kovács (198) used the fact that methyl violet must attain a certain concentration in the frog heart before it stops the beat, to estimate permeability changes in the cardiac tissue. The heart reecived 6 H.E.D. and was immersed in dye solution. Beating stopped in $20.5 \mathrm{~min}$., although the control required $28 \mathrm{~min}$. This difference is not striking, but experiments in which time of immersion was kept constant and the intensity of dye in the heart measured with a colorimeter gave results 
concordant with these. From such experiments, of course, no conclusions relevant to the permeability of the cell membrane can be drawn.

Gassul (116) irradiated (2 to 3 H.E.D.) explants of frog spleen in carmine-containing plasma. The reticular cells and, with stronger doses, the endothelial cells acquired a fine granular stain, although the controls took up no dye. The softer rays (those stopped by $3 \mathrm{~mm}$. of Al plus $0.5 \mathrm{~mm}$. of $\mathrm{Zn}$ ) were the most effective in causing increased penetration or accumulation of the dye.

C. Hoffmann (153) studied the effects of roentgen rays of varying hardness upon the penetration of trypan blue, light green, methyl red, and neutral red into Opalina ranarum in culture. Doses of 500 and $1000 \mathrm{r}$ were used. Irradiated cells accumulated stain more rapidly than control animals; hard and soft rays had the same effect.

In the experiments of Schmidt (331), Halberstädter and Wolfsberg (128, 129), Holtermann (157), and Tsebrikow (368) dyes are injected into whole irradiated animals, and there is determined the accumulation of these dyes in special tissues, particularly kidney epithelium. These results should not alone be considered as evidence for a change of tissue cell permeability after roentgen irradiation; if they imply a membrane effect at all, it is an increase, never a decrease, in permeability.

A number of workers have used electrical methods, which have the advantage of physical precision, but probably insuperable biological disadvantages - to use a change of electrical conductance or polarization resistance as a measure of permeability change, one must make a great number of simplifying assumptions, particularly about the state of the intercellular material and the concentration of free electrolyte within the cell. Thus the formula of Philippson, used by the two authors about to be considered, treats the tissue as a resistance and a capacity in series, with a shunt, and assumes that all cell membranes are lipoid in nature.

Mendeleef (259) irradiated guinea pigs with doses of $720 \mathrm{r}$ (4 ma., $188 \mathrm{kv}$., $0.5 \mathrm{Cu}+1.0 \mathrm{Al}$ ) and determined the electrical constants of liver tissue by the method of Philippson. The calculated results show a decrease in the ohmic and polarization resistance of the membranes which is interpreted as indicating increased cell permeability to ions. Tsebrikow $(367,368)$ used the same method with tissues of irradiated normal and cancerous mice. Her doses were 100 and $600 \mathrm{r}$ and she tried rays of varying hardness. In all cases irradiation of the animal caused an increase in tissue permeability, 40 per cent in normal tissue, 50 to 60 per cent in tumor tissue.

This latter result contradicts the conclusion of Waterman (382) that tumor cells and normal cells are affected by roentgen rays in an opposite manner. Waterman, however, compared rather acidic suspensions of isolated tumor cells with suspensions of lymphocytes. The ratio of the polarization resistance to the ohmic resistance (Waterman's 
" $P / W$ Konstant") was used as an index of permeability. The ray effect on this constant over a period of time undulates, but the immediate result is an increased "permeability" of lymphocytes and a decreased "permeability" of tumor cells.

Several applications of chemical methods of permeability determination remain to be considered. Kroetz (205) compared the rate of diffusion of chloride across normal frog skin with that across skin exposed to 10 to 30 H.E.D. In eight experiments, the average amount of $N / 100$ $\mathrm{AgNO}_{3}$ required to precipitate the chlorides that had crossed the membrane was $1.985 \mathrm{cc}$. for skin that had received $30 \mathrm{H}$.E.D., while the control value was 0.875 ce. Thus, in this series, irradiation caused a 198 per cent increase in penetration. Kovács $(198,199)$, using 4 H.E.D. in a frogskin preparation, could detect no increase in the rate of diffusion of hemoglobin.

Magath (248, 250), Magath and Kolomyetz (251), and Jalin (175) interpret data obtained with whole tissues as evidence for an increase of permeability in cell membranes upon roentgen irradiation. Earlier, Magath used the rate of swelling of normal and tumor tissue immersed in acidic buffer solutions as a measure of permeability. He gave normal and cancerous rabbits 5 to 6 H.E.D. The liver tissue of the normal irradiated animal swelled twice as fast as that of the control normal. Carcinoma tissue, however, showed a 20 per cent decrease in the rate of swelling when irradiated. Previous immersion in $\mathrm{CaCl}_{2}$ reversed both of these effects. The 1930 experiments, using phosphate production as a measure of permeability, gave identical relationships, although rat tissue was used, and a dose of $300 \mathrm{X}$. Jalin (175) found doses of $1 \mathrm{H}$ to accelerate the exosmosis of KI injected into rabbit muscle.

The most direct evidence for the permeability-increasing effect of roentgen rays comes from experiments on erythrocytes. Biologically, one of the most valuable of all the papers we shall consider is that of Lehmann and Wels (215) who, unfortunately, did not define their ray dose, but gave only tube voltage and amperage. After long irradiation, beef erythrocytes in dextrose were centrifuged off, and the exosmosed chloride determined in the solution. Irradiation caused an increase in the amount of diffusion of this ion from the cells. By the use of the hematocrit under the same conditions, they demonstrated that cell volume diminished more rapidly after irradiation than normally, further evidence of accelerated exosmosis. They also determined the total electrolyte diffusion by electrical-conductance measurements, and by comparing their results with the chloride determinations were able to show that both $\mathrm{NaCl}$ and $\mathrm{KCl}$ diffused out. Finally, they measured the rate of volume increase upon prolonged immersion of the cells in isotonic $\mathrm{NaCl}$; in one experiment, the average increase in the volume of the irradiated cells in $43 \mathrm{hr}$. was 49 per cent, the control value being 44.5 per cent. 
Koch (191) gave the blood of human patients $600 \mathrm{r}$ in vitro. Permeability changes were estimated by the hematocrit method and by chloride analysis. In most of the cases the results showed a permeability increase.

Saxe (322) measured the permeability to water of pig bladder upon irradiation with $3000 \mathrm{r}$. The bladder was stretched across the mouth of a simple osmometer. He obtained, in the one experiment mentioned, a considerable increase over the control in the rate of water penetration, but later work does not seem to confirm these results (122).

For plant cells there are data obtained by Williams (391) on her preparations of cells of Saxifraga umbrosa. Her method and the trend of her results are the same as in her radium experiments already considered. Roentgen rays caused increased permeability certainly to water and probably to other substances. As early as 1902 Seckt (340) had observed stomatal closure in cells of Tradescantia selloi under roentgen irradiation, which, by the criterion of Williams, is evidence of permeability increase.

On the whole, in spite of conflicting results, we should be inclined to believe that all types of radiation which affect living cells do cause a definite increase in the permeability of the plasma membrane. Nevertheless, in spite of the large number of papers already published, it is desirable that much more work be done in this field. The best methods have scarcely been used at all, and there is a dearth of certain and accurate information.

\section{SURFACE CHARGE}

Many writers have proposed changes in the electrical charges of protoplasmic colloids as the principal cause of the biological action of radiations $(33,63,233,235,262,400$, etc.). There is very little experimental evidence bearing on this view, which seems a reasonable inference from the well-known ionizing effect of all radiations. It must be pointed out that there is relatively little information of any sort concerning electrical charges either of cell surfaces or the surfaces of the cytoplasmic granules.

Young and Pingree (396) detected a retardation of the electrophoretic movement of cells of Sarcina flava, Sarcina rosea, and Bacillus prodigiosus suspended in glucose when the cells were exposed to the light of an incandescent bulb. Their results are not strictly quantitative, as the unequal distribution of the light through the circular cross section of the U-tubes used resulted in the formation of irregular migration surfaces.

A similar decrease in the negative charge of bacteria is reported by Lisse and Tittsler (239). These authors measured by the method of Falk the velocity of anodal migration of Escherichia coli suspended in water. Exposure to a 500-watt Mazda bulb caused a brief increase followed by a steady decrease in the rate of movement of the cells in the electrical field.

The effect of ultra-violet irradiation on the zeta potential of erythrocytes was measured by Falk and Reed (93). They irradiated dog blood 
in vivo through a quartz tube. The migration velocity in the experiment given changed from $12.4 \mu$ per sec. to $11.0 \mu$ per sec. after $1 \frac{1}{2} \mathrm{hr}$. exposure to the quartz mercury arc; the control remained practically constant.

Recently Lisse and Tittsler (240) have considered the action of the earbon arc (therapeutic B electrodes, 90 volts, $3 \mathrm{amp}$.) on the electrophoretic migration of cells of Escherichia coli suspended in water. They used both the method of Northrop and Kunitz and that of Falk, with some divergences in the results. When the Northrop-Kunitz chamber was used for the cataphoretic measurements, the authors observed with brief irradiation an increase in eataphoretic velocity; more severe treatment caused a consequent decrease. The first effect is described as "stimulating," the second as "lethal." One set of the results, in which the electrophoretic measurements were made within $10 \mathrm{hr}$. after irradiation, is reproduced from Table 10 of Lisse and Tittsler (see Table 3). Uncorrected values are given because of the great possibilities of experimental error in determining the correction factor from Smoluchowski's equation.

Table 3.-Effect of Carbon Arc on Migration Velocity of Escherichia coli (After Lisse and Tittsler, 200)

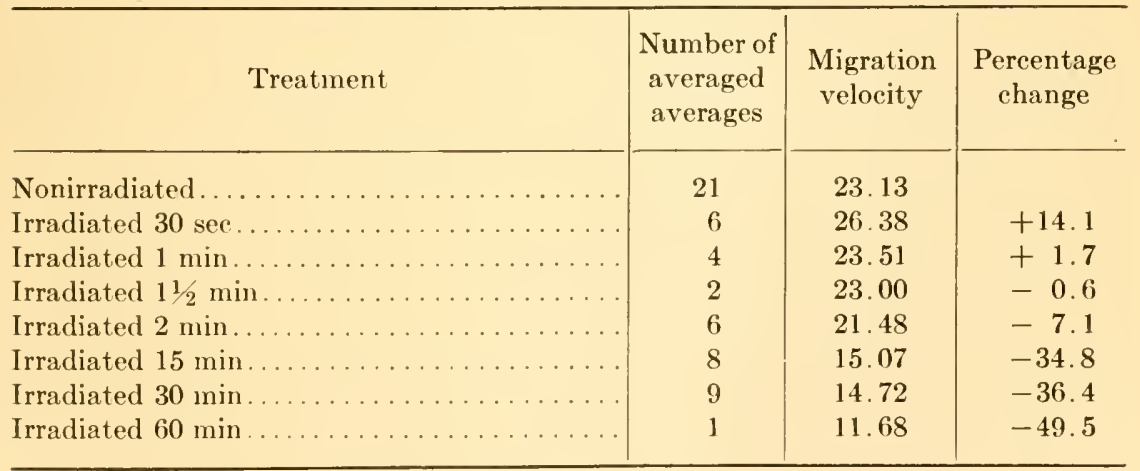

When the Falk capillary cell was used, other conditions being the same, the "stimulation" effect could not be demonstrated for shortirradiation treatments. There was a steady decrease in the charge. Lisse and Tittsler point out that the increase observed in the $\mathrm{pH}$ of the suspension might account to some extent for the change in migration velocity. They also note that the time elapsing between irradiation and the electrophoretic measurements is important but do not present complete irradiation experiments in which this factor is controlled.

There is evidence, therefore, from direct measurements of several workers, that visible light and ultra-violet rays cause a decrease in the surface charge of cells, bacteria in particular. There are no comparable data concerning the effects of roentgen rays and radium, except the recent report of Dozois, Tittsler, Lisse, and Davey (81). These workers 
exposed Escherichia coli to rays from a Coolidge tube at $30 \mathrm{kv}$., and 35 to 45 ma. With constant voltage, they express their doses in milliampere-minutes; these range from 245 to 3000 . The Falk capillary cell was used for electrophoretic measurements. They report totally negative results; no change in the charge on the cells. But the doses used also produced no effect on the viability of the organisms, as determined by direet counts.

Van Bonin and Bleidorn (31) attempted to determine the relation of roentgen-ray effects upon erythroeytes to their electrie eharge. They measured the effects of various concentrations of lanthanum ion, which is known to reduce the surface charge, upon the resistance of the cells to saponin hemolysis and osmotic hemolysis. Their figures show that lowering of the electric charge brings about proportional lowering of hemolytic resistance. But roentgen irradiation (1 to 8 erythema doses, filter $1 \mathrm{~mm}$. $\mathrm{Al}+0.5 \mathrm{~mm}$. $\mathrm{Zn}$ ) produced only a slight deerease in resistance to hypotonic solutions and no ehange in resistance to saponin. The authors conclude that roentgen rays do not act by lowering the surface charge of cells. The eontention is not proved unless the authors can show that their doses had some effect on the cells.

Straub and Gollwitzer-Meier (353, 354) disclaimed electrokinetic methods and attempted to deduce the relative surface charges of human blood cells in suspension from the irregularities of their $\mathrm{CO}_{2}$ dissociation curves. There seems to be no information coneerning radium effects upon surface charges of cells except these deductions. of Straub and Gollwitzer-Meier. The work of these authors and the experiment of Van Bonin and Bleidorn are obviously indirect. Dozois, Tittsler, Lisse, and Davey obtained negative results but used doses without any apparent biological effect. The study of this problem, of the effeet of roentgen and radium irradiation on electrical charges, presents a field virtually untouched.

A large literature has grown up around ehanges in the sedimentation velocity of blood corpuseles; this seems, if the law of Stokes holds, to depend on the degree of aggregation of the corpuscles and hence probably on their surface charge. This interpretation is maintained by Mond (262) who used ultra-violet rays and by Brummer $(49,50)$ experimenting with roentgen rays. Nevertheless, there is no consistency in the results. Some authors find an increase in the sedimentation veloeity, as Brummer, and Valeef (373), who used thorium $\mathrm{X}$ in vivo; Klein (1923) found that roentgen rays caused acceleration in some cases, retardation in others. Miculiez-Radecki (261) believes that the reaction depends on the interval between radiation and measurement. Mond (262) obtained both acceleration and retardation. Rubin and Glasser (319), after finding that the acceleration of sedimentation by roentgen rays was prevented by a filter which protected the suspensions from the heat of the apparatus, 
exposed the cells to heat comparable to that of the roentgen apparatus, and obtained the same acceleration of the sedimentation rate.

\section{EFFECTS ON PROTOPLASMIC COLLOIDS}

A living cell is more than a semipermeable membrane. Although cell physiologists so often discuss the osmotic membranes of cells and their changes in permeability, there can be no doubt that the interior protoplasm is at least equally important. It is commonly held that all types of stimulation cause an increased permeability of the plasma membrane, but very few authors have tried to explain how such an increase in permeability would affect the main mass of the protoplasm. According to some, electrical phenomena are involved, but the nature of such postulated electrical disturbances and how they affect the protoplasmic colloids are not at all clear. It might also be possible that with increased permeability, the ions surrounding the cell exert some particular action. No evidence for this latter view has been presented.

But whether or not it is possible to relate permeability change to a change in the interior, certain it is that the protoplasm itself is profoundly affected by stimulation in general and by radiation in particular. Radiologists have often sought to interpret protoplasmic effects in terms of the known action of rays on inanimate proteins. The fallacy of this type of argument has already been pointed out. It is certain, at least, that the colloidal properties of protoplasm are vastly different from those of any known protein, so that any attempt to interpret living substance in terms of protein must be made cautiously. The colloidal study of protoplasm is not easy. Microscopic observation alone is extremely uncertain, for complete coagulation of the cell contents is not necessarily accompanied by readily observable change. Not infrequently early authors who merely examined the general appearance of the protoplasm have mistaken liquefaction for coagulation and vice versa.

Fortunately, within recent years it has become possible to make reasonably accurate measurements of protoplasmic viscosity. These give indications of colloidal change. In some respects, however, viscosity studies of the living colloid are different from those of inanimate systems. In a test tube, coagulation is accompanied by a settling out of a precipitate. Within a tiny cell, no such settling out occurs. Either coagulation or gelation is indicated by a pronounced increase in protoplasmic viscosity. It is, however, not certain that the ordinary terminology of colloid chemistry is altogether appropriate for the living colloid. Protoplasm occurs in minute droplets; it is typically a suspension with 20 or 30 per cent or even more of suspended granular material. One can scarcely consider it in the same terms as a 1 per cent solution of egg albumen. But one can say very definitely that large changes in viscosity occur in the protoplasm, and that these are measurable. 
The measurement of protoplasmic viscosity has been discussed at considerable length by Heilbrunn (136). Two types of measurement are most in favor at the present time. With the aid of a centrifuge, protoplasmic granules are moved through the cell, and from the speed of this movement it is possible by Stokes's law to estimate the protoplasmic viscosity. It is a simple matter to compare the viscosity under various conditions. Absolute viscosity determinations are less easy and less certain. The rate of Brownian movement is also an index of protoplasmic viscosity. Both methods of viscosity determination have their serious limitations. In special cases, it may be possible that the viscosity measured by the two methods may be different. Thus, following vacuolization of a cell, granules within the vacuoles might show rapid Brownian movement, whereas centrifuge tests might indicate high viscosity. Finally, one may distinguish between the viscosity of the clear hyaline protoplasm and the viscosity of the protoplasm as a whole. The latter may be vastly greater, owing to the presence of a high concentration of granular material.

\section{VISIBLE LIGHT}

It is noteworthy that so few authors have studied the effect of visible light on the colloidal properties of protoplasm; especially is it surprising that no more work has been done on plant material. Using Ranunculus ficaria, Weber (387) compared the form and time of plasmolysis in cells of leaves exposed to darkness and light, respectively. The "light" leaves were in direct sunlight. From leaves in darkness plasmolysis is "convex" and rapid, whereas from those in the light it is "concave" and slow. Huber (166) also noted differences in the form of plasmolysis. Weber has shown that convex plasmolysis is associated with relatively low, and concave with high viscosity (Heilbrumn, 136); also that rapid plasmolysis is an indication of low viscosity. Hence he concludes that the viscosity is higher in the light than in darkness, but he is careful to note that the effect may be an indirect one and may be the secondary result of an action of light on other vital processes (e.g., transpiration or photosynthesis).

Gassul $(114,115)$ first showed that leucocytes and lymphocytes in spleen explants cytolyse more rapidly in the light than in the dark and accumulate lithium carmine stain at a greater rate. Similar studies by Earle (86) on leucocytes and fibroblasts in tissue culture are of considerable interest. He used tungsten filament lamps (15 to $200 \mathrm{w}$.), and studied the effect of these on the blood cells of cats, guinea pigs, and rabbits, as well as on fibroblasts obtained from chick embryos. When blood is illuminated, the leucocytes go through an interesting series of changes. At first there is a great decrease in viscosity, which is indicated by the "tremendously increased amplitude of Brownian movement of the 
granules within the cell." Various types of leucocytes differ, but in general liquefaction is followed by coagulation. This may involve disappearance of granules, and in fibroblasts there is a very striking vacuolization of the protoplasm. In most types of leucocytes the fat globules increase in size and number. All these phenomena are the very ones described by other authors as characteristic effects of ultra-violet or roentgen radiation. Unfortunately, however, Earle could not be certain that the appearances he noted in leucocytes and fibroblasts were really due to a direct action of the light on the cells in question. In the case of both leucocytes and fibroblasts, the presence of erythrocytes seemed to play an important role. The red blood cells are affected by the light more readily than the leucocytes, and it is quite possible that substances released from them are in large measure responsible for the effects observed in the other types of cells. Red blood cells are hemolyzed by light rays, an effect which seems to be due in large measure to an action on hemoglobin (225).

If the changes in leucocytes produced by illumination of blood are due to degenerative substances emerging from injured erythrocytes, this fact may be correlated with the theory that the action of roentgen rays is in large measure due to injury substances, or necrohormones (cf. Caspari, 55).

\section{ULTRA-VIOLET RAYS}

In interpreting the action of ultra-violet rays on protoplasm, various authors have stressed the fact that such rays may cause a coagulation of proteins. The effect of radiation on proteins is discussed in another section of this book (cf. Clark, pages 303-322). As pointed out previously, the "living colloid" is a much more complicated system than a protein solution. We shall here confine our attention to living protoplasm.

Virtually none of the authors whom we shall consider has reported the intensity used. Indeed, for the type of experiment usually performed this does not seem entirely possible at present; the International Conmittee for the Measurement of Ultra-violet Light has recently decided that no physical method of measurement that has yet been proposed will permit accurate comparison of doses from different lamps (see British Journal of Radiology $7: 119$. 1934).

Working on the theory that ultra-violet rays produced an effect by coagulating protein and that this effect varied with the $\mathrm{pH}$ (cf. Clark, 63), O'Donnell (278) studied the response of fish chromatophores to ultra-violet rays when the cells were bathed in solutions of varying $\mathrm{pH}$. She could detect no difference in one solution as compared with another, and hence could find no support for her simple theory. 
Cernovodeanu and Henri $(57,58)$ studied irradiated bacteria, protozoa, and leucocytes ultramicroscopically. They found a brilliant granular appearance which they considered identical with that of albumin coagulated with ultra-violet. The use of the ultramicroscope for the colloidal study of protoplasm is rather unsatisfactory (cf. Heilbrunn, 136, pages 38 to 39), so that the results of Cernovodeanu and Henri are not very convincing. Addoms (1) likewise employed the ultramicroscope to examine the protoplasm of wheat seedlings following irradiation with a mercury-vapor lamp. She also concluded that coagulation occurred.

In the course of some embryological studies, Schleip (330), among others, described effects on the protoplasm of Ascaris eggs which indicate a sharp increase in viscosity following irradiation with $\lambda 2800 \AA$ from a magnesium arc. Ruppert irradiated centrifuged eggs and determined the time necessary for the return of the granules which had been moved to one side of the cell. The time necessary for this return is much longer than the time required in the case of normal untreated eggs. Inasmuch as the return is almost certainly due to Brownian movement, there is rather clear evidence of an increase in viscosity.

Gibbs (118) used a mercury-vapor arc ( $\lambda 3126$ to $2378 \AA$ ) on Spirogyra cells and found that the rays caused first a liquefaction and then a coagulation of the protoplasm. He used the centrifuge method, which is apparently applicable to these cells (Heilbrunn, 136, pages 47 to 48). The results are clear-cut, although not expressed in quantitative units. Table 4 taken from Gibbs shows the decrease in protoplasmic viscosity caused by relatively short exposures.

Table 4.-Effect of Últra-violet Light on Spirogrra Protoplasm (Gibbs, 118)

\begin{tabular}{|c|c|c|c|}
\hline $\begin{array}{l}\text { Time of centri- } \\
\text { fuging, sec. }\end{array}$ & $\begin{array}{l}\text { Cells exposed to } \\
\text { unscreened arc for } \\
30 \mathrm{~min} .\end{array}$ & $\begin{array}{l}\text { Cells exposed to } \\
\text { are sereened with } \\
\text { "Noviol O" glass }\end{array}$ & Cells not exposed \\
\hline 45 & Slight displacement & Normal & Normal \\
\hline 60 & $\begin{array}{l}\text { Marked displacement } \\
\text { in some cells }\end{array}$ & Normal & Practically normal \\
\hline 75 & Marked displacement & $\begin{array}{l}\text { Very slight dis- } \\
\text { placement }\end{array}$ & $\begin{array}{l}\text { Very slight dis- } \\
\text { placement }\end{array}$ \\
\hline
\end{tabular}

Results somewhat similar to those of Gibbs were obtained by Heilbrunn and Young (138) for Arbacia eggs. In their experiments, however, they treated the cells first and then noted the viscosity at varying times after exposure. The centrifuge method was used, and the eggs in a thin layer were exposed to the radiation for $1 \mathrm{~min}$. Two minutes later, the protoplasmic viscosity was found to have dropped to about two-thirds of its original value. Then, after $15 \mathrm{~min}$., it rose to 3 or 4 
times its original value. The increase in viscosity begins at the cortex and travels inward. The values given above apply only to the central region of the cell.

Sea-urchin eggs respond to ultra-violet radiation by lifting off their outer membrane in a reaction characteristic of the fertilization process $(236,237)$. However, such a response does not occur (138) if the calcium is first removed by the addition of the oxalate ion. It has been found (11) that muscle does not show a contracture response upon stimulation with ultra-violet unless calcium ions are present. Apparently, therefore, the calcium ion plays a role in the effect of ultra-violet on protoplasm.

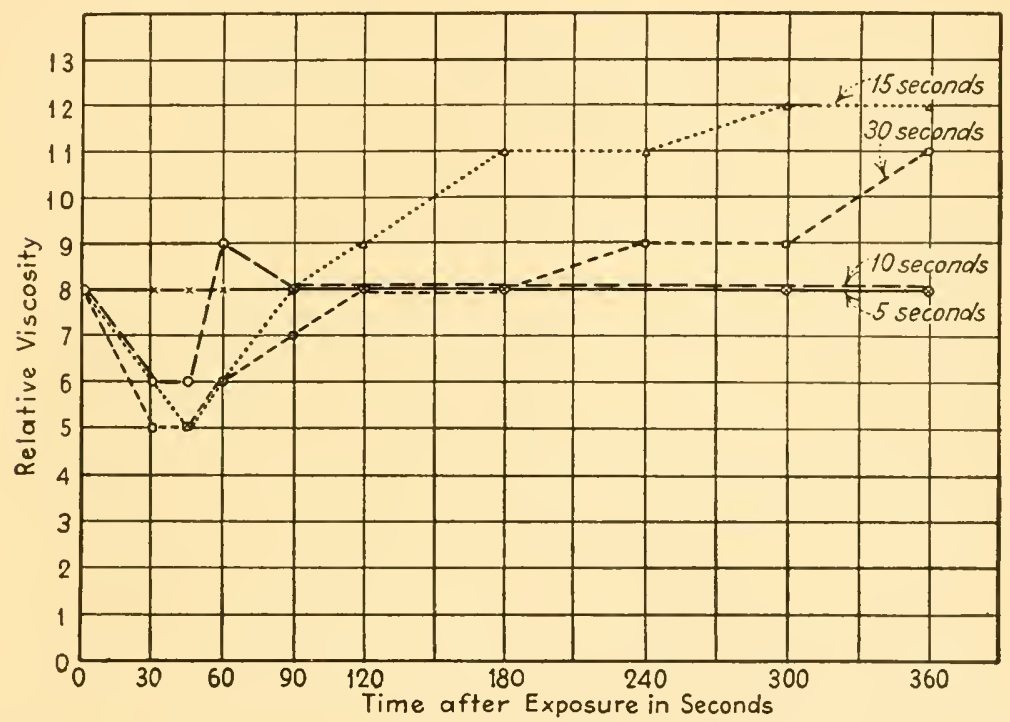

FIG. 2.-Viscosity of the plasmasol of Amoeba dubin at varying times after ultra-violet irradiation. (After Heilbrunn and Daugherty, 137.)

This importance of calcium is more clearly indicated by the work of Heilbrunn and Daugherty (137) on Amoeba. These authors irradiated amocbae with a Hanovia mercury-vapor lamp. By using two species of amoebae, they were able to determine viscosity change both in the main mass of the protoplasm and also in the outer gelatinous envelope or plasmagel. The main mass of the protoplasm (plasmasol) is first made more fluid and then more viscous by ultra-violet. The effect of various doses is shown in Fig. 2. An exposure of 5 sec. produced no change, while 10 sec. caused a transient liquefaction. Longer exposures caused liquefaction followed by a pronounced stiffening of the protoplasm. The fluid stage lasted only 1 to 2 min. The gelating effect of ultra-violet could be completely inhibited by immersion in sodium or ammonium oxalate solution. In the oxalate the viscosity increases somewhat 
(owing to the replacement of calcium by sodium ion), but there is no further increase upon radiation. Apparently, therefore, the observed gelation is dependent on the presence of the free calcium ion.

Whereas the interior protoplasm or plasmasol is gelled by ultra-violet, the outer cortex becomes more fluid. As far as can be judged from centrifuge tests (which in the case of an outer ring of material of variable width are not very accurate) the viscosity of the plasmagel decreases to about one-fourth of its original value. This liquefaction of the plasmagel is a characteristic effect produced by all sorts of stimulating agents. The same effect can be produced by the withdrawal of the calcium ion.

Heilbrunn and Daugherty therefore propose the theory that the primary effect of irradiation is the release of bound calcium from the cell cortex. The free calcium ion then enters the protoplasm proper, causing first liquefaction and then gelation. Such indeed is the known action of calcium ion on protoplasm, for a small addition of calcium ion produces liquefaction, whereas larger amounts of free calcium cause a peculiar coagulative reaction (136).

The theory of Heilbrunn and Daugherty is supported by the fact that irradiation actually does cause a release of calcium both from living cells and from proteins. Thus Nadson and Rochline-Gleichgewicht (269) report the formation of calcium oxalate in irradiated plant cells, and Clark (63) claims that ultra-violet releases calcium from combination with the proteins of the blood. Finally the recent work of Anslow and Foster (7) and of Anslow, Foster, and Klingler (8) shows clearly how ultra-violet radiation would act on the amino acid constituents of proteins so as to release bound cation from combination. These authors find that the rays break the carboxyl bond of the amino acid.

The relation of calcium ion to the colloidal chemistry of protoplasm is extremely interesting. This ion apparently bears the same relation to the protoplasmic colloid that it does to the blood. The coagulation of protoplasm is remarkably similar to the clotting of blood (136), and it is only reasonable to assume that this would be true, for cells in general are now known to contain all the materials involved in blood clotting $(100,200,201)$.

When a cell is torn, the exuding protoplasm forms a film about itself. Thus it clots just as blood does when it pours from a vessel. The reaction involved is termed the surface precipitation reaction and takes place only in the presence of free calcium. When a cell like a sea-urchin egg is torn or broken, not only does a film form at the edge of the emerging droplet, but numerous other films form within the droplet. Thus vacuoles are produced, and these vacuoles may occupy a part or all of the exuded drop or they may fill the entire cell. But neither the membrane at the surface of the emerging drop nor the vacuoles can appear in the absence of calcium. Hence calcium is involved in a species of reaction 
within the cell, the most characteristic feature of which is the appearance of numerous small vacuoles.

It is interesting to point out that ultra-violet radiation does cause a vacuolization reaction in diverse types of living systems, and as a matter of fact, vacuolization has eonstantly been described following all types of radiation. An early observation is that of Ogneff (279). Upon exposing experimental animals to a powerful earbon arc and studying the eyes cytologically, he noticed various necrotic changes in the cells of the corneal epithelium, chief among these being the gradual filling of the cell with small vacuoles ("Kügelchen") which seemed to appear first near the nucleus. (These effects may, however, have been due to infra-red, 156, 377.) Schulze (337) irradiated Rhoeo (=Tradescantia) hair cells, using $\lambda 2800 \AA$. He describes as the first and most pronounced effect of the radiation the appearance of many small vacuoles in the cytoplasm. As irradiation continued, these increased in size and number.

Bovie (34, page 14) has described vacuolization in amoeba protoplasm. Thus he says that following treatment of an amoeba with Schumann rays, "Under a high magnification (2200 diameters) the protoplasm was seen to be filled with fine vacuoles which were so numerous that it was converted into a fine froth."

The protoplasm of sea-urchin eggs is especially interesting because these cells have been used more than any others in physieal and colloidal studies of living substance. Upon excessive stimulation, the protoplasm of Arbacia eggs undergoes a violent transformation. Red pigment granules disappear, and numerous vacuoles fill the cell. Typically there is a marked increase in cell volume. The reaction is identical with that which oceurs when a cell is violently torn or broken (see above). The racuolization of sea-urehin eggs was noted by early workers, but its importance was first emphasized by Loeb (241) who referred to it as cytolysis. Ultra-violet rays cause a very complete cytolysis of seaurchin eggs $(133,236,356)$.

A characteristic type of cytolysis in Paramecium has been described by Hertel (142) in an interesting early paper. Other deseriptions of eytolysis in Paramecium include Thomaschewski (359), Cernovodeanu and Henri (57), Bovie (33, 34), Burge (53), Bovie and Daland (38), Rentschler (313), and Troisi (360). These different authors stressed various morphologieal appearanees, but the pieture as a whole is quite consistent. Hertel noted especially the lifting off of the pellicle here and there on the surface of the animal and its frequent rupture. This may be related to the liquefaction of the cortical protoplasm of Amocba, as described by Heilbrunn and Daugherty (137).

In review, it may be stated with some degree of confidence that ultra-violet rays cause first a liquefaction of the main mass of the protoplasm and then a pronounced stiffening. The liquefaction of the cell 
interior may be transitory, so that one is likely to overlook it unless viscosity determinations are made immediately after the rays begin to have effect. In the cortex of Amoeba, the rays cause a liquefaction which persists. This liquefaction of the cortex probably also involves a weakening of the very outer surface of the cell; hence the frequent descriptions, in the literature, of cell-membrane rupture following irradiation. The liquefaction of the cortex is apparently due to a release of calcium. In view of the fact that calcium is apparently the ion most important in preserving the semipermeability of cell membranes (cf. Lucké and McCutcheon, 244) and may very well enter into the substance of the plasma membrane, it is easy to understand why ultra-violet radiation (and indeed radiation in general) should increase the permeability of the cell.

If calcium is released from the cortex-and there are various indications in favor of this view-it would diffuse into the cell interior and cause first liquefaction, and then if enough of it were present, coagulation. Excess of free calcium in the cell interior causes the vacuolization reaction so common in all types of protoplasm (Heilbrunn, 136). This vacuolization reaction, as noted above, may be compared to the clotting of blood.

Thus we have a mechanism of extreme delicacy, one that can account for the radical colloidal changes observed, without recourse either to the insensitive process of ordinary coagulation or to a purely hypothetical mechanism such as special enzyme effects or point-heat effects. This reaction accounts for (though it does not explain) the morphological concomitants of radiation effects, and for the fact that the stimulating and injurious effects of rays in protoplasm are essentially the same as the effects of other effective physical and chemical agents. Presumably the protoplasmic clotting, like the blood clotting, involves complicated phenomena, and apparently thrombin-like substances are produced. Just as when blood clots, it produces substances which affect unaltered blood, so when the protoplasm of one cell clots, it may give off substances which affect neighboring cells. It is thus possible to interpret the necrohormone hypothesis which is popular with many radiologists (Caspari, 55).

\section{ROENTGEN RAYS AND RADYUM}

As far as present-day information goes, the effects of roentgen rays and of radium radiation on the colloids of the cell are in all respects essentially the same as those described for ultra-violet. Exactly the same cycle of viscosity change appears, and the coagulated protoplasm presents the same morphological appearance. Beyond much doubt the fundamental mechanism is identical. Indeed the more one studies the colloid chemistry of protoplasm, the more apparent it becomes that the protoplasmic colloid is so constituted as to be sensitive to various 
types of external agents and to respond to many diverse treatments in much the same manner.

In general, most authors are agreed that roentgen rays and radium radiation cause first a decrease in viscosity and then, on longer exposure, a sharp increase in viscosity or coagulation. As early as 1897, Lopriore found that roentgen rays caused an increase in the rate of protoplasmic streaming in leaf cells of Vallisneria spiralis. This was when the leaves were exposed for half an hour. After an hour's exposure, streaming stopped and the protoplasm became yellow, granular, and coarsely vacuolar. Seckt (340) found similar effects of roentgen rays on protoplasmic streaming in various plant cells, and the same type of phenomenon has also been described for two species of Amoeba (Schaudinn, 328).

Using radium, Zuelzer and Philipp (401), and more recently Petchenko (286), also described changes in the rate of amoeboid movement. The former authors used gamma and hard beta rays from preparations containing 40, 26, 15, and $13 \mathrm{mg}$. of radium, upon Amoeba diploidea, the latter studied Amoeba vahlkampfia with 5 and $10 \mathrm{mg}$. $\mathrm{RaBr}_{2}$. These studies agree; there is first a pronounced acceleration of the rate of streaming, indicating, according to Zuelzer and Philipp, a decreased viscosity. With prolonged irradiation, movement becomes much slower than the normal, the amoebae round up and become full of small vacuoles. These experiments indicate an initial decrease in viscosity followed by a sharp increase, but it must be remembered that the rate of protoplasmic streaming or of amoeboid movement is an uncertain index of protoplasmic viscosity. For the speed of movement depends not only on the riscosity but also on the unknown forces which propel the protoplasm.

In relatively recent years, more certain methods of viscosity determination have been used. Weber (386) radiated Spirogyra cells with 170 Holzknecht units but found no effect on viscosity when tested by the centrifuge method. After $20 \mathrm{hr}$., it is true, there was a viscosity increase, but this Weber interpreted as a secondary effect. Weber also radiated Phaseolus seedlings with 24 Holzknecht units, but he could detect no viscosity change. Doubtless the dosage used by Weber was too low for the types of protoplasm he studied.

Williams (1923) was able to find a very definite effect on the protoplasm of the stalk cells of Saxifraga umbrosa. Short exposures to either roentgen rays or to radium beta or gamma rays caused an increase in the rate of protoplasmic streaming and also an increase in the rate of Brownian movement of small particles in the protoplasm. Longer exposures caused slowing and stoppage of streaming. Following such long exposures there was extensive vacuolization.

Nakashima (272) irradiated isolated cells from the salivary glands of the snail Limnaea (40 kv., $10 \mathrm{ma} ., 6 \mathrm{hr} ., 30 \mathrm{~cm}$.) and observed an acceleration of Brownian movement in the protoplasmic granules. But 
he also observed a diminution of granular size and to this, rather than to lowered viscosity, he attributes the change in Brownian movement. With A moeba rostock, he likewise found increased Brownian movement.

Forbes and Thacher (102) treated the eggs of an annelid, Nereis, with beta rays of radium. They then fertilized the eggs and centrifuged them. Irradiated eggs showed somewhat less viscosity than controls. It may be noted that the centrifuge method is an excellent check on the Brownian movement method of determining viscosity, and vice versa, for if protoplasmic granules decrease in diameter, their Brownian movement would be accelerated, whereas their movement under the influence of centrifugal force would be greatly retarded.
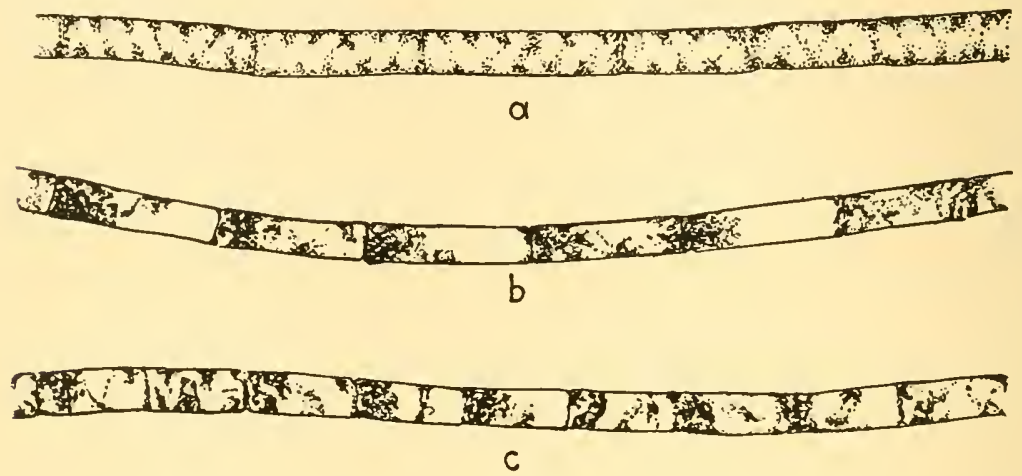

FIG. 3.-The effect of mild radium treatment on the viscosity of cells of Spirogyra varians. (After Feichtinger, 197.) (a) Control cells. No displacement of chromatophores by centrifuging. (b) Cells exposed to beta and gamma rays from $\mathrm{RaBr}_{2}$ preparation of $4 \mathrm{mg}$. radium equivalents. Chromatophores almost completely displaced by same centrifuge treatment as used on control (3a). (c) Cells treated with alpha rays from polonium preparation of $0.33 \mathrm{mg}$. radium equivalents. Same time of centrifuging as in $3 a, b$.

\section{Rochlin-Gleichgewicht (317) exposed cells of Elodea and Ptery-} gophyllum to radium emanation and found first an increase in rate of streaming and then a decrease. The results were interpreted as indicating first a decrease of protoplasmic viscosity, and then after longer or stronger dosage, an increase. The work of Rochlin-Gleichgewicht is of considerable interest and will be discussed further below.

Feichtinger (97) centrifuged Spirogyra cells following exposure to alpha, beta, and gamma rays. A number of polonium preparations were used as a source of alpha rays; an amount of $\mathrm{RaBr}_{2}$ considered equivalent in activity to $4 \mathrm{mg}$. of element was used in experiments with beta and gamma rays. Weak radiation caused a liquefaction of protoplasm, further exposure an increase in viscosity, and finally a coagulation (compare Figs. 3 and 4); Feichtinger used two species of Spirogyra. In the smaller, the alpha particles penetrated the whole cell, whereas in the larger species they penetrated only one-fifth of the diameter. In these larger cells, the effect seems to be propagated, for the result is 
uniform throughout the cell. However, in the application of the centrifuge method to Spirogyra cells, it must be remembered that the movement of chromatophores presumably depends on the viscosity of the layer of protoplasm directly adjacent to the cell wall (cf. Heilbrunn, 136, pages $47,48)$.

From the above, it may be concluded that although the number of workers is not great, their results are thoroughly consistent. The effect of radiation whether of roentgen rays, alpha, beta, or gamma rays, is to cause first a liquefaction, then a stiffening or coagulation. This is evidenced by the data on rate of protoplasmic streaming and more certainly by direct study of viscosity with either Brownian movement or centrifuge methods.
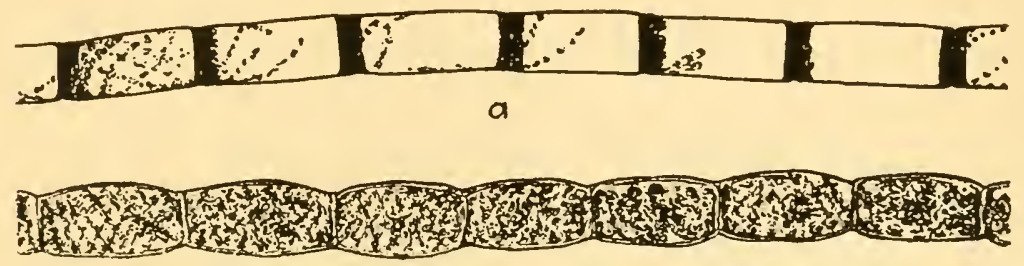

b

FIG. 4.-The effect of severe radium treatment on the viscosity of cells of Spirogyra clipsispora. (a) Control cells. Chromatophores displaced by centrifuging. (b) Cells exposed to beta and gamma rays from $\mathrm{RaBr}_{2}$ preparation of $50 \mathrm{mg}$. radium equivalents. Chromatophores not displaced by same centrifuge treatment as used on controls.

The final effect of radiation is to cause a coagulation. All authors are agreed on this except Jansson (177) who found increased Brownian movement in leucocytes following death. It is possible that Jansson may have observed movement of particles in vacuoles, and it should also be noted that an increase in the rolume of the cell such as Jansson observed would favor increased amplitude of Brownian movement.

The coagulation produced by roentgen rays or radium resembles that produced by ultra-violet rays, in that numerous vacuoles are typically produced. Numerous authors have described vacuolization phenomena. These include Nadson and Rochlin (269) on onion scale cells, Jansson (177) on myelocytes, Schubert (336) on chick-heart cells in tissue cultures, Johnson (180) and Komura (193) on plant cells, also Rochlin-Gleichgewicht on plant material, Gassoul $(112,113)$ on explants of frog spleen, etc. For other references see Table 5 .

It seems certain that roentgen rays and radium act in the same manner as ultra-violet rays (cf. Nadson and Stern, 271), and it is only logical to assume that any explanation which holds good for ultra-violet would also apply to these other types of radiation.

In this connection we should like to emphasize the interesting work of Rochlin-Gleichgewicht on the leaf cells of Elodea and Pterygophyllum. In this work a fine emanation needle was placed transversely across the 
cells so that different regions were at varying distances from the source. Exposures of $1 \mathrm{mc}$. and higher showed the following picture: Directly beneath the needle was a zone of necrosis in which cells were vacuolated and apparently coagulated and in which calcium oxalate crystals could be clearly distinguished. Beyond this, calcium oxalate crystals also appeared in a so-called transition zone in which the protoplasm was swollen and streaming movement had stopped. Farther still from the needle was a zone in which the protoplasm looked normal but streaming was accelerated. Beyond this the cells were normal. With lower doses $(0.08$ to $0.3 \mathrm{mc}$.), only the region beneath the needle is affected and this shows increased streaming. All the results were ascribed to the softer beta rays. Rochlin-Gleichgewicht attempts to interpret the results in terms of conventional colloid chemistry. The first effect of radiation is to increase the degree of dispersion and thus the protoplasmic viscosity is lowered. Then there is an increased swelling of cytoplasmic colloids which leads to an increase in viscosity; at this stage calcium oxalate crystals appear. Finally denaturation leads to dehydration, and this to vacuolization. To us this formal explanation does not seem so satisfactory as the scheme outlined in the last section to explain the effects of ultra-violet radiation. The results of Rochlin-Gleichgewicht fit in beautifully with this scheme.

It has been shown that roentgen rays and radium, like ultra-violet, commonly produce a vacuolization of the protoplasm. Such a vacuolization reaction is found not only after radiation but occurs in all types of protoplasm following the most diverse treatments; it is perhaps the most distinctive characteristic of living substance. The importance of the reaction was thoroughly emphasized by Heilbrunn (136) who regards it as a speeific colloid chemical reaction peculiar to living systems. The relation of the reaction to calcium and the surface precipitation reaction has already been pointed out. In Arbacia (sea urchin) egg protoplasm, both vacuolization and surface precipitation reactions are preceded by a breakdown of granular elements, and this breakdown is apparently related causally to both types of reaction. In particular, the red pigment granules of the Arbacia egg disappear. These red granules show all the reactions of mitochondria and may be considered as such. It is not surprising therefore to note that various cytologists have found that radiation has a destructive effect on mitochondria and other formed elements of the cytoplasm (cf. 168, 270, 379, 380). Such a breakdown of mitochondria, granules, etc., may well represent a first stage in the clotting or vacuolization process.

The vacuolization reaction as it oceurs in many types of protoplasm is but little understood. Relatively few workers have examined it experimentally. Doubtless it is a very complicated reaction, or series of reactions, and presumably, in its early stages at least, it involves counter- 
reactions which tend to bring the protoplasm back to normal. Perhaps, too, various types of vacuoles may be formed.

In the discussion of the effects of ultra-violet radiation, evidence was presented to show that the initial effect of the ultra-violet rays was to cause a release of calcium from the cortex of the cell. Students of roentgen rays and radium have demonstrated a release of calcium from the cell by chemical means. The work of Rochlin-Gleichgewicht (317) has already been noted. Adler and Wiederhold (3) find that following exposure to roentgen rays ( 1000 to $5000 \mathrm{r}$ ), the calcium content of the skin of rabbits may decrease as much as 57 per cent. Apparently there is an escape from other tissues as well as the skin, and Benassi (21) found an increase in blood calcium after exposure of rabbits to X-rays (cf. 6, 323). However, Kroetz (203) and Adler and Wiederhold (3) find a decrease in serum calcium following irradiation. The divergence is doubtless due to the fact that following X-ray treatment there is a huge increase in calcium eliminated from the body (K. and M. Adler, 2a). These authors conclude that the tissues must give off calcium following irradiation. That this release is from the cell cortex is indicated by the fact that there is apparently an increase in permeability following treatment with roentgen rays or radium (see section of this paper on Permeability), and also by the fact that following radiation, there is a general weakening of the outer membrane of the cell. Various authors describe rupture of the cell membrane following treatment with roentgen rays or radium, in Pelomyxa (399) and in yeast cells, both following radium treatment (266), and in bacteria after exposure to X-rays (181). As in the case of ultra-violet rays, we assume this weakening of the cortex or membrane of the cell to be associated with a loss of calcium, although other factors, such as an accumulation of fluid beneath the membrane, or a general increase in cell volume (Nadson, 265, 266) may also play a role.

In our attempt to find a general scheme which will explain the effects of all types of radiation on protoplasm, we have examined the literature from one angle rather than from all sides. We have also presented our theory as though it covered all the facts. This it can scarcely do. Protoplasm is extremely complex, both from a chemical and from a colloid chemical standpoint. The effects of radiation cannot be fitted into too simple a picture.

One of the most interesting observations we have not as yet considered is that of Nadson (266). He found that radium caused an increase in the number and size of fat droplets in exposed yeast cells. The same changes are produced by roentgen rays and by ultra-violet (Nadson and Stern, 271). Rochlin-Gleichgewicht also states that the final effect of radium emanation on cells of Elodea and Pterygophyllum is to cause a breakdown of protoplasmic fat protein combinations into 
fat and protein. Petchenko (286) also finds an increase in fat in Amoeba vahlkampfia after prolonged treatment with 5 or $10 \mathrm{mg}$. $\mathrm{RaBr}_{2}$. The fat was demonstrated in fixed preparations.

Some authors have attempted to show that the $\mathrm{pH}$ of the cell changes following irradiation. Hoffmann (153) found that the eiliate Opalina when stained with neutral red seemed to become more alkaline after treatment with roentgen rays. A similar observation had been made by Tschachotin (364) for sea-urchin eggs, and for frog and bird erythroeytes exposed to ultra-violet radiation. Lisse and Tittsler (240) also found an increase in the $\mathrm{pH}$ of bacterial suspensions after exposure to ultraviolet. Liechti (234) measured potentiometrically the subcutaneous $\mathrm{pH}$ of rabbits treated with roentgen rays (1.5 to 4.0 H.E.D.). He found a slight decrease in $\mathrm{pH}$, and he also obtained the same result with a human patient given 1 H.E.D. Magath (249) likewise found a decrease in tissue $\mathrm{pH}$ following exposure to roentgen rays. He studied the effect of 4 to 5 H.E.D. on chick embryos and mouse tumors, using both the quinhydrone electrode and colorimetric tests. When the chick embryos were treated in ovo, the skin mesenchyme and muscle showed a decrease in $\mathrm{pH}$, but not the liver or brain. Neither was there any effect on mouse liver or mouse cancer. All in all, the effect of radiation on cellular $\mathrm{pH}$ is not well established, so that no certain conclusion can be drawn from the statements in the literature.

\section{CONCLUSION}

It searcely seems possible to summarize all the results we have considered in any brief final statement. The following points have been selected because they seem to be reasonably certain and because they fit into an orderly seheme.

a. All types of radiation have the same general effect.

$b$. In spite of the uncertainty of permeability determinations and in spite of the fact that the more modern and the preferable methods of permeability study have been but little used in investigating the effect of radiation, the preponderance of opinion is rather strongly in favor of the view that radiation causes a substantial increase in permeability. This increase is caused by all types of radiation from visible light to gamma rays.

$c$. There is essential agreement among all those who have attempted a study of protoplasmic viscosity of irradiated cells. In both plant and animal cells, ultra-violet radiation, roentgen rays, and radium all cause first a liquefaction of the main mass of the protoplasm and then, with increase in exposure, a coagulation.

$d$. The coagulation produced by radiation is frequently evidenced morphologically by the appearance of vacuoles within the cell. Numerous authors have described vacuolization. Table 5 has been prepared 
to illustrate this point. The significance of the vacuolization reaction, its similarity to blood clotting, and its relation to calcium are discussed in the main body of this paper.

Table 5.-A Partial List of Descriptions of Vacuolization of Cells AFTER IRradiation

\begin{tabular}{|c|c|c|c|c|}
\hline Material & Ray & $\begin{array}{l}\text { Condi- } \\
\text { tion }\end{array}$ & Author & Date \\
\hline Saccharomyces. & Radium & Living & Nadson & 1922,1925 \\
\hline Saccharomyces. & Ultra-violet & Living & Nadson and Stern & 1931 \\
\hline Yeast cells.... & Radium & Living & Kotzareff and Chodat & 1923 \\
\hline Fusarium..... & Ultra-violet & Fixed & Bailey & 1932 \\
\hline Allium cepa.. & Roentgen & Living & $\begin{array}{l}\text { Nadson and Rochlin- } \\
\text { Gleichgewicht }\end{array}$ & 1926,1933 \\
\hline 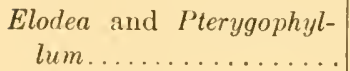 & Radium & Living & Rochlin-Gleichgen & 1930 \\
\hline Tradescantia............ & Ultra-violet & Living & Schulze & 1909 \\
\hline Vallisneria........... & Roentgen & Living & Lopriore & 1897 \\
\hline Saxifraga...... & Roentgen & Living & Williams & 1923 \\
\hline Saxifraga............ & Radium & Living & Williams & 1925 \\
\hline Crepis virens.......... & Radium & Fixed & Feichtinger & 1930 \\
\hline Vicia Faba... & Roentgen & Fixed & Komuro & 1925 \\
\hline 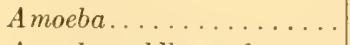 & Schumann & Living & Bovie & 1915 \\
\hline A moeba vahlkampfia... & Radium & Living & Petchenko & 1926 \\
\hline Colpidium.......... & Radium & Fixed & Meldolesi and Reverberi & 1930 \\
\hline Euplotes.... & Roentgen & Fixed & $\begin{array}{l}\text { Brown, Luck, Sheets, } \\
\text { and Taylor }\end{array}$ & 1933 \\
\hline A moeba diploidea..... & Radium & Living & Zuelzer and Philipp & 1924 \\
\hline Paramecium.... & Radium & Living & Zuelzer & 1905 \\
\hline Asterias egg.... & Ultra-violet & Living & Lillie and Baskervill & 1922 \\
\hline Arbacia egg... & Ultra-violet & Living & Heilbrunn and Young & 1930 \\
\hline Leucocytes.... . & Ultra-violet & Living & Eidenow & 1930 \\
\hline Lymphocytes..... & Roentgen & Fixed & Belot, Nahen, Cailliau & 1925 \\
\hline Myelocytes..... & Roentgen & Living & Jansson & 1927 \\
\hline Carcinoma..... & Radium & Fixed & Alter & 1919,1920 \\
\hline Nerve cells & Radium & & & \\
\hline Chick-heart explant. & $\begin{array}{l}\text { emanation } \\
\text { Roentgen }\end{array}$ & $\begin{array}{l}\text { Fixed } \\
\text { Living }\end{array}$ & $\begin{array}{l}\text { Knaffl-Lenz } \\
\text { Schubert }\end{array}$ & $\begin{array}{l}1912,1913 \\
1927\end{array}$ \\
\hline Frog-spleen explant. . & Roentgen & Living & Gassul & 1928 \\
\hline Chick-eye explant... & Roentgen & Living & Cox & 1931 \\
\hline Choroid of eye..... & Ultra-violet & Fixed & Ogneff & 1896 \\
\hline Chick fibroblasts.... & Visible & Living & Earle & 1928 \\
\hline Rat testis........... & Roentgen & Fixed & Adler & 1932 \\
\hline Chick heart in culture. & Radium & Living & Bisceglie and Bucciardi & 1929 \\
\hline Suprarenal gland...... & Roentgen & Fixed & Celotti & 1931 \\
\hline
\end{tabular}

e. The results on Amoeba indicate that ultra-violet radiation causes a liquefaction of the outer cortex or plasmagel with an apparent release of calcium. It is believed that the calcium released from the plasmagel diffuses to the interior of the cell and causes the internal liquefaction and 
ultimate coagulation of the main mass of the protoplasm. Experimental evidence in favor of this view is presented.

$f$. There is reason for believing that the plasma membrane of the cell is, in part at least, a calcium gel. Release of calcium would presumably liquefy or weaken such a gel. Hence it is understandable that radiation should break down the cell membrane. Moreover, in view of the fact that calcium, more than any other ion, is responsible for the semipermeability of the membrane, it is readily conceivable that release of calcium from the cortex of the cell or from the membrane might cause the permeability increase noted above.

g. The points brought out above are in accord with those observations which show that calcium is set free from plant and animal tissues after irradiation with ultra-violet, radium, or roentgen ray. A similar effect has been described for proteins and it may be interpreted in terms of a known action of rays on the carboxyl bond of amino acids.

\section{REFERENCES}

1. Addoms, RUTH M. Toxicity as evidenced by changes in the protoplasmic structure of root hairs of wheat. Amer. Jour. Bot. 14:147-166. 1927.

2. AdLer, K. Die biologische Wirkung der kurzwelligen Strahlen auf den Stoffwechsel der Zelle. Strahlentherapie 36:1-31. 1930.

2a. Adler, K., and M. Adler. Kalcium- und Kaliumstoffwechsel unter dem Einfluss der Röntgenstrahlen bei Kaninchen; Ausscheidung. Strahlentherapie $44: 481-488.1932$.

3. Adler, K., and O. Wiederhold. Kalcium- und Kaliumstoffwechsel unter dem Einfluss der Röntgenstrahlen bei Kaninchen I. Blutserum und Haut. Strahlentherapie $44: 383-392.1932$.

4. Alter, N. M. Histological changes in squamous cell carcinoma of the cervix of the uterus after radiation. Jour. Med. Res. 40:241-264. 1919-1920. (See also Ibid. 41 : 439-456.)

5. Andersen, H. C., and M. Fischer. Die Wirkung von $\alpha$-Strahlen auf Gewebekulturen. Strahlentherapie 48: 500-507. 1933.

6. Andersen, and G. Kohliann. Röntgenstrahlen und Mineralstoffwechsel. Fortschr. Geb. Röntgenstrahlen 30: 102-105. 1923.

7. Anslow, Gladys A., and Mary L. Foster. The influence of substitution groups on the visible and ultraviolet absorption spectra of amino acids and related substances. Jour. Biol. Chem. 97: 37-46. 1932.

8. Anslow, Gladys A., Mary L. Foster, and Charlotte Klinger. The absorption spectra of glycine solutions and their interpretation. Jour. Biol. Chem. 103 : 81-92. 1933.

9. Aschkinass, E., and W. Caspari. Ueber den Einfluss dissozierender Strahlen auf organisierte Substanz, insbesondere ueber die bacterienschädigende Wirkung der Becquerelstrahlen. Pflüger's Arch. Ges. Physiol. 86: 603-618. 1901.

10. AUERBach, L. Ueber die Einwirkung des Lichtes auf befruchtete Froscheiern. Centralbl. Med. Wiss. 8: 357-358. 1870.

11. Azuma, Y. Effects of $\mathrm{Ca}$ - and $\mathrm{K}$ - ions and ultra-violet rays upon involuntary muscles. Proc. Roy. Soc. [London] B. 100:431-439. 1926. (See also Ibid. 101: 24-29. 1927.)

12. Bailey, Alice A. Effects of ultraviolet radiations upon representative species of Fusarium. Botan. Gaz. 94: 225-271. 1932. 
13. Baldwin, W. M. A study of the combined action of X-rays and of vital strains upon Paramoecia. Biol. Bull. 39: 59-66. 1920.

14. BALDwiN, W. M. The effects produced by X-ray energy acting on frog ova at early developmental stages. Science 52: 229-230. 1930.

15. BARr, C. E. A stimulating effect of photocytolytic products of protoplasn. Jour. Med. Res. 44: 79-81. 1923-1924.

16. BARR, C. E., and W. T. Bovie. Ultraviolet cytolysis of protoplasm. Jour. Morphol. 38: 295-300. 1923.

17. BAYER, H. voN. Ueber die physiologische Wirkung der Becquerelstrahlen. Zeitschr. Allg. Physiol. 4 : 79-86. 1904.

18. Beauverie, J., and P. Cornet. Action des rayons ultra-violets sur la structure cellulaire dans la feuille et le bourgeon d'Elodea canadensis. Compt. Rend. Soc. Biol. [Paris] 102 : 775-777. 1929.

19. Becking, L. B., and M. I. Gregerson. The effect of light on the permeability of lecithin. Proc. Soc. Exp. Biol. and Med. 22:130-136. 1924.

20. Belot, J., L. Nahen, and Cailliau. Action des rayons X sur le lymphocytome (Etude clinique et histologique). Jour. Radiol. 9 : 305-315. 1925.

21. Benassi, E. Irradiazione röntgen della regione splenica e ricambio del calcio. Riv. Radiol. e Fis. Med. 2 : 311-328. 1930.

22. Benedicenti, A. Azione Farmacologica e Radioattiva. (A review.) Boll. Soc. Ital. Biol. Sper. $7: 363-424.1932$.

23. Bergonié, J. L., and Tribondeau. Action des rayons $\mathbf{X}$ sur les globules rouges du lapin. Arch. Elect. Med. 16: 597. 1908.

24. Bersa, E. Strahlenwirkung auf Protoplasma und Biokolloide. (A bibliography.) Protoplasma 1: 159-166. 1926.

25. Bersa, E. Strahlenwirkung auf Protoplasma und Biokolloide. (A bibliography.) Protoplasma 19:136-145. 1933.

26. Bie, V. Ist die bactericide Wirkung des Lichtes ein Oxydationsprozess? Mitt. Finsens Med. Lichtinst. 9: 5-75. 1909. (See also Ibid. 9: 75-146.)

27. Bisceglie, V., and G. Bucciardi. Le modificazioni fuzionali e strutturali degli espianti di cuore embrionale di pollo sottoposti all'azione di sostanze radioattive. Arch. Exp. Zellforsch. 7:444-483. 1929.

28. Blackman, V. H., and S. G. Paine. Studies in the permeability of the pulvinus of Mimosa pudica. Annals Bot. 32:69-86. 1918.

29. Вонм, G. Influence des rayons du radium sur les oeufs vierges et fécondes, et sur les premiers stades du développement. Compt. Rend. Acad. Sci. [Paris] 136 : 1085-1086. 1903. (See also Ibid. 136 : 1012-1013.)

30. Bohn, G., and Anna Drzewina. Action toxique du rouge neutre en présence de la lumiére. Compt. Rend. Soc. Biol. [Paris] 89: 386-388. 1923.

31. Bonin, G. von, and K. Bleidonx. Ueber die Resistenz der Erythrocyten nach Bestrahlung und nach Umladung. Strahlentherapie 12:549-555. 1921.

32. Bovie, W. T. The action of light on protoplasm. Amer. Jour. Trop. Dis. 2 : 506-517. 1915. (See also Amer. Soc. Trop. Med. [Boston] 9 : 225-235. 1914.)

33. Bovie. W. T. The visible effects of Schumann rays on protoplasm. Botan. Gaz. 59: 149-153. 1915.

34. Bovie, W. T. The action of Schumann rays on living organisıns. Botan. Gaz. 61: 1-29. 1916.

35. Bovie, W. T. An approximation of the value of the absorption index of fluorite rays in protoplasm. Jour. Med. Res. 39:239-249. 1919.

36. Bovie, W. T. Relation between physiological dominance and the biological effects of rays. Amer. Jour. Roentg. and Rad. Ther. 13:307-320. 1925. 
37. Bovie, W. T. Biophysies of ultraviolet light. Boston Med. and Surg. Jour. $195: 391-396.1926$.

38. Bovie, W. T., and G. A. Daland. New experiments on the sensitization of protoplasm to heat by exposure to light of short wave-length. Amer. Jour. Physiol. 66 : 55-66. 1923.

39. Bovie, W. T., and D. M. Hughes. The effects of quartz ultraviolet light on the division rate of paramecium. Jour. Med. Res. 39: 223-231. 1918. (See also lbid. 39 : 233-238.)

40. Bovie, W. T., and D. M. Hughes. Rate of recovery from the action of fluorite rays. Jour. Gen. Physiol. 1:323-329. 1918.

41. Bovie, W. T., and Alice Klein. Sensitization to heat due to exposure to light of short wave-lengths. Jour. Gen. Physiol. 1:331-336. 1918.

42. Brauner, L. Lichtkrümmung und Lichtwachstumsreaktion. Zeitsch. Bot. 14 : 497-547. 1922.

43. Brauner, L. Permeabilität und Phototropismus. Zeitsch. Bot. 16 : 113-132. 1924.

44. Brooks, Matilda, M. Studies on permeability VII. Effect of light of different wave lengths on the penetration of $2-, 6$ dibromo phenol indophenol and other dyes into Valonia. Protoplasma 1: 305-312. 1926.

45. Brooks, Matilda M. The effects of light, and other factors on the penetration of 2-, 6 dibromo phenol, indophenol and other dyes in to the living cell. Amer. Jour. Physiol. 76: 190. 1926. (See also Proc. Soc. Exp. Biol. and Med. 23: 576-577. 1926.)

46. Brooks, S. C. Methods of studying permeability of protoplasm to salts. Botan. Gaz. 64: 230-249. 1917.

47. Brown, M. G., J. M. Luck, G. Sheets, and C. V. TAylor. The action of X-rays on Euplotes taylori and associated bacteria. Jour. Gen. Physiol. 16: 397-406. 1933.

48. Brummer, K. Ueber den Einfluss kurzwelliger (Röıtgen-) Strahlen auf die Permeabilität der Haut. Dermat. Zeitsch. 45: 170-174. 1925.

49. Brummer, K. Weitere Beobachtungen über Permeabilitätsänderungen von Zellen unter Röntgenbestrahlung (Ein Beitrag zur Lehre von der Permeabilität.). Strahlentherapie 21: 447-451. 1926. (See also Ges. Deut. Naturf. und Aertze, Düsseldorf, 1926.)

50. Brummer, K. Die elektrischen Ladungen der Erythrozyten als Hauptfaktor der Ursache der Senkungsgeschwindigkeit. Strahlentherapie 22: 322-326. 1926.

51. Bruynoghe, R., and A. Dubors. Action du radium sur des protozoaires pathogènes en culture. Compt. Reud. Soc. Biol. [Paris] 93: 849-850. 1925.

52. Butrer, E. G. On some cellular reactions to X-radiations. Jour. Exper. Biol. 9: 107-116. 1932 .

53. Burge, W. E. Action of ultraviolet radiation in killing living cells such as bacteria. Amer. Jour. Physiol. 43 : 429-432. 1917.

54. Busck, G. Lichtbiologie. Eine Darstellung der Wirkung des Lichtes auf lebende Organismen. Mitt. Finsens Med. Lichtinst. 8: 1-144. 1904.

55. Caspari, W. Physiologie der Röntgen- und Radiumstrahlen. Bethe's Handbuch der Normalen und Pathologischen Physiologie 17:343-391. 1926.

56. Celotti, A. Ricerche istologiche sul comportamento delle surrenali e in particolaro modo della loro innervazione di fronte all'azione dei raggi X. Boll. Soc. Ital. Biol. Sper. 6 : 998-1000. 1931.

57. Cernovodeanu P., and V. Henri. Étude de l'action des rayons ultraviolets sur les microbes. Compt. Rend. Acad. Sci. [Paris] 150: 52-54. 1910. 
58. Cernovodeand P., and V. Henri. Action des rayons ultraviolets sur les microorganismes et sur différentes cellules. Étude microchimique. Compt. Rend. Acad. Sci. [Paris] 150: 729-731. 1910. (See also Ibid. 150: 549-551.)

59. Chambers, Helen, and S. Russ. The action of radium radiations upon some of the main constituents of normal blood. Proc. Roy. Soc. [London] B. 84: 124-136. 1911.

60. Charlton, C. F. The biological effect of radium. Radiology 7: 137-139. 1926.

61. Christiani, E. Die Senkungsreaktion der roten Blutkörperchen unmittelbar vor und nach Röntgenbestrahlungen. Strahlentherapie 43: 126-139. 1932.

62. Clark, Janet H. The physiological action of light. Physiol. Rev. 2: 277-309. 1922.

63. Clark, Janet H. The effect of ultraviolet light on the condition of calcium in the blood. Amer. Jour. Hyg. 3:481. 1923.

64. Cox, S. F. Sensitivity to X-rays of cells in vitro. Arch. Exp. Zellforsch. 11: 121-131. 1931.

65. Crowther, J. A. Some considerations relative to the action of X-rays on tissue cells. Proc. Roy. Soc., [London] B. 96: 207-211. 1924.

66. Chowther, J. A. The action of X-rays on Colpidium colpoda. Proc. Roy. Soc. [London] B. $100:$ 390-404. 1926. (See also Proc. Cambridge Phil. Soc. 1926, pp. 284-287. 1926.)

67. Dessauer, $\mathrm{F}$. The cause of the action of $\mathrm{X}$-rays and gamma rays of radium upon living cells. Jour. Radiol. 4: 411-415. 1923.

68. Dessader, F. Zur Erklärung der biologischen Strahlenwirkungen. Strahlentherapie $16: 208-221$. 1923-24.

69. Dessauer, F. Ueber die biologische Strahlenwirkung. Fortsch. Geb. Röntgenstr. 32 : 319-328. 1924.

70. Dessauer, F. Zur Frage der Grundvorgänges der biologischen Strahlenwirkung. Strahlentherapie 30:506-518. 1928.

71. Dessauer, F. Ueber die primäre Vorgänge der Strahleneinwirkung. Arch. Exp. Zellforsch. 11: 65-86. 1931.

72. Dessauer, F., and W. Caspari. Probleme der biologischen Strahlenwirkung. Punktwärme und Nekrohormonhypothese. Acta Radiol. 6: 241-271. 1926.

73. Dillewisn, C. van. Die Lichtwachstumsreaktion bei Avena. Dissertation. Utrecht, 1927.

74. Dobrovolskaïa-Zavadskaïa, N. A., and N. KoboziefF. L'action de l'émanation du radium sur les infusoires. Bull. Biol. France et Belgique 61: 384-396. 1927.

75. Dognon, A. Le mesure et l'action biologique des rayons $\mathrm{X}$ de différentes longeurs d'onde. Arch. Phys. Biol. 4: 87-182. 1925. (See also Compt. Rend. Acad. Sci. [Paris] 180: 694-697. 1925.)

76. Dognon, A. Influence of temperature upon biological action of X-rays. Arch. Physical Therapy 9: 55-59. 1928.

77. Dognon, A., and C. Piffault. Action comparée des rayons $\mathrm{X}$ et ultraviolets sur une protozoaire (paramécie). Compt. Rend. Soc. Biol. [Paris] 107: 15011503. 1931. (See also Ibid. 107: 1272-1273.)

78. Dognon, A., and C. Piffault. Le sensibilisation des paramécies aux rayons $\mathbf{X}$. Compt. Rend. Soc. Biol. 107 : 1503-1505. 1931.

79. Dognon, A., and J. C. Tsang. Le coefficient de température de l'action des rayons ultra-violets sur l'oeuf d'Ascaris. Compt. Rend. Soc. Biol. 98: 22-24. 1928.

80. Downes, A. H., and T. P. Blunt. The influence of light on protoplasm. Proc. Roy. Soc. [London] B. $28:$ 199-213. 1878. 
81. Dozois, K. P., R. P. Titrsler, M. W. Lisse, and W. P. Davey. The effect of low voltage $\mathrm{X}$-rays on the electrophoretic migration velocity, viability, and $\mathrm{pH}$ of Escherichia coli suspensions. Jour. Bacteriol. 24: 123-132. 1932.

82. Dreyer, G. Die Einwirkung des Lichtes auf Amöben. Mitt. Finsens Med. Lichtinst. 4:81-107. 1903; 5: 79-102. 1903.

83. Dreyer, G. Untersuchungen ueber die Einwirkung des Lichtes auf Infusorien. Mitt. Finsens Med. Lichtinst. 7 : 98-131. 1904.

84. Dreyer, G., and O. Hanssen. Sur le loi de la vitesse d'hémolyse des hématies sous l'action de la lumière, de la chaleur et de quelques corps hémolytiques. Compt. Rend. Acad. Sci. [Paris] 145 : 371-373. 1907.

85. Dyes, O. Grundlagen der Behandlung entzündlicher Krankeiten durch Roentgenbestrahlung. Strahlentherapie 47:160-178. 1933.

86. Earle, W. R. Studies upon effect of light on blood and tissue cells. I, II, III. Jour. Exp. Med. 48: 457-473, 667-681, 683-693. 1928.

87. Eckstein, A., and W. von Mollendorff. Histophysiologische Untersuchungen ueber den Einfluss der Bestrahlung mit der Quecksilberquarzlampe. Arch. Kinderheilk. 72: 205-218. 1923.

88. Efimoff, Alexandra, and W. W. Efimoff. Vitale Färbung und photodynamische Erscheinungen. Biochem. Zeitsch. 155: 376-380. 1925.

89. Eidenow, A. Irradiation of blood in vitro. Jour. Path. and Bact. 33: 769 782. 1930.

90. Ellinger, P., and M. Landsberger. Ueber den Mechanismus der katalytischen Komponente der Zellatmung und ihre Beeinflussung durch Röntgenstrahlen. Klin. Wochensch. 2 : 966-969. 1923.

91. Ewing, J. Radium therapy in cancer. Jour. Amer. Med. Assoc. 68: 12381247. 1917.

92. Ewing, J. Tissue reactions to radiation. Amer. Jour. Roentg. and Rad. Ther. 15 : 93-115. 1926.

93. FALK, T. S., and C. I. REed. Studies on the physiological action of light. VI. Alterations in cell electrophoretic potential produced by direct irradiation of the blood in vivo. Amer. Jour. Physiol. 75:616-617. 1926.

94. Falta, W. Chemische und biologische Wirkung der strahlenden Materie. Strahlentherapie $2: 357-367.1913$.

95. Feichtinger, Nora. Ueber die Einwirkung von $\alpha$ - und $\beta$-Strahlen auf das Protoplasma. Naturwissenschaften 18:252-257. 1930.

96. Feichtinger, Nora. Ueber die Einwirkung der $\alpha$ - und $\beta$-Strahlen auf das Protoplasma. Protoplasma 14:36-51. 1931.

97. Feichtinger, Nora. Viskositätsänderung des Protoplasmas als Folge radioaktiver Bestrahlung. Naturwissenschaften 21: 569-575, 589-591. 1933.

98. Fernad, A. Ueher den Mechanismus der Strahlenwirkung im Gewebe. Strahlentherapie 19 : 142-148. 1925.

99. Finsen, N. R. Ueber die Bedeutung der chemischen Strahlen des Lichtes für Medizin und Biologie. $84 \mathrm{pp.}$ Vogel; Leipzig.

100. Fischer, A. Natur des Thrombins. 1. Fraktionierung und Anreicherung. Biochem. Zeitsch. 264: 169-177. 1933.

101. Fitting, H. Untersuchungen über die Aufnahme von Salzen in die lebende Zelle. Jahrb. Wiss. Bot. 56: 1-64. 1915.

102. Forbes, A., and Catharine Thacher. Changes in the protoplasm of Nereis eggs induced by $\beta$ radiation. Amer. Jour. Physiol. 74: 567-578. 1925.

103. Forbes, H. S., and G. A. Daland. Further experiments on the sensitization to heat duc to exposure to short wave lengths. Influence of ozone. Amer. Jour. Physiol. 66: 50-54. 1923. 
104. Freytag, H. Zur Kenntnis der Ultraviolett-strahlenwirkung auf Blätter und Fruchtschalen. Beih. Botan. Zentralbl. 61: 408-436. 1933.

105. Fuller, H. F. Injurious effects of ultraviolet and infra red radiations on plants. Ann. Missouri Bot. Gar. 19: 79-86. 1932.

106. Gager, C. S. The effects of the rays of radium upon plants. Mem. New York Bot. Gard. 4: 1-278. 1908.

107. Ganbarov, G. Zur Frage der histologisehen Veränderungen des Karzinomgewebes nach Strahlung. Strahlentherapie 41: 531-542. 1931.

108. Gaxs, O. Ueber physikalisch-chemische Zustandsänderungen in gesunder und kranker Haut. Strahlentherapie 18:655-673. 1924.

109. Gass, O. Ueber die biologische Wirkung und die Angriffspunkte der Röntgenstrahlen. Areh. Dermat. und Syph. 155:64-67. 1928.

110. Gans, O., and H. Schlossuax. Ueber den Einfluss kurzwelliger (ultravioletter) Strahlen auf die Permeabilität der Haut. (Zugleich ein Beitrag zur Genese der Kalziumverschiebung.) Dermat. Wochensch. 80:469-475. 1925.

111. Gassoul, R. I. De l'action exercée par les rayons $\mathrm{X}$ sur les tissus vivants in vitro. III. Sur les modifications morphologiques apparaisant successivement dans les explanations sous l'influence des rayons X. Ann. Röntgenol. et Radiol. Leningrad $2: 341-356 . \quad 1926$.

112. Gassovi, R. I. L'influence des rayons $X$ sur les tissus virants in vitro. Ann. Roentgenol. et Radiol. 2 : 211-225. 1927.

113. Gassoul, R. I. De l'action exercée par les rayons $\mathrm{X}$ sur les tissus vivants "in vitro." Ann. Roentgenol. et Radiol. 2: 339-355. 1927.

114. Gassul, R. Ueber Tageslichtwirkung auf lebende Zellen in vitro. Arch. Exp. Zellforsch. 3 : 92-100. 1926.

115. Gassul, R. Ueber Strahlenwirkung auf lebende Zellen in vitro (Explantationsversuche). Klin. Wochensch. 5: 560-561. 1926.

116. Gassul, R. Ueber Röntgenstrahlenwirkung auf lebendes Gewebe in vitro (Morphologische Studien mittels Explantationsmethodik). Strahlentherapie 27 : 545-560. 1927.

117. Gaviati, A. Sulle alterazioni morfologiche e degenerative del sangue di animalsottoposti ai raggi $\mathrm{X}$, studiate col metodo della colorazione vitale. Haematologica $1: 273-297.1920$.

117a. Gellhorx, E. Das Permeabilitätsproblem. Berlin, 1929.

118. Gibss, R. D. Action of ultraviolet light on Spirogyra. Trans. Roy. Soc. Canada sec. V. 20: 419-426. 1926.

119. Gicklions, J. Ueber den Einfluss photodynamisch wirksamer Farbstoffösungen auf pflanzliche Zellen und Gewebe. Sitgungsb. K. Akad. Wiss. Wien. Math-Nat. Kl., Abt. 1, 123: 1221-1276. 1914.

120. Gjurić, D. M. Ueber einen Infrarotlichteffect am quergestreiften Muskel. Strahlentherapie $48: 397-400.1933$.

121. Glocker, R., and H. and M. LangendonfF. Zur Frage der "spezifischen" Wirkung der Kathodenstrahlen auf die Zelle. Naturwissensch. 19: 251. 1933.

122. Grauer, J. Die Änderung der biologischen Wirksamkeit von Membranen unter dem Einfluss von Roentgenstrahlen. Strahlentherapie 49: 118-131. 1934.

123. Groedel, F. M., and C. Schneider. Experimentelle Untersuchungen zur Frage der biologischen Wirkung der Röntgenstrahlen. Strahlentherapie 23: 411-446. 1926.

124. Guerrivi, G. Dell'influenza della luce filtrata attraverso schermi colorati sull'azione emolitica della clorofilla. Boll. Soc. Ital. Biol. Sper. 5: 567-569. 1930.

125. Guerrini, G. Sull'azione delle luci monocromatici. Boll. Soc. Ital. Biol. Sper. 5: 1098-1110. 1930. 
126. HaberLandT, F. Ueber die Einwirkung des elektrischen Lichtes auf vital gefärbte Froschleukozyten. Strahlentherapie 29: 161-171. 1928.

127. Halberstaedter, L., and A. Luntz. Die Wirkung der Radiumstrahlen auf Eudorina elegans. Arch. Protistenk. 68: 177-186. 1929.

128. Halberstädter, L., and O. Wolfsberg. Ueber die Einwirkung von Röntgenstrahlen auf die vitale Färbbarkeit der Gewebe. Fortsch. Geb. Röntgenstr. 29 : 545-551. 1922.

129. Halberstädter, L., and O. Wolfsberg. Funktionssteigerung und -schädigung von röntgenbestrahlten tierischen Geweben im Licht der Vitalfärbung. Zeitsch. Ges. Exp. Med. 32 : 367-377. 1923.

130. Hasselbach, K. A. Untersuchungen über die Wirkung des Lichtes aüf Blutfarbstoffe und rote Blutkörperchen wie auch über optische Sensibilisation für diese Lichtwirkungen. Biochem. Zeitsch. 19: 435-493. 1909.

131. Hausmann, W. Ueber Hämolyse durch Radiumstrahlen. Wien. Klin. Wochensch. 29: 1289-1290. 1916.

132. Hausmann, W. Ueber Strahlenhämolyse. Strahlentherapie 9: 46-80. 1919.

133. Hausmann, W., and W. Kerl. Zur Kenntnis der biologischen Radiumwirkung. Strahlentherapie 11: 1027-1033. 1920.

134. Hausmann, W., and W. E. Pauli. Ueber die Wirkung von Kathoden-strahlen auf Erythrocyten (Blutagarplatten). Strahlentherapie $30: 350-358.1928$.

135. Hebert, A., and A. Kling. De l'influence des radiations du radium sur les fonctions chlorophylliennes et respiratoires chez les végétaux. Compt. Rend. Acad. Sci. [Paris] 149 : 230-232. 1909.

136. Heilbrunn, L. V. The colloid chemistry of protoplasm. $356 \mathrm{p}$. Berlin, 1928.

137. Heilbrunn, L. V., and Kathryn Daugherty. The action of ultraviolet rays on amoeba protoplasm. Protoplasina 18: 596-619. 1933.

138. Hellbrunn, L. V., and R. A. Young. The action of ultraviolet rays on Arbacia egg protoplasm. Physiol. Zool. 3: 330-341. 1930.

139. Helber, E., and P. Linser. Experimentelle Untersuchungen über die Einwirkung der Röntgenstrahlen auf das Blut. München. Med. Wochensch. 62 : 689-691. 1905.

140. Henri, V. Comparison de l'action des rayons ultraviolets sur les organismes avec les réactions photochimiques simples et complexes. Mem. Soc. Biol. [Paris] $73:$ 323-325. 1912.

141. Henri. V., and A. Mayer. Action des radiations du radium sur les collö̈des, l'hémoglobine, les ferments, et les globules rouges. Compt. Rend. Acad. Sci. [Paris] 138 : 521-524. 1904.

142. Hertel, E. Ueber Beeinflussung des Organismus durch Licht, speziell durch die chemisch wirksamen Strahlen. Zeitsch. Allg. Physiol. 4: 1-43. 1904 .

143. Hertel, E. Ueber lichtbiologische Fragen. Zeitsch. Augenheilk. 26 : 393-397. 1911.

144. Hertwig, G. Das Radiumexperiment in der Biologie. Eine literarische Zusammenfassung. Strahlentherapie 11: 821-850. 1920.

145. Hertwig, O. Die Radiumkrankheit tierischer Keimzellen. Ein Beitrag zur experimentellen Zeugungs- und Vererbungslehre. Arch. Mikr. Anat. 77 : 1-164. 1911.

146. Herwerden, Margaret A. van. I. Omkeerhare Gelvorming in de levende Cel. II. Wijzigind in Doorgankelijkheid van het Protoplasma door Bestraling met Radium. Nederl. Tijdsch. Geneesk. 69: 1217-1221. 1925.

147. Hill, L., and A. Eidenow. The biological action of light. Proc. Roy. Soc. [London] B. 95 : 163-180. 1923. 
148. Hinrichs, Marie A. Modification of development on the basis of differential susceptibility to radiation. II. Arbacia and visible light following sensitization. Biol. Bull. 50: 1-16. 1926. (See also Ibid. 50: 473-489.)

149. Hinfichs, Marie A. Ultraviolet radiation; stimulation and inhibition in lower organisms. Proc. Soc. Exp. Biol. and Med. 26: 175-177. 1928.

150. Hoagland, D. R., and A. R. Davis. Further experiments on the absorption of ions by plants, including observations on the effects of light. Jour. Gen. Physiol. 6: 47-62. 1923.

151. Hoagland, D. R., P. L. Hibbard, and A. R. Davis. Influence of light, temperature and other conditions on the ability of Nitella cells to concentrate halogens in the cell sap. Jour. Gen. Physiol. 10:121-196. 1926.

152. Höвеr, R. Physikalische Chemie der Zelle und der Gewebe. $955 \mathrm{pp}$. Berlin, 1926.

153. Hoffunann, Carl. Strahlenhärte und biologische Wirkung. Strahlentherapie 43: 140-159. 1932.

154. Hoffmann, Curt. Ueber die Durchlässigkeit kernloser Zellen. Planta Arch. Wiss Bot. 4: 584-605. 1927.

155. HofFManN, V. Ueber Erregung und Lähmung tierischer Zellen durch Röntgenstrahlen. I. Experimentelle Untersuchungen an Froscheiern und -larven. Strahlentherapie 13: 285-298. 1922.

156. Hoffuann, W. Ueber die Lichtwirkung verschiedener Wellenlängen auf das Auge. I. Ultrarot. Strahlentherapie 34:190-196. 1929.

157. Holtermans, C. Ueber vitale Gewebefärbung unter dem Einfluss von Röntgenstrahlen. Strahlentherapie 17: 158-174. 1924. (See also Ibid. 17: 362365.)

158. Holthusen, H. Beiträge zur Biologie der Strahlenwirkung. Untersuchungen an Askarideneiern. Pflüger's Arch. Ges. Physiol. 187: 1-24. 1921.

159. Holthusen, H. Blutveränderungen durch Röntgenbestrahlung und deren Sensibilisierung. Strahlentherapie 14: 561-570. 1923.

160. Holthusen, H. Die Wirkung der Röntgenstrahlen in biologischer Hinsicht. Strahlentherapie 18 : 241-262. 1924.

161. Holthusen, H., and C. Zweifel. Einfluss der Quantengrösse auf die biologische Wirkung verschiedener Strahlenqualitäten; das Schädigungsbild von Ascaris megalocephala in Abhängigkeit von der Strahlenqualität. Strahlentherapie 43 : 249-272. 1932.

162. Holweck, F., and A. Lacassagne. Actions des rayons $\alpha$ sur Polytomella uvella. Détermination des "cibles" correspondant aux principales lésions observées. Compt. Rend. Soc. biol. [Paris] 107: 812-814. 1930.

163. Holweck, F., and A. Lacassagne. Essai d'interprétation quantique des diverses lésions produites dans les cellules par les radiations. Compt. Rend. Soc. Biol. [Paris] 107 : 814-817. 1930.

164. How ès, M. V. L'étude de la permeabilité. Considérations générales et revue critique des inethodes d'étude. Bull. Soc. Roy. Bot. Belg. 65:22-34. 1932.

165. Honjo, S. Ueber dic Einwirkung von Kathodenstrahlen auf die Nervenzellen mit ihren vergleichenden Studien mit der Röntgenstrahlen. [Japanese with German summary.] Okayama. Igak. Zasshi 42: 1272. 1930. Ibid. 43: 628. 1930.

166. Huber, B. Oekologische Probleme der Baumkrone. Planta 2: 476-488. 1926.

167. Hug, W. Calcium und Karzinom. Strahlentherapie 48: 118-124. 1933.

168. I Asswoine, G. L'ordre de succession des modifications morphologiques qui se produsait dans une cellule sous l'influence des rayons $\mathrm{X}$ et du radon. Ann. Roentgenol. et Radiol. 2 : 357-365. 1927. 
169. ILJin, W.S. The influence of slats on the alternation of concentration of cell-sap in plants. Studies Plant. Physiol. Lab. Prague 2 : 5-25. 1924.

170. Illino. Influenza di raggi $\mathrm{X}$ sugli elementi hematici in vivo ed in vitro. Clin. Med. Ital. 54 : 375-379. 1915.

171. IngBer, E. Intorno all Radioeccitazione e alla Radiolesione. [Contains a very rich bibliography.] Atti Ist. Bot. Univ. Pavia 2 : 173-268. 1931.

172. JacoBs, M. H. Permeability of the cell to diffusing substances. In Cowdry, General Cytology. pp. 97-165. 1924.

173. JacoBs, M. H. The permeability of the erythrocyte. Ergeb. Biol. 7: 1-55. 1931.

174. Jacobson, R. Ueber die Wirkung fluorescierender Stoffe auf Flimmerepithel. Zeitsch. Biol. 23 : 444-466. 1901.

175. JALIN, R. I. Ueber die Diffusionsosmoses der Jodionen im Muskel unter der Einwirkung der Röntgenstrahlen. Fortsch. Geb. Röntgenstr. 43 : 494-497. 1931.

176. JALler, CäciliE. Senkungsgeschwindigkeit der roten Blutkörperchen bei Röntgenbestrahlung in vitro. Deut. Med. Wochenseh. 50: 1080-1081. 1924.

177. Jansson, Gösta. Die Einwirkung der Röntgenstrahlen auf des Zellprotoplasma. Acta. Radiol. 8: 427-461. 1927.

178. JesioneK, A. Lichtbiologie. 177 p. Braunschweig, 1910.

179. Jodlbader, A. Die physiologische Wirkung des Lichtes. In Bethe's Handbuch der Normalen und Pathologischen Physiologie 17 : 305-342. 1926.

180. Johnson, EDNa L. Effects of X-rays upon growth, development, and oxidizing enzymes of Helianthus. Botan. Gaz. 82:373-402. 1926.

181. Jolkevitsch, A. J. Contribution à l'étude de l'action des rayons X sur les bacteries. Annales Roentgenol. et Radiol. 2 : 278-297. 1927.

182. Joseph, H., and S. Prowazek. Versuche ueber die Einwirkung von Röntgenstrahlen auf einige Organismen, besonders auf die Plasmatätigkeit. Zeitsch. Allg. Physiol. 1: 142-153. 1902.

183. Karczag, L. G. von Farkas, and G. Györgyi. Ueber die biologische Indifferenz der Röntgenstrahłen gegenüber künstlichen Gewebskulturen. Arch. Exp. Zellforsch. 4 : 206-211. 1927.

184. Keller, P. Die elektrophysiologischen Veränderungen der mit Ultraviolettlicht bestrahlten Haut. Strahlentherapie 39: 320-335. 1931.

185. Keller, P., and H. Rein. Polarisationsmessungen an der Haut nach Röntgenund Ultraviolettlichtbestrahlungen. Arch. Dermatol. und Syph. 155: 67-70. 1928.

186. Kine, E. N. Radiant energy and protoplasmic action. Physical Therap. 45: 581-587. 1927.

187. Kimura, N. The effects of X-ray irridation on living carcinoma and sarcoma cells in tissue cultures in vitro. Jour. Can. Res. 4: 95-135. 1919.

188. Kisselew, N. Veränderungen der Durchlässigkeit der Protoplasma der Schliesszellen in Zusammenhange mit stomataler Bewegung. Beih. Bot. Zentralbl. $41: 287-308.1925$.

189. KLlein, J. Untersuchungen über Senkungsgeschwindigkeit der Erythrozyten vor und nach Röntgenbestrahlungen. Strahlentherapie 16: 232-234. 1924.

190. Knaffl-Lenz, von E. Ueber die Wirkung der Radiumemanation. Wien. Klin. Wochenseh. 25: 441-442. 1912.

191. Kосн, L. Ueber das Verhalten des Chlors in den Erythroeyten nach Röntgenbestrahlung. Strahlentherapie 25: 470-475. 1927.

192. Kollath, W. Gewebsatmung und strahlende Energie. Strahlentherapie $\mathbf{3 5}$ : 444-488. 1930. 
193. Komoro, H. Cytological and physiologieal changes in Vicia faba irradiated with Roentgen rays. Botan. Gaz. 77:446-452. 1924. [For complete list of papers by Komuro ef. Ingber, 1931.]

194. Kondro, H. Microchemisehe und zytologisehe Befunde an röntgenbestrahltem Pflanzengewebe. I. Über tiefgefärbte zytoplasmatisehe Zellengruppen im Wurzelspitzengewebe von Vicia faba. Fortsch. Geb. Roentgens. 41 · 984-989. 1930.

195. Körnicke, M. Die Wirkung der Röntgenstrahlen auf die Pflanze. Handb. Ges. Anwend. Elektr. 3 : Teil 3., Heft 3, 1922.

196. Kotzareff, A., and F. Chodat. De l'aetion exereée par l'ćmanation du radium sur les levures. Compt. Rend. Soc. Phys. et Hist. Nat. Genève. 40: 36-39. 1923.

197. KovÁcs, K. Zur Biologie der Röntgenstrahlen. Strahlentherapie 26: 313328. 1927.

198. Kovács, K. Der Einfluss der Röntgenstrahłen auf die Diffusion und auf die Durehlässigkeit der Zellmembran. Strahlentherapie 30:77-85. 1928.

199. KovÁcs, K. Einige neuere Daten zu den physikochemisehen und biologischen Eigensehaften der Röntgenstrahlen. (Hungarian). Festseh. Bela Entz, pp. 182-190. 1928.

200. Kraus, F., and H. J. Fuchs. Über das Koagulin des Muskels. I. Zeitsch. Ges. Exp. Med. 64: 583-593. 1929.

201. Kraus, F. and H. J. Fuchs. Über das Koagulin des Muskels. II. Zeitseh. Ges. Exp. Med. 68: 245-257. 1929.

202. Kroetz, C. Die Bedeutung des physikalisch-chemischen Zustandes der Zellkolloide für ihre Strahlenempfindlichkeit. Biochem. Zeitseh. 137 : 372-396. 1923.

203. Kroetz, C. Die Bedeutung physikalisch-chemiseher und ehemiseher Änderungen der Zusammensetzung des Blutes unter Röntgeneinwirkung. Strahlentherapie 18: 545-555. 1924.

204. Kroetz, C. Gewebesbeeinflussung dureh Röntgenstrahlen. Strahlentherapie 22: 319-321. 1926. (See also Klin. Wochenseh. 4:631-632. 1925.)

205. Kroetz, C. Zur Biochemie der Strahlenwirkungen. VI. Der Röntgenstrahleneinfluss auf die Durchlässigkeit der überlebenden Froschhautmembran. Bioehem. Zeitsch. 191: 250-262. 1927.

206. Kroetz, C. Ueber die Lichtwirkung vom physikaliseh-chemischen Standpunkt aus. Strahlentherapie 28: 92-102. 1928.

207. Ккӧмеке, F. Ueber die Einwirkung der Röntgenstrahlen auf die roten Blutkörperchen. Strahlentherapie 22: 608-652. 1926. (See also Fortseh. Geb. Roentgenstr. Kongressh. 34:63-64. 1926.)

208. Krontowski, A. A., and E. G. Lebensohn. Ueber die Wirkung des Radiums auf den Energie liefernden Zellstoffwechsel im Verhältnis zu den Lebensäusserungen der Gewebe bei der Explantation und Transplantation. Areh. f. exper. Zellforseh. 13 : 407-411. 1932.

209. Krukenberg, H. Ein neuer Vorschlag zur Radiotherapie. München. Med. Wochensch. 60: 2112. 1913.

210. Lange, H., and M. Simon. Ucber Phosphorsäureausseheidung der Netzhaut bei Belichtung. Zeitsch. Physiol. Chem. 120:1-29. 1922.

211. LangendorfF, H., and M. Strahlenbiologische Untersuchungen an befruchteten Seeigeleiern. Strahlentherapie 42 : 793-799. 1931.

212. Laurens, H. The physiologieal effeets of radiation. Physiol. Rev. 8: 1-91. 1928.

213. Laurens, H. Physiological Effeets of Radiations. Amer. Chem. Soc. Monographs No. 62, 610 pp. Chemieal Catalog Co.; New York, 1933. 
214. Lazarenko, T., and M. Benenson. Zur Frage ueber den Einfluss der ultravioletten Strahlen auf das Wachstum der Nervenfasern in vitro. Arch. Exp. Zellforsch. 13 : 412-429. 1932.

215. Lehmann, F., and P. Wels. Die Wirkung der Röntgenstrahlen auf die Durchlässigkeit der roten Blutkörperchen für Elektrolyte. Pflüger's Arch. Ges. Physiol. 213 : 628-641. 1926.

216. Leitner, J. Die Wirkung der Röntgen- und Ultraviolettsstrahlen auf die Blutsenkungsreaktion. Fortsch. Geb. Röntgenstr. 41: 743-747. 1930.

217. Lepeschkin, W. W. Zur Kenntnis des Mechanismus der Variationsbewegungen. Ber. Deut. Bot. Ges. 26a: 724-735. 1908.

218. Lepeschkin, W. W. Zur Kenntnis der photonastischen Variationsbewegung und der Einwirkung des Beleuchtungswechsels auf die Plasmamembran. Beih. Botan. Zentralbl. 24 : 308-356. 1909.

219. Lepeschin, W. W. The constancy of living substance. Studies Plant. Physiol. Lab. Prague 1: 5-44. 1923.

220. Lepeschin, W. W. Mechanische Koagulation der lebenden Materie und Analogie zwischen Grundstoffen derselben und Explosivstoffen. Arch. Exp. Zellforsch. 4 : 212-245. 1927.

221. Lepeschkin, W. W. Light and the permeability of protoplasm. Amer. Jour. Bot. 17 : 953-970. 1930.

222. Lepeschin, W. W. Hemolysis and changes in resistance of erythrocytes produced by light. Protoplasma 14:11-27. 1931.

223. Lepeschkin, W. W. Influence of visible and ultraviolet rays upon the stability of protoplasm. Amer. Jour. Bot. 19: 547-558. 1932.

224. Lepeschkin, W. W. Changes of permeability of erythrocytes produced by light. Protoplasma 18: 243-259. 1933.

225. Lepeschin, W. W., and G. E. Davis. Hemolysis and the solar spectrum. Protoplasma 20:189-194. 1933.

226. Levy, Fr. Untersuchungen über den Einfluss ultravioletter Strahlen auf Sperma und Eier von Amphibien. Zeitsch. Allg. Physiol. 13 : 139-154. 1911.

227. Lewis, T., and Y. Zotrerman. Vascular reaction of skin to injury. VIII. Resistance of human skin to constant currents in relation to injury and vascular response. Jour. Physiol. 62 : 280-288. 1926-27.

228. Ley, R. Ueber die Sedimentierungsgeschwindigkeit der roten Blutkörperchen. Zeitsch. Ges. Exp. Med. 26: 59-68. 1922.

229. Lieber, G. D. Die physikalisch-chemische Wirkung von Röntgenstrahlen. Strahlentherapic 18: 536-544. 1924.

230. Lieber, G. D. Ueber die Einwirkung der Roentgenstrahlen auf den Organismus. Wiener Klin. Wochensch. 37: 979. 1924.

231. Lieber, G. D. Physikalisch-chemische Wirkung der Röntgenstrahlen im Organismus. Strahlentherapie 20: 93-102. 1925. (See also Verh. Deut. Roentg. Ges. 16: 73. 1925.)

232. Lieber, G. D. Physikalisch-chemische Wirkung der Röntgenstrahlen im Organismus. Strahlentherapie 29: 139-151. 1928.

233. Liechti, A. Untersuchungen über die Wirkung von Metallen als Sekundärstrahler. Klin. Wochensch. 3: 825-828. 1924.

234. Lıеснт, A. Ueber die Bedeutung der Beta-strahlen für die biologische Röntgenstrahlenwirkung. Acta Radiol. 5 : 385-394. 1926.

235. Liecilti, A. Zur Beeinflussung von bioelektrischen Potentialdifferenzen durch die Röntgenstrahlen. Biochem. Zeitsch. 171:240-255. 1926. (See also Klin. Wochensch. 5: 1911-1913. 1926.)

236. Lillie, R. S., and Margaret L. Baskervill. The actions of ultra violet rays on starfish eggs. Amer. Jour. Physiol. 61:57-71. 1922. 
237. Lillie, R. S., and Margaret L. Baskervill. The action of ultraviolet rays on Arbacia eggs, especially as affecting the response to hypertonic sea water. Amer. Jour. Physiol. 61:272-288. 1922.

238. Linser, P., and E. Herber. Experimentelle Untersuchungen über die Einwirkung der Röntgenstrahlen auf das Blut und Bemerkungen über die Einwirkung von Radium und ultraviolettem Lichte. Deut. Arch. Klin. Med. 83: 479-498. 1905.

239. Lisse, M. W., and R. P. Titrsler. Effect of irradiation on electrokinetic potential, agglutinability, lysis, and $\mathrm{pH}$ of Escherichia coli. Proc. Soc. Exp. Biol. and Med. 28: 811-813. 1931.

240. Lisse, M. W., and R. P. Titrsler. Effect of irradiation on electrophoretic velocity, viability, agglutinability, lysis, and $\mathrm{pH}$ of Escherichia coli. Penn. Agric. Exp. Sta. Tech. Bull. 276: 4-44. 1932.

241. Loeb, L. Ueber den Einfluss des Lichtes auf die Färbung und die Entwicklung von Eiern von Asterias in Lösungen verschiedener Farbstoffe. Roux' Arch. Entwicklungsmech. Organ. 23: 359-378. 1907. (See also Proc. Soc. Exp. Biol. and Med. 4: 94-96, 1907.)

242. Loes, L. The effects of Roentgen rays and radio-active substances on living cells and tissues. J. Cancer Res. 7: 229-282. 1922.

243. London, E. S. Das Radium in der Biologie und Medizin. 197 pp. Leipzig, 1911.

244. Lucké, B., and M. McCutcheon. The living cell as an osmometer and its permeability to water. Physiol. Rev. 12: 68-139.

245. Luntz, A. Ueber die Bedeutung des Zeitfaktors für die Wirkung der verzettelten Radiumbestrahlung auf Eudorina elegans; vorläufige Mitteilung. Strahlentherapie 41 : 132-134. 1931.

246. Lvoff, S. Zur Frage der Permeabilität der Spaltöffnungschliesszellen. (Russian with German summary.) Bull. Jard. Bot. Rep. Russe 25 : 1-149. 1926.

247. Magat, M. Problem of true tissue reaction after roentgen irradiation. Jour. Eksp. Biol. i. Med, 11(32): 243-251. 1929.

248. Magath, M. A. Einige Eigenschaften des röntgenbestrahlten Krebsgewebes nach Quellungsversuchen in vitro. Zeitsch. Krebsforsch. 25: 122-135. 1927.

249. MagATH, M. A. Zur Frage der Reakstionsverschiebung in röntgenbestrahlten Gewebe. Strahlentherapie 32: 534-549. 1929.

250. Magath, M. A. Änderungen der Zellpermeabilität durch Röntgenbestrahlung, gemessen an der Phosphatausscheidung überlebender Tumor- und Leberschnitte. Zeitsch. Krebsforsch. 31: 95-104. 1930. (See also Voprosi Onkologii 3, 1930.)

251. Magath, M. A., and M. J. Kolomijetz. Weitere Versuche über die Beziehungen zwischen der Röntgenstrahlen- und der Calciumwirkung auf Krebsgewebe. Zeitsch. Krebsforsch. 30:457-472. 1930.

252. Markowits, E. Zytologische Veränderungen an Paramoecium nach Bestrahlung mit Mesothorium. Arch. Zellforsch. 16: 238-248. 1922.

253. Martin, M. T., and M. A. Westbrook. Influence of ultra-violet radiation upon some plant cells. Jour. Exper. Biol. $7: 293-307.1930$.

254. Mascherpa, P. Emanazione di radio e fenomeni osmotici in vitro. Boll. Ital. Biol. Sper. 7 : 1181-1188. 1932. (See also lbid. $7: 91-94$.

255. Mascherpa, P., and A. Sgro. Emanazione di radio ed imbibizione muscolare. Boll. Soc. Ital. Biol. Sper. 8: 479-484. 1933.

256. Mavor, J. W., and D. M. DE Forest. Relative susceptibility to X-rays of eggs and sperm of Arbacia. Proc. Soc. Exp. Biol. and Med. 22:19-21. 1924.

257. Mayer, E. Die Wirkung von ultravioletten Strahlengemischen auf Gewebekulturen. Strahlentherapie 39: 148-193. 1930. 
258. Meldolesi, G., and G. Reverberi. Effetti della radio-emanazione sul ritmo riproducttivo e sulla morfologia di Colpidium colpoda. Boll. Ist. Zool. R. Univ. Roma 8 : 1-35. 1930.

259. Mendeleef, $P$. Action des irradiations sur l'organisme des cobayes. Compt. Rend. Soc. Biol. [Paris] 94: 1274-1277. 1926.

260. Mikulicz-RAdecki, F. von. Ueber die Veränderung der Blutkörperchensenkungsgeschwindigkeit im Gefolge von Röntgentiefbestrahlungen. Strahlentherapie 16 : 222-231. $1923-24$.

261. Mikulicz-RAdecki, F. voN. Röntgenbestrahlung und Blutkörperchensen kungsgeschwindigkeit. Zentralbl. Gynäk. 47 : 1075-1076. 1928.

262. Mond, R. Zur Theorie der Sedimentierung der roten Blutkörperchen. Der Einfluss der Bestrahlung mit ultraviolettem Licht. Arch. Ges. Physiol. 197 : 574-592. 1922.

263. Moppet, W. The lethal effect of ultraviolet light on normal and malignant tissues grown in vitro. Lancet 1926, 1: 907-908.

264. Mottram, J. C. Survival curves of cells under radiation. Jour. Cancer Res. 11: $130-134.1927$.

265. Nadson, G. A. Ueber die Wirkung des Radiums auf Hefepilze im Zusammenhang mit der Frage des Radiumeinflusses auf die lebendige Substanz im allgemeinen. Ann. Roentgenol. et Radiol., Leningrad 1:47-96. 1922.

266. Nadson, G. A. Ueber die Primärwirkung der Radiumstrahlen auf die lebendige Substanz. Biochem. Zeitsch. 155 : 381-386. 1925.

267. Nadson, G., and A. Jolkevitch. L'action combinée du radium et des agents chimiques sur les végétaux. Ann. Roentgenol. et Radiol. 2: 11-39. 1926.

268. Nadson, G. A., and E. J. Rochline. Sur l'action des rayons ultraviolets de la lampe de quartz sur la cellule chlorophyllifère. Vestnik rentgenologii i radiologii $6: 491-500.1928$.

269. Nadson, G., and Emilie Rochline-Gleichgewicht. Apparition des cristaux d'oxalate de calcium dans les cellules végétales sous l'influence de la radiation ultra-violette. Compt. Rend. Soc. Biol. [Paris] 98:363-365. 1928. (See also Ibid. 94: 249-251. 1926.)

270. Nadson, G., and E.-J. Rochlin. L'effet des rayons X sur le protoplasme, le noyau, et le chondriome de la cellule végétale d'aprés les observations sur le vivant. Protoplasma $20: 31-41.1933$.

271. Nadson, G., and C. Stern. De l'aetion des rayons ultra-violets et des rayons $\mathrm{X}$ sur la cellule des levures. Compt. Rend. Acad. Sci. U.S.S.R. 1931 A: 39-44. 1931.

272. NaKashima, Y. Einige Versuche zum Grundvorgänge der biologischen Strahlenwirkung. Strahlentherapie 24: 1-36. 1926.

273. Nasset, Elizabeth C., and C. A. Kofoid. Effects of radium and radium in combination with metallic sensitizers on Endamoeba dysenteriae in vitro. Univ. California Publ. Zool. 31: 387-416. 1928.

274. Nelson, C., and S. C. Brooks. The effect of infra-red light on subsequent fertilization of the eggs of certain marine invertebrates. Proc. Soc. Exp. Biol. and Med. 30 : 1007-1009. 1933.

275. Neumann, A. Ueber die Wirkung der Röntgenstrahlen auf die Leukozyten in vitro. Strahlentherapie 18: 74-84. 1924.

276. Nothmann-Zuckerkande, Helene. Ueber die Erregung der Protoplasmaströmung durch verschiedene Strahlenarten. Ber. Deut. Bot. Ges. 33: 301313. 1915 .

277. Nürnberger, L. Histologische Untersuchungen über die Einwirkung der Röntgenstrahlen auf das Zellprotoplasma. Zugleich ein Beitrag zur Kenntnis der Plastosomen. Virchow's Arch. Path. Anat. und Physiol. und Klin. Med. $246: 239-252.1923$. 
278. O'Donnell, A. M. A study of the effect of measured doses of ultra violet radiation on the irritability of melanophores. Amer. Jour. Physiol. 83 : 254-268. 1927.

279. Ogneff, J. Einige Bemerkungen über die Wirizung des elektrisehen Bogenlichtes auf die Gewebe des Auges. Areh. Ges. Physiol. 63 : 209-233. 1896.

280. PACKARD, C. The effects of beta and gamma rays on protoplasm. Jour. Exp. Zool. 19 : 323-353. 1915 .

281. PACKARD, C. The susceptibility of cells to radium radiations. Proc. Soc. Exp. Biol. and Med. 20: 226-227. 1923.

282. PaCkARD, C. The susceptibility of eells to radium radiations. Biol. Bull. 46 : 165-177. 1924.

283. PACKARD, C. The effect of light on the permeability of paramecium. Jour. Gen. Physiol. $7:$ 363-372. 1925.

284. PAUl, W., and A. Hartuans. Ueber die Wirkung von Kathodenstrahlen auf lebendes Gewebe. Areh. Mik. Anat. 103: 95-167. 1924.

285. PeкаReк, J. Absolute Viskositätsmessungen mit Hilfe der Brownischen Molekularbewegung. VII. Der Einfluss des Lichtes auf die Zellsaftviskosität. Protoplasma 20: 359-395. 1933.

286. Peтchenko, B. De l'influence des rayons du radium sur l'amibe. Annales Roentgenol. et Radiol. 2 : 40-50. 1926.

287. Peter, G. Zur Bedeutung der vaskulären Permeabilität für die Strahlenbehandlung maligner Tumoren. Strahlentherapie 26: 735-748. 1927.

288. Petry, E. Zur Mechanik der biologischen Wirkung der Röntgenstrahlen. Biochem. Zeitsch. 56:341-352. 1913.

289. Petry, E. Zur Kenntnis der Bedingungen der biologischen Wirkung der. Röntgenstrahlen. Biochem. Zeitsch. 119: 23-44. 1921; 128: 326-353. 1922.

290. Preiffer, H., and G. Bayer. Zur Kenntnis lichtkatalytiseher Wirkungen. Zeitseh. Ges. Exp. Med. 14 : 137-219. 1921.

291. Pincussen, L. Biologische Lichtwirkungen, ihre physikalisehen und ehemisehen Grundlagen. Ergeb. Physiol. 19: 79-289. 1921.

292. Pohle, E. A. Studies on the suspension stability of human blood. I. The velocity of sedimentation of erythrocytes in X-ray therapy eases. Radiology 5: 206-210. 1925.

293. Politzer, G. Ueber die spezifisehe Wirkung der Röntgenstrahlen. Strahlentherapie 27 : 533-544. 1927.

294. Pordes, F. Der Mechanismus der Röntgenwirkung. Ein Erklärungsversuch. Fortsch. Geb. Röntgenstr. 31: 287-297. 1923.

295. Pordes, F. Zum biologischen Wirkungsmechanismus der Röntgenstrahlen. Strahlentherapie 19: 307-324. 1925.

296. Prideaux, E. B. R., and F. O. Howitt. Electrophoretic velocity of gelatin and ovalbumin in different concentrations of their mixtures and the effect of ultra violet irradiation. Bioehem. Jour. 25: 391-402. 1931.

297. Pringsheim, N. Ueber Lichtwirkung und Chlorophyllfunktion in der Pflanze. Jahrb. Wiss. Bot. 12 : 288-437. 1881.

298. Pusey, W. A. The biologieal effects of radium. Seience 33:1001-1005. 1911.

299. Rajewsky, B. Die Wirkungen der kurzwelligen Strahlen auf Eiweisskörper. I. Biochem. Zeitseh. 227: 272-285. 1930.

300. RAYBAUD, L. De I'influence des radiations ultra-violettes sur les protoplasma. Compt. Rend. Soe. Biol. [Paris] 68: 381-382. 1910.

301. Redfield, A. C., and Elizabeth M. Bright. Temperature eoefficient of the action of $\beta$-rays upon the egg of Nereis. Jour. Gen. Physiol. 1:255-259. 1918.

302. Redfield, A. C., and Elizabeth M. Bright. A quantitative study of the effect of radium radiations upon the fertilization membrane of Nereis. Amer. Jour. Physiol. 46: 376-387. 1918. 
303. Redfield, A. C:, and Elizabeth M. Bright. The relative physiological effects of $\beta$-rays of different velocities. Jour. Gen. Physiol. 2: 31-47. 1919. (See also Ibid 2: 25-29.)

304. Redfield, A. C., and Elizabeth M. Bright. The physiological changes produced by radium rays and ultra-violet light in the egg of Nereis. Jour. Physiol. 55 : $61-85.1921$.

305. Redfield, A. C., and Elizabeth M. Bright. The effects of radium rays on metabolism and growth in seeds. Jour. Gen. Physiol. 4: 297-301. 1922.

306. Redfield, A. C., and Elizabeth M. Bright. Hemolytic action of radium emanation. Amer. Jour. Physiol. 65:312-318. 1923.

307. Redfield, A. C., and Elizabeth M. Bright. The physiological action of ionizing radiations. I, II. Amer. Jour. Physiol. 68: 54-61, 62-69. 1924.

308. Redfield, A. C. and Jeanne Wertheimer. The physiological action of ionizing radiations. IV. The comparison of $\beta$ - and X-rays. Amer. Jour. Physiol. 68 : 368-378. 1924.

309. Regelsberger, H. Ueber den Galvanismus der menschlichen Haut. I. Zum Problem der biologischen Strahlendosis. Zeitsch. Ges. Exp. Med. 42 : 159-171. 1924.

310. Regelsberger, H. Polarisationsmessungen an der menschlichen Haut. Fortsch. Geb. Röntgenstr. 41: 950-956. 1930.

311. Reiss, P. Sur l'action comparée des rayons $\mathrm{X}$ et du radium sur le noyau et le cytoplasme. Compt. Rend. Soc. Biol. [Paris] 92 : 1403-1406. 1925.

312. Reiss, $\mathrm{P}$. Une action physico-chimique des rayons $\mathrm{X}$ sur l'organisme. Compt. Rend. Soc. Biol. [Paris] 95 : 353-354. 1926.

313. Rentschler, L. B. Effects of ultraviolet light on paramecium. Science 73 : 480-481. 1931.

314. Richards, A. Experiments on X-radiation as a cause of changes in permeability. Amer. Jour. Physiol. 36: 400-419. 1915.

315. Richards, A., and D. J. Good. Notes on the effect of X-radiation on the development of Cumingia eggs. Biol. Bull. 37 : 209-221. 1919.

316. Rochlin, G., and Emilie Gleichgewicht. Zur Frage vom biologisehen Einfluss der Röntgenstrahlen. Fortsch. Geb. Röntgenstr. 33: 971-980. 1925.

317. Rochlin-Gleichgewicht, E. J. Effect of radon on chlorophyll containing cells. Vestnik Roentgen. i Radio. 8: 387-407. 1930.

318. Roskin, G. Die Wirkung der Radiumemanation auf Infusorien. Arch. Protistenk. 66:340-345. 1929.

319. Rubin, E. H., and O. Glasser. The effect of Roentgen radiation on the velocity of erythrocyte sedimentation. Amer. Jour. Roentg. 18: 520-527. 1927.

320. Salmonsen, C. J., and G. Dreyer. Recherches sur les effets physiologiques du radium. Compt. Rend. Acad. Sci. [Paris] 138: 1543-1545. 1904.

321. Saralegui, J. A. Estudio de la acción biologica de las rayos $\mathrm{X}$ subre la célula viviente. Semana Méd. [Buenos Aires] 32: 429-435. 1925.

322. SAXE, E. Die Änderung der biologischen Wirksamkeit von Membranen unter dem Einfluss von Röntgenstrahlen. Strahlentherapie 40: 125-132. 1931.

323. Schana, G., M. A. Gruschetzkaja, and E. J. Zwilichowskaja. Beitrag zur biologischen Wirkung der Röntgenstrahlen. (Wirkung der Röntgenstrahlung auf die Elektrolyte Kalium und Kalzium im Blutserum der Menschen.) Strahlentherapie $40: 111-124.1931$.

324. Scarth, G. W. Stomatal movement; its regulation and regulatory effect. Protoplasma 2 : 498-511. 1927.

325. Scarth, G. W. The influence of H-concentration on the turgor and movement of plant cells with special reference to stomatal behavior. Proc. Intern. Cong. Plant. Sci. 2 : 1151-1162. 1929. 
326. Scarth, G. W. Mechanism of the action of light and other factors on stomatal movement. Plant Physiol. 7 : 481-504. 1932.

327. Schaper, A. Experimentelle Untersuchungen über den Einfluss des Radiumstrahlen und der Radiumemanation auf embryonale und regenerative Entwicklungsvorgänge. Anat. Anz. 25: 298-314. 1904.

328. Schaudins, F. Ueber den Einfluss der Röntgenstrahlen auf Protozoen. Pflüger's Arch. 77 : 29-43. 1899.

329. Sснелк, S. Zur Lehre über den Einfluss der Farbe auf das Entwickelungsleben der Thiere. Mittl. Embryol. Inst. K. Univ. Wien 1:265-277. 1880.

330. Schle1p, W. Die Wirkung des ultravioletten Lichtes auf die morphologischen Bestandteile des Ascaris-Eies. Arch. Zellforsch. 17: 289-367. 1923.

331. Sснмитт, E. A. Experimentelle und histologische Untersuchung über den Einfluss der Röntgenstrahlen auf die vitale Färbbarkeit der Gewebe. Strahlentherapie $12: 517-548.1921$.

332. Schmidt-Nielson, S. Einige Erfahrungen ueber die Verwendbarkeit des Lichtes als Reagenz. Mitt. Finsens Med. Lichtinst. 10: 110-127. 1906.

333. Schneider, E. Die biologischen Wirkung der Röntgenstrahlen auf einzellige Lebewesen nach Untersuchungen am Paramaecium. Strahlentherapie 22: 92-106. 1926.

334. Schoenholz, L., and H. Hirsch. Histochemische Untersuchungen am Carcinom vor und nach der Bestrahlung. Strahlentherapie 34: 273-287. 1929.

335. Sсноцтz, W. Ueber die Einwirkung der Röntgenstrahlen auf Zellen. Zugleich ein Beitrag zur Darstellung der Mitosen durch Nuclealreaction. Deut. Med. Wochensch. 53 : 643-646. 1927.

336. Schubert, M. Biologische Röntgenstrahlenwirkung, ihre Erforschung mittels der Gewebeexplantationsmethode. Strahlentherapie 26 : 425-471. 1927.

337. Sсниlze, J. Ueber die Einwirkung der Lichtstrahlen von $280 \mu \mu$ auf Pflanzenzellen. Beih. Bot. Zentralbl. 25:30-80. 1909.

338. Schwarz, G. Ueber die Wirkung der Radiumstrahlen; eine physiologischchemische Studie am Hühnerei. Arch. Ges. Physiol. 100: 532-546. 1903.

339. Schwarz, G., and L. Zehner. Ueber einige biochemische Strahlungsreaktionen. Deut. Med. Wochensch. 38: 1776-1777. 1912.

340. Sескт, H. Ueber den Einfluss der X-strahlen auf den pflanzlichen Organismus. Ber. Deut. Bot. Ges. 20: 87-93. 1902.

341. Seide, J. Zur Kenntnis der biologischen Strahlenwirkung, Untersuchungen am Ascarisei mit Ultravioletten-, Röntgen-, und Radiumstrahlen. Zeitsch. Wiss. Zool. 124: 252-304. 1925.

342. Seıde, J. Der Angriffspunkt der Strahlen in der Zelle. Naturwissenschaften $16: 128-130.1928$.

343. Seyderhelm, R., and G. Opitz. Ueber der Einfluss der ultravioletten Bestrahlung auf die Beziehung zwischen Gift und Zellmembran. Strahlentherapie 28: 122-124. 1928.

344. Sonne, C., and P. Schultzer. On the capacity of ultra-violet light to increase the inorganic phosphorus of the blood serum in normal subjects (rabbits) and its mode of action. Acta Radiol. $7: 529-546.1926$.

345. Soper, W. B. Ueber das Verhalten des reticulo-endothelialen Zellapparates gegenüber der Bestrahlung und der Transplantation. Zeitsch. Exp. Path. und Ther. 16: 467-483. 1914.

346. Spear, F. G. Some effects of radium emanation upon tissue cultures in vitro. Arch. Exp. Zellforsch. 11: 119-120. 1931.

347. Spiegel-Adolf, Mona. Sekundäre Ultraviolettstrahlung und ihre biologische Wirkung. Klin. Wochensch. 9: 1615-1618. 1930. 
348. Stennstron, K. W. Review of the theories of the mechanism of action of radiation in the treatment of cancer. Jour. Cancer Res. 9: 190-203. 1925.

349. Stiles, W. Permeability. London, 1924.

350. Stoklasa, J. Die Dynamik der physiologisehen Wirkung der Radioaktivität auf das Protoplasma. Beitr. Biol. Pflanz. 18: 185-224. 1930.

351. Stoklasa, J., and J. PẼnkava. Biologie des Radiums und der radioaktiven Elemente. Berlin, 957 pp. 1932.

352. Strangeways, T. S. P., and H. E. H. OAKLey. The immediate changes observed in tissue cells after exposure to soft $\mathrm{X}$-rays while growing in vitro. Proc. Roy. Soc. [London] B 95 : 373-381. 1923.

353. Straub, H., and Klothilde Gollwitzer-Meyer. Blutgasanalysen XIII. Der. Einfluss von $\alpha$-Strahlen auf Hämoglobin und Blutkörperchen. Biochem. Zeitsch. 136: 128-139. 1923.

354. Straub, H., and Klothilde Gollwitzer-Meyer. Blutgasanalysen XIV. Der Einfluss kurzwelliger Energiebestrablung auf heterogene Systeme. Die Entladungespannung der Kolloide. Biochem. Zeitsch. 139: 302-320. 1923.

355. Strauss, O. Experimentelle Studien über gewisse biologische Strahlenwirkungen. Strahlentherapie 14: 81-92. 1922.

356. Тснанотіne, S. Les changements de la perméabilité de l'oeuf d'oursin localisés expérimentalement. Compt. Rend. Soc. Biol. [Paris] 84:464-466. 1921. (See also Ibid. 83: 1593-1595. 1920.)

357. Tсhahotine, S. Sur le mécanisme de l'action des rayons ultra-violets sur la cellule. Ann. Inst. Pasteur 35 : 321-325. 1921.

358. Tchahotine, S. Microperazioni cellulari col metodo di raggiopuntura microscopica ed altri metodi micrurgici. Boll. Soc. Ital. Biol. Sper. 3: 182-184. 1928.

359. Tomaschewski. Arbeiten aus der Lichtabteilung in der Klinik des Prof. Wellier Minolff. 60 pp. 1902. (Cited after Busck. First description of ultraviolet eytolysis in paramecium.)

360. Troisi, R. A. The effects of ultraviolet radiation on Paramecium. 1930. Master's Thesis, University of Pennsylvania.

361. Tröndle, A. Permeabilitätsänderungen und osmotischer Druck in den assimilierenden Zellen des Laubblattes. Ber. Deut. Bot. Ges. 27: 71-78. 1909.

362. Tröndle, A. Der Einfluss des Lichtes auf die Permeabilität der Plasmahaut. Jahrb. Wiss. Bot. 48: 171-280. 1910.

363. Tröndle, A. Ueber die diosmotischen Eigenschaften der Pflanzenzelle. Vierteljahrsh. Naturforsch. Ges. Zürich 61:465-493. 1916.

364. Tschachotin, S. Ueber Strahlenwirkung auf Zellen, speziell auf Krebsgeschwulstzellen und die Frage der chemischen Imitation derselben. München. Med. Wochensch. 59 : 2379-2381. 1912.

365. Tschachotin, S. Die mikroskopische Strahlenstichmethode, eine Zelloperationsmethode. Biol. Centralbl. $32: 623-630.1912$.

366. Tschassownikow, N. Ueber die Wirkung der Röntgenstrahlen auf den feinen Bau der Leberzellen beim Frosch. Virchow's Arch. Path. Anat. und Physiol. und Klin. Med. 269: 166-177. 1928.

367. Tsebrikow, Lydie. Sur le modification de la perméabilité des tissus vivants sous l'action des rayons de Roentgen. Arch. Internat. Méd. Exp. 3: 185-199. 1927.

368. Tsebrikow, Lydia. Sur le modification de la perméabilité de la membrane cellulaire sous l'effet combiné des rayons $\mathrm{X}$ et des catalyseurs photochimiques. Arch. Internat. Méd. Exp. 5: 641-648. 1930.

369. ULRICH. Lassen sich nach Röntgenbestrahlungen durch das Ultramikroskop Veränderungen der Erythrozyten nachweisen? Strahlentherapie 13 : 145-147. 1921. 
370. UMEDA, T. The action of light, X-rays, and radium on the movement of ciliated epithelium. (A study in tissue culture method.) (Japanese; English abstraet.) Acta Dermat. 10: 597-604. 1927.

371. UMEDA, T. The influence of ultraviolet rays on the movement of the epithelium of the urinary bladder in vitro. (Japanese; English abstract.) Acta Dermat. 14: 1-10. 1929.

372. Ursprung, A., and G. Blum. Ueber die Schädliehkeit ultravioletter Strahlen. 1917. Ber. deut. Bot. Ges. 35: 385-402. 1917.

373. Valeeff, I. Einfluss von Thorium $\mathrm{X}$ auf die Senkungsgesehwindigkeit der roten Blutkörperchen. Strahlentherapie $26: 363-378.1927$.

374. Vinteniberger, P. Sur le résultat des l'application d'une dose fort de rayons $\mathrm{X}$ à une région de la cellule ne renferment pas le noyau. Compt. Rend. Soc. Biol. [Paris] 99 : 1965-1967. 1928.

375. Vintemberger, P. Sur les effets d'application de rayons $\mathrm{X}$ localisces soit $\mathrm{au}$ protoplasme soit à la région nucléaire de la cellule. Compt. Rend. Soe. Biol. [Paris] 99 : 1968-1971. 1928.

376. Vintemberger, P. Sur les résultats de l'application d'une très fort dose de rayons $\mathrm{X}$ à diverses régions de l'oeuf de grenouille rousse. Compt. Rend. Soe. Biol. [Paris] 102 : 1055-1057. 1930. (See also Ibid. 102 : 1050-1052, 1053-1055.)

377. Vogt, A. Einige Messungen der Diathermansie des mensehlichen Augapfels und seiner Medien, sowie des menschliehen Oberlides nebst Bemerkungen zur biologisehen Wirkung des Ultrarot. Arch. Ophthalmologie 83: 99-113. 1912. (See also Ibid. 81: 155-188. 1912.)

378. Vollmar, Hildegard, and B. Rajewsky. Ein Beitrag zur Strahlenbiologie der Gewebekulturen. Strahlentherapie 48: 508-518. 1933.

379. Wail, S. S., and S. R. Frenkel. Ueber den Einfluss der Röntgenstrahlen auf das Zellplasma. Virehow's Areh. Path. Anat. und Physiol. und Ḱlin. Med. $257: 846-850 . \quad 1925$.

380. WAIL, S. S., and S. R. Frenkel. The effect of radium rays on the protoplasm of a cell. Ann. Roentg. et Radiol. 2 : 140-143. 1926.

381. Warren, S. L. The physiological effects of roentgen radiations upon normal body tissues. Physiol. Rev. 8: 92-129. 1928.

382. Waterman, N. Ueber physikalisehe Chemie des Carcinoms. Zeitseh. Krebsforseh. 27 : 228-240. 1928.

383. Waterman, N. P/W Konstante en Röntgenstrahlen. Nederlansch. Tijf. Geneesk. 72: 471-473. 1928.

384. Waterman, N. Struktur, Strahlung und Ferment. Arch. Exp. Zellforsch. 11: 89-92. 1931 .

385. Waterman, N. Zellstruktur, Strahlung und Ferment. Protoplasma 12 : 112-122. 1931.

386. Weber, F. Röntgenstrahlenwirkung und Protoplasmaviscosität. Pflüger's Areh. Ges. Physiol. 198: 644-647. 1923.

387. Weber, F. Plasmolysezeit und Lichtwirkung. Protoplasma 7: 256-258. 1929.

388. Weber, F. Plasmolyse und "Surface preeipitation reaction." Protoplasma 15: 522-531. 1932.

389. Weil, S. S., and J. G. Lrebersohn. Ueber die Wirkung der Röntgenstrahlen auf die Protoplasmastruktur während der sogenannten "latenten" Periode. Zentralbl. Allg. Path. und Path. Anat. 37:247-250. 1926.

390. WILLCOCK, E. G. The action of the rays from radium upon some simple forms of animal life. Jour. Physiol. 30: 449-454. 1904.

391. Williams, Maud. Observations on the action of $\mathrm{X}$ rays on plant cells. Ann. Bot. 37 : 217-223. 1923. 
392. Williams, MaUd. Some observations on the action of radium on certain plant cells. Ann. Bot. 39: 547-762. 1925.

393. WYND, F. L., and H. J. Fuller. Some effects of ultra violet radiation upon the $\mathrm{Ca}$ and $\mathrm{P}$ content of higher plants. Ann. Missouri Botan. Gard. 18 : 565-572. 1931.

394. Yамамото, T. Ueber den Einfluss der ultravioletten Strahlen auf die Resistenz der roten Blutkörperchen. Arb. Med. Univ. Okayama 2:219-224. 1930.

395. Yamasaki, I. Zur Kenntnis der Radiumhämolyse. Strahlentherapie 29: 363-366. 1929.

396. Young, S. W., and L. W. Pingree. Effect of light on the electrical charge of suspended particles. Jour. Phys. Chem. 17:657-674. 1913.

397. ZirkLe, R. E. Some effects of alpha radiation upon plant cells. Jour. Cell. and Compar. Physiol. 2 : 251-274. 1932.

398. ZirkLe, R. E. Modification of x-ray injury by carbon dioxide and ammonia. Amer. Jour. Physiol. 109: 115-116. 1934. (Abstr.)

399. Zuelzer, Margarete. Ueber die Einwirkung der Radiumstrahlen auf Protozoen. Arch. Protistenk. 5: 358-369. 1905.

400. Zuelzer, Margarete, and E. Philipp. Die Beeinflussung des kolloidalen Zustandes des Zellinhaltes von Protozoen durch Radiumstrahlen. Biol. Zentralbl. 45 : 557-572. 1924.

401. Zuelzer, Margarete, and E. Philipp. Der Beeinflussung des kolloidalen Zustandes des Zellinhaltes von Protozoen durch Radiumstrahlen. Strahlentherapie $20: 737-770.1925$.

402. Zuppinger, A. Radiobiologische Untersuchungen an Ascariseiern. Strahlentherapie 28: 639-757. 1928.

403. Zwaardemaker, H., and T. P. Feenstra. Die Wiederbelebung des Herzens, mittels weicher Röntgenstrahlen nachdem es durch die Entfernung seiner Permeablen Kaliumsalze zum Stillstand gebracht worden war. Strahlentherapie $36: 368-369.1920$.

404. Zycha, H. Ueber den Einfluss des Lichtes auf die Permeabilität von Blattzellen für Salze. Jahr. Wiss. Bot. 68: 499-548. 1928.

Manuscript received by the editor August, 1934. 



